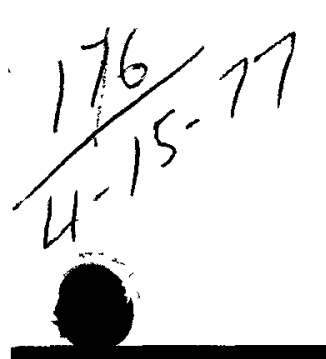

$$
\begin{gathered}
\text { GA-A } 14140 \\
\text { UC-77 } \\
\text { D2 } 903
\end{gathered}
$$

\title{
HTGR SPENT FUEL ELEMENT DECAY HEAT AND SOURCE TERM ANALYSIS
}

\author{
by \\ R. E. SUND, D. E. STRONG, and B. A. ENGHOLM
}

PORTIONS OF THIS REPORT ARE ILLEGIBLE. I has been reproduced from the best ava1lable

Prepared under copy to perait the broadest poss1blo ave13

Contract EY-76-C-03-0167

Project Agreement No. 53

for the San Francisco Operations Office

U.S. Energy Research and Development Administration

DATE PUBLISHED: FEBRUARY 1977

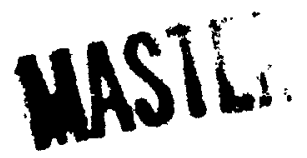




\section{NOTICE}

This report was prepared as an account of work sponsored by the United States Government. Neither the United States nor the United States Energy Research and Development Administration, nor any of their employees, nor any of their contractors, subcontractors, or their employees, makes any warranty, express or implied, or assumes any legal liability or responsibility for the accuracy, completeness or usefulness of any information, apparatus, product or process disclosed, or represents that its use would not infringe privately owned rights.

Printed in the United States of America

Available from

National Technical Information Service

U.S. Department of Commerce

5285 Port Roya1 Road

Springfield, Virginia 22161

Price: Printed Copy $\$ 12.50$; Microfiche $\$ 3.00$ 


\section{DISCLAIMER}

This report was prepared as an account of work sponsored by an agency of the United States Government. Neither the United States Government nor any agency Thereof, nor any of their employees, makes any warranty, express or implied, or assumes any legal liability or responsibility for the accuracy, completeness, or usefulness of any information, apparatus, product, or process disclosed, or represents that its use would not infringe privately owned rights. Reference herein to any specific commercial product, process, or service by trade name, trademark, manufacturer, or otherwise does not necessarily constitute or imply its endorsement, recommendation, or favoring by the United States Government or any agency thereof. The views and opinions of authors expressed herein do not necessarily state or reflect those of the United States Government or any agency thereof. 


\section{DISCLAIMER}

Portions of this document may be illegible in electronic image products. Images are produced from the best available original document. 


\title{
HTGR SPENT FUEL ELEMENT DECAY HEAT AND SOURCE TERM ANALYSIS
}

\author{
by \\ R. E. SUND, D. E. STRONG, and B. A. ENGHOLM
}

Prepared under

Contract EY-76-C-03-0167

Project Agreement No. 53

for the San Francisco Operations Office

U.S. Energy Research and Development Administration

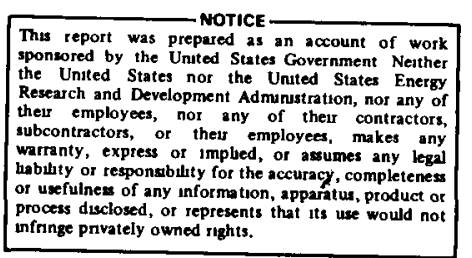

process disclosed, or represents that its use would not
infrnge pnvately owned rights. (n) 
0

-

- 


\begin{abstract}
Decay heat, gamma dose rates, and neutron source strengths were determined for spent fuel elements from a High-Temperature Gas-Cooled Reactor (HTGR). The calculations were based on curie values reported in General Atomic Report GA-A13886 for the earlier commercial version of a 3000-MW(t) HTGR utilizing the thorium-uranium four-year fuel cycle. The reactor core was designed for an average thermal power density of 8.5 watts per $\mathrm{cm}^{3}$ and a carbon-to-thorium atom ratio which varies between 210:1 and 240:1.

Calculations of decay heat, gamma dose rates, and neutron source strengths were made for spent fuel elements from the initial core and from representative nonrecycle and recycle reloads. The study was performed for decay times from 180 days to 10 years. Tables of the isotopic results are given for both the fertile and fissile particles in the fuel elements. In addition, ordered tables of the important isotopic contributors are presented. Graphical presentations of the results are shown and discussed; in addition, comparisons are made with previous determinations.
\end{abstract}


$$
\text { . }
$$ 
CONTENTS

ABSTRACT . . . . . . . . . . . . . . . . . . . . $i i 1$

1. INTRODUCTION . . . . . . . . . . . . . . . . . . . . 1

2. METHODS OF CALCULATION . . . . . . . . . . . . . 4

2.1. Decay Heat . . . . . . . . . . . . . . . . . 4

2.2. Gamma Dose Rates . . . . . . . . . . . . . 4

2.3. Neutron Source Strengths ............... 4

3. BASIC DATA DERIVATION. . . . . . . . . . . . . . . 5

3.1. Decay Energies for Decay Heat . . . . . . . . . . 5

3.2. Gamma Energy Spectra . . . . . . . . . . . . . 6

3.3. Neutron Emission ................. 7

4. SUMMARY RESULTS. . . . . . . . . . . . . . . . . 9

4.1. Decay Heat . . . . . . . . . . . . . . . 9

4.1.1. Decay Heat Versus Time After Discharge... . . 9

4.1.2. Comparison of Present Total Decay Heat Values

With Those From GA-LTR-4 . . . . . . . . 10

4.1.3. Alpha, Beta, and Gamma Decay Heat Versus Time. • 11

4.1.4. Isotopic Contributors to the Decay Heat. . . . . 11

4.2. Gamma Dose Rates . . . . . . . . . . . . . 12

4.2.1. Gamma Dose Rate Versus Time After Discharge. . . . 12

4.2.2. Comparison of Present Total Gamma Dose Rates to Those From GA-LTR-4 Data . . . . . . . . 13

4.2.3. Gamma Dose Rate by Source Energy Group Versus Time After Discharge . . . . . . . . . 13

4.2.4. Isotopic Contributors to Gamma Dose Rate . . . 14

4.3. Neutron Source Strengths . . . . . . . . . . . . 14

4.3.1. Neutron Source Versus Decay Time . . . . . . . 14

4.3.2. Isotopic Contributors to Neutron Source Strength . 15

5. CONCLUSIONS. . . . . . . . . . . . . . . . 16

6. REFERENCES . . . . . . . . . . . . . . . . . 19 
APPENDIX A: DEPARTURES FROM DATA PRESENTED IN REF. 1. . . . . . A-1

APPENDIX B: PERTINENT ENERGIES AND NEUTRON-YIELD VALUES . . . . . B-1

APPENDIX C: DESCRIPTION OF SFUEL CODE . . . . . . . . . . . . C-1

APPENDIX D: TABULATION OF RESULTS ............... . D-1

\section{FIGURES}

1. Decay heat vs. time after discharge: (left) fuel elements discharged at the beginning of reload 1 and originally loaded at reload 0 and (right) fuel elements discharged at the beginning of reload 5 and originally loaded at reload 1. . . . 21

2. Decay heat vs. time after discharge: (top) fuel elements discharged at the beginning of reload 10 and originally loaded at reload 6 and (bottom) fuel elements discharged at the beginning of reload 13 and originally loaded at reload 9. . .

3. Total decay-heat rate vs. time after discharge for fuel elements discharged at the beginning of reload 5 and originally loaded at reload 1 compared with the result from LTR-4 . . . . 23

4. Decay heat for alpha particles, beta particles, and gamma rays vs. time after discharge for fuel elements discharged at the beginning of reload 5 and originally loaded at reload 1. . . . . 24

5. Important isotopic contributors to decay heat for IS fertile particles and IS fissile particles in fuel elements discharged at the beginning of reload 1 and originally loaded at reload 0 .

6. Important isotopic contributors to decay heat for MS, 23RS, and 25RS fertile particles and MS fissile particles in fuel elements discharged at the beginning of reload 13 and originally loaded at reload 9. . . . . . . . . . . . . . .

7. Important isotopic contributors to decay heat for 23RS fissile particles and 25RS fissile particles in fuel elements discharged at the beginning of reload 13 and originally loaded at reload 9.........................

8. Gamma dose rate vs. time after discharge: (left) fuel elements discharged at the beginning of reload 1 and originally loaded at reload 0 and (right) fuel elements discharged at the beginning of reload 5 and originally loaded at reload 1. . . .

9. Gamma dose rate vs. time after discharge: (top) elements discharged at the beginning of reload 10 and originally loaded at reload 6 and (bottom) fuel elements discharged at the beginning of reload 13 and originally loaded at reload 9. . .

10. Total gamma dose rate vs. time after discharge for fuel elements discharged at the beginning of reload 5 and originally loaded at reload 1 compared with the result from LTR-4. . . . . . . 
11. Gamma dose rates by source energy group vs. time after discharge for MS fertile particles in fuel elements discharged at the beginning of reload 5 and originally loaded at reload 1. . . . 31

12. Gamma dose rates by source energy group vs. time after discharge for MS fissile particles in fuel elements discharged at the beginning of reload 5 and originally loaded at reload 1. . 32

13. Important isotopic contributors to gamma dose rate for IS fertile particles and IS fissile particles in fuel elements discharged at the beginning of reload 1 and originally loaded at reload 0. . . . . . . . . . . . . . . . . . . .

14. Important isotopic contributors to gamma dose rate for MS, 23RS, and 25RS fertile particles and MS fissile particles in fuel elements discharged at the beginning of reload 13 and originally loaded at reload 9.................. . . . 34

15. Neutron source strength vs, time after discharge: (top) fuel elements discharged at the beginning of reload 5 and originally loaded at reload 1 and (bottom) fuel elements discharged at the beginning of reload 10 and originally loaded at reload 6 . . .

16. Neutron source strength vs. time after discharge for fuel elements discharged at the beginning of reload 13 and originally loaded at reload 9................... 36

17. Important isotopic contributors to neutron source strength for MS, 23RS, and 25RS fertile particles and MS fissile particles in fuel elements discharged at the beginning of reload 13 and originally loaded at reload 9. . . . . . . . . . . . . . .

18. Important isotopic contributors to neutron source strength for 23RS fissile particles and 25RS fissile particles in fuel elements discharged at the beginning of reload 13 and originally loaded at reload $9 . . . . . . . . . . . .$.

\section{TABLES}

A-1. Isotopes omftted from present study on basis of curie-value criterion given in Section A.1.1. . . . . . . . . . . A-6

A-2. Decay chains in which curie value of daughter was set equal to that of parent. . . . . . . . . . . . . . . A A-7

A-3. List of curie values used in present calculations. . . . . . A-11

B-1. Half-1ives, decay energies, and neutron yields for important isotopes . . . . . . . . . . . . . . . . . . B-1

B-2. Gamma energy spectra . . . . . . . . . . . . . . B-7

B-3. Neutron yields for important 1sotopes. . . . . . . . . B-9 
TABLES (continued)

C-1. Fuel types accepted by SFUEL. . . . . . . . . . . . C-7

$\mathrm{C}-2$. Gamma energy groups and corresponding $\mathrm{MeV} / \mathrm{cm}^{2}-\mathrm{sec}$ to $\mathrm{rad} / \mathrm{hr}$ conversion factors. . . . . . . . . . . . . . C-8

C-3. SFUEL input instructions. . . . . . . . . . . . . . C-9

D-1. Decay heat for fuel elements discharged at beginning of reload 1 and originally loaded at reload $0 . . . . . . .$. D-1

D-2. Decay heat for fuel elements discharged at beginning of reload 5 and originally loaded at reload 1......... D-1

D-3. Decay heat for fuel elements discharged at beginning of reload 10 and originally loaded at reload $6 . . . . . .$. D-33

D-4. Decay heat for fuel elements discharged at beginning of reload 13 and originally loaded at reload 9....... . D-65

D-5. Gamma dose rates for fuel elements discharged at beginning of reload 1 and originally loaded at reload 0 . . . . . . D-97

D-6. Gamma dose rates for fuel elements discharged at beginning of reload 5 and originally loaded at reload 1 . . . . . . D-97

D-7. Gamma dose rates for fuel elements discharged at beginning of reload 10 and originally loaded at reload 6. . . . . D-157

D-8. Gamma dose rates for fuel elements discharged at beginning of reload 13 and originally loaded at reload 9. . . . . . D-217

D-9. Neutron source strengths for fuel elements discharged at beginning of reload 1 and originally loaded at reload 0 . . D-277

D-10. Neutron source strengths for fuel elements discharged at beginning of reload 5 and originally loaded at reload 1 . . D-277

D-11. Neutron source strengths for fuel elements discharged at beginning of reload 10 and originally loaded at reload 6. . D-289

D-12. Neutron source strengths for fuel elements discharged at beginning of reload 13 and originally loaded at reload 9. . D-301

D-13. Important isotopic contributors to decay heat . . . . . D-313

D-14. Important isotopic contributors to gamma dose rate . . . D-335

D-15. Important isotopic contributors to neutron source strength. . D-357 


\section{INTRODUCTION}

As part of the Thorium Utilization Program (contract number EY-76-C-03-0167, Project Agreement Number 53), the decay heat, gamma dose rates, and neutron source strengths were calculated for spent fuel elements from the earlier commercial version of a 3000-MW( $t$ ) High-Temperature GasCooled Reactor (HTGR). For the initial core, uranium enriched to $93 \%$ in $\mathrm{U}-235$ is used as the feed fissile material, and thorium fertile material is used for the production of bred U-233. The bred U-233 and unburned U-235, along with other accompanying uranium isotopes, can be recycled and used as fissile material, with enriched uranium added as makeup fuel. The fissile and fertile materials that are loaded into the core are segregated into two different types of fuel particles. Partial refueling is utilized, with approximately one-fourth of the core reloaded annually. More information on the fuel cycle is given in Report GA-A13886 (Ref. 1).

The present study is based on the curie values given in Ref. 1 for four selected reloads. The calculations in Ref. 1 assumed a 3000-MW(t) reactor, 3944 fuel elements, 8.5 watts per $\mathrm{cm}^{3}$ average power density, an $80 \%$ load factor, and a carbon-to-thorium atom ratio which varies between $210: 1$ and 240:1. The types of spent fuel and their designations are given below.

1. IS (Initial, Spent) fuel elements - spent initial-core fuel elements with the initial fissile and fertile materials described above.

2. MS (Makeup, Spent) fuel elements - spent makeup fuel elements which are used to reload the core after the initial operation and which are made from uranium enriched to $93 \%$ in $\mathrm{U}-235$ for the fissile particles and from thorium for the fertile particles. 
3. 23RS (U-233 Recycle, Spent) fuel elements - spent recycle fue1 elements which are made primarily from recovered U-233 material for the fissile particles and from thorium for the fertile particles.

4. 25RS (U-2 $3 \underline{5}$ Recycle, Spent) fuel elements - spent recycle fuel elements which are made from recovered U-235 material for the fissile particles and from thorium for the fertile particles.

The four reloads reported in Ref. 1 are:

1. Reload 1 - IS-type fuel elements discharged at the beginning of reload 1 after one year of operation at full power and $80 \%$ load factor.

2. Reload 5 - MS-type fuel elements (and IS-type elements) discharged at the beginning of reload 5 after four years of operation at full power and $80 \%$ load factor. Representative of makeup type fuel elements for nonrecycle reloads.

3. Reload 10 - only 23RS and 25RS fuel elements (no M-type elements used) discharged at the beginning of reload 10 after four years of operation at full power and $80 \%$ load factor.

4. Reload 13-MS, 23RS, and 25RS elements discharged at the beginning of reload 13 after four years of operation at full power and $80 \%$ load factor. Typical for an HTGR operating at equilibrium recycle.

Computations were performed separately for the fissile-particle inventory in a fuel element and for the fertile-particle inventory therein. In each case, results were obtained for each isotope that made a significant contribution. (See Sections A.1.1, and A.1.2. in Appendix A.) Results for each isotope were summed to obtain values for each chemical element. 
Calculations were performed for the five decay times given in Ref. 1, namely, 180 days, 1 year, 3 years, 5 years, and 10 years.

The method of calculation and basic data derivation are described in Sections 2 and 3, respectively. The results are described and depicted graphically in Section 4. Conclusions are presented in Section 5.

The appendices include Appendix A, a discussion of certain differences between Ref. 1 curie values and the values input to the computations for this report, and a list of all the curie values used in the present study; Appendix B, tabulations of pertinent energies and neutron-yield values for the important isotopes; and Appendix C, a description of the SFUEL code. In addition, detailed tabulations of the results are given in Appendix $D$, including a list of the most important isotopes for each case. 


\section{METHODS OF CALCULATION}

\subsection{DECAY HEAT}

The decay heat in units of watts per fuel element was calculated for representative cases. To obtain the decay heat, the isotope curie values given in Ref. 1 were multiplied by the number of $\mathrm{MeV}$ per disintegration for the alpha particles, beta particles, and gamma rays, and the results were then multiplied by appropriate conversion factors. (See Appendix A for changes in some curle values.) Decay-heat results for the isotopes were summed to obtain values for the chemical elements and the totals for the fuel elements. In addition, total alpha, beta, and gamma values were obtained for the fuel elements.

\subsection{GAMMA DOSE RATES}

Typical gamma dose rates were determined for the various cases. In this analysis, the curie values for the isotopes were combined with the number of $\mathrm{MeV}$ per disintegration for different gamma energy groups. The $\mathrm{MeV}$ per $\mathrm{cm}^{2}-\mathrm{sec}$ at one meter from the source was computed, and the appropriate conversion factors were used to calculate the dose rates for the different gamma energy groups.

\subsection{NEUTRON SOURCE STRENGTHS}

The neutron yields in neutrons per sec per fuel element were determined by multiplying the curie value for each isotope by the number of neutrons per curie-sec. The derivation of the neutron yield data is discussed in Section 3.3. 


\section{BASIC DATA DERIVATION}

\subsection{DECAY ENERGIES FOR DECAY HEAT}

The alpha, beta, and gamma energies used in the decay-heat calculations are given in Table $B-1$ in Appendix B. In most cases, the decay energies for the fission fragments were obtained from the information prepared by the Task Force on Fission-Product Data, as reported in Ref. 2. For a number of isotopes of interest, the decay energies in Ref. 2 were based on theory rather than experiment. In these cases, the Ref. 2 values were not used; instead the results usually were obtained from the compilation by Tobias (Ref. 3). Except for U-232, the decay energies in Table B-1 in Appendix $B$ for all isotopes with atomic numbers equal to or greater than 92 were obtained from C. W. Reich (Ref. 4). The isotopes in Table A-3 that were not available from Refs. 2, 3, or 4 were almost always obtained from Ref. 5. In a number of cases, information from Nuclear Data was used.

For the Ref. 2 and Ref. 3 compilations, the energies for the internalconversion electrons are included with the gamma energies; this procedure was also followed in the calculations with data from Ref, 5 and from Nuclear Data. The energies for the internal-conversion electrons are included in the beta energies for the data from Ref. 4. The latter data were not modified to the form of the other data, since the interna1-conversion electron energies from Ref. 4 were not listed separately. This difference in the input data should not affect the results significantly, because the energies for the internal-conversion electrons are typically small compared with the beta or gamma energies.

In the calculation of the average beta energy (excluding neutrino energy) from the end-point energy for the data given in Ref. 5 and in Nuclear Data, the data given in Ref. 6 were used for isotopes with atomic numbers less than 30 . These data include the effect of the atomic number. 
For isotopes with higher atomic numbers, the method described in Ref. 7 was used. (This method may be more readily available to the reader from Ref. 8.) The method gives a conservative result for isotopes with relatively high atomic numbers.

The alpha energies in Table B-1 in Appendix B include the nuclear recoil energies, which amount to roughly $2 \%$ of the values listed in the table.

Except for a number of the isotopes discussed in Appendix A.4., the half-lives listed in Table B-1 in Appendix B were those used in the calculation of the curie values given in Ref. 1. The half-lives are included here, since they were not given in Ref. 1, and since they are often important in identifying a particular isotope. In practically all of the cases, the half-lives are the same or close to the values given in the Eleventh Edition of the Chart of the Nuclides (Ref. 9). For Eu-154 and Eu-155, the half-life values used in Ref. 1 correspond to those given in Ref. 10.

\subsection{GAMMA ENERGY SPECTRA}

The gamma energy spectra for use in the gamma dose rate calculations are listed in Table B-2 in Appendix B. The break points between the gamma energy intervals are midway between the energies given at the top of Table $\mathrm{B}-2$. The data in the table do not include the energy from internal conversion electrons; in this respect, they differ from the total gamma energies in Table B-1 in Appendix B. Most of the data on the gamma spectra were taken directly from the PATH gamma-shielding code (Ref. 11), which in turn had been derived from Refs. 3 and 5. Some new gamma spectra were added, utilizing data in Refs. 3 and 5 and in Nuclear Data. Isotopes with small values for the total gamma energy or with very low-energy spectra are generally not included in the PATH code. In some cases, small differences in the values in Tables $\mathrm{B}-1$ and $\mathrm{B}-2$ are caused by the use of different references for the two sets of data. 


\subsection{NEUTRON EMISSION}

The most important sources of neutrons in the fuel are (1) spontaneous fission and (2) alpha decay followed by $(\alpha, n)$ reactions with carbon and oxygen. To obtain a list of important isotopes for neutron emission, the requirement of either alpha emission or spontaneous-fission decay was applied to the list which results from the criterion in Appendix A.1.1. on curie values. In addition, a number of isotopes were eliminated because their decay is predominantly by beta particles and, consequently, their $(\alpha, n)$ yields are relatively small. Also, $\mathrm{Cm}-243$ was not included, since its neutron contribution is small relative to the other curium isotopes. The resulting 1ist of isotopes is given in Table B-3 in Appendix B.

The calculations of the $(\alpha, n)$ yields were performed for the compositions of the fuel kernels. These are $\mathrm{UC}_{3} \mathrm{O}_{0.5}$ for the fissile kernels and $\mathrm{UO}_{2}$ for the fertile kernels. Other compositions in the vicinity of the alpha emitter would result in different ylelds. In the calculations, the thick-target carbon yields from Ref. 12 were used. The thick-target oxygen yields were determined from the expression $1.77 \times 10^{-11} \mathrm{E}^{4.84}$ neutrons per alpha particle, where $\mathrm{E}$ is the alpha energy in MeV. This expression is 0.81 of that given in Ref. 13, which, in turn is based on the results of Ref. 14. The factor of 0.81 was obtained from the ratio of the new $c(\alpha, n)$ yield from Ref. 12 at $5.5 \mathrm{MeV}$ to the $\mathrm{C}(\alpha, \mathrm{n})$ yield in Ref. 14.

The method given in Ref. 15 was used to convert from thick-target $(\alpha, n)$ yields to the yields for the fuel kernels. In these computations, the stopping power was taken to be proportional to $z(z+7)^{-1 / 2}(\operatorname{Ref} .13)$, where $Z$ is the atomic number. This expression, together with a stopping power of 0.60 for $\mathrm{Be}$ and 4.6 for $\mathrm{Pu}$ (Ref. 15), results in a stopping power equal to 0.83 for carbon and 1.03 for oxygen. The resulting neutron yield for $\mathrm{UC}_{3} \mathrm{O}_{0.5}$ is 0.37 of the yield for a thick carbon target, and the neutron yield for $\mathrm{UO}_{2}$ is 0.31 of the yield for a thick oxygen target. 
The neutron yields from $(\alpha, n)$ reactions are given in Table $B-3$ in Appendix B for the important isotopes.

The $(\alpha, n)$ yields calculated for the kernels are somewhat high because of fission fragments in the kernel in place of some of the uranium atoms. For the case of typical fission fragments entirely replacing the $U$ in $\mathrm{UC}_{3} \mathrm{O}_{0.5}$, the correction factor to get the neutron yield from the yield for a thick carbon target is 0.30 , as compared with 0.37 for $\mathrm{UC}_{3} \mathrm{O}_{0.5^{\circ}}$ On the other hand, for unprocessed fuel, the $(\alpha, n)$ yields in Table B-3 are somewhat low because of alpha particles that escape the kernel, which has a radius of $1.55 \times 10^{-2} \mathrm{~cm}$, and lose energy in the carbon coating. For instance, the range of alpha particles in going from $5.5 \mathrm{MeV}$ to the $(\alpha, n)$ threshold at $\sim 2.0 \mathrm{MeV}$ is 0.16 of the radius for the $\mathrm{UC}_{3} 0.5$ kernel.

The neutron yields from spontaneous fission are also given in Table B-3. The spontaneous-fission yields are given only when they are significant with respect to the $(\alpha, n)$ yields.

The total neutron yields are given in Tables B-1 and B-3. 


\section{SUMMARY RESULTS}

Tables D-1 through D-15 in Appendix D give the results. Figures are presented and discussed in this section, so that the results may be understood more clearly. In this report, time after discharge and decay time are considered to be synonymous with the time interval after shutdown for refueling. Also, when "reload 5 " is used, it refers to fuel that is discharged at the beginning of reload 5. A similar meaning applies to other reloads.

\subsection{DECAY HEAT}

\subsubsection{Decay Heat Versus Time After Discharge}

The decay-heat results versus time after discharge for the four different reloads are shown in Figs. 1 and 2 .

The decay heat for fertile particles which have been in the reactor for four years (reloads 5, 9, or 13) is much greater than that for fertile particles which have been in the reactor for one year (reload 1). The difference in the results is consistent with (1) the significantly larger amount of U-233 and the resultant larger fission rate in the fertile particles near the end of four years of operation, as compared with the conditions near the end of one year of operation and (2) the expected greater buildup of isotopes with long half-lives in the four-year fuel, as compared with the buildup in the one-year fuel.

At 180 days after discharge, the decay heat for U-235 fissile particles from reload 5 (for four-year operation) is lower than that from reload 1 (for one-year operation). The reasons for this are (1) the half-lives of the important isotopes for this decay time are typically much less than four years and (2) the U-235 in the fissile particles is mostly burned up 
after four years of operation, so that the fission rate near the end of the four-year operation is considerably lower than that near the end of the one-year operation. At ten years after discharge, isotopes that decay with long half-lives compared to four years are important. As expected, at this time after discharge, the decay heat for the U-235 fissile particles from reload 5 is considerably greater than that from reload 1.

The decay heat for the different types of fissile particles varies considerably at long times after discharge, as shown for reload 13 in Fig. 2 . The variations are caused mainly by the differences in the decay heat from Pu-238.

For fuel that was in the reactor for four years, the fertile-particle decay-heat values at 180 days after discharge are typically greater than the corresponding fissile-particle results. This again is caused by the large burnup of the U-235 in the fissile particles after four years of operation, so that the power near shutdown is lower than that for the fertile particles (with the bred U-233). At times greater than roughly one year after discharge, the fissile-particle results for four-year operation are greater than the fertile-particle results, as expected.

\subsubsection{Comparison of Present Total Decay Heat Values With Those from GA-LTR-4 (Ref. 16)}

The sum of the fertile and fissile decay-heat results for reload 5 of this study are compared with those from GA-LTR-4 (Ref 16) in Fig. 3. To obtain the decay-heat rates in percent, as shown in the figure, the decayheat values were divided by the average operating powers of the fuel elements. The two calculations have a number of differences which affect the results. In Ref. 16 the higher of the decay-heat rates from U-233 and U-235 fission fragments was used in determing the total decay-heat rates. Also, the decay-heat determinations for the fission fragments in Ref. 16 were based on operation of the fuel at constant power; in the present study, the power of the fuel originally loaded at reload 1 and discharged at the beginning of reload 5 decreases over the period that it is in the 
reactor. In addition, a number of differences exist in the calculations for the actinide nuclei. Because of the differences, the results of Ref. 16 should be somewhat higher than those of the present study at relatively short times after discharge. Also, the deviation between the results in percent should decrease for longer decay times. The results in Fig. 3 are consistent with these expectations.

In the above presentation, the reload 5 data were used to show a comparison between results of Ref. 16 and results that are representative of makeup fuel elements for non-recycle reloads. The MS results for reload 13, which is typical for an HTGR operating at equilibrium recycle, are three to five percent higher than those from reload 5. Consequently, the agreement between the MS results for reload 13 and the results from Ref. 16 is slightly better than the agreement shown in Fig. 3.

\subsubsection{Alpha, Beta, and Gamma Decay Heat Versus Time}

The alpha, beta, and gamma decay-heat results for reload 5 are given in Fig. 4. The alpha decay heat for the fertile particles increases with time after discharge for the time interval given in the figure. This is caused by the buildup of the Th-228 (with a 1.9-year half-life) from the decay of U-232 (with a 72-year half-life). The U-232 is produced in the reactor primarily from $U-233(n, 2 n)$ reactions. Th-228 has five daughters with significant alpha yields and with relatively short half-lives.

\subsubsection{Isotopic Contributors to the Decay Heat}

The major isotopic contributors to the decay heat for reloads 1 and 13 are shown in circular diagrams in Figs. 5, 6, and 7. The times given on the figures are the times after discharge. The percent contributions for the important isotopes in the fertile and fissile particles of reloads 5 and 10 are similar to the values for the corresponding particles in reload 13. Figures 5, 6, and 7 were drawn starting from a line corresponding to the positive $\mathrm{x}$ axis and proceeding counterclockwise according to the most important isotopes. Isotopes that are mothers and daughters are shown next 
to each other and are separated by dashed lines; also, the mother isotope is always shown first. For instance, $\operatorname{Pr}-144$ (with a half-life of 17.3 minutes) is a daughter of Ce-144 (with a half-life of 284 days). After a chemical separation of the two elements, the $\operatorname{Pr}-144$ would soon grow back into the cerium sample. The decay-heat results show that Pr-144 is the most important isotope at 180 days after discharge. Ce-144, with a much smaller percent contribution, is given first in the figures, and $\operatorname{Pr}-144$ is given second in a counterclockwise direction.

As shown in Figs. 6 and 7 for reload 13, Pu-238 contributes 64\%, 16\%, and $2.4 \%$ of the decay heat at 10 years after discharge for 25RS, MS, and 23RS fissile particles, respectively. The $\mathrm{Pu}-238$ is produced mainly from $\mathrm{U}-235$ and U-236; differences in the amounts of U-235 and U-236 in the three types of fissile particles loaded into the reactor explain the variations in the importance of $\mathrm{Pu}-238$.

\subsection{GAMMA DOSE RATES}

\subsubsection{Gamma Dose Rate Versus Time After Discharge}

The gamma dose rates versus time after discharge are shown in Figs. 8 and 9. The calculations assume that all of the isotopes from one fuel element are concentrated into a point source in a vacuum, with the detector one meter away. The characteristics of the curves in Figs. 8 and 9 are generally similar to the decay-heat curves in Figs. 1 and 2 . There is a difference, however, in the fissile-particle curves. For the decay-heat results, the fissile-particle curves for reload 13 have different shapes because of differences in the $\mathrm{Pu}-238$ contributions, as discussed above. For the gamma dose rates, the Pu-238 contributions are negligible, and the fissile-particle curves for reload 13 have similar shapes. 
4.2.2. Comparison of Present Total Gamma Dose Rates to Those from GA-LTR-4 Data

In Fig. 10 the sum of the fertile and fissile gamma dose rates for a fuel element from reload 5 of this study are compared with those derived from the GA-LTR-4 data (Ref. 16) on U-235 fission fragments. Both sets of data in the figure assume a point source in a vacuum, with the detector one meter away. Before plotting the Ref. 16 results, they were divided by 1.05 to account for the difference in the power per fuel elements. Because there were only two Ref. 16 data points in the time region covered in Fig. 10, the shape of the curve was drawn approximately like that for the present results. The differences in the power of the fuel versus operating time, as described in Section 4.1.2, should cause the Ref. 16 results for relatively short times after discharge to be slightly higher than the present results; at longer times, the two results should approach each other. The data in Fig. 10 are generally consistent with these expectations. The slightly lower Ref. 16 result at approximately 3 years after discharge is probably caused by differences in the important fission-fragment yields for $\mathrm{U}-233$ and $\mathrm{U}-235$ and by differences in the calculational procedures.

\subsubsection{Gamma Dose Rate by Source Energy Group Versus Time After Discharge}

The gamma dose rates for various energy groups are shown versus time after discharge in Figs. 11 and 12. The data are for a fuel element from Reload 5.

Note that the gamma source spectrum for fisslle particles appreciably softens with decay time and also decreases in absolute magnitude. In the case of the fertile particles, buildup of U-232 daughter products, as indicated by the $2.2-$ to $2.65-\mathrm{MeV}$ curve in Fig. 11 , counteracts any softening of the spectrum. 


\subsubsection{Isotopic Contributors to Gamma Dose Rate}

The important isotopic contributors to the gamma dose rates are shown in Figs. 13 and 14 for reloads 1 and 13. The fissile-particle results for the 23RS and 25RS fuel from reloads 10 and 13 and for the MS fuel from reload 5 are similar to the MS fissile-particle results shown in Fig. 14 for reload 13. Also, the fertile-particle results for reloads 5 and 10 are similar to the fertile-particle results shown in Fig. 14 for reload 13.

The Cs-134 contributions for the three types of fissile particles in reload 13 (for four-year operation) are much greater than the Cs-134 contribution for the fissile particles in reload 1 (for one-year operation). These changes in the contributions are expected for the different operating times, because Cs-134 is produced in a two-step process, namely, the production of Cs-133 from fission and the subsequent neutron capture to produce $\mathrm{Cs}-134$.

\subsection{NEUTRON SOURCE STRENGTHS}

\subsubsection{Neutron Source Versus Decay Time}

The neutron yields versus time are shown in Figs. 15 and 16 . Because some of the important isotopes for neutron yields are produced in the reactor after many neutron captures, the curie values for these isotopes have large uncertainties. In particular, the time steps used in Ref. 1 give rise to inaccurate curie values for those isotopes in reload 1 that are produced after a number of neutron captures (as, for instance, the curium 1sotopes); consequently, no figure is shown in this report on the neutron data for reload 1. In addition, the values given in Appendix D Tables D-11 and D-12 for 25RS fissile particles have a high degree of uncertainty; therefore, neutron source strengths for these particles are not shown in the figures. If one considers the locations of the 25RS fuel elements in the reactor as well as the other relevant input information, the 25RS fissile particles are expected to have neutron yields that are a factor of two or more higher than those from MS fissile particles. 
Very large differences occur in the neutron yields for the different types of particles in the spent fuel, because the curie values for the important isotopes vary greatly with the composition of the fuel before it is loaded into the reactor. These effects are discussed in Section 4.3.2.

\subsubsection{Isotopic Contributors to Neutron Source Strength}

The important isotopic contributors are given in Figs. 17 and 18 for reload 13. The 25RS fissile data on important isotopic contributors have a relatively high uncertainty. The percent contributions for the important isotopes in the fertile and fissile particles of reloads 5 and 10 are similar to the values for the corresponding particles in reload 13 . Depending upon the type of fuel and the time after discharge, the important isotopes are $\mathrm{Cm}-244, \mathrm{Cm}-242, \mathrm{Pu}-238$, and $\mathrm{U}-232$ along with the daughters of $\mathrm{U}-232$. The curium isotopes are produced mainly from U-238; $\mathrm{Pu}-238$ is produced mainly from U-235 and U-236; and U-232 is produced mainly from U-233. Because of the relatively large amount of $\mathrm{U}-236$ and $\mathrm{U}-238$ in the recycle U-235 fissile particles that are loaded into the core, the 25RS fissile particles that are discharged from the core are expected to have relatively large curie values for $\mathrm{Pu}-238, \mathrm{Cm}-242$, and $\mathrm{Cm}-244$, and the total neutron yield for the 25RS fissile particles is expected to be relatively high compared with the yield for MS fissile particles. Of the three isotopes, the data indicate that $\mathrm{Cm}-244$ is by far the most important for the neutron production in the 25RS fissile particles. Also, the $\mathrm{Pu}-238, \mathrm{Cm}-242$, and $\mathrm{Cm}-244$ curie values and the resultant neutron yields for the MS fissile particles are higher than those for the 23RS fissile particles. For the MS fissile particles, Cm-244 contributes $75 \%$ of the yield at 180 days after discharge and $80 \%$ of the yield at 10 years after discharge. For the 23RS fissile particles, Pu-238 and the daughters of U-232 are important contributors. The fertile particles have no uranium when the fuel is loaded into the core; consequently, the $\mathrm{Pu}-238, \mathrm{Cm}-242$, and $\mathrm{Cm}-244$ curie values at discharge are small. For the fertile particles, U-232 and the daughter isotopes of U-232 are the major neutron sources. 


\section{CONCLUSIONS}

The present study has produced a large collection of data on decay heat, gamma dose rates, and neutron sources from spent HTGR fuel of various types and operational modes. The reader will draw many of his own conclusions from the detailed information presented in Tables $D-1$ through $\mathrm{D}-15$ in Appendix D. The safety analyst may be interested in cases where such nuclides as $\mathrm{Pu}-238$ or $\mathrm{Sr}-90$ are the predominant contributors. Radiation protection and shielding engineers will utilize the gamma dose rates and neutron source strengths. Those involved with process design and heat removal systems will have the most interest in decay-heat values.

An extensive assortment of graphical and pictorial material has been prepared to complement the detailed tabular data. Perusal of Figs. 1 through 18 leads to the following conclusions:

1. The decay heat rate from spent fuel after four-year operation, as determined in this study, displays good agreement with results published in licensing report GA-LTR-4 (Ref. 16). Considering the different assumptions, agreement with proposed ANSI Standard 18.6 (Ref. 17) is also satisfactory, even for 10 years decay.

2. For decay times exceeding approximately one year, the fissile particles in a fuel element always produced more decay heat than the fertile particles.

3. Spent U-235 recycle fissile particles generate significantly more decay heat than spent $U-233$ recycle fissile or spent nonrecycle fissile particles at long decay times. 
4. For time after discharge from one to five years the percent decay heat for a non-recycle fuel element can be approximately fit by the expression $0.024 t^{-0.8}$, where $t$ is the decay time in years.

5. Beta particles produce the largest share of the decay heat -about two-thirds, for one-year decay time.

6. For all spent, four-year particles except U-235 recycle fissile, major isotopic contributors to decay heat are $\operatorname{Pr}-144$ at 180 days, Cs-134 at 3 years, and $\mathrm{Y}-90$ at 10 years. For spent U-235 recycle fissile particles, $\mathrm{Pu}-238$ is the most important isotope at decay times of 3 and 10 years.

7. Gamma dose rates also display good agreement with corresponding Ref. 16 calculations. The gamma dose rate very roughly drops a factor of 10 from a decay time of 180 days to a decay time of 10 years.

8. Unshielded gamma dose rates after one-year decay are caused mainly by cesium -- Cs-134 or $\mathrm{Cs}-137 / \mathrm{Ba}-137 \mathrm{~m}$. With heavy shielding, it is probable that $\operatorname{Pr}-144$, which emits a hard gamma ray, would become increasintly important.

9. The neutron yields vary considerably for the different types of spent fissile particles. Decay time has little effect on reducing the neutron level. $\mathrm{Cm}-244$ is the major contributor for spent non-recycle fissile particles and for spent $U-235$ recycle fissile particles. Pu-238 and the daughters of U-232 are important contributors for spent U-233 recycle fissile particles. 
The Ref. 1 methods, SFUEL code, and the physics input data could be utilized for future studies in several areas. Decay times longer than 10 years should be studied. Two- and three-year fuel that is loaded into the inftial core should be evaluated. Plutonium feed or recycle fuel could be treated. The methods could be extended to LWR fuel for use by Barnwell. Even if no new fuel types are investigated, one- or two-year updates of the present study are recommended, to incorporate new physics data as they become available. 


\section{REFERENCES}

1. Hamilton, C. J., N. D. Holder, V. H. Pierce, and M. W. Robertson, "HTGR Spent Fuel Composition and Fuel Element Block Flow," ERDA Report GA-A13886, General Atomic, July 1, 1976.

2. England, T. R., and R. E. Schenter, "ENDF/B-IV Fission-Product Files: Summary of Major Nuclide Data," LA-6116-MS, October 1975.

3. Tobias, A., "Data for the Calculation of Gamma Radiation Spectra and Beta Heating from Fission Products (Revision 3)," RD/B/M2669, June, 1973.

4. Reich, C. W., Aerojet Nuclear Company, private communication, July 1976.

5. Lederer, C. M., J. M. Hollander, and I. Perlman, Table of Isotopes, Sixth Edition, John Wiley and Sons, Inc., New York, New York (1967).

6. Dillman, L. T., J. Nuc. Medicine 10, supplement 2, pamphlet 4, p. 7., March 1969.

7. England, T. R., "An Investigation of Fission Product Behavior and Decay Heating in Nuclear Reactor," Ph. D. Thesis, University of Wisconsin, August 1969.

8. Goldsmith, M., and K. Shure, "On the Average Energies of $\beta$ - Particles Emitted by Fission Products," Nuc1. Sci. and Eng. 60, 322 (1976).

9. "Chart of the Nuclides," Eleventh Edition, Revised to April 1972, Prepared by N. E. Holder and F. W. Walker, Knolls Atomic Power Laboratory .

10. "Chart of the Nuclides," modified by C. A. Rohrmann, Battelle-Northwest, May 1969.

11. Alexander, J. H., S. S. Clark, and B. A. Engholm, "The PATH GammaShielding Code," Nuc1. Sci.\& Eng. 27, 2, 490 (February, 1967).

12. Bair, J. K., "Absolute Neutron Yields from Alpha-Particle Interactions with Thick Targets of Natural Carbon," Nucl. Sc1 \& Eng. 51, 83 (1973). 
13. Rushton, J. E., J. D. Jenkins, and S. R. McNeany, "Nondestructive Assay Techniques for Recycled U-233 Fuel for High-Temperature GasCooled Reactors," Nuc1. Materials Management 4, 18 (1975).

14. Gorhkov, G. V., V. A. Zyabkin, and O. S. Tsvetkov, "Neutron Yields from the Reaction $(\alpha, n)$ in $\mathrm{Be}, \mathrm{B}, \mathrm{C}, 0, \mathrm{~F}, \mathrm{Mg}, \mathrm{SI}$ and Granite Irradiated with Polonium $\alpha$ Particles," Soviet Atomic Energy 13, 654 (1963).

15. Argonne National Laboratory, "Reactor Physics Constants," ANL-5800, p. 711, Ju1y 1963.

16. Sund, R. E., "Afterheat Calculations for the HTGR," General Atomic Report Gulf-GA-A12499-A (GA-LTR-4), Amendment A, July 1974.

17. Proposed ANSI Standard 18.6, "Decay Energy Release Rates following Shutdown of Uranium Fueled Thermal Reactors."

18. Augustson, R. H., and T. D. Reilly, "Fundamentals of Passive NonDestructive Assay of Fissionable Material," LA-5651-M (September 1974).

19. Bailey, H.S., R. N. Evatt, G. L. Gyorey, and C. P. Ruiz, "Neutron Shielding Problems in the Shipping of High Burnup Thermal Reactor Fue1," Nuc1. Technology 17, 217 (1973).

20. Halpern, I., "Nuclear Fission," Ann. Rev. Nucl. Sci. (Annual Reviews, Inc., Palo Alto, California, 1959), Vol. 9, p. 247. 


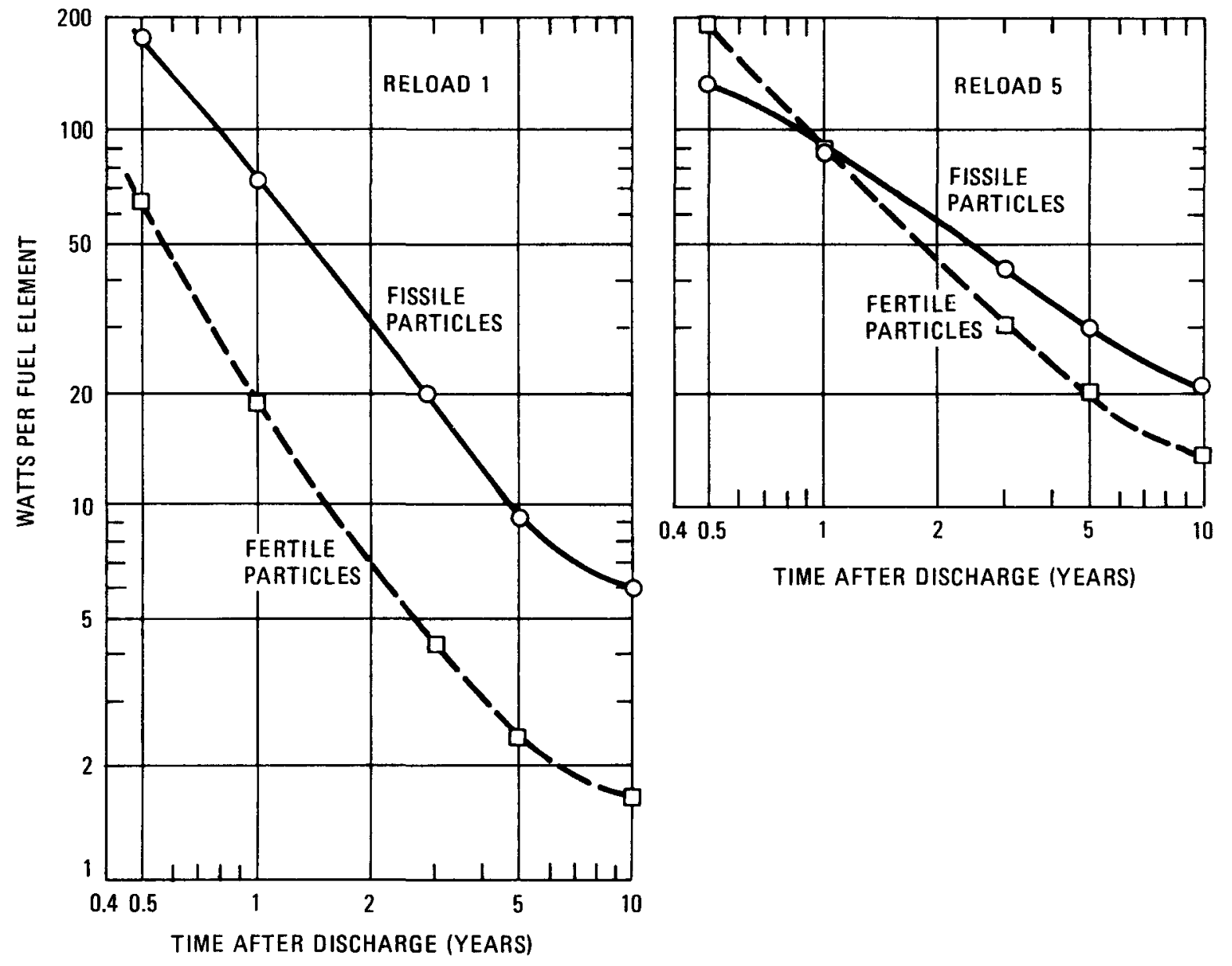

Fig. 1. Decay heat vs. time after discharge: (left) fuel elements discharged at the beginning of reload 1 and originally loaded at reload 0 and ( $r$ ight) fuel elements discharged at the beginning of reload 5 and originally loaded at reload 1 

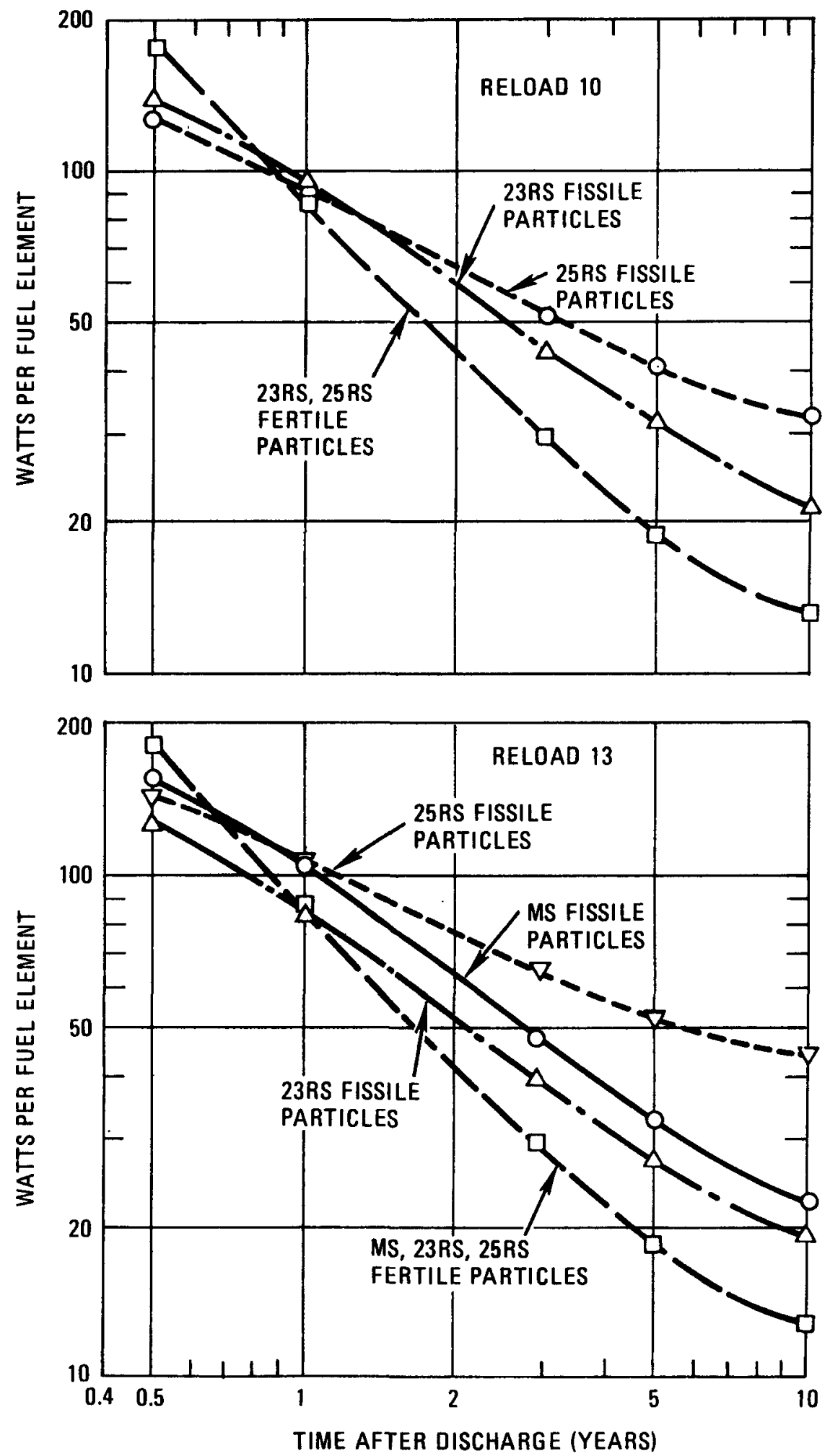

Fig. 2. Decay heat vs. time after discharge: (top) fuel elements discharged at the beginning of reload 10 and originally loaded at reload 6 and (bottom) fuel elements discharged at the beginning of reload 13 and originally loaded at reload 9 


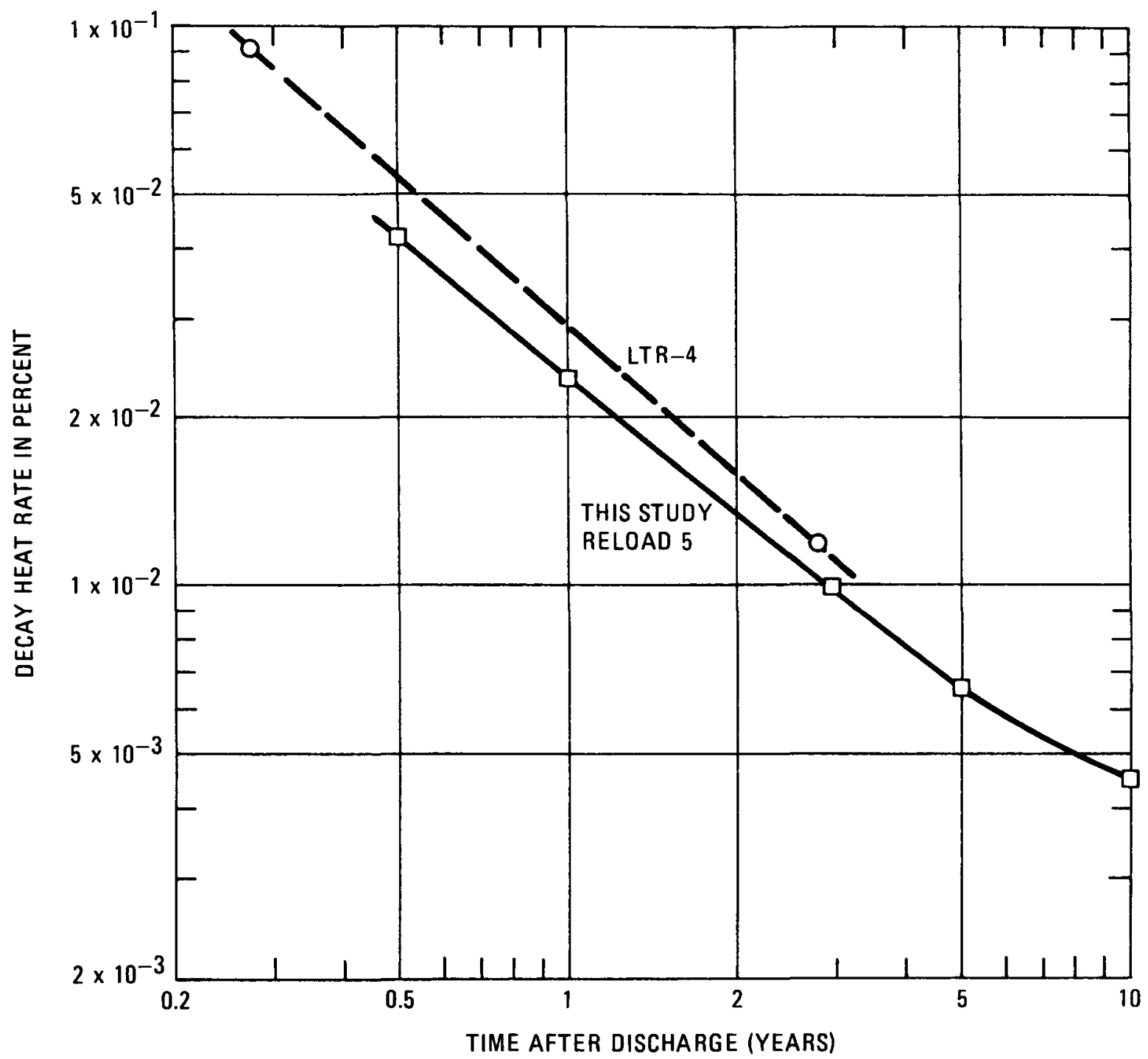

Fig. 3. Total decay-heat rate vs. time after discharge for fuel elements discharged at the beginning of reload 5 and originally loaded at reload 1 compared with the result from LTR-4 (Ref. 16) 

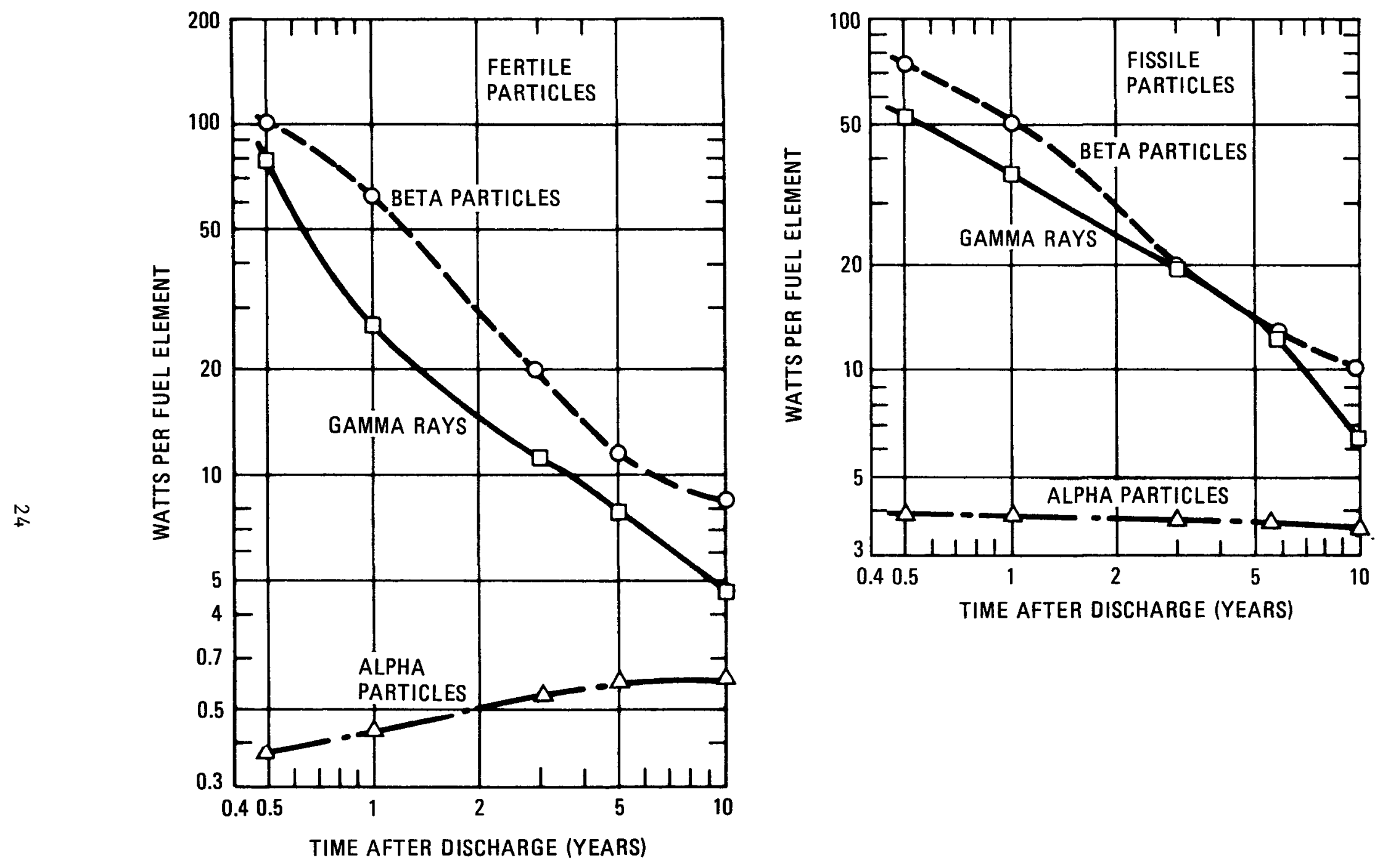

Fig. 4. Decay heat for alpha particles, beta particles, and gamma rays vs. time after discharge for fuel elements discharged at the beginning of reload 5 and originally loaded at reload 1 

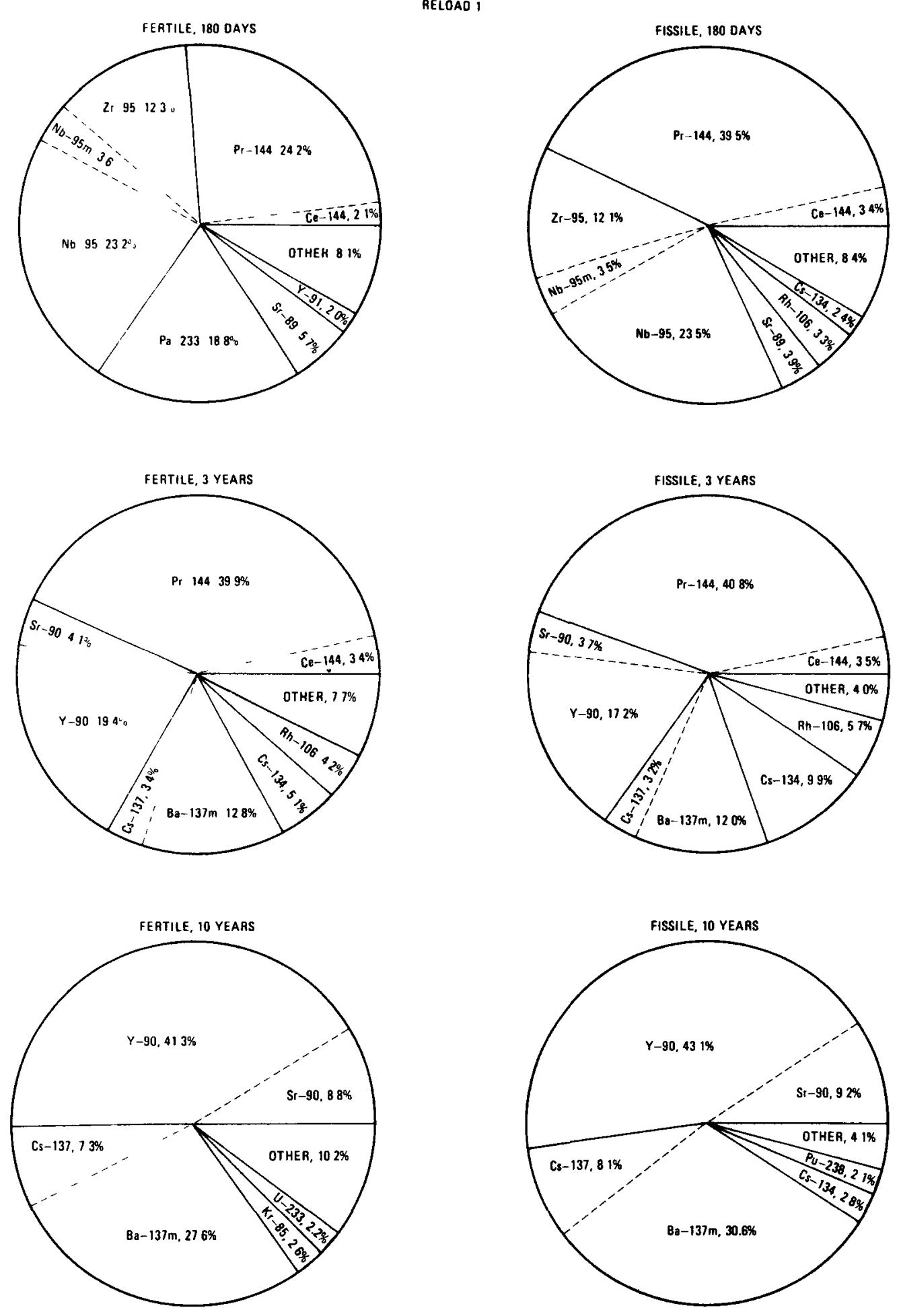

Fig. 5. Important isotopic contributors to decay heat for Is fertile particles and IS fissile particles in fuel elements discharged at the beginning of reload 1 and orlginally loaded at reload 0 
RELOAD 13
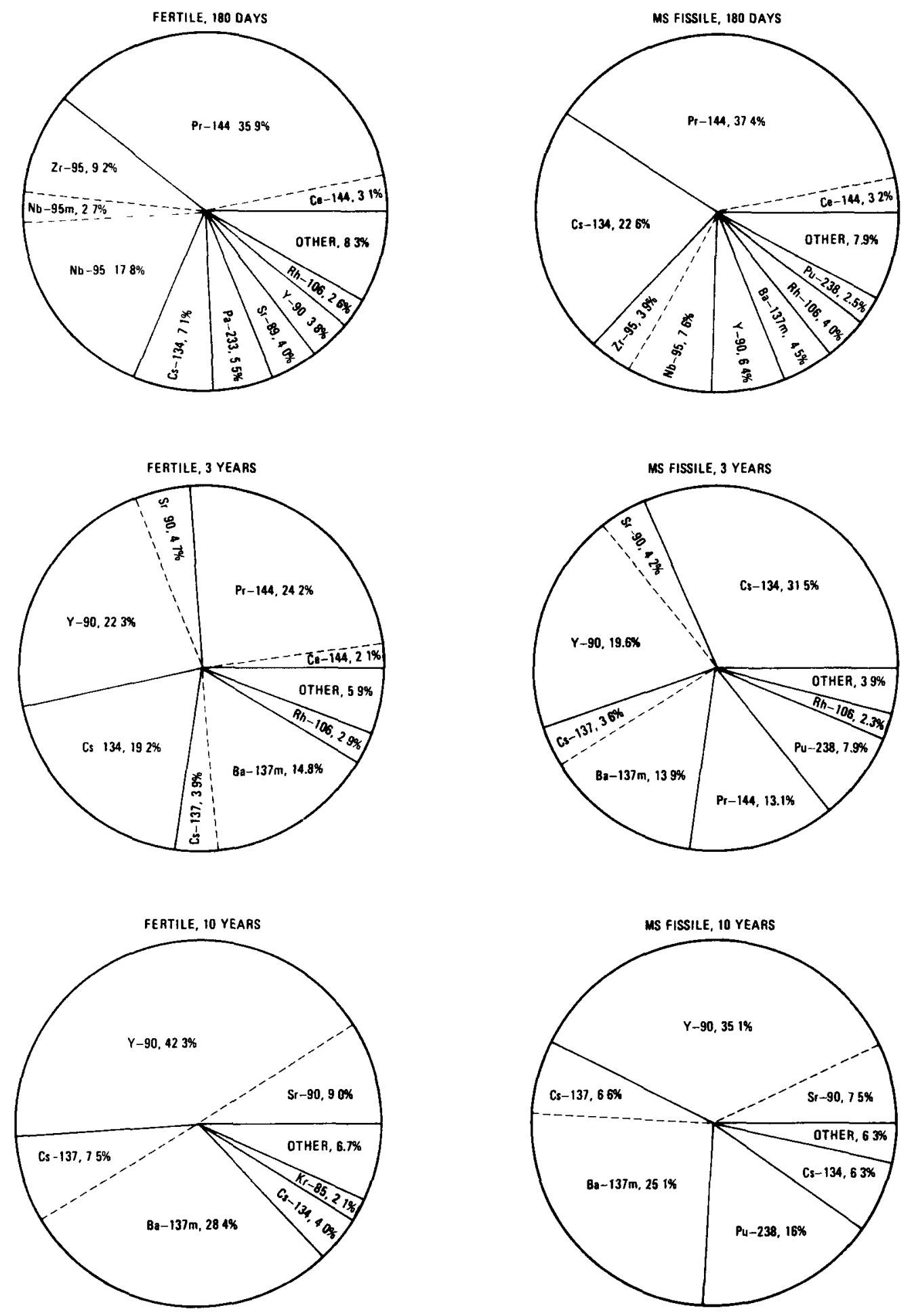

Fig. 6. Important isotopic contributors to decay heat for MS, 23RS, and 25RS fertile particles and MS fissile particles in fuel elements discharged at the beginning of reload 13 and originally loaded at reload 9 

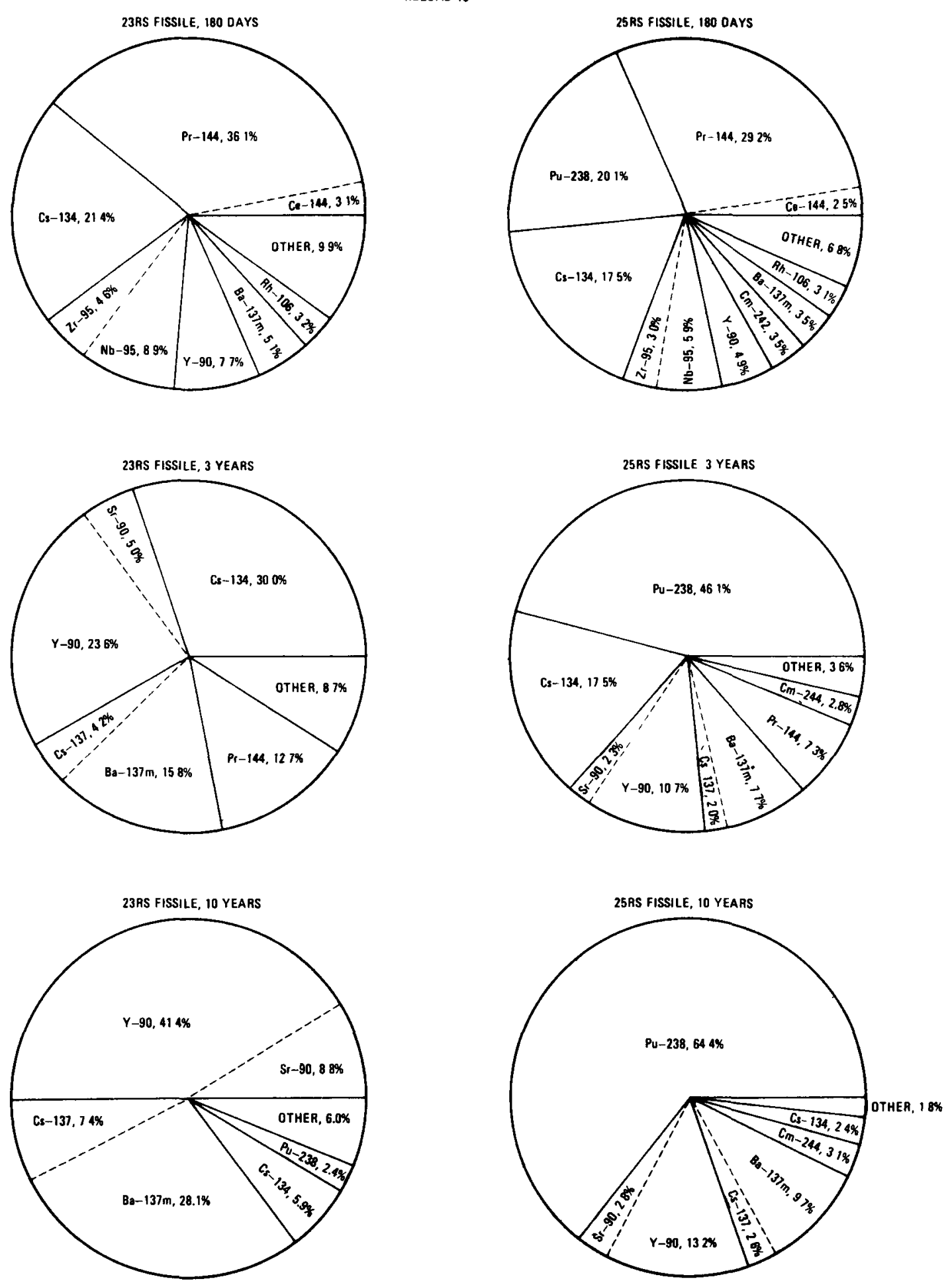

F1g. 7. Important isotopic contributors to decay heat for 23RS f1ssile particles and 25RS fissile particles in fuel elements discharged at the beginning of reload 13 and originally loaded at reload 9 


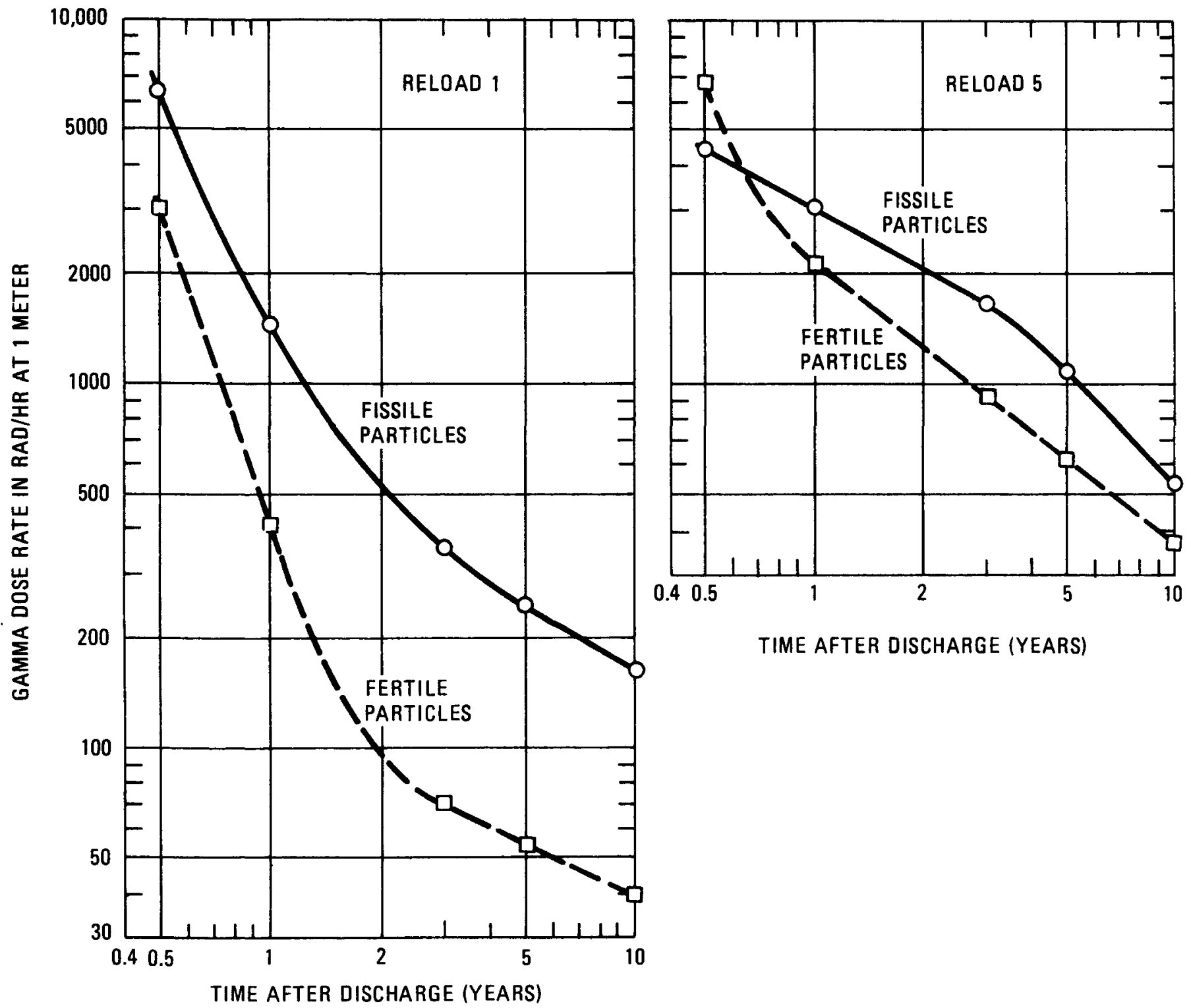

Fig. 8. Gamma dose rate vs. time after discharge: (left) fuel elements discharged at the beginning of rełoad 1 and originally loaded at reload 0 and (right) fuel elements discharged at the beginning of reload 5 and originally loaded at reload 1 

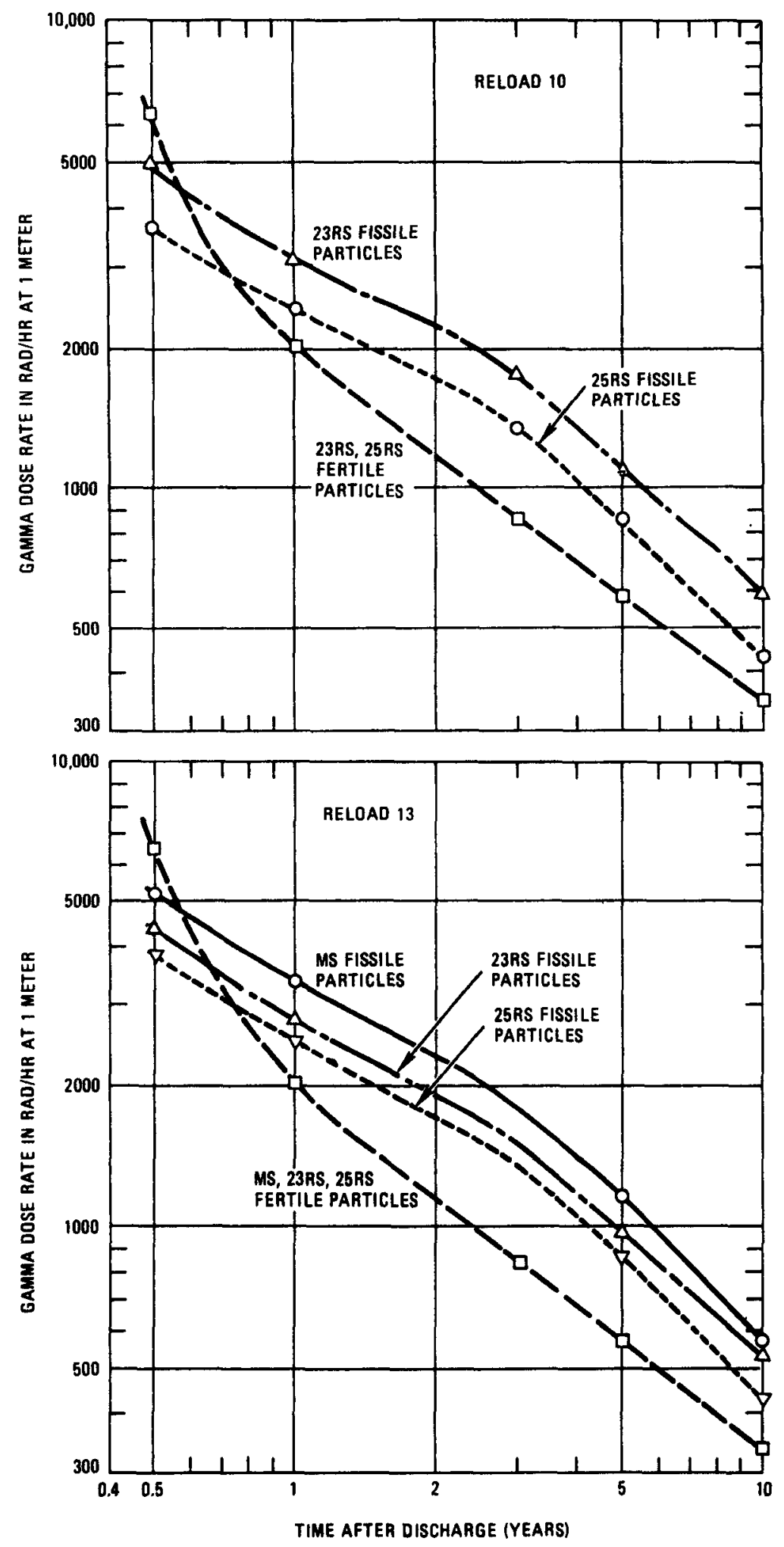

Fig. 9. Gamma dose rate vs. time after discharge: (top) fuel elements discharged at the beginning of reload 10 and originally loaded at reload 6 and (bottom) fuel elements discharged at the beginning of reload 13 and originally loaded at reload 9 


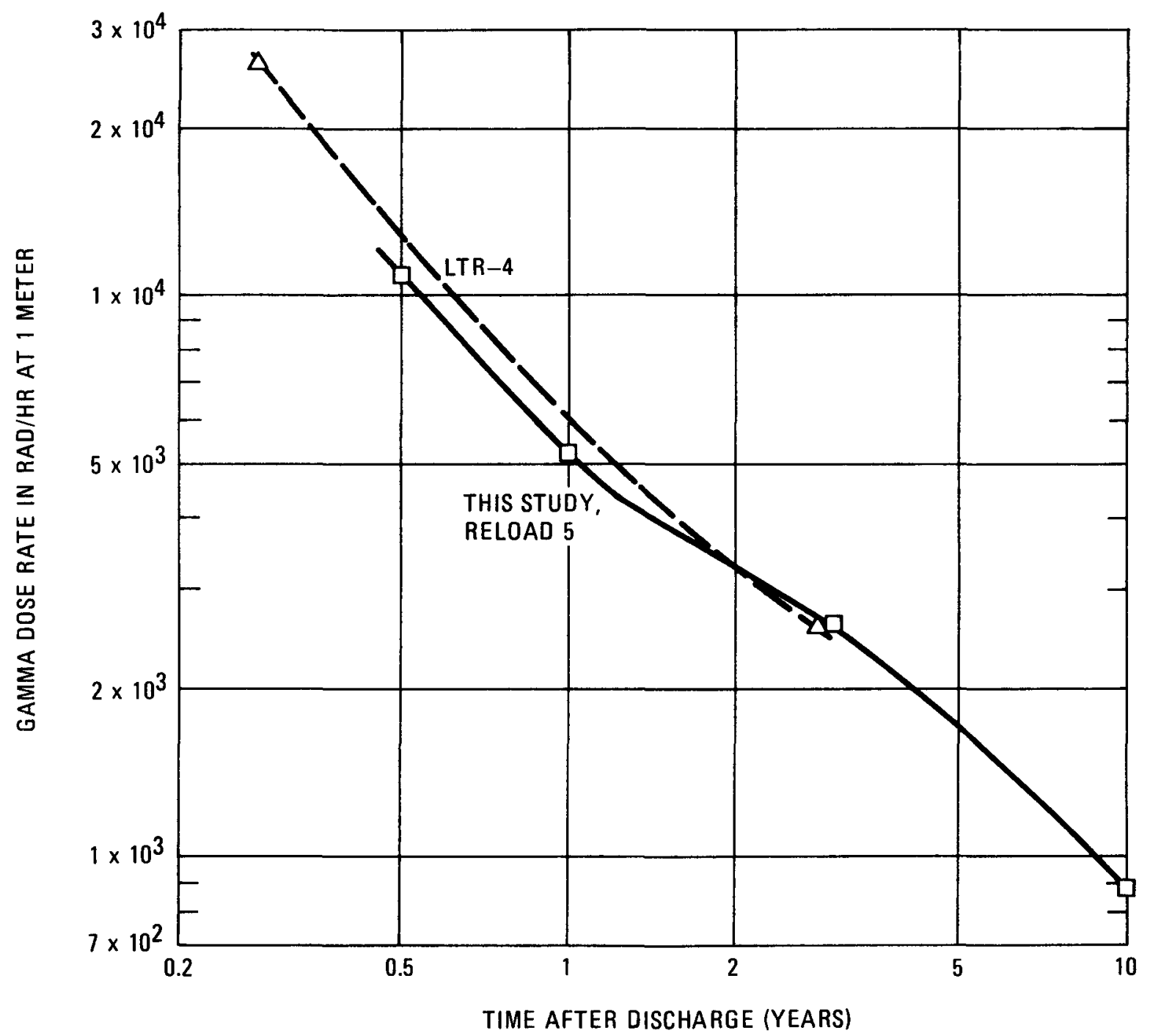

Fig. 10. Total gamma dose rate vs. time after discharge for fuel elements discharged at the beginning of reload 5 and originally loaded at reload 1 compared with the result from LTR-4 (Ref. 16) 


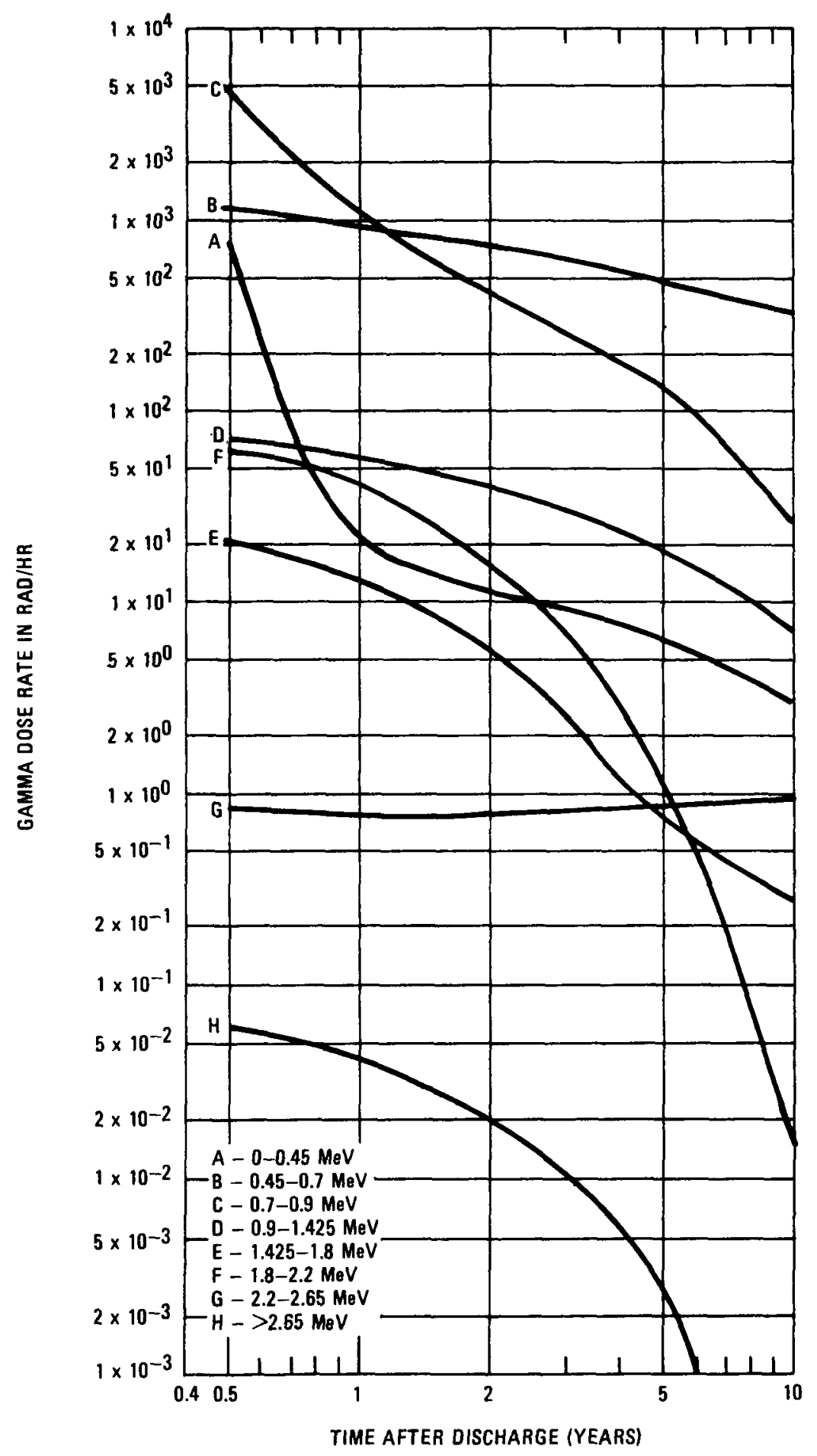

Fig. 11. Gamma dose rates by source energy group vs. time after discharge for MS fertile particles in fuel elements discharged at the beginning of reload 5 and originally loaded at reload 1 


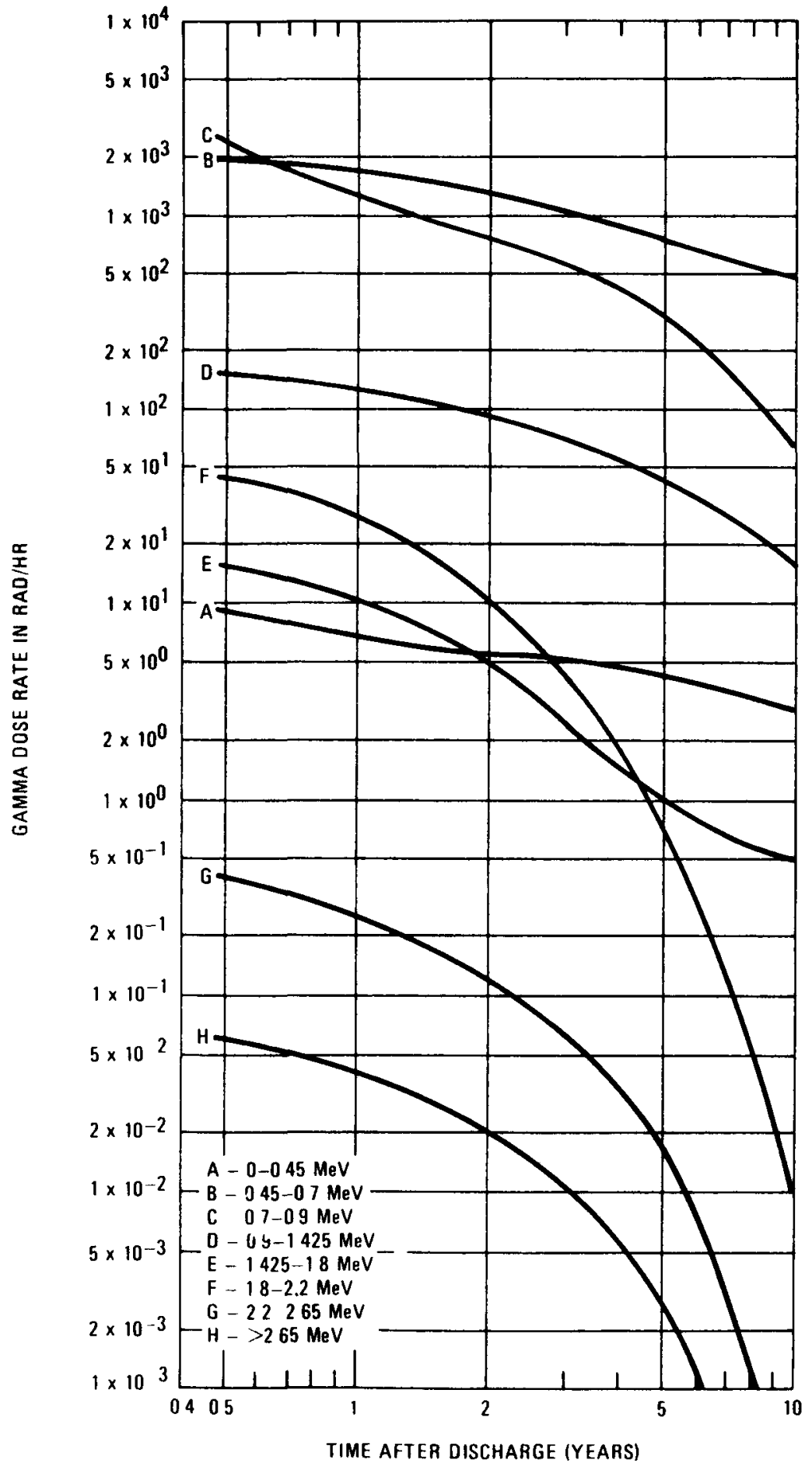

Fig. 12. Gamma dose rates by source energy group vs. time after discharge for MS fissile particles in fuel elements discharged at the beginning of reload 5 and originally loaded at reload 1 


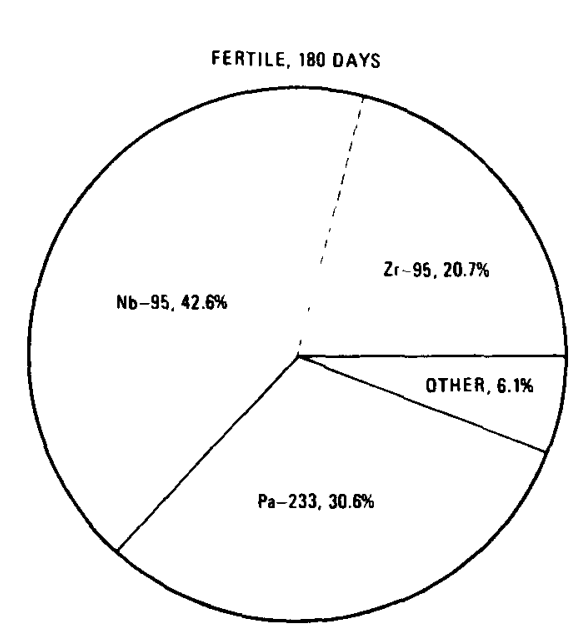

RELOAO 1
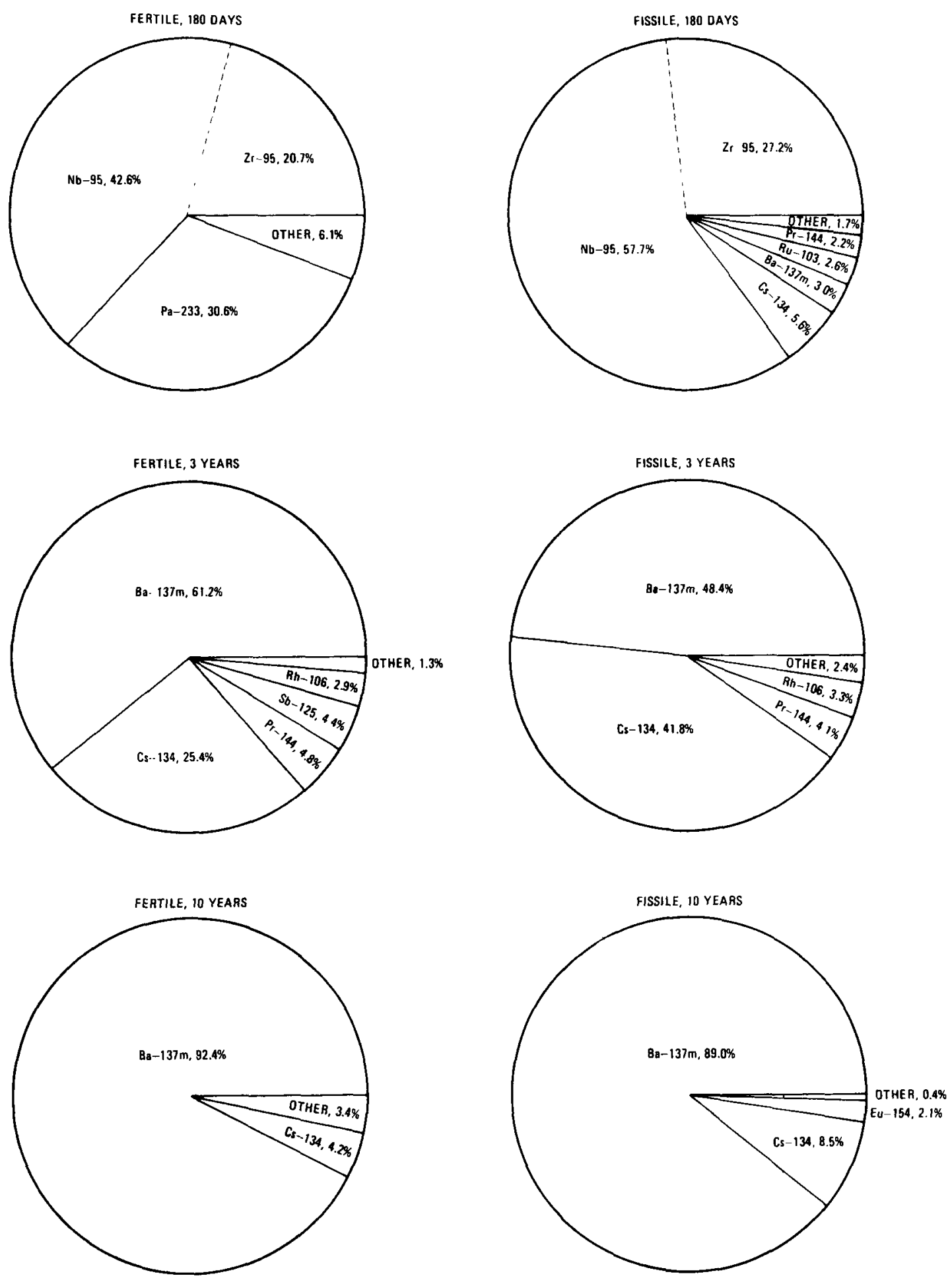

Fig. 13. Important 1sotopic contributors to gamma dose rate for IS fertile particles and IS fissile particles in fuel elements discharged at the beginning of reload 1 and originally loaded at reload 0 

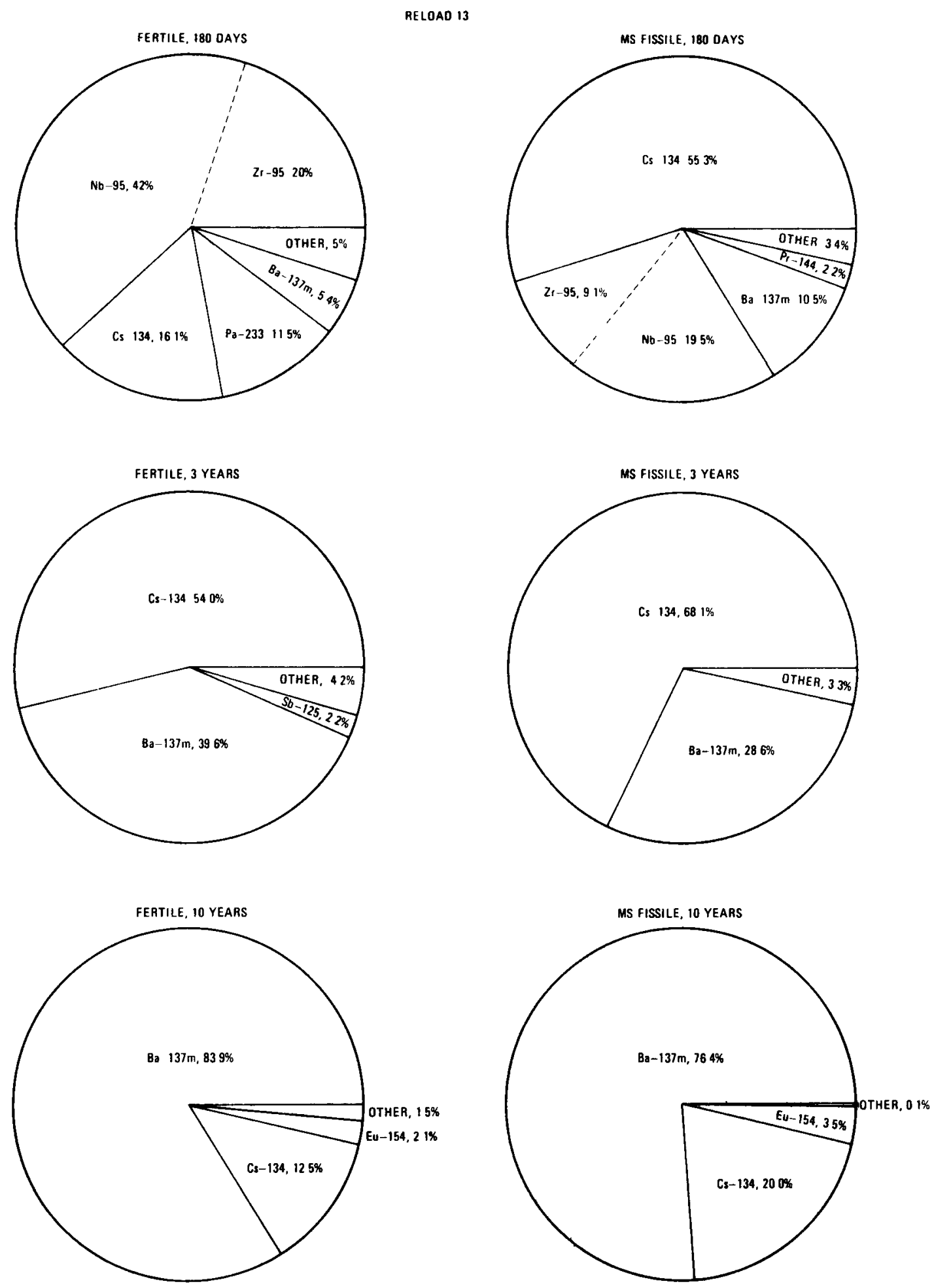

Fig. 14. Important isotopic contributors to gamma dose rate for MS, 23RS, and 25RS fertile particles and MS fissile particles in fuel elements discharged at the beginning of reload 13 and originally loaded at reload 9 


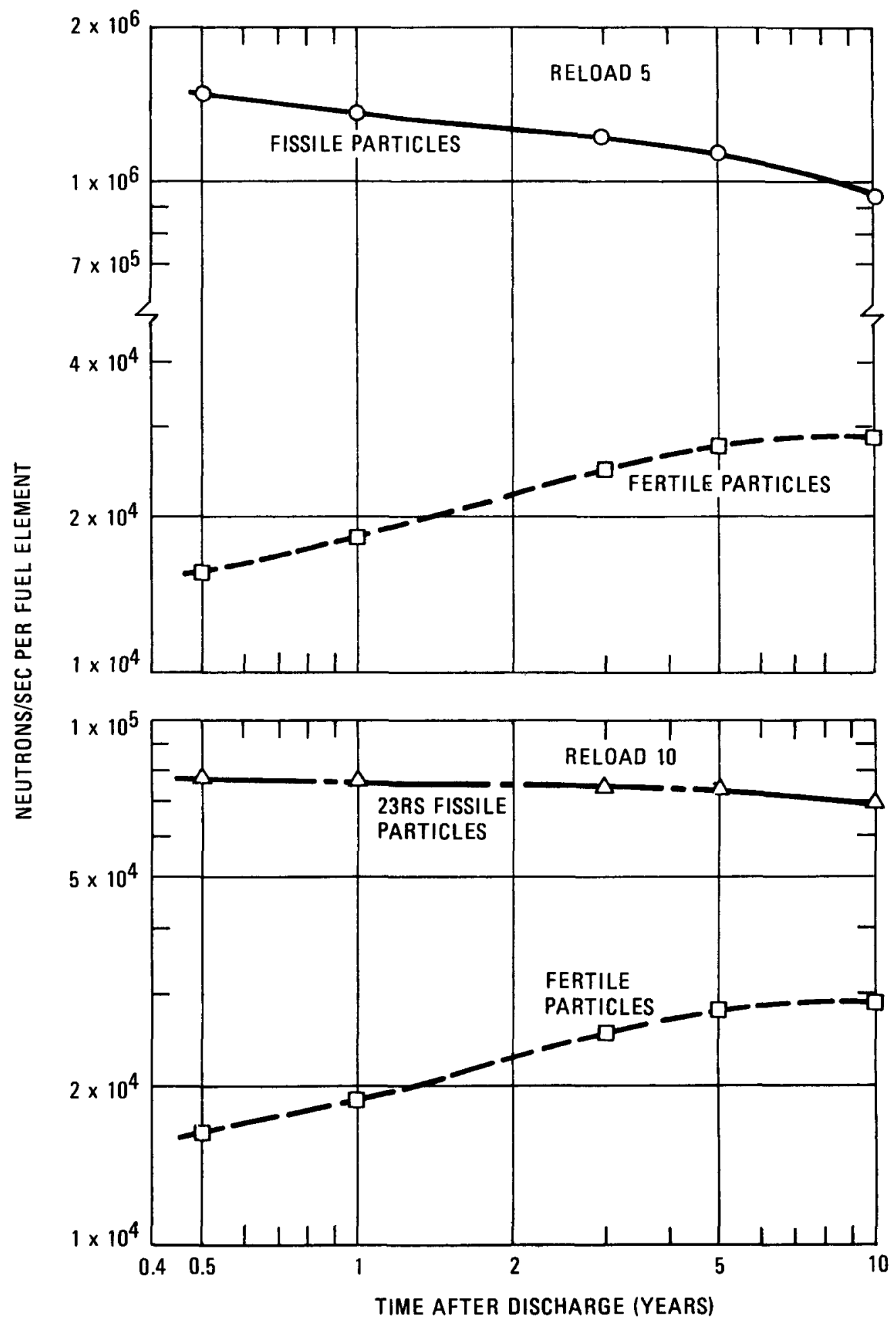

Fig. 15. Neutron source strength vs. time after discharge: (top) fuel elements discharged at the beginning of reload 5 and originally loaded at reload 1 and (bottom) fuel elements discharged at the beginning of reload 10 and originally loaded at reload 6 . (See text for comments on 25RS particles.) 


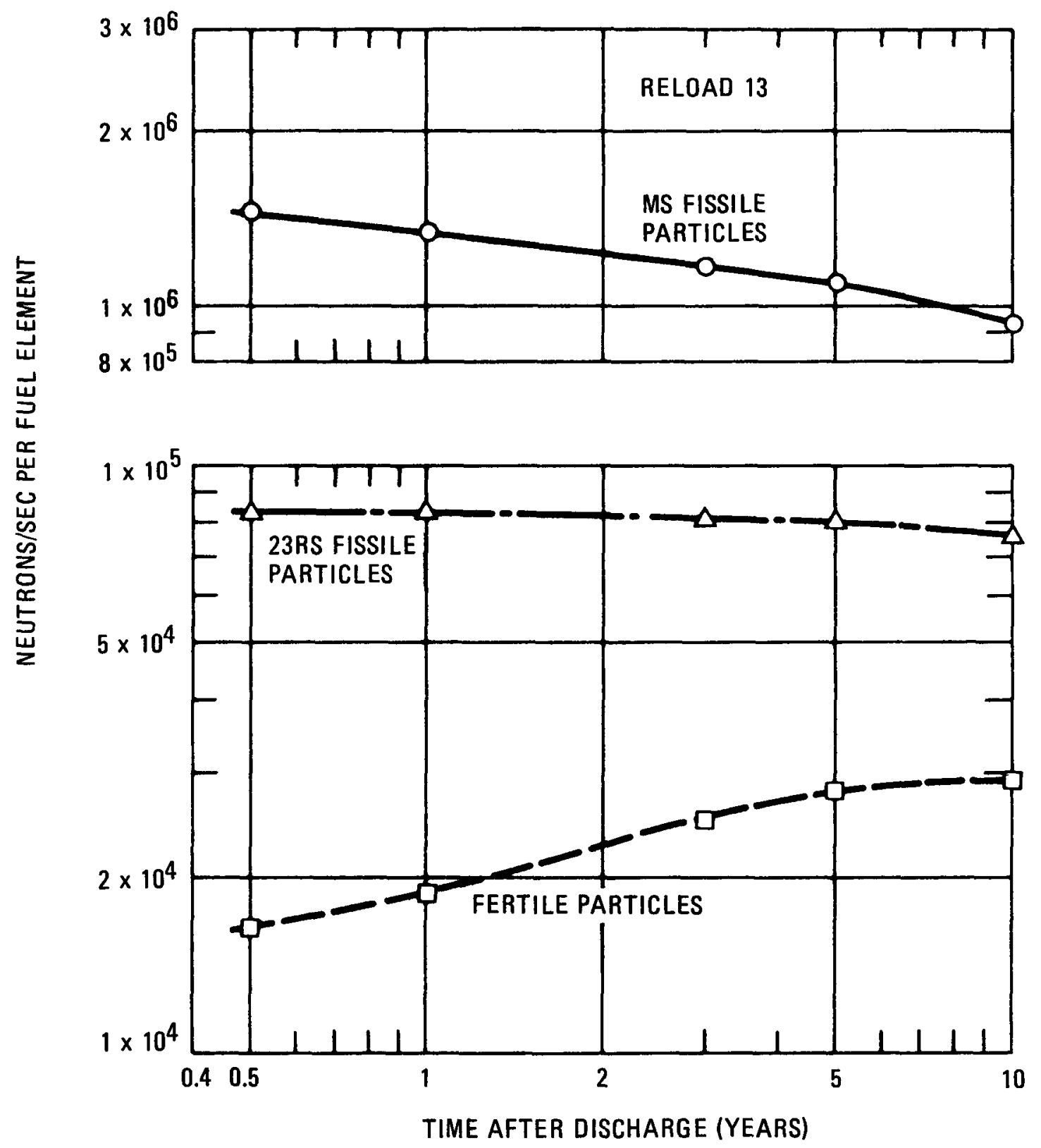

F1g. 16. Neutron source strength vs. time after discharge for fuel elements discharged at the beginning of reload 13 and originally loaded at reload 9. (See text for comments on 25RS particles.) 

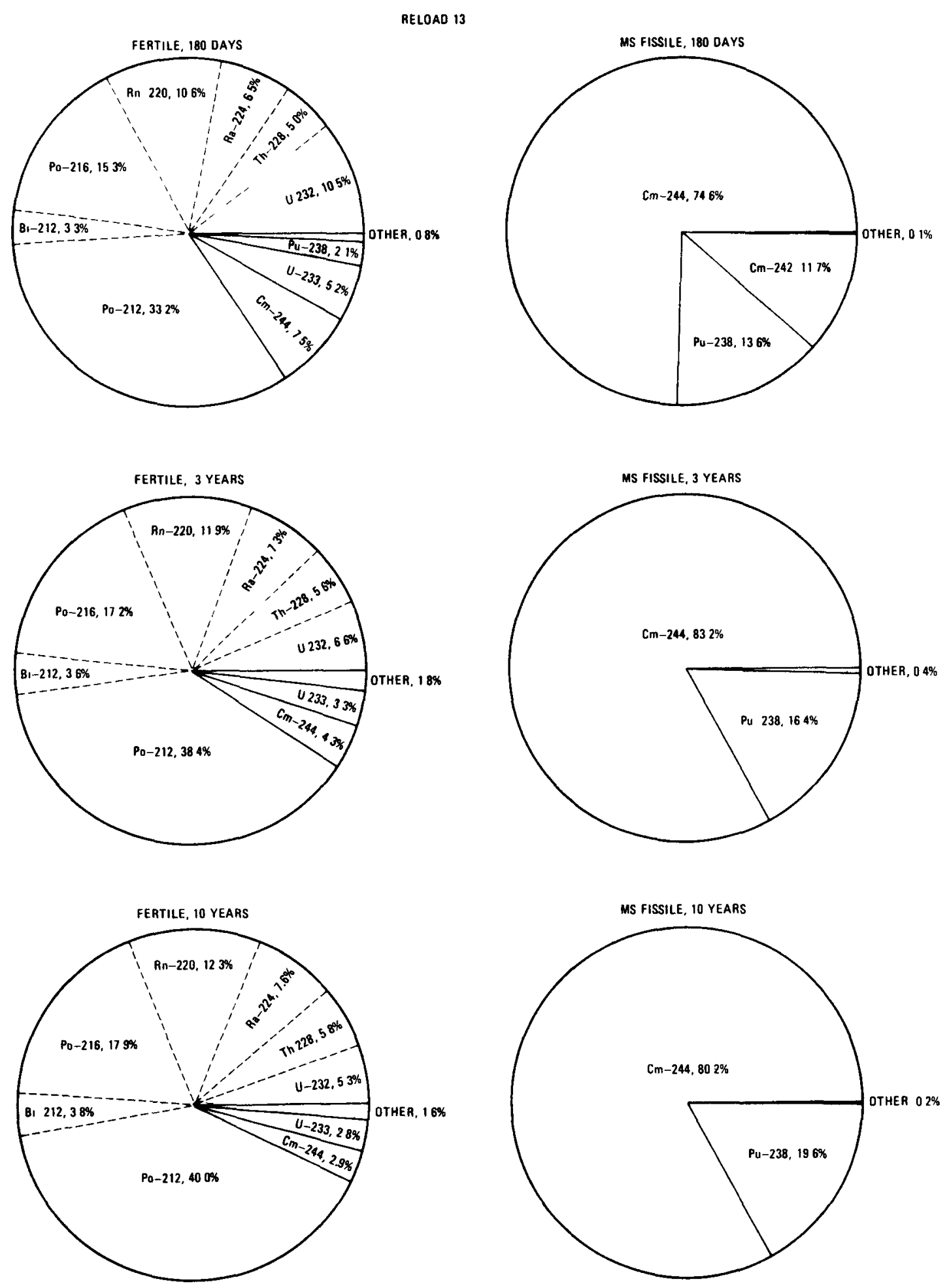

Fig. 17. Important isotopic contributors to neutron source strength for MS, 23RS, and 25RS fertile particles and MS fissile particles in fuel elements discharged at the beginning of reload 13 and originally loaded at reload 9 
RELOAD 13
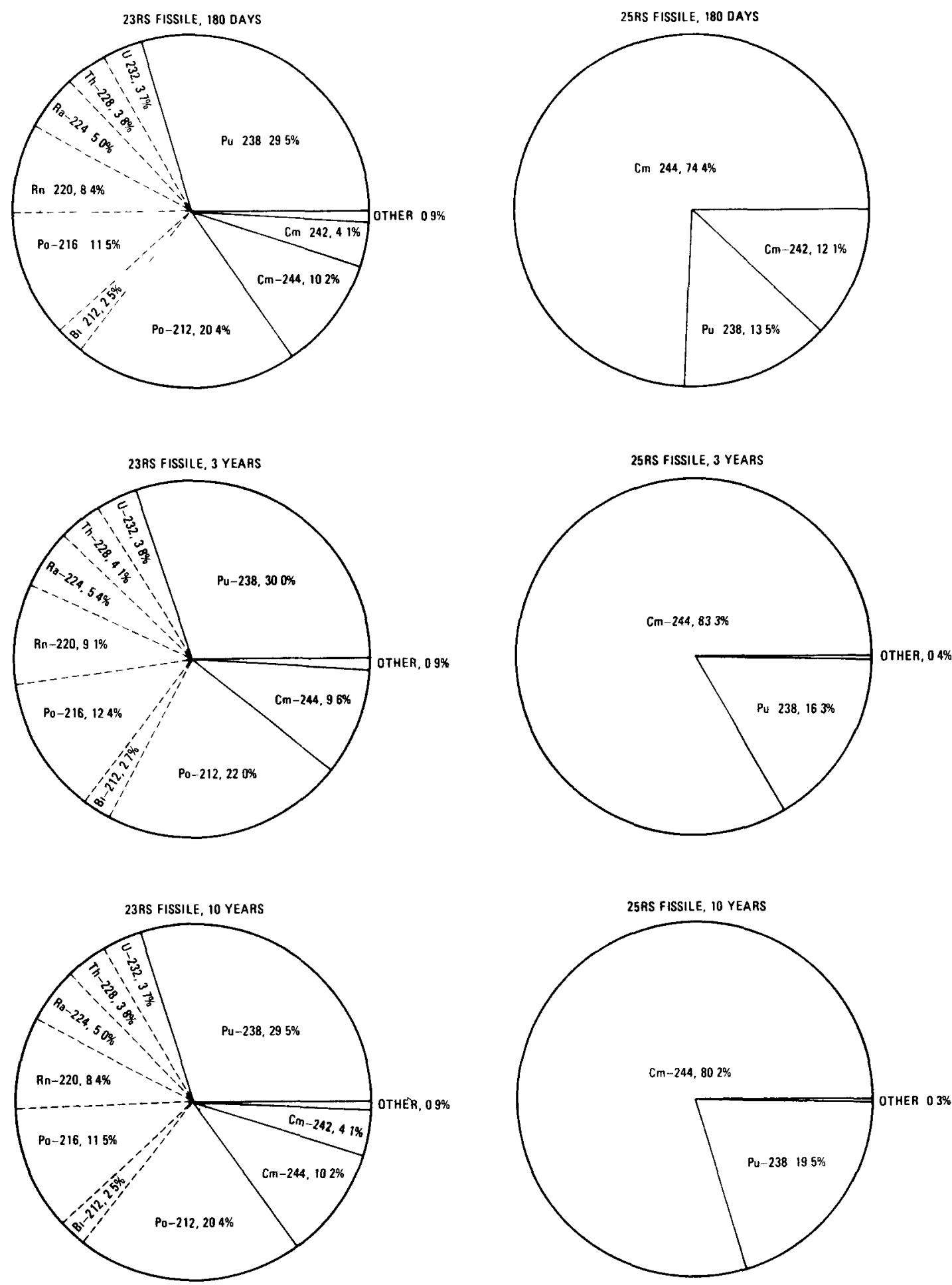

Fig. 18. Important isotopic contributors to neutron source strength for 23RS fissile particles and 25RS fissile particles in fuel elements discharged at the beginning of reload 13 and originally loaded at reload 9 
APPENDIX A

DEPARTURES FROM DATA PRESENTED IN REF. 1 
•

-

• 
APPENDIX A

DEPARTURES FROM DATA PRESENTED IN REF. 1

As discussed in Section 1 and 2, curle values as printed in Ref. 1 were directly entered into the SFUEL code for conversion to decay heat, gamma dose rates, and neutron sources. Exceptions to this procedure, detailed in this appendix, fall into four main categories: omission of data, addition of data, simplification of data, and correction of data.

\section{A. 1. OMISSION OF DATA}

A.1.1. Many isotopes were found to have insignificant curie values relative to other isotopes of the same chemical element, for all fuel types and decay times. The criterion adopted was that any isotope would be dropped if its curie value was always less than $10^{-3}$ of the largest isotopic contributor in an element. A complete list of such omitted isotopes is given in Table $\mathrm{A}-1$.

A.1.2. Very low curie values of remaining isotopes, for any fuel types or decay times, were, in many cases, set equal to zero. The criterion was generally taken as $<10^{-10}$ curie. In the cases of bromine, phosphorous, oxygen, nitrogen, boron, and lithium isotopes, the entire elements were eliminated because the curie values in Ref. 1 were zero for all their isotopes.

\section{A.2. ADDITION OF DATA}

A.2.1. The Isotope "In-114" listed in Ref. 1 was intended to be In-114m. Its short-lived daughter, In-114, was added to the list. 


\section{A.3. SIMPLIFICATION OF DATA}

A.3.1. There are several decay chains comprising long-lived parents and short-lived daughters which, by 180 days decay time, are in radioactive equilibrium, i.e., the curie values of each isotope in the chain are equal. We11-known examples include major parts of the thorium, actinium, and neptunium series.

The stepping method used in Ref. 1 for solving the decay equations could not provide accuracy in curie values of some daughters better than $\pm 5 \%$. Hence, it is noticed that the curie vaiues of some short-lived daughters are not exactly equal to those of their long-lived parents. It was felt that, for the present study, simplification and increased accuracy could be achieved by intentionally setting some daughter curie values equal to their parents. The cases in which this procedure was followed are listed in Table A-2. (The same procedure was followed for several other cases; these are 1isted separately in Section A.4, since the curie values sometimes differed by a large factor.)

A.3.2. Only four significant figures are carried in the decay heat, gammaray dose rate, and neutron source values printed here (as compared with five significant figures in Ref. 1). Even though four significant figures are given, in most cases the data are not accurate to this extent.

A.3.3. Several columns of data in Ref. 1 are all zero, $1 . e$. , for spent recycle fuel discharged at the beginning of reload 1 or 5 or spent makeup fuel discharged at the beginning of reload 10. These columns were omitted from the present report.

\section{A.4. CORRECTION OF DATA}

Errata sheets to Ref. 1 were issued for A.4.2. below. By the time this report is 1ssued, errata sheets to Ref. 1 may have been issued for other statements below. 
A.4.1. Because of the decay chain Am-242m (152 years) $\rightarrow$ Am-242 (16 hours) $\rightarrow$ Cm-242 (163 days), the curie value of $\mathrm{Cm}-242$ for the times of interest in the present study should always be equal to or greater than the curie value of Am-242m. For a number of relatively long decay times, however, this was not true for the data in Ref. 1. Therefore, the curie value of $\mathrm{Cm}-242$ was set equal to the curie value of $\mathrm{Am}-242 \mathrm{~m}$ in these cases.

A.4.2. Curie values for U-232 were all low by a factor of $10^{6}$ in Ref. 1 . These were therefore multiplied by $10^{6}$ for the present study.

A.4.3. The curie value for $\mathrm{Pa}-234$ should equal that for Th-234 for the decay times in this study. In some cases, the curie values in Ref. 1 for these isotopes differed by a factor of 10 . Therefore, the Pa-234 curie values were corrected for the present study.

A.4.4. Curie values for Ra-228 were all high by a factor of 10 in Ref. 1 . These were therefore multiplied by $10^{-1}$ for the present study.

A.4.5. The Ac-228 curie values in Ref. 1 were sometimes incorrect. For this study, the Ac-228 curie values were set equal to the corrected Ra-228 curie values.

A.4.6. Curie values for Th-227 were all high by a factor of 10 in Ref. 1 . After multiplying these by $10^{-1}$ it was found that the criterion of Section A.1.1. applied; Th-227 was therefore dropped from consideration.

A.4.7. After the U-232 curie values in Ref. 1 were corrected (see Section A.4.2), it was found that some of the curie values for the Th-228 daughter must be in error for the U-233 fissile particles. Corrections were made using the following formulas: 


$$
\begin{aligned}
& C i_{B}(T)=C i_{A}(0) \frac{\lambda_{B}}{\lambda_{B}^{*}-\lambda_{A}^{*}}\left(e^{-\lambda_{A}^{*} T}-e^{-\lambda_{B}^{*} T}\right)+C i_{B}(0) e^{-\lambda_{B}^{*} T} \\
& C i_{B}\left(t_{S}\right)=C_{A}(T) \frac{\lambda_{B}}{\lambda_{B}-\lambda_{A}}\left(e^{-\lambda_{A} t} s-e^{-\lambda_{B} t}\right)+C i_{B}(T) e^{-\lambda_{B} t_{S}}
\end{aligned}
$$

$$
\text { where } \begin{aligned}
\mathrm{Ci}_{A}(0) & =\text { curies of } \mathrm{U}-232 \text { loaded, } \\
\mathrm{Ci}_{B}(0) & =\text { curies of } \mathrm{Th}-228 \text { loaded, } \\
\mathrm{Ci}_{A}(\mathrm{~T}) & =\text { curies of } \mathrm{U}-232 \text { discharged, } \\
\mathrm{Ci}_{B}(\mathrm{~T}) & =\text { curies of } \mathrm{Th}-228 \text { discharged, } \\
\mathrm{Ci}_{B}\left(\mathrm{t}_{\mathrm{s}}\right) & =\text { curies of } \mathrm{Th}-228 \text { at decay time } \mathrm{t}_{\mathrm{s}}, \\
\mathrm{T} & =4 \text { years } \\
\lambda_{A} & =3.05(-10) \mathrm{sec}^{-1}, \\
\lambda_{B} & =1.15(-8) \mathrm{sec}^{-1}, \\
\lambda_{A}^{*} & =7.608(-9) \mathrm{sec}^{-1}, \\
\lambda_{B}^{*} & =1.88(-8) \mathrm{sec}^{-1},
\end{aligned}
$$

A.4.8. The curie values for $\mathrm{Bi}-213, \mathrm{Po}-213$, and $\mathrm{Pb}-209$ in Ref. 1 were incorrect. These were therefore set equal to the curie values for At-217. (The curie value for Po-213 should equal 0.978 of that for Bi-213, hut in this calculation, we approximated 0.978 with 1.0. )

A.4.9. An incorrect branching ratlo used in Ref. 1 resulted in substantial $\mathrm{Pb}-206 \mathrm{~m}$ activities when they should have been negligible. This correction qualified $\mathrm{Pb}-206 \mathrm{~m}$ to be eliminated on the basis of Section A.1.1.

A.4.10. The La-137 curie values in Ref. 1 are too high by a factor of 10 . These were therefore multiplied by $10^{-1}$ for the present study.

A.4.11. Curle values of Tc-99 were low by a factor of 5.695 in Ref. 1 . These were therefore multiplied by 5.695 for the present study. 
A.4.12. The isotope "Ag-108" in Ref. 1 was intended to be $\mathrm{Ag}-108 \mathrm{~m}$. $\mathrm{Ag}-108$ was not added to the list, since the decay-heat values and gamma dose rates in this study for $\mathrm{Ag}-108$ are small relative to those for $\mathrm{Ag}-108 \mathrm{~m}$.

A complete list of the curie values used in the present study, with al1 of the changes given above except for the change discussed in A.1.2., is given in Table A-3. The change discussed in A.1.2. was performed in the calculations. 
TABLE A-1

ISOTOPES OMITTED FROM PRESENT STUDY ON BASIS OF CURIE-VALUE CRITERION GIVEN IN SECTION A.1.1.

\begin{tabular}{|c|c|c|c|c|c|}
\hline $\mathrm{Cm}-245$ & $\mathrm{~Pb}-206$ & $S m-147$ & $\mathrm{Xe}-131$ & $\mathrm{Ag}-109 \mathrm{~m}$ & $Y-92$ \\
\hline $\mathrm{Am}-244$ & $\mathrm{~Pb}-207 \mathrm{~m}$ & $\mathrm{Sm}-148$ & $\mathrm{Xe}-132$ & $\mathrm{Ag}-109$ & $Y-93$ \\
\hline $\mathrm{Pu}-236$ & $\mathrm{~Pb}-207$ & $S m-149$ & $\mathrm{Xe}-133 \mathrm{~m}$ & $\mathrm{Ag}-111 \mathrm{~m}$ & $\mathrm{Sr}-86$ \\
\hline $\mathrm{Pu}-242$ & $\mathrm{~Pb}-208$ & $\mathrm{Sm}-150$ & $\mathrm{Xe}-133$ & $\mathrm{Ag}-111$ & $\mathrm{Sr}-87$ \\
\hline $\mathrm{Pu}-243$ & $\mathrm{~Pb}-210$ & $\mathrm{Sm}-152$ & $\mathrm{Xe}-134$ & $\mathrm{Ag}-112$ & $\mathrm{Sr}-88$ \\
\hline$P u-244$ & $\mathrm{~Pb}-214$ & $\mathrm{Sm}-153$ & $\mathrm{Xe}-135 \mathrm{~m}$ & $P d-104$ & Sr -91 \\
\hline$N p-236$ & $\mathrm{~T} 1-205$ & $\mathrm{Sm}-154$ & $\mathrm{Xe}-135$ & $\mathrm{Pd}-105$ & $\mathrm{Rb}-85$ \\
\hline$N p-238$ & $\mathrm{~T} 1-206$ & $\mathrm{Sm}-156$ & $\mathrm{Xe}-136$ & $P d-106$ & $\mathrm{Kr}-82$ \\
\hline$N p-239$ & $\mathrm{~T} 1-207 \mathrm{~m}$ & $P m-144$ & $I-127$ & $P d-108$ & $\mathrm{Kr}-83 \mathrm{~m}$ \\
\hline$N p-240$ & $\mathrm{~T} 1-209$ & $P m-145$ & I-132 & $\mathrm{Pd}-109$ & $\mathrm{Kr}-83$ \\
\hline $\mathrm{Np}-241$ & $\mathrm{~T} 1-210$ & $\mathrm{Pm}-146$ & $I-133$ & $\mathrm{Pd}-110$ & $\mathrm{Kr}-84$ \\
\hline $\mathrm{U}-230$ & Dy-156 & $\mathrm{Pm}-148$ & $I-135$ & $P d-111 m$ & $\mathrm{Kr}-85 \mathrm{~m}$ \\
\hline $\mathrm{U}-239$ & Dy -157 & $\mathrm{Pm}-149$ & $\mathrm{Te}-125$ & $\mathrm{Pd}-111$ & $\mathrm{Kr}-86$ \\
\hline$U-240$ & Dy-158 & $P m-151$ & $\mathrm{Te}-128$ & $\mathrm{Pd}-112$ & $\mathrm{Br}-79$ \\
\hline $\mathrm{Pa}-230$ & Dy -160 & $\mathrm{Nd}-142$ & $\mathrm{Te}-130$ & Rh-103 & $\mathrm{Br}-81$ \\
\hline $\mathrm{Pa}-232$ & $D y-161$ & $\mathrm{Nd}-143$ & $\mathrm{Te}-131 \mathrm{~m}$ & $\mathrm{Rh}-105 \mathrm{~m}$ & $\mathrm{Br}-82$ \\
\hline $\mathrm{Pa}-234 \mathrm{~m}$ & Dy -162 & $\mathrm{Nd}-144$ & $\mathrm{Te}-131$ & $\mathrm{Rh}-105$ & $\mathrm{Br}-83$ \\
\hline $\mathrm{Pa}-235$ & Dy -163 & $\mathrm{Nd}-145$ & $\mathrm{Te}-132$ & $\mathrm{Ru}-$ & $\mathrm{Se}-80$ \\
\hline Th-226 & Dy -164 & $\mathrm{Nd}-146$ & $\mathrm{Sb}-121$ & $\mathrm{Ru}-1$ & $\mathrm{Se}-82$ \\
\hline Th-227 & $\mathrm{Tb}-155$ & $\mathrm{Nd}-148$ & $\mathrm{Sb}-122$ & $\mathrm{Ru}-$ & $\mathrm{Ni}-58$ \\
\hline Th-233 & $\mathrm{Tb}-156$ & $\mathrm{Nd}-150$ & $\mathrm{Sb}-123$ & $\mathrm{Ru}-$ & $\mathrm{Ni}-60$ \\
\hline Th-235 & $\mathrm{Tb}-159$ & $\operatorname{Pr}-141$ & $\mathrm{Sb}-$ & $\mathrm{Ru}-$ & $\mathrm{Ni}-61$ \\
\hline$A c-229$ & $\mathrm{~Tb}-161$ & $\operatorname{Pr}-142$ & & $\mathrm{Ru}-$ & $\mathrm{Ni}-62$ \\
\hline $\mathrm{Ra}-222$ & $\mathrm{~Tb}-162$ & $\operatorname{Pr}-143$ & $\mathrm{Sb}-$ & Mo-s & $\mathrm{Ni}-64$ \\
\hline $\mathrm{Ra}-226$ & $\mathrm{~Tb}-163$ & $\operatorname{Pr}-145$ & $\mathrm{Sn}-115$ & Mo- & $\mathrm{C} 1-35$ \\
\hline $\mathrm{Ra}-227$ & $\mathrm{~s}-152$ & $\mathrm{Ce}-136$ & $117 \mathrm{~m}$ & Mo-s & $\mathrm{C} 1-37$ \\
\hline $\mathrm{Ra}-229$ & $\mathrm{Gd}-154$ & $\mathrm{Ce}-137$ & Sn-117 & Mo-98 & $\mathrm{S}-32$ \\
\hline $\operatorname{Fr}-224$ & $G d-155$ & $\mathrm{Ce}-138$ & $S n-119$ & Mo-9 & $s-33$ \\
\hline $\mathrm{Rn}-218$ & $d-156$ & $\mathrm{Ce}-139$ & $S n-120$ & Mo- 100 & S-34 \\
\hline$R n-222$ & $d-157$ & $\mathrm{Ce}-140$ & $S n-121$ & $\mathrm{Nb}-93$ & $s-36$ \\
\hline$R n-223$ & $d-158$ & $\mathrm{Ce}-142$ & $S n-125$ & $\mathrm{Nb}-96$ & $P-33$ \\
\hline$R n-224$ & Gd-159 & $\mathrm{Ce}-143$ & In- 113 & $\mathrm{Nb}-97 \mathrm{~m}$ & $0-16$ \\
\hline At -219 & $\mathrm{Gd}-160$ & $\mathrm{La}-138$ & In-115m & $\mathrm{Nb}-97$ & $0-17$ \\
\hline Po-209 & $\mathrm{Gd}-162$ & La-139 & In-115 & $\mathrm{Zr}-90$ & $N-14$ \\
\hline Po-211 & $\mathrm{Eu}-149$ & $\mathrm{Ba}-135$ & $\mathrm{Cd}-110$ & $\mathrm{Zr}-91$ & $N-15$ \\
\hline Po-214 & $\mathrm{Eu}-150$ & $\mathrm{Ba}-136$ & $C d-111$ & $\mathrm{Zr}-92$ & $C-12$ \\
\hline Po-218 & $\mathrm{Eu}-151$ & $\mathrm{Ba}-137$ & $\mathrm{Cd}-112$ & $\mathrm{Zr}-94$ & $C-13$ \\
\hline Bi-209 & $\mathrm{Eu}-153$ & $\mathrm{Ba}-138$ & $\mathrm{Cd}-113$ & Zr-96 & $B-10$ \\
\hline$B i-210$ & $\mathrm{Eu}-157$ & $C s-133$ & $\mathrm{Cd}-114$ & $\mathrm{Zr}-97$ & $B-11$ \\
\hline Bi-214 & $\mathrm{Sm}-144$ & $\mathrm{Cs}-135$ & $\mathrm{Cd}-115$ & $Y-89$ & Li-6 \\
\hline $\mathrm{Pb}-206 \mathrm{~m}$ & $\mathrm{Sm}-146$ & $\mathrm{Cs}-136$ & $\mathrm{Ag}-107$ & $\mathrm{Y}-91 \mathrm{~m}$ & Li-7 \\
\hline
\end{tabular}


TABLE A-2

DECAY CHAINS IN WHICH CURIE VALUE OF DAUGHTER WAS SET EQUAL TO THAT OF PARENT

\author{
Parent \\ (Curie value \\ from Ref. 1)
}

\author{
Daughter(s) \\ (Curie value(s) set equal \\ to parent curie value)
}

$\begin{array}{ll}\text { Am-242m } & \mathrm{Am}-242 \\ \mathrm{U}-235 & \mathrm{Th}-231 \\ \mathrm{Th}-228 & \mathrm{Ra}-224, \mathrm{Rn}-220, \mathrm{Po}-216, \mathrm{~Pb}-212 \\ & \mathrm{Bi}-212, \mathrm{Po}-212(\mathrm{a}) \mathrm{T} 1-208(\mathrm{a}) \\ \mathrm{Th}-229 & \mathrm{Ra}-225, \mathrm{Ac}-225, \mathrm{Fr}-221, \mathrm{At}-217 \\ & \mathrm{Bi}-213, \mathrm{Po}-213, \mathrm{~Pb}-209 \\ \mathrm{Ra}-223 & \mathrm{Rn}-219, \mathrm{Po}-215, \mathrm{~Pb}-211, \mathrm{Bi}-211, \\ & \mathrm{~T} 1-207 \\ \mathrm{Ce}-144 & \mathrm{Pr}-144 \\ \mathrm{Ba}-140 & \mathrm{La}-140 \\ \mathrm{Cs}-137 & \mathrm{Ba}-137 \mathrm{~m} \\ \mathrm{Te}-129 \mathrm{~m} & \mathrm{Te}-129 \\ \mathrm{Te}-127 \mathrm{~m} & \mathrm{Te}-127 \\ \mathrm{In}-114 \mathrm{~m} & \mathrm{In}-114 \\ \mathrm{Ru}-103 & \mathrm{Rh}-103 \mathrm{~m} \\ \mathrm{Sr}-90 & \mathrm{Y}-90 \\ & \end{array}$

(a) Because of branching for $B i-212$, curie values for Po-212 and T1-208 were set equal to 0.64 and 0.36 , respectively, of curie values for $\mathrm{Th}-228$. 
•

-

0 
TABLE A-3

LIST OF CURIE VALUES USED IN PRESENT CALCULATIONS

NOTE: Table A-3 consists of 45 pages of computer print-out (pages A-11 through A-55). 
•

-

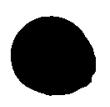


THIS SEGMENT WAS OISCHARGEO AT THE BEGINNING OF RELOAD 1 AND WAS
THIS SEGMENT WAS OISCHARGED AT THE BEG ORIGIMALLY LOAOED AT RELOAO

CURIE VALUES USED PER F.E. AT IIME 180 DAYS AFTER OISCHARge

IS FRESH U-235 F.E.

FERTILE PTL: FISSILE PTL.

NUCLIDE

$\mathrm{CH}-242$

$\mathrm{CH}-243$

$\mathrm{CH}-244$

$A H-241$
$A H-242 M$

$A H-242$

$A H-243$

PU -238

PU -239

$P U-240$

$P U-241$

$U-23$

$u-23$

$u-235$

$0-236$
$0-237$
$0-238$

0
$0-237$
$U-238$

$P A-231$

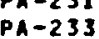

$P A-234$

TH-228

$T H-229$

$T H-230$

IH-231

TH-232

$\mathrm{TH}-234$

AC-225

AC- 227

AC-228

$R A-223$

$R A-224$

$R A-225$

$R A-228$

RR

$F R-223$

RN

$R N-220$

AT -217

PO- 210

$P O-212$

$P O-215$

$P O-216$

$B I-211$

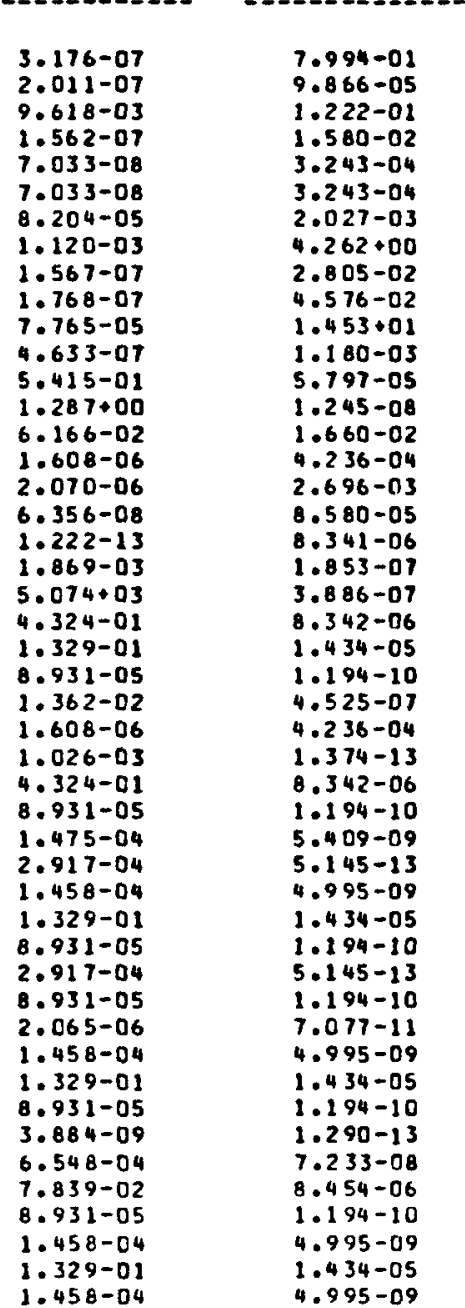

MS FRESH U-235 F.E.

FERIILE PTL.

FISSILE PTL.

CO

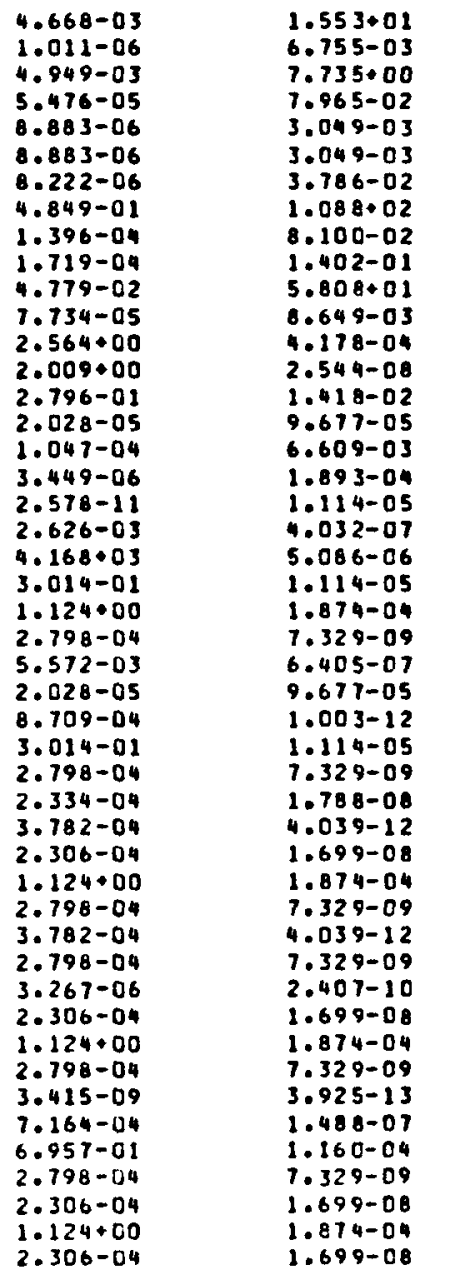




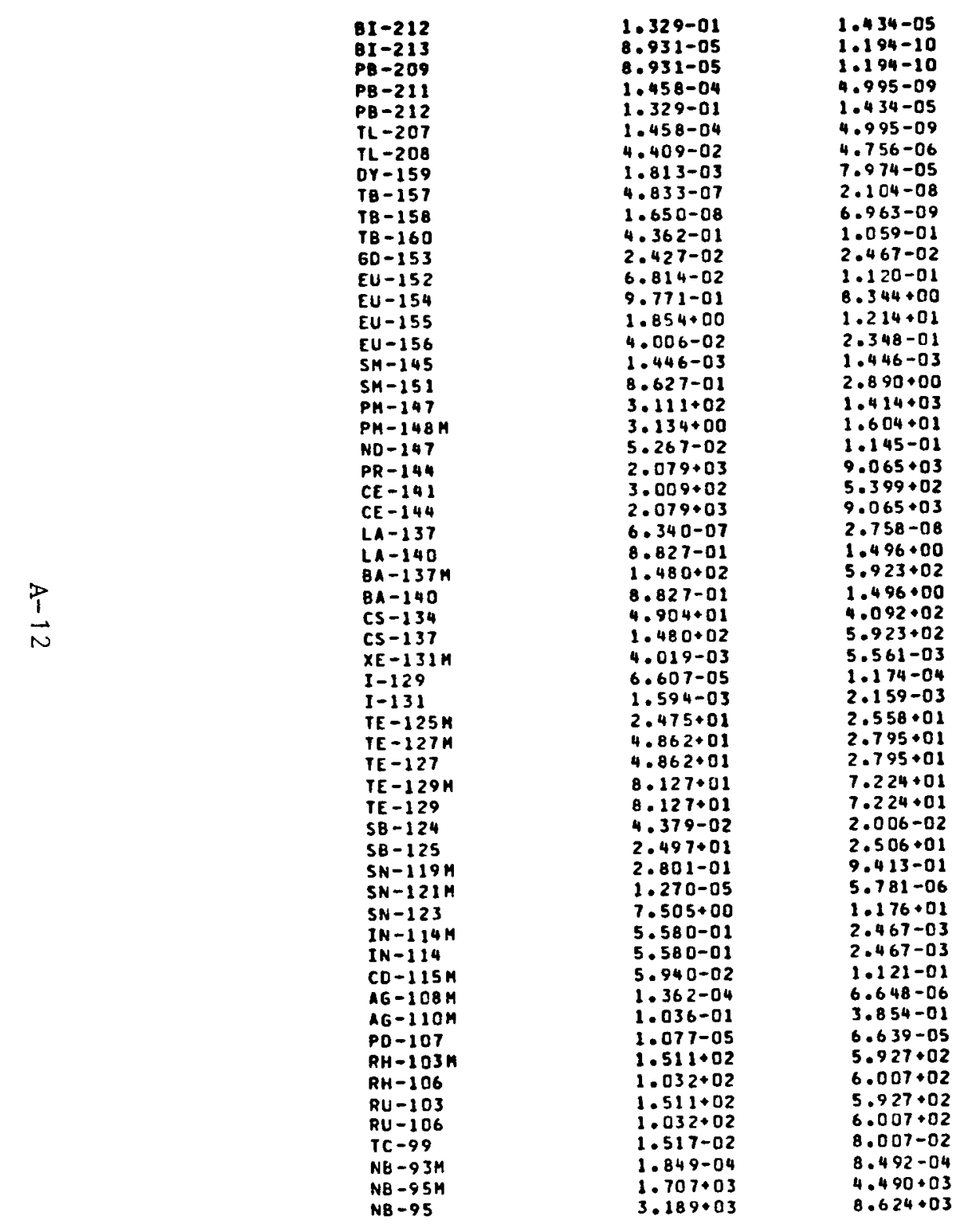

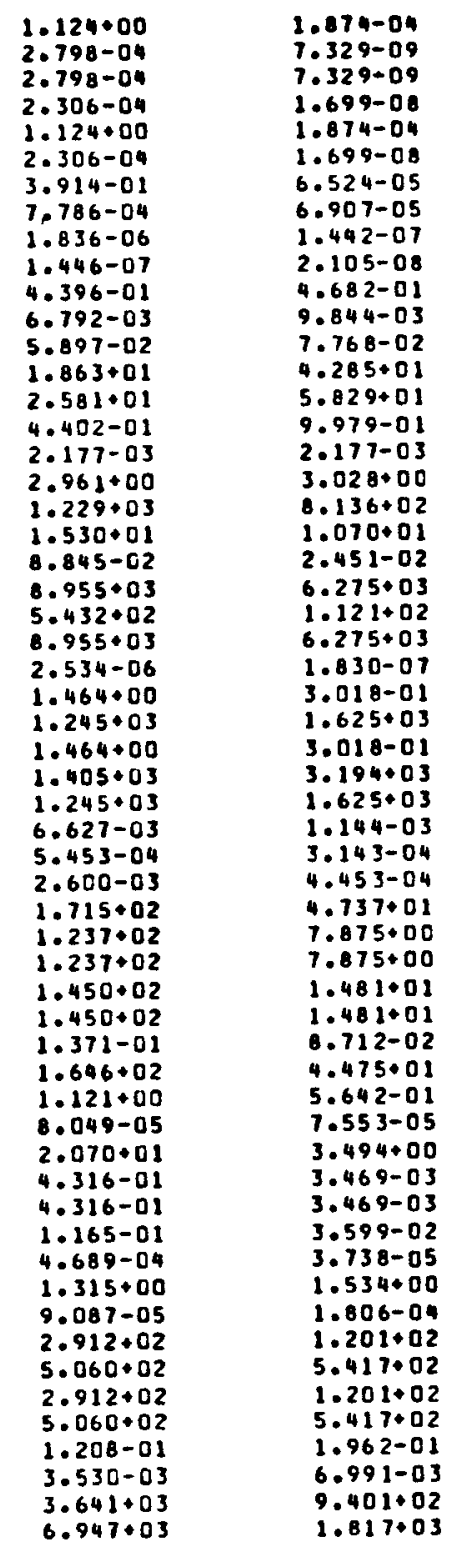




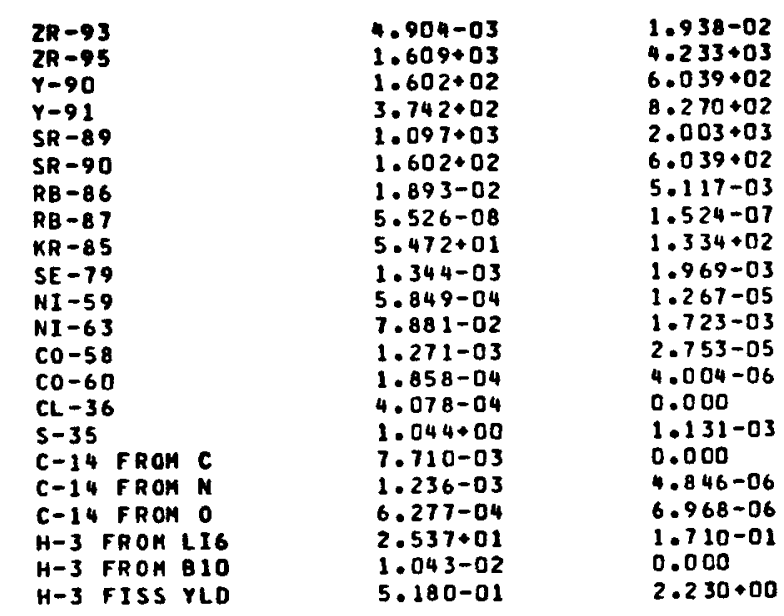

$\begin{array}{ll}1.197-02 & 5.487-02 \\ 3.433+03 & 8.863+02 \\ 1.340+03 & 1.642+03 \\ 7.666+02 & 1.719+02 \\ 2.174+03 & 4.070+02 \\ 1.340+03 & 1.642+03 \\ 4.292-02 & 1.344-02 \\ 4.657-07 & 4.235-07 \\ 3.462+02 & 2.127+02 \\ 8.945-03 & 3.520-03 \\ 2.023-03 & 7.284-05 \\ 2.676-01 & 9.640-03 \\ 1.141-03 & 4.109-05 \\ 5.500-04 & 1.991-05 \\ 1.321-03 & 0.000 \\ 9.636-01 & 1.745-03 \\ 2.709-02 & 0.000 \\ 4.352-03 & 2.901-05 \\ 2.252-03 & 4.080-05 \\ 3.277+01 & 3.669-01 \\ 5.302-02 & 0.000 \\ 4.212+00 & 5.724+00\end{array}$


THIS SEGMENT WAS OISCHARGED AT THE BEGINNING OF RELOAD 1 AND WAS

ORIGINALLY LOAOEO AT RELOAD O

CURIE VALUES USED PER F.E. AT TIME

THIS SEGMENT WAS DISCHARGEO AT THE BEGINNING OF RELOAD 5 AND

reAR aFter DISCHaRge

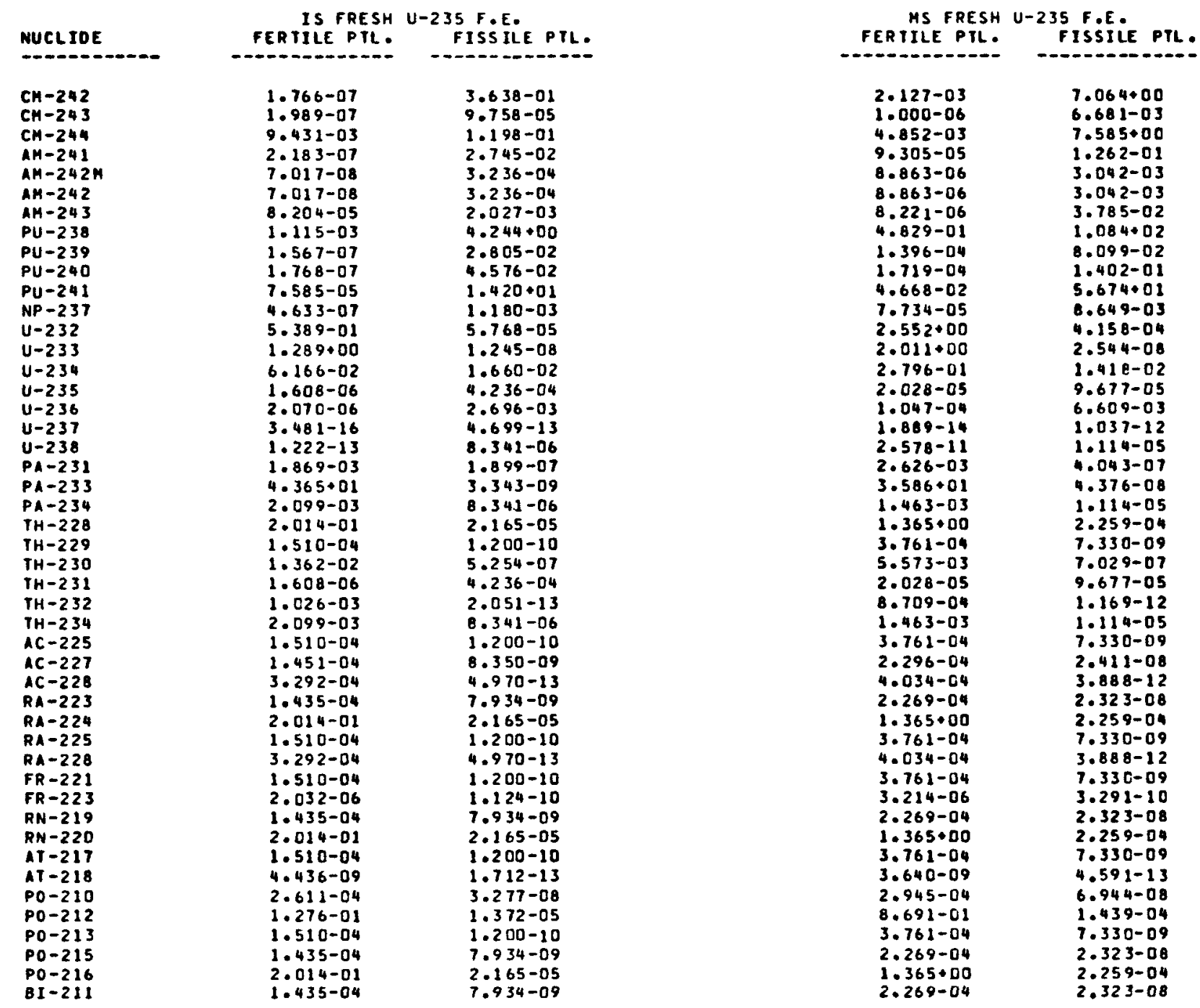




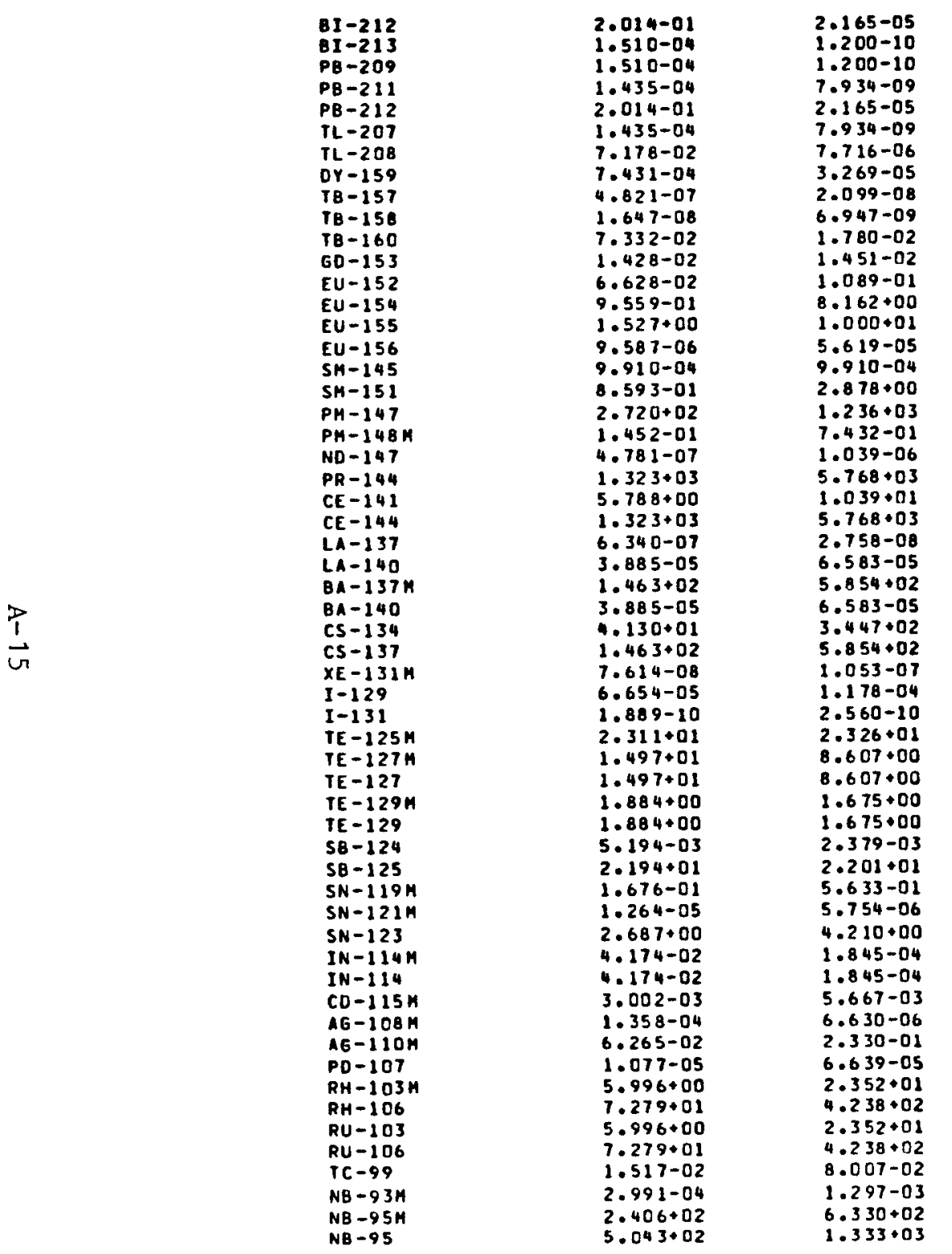

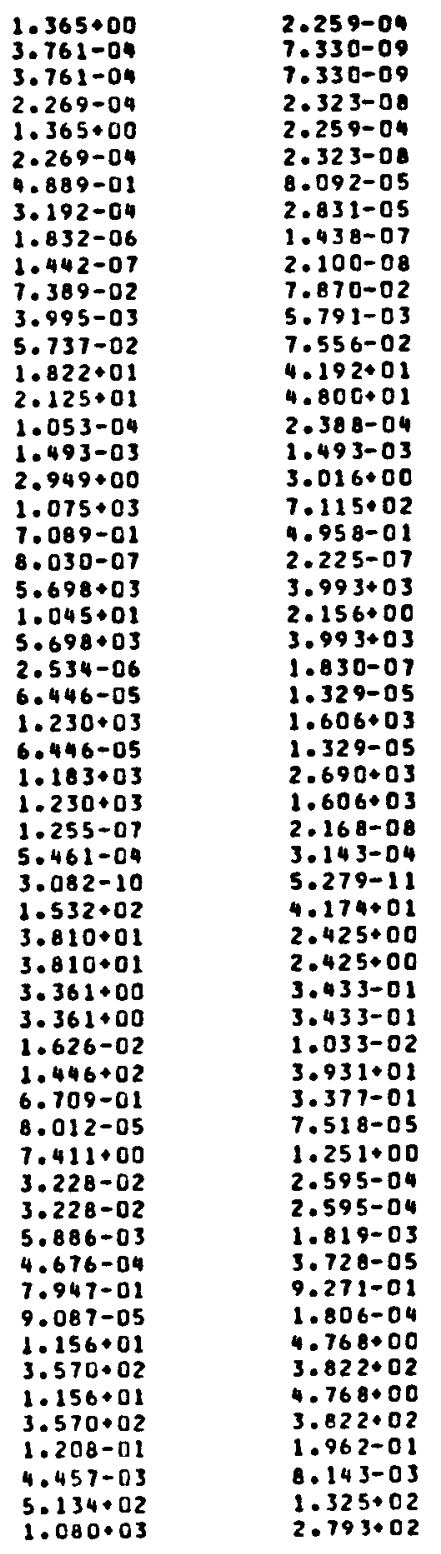




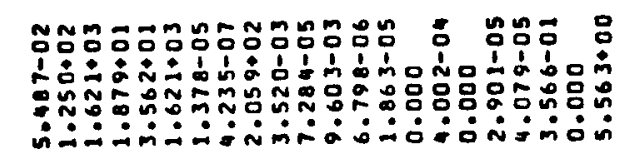

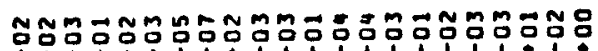

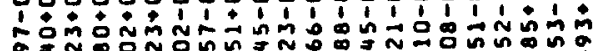

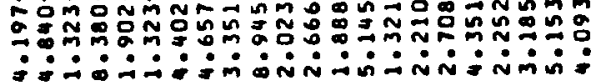

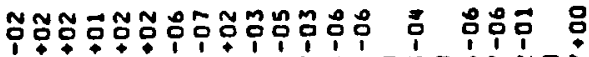

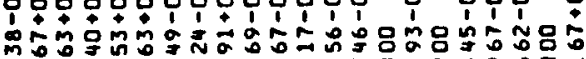

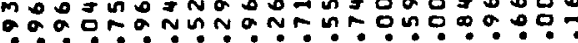

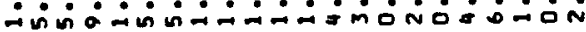

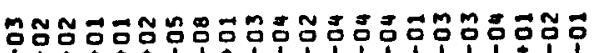

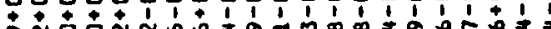

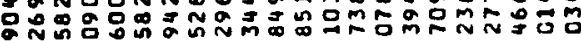

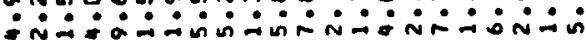

\section{(1)}

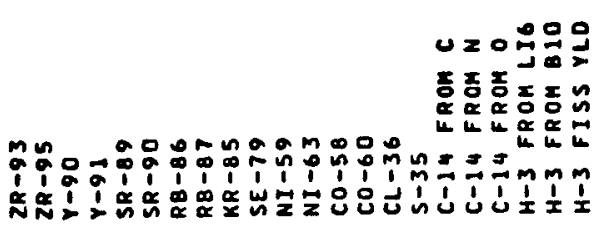

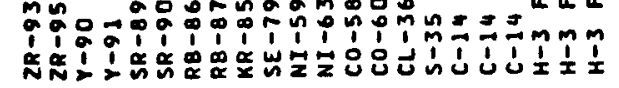


THIS SEGMENT WAS OISCHARGEO AT THE BEGINNING OF RELOAD I AND YAS ORIGINALIY LOF REL RELOAOO

CURIE VALUES USED PER F.E. AT TIME 3 VEARS after DISCHarge

THIS SEGMENT YAS OISCHARGEO AT THE BEGINNING OF RELOAD 5 ANO UAS

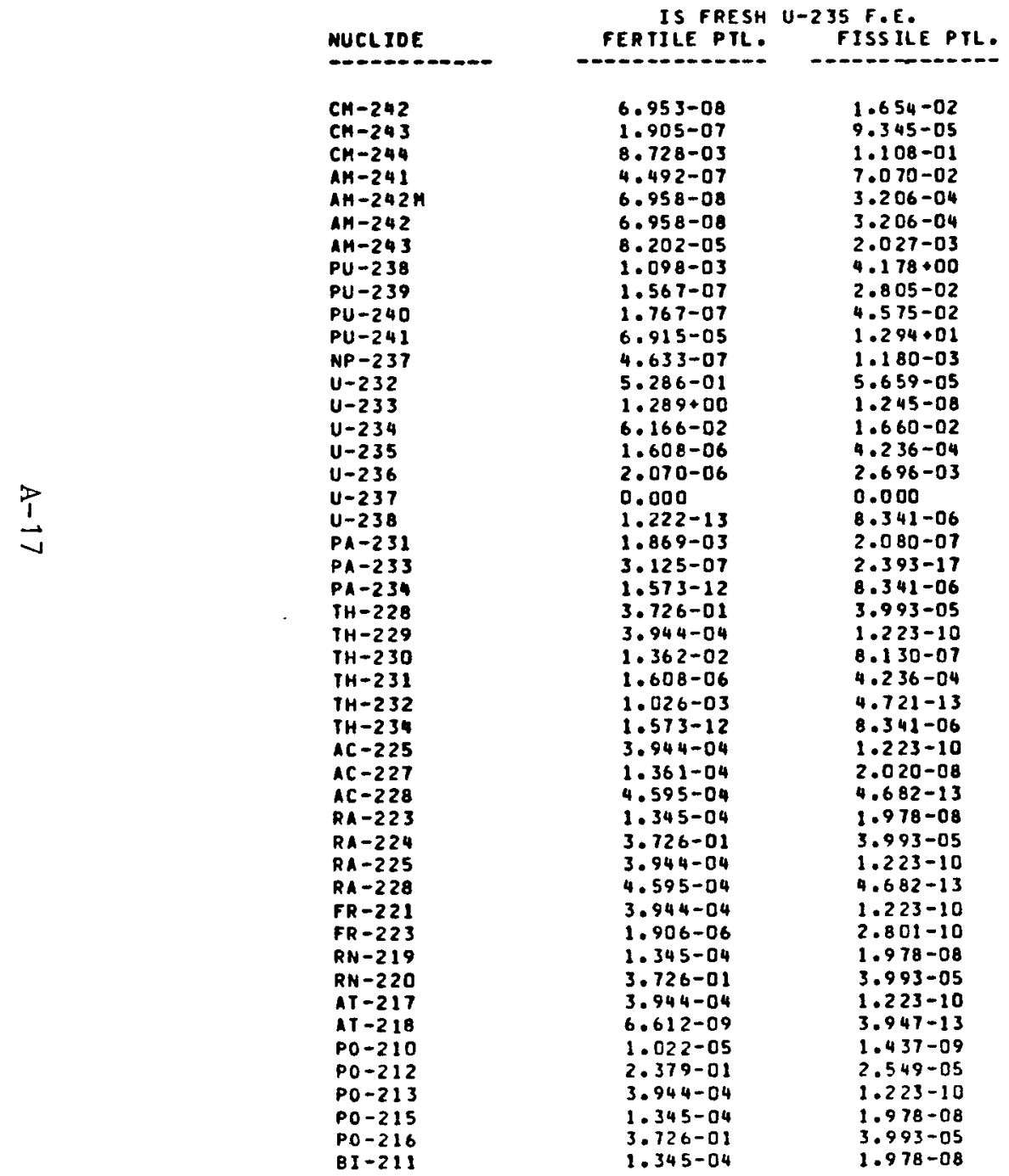

MS FRESH U-235 F.E.
FERTILE PTL. FISSILE PTL.

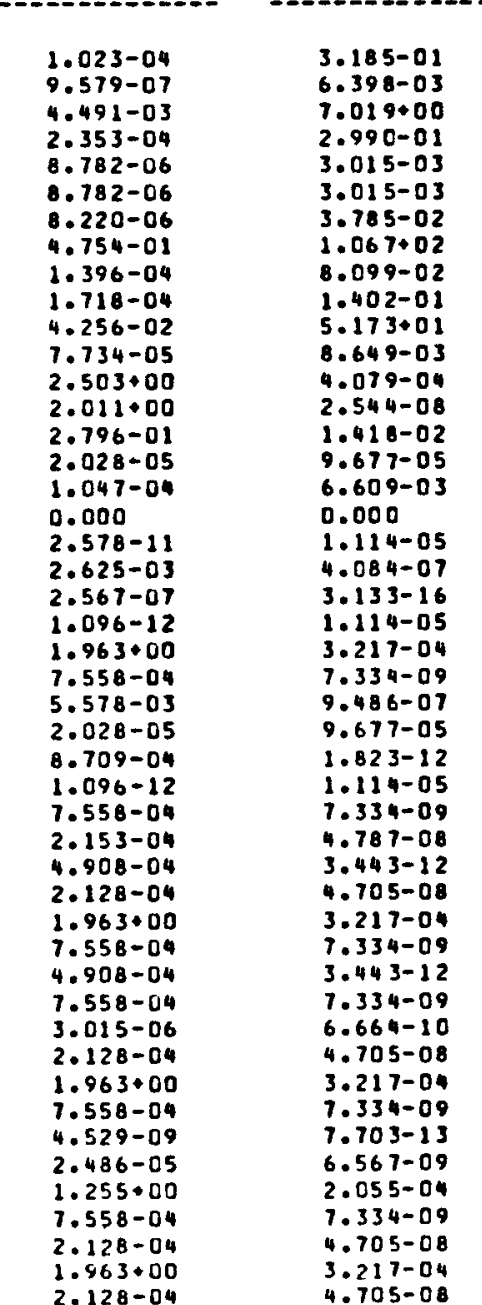




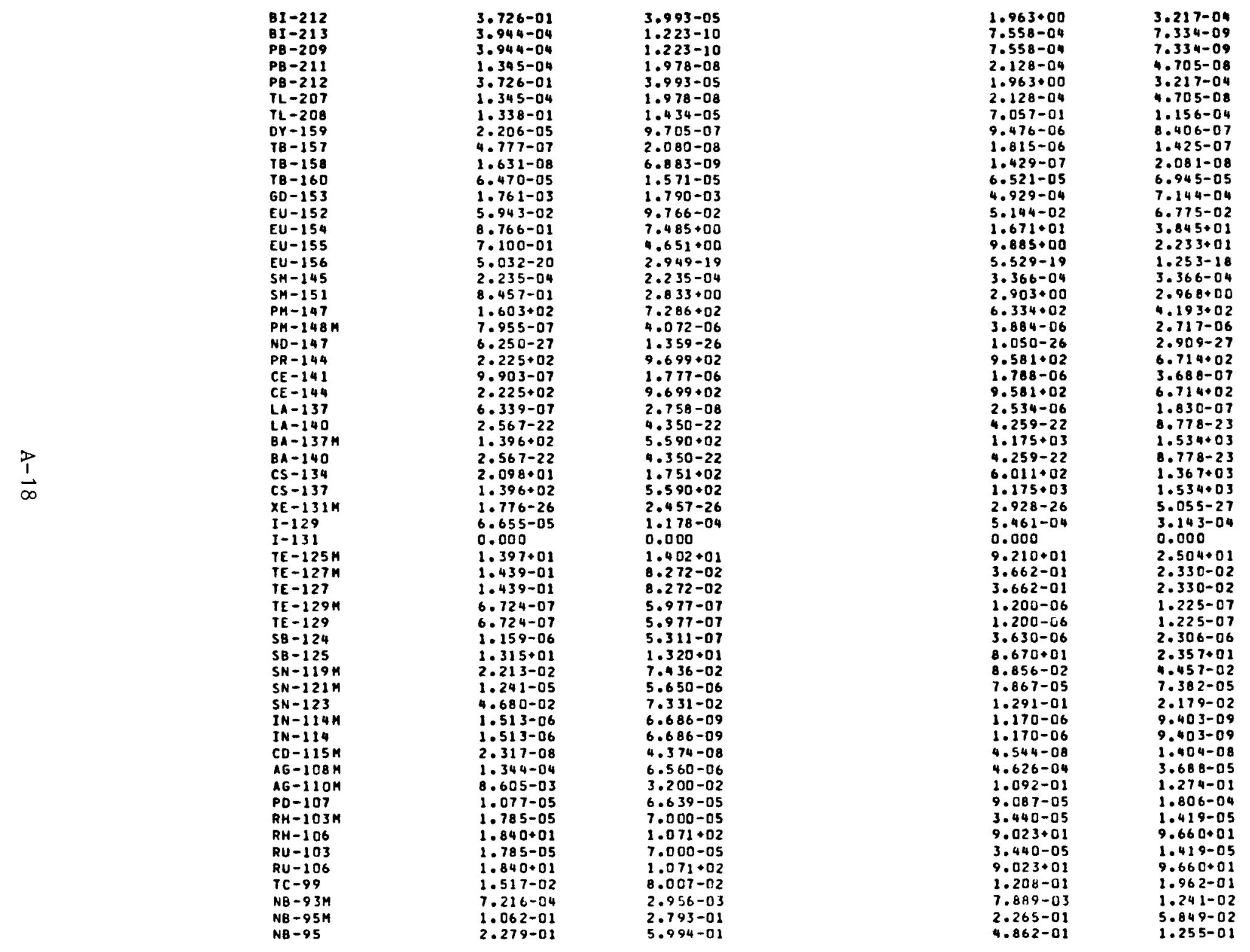




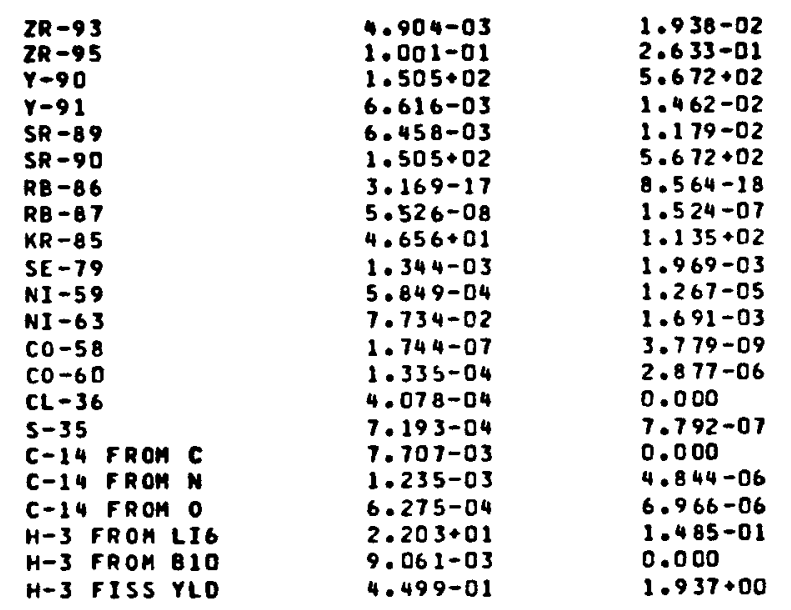

$\begin{array}{ll}4.197-02 & 5.487-02 \\ 2.136-01 & 5.514-02 \\ 1.259+03 & 1.542+03 \\ 1.355-02 & 3.039-03 \\ 1.280-02 & 2.396-03 \\ 1.259+03 & 1.542+03 \\ 7.183-17 & 2.249-17 \\ 4.657-07 & 1.235-07 \\ 2.946+02 & 1.180+02 \\ 8.945-03 & 3.520-03 \\ 2.023-03 & 7.284-05 \\ 2.626-01 & 9.460-03 \\ 1.566-07 & 5.639-09 \\ 3.952-04 & 1.431-05 \\ 1.321-03 & 0.000 \\ 6.639-04 & 1.202-08 \\ 2.708-02 & 0.000 \\ 4.350-03 & 2.900-05 \\ 2.251-03 & 4.078-05 \\ 2.846+01 & 3.186-01 \\ 4.605-02 & 0.000 \\ 3.658+00 & 4.971+00\end{array}$


THIS SEGMENT HAS DISCHARGED AT

THE BEG INNING OF RELOAD I AND WAS

ORIGINALLY LOAOED AT RELLAD O

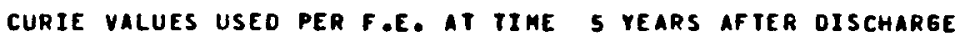

THIS SEGHENT WAS OISCHARGE D AT

THE BEGINNING OF RELOAO 5 AND VAS

ORIGINALIY LOAOED REL REL
NUCLIDE

\section{CK-242}

$\mathrm{CM}-293$

$\mathrm{CH}-244$
$A M-241$

$A M-242 \mathrm{H}$

$A M-242$

$A H-243$
$P U-238$

PU -239

PU -240

$P U-241$

$u-232$

$u-233$

$u-234$
$u-235$

$u-236$

$u-237$

$U-238$

$P A-231$

$P A-233$

-1
$P A-228$

$T H-229$

$T H-230$

$T H-231$

$\mathrm{TH}-232$
$\mathrm{TH}-234$

$T H-234$
$A C-225$

ac -227

AC- 228

$R A-223$

$R A-224$

$R A-22 B$

FR -221

$F R-223$

RN-219

RN -220

AT -218

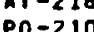

PT
$P O-210$
$P O-212$

$P O-212$

$P O-213$
$P O-215$

$P O-215$
$P O-216$
IS FRESH U-235 F.E.

\begin{tabular}{|c|c|}
\hline $\begin{array}{l}6.890-08 \\
1.824-07 \\
8.078-03 \\
6.589-07 \\
6.890-08 \\
6.890-08 \\
8.201-05 \\
1.081-03 \\
1.567-07 \\
1.767-07 \\
6.305-05 \\
4.633-07 \\
5.185-01 \\
1.289-00 \\
6.166-02 \\
1.608-06 \\
2.070-06 \\
0.000 \\
1.222-13 \\
1.868-03 \\
2.241-15 \\
1.180-21 \\
4.502-01 \\
6.378-04 \\
1.362-02 \\
1.608-06 \\
1.026-03 \\
1.180-21 \\
6.378-04 \\
1.277-04 \\
5.654-04 \\
1.262-04 \\
4.502-01 \\
6.378-04 \\
5.654-04 \\
6.378-04 \\
1.787-06 \\
1.262-04 \\
4.502-01 \\
6.378-04 \\
8.785-09 \\
3.540-00 \\
2.879-01 \\
6.378-04 \\
1.262-04 \\
4.502-01 \\
1.262-04\end{array}$ & $\begin{array}{l}9.959-04 \\
8.948-05 \\
1.026-01 \\
1.100-01 \\
3.177-04 \\
3.177-04 \\
2.027-03 \\
4.113-00 \\
2.805-02 \\
4.574-02 \\
1.180-01 \\
1.180-03 \\
5.551-05 \\
1.245-08 \\
1.660-02 \\
4.236-04 \\
2.696-03 \\
0.000 \\
8.341-06 \\
2.261-07 \\
1.716-25 \\
8.341-06 \\
4.821-05 \\
1.247-10 \\
1.101-06 \\
4.236-04 \\
7.391-13 \\
8.341-06 \\
1.247-10 \\
3.245-08 \\
4.947-13 \\
3.201-08 \\
4.821-05 \\
1.247-10 \\
4.947-113 \\
1.247-10 \\
4.534-10 \\
3.201-08 \\
4.821-05 \\
1.247-10 \\
7.099-13 \\
0.000 \\
3.083-05 \\
1.247-10 \\
3.201-08 \\
4.821-05 \\
3.201-08\end{array}$ \\
\hline
\end{tabular}

HS FRESH U-235 F.E.

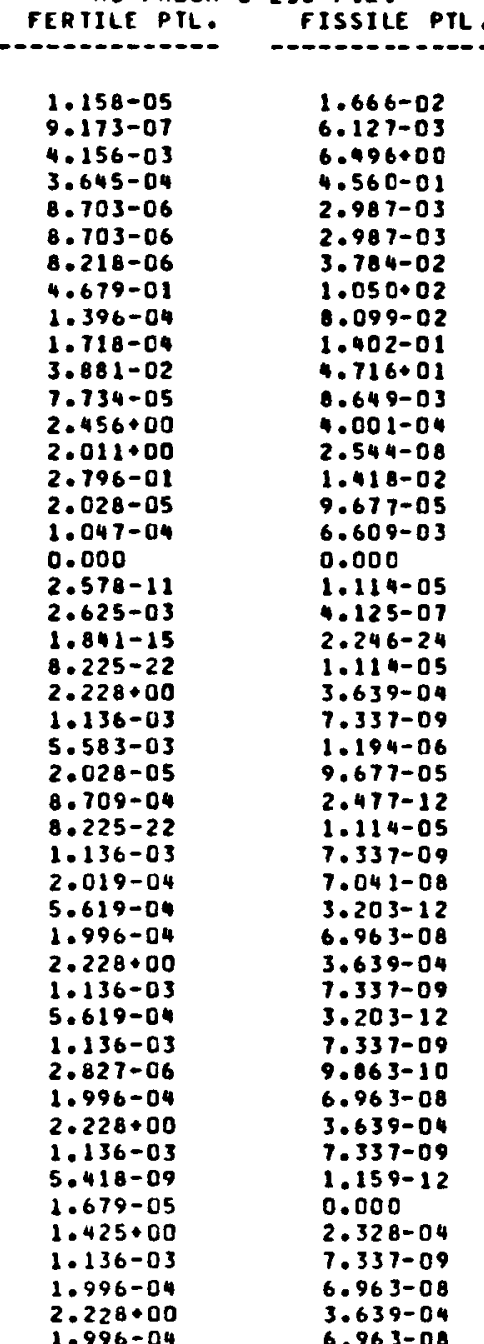




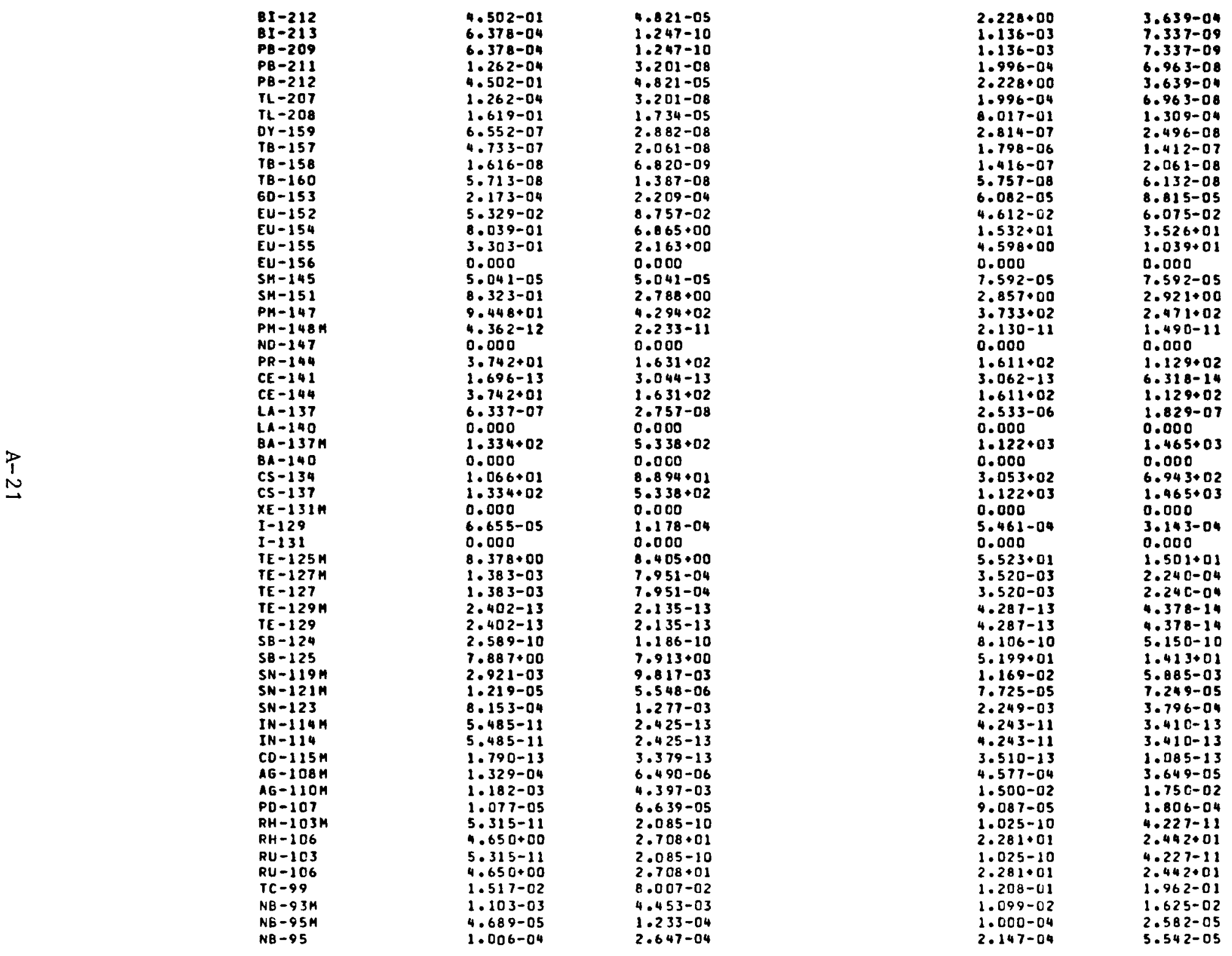




$\begin{array}{lll}2 R-93 & 4.904-03 & 1.938-02 \\ 2 R-95 & 4.420-05 & 1.163-04 \\ Y-90 & 1.431+02 & 5.395+02 \\ Y-91 & 1.071-06 & 2.367-06 \\ S R-89 & 4.347-07 & 7.936-07 \\ S R-90 & 1.431+02 & 5.395+02 \\ R B-86 & 0.000 & 0.000 \\ R B-87 & 5.526-08 & 1.524-07 \\ K R-85 & 4.093+01 & 9.979+01 \\ S E-79 & 1.344-03 & 1.969-03 \\ N I-59 & 5.849-04 & 1.267-05 \\ N I-63 & 7.618-02 & 1.666-03 \\ C O-58 & 1.448-10 & 3.136-12 \\ C O-60 & 1.025-04 & 2.210-06 \\ C L-36 & 4.078-04 & 0.000 \\ S-35 & 2.162-06 & 2.342-09 \\ C-14 \text { FROM C } & 7.705-03 & 0.000 \\ C-14 \text { FROM N } & 1.235-03 & 4.843-06 \\ C-14 \text { FROM O } & 6.274-04 & 6.964-06 \\ H-3 \text { FROH LI6 } & 1.969+01 & 1.327-01 \\ H-3 \text { FROM } 810 & 8.097-03 & 0.000 \\ H-3 \text { FISS YLD } & 4.020-01 & 1.731 .00\end{array}$

$\begin{array}{ll}1.197-02 & 5.487-02 \\ 9.430-05 & 2.435-05 \\ 1.197+03 & 1.467-03 \\ 2.194-06 & 4.918-07 \\ 8.615-07 & 1.613-07 \\ 1.197+03 & 1.467 .03 \\ 0.000 & 0.000 \\ 4.657-07 & 4.235-07 \\ 2.590+02 & 1.591+02 \\ 0.945-03 & 3.520-03 \\ 2.023-03 & 7.284-05 \\ 2.587-01 & 9.318-03 \\ 1.300-10 & 4.681-12 \\ 3.036-04 & 1.099-05 \\ 1.320-03 & 0.000 \\ 1.996-06 & 3.614-09 \\ 2.707-02 & 0.000 \\ 4.349-03 & 2.899-05 \\ 2.250-03 & 4.077-05 \\ 2.543001 & 2.847-01 \\ 4.115-02 & 0.000 \\ 3.269+00 & 4.442000\end{array}$


THIS SEGMENT HAS DISCHARGED AT THE BEGINNING OF RELOAD I ANO YAS ORIGINALLY LOADED AT RELOAD O
THIS SEGMENT MAS DISCHAREED AT THE BEGINNING OF RELOAD 5 AND WAS ORIGINALLY LOADEO AT REL OAD 1

CURIE VALUES USED PER F.e. at TIME 10 VeARs after OISCHARge

\begin{tabular}{|c|c|c|}
\hline RUCLIDE & $\begin{array}{l}\text { IS FRESH } \\
\text { FERTILE PIL }\end{array}$ & $\begin{array}{l}\text { U-235F FE: } \\
\text { FISSILE PTL. }\end{array}$ \\
\hline $\mathrm{CM}-242$ & $6.735-08$ & $3.106-04$ \\
\hline $\begin{array}{l}C M-243 \\
C H-244\end{array}$ & $\begin{array}{l}1.637-07 \\
6.656-03\end{array}$ & $\begin{array}{l}8.030-05 \\
8.453-02\end{array}$ \\
\hline$A M-241$ & $1.102-06$ & $1.931-01$ \\
\hline$A H-242 H$ & $6.735-08$ & $3.106-04$ \\
\hline$A M-242$ & $6.735-08$ & $3.106-04$ \\
\hline$A M-243$ & 8.197-05 & $2.026-03$ \\
\hline$P U-238$ & $1.039-03$ & $3.953+00$ \\
\hline$P U=239$ & $1.567-07$ & $2.804-02$ \\
\hline$P U-240$ & $1.766-07$ & $4.572-02$ \\
\hline$P U-241$ & $5.004-05$ & $9.365+00$ \\
\hline$N P-237$ & $4.633-07$ & $1.180-03$ \\
\hline$u-232$ & $4.942-01$ & $5.290-05$ \\
\hline$U-233$ & $1.289+00$ & $1.245-08$ \\
\hline$u-234$ & $6.166-02$ & $1.660-02$ \\
\hline$u-235$ & $1.608-06$ & $4.236-04$ \\
\hline$u-236$ & $2.070-06$ & $2.696-03$ \\
\hline$u-237$ & 0.000 & 0.000 \\
\hline $\mathrm{U}-238$ & $1.222-13$ & $8.341-06$ \\
\hline$P A-231$ & $1.868-03$ & $2.712-07$ \\
\hline$P A-233$ & 0.000 & 0.000 \\
\hline$P A-234$ & 0.000 & $8.341-06$ \\
\hline$T H-228$ & $4.944-01$ & $5.293-05$ \\
\hline $1 H-229$ & $1.246-03$ & $1.305-10$ \\
\hline$T H-230$ & $1.362-02$ & $1.820-06$ \\
\hline$T H-231$ & $1.608-06$ & $4.236-04$ \\
\hline$T H-232$ & $1.026-03$ & $1.406-12$ \\
\hline$T H-234$ & 0.000 & $8.341-06$ \\
\hline$A C-225$ & $1.246-03$ & $1.305-10$ \\
\hline$A C-227$ & $1.087-04$ & $6.458-08$ \\
\hline$A C-228$ & $7.515-04$ & $7.390-13$ \\
\hline$R A-223$ & $1.075-04$ & $6.412-08$ \\
\hline$R A-224$ & $4.944-01$ & $5.293-05$ \\
\hline$R A-225$ & $1.246-03$ & $1.305-10$ \\
\hline$R A-228$ & $7.515-04$ & $7.390-13$ \\
\hline$F R-221$ & $1.246-03$ & $1.305-10$ \\
\hline$F R-223$ & $1.522-06$ & $9.082-10$ \\
\hline$R N-219$ & $1.075-04$ & $6.412-08$ \\
\hline$R N-220$ & $4.944-01$ & $5.293-05$ \\
\hline$A T-217$ & $1.246-03$ & $1.305-10$ \\
\hline$A T-21 B$ & $1.421-08$ & $1.898-12$ \\
\hline PO-210 & $2.844-06$ & 0.000 \\
\hline PO-212 & $3.164-01$ & $3.387-05$ \\
\hline$P O-213$ & $1.246-03$ & $1.305-10$ \\
\hline$P O-215$ & $1.075-04$ & $6.412-08$ \\
\hline$P O-216$ & $4.944-01$ & $5.293-05$ \\
\hline BI -211 & $1.075-04$ & $6.412-08$ \\
\hline
\end{tabular}
HS FRESH U-235 F.E.
FERTILE PTL. FISSILE PTL.

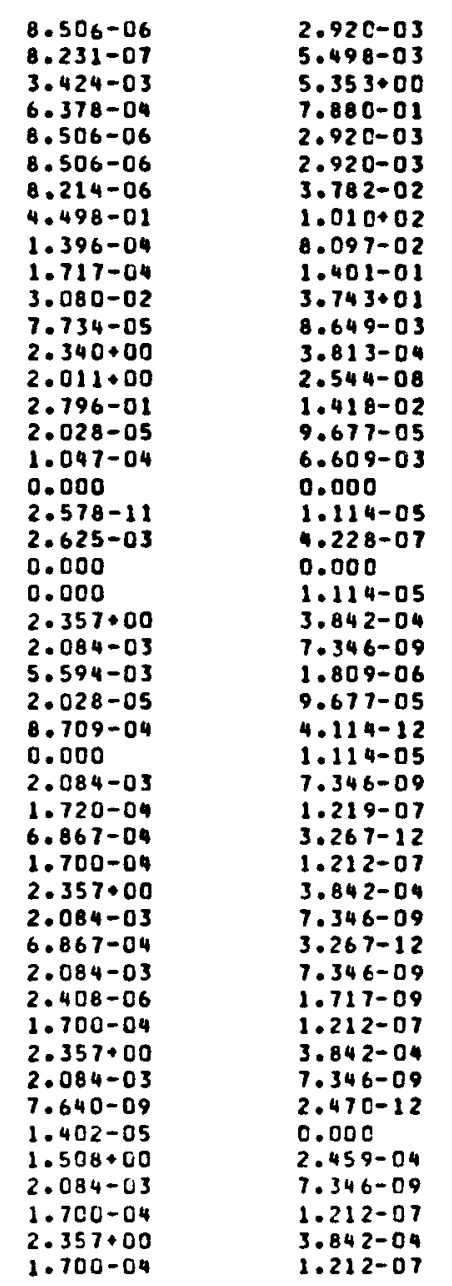




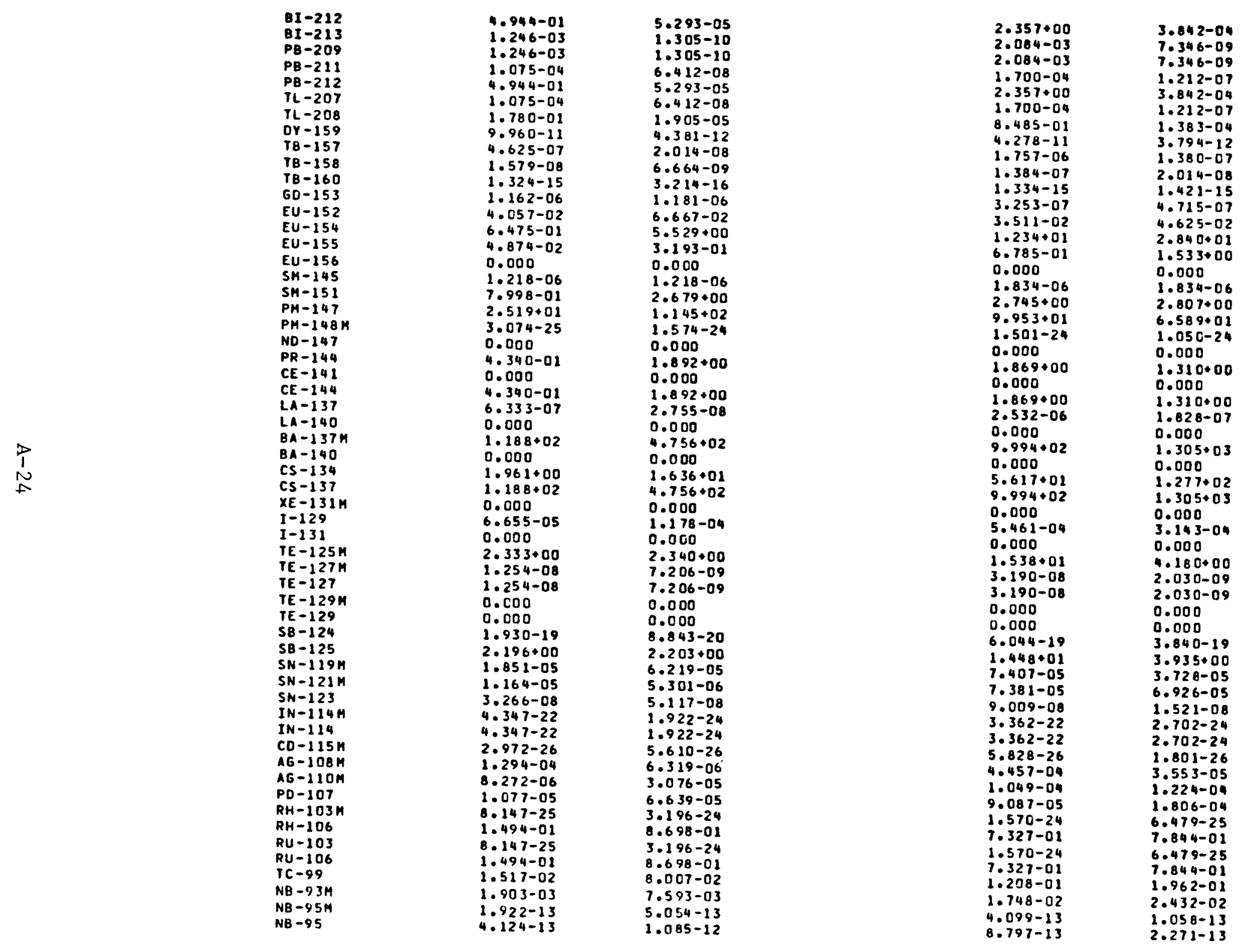




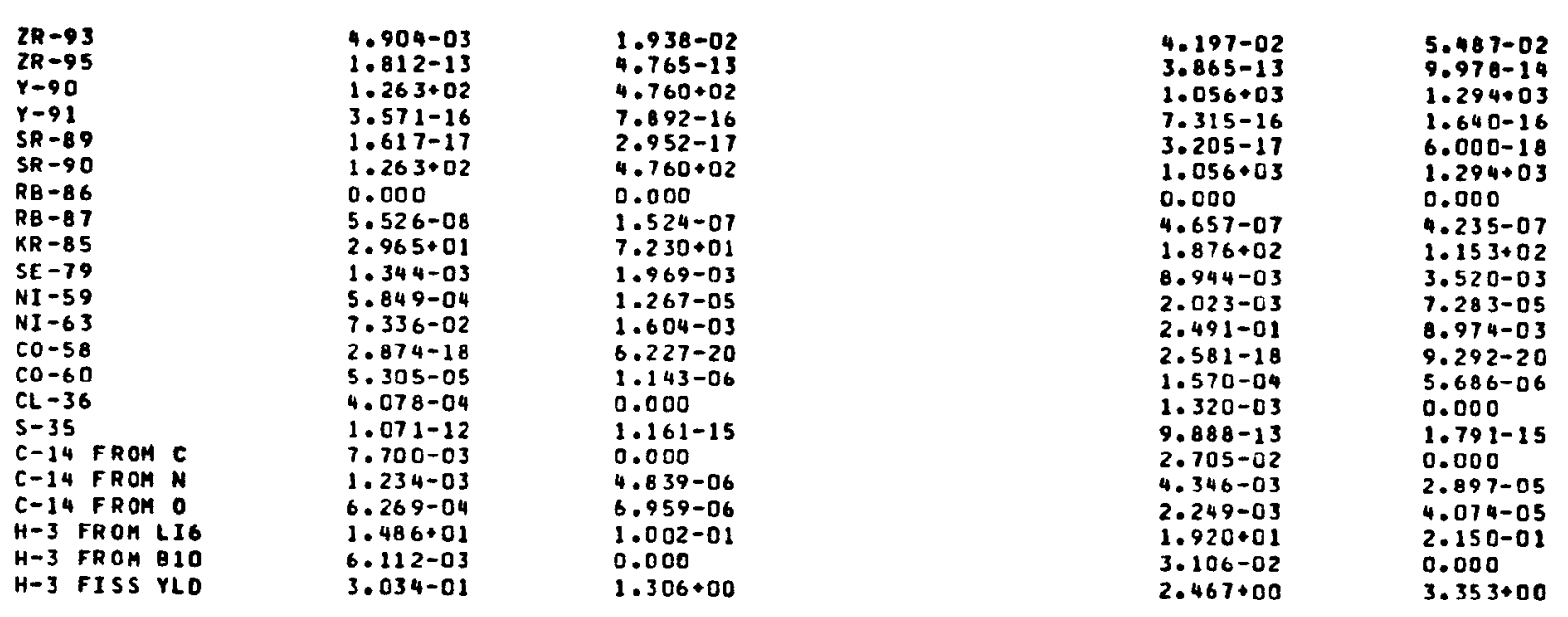


THIS SEGMENT WAS OISCHARGED AT THE BEgINNING OF RELOAO 10 AND WAS ORIGIMALLY LOADED AT RELOAD 6

CURIE VALUES USED PER F.E. AT TIME 180 DAYS AFTER DISCHARGE

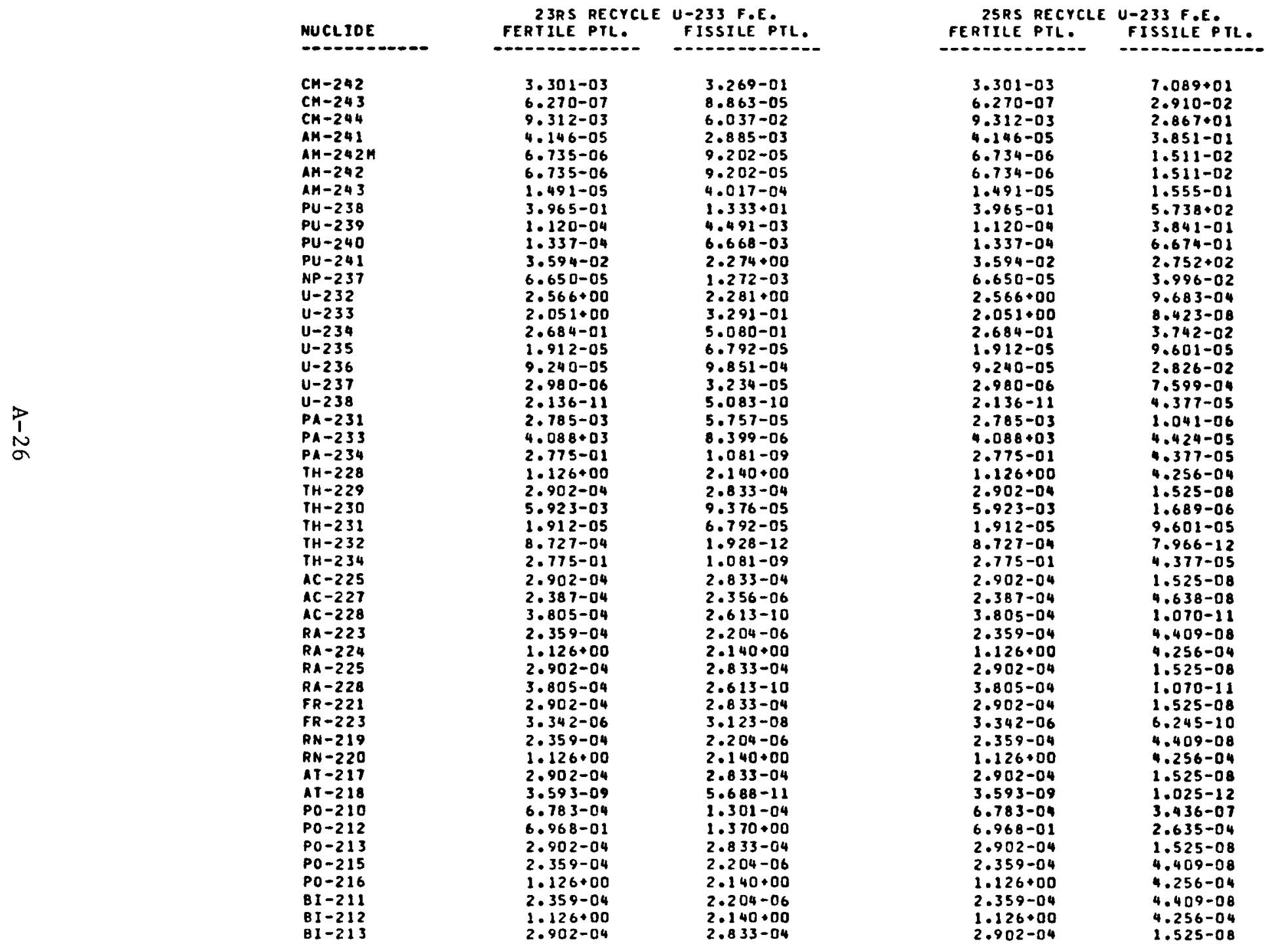




\begin{tabular}{|c|c|c|}
\hline $\begin{array}{l}P B-209 \\
P B-211 \\
P B-212 \\
T L-207 \\
T L-208 \\
D Y-159 \\
T B-157 \\
T B-158 \\
T B-160\end{array}$ & $\begin{array}{l}2.902-04 \\
2.359-04 \\
1.126+00 \\
2.359-04 \\
3.919-01 \\
7.835-04 \\
1.813-06 \\
1.380-07 \\
4.281-01\end{array}$ & $\begin{array}{l}2.833-04 \\
2.204-06 \\
2.140=00 \\
2.204-06 \\
7.704-01 \\
1.028-04 \\
5.856-07 \\
1.958-07 \\
4.528-01\end{array}$ \\
\hline $\begin{array}{l}G D-153 \\
E U-152 \\
E U-154\end{array}$ & $\begin{array}{l}7.321-03 \\
6.106-02 \\
1.777+01\end{array}$ & $\begin{array}{l}1.238-C 2 \\
7.318-02 \\
3.655+01\end{array}$ \\
\hline $\begin{array}{l}E U-155 \\
E U-156 \\
S H-145\end{array}$ & $\begin{array}{l}2.464+01 \\
3.962-01 \\
2.143-03\end{array}$ & $\begin{array}{l}4.967+01 \\
7.996-01 \\
3.164-03\end{array}$ \\
\hline $\begin{array}{l}S M-151 \\
P M-147 \\
P M-148 M\end{array}$ & $\begin{array}{l}2.958+00 \\
1.200+03 \\
1.530+01\end{array}$ & $\begin{array}{l}2.804+00 \\
8.465+02 \\
1.131+01\end{array}$ \\
\hline $\begin{array}{l}\text { ND }-147 \\
P R-144 \\
C E-141\end{array}$ & $\begin{array}{l}8.511-02 \\
8.596+03 \\
5.238+02\end{array}$ & $\begin{array}{l}3.039-02 \\
6.592+03 \\
1.681+02\end{array}$ \\
\hline$C E-144$ & $8.596+03$ & $6.592 \cdot 03$ \\
\hline $\begin{array}{l}1-137 \\
-140\end{array}$ & $2.497-06$ & $\begin{array}{l}1.101-06 \\
4.370-01\end{array}$ \\
\hline $\begin{array}{l}L A-140 \\
B A-137 M\end{array}$ & $\begin{array}{l}1.411+00 \\
1.183+03\end{array}$ & $\begin{array}{l}4.370-01 \\
1.925+03\end{array}$ \\
\hline $\begin{array}{l}8 A-140 \\
C 5-134\end{array}$ & $\begin{array}{l}1.411+00 \\
1.302+03\end{array}$ & $\begin{array}{l}4.370-01 \\
3.083+03\end{array}$ \\
\hline $\begin{array}{l}C S-137 \\
X E-1314\end{array}$ & $\begin{array}{l}1.183+03 \\
6.389-03\end{array}$ & $\begin{array}{l}1.925+03 \\
1.877-03\end{array}$ \\
\hline $1-129$ & $5.200-04$ & $7.940-04$ \\
\hline $1-131$ & $2.506-03$ & $7.316-04$ \\
\hline$T E-125 \mathrm{M}$ & $1.639+02$ & $1.962+02$ \\
\hline TE - $127 \mathrm{M}$ & $1.196+02$ & $3.733+01$ \\
\hline $\begin{array}{l}T E-127 \\
T E-129 M\end{array}$ & $1.196+02$ & $\begin{array}{l}3.733+01 \\
3.633+01\end{array}$ \\
\hline $\begin{array}{l}\text { TE }-129 M \\
T E-129\end{array}$ & $1.400+02$ & $\begin{array}{l}3.633+01 \\
3.633+01\end{array}$ \\
\hline $\begin{array}{l}T E-129 \\
S B-124\end{array}$ & $1.400+02$ & $\begin{array}{l}3.633+01 \\
1.756-01\end{array}$ \\
\hline $\begin{array}{l}5 B-124 \\
S B-125\end{array}$ & $\begin{array}{l}1.292-01 \\
1.573+02\end{array}$ & $\begin{array}{l}1.756-01 \\
1.853+02\end{array}$ \\
\hline SN-119N & $1.078+00$ & $7.273-01$ \\
\hline$S N-121 \mathrm{~K}$ & $7.661-05$ & $8.974-05$ \\
\hline$S N-123$ & $1.997+01$ & $7.618+00$ \\
\hline$I N-114 \mathrm{M}$ & $4.225-01$ & $5.015-03$ \\
\hline $1 N-114$ & $4.225-01$ & $5.015-03$ \\
\hline$C D-115 N$ & $1.123-01$ & $4.905-02$ \\
\hline$A G-108 M$ & $4.530-04$ & $5.333-05$ \\
\hline $16-110 M$ & $1.234+00$ & $2.039+00$ \\
\hline$P D-107$ & $8.640-05$ & $1.469-04$ \\
\hline$R H-103 M$ & $2.799+02$ & $1.224+02$ \\
\hline RH-106 & 4.846 .02 & $4.585+02$ \\
\hline$R U-103$ & $2.799+02$ & $1.224+02$ \\
\hline$R U-106$ & 4.846002 & $4.585+02$ \\
\hline TC -99 & $1.153-01$ & $1.820-01$ \\
\hline$N B-93 H$ & $3.326-03$ & $8.376-03$ \\
\hline NB $-95 M$ & 3.510 .03 & 1.269003 \\
\hline NB - 95 & $6.697+03$ & 2.446 .03 \\
\hline $2 R-93$ & $3.990-02$ & $6.593-02$ \\
\hline & 3.309 .03 & \\
\hline
\end{tabular}

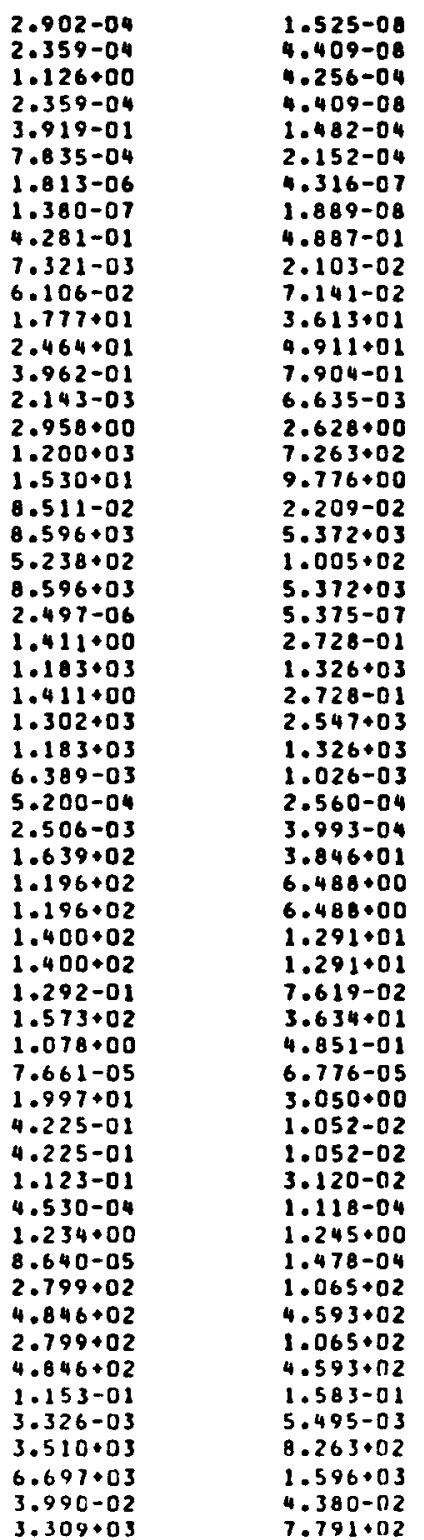




$\begin{array}{lll}Y-90 & 1.275+03 & 2.053+03 \\ Y-91 & 7.391+02 & 2.522+02 \\ S R-89 & 2.097+03 & 6.638+02 \\ S R-90 & 1.275+03 & 2.053+03 \\ R B-86 & 4.050-02 & 2.711-02 \\ R B-87 & 4.433-07 & 7.064-07 \\ K R-85 & 3.353+02 & 3.876+02 \\ S E-79 & 8.664-03 & 1.038-02 \\ N I-59 & 1.893-03 & 1.006-04 \\ N I-63 & 2.506-01 & 1.333-02 \\ C O-58 & 1.101-03 & 5.852-05 \\ C O-60 & 5.154-04 & 2.756-05 \\ C L-36 & 1.243-03 & 0.000 \\ \text { S-35 } & 9.093-01 & 2.432-03 \\ C-14 \text { FROM C } & 2.530-02 & 0.000 \\ C-14 \text { FROH N } & 4.079-03 & 4.016-05 \\ C-14 \text { FROH O } & 2.091-03 & 5.629-05 \\ H-3 \text { FROM LI6 } & 3.269+01 & 5.406-01 \\ H-3 \text { FROM B1D } & 6.380-02 & 0.000 \\ H-3 \text { FISS YLD } & 4.006+00 & 6.335+00\end{array}$

$\begin{array}{ll}1.275+03 & 1.311+03 \\ 7.391+02 & 1.504+02 \\ 2.097+03 & 3.554+02 \\ 1.275+03 & 1.311+03 \\ 4.050-02 & 9.977-03 \\ 4.433-07 & 3.380-07 \\ 3.353+02 & 1.767+02 \\ 8.664-03 & 2.899-03 \\ 1.893-03 & 2.110-04 \\ 2.506-01 & 2.796-02 \\ 1.101-03 & 1.227-04 \\ 5.154-04 & 5.779-05 \\ 1.243-03 & 0.000 \\ 9.093-01 & 5.100-03 \\ 2.530-02 & 0.000 \\ 4.079-03 & 8.421-05 \\ 2.091-03 & 1.180-04 \\ 3.269+01 & 1.133+00 \\ 6.380-02 & 0.000 \\ 4.006+00 & 4.580+00\end{array}$


THIS SEGMENT YAS OISCHARGED AT THE BEgINNING OF RELOAO 10 AHD WAS ORIGIMALLY LOAOED AT RELOAO Curie values used per f.e. at time 1 year after discharge

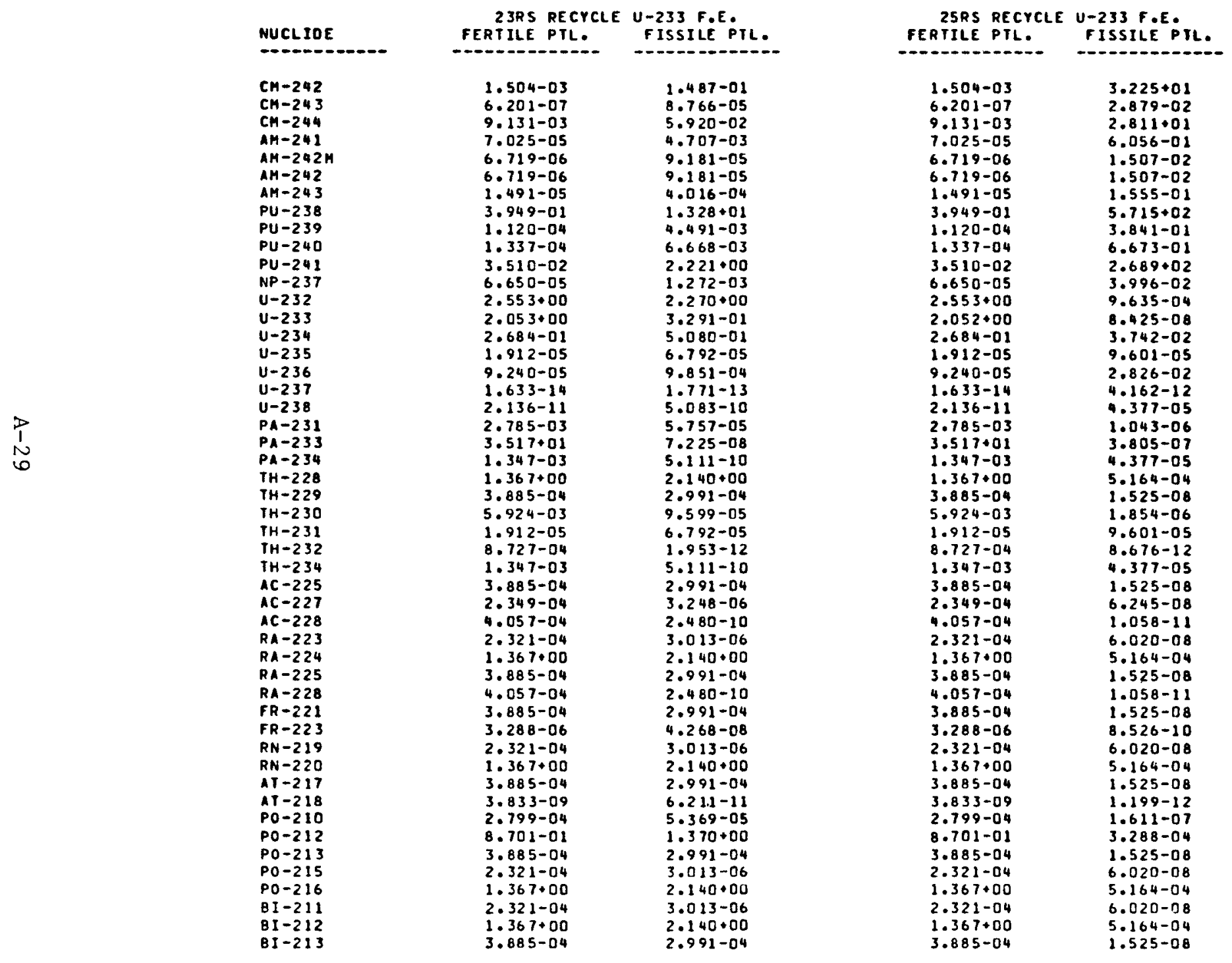




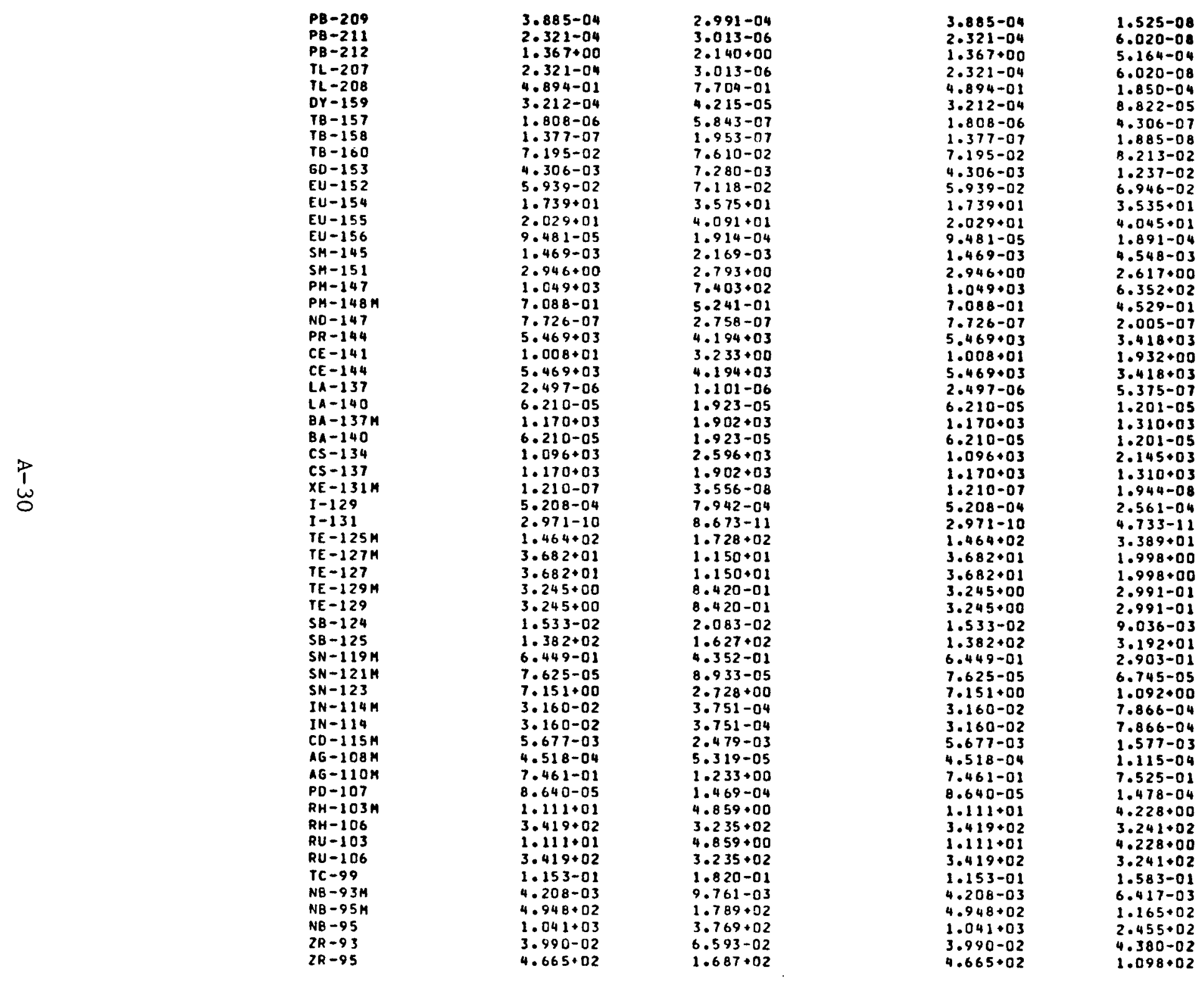




$\begin{array}{ll}Y-90 & 1.259+03 \\ Y-91 & 8.079+01 \\ S R-89 & 1.835+02 \\ S R-90 & 1.259+03 \\ R B-86 & 4.154-05 \\ R B-87 & 4.433-07 \\ K R-85 & 3.246+02 \\ S E-79 & 8.664-03 \\ N I-59 & 1.893-03 \\ N I-63 & 2.496-01 \\ C O-58 & 1.821-04 \\ C O-60 & 4.821-04 \\ C L-36 & 1.243-03 \\ S-35 & 2.085-01 \\ C-14 \text { FROM C } & 2.530-02 \\ C-14 \text { FROM N } & 4.078-03 \\ C-14 \text { FROH O } & 2.090-03 \\ H-3 \text { FROM LI6 } & 3.177+01 \\ H-3 \text { FROH B1O } & 6.201-02 \\ H-3 \text { FISS YLD } & 3.894+00 \\ & \end{array}$

$2.027+03$
$2.756+01$
$5.809+01$
$2.027+03$
$2.781-05$
$7.064-07$
$3.751+02$
$1.038-02$
$1.006-04$
$1.328-02$
$9.683-06$
$2.578-05$
0.000
$5.577-04$
0.000
$4.016-05$
$5.628-05$
$5.254-01$
0.000
$6.156+00$

$1.295 \cdot 03$

$8.079+01 \quad 1.644001$

$\begin{array}{ll}3.110+01 \\ 1.259+03 & 1.295 .03\end{array}$

$4.154-05 \quad 1.295003$

$4.433-07 \quad 3.380-07$

$3.246+02 \quad 1.710 .02$

8.664-03 2.899-03

$1.893-03 \quad 2.110-04$

$2.496-01 \quad 2.785-02$

$1.821-04 \quad 2.030-05$

$4.821-04 \quad 5.405-05$

$1.243-03 \quad 0.000$

2.085-01 1.169-03

$2.530-02 \quad 0.000$

$4.078-03 \quad 8.420-05$

$2.090-03 \quad 1.180-04$

3.177 .01

$6.201-02$

$1.102 \cdot 00$

0.000 
THIS SEGMENT WAS DISCHARGEO AT THE BEgINNING OF RELOAO 10 AND WAS ORIGINALLY LOADED AT RELOAD

CURIE VALUES USED PER F.e. AT TIHE 3 YeARS AFTER OISCharge

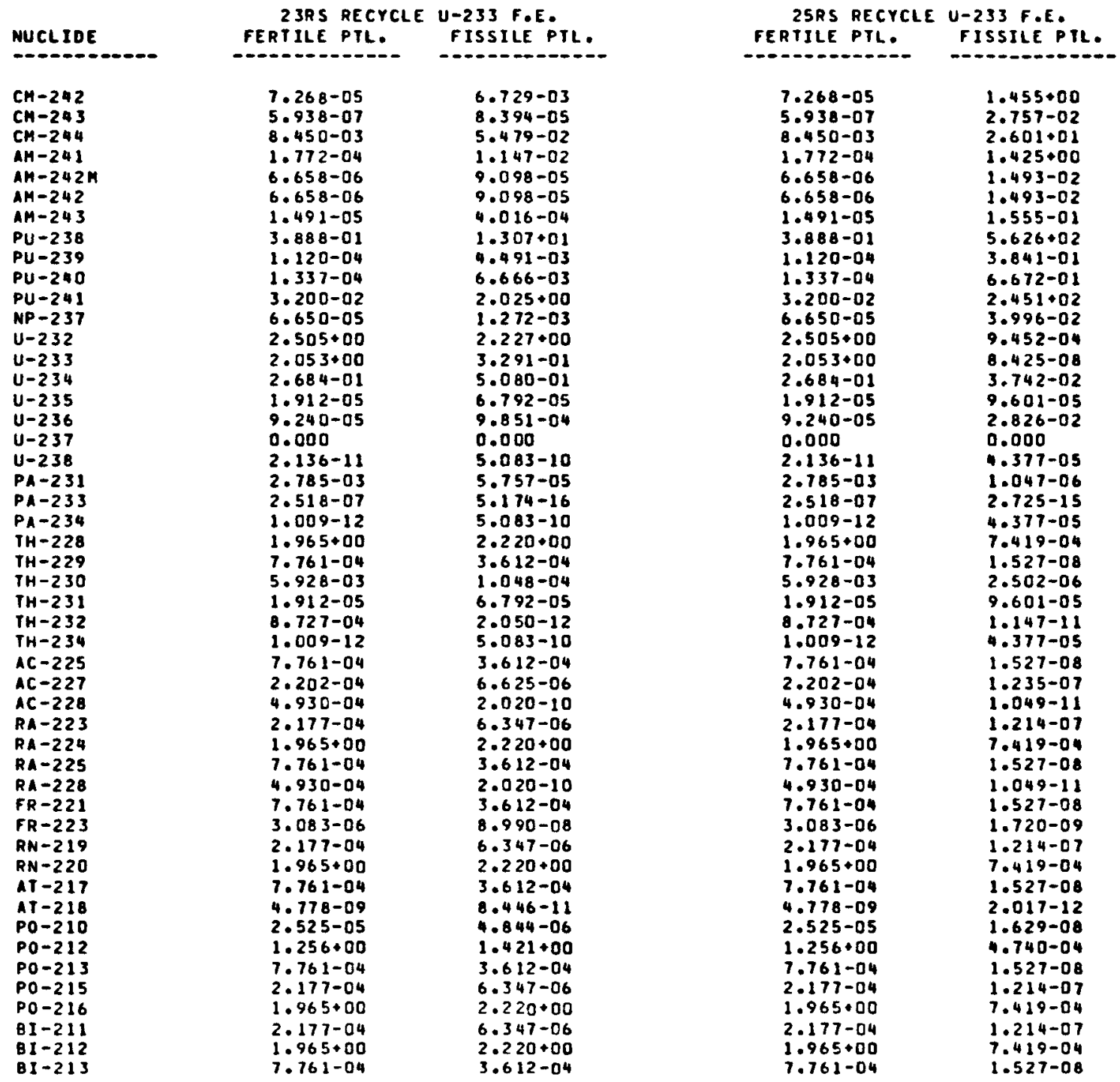




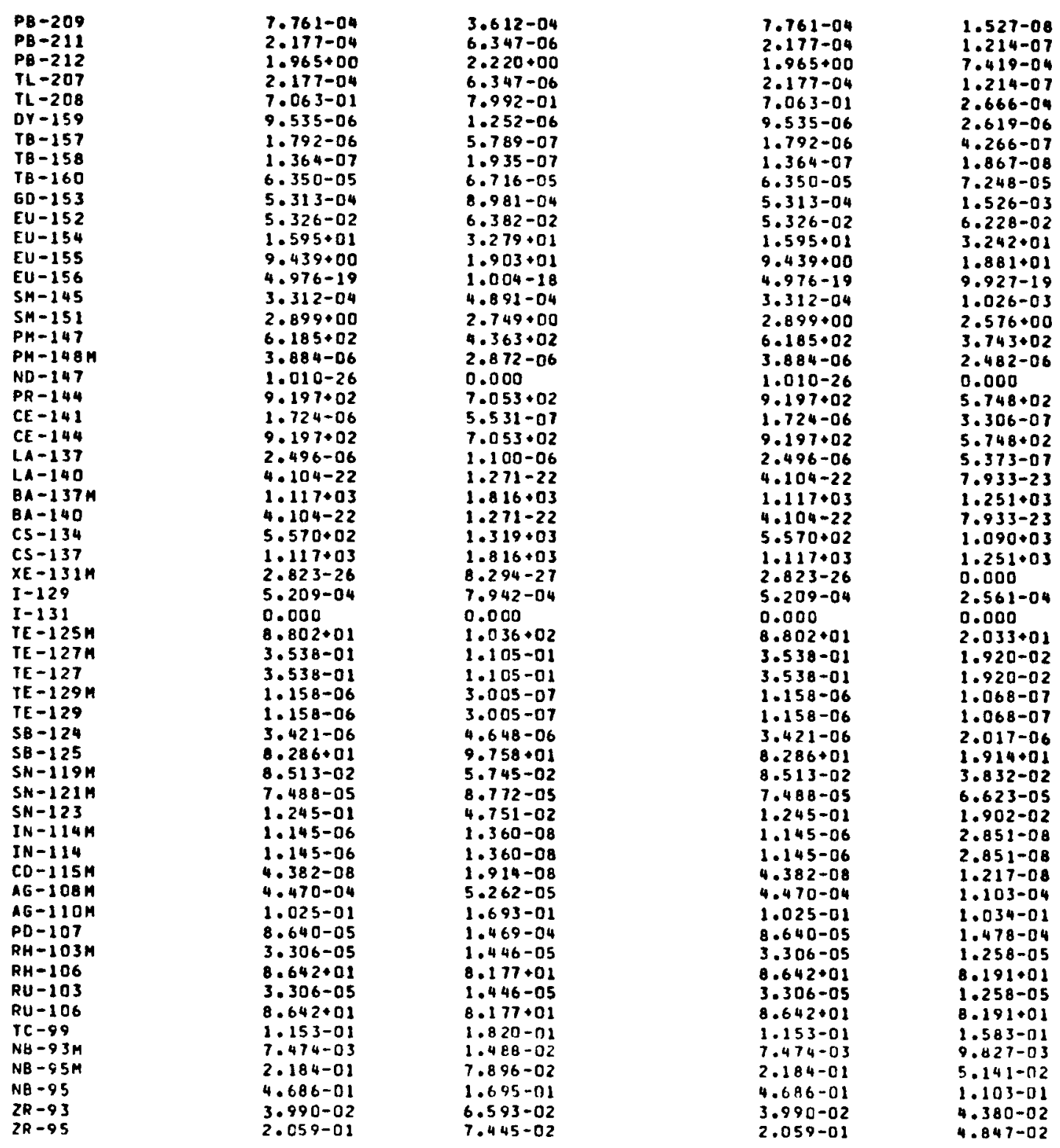




\begin{tabular}{|c|c|c|c|}
\hline $\begin{array}{l}r-90 \\
y-91\end{array}$ & & $\begin{array}{l}1.197+03 \\
1.307-02\end{array}$ & $\begin{array}{l}1.928+03 \\
4.459-03\end{array}$ \\
\hline$S R-89$ & & $1.234-02$ & $3.908-03$ \\
\hline$S R-90$ & & $1.197+03$ & $1.928+03$ \\
\hline$R B-86$ & & $6.778-17$ & $4.537-17$ \\
\hline$R B-87$ & & $4.433-07$ & $7.064-07$ \\
\hline$K R-85$ & & $2.853+02$ & $3.297+02$ \\
\hline$S E-79$ & & $8.664-03$ & $1.037-02$ \\
\hline NI -59 & & $1.893-03$ & $1.006-04$ \\
\hline$N 1-63$ & & $2.459-01$ & $1.308-02$ \\
\hline$c 0-58$ & & $1.511-07$ & $8.033-09$ \\
\hline$c 0-60$ & & $3.703-04$ & $1.980-05$ \\
\hline $\mathrm{CL}-36$ & & $1.243-03$ & 0.000 \\
\hline$s-35$ & & $6.265-04$ & $1.676-06$ \\
\hline$c-14$ & FROH C & $2.529-02$ & 0.000 \\
\hline$c-14$ & FROM N & $4.077-03$ & $4.015-05$ \\
\hline$c-14$ & FROH O & $2.090-03$ & $5.627-05$ \\
\hline$H-3 F$ & FROH LI6 & $2.839+01$ & $4.695-01$ \\
\hline $\begin{array}{ll}H-3 & F \\
H-3 & F\end{array}$ & $\begin{array}{l}\text { FROM BIO } \\
\text { FISS YLO }\end{array}$ & $\begin{array}{l}5.541-02 \\
3.479+00\end{array}$ & $\begin{array}{l}0.000 \\
5.502+00\end{array}$ \\
\hline
\end{tabular}

$\begin{array}{ll}1.197-03 & 1.231+03 \\ 1.307-02 & 2.659-03 \\ 1.234-02 & 2.092-03 \\ 1.197-03 & 1.231003 \\ 6.778-17 & 1.670-17 \\ 4.433-07 & 3.380-07 \\ 2.853+02 & 1.503+02 \\ 8.664-03 & 2.898-03 \\ 1.893-03 & 2.110-04 \\ 2.459-01 & 2.743-02 \\ 1.511-07 & 1.684-08 \\ 3.703-04 & 4.152-05 \\ 1.243-03 & 0.000 \\ 6.265-04 & 3.514-06 \\ 2.529-02 & 0.000 \\ 4.077-03 & 8.418-05 \\ 2.090-03 & 1.180-04 \\ 2.839+01 & 9.844-01 \\ 5.541-02 & 0.000 \\ 3.479000 & 3.978+00\end{array}$


THIS SEgMENT WAS OISCHARgED AT THE BEgINNING OF RELOAD 10 anO VAS ORIGIMALLY LOADED AT RELOAD 6

CURIE VALUES USED PER F.e. AT TIME 5 years aFter discharge

\begin{tabular}{|c|c|c|c|c|}
\hline $\begin{array}{l}\text { NUCLIDE } \\
\text { NUES }\end{array}$ & $\begin{array}{l}\text { 23RS RECYCLE } \\
\text { FERTILE PTL. }\end{array}$ & $\begin{array}{l}\text { U-233 F.E. } \\
\text { FISSILE PIL. }\end{array}$ & $\begin{array}{l}\text { 25RS RECYCLE } \\
\text { FERTILE PTL. }\end{array}$ & $\begin{array}{l}\text { U-233 FEE. } \\
\text { FISSILE PIL. }\end{array}$ \\
\hline $\begin{array}{l}\mathrm{CH}-242 \\
\mathrm{CH}-243\end{array}$ & $\begin{array}{l}8.561-06 \\
5.686-07\end{array}$ & $\begin{array}{l}3.737-04 \\
8.038-05\end{array}$ & $\begin{array}{l}8.561-06 \\
5.686-07\end{array}$ & $\begin{array}{l}7.705-02 \\
2.640-02\end{array}$ \\
\hline$C H-244$ & $7.820-03$ & $\begin{array}{l}8.070-02 \\
5.070-02\end{array}$ & $7.820-03$ & $2.408+01$ \\
\hline$A M-241$ & $2.744-04$ & $1.762-02$ & $2.744=04$ & $2.169+00$ \\
\hline$M M-242 M$ & $6.597-06$ & $9.015-05$ & $6.597-06$ & $1.480-02$ \\
\hline$A M-242$ & $6.597-06$ & $9.015-05$ & $6.597-06$ & $1.480-02$ \\
\hline$A H-243$ & $1.491-05$ & $4.015-04$ & $1.491-05$ & $1.554-01$ \\
\hline$P U-238$ & $3.827-01$ & $1.287+01$ & $3.827-01$ & $5.538 \times 02$ \\
\hline$P U-239$ & $1.120-04$ & $4.490-03$ & $1.120-04$ & $3.841-01$ \\
\hline$P U-240$ & $1.337-04$ & $6.665-03$ & $1.337-04$ & $6.670-08$ \\
\hline$P U-241$ & $2.918-02$ & $1.846+00$ & $2.918-02$ & $2.235+02$ \\
\hline NP -237 & $6.650-05$ & $1.272-03$ & $6.650-05$ & $3.996-02$ \\
\hline$v-232$ & $2.457+00$ & $2.184+00$ & $2.457+00$ & $9.271-0.4$ \\
\hline$u-233$ & $2.052+00$ & $3.291-01$ & $2.052+00$ & $8.425-08$ \\
\hline$u-234$ & $2.684-01$ & $5.079-01$ & $2.684-01$ & $3.742-02$ \\
\hline$u-235$ & $1.912-05$ & $6.792-05$ & $1.912-05$ & $9.601-05$ \\
\hline$u-236$ & $9.240-05$ & $9.851-04$ & $9.240-05$ & $2.826-02$ \\
\hline$u-237$ & 0.000 & 0.000 & 0.000 & 0.000 \\
\hline$v-238$ & $2.136-11$ & $5.083-10$ & $2.136-11$ & $4.377-05$ \\
\hline$P A-231$ & $2.785-03$ & $5.757-05$ & $2.785-03$ & $1.051-06$ \\
\hline$P A-233$ & $1.805-15$ & $3.709-24$ & $1.805-15$ & $1.954-23$ \\
\hline$P A-234$ & $7.572-22$ & $5.083-10$ & $7.572-22$ & $4.377-05$ \\
\hline$T H-228$ & $2.230+00$ & $2.220 \cdot 00$ & $2.230+00$ & $8.416-04$ \\
\hline TH-229 & $1.163-03$ & $4.233-04$ & $1.163-03$ & $1.528-08$ \\
\hline$T H-230$ & $5.933-03$ & $1.136-04$ & $5.933-03$ & $3.150-06$ \\
\hline$T H-231$ & $1.912-05$ & $6.792-05$ & $1.912-05$ & $9.601-05$ \\
\hline$T H-232$ & $8.727-04$ & $2.148-12$ & $8.727-04$ & $1.427-11$ \\
\hline $\mathrm{TH}-234$ & $7.572-22$ & $5.083-10$ & $7.572-22$ & $4.377-05$ \\
\hline$A C-225$ & $1.163-03$ & $4.233-04$ & $1.163-03$ & $1.528-08$ \\
\hline$A C-227$ & $2.066-04$ & $9.792-06$ & $2.066-04$ & $1.810-07$ \\
\hline$A C-228$ & $5.640-04$ & $1.647-10$ & $5.640-04$ & $1.095-11$ \\
\hline$R A-223$ & $2.042-04$ & $9.683-06$ & $2.042-04$ & $1.790-07$ \\
\hline$R A-224$ & $2.230+00$ & $2.220+00$ & $2.230+00$ & $8.416-04$ \\
\hline$R A-225$ & $1.163-03$ & $4.233-0.4$ & $1.163-03$ & $1.528-08$ \\
\hline$R A-228$ & $5.640-04$ & $1.647-10$ & $5.640-04$ & $1.095-11$ \\
\hline$F R-221$ & $1.163-03$ & $4.233-04$ & $1.163-03$ & $1.528-08$ \\
\hline$F R-223$ & $2.892-06$ & $1.371-07$ & $2.892-06$ & $2.536-09$ \\
\hline$R N-219$ & $2.042-04$ & $9.683-06$ & $2.042-04$ & $1.790-07$ \\
\hline$R N-220$ & $2.230+00$ & $2.220+00$ & $2.230+00$ & $8.416-04$ \\
\hline AT -217 & $1.163-03$ & $4.233-04$ & $1.163-03$ & $1.528-08$ \\
\hline$A T-218$ & $5.723-09$ & $1.096-10$ & $5.723-09$ & $3.039-12$ \\
\hline$P O-210$ & $1.752-05$ & 0.000 & $1.752-05$ & 0.000 \\
\hline$P O-212$ & $1.426+00$ & $1.421+00$ & $1.426+00$ & $5.383-04$ \\
\hline$P O-213$ & $1.263-03$ & $4.233-04$ & $1.163-03$ & $1.528-08$ \\
\hline$P O-215$ & $2.042-04$ & $9.683-06$ & $2.042-04$ & $1.790-01$ \\
\hline$P O-216$ & $2.230+00$ & $2.220+00$ & $2.230+00$ & $8.416-04$ \\
\hline $81-211$ & $2.042-04$ & $9.683-06$ & $2.042-04$ & $1.790-07$ \\
\hline BI -212 & & $2.220+00$ & $2.230+00$ & $8.416-0.4$ \\
\hline$B I-213$ & $1.163-03$ & $4.233-04$ & $1.163-03$ & $1.528-08$ \\
\hline
\end{tabular}




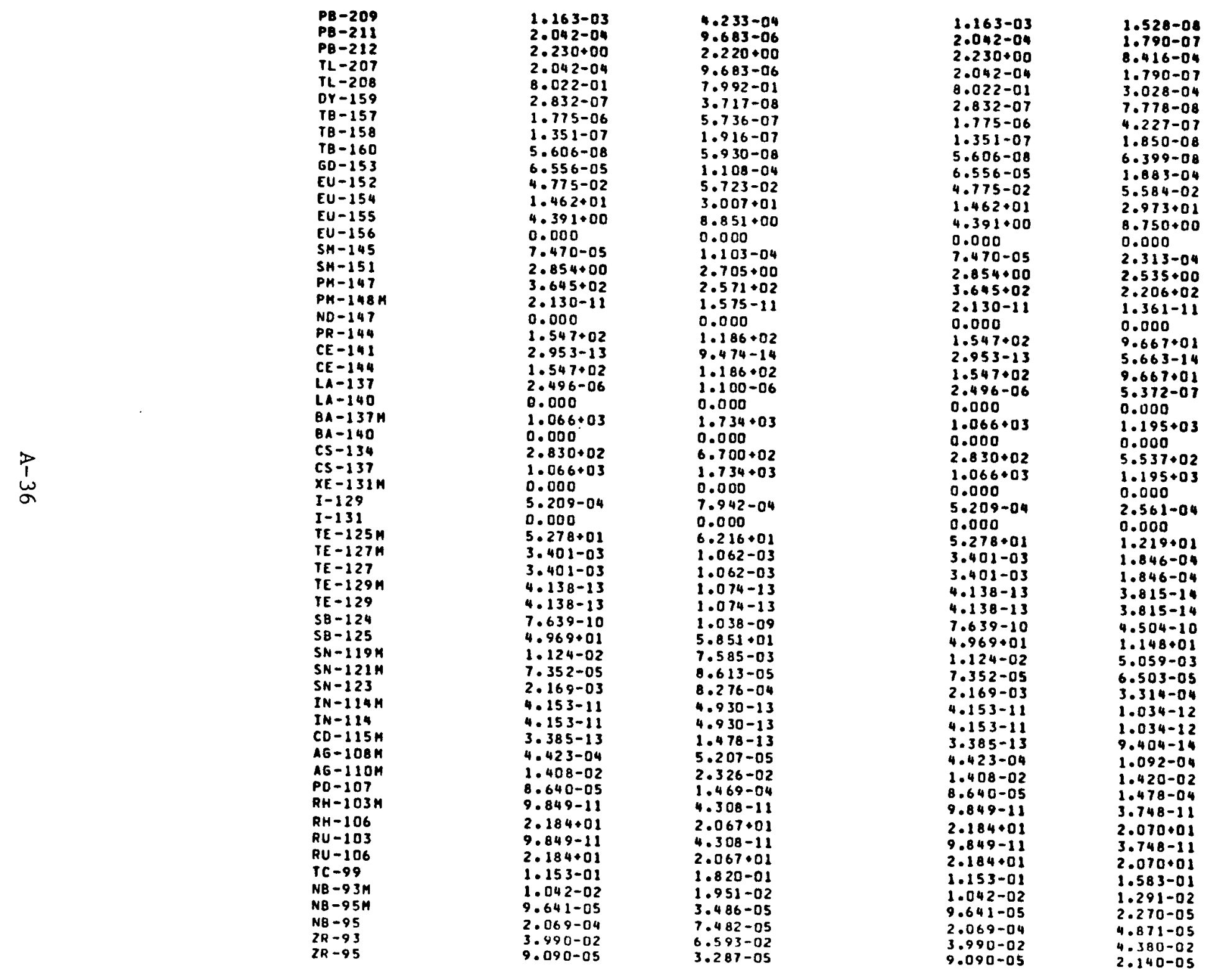




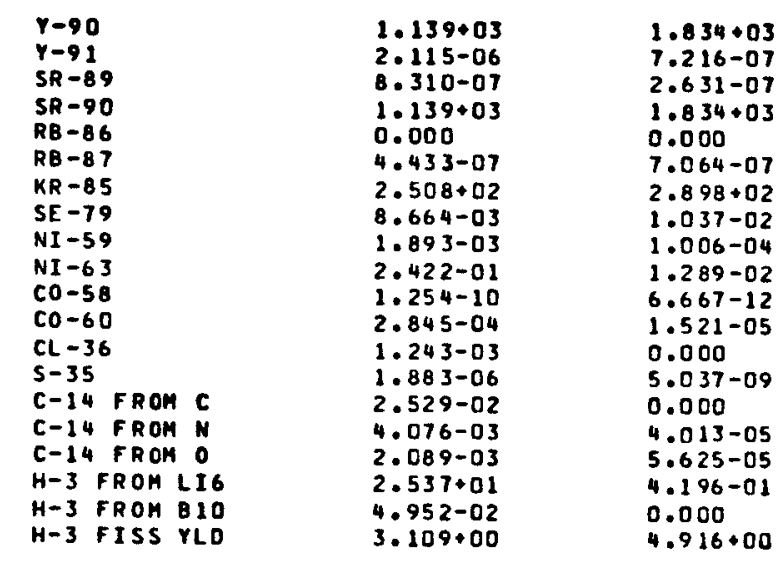

$\begin{array}{ll}1.139+03 & 1.171003 \\ 2.115-06 & 1.304-07 \\ 0.310-07 & 1.408-07 \\ 1.139+03 & 1.171+03 \\ 0.000 & 0.000 \\ 4.433-07 & 3.380-07 \\ 2.508+02 & 1.321+02 \\ 8.664-03 & 2.898-03 \\ 1.893-03 & 2.110-04 \\ 2.422-01 & 2.702-02 \\ 1.254-10 & 1.398-11 \\ 2.845-04 & 3.190-05 \\ 1.243-03 & 0.000 \\ 1.883-06 & 1.056-08 \\ 2.529-02 & 0.000 \\ 4.076-03 & 8.415-05 \\ 2.089-03 & 1.179-04 \\ 2.537001 & 8.797-01 \\ 4.952-02 & 0.000 \\ 3.109+00 & 3.555+00\end{array}$


THIS SEGHENT WAS OISCHARGE AT THE BEgINNING OF RELOAO 10 aND WAS ORIGINALLY LOADE AT RELOAD 6 CURIE VALUES USE PER F.E. AT TIME 10 YeARS AFter OISCHARGE

NUCLIDE

CK -242

$\mathrm{CM}-243$

$\mathrm{CH}-244$

$A M-241$
$A M-242 M$

$A M-242$

$A H-243$
$P U-238$

$P U-2438$
$P U-239$

$P U-239$
$P U-240$

$P U-241$
NP -237

NP -237

$u-232$

$0-233$

$u-235$

$u-236$
$U-237$
$U-238$

$U-238$

$P A-231$
$P A-233$

$P A-234$

TH-228

$T H-229$

TH-230

$T H-231$

$\mathrm{TH}-231$
$\mathrm{TH}-234$

AC -225

$A C-225$
$A C-227$

AC -228

$A C-228$

$A A-223$
$R A-224$
$R A-225$

$R A-223$
$R A-224$
$R A-225$

$R A-228$

$R R-221$
$F R-223$

$F R-223$
$R M-219$

$R N-219$
$R N-220$

AT -217

AT-218

PO- 210

$P O-212$

$P O-213$

$P O-215$

PO- 216

$8 I-211$

$B I-212$
$B I-213$
23RS RECYCLE U-233 FEE. 23RS RECYCLE U-233 F.E.
FERTILE PIL. FISSILE PIL.

$5.103-07$

$6.443-03$

$6.449-06$

$6.449-06$

$6.449-06$
$1.490-05$

$3.678-01$

$1.120-04$

$1.336-04$

2. $316-02$

$2.342+00$

$2.052+00$

$2.684-01$

$1.912-05$

$9.240-05$

0.000
$2.136-1$

$2.136-11$
$2.785-03$

0.000

0.000

$2.358+00$
$2.132-03$

$2.132-03$
$5.944-03$

1. $912-05$

$0.727-04$

0.000

1.759-04

$6.887-04$

$1.739-04$

$2.358+00$

$2.132-03$

$6.887-04$

$2.132-03$
$2.463-06$

$1.739-04$

$2.358+00$

2.132-03

8.084-09

$1.464-05$
$1.509+00$

$1.509+00$

2.132-03

$1.739-04$
$2.358+00$

1.739-04

$2.358+00$
$2.132-03$
$8.812-05$

7.213-05

$4.178-02$

.

$8.812-05$

$4.013-04$

1. $237+01$

$1.237+01$
$4.490-03$

6.661-03

$1.465+00$

$1.272-03$

$2.081+00$

3.291-01

5.079-01

6.792-05

$9.851-04$

0.000

$5.083-10$
$5.757-05$

0.000

5.083-10

$2.120+00$

$5.784-04$

$1.356-04$

$6.792-05$

$2.392-12$

$5.784-04$

$9.908-11$

$900-11$

$2.120+00$

$5.784-04$

$9.908-11$

$5.784-04$

2.377-07

$1.678-05$

$2.120 \cdot 00$

$5.784-04$

1. $844-10$

0.000

$1.357+00$

$5.784-04$

$1.678-05$

$2.120+00$

$1.678-05$

$2.120+00$
$5.784-04$ 25RS RECYCLE U-233 F.E.
FERTILE PIL. FISSILE PIL.

$6.449-06$

5.103-07

$6.443-03$
$4.798-04$

$6.449-06$

$6.449-06$

$1.490-05$

$3.678-01$
$1.120-04$

$1.336-04$

$2.316-02$

$6.650-05$
$2.342+00$

$2.342+00$
$2.052+00$

$2.684-01$

$1.912-05$

$9.240-05$

0.000

$2.136-11$
$2.785-03$

0.000

0.000

$2.350+00$

$2.132-03$

$5.944-03$
$1.912-05$

$1.912-05$
$8.727-04$

$1.727-04$
0.000

$2.132-03$

$1.759-04$

$6.887-04$

2.358400

$2.132-03$

$6.887-04$

2.887-04

$2.132-03$
$2.463-06$

$1.739-04$

$2.358+00$

2.132-03

8.084-09

$1.464-05$

$1.509+00$

2.132-03

$1.739-04$

$2.358+00$

$1.739-04$

$2.358+00$
$2.132-03$

$1.447-02$
$1.4469-02$
2.369401
1.98401
$3.742+00$
$1.447-02$
$1.447-02$
$1.554-01$
$5.323+02$
$3.840-01$
$6.667-01$
$1.774+02$
$3.996-02$
$8.836-04$
$8.425-08$
$3.742-02$
$9.601-05$
$2.826-02$
0.000
$4.377-05$
$1.061-06$
0.000
$4.377-05$
$8.899-04$
$1.531-08$
$4.771-06$
$9.601-05$
$2.127-11$
$4.377-05$
$1.531-08$
$3.107-07$
$1.332-11$
$3.090-07$
$8.899-04$
$1.531-08$
$1.382-11$
$1.531-08$
$4.337-09$
$3.090-07$
$8.899-04$
$1.531-08$
$6.489-12$
0.000
$5.696-04$
$1.531-08$
$3.090-07$
$8.899-04$
$3.090-07$
$8.899-04$
$1.531-08$

.53




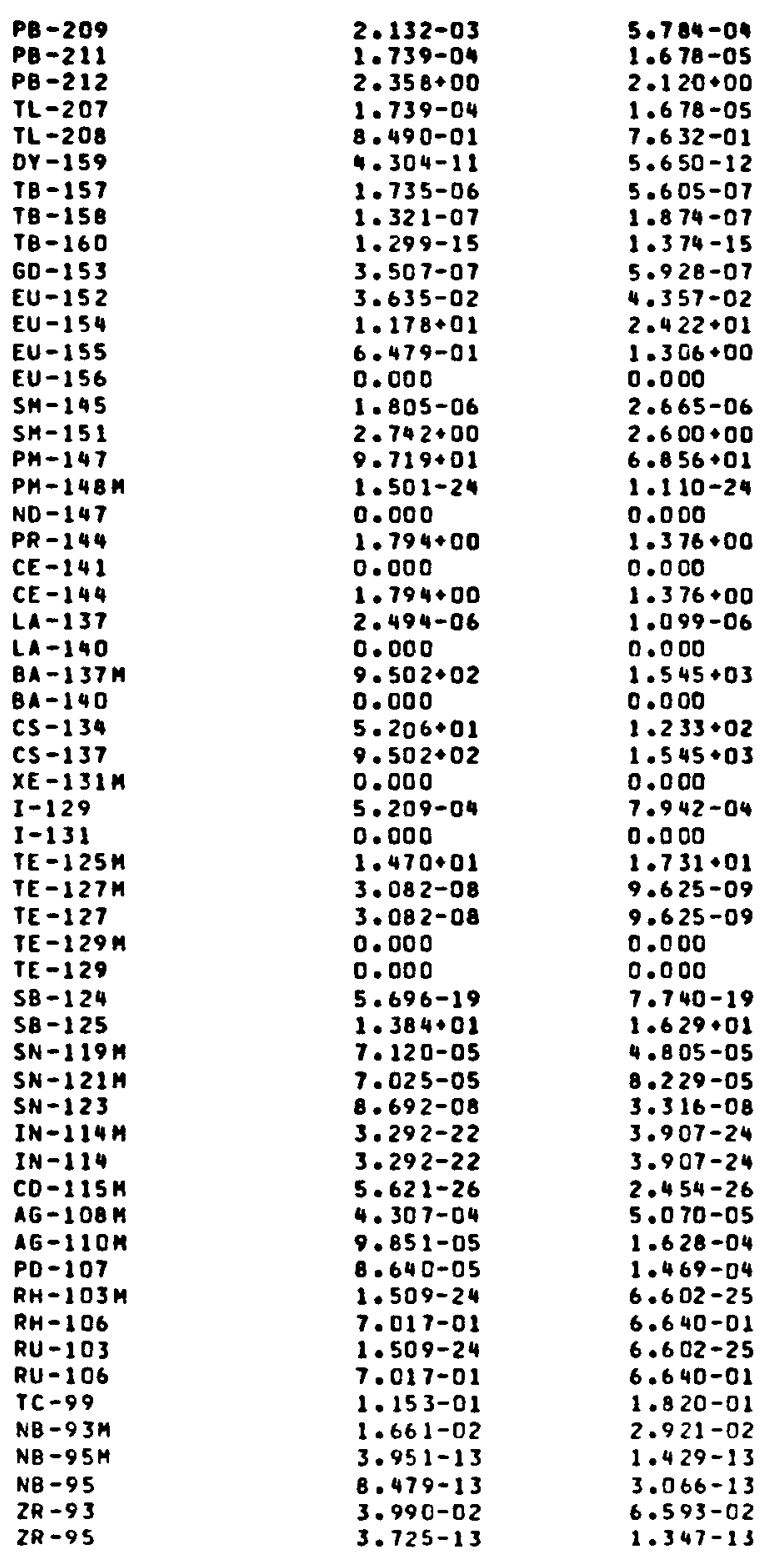

$\begin{array}{ll}2.132-03 & \\ 1.739-04 & 1.531-08 \\ 2.358-00 & 3.090-07 \\ 1.739-04 & 8.899-04 \\ 8.490-01 & 3.090-07 \\ 4.304-11 & 3.204-04 \\ 1.735-06 & 1.182-11 \\ 1.321-07 & 4.130-07 \\ 1.299-15 & 1.808-08 \\ 3.507-07 & 1.483-15 \\ 3.635-02 & 1.007-06 \\ 1.178+01 & 4.251-02 \\ 6.479-01 & 2.394+01 \\ 0.000 & 1.291+00 \\ 1.805-06 & 0.000 \\ 2.742+00 & 5.589-06 \\ 9.719+01 & 2.436+00 \\ 1.501-24 & 5.882+01 \\ 0.000 & 9.592-25 \\ 1.794+00 & 0.000 \\ 0.000 & 1.121+00 \\ 1.794+00 & 0.000 \\ 2.494-06 & 1.121+00 \\ 0.000 & 5.369-07 \\ 9.502+02 & 0.000 \\ 0.000 & 1.064+03 \\ 5.206-01 & 0.000 \\ 9.502+02 & 1.019+02 \\ 0.000 & 1.064+03 \\ 5.209-04 & 0.000 \\ 0.000 & 2.561-04 \\ 1.470001 & 0.000 \\ 3.082-08 & 3.395+00 \\ 3.082-08 & 1.673-09 \\ 0.000 & 1.673-09 \\ 0.000 & 0.000 \\ 5.696-19 & 0.000 \\ 1.384+01 & 3.358-19 \\ 7.120-05 & 3.196+00 \\ 7.025-05 & 3.205-05 \\ 8.692-08 & 6.213-05 \\ 3.292-22 & 1.328-08 \\ 3.292-22 & 8.193-24 \\ 5.621-26 & 8.193-24 \\ 4.307-04 & 1.561-26 \\ 9.851-05 & 1.063-04 \\ 8.6400-05 & 9.937-05 \\ 1.509-24 & 1.478-04 \\ 7.017-01 & 5.745-25 \\ 1.509-24 & 6.651-01 \\ 7.017-01 & 5.745-25 \\ 1.153-01 & 6.651-01 \\ 1.661-02 & 1.583-01 \\ 3.951-13 & 1.936-02 \\ 8.479-13 & 9.302-14 \\ 3.990-02 & 1.996-13 \\ 3.725-13 & 4.380-02 \\ & 8.770-14 \\ & \end{array}$




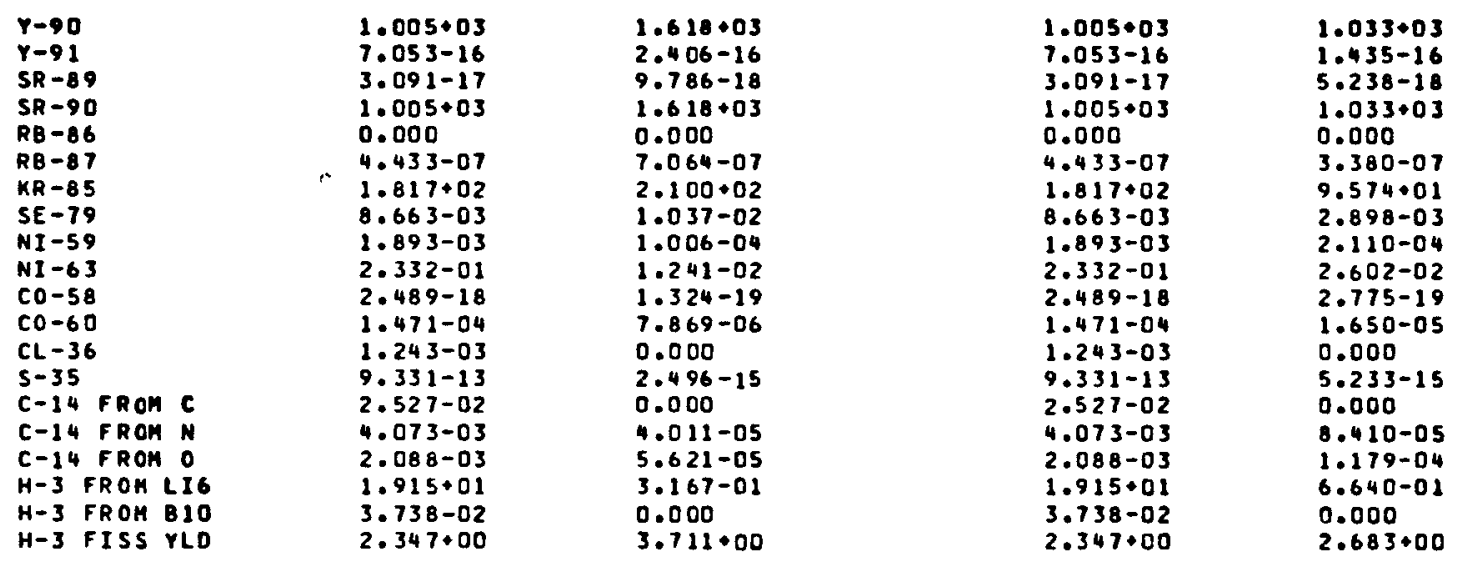




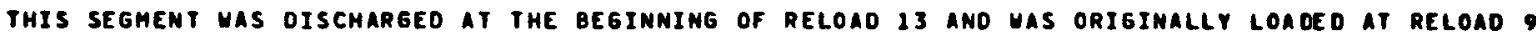
CURIE VRLUES USED PER F.E. AT IIHE 180 DAYS AFTER DISCHARGE

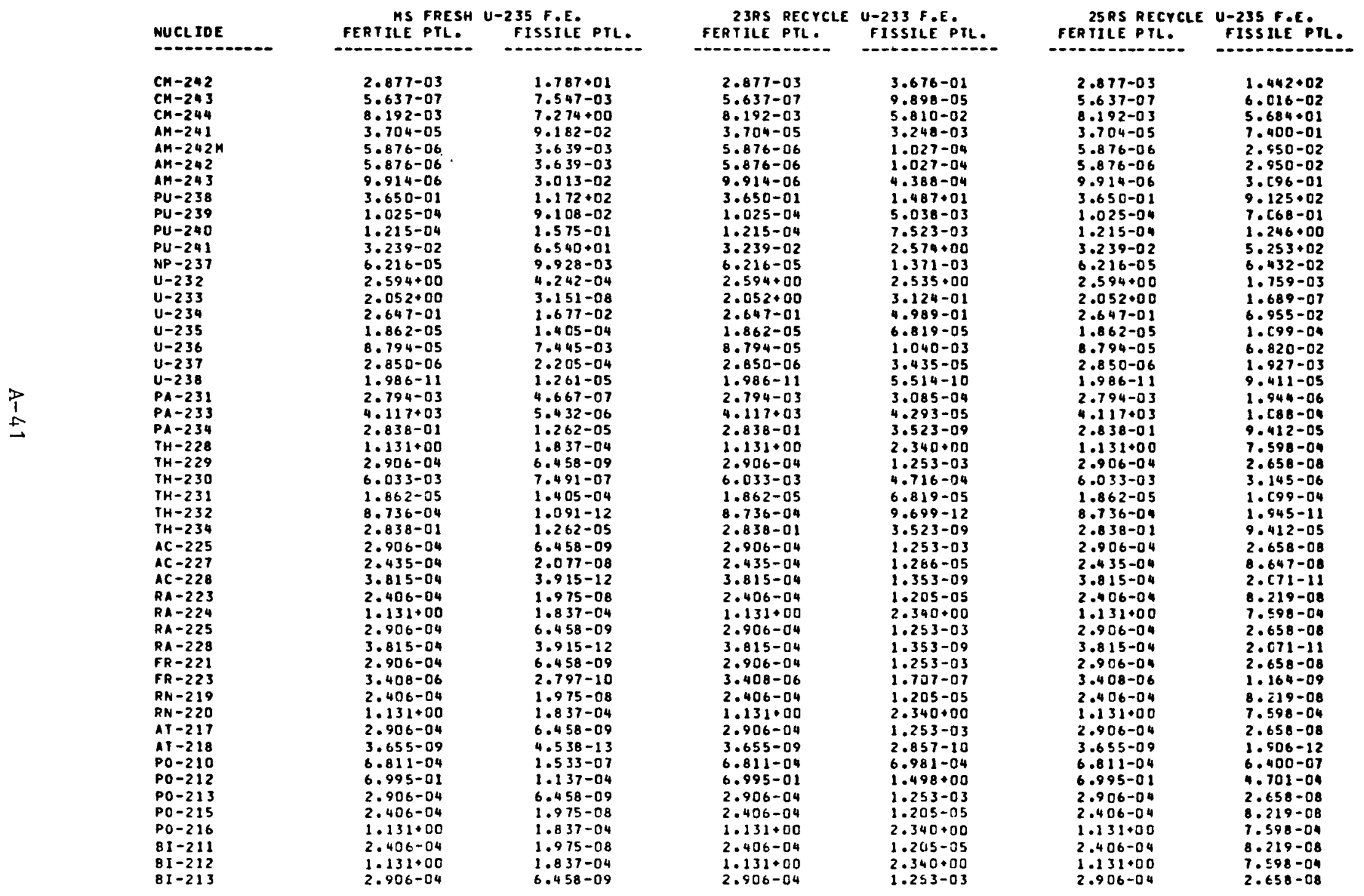




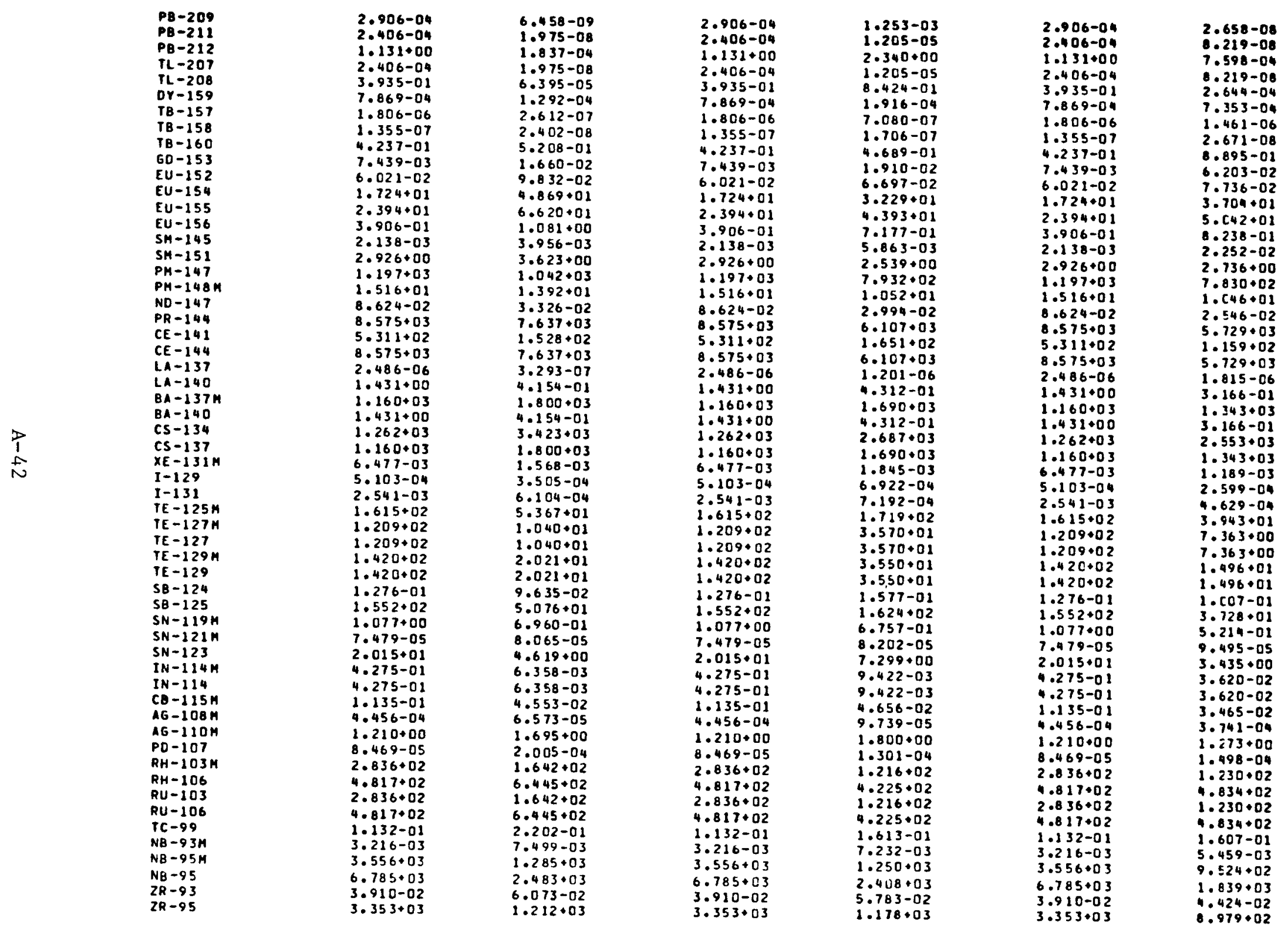




\begin{tabular}{|c|c|c|c|}
\hline $\begin{array}{l}Y-90 \\
Y-91 \\
S R-B 9\end{array}$ & & $\begin{array}{l}1.250+03 \\
7.492+02 \\
2.126+03\end{array}$ & $\begin{array}{l}1.820+03 \\
2.346+02 \\
5.574+02\end{array}$ \\
\hline$S R-90$ & & $1.250+03$ & $1.820+03$ \\
\hline$R B-86$ & & $4.080-02$ & $1.424-02$ \\
\hline RB -87 & & $4.347-07$ & $4.702-07$ \\
\hline$K R-85$ & & $3.317+02$ & $2.524+02$ \\
\hline$S E-79$ & & $8.562-03$ & $4.152-03$ \\
\hline N1 -59 & & $1.825-03$ & $1.216-04$ \\
\hline$N I=63$ & & $2.418-01$ & $1.612-02$ \\
\hline $\operatorname{co-58}$ & & $1.111-03$ & $7.404-05$ \\
\hline co-60 & & $4.988-04$ & $3.342-05$ \\
\hline$C L-36$ & & $1.202-03$ & 0.000 \\
\hline$s-35$ & & $9.188-01$ & $3.079-03$ \\
\hline$c-14$ & FROM C & $2.438-02$ & 0.000 \\
\hline$c-14$ & FROM $N$ & $3.939-03$ & $4.859-05$ \\
\hline$c-14$ & FROH O & $2.008-03$ & $6.796-05$ \\
\hline$H-3 F$ & FROH LI6 & $3.265+01$ & $6.765-01$ \\
\hline$H-3 F$ & FROM BIO & $6.387-02$ & 0.000 \\
\hline & & & \\
\hline
\end{tabular}

$\begin{array}{ll}1.250+03 & 1.801+03 \\ 7.492+02 & 2.478+02 \\ 2.126+03 & 6.523+02 \\ 1.250+03 & 1.801+03 \\ 4.080-02 & 2.437-02 \\ 4.347-07 & 6.173-07 \\ 3.317+02 & 3.470+02 \\ 8.562-03 & 9.220-03 \\ 1.825-03 & 1.802-04 \\ 2.418-01 & 2.389-02 \\ 1.111-03 & 1.097-04 \\ 4.988-04 & 4.953-05 \\ 1.202-03 & 0.000 \\ 9.188-01 & 4.563-03 \\ 2.438-02 & 0.000 \\ 3.939-03 & 7.201-05 \\ 2.008-03 & 1.007-04 \\ 3.265+01 & 1.002+00 \\ 6.387-02 & 0.000 \\ 3.931+00 & 5.578+00\end{array}$

$1.250+03$
$7.492+02$
$2.126+03$
$1.250+03$
$4.080-02$
$4.347-07$
$3.317+02$
$8.562-03$
$1.825-03$
$2.418-01$
$1.111-03$
$4.988-04$
$1.202-03$
$9.188-01$
$2.438-02$
$3.939-03$
$2.008-03$
$3.265+01$
$6.387-02$
$3.931+00$

$2.325+03$

$1.734+02$
$4.108+02$

$1.325+03$

1. $020-02$

3.418-07

$1.834+02$

$2.999-03$

$6.923-04$

$9.176-02$

$.215-04$
$1.903-04$

$1.903-04$

0. 1000

$1.753-02$

$2.766-04$
$3.869-04$

$3.869-04$

0.000

H-3 FISS YLD

$6.387-02$
$3.931+00$

$4.636+00$ 
THIS SEGMENT WAS DISCHARGED AT THE BEGINNING OF RELOAD 13 AND YAS ORIGIMALLY LOADEO AT RELOAO 9

CURIE VALUES USEO PER F.E. AT TIME 1 YEAR AFTER DISCHARGE

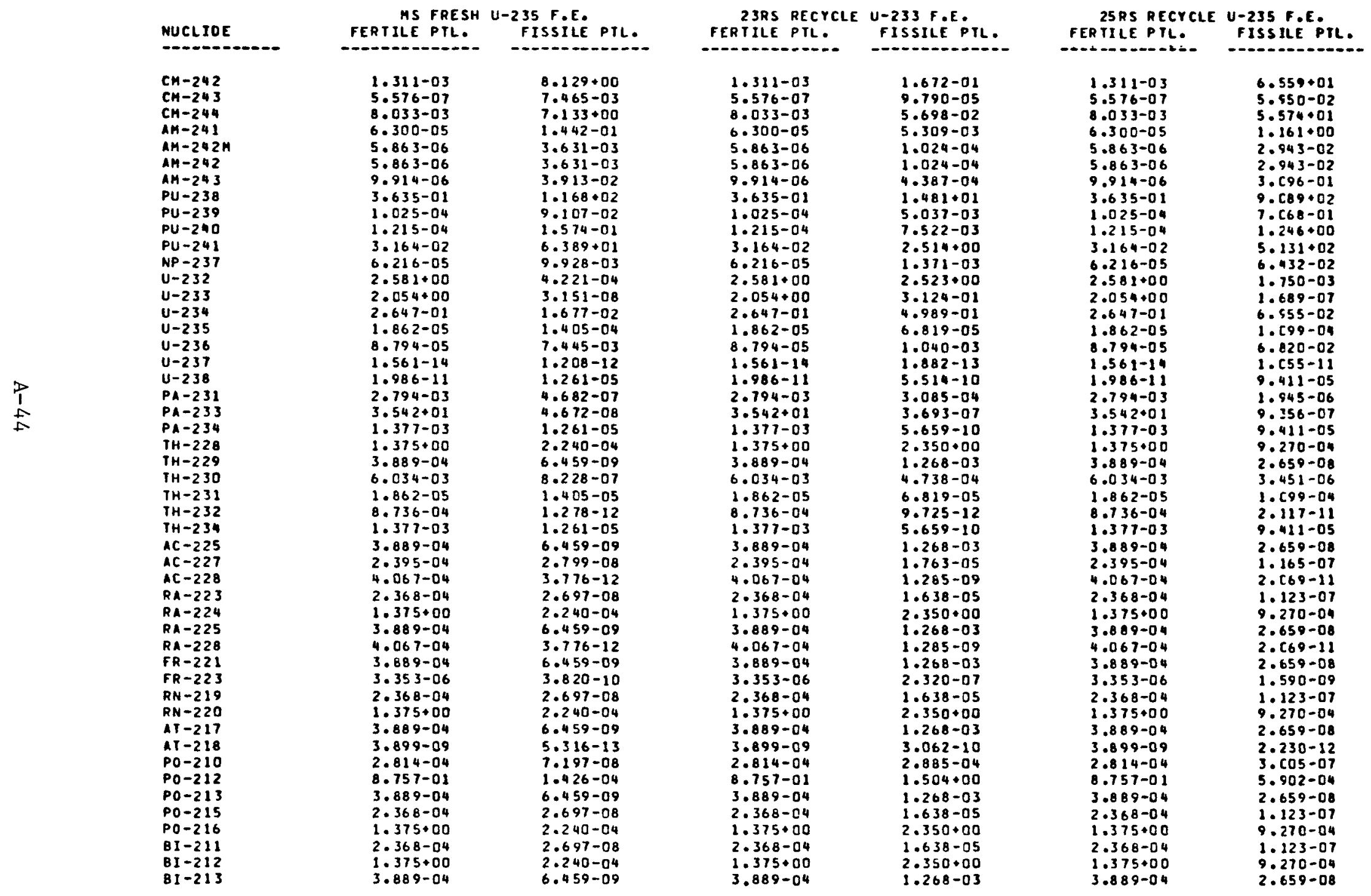




\begin{tabular}{|c|c|c|c|c|c|c|}
\hline $\begin{array}{l}P B-209 \\
P B-211\end{array}$ & $\begin{array}{l}3.889-04 \\
2.368-04\end{array}$ & $\begin{array}{l}6.459-09 \\
2.697-08\end{array}$ & $\begin{array}{l}3.889-04 \\
2.368-04\end{array}$ & $\begin{array}{l}1.260-03 \\
1.638-05\end{array}$ & $\begin{array}{l}3.889-04 \\
2.368-04\end{array}$ & $\begin{array}{l}2.659-08 \\
1.123-07\end{array}$ \\
\hline$P_{B}-212$ & $1.375+00$ & $2.240-04$ & $1.375+00$ & $2.350+00$ & $1.375+00$ & \\
\hline $\begin{array}{l}T L-207 \\
T L-208\end{array}$ & $\begin{array}{l}2.368-04 \\
4.926-01\end{array}$ & $\begin{array}{l}2.697-08 \\
8.021-05\end{array}$ & $\begin{array}{l}2.368-04 \\
4.926-01\end{array}$ & $\begin{array}{l}1.638-05 \\
8.460-01\end{array}$ & $\begin{array}{l}2.368-04 \\
4.926-01\end{array}$ & $\begin{array}{l}1.123-07 \\
3.320-04\end{array}$ \\
\hline or -159 & $3.225-04$ & $5.294-05$ & $3.225-04$ & $7.053-05$ & $3.225-04$ & $3.1_{4}-0_{4}$ \\
\hline$T B-157$ & $1.802-06$ & $2.606-07$ & $1.802-06$ & $7.063-07$ & $1.802-06$ & $1.457-06$ \\
\hline $\begin{array}{l}18-158 \\
18-160\end{array}$ & $\begin{array}{l}1.351-07 \\
7.121-02\end{array}$ & $\begin{array}{l}2.396-08 \\
8.754-02\end{array}$ & $\begin{array}{l}1.351-07 \\
7.121-02\end{array}$ & $\begin{array}{l}1.702-07 \\
7.882-02\end{array}$ & $1.351-07$ & $\begin{array}{l}2.665-08 \\
1.495-01\end{array}$ \\
\hline$G 0-153$ & $4.376-03$ & $9.764-03$ & $4.376-03$ & $1.123-02$ & $4.376-03$ & $3.649-02$ \\
\hline$E U-152$ & $5.856-02$ & $9.564-02$ & $5.856-02$ & $6.514-02$ & $5.856-02$ & $7.525-02$ \\
\hline$E U-154$ & 1.687 .01 & 4.763 .01 & $1.687+01$ & 3.159 .01 & $1.687+01$ & $3.624+01$ \\
\hline$E U-155$ & $1.972+01$ & $5.452+01$ & $1.972+01$ & $3.618+01$ & $1.972+01$ & $4.153+01$ \\
\hline$E U-156$ & $9.346-05$ & $2.587-04$ & $9.346-05$ & $1.717-04$ & $9.346-05$ & $1.971-04$ \\
\hline$S M-145$ & $1.466-03$ & $2.712-03$ & $1.466-03$ & $4.019-03$ & $1.465-03$ & $1.544-02$ \\
\hline$S H-151$ & $2.915+00$ & $3.608+00$ & $2.915+00$ & $2.529+00$ & $2.915+00$ & $2.725+00$ \\
\hline$P H-147$ & $1.047+03$ & $9.112 \div 02$ & 1.047 .03 & $6.937+02$ & $1.047+03$ & $6.847+02$ \\
\hline$P M-148 H$ & $7.026-01$ & $6.450-01$ & $7.026-01$ & $4.874-01$ & $7.026-01$ & $4.844-01$ \\
\hline NO -147 & $7.829-07$ & $3.020-07$ & $7.829-07$ & $2.718-07$ & $7.829-07$ & $2.311-07$ \\
\hline$P R-144$ & $5.456+03$ & $4.860+03$ & $5.456+03$ & $3.886+03$ & 5.456 .03 & 3.645 .03 \\
\hline$C E-141$ & $1.022+01$ & $2.939+00$ & $1.022+01$ & $3.175+00$ & $1.022+01$ & $2.230+00$ \\
\hline$C E-144$ & $5.456+03$ & 4.860003 & $5.456+03$ & $3.086+03$ & 5.456 .03 & 3.645 .03 \\
\hline$[A-137$ & $2.486-06$ & $3.293-07$ & $2.486-06$ & $1.201-06$ & $2.486-06$ & $1.815-06$ \\
\hline$L A-140$ & $6.296-05$ & $1.828-05$ & $6.296-05$ & $1.898-05$ & $6.296-05$ & 1. 393-05 \\
\hline$B A-137 A$ & $1.147+03$ & $1.779+03$ & 1.147 .03 & $1.670+03$ & $1.147+03$ & $1.327+03$ \\
\hline$B A-140$ & $6.296-05$ & $1.828-05$ & $6.296-05$ & $1.898-05$ & $6.296-05$ & $1.393-05$ \\
\hline$c s-134$ & $1.063+03$ & $2.083+03$ & $1.063+03$ & 2.263 .03 & $1.063+03$ & $2.150+03$ \\
\hline$C S-137$ & 1.147 .03 & $1.779+03$ & $1.147+03$ & $1.670 \cdot 03$ & $1.147+03$ & $1.327+03$ \\
\hline$X E-131 H$ & $1.227-07$ & $2.971-08$ & $1.227-07$ & $3.496-08$ & $1.227-07$ & $2.253-08$ \\
\hline $1-129$ & $5.111-04$ & $3.506-04$ & $5.111-04$ & $6.924-04$ & $5.111-04$ & $2.600-04$ \\
\hline $1-131$ & $3.012-10$ & $7.235-11$ & $3.012-10$ & $8.526-11$ & $3.012-10$ & $5.487-11$ \\
\hline IE $-125 \mathrm{~K}$ & $1.444+02$ & $4.733+01$ & $1.444+02$ & $1.514 \cdot 02$ & $1.444+02$ & $3.476+01$ \\
\hline$T E-127 \mathrm{H}$ & 3.722 .01 & $3.204+00$ & $3.722+01$ & $1.099+01$ & $3.722+01$ & 2.267 .00 \\
\hline$T E-127$ & $3.722+01$ & $3.204+00$ & $3.722+01$ & $1.099+01$ & 3.722 .01 & $2.267+00$ \\
\hline TE-129M & $3.292+00$ & $4.685-01$ & $3.292+00$ & $8.229-01$ & 3.292000 & $3.468-01$ \\
\hline$T E-129$ & $3.292+00$ & $4.685-01$ & $3.292+00$ & $8.229-01$ & 3.292000 & $3.468-01$ \\
\hline$S B-124$ & $1.513-02$ & $1.143-02$ & $1.513-02$ & $1.870-02$ & $1.513-02$ & $1.195-02$ \\
\hline$S B-125$ & $1.363+02$ & $4.458+01$ & $1.363+02$ & $1.426+02$ & $1.363+02$ & $3.274+01$ \\
\hline$S N-119 M$ & $6.446-01$ & $4.165-01$ & $6.446-01$ & $4.044-01$ & $6.446-01$ & $3.120-01$ \\
\hline$S N-121 M$ & $7.444-05$ & $8.028-05$ & $7.444-05$ & $8.164-05$ & $7.444-05$ & $9.451-05$ \\
\hline$S N-123$ & $7.216+00$ & $1.654+00$ & $7.216+00$ & $2.613+00$ & $7.216+00$ & $1.230+00$ \\
\hline$I N-114 M$ & $3.198-02$ & $4.756-04$ & $3.198-02$ & $7.048-04$ & $3.198-02$ & $2.708-03$ \\
\hline $1 N-114$ & $3.198-02$ & $4.756-04$ & $3.198-02$ & $7.048-04$ & $3.198-02$ & $2.708-03$ \\
\hline CO-115H & $5.737-03$ & $2.301-03$ & $5.737-03$ & $2.353-03$ & $5.737-03$ & $1.751-03$ \\
\hline$A G-108 M$ & $4.444-04$ & $6.555-05$ & $4.444-04$ & $9.712-05$ & $4.444-04$ & $3.731-04$ \\
\hline$A G-11 O M$ & $7.316-01$ & $1.025+00$ & $7.310-01$ & $1.088+00$ & $7.316-01$ & $7.696-01$ \\
\hline$P D=107$ & $8.469-05$ & $2.005-04$ & $8.469-05$ & $1.301-04$ & $8.469-05$ & $1.498-04$ \\
\hline RH-103H & $1.120+01$ & $6.519+00$ & $1.126+01$ & $4.827+00$ & $1.126+01$ & $4.881+00$ \\
\hline$R H-106$ & $3.399+02$ & $4.547+02$ & $3.399 \cdot 02$ & 2.981 .02 & $3.399+02$ & $3.411+02$ \\
\hline$R U-103$ & $1.126+01$ & $6.519+00$ & $1.126+01$ & $4.827+00$ & $1.126+01$ & $4.881+00$ \\
\hline$R U-106$ & $3.399+02$ & $4.547+02$ & $3.399+02$ & $2.981+02$ & $3.399+02$ & $3.411+02$ \\
\hline TC-99 & $1.132-01$ & $2.202-01$ & $1.132-01$ & $1.613-01$ & $1.132-01$ & $1 . t 07-01$ \\
\hline NB $-93 M$ & $4.082-03$ & $8.780-03$ & $4.082-03$ & $8.449-03$ & $4.082-03$ & $6.393-03$ \\
\hline$N B-95 \mathrm{H}$ & $5.014+02$ & $1.812 \div 02$ & $5.014+02$ & 1.762 .02 & $5.014 \cdot 02$ & $1.343+02$ \\
\hline NB - 95 & $1 . \mathrm{CS}_{4}+03$ & $3.819+02$ & $1.054+03$ & $3.712+02$ & 1.054 .03 & 2.829 .02 \\
\hline $2 R-93$ & $3.910-02$ & $6.073-02$ & $3.910-02$ & $5.763-02$ & $3.910-02$ & $4.424-02$ \\
\hline $2 R-95$ & 4.727 .02 & $1.709+02$ & $4.727+02$ & 1.661 .02 & 4.727 .02 & 1.266 .02 \\
\hline
\end{tabular}




$\begin{array}{lll}Y-90 & 1.234+03 & 1.797+03 \\ Y-91 & 8.189+01 & 2.564+01 \\ S R-89 & 1.861+02 & 4.878+01 \\ S R-90 & 1.234+03 & 1.797+03 \\ R B-86 & 4.185-05 & 1.460-05 \\ R B-87 & 4.347-07 & 4.702-07 \\ \text { KR-85 } & 3.211+02 & 2.443+02 \\ S E-79 & 8.562-03 & 4.152-03 \\ N I-59 & 1.825-03 & 1.216-04 \\ N 1-63 & 2.409-01 & 1.606-02 \\ C O-58 & 1.839-04 & 1.225-05 \\ C O-60 & 4.665-04 & 3.126-05 \\ C L-36 & 1.202-03 & 0.000 \\ S-35 & 2.107-01 & 7.061-04 \\ C-14 \text { FROM } & 2.438-02 & 0.000 \\ C-14 \text { FROM N } & 3.939-03 & 4.859-05 \\ C-14 \text { FROM O } & 2.008-03 & 6.795-05 \\ H-3 \text { FROH LI6 } & 3.174+01 & 6.575-01 \\ H-3 \text { FROM B10 } & 6.208-02 & 0.000 \\ H-3 \text { FISS YLO } & 3.820+00 & 6.183+00\end{array}$

$1.234+03$
$8.189+01$
$1.861+02$
$1.234+03$
$4.185-05$
$4.347-07$
$3.211+02$
$8.562-03$
$1.825-03$
$2.409-01$
$1.839-04$
$4.665-04$
$1.202-03$
$2.107-01$
$2.438-02$
$3.939-03$
$2.008-03$
$3.174+01$
$6.208-02$
$3.820+00$

1.779 .03

$2.709+01$
$5.708+01$

1.770 .01

$2.500-05$

$6.173-07$

$3.359 \cdot 02$

$9.220-03$

$1.802-04$

$2.380-02$

$1.815-05$

$4.632-05$

0.000

$1.046-03$

0.000

7.201-05

$1.007-04$

0.000

5.421 .00

$\begin{array}{ll}1.234003 & 1.309+03 \\ 8.189+01 & 1.895+01 \\ 1.861002 & 3.595+01 \\ 1.234+03 & 1.309+03 \\ 4.185-05 & 1.146-05 \\ 4.347-07 & 3.418-07 \\ 3.211+02 & 1.775+02 \\ 8.562-03 & 2.999-03 \\ 1.825-03 & 6.923-04 \\ 2.409-01 & 9.141-02 \\ 1.839-04 & 6.574-05 \\ 4.665-04 & 1.779-04 \\ 1.202-03 & 0.000 \\ 2.107-01 & 4.020-03 \\ 2.438-02 & 0.000 \\ 3.939-03 & 2.766-04 \\ 2.008-03 & 3.868-04 \\ 3.174+01 & 3.743+00 \\ 6.208-02 & 0.000 \\ 3.820+00 & 4.506+00\end{array}$


THIS SEGMENT WAS OISCHARGED AT THE BEgINNING OF RELOAD 13 ANO WAS ORIGINALLY LOADEO AT RELOAD 9 CURIE VALUES USEO PER F.E. AT IIME 3 VEARS AFTER OISCHARGE

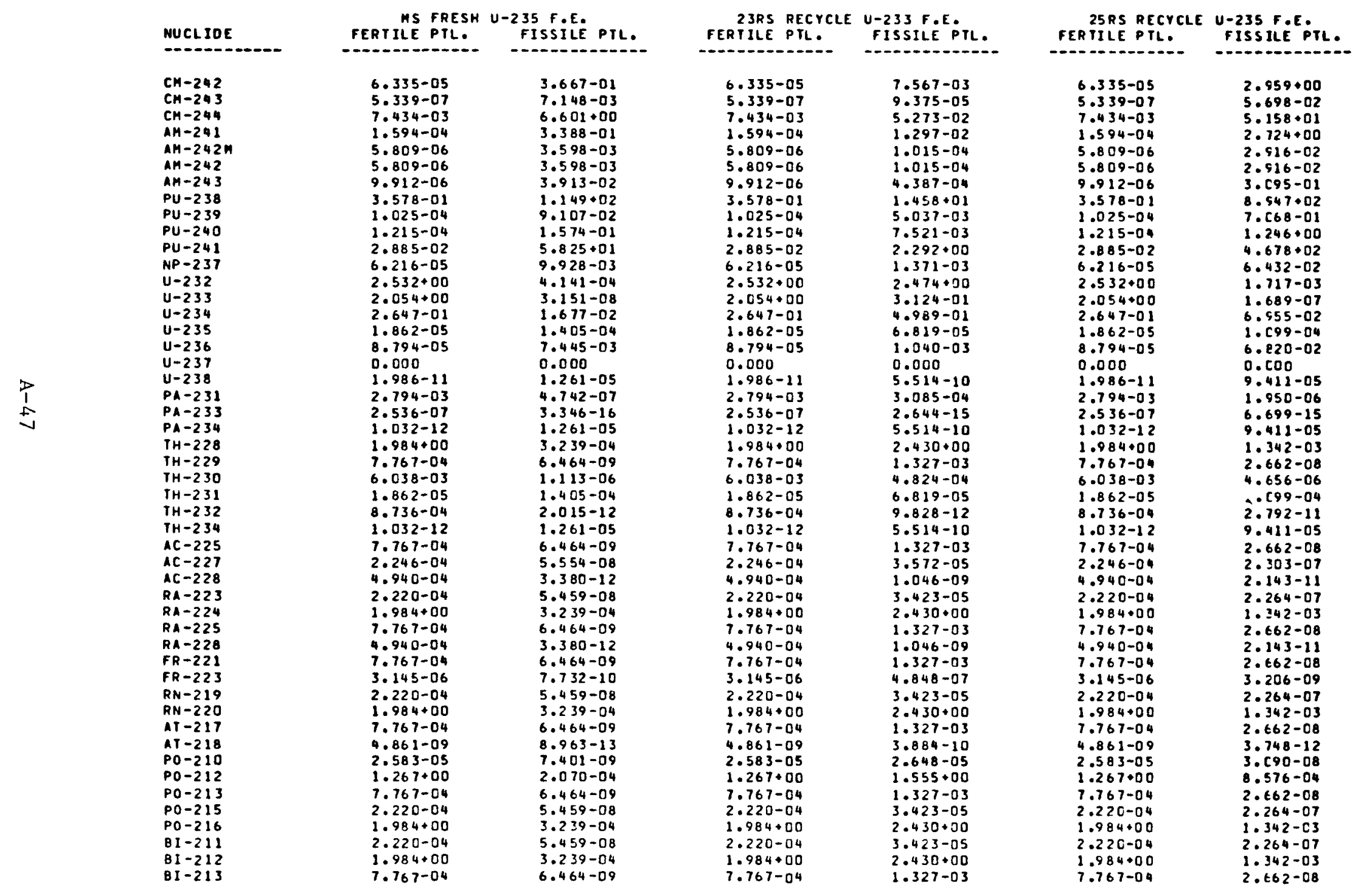




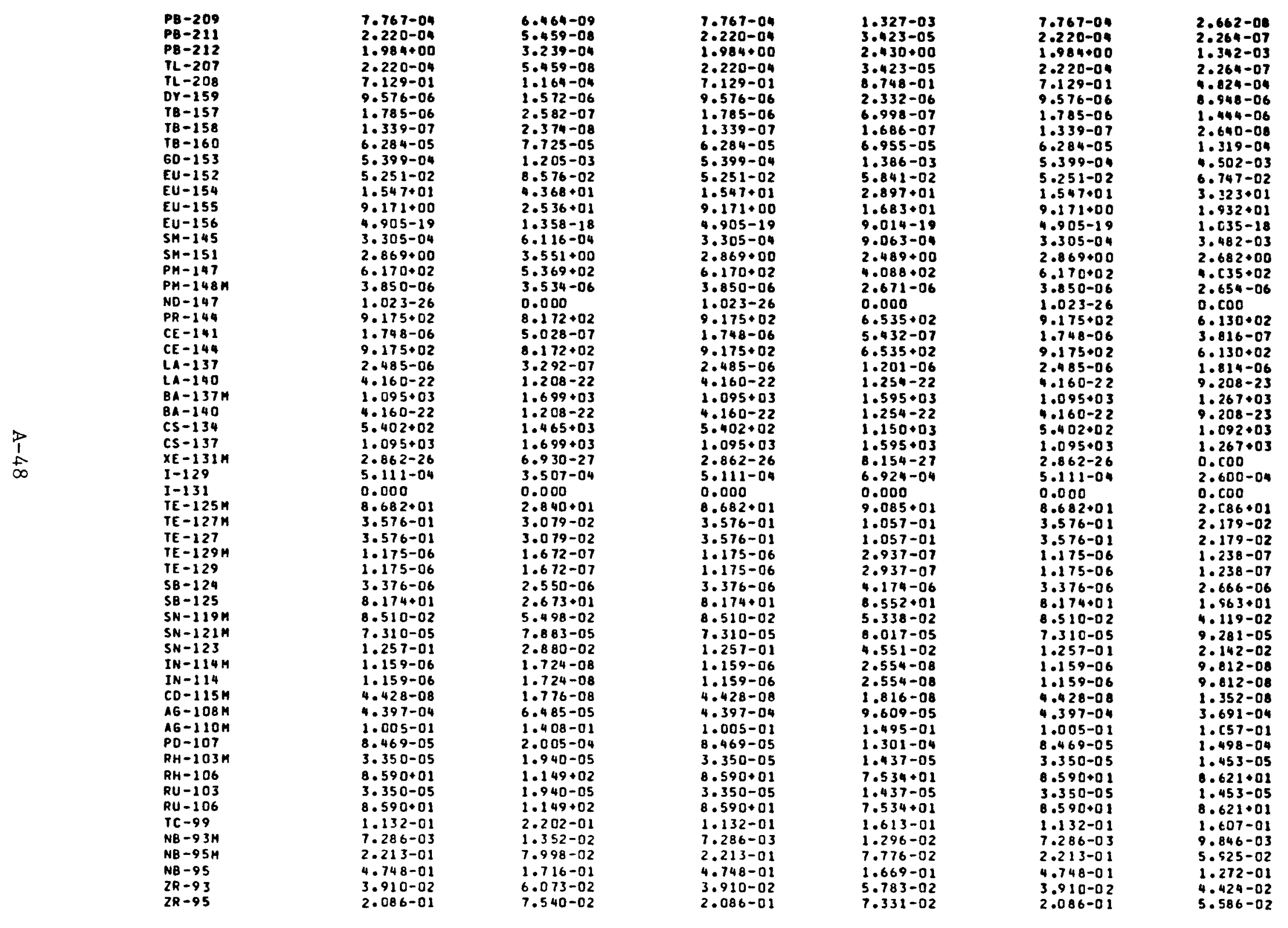




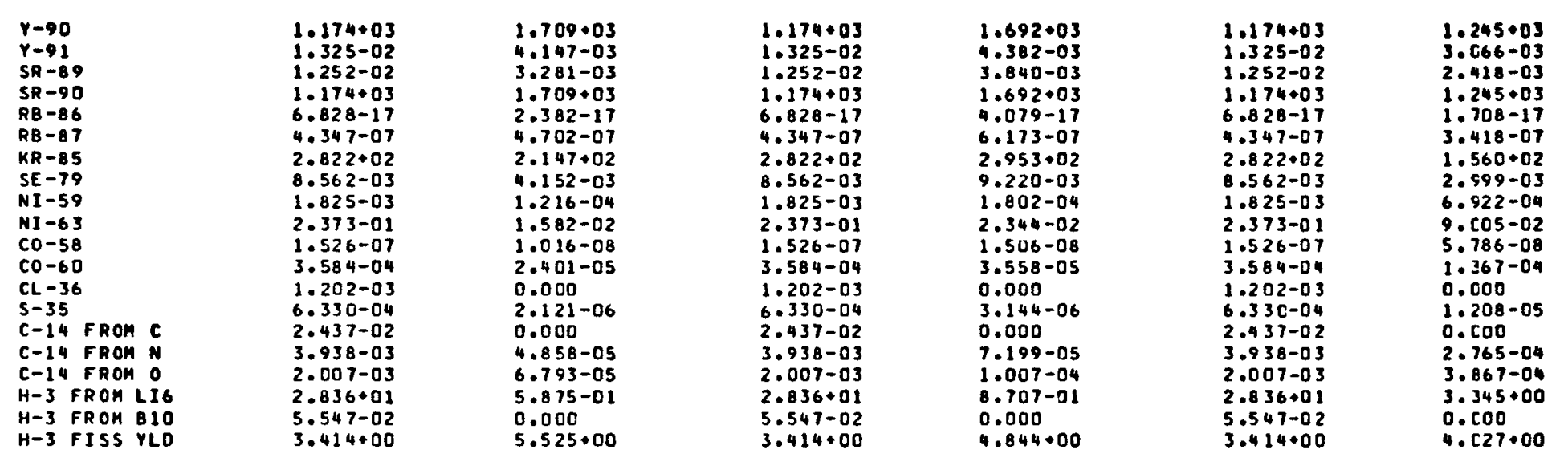


THIS SEGMENT WAS OISCHARgED AT THE BEgINNING OF RELOAO 23 AND WAS ORIGINALLY LOADED AT RELOAD

CURIE VALUes useo per F.e. AT IIHE 5 vears after discharge

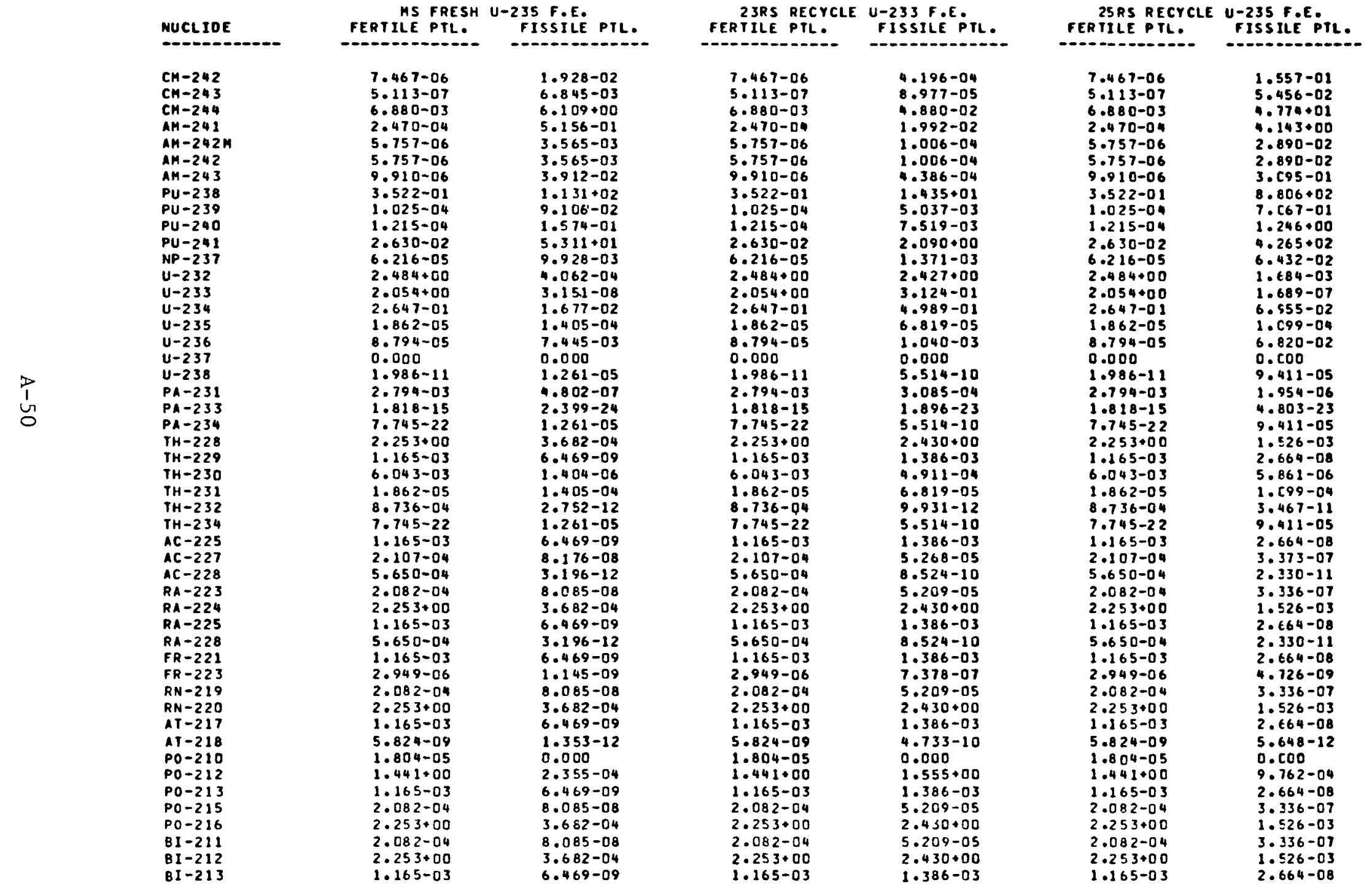




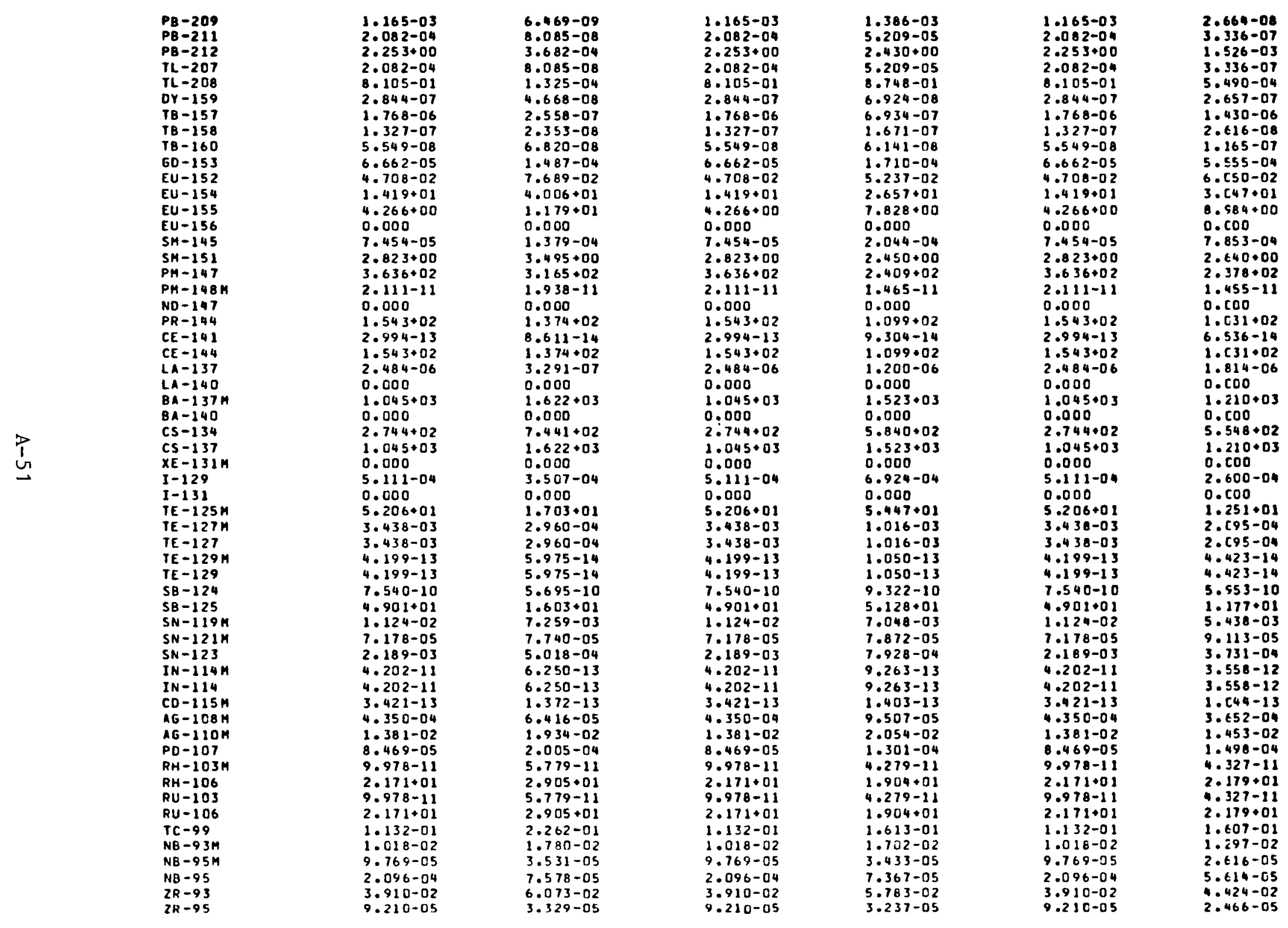




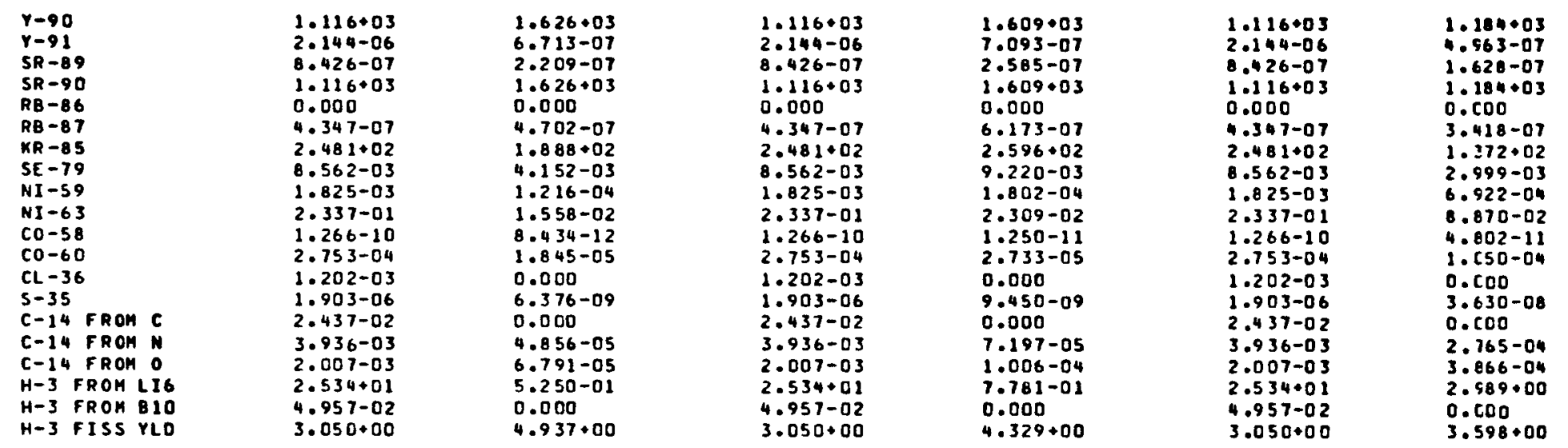




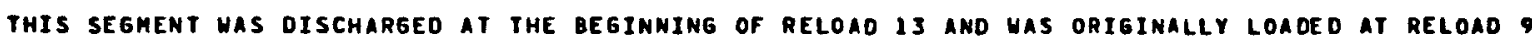

CURIE VALUES USED PER F.E. AT TIME 10 YEARS AFTER DISCHARGE

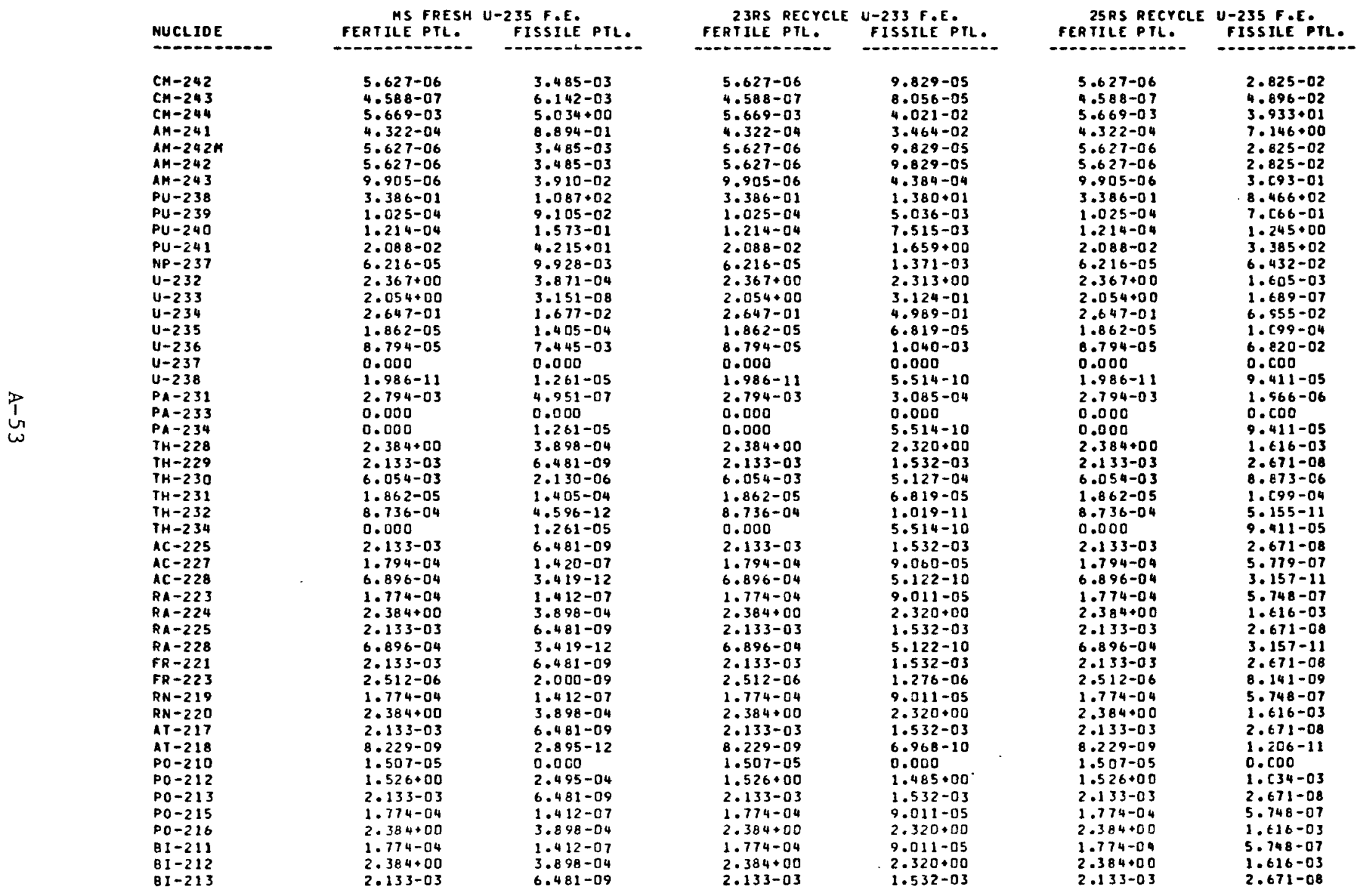




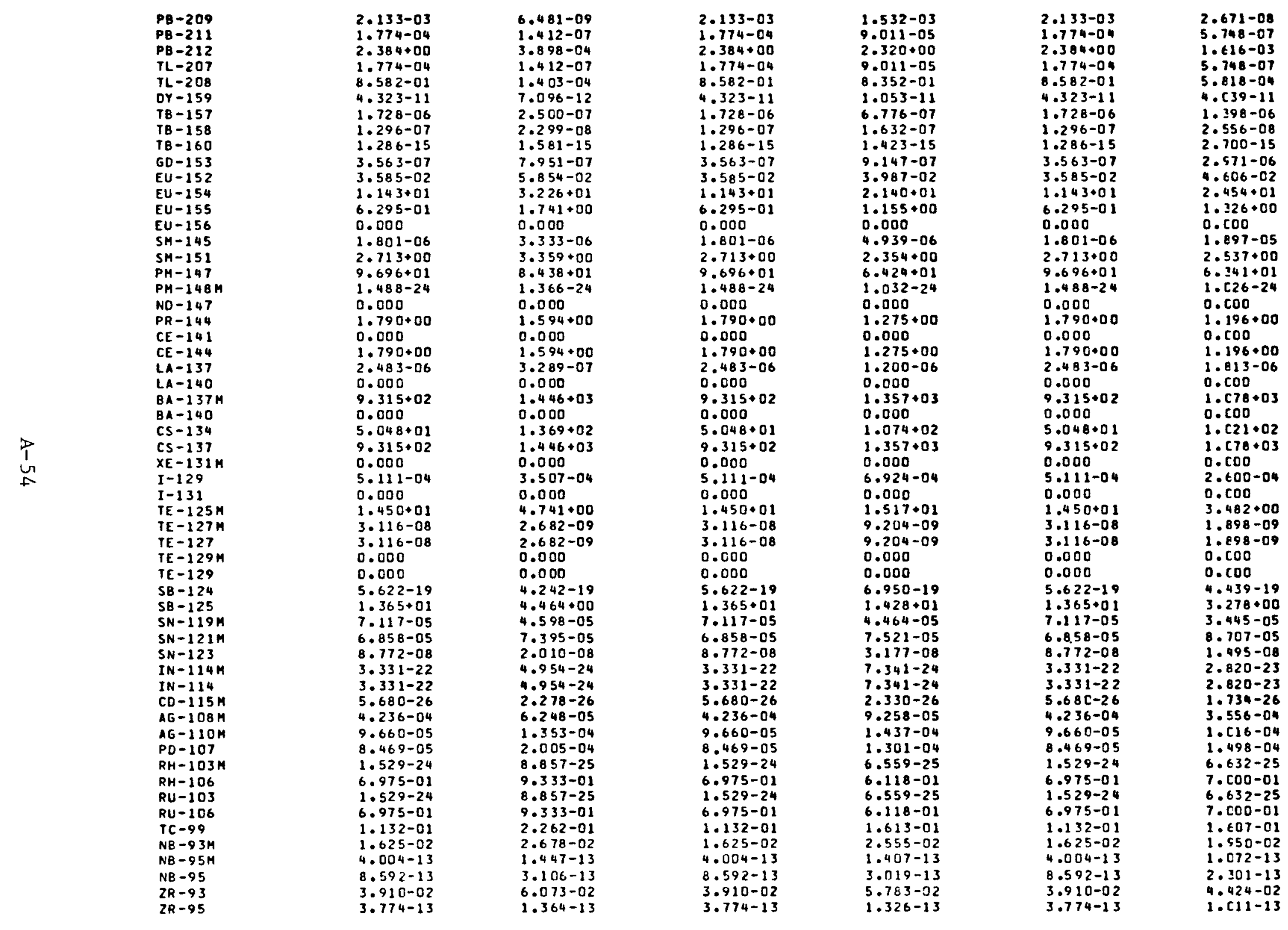




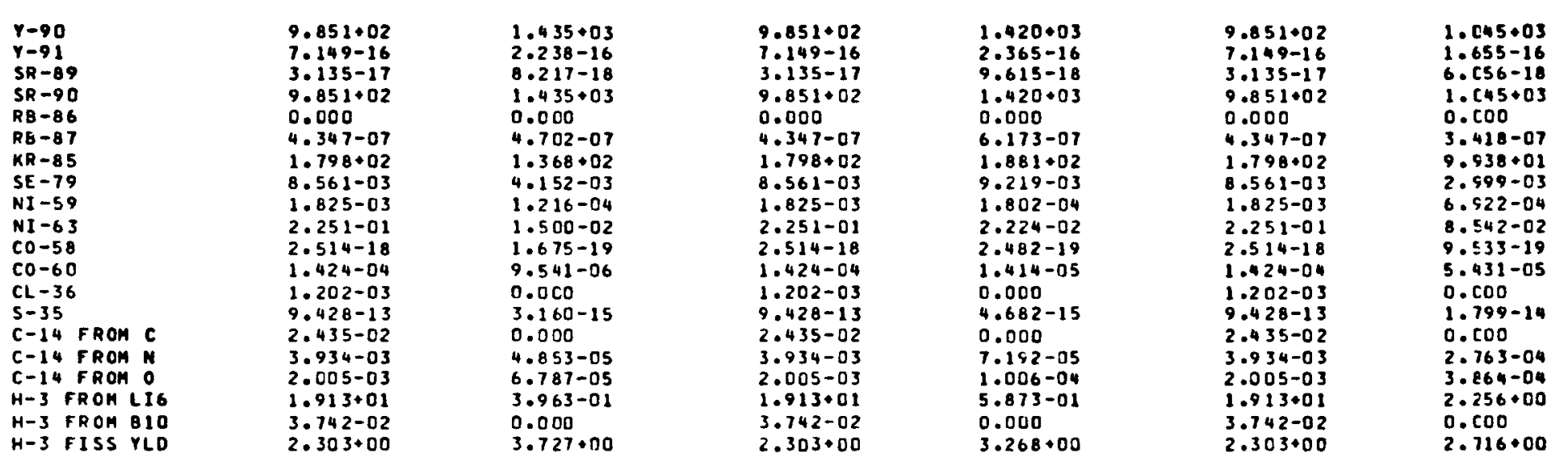


APPENDIX B

PERTINENT ENERGIES AND NEUTRON-YIELD VALUES 
TABLE B-1

HALF-LIVES, DECAY ENERGIES, AND NEUTRON YIELDS FOR IMPORTANT ISOTOPES ${ }^{(a)}$

\begin{tabular}{|c|c|c|c|c|c|c|c|}
\hline NUCL IDE & HALF LIFE & $\begin{array}{l}\text { DECAY CONST. } \\
\text { (1/SECI }\end{array}$ & $\begin{array}{l}\text { ALPHA } \\
\text { ENERGY } \\
\text { IMEVI } \\
\end{array}$ & $\begin{array}{c}\text { BETA } \\
\text { AVERAGE } \\
\text { ENERGY (MEY) } \\
\end{array}$ & $\begin{array}{l}\text { GAMHA } \\
\text { ENERGY } \\
\text { INEVI }\end{array}$ & $\begin{array}{l}\text { NEUTRONS } \\
\text { PER SEC-CI } \\
\text { IFERTILE PTL, }\end{array}$ & $\begin{array}{l}\text { MEUTRONS } \\
\text { PER SEC-CI } \\
\text { IF I SSILE PTL) }\end{array}$ \\
\hline $\mathrm{CH}-242$ & 1.630 .020 & $4.921-08$ & $6.207 \cdot 00$ & $6.300-03$ & $1.720-03$ & 8.230 .03 & 9.410 .03 \\
\hline CH -243 & $3.200 .01 \mathrm{~V}$ & $6.862-10$ & $5.929+00$ & $9.530-02$ & $1.960-01$ & & \\
\hline$C M-244$ & $1.790 .01 \mathrm{Y}$ & $1.227-09$ & $5.839+00$ & $5.550-03$ & $1.520-03$ & $1.460 * 05$ & 1.470 .05 \\
\hline$A H-241$ & $4.330+02 \gamma$ & $5.072-11$ & $5.567+00$ & $1.470-02$ & $2.620-02$ & $7.800 \bullet 02$ & 1.510003 \\
\hline$A M-242 M$ & $1.520002 Y$ & $1.445-10$ & $2.500-02$ & $3.020-02$ & $4.660-03$ & & \\
\hline$A H-242$ & $1.602+01 \mathrm{H}$ & $1.202-05$ & 0.000 & $1.730-01$ & $8.600-02$ & & \\
\hline$A H-243$ & $7.370 .03 \mathrm{Y}$ & $2.980-12$ & $5 \cdot 348+00$ & $1.250-02$ & $5.510-02$ & $6.500 \cdot 02$ & 1.230003 \\
\hline$P U-238$ & $8.780 .01 \mathrm{Y}$ & $2.501-10$ & $5.577+00$ & $6.700-03$ & $1.540-03$ & $9.300 \cdot 02$ & 1.660 .03 \\
\hline$P U-239$ & $2.439+04 \mathrm{~V}$ & $9.004-13$ & $5.235+00^{\prime}$ & $3.100-03$ & $6.500-04$ & $5.900 \bullet 02$ & $1.120 \cdot 03$ \\
\hline$P U-240$ & $6.540+03 \mathrm{~V}$ & $3.358-12$ & $5.250+00$ & $6.800-03$ & $1.470-03$ & $5.080+03$ & 5.610 .03 \\
\hline$P U-241$ & $1.500 .02 \mathrm{r}$ & $1.464-09$ & $1.200-04$ & $5.230-03$ & $1.000-04$ & & \\
\hline NP - 237 & $2.140 .06 \mathrm{Y}$ & $1.026-14$ & $4.865+00$ & $3.760-02$ & $3.180-02$ & $4.020 \cdot 02$ & $7.700 \cdot 02$ \\
\hline$v-232$ & $7.200+01 \mathrm{Y}$ & $3.050-10$ & $5 \cdot 390+00$ & 0.000 & $2.000-02$ & $6.500+02$ & $1.230 \cdot 03$ \\
\hline $0-233$ & $1.580 .05 Y$ & $1.390-13$ & $4.893+00$ & $3.170-03$ & $9.100-04$ & $4.020 \cdot 02$ & $7.700 * 02$ \\
\hline$u-234$ & $2.440+05 \mathrm{Y}$ & $9.000-14$ & $4.856+00$ & $1.220-02$ & $1.880-03$ & $4.020 \div 02$ & $7.700+02$ \\
\hline$v-235$ & $7.040 .08 \mathrm{Y}$ & $3.119-17$ & $4.471+00$ & $2.110-02$ & $1.520-01$ & $5 \cdot 380 \bullet 02$ & $9.040 \cdot 02$ \\
\hline$U-236$ & $2.342 .07 \mathrm{Y}$ & $9.377-16$ & $4.570+00$ & $8.200-03$ & $1.350-03$ & $3.820 \cdot 02$ & $7.270 \cdot 02$ \\
\hline$u-237$ & $6.750+00 \mathrm{D}$ & $1.188-06$ & 0.000 & $1.650-01$ & $1.430-01$ & & \\
\hline$U-238$ & $4.470+09 Y$ & $4.913-18$ & $4.266+00$ & $7.000-03$ & $1.140-03$ & $4.510 \cdot 04$ & $4.550+04$ \\
\hline$P A-231$ & $3.250 .04 Y$ & $6.757-13$ & $5.050 \cdot 00$ & 0.000 & $8.800-02$ & $4 \cdot 900 \cdot 02$ & $8.800 \cdot 02$ \\
\hline$P A-233$ & $2.700+010$ & $2.971-07$ & 0.000 & $6.336-02$ & $3.499-01$ & & \\
\hline$P A-234$ & $6.750+00 \mathrm{H}$ & $2.852-05$ & 0.000 & $2.042-01$ & $1.591 \cdot 00$ & & \\
\hline
\end{tabular}

(a) See Appendix $\mathrm{C}$ for method of conversion to decay heat and neutron source strengths. 
TABLE B-1 (Continued)

\begin{tabular}{|c|c|c|c|c|c|c|c|}
\hline MUCLIDE & HALF LIFE & $\begin{array}{l}\text { DECAY CONST. } \\
\text { (1/SECI }\end{array}$ & $\begin{array}{l}\text { ALPHA } \\
\text { ENERGY } \\
\text { (HEV) }\end{array}$ & $\begin{array}{c}\text { BETA } \\
\text { AVERAGE } \\
\text { ENERGY (MEV) }\end{array}$ & $\begin{array}{l}\text { GAMMA } \\
\text { ENERGY } \\
\text { (HEY) }\end{array}$ & $\begin{array}{l}\text { HEUTAONS } \\
\text { PER SEC-CI } \\
\text { IFERTILE PIL) }\end{array}$ & $\begin{array}{l}\text { NEUTRONS } \\
\text { PER SEC-CI } \\
\text { IFISSILE PTL) }\end{array}$ \\
\hline$I H-228$ & $1.910+00 Y$ & $1.150-08$ & $5.500 \cdot 00$ & 0.000 & $2.400-02$ & $7.100 * 02$ & $1.370+03$ \\
\hline $1 H-229$ & $7: 340+\overline{0} 3 \mathrm{Y}$ & $2.992-12$ & $4.900 \cdot 00$ & 0.000 & $2.100-01$ & $4.450 \cdot 02$ & $8.100+02$ \\
\hline TH-230 & $8.000+04 Y$ & $2.745-13$ & $4.750 \cdot 00$ & 0.000 & $1.600-02$ & $3.630+02$ & $7.300+02$ \\
\hline IH-231 & $2.552+01 \mathrm{H}$ & $7.543-06$ & 0.000 & $8.200-02$ & $9.900-02$ & & \\
\hline $1 H-232$ & $1.400+10 Y$ & $1.569-18$ & $4.060 \cdot 00$ & 0.000 & $1.400-02$ & $1.660 \cdot 02$ & $5.800 \cdot 02$ \\
\hline$T H-234$ & 2.410 .010 & $3.328-07$ & 0.000 & $4.453-02$ & $3.043-02$ & & \\
\hline$A C-225$ & $1.000+010$ & $8.021-07$ & $5.900+00$ & 0.000 & $3.400-02$ & $1.010 \cdot 03$ & 1.920 .03 \\
\hline$A C-22 T$ & $2.160+01 \mathrm{r}$ & $1.017-09$ & $7.100-02$ & $9.680-03$ & $5.705-03$ & & \\
\hline$A C-228$ & $6.130 .00 \mathrm{H}$ & $3.140-05$ & 0.000 & $4.118-01$ & $1.048+00$ & & \\
\hline$R A-223$ & $1.143+01 \mathrm{D}$ & $7.017-07$ & $5.770+00$ & 0.000 & $2.060-01$ & $9.200+02$ & 1.780 .03 \\
\hline$R A-224$ & $3.640+000$ & $2.204-06$ & $5.770+00$ & 0.000 & $1.300-02$ & $9.200 \cdot 02$ & 1.780 .03 \\
\hline$R A-225$ & $1.480+010$ & $5.419-07$ & 0.000 & $1.084-01$ & $2.520-02$ & & \\
\hline$R A-228$ & $6.700+00 \mathrm{Y}$ & $3.278-09$ & 0.000 & $1.419-02$ & 0.000 & & \\
\hline$F R-221$ & $4.800000 \mathrm{~m}$ & $2.406-03$ & $6.420+00$ & 0.000 & $3.600-02$ & $1.500 \cdot 03$ & 3.010 .03 \\
\hline$F R-223$ & $2.200+01 \mathrm{M}$ & $5.250-04$ & 0.000 & $3.745-01$ & $9.866-02$ & & \\
\hline$R N-219$ & $4.000+00 \mathrm{~s}$ & $2.733-01$ & $6.880+00$ & 0.000 & $6.200-02$ & 2.020 .03 & 3.830 .03 \\
\hline$R N-220$ & $5.530 .01 \mathrm{~S}$ & $1.253-02$ & $6.400+00$ & 0.000 & $4.000-03$ & $1.500 \cdot 03$ & 3.010 .03 \\
\hline$A T-217$ & $3.200-02 \mathrm{~s}$ & $2.166+01$ & $7.200+00$ & 0.000 & 0.000 & $2.680+03$ & $4.930+03$ \\
\hline$A T-218$ & $1.500+00 \mathrm{~s}$ & $4.620-01$ & $6.820 \cdot 00$ & $1.000-03$ & $3.000-03$ & $2.020 \cdot 03$ & $3.830+03$ \\
\hline$P O-210$ & $1.383+02 \mathrm{D}$ & $5.800-08$ & $5.410+00$ & 0.000 & 0.000 & $6.500+02$ & $1.230+03$ \\
\hline$P O-212$ & $3.040-07 \mathrm{~s}$ & $2.280+06$ & $8.950+00$ & 0.000 & 0.000 & $7.600+03$ & $1.140+04$ \\
\hline$P O-213$ & $4.200-06 \mathrm{~s}$ & $1.650+05$ & 8.540 .00 & 0.000 & 0.000 & $6.040 \div 03$ & $1.000+04$ \\
\hline
\end{tabular}


TABLE B-1 (Continued)

\begin{tabular}{|c|c|c|c|c|c|c|c|}
\hline MUCL IOE & HALF LIFE & $\begin{array}{l}\text { DECAY CONST. } \\
\text { II/SECI }\end{array}$ & $\begin{array}{l}\text { ALPHA } \\
\text { ENERGY } \\
\text { (HEV) }\end{array}$ & $\begin{array}{l}\text { BETA } \\
\text { AVEAAGE } \\
\text { ENERGY (HEV) }\end{array}$ & $\begin{array}{l}\text { GAHMA } \\
\text { EMERGY } \\
\text { (MEY) }\end{array}$ & $\begin{array}{l}\text { NEU TRON S } \\
\text { PER SEC-CI } \\
\text { IFERTILE PTL) }\end{array}$ & $\begin{array}{l}\text { NEUIRONS } \\
\text { PER SEC-CI } \\
\text { IF I SSILE PILI }\end{array}$ \\
\hline$P 0-215$ & $1.780-03 \mathrm{~s}$ & $3.893+02$ & $1.520 \cdot 00$ & 0.000 & 0.000 & $3.270 \cdot 03$ & $6.020 \times 03$ \\
\hline$P 0-216$ & $1.450-015$ & $4.779+00$ & $6.910 \cdot 00$ & 0.000 & 0.000 & $2 \cdot 170 \cdot 03$ & $4.110+03$ \\
\hline BI-211 & $2.160+00 \mathrm{H}$ & $5.347-03$ & $6.670 \cdot 00$ & 0.000 & $5.600-02$ & $1.880 \cdot 03$ & $3.560 \cdot 03$ \\
\hline $81-212$ & $6.060+01 \mathrm{M}$ & $1.906-04$ & $2.225+00$ & $5.333-01$ & $1.220-01$ & $4.610+02$ & $8.900 \bullet 02$ \\
\hline$B 1-213$ & $4.700+01 \mathrm{M}$ & $2.457-04$ & $1.287-01$ & $4.662-01$ & $1.519-01$ & & \\
\hline$P B-209$ & $3.300 * 00 \mathrm{H}$ & $5.833-05$ & 0.000 & $2.078-01$ & 0.000 & & \\
\hline PB-211 & $3.610 .01 \mathrm{H}$ & $3.199-04$ & 0.000 & $4.870-01$ & $6.289-02$ & & \\
\hline$P B-212$ & $1.064 .01 \mathrm{H}$ & $1.809-05$ & 0.000 & $1.095-01$ & $2.143-01$ & & \\
\hline$T L-207$ & $4.790+00 \mathrm{M}$ & $2.411-03$ & 0.000 & $5.398-01$ & $1.435-03$ & & \\
\hline$T L-208$ & $3.100+00 \mathrm{H}$ & $3.726-03$ & 0.000 & $6.084-01$ & $3.415+00$ & & \\
\hline or -159 & $1.440+020$ & $5.570-08$ & 0.000 & 0.000 & $6.508-02$ & & \\
\hline $18-157$ & $1.500 .02 \gamma$ & $1.464-10$ & 0.000 & 0.000 & $5.000-02$ & & \\
\hline $18-158$ & $1.500002 Y$ & $1.464-10$ & 0.000 & $3.989-02$ & $7.960-01$ & & \\
\hline$T B-160$ & $7.200+010$ & $1.114-07$ & 0.000 & $2.100-01$ & 1.191400 & & \\
\hline$G D-153$ & 2.420 .020 & $3.314-08$ & 0.000 & 0.000 & $1.408-01$ & & \\
\hline$E U-152$ & $1.270 .01 \mathrm{~V}$ & $1.729-09$ & 0.000 & $8.900-02$ & $1.195+00$ & & \\
\hline$E U-154$ & $1.600001 \mathrm{~V}$ & $1.372-09$ & 0.000 & $2.470-01$ & $1.250+00$ & & \\
\hline$E U-155$ & $1.810 .00 \gamma$ & $1.213-08$ & 0.000 & $4.470-02$ & $8.150-02$ & & \\
\hline$E U-156$ & 1.540 .010 & $5.208-07$ & 0.000 & $4.300-01$ & $1.318+00$ & & \\
\hline$S M-145$ & $3.400 .02 \mathrm{D}$ & $2.359-08$ & 0.000 & 0.000 & $1.012-01$ & & \\
\hline$S H-151$ & 8.710 .017 & $2.521-10$ & 0.000 & $1.950-02$ & $4.000-04$ & & \\
\hline$P H-147$ & $2.622+00 \mathrm{Y}$ & $8.375-09$ & 0.000 & $6.300-02$ & $1.000-04$ & & \\
\hline
\end{tabular}


TABLE B-1 (Continued)

\begin{tabular}{|c|c|c|c|c|c|c|c|}
\hline MUCL IDE & HALF LIFE & $\begin{array}{l}\text { DECAY CONST. } \\
\text { (1/SECI }\end{array}$ & $\begin{array}{l}\text { ALPHA } \\
\text { ENERGY } \\
\text { (MEV) }\end{array}$ & $\begin{array}{l}\text { BETA } \\
\text { AVERAGE } \\
\text { ENERGY IMEV) }\end{array}$ & $\begin{array}{l}\text { GAMHA } \\
\text { ENERGY } \\
\text { (MEV) }\end{array}$ & $\begin{array}{l}\text { NEUTROH S } \\
\text { PER SEC-CI } \\
\text { IFERTILE PTL) }\end{array}$ & $\begin{array}{l}\text { NEUTAONS } \\
\text { PER SEC-CI } \\
\text { IF I SSILE PILI }\end{array}$ \\
\hline$P K-1484$ & 4.180 .010 & $1.919-07$ & 0.000 & $1.470-01$ & $2.009+00$ & & \\
\hline ND -147 & 1.110 .010 & $7.226-07$ & 0.000 & $2.420-01$ & $1.190-01$ & & \\
\hline$P R-144$ & $1.727+01 \mathrm{M}$ & $6.688-04$ & 0.000 & $1.263+00$ & $3.100-02$ & & \\
\hline$C E-141$ & $3.250 .01 \mathrm{D}$ & $2.468-07$ & 0.000 & $1.590-02$ & $7.170-02$ & & \\
\hline CE-144 & $2.840+020$ & $2.824-08$ & 0.000 & $0.300-02$ & $2.890-02$ & & \\
\hline$\langle A-13\rangle$ & $6.000+04 r$ & $3.660-13$ & 0.000 & 0.000 & $3.700-02$ & & \\
\hline$L A-140$ & $4.020 \div 01 \mathrm{H}$ & $4.789-06$ & 0.000 & $5.170-01$ & $2.300 \cdot 00$ & & \\
\hline$B A-137 A$ & $2.550 .00 \mathrm{H}$ & $4.529-03$ & 0.000 & 0.000 & $0.620-01$ & & \\
\hline$B A-140$ & $1.280+010$ & $6.266-07$ & 0.000 & $2.800-01$ & $2.170-01$ & & \\
\hline $\operatorname{cs}-134$ & $2.050+00 \mathrm{Y}$ & $1.071-08$ & 0.000 & $1.610-01$ & $1.580 \cdot 00$ & & \\
\hline$(5-13)$ & $3.000+01 \gamma$ & $7.320-10$ & 0.000 & $1.740-01$ & 0.000 & & \\
\hline$X E-1314$ & 1.180 .010 & $6.797-07$ & 0.000 & 0.000 & $1.680-01$ & & \\
\hline$I-129$ & $1.600007 \gamma$ & $1.372-15$ & 0.000 & $6.240-02$ & $4.000-02$ & & \\
\hline $1-131$ & $8.050+000$ & $9.964-07$ & 0.000 & $1.860-01$ & $3.890-01$ & & \\
\hline$T E-125 n$ & $5.800 .01 \mathrm{D}$ & $1.383-07$ & 0.000 & 0.000 & $2.440-01$ & & \\
\hline$T E-127 M$ & 2.090 .020 & $7.359-08$ & 0.000 & $6.100-03$ & $8.800-02$ & & \\
\hline$T E-127$ & $9.400+00 \mathrm{H}$ & $2.048-05$ & 0.000 & $2.270-01$ & $5.170-03$ & & \\
\hline TE-129n & $3.410+010$ & $2.352-07$ & 0.000 & $2.140-01$ & $9.660-02$ & & \\
\hline $1 E-129$ & $6.870+01 \mathrm{M}$ & $1.681-04$ & 0.000 & $5.340-0 i$ & $7.290-02$ & & \\
\hline $58-124$ & $6.020+01 \mathrm{D}$ & $1.332-07$ & 0.000 & $3.720-01$ & $1.879+00$ & & \\
\hline $58-125$ & $2.710+00 Y$ & $6.103-09$ & 0.000 & $8.690-02$ & $4.520-01$ & & \\
\hline$S N-119 M$ & $2.500+020$ & $3.208-08$ & 0.000 & 0.000 & $8.900-02$ & & \\
\hline
\end{tabular}


TABLE B-1 (Continued)

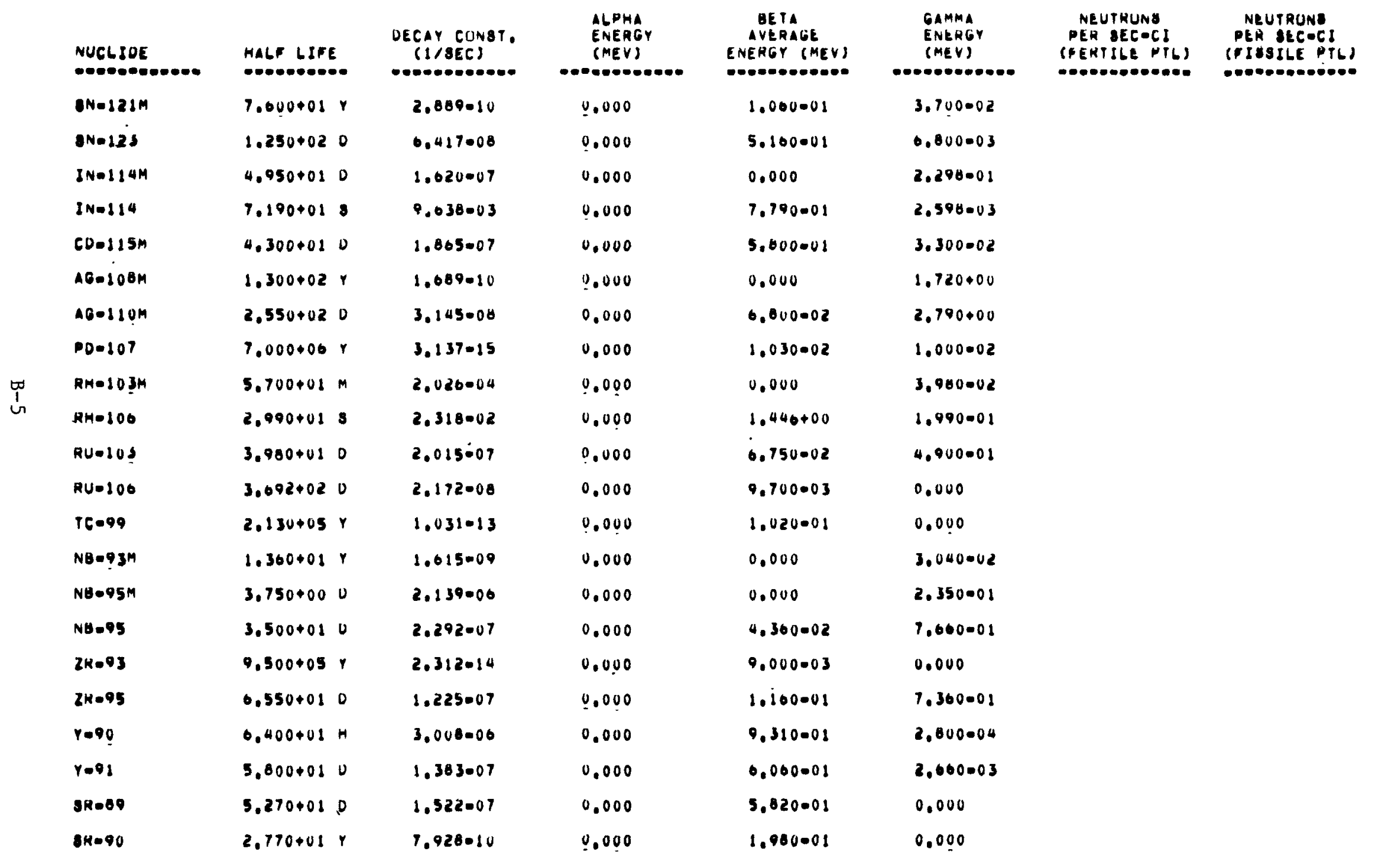


TABLE B-1 (Continued)

\begin{tabular}{|c|c|c|c|c|c|c|c|}
\hline NUCRIDE & $\begin{array}{l}\text { HALF LIFE } \\
-\end{array}$ & $\begin{array}{l}\text { DECAY CONST. } \\
\text { I1/SECI }\end{array}$ & $\begin{array}{l}\text { ALPHA } \\
\text { ENERGY } \\
\text { (MEV) } \\
\end{array}$ & $\begin{array}{l}\text { BETA } \\
\text { AVERAGE } \\
\text { ENERGY IMEV) }\end{array}$ & $\begin{array}{l}\text { GAMHA } \\
\text { ENERGY } \\
\text { IMEVI }\end{array}$ & $\begin{array}{l}\text { NEUTRONS } \\
\text { PER SEC-CI } \\
\text { IFERTILE PILI }\end{array}$ & $\begin{array}{l}\text { NEUTRONS } \\
\text { PER SEC-CI } \\
\text { IFISSILE PILI }\end{array}$ \\
\hline$R 8-86$ & $1.866+010$ & $4.298-07$ & 0.000 & $6.700-01$ & $9.430-02$ & & \\
\hline$R B-B 7$ & $4.800 \cdot 10 \mathrm{r}$ & $4.575-19$ & 0.000 & $7.851-02$ & 0.000 & & \\
\hline$K R-85$ & $1.076+01 \mathrm{Y}$ & $2.041-09$ & 0.000 & $2.510-01$ & $2.230-03$ & & \\
\hline$S E-79$ & $6.500+04 \gamma$ & $3.378-13$ & 0.000 & $4.200-02$ & $1.000-04$ & & \\
\hline NI -59 & $8.000004 \mathrm{Y}$ & $2.745-13$ & 0.000 & 0.000 & $8.000-03$ & & \\
\hline$N 1-63$ & $9.200001 \mathrm{Y}$ & $2 \cdot 387-10$ & 0.000 & $1.760-02$ & 0.000 & & \\
\hline co-58 & 7.240 .010 & $1.123-07$ & 0.000 & $3.020-02$ & $9.816-01$ & & \\
\hline $\operatorname{co-60}$ & $5.260 .00 \mathrm{Y}$ & $4.175-09$ & 0.000 & $9.450-02$ & $2.505+00$ & & \\
\hline$C L-30$ & $3.01000 \mathrm{~s}$ & $1.153-14$ & 0.000 & $2.730-01$ & 0.000 & & \\
\hline$s-35$ & $8.720+01.0$ & $9.198-08$ & 0.000 & $4.920-02$ & 0.000 & & \\
\hline$c-14$ & $5.000+03 Y$ & $4.392-12$ & 0.000 & $4.930-02$ & 0.000 & & \\
\hline$H-3$ & $1.233+01 \mathrm{Y}$ & $1.781-09$ & 0.000 & $5.690-03$ & 0.000 & & \\
\hline
\end{tabular}


TABLE B-2

GAMMA ENERGY SPECTRA

GAHMA ENERGY RELEASE TABLE (MEV PER DISINTEGRATION)

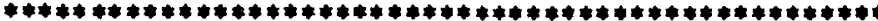

PHOTON ENERGY (MEV) (a)

\begin{tabular}{|c|c|c|c|c|c|c|c|c|c|c|c|c|c|c|}
\hline ISOTOPE & HALF LIF & & 0.1 & 0.3 & 0.6 & 0.8 & 1.0 & 1.25 & 1.6 & 2.0 & 2.4 & 2.9 & 3.5 & TOTAL \\
\hline$U-235$ & $7.040+08$ & $r$ & .0400 & .1000 & .0000 & .0000 & .0000 & .0000 & .0000 & .0000 & .0000 & .0000 & - $C 000$ & .1400 \\
\hline$P A-233$ & $2.700+01$ & 0 & .0484 & .3015 & .0000 & .0000 & .0000 & .0000 & .0000 & .0000 & .0000 & .0000 & .0000 & .3499 \\
\hline$P A-234$ & $6.750+00$ & H & .0000 & .0800 & .0800 & .0400 & .7800 & .0600 & .1500 & .0000 & .0000 & .0000 & .0000 & 1.1900 \\
\hline$A C-228$ & $6.130+00$ & H & .0000 & .0400 & .0200 & .0500 & .4700 & .0000 & .1600 & .0400 & .0000 & .0000 & .0000 & .7800 \\
\hline$R A-223$ & $1.143+01$ & 0 & .0200 & .0500 & .0000 & .0000 & .0000 & .0000 & .0000 & .0000 & .0000 & .0000 & .0000 & .0700 \\
\hline$R N-219$ & $4.000+00$ & $\mathbf{s}$ & .0000 & .0500 & .0000 & .0000 & .0000 & .0000 & .0000 & .0000 & .0000 & .0000 & .0000 & .0500 \\
\hline BI-211 & $2.160+00$ & M & .0000 & .0500 & .0000 & .0000 & .0000 & .0000 & .0000 & .0000 & .0000 & .0000 & .0000 & .0500 \\
\hline BI-212 & $6.060+01$ & M & .0000 & .0000 & .0000 & .0600 & .0100 & .0000 & .0300 & .0000 & .0000 & .0000 & .0000 & .1000 \\
\hline BI-213 & $4.700 \cdot 01$ & M & .0000 & .1400 & .0000 & .0000 & .0100 & .0000 & .0000 & .0000 & .0000 & .0000 & .0000 & .1500 \\
\hline$P B-211$ & $3.610 \cdot 01$ & $M$ & .0000 & .0300 & .0000 & .0300 & .0000 & .0000 & .0000 & .0000 & .0000 & .0000 & .0000 & .0600 \\
\hline$P B-212$ & $1.064+01$ & H & .0000 & .1200 & .0000 & .0000 & .0000 & .0000 & .0000 & .0000 & .0000 & .0000 & $\cdot \cos 0$ & .1200 \\
\hline$T L-208$ & $3.100+00$ & M & .0000 & .0200 & .6200 & .1200 & .0000 & .0000 & .0000 & .0000 & 2.6100 & .0000 & - $C 000$ & 3.3700 \\
\hline$T B-158$ & $1.500 \cdot 02$ & $Y$ & .0000 & .0090 & .0000 & .0770 & .6150 & .0200 & .0000 & .0000 & .0000 & .0000 & .0000 & .7210 \\
\hline $78-160$ & $7.200+01$ & D & .0000 & .1200 & .0000 & .2900 & .3100 & .2400 & .0000 & .0000 & .0000 & .0000 & .0000 & .9600 \\
\hline$E U-152$ & $1.270+01$ & $Y$ & .0556 & .1384 & .0222 & .1432 & .4296 & .3492 & .0134 & .0000 & .0000 & .0000 & .0000 & 1.1516 \\
\hline$E U-154$ & $1.600+01$ & $r$ & .0505 & .0206 & .0532 & .2943 & .2911 & .4621 & .0376 & .0000 & .0000 & .0000 & .0000 & 1.2094 \\
\hline$E U-156$ & $1.540+01$ & D & .0000 & .0000 & .0500 & .1500 & .1400 & .3800 & .0000 & .4800 & .0500 & .0000 & .0000 & 1.2500 \\
\hline$P H-148 M$ & $4.180+01$ & D & .0057 & .1539 & 1.2056 & .2235 & .3687 & .0000 & .0000 & .0000 & .0000 & .0000 & .0000 & 1.9574 \\
\hline NO-147 & $1.110+01$ & D & .0264 & .0157 & .0685 & .0000 & .0000 & .0000 & .0000 & .0000 & .0000 & .0000 & .0000 & .1106 \\
\hline$P R-244$ & $1.727 \cdot 01$ & H & .0000 & .0000 & .0105 & .0002 & .0000 & .0001 & .0043 & .0162 & .0000 & .0000 & .0000 & .0313 \\
\hline
\end{tabular}

(a) The break points between the gamma energy intervals are mid-way between the photon energy values. 
TABLE B-2 (Continued)

\begin{tabular}{|c|c|c|c|c|c|c|c|c|c|c|c|c|c|c|}
\hline ISUTUPE & HALF LIFE & & 0.1 & 0.3 & 0.6 & 0.8 & 1.0 & 1.25 & 1.0 & 2.0 & 2.4 & 2.9 & $\$ .5$ & IUTAL \\
\hline$\bullet \bullet \bullet \bullet$ & 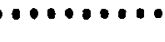 & $\cdots$ & $\cdots \cdots$ & •... & $\ldots \ldots$ & $\cdots \cdots$ & $\cdots \cdots$ & $\cdots \cdots$ & $\cdots \cdots$ & $\cdots \cdots$ & $\cdots \cdots$ & $\cdots \cdots$ & $\cdots \cdots$ & $\cdots \cdots$ \\
\hline $6 A-140$ & $4.020+01$ & $H$ & .0010 & .0440 & .2243 & .2027 & .0900 & .0027 & 1.5281 & .0000 & .1078 & .0028 & .0001 & $2, \$ 113$ \\
\hline$B A-137 M$ & $2.550+00$ & m & .0023 & .0000 & .5597 & .0000 & .0000 & .0000 & .0000 & .0000 & .0000 & .0000 & .0000 & .2620 \\
\hline$B A=140^{\circ}$ & $1.280+01$ & 0 & .0100 & .0340 & .1319 & .0000 & .0000 & .0000 & .0000 & .0000 & .0000 & .0000 & .0000 & .1625 \\
\hline $\cos -134$ & $2.050+00$ & $r$ & .0000 & .0001 & .7252 & .7049 & .0109 & .0086 & .0004 & .0000 & $.000 n$ & .0000 & .0000 & 1,0701 \\
\hline $\mid-131$ & $8.050+00$ & 0 & .0039 & .3186 & .0459 & .0123 & .0000 & .0000 & .0000 & .0000 & .0000 & .0000 & .0000 & .0807 \\
\hline$T E-129 \mathrm{~m}$ & $3.410+01$ & 0 & .0000 & .0000 & .0718 & .0222 & .0013 & .0003 & .0000 & .0000 & .0000 & .0000 & .0000 & .0962 \\
\hline$T E=129$ & 0.870401 & $M$ & .0004 & .0054 & .0578 & .0033 & .0125 & .0005 & .0000 & .0000 & .0000 & .0000 & .0000 & .0859 \\
\hline $38=124$ & $0.020+01$ & 0 & .0000 & .0000 & .0300 & .1100 & .0400 & .0000 & .8400 & .1200 & .0000 & .0000 & .0000 & 1.9000 \\
\hline$S B-125$ & $2.710+00$ & $r$ & .0150 & .1390 & .2755 & .0000 & .0000 & .0000 & .0000 & .0000 & .0000 & .0000 & .0000 & .4281 \\
\hline$C D-115 M$ & $4.300+01$ & $D$ & .0000 & .0000 & .0000 & .0000 & .0200 & .0100 & .0000 & .0000 & .0000 & .0000 & .0000 & .0300 \\
\hline $16-108 M$ & $1.300+02$ & $r$ & .0050 & .38 uv & $.55^{\circ} 0$ & .0446 & .0090 & .0000 & .0000 & .0000 & .0000 & .0000 & .0000 & 1.0970 \\
\hline $16-110 \mathrm{M}$ & $2.550+02$ & $D$ & .0042 & .0158 & .7617 & 1.0315 & .3195 & .3642 & .2952 & .0020 & .0022 & .0000 & .0000 & 2.7903 \\
\hline$R H=100$ & $2.990+01$ & $\mathbf{s}$ & .0000 & .0006 & .1709 & .0038 & .0160 & .0055 & .0040 & .0016 & .0017 & .0003 & .0000 & .0044 \\
\hline$R U=103$ & $3.980+01$ & 0 & .0002 & .0025 & .4828 & .0000 & .0000 & .0000 & .0000 & .0000 & .0000 & .0000 & .0000 & .4855 \\
\hline$N 8-95$ & $3.500+01$ & 0 & .0000 & .0000 & .0000 & .7051 & .0000 & .0000 & .0000 & .0000 & .0000 & .0000 & .0000 & $9 ! 651$ \\
\hline $2 R=95$ & $0.550+01$ & $v$ & .0000 & .0014 & .0000 & .7347 & .0000 & .0000 & .0000 & .0000 & .0000 & .0000 & .0000 & ? ?361 \\
\hline$R A-86$ & $1.866+01$ & $D$ & .0000 & .0000 & .0000 & .0000 & .0900 & .0000 & .0000 & .0000 & .0000 & .0000 & .0000 & 90900 \\
\hline $\cos 58$ & $7.140+01$ & 0 & .0000 & .0000 & .1530 & .8062 & .0000 & .0000 & .0104 & .0000 & .0000 & .0000 & .0000 & .9696 \\
\hline 60.60 & $5.200+00$ & $Y$ & .0000 & .0000 & .0000 & .0000 & .0000 & 2.5000 & .0000 & .0000 & .0000 & .0000 & .0000 & 2.9000 \\
\hline
\end{tabular}


TABLE B-3
NEUTRON YIELDS FOR IMPORTANT ISOTOPES

\begin{tabular}{|c|c|c|c|c|c|c|c|c|c|c|}
\hline \multirow[b]{2}{*}{ Isotope } & \multirow{2}{*}{$\begin{array}{l}\text { Approximate } \\
\text { Energy of } \\
\text { Main } \\
\text { Alpha } \\
\text { Particle } \\
\text { (MeV) }\end{array}$} & \multirow{2}{*}{$\begin{array}{c}\text { Intensity } \\
\text { of Alphia } \\
\text { Particles } \\
\text { Per } \\
\text { Disintegration } \\
(\%)\end{array}$} & \multirow[b]{2}{*}{$\begin{array}{l}\text { Total } \\
\text { Half-Life } \\
\text { (years) }\end{array}$} & \multirow{2}{*}{$\begin{array}{c}\text { Spontaneous } \\
\text { Fission } \\
\text { Half-Life (b) } \\
\text { (years) }\end{array}$} & \multirow{2}{*}{$\begin{array}{l}\text { Number } \\
\text { of Veutrons } \\
\text { Per } \\
\text { Spontaneous } \\
\text { Fission (b) }\end{array}$} & \multicolumn{2}{|c|}{$\begin{array}{c}\text { Yield From }(\alpha, \mathrm{n}) \\
\text { Reactions } \\
\text { (neutrons per sec-curre) }\end{array}$} & \multirow{2}{*}{$\begin{array}{c}\text { Yold from } \\
\text { Spontaneous } \\
\text { Fission } \\
\text { (neutrons per } \\
\text { sec-curie) }\end{array}$} & \multicolumn{2}{|c|}{$\begin{array}{l}\text { Total Neutron Yield } \\
\text { (neutrons per sec-curfe) }\end{array}$} \\
\hline & & & & & & $\begin{array}{c}\text { Fertile } \\
\text { Particles } \\
\left(\mathrm{UO}_{2}\right)(\mathrm{c})\end{array}$ & $\begin{array}{c}\text { Fissile } \\
\text { Particles } \\
\left(\mathrm{UC}_{3} \mathrm{O}_{0.5}\right)(\mathrm{c}) \\
\end{array}$ & & $\begin{array}{c}\text { Fertile } \\
\text { Particles } \\
\left(\mathrm{UO}_{2}\right)(\mathrm{c}) \\
\end{array}$ & $\begin{array}{c}\text { Fissile } \\
\text { Particles } \\
\left(\mathrm{Uc}_{30} \mathrm{O}_{0.5}\right)^{(\mathrm{C})} \\
\end{array}$ \\
\hline $\begin{array}{l}\mathrm{Cm}-242 \\
\mathrm{Cm}-244\end{array}$ & $\begin{array}{l}6.1 \\
5.8\end{array}$ & $\begin{array}{l}100 \\
100\end{array}$ & $\begin{array}{l}4.46-1 \\
1.79+1\end{array}$ & $\begin{array}{r}7.2+6 \\
21.4+7\end{array}$ & $\begin{array}{l}n_{3} \\
n_{3}\end{array}$ & $\begin{array}{l}1.28+3 \\
1.01+3\end{array}$ & $\begin{array}{l}2.46+3 \\
1.92+3\end{array}$ & $\begin{array}{l}6.95+3(d) \\
1.45+5(d)\end{array}$ & $\begin{array}{l}8.23+3 \\
1.46+5\end{array}$ & $\begin{array}{l}9.41+3 \\
1.47+5\end{array}$ \\
\hline $\begin{array}{l}\text { Am:-241 } \\
\text { Am-243 }\end{array}$ & $\begin{array}{l}5.5 \\
5.3\end{array}$ & $\begin{array}{l}100 \\
100\end{array}$ & $\begin{array}{l}4.33+2 \\
7.37+3\end{array}$ & $2,+14$ & 2.3 & $\begin{array}{l}7.80+2 \\
6.50+2\end{array}$ & $\begin{array}{l}1.51+3 \\
1.23+3\end{array}$ & $\begin{array}{c}\text { Sma11 } \\
\text { Assumed sma11 }\end{array}$ & $\begin{array}{l}780+2 \\
6.50+2\end{array}$ & $\begin{array}{l}1.51+3 \\
1.23+3\end{array}$ \\
\hline $\begin{array}{l}\mathrm{Pu}-238 \\
\mathrm{Pu}-239 \\
\mathrm{Pu}-240\end{array}$ & $\begin{array}{l}5.5 \\
5.2 \\
5.2\end{array}$ & $\begin{array}{l}100 \\
100 \\
100\end{array}$ & $\begin{array}{l}8.78+1 \\
2.44+4 \\
6.54+3\end{array}$ & $\begin{array}{l}4.9+10 \\
5.5+15 \\
1.17+11(e)\end{array}$ & $\begin{array}{l}2.26 \\
2.17\end{array}$ & $\begin{array}{l}7.80+2 \\
5.90+2 \\
5.90+2\end{array}$ & $\begin{array}{l}1.51+3 \\
1.12+3 \\
1.12+3\end{array}$ & $\begin{array}{l}1.5+2(\mathrm{~d}) \\
\operatorname{Sma11} \\
4.49+3\end{array}$ & $\begin{array}{l}9.30+2 \\
5.90+2 \\
5.08+3\end{array}$ & $\begin{array}{l}1.66+3 \\
1.12+3 \\
5.61+3\end{array}$ \\
\hline$N_{p}-237$ & 4.8 & 100 & $2.14+6$ & $>1 \quad+18$ & & $4.02+2$ & $7.70+2$ & Smal1 & $4.02+2$ & $7.70+2$ \\
\hline $\begin{array}{l}v-232 \\
v-233 \\
v-234 \\
v-235 \\
U-236 \\
v-238\end{array}$ & $\begin{array}{l}5.3 \\
4.8 \\
4.8 \\
4.4 \\
4.5 \\
4.2\end{array}$ & $\begin{array}{l}100 \\
100 \\
100 \\
100 \\
100 \\
100\end{array}$ & $\begin{array}{l}7.2+1 \\
1.58+5 \\
2.44+5 \\
7.04+8 \\
2.34+7 \\
4.47+9\end{array}$ & $\begin{array}{cl}8 & +13 \\
23 & +17(\mathrm{f}) \\
2 & +16 \\
1.9+17 \\
2 & +16 \\
2 & +19(\mathrm{~g})\end{array}$ & $\begin{array}{l}22 \\
22 \\
\sim 2 \\
1.95\end{array}$ & $\begin{array}{l}6.50+2 \\
4.02+2 \\
4.02+2 \\
2.64+2 \\
2.95+2 \\
2.11+2\end{array}$ & $\begin{array}{l}1.23+3 \\
7.70+2 \\
7.70+2 \\
6.30+2 \\
6.40+2 \\
5.90+2\end{array}$ & $\begin{array}{r}\text { Smal1 } \\
\text { Smal1 } \\
\text { Smal1 } \\
2.74+2 \\
8.66+1 \\
4.49+4\end{array}$ & $\begin{array}{l}6.50+2 \\
4.02+2 \\
4.02+2 \\
5.38+2 \\
3.82+2 \\
4.51+4\end{array}$ & $\begin{array}{l}1.23+3 \\
7.70+2 \\
7.70+2 \\
9.04+2 \\
7.27+2 \\
4.55+4\end{array}$ \\
\hline $\mathrm{Pa}-231$ & 5.0 & 100 & $3.25+4$ & $\geq 1+16(\mathrm{f})$ & & $4.90+2$ & $8.80+2$ & Small & $490+2$ & $8.80+2$ \\
\hline $\begin{array}{l}\text { Th-228 } \\
\text { Th-229 } \\
\text { Th- } 230 \\
\text { Th-232 }\end{array}$ & $\begin{array}{l}5.4 \\
4.9 \\
4.7 \\
4.0\end{array}$ & $\begin{array}{l}100 \\
100 \\
100 \\
100\end{array}$ & $\begin{array}{l}1.91 \\
7.34+3 \\
7.7+4 \\
1.40+10\end{array}$ & $\begin{array}{l}\geq 1.5+17 \\
\geq 1 \quad+21\end{array}$ & & $\begin{array}{l}7.10+2 \\
4.45+2 \\
3.63+2 \\
1.66+2\end{array}$ & $\begin{array}{l}1.37+3 \\
8.10+2 \\
7.30+2 \\
5.80+2\end{array}$ & $\begin{array}{l}\text { Assumed sma11 } \\
\text { Assumed sma11 } \\
\text { Sma11 } \\
\text { Small }\end{array}$ & $\begin{array}{l}7.10+2 \\
4.45+2 \\
3.63+2 \\
1.66+2\end{array}$ & $\begin{array}{l}1.37+3 \\
8.10+2 \\
7.30+2 \\
5.80+2\end{array}$ \\
\hline $\mathrm{Ac}-225$ & 5.8 & 100 & $2.74-2$ & & & $1.01+3$ & $1.92+3$ & Assumed sma11 & $1.01+3$ & $1.92+3$ \\
\hline $\begin{array}{l}\mathrm{Ra}-223 \\
\mathrm{Ra}-224\end{array}$ & $\begin{array}{l}5.7 \\
5.7\end{array}$ & $\begin{array}{l}100 \\
100\end{array}$ & $\begin{array}{l}3.13-2 \\
9.97-3\end{array}$ & & & $\begin{array}{l}9.20+2 \\
9.20+2\end{array}$ & $\begin{array}{l}1.78+3 \\
1.78+3\end{array}$ & $\begin{array}{l}\text { Assumed smal1 } \\
\text { Assumed sma11 }\end{array}$ & $\begin{array}{l}9.20+2 \\
920+2\end{array}$ & $\begin{array}{l}1.78+3 \\
1.78+3\end{array}$ \\
\hline$F_{r}-221$ & 6.3 & 100 & $9.13-6$ & & & $1.50+3$ & $3.01+3$ & Assumed small & $1.50+3$ & $3.01+3$ \\
\hline $\begin{array}{l}\mathrm{Rn}-219 \\
\mathrm{Rn}-220\end{array}$ & $\begin{array}{l}6.7 \\
6.3\end{array}$ & $\begin{array}{l}100 \\
100\end{array}$ & $\begin{array}{l}1.25-7 \\
1.76-6\end{array}$ & & & $\begin{array}{l}2.02+3 \\
1.50+3\end{array}$ & $\begin{array}{l}3.83+3 \\
3.01+3\end{array}$ & $\begin{array}{l}\text { Assumed small } \\
\text { Assumed small }\end{array}$ & $\begin{array}{l}2.02+3 \\
1.50+3\end{array}$ & $\begin{array}{l}3.83+3 \\
3.01+3\end{array}$ \\
\hline $\begin{array}{l}\text { At }-217 \\
\text { At }-218\end{array}$ & $\begin{array}{l}7.1 \\
6.7\end{array}$ & $\begin{array}{r}100 \\
\sim 100\end{array}$ & $\begin{array}{l}1.01-9 \\
6 . \quad-8\end{array}$ & & & $\begin{array}{l}2.68+3 \\
2.02+3\end{array}$ & $\begin{array}{l}4.93+3 \\
3.83+3\end{array}$ & $\begin{array}{l}\text { Assuned small } \\
\text { Assumed small }\end{array}$ & $\begin{array}{l}2.68+3 \\
2.02+3\end{array}$ & $\begin{array}{l}4.93+3 \\
3.83+3\end{array}$ \\
\hline $\begin{array}{l}\text { Po- } 210 \\
P_{O}-212 \\
P_{0}-213 \\
P_{0}-215 \\
P_{0}-216\end{array}$ & $\begin{array}{l}5.3 \\
8.8 \\
8.7 \\
7.4 \\
6.8\end{array}$ & $\begin{array}{r}100 \\
100 \\
100 \\
2100 \\
100\end{array}$ & $\begin{array}{l}3.79-1 \\
9.5-15 \\
13-13 \\
5.6-11 \\
4.9-9\end{array}$ & & & $\begin{array}{l}6.50+2 \\
7.60+3 \\
6.04+3 \\
3.27+3 \\
2.17+3\end{array}$ & $\begin{array}{l}1.23+3 \\
1.14+4 \\
1.00+4 \\
6.02+3 \\
4.11+3\end{array}$ & $\begin{array}{l}\text { Assumed small } \\
\text { Assumed small } \\
\text { Assumed small } \\
\text { As sumed small } \\
\text { Assumed small }\end{array}$ & $\begin{array}{l}6.50+2 \\
7.60+2 \\
6.04+3 \\
3.27+3 \\
2.17+3\end{array}$ & $\begin{array}{l}1.23+3 \\
1.14+4 \\
1.00+4 \\
6.02+3 \\
4.11+3\end{array}$ \\
\hline $\begin{array}{l}\mathrm{B}_{1}-211 \\
\mathrm{~B}_{1}-212\end{array}$ & $\begin{array}{l}6.6 \\
6.1\end{array}$ & $\begin{array}{r}\sim 100 \\
36\end{array}$ & $\begin{array}{l}4.07-6 \\
1.15-4\end{array}$ & & & $\begin{array}{l}1.88+3 \\
4.61+2\end{array}$ & $\begin{array}{l}3.56+3 \\
8.90+2\end{array}$ & $\begin{array}{l}\text { Assumed small } \\
\text { Assuned small }\end{array}$ & $\begin{array}{l}1.88+3 \\
4.61+2\end{array}$ & $\begin{array}{l}3.56+3 \\
8.90+2\end{array}$ \\
\hline
\end{tabular}

(a) From Ref. 9 .

(b) Information is given in these columns for some of the isotopes to evaluate the neutron yields from spontaneous fission. The spontaneous fission half-lives are obtained from Ref. 5 except where noted. Except for the curium 1 sotopes, the number of neutrons per spontaneous fission were obtalned from Ref. 18

(c) Or for another heavs nuclide in place of uranium.

(d) From Ref. 19.

(e) From Ref. 18.

(f) From Ref. 20.

(h) From Ref. 13. 
APPENDIX C

DESCRIPTION OF SFUEL CODE 
APPENDIX C

DESCRIPTION OF SFUEL CODE

The SFUEL computer code was developed to calculate decay heat, gamma dose rates, and neutron source terms from the curie values for the contributing isotopes and from other basic input information. The code was designed to provide versatility while keeping input easy and to a minimum.

SFUEL will handle up to seven fuel types made up of two particle types each, 53 different chemical elements with 129 isotopes, and five decay times. Specific information on fuel types, elements, and decay times is given in Table $\mathrm{C}-1$.

Basic input information for each isotope includes half-life, disintegration energies, neutron conversion factors, gamma energy spectra, and curie values. Using this information, SFUEL will calculate and print out a variety of information for the individual isotopes and chemical elements in a fuel element: decay heat in watts, gamma dose rates in rad per $\mathrm{hr}$ at one meter away, neutron source terms in neutrons per sec, and the 10 highest contributing isotopes and elements for each case, as well as the input data.

C. 1. MAIN PROGRAM - SFUEL

The main program serves as a block-data routine for the chemical element names and their corresponding symbols, and reads in data and calls the subroutines needed for the desired calculations noted on an option card. The main program calculates the decay constant for each isotope, using the half-life, converting it to seconds, and using the expression

$$
\frac{0.693}{\text { half-1ife in seconds }}
$$




\section{C.2. SUBROUTINE - WATTS}

If the heating rate in watts is desired, this subroutine is called. Subroutine iterations are done for each decay time. For each isotope in the fertile or fissile particles of a fuel element, the heating rate is first calculated according to disintegration type (alpha particle, beta particle, and gamma ray) by using the appropriate curie values, the alpha, gamma, and beta decay energies in MeV/disintegration, and the equation

$$
\begin{aligned}
\frac{\text { watts }}{\text { fuel element }}= & \frac{\text { curies }}{\text { fuel element }} \times \frac{\mathrm{MeV}}{\text { disintegration }} \\
& \times\left(3.7 \times 10^{10} \frac{\mathrm{disintegrations}}{\mathrm{sec}-\text { curie }} \div 6.25 \times 10^{12} \frac{\mathrm{MeV} / \mathrm{sec}}{\mathrm{watt}}\right)
\end{aligned}
$$

The watts for each disintegration type are then summed to find total watts for each isotope.

After the heating rate is found for each isotope, the results for like disintegration types are summed to determine the alpha, beta, and gamma heating rates for both the fertile and the fissile particles in a fuel element. Total heating rates for the fertile and fissile particles are then found by summing the total of each disintegration type. A percent contribution from gamma rays is determined by dividing the total gamma heating by the total heating and multipling by 100 .

The decay heat in watts is then printed. A calculation is performed to determine if any isotope has a curie value less than or equal to $10^{-10}$ at a particular decay time for all fuel and particle types. For any of these isotopes, printing is suppressed to eliminate negligible values. In conjunction with the printing, SFUEL determines which isotopes are associated with each chemical element, sums the heating rate from the associated isotopes, and determines the percent contribution from gamma rays for the element. After each group of isotopes associated with an element is printed, the element name, element heating, and percent contribution by gamma rays are printed. Elements are not printed unless at least one associated isotope 
has a sufficient curie value to be printed. For any type of fuel, after all isotopes and associated elements at a given time are printed, the grand total heating rate for that fuel and particle type is printed along with the tota1 alpha, beta, and gamma heating rates.

After heating rates for all fuel and particle types at a given decay time are calculated, SFUEL reorders the isotopes and elements into separate tables according to their contribution to the heating rate for that time, fuel type, and particle type. The 10 highest contributors by isotope and by element, their heating rates, and their percent contributions to the total heating rates are then stored for later use by SFUEL.

C.3. SUBROUTINE - DOSE

If the gamma dose rate in rad/hr at one meter away from the isotopes in a fuel element is desired, this subroutine is called. Iterations of the entire subroutine are done for each decay time. For each isotope that has a gamma energy spectrum input to SFUEL, the dose rate for each fuel and particle type and for each decay time is calculated at each of 11 gamma energy groups $\left(E_{i}\right)$ using the equation

$\frac{\frac{\mathrm{rad}}{\mathrm{hr}}\left(E_{i}\right)}{\text { fuel element }}=\frac{\left(\frac{\text { curies }}{\text { fuel element }} \times 3.7 \times 10^{10} \frac{\text { disintegration }}{\text { sec-curie }}\right)\left(\frac{\mathrm{MeV}}{\text { disintegration }}\left(E_{i}\right)\right)}{\frac{\mathrm{MeV} / \mathrm{cm}^{2}-\mathrm{sec}}{\mathrm{rad} / \mathrm{hr}}\left(E_{i}\right) \times 4 \pi 100^{2} \mathrm{~cm}^{2}}$,

where $\mathrm{MeV} /$ disintegration $\left(E_{i}\right)$ is the gamma energy spectrum input to SFUEL. The accepted energy groups and their corresponding conversion factors of $\mathrm{MeV} / \mathrm{cm}^{2}-\mathrm{sec}$ to $\mathrm{rad} / \mathrm{hr}$ are given in Table $\mathrm{C}-2$.

The total $\mathrm{rad} / \mathrm{hr}$ for the isotope is then found from

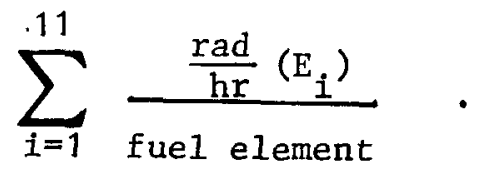


The gamma dose rates are then printed. If an isotope has a curie value greater than $10^{-10}$ for any fuel or particle type at a given decay time and has a gamma energy spectrum with at least one non-zero value, the isotope and the corresponding dose rates are printed. Only energy groups with non-zero values are printed. In conjunction with the printing, SFUEL determines which isotopes are associated with each chemical element and then sums the dose rates from these isotopes. After information for the isotopes of an element is printed, the element name, element dose rate by gamma energy group, and total dose rate for the element are printed. An element is included in the table only if at least one associated isotope is printed. Gamma energy groups with zero values for the elements are not printed.

At the conclusion of each decay time for each fuel and particle type, the isotopic dose rates for the gamma groups are summed to obtain total dose rates for that fuel and particle type. These numbers are printed if they are non-zero values.

The total dose rates from isotopes and elements are then reordered in their respective tables to reflect their contribution to the dose rate for that time and fuel type. The percent each contributes to the total is then determined. The 10 highest isotope contributors, 10 highest element contributors, their dose rates, and their percent contributions to the dose rates are then stored for later use by SFUEL.

\section{C.4. SUBROUTINE - NEUTRO}

If the neutron source in $\mathrm{n} / \mathrm{sec}$ is desired, this subroutine is called. Iterations of this subroutine are done for each decay time. For each fuel and particle type, and each isotope that has a neutron source associated with it for fertile and/or fissile particles, the neutron yield is calculated using the equation

$$
\frac{\text { neutrons }}{\text { sec-fuel element }}=\frac{\text { curies }}{\text { fuel element }} \times \frac{\text { neutrons }}{\text { sec-curie }} .
$$


These resultant source terms are summed by fuel and particle type to find the total neutron yields for the fertile and fissile particles for each fuel type.

The neutron source strengths are then printed. If an isotope has a curie value greater than $10^{-10}$ for any fuel and particle type at a given decay time and has a non-zero value for the fertile or fissile particle neutron yield, the isotope and the neutron source strengths are printed. In conjunction with the printing, SFUEL determines which isotopes are associated with each chemical element, sums the neutron source strengths from these isotopes, and prints the element name and element neutron source strength. An element is included in the table only if at least one associated isotope is printed.

SFUEL then calculates the percent each isotope and each element contribute to the total neutron source for each fuel and particle type. It then reorders the isotopes and elements in separate tables according to their contribution to the neutron source for each fuel and particle type. The 10 highest isotope contributors, 10 highest element contributors, their neutron yields, and their percent contributions to the total neutron yields for each decay time are then stored for later use by SFUEL.

\section{C.5. SUBROUTINE - TABLE}

This subroutine is called if any of the auxiliary tables are desired.

The first table to be printed by TABLE is entitled "Gamma Energy Release Table." It prints the data read in without any calculations. The isotope name, half-life, and its gamma energy spectrum are printed. Only isotopes with a non-zero entry in the input spectrum are printed.

The second table to be printed by TABLE is entitled "Half-Lives and Energies of Radioactive Nuclides in HTGR Spent Fuel Elements." The only item printed in this table that is calculated by SFUEL is the decay constant, which is determined in the main program. For each isotope input 
to the code, this table lists the name of the isotope, half-1ife, decay constant, alpha, beta, and gamma energies, and the fertile and fissile particle conversion from curies to neutrons per second.

The third table to be printed in TABLE gives the 10 isotopes and 10 elements with the highest contributions to each of the three main SFUEL areas: decay heat, gamma dose rate, and neutron yield for each decay time, fuel, and particle type. The percent each contributes to the total is given, along with the individual heating rate, dose rate, or neutron yield.

The last table to be printed gives the isotope name and curie values input to SFUEL for each isotope, decay time, fuel, and particle type. No calculations were involved to print any of the information in this table.

\section{C.6. MAP}

SFUEL is segmented and mapped to reduce the storage requirements of the code. Only the main program and a sorting routine are always available during program execution. The remaining subroutines are available only while being used, with the exception of NEUTRO and TABLE, which are available concurrently with each other.

\section{C.7. RUN INSTRUCTIONS}

Detailed input instructions are found in Table C-3. 
TABLE $C-1$

FUEL TYPES ACCEPTED BY SFUEL

\begin{tabular}{l|c|c}
\hline $\begin{array}{c}\text { Fuel Types, } \\
\text { Fissile \& Fertile }\end{array}$ & $\begin{array}{c}\text { Discharged at } \\
\text { Beginning of Reload }\end{array}$ & $\begin{array}{c}\text { Originally Loaded } \\
\text { at Reload }\end{array}$ \\
\hline IS Fresh U-235 & 1 & 0 \\
MS Fresh U-235 & 5 & 1 \\
23RS Recycle U-233 & 10 & 6 \\
25RS Recyc1e U-235 & 10 & 6 \\
MS Fresh U-235 & 13 & 9 \\
23RS Recycle U-233 & 13 & 9 \\
25RS Recycle U-235 & 13 & 9 \\
\hline
\end{tabular}


TABLE C-2

GAMMA ENERGY GROUPS AND CORRESPONDING MEV/ $\mathrm{CM}^{2}-\mathrm{SEC}$

TO RAD/HR CONVERSION FACTORS

\begin{tabular}{c|c}
\hline $\begin{array}{c}\text { Midpoint of } \\
\text { Energy Group } \\
(\mathrm{MeV})\end{array}$ & $\begin{array}{c}\text { Conversion } \\
\text { Factor } \\
\left(\frac{\mathrm{MeV} / \mathrm{cm}^{2}-\mathrm{sec}}{\mathrm{rad} / \mathrm{hr}}\right)\end{array}$ \\
\hline 0.1 & $5.9 \times 10^{5}$ \\
0.3 & $5.54 \times 10^{5}$ \\
0.6 & $5.44 \times 10^{5}$ \\
0.8 & $5.53 \times 10^{5}$ \\
1.0 & $5.77 \times 10^{5}$ \\
1.25 & $5.96 \times 10^{5}$ \\
1.6 & $6.28 \times 10^{5}$ \\
2.0 & $6.81 \times 10^{5}$ \\
2.4 & $7.24 \times 10^{5}$ \\
2.9 & $7.78 \times 10^{5}$ \\
3.5 & $8.15 \times 10^{5}$ \\
\hline
\end{tabular}


TABLE C-3

SFUEL INPUT INSTRUCTIONS

\begin{tabular}{lllll} 
Card & Format & Cols. & Name & \multicolumn{1}{c}{ Description } \\
1 & $12 \mathrm{~A} 6$ & $1-72$ & TITLE & $\begin{array}{l}\text { Problem Title } \\
\text { Calculate heating rate in watts } \\
0=\text { Yes, } 1=\text { No }\end{array}$ \\
& $4 \mathrm{II}$ & 1 & IWA & $\begin{array}{l}\text { Calculate dose rate in } \mathrm{rad} / \mathrm{hr} \\
0=\text { Yes, } 1=\text { No }\end{array}$ \\
& 2 & IDO & $\begin{array}{l}\text { Calculate neutron source in } \mathrm{n} / \mathrm{sec} \\
0=\text { Yes, } 1=\text { No } \\
\text { Print out auxiliary tables } \\
0=\text { Yes, } 1=\text { No }\end{array}$
\end{tabular}

Repeat cards 3 thru 8 for each isotope

$2 \mathrm{~A} 6 \quad 1-12$ ISO Isotope name

E11.5 13-23 HALIFE Numeric portion of half-life

A1 24 SYM Half-life unit (S, M, H, D, Y)

3E12.6 25-36 TALPHA Total alpha disintegration energy

37-38 TBETA Average beta disintegration energy

49-60 TGAMMA Total gamma disintegration energy

4 2E12.6 1-12 FENEUT Neutrons/sec-curies for fertile particles

13-24 FINEUT Neutrons/sec-curies for fissile particles

$5 \quad 11 \mathrm{~F} 6.4 \quad$ GSPECT Gamma energy spectrum in MeV/disintegration using the following $\mathrm{MeV}$ energy ranges:
$1-6$
$0-0.2$
$7-12$
$0.2-0.45$
$13-18$
$0.45-0.7$
$19-24$
$0.7-0.9$
$25-30$
$0.9-1.125$
$31-36$
$1.125-1.425$
$37-42$
$1.425-1.8$
43-48
$1.8-2.2$ 
TABLE C-3 (Continued)

$\begin{array}{cccc}\text { Card } & \text { Format } & \text { Cols. Name } & \text { Description } \\ (5 \text { cont. }) & 49-54 & & 2.2-2.65 \\ & 55-60 & 2.65-3.2 \\ & 61-66 & E>3.2\end{array}$

Repeat cards 6 through 8 for each desired decay time. Decay times accepted by SFUEL are (in order): 180 days, 1 year, 3 years, 5 years, and 10 years. There must be a minimum of one decay time. The maximum number of times accepted are five.

6

$6 \mathrm{E} 12.6 \quad 1-12 \quad$ CURIES

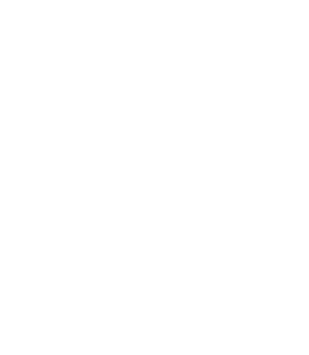

13-24 CURIES

25-36 CURIES

37-48 CURIES Curies from MS fresh U-235 discharged at reload 5 - fissile particle

49-60 CURIES Curies from 23RS recycle U-233 discharged at reload 13 - fertile particle

61-72 CURIES Curies from 23RS recycle U-233 discharged at reload 13 - fissile particle

$7 \quad 6 \mathrm{E} 12.6 \quad 1-12 \quad$ CURIES

Curies from 25RS recycle U-235 discharged at reload 10 - fertile particle

13-24 CURIES Curies from 25RS recycle U-235 discharged at reload 10 - fissile particle

25-36 CURIES Curies from MS fresh U-235 discharged at reload 13 - fertile particle

37-48 CURIES Curies from MS fresh U-235 discharged at reload 13 - fissile particle

49-60 CURIES Curies from 23RS recycle U-233 discharged at reload 13 - fertile particle

61-72 CURIES Curies from 23RS recycle U-233 discharged at reload 13 - fissile particle

$8 \quad 2 \mathrm{E} 12.6 \quad 1-12 \quad$ CURIES

Curies from 25RS recycle U-235 discharged at reload 13 - fertile particle

13-24 CURIES Curies from 25RS recycle U-235 discharged at reload 13, fissile particle 


\section{TABLE C-3 (Continued)}

\begin{tabular}{ccccc} 
Card & Format & Cols. & Name & \multicolumn{2}{c}{ Description } \\
$(8$ cont.) & I1 & 25 & FLAG1 & $\begin{array}{l}\text { Indicates this is last decay time to be } \\
\text { given for this isotope: } 0=\text { No, } 1=\text { Yes }\end{array}$ \\
9 & & & $\begin{array}{l}\text { Two blank cards follow last data card } \\
\text { for entire deck }\end{array}$
\end{tabular}


APPENDIX D

TABULATION OF RESULTS 
DECAY HEAT 
TABLE D-1

DECAY HEAT FOR FUEL ELEMENTS DISCHARGED AT

BEGINNING OF RELOAD 1 AND ORIGINALLY LOADED AT RELOAD 0

and

TABLE D-2

DECAY HEAT FOR FUEL ELEMENTS DISCHARGED AT BEGINNING OF RELOAD 5 AND ORIGINALLY LOADED AT RELOAD 1

\footnotetext{
NOTE: Tables $\mathrm{D}-1$ and $\mathrm{D}-2$ consist of 29 pages of computer print-out, with the two tables appearing side by side (pages $\mathrm{D}-3$ through $\mathrm{D}-31$ ).
} 
0

-

0 
IMIS SEGMENT WAS UISCMARGEO AT

THE GEGINNING UF MELHAD I AND WAS

URIGINALLY LUADEL aT HELUAD $U$
THIS SEGMENT WAS DISCHAHGEU AT

HE BEGINAING UF RELUAL S AND WAS

UKIGINALLY LUADEU AT RELUAU I

WECAY HEAT IN MATIS RER FEE. AT TINE IBU UAYS ABTER DISCHAHGE

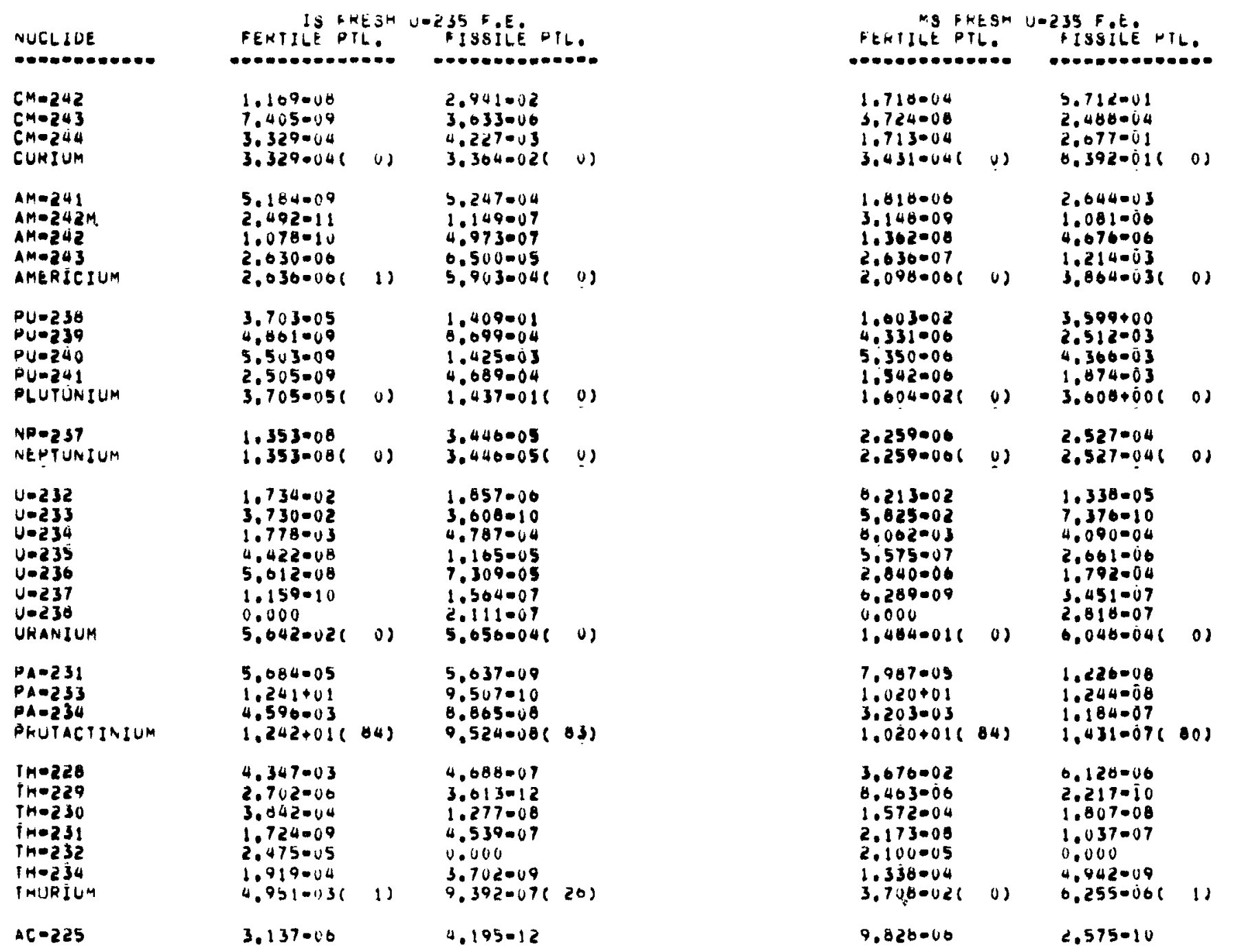




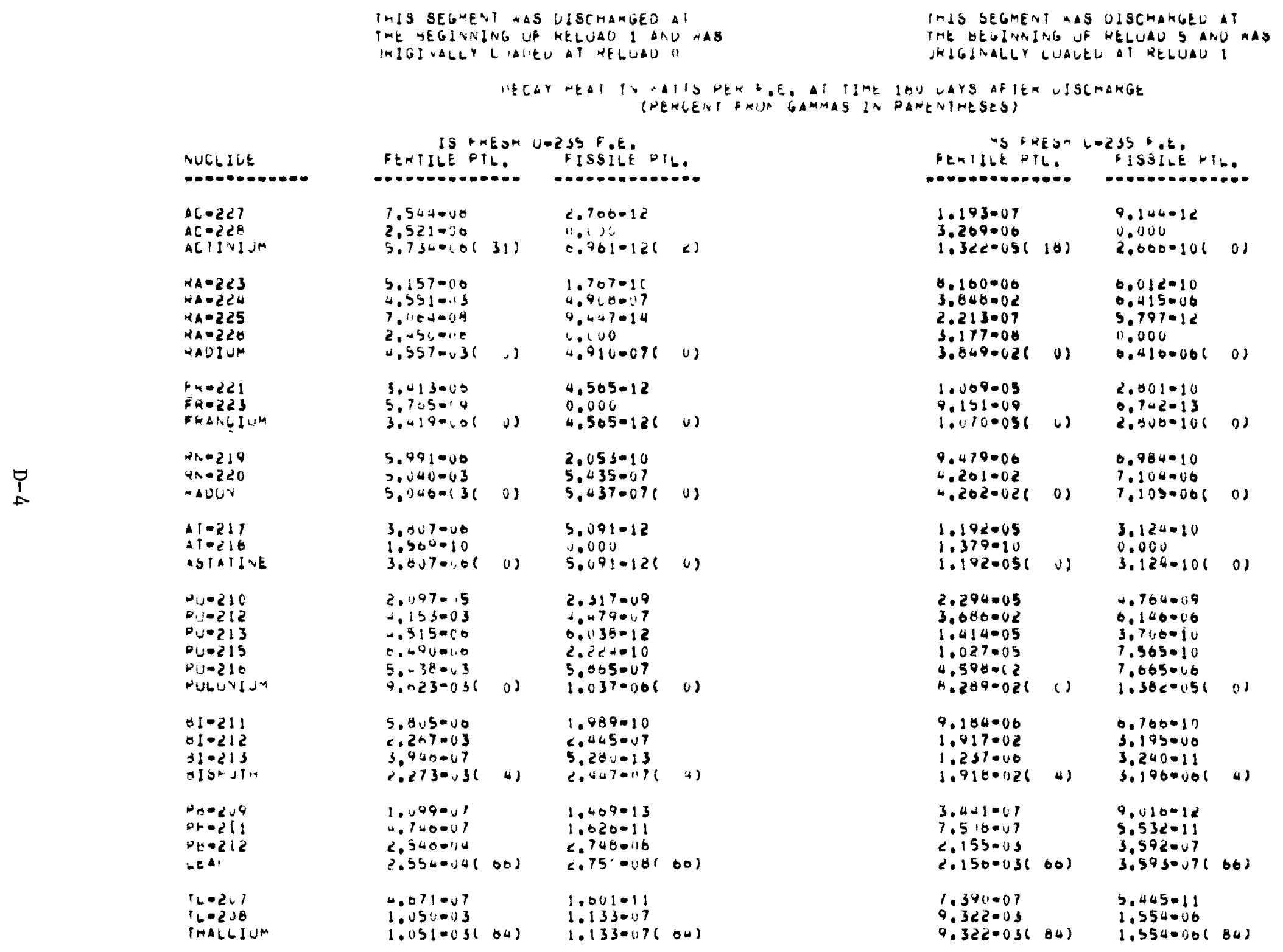




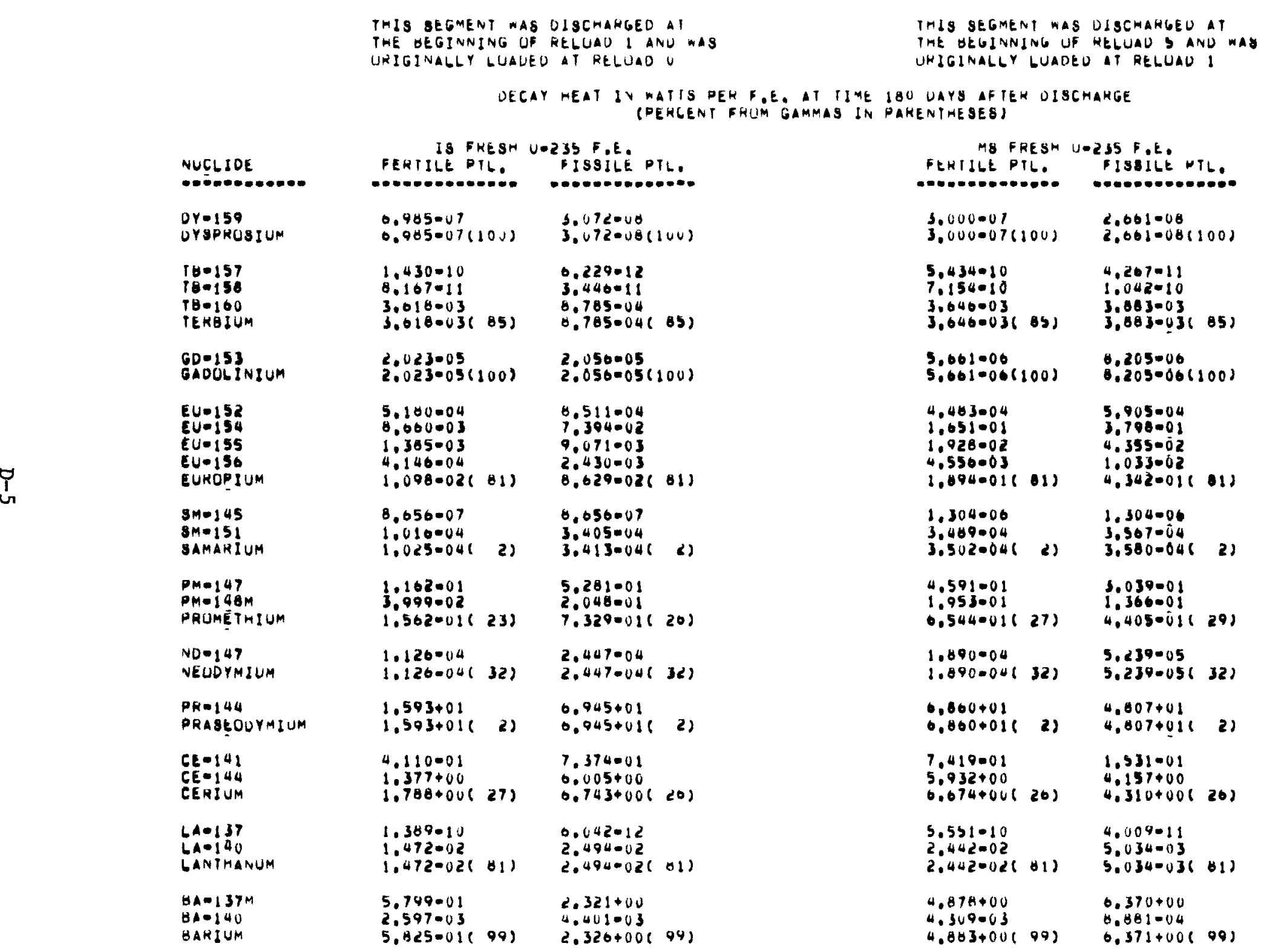


THIS SEGMENT WAS UISCMAKGEO AI

THE GEGINNING UF RELOAU I AND WAS

URIGINALLY LUADED AT RELUAD U
THIS SEGMEVT MAS OISCHAHGEU AT THE HEGINNING UR RELUAD S AND MAD

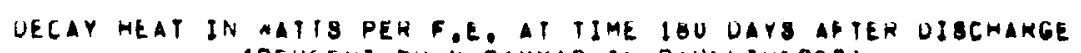
(PERGENT FRUM GAMMAS IN PAKENTMESES)

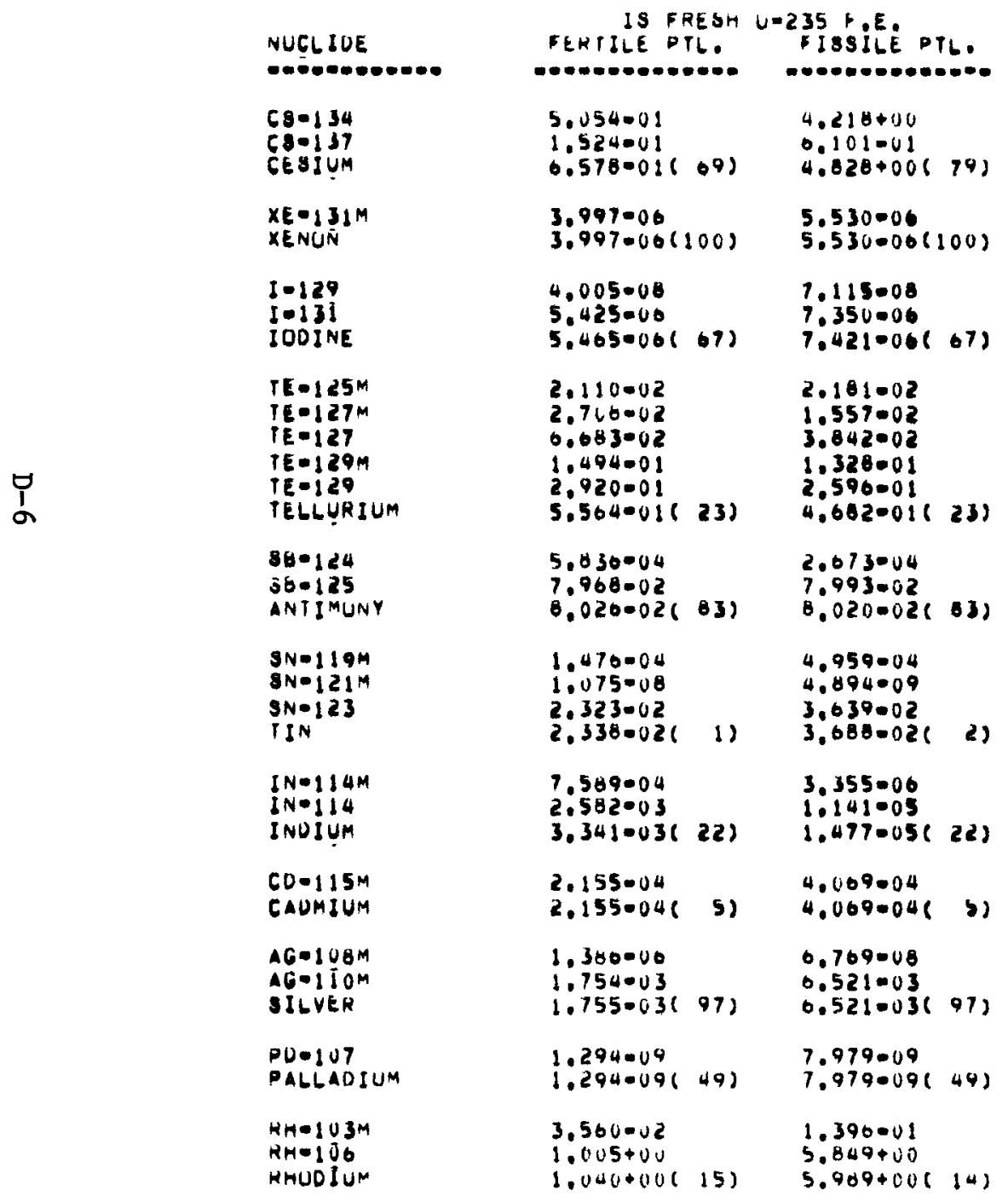

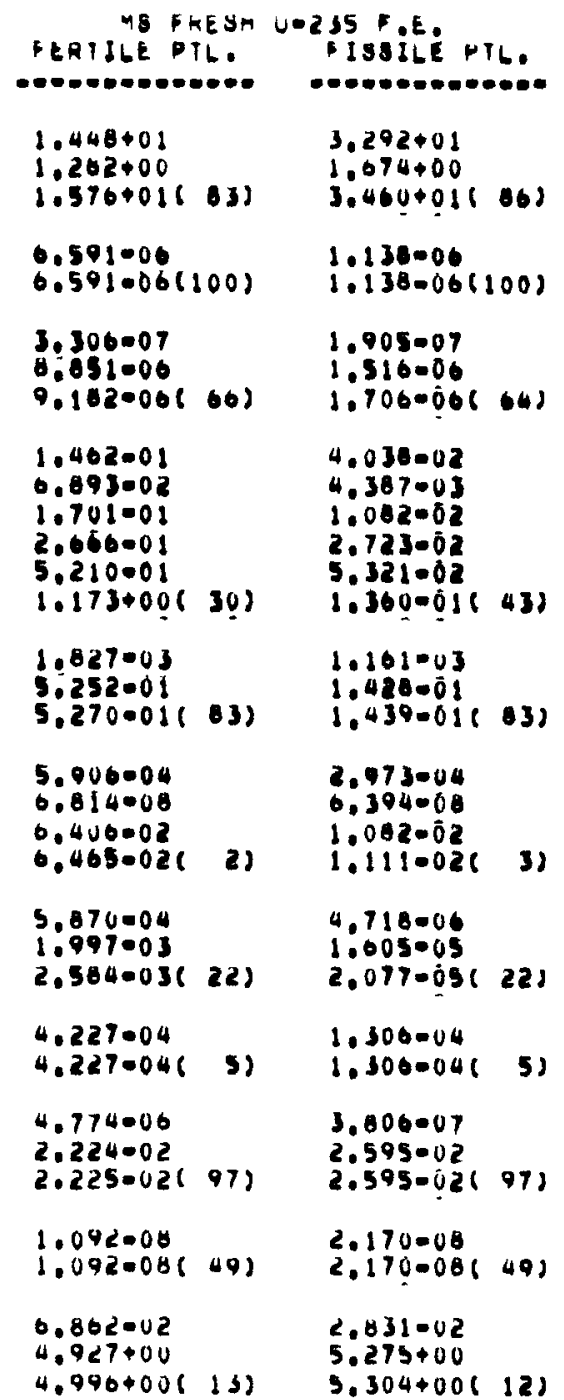


THIS SEGMENT WAS DISCMAMGEU AT THE HEGINNING OF RELUAD I INO WAS URIGINALLY LUADED AT RELOAD O
TMIS SEGMENT MAS DISCHARGEO AT THE GEGINMING OF RELOSAU SANO WAO WRIGINALLY LUADEO AI RELUAD I DECAY HEAT IA WAIIS PER E.E. AI TIME I8U UAYS AFIER OISCHARGE

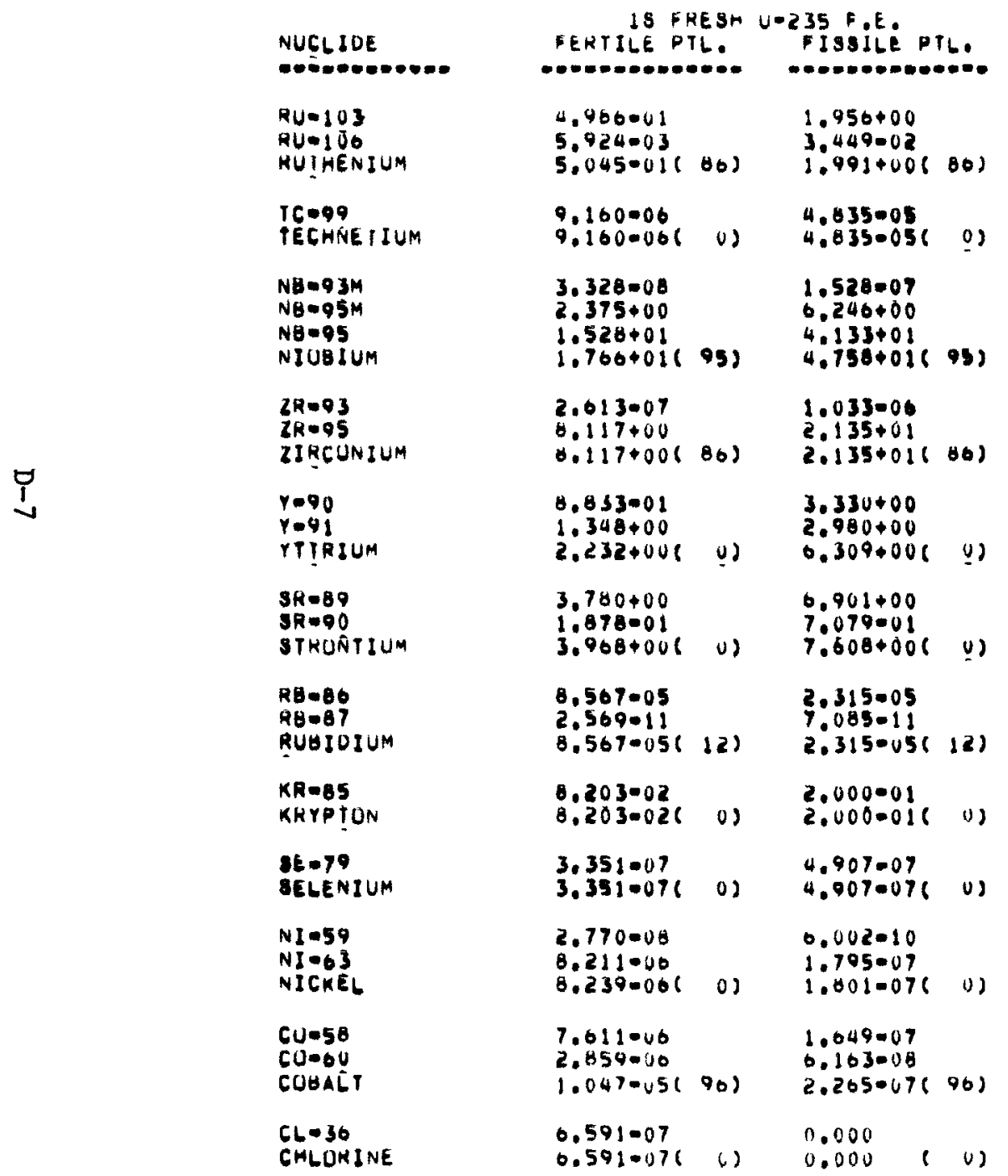

\begin{tabular}{|c|c|c|c|}
\hline $\begin{array}{l}9.012001 \\
2.905=02 \\
9.9030011\end{array}$ & 851 & $\begin{array}{l}3.965001 \\
3.111002 \\
4.2760016\end{array}$ & (1) \\
\hline $\begin{array}{l}7.294005 \\
7.2940 .051\end{array}$ & 0) & $\begin{array}{l}1.105=04 \\
1.185=046\end{array}$ & 0) \\
\hline $\begin{array}{l}6.353=07 \\
5.066+00 \\
3.330+01 \\
3.036+016\end{array}$ & 95) & $\begin{array}{l}1.250-00 \\
1.308+00 \\
8.706+00 \\
1.002+016\end{array}$ & 951 \\
\hline $\begin{array}{l}2.236000 \\
1,732+01 \\
1.732+016\end{array}$ & 861 & $\begin{array}{l}2.923-00 \\
4.471+00 \\
4.471+006\end{array}$ & 100 \\
\hline $\begin{array}{l}7.389+00 \\
2,762+00 \\
1.015+016\end{array}$ & (1) & $\begin{array}{l}9.052+00 \\
6.103001 \\
9.671+006\end{array}$ & 01 \\
\hline $\begin{array}{l}7.491+00 \\
1.571+00 \\
9.002+006\end{array}$ & $0)$ & $\begin{array}{l}1.402+00 \\
1.924+00 \\
3.327+006\end{array}$ & 03 \\
\hline $\begin{array}{l}1.942=04 \\
2.964=10 \\
1.942=046\end{array}$ & (2) & $\begin{array}{l}0.080=05 \\
1.968-10 \\
0.080-056\end{array}$ & (12) \\
\hline $\begin{array}{l}5.191=01 \\
5.1010016\end{array}$ & 0) & $\begin{array}{l}3.189001 \\
3.1890016\end{array}$ & $0)$ \\
\hline $\begin{array}{l}2.229000 \\
2.2290061\end{array}$ & נִ & $\begin{array}{l}8.773=07 \\
0.773-076\end{array}$ & $0)$ \\
\hline $\begin{array}{l}9,582=08 \\
2,780005 \\
2,790-056\end{array}$ & 01 & $\begin{array}{l}3.450=09 \\
1.004=06 \\
1.008=006\end{array}$ & $0)$ \\
\hline $\begin{array}{l}0,836=00 \\
8,464=06 \\
1,530=051\end{array}$ & $90)$ & $\begin{array}{l}2.461-07 \\
5.065-07 \\
5.520=076\end{array}$ & $(90)$ \\
\hline $\begin{array}{l}154006 \\
1540006\end{array}$ & 0) & $\begin{array}{l}0.000 \\
0.000\end{array}$ & 08 \\
\hline
\end{tabular}


THIS SEGMENT WAS DISCMARGEU AI

THE OEGINNING UF RELUAD I ANO WAS

URIGINALLY LUADEO AT HELOAD U

THIS SEGMENT MAS UISCHARGEO AT

THE BEGINAING UF RELUAL S AND WAS

UHIGINALLY LUADEU AT RELLIAO I

DECAY HEAT IN WATTS PER F.E. AT TIHE 1 GO DAYS AFIER UISCHAHGE (PEKCENT FRUM GAMMAS IN PAKENIMESES?

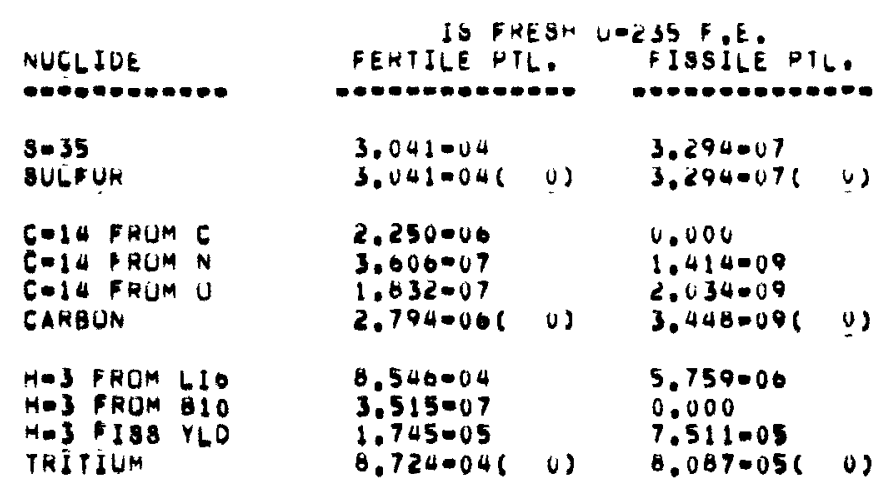

$\underset{1}{1}$

$\begin{array}{lll}\text { ALL ELEMENTS } & & \\ \text { TUTAL BETA } & 2.870+01 & 9.937+01 \\ \text { TOFAL GAMMA } & 3.713+01 & 7.64401 \\ \text { TUTAL ALPHA } & 8.247002 & 1.778001 \\ \text { GRAND TUTAL } & 6.591+01(50) & 1.700+02(43)\end{array}$

\begin{tabular}{|c|c|c|c|}
\hline $\begin{array}{l}2.807=04 \\
2.807=046\end{array}$ & u) & $\begin{array}{l}5.083=07 \\
5.0030076\end{array}$ & 01 \\
\hline $\begin{array}{l}7.905=00 \\
1.270=06 \\
0.572=07 \\
9.8520006\end{array}$ & (v) & $\begin{array}{l}0.000 \\
8.467-09 \\
1.191-00 \\
2.037-080\end{array}$ & $0 \mathrm{~s}$ \\
\hline $\begin{array}{l}1.104003 \\
19786006 \\
1.419004 \\
1.2480031\end{array}$ & 0) & $\begin{array}{l}1.236-05 \\
0.000 \\
1.926-04 \\
2.052-046\end{array}$ & 01 \\
\hline
\end{tabular}

3.800001

$3.800001(40)$
$1.059+02$
$0.432+01$
3.00001
$3.443+00$

$1.325+026402$
$7.444+01$ 
IMIS SEGMENT MAS UISCHAKGEO AI THE GEGINNING OF RELUAD I ANU WAS JRIGINALLY LUADEO AT RELUAD U
THIS SEGMENT WAS DISCHAHGEV AT THE BEGINNING UF RELUAO 5 ANU WAS THE GEGINATING UF RELUAU S ANU

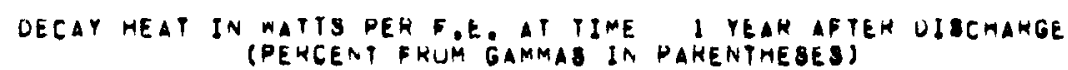

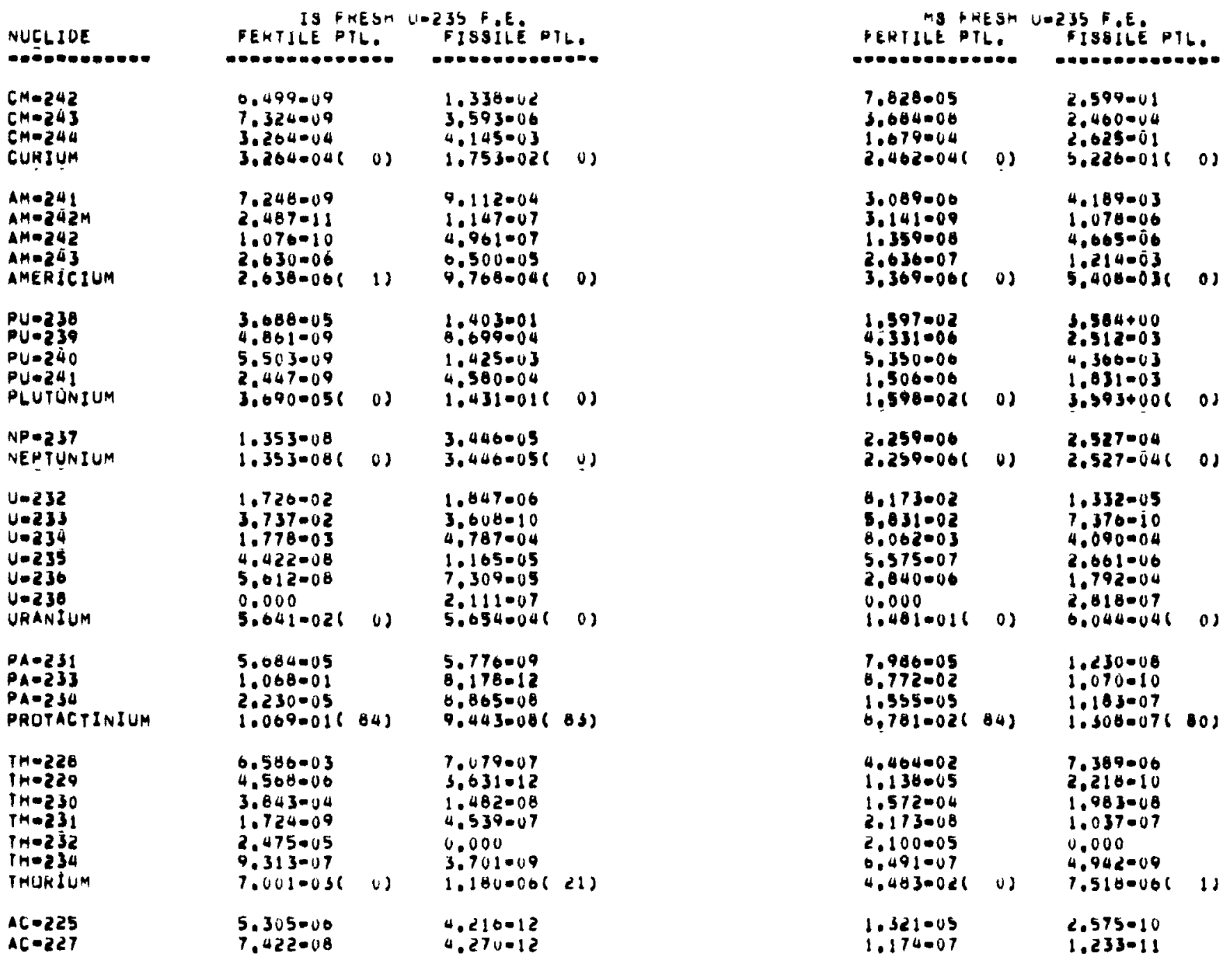


THIS SEGMENT MAS DISCMATGEO AI THE BEGINAING JF KELUAO I ANU AAS

OHE IGINALLY LUAOEU AT RELUAOU

Lecar heat IN mat's PEH F.E. AT IIME

D YEAK AF TEH DISCAARGE

(PERCEAT PMOM GAMMAS IN PARENTHESES)

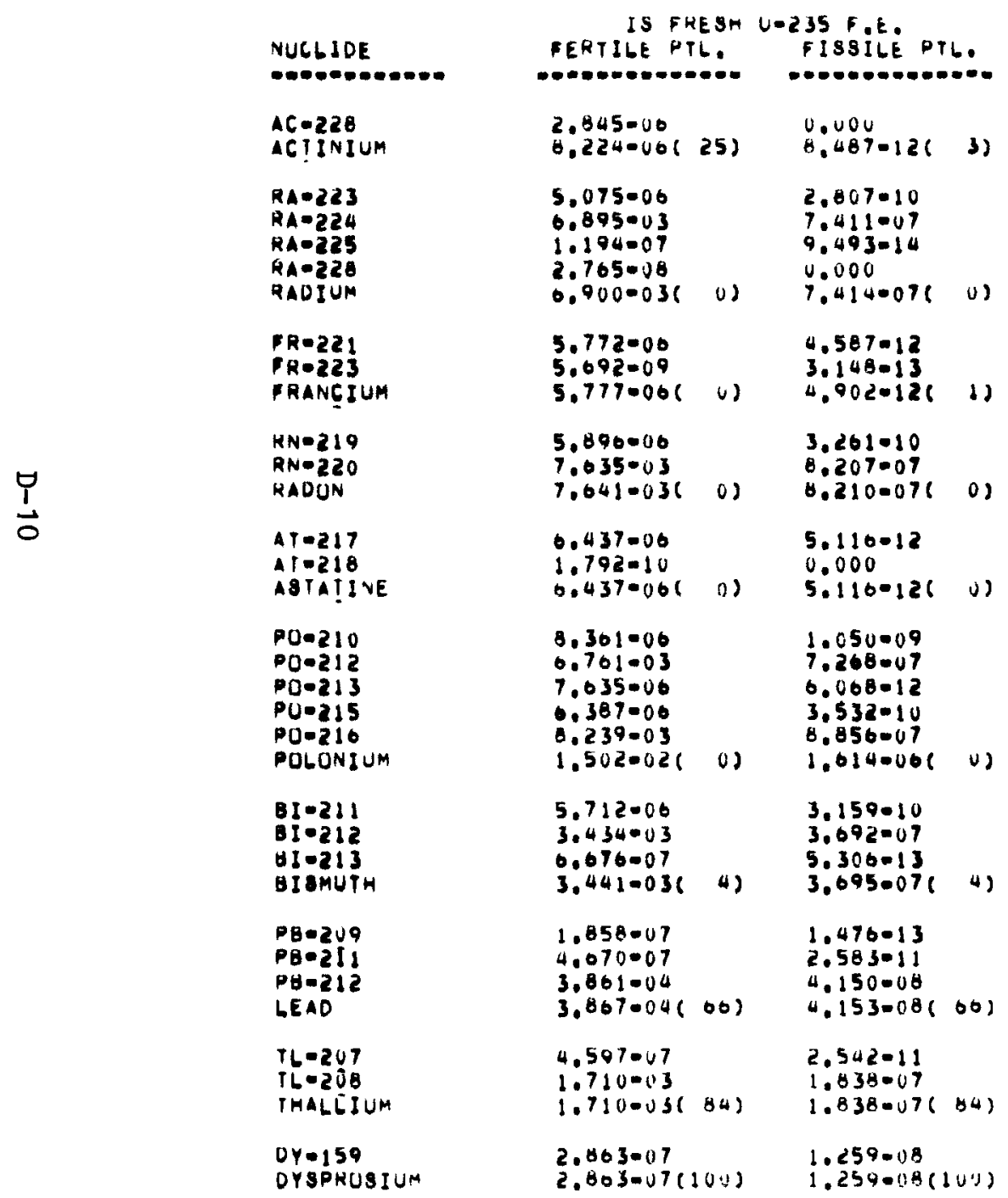

MS RKESM U-235 B.E. FERTILE VTL. FISSILE NTLO

\begin{tabular}{|c|c|c|c|}
\hline $\begin{array}{l}3.007 .00 \\
1.0410056\end{array}$ & 15) & $\begin{array}{l}0.000 \\
2.008-106\end{array}$ & 01 \\
\hline $\begin{array}{l}8.028006 \\
4.673002 \\
2.074007 \\
3.380000 \\
4.6740026\end{array}$ & 0) & $\begin{array}{l}0.220-10 \\
7.735000 \\
5.708-12 \\
0.000 \\
9.7360068\end{array}$ & (1) \\
\hline $\begin{array}{l}1.457005 \\
9.003=04 \\
1.4380056\end{array}$ & $0)$ & $\begin{array}{l}2.802-10 \\
9.210-13 \\
2.811-106\end{array}$ & 01 \\
\hline $\begin{array}{l}9.326006 \\
3.175002 \\
5.1700021\end{array}$ & 0) & $\begin{array}{l}0.548-10 \\
8.560-06 \\
0.567-061\end{array}$ & 01 \\
\hline $\begin{array}{l}2.603=05 \\
1.491-10 \\
1.003=058\end{array}$ & $0)$ & $\begin{array}{l}3.124-10 \\
0.000 \\
3.124-101\end{array}$ & 01 \\
\hline $\begin{array}{l}9.452-006 \\
0.605002 \\
1.901005 \\
1.010005 \\
5.504002 \\
1.0190016\end{array}$ & 0) & $\begin{array}{l}2.224009 \\
7.622000 \\
3.706010 \\
1.034009 \\
9.243-06 \\
1.687=056\end{array}$ & 01 \\
\hline $\begin{array}{l}9.036000 \\
2.328002 \\
1.662006 \\
2.3290026\end{array}$ & 4) & $\begin{array}{l}9.251-10 \\
3.853-106 \\
3.240-11 \\
3.854-066\end{array}$ & (4) \\
\hline $\begin{array}{l}4.020007 \\
7.367 .07 \\
2.617 .03 \\
2.016 .036\end{array}$ & 60) & $\begin{array}{l}9.017-12 \\
7.563-11 \\
4.331-07 \\
4.332-076\end{array}$ & 061 \\
\hline $\begin{array}{l}7.271 .07 \\
1.164 .02 \\
1.100 .021\end{array}$ & 641 & $\begin{array}{l}1.444-11 \\
1.427=06 \\
1.927-066\end{array}$ & 841 \\
\hline $\begin{array}{l}1.230007 \\
1.230-07\end{array}$ & & $\begin{array}{l}1.091=08 \\
1.091-06(1\end{array}$ & \\
\hline
\end{tabular}


THIS SEGMENT WAS DISCMARGEO AI THE GEGINNING UF RELUAL I ANU WAS

$$
\begin{aligned}
& \text { UECAY HEAT IN WATIS PER F.E AT TIME L YEAR AFTEK OISCMAHGE } \\
& \text { (PEKLENT FRUIM GAMMAS IN PARENIHESES) }
\end{aligned}
$$

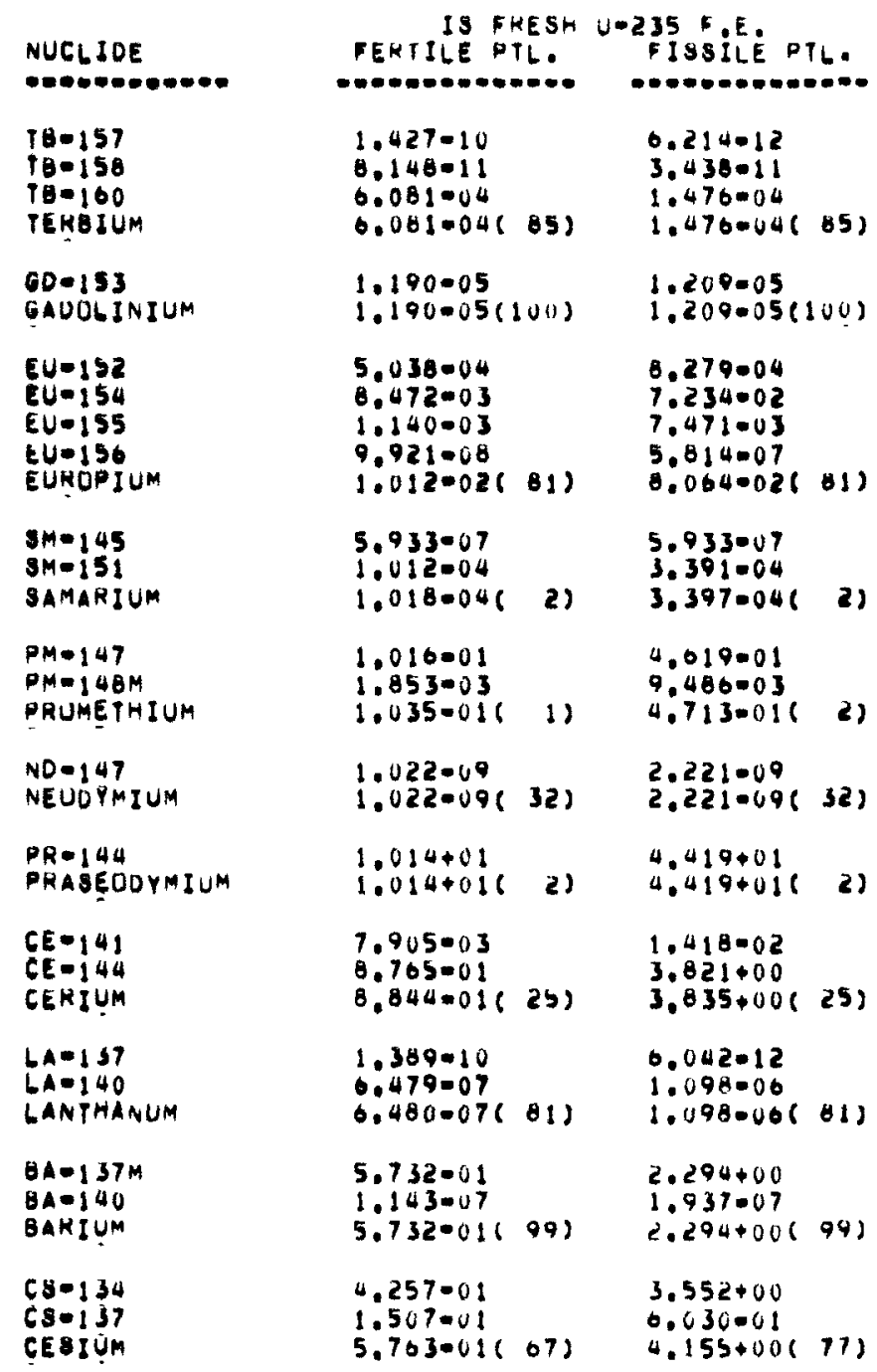

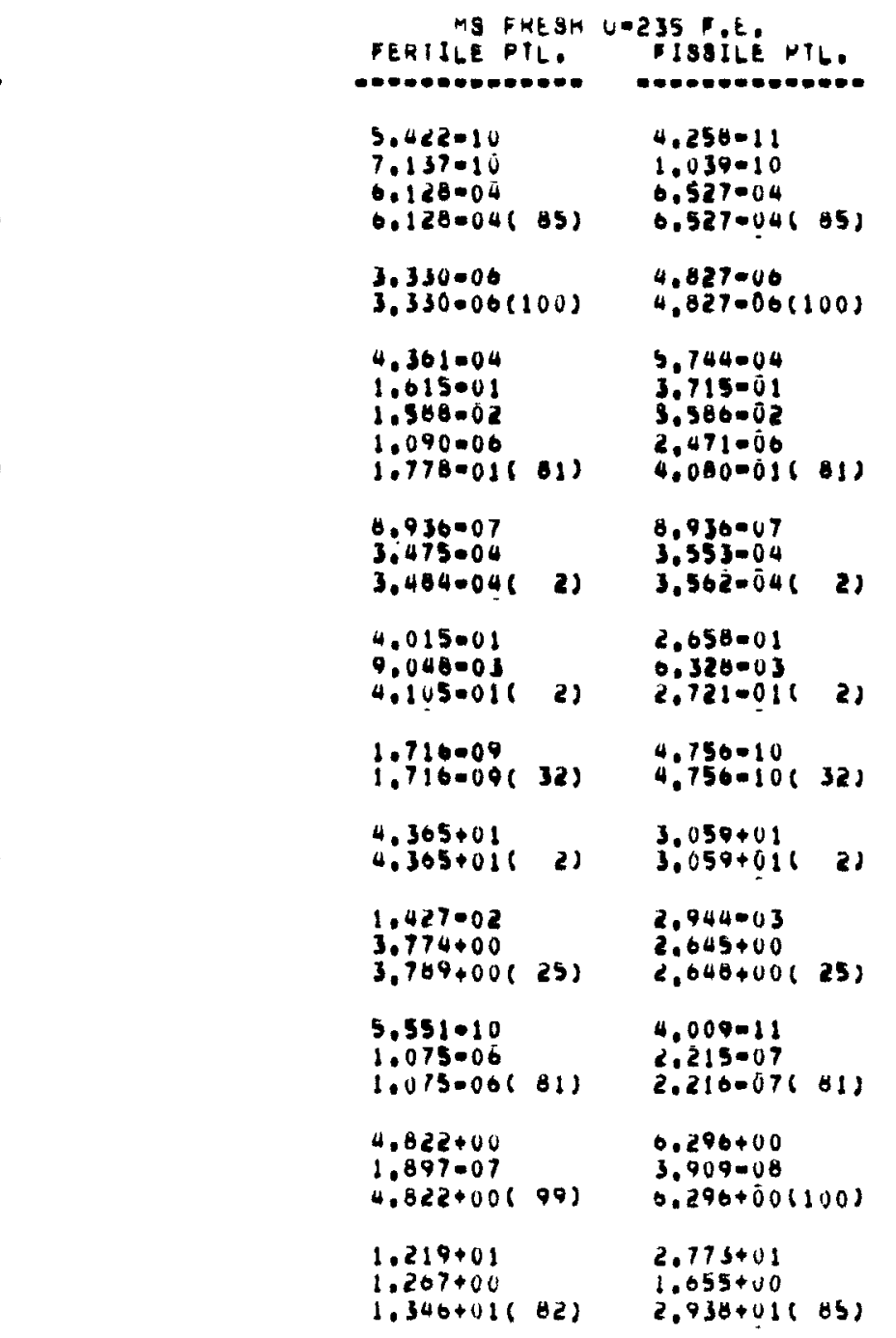

IMIS SEGMENI WAS DISCMAKGEU AT THE BEGINNING UP KELUAD S ANU MAS URIGINALLY LUAUEU AI RELUADI 
THIS SEGMENT WAS OISCHARGED AI

THE BEGINAING UF RELUAO I ANU MAS

DRIGINALLY LUADED AT RELUAD 0
THIS SEGMENT MAS UISCHAHGEO AT THE BEGINNING UF RELUAD O ANO WAB UHIGINALLY LUADEO AT RELUAD I

DECAY HEAT IN WATIS PER F.L. AT TIME I YEAH AFIEN DISCHARGE (PEHCENT FRUM GAMMAS IN PARENTHEBES)

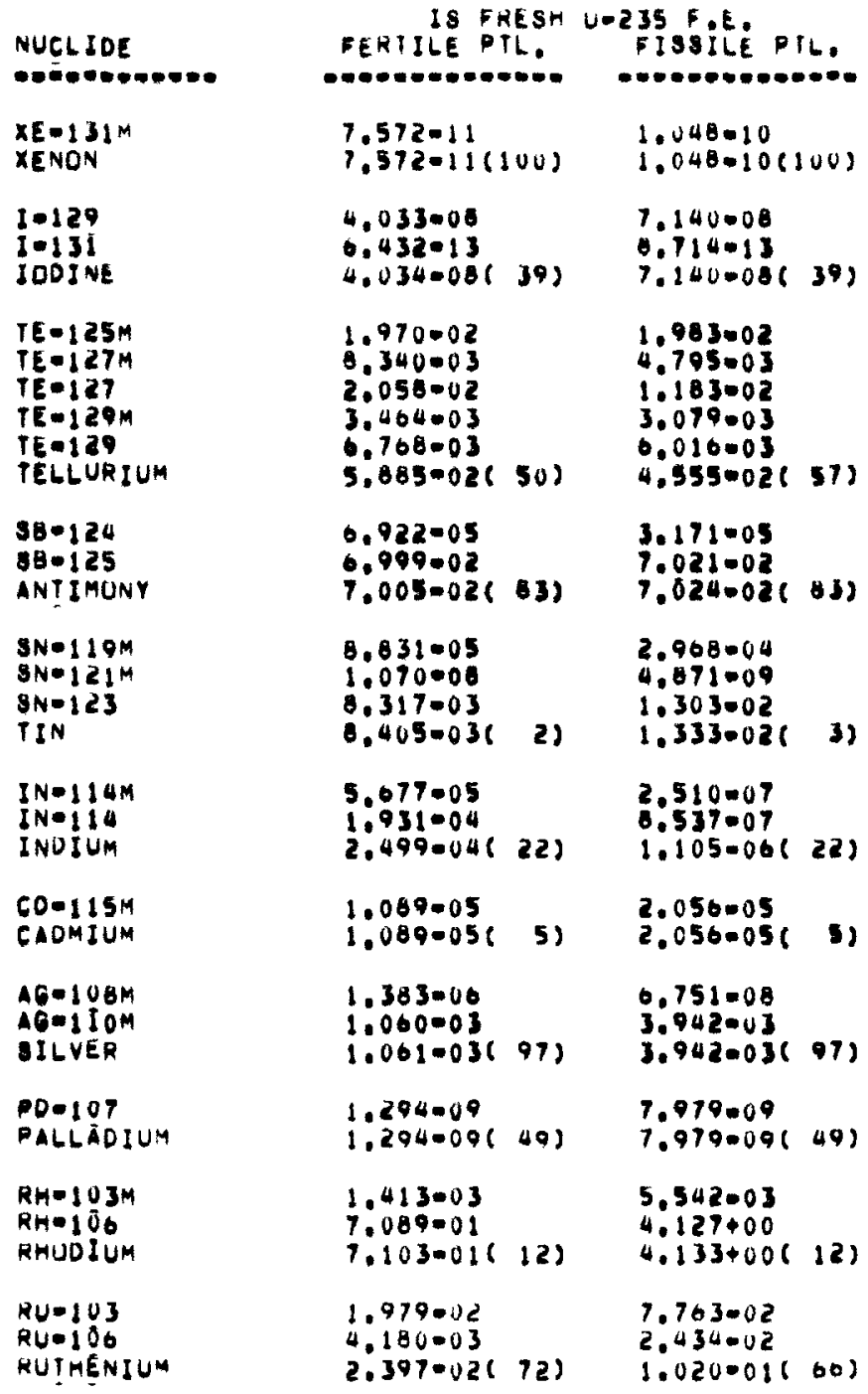

\begin{tabular}{|c|c|c|c|}
\hline $\begin{array}{l}49-10 \\
49-10(1)\end{array}$ & 1008 & $\begin{array}{l}2.156-11 \\
2.156-116\end{array}$ & 1000 \\
\hline $\begin{array}{l}3.311=07 \\
1.049012 \\
3.3110076\end{array}$ & 30) & $\begin{array}{l}1.906=07 \\
0.000 \\
1.906-076\end{array}$ & 301 \\
\hline $\begin{array}{l}1.300001 \\
2.123=02 \\
5.237=02 \\
6.180003 \\
1.200=02 \\
2.2250011\end{array}$ & 69 & $\begin{array}{l}3.558=02 \\
1.351=03 \\
3.333=03 \\
6.312=04 \\
1.2335=03 \\
4.213=028\end{array}$ & \\
\hline $\begin{array}{l}2.167-04 \\
4.613001 \\
4.0150016\end{array}$ & 631 & $\begin{array}{l}1.377-04 \\
1.254 .01 \\
1.255-016\end{array}$ & (4) \\
\hline $\begin{array}{l}3,535=04 \\
0.763=08 \\
2.294=02 \\
2.3290026\end{array}$ & 2) & $\begin{array}{l}1.779-04 \\
0.364008 \\
3.872003 \\
4.050-031\end{array}$ & $5)$ \\
\hline $\begin{array}{l}4,391005 \\
1,494004 \\
1,9330046\end{array}$ & 223 & $\begin{array}{l}3.520007 \\
1.200006 \\
1.5530006\end{array}$ & (22) \\
\hline $\begin{array}{l}2.136005 \\
2.1360056\end{array}$ & 3) & $\begin{array}{l}0.601=06 \\
6.601-061\end{array}$ & 5) \\
\hline $\begin{array}{l}4.761=06 \\
1.345=02 \\
1.3450026\end{array}$ & 971 & $\begin{array}{l}3.796=07 \\
1.560=02 \\
1.569=021\end{array}$ & $(97)$ \\
\hline $\begin{array}{l}1.002008 \\
1.0020086\end{array}$ & 491 & $\begin{array}{l}2.170008 \\
2.1700086\end{array}$ & $(40)$ \\
\hline $\begin{array}{l}2.723-03 \\
3.477+00 \\
3.479+006\end{array}$ & (2) & $\begin{array}{l}1.124=03 \\
3.722+000 \\
3.723+001\end{array}$ & ( 12$)$ \\
\hline $\begin{array}{l}3.815002 \\
2.050=02 \\
5.8050026\end{array}$ & 573 & $\begin{array}{l}1.574002 \\
2.105-02 \\
3.7690026\end{array}$ & 36 \\
\hline
\end{tabular}


THIS SEGMENT WAS LISCHARGED AI THE BEGINNING UF RELUAD I ANU WAS URIGINALLY LUADEO AT RELUAD U
TMIS SEGMENT MAS UI8CMARGEU AT THE BEGINNING UF RELUAD S AND WAS URIGINALLY LUADEU AT RELUAU I

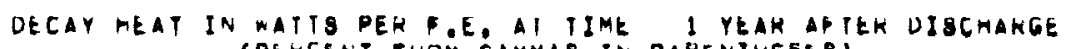
(PERGENT FKOM GAMMAS IN PARENTMESES)

\begin{tabular}{|c|c|c|c|c|}
\hline 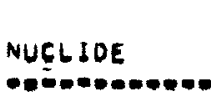 & $\begin{array}{r}\text { IS F } \\
\text { FERTILE } \\
\text { WO-OO-OE- }\end{array}$ & $\begin{array}{l}\text { RESH } \\
\text { TL. } \\
-\infty=-\end{array}$ & 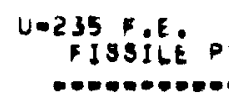 & TL. \\
\hline $\begin{array}{l}\text { TC- } 99 \\
\text { TECHNETIUM }\end{array}$ & $\begin{array}{l}9.160000 \\
9.1600066\end{array}$ & u) & $\begin{array}{l}4.835 .05 \\
4.8350051\end{array}$ & (0) \\
\hline $\begin{array}{l}\text { NB=93M } \\
\text { NBE-95M } \\
\text { NB-95 } \\
\text { NIUBIUM }\end{array}$ & $\begin{array}{l}5.382-08 \\
3.348=01 \\
2.417+00 \\
2.752+001\end{array}$ & 95) & $\begin{array}{l}2.335=07 \\
8.806=01 \\
0.387+00 \\
7.268+006\end{array}$ & (95) \\
\hline $\begin{array}{l}\text { ZR-93 } \\
\text { ZR=95 } \\
\text { ZIRCUNIUM }\end{array}$ & $\begin{array}{l}2.013=07 \\
1.144+00 \\
1.144+008\end{array}$ & $86)$ & $\begin{array}{l}1.033=06 \\
3.010+00 \\
3.010+006\end{array}$ & $(80)$ \\
\hline $\begin{array}{l}Y=90 \\
Y=91 \\
Y P T R I U M\end{array}$ & $\begin{array}{l}0.721=01 \\
1.474001 \\
1.020+006\end{array}$ & 0) & $\begin{array}{l}3.288+00 \\
3.257-01 \\
3.613+001\end{array}$ & 0) \\
\hline 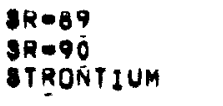 & $\begin{array}{l}3.308=01 \\
1.8540 .01 \\
5.102=018\end{array}$ & 0) & $\begin{array}{l}0.038001 \\
6.900-01 \\
1.303+001\end{array}$ & 00 \\
\hline $\begin{array}{l}\text { RB }-86 \\
\text { RB }=87 \\
\text { HUBIDIUM }\end{array}$ & $\begin{array}{l}8.787=08 \\
2.569-11 \\
8.789 .086\end{array}$ & (2) & $\begin{array}{l}2.375=08 \\
7.085011 \\
2.382-081\end{array}$ & (2) \\
\hline $\begin{array}{l}\text { KR=e5 } \\
\text { KRYPION }\end{array}$ & $\begin{array}{l}7.940002 \\
7.9400026\end{array}$ & 08 & $\begin{array}{l}1.930001 \\
1.9360011\end{array}$ & $0)$ \\
\hline $\begin{array}{l}\text { SER } \\
\text { EELETIUM }\end{array}$ & $\begin{array}{l}3.351=07 \\
3.351=076\end{array}$ & o) & $\begin{array}{l}4.907=07 \\
4.907=071\end{array}$ & $0)$ \\
\hline $\begin{array}{l}\text { NI }=59 \\
\text { NIDG } \\
\text { NICKEL }\end{array}$ & $\begin{array}{l}2.770=08 \\
8.180006 \\
8.2000006\end{array}$ & 0) & $\begin{array}{l}0.002-10 \\
1.788 .09 \\
1.7940071\end{array}$ & 0) \\
\hline $\begin{array}{l}C 0=58 \\
C 0=60 \\
C O B A T T\end{array}$ & $\begin{array}{l}1.259=06 \\
2.674=06 \\
3.9340006\end{array}$ & 96) & $\begin{array}{l}2.729008 \\
5.764008 \\
8.493-081\end{array}$ & $90)$ \\
\hline $\begin{array}{l}\text { CL-36 } \\
\text { CHLONINE }\end{array}$ & $\begin{array}{l}6.591=07 \\
6.591=076\end{array}$ & $0)$ & $\begin{array}{l}0.000 \\
0.000\end{array}$ & v) \\
\hline $\begin{array}{l}s=35 \\
\text { SULFUR }\end{array}$ & $\begin{array}{l}0.973=05 \\
0.973=056\end{array}$ & u) & $\begin{array}{l}7.554008 \\
7.5540 .086\end{array}$ & (u) \\
\hline
\end{tabular}

MS FKEST VEZ 35 FOE PERTILE PIL. BSBILE HIL.

7.294 .05 7.2940051

$1.185=04$

$8.022 \cdot 07$

$7.142=01$

$5.175+000(92)$

2.236006

$2.441+00$
$2.441+00(86)$

$7.296+00$

$\begin{array}{llll}3.019001 & 0.769-02 \\ 7.598+00( & 0) \quad 9.005+008 & 08\end{array}$

$\stackrel{P}{\omega}$

\begin{tabular}{|c|c|c|c|}
\hline $\begin{array}{l}6,555-01 \\
1.551+00 \\
2.207+006\end{array}$ & 08 & $\begin{array}{l}1.227-01 \\
1.000000 \\
2.023+006\end{array}$ & $0)$ \\
\hline $\begin{array}{l}1.992=07 \\
2.164=10 \\
1.9940095\end{array}$ & 121 & $\begin{array}{l}0.236=00 \\
1.968-10 \\
6.2560006\end{array}$ & (2) \\
\hline $\begin{array}{l}5.024001 \\
5.0240016\end{array}$ & Q9 & $\begin{array}{l}3.087=01 \\
3.087=016\end{array}$ & O J \\
\hline $\begin{array}{l}2.229000 \\
2.229=066\end{array}$ & (a) & $\begin{array}{l}0.773=07 \\
4.773=076\end{array}$ & 01 \\
\hline $\begin{array}{l}9.582000 \\
2.777-05 \\
2.787-056\end{array}$ & 01 & $\begin{array}{l}3.450009 \\
1.001=00 \\
1.0040068\end{array}$ & $0)$ \\
\hline $\begin{array}{l}1.131000 \\
7.917=06 \\
9.040=006\end{array}$ & 401 & $\begin{array}{l}4.072=08 \\
2.860007 \\
3.274=070\end{array}$ & 961 \\
\hline $\begin{array}{l}2.1340006 \\
2.1340001\end{array}$ & 08 & $\begin{array}{l}0.000 \\
0.000\end{array}$ & 01 \\
\hline $\begin{array}{l}0.436005 \\
0.4360051\end{array}$ & 01 & $\begin{array}{l}1.160-07 \\
1.1600=076\end{array}$ & 0) \\
\hline
\end{tabular}


THIS SEGMENT WAS DISCMARGED AT

THE BEGINNING UF RELUAD I ANL WAS

OHIGINALLY LOADED AT RELUAD O

DECAY HEAT IN WATIS DER F.E. AT TIME I YEAR AFTER UISCHAKGE (PERCENT FROM GAMMAS IN DARENTHESES)

NUGLIDE

$C=14$ FROM $C$

Col 14 ROM

COI4 FROM

CARBUN

HaJ FROM LI6

MOS BROM BIO

Hab FISB YLD

TRITIUM

ALG ELEMENTS

TOTAL BETA

TUTAL GAMMA

TOTAL ALPAA

GRAND TOTAL
IS GRESH U-235 F.E.

FERTILE PTL. FISSILE PIL.

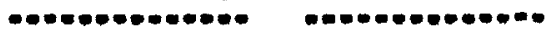

2.250000

3.600007

2.704000

0.000

1.414009

2.034009

0.306004

3.410007

$1.096-05$
6.479004

\section{$5.507-06$ \\ 0.000 \\ 7.300005 \\ Q)}

$1.344+01$

$5.341+00$
$9.592+02$

$5.695+01$

$1.783+01$

$1.887+01(28)$

1.61500

$7.494001(23)$
THIS SEGMENT MAS OISCHARGED AT ITE DEGINNING OF RELUAD 5 AND MAS UMIGINALLY LUADEO AT RELUAD I
FE MAESM

9 PRESN U.235 F.t

FERTILE PTL.

TISBILE PTL.

7.90500

7.905006
1.270000

0.571007

\subsection{0}

1.191008
$2.037-086$

$1.073 \times 03$

1.736006
1.379004

1.201 .05

0.000

$1.212=036$

$1.874=0$

1,9940045 od

$0.242+01$

2.690 .01

4.270001

$5.120+01$

3,384401

$4.114+00$

$8.974001(29) \quad 9.115+01639$ 
THIS OEGMENT WAS OISCMARGED AT
TME BEGINNING OF RELUAD I ANO WAS
UHIGINALLY LUADEO AT RELUAD O

decar meat in matrs per oge at IIME

D YEARS AFTEK OISCMAKGE

THIS SLGMENT WAS DISCHARGEU AT THE BEEINNING UF RELUAO S ANO WAE UKIGINALLY LUAUEU AI RELUAU I

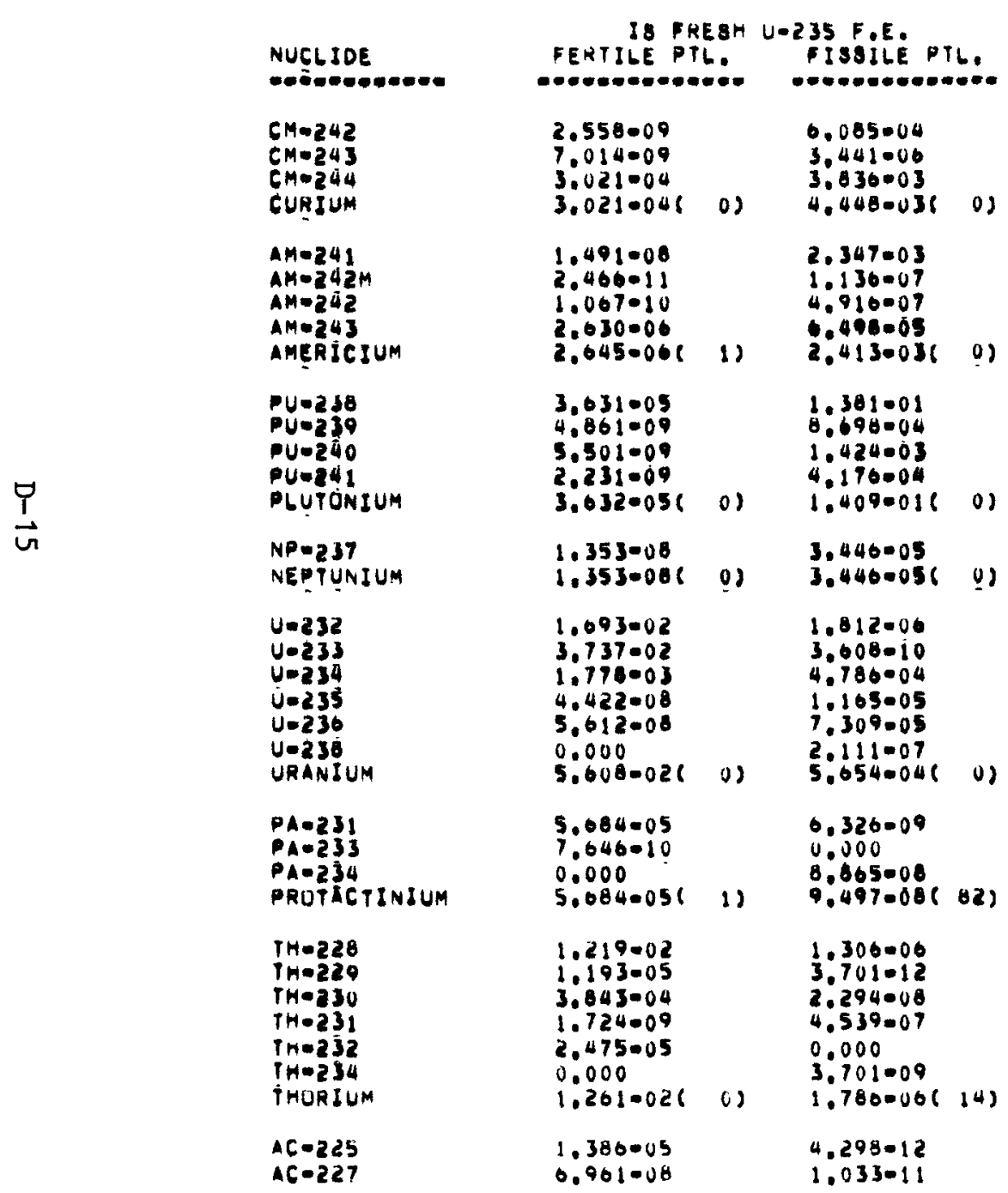

Mg FKESH U.Z35 F.E PERTILE PTL. CISSILE PTL, Cocecocos

$\begin{array}{ll}3.763006 & 1.172002 \\ 3.527-08 & 2.356004\end{array}$

$1.554004 \quad 2.429001$

$1.59200460) \quad 2.54900280)$

$7.611000 \quad 9.927 .03$

$\begin{array}{ll}3.112009 & 1.068006 \\ 1.347008 & 4.623006\end{array}$

1.347008 1.62J006

0.0910068 0) 1.115.02( 01

$1.572002 \quad 5,520400$

$4.331000 \quad 2.512-03$

$5.349006 \quad 4.365-03$

$1,5730026$ of 3,53740060$)$

$\begin{array}{llll}2.259006 & 2.527004 \\ 2.2590006 & 0) \quad 2.527-040\end{array}$

$0.017 .02 \quad 1.300005$

$5.831 .02 \quad 7.370-10$

$8.062=03 \quad 4.090-04$

$5,575=07 \quad 2.601006$

2,818007

0.0000
1.465011

$7.900005 \quad 1.2420 .08$

$6.201-10$

$\begin{array}{lll}0.000 & 1.183007 \\ 7.980005(1) & 1.308007(00)\end{array}$

$0.421 .02 \quad 1.052005$

$2.260005 \quad 2.219-10$

$\begin{array}{ll}1.574008 & 2.677006 \\ 2.173008 & 1.037 .07\end{array}$

$\begin{array}{ll}2.173000 & 1.037007 \\ 2.100005 & 0.000\end{array}$

$\begin{array}{ll}2,100005 & 0.000 \\ 0.000 & 4.042009\end{array}$

$0.0001-02(0) \quad 1.065-056 \quad 0)$

$\begin{array}{lll}1 C-227 & 0.901-08 & 1.033-11\end{array}$

$2.655005 \quad 2.576-10$

$\begin{array}{ll}2.101001 & 2.576-10 \\ 2.448-11\end{array}$ 
THIS SEGMENT "AS OISCHAKGEO AI THE BEGINNING OF RELOAO I AND WAS URIGINALLY LOADED AT RELUADO

WAS

IMIS SEGMENT WAS OIBCHAKGED AT TME BEGIANING UP RELOAD S AND MAS URIGINALLY LUADEO AT FELOADI

DECAY MEAT IN MAIIS PER F,E AT TIME I YEARS AFTEN DIBCHAKGE (PEKCENT FROM GAMMAS IN PAHENTHESES)

\begin{tabular}{|c|c|c|c|c|}
\hline NUCLIDE & 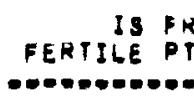 & RESH & 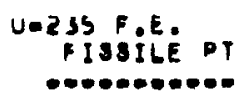 & Th. \\
\hline $\begin{array}{l}A C-228 \\
\text { ACIINIUM }\end{array}$ & $\begin{array}{l}3.971=06 \\
1.9900051\end{array}$ & (b) & $\begin{array}{l}0.000 \\
1.4030116 .\end{array}$ & 4) \\
\hline $\begin{array}{l}R A-223 \\
R A=-224 \\
R A=-225 \\
R A-228 \\
\text { RADIUM }\end{array}$ & $\begin{array}{l}4.760-06 \\
1.270002 \\
3.120007 \\
3.059008 \\
1.270-026\end{array}$ & 0) & $\begin{array}{l}0.997-10 \\
1.367=06 \\
9.678-14 \\
0.000 \\
1.368-001\end{array}$ & ? \\
\hline $\begin{array}{l}\text { PRE-221 } \\
\text { PRE-2Z3 } \\
\text { PRANCIUM }\end{array}$ & $\begin{array}{l}1.508=05 \\
5.338=09 \\
1.508=051\end{array}$ & $0)$ & $\begin{array}{l}4.670-12 \\
7.846-13 \\
5.401=126\end{array}$ & 3) \\
\hline $\begin{array}{l}R N=219 \\
R N=220 \\
R A D O N\end{array}$ & $\begin{array}{l}5.529006 \\
1.413002 \\
1.413=021\end{array}$ & D) & $\begin{array}{l}8.128=10 \\
1.514=06 \\
1.5150006\end{array}$ & 0) \\
\hline $\begin{array}{l}\text { AT-217 } \\
\text { ATE-2IB } \\
\text { ASTAIINE }\end{array}$ & $\begin{array}{l}1.681 .05 \\
2.671 .10 \\
1.681 .056\end{array}$ & 01 & $\begin{array}{l}5.215-12 \\
0.000 \\
5.215-126\end{array}$ & 08 \\
\hline 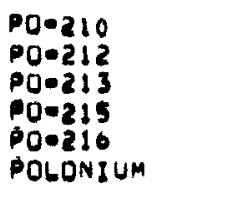 & $\begin{array}{l}3.273=07 \\
1.260002 \\
1.994005 \\
5.990=00 \\
1.524002 \\
2.787=021\end{array}$ & 01 & $\begin{array}{l}4.603-11 \\
1.351-06 \\
6.186-12 \\
8.804-10 \\
1.633-06 \\
2.985-068\end{array}$ & 08 \\
\hline $\begin{array}{l}8 I-211 \\
8 I-515 \\
8 I-213 \\
\text { BISMUTH }\end{array}$ & $\begin{array}{l}5.357 .06 \\
6.354003 \\
1.744 .06 \\
6.361 .036\end{array}$ & 4) & $\begin{array}{l}7.875-10 \\
6.809007 \\
5.409-13 \\
6.8170076\end{array}$ & (4) \\
\hline $\begin{array}{l}P B-209 \\
P B-2 I 1 \\
P B=212 \\
\text { LEAD }\end{array}$ & $\begin{array}{l}4.852-07 \\
4.380-07 \\
7.143-04 \\
7.152-046\end{array}$ & (6)) & $\begin{array}{l}1.505-13 \\
6.438=11 \\
7.654-08 \\
7.661=068\end{array}$ & $(60)$ \\
\hline $\begin{array}{l}\text { TLE-20T } \\
\text { PL-20 } \\
\text { THALLIUM }\end{array}$ & $\begin{array}{l}4.311=07 \\
3.189003 \\
3.1870036\end{array}$ & 841 & $\begin{array}{l}0.337=11 \\
3.415007 \\
3.4100076\end{array}$ & $84)$ \\
\hline $\begin{array}{l}\text { OY- } 159 \\
\text { OYSPRUSIUM }\end{array}$ & $\begin{array}{l}8.501=09 \\
8.501=091\end{array}$ & 1001 & $\begin{array}{l}3.739-10 \\
3.730-10(1\end{array}$ & $(100)$ \\
\hline
\end{tabular}

MB PRESM U-235 F.E PERTILE PIL. FISSILE HIL. $4.242-00$ $3.090005(10) \quad 2.821 .106 \quad 13$

7.529000

1.604009

.722002

.976007

4.122000

$5,801=12$

\begin{tabular}{|c|c|c|}
\hline $\begin{array}{l}2.889005 \\
8.443=00 \\
2.890-058\end{array}$ & 07 & $\begin{array}{l}2,803-10 \\
1,866-12 \\
2,822-106\end{array}$ \\
\hline
\end{tabular}

\section{$8.747 .06 \quad 1.933009$}

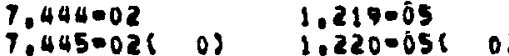

$3.222 .05 \quad 3.126-10$

$1.630-10 \quad 0.000$

$3.222-038$ 0) $3.126-106$ o)

$7.962=07$

$0,647-02$

$3,821=05$

9,475006

1.408001

0.470000

3.340002

3.3500026

2. $103=10$

$2.103-10$

$3.708-10$

3.004009

1.310005

$2.40500 \hat{56}$ 0)

9.298 .07

6.928007

0.704003

$3.706003(60)$

1.073009

$3.242=11$

$5.487 .00($ a)

0.819007

$1.681 .02(64)$

$0.021=12$

$1.531-10$

6.160007

$0.168 .07(66)$

3.651009
$3.051=00(100)$

$1.507-10$

$2,753=06$
$2,753=06(\quad 4)$ 


\begin{tabular}{|c|c|c|c|c|}
\hline \multirow[b]{3}{*}{ NUCLIDE } & \multicolumn{2}{|c|}{$\begin{array}{l}\text { THIS SEGMENT AAS UIBCHARGED AT } \\
\text { THE BEGINNING OF RELOAD I ANO WAS } \\
\text { ORIGINALLY LOAOEO AT RELUAO U }\end{array}$} & \multicolumn{2}{|c|}{ 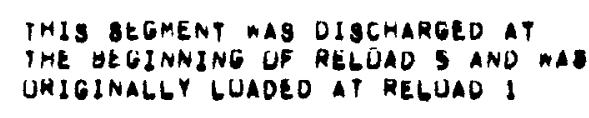 } \\
\hline & \multicolumn{4}{|c|}{ 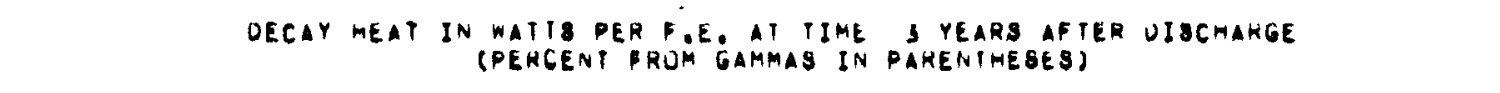 } \\
\hline & 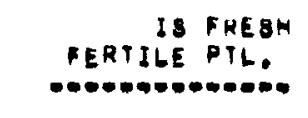 & 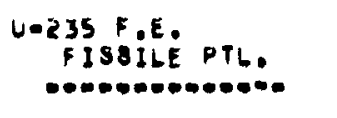 & 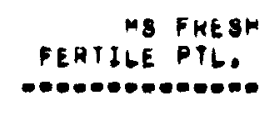 & 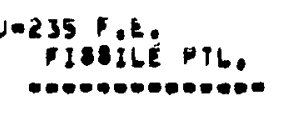 \\
\hline 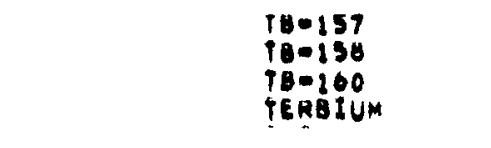 & $\begin{array}{l}1.414=10 \\
8: 073011 \\
3: 306007 \\
3.369007(85)\end{array}$ & $\begin{array}{l}0.157-12 \\
3.400011 \\
1.00009 \\
1.303007(85)\end{array}$ & $\begin{array}{l}5.372010 \\
7.071010 \\
5.408009 \\
5.421007(85)\end{array}$ & $\begin{array}{l}4.218-11 \\
1.030=10 \\
5.900=07 \\
5.701=076853\end{array}$ \\
\hline $\begin{array}{l}\text { GD.153 } \\
\text { GADOLIINZUM }\end{array}$ & 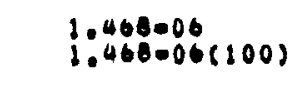 & $\begin{array}{l}1.402=00 \\
1: 402006(100)\end{array}$ & 4.109007 & $\begin{array}{l}5.9550 .07 \\
5.955007(100)\end{array}$ \\
\hline $\begin{array}{l}E U=152 \\
E U=154 \\
E U=155 \\
\text { EUKOPIUM }\end{array}$ & $\begin{array}{l}4.518004 \\
7.709=03 \\
5.305=04 \\
8.751=03(82)\end{array}$ & $\begin{array}{l}7.423=04 \\
6.6340 .02 \\
3.4750 .03 \\
7.055=02(82)\end{array}$ & $\begin{array}{l}3.910004 \\
1: 401001 \\
1.30003 \\
1.559001(02)\end{array}$ & $\begin{array}{l}5,150004 \\
3,409001 \\
3,0680.02 \\
3,570.01(102)\end{array}$ \\
\hline $\begin{array}{l}\operatorname{gM}=145 \\
\operatorname{MM}=152 \\
\text { SAMAKIUM }\end{array}$ & $\begin{array}{l}1.338007 \\
90963=05 \\
0,9760051\end{array}$ & $\begin{array}{l}1.330=07 \\
3: 337=04 \\
3.330=04(\quad 2)\end{array}$ & $\begin{array}{l}2.015007 \\
3.420=04 \\
3.4220046 \quad 21\end{array}$ & $\begin{array}{l}2.015=07 \\
3.097=04 \\
3.490004(2)\end{array}$ \\
\hline $\begin{array}{l}M M-147 \\
M-149 M \\
\text { PRDMETHIUM }\end{array}$ & $\begin{array}{l}5.989-02 \\
1.015=00 \\
5.989=02(0)\end{array}$ & $\begin{array}{l}2.722001 \\
5.100008 \\
2.722=016 \quad 01\end{array}$ & $\begin{array}{l}2.300001 \\
4.938008 \\
2.3060016 \quad 08\end{array}$ & $\begin{array}{l}1.560001 \\
3.467080 \\
1.566=0110\end{array}$ \\
\hline $\begin{array}{l}\text { PRAS } 44 \\
\text { PRASEOOYMIUM }\end{array}$ & $\begin{array}{l}1.704+00 \\
1.704+00(2)\end{array}$ & $\begin{array}{l}7.430+00 \\
7.430+00(\quad 2)\end{array}$ & $\begin{array}{l}7.340+00 \\
7: 340+006 \quad 2)\end{array}$ & $\begin{array}{l}5.143+00 \\
5.143+001 \quad 23\end{array}$ \\
\hline $\begin{array}{l}\text { CEDI41 } \\
\text { CE-144 } \\
\text { CERIOH }\end{array}$ & $\begin{array}{l}1.352000 \\
1.474=01 \\
1.474001625)\end{array}$ & $\begin{array}{l}2.427=09 \\
6.425=02 \\
0.425=01(25)\end{array}$ & $\begin{array}{l}2.442000 \\
0,347001 \\
0.347=01(25)\end{array}$ & $\begin{array}{l}5.038-10 \\
4.040002 \\
4.440=016253\end{array}$ \\
\hline $\begin{array}{l}\text { LAPI3T } \\
\text { BANTHANUM }\end{array}$ & $\begin{array}{l}1.388-100 \\
1.380-10(100)\end{array}$ & $\begin{array}{l}0.040-12 \\
6.040012(100)\end{array}$ & $\begin{array}{l}5.550010 \\
5.550-10(100)\end{array}$ & $\begin{array}{l}4.000-11 \\
4.000-11(100)\end{array}$ \\
\hline $\begin{array}{l}\text { BA-1374 } \\
\text { BARIUMM }\end{array}$ & $\begin{array}{l}5,473-01 \\
5,473-01(100)\end{array}$ & $\begin{array}{l}2.191+00 \\
2.191+00(100)\end{array}$ & $\begin{array}{l}4.604+00 \\
4.004+00(100)\end{array}$ & $\begin{array}{l}0.011+00 \\
6.011+00(100)\end{array}$ \\
\hline 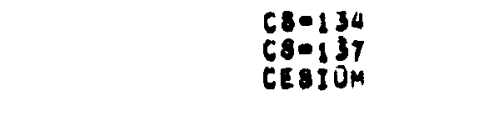 & $\begin{array}{l}2,102-01 \\
1,430=01 \\
3,601001(54)\end{array}$ & $\begin{array}{l}1.805+00 \\
5.758=01 \\
2.380+00(00)\end{array}$ & $\begin{array}{l}0.195+00 \\
1.210+00 \\
9.405+00(75)\end{array}$ & $\begin{array}{l}1.409+01 \\
1.580+00 \\
1.507+0.1(B 1)\end{array}$ \\
\hline $\begin{array}{l}\text { J-129 } \\
300 I N E\end{array}$ & $\begin{array}{l}4.034008 \\
4.034008(39)\end{array}$ & $\begin{array}{l}7.141=08 \\
7.141=08(34)\end{array}$ & $\begin{array}{l}3.311007 \\
3.311=07(39)\end{array}$ & $\begin{array}{l}1.900-107 \\
1.900-076(30)\end{array}$ \\
\hline
\end{tabular}




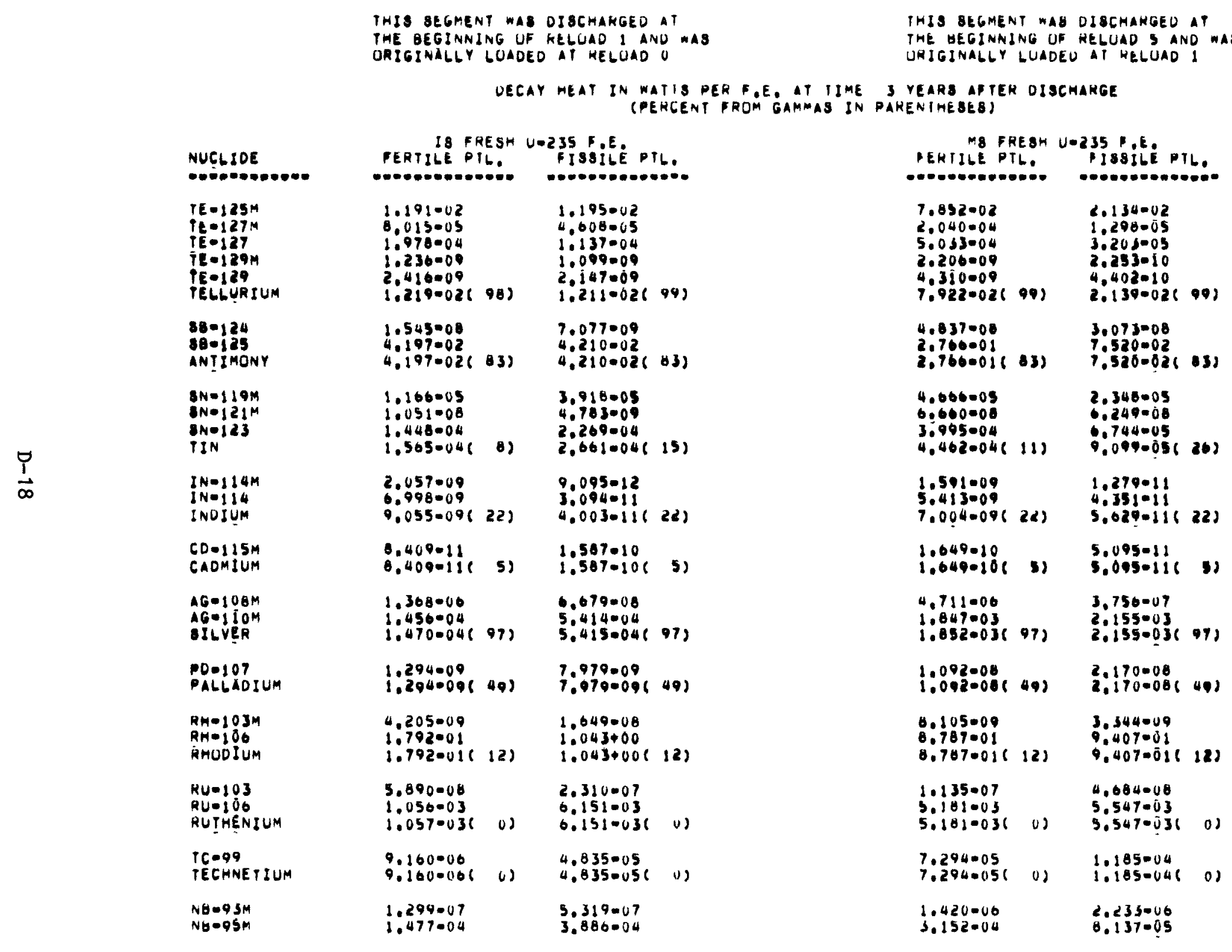


THIS SEGMENT WAS DISCHARGED AT
THE BEGINNING OF RELOAD I ANO WAS
URIGINALLY LUADEO TT RELOADO

degaY meAT IA WATTS PER F,E. AT TIME

A WAITS PER FEE LAT TIME J YEARS AFTER DISCHAKGE
(PERCENTEROM GAMMAS IN PARENTHESES)

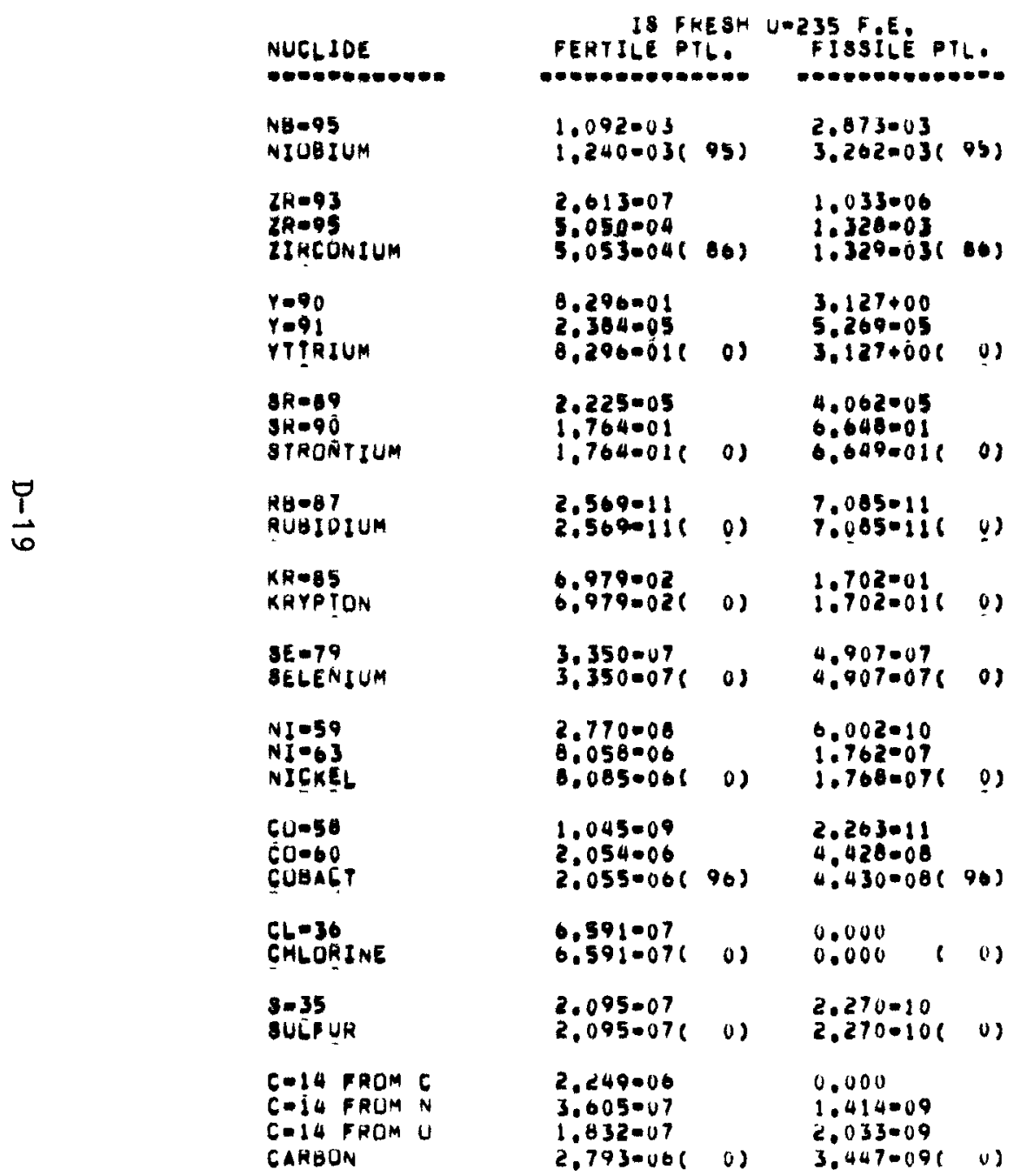

THIS SEGMENT WAS DISCHARGED AT TME BEGINAING OF RELULU S AND WAS ORIGSNALLY GUAOLU AT RELUADO

\begin{tabular}{|c|c|c|c|}
\hline $\begin{array}{l}2.330 .03 \\
2.647-036\end{array}$ & $95)$ & $\begin{array}{l}0.010=04 \\
0.852=041\end{array}$ & $95)$ \\
\hline $\begin{array}{l}2.230006 \\
1.077003 \\
1.0000031\end{array}$ & (6) & $\begin{array}{l}2.923000 \\
2.781=04 \\
2.011=041\end{array}$ & 853 \\
\hline $\begin{array}{l}0,940+00 \\
4.884005 \\
0,040+000\end{array}$ & 02 & $\begin{array}{l}8.501+00 \\
1.095=05 \\
8.501+0006\end{array}$ & (1) \\
\hline $\begin{array}{l}4.409005 \\
1.476000 \\
1.4760001\end{array}$ & 08 & $\begin{array}{l}1.255006 \\
1.807+00 \\
1.007 .006\end{array}$ & 01 \\
\hline $\begin{array}{l}2.164=10 \\
2.104-108\end{array}$ & Q) & $\begin{array}{l}2,960-10 \\
1.960-106\end{array}$ & of \\
\hline $\begin{array}{l}4.410=01 \\
4.416=016\end{array}$ & 0) & $\begin{array}{l}1.769001 \\
1.7690016\end{array}$ & 01 \\
\hline $\begin{array}{l}2.229000 \\
2.220-061\end{array}$ & 01 & $\begin{array}{l}8.773=09 \\
8.7730076\end{array}$ & 01 \\
\hline $\begin{array}{l}9.582=08 \\
2.730-05 \\
2.745=051\end{array}$ & Q) & $\begin{array}{l}3.450=09 \\
9.8560 .07 \\
9.091-071\end{array}$ & 0) \\
\hline $\begin{array}{l}9.383=10 \\
0.081=06 \\
6.082=061\end{array}$ & $96)$ & $\begin{array}{l}3.378-11 \\
2.202007 \\
2.2020076\end{array}$ & $96)$ \\
\hline $\begin{array}{l}2.134006 \\
2.1340068\end{array}$ & $\underline{0}$ & $\begin{array}{l}0.000 \\
0.000\end{array}$ & 01 \\
\hline $\begin{array}{l}1.034=07 \\
1.934=076\end{array}$ & $0)$ & $\begin{array}{l}3,502-10 \\
3,502-106\end{array}$ & 01 \\
\hline $\begin{array}{l}7,902006 \\
1.270006 \\
6,570=07 \\
9.829=061\end{array}$ & $0)$ & $\begin{array}{l}0.000 \\
8.464=09 \\
1.190=08 \\
2.037=086\end{array}$ & (1) \\
\hline
\end{tabular}


THIS SEGMENT MAS DISCHARGEO AI

THE BEGINNING UE RELUAD I AND WAS

URIGINALLY LUADED AT RELUAD O

THIS SEGMENT WAS DISCMAKGEU AT

THE GEGINNING UF RELUAD S ANO WAS

DECAY hEAT IN WATIS PEA F.E. AT IIME 3 YEARS AFTEM DISChaRge (DENCENT FROM GAMMAS IN PAHENIAESES

\begin{tabular}{|c|c|c|}
\hline NUCLIDE & $\begin{array}{l}\text { IS FRESH } \\
\text { FERTILE PTL. }\end{array}$ & 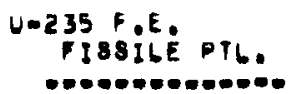 \\
\hline $\begin{array}{l}\text { HoJ FRUM LIO } \\
M=3 \text { ROM } \\
H=3 \text { FISS YLO } \\
\text { TRITIUM }\end{array}$ & $\begin{array}{l}7.422=04 \\
3.052=07 \\
1.515=05 \\
7.577=046\end{array}$ & $\begin{array}{l}5.002000 \\
0.000 \\
6.523 .05 \\
7.0240 .051\end{array}$ \\
\hline
\end{tabular}

MS RHESM VOZZ35 F.E. FERTILE PTL. PISSILE PTL. .

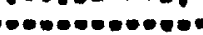

9.387 .04

1.531 .06

1.232 .04

1.073005

0.000

.674004
1.7820048

o)

ELEMENTS

TOTAL BETA

TOTAL GAMMA

POTAL ALPHA

$3.242+00$

0.049001

1.286 .01

$1.365+01$

$4.412+00$

$1.821 .01(24)$

$\begin{array}{ll}1.937+01 & 1.978+01 \\ 1.114+01 & 1.953+01 \\ 5.40501 & 3.796+00 \\ 3.105+01(35) & 4.318+01(45)\end{array}$


PHIS SEGMENT MAS DISCMARGED AI THE GEGINNING UF RELUAU I ANU MAS URIGINALLY GUADED AT RELUADO
THIS SEGMENT MAS UISCMARGED AT TME UEGINNING UF RELUAD S AND MAS URIGINALLY LUADEU AT RELUAU I

UecaY HeAT IN MATIS PER Fok. AT TIME S YEAKS AFTEK UISCMAKGE (DERCENT FROM GAMMAS IN PAKENTHESES)

NUCLIOE

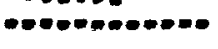

$C M=242$

$C M-2^{\pi} 3$

CURIUM

$1 M=241$

$A M-242 M$

$A M=242$
$A M=243$

AMO
AMERICIUM

$P U=238$

$P U=239$
$P U=240$

Pu=24!

Plutunium

NP. 239

NEHTUNIUM

$u=232$
$U=233$

$\mathrm{N}=\mathrm{2} 3$

$v-235$

$4-236$

Ne.538

URANIUM

$P A-231$
$P A-234$

prutactinIUM

$T H \cdot 220$

$T H=229$

IH- $3 \mathrm{HO}$

$T H=23 !$
$T H=232$

$T H=232$
$T H=234$

THORIUM

$\triangle C=225$
$A C=227$

$A C-227$
$A C-228$

AC C 228
ACTINIUM

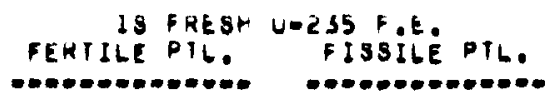

2.535004

2.535009

0.716009

2.796004

$2.790-046$

$3.004-05$

3.295006

3.551 .03
3.590 .036

2.188008

$2.442-11$

1.056010

2.029 .06

2.651 .006

3.652 .03

1.126007

4.872007

$0.497-05$

3,574005
4,860009

5.500009

2.034 .09

3.57500560

1.353008
1.353008

1.001 .02

$3,737=02$

$1.778=03$

4.422 .08

$5,612=08$

0.000

5.575 .021

$3.717 .03(0)$

1.300001

8.698 .04

1.424 .03

3.807 .04

1.387 .01602

3.440005

3.44600560

1.778 .06

4.786004

1.165005

7.309 .05

2.111 .07

$5.654 .046 \quad 0$

5.683 .05

0.000

$5.683005(1)$

0.876009

8.865 .08

$9.552008(82)$

1.472002
1.929005

3.803004

1.724 .09

$1.724-09$
$2.475-05$

0.000

$1.315-026 \quad 08$

1.571 .06

$3.772-12$

$4.539-07$

0.000

3.701 .09

3.701009
$2.065000(12)$

$2.241 \cdot 05$

$6.528=00$

4.880000

$4.380-12$
$1.059 \cdot 11$

$1.059 \cdot 1$
0.000

2.097 .1163
MS FKESt U-zas E

FERIILE PIL. FISSILE NTL.

4.201 .07

3,378000

$1.210005 \quad 1.514002$

$3.004009 \quad 1.059006$

$1.334008 \quad 4.561-00$

$2.635007 \quad 1.213 .03$

1.2380056 os $1.036-028$ os

$1.547002 \quad 3.473400$

5.348000

1.252 .06

$2,512-03$

$3,461+0060$

$2.259006 \quad 2.527004$

$2.250-068$ 0) 2.527-0.04 03

$7.804002 \quad 1.201 .05$

$5,831002 \quad 7.376-10$

$0.062003 \quad 4.090004$

$5.575=07 \quad 2.661-06$

$2.840006 \quad 1,792004$

0.000

$1.450001605 \quad 6.039=046$

$7.986005 \quad 1.255=08$

$0.00005 \quad 1.255000$

$7.986-05($ i) $1.309007(60)$

$7.287002 \quad 1.190005$

$3.435 .05 \quad 2.220-10$

$1.575=04$

1.037007

2.100005

0

$7.308-02(0) \quad 1.2040056 \quad 0)$

\$.989.05 $\quad 2.577 .10$

$1.033001 \quad 3.001=1$

$\begin{array}{ll}4.850006 & 0.000 \\ 4.4850056 \text { os } & 2.438-106 \quad 11\end{array}$ 
IMIS SEGMENT MAS DISGTARGEU AI TME GEGIANING UF RELOAD I ANU WAS ORIGINALLY LUADED AT RELOAD O
THIS SEGMENT WAS DISCHARGED AT THE OEGINNING UF RELUAD 5 AND WAS UHIGINALGY LUADEO AT REGUAU I

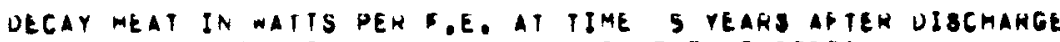
(PERCENT FROM GAMMAS IN PARENTHESES)

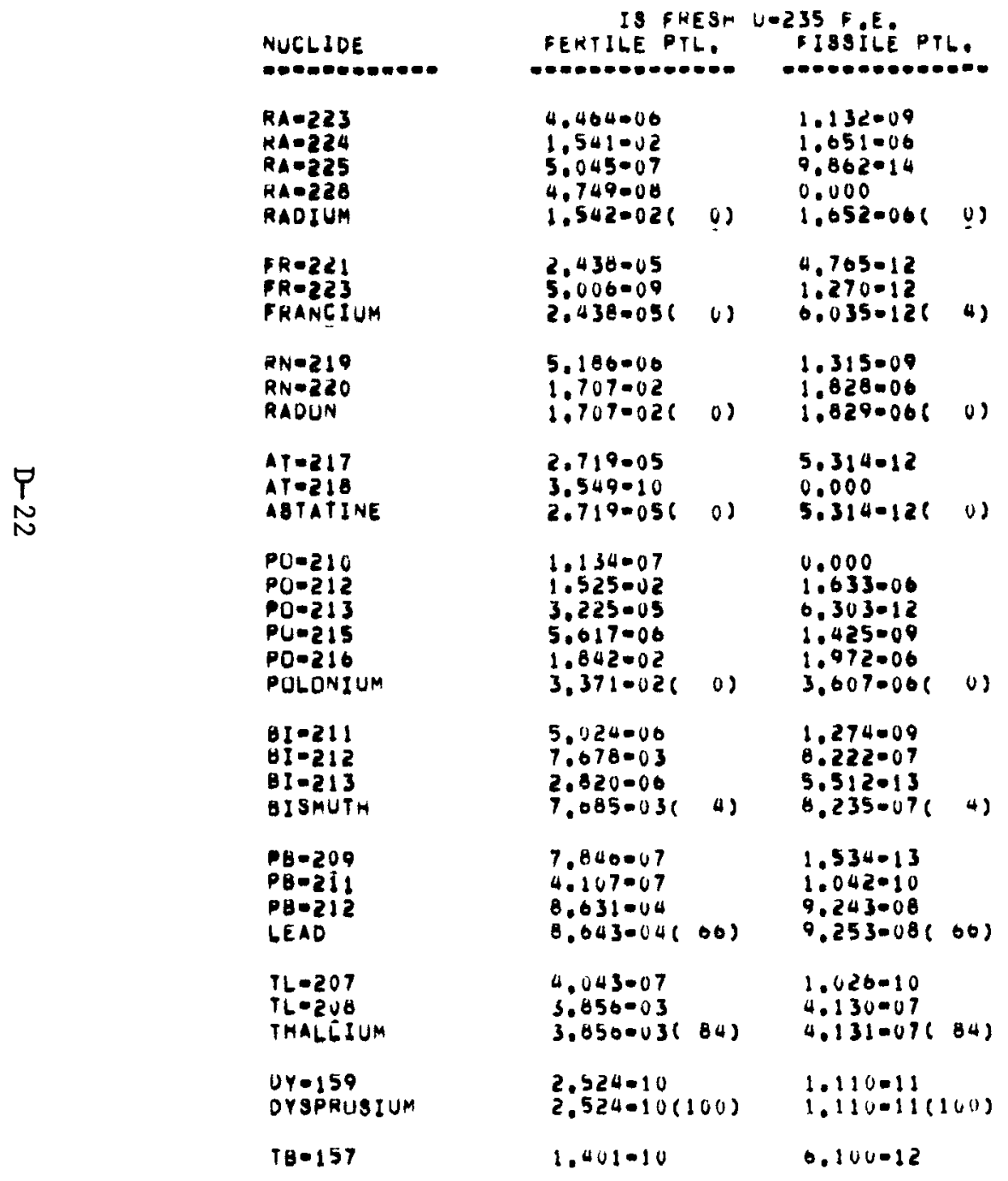

MS FHESH U-235 F.

PEKTILE PTL. FIS8ILE NIL,

$7.061006 \quad 2.463009$

$7.629 .02 \quad 1.246 .05$

4.71000

$\begin{array}{lll}4.719008 & 0.000 \\ 7.6290026 & 01 & 1.2460058\end{array}$

$4.340005 \quad 2.004-10$

$\begin{array}{lll}7.919009 & 2.763-12 \\ 4.3410056 & 0) \quad 2.832-106 & 01\end{array}$

$0.202006 \quad 2.802009$

$\begin{array}{llll}8.448002 & 1.380005 \\ 8.4490021 & 0) & 1.3800056 & 01\end{array}$

$4.640005 \quad 3.127=10$

$\begin{array}{lll}2.109010 & 0.000 \\ 4.840005(\text { o) } & 3.127-106 & 0\end{array}$

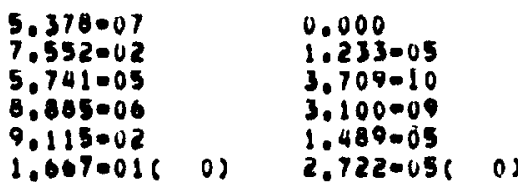

1.007001 ( o) 2.722.056

$7.947000 \quad 2.775000$

0.206006

$3.801 .02($ 4) 6.209-06( a)

$1.397-00 \quad 9.025-12$

$\begin{array}{ll}1.397-00 & 9.025-12 \\ 0.497-07 & 2.267-10\end{array}$

$4.272-03 \quad 6.970=07$

$4.274003(60) \quad 0.979007(66)$

$6.395007 \quad 2.231 .10$

$\begin{array}{ll}1.909002 & 3.118006 \\ 1.909002(84) \quad 3.118000(84)\end{array}$

$1.084-10 \quad 9.617-12$

$1.084010(100) \quad 9.617-12(100)$

$5.322-10 \quad 4.100-1$ 
THIS SEGMENT NAS DISCHARGEO A I
TME GEGINAING OB HELGAO I ANO WAS
LEIGINALLY LUADED AT RELUAD O

DeCAY MEAT IN WATIS PER F.E. AT TIME S YEARS AFIEK UISCHARGe

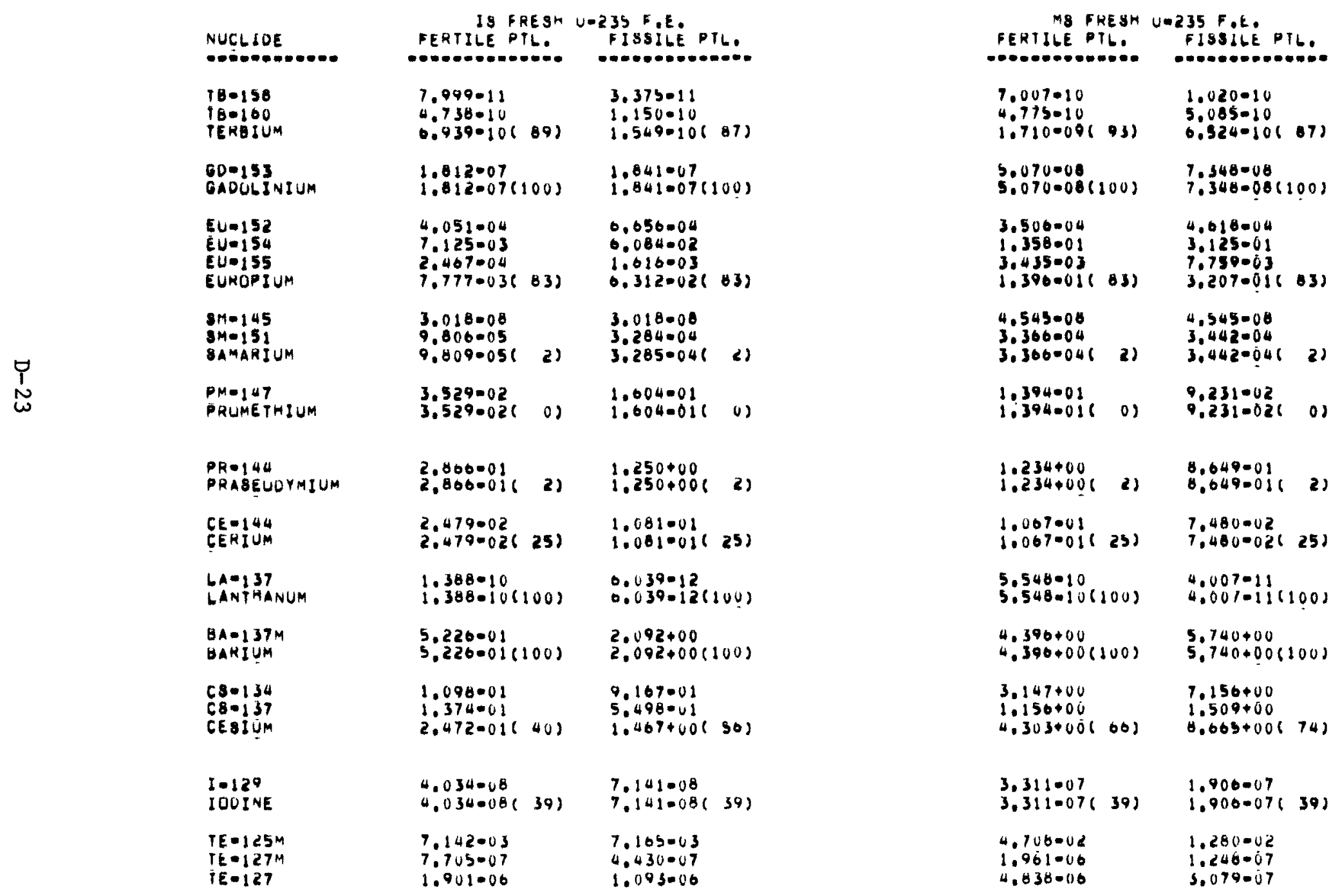

TMIS SEGMENT WAS DISCMAKGEU AT

THE DEGINAJNG LF GELUAOS AND aAS

URIGINALLY GUADEU AT RELUAD 1 (PEKCENT FRUM GAMMAS IN FAKENIMESES) 


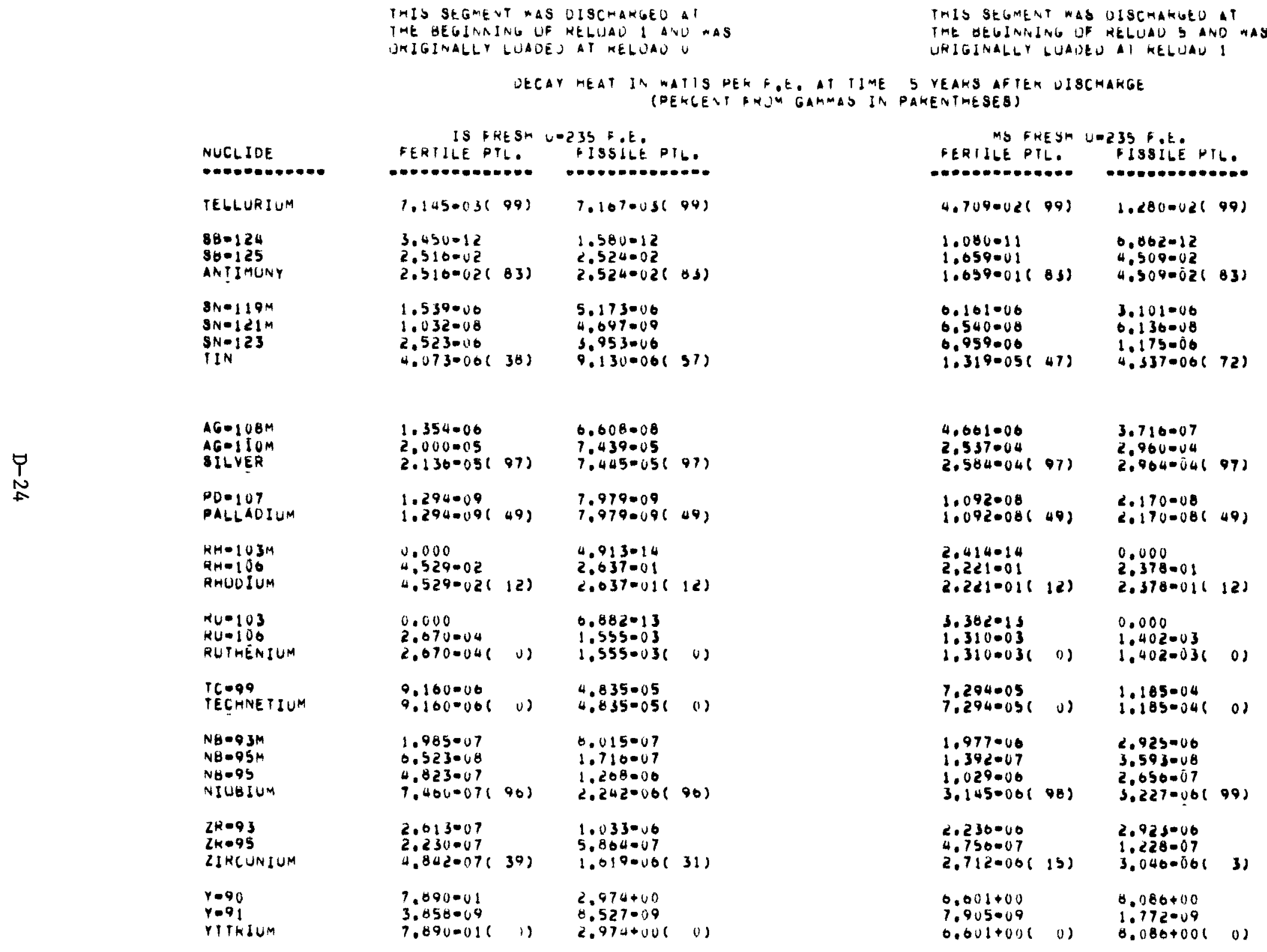


THIS SEGMENT NAS LISCMAHGED AI

THE BEGIANING UP MELUAD I AND A AS

UEIGIVALLY LUAUEO AT MELUAD U
TMIS SEGMEAI WAS UISCMARGES AT TME BEGINNING UF RELUAD S AND MAS URIGINALLY LUADED AT KELUAU I

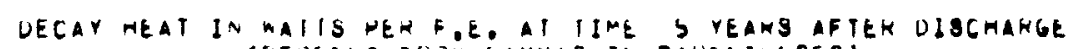
(PERGENT GRIM GAMMAS IV PAKERIMESES)

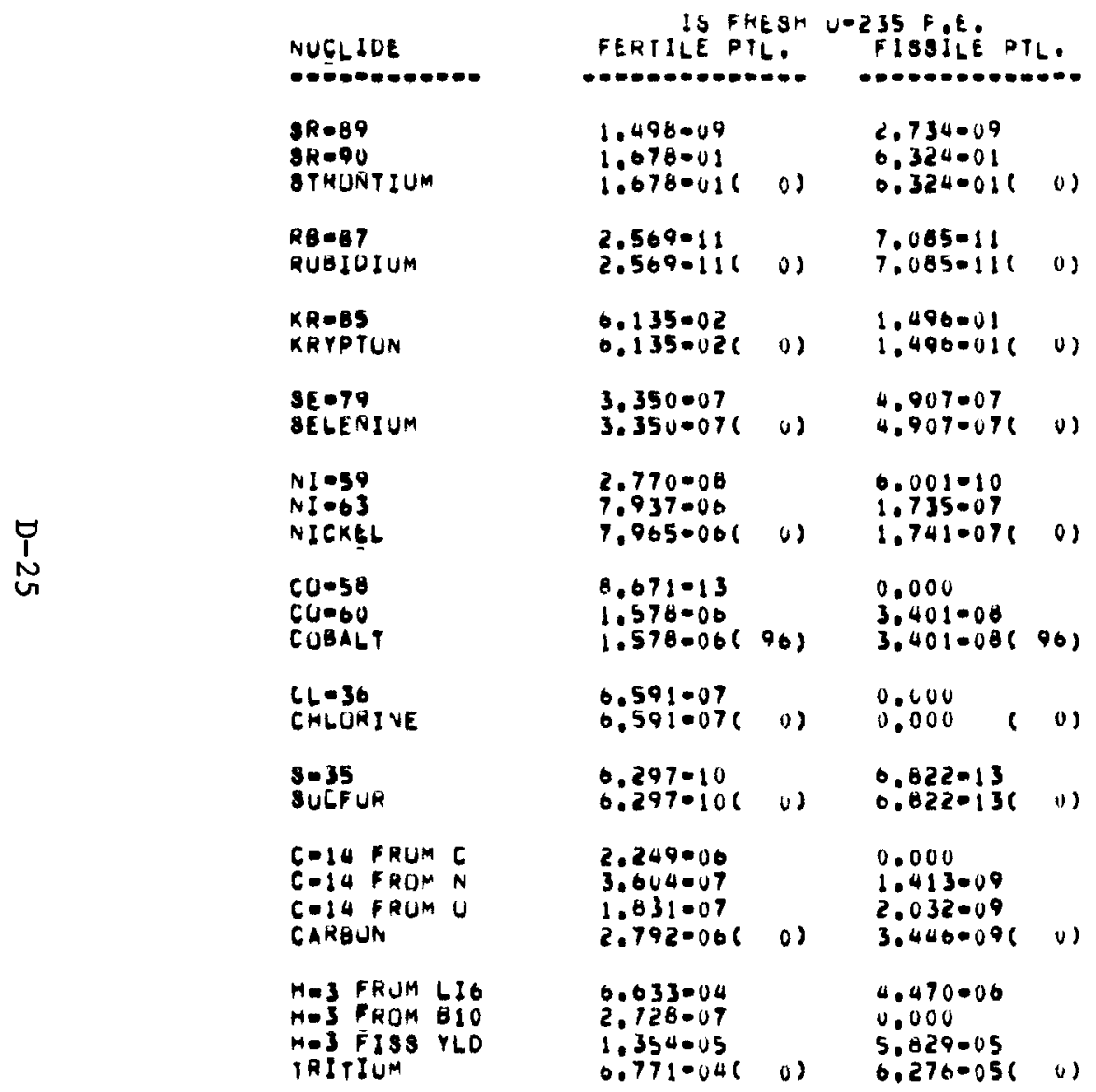

\begin{tabular}{|c|c|c|c|}
\hline $\begin{array}{l}2.908=09 \\
1.003+00 \\
1.003+006\end{array}$ & (u) & $\begin{array}{l}3.537-10 \\
1.719+00 \\
1.714+006\end{array}$ & C) \\
\hline $\begin{array}{l}2.104-10 \\
2.164-106\end{array}$ & $0)$ & $\begin{array}{l}1.968-10 \\
1.968-106\end{array}$ & D) \\
\hline $\begin{array}{l}3.802=01 \\
3.802-016\end{array}$ & 0) & $\begin{array}{l}2.385001 \\
2.3850016\end{array}$ & 01 \\
\hline $\begin{array}{l}2.229000 \\
2.2290006\end{array}$ & 01 & $\begin{array}{l}8.773007 \\
8.7730076\end{array}$ & D) \\
\hline $\begin{array}{l}9.582-08 \\
2.695005 \\
2.705-056\end{array}$ & 01 & $\begin{array}{l}3.450=09 \\
9.709-07 \\
9.7430076\end{array}$ & 0) \\
\hline $\begin{array}{l}7.787-13 \\
4.672-06 \\
4.072-066\end{array}$ & $96)$ & $\begin{array}{l}0.000 \\
1.092-07 \\
1.092-076\end{array}$ & $96)$ \\
\hline $2.134=06$ & 0) & $\begin{array}{l}0,000 \\
0,000\end{array}$ & 0) \\
\hline $\begin{array}{l}.812-10 \\
.812-106\end{array}$ & 01 & $\begin{array}{l}1.053-12 \\
1.053-126\end{array}$ & 0) \\
\hline $\begin{array}{l}7.900006 \\
1.269006 \\
6.508007 \\
9.8260006\end{array}$ & 0) & $\begin{array}{l}0.000 \\
0.462-09 \\
1.190-00 \\
2.0360006\end{array}$ & 0) \\
\hline $\begin{array}{l}8.567 .04 \\
1.380006 \\
1.101=04 \\
9.0620046\end{array}$ & & $\begin{array}{l}9.592=00 \\
0.000 \\
1.496=04 \\
1.5920 .046\end{array}$ & 01 \\
\hline
\end{tabular}


THIS SEGMENT WAS DIBCHARGEO AT TME BEGINAIVG LF KELOAO I ANU MAS TRE BEGINAIVG LE KELOAO I ANU OA

ma

THIS BEGMENT MAS DISCHARGEU AT THE BEGINNING OF RELUAU Y AND AAS

THE GEGINNING DF RELUAU S AND

DECAY MEAT IN WATIS PER F.E. AT TIME 5 YEARS AFIEN DISCHAKGE (PERGENT FROM GAMMAS IN PARENTHESES)

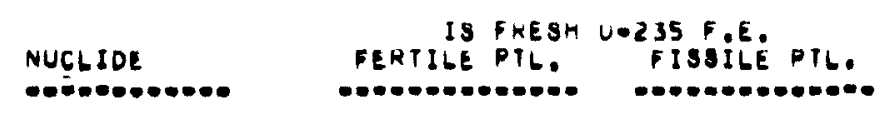

ALL ELEMENTS
TOTAL BETA
TUTAL GAMMA
TUTAG ALPMA
ORAND TOTAL
$1.547+00$
6.810001

$6.810=01$

$1.433-01$
$2.371+00(28$
$0.098+00$
$3.097+00$

$1.400=01$

$9.341+00(33)$

MS FRESM U.235 F.E.

PERTILE PTL. PISSILE PTLE

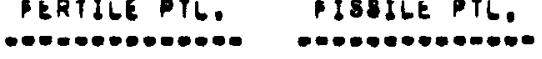

$\begin{array}{ll}1.152+01 & 1.348+01 \\ 7.606+00 & 1.265+01 \\ 5.099001 & 3.717+00 \\ 1.977+01(30) & 2.952+016\end{array}$




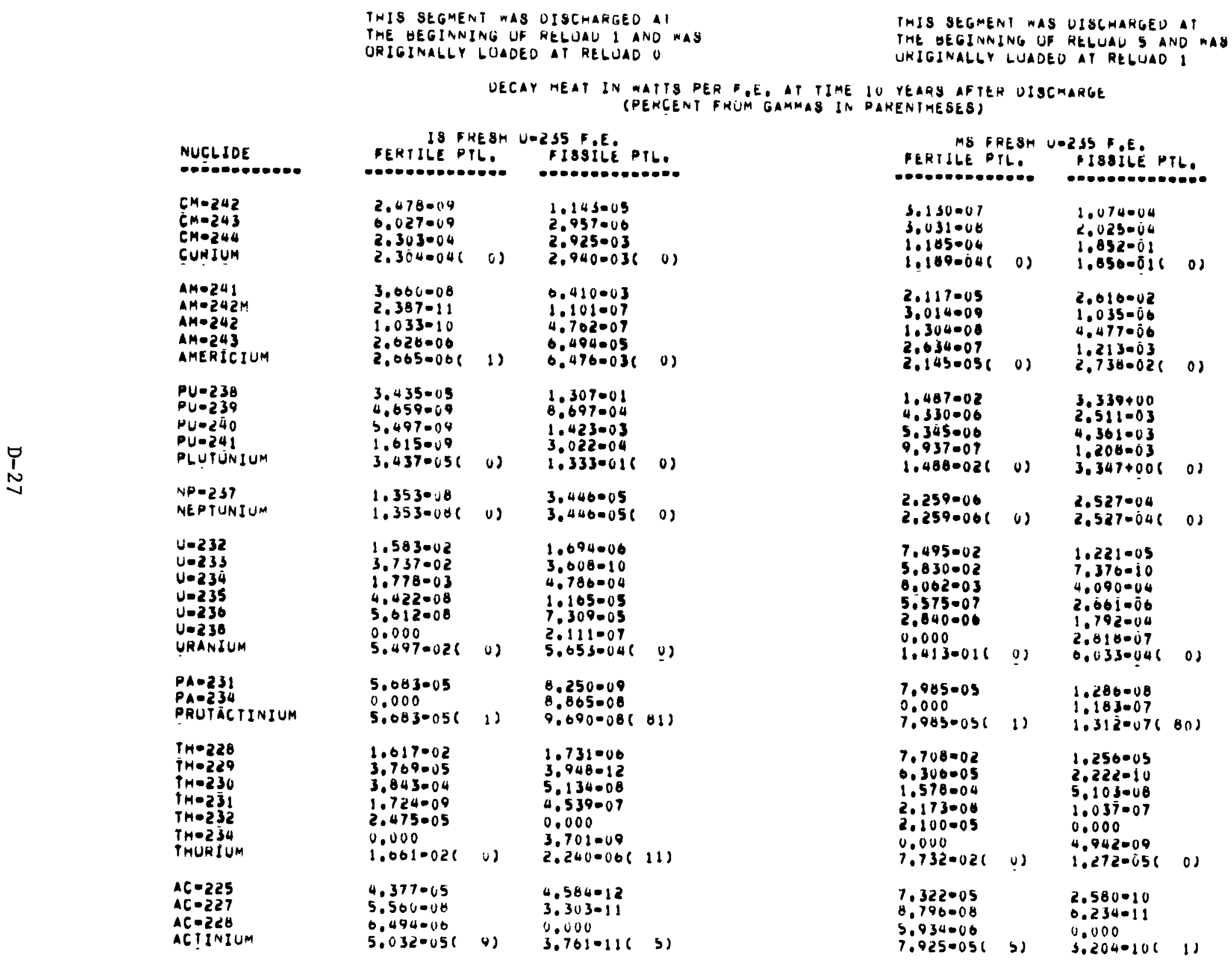


THIS SEGMENT AAS DISCMARGED AT

URIGINALLY LLADEL OT KELUAD O
IMIS SEGMENT WAS DISCHAHGEU AT THE OEGINNING UF RELUAD 5 AND AAS

GIKIGINALLY LUAOED AT HELUAD I

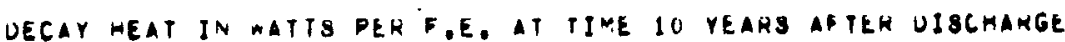
(PEKLENT FRUM GAMMAS IN PAHENTMESES)

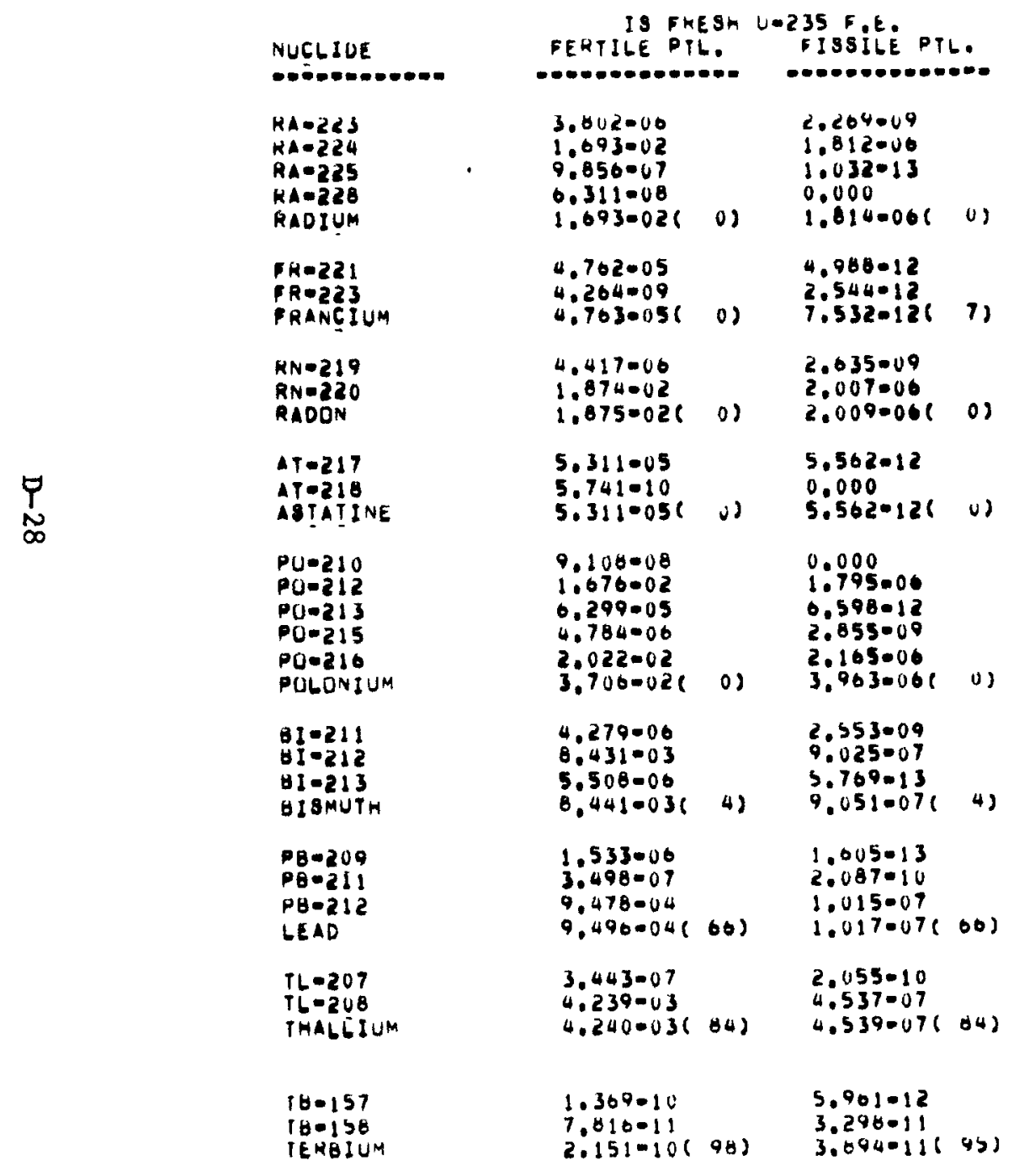

MS F KESH U-235 F,E

EERTILE PTL FIS8ILE NTL.

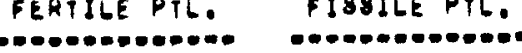

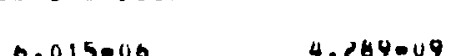

0.015000

4.284009 $1.049000 \quad 3.810-12$ 0.000

$6,070-02(0) \quad i .316-056 \quad 0)$

$7.960005 \quad 2.007-10$ $\begin{array}{llll}0.745-09 & & 4.810-12 & \\ 7.967-05( & 0) & 2.856-108 & 01\end{array}$

$0.987 .00 \quad 4.982=09$

$0.935002 \quad 1.456005$ $0.930-02($ 0) $\$ .457-0510)$

$6.685005 \quad 3.131010$ $\begin{array}{llll}3.087-10 & 0.000 \\ 8.085-056 & 0) \quad 3.131-106 & 08\end{array}$

$4.449007 \quad 0.000$

$7.992 .02 \quad 1.303 .05$

$\begin{array}{ll}1.054004 & 3.714010 \\ 7.56900 & 5.397-09\end{array}$

$9.641003 \quad 1.572=05$

1.7640016 o) 2.87500560

$0.709=06 \quad 4.027 .09$

$4.019002 \quad 0.551006$

9.214006 (3) $3.247-11$
$4.021002(550-061$

$4.021002($ a) 6.550006

$\begin{array}{ll}2.564000 & 9.036-12 \\ 5.534007 & 3.940-10\end{array}$

9.534007
4.516 .03

$\begin{array}{ll}4.516003 & 7.365007 \\ 4.521003(00) \quad 7.369-07(66)\end{array}$

5.447 .07

2.021 .02

$2.021 .02(64)$

$3.804 \cdot 10$

3.294006

$3.294-0.01845$

5.201 .10

$0.847-10609$

4.084 .11

$9.969-11$

$1,405-10(96)$ 
THIS SEGMENT WAS OISCHARGED I PHE GEGINNING UF RELUAD I AND WAS THE OEGINNING OF RELUAO I AND
THIS SEGMENT WAS OISCHAKGEO AT

TAE HEGINNING UF RELUAD 5 AND WAS URIGJNALGY LUADEU AT RELUAU?

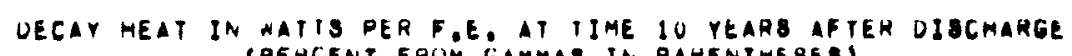
SPEKGENT GRUM GAMMAS IN PAKENTMESES

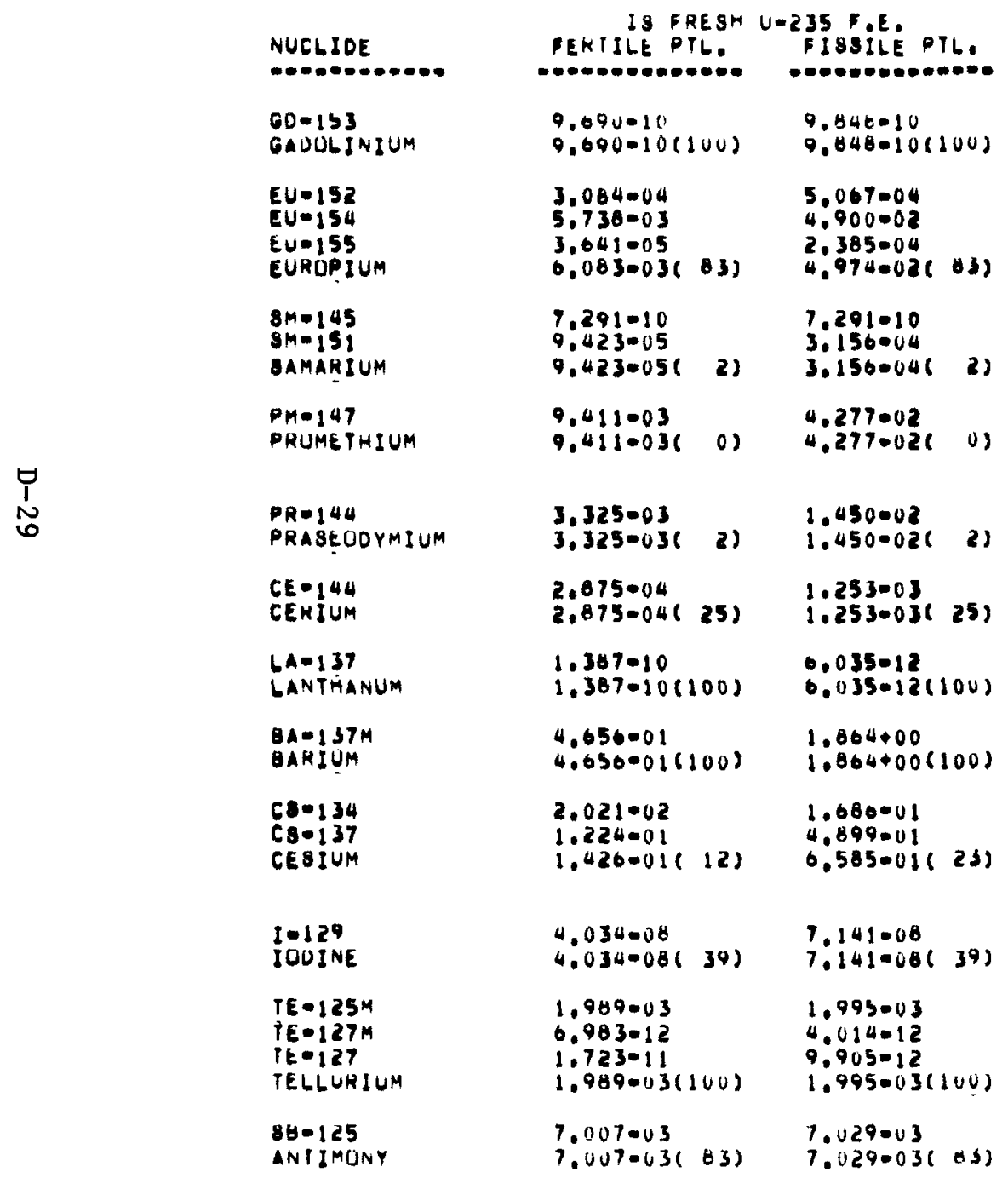


THIS SEGMENT WAS DISCMARGED AT

THE BEGINAING OF RELOAD I AND WAS

UHIGINALLY LOADED IT RELUAD

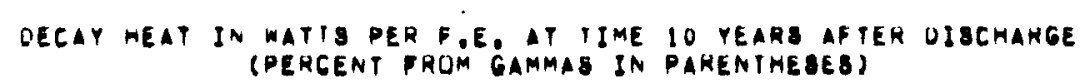

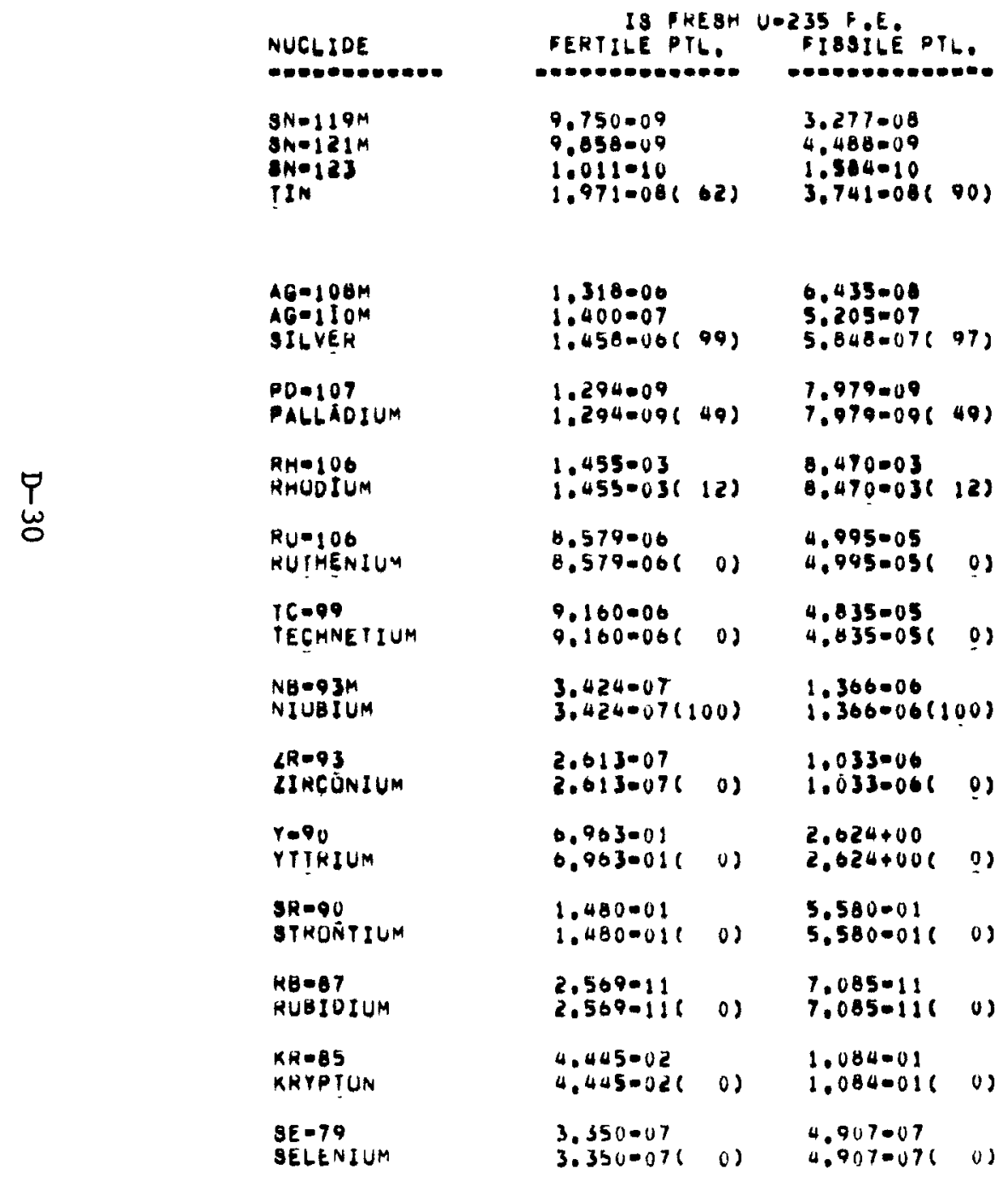

THIS BEGMENT MAS DIOCHARGEU AT

TME OEGINNING OF RELUAD S AND MAS ORI INALLY LUAOED AT RELOAO I

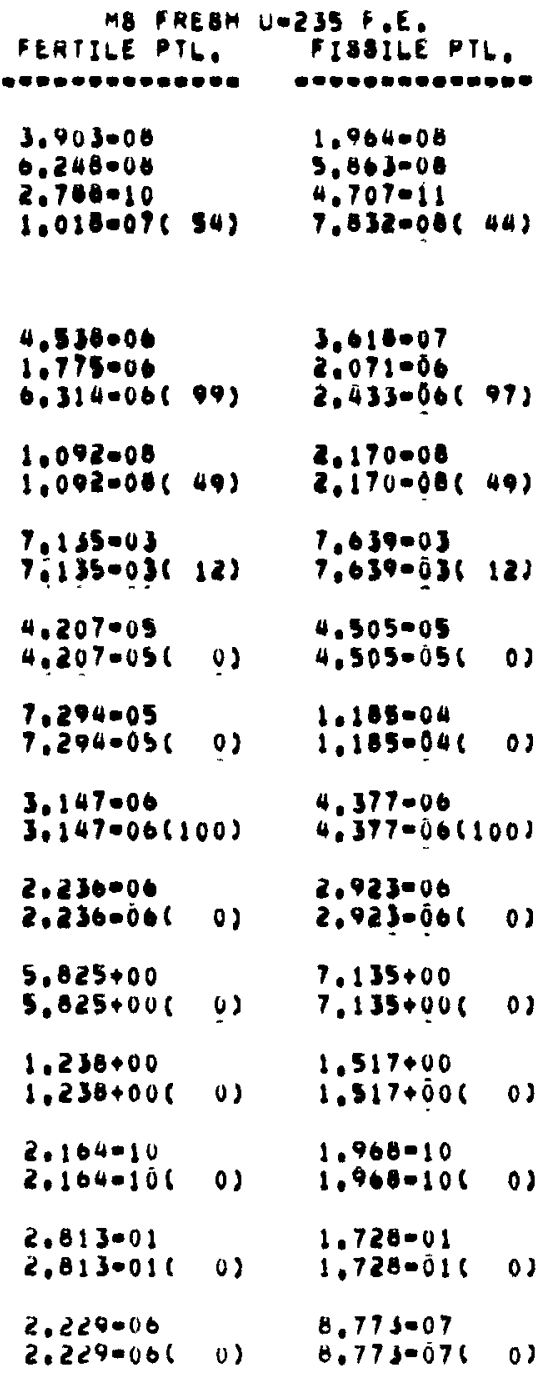


THIS SEGMENT WAS DISCMARGED AT THE BEGINNING OF HELOAD I ANO WAS UKIGINALLY GOADED AT RELUAD O

THIS SEGMENT WAS DIBCHAKGED AT THE GEGINNING OP RELUADS AND WAS UKIGINALLY LUADED AT RELUAO I

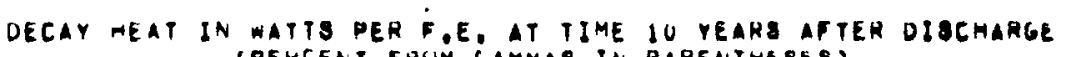

NUCLIOE

NI $\log 9$

$n I=0,4$

NIGKEL

COEBO
COBALT

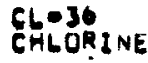

Cola FRUM $C$

C.II FROM N

C-14 FROM

CARGON

$\stackrel{\leftrightarrow}{\omega}$

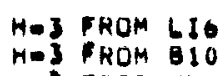

Hol FISS YLD

TAITIUM

ALL ELEMENTS

TOTAL BETA

TOTAL GAMMA

GRANO TOTAL
(PEKCENT FRUM GAMMAS IN PARENTHESES)

IS FRESH U.235 FE.

BERTILE PTL UTE FISSILE PTL.

$0.001-10$

1.671 .09
$1.677-076$

7.6710066

1.759 .00

$0.163=07$
$8.103=07(96)$

$1.759008(96)$

$0.590-07$

0.000

( 0$)$

$2.247-06$
3.602007

3.602007

2.790 .060

0.000

$5.007 \cdot 00$

2,031009

2.059 .07

$1.022-05$

5.1110045

3.374006

0.000

4.401 .05

$4.7300058 \quad 0$

ME FRESH U-23S F.E.

FERTILE PTLO FISAJLE NTL. oconoverocecon

9.501000
2.595005

$2.545005 \quad 3.449-09$

$2.005=056$ os $\quad 8,304=06760)$

2.417000

$2.417000(96)$

$0.749-08$

$\begin{array}{llllll}2.134000 & 0.000 \\ 2.134006(\text { os } & 0.000 & (0)\end{array}$

1.80500

1,260008

2.0350008 o

0.467004

1.046006

$7.3090046 \quad 0$

$7.241-06$

0,000

1.12900

.202-0̃4s

$\begin{array}{ll}1.032+00 & 3.070+00 \\ 5.027001 & 2.070+00 \\ 1.511001 & 1.428001 \\ 1.086+00(29) & 6.083+00(34)\end{array}$

$0.520+00$

$4.615+00$

41000

1.375001( 33)

$1.038+01$
$0.341+00$

$354+00$

$3.047+01(31)$ 
0

-

0 
TABLE D-3

DECAY HEAT FOR FUEL ELEMENTS DISCHARGED AT BEGINNING OF RELOAD 10 AND ORIGINALLY LOADED AT RELOAD 6

NOTE: Table D-3 consists of 29 pages of computer print-out (pages D-35 through $\mathrm{D}-63$ ). 
-

-

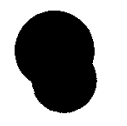


THIS SEGMENT MAS DISCHARGED AT THE BEGINNJNG OF KELUIAU IO ANU MAS URIGINALGY GUAOEO AT KELUAU O

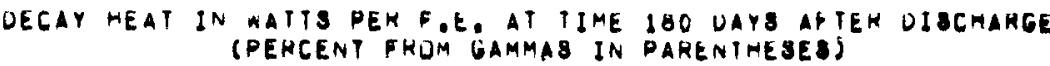

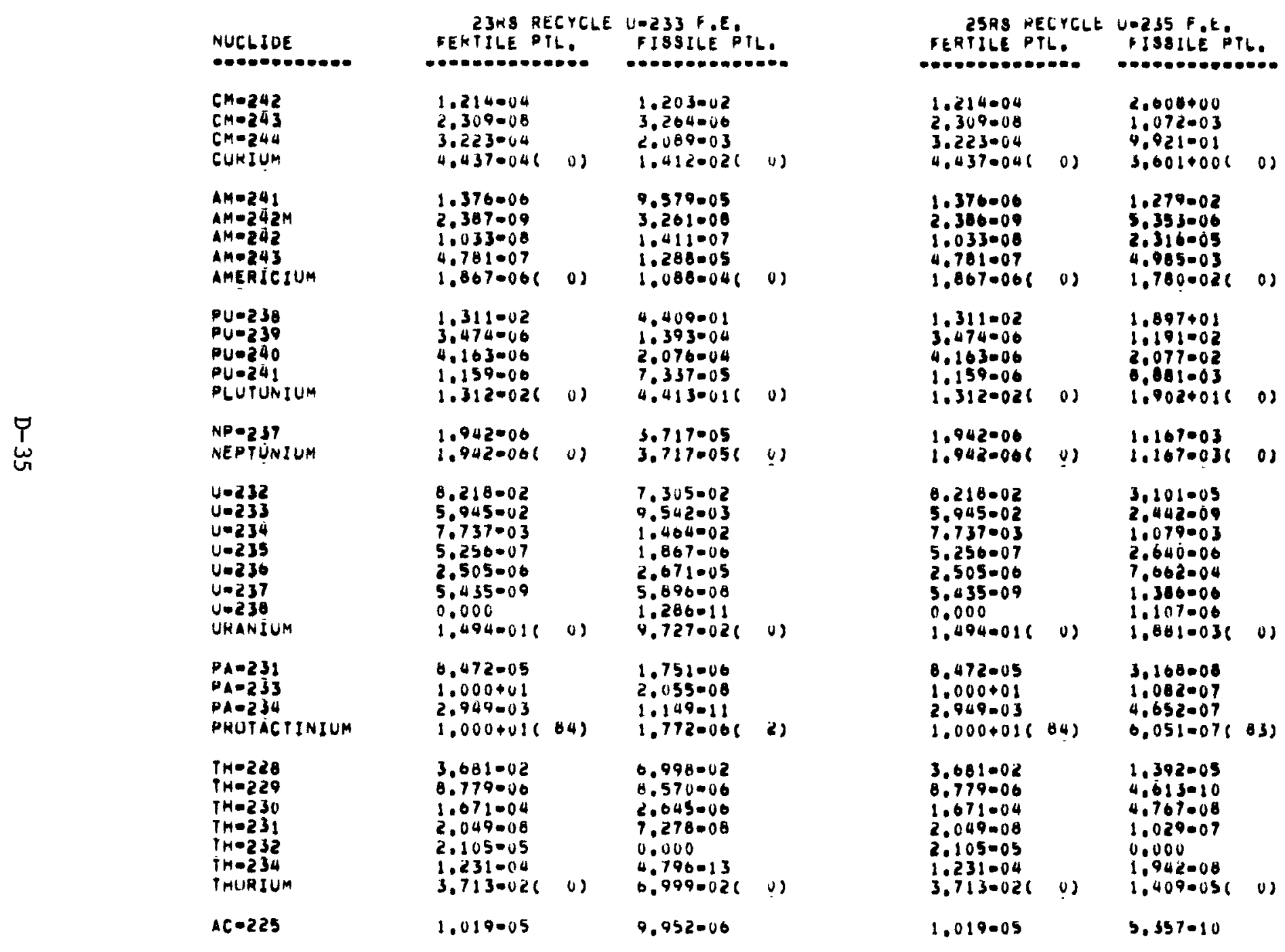




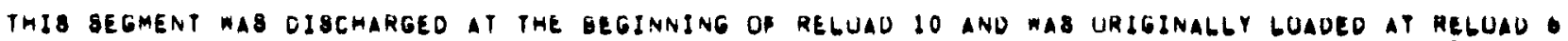
DECAY HEAT IN MATIS PEN FE D TI TIME 180 DAYS AFIER OISCHARGE (HENCENT FUM GAMMAS IN PARENTHESES)

\begin{tabular}{|c|c|c|c|c|c|}
\hline & $\begin{array}{l}\text { NUCLIDE } \\
\text { WDE }\end{array}$ & 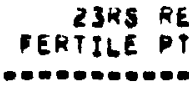 & $\begin{array}{l}\text { ECYCLE } \\
\text { Th. } \\
\text { T. }\end{array}$ & 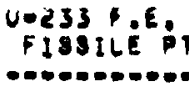 & It. \\
\hline & $\begin{array}{l}A C=227 \\
A C=22 B \\
A C I I N Z U M\end{array}$ & $\begin{array}{l}1.221=07 \\
3.280-06 \\
1.301-051\end{array}$ & 17 & $\begin{array}{l}1.205009 \\
2.258-12 \\
9.954-006\end{array}$ & $0)$ \\
\hline & $\begin{array}{l}\text { RA-2Z3 } \\
\text { RA-224 } \\
R A=225 \\
\text { RA-228 } \\
\text { RADIUM }\end{array}$ & $\begin{array}{l}8.346006 \\
3.854002 \\
2.295-07 \\
3.196000 \\
3.854=026\end{array}$ & $0)$ & $\begin{array}{l}7.799=08 \\
7.326-02 \\
2.241009 \\
2.194-14 \\
7.326-021\end{array}$ & נט \\
\hline & $\begin{array}{l}F R-221 \\
F K-223 \\
\text { FRANEIUM }\end{array}$ & $\begin{array}{l}1.100=05 \\
9.360009 \\
1.110 .051\end{array}$ & 01 & $\begin{array}{l}1.083=05 \\
8.749-11 \\
1.083=056\end{array}$ & 0) \\
\hline & $\begin{array}{l}\text { RNEZ219 } \\
\text { RN=220 } \\
\text { RADON }\end{array}$ & $\begin{array}{l}9.096=00 \\
4.267-02 \\
4.268 .026\end{array}$ & 0) & $\begin{array}{l}9.060000 \\
8.113=02 \\
6.113=021\end{array}$ & $0)$ \\
\hline$\vec{\sigma}$ & $\begin{array}{l}\text { AT-217 } \\
\text { AT-216 } \\
\text { ASIAIINE }\end{array}$ & $\begin{array}{l}1.237-05 \\
1.452-10 \\
1.237-056\end{array}$ & (v) & $\begin{array}{l}1.208005 \\
0.000 \\
1.2080056\end{array}$ & uJ \\
\hline & $\begin{array}{l}P U=210 \\
P U=212 \\
P U=213 \\
P U=215 \\
\text { POE-210 } \\
\text { POLONIUM }\end{array}$ & $\begin{array}{l}2.172=05 \\
3.692002 \\
1.467=05 \\
1.050=05 \\
4.605=02 \\
8.301=026\end{array}$ & 0) & $\begin{array}{l}4.165=06 \\
7.250 .02 \\
1.432005 \\
9.814008 \\
8.754=02 \\
1.601=016\end{array}$ & 01 \\
\hline & $\begin{array}{l}6 I-211 \\
0 I-215 \\
4 I-213 \\
B I S M U T H\end{array}$ & $\begin{array}{l}9.394006 \\
1.919002 \\
1.283=06 \\
1.921 .026\end{array}$ & 4) & $\begin{array}{l}6.776 .08 \\
3.649 .02 \\
1.252006 \\
3.6490026\end{array}$ & 4) \\
\hline & $\begin{array}{l}P B-209 \\
P B+211 \\
P B-212 \\
L E A D\end{array}$ & $\begin{array}{l}3.570=07 \\
7.680 .07 \\
2.158003 \\
2.1590036\end{array}$ & (6) & $\begin{array}{l}3.485=07 \\
7.176009 \\
4.102=03 \\
4.103=031\end{array}$ & (6)) \\
\hline & $\begin{array}{l}\text { TL-207 } \\
\text { TL-208 } \\
\text { THALCIUM }\end{array}$ & $\begin{array}{l}7.559=07 \\
9.334=03 \\
9.334=036\end{array}$ & $84)$ & $\begin{array}{l}7.063=09 \\
1.835=02 \\
1.8350 .026\end{array}$ & 842 \\
\hline
\end{tabular}

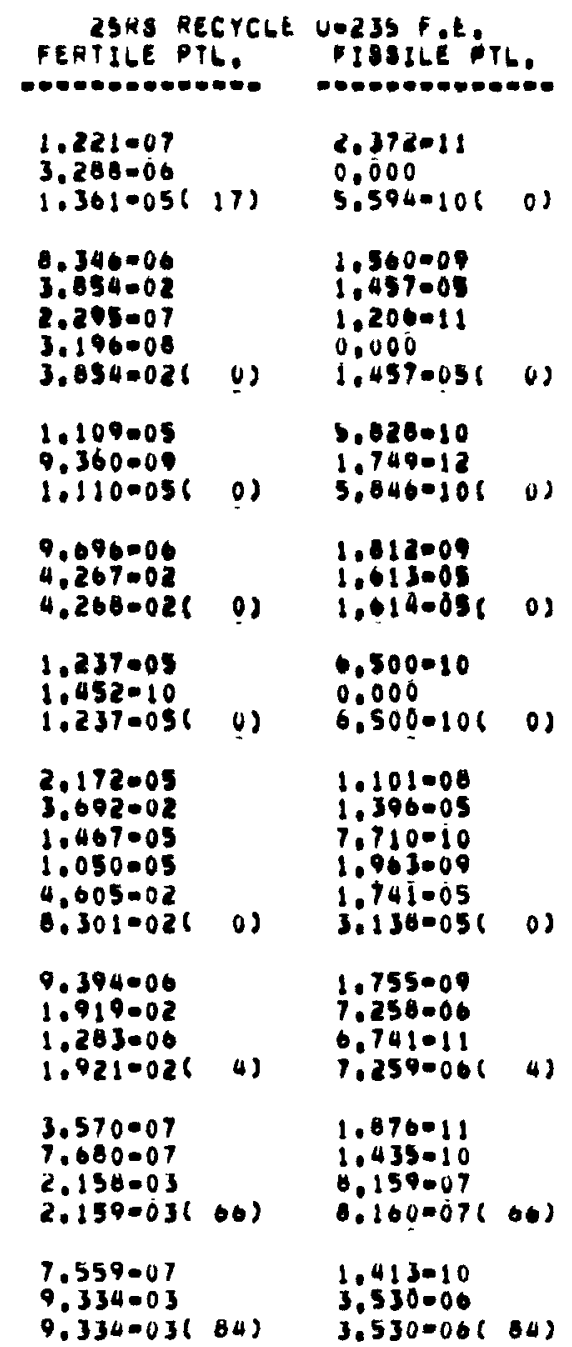




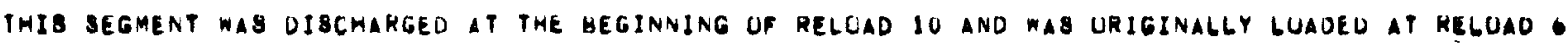

UECAY HEAT IN WATTS PEN F.E. AT IIME 1 OO DAYS APIER DISCMAHGE

(PEKCENT GRUM GAMMAS IN PARENTHESES)

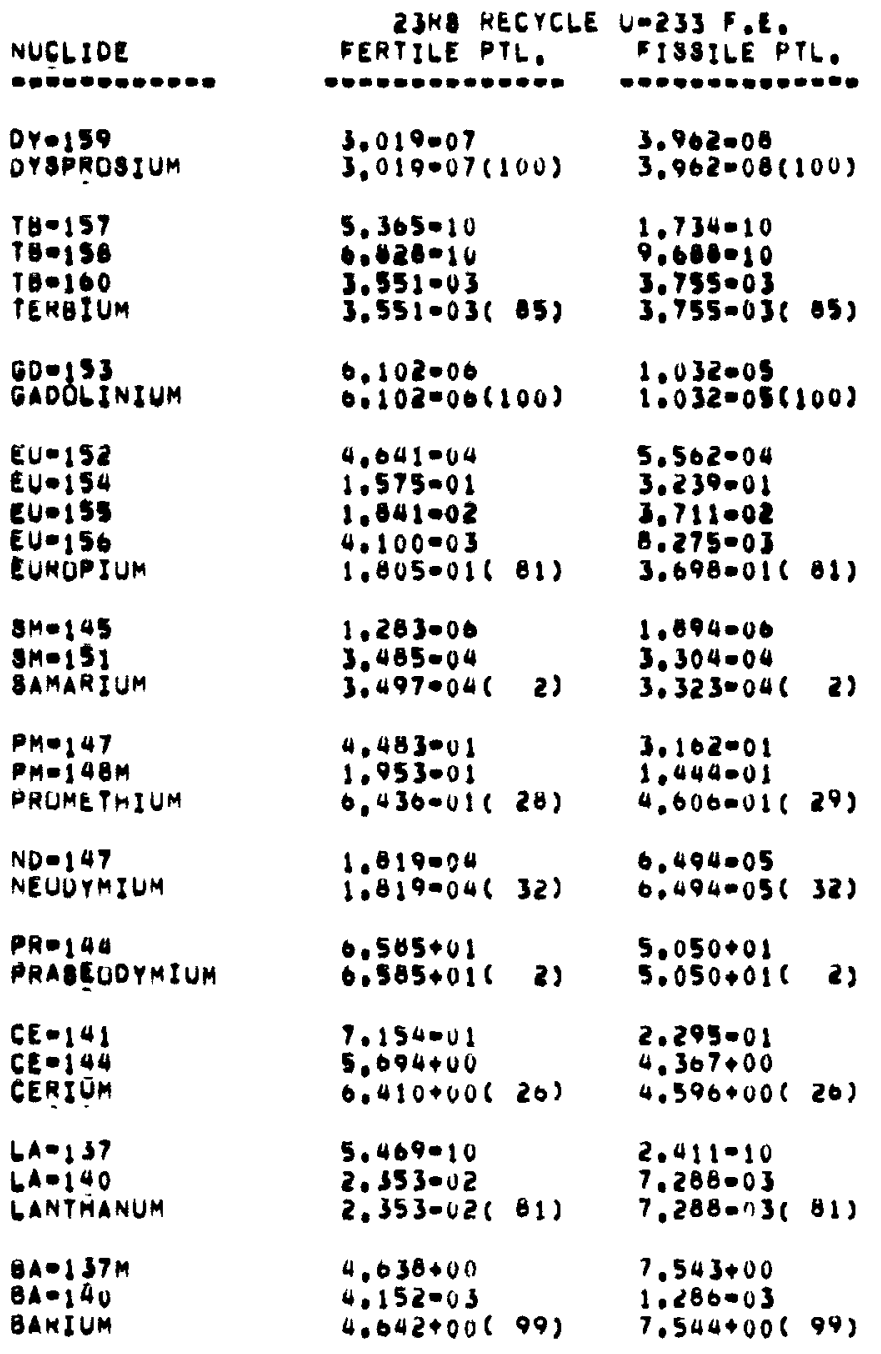

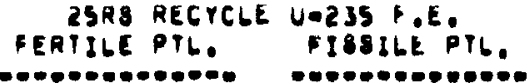

3.019007
$3.019007(100) \quad 8.292008$

$5.365 \cdot 10 \quad 1.2780 .10$

$0.828 .10 \quad 9.347-11$

3.551003
$3.551 .03(05) \quad 4.053003$
$4.053003($ es)

$0.102006 \quad 1.753005$

$6.102006(100) \quad 1,753005(100)$

4.641 .04

1.575001

$1.041=02$

3.202001

$1,8050016012 \quad 3,656001(01)$

早

\section{$1.263=06$}

$3.485=04$

\subsection{0}

4.483001

1.953 .01

$0.436001(28)$

2.715001

1.348001

1.819004

$1.810004(32) \quad 4.720005(32)$

0.585401

$6.565+011$

$4.115 * 01$

$4.115+01621$

7.154001

$6.410+00(26) \quad 3.696+00(26)$

$5.409-10$

$1.177-10$

$5.409-10$
$2.353-02$

$\begin{array}{ll}2.353-02 & 4.549-03 \\ 2.353 .02(81) & 4.549-03(81)\end{array}$

$4.638+00$

4.152 .03

$4.642400(99)$
$5.196+00$

8,025004
$5.197+00(99)$ 


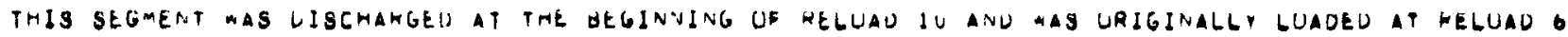
DECAY MEAT IN MATTS PER BE. AT IIME 180 DAYS APIEK UJBCAARGE (PERCENT FMOM GAMMAS IN PAKENTHESES)

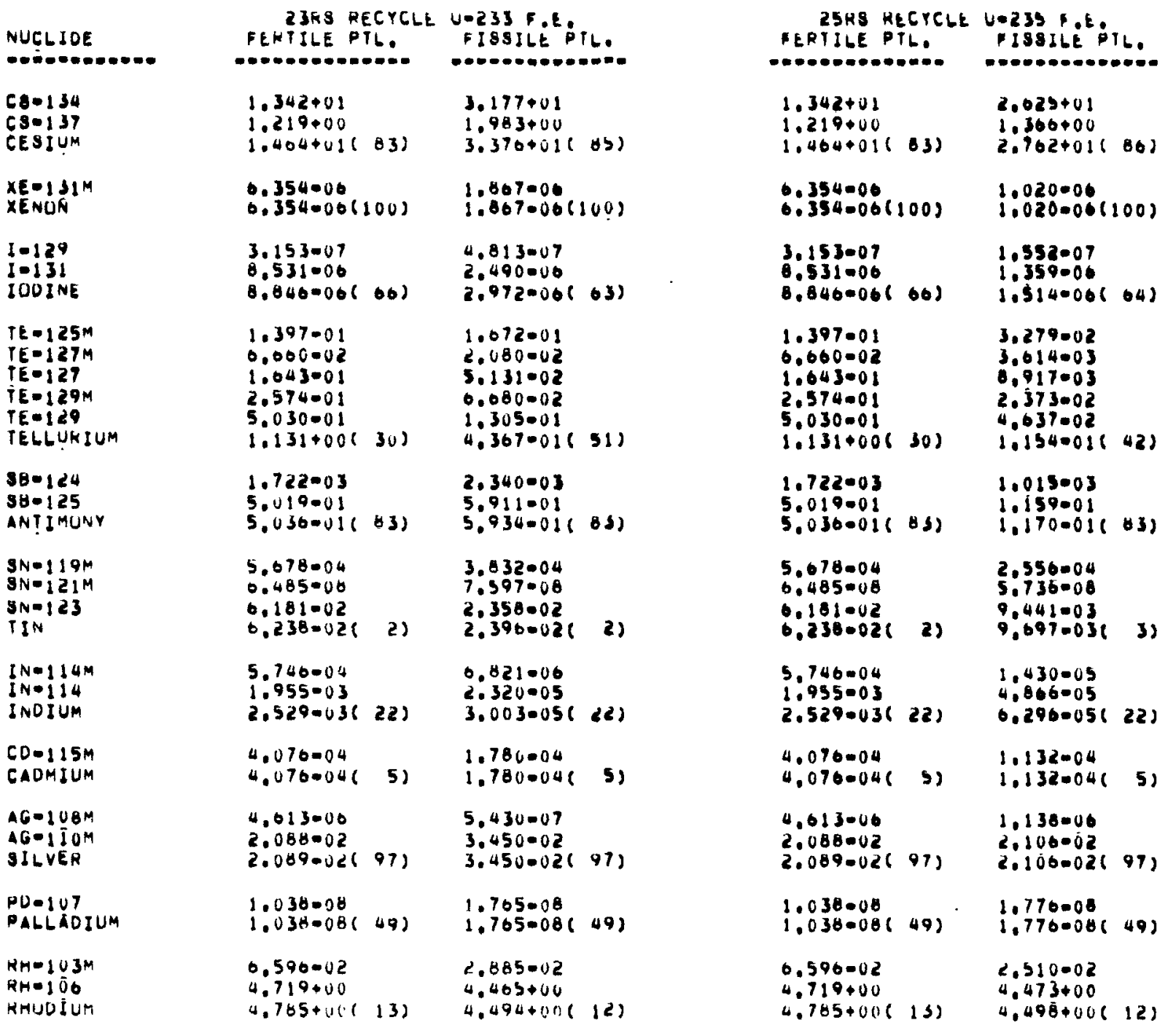




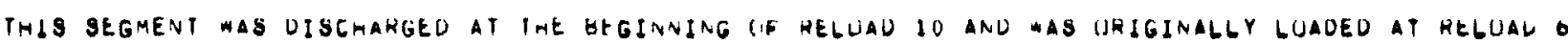
DECAY MEAT IN MATTS PER BE. AT IIME 280 DAYS APIEK UISCMARGE
(PERCENT RUM GAMMAS IN PARENTHESES)

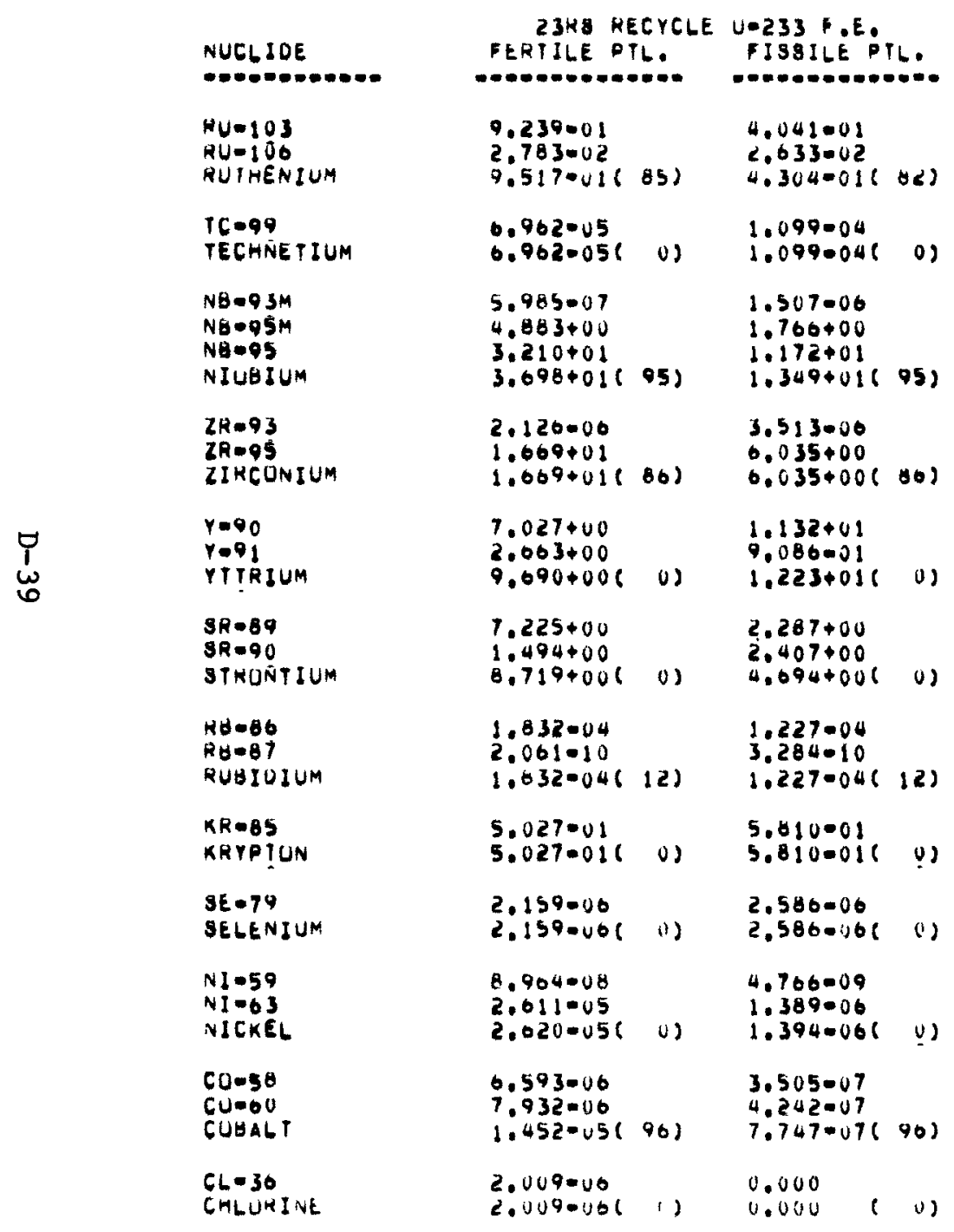

25HS RECYCLE U-235 F.E

PERTILE PTL. FISSILE PTL.

-

9.239001
2.783002
9,5170016

$9,517001683) \quad 3,7800016012$

$0.962=05 \quad 9.554 .05$

6.962 .056 os $9.559=056$

$5.985=07 \quad 9.889007$

$4.883400 \quad 1.150+00$

$\begin{array}{ll}3.628+01 & \\ 3.69+016 & 8.652+801+00(95)\end{array}$

$3.696+01895) \quad 0.808+00(25$

$2.120006 \quad 2.334=06$

$\begin{array}{ll}1.669+01 & 3,930+00 \\ 1.609+01(66) & 3,930+00(86)\end{array}$

$7.027+00 \quad 7.229+00$

$\begin{array}{llll}2.663000 & & 5.419001 \\ 9.690+00( & 0) & 7.778+00( & 0\end{array}$

$7.225400 \quad 1.224+00$

$\begin{array}{llll}1.494+00 & & 1.537+00 \\ 8.719+00(\quad 0) \quad 2.761+006 & 0)\end{array}$

$1.832004 \quad 4.514005$

$2.061 .10 \quad 1.571-10$

$1.832004(12) \quad 4.514005(12)$

$\begin{array}{llll}5.027 .01 & 2.049 .01 \\ 5.027 .016 & 0) \quad 2.049 .01( & 0)\end{array}$

$2.159006 \quad 7.224007$

$\begin{array}{llll}2.159006 & \\ 2.159 .00(0) & 7.2240007(0)\end{array}$

$8.964008 \quad 9.994009$

$2.611 .05 \quad 2.913=06$

$2.620003(0) \quad 2.923006($

$0.593006 \quad 1.350 .07$

$\begin{array}{ll}7.932 .06 & \\ 1.052 .05(96) & 1.024006(96)\end{array}$

$\begin{array}{lll}2.009006 & 0.000 \\ 2.009000(0) & 0.000(0)\end{array}$ 


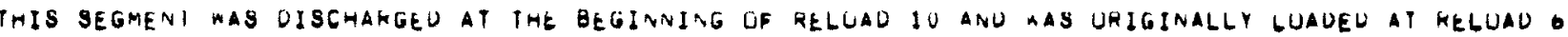

DECAY HEAT IN MATTS PER Fe GT TIME I HO OAYS AFIER DIBCHAPGE (PENCENT FROM GAMMAS IN PAKENIMESES)

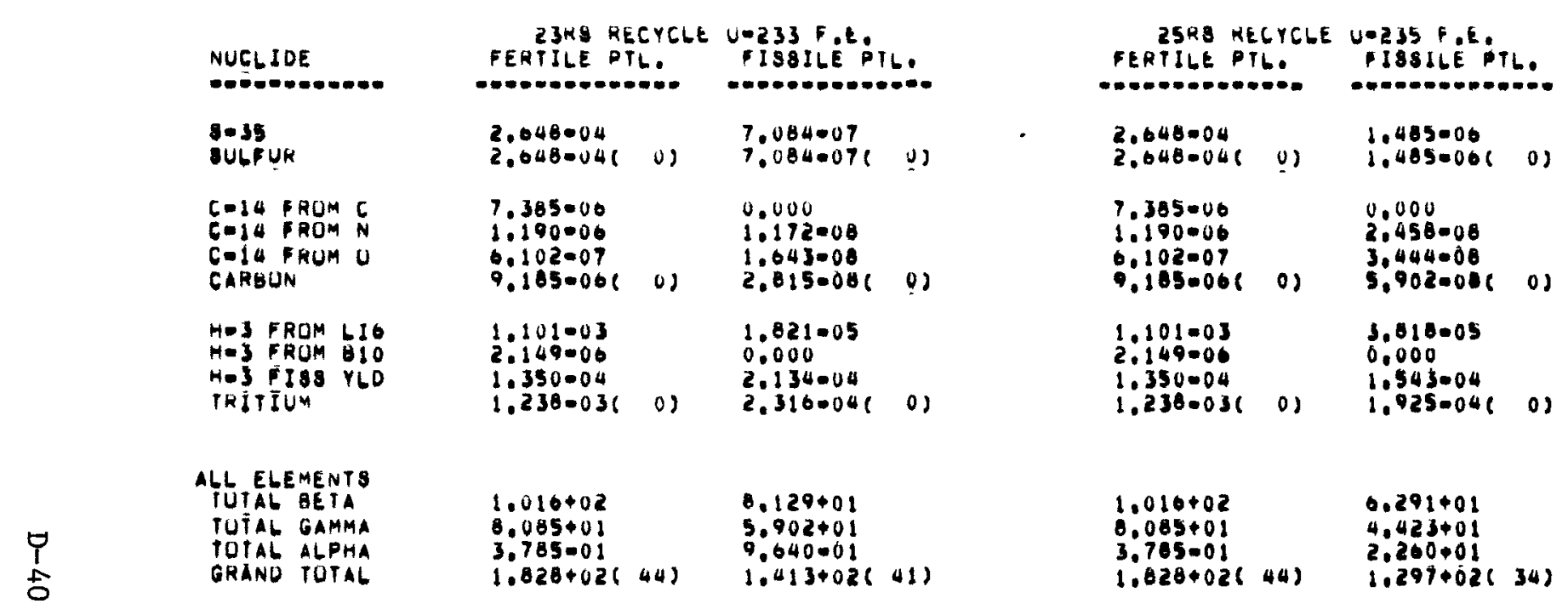


THIS SEGMENT WAS DISCHARGED AT THE GEGINNING UR HELUAD 10 ANU MAS URIGINALLY LUADEO AT RELUAU O

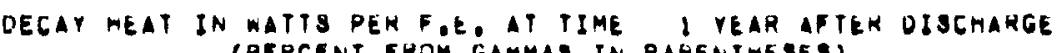
(PERCENT FROM GAMMAS IN PARENTMESES)

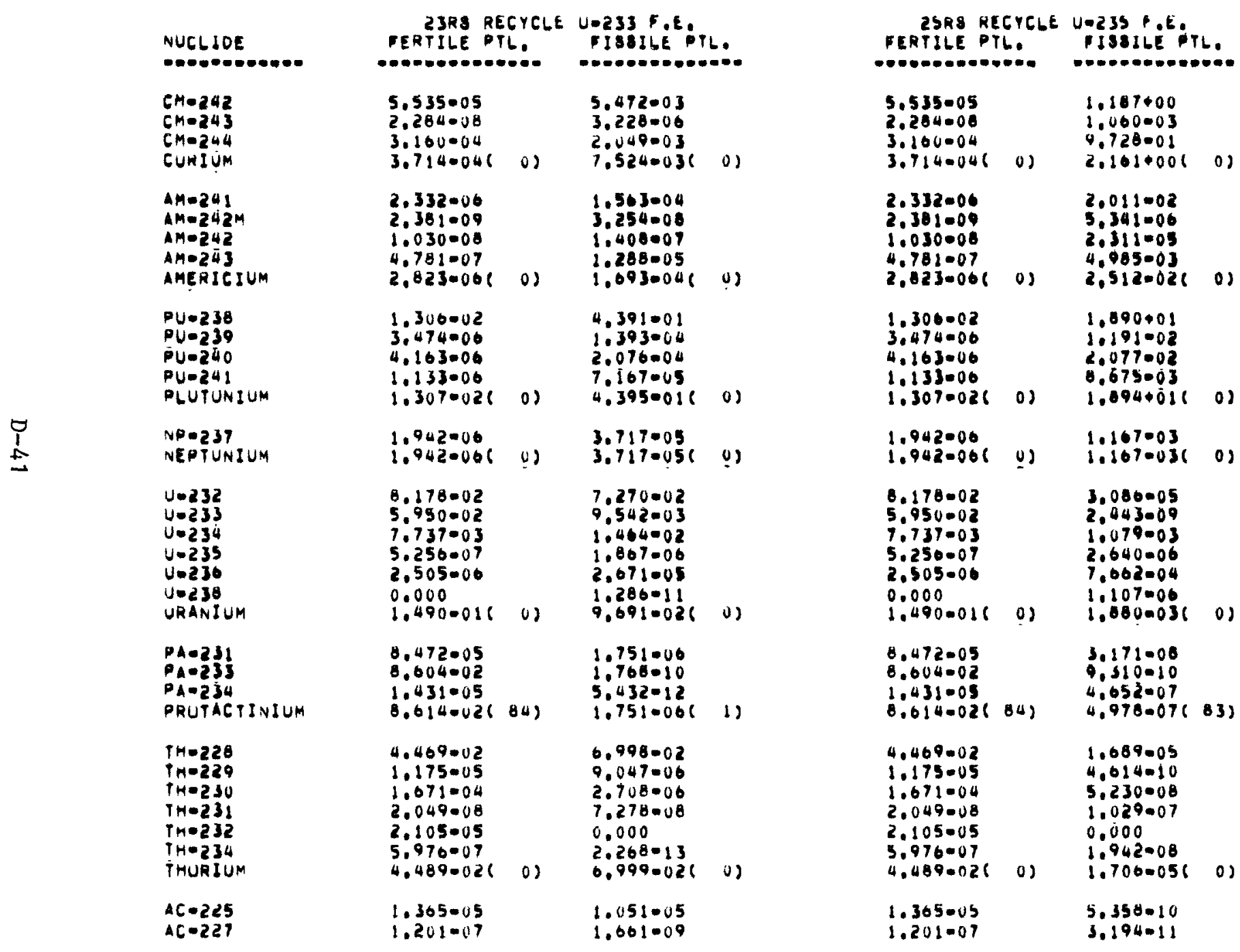




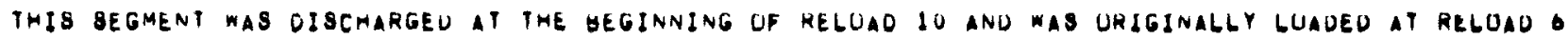
DECAY MEAT IN WATTS PER F.E. AI TIME I VEAR AFTER UISGMARGe (PERCENT PHOM GAMMAS IA PARENTHESEO)

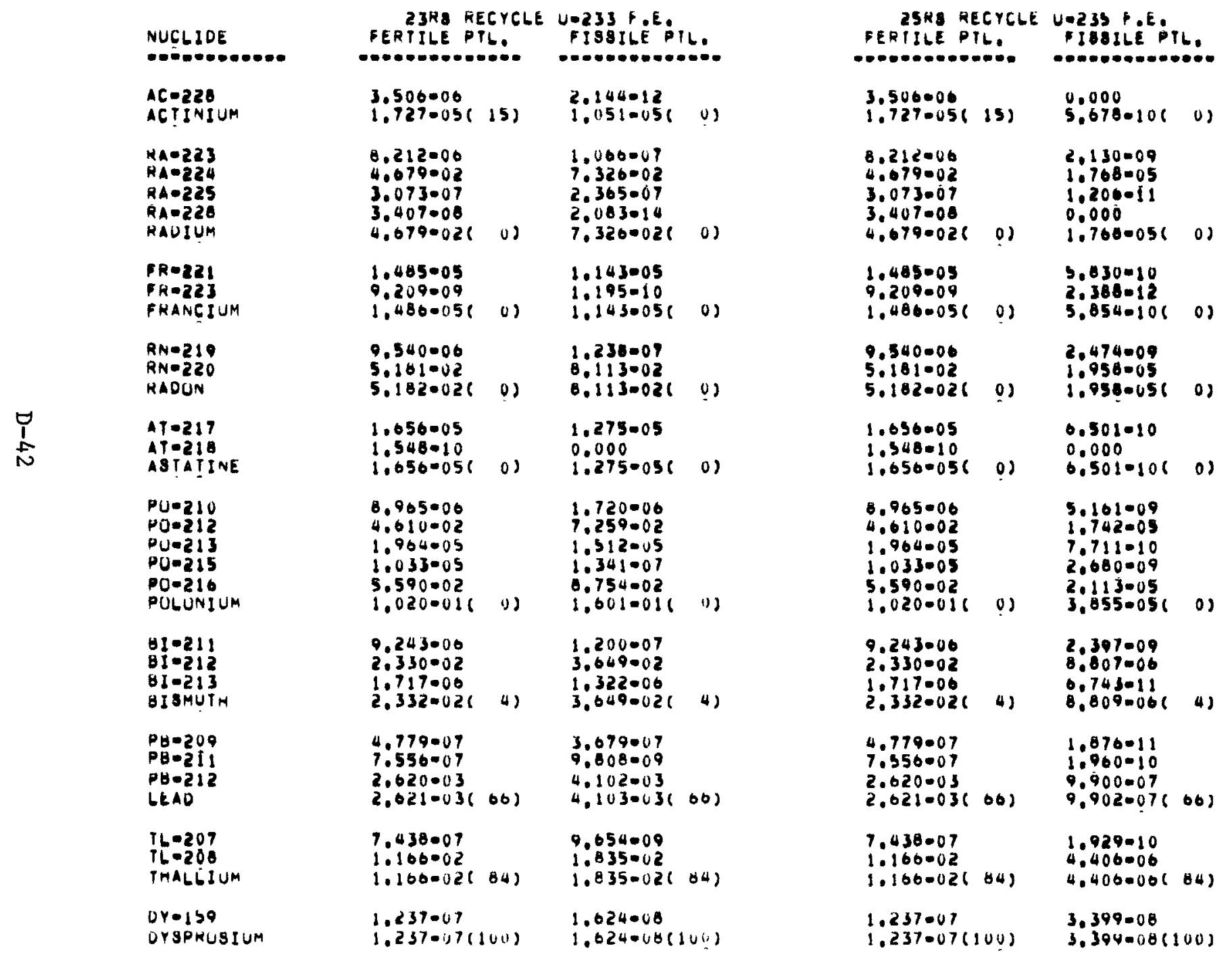




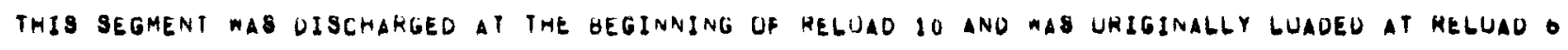
DECAY MEAT IN WATTS PER F.E. AT TIME J YEAR AFIEN OISCHAKGE

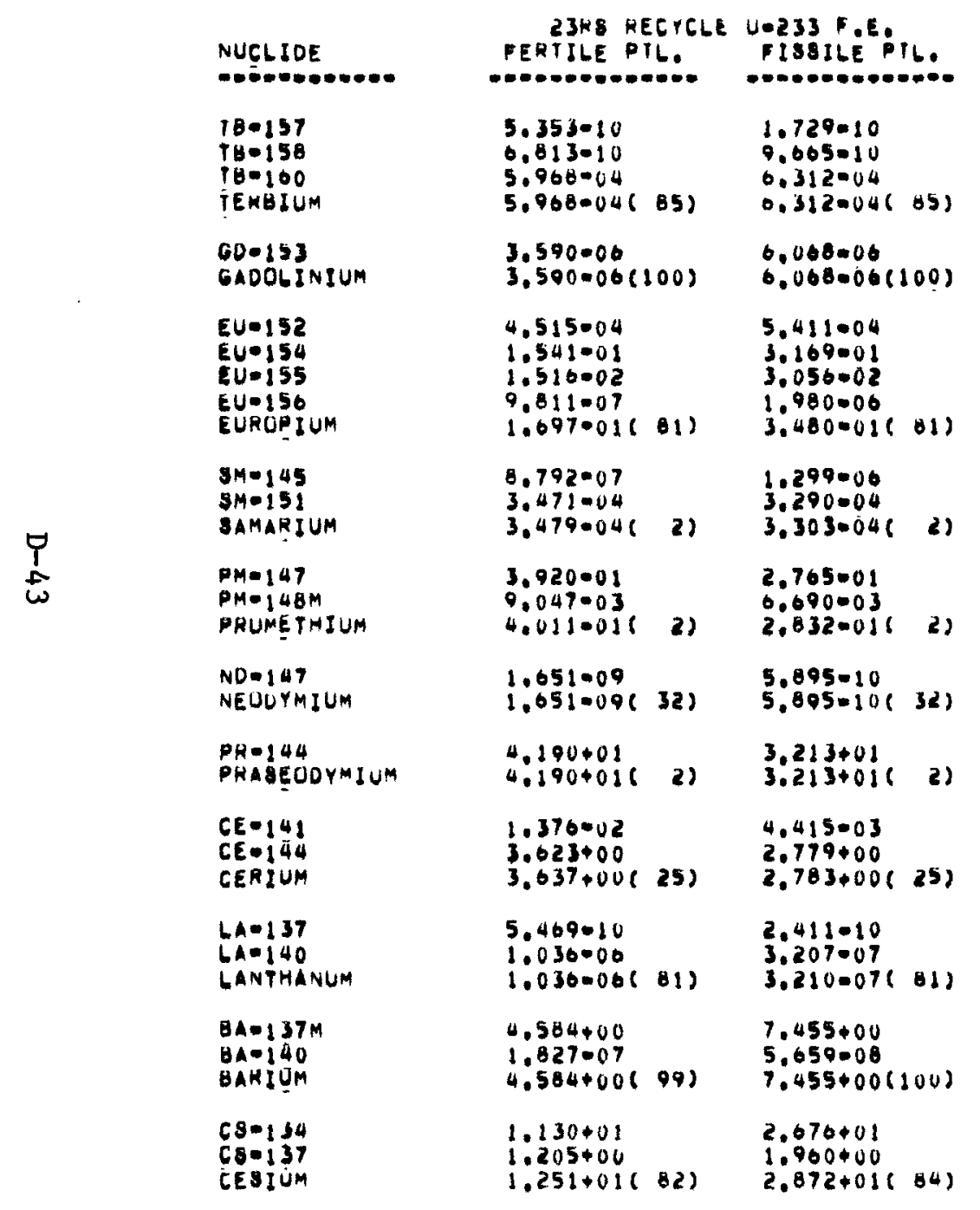

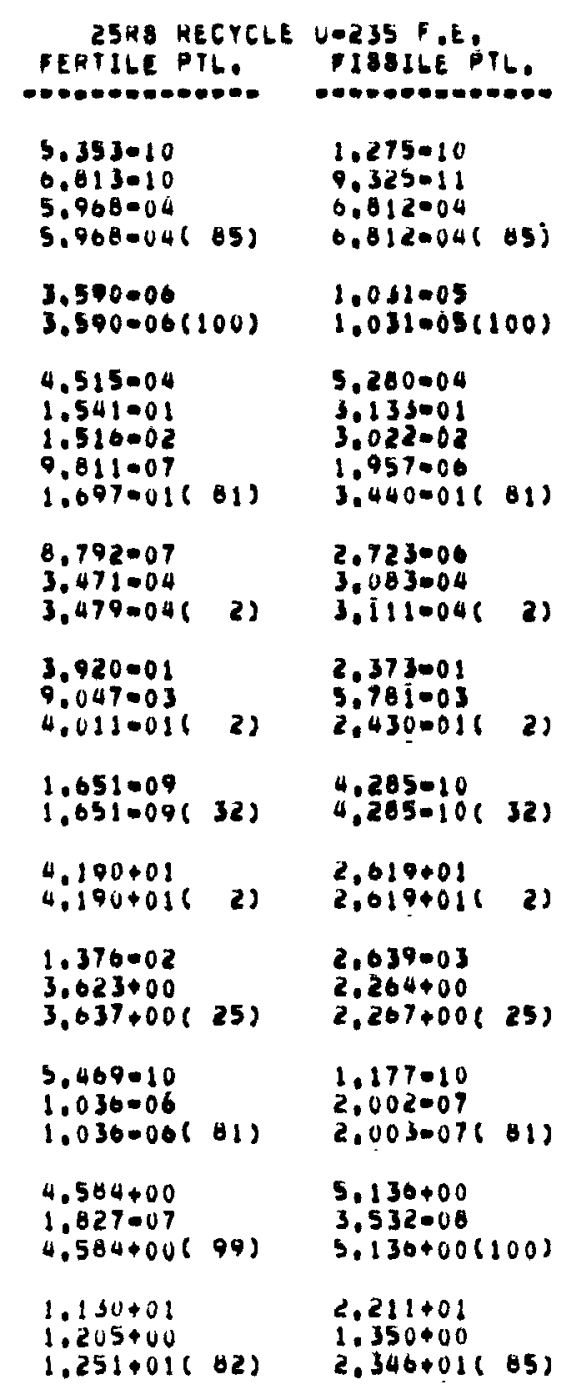




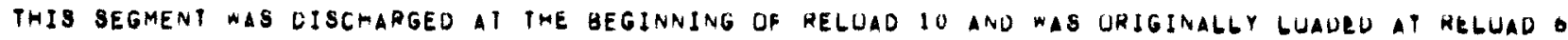
DECAY HEAT IN WATTS PER FEE AT TIME I YEAR AFTEK DISCHARGE

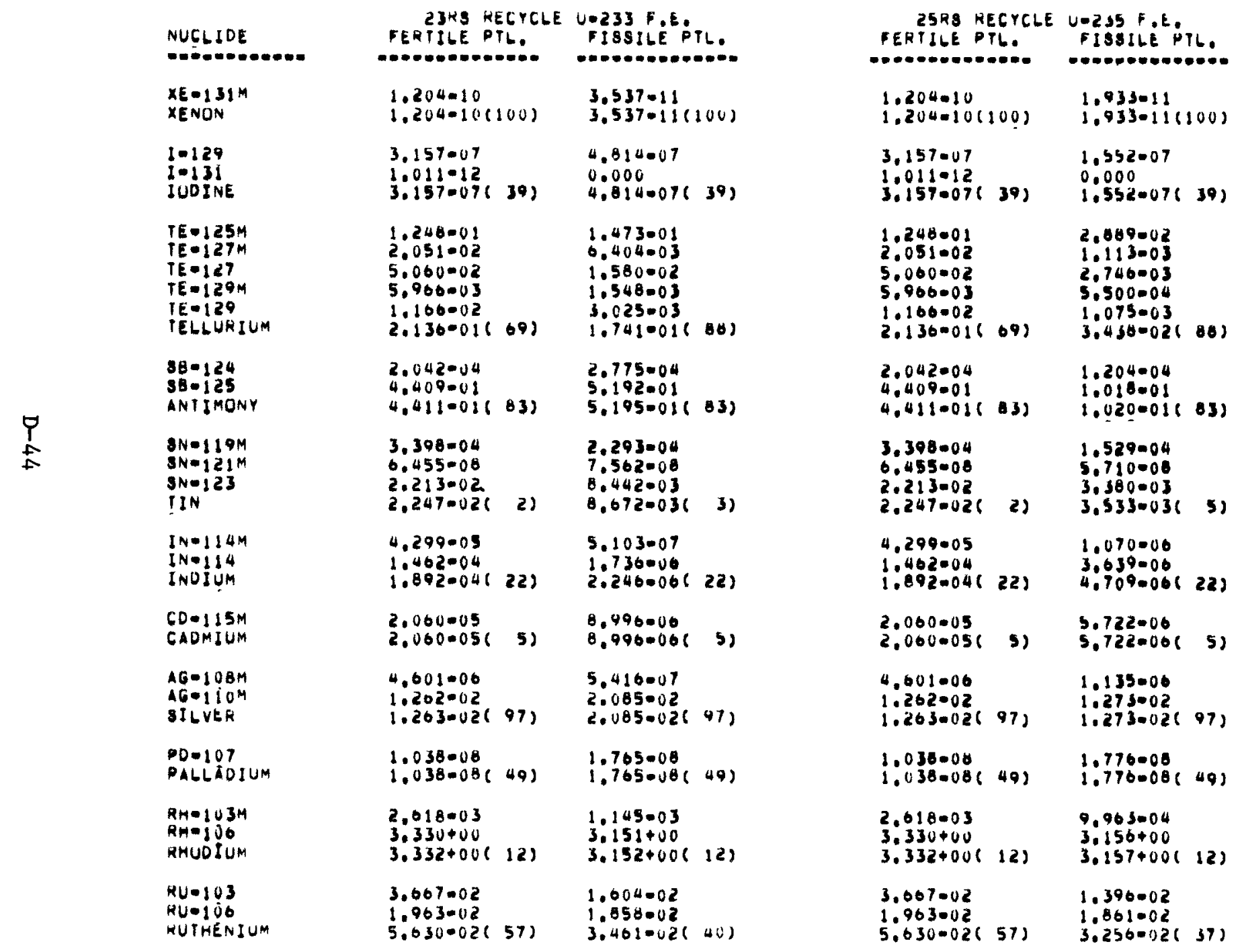


TMIS SEGMENT MAS DISCHAKGED AT THE GEGINNING UR RELUAD IO ANU WAS UKIGINALLY LUADEU AT RELUAD G

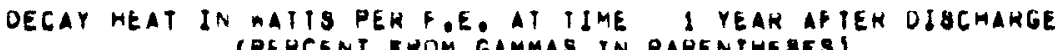
(PERCENT PROM GAMMAS IN PARENTHESES)

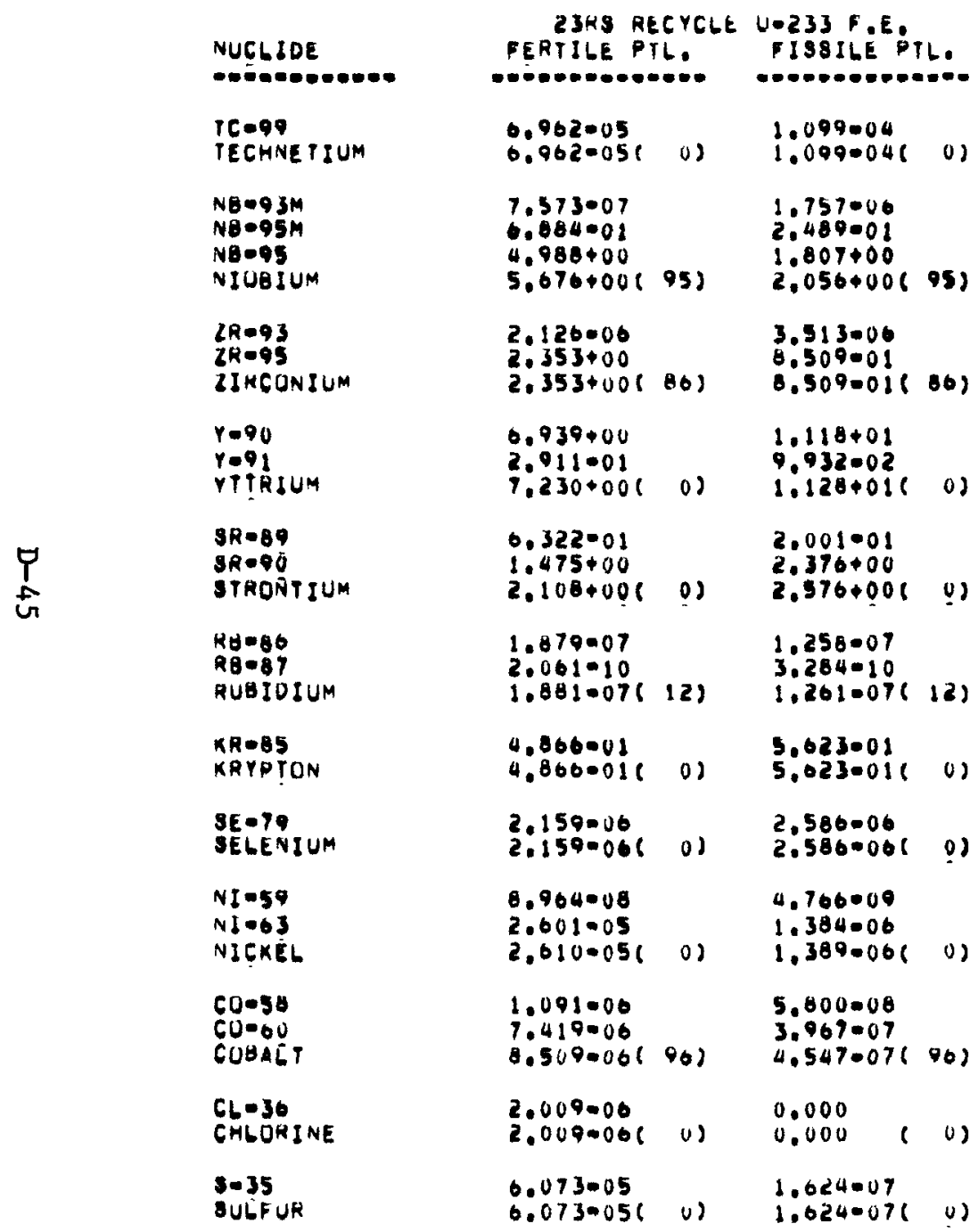

\begin{tabular}{|c|c|c|c|}
\hline $\begin{array}{l}\text { 25RS KE } \\
\text { FERTILE PT }\end{array}$ & $\begin{array}{l}\text { ECYGLE } \\
\text { TL. } \\
\text { E. }\end{array}$ & $\begin{array}{l}\text { U.235 F.E. } \\
\text { FISILE PT }\end{array}$ & Th. \\
\hline $\begin{array}{l}0.962-05 \\
0.962-056\end{array}$ & $0)$ & $\begin{array}{l}9,5590.05 \\
9,550=056\end{array}$ & $0)$ \\
\hline $\begin{array}{l}7.573=07 \\
6.884001 \\
4.908+00 \\
5.6704006\end{array}$ & $95)$ & $\begin{array}{l}1.155000 \\
1.021001 \\
1.177+00 \\
1.339+001\end{array}$ & 95) \\
\hline $\begin{array}{l}2.120000 \\
2.353+00 \\
2.353+008\end{array}$ & (6) & $\begin{array}{l}2.334006 \\
5.540=01 \\
5.3400016\end{array}$ & $0(1)$ \\
\hline $\begin{array}{l}0.939+00 \\
2.911001 \\
7.230+006\end{array}$ & $0)$ & $\begin{array}{l}7.138+00 \\
5.123002 \\
7.107+001\end{array}$ & 08 \\
\hline $\begin{array}{l}0.322001 \\
1.475+00 \\
2.1000008\end{array}$ & $0)$ & $\begin{array}{l}1.071001 \\
1.518+00 \\
1.025+006\end{array}$ & $0)$ \\
\hline $\begin{array}{l}1.879-07 \\
2.061-10 \\
1.0810071\end{array}$ & (2) & $\begin{array}{l}4.630008 \\
1.571010 \\
4.6460086\end{array}$ & (2) \\
\hline $\begin{array}{l}4.800001 \\
4.8660011\end{array}$ & $0)$ & $\begin{array}{l}2,563001 \\
2,5630016\end{array}$ & 08 \\
\hline $\begin{array}{l}2.159006 \\
2.1500061\end{array}$ & (ט) & $\begin{array}{l}7.2240 .07 \\
7.2240 .076\end{array}$ & $0)$ \\
\hline $\begin{array}{l}8.904008 \\
2.601005 \\
2.610 .056\end{array}$ & $0)$ & $\begin{array}{l}9.994=09 \\
2.902=06 \\
2.912=066\end{array}$ & $0)$ \\
\hline $\begin{array}{l}1.091006 \\
7.419006 \\
8.5090061\end{array}$ & $96)$ & $\begin{array}{l}1.210007 \\
8.310007 \\
9.5340076\end{array}$ & $\left.9_{0}\right)$ \\
\hline $\begin{array}{l}2.009006 \\
2.0090061\end{array}$ & u) & $\begin{array}{l}0.000 \\
0.000\end{array}$ & 08 \\
\hline $\begin{array}{l}0.073-05 \\
6.0730051\end{array}$ & (0) & $\begin{array}{l}3.400007 \\
3.4060076\end{array}$ & $0)$ \\
\hline
\end{tabular}


THIS BEgMENT WAS OISCMARGED AT THE GEGINNING UP RELUAO IO ANU WAS URIGINALLY LUADEU AT RELOAD DECAY HEAT IN MATTS PER F.E. AI TIME I YEAR AFTER UISCHAHGE (PEREENT PROM GAMMAS IN PARENIHESESS

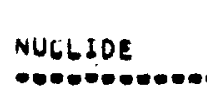

Cola FROM 5

Cola FROM 5 Cola FROM N
ColO FROM O

COIU FRO
CAHBUN

HoJ FrOM LIO Hej FROM Bio Th 158 YLO TRITIUM

\section{ALL ELEMENTS} TOTAG BETA

TOPAL GAMMA

TUTAL ALPHA

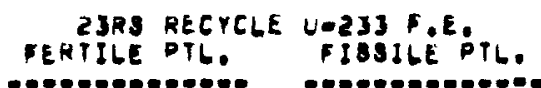

7.304000

$1.190-06$

0.101 .07

0.1850008 ud

1.070003

2,089006

1.312004

$1.204003($ u)

FIBSILE PIL.

0.000

1.172000

1.643008

2.0150006 प

1.770005

0.000

2.07400

$5.980+01$

$2.543+01$

4.254001

$0.500+01(29)$

$5.573+01$

$3.725+01$

9.5540 .01

$9.394+01(39)$
2.251.046 0

2SRS RECYCLE U:235 F.E. FERIILE PTL. FISILE PTLO

7.384000

$1.190=06$

$0.101=07$

9.1850068 (4)

0.000

2.457 .00

3.444008

5.90200860

1.070003

2.084006

1.312004

1.2040036 os

3.711005

0.000

1.499004

1.070004508

$\stackrel{P}{\stackrel{p}{a}}$

$\begin{array}{ll}5.980+01 & 4.300+01 \\ 2.543+01 & 2.00901 \\ 4.254001 & 2.109+01 \\ 8.560+01(29) & 9.308+01(31)\end{array}$




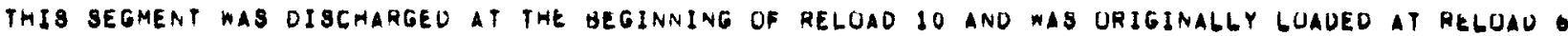

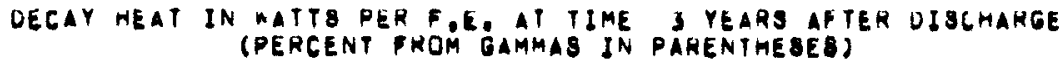

NUCL JOE

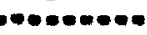

$C_{C M-243}$

$C M-243$
$C M=244$

CURIUM

$A M=241$
$A M=242 M$

$A M-242 M$
$A M+24 ?$

$A M+242$
$A M-243$

AMERICIUM

PUन 38

$P U=239$
$P U=240$

$P U=240$
$P U=241$

PLuTúnium

$\stackrel{8}{1}$

NP- 237

U.232

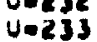

verzá

$4-235$

U.236

$U-230$

UHANIUM

$P A-23 !$

PA-23S

PRITACTINIUM

$1 \mathrm{H}=228$

$1+1+-229$

TM=230

THe.23

$\{n=23$

TH०e 34

THURIUM

$A C-225$
$A C-227$

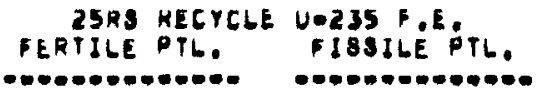

\begin{tabular}{|c|c|c|}
\hline $\begin{array}{l}2.674000 \\
2.167=08 \\
2.924004 \\
2.0510046\end{array}$ & $0)$ & $\begin{array}{l}5.555002 \\
1.015003 \\
9.003001 \\
9.5490016\end{array}$ \\
\hline $\begin{array}{l}5.883=06 \\
2.359009 \\
1.021000 \\
4.780007 \\
0.3740 .066\end{array}$ & 0) & $\begin{array}{l}4.729=02 \\
5.293=06 \\
2.290005 \\
4.904003 \\
5.231=026\end{array}$ \\
\hline
\end{tabular}

$1.285002 \quad 1.060+01$

$3.474006 \quad 1.191=02$

$4.162006 \quad 2.097002$

$\begin{array}{llll}1.033006 & 7.909003 \\ 1.286002(\text { o) } & 1.8640016 & 0)\end{array}$

$1.942006 \quad 1.167003$

1.9420066 o) 1.16700360$)$

$0.022002 \quad 3.027 .05$

$5.050002 \quad 2.043009$

$7,737=03 \quad 1.070003$

$5.256007 \quad 2.640006$

$2,505006 \quad 7.662004$

$0.000 \quad 1.107=00$

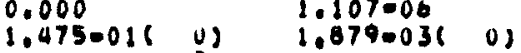

$0.471005 \quad 3.183=00$

$6.161 .10 \quad 0.008$

$\begin{array}{ll}0.161-10 & 0.000 \\ 0.000 & 4.052-07\end{array}$

$\begin{array}{lll}0.000 & 4.052007 \\ 8.471-05(\text { i) } & 4.970 .07(3)\end{array}$

$0.420002 \quad 2.420005$

$2.348005 \quad 4.616010$

$1.673004 \quad 7.060008$

$2.049008 \quad 1.029007$

$2.105005 \quad 0.000$

$0.000 \quad 1,942008$

$0.447-02(0) \quad 2.445 .056 \quad 0)$

$\begin{array}{ll}2.726005 & 5.363-10 \\ 1.126007 & 6.316011\end{array}$ 


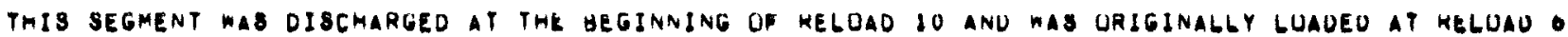

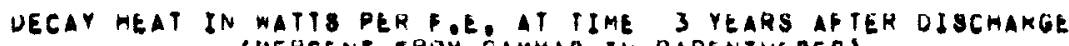
(PERCENT PROM GAMMAS IN DARENTHESES)

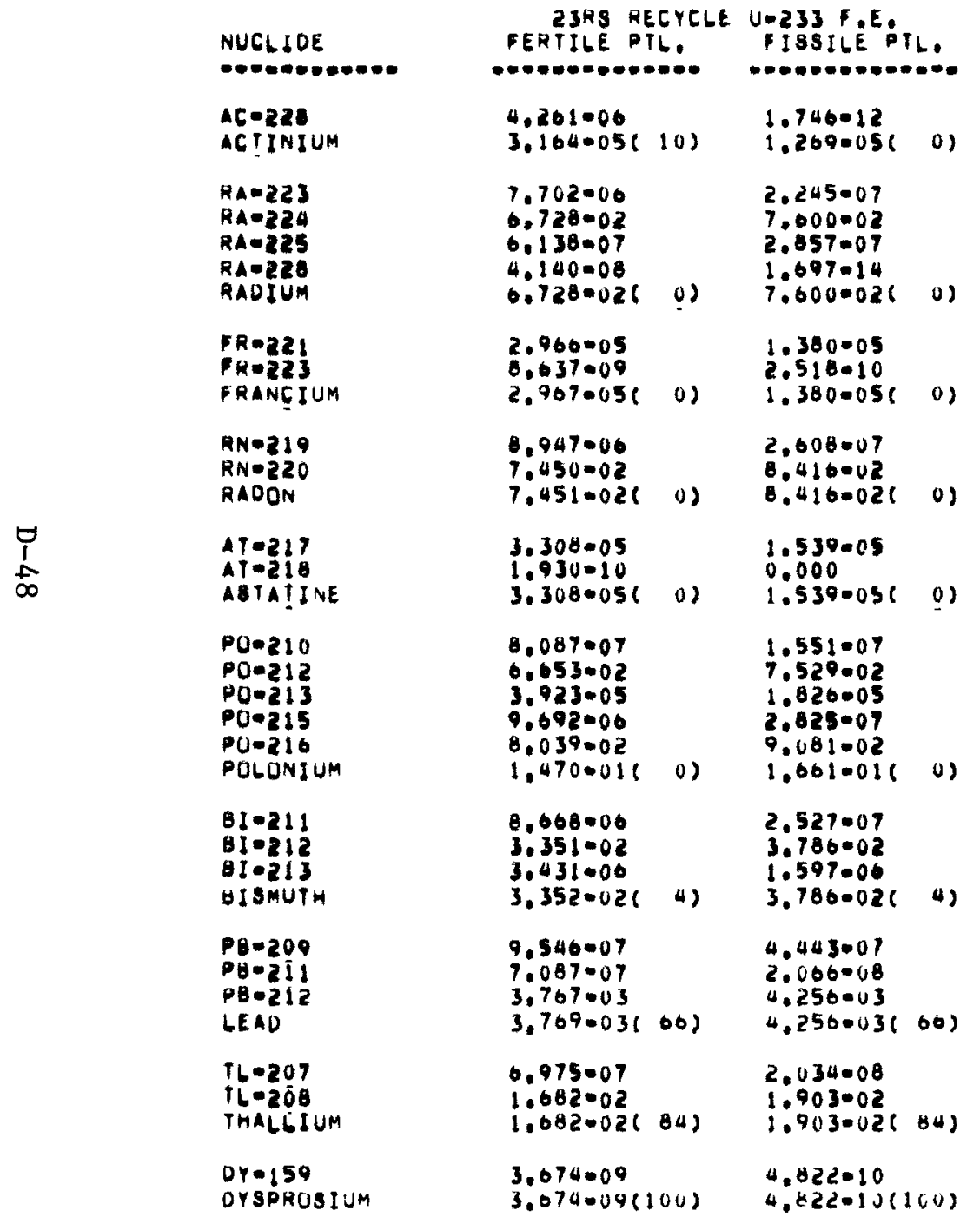

$25 \% 8$ RECYCLE U.235 F.E. FERTILE PTL, FIS8ILE PIL.

$4.201006 \quad 0.000$

$3.164005(10) \quad 5.904 .1011$

$7.702006 \quad 4.295009$

$6.728 .02 \quad 2.540-05$

$6.136007 \quad 1.207-11$

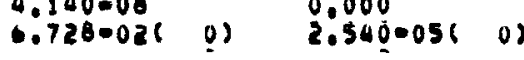

$2.906005 \quad 5.835-10$

$\begin{array}{lll}0.637 .09 & & 5.816012 \\ 2,967.05( & 0) & 5.883010(0)\end{array}$

$8.047000 \quad 4.989009$

$\begin{array}{llll}7.450 .02 & 2.813005 \\ 7.451 .026 & 0) & 2.8130056 & 0\end{array}$

$3,308005 \quad 0,507 \cdot 10$

$\begin{array}{llll}1.030-10 & 0.000 \\ 3.300-056 & \text { us } & 6.507-106 & 0\end{array}$

$8.087-07 \quad 5.216010$

$0.053 .02 \quad 2.511 .05$

9.023005

$8.039 .02 \quad 3.035 .05$

$1.470 .016 \quad 0) \quad 5.547 .0560)$

$8.668006 \quad 4.834-00$

$0.060006 \quad 4.634000$

$3.431=06$

3.352 .026 4) $\quad 1.266-05(4)$

$9.546007 \quad 1.078-11$

$7.087-07 \quad 3.952-10$

$\begin{array}{ll}3.767-03 & 1.422=06 \\ 3,769-03(66) & 1.423-06(66)\end{array}$

$0.975-07 \quad 3.890-10$

$1.082=02$

$1.682002(84) \quad 6.350 .06(84)$

$\begin{array}{ll}3.674-09 & 1.009009 \\ 3.674-09(100) & 1.004009(100)\end{array}$ 


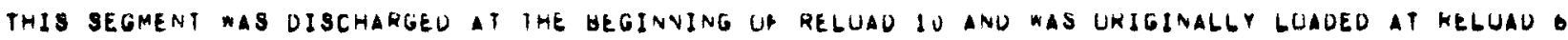

DECAY HEAT IN WATTS PER F.E AT IIME 3 YEARS AFIER UJSCHAKGE

(PERCENT FOM GAMMAS IN PARENTMEBESS

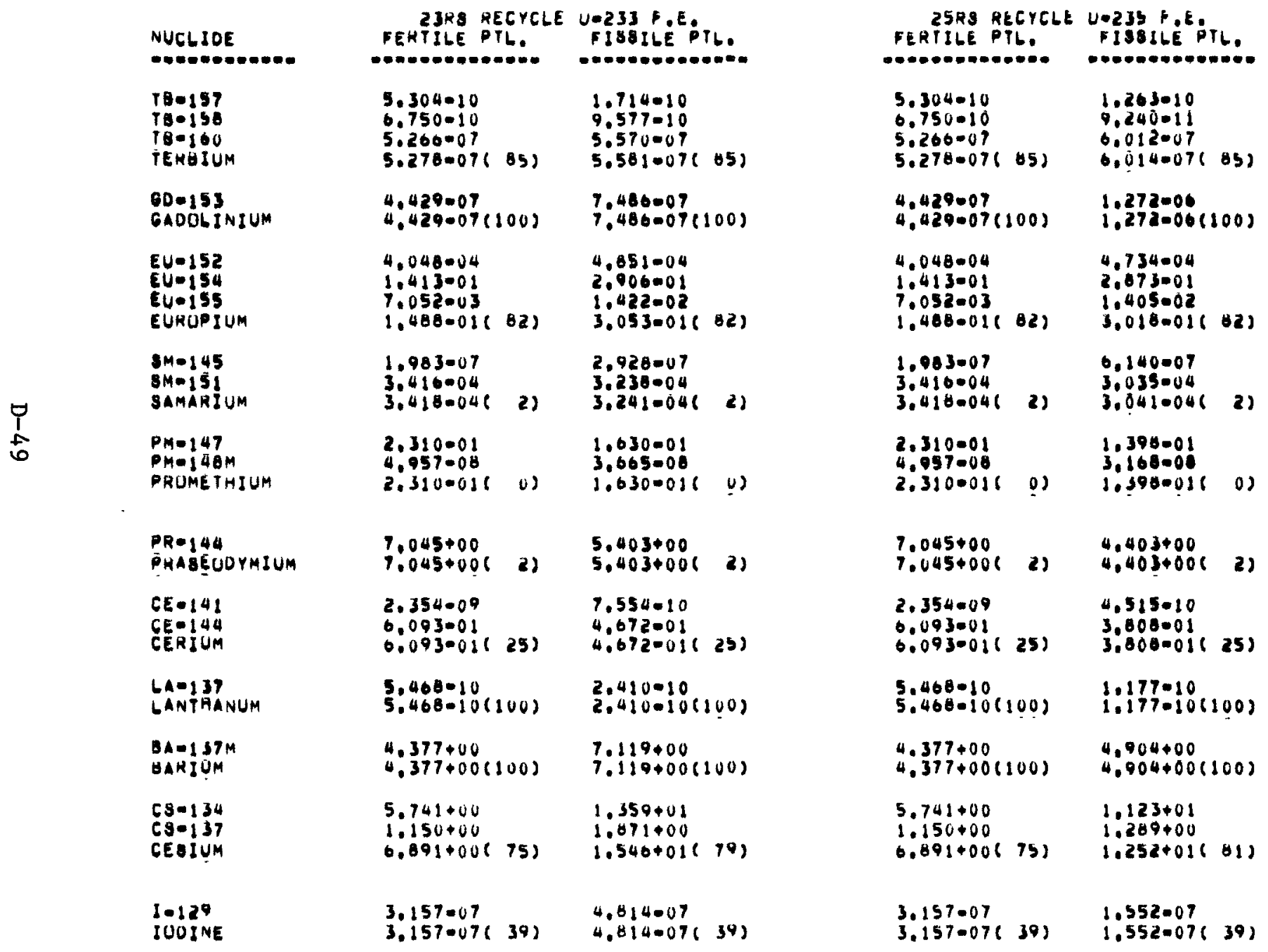




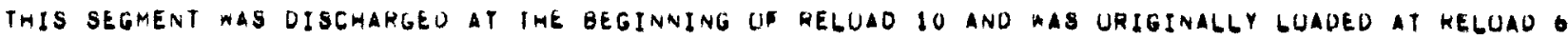
DECAY HEAT IN MATTS PEK F.E. AT TIME 3 YEARS AFIEK UISCMAKGE (PERCENT FRUM GAMMAS IN PARENTMESES'

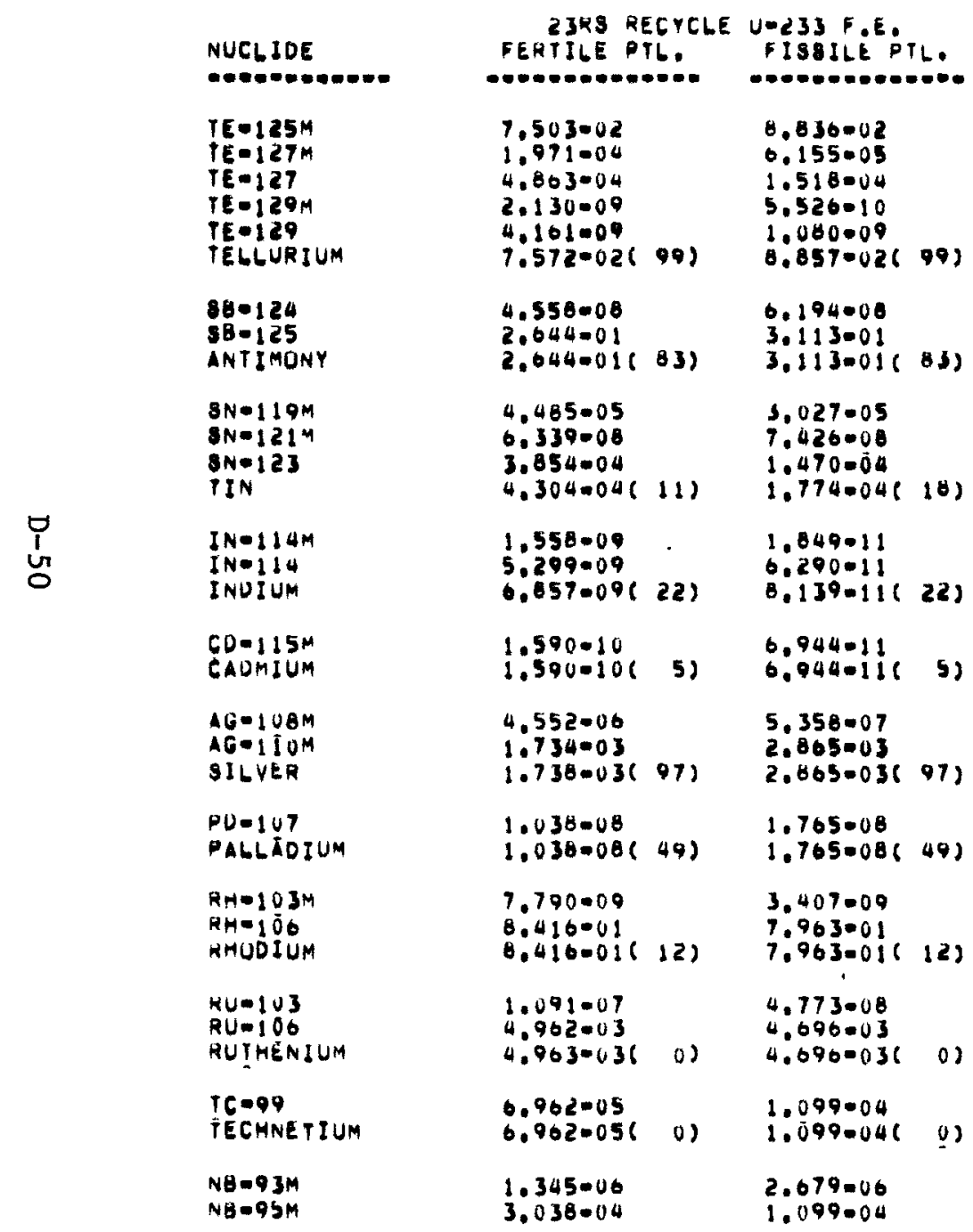

\begin{tabular}{|c|c|c|c|}
\hline $\begin{array}{l}25 \mathrm{~K} \\
\text { FERTILE }\end{array}$ & EYCLE & $\begin{array}{c}\text { U-23S P EE } \\
\text { PSSILE }\end{array}$ & ith. \\
\hline $\begin{array}{l}7.503002 \\
1.991004 \\
4.063004 \\
2.130=09 \\
4.161=09 \\
7.5720028\end{array}$ & $99)$ & $\begin{array}{l}1.733002 \\
1.070005 \\
2.039005 \\
1.963010 \\
3.636010 \\
1.7370026\end{array}$ & \\
\hline $\begin{array}{l}4.558008 \\
2.644001 \\
2.0440016\end{array}$ & $(3)$ & $\begin{array}{l}2.087 .08 \\
6.107 .02 \\
6.107 .026\end{array}$ & 63 \\
\hline $\begin{array}{l}4.455003 \\
6.339008 \\
3.854=04 \\
4.3040046\end{array}$ & (11) & $\begin{array}{l}2.019005 \\
5.607=08 \\
5.087-05 \\
7.9120056\end{array}$ & \\
\hline $\begin{array}{l}1.558=09 \\
5.299-09 \\
6.857-096\end{array}$ & (22) & $\begin{array}{l}3,677=11 \\
1.319-10 \\
1.707=100\end{array}$ & \\
\hline $\begin{array}{l}1.590-10 \\
1.500-106\end{array}$ & 5) & $\begin{array}{l}4.417-11 \\
4.417-116\end{array}$ & 5) \\
\hline $\begin{array}{l}4.552 .06 \\
1.734003 \\
1.7360036\end{array}$ & 971 & $\begin{array}{l}1.123006 \\
1.749003 \\
1.7500036\end{array}$ & \\
\hline $\begin{array}{l}1.038 .08 \\
1.038 .086\end{array}$ & 491 & $\begin{array}{l}1.770008 \\
1.7700085\end{array}$ & 498 \\
\hline $\begin{array}{l}7.790=09 \\
8.410001 \\
8.4160 .016\end{array}$ & (2) & $\begin{array}{l}2,965009 \\
7.977=01 \\
7.977-016\end{array}$ & \\
\hline $\begin{array}{l}1.091=07 \\
4.962 .03 \\
4.9630036\end{array}$ & (0) & $\begin{array}{l}4.153=08 \\
4.703=03 \\
4.7040036\end{array}$ & 08 \\
\hline $\begin{array}{l}0.962-05 \\
6.962-051\end{array}$ & (ט) & $\begin{array}{l}9.559005 \\
9.559 .056\end{array}$ & 08 \\
\hline $\begin{array}{l}1.3450 .06 \\
3.038=04\end{array}$ & & $\begin{array}{l}1.769=06 \\
7.152=05\end{array}$ & \\
\hline
\end{tabular}




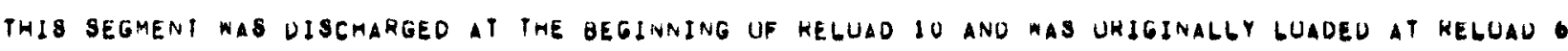

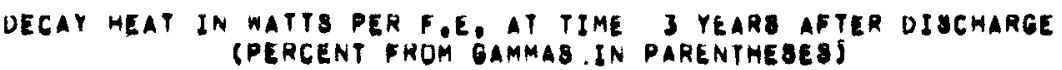

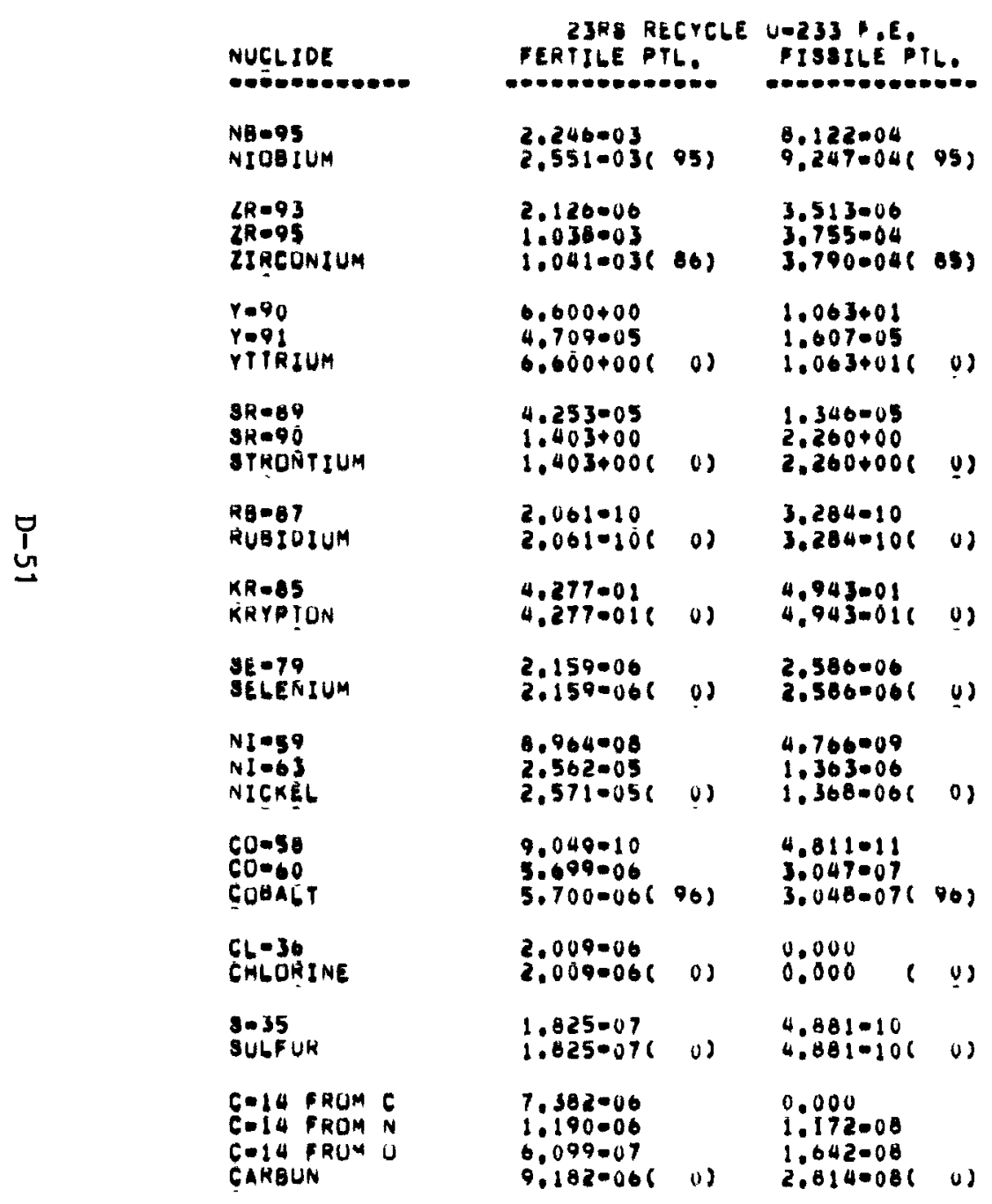

$25 R 8$ RECYCLE U-Z3S F.E. FETILE PTL. FISILE OTL.

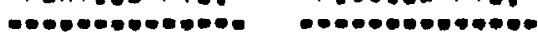
2.240 .03
$2.551 .03(95) \quad 3.200004$
$6.021004(95)$ $2.126006 \quad 2.334006$ $\begin{array}{ll}1.038003 & 2,3030004 \\ 1.041003(00) & 2,460004(88)\end{array}$

$0.000 \div 00 \quad 0.789+00$ $\begin{array}{llll}4.700005 & 9.581006 \\ 6.000+00( & 0) & 6.7890006 & 0)\end{array}$

$4.253005 \quad 7.208006$ $1.403+00 \quad 1.443+00$ $1.403+006$ Q1 $1.443+00608$ $2.061 .10 \quad 1.571-10$ 2.0610100 9) $1.571-106$ os $\begin{array}{llll}4.277-01 & & 2.253=01 \\ 4.277-016 & 0) \quad 2.253-016 & 01\end{array}$ $\begin{array}{llll}2.159000 & & 7.224-07 \\ 2.1590006 & 0) & 7.224007( & 01\end{array}$

$8.964000 \quad 9.994009$

9.960 .06
$2.562005 \quad 2.898006$
$2,571005(0) \quad 2,868006(0)$

$9.049-10 \quad 1.009-10$ $\begin{array}{ll}5.699006 & 6.390007 \\ 5.700-06(96) & 6.391=07(96)\end{array}$

$2.009000 \quad 0.000$ 2.0000006 9) 9.000 ( 05

$\begin{array}{llll}1.825007 & 1.023009 & \\ 1.825 .07( & 0) \quad 1.023009( & 0)\end{array}$

$\begin{array}{ll}7.382006 & 0.000 \\ 1.190006 & 2.05700\end{array}$
$0,099.07 \quad 3.443=08$

9.182006 ( 0) 5,900008602 


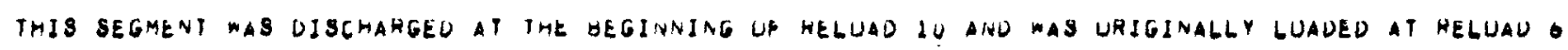
DECAY MEAT IN WATTS PEN FiE. AT TIME J YEARS AFIER UISCHAKGE

\begin{tabular}{|c|c|c|}
\hline $\begin{array}{l}\text { NUCG JOE } \\
\text { SEC }\end{array}$ & $\begin{array}{l}\text { 23R8 RECYCLE } \\
\text { FERTILE PIL. } \\
\text { OOO }\end{array}$ & 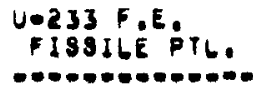 \\
\hline $\begin{array}{l}\text { HeJ FROM LIO } \\
H=3 \text { HOM BIO } \\
H=3 \text { FISS YLD } \\
\text { TRITIUM }\end{array}$ & $\begin{array}{l}9.564=04 \\
1.867-06 \\
1.172=04 \\
1.076-031\end{array}$ & $\begin{array}{l}1.582005 \\
0.000 \\
1.853-04 \\
2.0110040\end{array}$ \\
\hline
\end{tabular}

\begin{tabular}{|c|c|}
\hline $\begin{array}{l}\text { 25HS RECYCLE } \\
\text { FERTILE PTL: } \\
\text { E.- }\end{array}$ & $\begin{array}{l}\text { UE235 P.E. } \\
\text { FISSILE PTL. }\end{array}$ \\
\hline $\begin{array}{l}9.564=04 \\
1.867=06 \\
1.172=04 \\
1.076=036\end{array}$ & $\begin{array}{l}3.310005 \\
0.000 \\
i .340 .04 \\
1.07 \mathrm{j} .048\end{array}$ \\
\hline
\end{tabular}

ALL ELEMENTS
TOTAL OETA
TOTAL GAMMA
TUTAL ALPHA

$\begin{array}{ll}1.849+01 & 2.311+01 \\ 1.047001 & 2.044401 \\ 5.391 .01 & 9.574001 \\ 2.950401(35) & 4.451+01(45)\end{array}$

$\begin{array}{ll}1.849+01 & 1.030401 \\ 1.047001 & 1.57301\end{array}$

5.391001

$2.950+01(35) \quad 3.164+01630)$ 


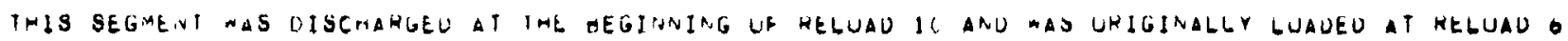

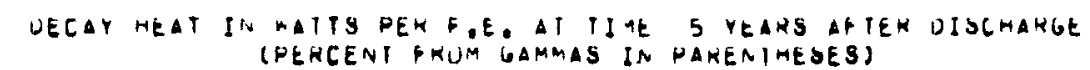

NUCLIUE

$C M-242$
$C M=243$

$C M=243$
$C M=244$

CUNIUM

$A M=241$
$M M=242 M$

$A M=242 M$
$M M=24 ?$

$A M-242$
$A M=243$

AMERICIUM

PU-238

PU
PU 239
PU:

$P U=24$

PLUTUNJUM

$\stackrel{p}{u}$
NPEZ37
NEPTUNIUM

$u=232$
$U-233$

$\mathrm{N} \cdot 234$

$u=235$

$4=236$

$U-230$

URANIUM

$P A=231$
$P A-234$

PRUTAGIINIUM

IH- 228

TH- 229

$T H=230$

$1 \mathrm{in}=2 \mathrm{SI}$

$T H-232$
$T H-234$

THEZ 34
IMUR IUM

AC- 225

$A C-227$

$A C-25$

acijingum

(PERCENT FRUM GAMMAS IN PAKENIMESES)

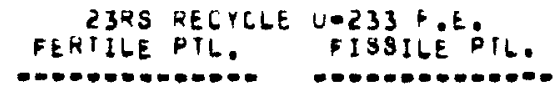

3.150007

2.094009

$2.707-04$

2.7100040

1.375 .05

1.755003

1.711003611

9.108006

2.338 .09

$1.012=08$

$4.779-07$

5.850 .04

3.195008

1.382 .07

1.287 .05

$5.980004(0)$

$1.265=02$

$3.474-116$

$4.161=06$

$0.414-07$

$1.260=026$

1.255 .01

$2.075=04$

5.457 .05

$4.259001($ N)

1.942006
1.9420001

$3.717-05$

7.869002

$5.950-02$

$7.737 \cdot 03$
$5.250-07$

2.505006

0.000

1.459 .011

0.4710 .05

0.0700

8.471 .058

$0.995-012$

$9.542-03$

1.464002

$.067-00$

2.071005

$9.417-026$ u

1.751000

$5.402-12$

1.7510000

$7.292-02$

3.520005

1.674004

$2.049=06$

2.105005

0.000
7.3140020

4.087 .05

1.050007

4.874 .06

4.505 .05(

\section{$7.200 \cdot 02$}

1.280005

3.205006

7.278008

0.000
$2.250-13$

$7.201 .02(0)$

$1.487-05$

$5.007=09$

$1.423-12$

$1.487005($ 11)

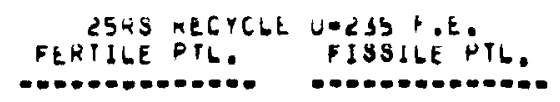

3.150001

3.090008
5.7070 .04

2.707004

$2.710=041$

\subsection{5}

0

0.5700016 al

9.108006

$2,338-09$

1.012008

4.779 .07

9,59900010

7.199 .02

5.244006

$2.209-05$

4.985 .03

$1.205=02$

3.474000
4.101006

$\begin{array}{ll}4.161006 & 2.070002 \\ 0.414007 & 7.211003\end{array}$

$1.206-02($ u) $1.835+016$ o

1.942006
$1.942006($ u) $\quad 1.167003$
1.1070036

$7.869002 \quad 2.969005$

5.950002

7.737 .03

5.256007

$2.505=00$

0.000

1.450 .016 (4)

8.471 .05

0.000

$0.00005(1)$

$7.292=02$

3.520 .05

$1.674=04$

$2.049=08$

$2.105-05$

0.000

$7,314=026 \quad 0)$

1.079003

2.640006

1.002004

1.107006

$1.879-036 \quad 0)$

\section{$\$, 190008$}

$4.652-07$

$4.971007(83)$

$4.087=05$

$1.056-07$

$4.674=110$

$4.385-.25(8)$
$2.752-05$

4.622010

0.889008

$1.029-07$

0.000

1.942008

$2.773005(0)$

5.367 .10

9.258011

0.000

$6.293-106 \quad 1$ 


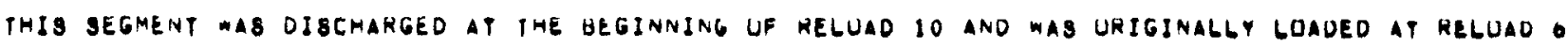

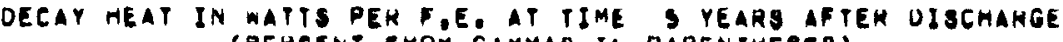
(PEHCENT FKOM GAMMAS IN PARENTHEBES)

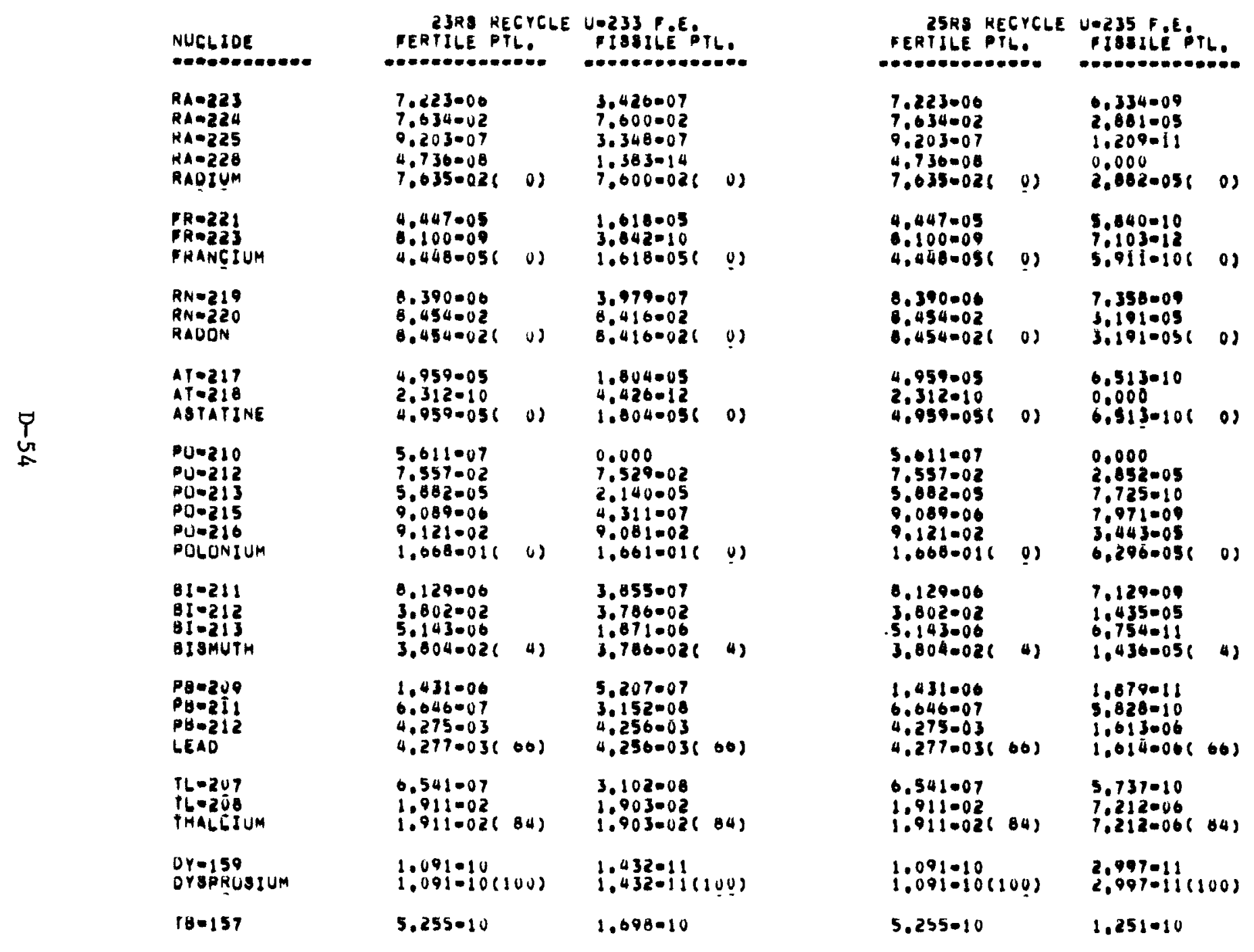




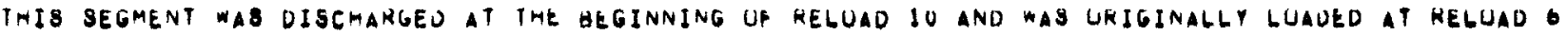
DECAY HEAT IN WATTS PEA E.E. AT TIME S YEARS AFTER DIUCMARge (PERGENT FRUM GAMMAS I.N PARENTHEBES)

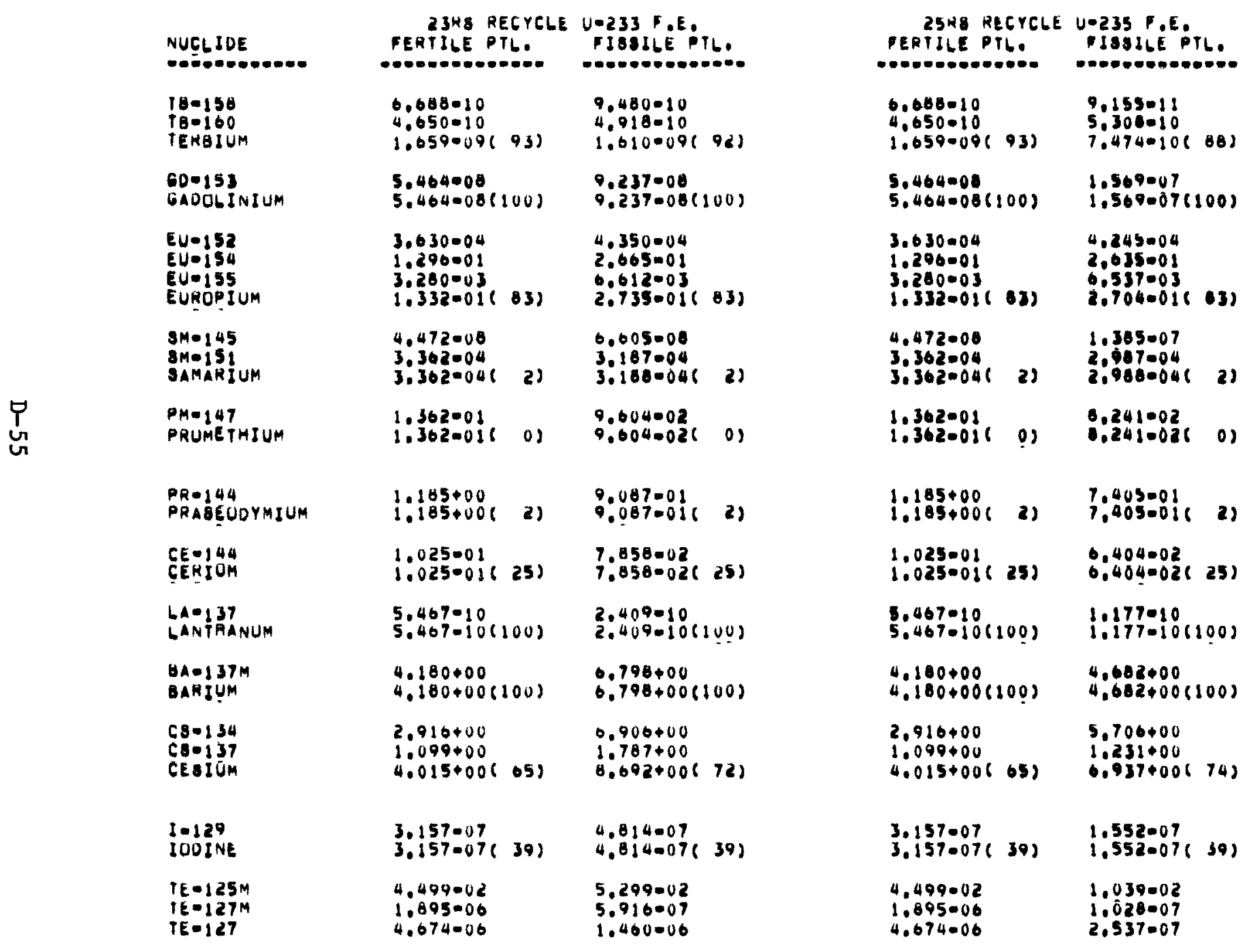


THIS SEGMENT WAS DIBCHARGED AT THE BEGINNING OF RELUAD 10 AND WAS URIGINALLY LUADED AT RELOAD G DECAY HEAT IN WATTS PEN FEE GAT TIME S YEARS AFIEN DISCHARGE

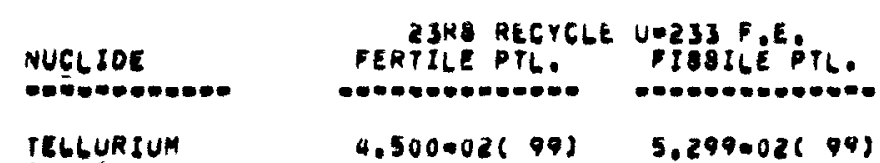

TELGURIUM

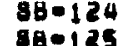

ANTIMUNY

SN-119M

$3 N=1213$
$3 N=12$

TIN

$10.100 \mathrm{M}$

\section{AGOIIOM}

SILVER

$\not$
un

\section{PDeiUR
PALLIOLIUM}

RHOLOE

$R U=106$

RUTHENIUM

rCe99

TECHNETIUM

NB-93M

NB-95M

NB-95

NJUBIUM

2R०9S

ZRe-95

ZIREONIUM

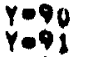

YTIRIUM

$3 R \oplus 89$
$1.018-11 \quad 1.383011$

$1.585 .01 \quad 1.867 .01$

i.seg-oic (3) $\quad 1.8670016$ b3)

$5.922=06$

$6.224-08$

6.715006

$1.270005(47)$

3.997000

1.292000

3.501 .00

$0.631-061018$

$4.503006 \quad 5.302 .07$

2.382004
$2.427 .04(97) \quad 3.936004$
$3.941004(97)$

$\begin{array}{ll}1.038008 & 1.765=09 \\ 1.038008(49) & 1,765000(49)\end{array}$

2.127001
$2.127001(12) \quad 2.013=01$
$2.013001(2)$

$1,765000(49)$

$1.254=03$

$\begin{array}{ll}1.167-03 \\ 1.167-036 & 0)\end{array}$

$1.254=036$ o

1.099004

$1.0990046 \quad 09$

0.962 .056

3.512000

$4.850=06$

$3,586=07$

1.341007

9.917009

$3,002000(98) \quad 3.919000(99)$

2.126000

$3.513=00$

$4.585=07$

$2.584006(25) \quad 3.078006(3)$

$6.278+00$
7.621 .09

$0.278+006$

$1.011+01$
2.600009

$1.011+0210$

$2.863 \cdot 09$

$9.063 \cdot 10$
2SRS RECYCLE

FERTILE PTL. PIBSILE OTL.

$4.500002(99) \quad 1.039002699)$

$1.016-11 \quad 0.001012$

$1.505001 \quad 3.662002$

$5.922006 \quad 2.000006$

$0.224008 \quad 5.505000$

1.0200d

$1.270005(47) \quad 3.746000(71)$

\section{$4.503006 \quad 1.182006$}

$\begin{array}{ll}2.382004 & 2.403004 \\ 2.427004(97) & 2.4140046972\end{array}$

1.030009

$1.036000(49) \quad 1.776060(49)$

$2.127-01, \quad 2.010001$

$2.1270018(2) \quad 2.016001(12)$

$\begin{array}{llll}1.254003 & 1.189003 \\ 1.254003( & 0) & 1.1890036 & 0)\end{array}$

$\begin{array}{llll}0.962005 & & 9.559005 \\ 0.962005(0) & 9.559005(0)\end{array}$

1.870006

1.301 .07

$9.919=07$

$3.002006(98)$

2.126000

$2.504006\left(\right.$ (5) $\quad \begin{array}{l}1.079007 \\ 2.442006(3)\end{array}$

$0.278+00$

$6.278+008$ 0.) $\quad 0.450 .0060$ )

$2.063009 \quad 4.852 \cdot 10$ 


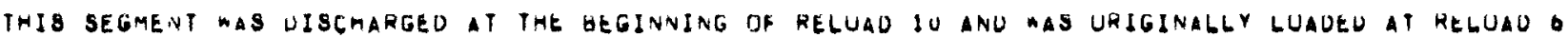

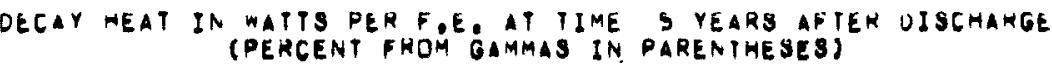

NUCL JDE

$8 R=90$

BRTRONTIUM
BTRO

$R 日=87$

RUSIOIUM

KA-BS

KAYPTON

SEDT9

BELENIUM

NI०59

NI๑6

ษ

co-5s

coBAt

CLe30

GHLORINE

S-35
SULFUK

C-14 RRUM C

C-I 14 FROM N

C-14 FRUM U

CEIL FR
CAKBON

Hes FROM LIt

HOJ FROM BIO
M-J FISB YLD

HES FITUM
TRE TUM

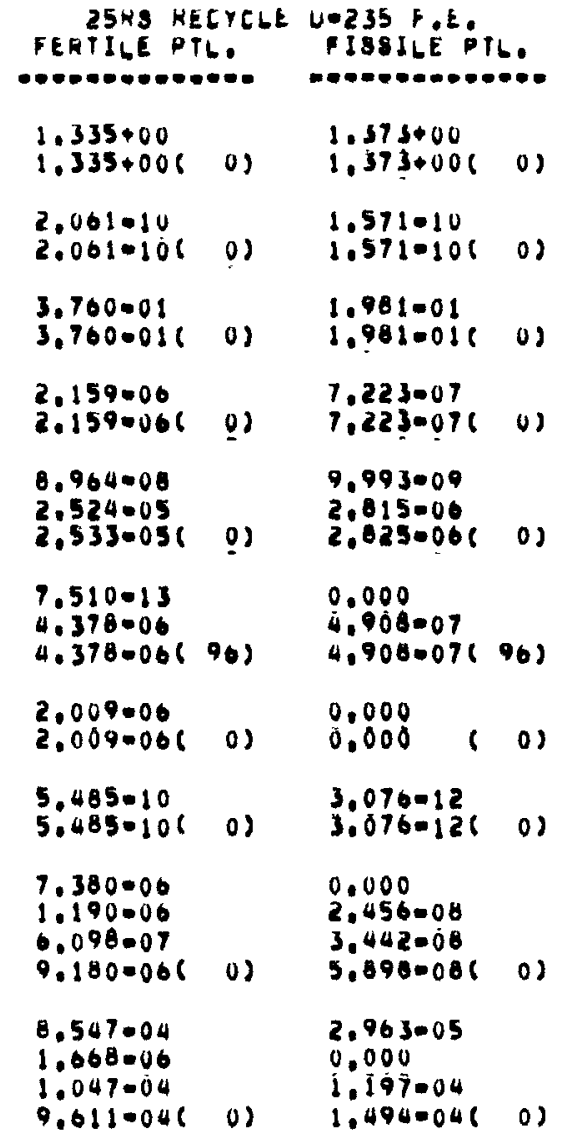




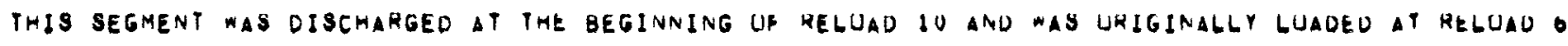
DECAY HEAT IN WATTS PEH F.E. AT TIME S YEARS AFIEK OISCHAHGE

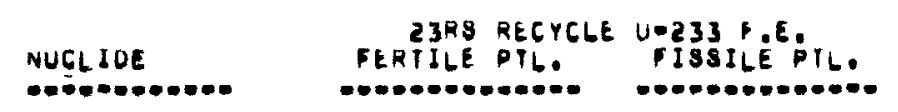

ALL ELEMENTS

TOTAL BETA

ToTAL GAMMa

TUTal alPma

GRAND TOTAL

$\begin{array}{ll}1.097+01 & 1.042+01 \\ 7.223+00 & 1.360+01 \\ 5.854001 & 9.491-01 \\ 1.878+01(38) & 3.097+01(43)\end{array}$

25HS KECYCLE UE233 F.K. FERIJLE PTL. FISSILEPTL.

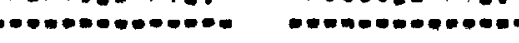

$1.097+01$

$7.223+00$
$5.884-01$

$1.876+01(38)$

$$
\begin{aligned}
& 1.090+01 \\
& 1.020+01 \\
& 1.925+01 \\
& 4.032+016253
\end{aligned}
$$




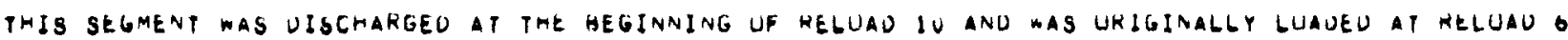

DECAY HEAT IN RATIS PER F.E. AT TIME IU YEARS APTEK DISCMAHGE (PERCENT SKUM GAMMAB IN PARENTHEBES)

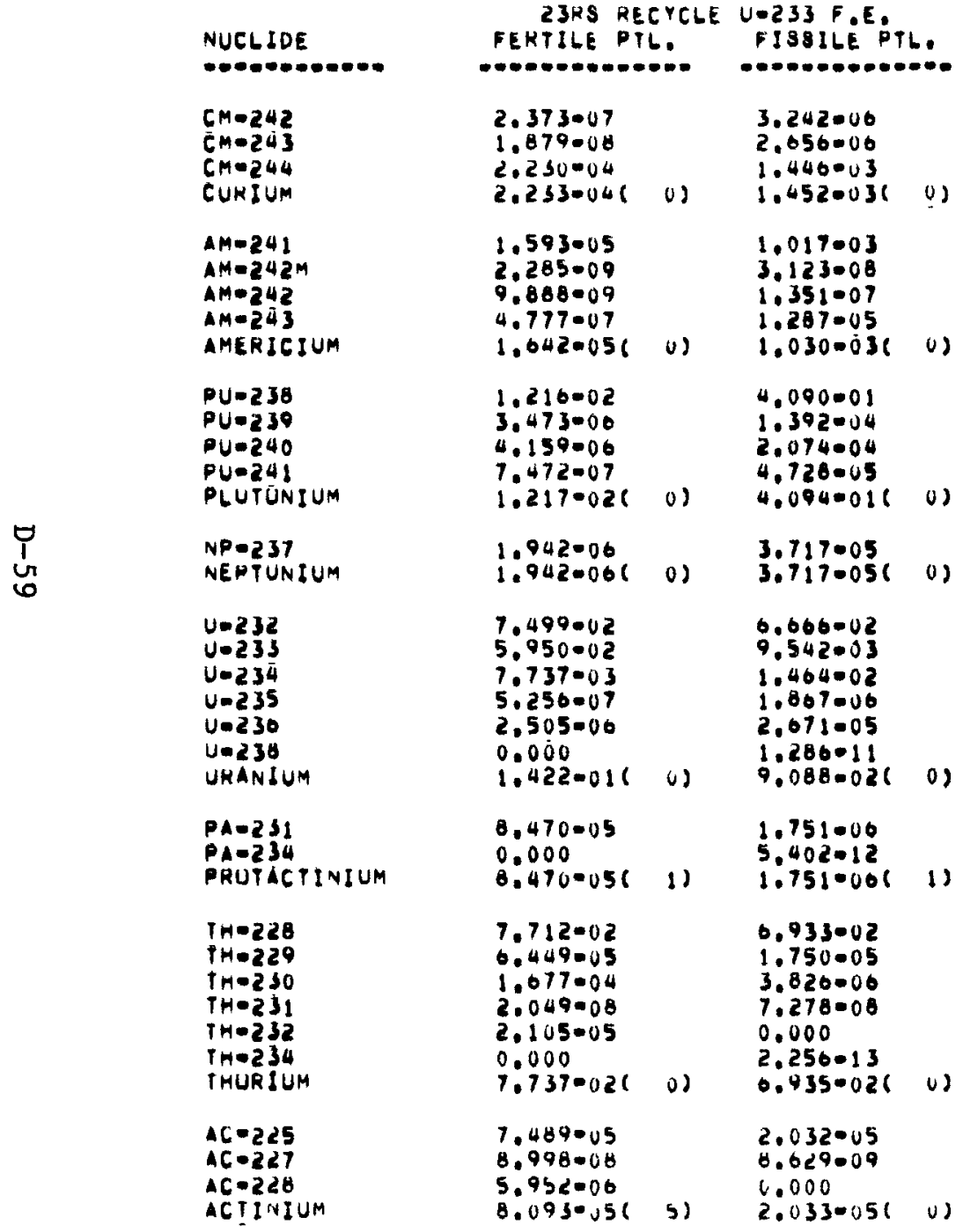

25HS RECYCLE UA23S F.E. PERTILE PTL. FISSILE PIL.

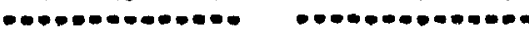

\begin{tabular}{|c|c|c|}
\hline $\begin{array}{l}2.373=07 \\
1.879=08 \\
2.230=04 \\
2.233=046\end{array}$ & (v) & $\begin{array}{l}5,322004 \\
0.725004 \\
0.805=01 \\
0.879011\end{array}$ \\
\hline $\begin{array}{l}1.593005 \\
2.285=09 \\
9.880=09 \\
4.777=07 \\
1.6420056\end{array}$ & (0) & $\begin{array}{l}1.242001 \\
5.120006 \\
2.218005 \\
4.981003 \\
1.2920011\end{array}$ \\
\hline $\begin{array}{l}1.216002 \\
3.473006 \\
4.159000 \\
7.472007 \\
1.2170026\end{array}$ & (0) & $\begin{array}{l}1.700+01 \\
1.191002 \\
2.075=02 \\
5.723=03 \\
1.7644011\end{array}$ \\
\hline
\end{tabular}

$\begin{array}{lll}1.942006 \\ 1.9420066 & 0) \quad 1.167-03 \\ 1.167 .036 & 0\end{array}$

$7.499 .02 \quad 2.030 .05$

$5.950002 \quad 2.442009$

$7.737 .03 \quad 1.079003$

$5.250 .07 \quad 2.640 .06$

$2.505000 \quad 7.602004$

0.000

1.0220018 0) 1.87700360$)$

$0.470-05$

$3.227=08$

$\begin{array}{ll}0.000 & 4.052007 \\ 8.070-05(\text { 1) } & 4.074007(82)\end{array}$

$7.712002 \quad 2.910005$

$6.449005 \quad 4.632010$

$1.077 .04 \quad 1.346=07$

$2.069000 \quad 1.029007$

2.105005

$\begin{array}{llll}0.000 & 1.942008 \\ 7.737002( & 0) \quad 2.9300056 & 02\end{array}$

$7.489005 \quad 5.379 .10$

$8.998000 \quad 1.589=10$

$\begin{array}{llll}3.952006 & & 0.000 \\ 8.093-05(\text { 5) } & 6.900-106 \quad 1\end{array}$ 


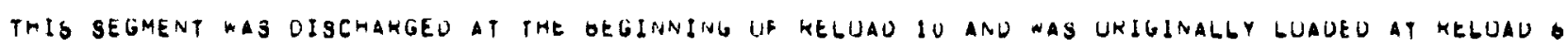

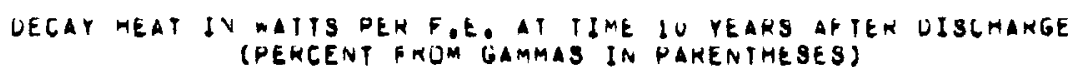

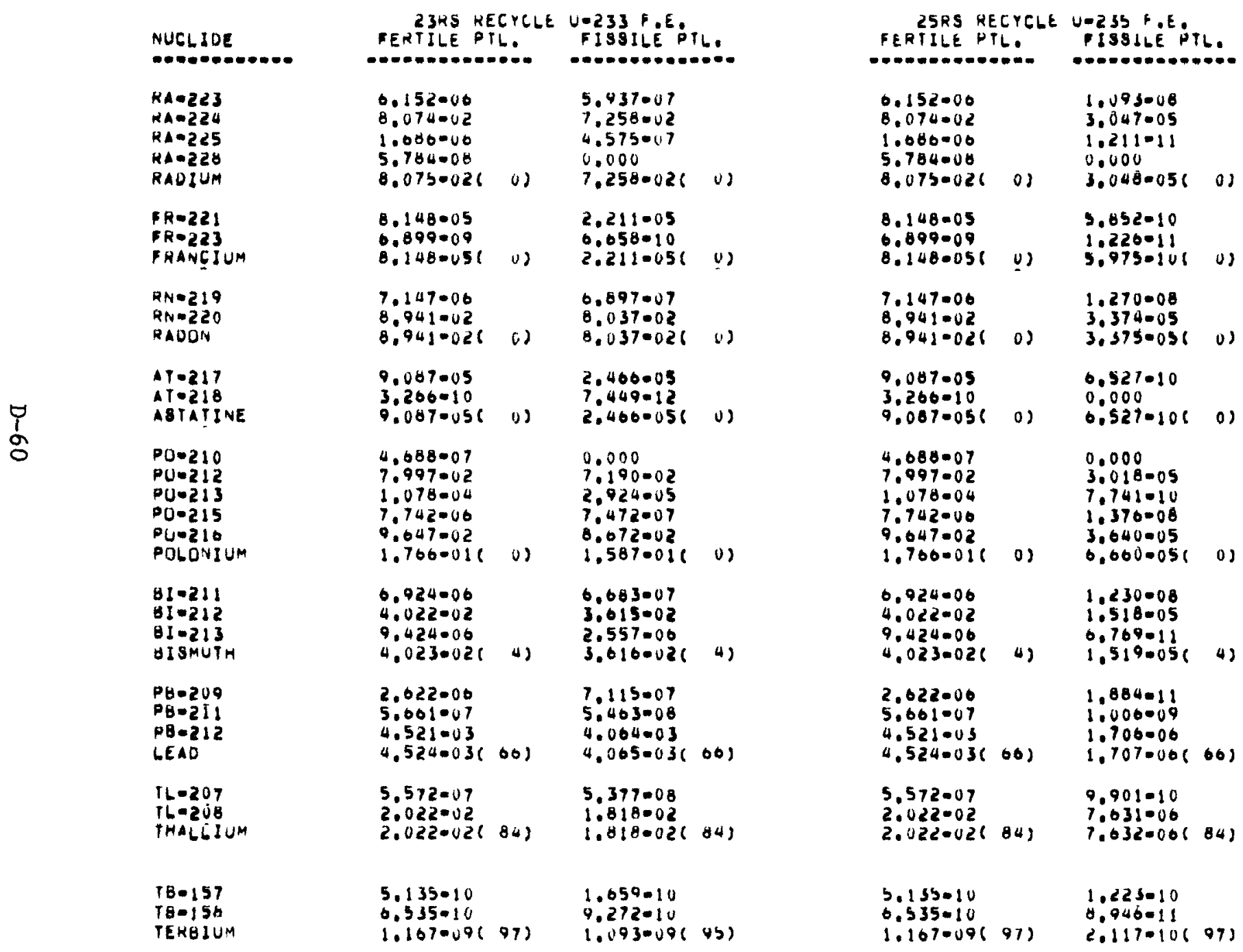


THIS SEGMENI MAS DISCHARGED AT THE BEgINNING OF RELUAD IO AND MAS URIGINALLY GUAUEO AT KELUAU G DECAY HEAT IN WAITS PER Fok AT TIME IO YEARS aFTER UISCHARGE

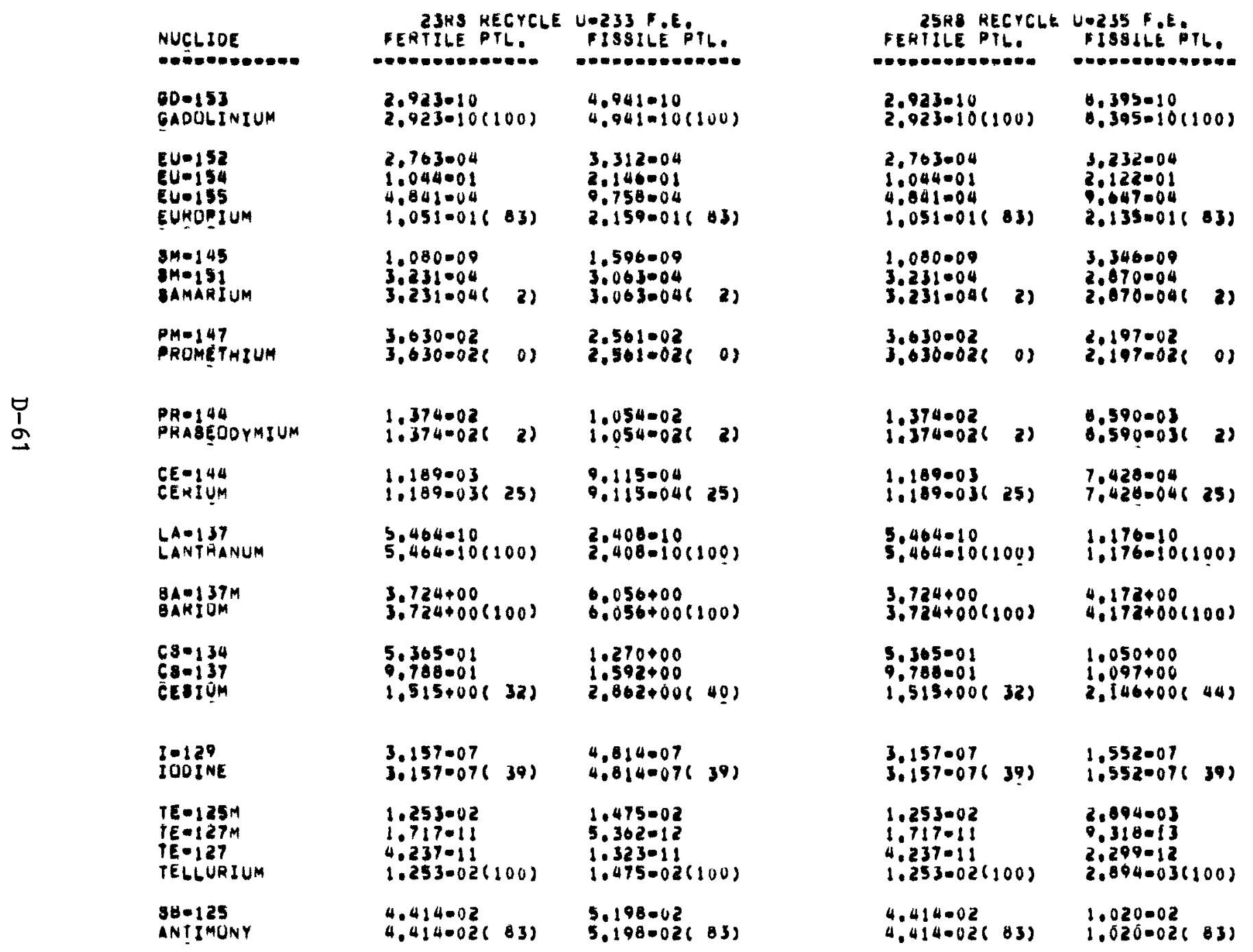




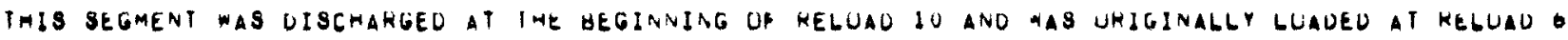
DECAY WEAT IN MATTS PEK F.E. AT TIME IU YEARS AFTER DISCHAHGE (PERCENT RUM GAMMAS IN PAMENTHESES)

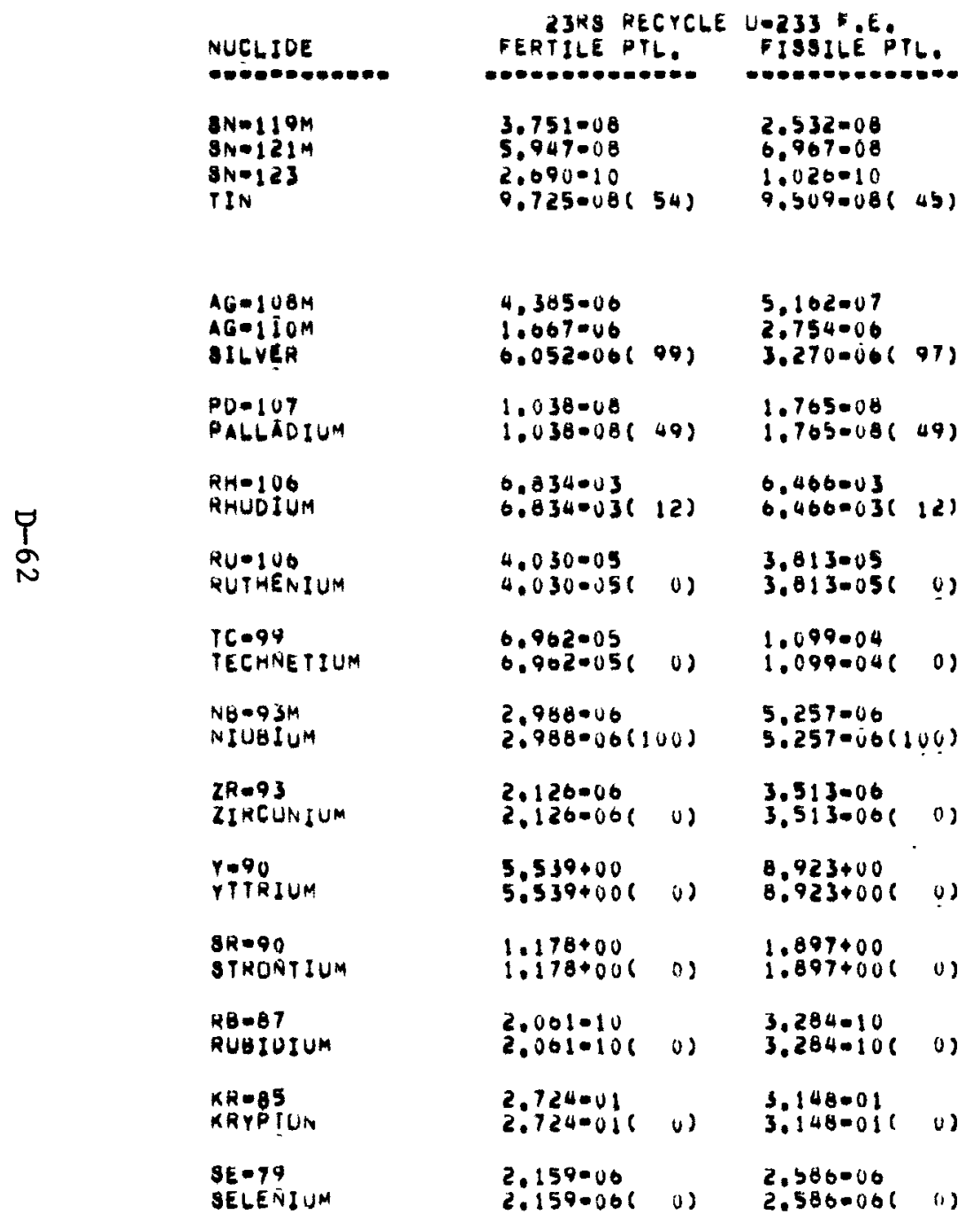

\begin{tabular}{|c|c|c|c|}
\hline $\begin{array}{r}25 R 8 \\
\text { EERTILE }\end{array}$ & Crelet & 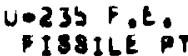 & \\
\hline $\begin{array}{l}3.751=08 \\
5.947-08 \\
2.090-10 \\
9.725=086\end{array}$ & $54)$ & $\begin{array}{l}1.689000 \\
5.260006 \\
4.109-11 \\
6.9530086\end{array}$ & $4(5)$ \\
\hline $\begin{array}{l}4.305=06 \\
1.067=06 \\
6.052=066\end{array}$ & 991 & $\begin{array}{l}1.082006 \\
1.681006 \\
2.7640061\end{array}$ & $98)$ \\
\hline $\begin{array}{l}1.038008 \\
1.036 .086\end{array}$ & (9) & $\begin{array}{l}1.776000 \\
1.7760001\end{array}$ & 49) \\
\hline $\begin{array}{l}0.8340 .03 \\
0.8340 .036\end{array}$ & (2) & $\begin{array}{l}0.477-03 \\
6.477-036\end{array}$ & 12) \\
\hline $\begin{array}{l}4.030=05 \\
4.030=051\end{array}$ & $0)$ & $\begin{array}{l}3.829005 \\
3.819-051\end{array}$ & 0) \\
\hline $\begin{array}{l}6.962=05 \\
6.9020 .051\end{array}$ & 0) & $\begin{array}{l}9.559 .05 \\
9.559 .051\end{array}$ & 0) \\
\hline $\begin{array}{l}2.9880 .06 \\
2.9880066\end{array}$ & 1001 & $\begin{array}{l}3,485=06 \\
3,485=061\end{array}$ & 1008 \\
\hline $\begin{array}{l}2.126006 \\
2.1260 .061\end{array}$ & 01 & $\begin{array}{l}2.334 .06 \\
2,334.061\end{array}$ & $0)$ \\
\hline $\begin{array}{l}5.539+00 \\
5.539+006\end{array}$ & 01 & $\begin{array}{l}5,690400 \\
5.698+008\end{array}$ & 01 \\
\hline $1: 178+000$ & 01 & $\begin{array}{l}1.211+00 \\
1.211+006\end{array}$ & 01 \\
\hline $\begin{array}{l}2.061=10 \\
2.061=106\end{array}$ & $0)$ & $\begin{array}{l}1.571-10 \\
1.571-106\end{array}$ & 0) \\
\hline $\begin{array}{l}2.724 .01 \\
2.724 .016\end{array}$ & v) & $\begin{array}{l}1.435001 \\
1.435=016\end{array}$ & 0) \\
\hline $\begin{array}{l}2.159006 \\
2.1590061\end{array}$ & u) & $\begin{array}{l}7.225007 \\
7.223=076\end{array}$ & $0)$ \\
\hline
\end{tabular}




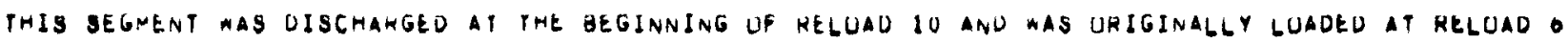

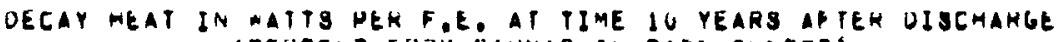
TPERCENT FHOM GAMMAS IN PARENTHESES

NUGL JOE

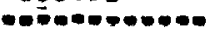

$N]=59$

$N I=63$

NICKEL

CODEO

CLE-36

C-14 FROM C

E-IU FROM N C-IL FROM O CARBUN

$\varphi$
$\sigma$
H.J PROM LLO
M-3 PROM BiO
Maj PISA YLD

TRITIUM

ALL ELEMENTS

TOTAL BETA

TUTAL GAMMA

TOTAL ALPHA

GRANO TOTAL
$23 K S$ RECYCLE U-233 FOE. FERTILE PTLE FISILE PTL.

8.904008
2.430 .05

$\begin{array}{llll}2.430 .05 & 1.293006 \\ 2.439-45(\text { u) } & 1.298 .008\end{array}$

2.204000

$2,264006(90)$

$1.211=07$

$1,211007(90)$

2.009006

$2,000=0060$

0.000
0.000

$(0)$

$7.375=06$

1.189000

6.093007

$9.173=06($ v)

0.000

$1.171-08$
1.641008

$2.8110086 \quad 0$

0.452004

1.259006

$7.255 .04(0)$

$1.067=05$

0.000

1.250 .04

$1.357-046 \quad 0$

6.108+00

$4.377+00$

$0.092-01$

$1.309+01633)$

$1.294+01$

$0,103-01$

$2.132+01635$
2SRS RECYCLE UW235 F.E

FERTILE PTL. MISILE PTL.

\section{$8.904008 \quad 9.995009$}

$\begin{array}{lll}2.430005 & 2.711006 \\ 2.439-05(\text { u) } & 2.721000(\end{array}$

$2.204000 \quad 2.539007$

$2,264000(96) \quad 2,539007(96)$

$\begin{array}{lll}2.009006 & & 0.000 \\ 2.0090006 & 0) & 0.000\end{array}$

$(0)$

7.375000

1.189006

$0.093=07$

9.1730001

0.000

$2,450.08$

3,440008

$5.894008(0)$

0.452004
$1.259-06$

$2.237 \cdot 05$

0.000

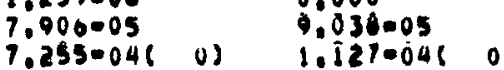

$0.345+00$

$5.324+00$

0.002001

$1.309+01(33) \quad 3.210001(26)$ 
0

-

0 
TABLE D-4

DECAY HEAT FOR FUEL ELEMENTS DISCHARGED AT BEGINNING OF RELOAD 13 AND ORIGINALLY LOADED AT RELOAD 9

NOTE: Table D-4 consists of 29 pages of computer print-out (pages 67 through 95). 
-

-

8 


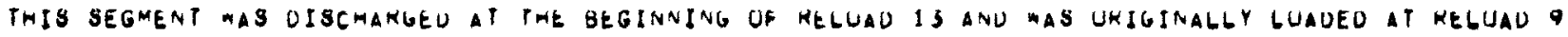

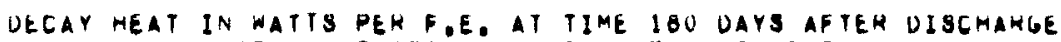
(PEKLENT FROM GAMMAS IN PAKENTHESES)

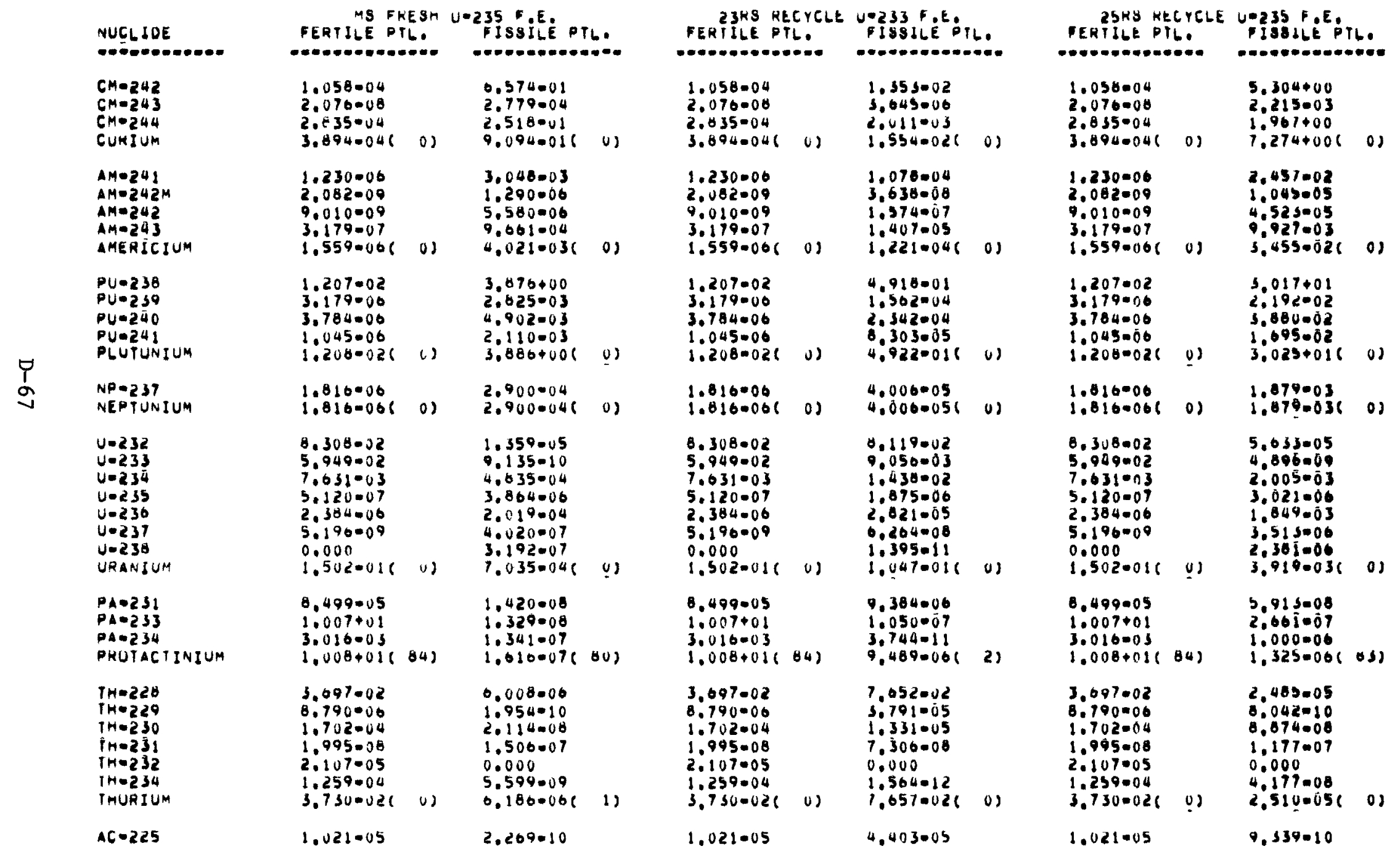




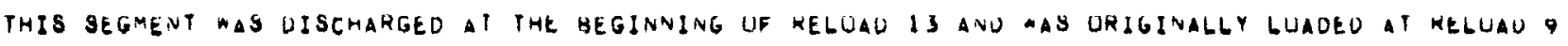
DECAY HEAT IN WATIS PER FE. AT IIME 180 DAYS AFTEN OISCHAKGE

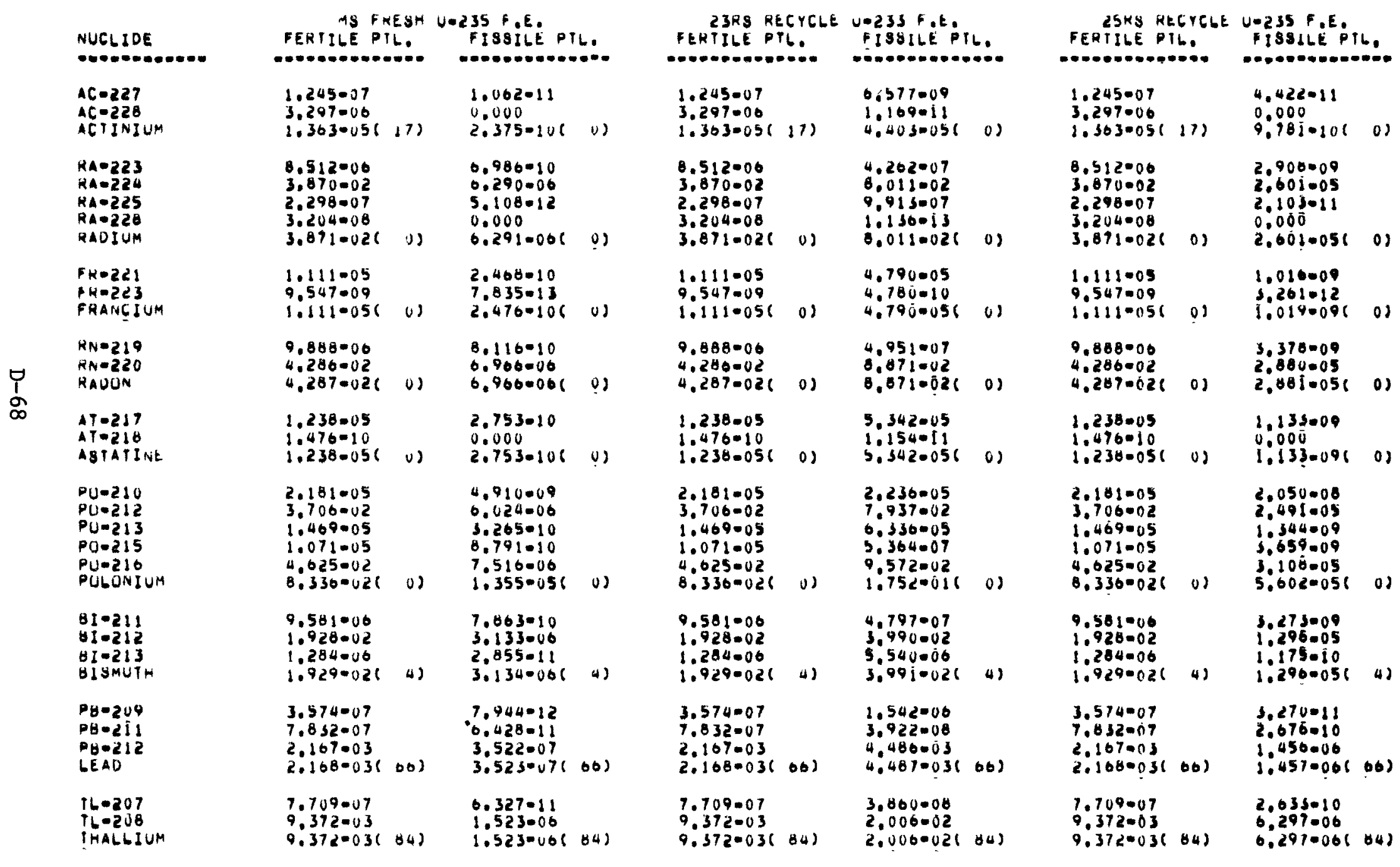




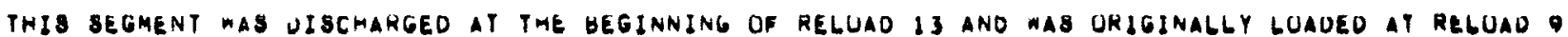

DECAY HEAT IN WATIS PER F.E. AT TIME 180 DAYS AFIEK DISCHAKGE (PEKCENT ROM GAMMAS in PAHENIHESES)

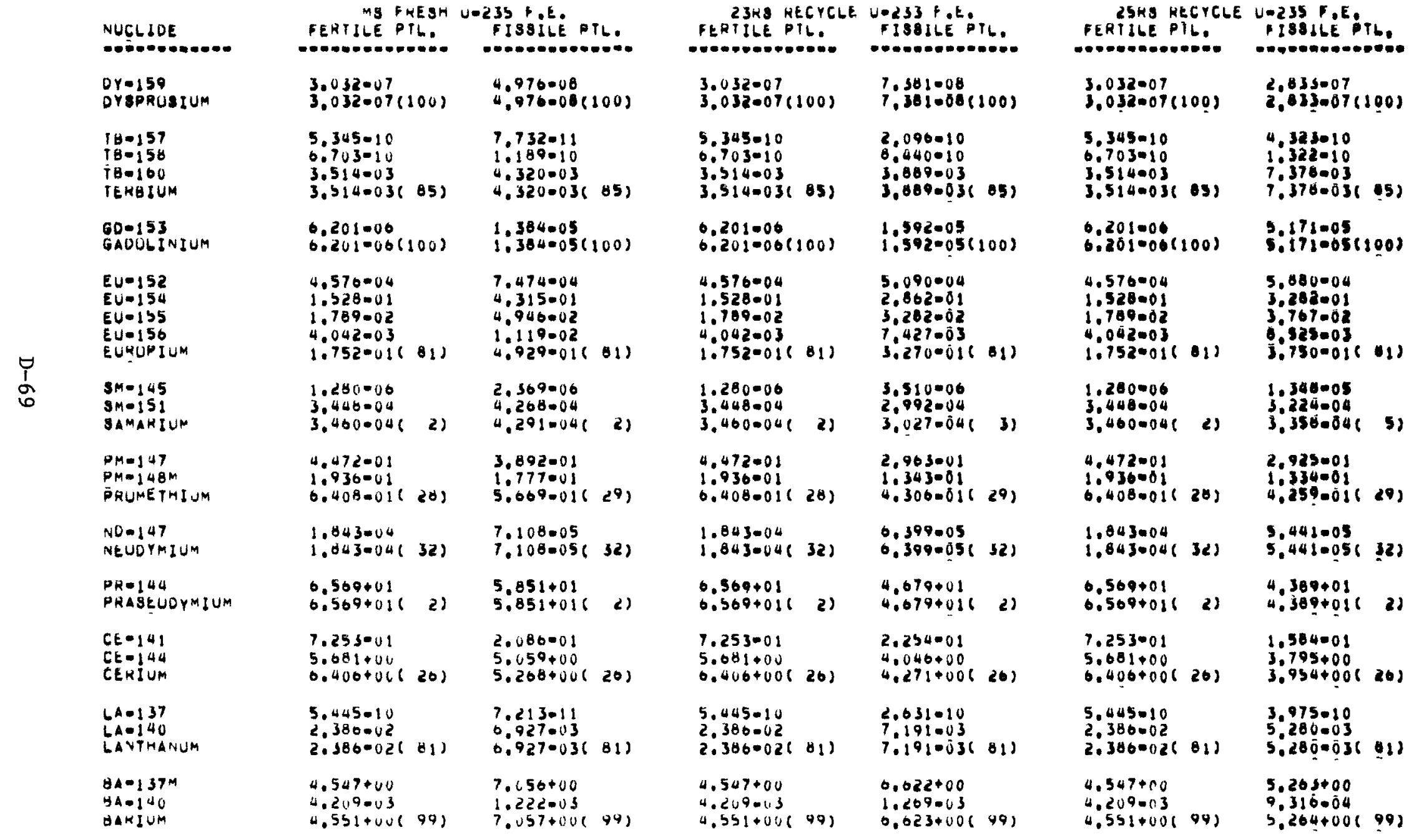




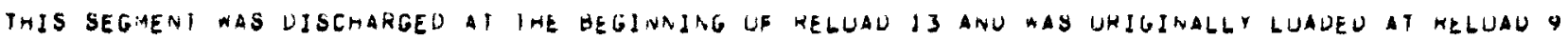
DECAR MEAT IN MATIS PER F.E. AT TIME 1 SU LAYS AFTER UISCHAMGE (PEHCENT FROM GAMMAS IN PAGENTMESES)

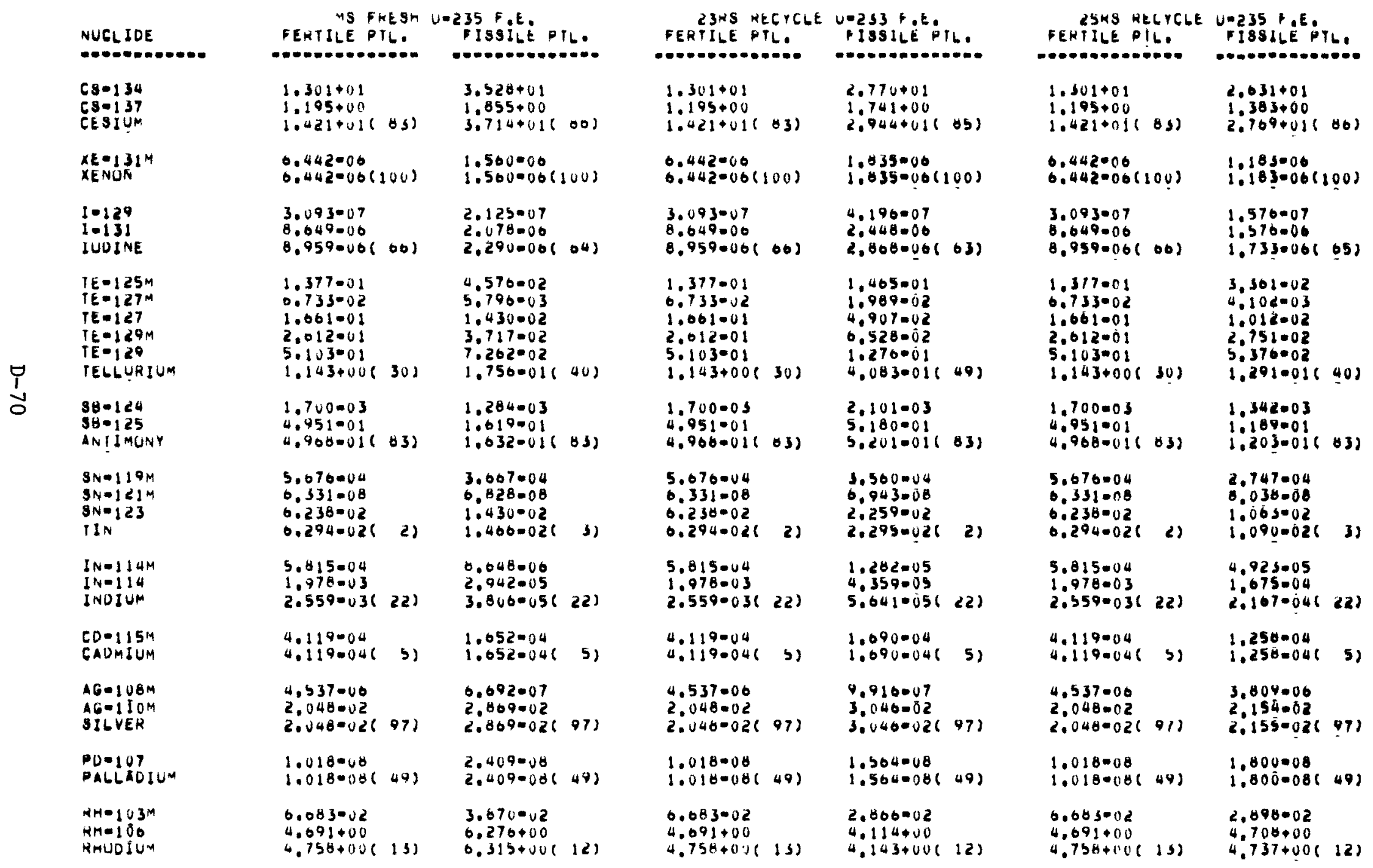




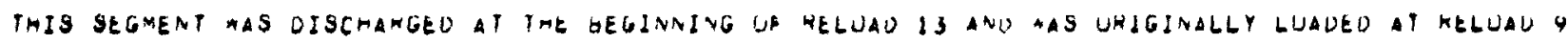

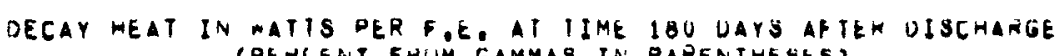
(PERLENT FRUM GAMMAS IN PARENTHESES)

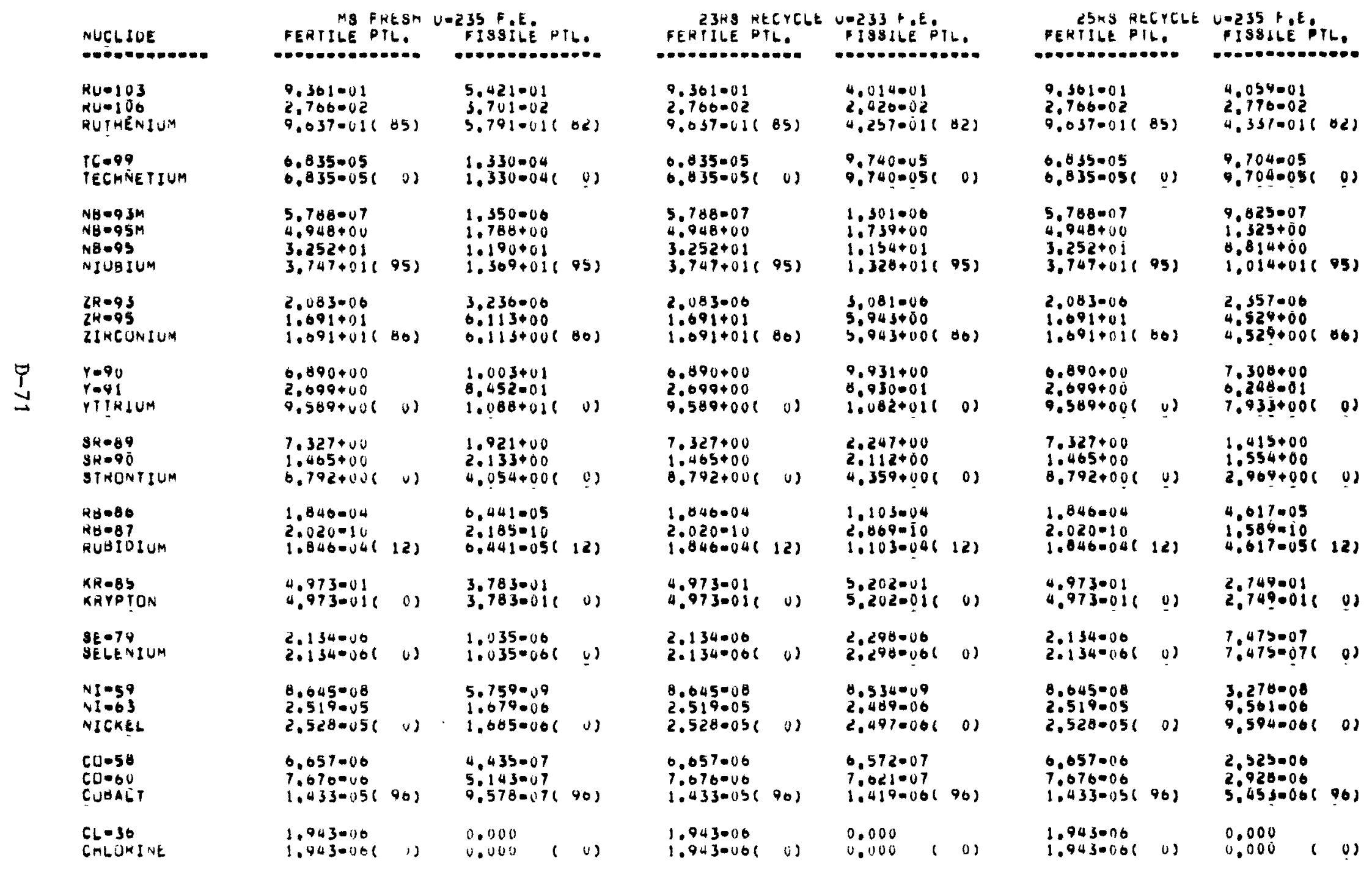




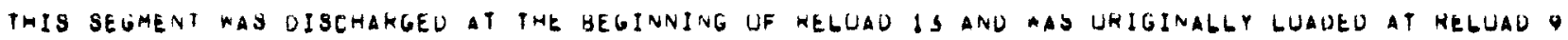

decar heat in watis per F.e. at time lou vaYs aflen uischakge (PEREENT GROM GAMMAS IN PAKENTHESES)

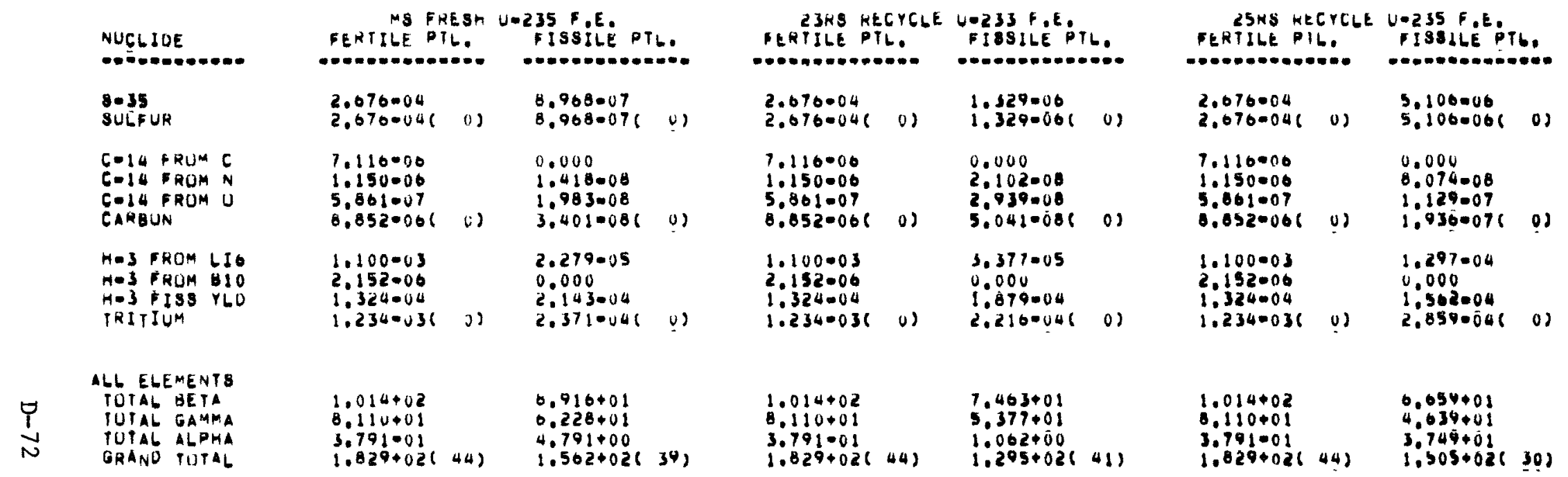




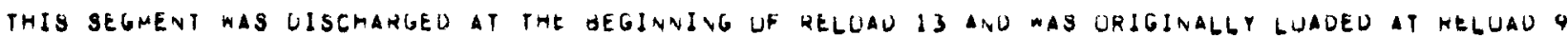

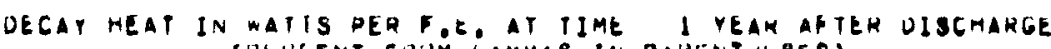
(PEHGENT FRUM GaMmas IN PAHENTHESES)

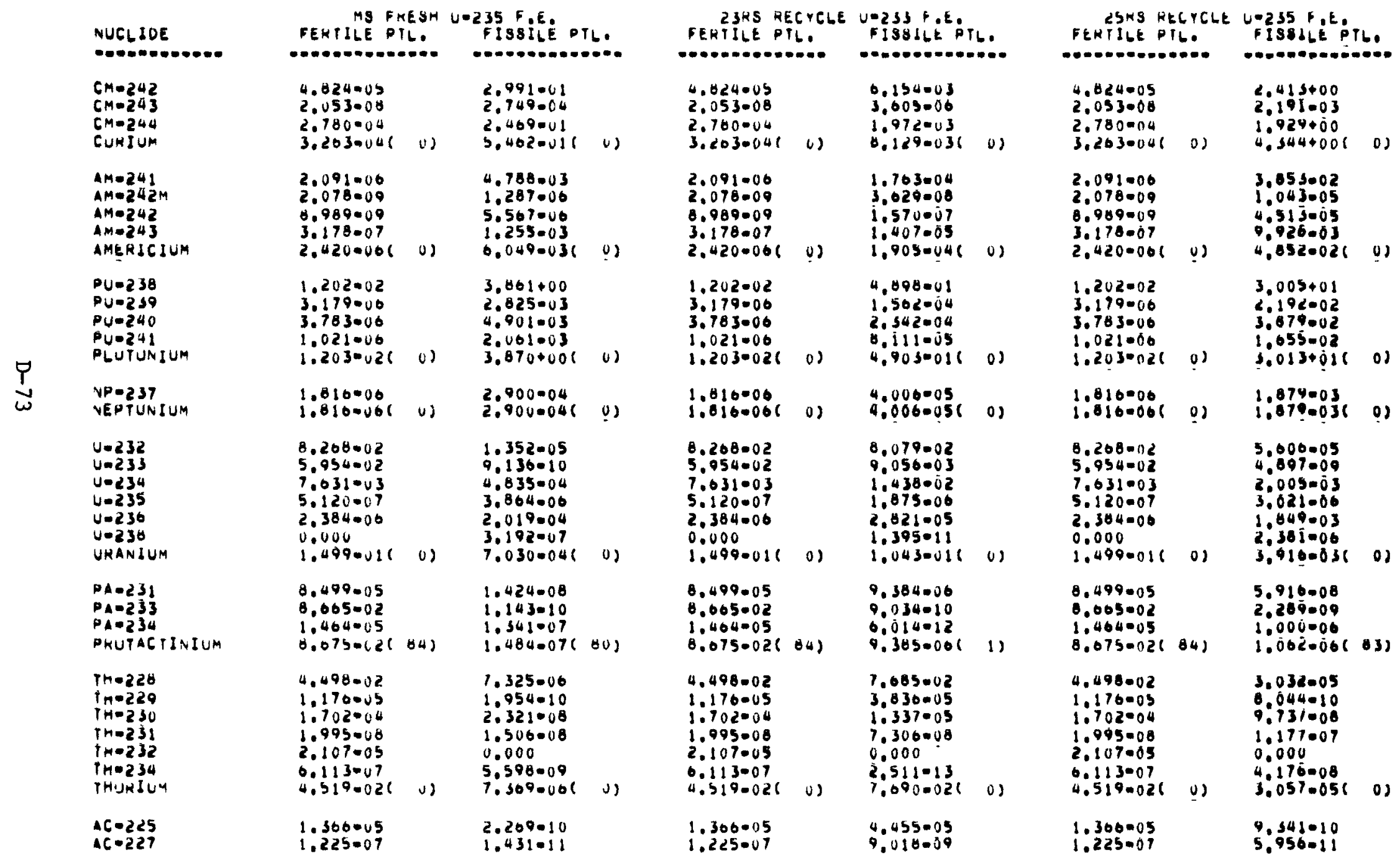




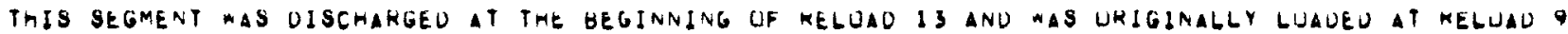

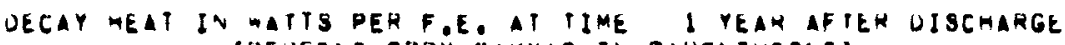

(PERCENT FROM GAMMAS IN PARENTHESE

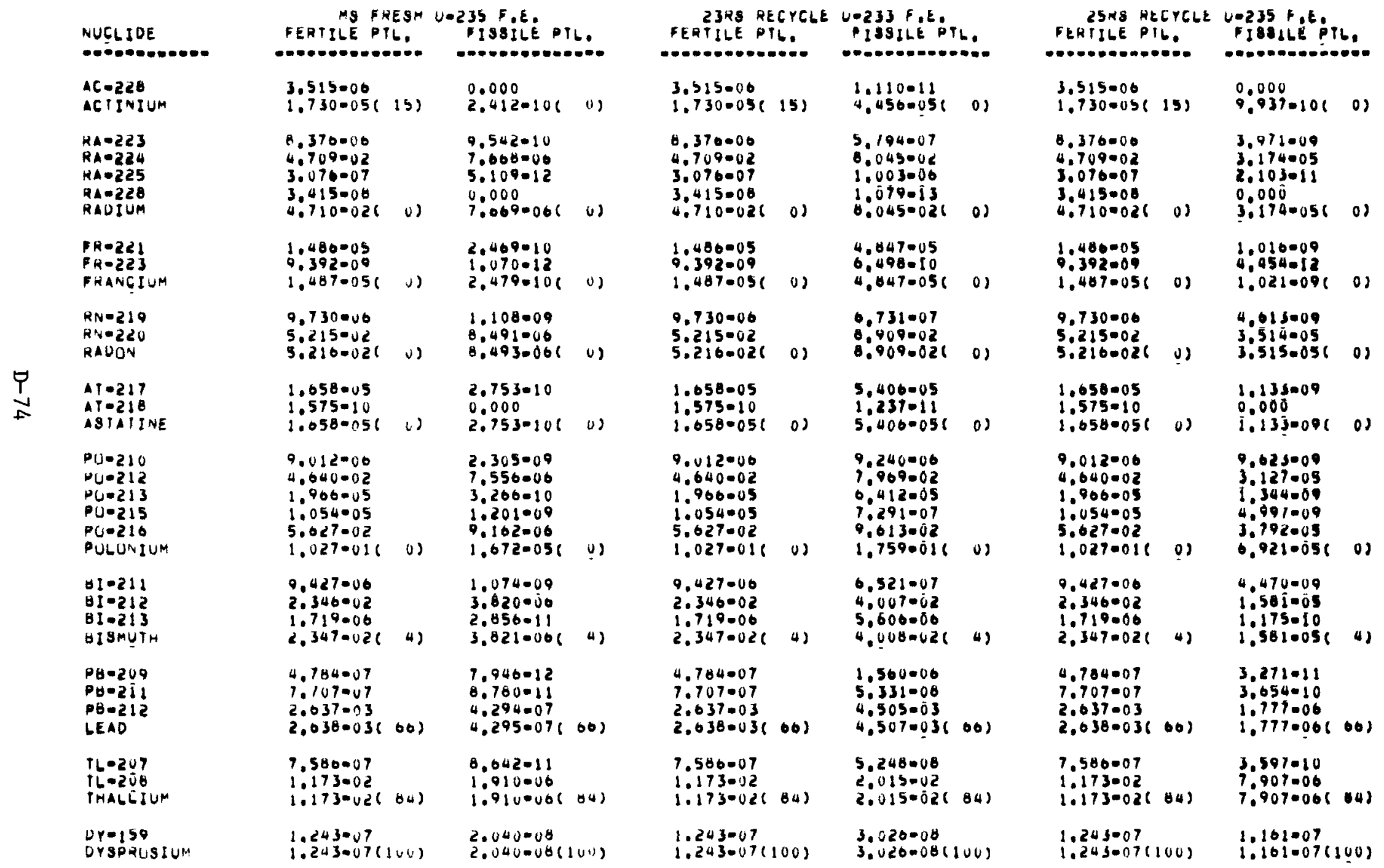




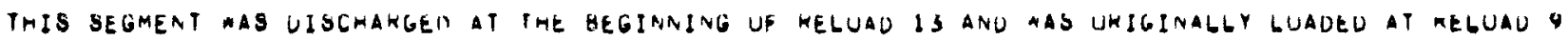

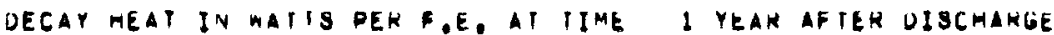
(PERIENT BRUM GAMMAS IN PARENIHESES)

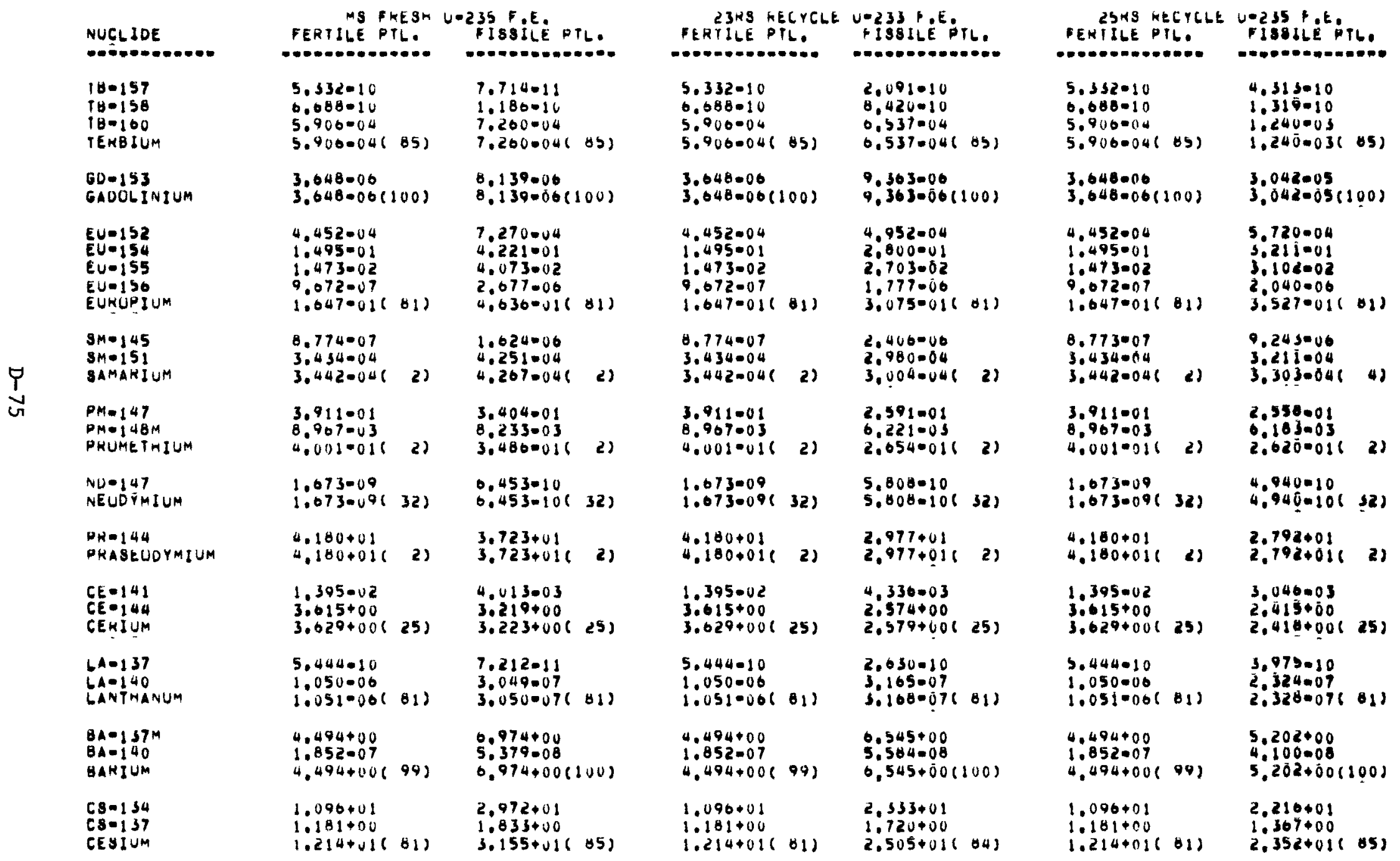




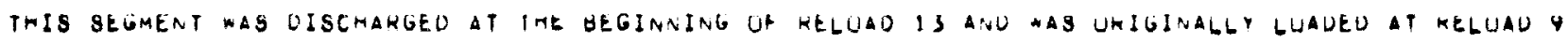
DECAY MEAT IN WATTS PER F.E. AT IIME 1 YEAN AFIEN DISCMAHGE (PERLENT FRUM GAMMAS IN DAHENIMESES)

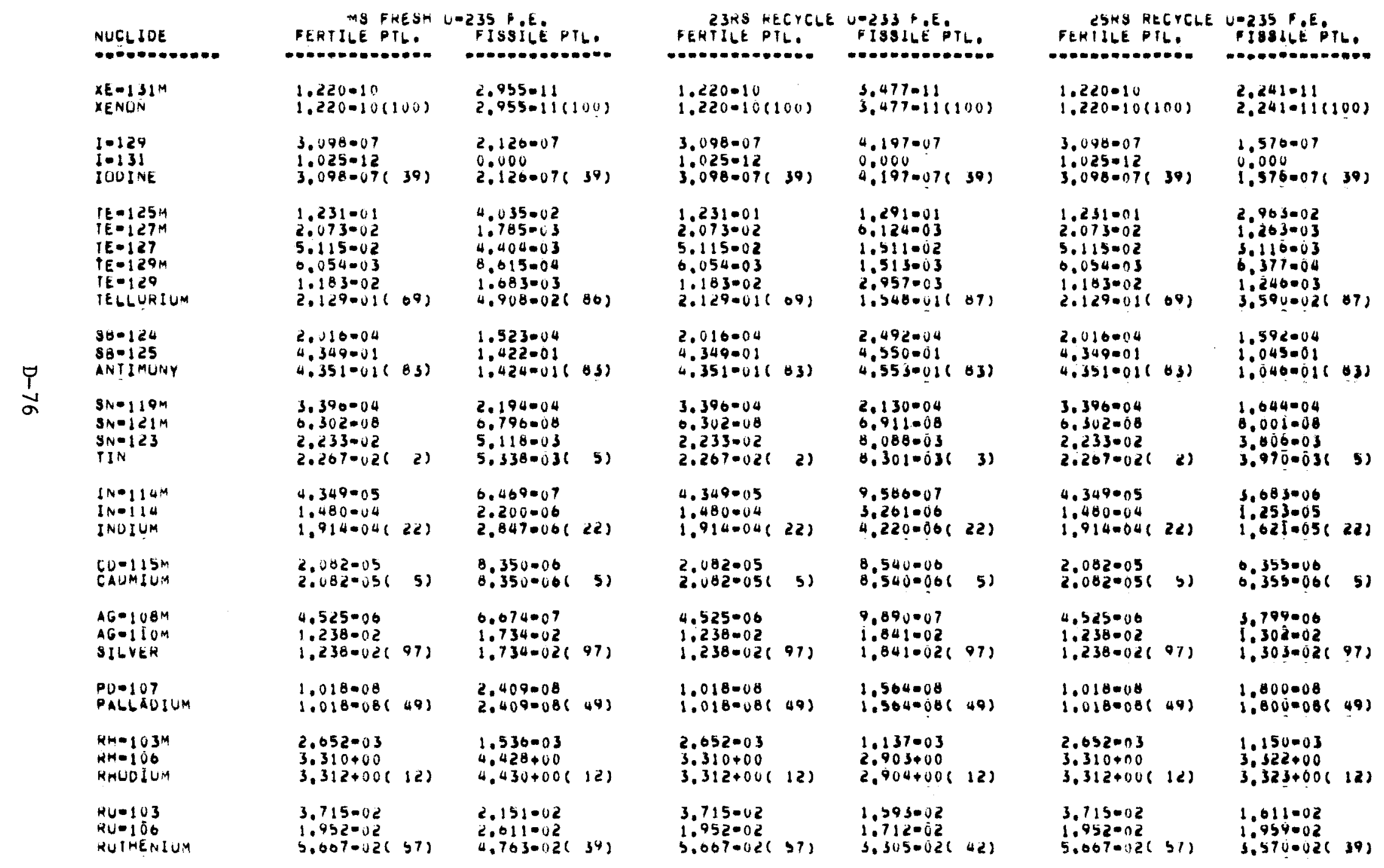




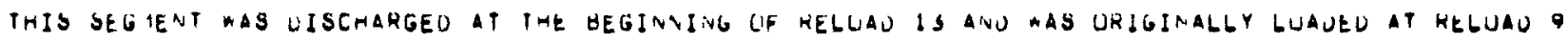

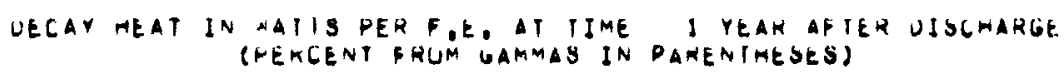

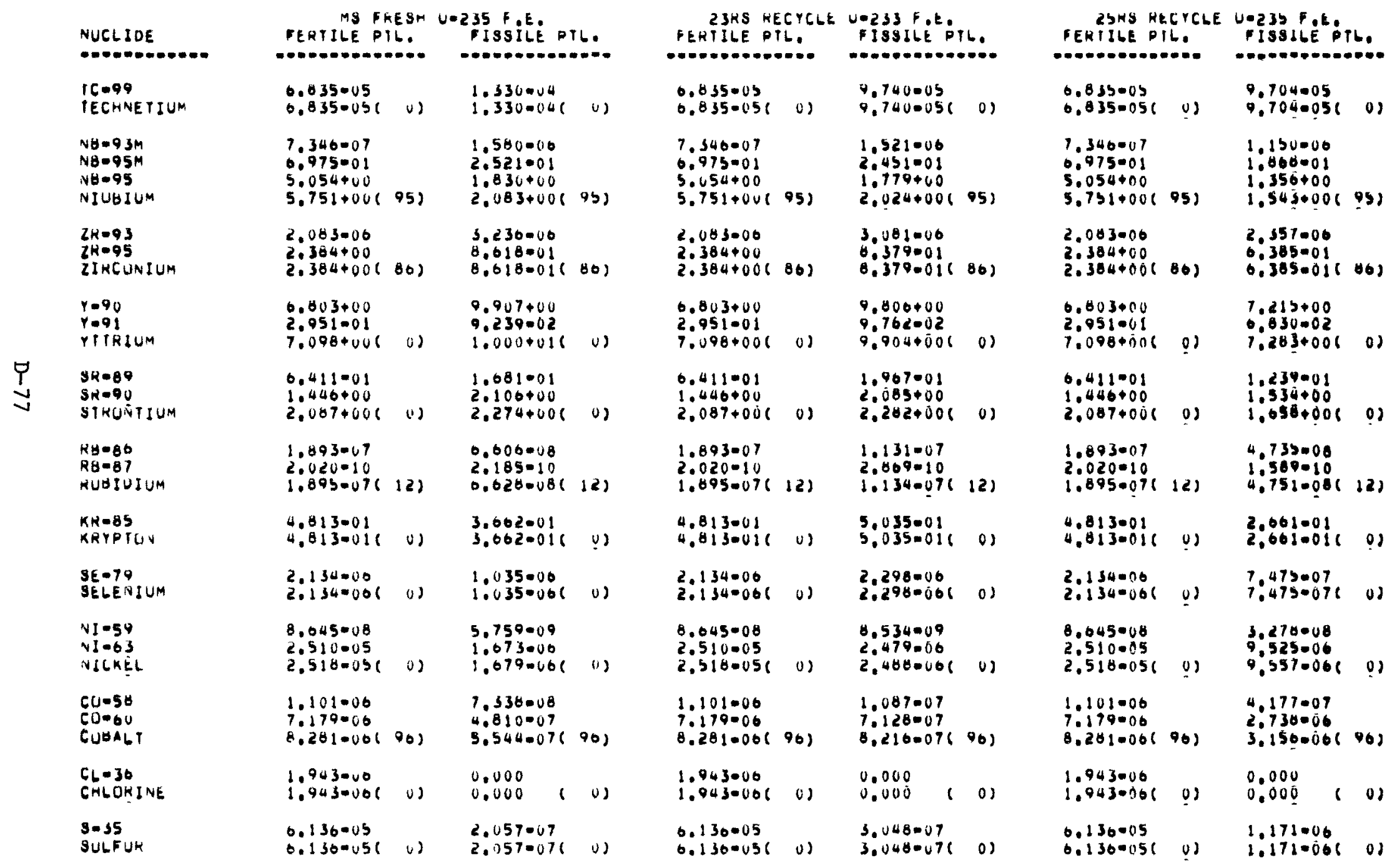




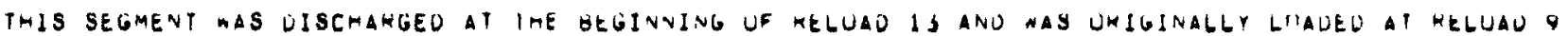

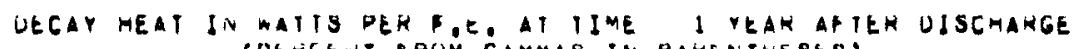
(PERGEIUT ROM GAMMAS IN PAKENTHEBES)

NUCLIDE

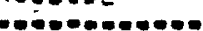

C-14 Favm Col 14 FriUM $N$ Col 14 FRUM U CAHBUN

M-3 FRUM LIO H. 3 FROM BIO $M=3$ FISS YLO IRITIUM

ALL ELEMENTS IUIAL BETA

TOTAL GAMMA

TOTAL ALPMA
MS FHESH V-235 F.E. FERTILE PTL. FISSILE PTL.

7.110000 1.150006 3.860007

1.009 .03

$2.091=00$

1.287 .04

$1.200-031$

0.000
1.418008
$1.985=08$
$3.401000(0)$
$2.215=05$
0.000
2.083004
$2.304004(0)$

$23 K S$ RECYCLE
FERIILE PTL.

$U-233 F, E$.

I ISILE PTL. C............

0.000

$? .116000$

1.150000

$8.851=006$ us 5.041 .0080 (

1.069 .03

2.091 .00

$1.287=04$

$1.200-036$

\section{$3.282=05$}

0.000

1.820004

$2.1540046 \quad 01$

$0)$

$5.947+01$

$2,512+01$

$4.209-01$

$0.055+01$

$3.953+0$

$1.045+02(37)$

$5.947+01$

$2.512+01$

4.269001

$0.502+01(29)$

$5.474+01$

$5.204+01$

$1.055+00$
$8.473+01(50)$
DSHS RLCYCLE UA235 F, FENIILE PIL, FISSILEPTL,

\section{$7.116006 \quad 0.000$}

5.130006

$8.851 .0060) \quad 1.930007(0)$

1.069 .03

2.091 .06

$1.287 \cdot 04$
1.2000031

$1.261=04$

0.000

1.510004
2.7700046

2.77.004

$\begin{array}{ll}5.947+01 & 4.317+01 \\ 2.512+01 & 2.949+01 \\ 4.269011 & 3.446+01 \\ 8.502+01(29) & 1.095+02627\end{array}$




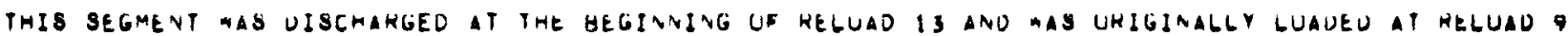

DECAY mEAT IN MAIIS PER FoE. AT TIME J YEARS AFIEK DIBCHARGE (PERCENT FRUM GAMMAS IN PIKENTHESES)

\begin{tabular}{|c|c|c|c|c|c|c|c|c|c|c|c|c|}
\hline NUELIDE & $\begin{array}{r}\text { MS FR } \\
\text { FERTILE PI }\end{array}$ & & $\begin{array}{l}\text { W-235 FIE. } \\
\text { FISSILE }\end{array}$ & TL. & $\begin{array}{r}\text { Z3RS RE } \\
\text { FERTILE PI }\end{array}$ & EYCLE & $\begin{array}{l}\text { UE-233 P.E. } \\
\text { FISSILE PI }\end{array}$ & & FERTILE RE & CYCLE & 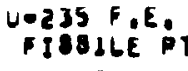 & Th. \\
\hline $\begin{array}{l}C M=242 \\
C M-243 \\
C M=244 \\
C U N J U M\end{array}$ & $\begin{array}{l}2.331000 \\
1.900008 \\
2.573004 \\
2.5960041\end{array}$ & (v) & $\begin{array}{l}1.340 .02 \\
2.632004 \\
2.265 .01 \\
2.4220016\end{array}$ & (1) & $\begin{array}{l}2.331006 \\
1.900008 \\
2.573004 \\
2.5960046\end{array}$ & (1) & $\begin{array}{l}2.764004 \\
3.452006 \\
1.023003 \\
2.1070031\end{array}$ & 01 & $\begin{array}{l}2.331000 \\
1.960008 \\
2.573004 \\
2.5400041\end{array}$ & u) & $\begin{array}{l}1.089001 \\
2.096003 \\
1.785+00 \\
1.890+001\end{array}$ & 0) \\
\hline $\begin{array}{l}A M-241 \\
A M-242 M \\
A M-242 \\
A M=243 \\
A M E R I C I U M\end{array}$ & $\begin{array}{l}5.292000 \\
2.059009 \\
8.907009 \\
3.178=07 \\
5.6210006\end{array}$ & Q) & $\begin{array}{l}1.125002 \\
1.275006 \\
5.517000 \\
1.254=03 \\
1.251=026\end{array}$ & 01 & $\begin{array}{l}5.2920 .06 \\
2.059009 \\
8.907 .09 \\
3.178007 \\
5.621 .006\end{array}$ & $0)$ & $\begin{array}{l}4.300004 \\
3.590008 \\
1.5500 .07 \\
1.400005 \\
4.4480 .041\end{array}$ & 01 & $\begin{array}{l}5.292006 \\
2.059009 \\
8.907=09 \\
3.178007 \\
5.621=001\end{array}$ & 01 & $\begin{array}{l}9.042002 \\
1.033005 \\
4.472005 \\
9.924003 \\
1.0040016\end{array}$ & 0) \\
\hline $\begin{array}{l}P U=238 \\
P U=239 \\
P U=240 \\
P U=241 \\
\text { PLUTUNIUM }\end{array}$ & $\begin{array}{l}1.183 .02 \\
3.179006 \\
3.783000 \\
9.308-07 \\
1.180 .026\end{array}$ & $0)$ & $\begin{array}{l}3.800+00 \\
2.824003 \\
4.900003 \\
1.879003 \\
3.8100001\end{array}$ & (u) & $\begin{array}{l}1.183002 \\
3.179006 \\
3.783006 \\
9.308007 \\
1.1840021\end{array}$ & u) & $\begin{array}{l}4.021001 \\
1.562004 \\
2.341004 \\
7.3950 .5 \\
4.0260011\end{array}$ & 01 & $\begin{array}{l}1.183002 \\
3.179006 \\
3.783006 \\
9.308007 \\
1.1840026\end{array}$ & 01 & $\begin{array}{l}2.950+01 \\
2.192002 \\
3.870002 \\
1.509002 \\
2.960+011\end{array}$ & Q) \\
\hline $\begin{array}{l}\text { NPE } 237 \\
\text { NEPTUNIUM }\end{array}$ & $\begin{array}{l}1.810006 \\
1.8100060\end{array}$ & (4) & $\begin{array}{l}2,900=04 \\
2.9000046\end{array}$ & (1) & $\begin{array}{l}1.810=06 \\
1.8100066\end{array}$ & $0)$ & $\begin{array}{l}4.000005 \\
4.000-056\end{array}$ & 01 & $\begin{array}{l}1.816=06 \\
1.810=006\end{array}$ & 01 & $\begin{array}{l}1.879003 \\
1.8790031\end{array}$ & $0)$ \\
\hline $\begin{array}{l}U=232 \\
U-233 \\
U=234 \\
U=235 \\
U=230 \\
U=238 \\
U R A N I U M\end{array}$ & $\begin{array}{l}5.110 .02 \\
5.954=02 \\
7.031 .03 \\
5.120007 \\
2.384 .06 \\
0.000 \\
1.483=011\end{array}$ & 0) & $\begin{array}{l}1.326005 \\
9.136-10 \\
4.835=04 \\
3.804006 \\
2.019=04 \\
3.192007 \\
7.028=048\end{array}$ & (1) & $\begin{array}{l}8.110 .02 \\
5.954 .02 \\
7.031003 \\
5.120 .07 \\
2.384 .00 \\
0.000 \\
1.483 .016\end{array}$ & 0) & $\begin{array}{l}1.023002 \\
9.056003 \\
1.030002 \\
1.075000 \\
2.821005 \\
1.305011 \\
1.027011\end{array}$ & 01 & $\begin{array}{l}8.110002 \\
5.954002 \\
7.631003 \\
5.120007 \\
2.384000 \\
0.000 \\
1.4830016\end{array}$ & $0)$ & $\begin{array}{l}3.499=05 \\
4.891009 \\
2.005003 \\
3.021006 \\
1.849003 \\
2.301=00 \\
3.9150031\end{array}$ & 0) \\
\hline $\begin{array}{l}P A-231 \\
P A-233 \\
P A=234 \\
\text { PRUTACTINIUM }\end{array}$ & $\begin{array}{l}6.499-05 \\
6.205 .10 \\
0.000 \\
8.499 .051\end{array}$ & 1) & $\begin{array}{l}1.442008 \\
0.000 \\
1.341 .07 \\
1.4850071\end{array}$ & Bus & $\begin{array}{l}0.499-05 \\
6.205 .10 \\
0.000 \\
8.4990056\end{array}$ & (1) & $\begin{array}{l}9.384000 \\
0.000 \\
5.801-12 \\
9.3840006\end{array}$ & 11 & $\begin{array}{l}8.499=05 \\
6.205010 \\
0.000 \\
0.499=056\end{array}$ & 1) & $\begin{array}{l}5.930-08 \\
0.000 \\
1.000006 \\
1.0590068\end{array}$ & $(3)$ \\
\hline $\begin{array}{l}I M-228 \\
T H=229 \\
T M=230 \\
\text { IM- } 31 \\
\text { IH-232 } \\
\text { IM-234 } \\
\text { IMUKIUM }\end{array}$ & $\begin{array}{l}0.487=02 \\
2.350005 \\
1.704=04 \\
1.995=08 \\
2.107-05 \\
0.000 \\
6.5090026\end{array}$ & (1) & $\begin{array}{l}1.059005 \\
1.955010 \\
3.141 .08 \\
1.506=07 \\
0.000 \\
5.598009 \\
1.0780056\end{array}$ & (1) & $\begin{array}{l}0.487 .02 \\
2.350005 \\
1.704-114 \\
1.995008 \\
2.107-15 \\
6.000 \\
6.509-026\end{array}$ & $0)$ & $\begin{array}{l}7.947=02 \\
4.014005 \\
1.361=05 \\
7.306008 \\
0.000 \\
2.447-13 \\
7.952-026\end{array}$ & 01 & $\begin{array}{l}0.487002 \\
2.350 .05 \\
1.704004 \\
1.995=08 \\
2.107 .05 \\
0.000 \\
0.5090 .026\end{array}$ & us & $\begin{array}{l}4.390=05 \\
8.052-10 \\
1.314007 \\
1.1770 .07 \\
0.000 \\
4.176008 \\
4.4190056\end{array}$ & (0) \\
\hline $\begin{array}{l}1 c-225 \\
1 C-227\end{array}$ & $\begin{array}{l}2.729=05 \\
1.149=07\end{array}$ & & $\begin{array}{l}2.271-10 \\
2.841-11\end{array}$ & & $\begin{array}{l}2.729=05 \\
1.149=07\end{array}$ & & $\begin{array}{l}4.062-05 \\
1.027-00\end{array}$ & & $\begin{array}{l}2.729=05 \\
1.149=07\end{array}$ & & $\begin{array}{l}9.351-10 \\
1.178-10\end{array}$ & \\
\hline
\end{tabular}


IMIS SEGMENT MAS UISGHARgEO AT THE BEGIVNING UF HELUAD I3 ANU NAS UKIGINALLY GUADEU AT WEGUAD Q

LECAY MEAT IN WATIS PER FiE. AT TIME S YEAHS AFIEH UISCHARGE

\begin{tabular}{|c|c|c|c|c|c|c|c|}
\hline $\begin{array}{l}\text { NUCL IDE } \\
\text { NOA }\end{array}$ & $\begin{array}{l}\text { YS FHEST } \\
\text { FERTILE PTLD. } \\
\text {-OOOO-OO-OOE. }\end{array}$ & 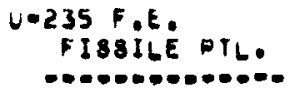 & 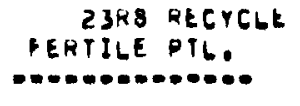 & $\begin{array}{l}\text { UE2S3 F.E. } \\
\text { FISSILE PIL. }\end{array}$ & 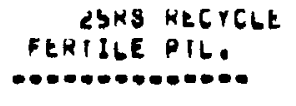 & 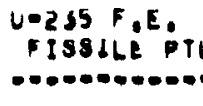 & L. \\
\hline $\begin{array}{l}A C=226 \\
A C I I N I U M\end{array}$ & $\begin{array}{l}4.209006 \\
3.167-051102\end{array}$ & $\begin{array}{l}0.000 \\
2.555 .101\end{array}$ & $\begin{array}{l}4.269000 \\
3.167 .05(10)\end{array}$ & $\begin{array}{l}9.040-12 \\
4.603-051\end{array}$ & $\begin{array}{l}4.269006 \\
3.167005(10)\end{array}$ & $\begin{array}{l}0.000 \\
1.0530008\end{array}$ & 11 \\
\hline $\begin{array}{l}H A-223 \\
H A=224 \\
R A=225 \\
R A=228 \\
R A U I U A\end{array}$ & $\begin{array}{l}7.8550 .16 \\
0.791002 \\
0.104007 \\
4.148008 \\
0.7920021\end{array}$ & $\begin{array}{l}1.931-09 \\
1.109005 \\
5.113012 \\
0.000 \\
1.1090056\end{array}$ & $\begin{array}{l}7.655006 \\
0.791002 \\
0.144007 \\
4.148-00 \\
6.792-021\end{array}$ & $\begin{array}{l}1.211006 \\
8.319002 \\
1.050006 \\
0.785014 \\
0.3190026\end{array}$ & $\begin{array}{l}7.855006 \\
0.791002 \\
0.144007 \\
4.140008 \\
0.7920021\end{array}$ & $\begin{array}{l}0.000009 \\
4.595005 \\
2.105011 \\
0.000 \\
4.590-056\end{array}$ & 03 \\
\hline $\begin{array}{l}F R=221 \\
F R=223 \\
F R A N E I U M\end{array}$ & $\begin{array}{l}2,909=05 \\
8,808=09 \\
2,970=056\end{array}$ & $\begin{array}{l}2.471-10 \\
2.166-12 \\
2.092-106\end{array}$ & $\begin{array}{l}2.909=05 \\
8.808-09 \\
2.970=656\end{array}$ & $\begin{array}{l}5.0720 .05 \\
1.358000 \\
5.0720056\end{array}$ & $\begin{array}{l}2,909005 \\
8,808009 \\
2.9700051\end{array}$ & $\begin{array}{l}1.017=09 \\
8,080012 \\
1.026=096\end{array}$ & 08 \\
\hline $\begin{array}{l}\text { RN=219 } \\
\text { RN-220 } \\
\text { RADUN }\end{array}$ & $\begin{array}{l}9.125006 \\
7.521 .02 \\
7.521 .026\end{array}$ & $\begin{array}{l}2.243009 \\
1.228005 \\
1.2280 .056\end{array}$ & $\begin{array}{l}9.125006 \\
7.521=02 \\
7.521=026\end{array}$ & $\begin{array}{l}1.407000 \\
9.213002 \\
9.2130021\end{array}$ & $\begin{array}{l}9.125000 \\
7.521002 \\
7.5210 .02(\quad 01\end{array}$ & $\begin{array}{l}9.30 d=09 \\
\$ .009005 \\
5.0900056\end{array}$ & $0)$ \\
\hline $\begin{array}{l}\text { ATE-217 } \\
\text { ATEZIR } \\
\text { ASTATIVE }\end{array}$ & $\begin{array}{l}3.311-05 \\
1.964-10 \\
3.311-051\end{array}$ & $\begin{array}{l}2.755=10 \\
0.000 \\
2.755=106\end{array}$ & $\begin{array}{l}3.311=05 \\
1.964-10 \\
3.311=056\end{array}$ & $\begin{array}{l}5.650-05 \\
1.560-11 \\
5.650-056\end{array}$ & $\begin{array}{l}3.311=05 \\
1.964-10 \\
3.311=05(\quad 0)\end{array}$ & $\begin{array}{l}1.135009 \\
0.000 \\
1.1350096\end{array}$ & $0)$ \\
\hline $\begin{array}{l}P U-210 \\
P U=212 \\
P D=213 \\
P U=215 \\
P U=210 \\
P U L O N I U M\end{array}$ & $\begin{array}{l}8.272=07 \\
6.715=02 \\
3.927-05 \\
0.884=00 \\
0.115=02 \\
1.483=016\end{array}$ & $\begin{array}{l}2.370=10 \\
1.097=05 \\
3.268=10 \\
2.430-09 \\
1.325-05 \\
2.422=056\end{array}$ & $\begin{array}{l}8.272007 \\
6.715002 \\
3.927 .05 \\
9.884=00 \\
8.115002 \\
1.4830011\end{array}$ & $\begin{array}{l}8.482=07 \\
8.239=02 \\
6.709-05 \\
1.524000 \\
9.940-02 \\
1.8190011\end{array}$ & $\begin{array}{l}8.272=07 \\
6.715002 \\
3.927 .05 \\
9.884006 \\
8.115002 \\
1.403=018\end{array}$ & $\begin{array}{l}9.093-10 \\
4.5440 .05 \\
1.346-09 \\
1.008008 \\
5.491 .05 \\
1.004=041\end{array}$ & 0) \\
\hline 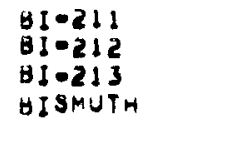 & $\begin{array}{l}8.841=00 \\
3.383=02 \\
3.434 .00 \\
3.384 .02(\end{array}$ & $\begin{array}{l}2.174009 \\
5.524006 \\
2.850-11 \\
5.5200068\end{array}$ & $\begin{array}{l}3.641=06 \\
3.383=02 \\
3.434 .06 \\
3.384=021\end{array}$ & $\begin{array}{l}1.563006 \\
4.144002 \\
5.860 .06 \\
4.1450 .021\end{array}$ & $\begin{array}{l}8.841 .00 \\
3.383 .02 \\
3.434 .06 \\
3.384 .02(\end{array}$ & $\begin{array}{l}9.015=09 \\
2.289005 \\
1.171=10 \\
2.2900051\end{array}$ & 4) \\
\hline $\begin{array}{l}P Y-209 \\
P B-211 \\
P B=212 \\
G E A D\end{array}$ & $\begin{array}{l}9.555=07 \\
7.228007 \\
3.803=03 \\
3.804=03606)\end{array}$ & $\begin{array}{l}7.952-12 \\
1.777-10 \\
0.210 .07 \\
6.212007(00)\end{array}$ & $\begin{array}{l}9.555007 \\
7.228007 \\
3.803003 \\
3.804003(06)\end{array}$ & $\begin{array}{l}1.032-06 \\
1.114007 \\
4.658003 \\
4.060003(06)\end{array}$ & $\begin{array}{l}9.555=07 \\
7.228=07 \\
3.803=03 \\
3.804=03(60)\end{array}$ & $\begin{array}{l}3,274-11 \\
7,368=10 \\
2,373=06 \\
2,374=061\end{array}$ & $00 \mathrm{~J}$ \\
\hline $\begin{array}{l}\text { TLE-207 } \\
\text { TL-208 } \\
\text { THALEIUM }\end{array}$ & $\begin{array}{l}1.114007 \\
1.698002 \\
1.690002(84)\end{array}$ & $\begin{array}{l}1.749-10 \\
2.772=06 \\
2.772006(\text { b4) }\end{array}$ & $\begin{array}{l}7.114007 \\
1.698-02 \\
1.698002(84)\end{array}$ & $\begin{array}{l}1.097=07 \\
2.083002 \\
2.083=02(84)\end{array}$ & $\begin{array}{l}7.114007 \\
1.098002 \\
1.608002(64)\end{array}$ & $\begin{array}{l}7.252-10 \\
1.149-05 \\
1.149-056\end{array}$ & 843 \\
\hline $\begin{array}{l}\text { OY- } 159 \\
\text { OYSPRLSIUM }\end{array}$ & $\begin{array}{l}3.089-09 \\
3.089000(100)\end{array}$ & $\begin{array}{l}0.050-10 \\
0.050-10(1 \cup v)\end{array}$ & $\begin{array}{l}3.089=09 \\
3.089=09(100)\end{array}$ & $\begin{array}{l}8.983-10 \\
8.963-10(100)\end{array}$ & $\begin{array}{l}3.689009 \\
3.089 \cdot 19(160)\end{array}$ & $\begin{array}{l}3,440009 \\
3,408009(1\end{array}$ & 1003 \\
\hline
\end{tabular}




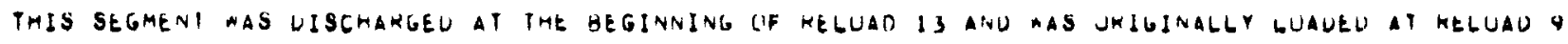

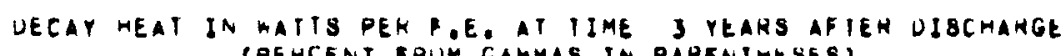
(PENCENT RUM GAMMAS IN PARENIMESES)

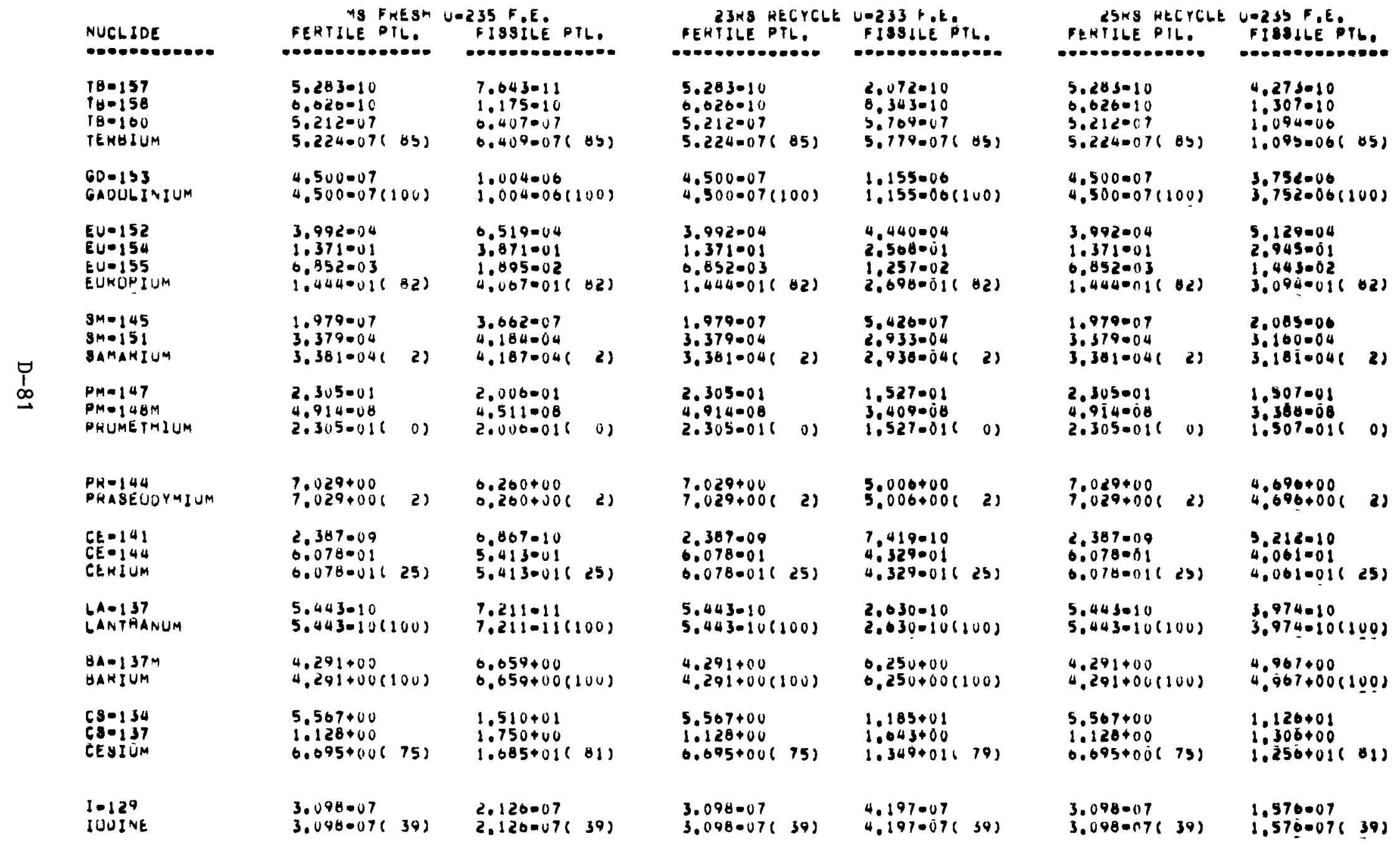




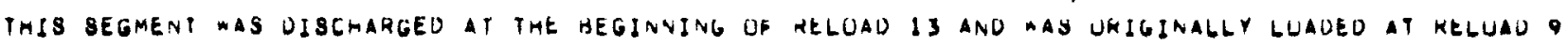

DECAY MEAT IN WATIS PER F.E. AT TIME 3 YEARS AFIER DISCMAMGE (PERCENT FROM GAMMAS IN PAKENTHEBES)

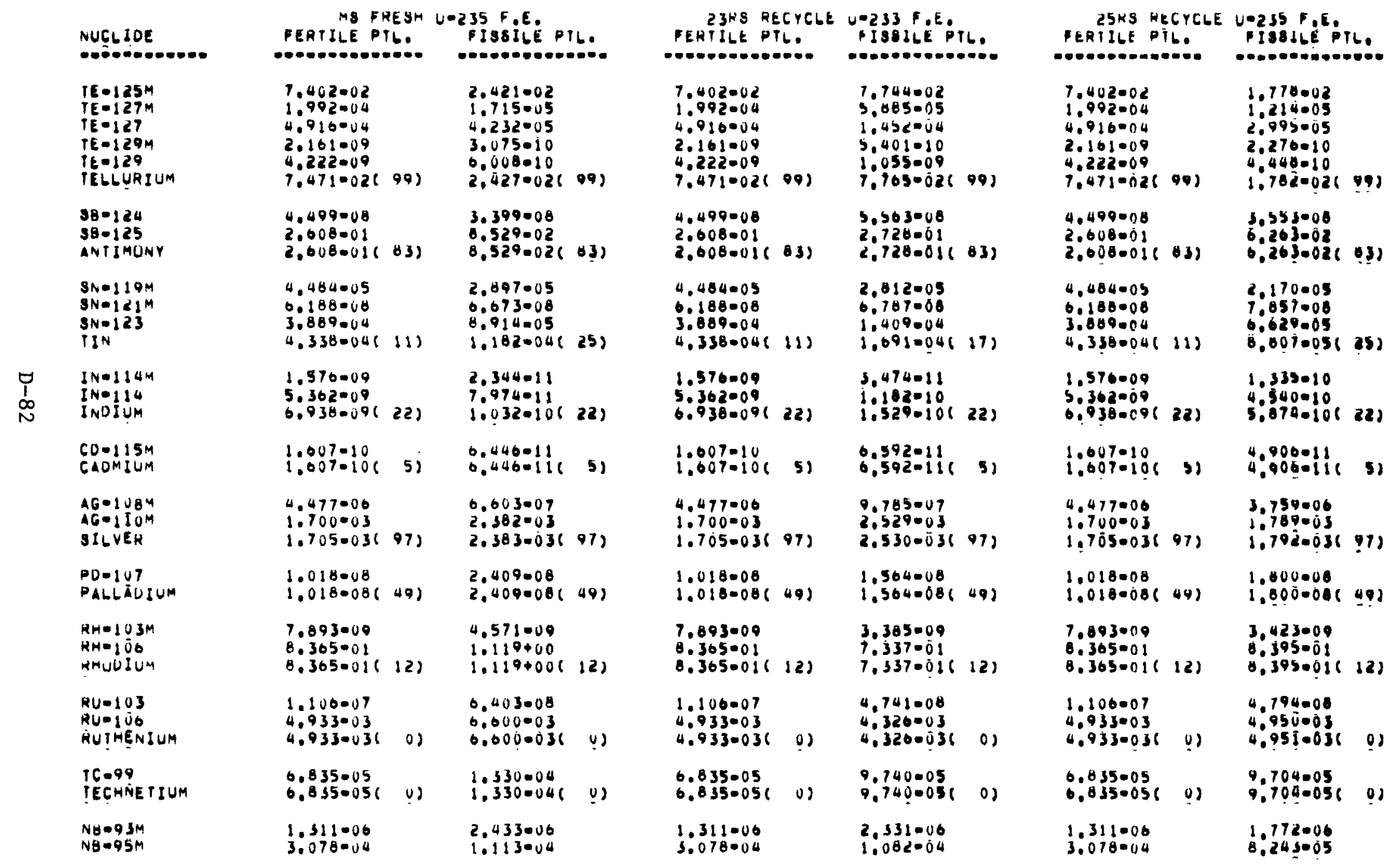




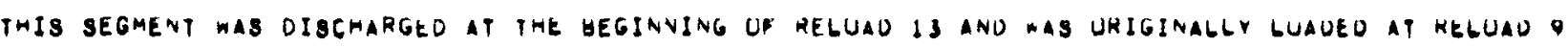
DEGAY MEAT IN MAITS PER FiE. AT IIME J YEARS AFTER DISCHARGE

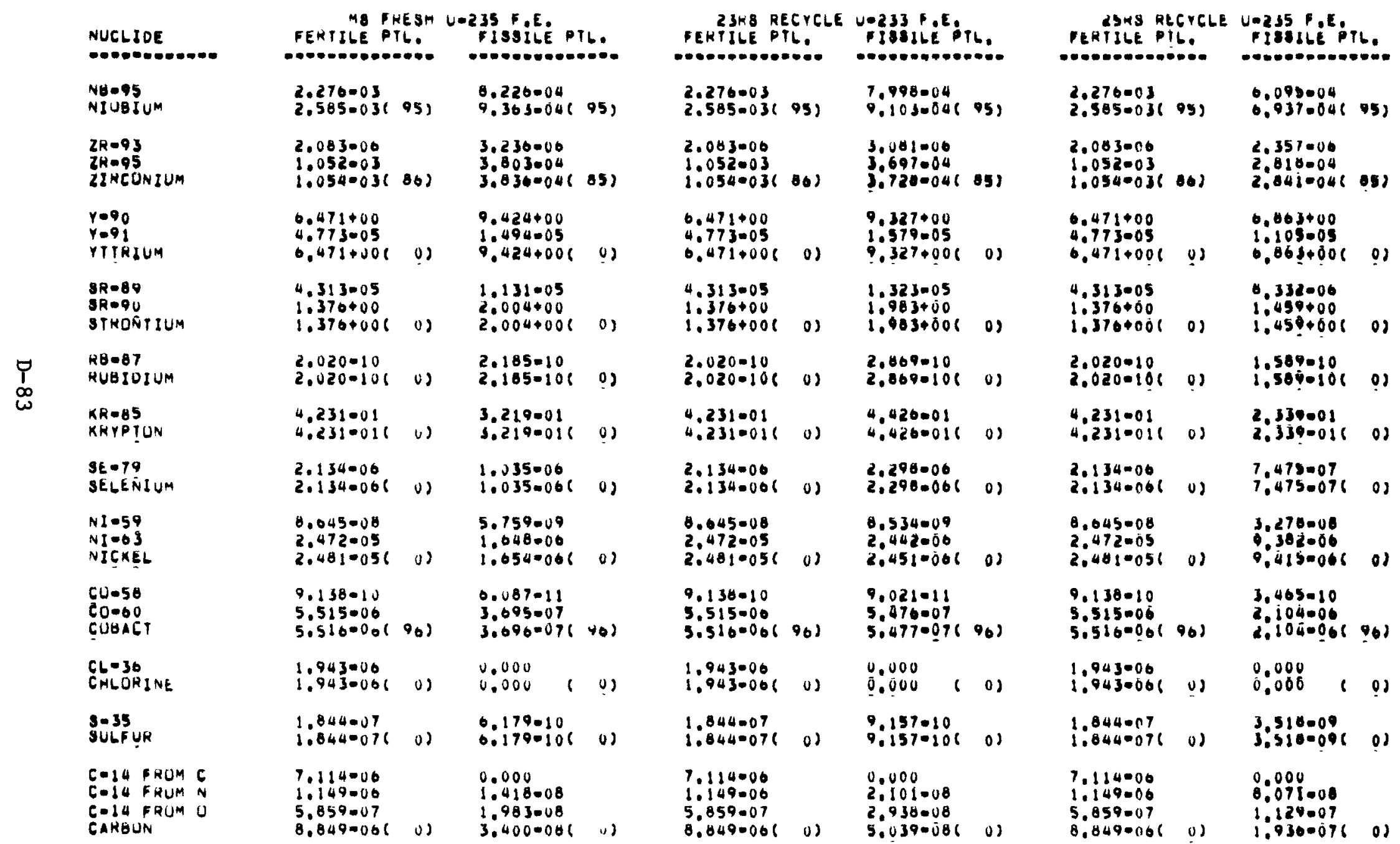




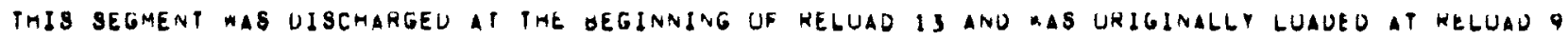
UECAY MEAT IN MATIS PER ERE. AT IIME J YEAKS AFIEN DISCMAKGE

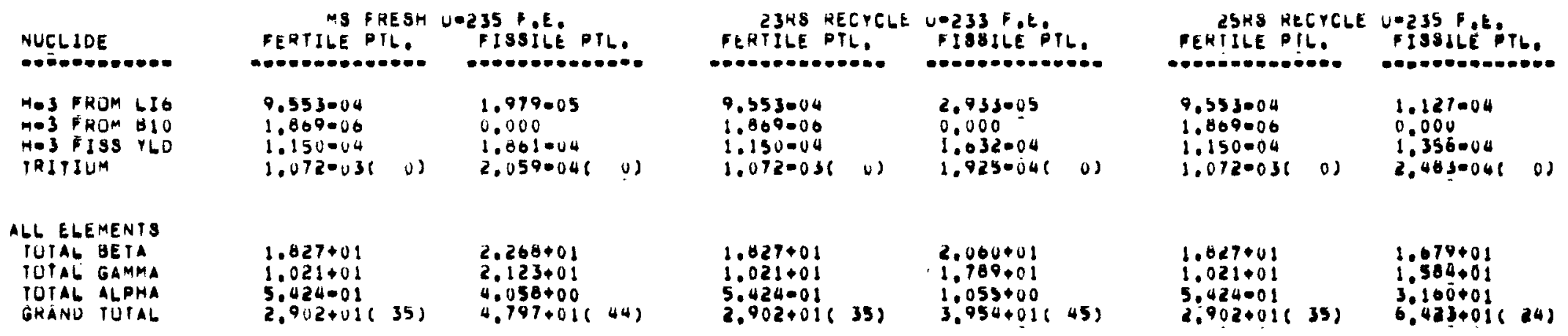


THIS BEGMENT WAS DISEMARGED AT THE GEGINNING UP RELUAD I3 AND WAS URIGINALLY LUADED AT HELUAD

DECAY HEAT IN WATIS PER F.E. AT IIME S YEARS AFIEA UISGHARGE

(PERCENT FROM GAMMAS IN PARENIHESES)

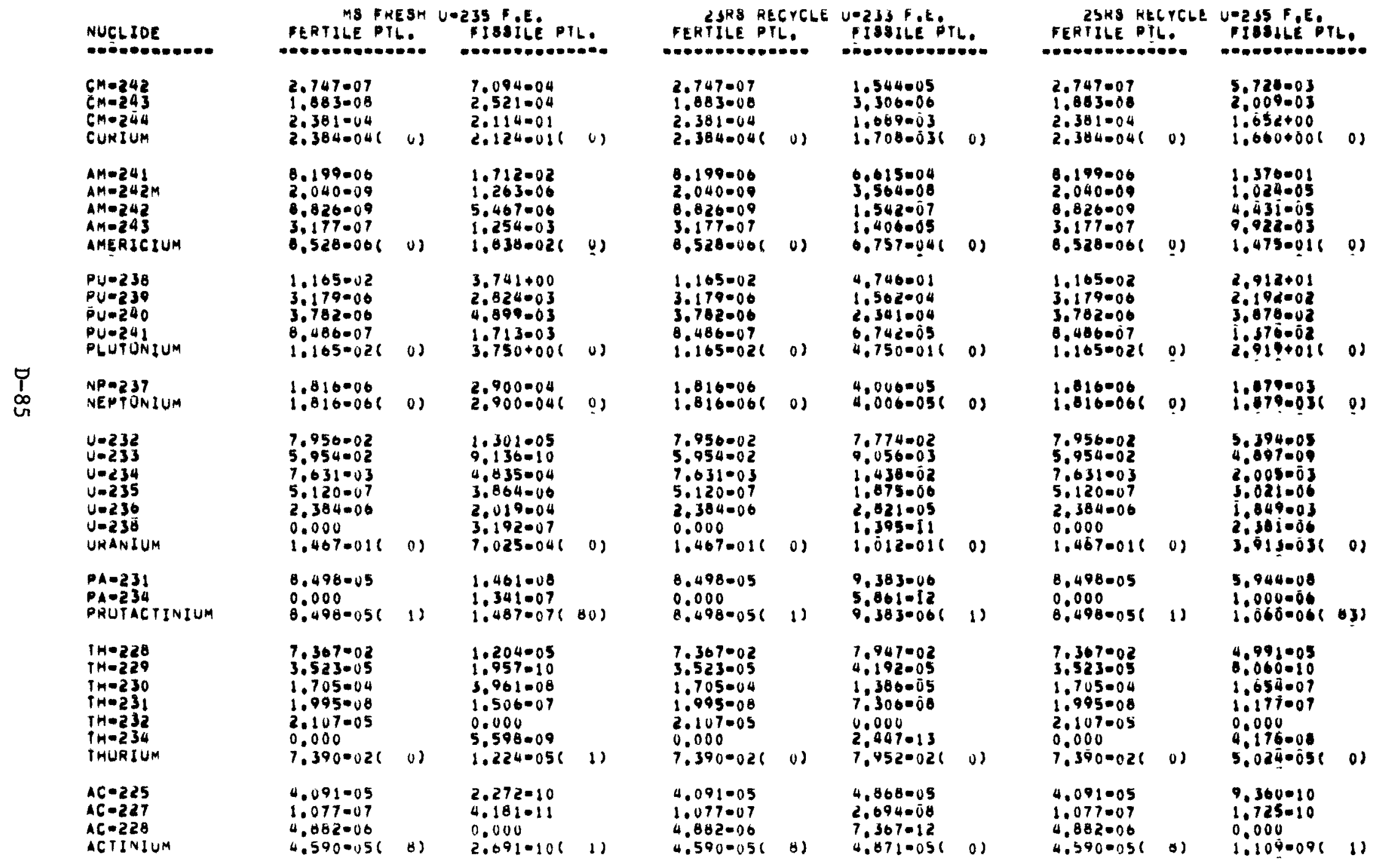


THIS SEGMENT MAS UISCHARGEO AT THE GEgINNING UF KELUAD IS ANU WAS URIGINALLY LUADEU AT KELUAU Q

DECAY MEAT IN WATIS PER F.E. AT IIME S YEARS AFTEN UISCHARGE (PERCENT FROM GAMMAS IN PAHENTHESES)

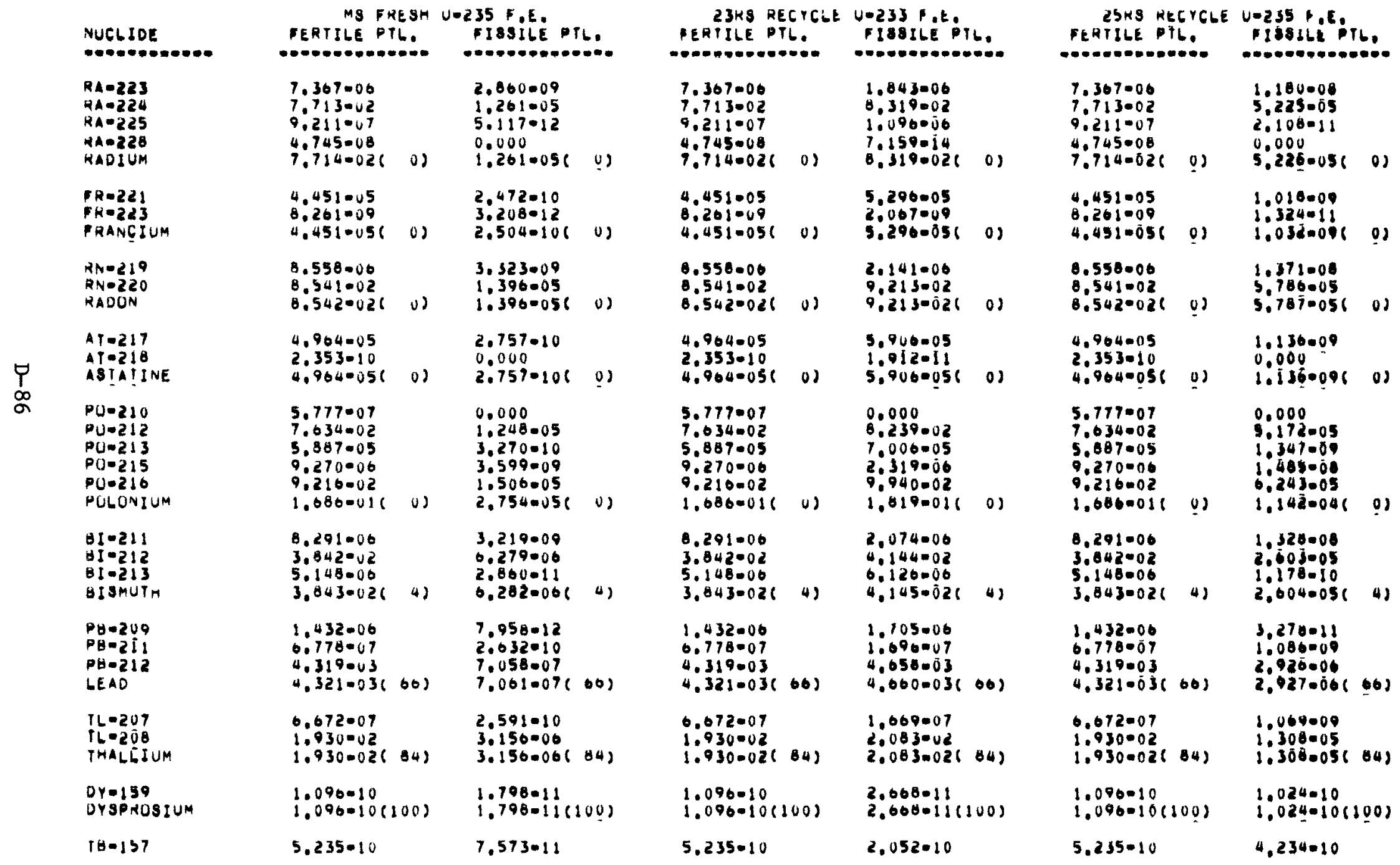


THIS SEgMENT WAS OISCMARgED AT THE OEgINNING UF RELUAD I3 AND MAS URIGINAGLY LUADED AT RELUAO DECAY HEAT IN MATIS PER FEE AT TIME S YEARS AFTEH DISCHAKGE

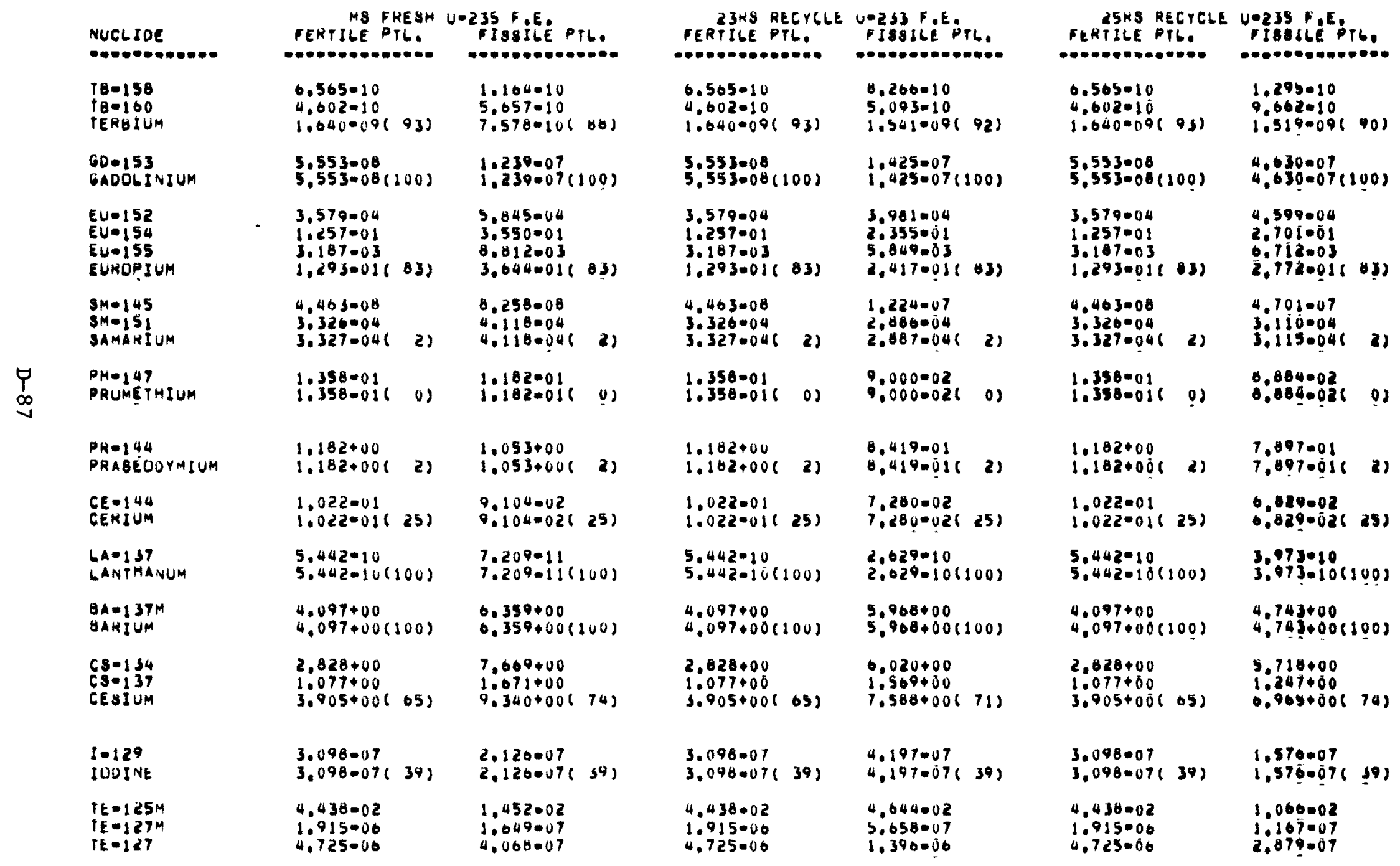




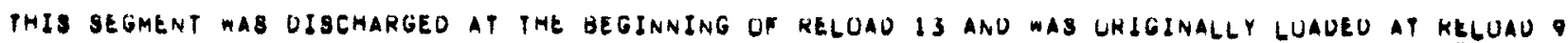
DECAY heAT IN WAIIS PER P.E. AT TIME 5 YEARS AFTER DISChARgE

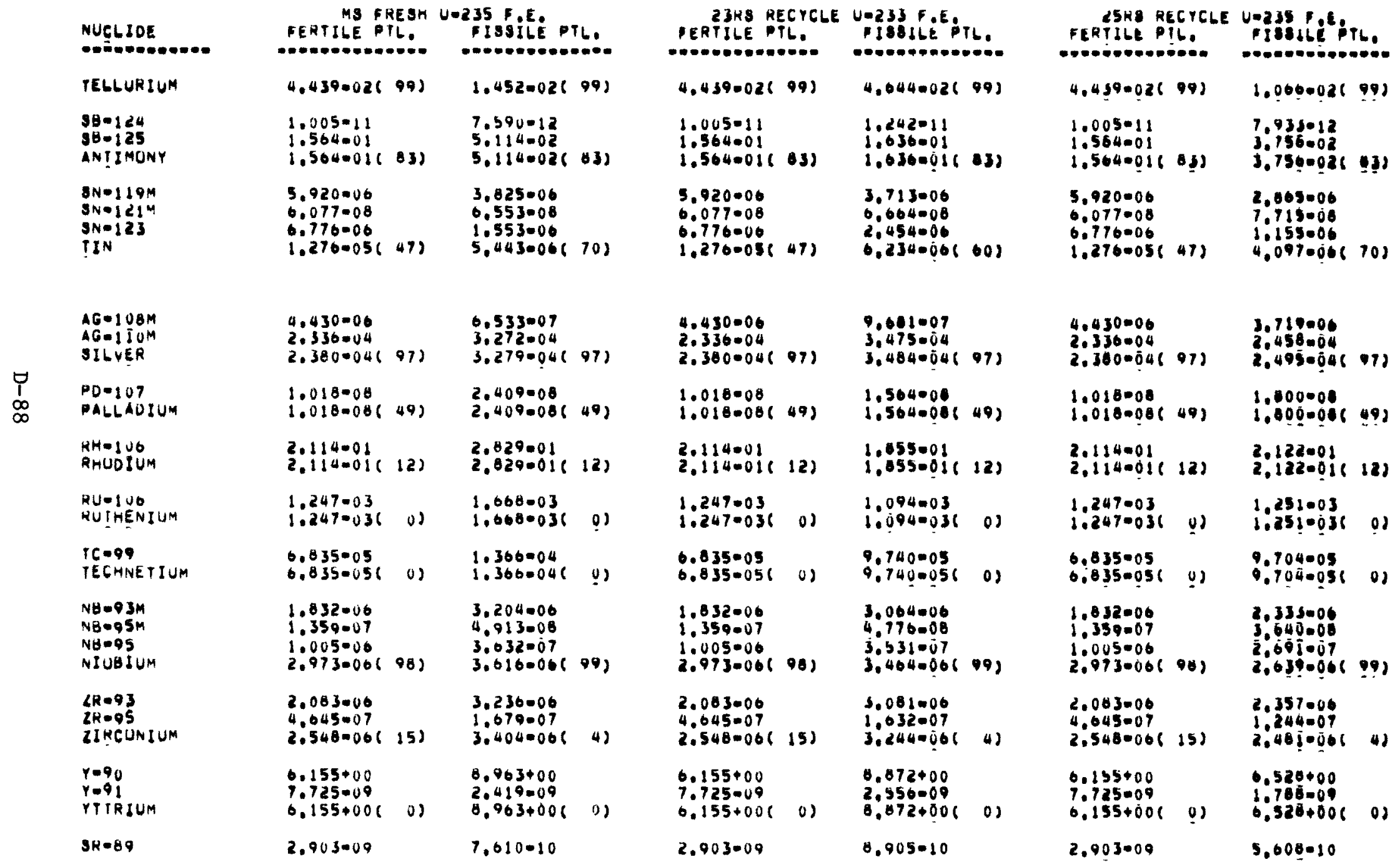




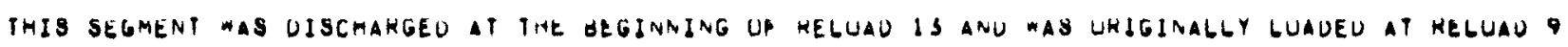
oEcaY HEAT IN WATIS PER DE. AT TIME S YEAHS AFIEK DISCHAMGE

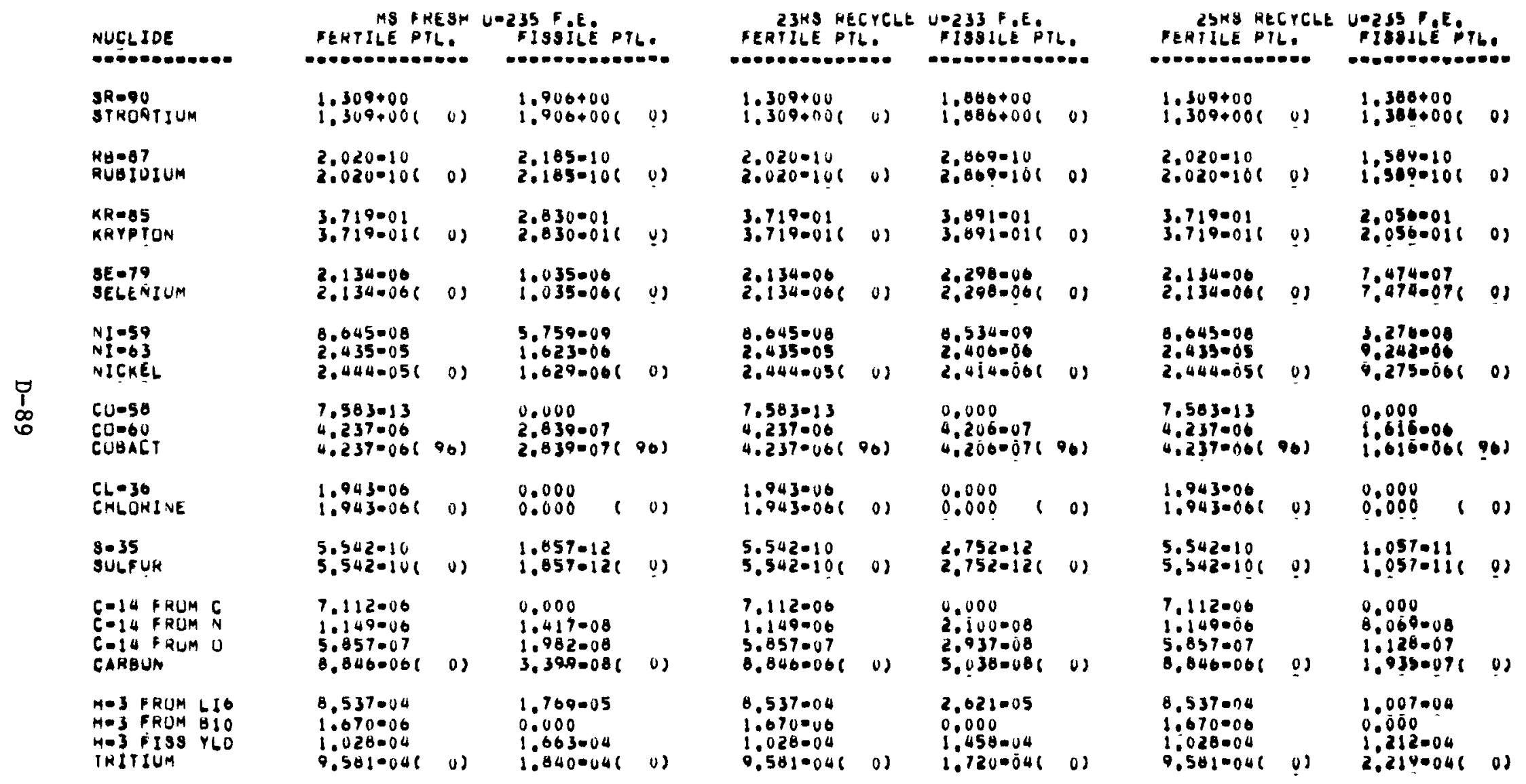




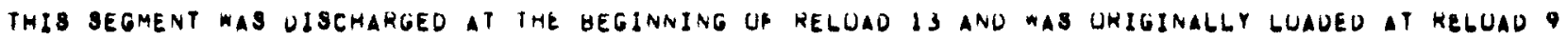

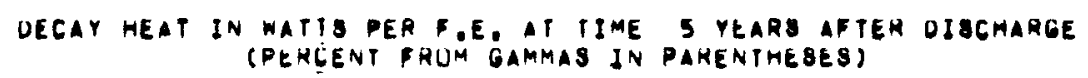

\begin{tabular}{|c|c|c|c|c|c|c|}
\hline 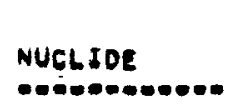 & 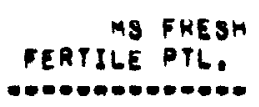 & 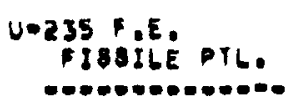 & $\begin{array}{l}\text { 23K8 RECYCLE } \\
\text { FERTILE PTL. } \\
\text { WEOWEOEDE. }\end{array}$ & 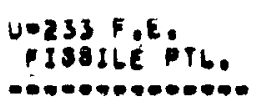 & 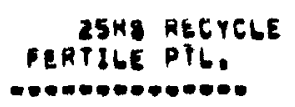 & 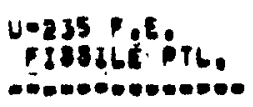 \\
\hline $\begin{array}{l}\text { LL ELEMENTS } \\
\text { TOIAL BETA } \\
\text { TOTAL GAMMA } \\
\text { TOTAL ALPMA } \\
\text { GRANO TOTAL }\end{array}$ & $\begin{array}{l}1.078+01 \\
7.055+00 \\
5.926001 \\
1.843+01(38)\end{array}$ & $\begin{array}{l}1.507+01 \\
1.377+01 \\
3.974000 \\
3.281+01(41)\end{array}$ & $\begin{array}{l}1.076+01 \\
7.055+00 \\
5.926001 \\
1.043+01(38)\end{array}$ & $\begin{array}{l}1.440+01 \\
1.191+01 \\
1.046+00 \\
2.743+01643)\end{array}$ & $\begin{array}{l}1.078+01 \\
7.055+00 \\
5.926001 \\
1.043+016 \quad 303\end{array}$ & $\begin{array}{l}1.110+01 \\
1.028+01 \\
3.095+01 \\
5.232+01(29\end{array}$ \\
\hline
\end{tabular}




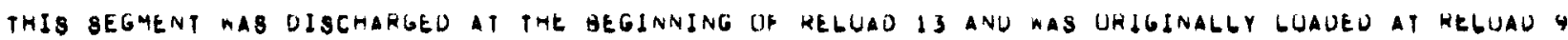

DEgAY meat IN WATTS PER F.E. AT TIME IO YEARS AFtEK UISCHAKGE

\begin{tabular}{|c|c|c|c|c|c|c|c|c|c|c|c|c|}
\hline $\begin{array}{l}\text { NUCLIDE } \\
\text { NCLW }\end{array}$ & $\begin{array}{r}\text { Mg FR } \\
\text { FERTILE PT }\end{array}$ & & 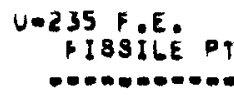 & & 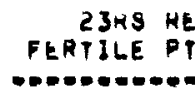 & $\begin{array}{l}\text { CYCLE } \\
6 . \\
6-\infty\end{array}$ & 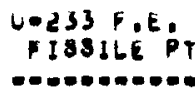 & & $\begin{array}{r}\text { 25KS RE } \\
\text { FERTILE PI }\end{array}$ & $\begin{array}{l}\text { GCLE } \\
6 . \\
6 .\end{array}$ & 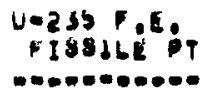 & Th. \\
\hline $\begin{array}{l}C M-242 \\
C M=243 \\
C M-244 \\
C U H I U M\end{array}$ & $\begin{array}{l}2.070=07 \\
1.090-08 \\
1.902004 \\
1.9040041\end{array}$ & (1) & $\begin{array}{l}1.262004 \\
2.202004 \\
1.7420 .01 \\
1.740 .018\end{array}$ & (v) & $\begin{array}{l}2.070=07 \\
1.090-08 \\
1.962004 \\
1.964-048\end{array}$ & u) & $\begin{array}{l}3.017=06 \\
2.967=00 \\
1.392003 \\
1.398-031\end{array}$ & 01 & $\begin{array}{l}2.070007 \\
1.690008 \\
1.962004 \\
1.9640041\end{array}$ & 0) & $\begin{array}{l}1.059003 \\
1.803003 \\
1.301+00 \\
1.364+006\end{array}$ & 01 \\
\hline $\begin{array}{l}A M=241 \\
A M=242 M \\
M M=242 \\
M M=243 \\
M M E R I C I U M\end{array}$ & $\begin{array}{l}1.435=05 \\
1.994-09 \\
8.027=09 \\
3.176007 \\
1.468-051\end{array}$ & 08 & $\begin{array}{l}2.953002 \\
1.235006 \\
5.343000 \\
1.250 .03 \\
3.079-026\end{array}$ & U) & $\begin{array}{l}1.435=05 \\
1.994=09 \\
8.627=09 \\
3.176007 \\
1.4680056\end{array}$ & 01 & $\begin{array}{l}1.150=03 \\
3.483008 \\
1.507=07 \\
1.405005 \\
1.1040036\end{array}$ & 01 & $\begin{array}{l}1.435=05 \\
1.904=09 \\
8.027-09 \\
3.176-07 \\
1.468 .058\end{array}$ & U) & $\begin{array}{l}2.372001 \\
1.001005 \\
4.331=05 \\
9.910003 \\
2.0720016\end{array}$ & 01 \\
\hline $\begin{array}{l}P U=238 \\
P U=239 \\
P U=240 \\
P U=241 \\
\text { PLUTUNIUM }\end{array}$ & $\begin{array}{l}1.120=02 \\
3.178000 \\
3.780006 \\
0.736=07 \\
1.120=026\end{array}$ & $0)$ & $\begin{array}{l}3.590+00 \\
2.824003 \\
4.097=03 \\
1.360=03 \\
3.605+006\end{array}$ & $0)$ & $\begin{array}{l}1.120=02 \\
3.1780 .00 \\
3.780000 \\
0.736=07 \\
1.120=021\end{array}$ & 0) & $\begin{array}{l}4.3020 .01 \\
1.5620 .04 \\
2.3390 .04 \\
5.351 .05 \\
4.566=016\end{array}$ & 01 & $\begin{array}{l}1.120=02 \\
3.178006 \\
3.780000 \\
6.736007 \\
1.120=026\end{array}$ & 01 & $\begin{array}{l}2.790+01 \\
1.191002 \\
3.876002 \\
1.092002 \\
2.806+016\end{array}$ & 01 \\
\hline $\begin{array}{l}\text { YP-237 } \\
\text { NEPTONIUM }\end{array}$ & $\begin{array}{l}1.810000 \\
1.810-001\end{array}$ & 0) & $\begin{array}{l}2.900=04 \\
2.9000046\end{array}$ & $\underline{v)}$ & $\begin{array}{l}1.816000 \\
1.8160006\end{array}$ & 0) & $\begin{array}{l}4.006=05 \\
4.0000051\end{array}$ & 01 & $\begin{array}{l}1.816006 \\
1.8160066\end{array}$ & o) & $\begin{array}{l}\{.879003 \\
1.8790036\end{array}$ & o) \\
\hline $\begin{array}{l}U=232 \\
U=233 \\
U=234 \\
U=235 \\
U=230 \\
U=238 \\
U R A N I U M\end{array}$ & $\begin{array}{l}7.582-02 \\
5.954=02 \\
7.031=03 \\
5.120=07 \\
2.384=00 \\
0.000 \\
1.430=016\end{array}$ & $0 \mathrm{~J}$ & $\begin{array}{l}1.240=05 \\
9.136=10 \\
4.834=04 \\
3.864006 \\
2.019=04 \\
3.192=07 \\
7.019-046\end{array}$ & (v) & $\begin{array}{l}7.582 .02 \\
5.954=02 \\
7.631=03 \\
5.120-07 \\
2.384 .06 \\
0.000 \\
1.430 .011\end{array}$ & 08 & $\begin{array}{l}7.409-02 \\
9.055003 \\
1.438-02 \\
1.875-06 \\
2.821005 \\
1.395-11 \\
9.755=026\end{array}$ & 09 & $\begin{array}{l}7.582=02 \\
5.954=02 \\
7.631=03 \\
5.120=07 \\
2.384=06 \\
0.000 \\
1.430=016\end{array}$ & 03 & $\begin{array}{l}5.141=05 \\
4.807=00 \\
2.005=03 \\
3.021006 \\
1.649=03 \\
2.381006 \\
3.0110036\end{array}$ & 08 \\
\hline $\begin{array}{l}P A-2 B I \\
P A=23 A \\
\text { PRUIACTINIUM }\end{array}$ & $\begin{array}{l}8.4980 .05 \\
0.000 \\
8.4980056\end{array}$ & 12 & $\begin{array}{l}1.500008 \\
1.341007 \\
1.4910076\end{array}$ & 791 & $\begin{array}{l}8.498-05 \\
0.000 \\
8.498-056\end{array}$ & 11 & $\begin{array}{l}9.583=06 \\
5.861=12 \\
9.383=066\end{array}$ & 1) & $\begin{array}{l}8.498005 \\
0.000 \\
8.4980 .056\end{array}$ & 1) & $\begin{array}{l}5.979000 \\
1.000000 \\
1.0600060\end{array}$ & 035 \\
\hline $\begin{array}{l}T H=228 \\
T H=229 \\
T H=230 \\
T H=231 \\
T H=232 \\
T M=234 \\
\text { THURIUM }\end{array}$ & $\begin{array}{l}7.796=02 \\
6.454=05 \\
1.708=04 \\
1.995=08 \\
2.107-05 \\
0.000 \\
7.022=026\end{array}$ & 0) & $\begin{array}{l}1.275005 \\
1.961-10 \\
0.010008 \\
1.500007 \\
0.000 \\
5.598009 \\
1.290-056\end{array}$ & 1) & $\begin{array}{l}7.796002 \\
0.454-05 \\
1.708-04 \\
1.995-08 \\
2.107-05 \\
0.000 \\
7.822=021\end{array}$ & 01 & $\begin{array}{l}7.587=02 \\
4.6360 .05 \\
1.046005 \\
7.306008 \\
0.000 \\
2.447-13 \\
7.5930026\end{array}$ & 01 & $\begin{array}{l}7.790-02 \\
6.450-05 \\
1.700=04 \\
1.995=08 \\
2.107=05 \\
0.000 \\
7.822-026\end{array}$ & 03 & $\begin{array}{l}5.285=05 \\
8.0811010 \\
2.505007 \\
1.179007 \\
0.000 \\
4.176000 \\
5.3200051\end{array}$ & 02 \\
\hline $\begin{array}{l}A C=225 \\
A C=227 \\
A C=228 \\
A C I I V I U M\end{array}$ & $\begin{array}{l}7.494-05 \\
9.177-08 \\
5.960-06 \\
0.100-056\end{array}$ & 5) & $\begin{array}{l}2.277-10 \\
7.200-11 \\
0.000 \\
3.003-106\end{array}$ & (2) & $\begin{array}{l}7.494=05 \\
9.177-08 \\
5.900=00 \\
8.100=056\end{array}$ & 5) & $\begin{array}{l}5.364=05 \\
4.635=08 \\
4.427=12 \\
5.568=056\end{array}$ & 0) & $\begin{array}{l}7.494=05 \\
9.177008 \\
5.900006 \\
8.10001156\end{array}$ & ()) & $\begin{array}{l}9.584-10 \\
2.955-10 \\
0.000 \\
1.234=096\end{array}$ & 2) \\
\hline
\end{tabular}




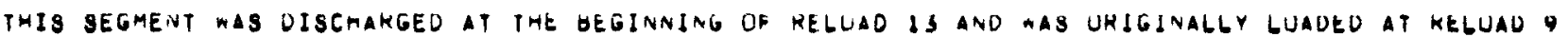

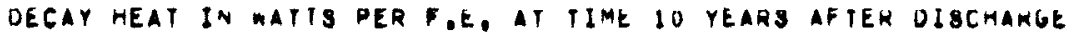
(PEKGENT PRuM Gammas ia pakentheses)

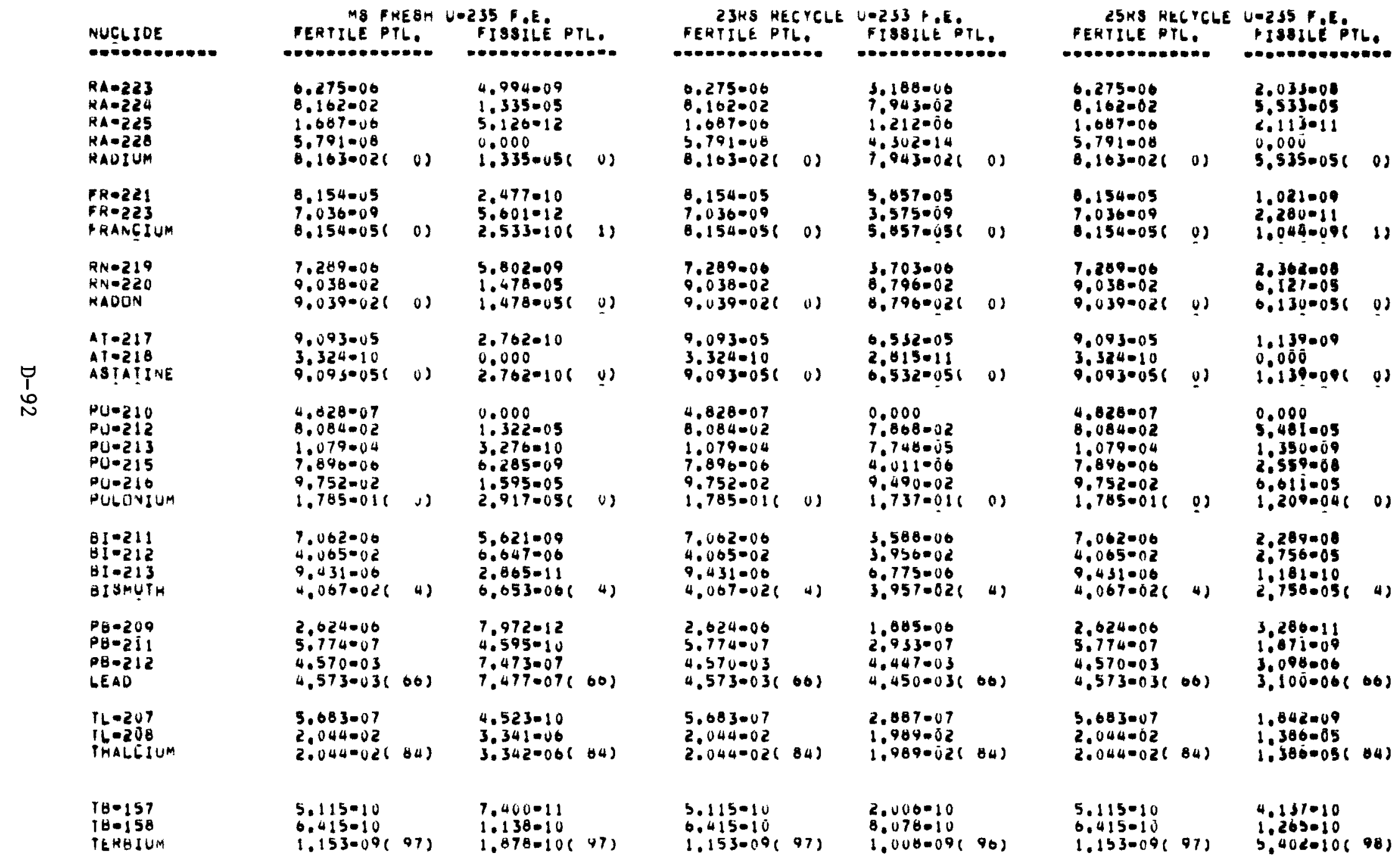




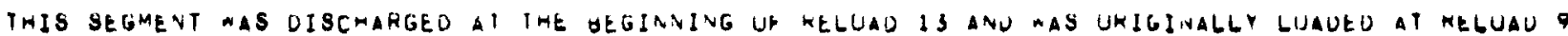

DECAY HEAT IN MAIIS DER F.E. AT IIME IU YEAKS AFIEK UISCHARgE (PERLENT FRIJM GAMMAS IN PAHENIMESES)

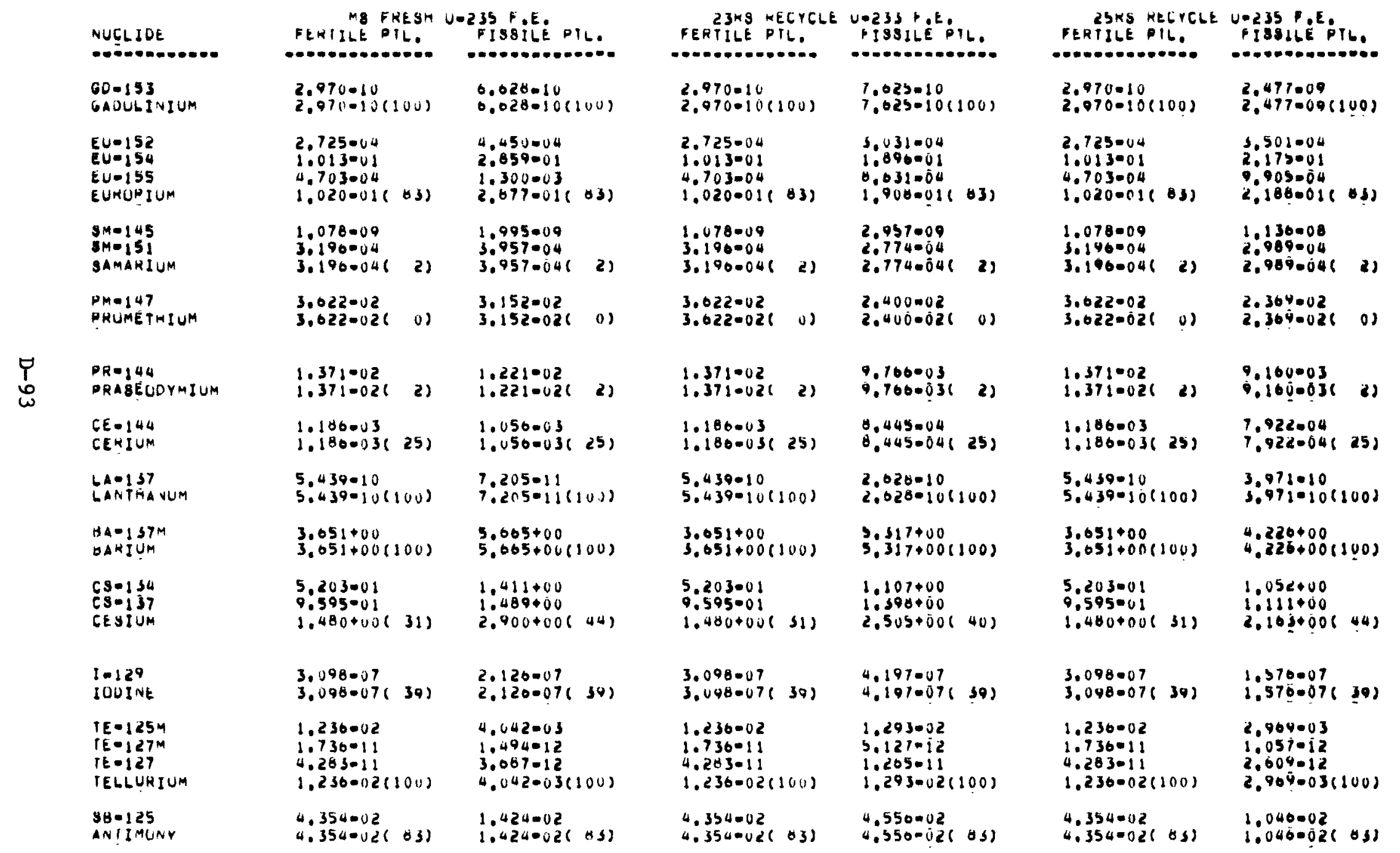


THIS SEgME⿰亻⿱

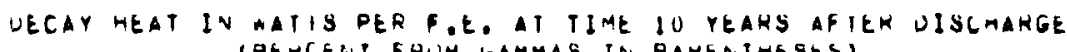

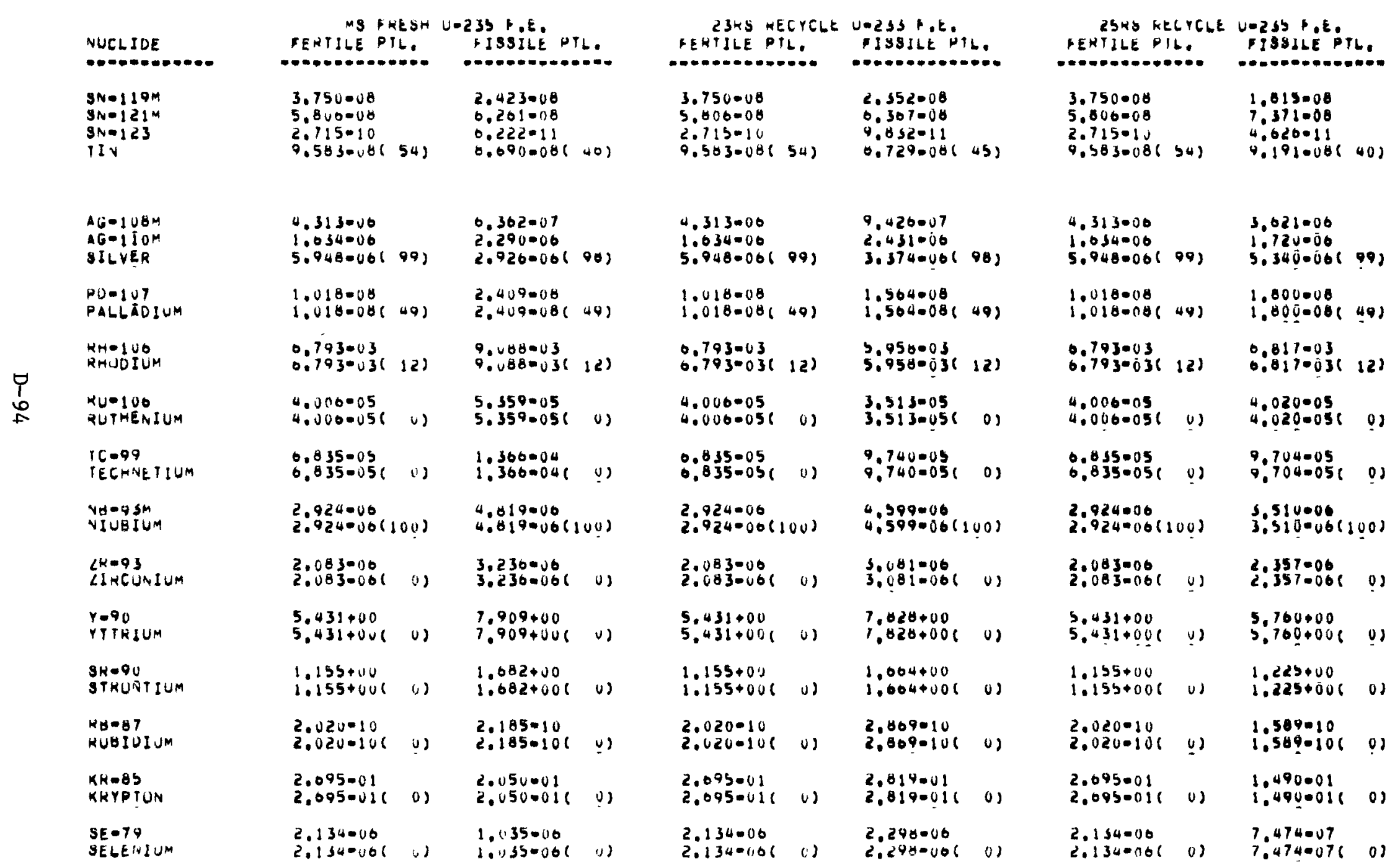




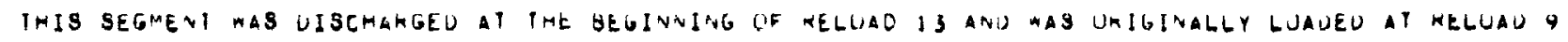

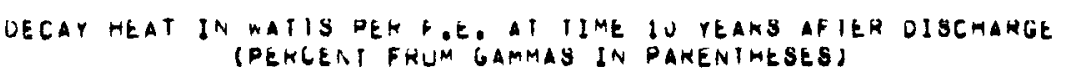

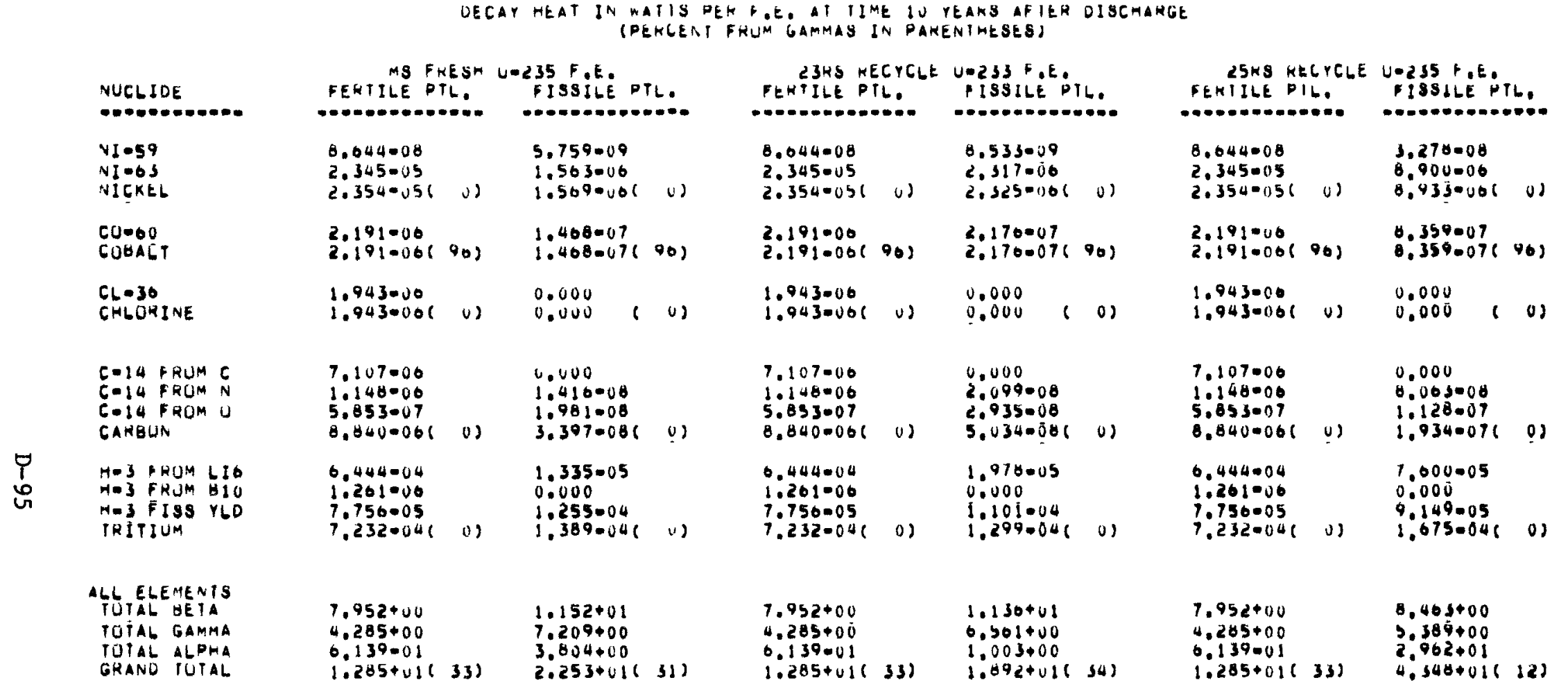




\section{GAMMA DOSE RATES}


TABLE D-5

GAMMA DOSE RATES FOR FUEL ELEMENTS DISCHARGED AT BEGINNING OF RELOAD 1 AND ORIGINALLY LOADED AT RELOAD 0

and

TABLE D-6

GAMMA DOSE RATES FOR FUEL ELEMENTS DISCHARGED AT BEGINNING OF RELOAD 5 AND ORIGINALLY LOADED AT RELOAD 1

NOTE: Tables $D-5$ and $D-6$ consist of 58 pages of computer print-out, with the two tables appearing side by side (pages D-99 through D-156. 
•

-

8 
THIS SEGMENT WAS OISGHARGEU AT

THE BEGINNING UF RELOAD I ANU MAS

QRIGINALLY LUADED AT RELUAO O
THIS SEGMENT MAS UISCHARGED AT

THE BEGINNING UF RELUAD 5 AND WAE

UNIGIMALLY LOADEO AT MELUAD I
NUCLIDE AND GAMMA ENERGY IN MEV

-000000000.

U. 235

$0 \div \frac{1}{3}$

TU:TAL

URANIUM

0.1

Tós

$P A=233$

0.1

$Y$
0

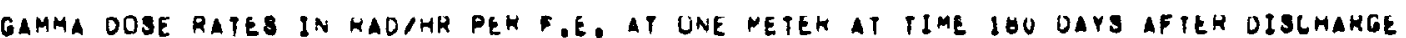

18 FKE

FERTILE PIL. FISILE PTL

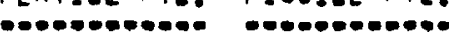

$\begin{array}{ll}3.211-08 & 8.456-06 \\ 8.549-08 & 2.251-05 \\ 1.176007 & 3.097-05 \\ & \\ 3.211008 & 0.456006 \\ 8.540-08 & 2.251=05 \\ 1.170007 & 3.09700\end{array}$

$\begin{array}{ll}1.226+02 & 9.386009 \\ 0.131 .02 & 0.227=08\end{array}$

$\begin{array}{ll}0.131002 & 0.227008 \\ 0.356+02 & 7.165000\end{array}$

$1.839 .02 \quad 3.547 .07$

$1.072-02 \quad 3.01207$
$0.210-03$

$9.210=03 \quad 1.777=07$

1.721 .01
1.282002

$3.041 .02 \quad 5.060007$

$2.017001 \quad 5.040000$

i.̈TAL

PROTACTINIUM

0.1

0.3

0.6

0.8

$1: 0$

IJTAL

$\begin{array}{ll}1.220+02 & 9.386-00 \\ 0.131+02 & 4.109007 \\ 1.872-02 & 3.612007 \\ 9.210003 & 1.777007 \\ 1.721-01 & 3.320006 \\ 1.282002 & 2.473007 \\ 3.041-02 & 5.860007 \\ 9.359+02 & 5.119006\end{array}$

MS FHESH U-235 D.E.

FEHTILE PTL. FISSILE PTL

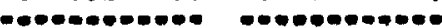

$\begin{array}{ll}4.048007 & 1.932006 \\ 1.078006 & 5.143006 \\ 1.483006 & 7.075006 \\ & \\ 4.048007 & 1.932-00 \\ 1.078006 & 5.143006 \\ 1.483006 & 7.075=00\end{array}$

$1.007+02 \quad 1.229007$

$\begin{array}{ll}0.699+02 & 8.131=07 \\ 7.680+02 & 9.379007\end{array}$

$1.201 .02 \quad 4.735007$

$1.305002 \quad 4.022007$

$6.419003 \quad 2.372007$

$1.200001 \quad 4.433006$

$8.934003 \quad 3.301007$

$\begin{array}{ll}2.120002 & \$ .832-07 \\ 1.824-01 & 6.730-06\end{array}$

$1.007+02 \quad 1.229007$

$0.679+42 \quad 1.289000$

$1.305002 \quad 4.822007$

$0.419003 \quad 2.372 .07$

1.200001

1.93003
$2.120 .02 \quad 7.301007$

2.120002
7.0802 


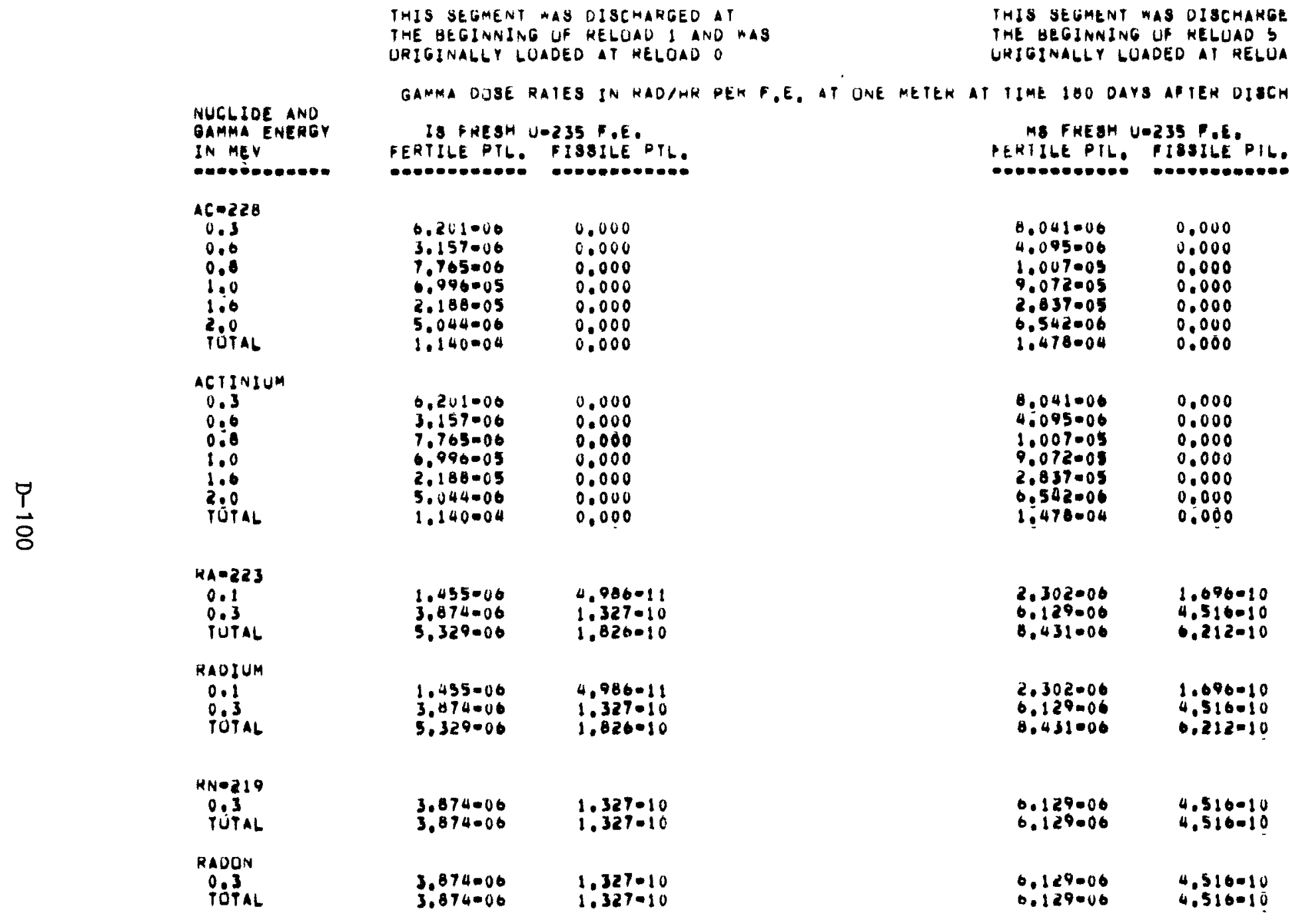


TMIS SEGMENT HAS UISCMARGEU AT

THE BEGINNING UF RELOAD I AND NAS ONIGINALLY LUADED AT KELUAD O

GAMMA DUSE RATES IN RADIHK DEH .E. AT DNE METER AT TIME 180 DAYS AFTER DISCHARGE NUCLIDE AND

$\stackrel{\theta}{0}$
THIS SEGMENT WAS UISCHAKGEO AT

IHE OEGITNING OF RELUAD 5 AND WAS

URIGINALLY LOADEO AT RELOAD I

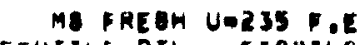 FEKTILE PTL. FISSILE PIL.

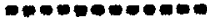

$2.082 .05 \quad 5.454 .10$ IN MEV

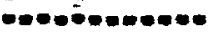

010511

Pótal

$8 !=212$

0.8

1.0

TOTA6

$8[-213$

0.3

TUTAL

IS FRESH U-235 EDE.
FERTILE PIL. FISSILE PTL.

$\begin{array}{ll}3.874006 & 1.327-10 \\ 3.874006 & 1.327-10\end{array}$

4.247 .03

6.784 .04

1.870 .03

$6.795=03$

4.580007

7.316008

$2.017=07$

$6.645=06$

4.557 .07

$0.086-12$

$9.496-12$

1.052005

4.247003

$6,780.04$

$6.806=03$

$1.416 \cdot 10$

7.31000

3110000

2.017007
$1.330=07$

PB-E11

0.3

TÓTAL

$P B=212$

0.3
TUTAL

LEAD

0.8

TUTAL
$2.324-06 \quad 7.965-11$

$2,329.06 \quad 7.979 .11$

.053006

8.478 .03

$0,478.03$

9.144007
9.144007

0.481 .03

2.329000

9.144007

$7.970-11$ $\begin{array}{ll}0.129006 & 4.516010 \\ 0.129006 & 4.5100110\end{array}$

$3.591 .02 \quad 5.980=06$

$5.736-03 \quad 9.562007$

$1.581 .02 \quad 2.636006$

$\begin{array}{ll}3.420005 & 3.740-11 \\ 2.820 .10\end{array}$

2.694005

3.591002

$5.737=0$

5.748 .02

$9.969-10$

$5,986=06$

0.562 .07

$2.636=00$

9.570006

$3.077 .06 \quad 2.709-10$

$3.684 .06 \quad 2.714010$

$7.301 .06 \quad 5.424-10$

$7.169 .02 \quad 1.195-05$

$7.109002 \quad 1.195005$

$7.100002 \quad 1.195005$

$\begin{array}{ll}3.684006 & 2.714-10 \\ 7.169002 & 1.195-05\end{array}$ 


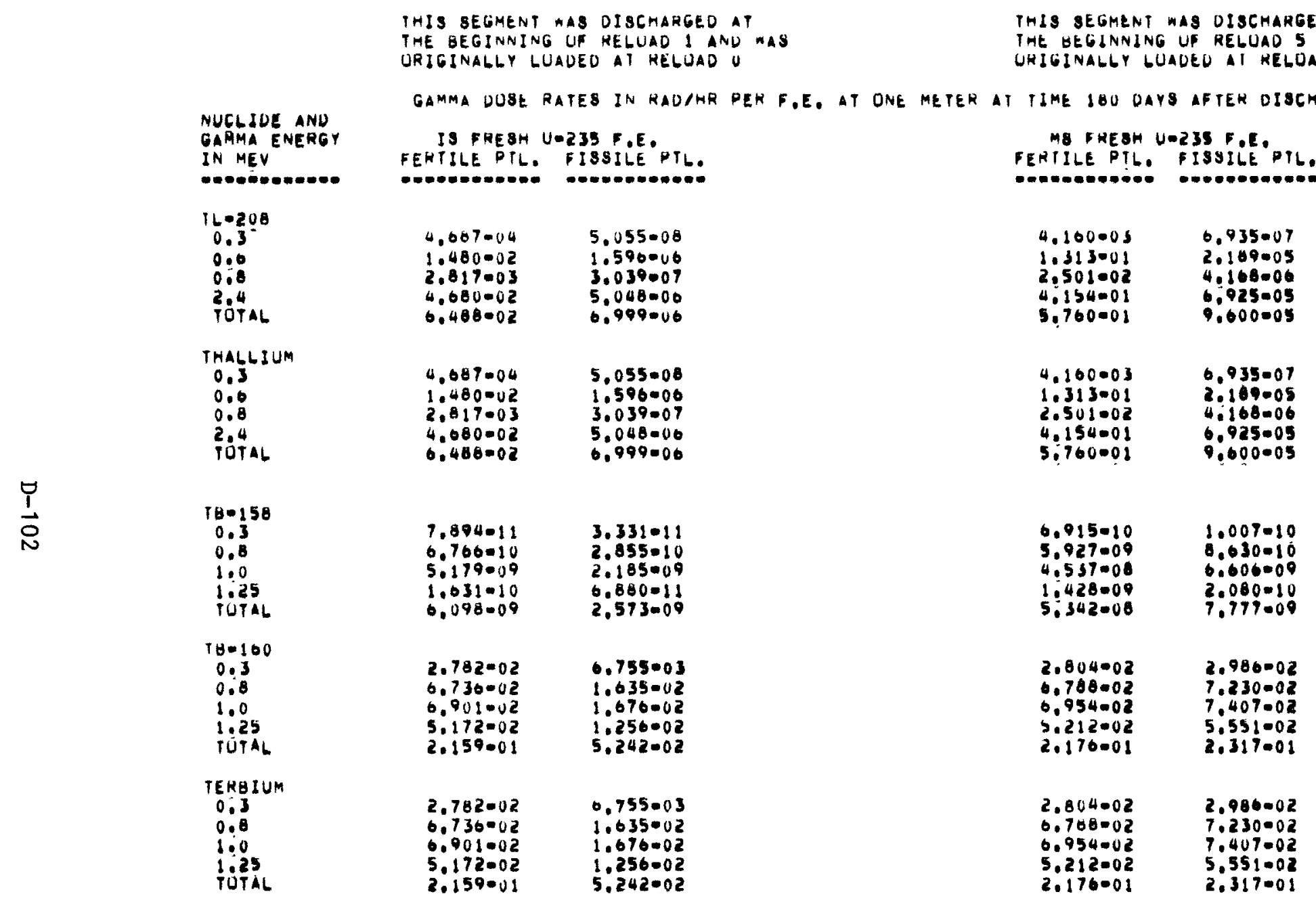


IMIS SEGMENT WAS DIBCHARGEO A

THE BEGINAING UR RELUAD I AND WAS

THIS SEGMENT WAS DISCHAKGEO AT URIGINALLY LOADEU AT RELUAD O

IAE BEGINNING UF RELUAD S AND MAS

URIGINALLY LUADEO AT RELUAD

NUCLJUE AND OAMMA ENERG IN MES $Y$

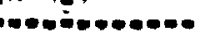

$E 0.152$
0.1
0.3
0.6
0.8
1.0
1.25
1.6
10746

$\stackrel{\bullet}{1}$

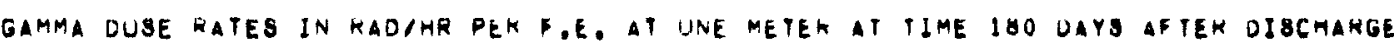

IS FRESH UOS35 FIE
PERTILEPTL. FISSILE PIL.

1.891 .03

5.012003

5.195 .03

1.494002

1.176002

$4.281=04$

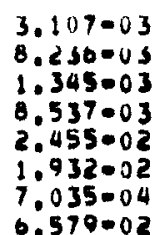

2.463002

$1.070=02$

2.814002

1.531 .01

$1.451=0$ !

2.231 .01

$1.723=02$

$6.020-01$

1.084003

3.200003

$2.862=03$

$7.521-03$

. $314=03$

2.380002

$2.052=02$

1.571 .02

3.004 .02

1.615001

1.629001

$2.423-01$

1.765 .02

$0.314=03$

0.146004

0.059001

\section{$2.103=01$ \\ 2.403009 \\ $1.307+00$ \\ $1.239+00$ \\ $1.005+00$ \\ $1.471-01$ \\ 5. $140+00$}

$0.354 \cdot 03$

1.875002

1.677 .02

4.408 .02

4.073002

1.795001

2.134 .01

9.95600

2.470 .01

$1.335+00$

$1.281+00$

$1.968+00$

1.476 .0

$4.873=02$

4.774003

$5.346+00$
MS FRESH U-235 F.E FERIILE PIL. FISSILE PIL.

\begin{tabular}{|c|c|}
\hline $\begin{array}{l}1.036003 \\
4.338003 \\
7.080004 \\
4.497-03 \\
1.293002 \\
1.017002 \\
3.705004 \\
3.465=02\end{array}$ & $\begin{array}{l}2.155=03 \\
5.714001 \\
9.334004 \\
5,923003 \\
1.703002 \\
1.340002 \\
4.800004 \\
4.564=02\end{array}$ \\
\hline $\begin{array}{l}4.094001 \\
2.039001 \\
5.363001 \\
2.910+00 \\
2.767000 \\
4.252+00 \\
3.284001 \\
1.148+01\end{array}$ & $\begin{array}{l}1.080000 \\
4.692001 \\
1.234+00 \\
6.715+00 \\
0.360+00 \\
9.783+00 \\
7.554001 \\
2.640401\end{array}$ \\
\hline $\begin{array}{l}1.191002 \\
3.510002 \\
3.145002 \\
8.265002 \\
9.130002 \\
0.952003 \\
2.615001\end{array}$ & $\begin{array}{l}2.700002 \\
7.969002 \\
7.129=02 \\
1.073001 \\
2.0711001 \\
2.029002 \\
5.927=01\end{array}$ \\
\hline
\end{tabular}

$\begin{array}{ll}4.710001 & 1.082+00 \\ 2.083001 & 4.749001 \\ 5.489 .01 & 1.202+00 \\ 2.958+00 & 0.800+00 \\ 2.811+00 & 0.454+00 \\ 4.345+00 & 9.984+00 \\ 3.287-01 & 7.55901 \\ 9.130-02 & 2.071001 \\ 0.952003 & 2.029002 \\ 1.177+01 & 2.704+01\end{array}$




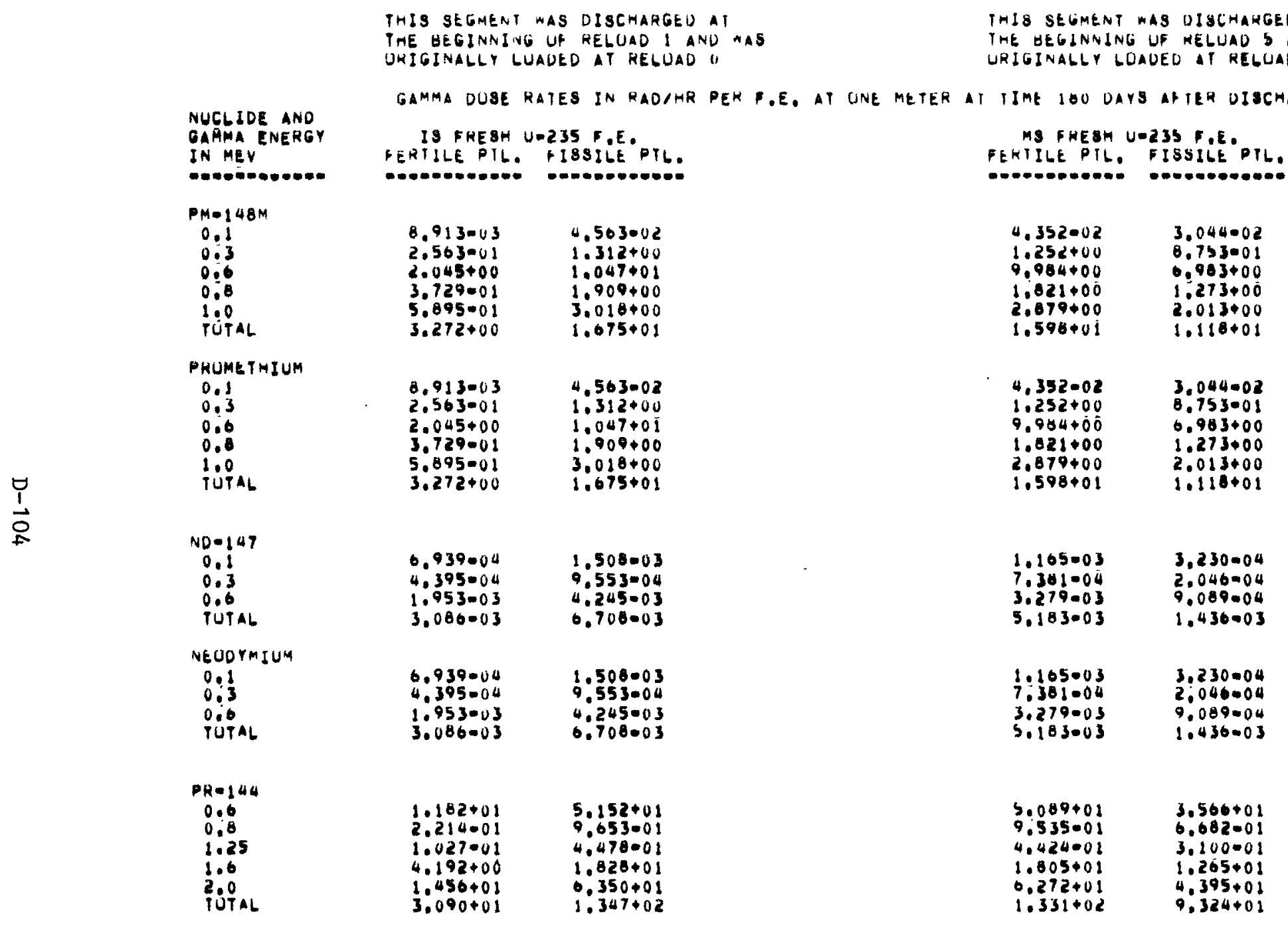




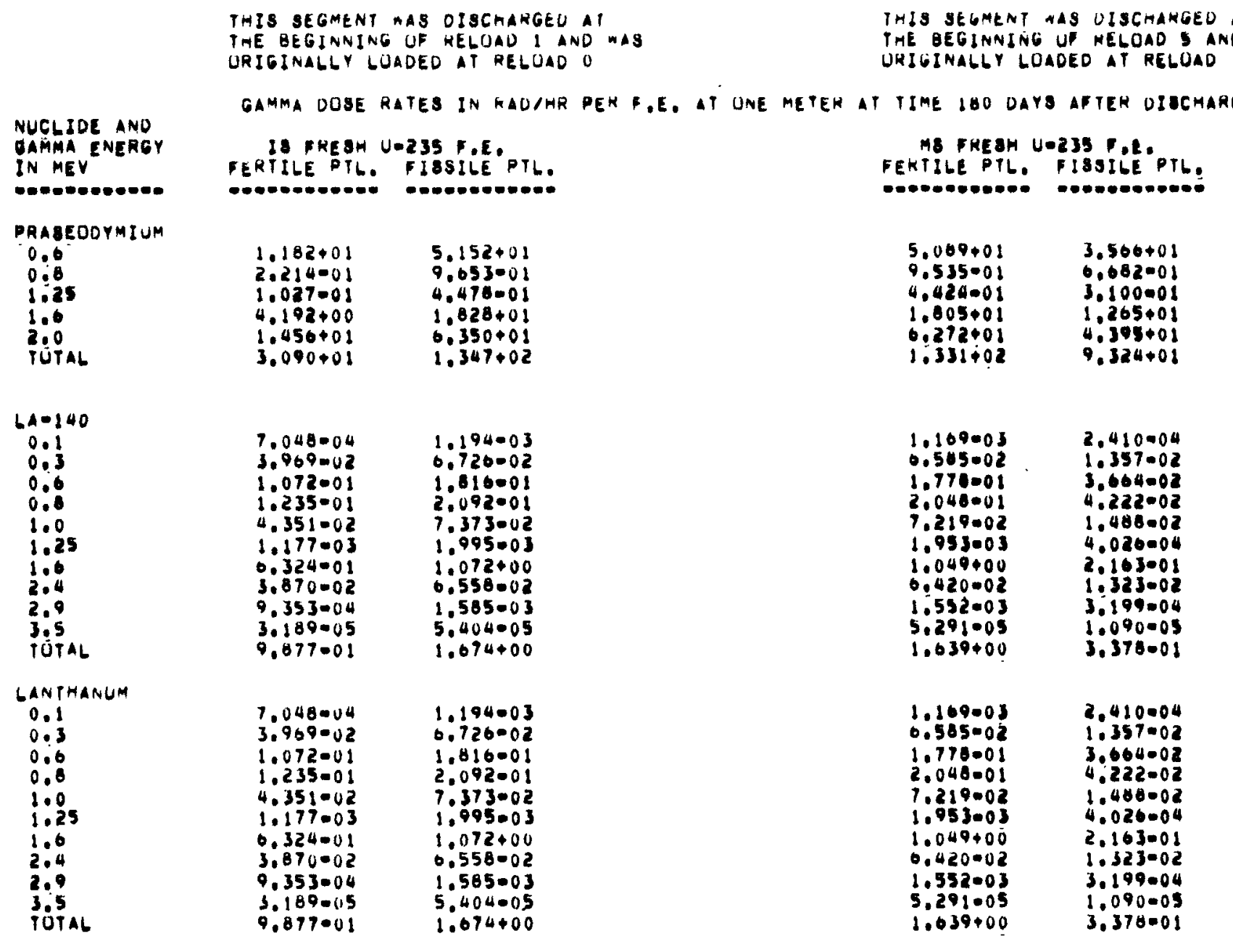




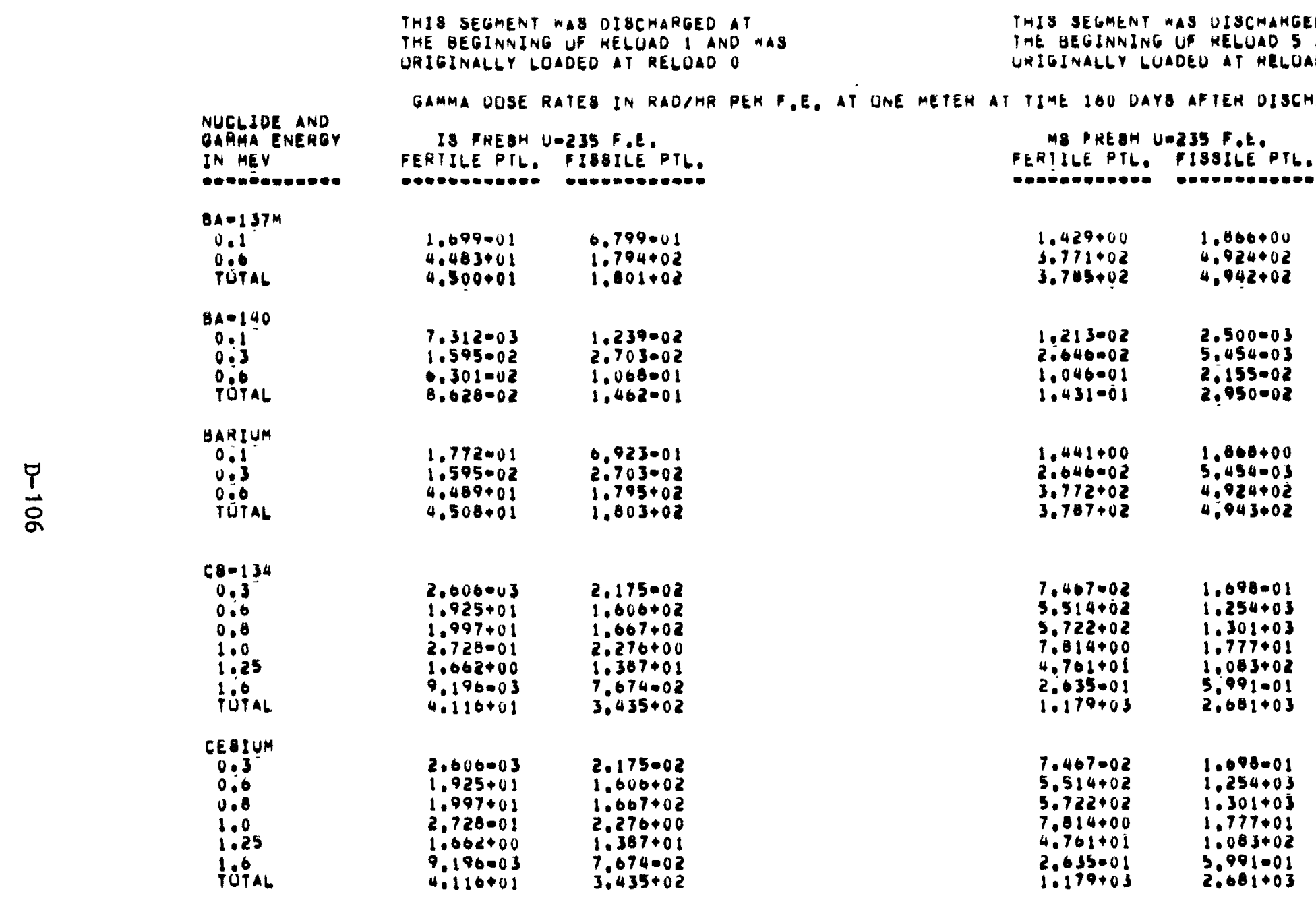




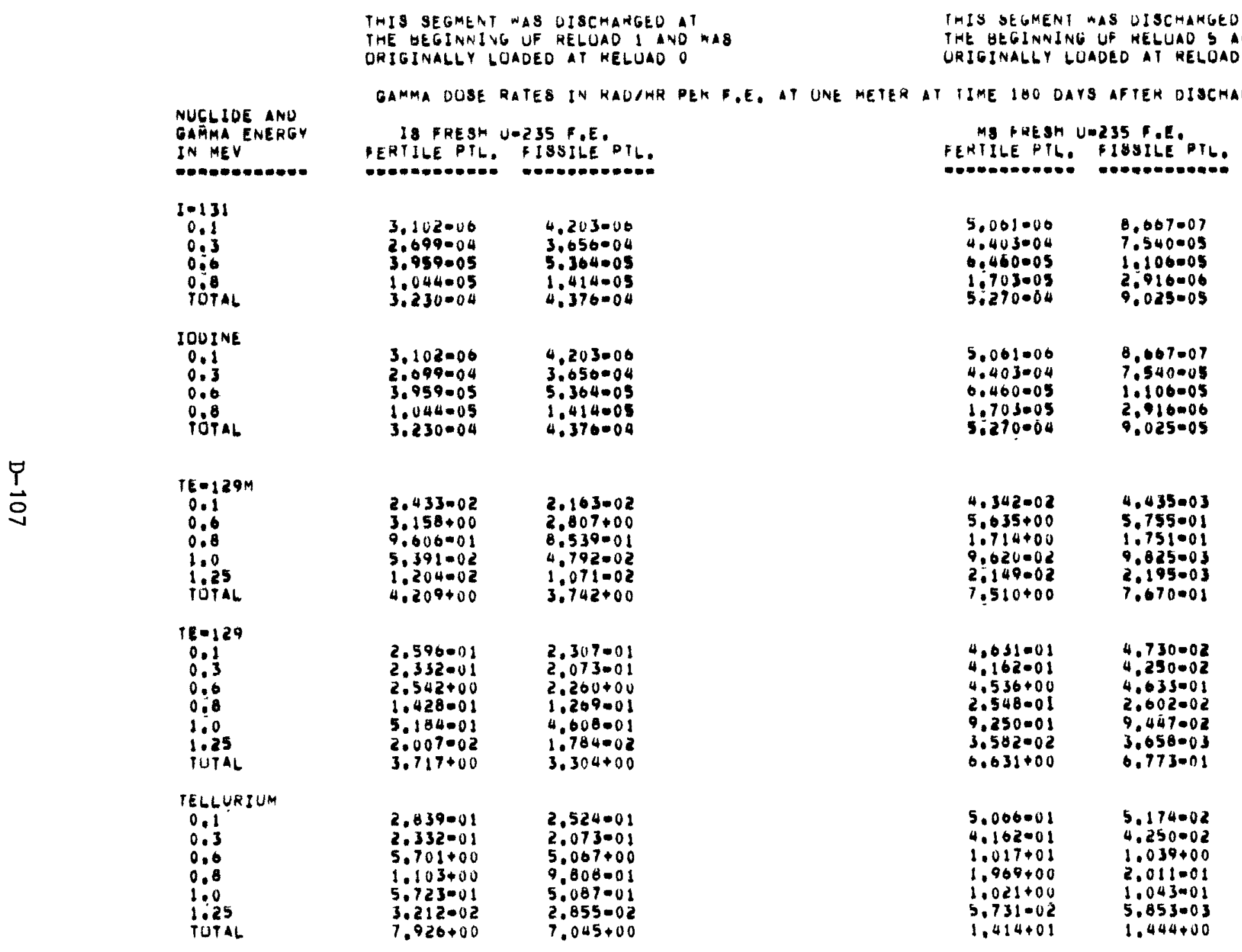




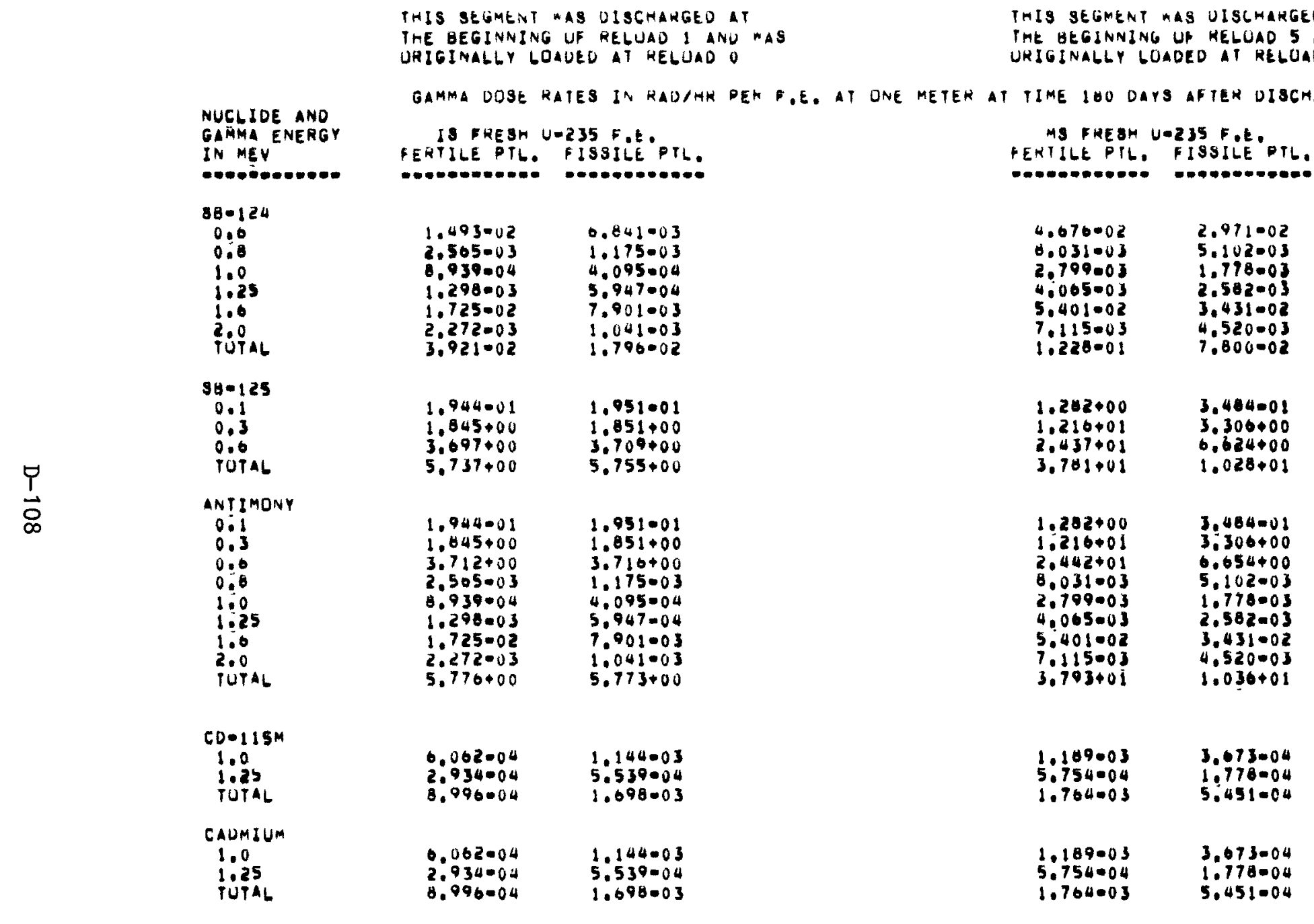




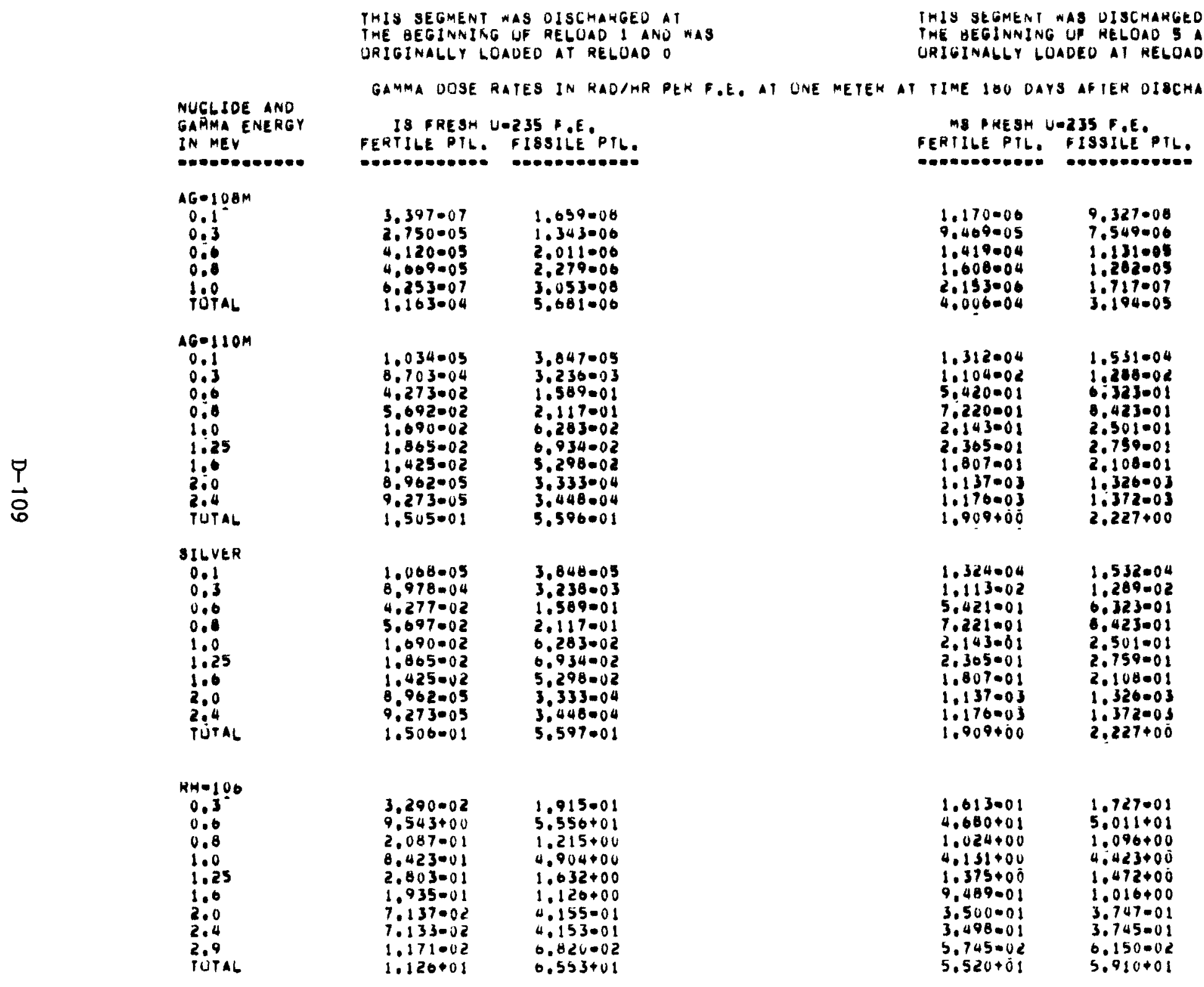




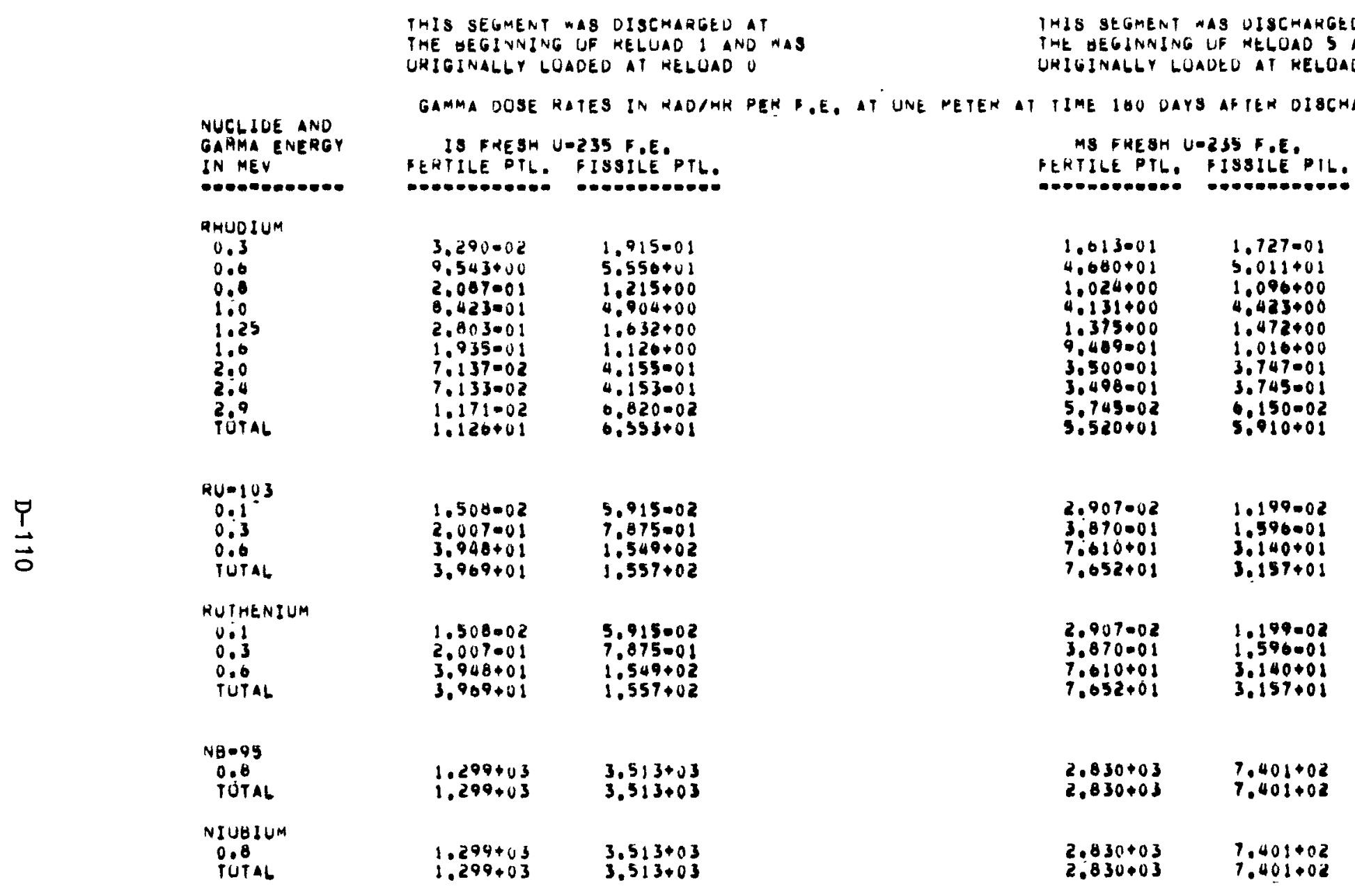


THIS SEGMENT MAS DISCMARGED AT

THE BEGINNING OP RELOAD I AND MAS

URIGINALLY LOADED AT RELOADO
THIS SEGMENT WAS DISCHAKGED AT THE BEgINNJNG of Keluad 5 and wa URIGINALLY GUADED AT RELOAD I
NUCLJDE ANO

IN MEY ENERG

IN MEV

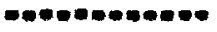

$28=95$

0.3

Tótal

ZIRCUNIUM

0.3

POTAL

Ro-80

I: 0

$\stackrel{\bullet}{\Xi}$

TOOTAL

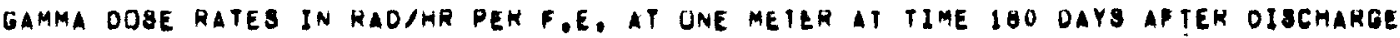

I8 FRESH U

FERTILE PIL. FISSILE PTL.

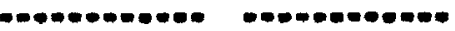

$\begin{array}{ll}1.197+00 & 3.149+00 \\ 0.295+02 & 1.656+03\end{array}$

$\begin{array}{ll}1.295+02 & 1.656+03 \\ 0.307+02 & 1.659+03\end{array}$

$1.197+00 \quad 3.149+00$

$0.295+02 \quad 1.656+03$

$1.659+03$

$0.696004 \quad 2.350004$

$0.696004 \quad 2.350004$

$\begin{array}{ll}8.696004 & 2.350004 \\ 8.696004 & 2.350004\end{array}$

$\cos 56$

0.8

TO:

$60-60$

T.25

TOBAL

0.6

1.25

TUTAL
MS FHESM UAZ35 O.E.

FERTILE PTL BSILE PTL.

$\begin{array}{ll}2.554000 & 0.595001 \\ 1.343003 & 3.407+02\end{array}$

$1.346003 \quad 3.474002$

$2,554+00 \quad 0,595=02$

$1.343+03 \quad 3,467+02$

$1036003 \quad 3.474+02$

$1.971=03 \quad 0.172000$

$1,971=03 \quad 6.172004$

$\begin{array}{ll}1.971-03 & 0.172=04 \\ 1.971-03 & 6.172004\end{array}$

\begin{tabular}{|c|c|}
\hline $\begin{array}{l}9.451=05 \\
4.899004 \\
5.565=06 \\
5.900004\end{array}$ & $\begin{array}{l}3.403=00 \\
1.764005 \\
2.003=07 \\
2.124005\end{array}$ \\
\hline $\begin{array}{l}6.793=04 \\
6.793=04\end{array}$ & $\begin{array}{l}2.460-05 \\
2.460=05\end{array}$ \\
\hline $\begin{array}{l}9.451005 \\
4.899004 \\
0.193004 \\
5.565000 \\
1.269003\end{array}$ & $\begin{array}{l}3.403=06 \\
1.764-05 \\
2.400=05 \\
2.003=07 \\
4.584=05\end{array}$ \\
\hline
\end{tabular}


THIS SEGMENI WAS DISCHAKGED AT

THE BEGINAING UF RELUAD I AND MAS URIGINALLY LOADED AT RELOAD U

GAMMA DOSE RATES IN FADIHR PEK F.E. AT UNE METER AT

GAMMA ENERG

IN MEV

FERTILE PTL. FI8SILE PTL.

ALL ELEMENTS

0.12

0.8

1.0

2.0

2.0
2.4
2.9

2.

TOTAL

i
IS SRESH UNZ35 F.E.

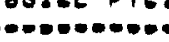

IMIS SEGMENT WAS UISCMARGED AT THE BEGINNING UF RELUAD 5 ANO WAB

UHE GINALLY LUADED I RELUAD I

TIME 180 DAYS AFTER OISCHARGE

MS FRESH U 235 FE. FERTILE PIL. FISSILE PTL.

$\begin{array}{ll}1.233+02 & 1.461+00 \\ 8.170+02 & 7.726+00 \\ 1.367+02 & 0.220+02 \\ 1.951+03 & 5.342+03 \\ 2.745+00 & 1.214+01 \\ 2.406+00 & 1.803+01 \\ 5.109+00 & 2.076+01 \\ 1.465+01 & 6.396+01 \\ 1.577-01 & 4.860001 \\ 1.265-02 & 0.078002 \\ 3.189-05 & 5.404005 \\ 3.053+03 & 6.089+03\end{array}$

$1.045+02$

$0.053+02$

$1.140+03$

$4.755+03$

$1.914+01$

$5.414+01$

$2.091+01$

$6.317+0$

6.390001

5,900002

5,291009

$6,852+03$

$3.393+00$

$5.932+00$

$1.800+03$

$2.399+03$

$3.110+01$

$1.204+02$

$1.548+01$

$4.454+01$

4.095001

0.102 .02

1.090 .05

$4.500+03$ 
THIS SEGMENT WAS DISCHAHGED AT THE BEGINNING UF RELUAD I AND WAS URIGINALLY LOADEO AT KELUADO
IMIS SEGMENT MAS DISCMAKGEO AT THE HEGINNING UF MELUAD 5 ANU WAO URIGINALLY LOADEO AT RELUADI
NUCLIDE AND GAMMA ENERGY IN MEY

\section{-}

$v=235$

0.1
0.3
Tótal

URANIUM

0.1

TOTIAL

$04=233$

0.1

TOTAL

0.234

0.3

0.6

0.0

1.25

TUTAL

PRUTACTINIUM

0.1

$0: 3$

0.6

1.25

1.6

I reah after dischahge

IS FKESH U.235 F.E.

FERTILE PIL. FISSILE PTL.

\section{$3.211=08$ \\ $8,549=08$ \\ 8.450000 \\ 2.251 .05 \\ 3.211 .08 \\ 8.509000 \\ $8.456=06$ \\ 2.251 .05}

$1.054+00$

0.048

$6.074-11$

$5.356-10$

$6.164-10$

$8.923=05$

$9.087-05$

4.470005
$8.353=04$

$6.221=05$

1.47600

1.270003

$1.054+00$

$6.894+00$

$9.087-05$

4.470 .05

$6.353=04$

0.221 .05

1.476004

3.546007

$3.612=07$

1.776007

3.320006

2.472007

$5.866-07$

$5.047=06$

8.074 .11

$3.552 \cdot 07$

3.612007

1.776007

$3.320=06$

2.472 .07

$5.866=07$

5.048006
MS EKESH U-235 F.E. PERTILE PTL, FISSILE PTL.

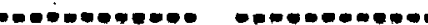

$4.048007 \quad 1.932=06$

$1.043000 \quad 3.143006$

$4.048 .07 \quad 1.932006$

$\begin{array}{ll}9.0078006 & 5.143000 \\ 1.083006 & 7.075006\end{array}$

$\begin{array}{ll}0.600001 & 1.057009 \\ 5.745000 & 7.011009\end{array}$

$0.611+00 \quad 8.068=09$

$0.220005 \quad 4.73507$

$0.334=05$

5.115005

5.822004

4.336 .05

1.029004

$4.822-07$

$2.312=07$

4.432 .06

$3.301=07$

$7.832 \cdot 07$

$6.738=06$

8.600001

$5.745+0$

$0.334=0$

3,115005

5,822004

4.330 .05

1.029004

$6.612+00$

1.057 .09

$4.805=07$

$4.022=07$

2,372007

4.432006

3.301 .07

7.832 .07

$6.747-06$ 


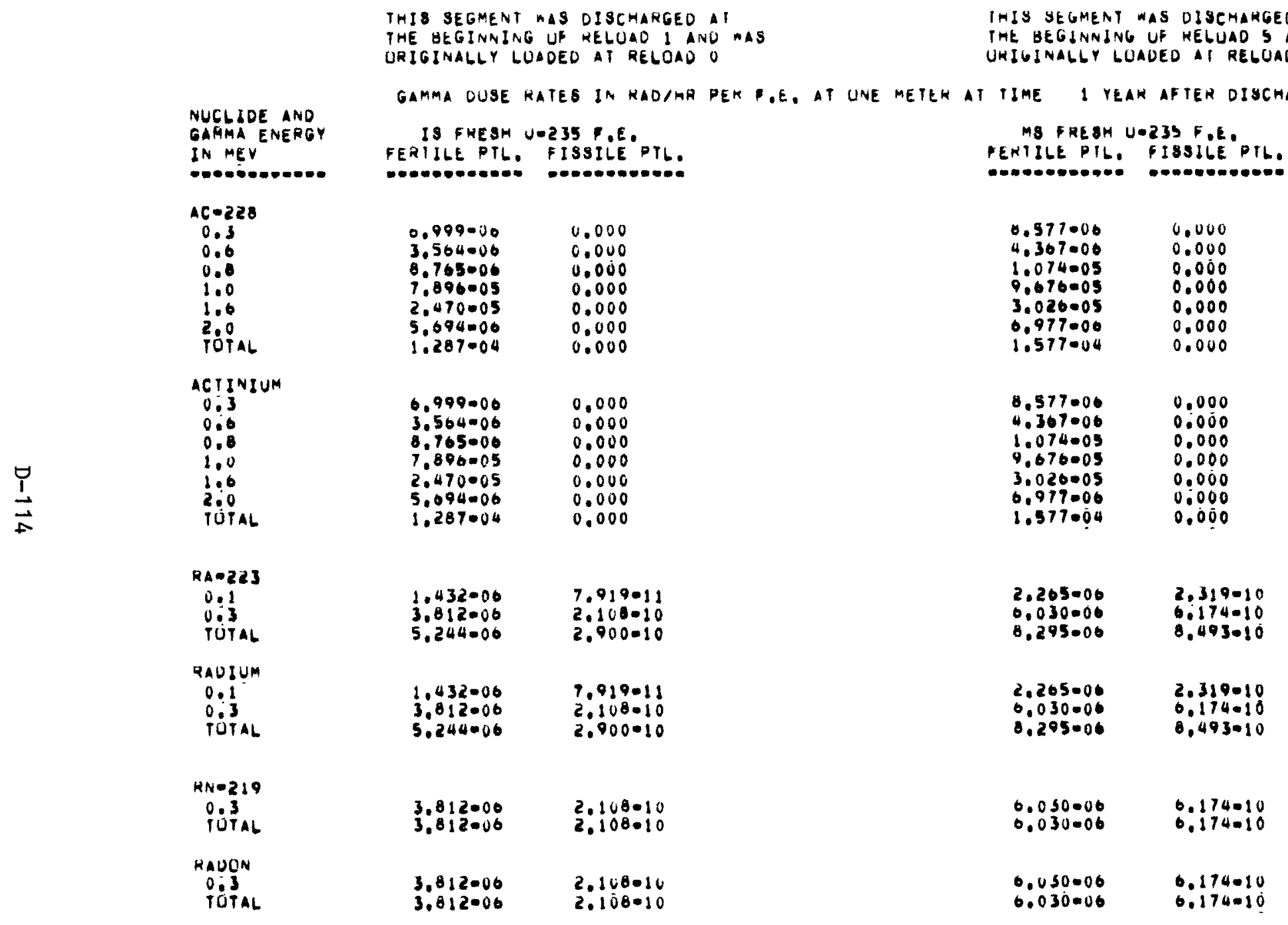


IHIS SEGMENT AAS DISCHAKGED AT

IHE DEGINNING UF RELOAO I AND WAS OAIGINALLY LUADED TI RELUAD

GAMMA DUSE RAIES IN RADIHR PEK F.E. AT UNE METER A

NUCLIOE AND GAAMA ENERGY IN MEV

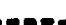

IS FREBH U-235 F.E. FERTILE PTL. FISSILE PTL.

\section{$B+211$}

TUTAL

$1=212$

1.0

TUTAL

$B\{-213$

0.3

1.:0

BgsMUTH

0.3
0.8
1.0

1.0

tótAL

\section{$P B=211$}

0.3
0.8

TOTA

$P B-212$
0.3

0.3
TUTAL

LEAD
0.3
0.0

TUTAL
$3.812=00$

$3.812=06$

$2.108 \cdot 1$

$2.108-10$

0.434003

$1.028=03$

2.633003
1.029002

0.916007

$1.105=07$

1.107 .06

1.124005
7.700007

$1.201 .05 \quad 9.543-12$

$1.505=05$

$0.434=03$

1.028 .03

$2.833=03$

\section{$2.198-10$}

1.105007

1.107006

2.287 .06
2.291 .06

$2.291=06$
$4.579=06$

$1.265 \cdot 10$

$1.267-10$

$2.532 \cdot 10$

$1.284=02$
$1.284=02$

$1.381=00$
$1.381=00$

1.285002

2.291006
1.285002

$1.381=06$

$1.267=10$
1.381006
THIS SEGMENT WAS UISCHAKGED AT

THE BEGINNING UF KELUAD 5 ANO WAB

URIGINALLY LUADED AT RELOAD

Turab

\section{MS FRESM U 235 F.E. FERTILE PIL. FIBSILE PIL.

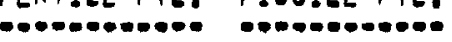

\begin{tabular}{|c|c|}
\hline $\begin{array}{l}0.0330000 \\
0.030006\end{array}$ & $\begin{array}{l}0.174010 \\
0.174-10\end{array}$ \\
\hline $\begin{array}{l}4.361 .02 \\
0.960003 \\
1.920002 \\
0.978002\end{array}$ & $\begin{array}{l}7.218006 \\
1.153006 \\
3.178006 \\
1.155005\end{array}$ \\
\hline $\begin{array}{l}2.798005 \\
1.919006 \\
2.990=05\end{array}$ & $\begin{array}{l}5.454-10 \\
3.741-11 \\
5.828-10\end{array}$ \\
\hline $\begin{array}{l}3.401005 \\
4.361-02 \\
6.968-03 \\
1.920002 \\
0.981 .02\end{array}$ & $\begin{array}{l}1.163000 \\
7.218000 \\
1.155006 \\
3.178006 \\
1.155005\end{array}$ \\
\hline $\begin{array}{l}3.618006 \\
3.625006 \\
7.243006\end{array}$ & $\begin{array}{l}3.704-10 \\
3.711-10 \\
7.416-10\end{array}$ \\
\hline $\begin{array}{l}8.706002 \\
8,700-02\end{array}$ & $\begin{array}{l}1.441005 \\
1.441005\end{array}$ \\
\hline $\begin{array}{l}8.707-02 \\
3.625000 \\
8.707-1.2\end{array}$ & $\begin{array}{l}1.441=05 \\
3.711=10 \\
1.441005\end{array}$ \\
\hline
\end{tabular}




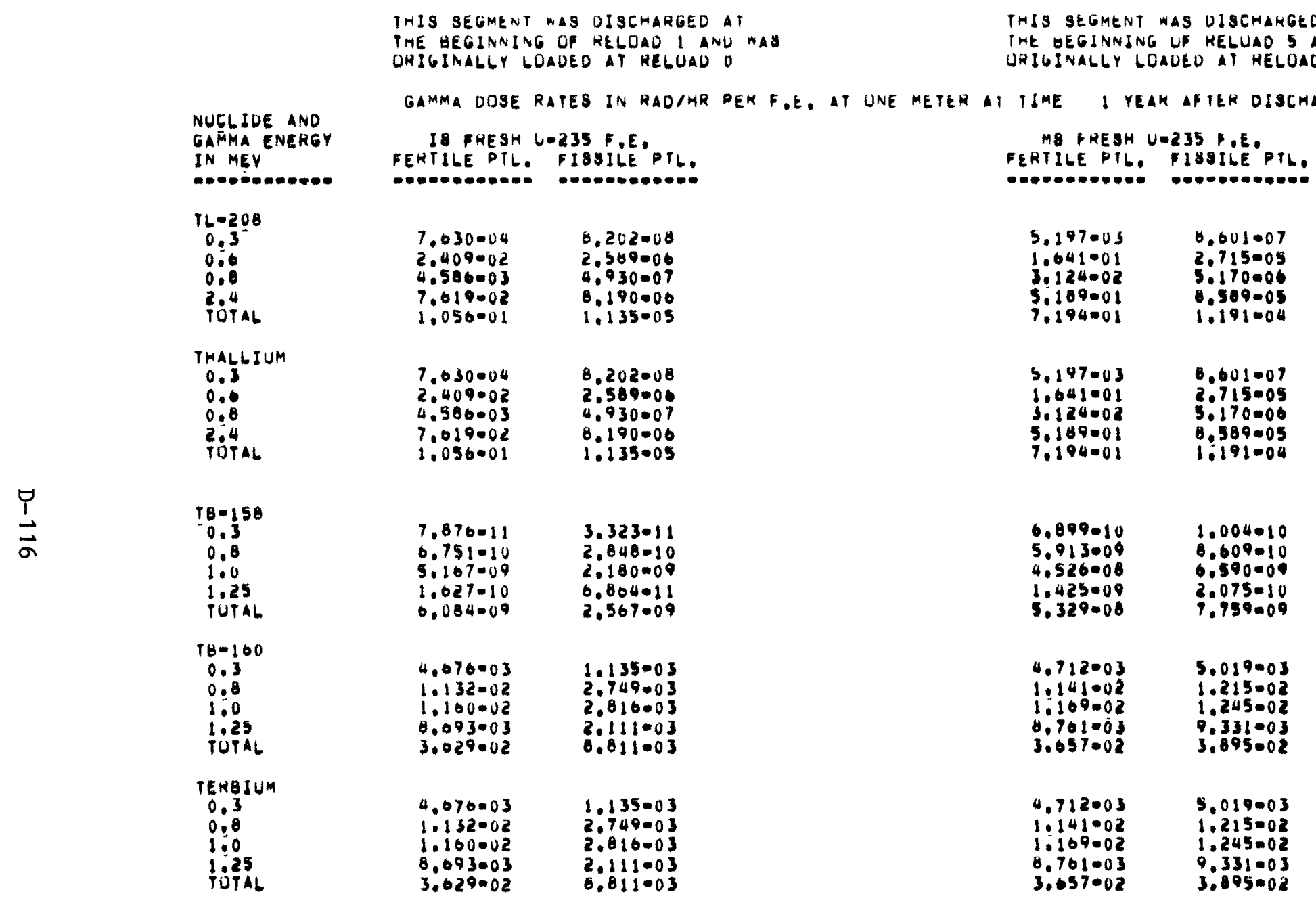


THIS SEGMENT WAS UISCHARGED AT TAE BEGINNING UF RELOAD I AND MAS

IMIS SEGMENT MAS DISCHARGED AT THE BEGINNING LF KELUAD 5 ANO WAS ORIGINALGY LUADED AI RELUAD

UHIGINALLY LOAOED AT RELOAD I

NUELJOE AND GAMMA ENERGY IN MEV 0000.000 .0

EU- 152

0.1

0.3

0.8

1.00

1.25

Tótal

LU 154
GAMMA DUSE FATES IN RADIHR PEK F.E. AT UNE METER AT FERTILE PTL, FISSILE PTL.

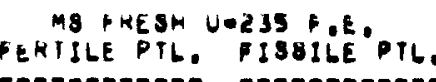

$\begin{array}{ll}1.839-03 & 3.022003 \\ 4.870003 & 8.012003 \\ 7.904004 & 1.309003 \\ 5.054003 & 0.305003 \\ 1.453-02 & 2.388002 \\ 1.143002 & 1.879002 \\ 4.164004 & 6.843004 \\ 3.895002 & 6.400 .02\end{array}$

$\begin{array}{ll}1.592003 & 2.097003 \\ 4.220003 & 5.558003 \\ 0.893004 & 9.079=04 \\ 4.374003 & 5.701=03 \\ 1.258002 & 1.056=02 \\ 9.896003 & 1.304002 \\ 3.004=04 & 4.947004 \\ 3.391002 & 4.440=02\end{array}$

2,409002

1.047 .02

$2.752-02$

$1.490-01$

$1.420=01$

2.182001

$1.085-02$
$5,889-11$

2.057001
8.936002

2.350001

$1.279+00$

$1.212+00$

$1.663+00$

1.439 .01

TUTAL

$E \cup=156$

0.6
0.8
1.0
1.25
2.0
2.4
1.01

2.594007

$7.657-07$

$0.849-07$

1.800000

1.990000

1.949007

$5.029+00$

1.521 .06

4.487 .06

4.014000

1.055005

1.166005

1.142006

EUHUFIUM

0.1
0.3
0.6
0.8
1.0
1.25
1.6
2.0
2.4
$101 A L$

$\begin{array}{ll}2.593-02 & 2.087-01 \\ 1.534002 & 9.738002 \\ 2.832002 & 2.363001 \\ 1.340-01 & 1.287+00 \\ 1.505-01 & 1.230001 \\ 2.297-01 & 1.082000 \\ 1.727-02 & 1.046001 \\ 1.490-00 & 1.100=05 \\ 1.949-07 & 1.142000 \\ 0.279001 & 5.093+00\end{array}$

\section{$4.592-01 \quad 1.057+00$}

$\begin{aligned} & 1.995 .01 \\ & 5.247001\end{aligned} \quad 1.500-01$

$2.855+00 \quad 0.509400$

$2.707+00 \quad 0.227+00$

$4.100+00 \quad 9.570+00$

$\begin{array}{ll}3.212001 & 7.390001 \\ 1.123+01 & 2.583001\end{array}$

2.851000

0.414006

7.526006

$1.978 \cdot 05$

2.180005

2.142006

0.257005

4.608001
$2.057-01$

2.057001
$5.250-01$

$2.800+00$

$2.714+0$

$4.170+00$

3.210001

$2.18600 \mathrm{~s}$

$2.142-06$

$1.120+01$

$$
\begin{aligned}
& 0.462=00 \\
& 1.907=05 \\
& 1.700=05 \\
& 4.483005 \\
& 4.956005 \\
& 4.856006 \\
& 1.418004 \\
& \\
& 1.059+00 \\
& 4.645=01 \\
& 1.208+00 \\
& 0.515+00 \\
& 0.244000 \\
& 0.583+00 \\
& 7.395=01 \\
& 4.956-05 \\
& 4.856006 \\
& 2.587+01
\end{aligned}
$$




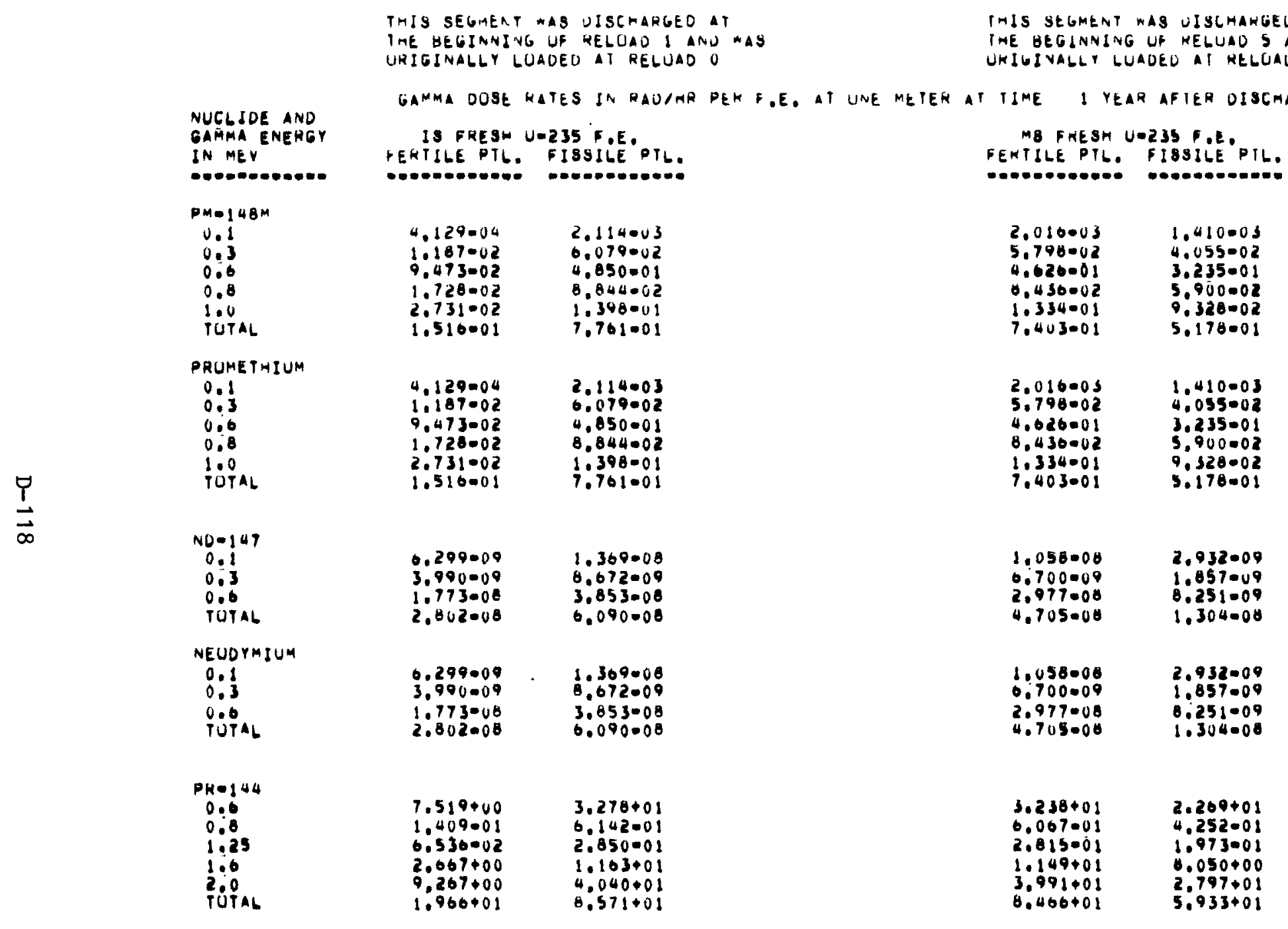




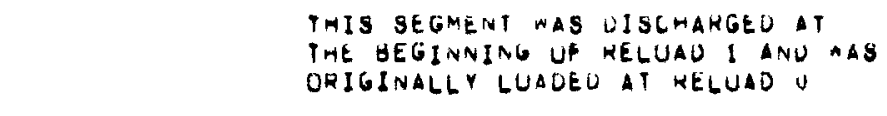

NUCLIOE AND

GAMMMI ENERGY

IN MEV

PRAsecormIUM

PRASE
$0.6^{\circ}$
0.8

0.8

1.06

TUT AL

$L A=140$

0.1
0.13
0.06
0.8
1.00
1.25
1.16
2.14
2.09
3.5
TUTAL

TUYAL

\section{LANTMANUM}

$0: 1$
$0: 3$
$0: 0$
$0: 8$
$1: 0$
$1: 25$
$1: 6$
$2: 0$
$2: 9$
$3: 5$
$T 0 Y A L$
ORIGINALLY LUADEU AT KELUAD U FENTILE PIL. FISSILE PIL.

$\begin{array}{ll}7.519+00 & 3.278+01 \\ 1.409001 & 0.142001 \\ 6.530002 & 2.850=01 \\ 2.067+00 & 1.163+01 \\ 0.267+00 & 4.040+01 \\ 1.966+01 & 8.571+01\end{array}$

3.102000 1.747 .00

4.716000

5.434006

$3.557=00$

$2.960=06$

7.992006
9.208 .06

$3.245=06$

8.781 .08

4.717005

2.886 .06

0.970008

2.78300

1.703006

$4.117=08$

$1.367=05$

4.347 .05

5.257008

5.257008
2.960006

$7.992=06$

$9.208-06$

$3.245=06$

8.781 .08

$4.717=05$

2.886006

6.976008

.378009

4.117008

1.403009

7.367 .05

$\begin{array}{ll}3.102008 & 5.257008 \\ 1.747006 & 2.960006 \\ 4.716006 & 7.992006 \\ 5.434000 & 9.208006 \\ 1.915000 & 3.245006 \\ 5.182008 & 8.781008 \\ 2.783005 & 4.717005 \\ 1.703006 & 2.886006 \\ 4.117008 & 6.976008 \\ 1.403009 & 2.378000 \\ 4.347005 & 7.367-05\end{array}$

TMIS SEGMENT WAS LISCMAKGEO IT

GAMMA UOSE HATES IN RADIHA PEK F,E, AI UNE METER

TMIS SEGMENI WAS UISCHAKGEU AT TE BEGINVING UF RELUAD 5 ANU MAS

I TIME I TEAN AFIER DISCMAKGE MS FKESH UA235 F,E PEHILE PIL. FISSILE PIL.

$\begin{array}{ll}3.296+01 & 2.269+01 \\ 0.007001 & 4.252001 \\ 2.815001 & 1.973001 \\ 1.149+01 & 8.050+00 \\ 3.991+01 & 2.797+01 \\ 8.466+01 & 5.933+01\end{array}$

\begin{tabular}{|c|c|}
\hline $\begin{array}{l}5.147000 \\
2.898000 \\
7.825006 \\
9.010006 \\
3.177006 \\
8.596008 \\
4.618005 \\
2.826000 \\
6.830008 \\
2.329000 \\
7.213005\end{array}$ & $\begin{array}{l}1.061000 \\
5.073=07 \\
1.013000 \\
1.058000 \\
6.549007 \\
1.712000 \\
9.518000 \\
5.824007 \\
1.408000 \\
4.799=10 \\
1.487005\end{array}$ \\
\hline $\begin{array}{l}5.147008 \\
2.898006 \\
7.825006 \\
9.016006 \\
3.177006 \\
8.598000 \\
4.618=05 \\
2.826006 \\
0.830008 \\
2.329=09 \\
7.213005\end{array}$ & $\begin{array}{l}1.001=00 \\
5.973=07 \\
1.613=06 \\
1.858-06 \\
6.549=07 \\
1.772000 \\
9.518=06 \\
5.824=07 \\
1.408=00 \\
4.799=110 \\
1.487=05\end{array}$ \\
\hline
\end{tabular}




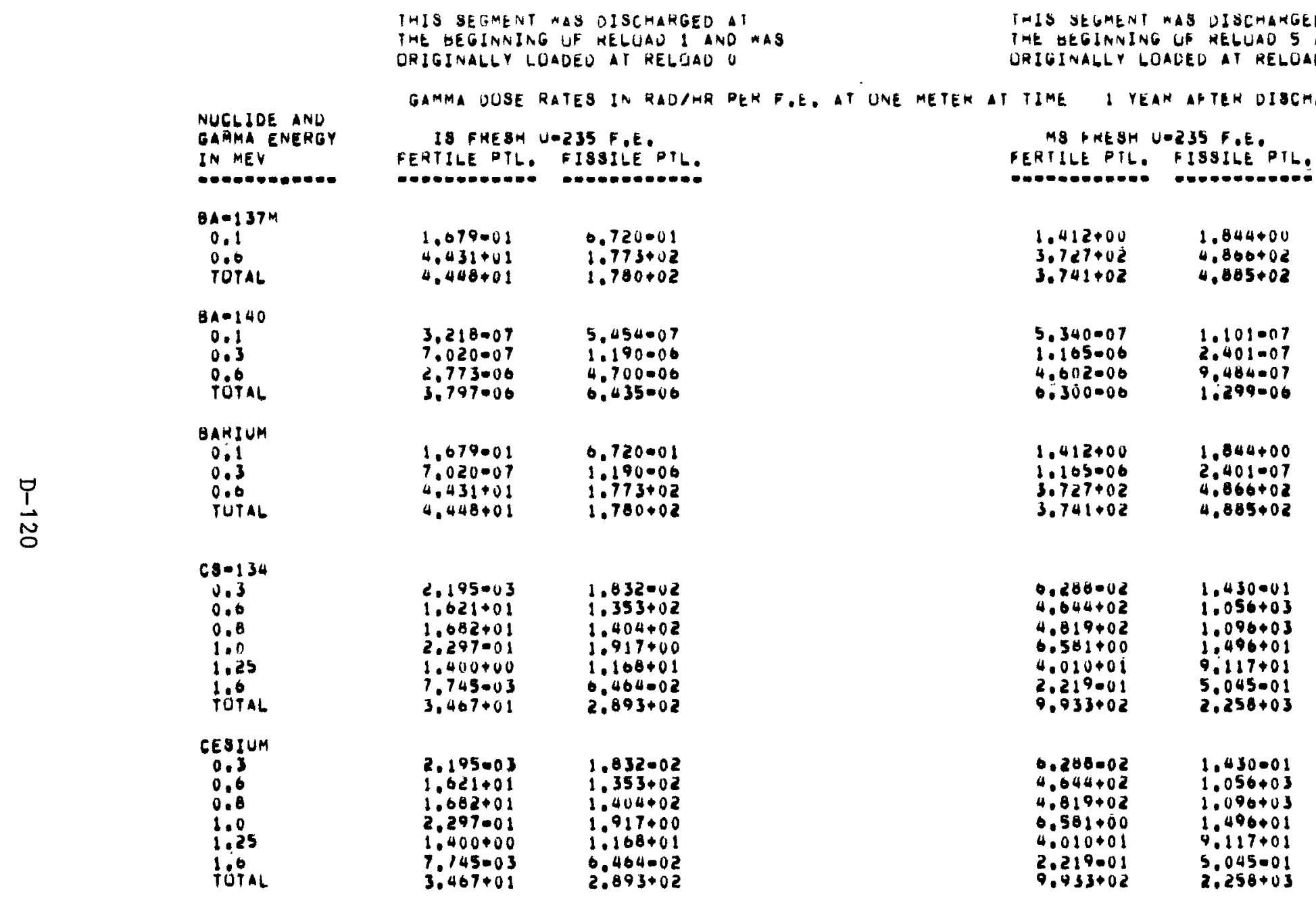


THIS BEGMENT NAS DISCMAHGEO AT THE DEGINNING UF KELUAD I AND MAS

IHIS GEGMENI NAS DISCNAKGEU AI THE HEGIMYING UF RELUAD 5 ANU WAS UHIGINALLY LUAUEU AI RELUAO U

URIGINALLY LUAUEU AI KELUAO I

NUGLIDE AND

gamma GuSE KaTES IN haU/Hr PEK F.E. AT UNE METEK a

I TIME I reak after uISCMakge OAMMAL ENERGY IN MEV

IS FRESH U.235 F.E

FEATILE PTL. FISSILE PIL.

\section{$\lfloor-13\rfloor$}

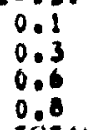

IÖTAL

IUDINE

0.1

0.6

0.8

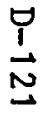

$\begin{array}{ll}3.077-13 & 4.982-13 \\ 3.199-11 & 4.334-11 \\ 4.694-12 & 6.350-12 \\ 1.237-12 & 1.676-12 \\ 3.829-11 & 5.180-11 \\ & \\ 3.677-13 & 4.982-13 \\ 3.199-11 & 4.334-11 \\ 4.694-12 & 0.359-12 \\ 1.237-12 & 1.676-12 \\ 3.829-11 & 5.180-11\end{array}$
1.321 .02
$2.327-02$

$2.227-02$

$1.250=03$

2.792004

$0.017=03$
5.406003

$5.693=02$

$3.310-03$

$1.202=02$

$4.053=00$
$8.015=02$

$6.381=03$

5.406003

$1.321=01$

$2.558=02$

$1.327-02$
$7.445-04$

$1.837=01$
5.014004

0.507 .02

900

$1.1111=03$

$8,673=02$

5.348 .03

4.806003

5.238002

$2.902-03$

1.068 .02

1.130 .04
7.658 .02

$5.850 \cdot 03$

4.806003

$1.175=01$

$2.273-02$

1.179 .02

6.618004

$1.033-01$
MS FRE SM U-235 F.E.

FENILL PTL. FISSILE PTL.

$\begin{array}{ll}5.949-13 & 0.000 \\ 3.219011 & 0.000 \\ 7.650012 & 0.000 \\ 2.019012 & 0.000 \\ 0.247-11 & 0.000\end{array}$

$5.999-13 \quad 0.000$

$\begin{array}{ll}5.219-11 & 0.000 \\ 7.658012 & 0.000\end{array}$

$2.019012 \quad 0.000$

$0.247-11 \quad 0.000$

$1.006003 \quad 1.028004$

$1.300001 \quad 1.334002$

$3.973002 \quad 4.058003$

$\begin{array}{ll}2.250003 & 2.277=04 \\ 4.961004 & 5.086005\end{array}$

1.741001

1.013002
$9.640-03$$\quad 9.090003$

$1.051001 \quad 1.074002$

$5.900003 \quad 0.032004$

$2.104002 \quad 2.190003$

$8.302004 \quad 8.180003$

$\begin{array}{ll}8.362004 & 0.480=05 \\ 1.537 .01 & 1.570 .02\end{array}$

$1.174002 \quad 1.199005$

$9.640003 \quad 9.852004$

$2.358001 \quad 2.408 .02$

$4.563002 \quad 4.661003$

$2.367 .02 \quad 2.417-03$

$1.328 .03 \quad 1.357 .04$

$3.278 .01 \quad 3.348 .02$ 
THIS SEGMENT WAS UISCHARGEO AT

THE BEGINNING OF RELUAL I ANU MAS

THIS SEGMENT MAS UISCHAKGED AT

TAE BEGINNING UF RELUAD 5 ANU WAS

URIGINALLY LOADEO AT RELISAD U

URIGINALLY LUAOED II KELUAO I

NUGLSOE AND OAPMA ENERGY

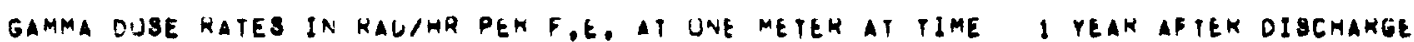

IN MEV

-

$98 \cdot 124$

0.0

1.0

1.25

1.60
2.0

TUTAL

$38-125$

0.13

TUTAL

$\stackrel{⿱}{N}$

ANIIMONY

0.1

0.8

1.25

1.6

TUTAL

18 FRESM U-235 F.E.

FERTILE PTL. FI83ILE PIL.

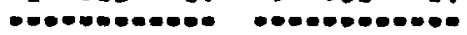

$\begin{array}{ll}1.771003 & 8.114004 \\ 3.042004 & 1.394004 \\ 1.000004 & 4.857005 \\ 1.540-04 & 7.053005 \\ 2.046-03 & 9.371004 \\ 2.095004 & 1.235004 \\ 4.650003 & 2.130003\end{array}$

1.700001
$1.621+00$

$3.247+00$

1,713001

$.020+00$
$3.258+00$

$5.055+00$

1.708001

$1.713-01$

$1.621+00$

$1.626+00$
$3.259+00$

$3.259+00$

4.857 .05

$7.053=05$

1.042 .04

1.000004

$2.046-03$

9.371 .04

$1.235=04$

$60=115 M$

1.00

I.25

3.057 .00

$3.064=05$

4.547 .05

$5.703=05$

0.502005

$3,064=05$

$1.483-05$
$4.547-05$

$5.783-05$

CADMIUA

1.0
1.25

2.799 .05
8.582 .05

MS FHESH U-Z3S F.E.

FEHTILE PIL. FIESILE PTL.

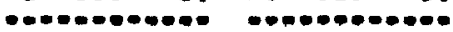

$\begin{array}{ll}5.540003 & 3.523003 \\ 4.525004 & 6.052004 \\ 3.320004 & 2.109004 \\ 4.821004 & 3.063004 \\ 0.405003 & 4.069003 \\ 8.430004 & 5.361004 \\ 1.450002 & 9.251=03\end{array}$

$1.126+00$
$1.000+01$

$\begin{array}{ll}1.000+01 & 2.904+00 \\ 2.141+01 & 5.819+00\end{array}$

$\begin{array}{ll}2.141+01 & 5.819+00 \\ 3.321+01 & 9.029+00\end{array}$

$1.120+00 \quad 3.060=01$

$1.068+01 \quad 2.904+00$

$2.141+01 \quad 5.822+00$

$3.320004 \quad 2.052004$

$4.821004 \quad 3.063004$

$0.405003 \quad 4.009003$

$8.438 .04 \quad 5.361 .04$

$3.323+01 \quad 9.038+00$

$6.007009 \quad 1.856005$

$\begin{array}{ll}2.908005 & 8.986006 \\ 8.015-05 & 2.755005\end{array}$

0.007005
$2.008=05$

$2.908=05$
$0,915=05$

1.856005
8.986000

2.755 .05 
THIS SEGMENT WAS DISCMARGEU AT

THE OEGINNING UR RELUAD I ANO MAS

ORIGINALLY LOADED AT HELUAD O
TMIS SEGMENT WAS OISCMARGEU AT

THE BEGINNING UP RELUAD 5 AND MAS

UKIGINALLY LUADEO AT KELUAD I

\begin{abstract}
NUCLIDE ANO GAPMA ENERGY
\end{abstract}

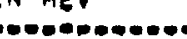

$16=108 \mathrm{M}$

0.1

0.1
0.0
0.8

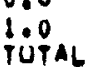

$0.110 M$

0.1
0.03
0.10
0.8

0.0

100

1.25

2.0

TÓTAL

SILVEK

0.1

0.3

0.6

1.0

1.25

1.6

2.0

TUTAL

$R H=106$
0.3
0.0
0.8
1.0
1.25
1.0
2.0
2.4
2.9
$101 A L$

gAMMA DOSE RATES IN RADIMR REK F.E. AT UNE METER AI TIME

I year apiek ojschakge

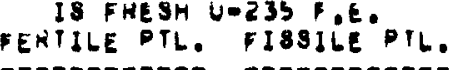

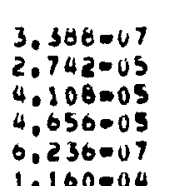

1.054 .08

1.339006

2.006006

$2.273-06$

3.045008

5.605000

0.253006

$5,261=04$
$2,583-02$

3,449002

1.0727002

8.012003
$5.417=05$

$5.605-05$

$9.097-02$

$0.591=06$

$5.535=06$

$3.445-02$

$1.021-02$

$1,127-02$

$0.612=03$
$5.417=05$

$5,017=05$

$9.109=02$

$2.325=05$

1.956003

$9.005-02$

$1.279-01$

3.798002

$4.192=02$

3.203 .02

3.015004

$2.004=06$

2.004000
3,363001

$2.327-05$

1.958003

9.605002

1.20001

3.798002

1.192002

3.203002

2.015004

2.084004

3. 383001

$1.351-01$

$3.920+01$

$6.733+0$

1.47300

5.943001

$1.978-0$

$1.365=0$

$5.035=12$
$5.032=02$

$8.204-03$

$7.941+v u$

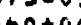

$3.400+00$

$9.908=01$

$2.932-01$

$2.930-01$

$4.812=02$

$4.623+111$
MS FRESH UEZ3S DE.

FENIILE PTL. FISSILE PTL.

1.167000
9.444005
1.415000
1.0033004
2.147000
3.990004

9.302000

7,529000

$1.128-05$

1.712007

3.1860 .05

7.932005

6.673003
3.270001
4.0304001

4.364001

1,296001

1.43000

1.092001

0.87200

7.11000

$1.154+00$

9.253005

.705003

3.022001

3.092001

1.512001

1.064001

1.274001

8.017004

$1.346+00$

$8.048=05$

0,70800

3.27800

4.36600

1.290001

1.030001

1.092001

6,872004

7.11000

$1.150+00$

9.263005

7.793005

3.022001

5.092001

1.512001

1.008001

$1.274=01$

8.017004

0.295004

$1.346+00$

1.158001

$3.302+01$

$7.223=01$
$3.915+00$

$2.915+00$

9.700001

$0.695=01$

$2.470=01$

$4.053=0$

$3.895+01$

1.219001 $3.535 \times 01$ $7.733=01$ $3.121+00$ $1.039+00$ 7.168001

$2.644=01$

2.642 .01

$4.339=02$

$4.170+01$ 


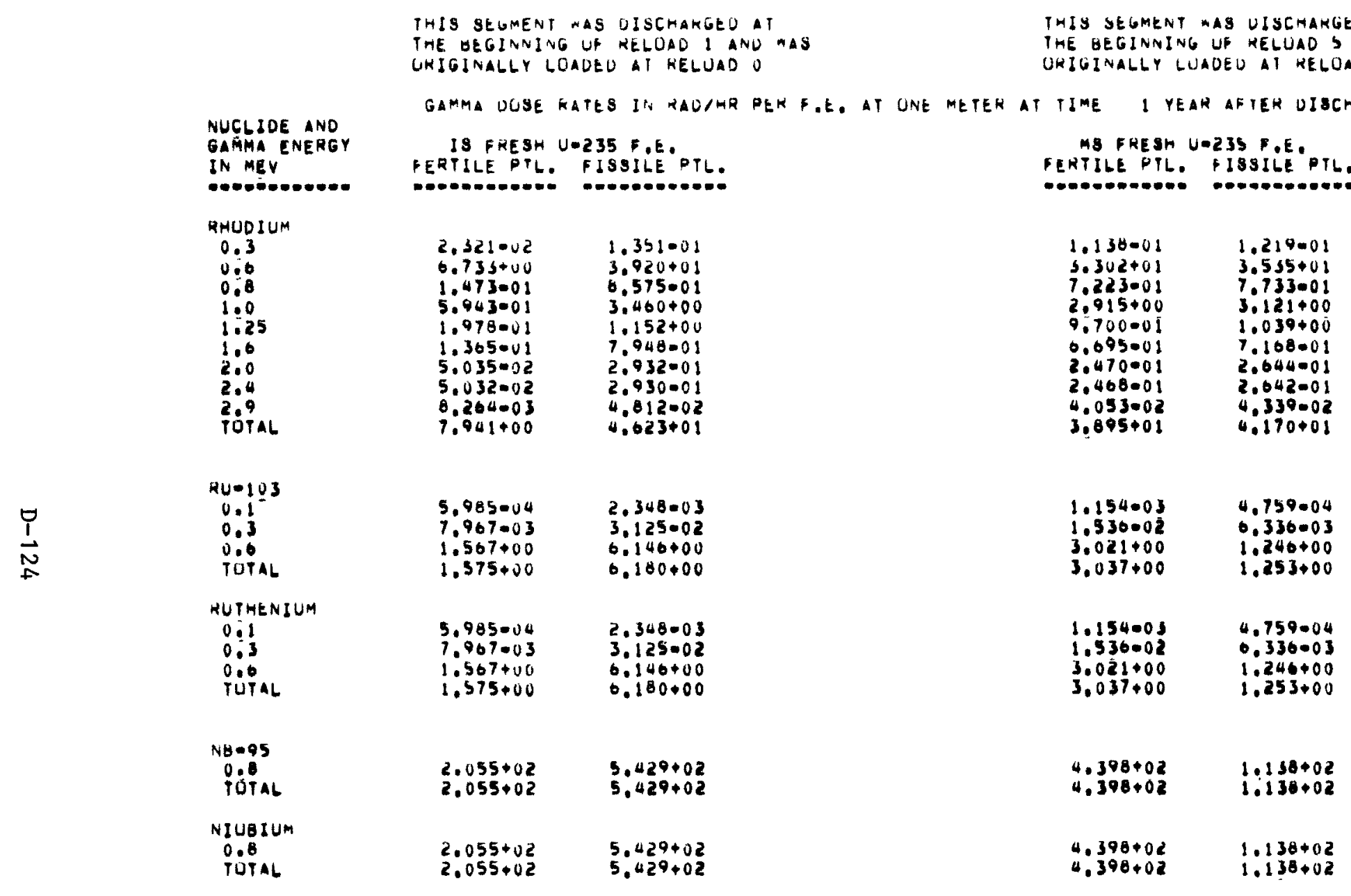




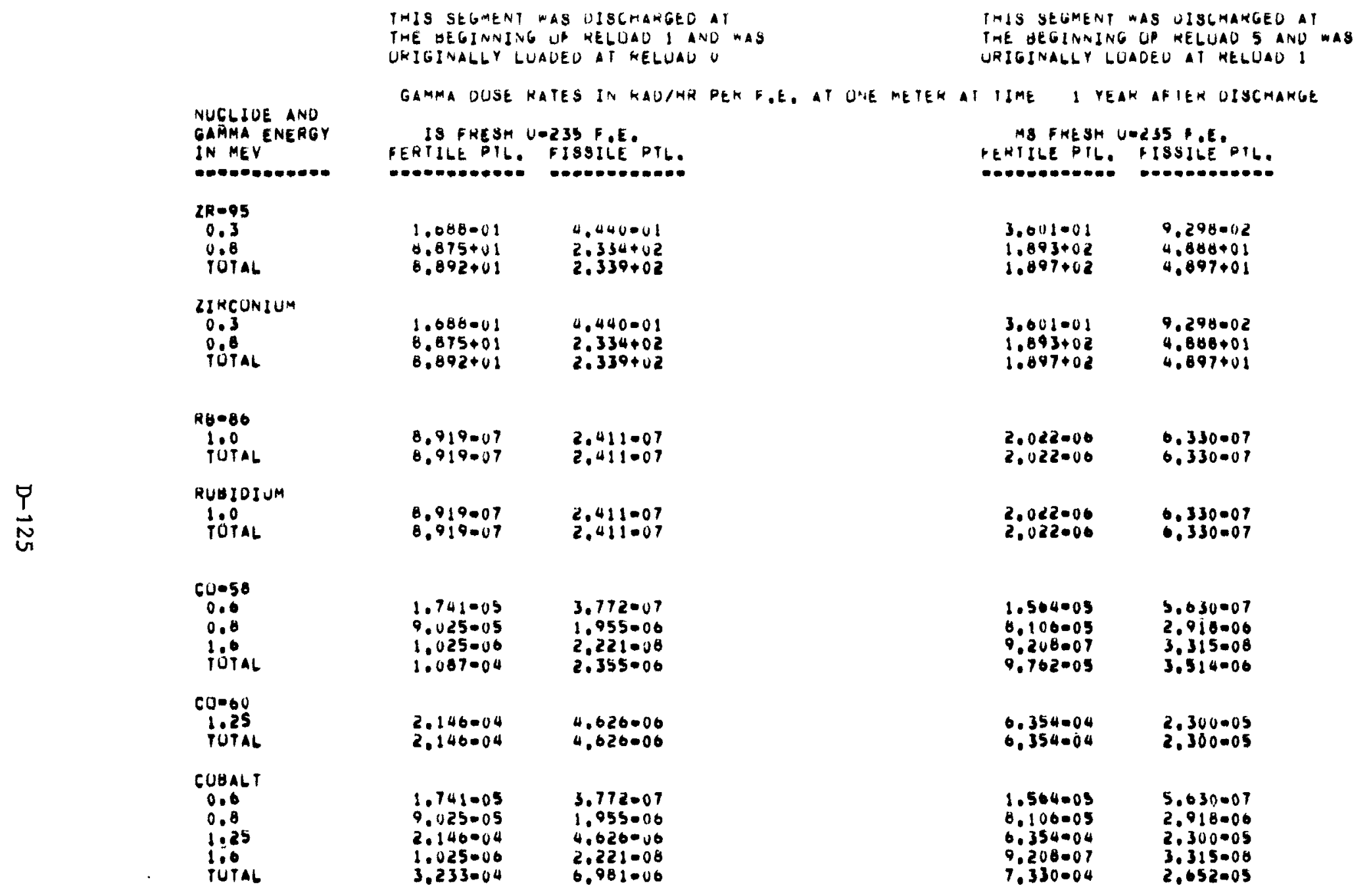


THIS SEGMENI MAS DISCMAKGED AI

THE BEGIINNING UF RELUAO I ANO WAS

URIGINALLY LUAUEO IT KELUAD O

GAMMA DUSE WATES IN FADIHR PEK F,E. AT UNE METER a

IS FRESH W-235 F,E.

FERTILE PIL. FISSILE PTL.

GAMMMA ENERG IN MEY

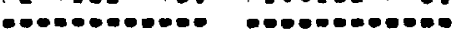

ALL ELEMENTS

0.1

0.3

$1: 0$

$\frac{1}{2}: 6$

2.4

2.9

$\frac{P}{N}$

$\begin{array}{ll}1.420+00 & 1.002+00 \\ 8.868+00 & 2.421+00 \\ 7.989+01 & 3.949+02 \\ 3.116+02 & 9.197+02 \\ 1.045+00 & 0.000+00 \\ 1.914+00 & 1.504+01 \\ 2.843+00 & 1.207+01 \\ 9.318+00 & 4.070+01 \\ 1.206001 & 2.93201 \\ 8.264003 & 4.812002 \\ 1.403009 & 2.378009 \\ 4.170+02 & 1.394+03\end{array}$

IMIS SEGMENT AAS UISEMARGED AT

THE GEGINNING UP HELUAD 5 AND MAS

UHIGINALLY LUAUED AT HELUAD !
MB PRESH U.235 F.E. FEKTILE PIL, FISSILE PILO

$\begin{array}{ll}3.860+00 & 3.212400 \\ 1.730+01 & 3.787+00 \\ 9.287+02 & 1.010+03 \\ 1.110+03 & 1.267+03 \\ 1.252+01 & 2.459+01 \\ 4.567+01 & 1.022402 \\ 1.284001 & 1.014+01 \\ 4.016+01 & 2.823+01 \\ 7.665001 & 2.65201 \\ 4.053002 & 4.339002 \\ 2.329009 & 4.799010 \\ 2.178+03 & 3.040+03\end{array}$




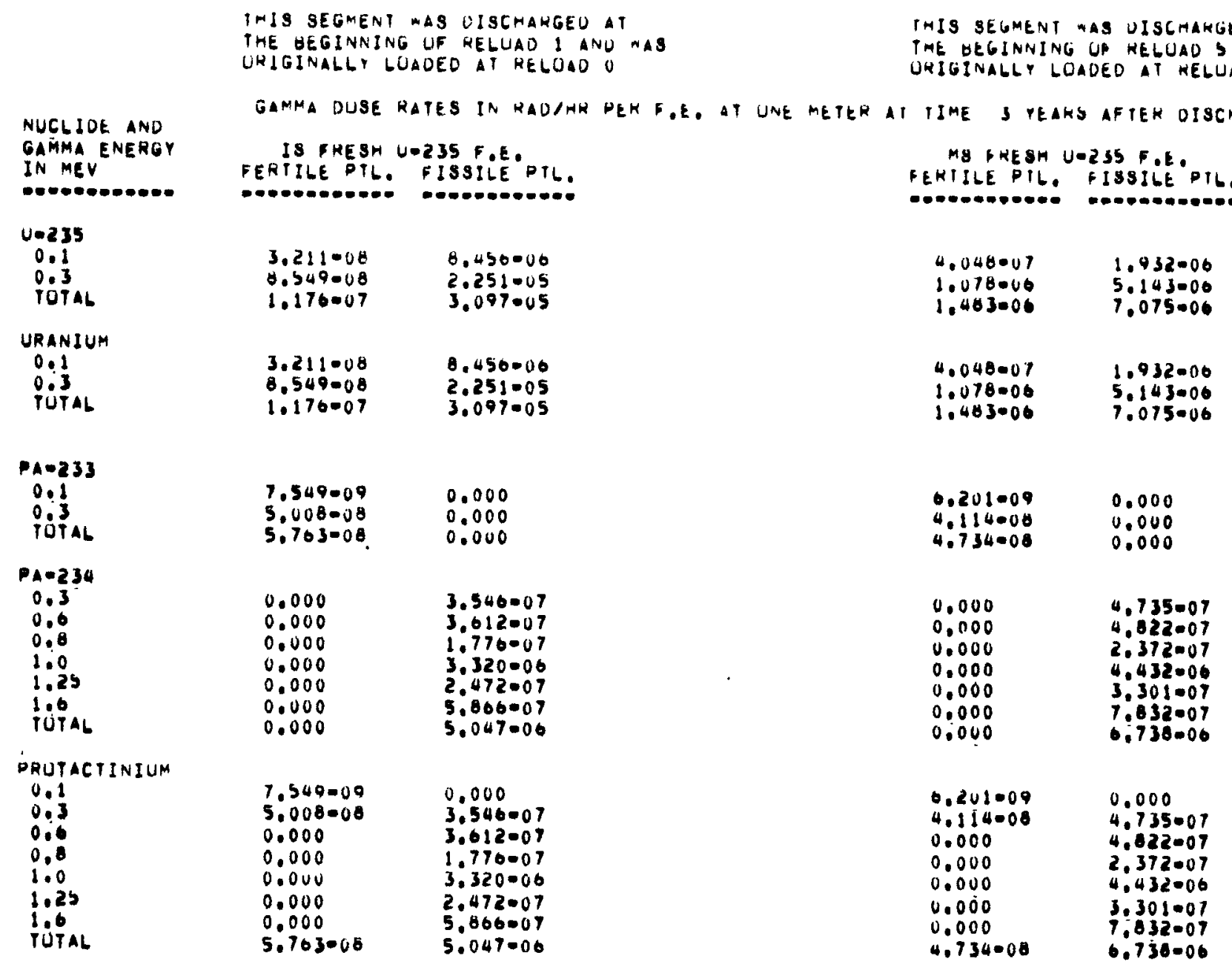


THIS SEGMENT WAS UISCTARGEO A

THE HEGINAING UF TELOAO I AND WAS

ORIGINALLY GUAOED AT RELUAU O

NUCLIDE ANO GAPMA ENERGY IN MEV

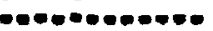

\section{$A C-22 \theta$}

0.3

0.6

1.0

2.0

UTAL

ACIINIUM

0.3

0.6

1.0

1.0
2,0

$\underset{\infty}{\stackrel{1}{N}}$

GAMMA DOSE RATES IN KAJ

FERIILE PTL. FISSILE PTL.

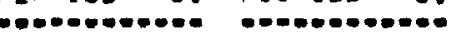

$\begin{array}{ll}9.709000 & 0.000 \\ 4.974000 & 0.000 \\ 1.223005 & 0.000 \\ 1.102004 & 0.000 \\ 3.447-05 & 0.000 \\ 7.947-00 & 0.000 \\ 1.796004 & 0.000\end{array}$

$9.769=00$

4.974006

1.223 .05

3.447 .05

7.947 .06

$1.790=04$

0.000
0.000

0.000
0.000

0.000

0.000

0.000

0.000
$4 A-223$

0.13

Toral

RADIUM

0.1

TÓTAL

HNa. 19

0.3

rorat

RADUN

Tótal $\begin{array}{ll}1.343006 & 1.974010 \\ 3.575006 & 5.255010\end{array}$

$\begin{array}{ll}4.97000 & 5.255010 \\ 4.918000 & 7.229010\end{array}$

$1.343000 \quad 1.974010$

$\begin{array}{ll}1.575006 & 5.255 \cdot 10 \\ 4.918006 & 9.229 \cdot 10\end{array}$

$\begin{array}{ll}3.575000 & 5.255 \cdot 10 \\ 3.575006 & 5.255 \cdot 10\end{array}$

$\begin{array}{ll}3.575000 & 5.255 \cdot 10 \\ 3.575000 & 5.255-10\end{array}$
IHIS SEGMENT "AS DISCHARGEU AT

TME UEGINNING UR HELUAO 5 ANU WAS

URIGINALG" GUAUEO AI RELIIAU I
MS FHESH U-235 P.E.

FENILLE PTL, FISYILE PIL.

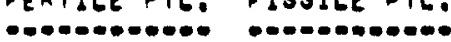

$1.04304 y$

$3.313040 \quad 0.000$

$1.307 .05 \quad 0.000$

1.1770000 .000

$3.602005 \quad 0.000$

0.000

$1.918004 \quad 0.000$

$1.043005 \quad 0.000$

$3.313006 \quad 0.000$

$1.307 .05 \quad 0.000$

$1.177004 \quad 0.000$

$3.682005 \quad 0.000$

$8.489 .06 \quad 0.000$

$1.918004 \quad 0.000$

$2.124006 \quad 0.696010$

$5.656006 \quad 1.250009$

$7,780006 \quad 1.720009$

$2.124000 \quad 4.690010$

$\begin{array}{ll}5.656006 & 1.250009 \\ 7.700006 & 1.720009\end{array}$

$5.050000 \quad 1.250009$

$5.650000 \quad 1.250009$

$5.656006 \quad 1.250009$ 


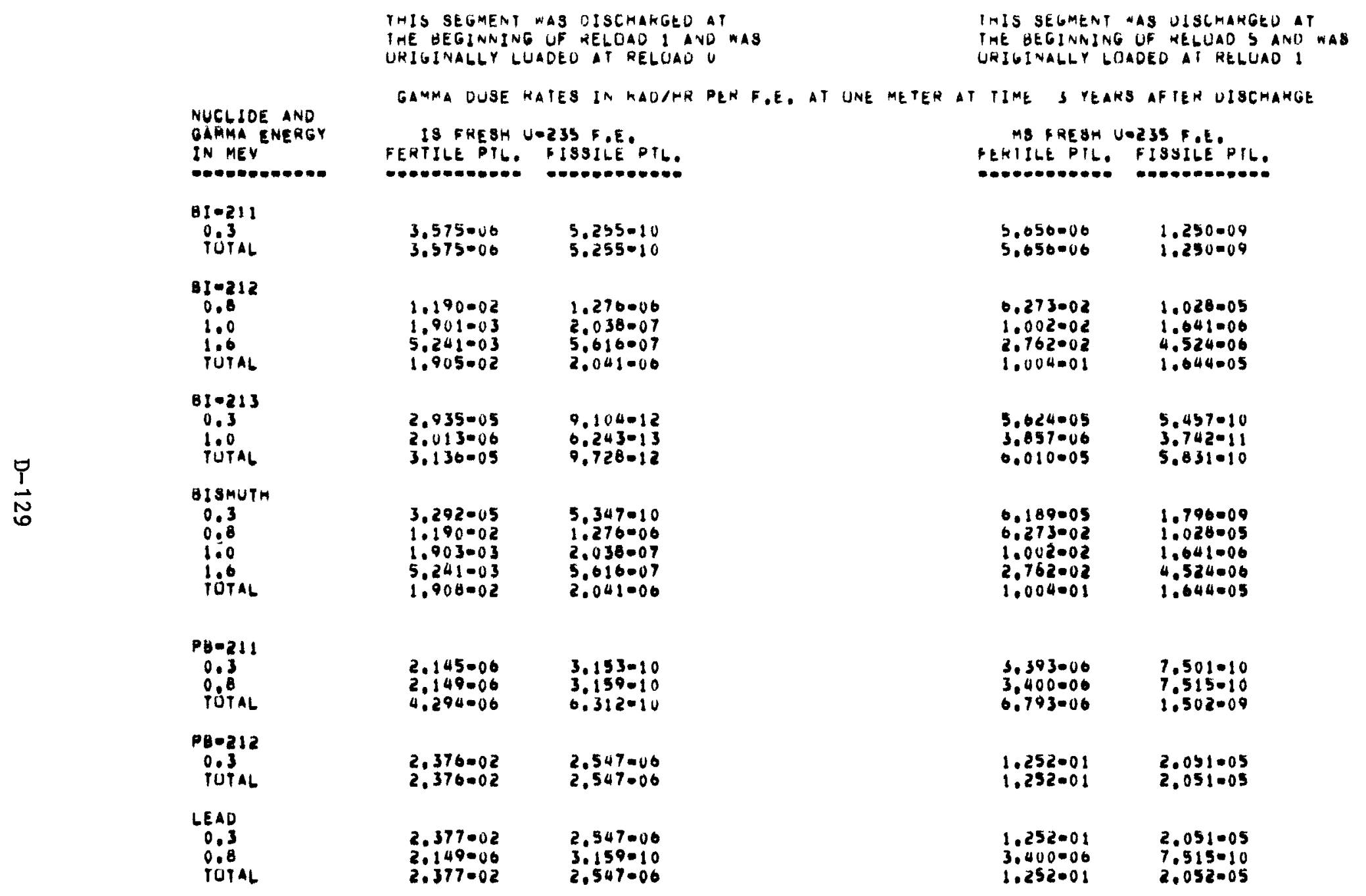




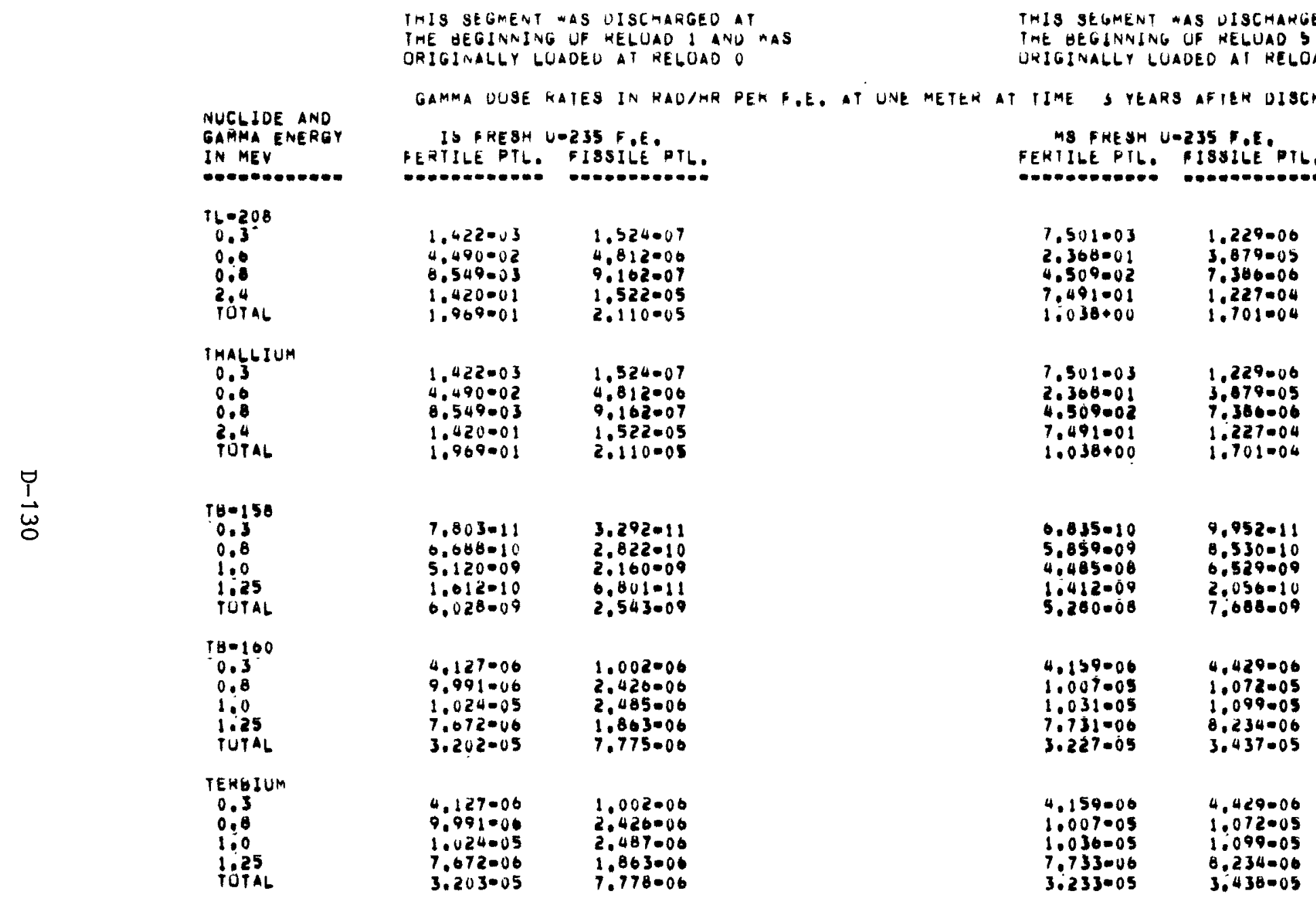


THIS SEGMENT MAS DISCMARGEO AT

THE OEGINNING UF RELUAD I ANO AAS URIGINALGY LOADED AT RELOADO

NUCLIDE AND GATMAR EMERG IN MEV

IN ME-

\section{$E \cup=152$}

0.152
$0: 3$
$0: 6$
$0: 8$
100
$1: 25$
$1: 0$
TOTAL

TOTAL

$54=154$

0.1
0.3
0.6
0.8
1.0
1.25
1.6
TUTAL

EUKOPIUM

0.1
0.3
0.6
0.8
1.0
1.25
1.0

TÚt

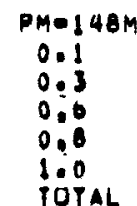

\section{FRESM U-235 F.E.}

FERTILE PTL. FIBSILE PTLO

$1.649=03$
$4.372=03$
$7.141-04$
$4.531=03$
1.303002
$1.025=02$
3.734004
$3.492-02$

2.710 .03

7.183003

$1.173=03$

$7.446=03$

2.141002

.605002
6.135004

5.7360 .02

$2.209-02$

9.598003

2.524 .02

1.374001

3.00100

1,545002

$5,401=01$

1.886001

0.15500

$1.173+00$

$1.113+00$

$1.709+00$

$1.320=0$

1.320001
$4.612+00$

2.374002
$1.397-02$
2.596002
1.419001
1.432001
2.104001
1.583002
5.750001

1.914001

8,914002

$2.167=0$

$1.180+00$

$1.133+00$

1.726400

$1.326=01$
$4.009+00$

2.263009
0.500000
5.191007
9.466008
$1.497-07$
8.307007

$1.158-08$

$3.331=0$

$2.657=00$

4.840007

7.062 .07

$8.307=07 \quad 4.253=06$

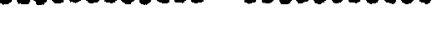

THIS SEGMENT WAS DISEHAKGED AT THE OEGINNING UF HELOADS AND WAS URIGINALLY LOADED II RELOAD I

MS PRESM UEZ35 R.E. FERTILE PTL. FISSILE PIL.

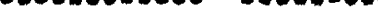

\begin{tabular}{|c|c|}
\hline $\begin{array}{l}1.427003 \\
3.704003 \\
6.180004 \\
3.922003 \\
1.128002 \\
6.874003 \\
3.232004 \\
5.022002\end{array}$ & $\begin{array}{l}1.880=03 \\
4.984003 \\
8.141004 \\
5.160003 \\
1.485002 \\
1.169=02 \\
4.257=04 \\
3.991002\end{array}$ \\
\hline $\begin{array}{l}4.211-01 \\
1.829001 \\
4.812001 \\
2.018+00 \\
2.402+00 \\
3.815+00 \\
2.040-01 \\
1.030+01\end{array}$ & $\begin{array}{l}9.689+01 \\
4.209-01 \\
1.107+00 \\
6.024+00 \\
5.711100 \\
.777+00 \\
0.777-01 \\
2.369+01\end{array}$ \\
\hline $\begin{array}{l}4.225001 \\
1.867001 \\
4.818001 \\
2.622+00 \\
2.493+00 \\
3.824400 \\
2.049001 \\
1.033+01\end{array}$ & $\begin{array}{l}9.708001 \\
4.259-01 \\
1.108+00 \\
0.029+00 \\
5.726+00 \\
8.788+00 \\
6.782001 \\
2.373+01\end{array}$ \\
\hline
\end{tabular}

$\begin{array}{ll}1.105008 & 7.728009 \\ 3.177007 & 2.222007 \\ 2.535006 & 1.773-06 \\ 4.622007 & 3.233=07 \\ 7.308=07 & 5.111=07 \\ 4.056006 & 2.037=06\end{array}$


THIS SEGMENT HAS UISGHAKGEU AI

THE BEGINNING UF RELUAO I ANU MAS

URIGINALLY LOADES AI RELUAD U

THIS SEGMENT MAS DISCHAKGED AT

TAE BEGINVING UR RELUAD S AND WAB

UNIGINALLY LOADEO AT RELUAU I

NUCLIOE AND
GARMA ENERGY GARMA ENERG IN MEV

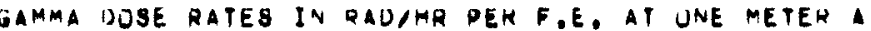

18 FRESH U.235 F.E.

FERTILE PTL. FISSILE PTL.

PRUMETHIUM

0.1

0.6

0.8

Tutal

\section{$P R=144$}

0.6

1.25

1.0

PÚTAL

PRASEDOYMIUM

0.6

1.25

1.6

TOTAL

$B 4=137 M$

8.0 .1374
0.1
0.6

TOTAL

BARIUM

0.1

0.6

$\begin{array}{ll}2.203009 & 1.158008 \\ 0.500008 & 3.331-07 \\ 5.191007 & 2.657-00 \\ 9.466-08 & 4.846007 \\ 1.497-07 & 7.662-09 \\ 8.307-07 & 4.253-00\end{array}$

$1.204400 \quad 5.512400$

$\begin{array}{ll}1.309002 & 1.033001 \\ 1.090002 & 4.09002\end{array}$

$4.486001 \quad 1.955+00$

$1.558+00 \quad 0.794+00$

$3.300+00 \quad 1.041+01$

$1.204+00$

$2,309=02$

$1,099-02$

4.486001

$1.558+00$

$5,512+00$

$1.033=01$

$4.792 \cdot 02$

$1.955+00$

$1.741+01$

$1.603001 \quad 6.416001$
4.230001

$\begin{array}{ll}4.230+01 & 1.693+02 \\ 4.247+01 & 1.700+02\end{array}$

$1.005=01$

$4.230+01$

$4.247+01$

$1.693+02$
$1.700+02$

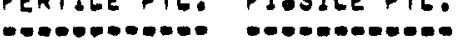

MS FRESH UEZ35 F.E FERTILE PIL. FISSILE PTL. Cococonovas

\begin{tabular}{|c|c|}
\hline $\begin{array}{l}1.1 \nu 5000 \\
3.197-07 \\
2.535000 \\
4.622007 \\
7.300007 \\
4.050000\end{array}$ & $\begin{array}{l}7.720=09 \\
2.222007 \\
1.773=00 \\
3.233007 \\
5.111007 \\
2.837=00\end{array}$ \\
\hline
\end{tabular}

$5.445+00$

1.020001

4.733002

$1.932+00$
$6,711+00$

$1.424+01$

7.150002

$1.354+00$

$4.703+00$

$9.977+00$

$5.445+00 \quad 3.816+00$

$1.020001 \quad 7.150002$

$4.733002 \quad 3.317002$

$1.932+00 \quad 1.354+00$

$0.711+00 \quad 4.703+00$

$1.424+01 \quad 9.977400$

$1.348+00 \quad 1.701+00$

$3.559+02 \quad 4.647+02$

$3.572+02 \quad 4.664+02$

$1.346+00 \quad 1.761+00$

$\begin{array}{ll}3.559+02 & 4.647+02 \\ 3.572+02 & 4.064+02\end{array}$ 
THIS SEGMENT WAS OISCHARGEO AT

TME GEGINNING UH RELUAO I ANU MAS

UHIGINALLY LUADED AI KELUAD U NUCLDDE ANO IN MEV JN MEV

\section{$c 8=134$}

0.3

0.8

0.8

$1 \div 25$

fótal

CEBIUM

0.3

0.6

0.8

1.0

1.6

TOTAL

TE- $129 M$

0.1

0.6

1.25

TÜTAL

$1 E \cdot 120$

0.1

0,3

0.6

0.0

1.0

POPAL
I8 FRE UH UA235 E.E.

FERTILE PTL. FISSILE PTLO

$\begin{array}{ll}1.115-03 & 9.305-03 \\ 8.235+00 & 0.872+01 \\ 0.944+00 & 7.130+01 \\ 1.167-01 & 9.730-01 \\ 9.110001 & 5.933+00 \\ 3.935-03 & 3.283+02 \\ 1.701+01 & 1.470+02\end{array}$

$1 \cdot 115003$

$8.544+00$

$1.167-01$

$7.110=01$

$3.935=03$

$1.761+01$

$2.013 \cdot 10$

2.613008

$0.000-10$

$3,482 \cdot 06$

$2.148-09$

$1.930=09$
$2.103=08$

$2.103=08$
$1.101-09$

$1.181=09$
$4.289-09$

1.061 .10

3.075 .08

\section{$9.305-03$}

$0.072+01$

$7.130+01$

$9.738=01$

$5,933+00$

3.283 .02

$1.470+02$

$1.790 \cdot 10$

$2.323 \cdot 08$

3.06400

3.965010

3.095008

$$
\begin{aligned}
& 1.909-09 \\
& 1.715009 \\
& 1.870-08 \\
& 1.050-09 \\
& 3.812-09 \\
& 1.476-10 \\
& 2.733-08
\end{aligned}
$$

GAMMa UOSE RATES IN RAOIHR PEN F.E. AT UNE METEK IT THE HEGINNING UF HELUAD 5 ANO MAS URIGINALLY LUADEO AI RELUAD I
MS FRESM UTZ35 F.E. FENTILEPTL. FISSILE PTL.

$\begin{array}{ll}3.194002 & 7.263-02 \\ 2.359+02 & 5.364+02 \\ 2.440+02 & 5.500+02 \\ 3.343+00 & 7.001+00 \\ 2.037+01 & 4.631+01 \\ 1.127-01 & 2.503001 \\ 5.040+02 & 1.147+03 \\ & \\ 3.194002 & 9.263002 \\ 2.359+02 & 5.364+02 \\ 2.448+02 & 5.560+02 \\ 3.343+00 & 7.601+00 \\ 2.037+01 & 4.631+01 \\ 1.127-01 & 2.563+01 \\ 5.046+02 & 1.147+03\end{array}$

$3.592-10 \quad 3.009-11$

$4.6620 .08 \quad 4.762009$

$1.418008 \quad 1.448-00$

1.770 .10

1.44800

$0.126-11$

0.340009

$\begin{array}{ll}3.832009 & 3.913-10 \\ 3.443009 & 3.517-10 \\ 3.153=08 & 3.833=09 \\ 2.108 .09 & 2.153 .10 \\ 7.652009 & 7.816-10 \\ 2.963-10 & 3.027-11 \\ 5.480 .08 & 5.603 .09\end{array}$


THIS SEGMENT WAS OISCHAKGED AT

URIGINALLY LUADED AT KELUAN U
THIS SEGMENT WAS DISCHAKGED AT THE GEGINNING UF WELOAD 5 ANO WAS

URIGINALLY LUAUED AT RELUAU I

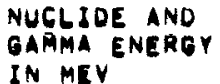

IN MEV

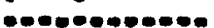

TELLURIUM

0.1

0.3

1.05

TUTAL

\section{$58 \cdot 124$}

0.6

$\underset{⿱ 亠}{\omega}$
GAMM DUSE RAIES IN HAOINR PEK G.E. AT UNE METEH AT

18 FKESH UW 235 F.E. FEKTILE PTL. IS8ILE PTL.
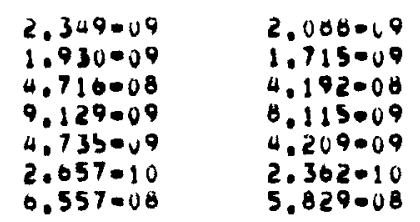

$3.953-07$

6.789 .08

2.360008

3.436008

$4.565 \cdot 07$

6.014000

1.038006

$1.024-01$

$9.718-01$

$1.947+00$

$3.021+00$

$1.024=01$

9.718001

1.947400

0.78900

2.366008

3.436000

$4.565=07$

$3.021+00$
1.811007

3.110008

1.084006
1.574008

1.574008
2.091007

2.755000

4,755007

$1.027-01$

$1.953+00$

$3.031+00$

$1.027-01$

9.749001

$1.953+00$

.110-08

1.060008

1.574000

$2.091=07$

2.755008
$3.031+00$
MS FRE PERTILE PIL, FI8SILEPTL.

$\begin{array}{ll}4.191009 & 4.280-10 \\ 3.443009 & 3.517-10 \\ 0.415008 & 8.595009 \\ 1.629-00 & 1.604009 \\ 8.446009 & 8.629010 \\ 4.741010 & 4.843-11 \\ 1.170007 & 1.195008\end{array}$

1.238 .00

2.12000

$7,409=05$

$1.070=0 ?$

1.430006

1.803007

3.250000

0.750001

$0.405+00$

$1.283+01$

$1.992+01$

$7.003 \cdot 07$

1.351 .07

4.707 .00

0.836000

0.002 .07

1.196 .07

$2.065-06$

0.750 .01

$0.405+00$

$1.203+01$

2.12600

1.07000

1.07000

1.430006

1.083007

1.655001

1.741 .00

$3.489+00$

1.055001

$1.741+00$

$3.489+00$

$1.351=07$

$4.707-08$

$0.836=08$

$9.082=07$

1.196007
3.414400 


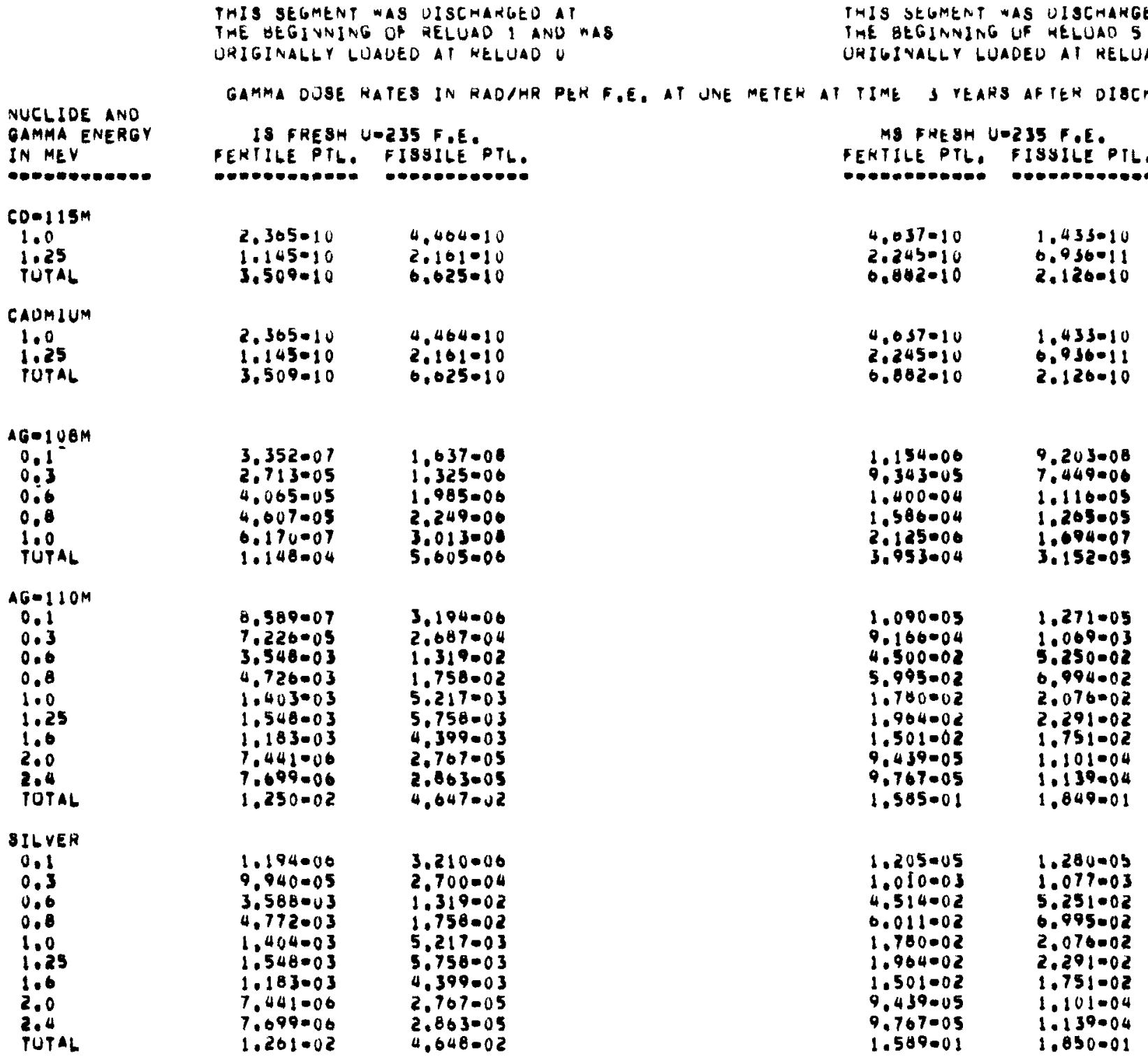




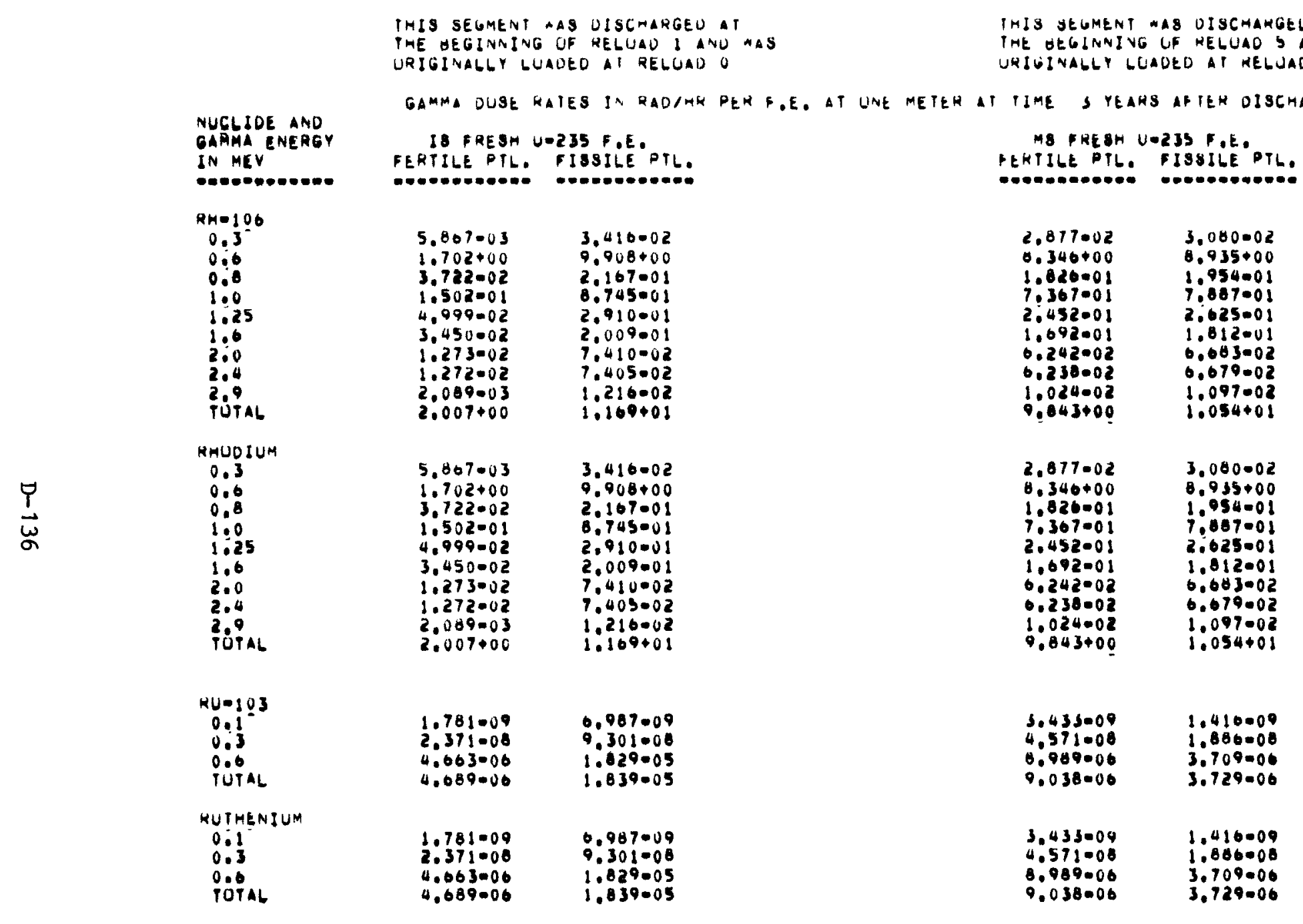




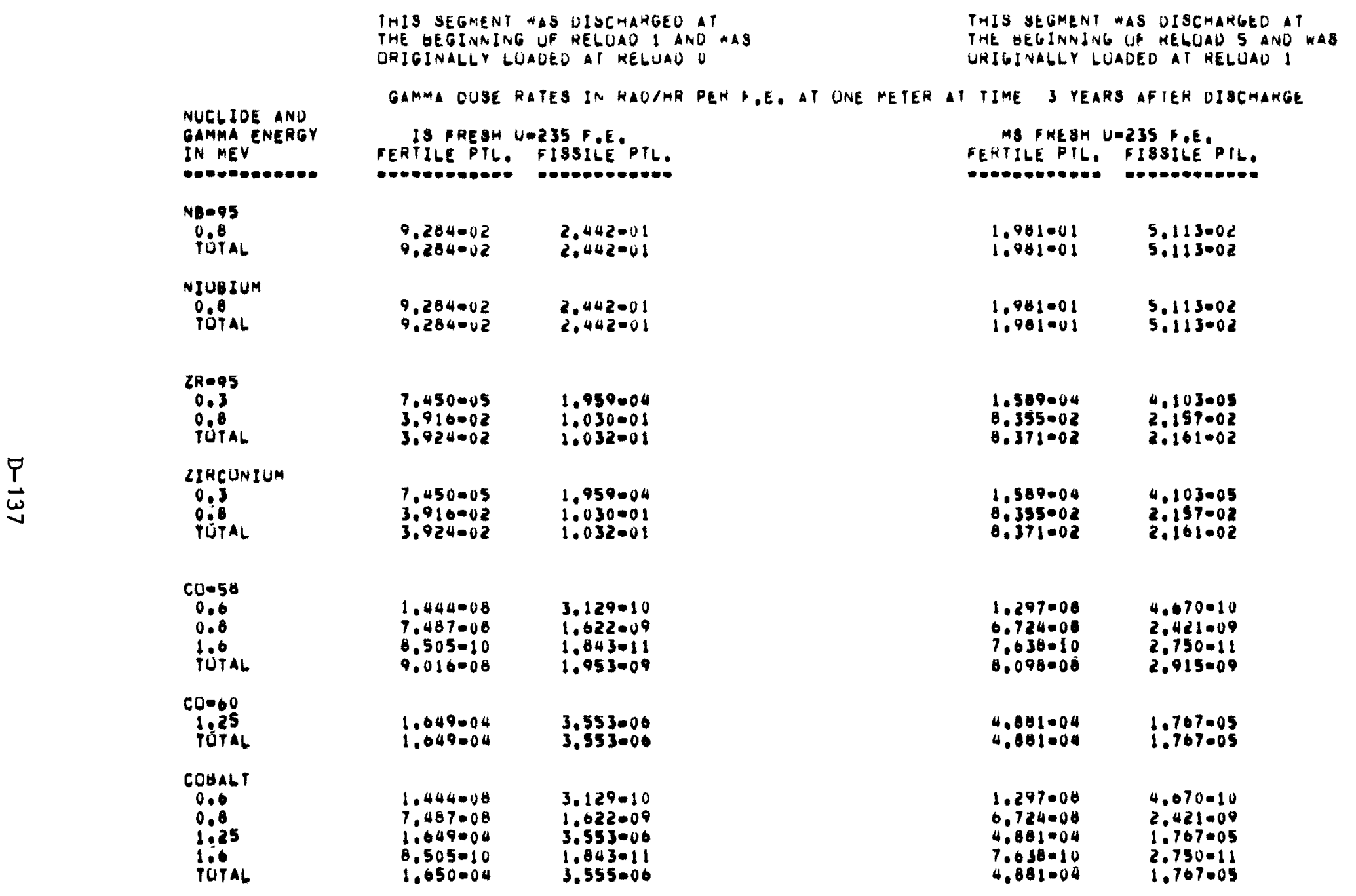


IHIS SEGMENT MAS OISCMAKGED AT

THE GEGINAING OF RELUAD 1 AND WAS

URIGINALLY LUADED AT RELUAD

NUCLIDE ANU GAMAA ENERG IN MEV

gamma dUSE Rates IN RADINR DEK E.E. AT UNE METEK a

IS FHESH $N=235$ F.E.

FERTILEPTL, FISSILE PIL.

ALL ELEMENTS

0.13

0.8

0.8

1.0

1.6

$2: 4$

IÓTAL

$\underset{\infty}{\stackrel{\varphi}{w}}$

$\begin{array}{ll}2.864-01 & 9.357-01 \\ 1.018+00 & 1.108+00 \\ 5.553+01 & 2.557+02 \\ 8.904+00 & 7.317+01 \\ 4.136-01 & 2.987+00 \\ 9.841-01 & 8.000+00 \\ 5.093-01 & 2.320+00 \\ 1.571+00 & 0.868+00 \\ 1.547-01 & 7.410-02 \\ 2.089-03 & 1.216=02 \\ 6.937+01 & 3.511+02\end{array}$

IMIS SEGMENT ABS UISGMARGEL AI

ITE BEGINNING UF RELOAO S AND WAB

IIME S YEARS APTER UISCMARGE

MS FHESH UEZSS P.E. PERTILEPTL. FISSILE PTLO and

$\begin{array}{ll}2.446+00 & 2.915+00 \\ 6.787+00 & 2.272+00 \\ 0.192+02 & 1.018+03 \\ 2.481+02 & 5.630+02 \\ 0.041+00 & 1.414+01 \\ 2.451+01 & 5.542+01 \\ 2.551+00 & 2.487+00 \\ 6.713+00 & 4.770+00 \\ 0.115001 & 0.702002 \\ 1.024002 & 1.097002 \\ 9.178+02 & 1.664+03\end{array}$

$9.178+02$

$.664+03$ 


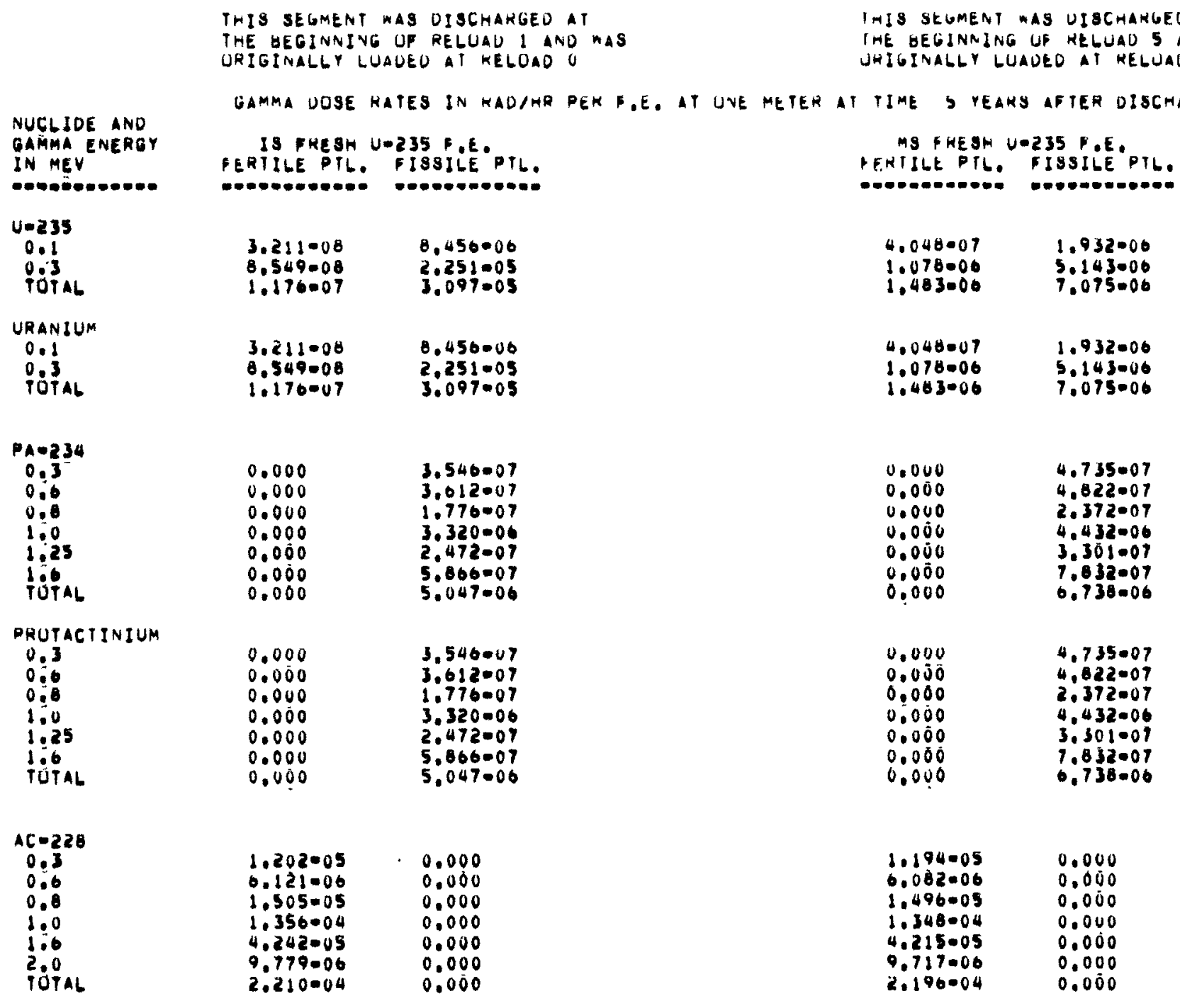


THIS SEGMENT MAS DISCHARGEO AT

THE GEGINNING OF RELOAO I ANU MAS

URIGINALGY LUADEO AT RELUAD O
IHIS SEGMENT WAS UISCMANGEO AT

THE GEGINNING UF RELUAU 5 AND WAB

URIGINALLY LOAUED AT RELOAD I

NUCLJUE AND OAMMA ENERGY IN MEV

gamma dUSE Rates IN RAD/HR PEK F.e. at UNE METEN a

18 PRESH UN235 F.E. CERTILE PTL. FISSILE PTL.

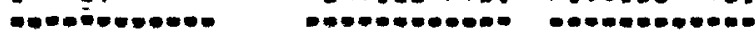

\section{ACTINIUM}

0.3

0.6

1.0

2.0

TOTAL

1.202005
6.121006
$1.505=05$
$1.356=04$
0.242005
$9.779=00$
2.210004

0.000

0.000

0.000

0.000

0.000

0.000

0.000

$44-223$

0.1

$\stackrel{\text { I }}{\vec{f}}$

TUTAL

RADIUM

0.13

fótal

RN-319

0.3

rutal

TOTAL

$1=211$

0.3

$8]-212$

0.8

$1: 0$

IU⿴囗十⺝

$\begin{array}{ll}1.259006 & 3.195=10 \\ 3.353006 & 0.506-10 \\ 4.612006 & 1.170-09 \\ & \\ 1.259006 & 3.195-10 \\ 3.353006 & 8.506-10 \\ 4.012006 & 1.170-09\end{array}$

$3.353006 \quad 0.506-10$

$3.353=06$

$8,506-10$

$3.353=06$

8.500010

$8.506-10$

$3.353=06$

$6.506-10$

1.438 .02

2.297003

$2.301=02$

1.540006

2.460 .07

$0.762=07$
$2.404-06$
MS FREOM U-235 F.E. FERIILE PIL, FISSILE PIL.

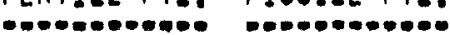

$\begin{array}{ll}1.194005 & 0.000 \\ 0.082006 & 0.000 \\ 1.096005 & 0.0000 \\ 1.348004 & 0.000 \\ 4.215005 & 0.000 \\ 9.717006 & 0.000 \\ 2.106004 & 0.000\end{array}$

$\begin{array}{ll}1.992000 & 0.950010 \\ 5.304006 & 1.850000\end{array}$

$\begin{array}{ll}5.304006 & 1.850009 \\ 7.296006 & 2.545009\end{array}$

$1.992000 \quad 0.95001$

$\begin{array}{ll}5.304006 & 1.050000 \\ 7.290006 & 2.545009\end{array}$

$5.304006 \quad 1.050009$

$3.304006 \quad 1.850009$

$5.304006 \quad 1.950009$

$7.119 .02 \quad 1.163 .05$

$1.137 .02 \quad 1.857 .00$

$\begin{array}{ll}3.134002 & 5.119000 \\ 1.150001 & 1.800005\end{array}$ 
IHIS SEGMENT NAS OISCHANGED AT

THE BEGINNING OF RELUAD I AND WAS

TRE BEGIGINALLY LOADEU AT HELUADU

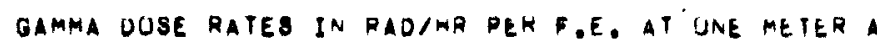

NUCL IOE AND GAMMA ENERGY IN $M E V$

IN Mer

\section{$0: \frac{1}{0.3} 13$}

1.0

EISMUTH

0.3

0

100

TOTAL

$\stackrel{\oplus}{\rightarrow}$

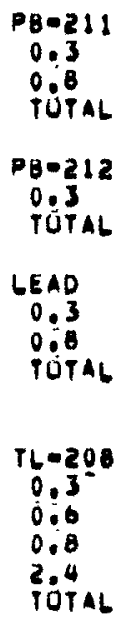

IS FRESH U-2J5 F.E.

FERTILE PTL. FISSILE PTL.

\section{$4.746-05$ \\ $9.277 \cdot 12$}

3.255006

$0.362 \cdot 133$

$5.081=05$

1.438002

2.301 .03

$0.333=03$
2.307002

$8.598 \cdot 10$

1.540006

2.460007
0.782007

$2.465=06$

2.012006

$2.015=00$

\section{$5.103-10$}

$5.113+10$

$2.871=02$
$2.871=02$

$3.075=06$

3.075006

2.872 .02

2.015000

3.075006

$5.113-10$

$2.672-02$

$1.721-03$

5.4330 .02

$1.034=02$

$2.382-01$
1.843007

5.819006

1.108 .06

1.841 .05

2.552005
IMIS SEGMENT WAS UISCHAKGEO AT

IHE BEGINNING UF RELUAD 5 ANU WAS

UHIGINALG LUAUED AT HELUAD I

T TIME S YEARS aFTEN DISCHARge

MS FRE 8 N U.235 P.R

PERTILE PIL, PISSILE PIL.

$\begin{array}{ll}0.449005 & 5.459-10 \\ 5.794000 & 3.740 .11\end{array}$

$9.026005 \quad 5.834010$

$8.979005 \quad 2.396009$

$9.119002 \quad 1.163005$

$1.138002 \quad \begin{aligned} & 1.857-06 \\ & 1.134002\end{aligned}$

$1.100001 \quad 1.800005$

$\begin{array}{ll}3.182006 & 1.110009 \\ 3.1186006 & 1.112009 \\ 0.370006 & 2.222009\end{array}$

$1.421 .01 \quad 2.321005$
$1.421 .01 \quad 2.321 .05$

$1.421 .01 \quad 2.321 .05$

$\begin{array}{ll}3.188006 & 1.122009 \\ 1.421 .01 & 2.321005\end{array}$

$\begin{array}{ll}0.522003 & 1.391006 \\ 2.600001 & 4.393005 \\ 5.122002 & 0.363006 \\ 8.510001 & 1.389004 \\ 1.180000 & 1.920004\end{array}$




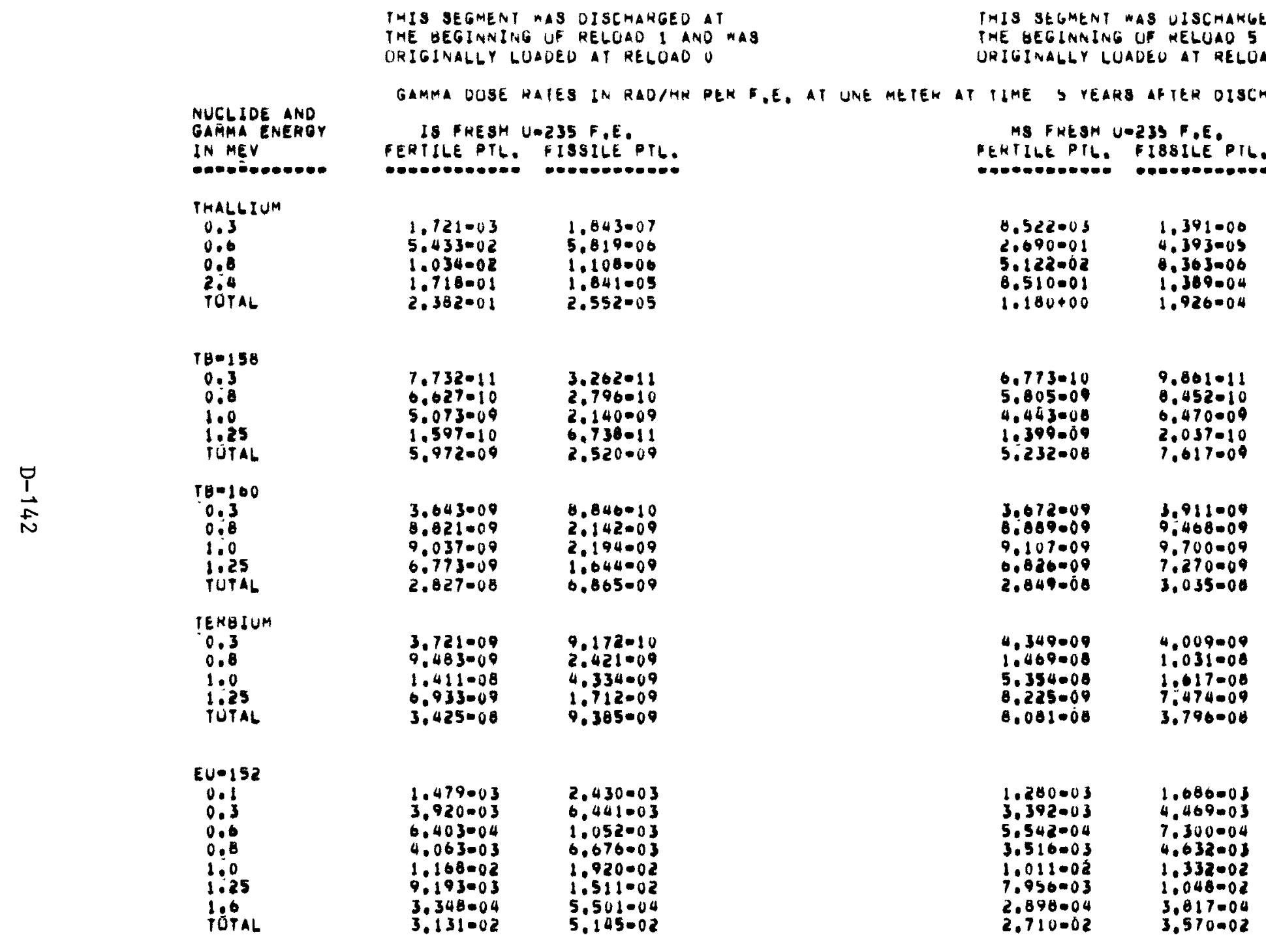


THIS SEGMENT HAS OISCHAKGED AT THE DEGINNING UF RELUAU I AND MAS

IMIS SEEMENT MAS UISCHAKGED AT ORIGINALLY LUADEU AT GELUAU U

TME BEGINNING UP HELUAD 5 ANU WAS

NUCL IDE AND

GATMA ENERGY GAKMA DUSE RATES IN RAUITR PI

GRIGINALLY LUAUEU AT RELUAD I

IN MEV

IS RESH U. $235 \%$.E.

FERTILE PIL. FISSILE PIL.

\section{$E v-154$}

0.1
0.3
0.6
0.8
$1: 0$
$1: 25$
1.6
10746

$8.802 \cdot 03$

$2.315=02$

1.260001

1.83500

$1.417=02$
$4.953=01$

$1.730=0 !$

7.510002

.977002

$1.076+00$

1.020400

$1.507+00$

1.210001

EUNOPIUM

$\begin{array}{ll}2.174002 & 1.754-01 \\ 1.272-02 & 8.160002 \\ 2.379-02 & 1.987001 \\ 1.300-01 & 1.082+00 \\ 1.311-01 & 1.039+00 \\ 1.927-01 & 1.582+00 \\ 1.451-02 & 1.216001 \\ 5.260-01 & 4.201+00\end{array}$

$3.984=03$

$1.848=03$
$7.543-02$

2.051 .0

5.560001

9.270001

$1.737=02$

8.050003
3.269001

$1.143+00$

$2.424+00$

PRABEODYMIUA

0.6

0,8

1.25

$3: 0$

Tótal

$\begin{array}{ll}2.126001 & 9.270001 \\ 3.984 .03 & 1.737 .02 \\ 1.848 .03 & 8.058003 \\ 7.543002 & 3.289-01 \\ 2.621 .01 & 1.143+00 \\ 5.560 .01 & 2.424+00\end{array}$

ME SKESH U-235 F.E.

PERIILE PIL. FISIILE PIL.

$\begin{array}{ll}3.802001 & 8.885001 \\ 1.070001 & 3.800=01 \\ 4.412001 & 1.015+00 \\ 2.401+00 & 5.525+00 \\ 2.276+00 & 5.237+00 \\ 3.498+00 & 0.049+00 \\ 2.701001 & 6.215001 \\ 9.401+00 & 2.172+01\end{array}$

$3.875001 \quad 0.902008$

$1.712001 \quad 3,905002$

$4.418001 \quad 1.016000$

$2.405400 \quad 5.529000$

$3.300+00 \quad 8.059+00$

$\begin{array}{ll}2.704001 & 0.219001 \\ 0.468+00 & 2.176+01\end{array}$

$9.137 .01 \quad 0.417 .01$

$1.716002 \quad 1.202002$

$7.900003 \quad 5.578005$
$3.240 .01 \quad 7.370001$

$1.129+00 \quad 7.908001$

$3.394+00$

$9.157 .01 \quad 0.417-01$

$1.716=02 \quad 1.202002$

3.94001

$3.240 .01 \quad 2.276001$

$\begin{array}{ll}1.129+00 & 1.900001 \\ 2.394+00 & 1.678+00\end{array}$ 


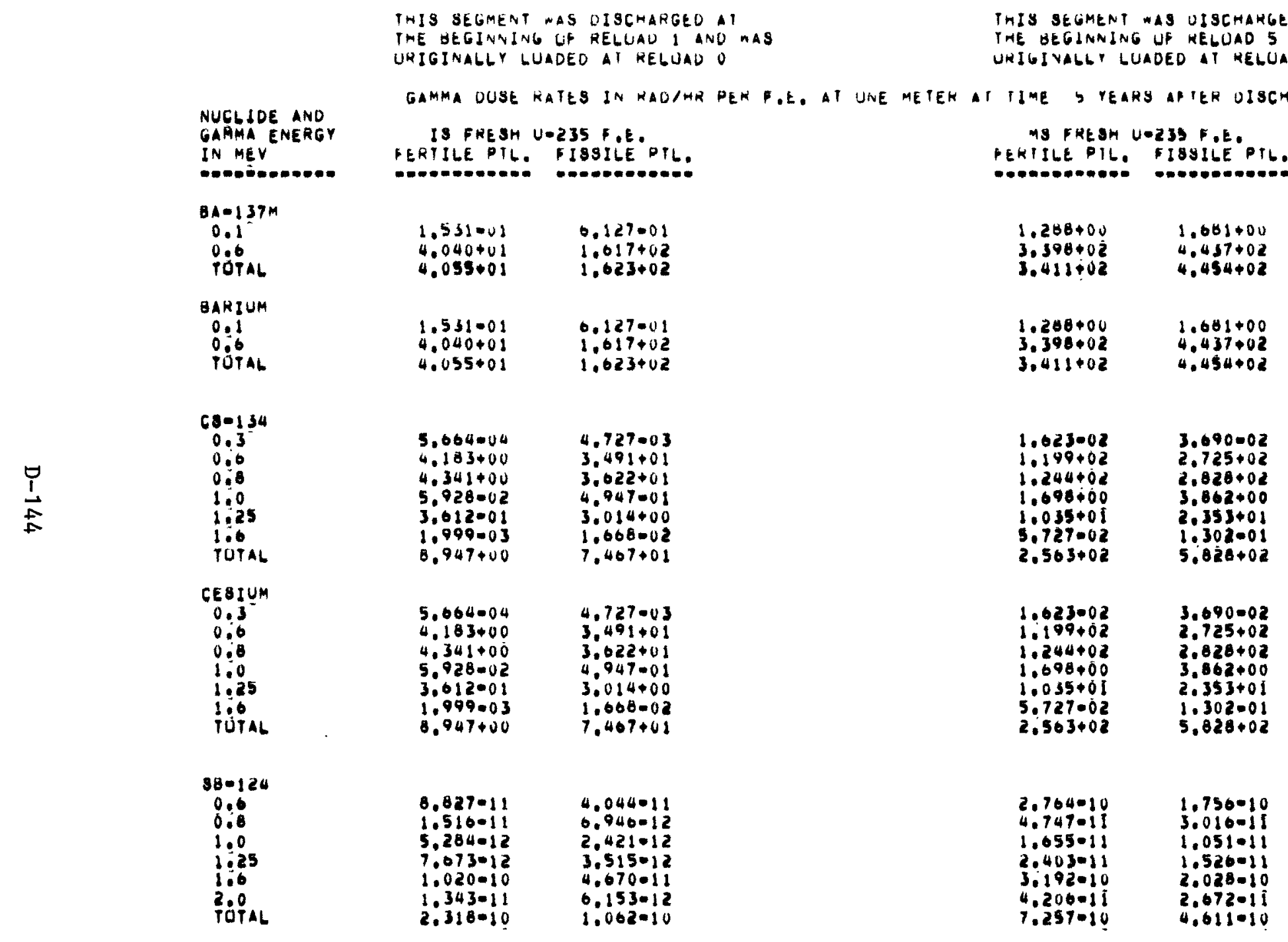


THIS SEGMENT MAS UISCHARGEO AI

THE GEGINNING OF RELUAO I AVU WAS

URIGINALLY LUAUED AT HELUAD O
THIS SEGMENT WAS DISCHAHGEO AT THE BEGINNING UP RELUAD S ANU WAS

WRIGINALLY LUADEL AI HELUAD I
NUCLIOE AND GAMMA ENERGY IN MEV

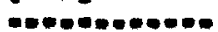

$38-125$

0.13

TÓGAL

ANIIMUNY

$0: 1$
$0: 3$
$0: 6$
$0: 8$
$1: 0$
$1: 25$
100
$2: 0$
$P O T A L$

$\underset{i}{i}$

\section{$46-108 \mathrm{M}$}

0.1009
0.19
0.3
0.6

0.8

100

$G=11014$

$0=110$
0.1
0.3
0.6
0.8
1.0
1.25
6.6
$2: 0$
2.4
TUTAL

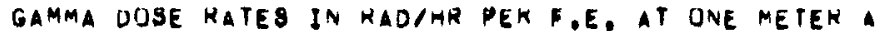

IS FRESM UDZZ35 F.E.

FERTILE PTL. FISSILE PTL.

$0.141=02$

5.827 .01
$1.168+00$
1.0912000

$1.812+00$

0.100002
5.840001

5.840001
$1.171+00$

$1.818+00$

$0.141=02$

5.827001

$1.108+00$

$1.516-11$
$5.284-12$

7.673-12

$1.020-10$

1.343011
$1.812+00$

0.160002

5.846001

$1.171+00$

$6.946-12$

$2.421-12$

3.515012

$4.670 \cdot 11$

0.153012

$1.810+00$

$3.317-07$

4.022005

$4.556=05$

$6.105 \cdot 07$

$1.136-04$

1.311006

1.964006

2.981008

5.540006

1.280007

9.920006

$4.874=04$

6.493004

1.928004

2.127 .04

$1.025=04$

1.022006

1,717003
4.386007

3.092005

3.69205

1.813003

2.415003

7100004

7.910004

6.044004

3.802006

$6.384-03$
MS PKESH U-235 P.E. FEKIILE PTL, FISYILE PTL.

$\begin{array}{ll}4.048001 & 1.100001 \\ 3.841+00 & 1.044000 \\ 7.696+00 & 2.092000 \\ 1.194+01 & 3.246+00\end{array}$

$0.040001 \quad 1.100001$

$3.841+00 \quad 1.044000$

$7.696400 \quad 2.092400$

$4.747 .11 \quad 3.026011$

$1.055011 \quad 1.051 .11$

$2.403 .11 \quad 1.526-11$

$3.192010 \quad 2.028-10$

$4.206-11 \quad 2.672-11$

$1.194+01 \quad 3.246000$

$1.142006 \quad 9.106=00$

$9.245005 \quad 9.370006$

$1.365004 \quad 1.104005$

$1.570004 \quad 1.251 .05$

$\begin{array}{ll}2.102006 & 1.676=07 \\ 3.911004 & 3.118=08\end{array}$

$1.497-06 \quad 1.746006$

$1.259004 \quad 1.469004$

$0.183003 \quad 7.213=03$

$8.236003 \quad 9.609003$

$2.445003 \quad 2.853003$

$2.698 .03 \quad 3.148 .03$

$2.062003 \quad 2.405003$

$1.297 .05 \quad 1.513005$

$\begin{array}{ll}1.342005 & 1.565005 \\ 2.170002 & 2,541.02\end{array}$ 
INIS SEGMENT HAS DISCHAMGED AT

TME GEGINVING UF RELUA I AND NAS

URIGINALLY LUADED AT RELUAD

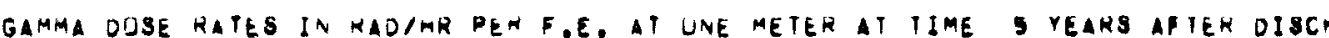

NUCLLIUE AND GAMMA ENERG IN MEV

000000000

\section{SILVER}

0.1

0.3

0.8

1.025

1.6

3.0

TUTAL

$\frac{P_{1}}{\stackrel{5}{a}}$
IS FHESH U-235 F.E.

FERTILE PTLO FISSILE PIL

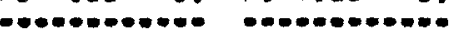

4.497 .07

3.077 .05

5.276004

1.934 .04

2.127 .04

$1.025=04$

1.022000

$1.058=00$

1.830003

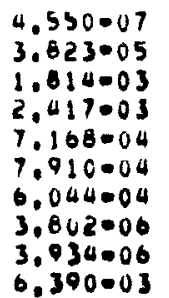

$1.483=03$

$4.301=01$

9.409003

$3.797-02$

$1.264=02$

$8,721-03$

$3.217=03$

5,280004

$5,073.01$

1.483 .03

4.301 .01

9.409003

3.797.02

$1.204-112$

8.721003

$3.217-03$

$3.215=03$

5.280 .04

5.073001
0.634 .03

$2.504+00$

5.470 .02

2.211001

$7.337=02$

5.070 .02

1.073002

1.072 .02

$3,074=03$
$2,954+00$

$8.634-03$

$2.504+00$

$5.498-02$

2.211001

7.357002

3.078 .02

$1.873=02$

1.872002

3.074003
IHIS SEGMENT MAS UISCTAKGEU AI

THE BEGINAING UP RELUAU 5 AND WAS

URILINALLY LUAOEU IT RELUAD

TÓTAL

MS FRESH U-235 TE. PEHTILE PIL. PIBILE PIL.

$\begin{array}{ll}2.659000 & 1.037-00 \\ 2.104004 & 1.543004 \\ 0.321003 & 7.224003 \\ 0.393003 & 9.621003 \\ 2.447003 & 2.853003 \\ 2.698003 & 3.148003 \\ 2.062003 & 2.405003 \\ 1.207005 & 1.513005 \\ 1.342005 & 1.565005 \\ 2.217002 & 2.54002\end{array}$

$\begin{array}{ll}7.273003 & 7.707003 \\ 2.110000 & 2.259+00 \\ 4.614002 & 4.940002 \\ 1.802001 & 1.994001 \\ 6.197-02 & 6.635002 \\ 4.277002 & 4.570002 \\ 1.576002 & 1.689002 \\ 1.577002 & 1.680002 \\ 2.589003 & 2.772003 \\ 2.480+00 & 2.664000\end{array}$

7.273003

$2.110+00$

4.614002

1.862001

6.197002

6.197 .02

.277 .02
1.576002

1.577002

2.589003

$2,488+00$
$7.787-03$

$2.259+00$

1.994001

$0.635=02$

$4.579-02$

1.689 .02

1.680 .02

2.772003

$2.664+00$ 
THIS SEGMENT MAS DISCHAHGEO AT

THE BEGINAING UF RELUAD I ANO WAS

URIGINALLY LUADEU AT RELUAU U

THIS SEGRENT HAS OISCMAHGED AT

ME OEGLNNING UF RELUAO 5 ANU WAS

URIGINALLY LUA
NUCLIDE ANO

OAMMMA ENERG

IN MEV

GAMMA DUSE RATES IN RAOINR REK F,E. AT UNE METER AI

IS FRESH U-Z3S F,E.

FERIILE PIL. FISILE PTL.

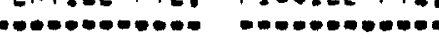

Rบo 103

0.1

0.3

TÓTAL

RUTHENIUM

0.1

TÓTAL

NB. 95

TOTAL

$\stackrel{\varphi}{\overrightarrow{0}}$

NIUBIUM

Tơ

0.000

0.000
0.000

0.000

2.081014

$5: 449011$

5.478 .11

0.000

0.000

0.000

2.081014

5.409011

$5,470.11$

$4.099-05$

1.078004
1.078004

$0.099005 \quad 1.078004$

1.078 .04

3.280 .08

1.729 .05

8.651 .08

48.95
0.3

Tótal

ZINCUNIUM

0.3

0.8
TUTAL

$1.732=05$

4.5080 .03

$.557=05$

3.289008

1.729005

0.651 .08

4.548 .05

$4.557-05$

co-50

0.6

iótal

$\begin{array}{ll}1.199-11 & 0.000 \\ 0.214011 & 0.000 \\ 7.059-113 & 0.000 \\ 7.483-11 & 0.000\end{array}$

MS PREgH UDZ35 F.E.

PERTILE PIL. PISSILE PIL.

op-eotomen

$\begin{array}{ll}1.023014 & 0.000 \\ 1.362-13 & 0.000 \\ 2.6760 .11 & 0.000\end{array}$

$\begin{array}{ll}2.676011 & 0.000 \\ 2.692 .11 & 0.000\end{array}$

$1.023-14 \quad 0.000$

$\begin{array}{ll}1.362013 & 0.000 \\ 2.678011 & 0.000\end{array}$

$2.692-11 \quad 0.000$

$\begin{array}{ll}0.743005 & 2.258=05 \\ 0.745005 & 2.258005\end{array}$

$0.745005 \quad 2.258005$

$\begin{array}{ll}0.745005 & 2.250005 \\ 0.745-05 & 2.250-0.05\end{array}$

$\begin{array}{ll}7.017000 & 1.811=08 \\ 3.689005 & 9.524006\end{array}$

$\begin{array}{ll}3.689005 & 9,524=06 \\ 3.696005 & 9,542=06\end{array}$

$7.017008 \quad 1.811004$

$3.089005 \quad 9.524=06$

$3.696005 \quad 9.542006$

$\begin{array}{ll}1.077-11 & 0.000 \\ 5.501 .11 & 0.000 \\ 6.359-13 & 0,000 \\ 6.721011 & 0.000\end{array}$


THIS SEGMENY WAS UISCHARGEO AT

THE HEGINNING OF RELUAD 1 ANO WAS

GAMMA DOSE RATES IN RADIHR PEM F.E. AT UNE METEK AT TIM

IS FRESH U-235 F.E.

NUCLIOE AND

GAAMA ENERO

IN MEV

60.60
1.25

TÓTAL

COBALT

0.6

1.25

Tớt

$\underset{\infty}{\frac{1}{\infty}}$

ALL ELEMENIS

0,1

0.6

0.8

$\prod_{1025}$

1.0

2.4

IOIAL
FERTILE PTL. FISSILE PIL.

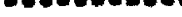

$1.267=04$

$1.267=04$

2.730000

$1.199 \cdot 11$

$6.214-11$

$1.267-04$

7.059013

1.267000

0.000

2.730 .06

0.000

$2.730 \cdot 06$

$2.362 \cdot 01$

6.280001

$4.647+01$

$4.510+00$

$2.310-01$

1.072001

1.072001

2.653001

1.751001

$5,319+01$

0.497001

$0.796=01$

$2.014+02$

$3.755+00$

$1.755+00$

5.6700

5.185001

$1.161+00$

1.874002

$2.485+02$
THIS IEGMENT HAS OISCMARGEO AT

IHE BEGINNING OP HELOAD 5 MND WA

UAIGINALLY LOADED AT KELOAD I

TME 5 YEARS APIER DISCMAMGE

MS FRESH U-23S P. E. FERTILE PTL. BIOSILE PTL.

\subsection{9 .04 \\ 1,350005}

$3.740004 \quad 1,350005$

$1.077-11 \quad 0.000$

$5.581-11 \quad 0.000$

0.330 .1300 .000

3,749004

$2.000+00 \quad 2.601+00$

$4.189+00 \quad 1.479+00$

$4.711+02 \quad 7.222+02$

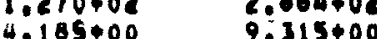

$1.189+0010.315400$

T.

$? .24001$

$3.166+01$

$1.028+00$

0.076001

$\begin{array}{ll}8.667-01 & 1.704002 \\ 2.589 .03 & 2.772003 \\ 0.252+02 & 1.050003\end{array}$

$6.252+02 \quad 1.056+03$ 


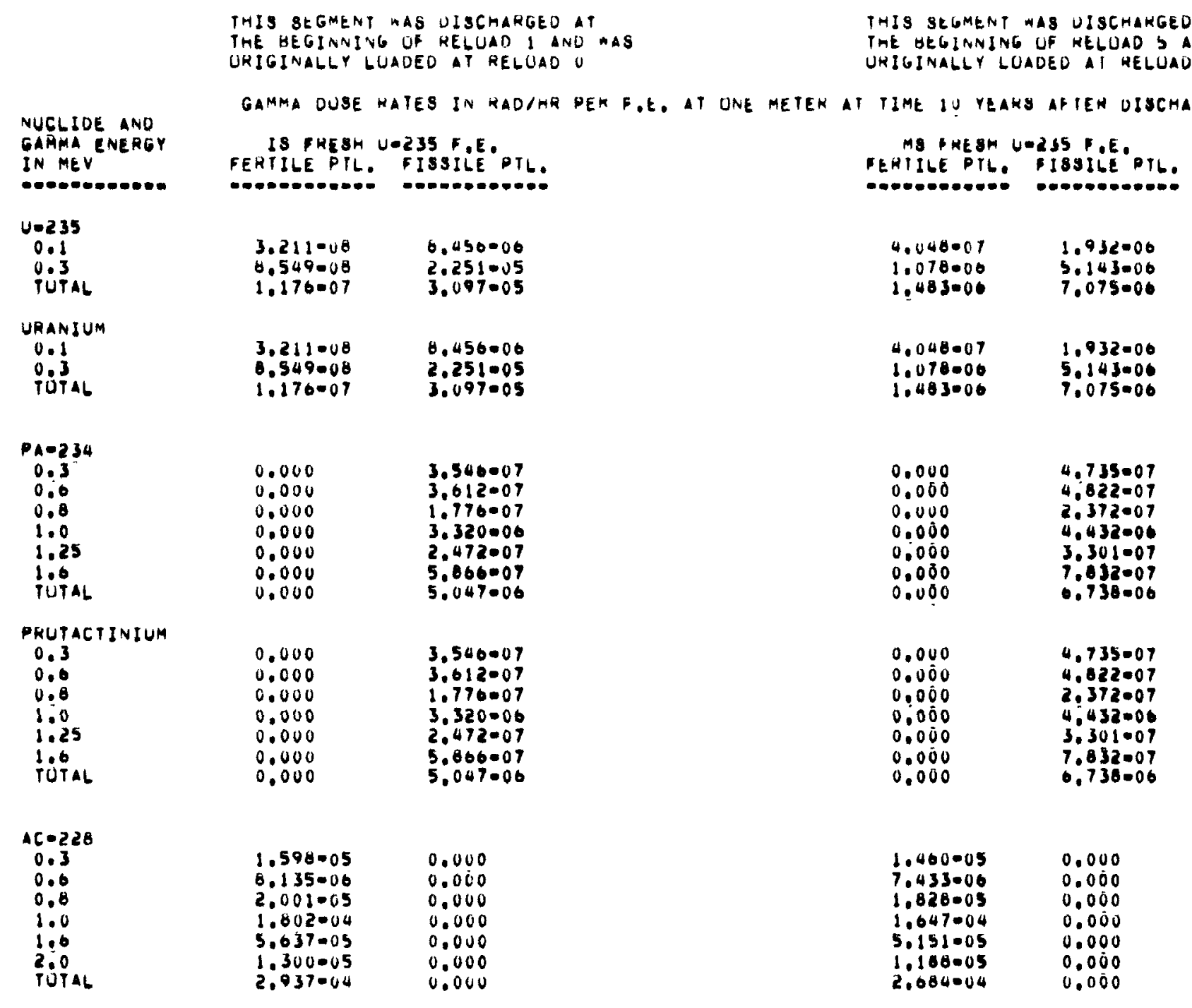




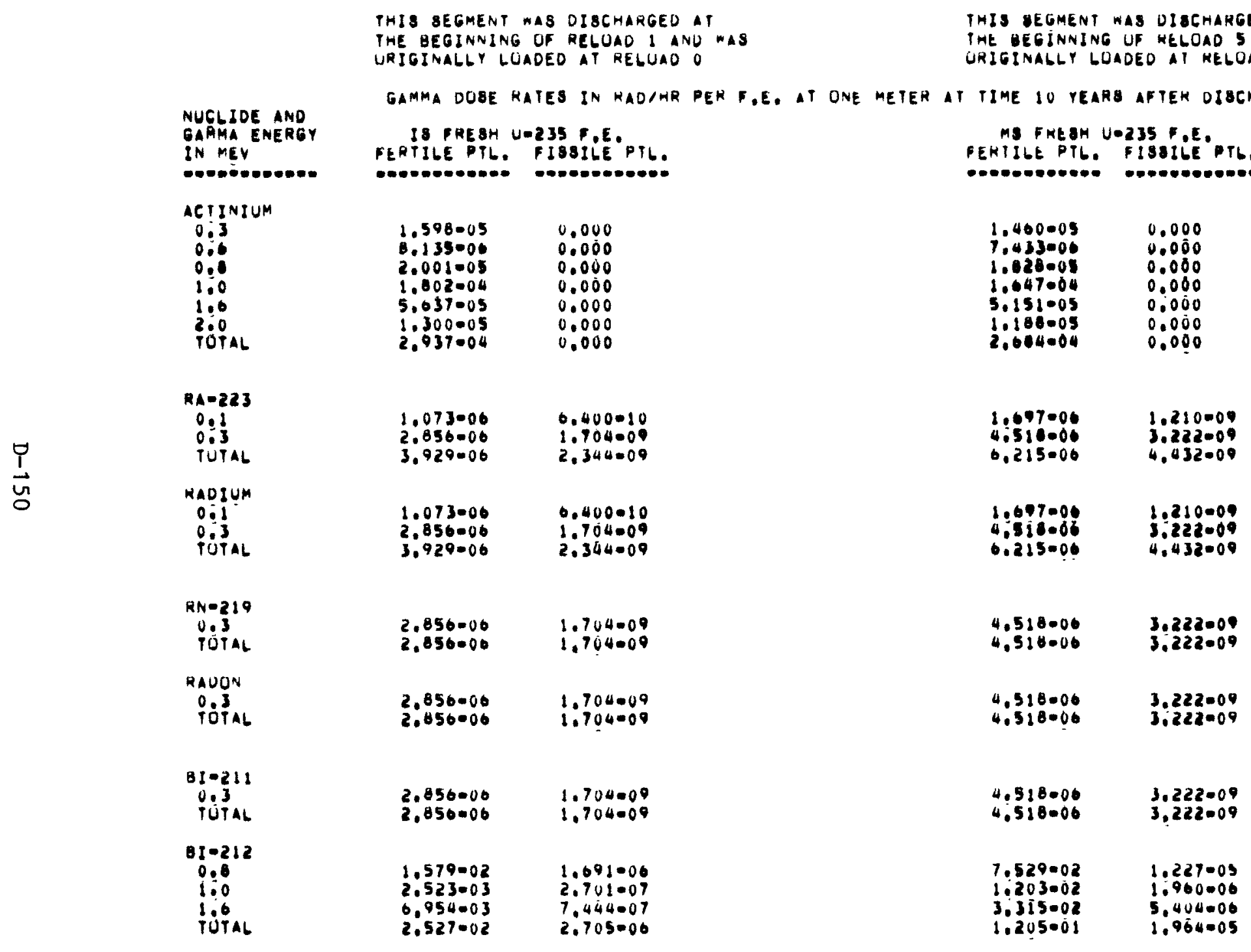




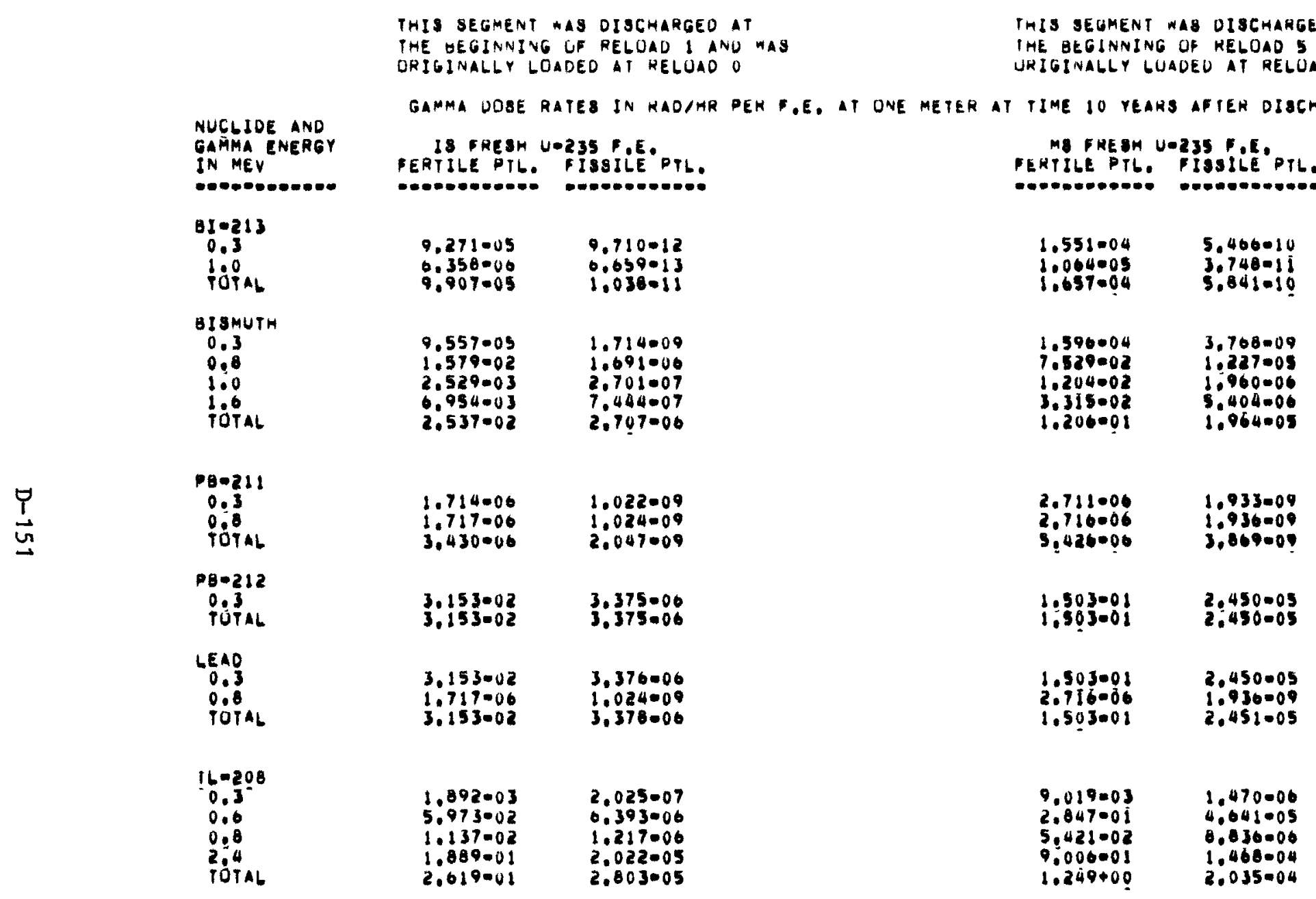


THIS SEGMENT WAS OISCMARGED AT TME BEGINNIAG OF RELUAD I AVO MAS ORIGINALLY LOADED AT RELUAO O

IS FRESH UEZ3S F.E.

NUSLSDE AND IN MEY

\section{THALLIUM}

0.3

0.6

rotal

$T B=158$

$18=15$
0.3
0.8

$1: 0$

$1: 0$

$\stackrel{\vec{N}}{\mathrm{u}}$

PERTILE PIL. FISBILE PTL.

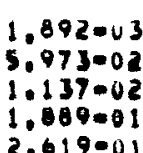

2.025007

6.393004

1.217 .06

2.022005

$2.803=05$

$7,555 \cdot 11$

$0.476-10$

4.957000

$1.501-10$

$3.108-11$

$2.732-10$

2.091009

$0,584.11$

$5,836=0$

$7.555-11$

$3.188-11$

2.732 .10
$0.476-10$

1.561 .10

0.584 .11

2.462 .09

\section{EU-152}

0.15
$0: 3$
$0: 6$
$0: 8$
100
1025
$1074 L$
TOTAL

Cue 154

0.1
$0: 3$
$0: 6$
0.8
$1: 0$
1.25
106
10741

1.126003

2.984 .03

$4.675=04$

3.093003

8.894003
6.999003

$6.999-03$

2.549004
2.384002

1.850003

4.904 .03

8.010004

$5.083=03$

$1.461=01$

1.150002

$3.917=02$

1.032 .02

7.089003

1.864002

1.393001

6.053002
1.592 .01

$1.592-01$

8.663 .01
8.213 .01

$0.018=05$

$1.47800 !$

$.262+000$
$9.746-02$

$3.400+00$

1.141002
THIS SEGMENT WAS OISCHAKGED AT TME BEGINNING OF RELOAD 5 AND WAS ORIGINALGY LUADEO AT RELOAO

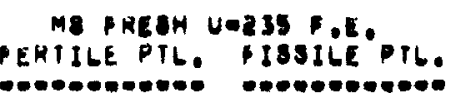

$\begin{array}{ll}9.019003 & 1.470000 \\ 2.847001 & 4.041 .05 \\ 5.421002 & 6.836006 \\ 9.006001 & 1.460004 \\ 1.209+00 & 2.035004\end{array}$

$1.240+00 \quad 2.035004$

$\begin{array}{ll}0.638010 & 9.636-11 \\ 5.672009 & 0.259-10 \\ 4.342000 & 6.321009 \\ 1.367009 & 1.990-10 \\ 5.112008 & 7.443009\end{array}$

\begin{tabular}{|c|c|}
\hline $\begin{array}{l}6.918010 \\
5,672009 \\
4.342000 \\
1.307009 \\
5.112=08\end{array}$ & $\begin{array}{l}9.630-11 \\
0.256-10 \\
0.32200 \\
1.900-10 \\
1.443008\end{array}$ \\
\hline
\end{tabular}

$\begin{array}{ll}9.743004 & 1.283003 \\ 2.983003 & 3.402003 \\ 4.219004 & 5.557004 \\ 2.677003 & 3.520003 \\ 7.697003 & 1.014002 \\ 6.057003 & 9.979003 \\ 2.206004 & 2.906004 \\ 2.063002 & 2.718002\end{array}$

$\begin{array}{ll}3.110001 & 7.150001 \\ 1.351001 & 3.109001 \\ 3.554001 & 8.176001 \\ 1.934+00 & 4.450000 \\ 1.833+00 & 4.218000 \\ 2.818+00 & 0.482+00 \\ 2.176001 & 5.006001 \\ 7.004+00 & 1.749+01\end{array}$


THIS SEGMENT WAS OISCMARGED AT

THE GEGINNING OF RELUAD I ANU MAS

THIS SEGMENT MAS DISCMARGED AT

THE BEGINNSNG GP RELUAD 5 ANO WAS

ORIGINALLY LOADEO AT RELUAD O

ORIGINALLY LOADED AT RELUAD I

NUCLLDE ANO

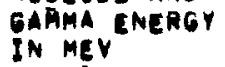

IN MEY

EUROE zUM

SUROQ⿱一⿻上丨)

$0: 3$

0.8

109

1.23

tótal

$P R=14 a$

0.6

0.8

2.0

TótAL

PRABEOOYMIUM

0.6

0.8

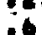

TOPAL

$0 A-137 m$

0.1

TOTAL

BAKIUM

0.1

PótAL

GAMMA DOSE RATES IN RADIHE PEK B. AT ONE METEK AI TIME 10 YEARS ARTEK DISCMARGE

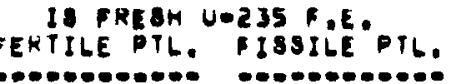

$1.744=0$

1.007002

1.913002

1.046001

1.051001

1.508001

1.167 .02

$4.228 \cdot 01$

$2.467-03$

4.622 .05

2.14400

3.750004

3,040003

6.449003

$2.407-03$

$4.622=05$

2.144005

8.750004

3.040 .03

$0.449=03$

$1.364=0 !$
$3.509+0 !$

$3.013+01$

5.459 .01

$1.041+02$

1.364001

$3.599+01$

5.439001

$1.441+02$
$1.446+02$

1.075002

2,015000

3.813 .03

1.325002
MS FHE CERIILE PTL. FIDSILE PIL

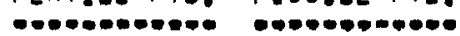

$\begin{array}{ll}3.120001 & 7.169001 \\ 1.377001 & 3.143001 \\ 3.558001 & 8.102001 \\ 1.937000 & 4.453+00 \\ 1.041+60 & 4.220+00 \\ 2.024000 & 0.490+00 \\ 2.170001 & 5.009008 \\ 7.625+00 & 1.752+01\end{array}$

$1.002=02$

990004

$9234=05$

3,760003

1.309002

2,797002

.002002

1,990004

9,234005

3,768003

1,309002
$2,797=02$

7.444 .03

1.395000

0.01205

2.642003

$9: 974003$

7.444003

$1.305=04$

$0.471 \cdot 05$

2.648 .03

9.214003

$\begin{array}{ll}1.147+00 & 1.490+00 \\ 3.026+02 & 3.953+02 \\ 3.039+02 & 3.968+02\end{array}$

$1.147+00$

$147+00$
$3.028+02$
3.039402

$3,028+02$

$1.498+00$

$3.953+02$

$3.953+02$ 
THIS SEGMEAT WAS DISCHARGED AT

UAIGINALLY LUADED AT RELOAD O
INIS SEGMENT AAS DISCMAKGEO AT TAE BEGINNING OF TELUAD S AND WAE URIGINALLY LUADED AI HELUAD I
NUCLJDE AND OARMR ENERO IN MEV

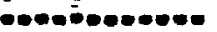

\section{$\cos 134$}

0.3

0.6

$\log _{0} 05$

1.5.

10\%4

CEIUM

0.3

0.6

$\frac{\theta}{u}$

Tótal

\section{$88 \cdot 125$}

0.1

TÓTAL

ANTIMONY

0.1

TOTAL

$1 G+108 M$

0.1
0.3
0.6
0.8
1.0
TOTAL

IE FRESH UDZ33 F,E.

FERTILE PTL. FISSILE PTL.

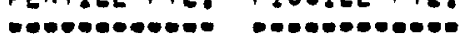

ERTILE PTH.

FISILE PTL.

$\begin{array}{ll}1.042004 & 8.697-04 \\ 7.690001 & 0.423+00 \\ 7.985001 & 6.600+00 \\ 1.091002 & 9.101002 \\ 0.045002 & 5.545001 \\ 3.077=04 & 3.009=03 \\ 1.600+00 & 1.370+01\end{array}$

2.905003

$2.205+01$

3.280401

उ. 12400

$1.904+00$

$1.053=02$

$4.710+01$

$0.788-03$

$3.013+01$

$5.202+01$

4.104001

$4.328+00$
$2.395=02$

$2.395-02$

$.072+02$

$1.042004 \quad 8.697004$

$7.096-01 \quad 0.423+00$

7.985001

$1.091 .02 \quad 9.101002$

$0.645 .02 \quad 5.545 .01$

$3.677=04 \quad 3.069-03$

$1.646+00$

$1.374+01$

2.98500

$2.205+0 j \bar{j}$

$2.280+01$

3.124001

1.904400

1.053002

0.780 .03

$5.013+01$

$5.202+01$

$7.104-01$

$4,328+00$

$2.395-02$

$1,012+02$

1.710002

1.715002

1.628 .01

3.262001

3.251001

5.001 .01

1.710002

1.023001

1.715002

1.028 .01

$3.262-01$

$5.045-01$

5.061 .01

3.230007

2.614 .05

3,910005

$4.438-05$

$5.944 \cdot 07$

1.100004

$1.577=08$

1.276006

$1.912=06$

2.167006

$2.902-08$

5,000000

$1.127001 \quad 3.064002$

$2.143000 \quad 5.826001$

$3.325+000$

$1.127-01 \quad 3.064002$

$1.069+00$

$3,325+00$

2.907 .01

5.820001
9.039001

1.122006

9.001005

1,308004

2.528004

$2.047=06$
$3.808=04$

8.806000

$7.176=00$

1.075005

$1.218=05$

1.632 .07

$3.050=05$

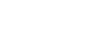


THIS DEGMENT MAS DISCHARGEO AT

TME BEGINNING OF RELOAD I AND MAS

THIS SEGMENT WAS DISCHAKGEO AT

URIGINALLY LUADED AT RELUADO

THE BEGINNING OF RELUAD 5 ANU WA

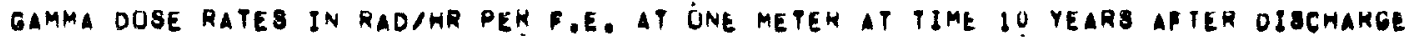

NUCLIDE AND GAMMA ENEAOY

IN MEV

IS FRESH U-235 F.E.

FERTILE PTL. FISIILE PIL.

MS FHESH U-235 B.

FERTILE PTL,

IOSILE PIL.

\section{$\triangle 0 \cdot 110 \mathrm{M}$}

0.13

$0: 3$

$1: 0$

1.25

$2: 0$

34

TƯTAL

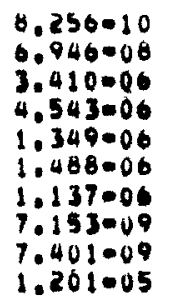

$3.070=09$

2.583007

1.268 .05

5.015006

$5,535-06$

4.229006

$2.660-08$

$2.752-08$

4.467 .05

8ILVER
0.1
0.3
0.6
0.8
1.00
1.25
1.6
2.0
2.04
TOTAL

3.238 .07

$2.621-05$

$4.257=05$

4.892005

1.943006

1.488006

$1.137=06$

$7.401=0$

1.064000

1.535006

$1.459-05$

5.044006

$5.535=06$

4.229 .06

2.660008

$2.752=08$

$5.007-05$

8.811007

4.320005

5.763005

$1.711-05$

1.880005

1.442005

9.07300

0.38800

1.524004

$1.222=08$

$1.020-06$

5.047005

$6.723-09$

$1.996-05$

2.203005

$1.663=05$

$1.050-0$ ?

$1.095-07$

1.710.04

$1.123000 \quad 1.009007$

9.089005

1.701 .04

2.105004

1,915005

1.868005

$1,442=05$

$9.073=00$

9.368000

., 204006

$7.942 \cdot 03$

2,012-05

$2.203-05$

1.663 .05

1.059007

$1.095-07$

2.081004

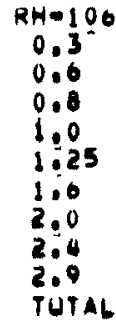

$4.764=05$
$1.382=02$
$3.023=04$
$1.220-03$
$1.059-04$
$2.802-04$
$1.033=04$
$1.033=04$
$1.696-05$
$1.630=02$

$2.774=04$
$8.045=02$

$1.700-03$

$7.102-03$

2.363 .03

1.631 .03

6.017 .04

6.013004

$9.013+04$

9.489002

2.330004

6.717002

$1.082-03$

5.902003

1.991003

$\{.374003$

5.008 .04

5,005004

8.319005

$7.993=02$

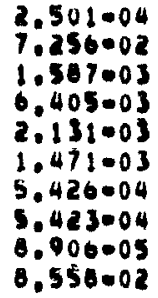




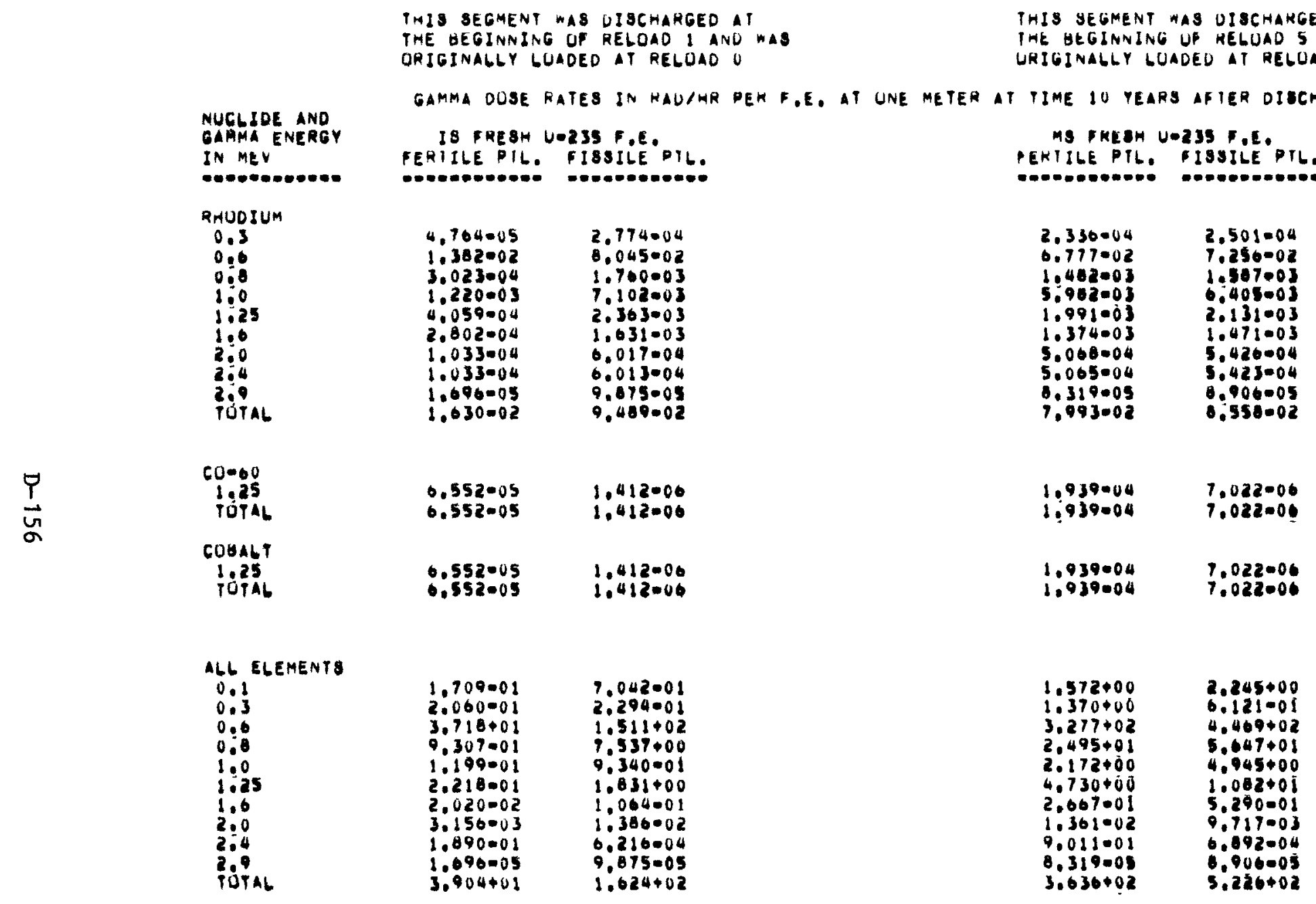


TABLE D-7

GAMMA DOSE RATES FOR FUEL ELEMENTS DISCHARGED AT BEGINNING OF RELOAD 10 AND ORIGINALLY LOADED AT RELOAD 6

NOTE: Table $D-7$ consists of 58 pages of computer print-out (pages D-159 through D-216). 
$\bullet$

•

8 


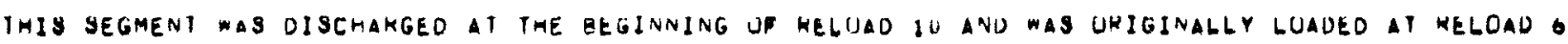
NUCLJOE AND

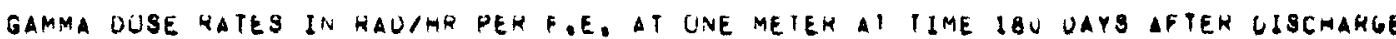

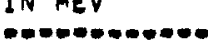

40235

0.1

Tutal

URANJUM

0.1

TÓTAL

$P A \cdot 233$

$0.1=$

TOTAL

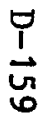

$P A=234$

0.0 GAMMA ENERGY IN MEV

$23 R S$ MECYCLE U-233 R.E.

FERTILE PTL. FISSILE PIL.

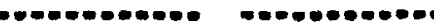

3.820007

1.010000

$1.350=00$

3.610000
4.965000

3.816007

1.010000

1.356006

1.610000
4.965000

$2.029-07$

0.8

1.0

1.25

TÜTAL

PRUTACIINIUM

0.1
0.3
0.6
0.8
1.0
1.25
1.0
TOTAL

0.1

0.6

$1: 0$
1.346000

$1.549=06$

$9.874+01$

$6.551+02$
$7.538+02$

$1.180=02$

1.201002

5.910003

$1.104-01$

0.225003

$1.951=02$

1,67901

$9.874+01$

$0.551+02$

1.201002
$3.910-03$

1,104001

1.104001
$8.225-03$

$1.951=02$

$7,540+02$

$4.595-11$

4.680 .11

$2.302-11$

$3.204-11$

$3.204-1$

$0.601=11$

$2.029=07$

1.340000

$1.346=06$
$4.680-11$

$2,302-11$

$4.302-10$

$3.204-11$

$7.001-11$

1.549000
25RS RECYCLE U.235 F.E.

FERTILE PTL. FISYILE PTL.

3.816007

$\begin{array}{ll}1.398006 & 5.103006 \\ 1.019006\end{array}$

$3.016007 \quad 1.916006$

$\begin{array}{ll}1.016006 & 5.103006 \\ 1.308006 & 7.019006\end{array}$

1.398006

$9.074001 \quad 1.009000$

$6.551+02 \quad 9.089=06$

$7.538+02 \quad 8.157006$

$1.180=02 \quad 1.861006$

$1.201002 \quad 8.895=06$

9.323007

1.10400103

$1.225003 \quad 1.297006$

$1,690001 \quad 2.649005$

$9.874+01 \quad 1.069006$

$0.551+02$

1.895000

10104001

$1.104001 \quad 1.742 .05$

$0.225=03 \quad 1.297006$

$\begin{array}{ll}1.951002 & 3.078006 \\ 7.540+02 & 3.464=05\end{array}$ 


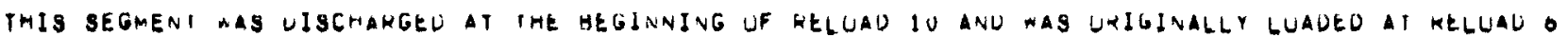
NUCLIDE ANO GAMMA ENERGY IN MEV

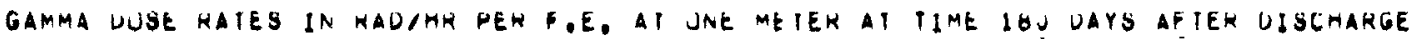

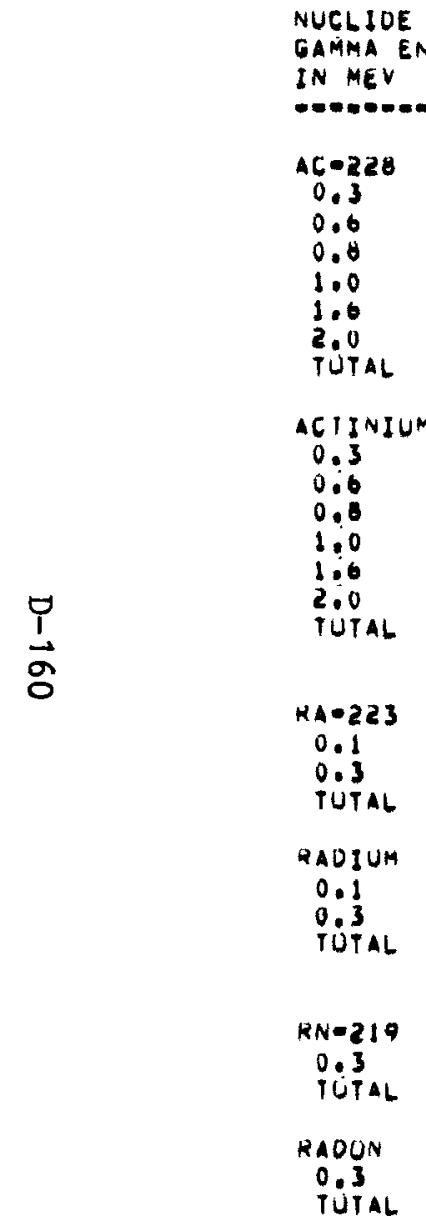

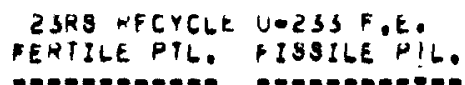

$8.089 \cdot 06$

$4.119-0$

1.013 .05

0.126005

2.854 .05

0.581 .06

1.487 .04

$8.089=06$

$4.119=0$

1.013005

$9.120-05$

$2.854-05$

6.581 .06

1.087 .04

$2.355=06$

$0.269=06$

8.024006

2.355006
0.269000

0.269000
8.024000

6.200 .06

$0.209=06$

6,209000

6.269006
$2.200=00$

3.858000
$8.058=00$

2.200008

$5.858=06$
8.0580 .06

5.858000
$5,858.00$

$5.555-12$

$2.829-12$
$0.950-12$

$0.267-11$

$1.960-11$

$1.021-10$

$5,555-12$

0.956012

$6.207-1$

$1.960-11$

1.021010

$5.858=06$

$5.858=08$
2SRS RECYCLE U-235 F.E.

FERIILE PIL. FISSILE PIL.

$8.089=00$

$4.119000 \quad 0.000$

$1.013005 \quad 0.000$

$9.126005 \quad 0.000$

$2.854005 \quad 0.000$

$\begin{array}{ll}0.581006 & 0.000 \\ 1.489004 & 0.000\end{array}$

$8.089000 \quad 0.000$

$0.119 .00 \quad 0.000$

$1.013005 \quad 0.000$

$2.854005 \quad 0.000$

$0.581 .06 \quad 0.000$

$1.407 .04 \quad 0.000$

$2.355006 \quad 4.400-10$

$6.269000 \quad 1.172009$

0.62400

$2.355-00 \quad 4.400-10$

$0.269 .06 \quad 1.172009$

$8.624=06 \quad 1.612009$

6.269000

$0,269006 \quad 1.172009$

$0.269000 \quad 1.172009$

$0.269000 \quad 1.172009$ 
THIS SEGMENI WAS DISCHaRgEO at THE bEgLNNING UF RELOAO IO ANO WAS ORIGINALLY LUADED AT KELOAO G NUCLIDE AND
GAMMA ENERGY IN MEV

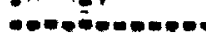

\section{$B+211$}

TOTAL

$1+212$

0.8

1.0

TuTAL

$8[-213$

0.3

TÚTAL

$\stackrel{\varphi}{\vec{a}}$

BI IMUTH

$0.3^{\circ}$
0.8
1.0
1.6

gAMMA DUSE RATES IN KADIHR PER F.R. AT ONE METER AI IIME IBU UAYS ARTEK DISCHARGE

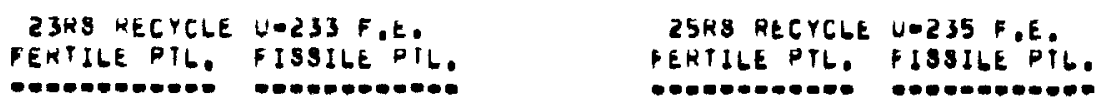

$25 R 3$ RECYCLE U-235 F.E.

PERTILE PTL, DSSILE PIL.

$0.269=00$

$5.858 \cdot 08$

$5.856=0 \%$

0.209006

0.269000

$P B-511$

0.3

PŨal

$P g=212$

PŨTAL

LEAO

0.3

PÚTAL

\subsection{2}

$5.744 .03 \quad 1.092002$

$\begin{array}{ll}1.583002 & 3.010002\end{array}$

2.159005

1.481006

2.108005

$1.446-00$

2.307005

$\begin{array}{ll}2.780005 & 2.114005 \\ 3.590002 & 6.036002\end{array}$

$5.745003 \quad 1.092=02$

. 58300.

3001000

3.010002

$5.750-02$

\subsection{0}

$\begin{array}{ll}3.596002 & 1.300005 \\ 5.744003 & 2.192006\end{array}$

$1.583002 \quad 5.986006$

$2.159005 \quad 1.135009$

$\begin{array}{ll}1.481006 & 7.782011 \\ 2.307-05 & 1.213009\end{array}$

$2.786005 \quad 2.306=09$

1.360005

$.745003 \quad 2.172000$

$\begin{array}{ll}1.583002 & 5.986006 \\ 5.756002 & 2.176005\end{array}$

$3.762000 \quad 3.515008 \quad 3.762-06 \quad 7.029=10$

$3.768=06 \quad 3.521000 \quad 3.768006 \quad 7.042010$

$7.530006 \quad 1.407009$

$7.179-02 \quad 2.7140 .05$

$7.179002 \quad 2.714005$

$7.179 .02 \quad 2.714005$

$\begin{array}{ll}3.760006 & 7.042010 \\ 7.179002 & 2.714005\end{array}$ 
THIS SEGMENT WAS DISCHARGEO AT THE BEgINNING OF RELUAO 10 ANO WAS UKIGINALGY LUADEO AT RELOAO 6 NUCLIOE AND GAPMA ENERGY IN $M E Y$

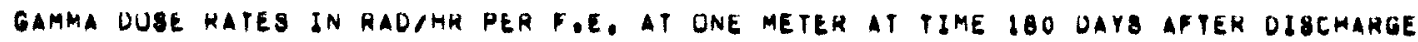

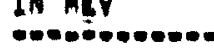

\section{T6-200}

0.3

0.3

:0

THALLIUM

0.3

0.6

9.4

Tótal

$\frac{⿱}{\vec{N}}$

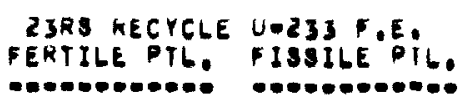

4.106003

1.315001

2.504002

5.160001

4.160003

1.315001

$2.504-02$

4.160 .01

$5.769-01$

$6.000-10$

5.657 .00

4.331 .00

5.099 .08

$2.730=02$
6.610 .02

0.610 .02
$0.772-02$

6.772002
5.076002

$5.076-02$
2.119 .01

$2,730.02$

0.010002

6.772002

5.076002

2. $110-01$
0.189003

2.585001

4,922002

$0.277=01$

$1.134+00$

$8.189=03$

2.585001

$0.922=02$

$1.134+00$

$9.365-10$

0.02600

6.14400

7.234000

$2.888-02$

$6,991-02$

$7.163-02$

5.369002

2.241051

2.808002

$6.99100 \mathrm{C}$

$7.163=02$

$5,369-0$

$2.241-01$
2SRS RECYCLE UA2JS E.E.
FERTILE PTL. FI8UILE PTL.

$\begin{array}{ll}4.166003 & 1.575000 \\ 1.315001 & 4.973=05 \\ 2.504002 & 9.409000 \\ 4.160001 & 1.573004 \\ 5.767-01 & 2.181004\end{array}$

$5.76701 \quad 2.181004$

$4.100003 \quad 1.575000$

$1.315001 \quad 4.073005$

$2.504002 \quad 9.469006$

$4.160002 \quad 1.373=0$

2.181004

$0.600-10 \quad 9.035-11$

$3.657=09$

$4.331-90 \quad 5.9200$

$5,000-05$

6.970 .09

$2.730002 \quad 3.117002$

$6.010002 \quad 7.545002$

6.77a.0e 1.73000

$2.119 .01 \quad 2.410 .01$

$2.730002 \quad 3.117=02$

$0.010002 \quad 7.545002$

$6.772 .02 \quad 7.730 .08$

$5.076002 \quad 5,794002$

$2.119001 \quad 2.419=02$ 
THIS BEGMENT WAS DISCMARGEU AT THE BEGINNING UF RELUAD IU AND WAS UHIGINALLY LOADED AT RELUAD G

NUCLIDE AND

IN MEY

INo.0.0.0.0.

$54 \cdot 152$

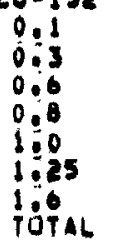

Cue 154

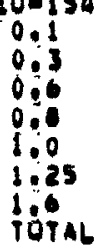

$\frac{p}{\sigma}$

GAMMA DUSE RATES IN RAUJHK PER FeE. AT UNE METER AT IIME 180 UAYS APTER DIBCMAROE

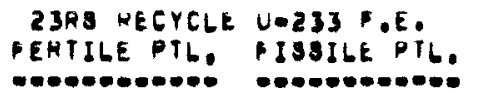

1.694003

4.491 .03

7.337 .04

4.056003

$\prod_{1.05300}^{1.00}$

$3,836-04$

$3,508-02$

4.479001

1.946001

5.118001

$2.785+00$

$2.640 \div 00$

$4.057+00$

3.133001
$1.095+01$

1.072002
3.104002

2.830002

2.830002
7.437 .02

a. $228-02$

0.056003

$2,353-01$

4.496001

$1.991-01$

$5.232=01$

$2.821+00$

$2.662+00$

$4.142+0$

$3.137-01$

0.222002

$1.122+0$

\subsection{3 \\ 5.363003 \\ 5.590005 \\ 1.604002 \\ $1.262=02$ \\ 4.300002 \\ $9.210-01$ \\ $4.001-01$ \\ $1.052+00$ \\ $5.727+00$ \\ $8.343+0$ \\ 0.052008}

\subsection{4 .02 \\ 5.713002 \\ 5.713002
1.501001 \\ 1.059001 \\ 1.059001 \\ 1.620002
4.750001}

0.231001

$4.055-01$

$1.075+00$

$5.796+00$

$8.500+00$

0.447 .01

1.659 .0

1.626002
$2.303+01$
25RS RECYCLE U-235 F.E.

FERTILE PTLE FI8SILE PTL

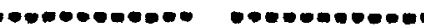

\begin{tabular}{|c|c|}
\hline $\begin{array}{l}1.094003 \\
4.491=03 \\
7.337=04 \\
4.056003 \\
1.339002 \\
1.033002 \\
3.836=04 \\
3.580 .02\end{array}$ & $\begin{array}{l}1.981=03 \\
5.252=03 \\
6.580-04 \\
5.444-03 \\
1.505002 \\
1.232002 \\
4.486-04 \\
4.196002\end{array}$ \\
\hline $\begin{array}{l}4.479=01 \\
1.946001 \\
5.110001 \\
2.785+00 \\
2.640+00 \\
1.057+00 \\
3.133001 \\
1.095+01\end{array}$ & $\begin{array}{l}9.106001 \\
3.950001 \\
1.040+00 \\
5,602+00 \\
5: 367+60 \\
6249+00 \\
6: 370001 \\
2,226+01\end{array}$ \\
\hline
\end{tabular}

$\begin{array}{ll}1.072002 & 2.159-02 \\ 3.164002 & 6.313=02 \\ 2.830002 & 5.647-02 \\ 7.437-02 & 1.484-01 \\ 8.220002 & 1.640001 \\ 8.056003 & 1.607002 \\ 2.353001 & 4.605=01\end{array}$

$4,496=0$

1.991 .01

5.232001

$3.621+00$

$2,682+00$

$3.137=0$

0.222002

8.056 .03

$1.122+01$
0.120001 $4.008=01$ $1.063+00$ $5.730+00$ $5.439+00$ $8.409+00$ $6,374=01$ 1.640001 $1.607-02$ $2.217+01$ 
THIS BEGMENT WAS OIBCHARGEO AT THE BEGSNNING OF RELUAO IO ANO WAB UHIGINALLY LUADEO AT RELOAD G NUCLIDE AND IN MEY ENERGY

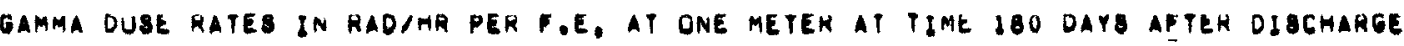

\begin{tabular}{|c|c|c|c|c|}
\hline $\begin{array}{l}P M=140 M \\
0.1 \\
0.3 \\
0.0 \\
0.8 \\
\text { PO: } \\
\text { TOLAL }\end{array}$ & $\begin{array}{l}4.352-02 \\
1.251+00 \\
9.984+00 \\
1.821+00 \\
2.879+00 \\
1.596+01\end{array}$ & $\begin{array}{l}3.218002 \\
9,253001 \\
1.382+00 \\
1.346+00 \\
2.128+00 \\
1.161+01\end{array}$ & $\begin{array}{l}4.352-02 \\
1.251+00 \\
9.984+00 \\
1.821+00 \\
2.879+00 \\
1.596+01\end{array}$ & $\begin{array}{l}2.781002 \\
7.996001 \\
6.379+00 \\
1.163+00 \\
1.030400 \\
1.021+01\end{array}$ \\
\hline $\begin{array}{l}\text { PRUMETHIUM } \\
0.1 \\
0.1 \\
0.6 \\
0.8 \\
1.0 \\
\text { TOTAL }\end{array}$ & $\begin{array}{l}4.352-02 \\
1.251+00 \\
9.984+00 \\
1.821+00 \\
2.079+00 \\
1.590+01\end{array}$ & $\begin{array}{l}3.210002 \\
9.253001 \\
7.302+00 \\
1.340+00 \\
2.128+00 \\
1.181+01\end{array}$ & $\begin{array}{l}4.312002 \\
1.251+00 \\
9.904+00 \\
1.821+00 \\
2.879+00 \\
1.596+01\end{array}$ & $\begin{array}{l}2.711002 \\
7.996001 \\
6.379+00 \\
1.103+00 \\
1.830+00 \\
1.021+01\end{array}$ \\
\hline $\begin{array}{l}\text { ND }=147 \\
0.1 \\
0.3 \\
0.6 \\
T O T A L\end{array}$ & $\begin{array}{l}1.121003 \\
7.102004 \\
3.156003 \\
4.987003\end{array}$ & $\begin{array}{l}4,003=04 \\
2,536=04 \\
1.127 .03 \\
1.701003\end{array}$ & $\begin{array}{l}1.121=03 \\
7.102004 \\
3.196083 \\
4.987003\end{array}$ & $\begin{array}{l}2.910004 \\
1: 863004 \\
8,169004 \\
1,294003\end{array}$ \\
\hline $\begin{array}{l}\text { NEUDYMIUM } \\
0.1 \\
0.3 \\
0: 0 \\
\text { TOTAL }\end{array}$ & $\begin{array}{l}1.121=03 \\
7.102=04 \\
3.156=03 \\
4.987-03\end{array}$ & $\begin{array}{l}4.003004 \\
2.536004 \\
1.127=08 \\
1.781003\end{array}$ & $\begin{array}{l}1.121003 \\
7.102004 \\
3.150003 \\
4.967003\end{array}$ & $\begin{array}{l}2.910=04 \\
1.843004 \\
6.209004 \\
1.290-03\end{array}$ \\
\hline $\begin{array}{l}P R=144 \\
0.6 \\
0.8 \\
1.25 \\
1.6 \\
2.0 \\
\text { TOTAL }\end{array}$ & $\begin{array}{l}4.885+01 \\
9.154-01 \\
4.247=01 \\
1.733+01 \\
6.021+01 \\
1.277+02\end{array}$ & $\begin{array}{l}3.746+01 \\
9.020001 \\
3.257=01 \\
1.329+01 \\
4.617+01 \\
9.795+01\end{array}$ & $\begin{array}{l}4.065+01 \\
9.154=01 \\
4.247=01 \\
1.733+01 \\
0.021+01 \\
1.277+02\end{array}$ & $\begin{array}{l}3.053+01 \\
5.721001 \\
2.654-01 \\
1.003+01 \\
3.763+01 \\
7.903+01\end{array}$ \\
\hline
\end{tabular}




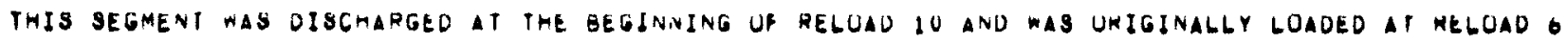
NUCLIDE AND
GAMMA ENERGY

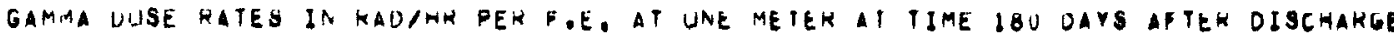
IN MEV

IN MEV

PRABEUOYMIUM

0.6

1.25

1.6

2.0

TUTAL

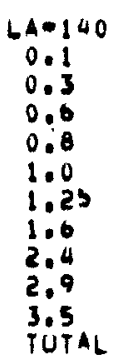

LANTMANUM
0.1
0.3
0.0
0.8
1.0
1.25
1.6
2.4
2.9
3.5
TUTAL

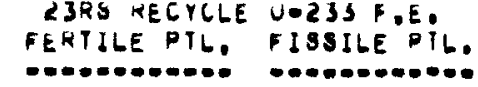

$4.885+61$

$4.247-0$

$1.733+0$

$0.021+01$

1.

$1.127-03$

0.344 .02

$1.713=01$

1.974001

6.955 .02

1.882 .03

$1.011+00$

6.18000

$1.495-03$

5.0980 .05

$1.579+00$

1.127003

$0.344-02$

1.713001

$1.974=01$

$0.955-v 2$

$1.082=03$

$1.011+00$

6.186002

1.495003

$5.098-05$

5.098005
$1.579+00$

$$
\begin{aligned}
& 3.746+01 \\
& 7.020+01 \\
& 3.257=01 \\
& 1.329+01 \\
& 4.617+01 \\
& 9.795+01
\end{aligned}
$$

3.489004

$1.965-02$

$3.305=02$

$6.112=02$

2.154 .02

5,829004

3.131001

1.910002

4.631 .04

1.579005

$4.890-01$

$3.489-04$

$1.905-02$

$5.305=02$

0.112002

2.1540.02

3,131001

1.916002

1.916002
4.631004

\begin{tabular}{|c|c|}
\hline $\begin{array}{l}1.127-03 \\
6.344002 \\
1.713001 \\
1.974001 \\
6.955002 \\
1.882003 \\
1.0111000 \\
6.180002 \\
1.495003 \\
5.098005 \\
1.579+00\end{array}$ & $\begin{array}{l}2.178004 \\
1.226002 \\
3.311002 \\
3.815002 \\
1.344002 \\
3.638004 \\
1.954001 \\
1.190002 \\
2.890004 \\
1.854000 \\
3.052001\end{array}$ \\
\hline
\end{tabular}

$1.579-05$

$4.890-01$
2SRS RECYCLE U.235 F.E.

FERTILE PIL. FISSILE PTL.

$\begin{array}{ll}4.885+01 & 3.053+01 \\ 9.154001 & 5.721=01 \\ 4.247-01 & 2.054001 \\ 1.733+01 & 1.003+01 \\ 6.021+01 & 3.763+01 \\ 1.277+02 & 7.903+01\end{array}$

$1.127-03$

0.344002

1.713 .01

1.974001

$6.955-02$

$1.011+00$

$1.011+00$

0.180002

$5,098-05$

5.098005
$1.579+00$
2.1780104

3.311 .02

$3.815-02$

$1.344-02$

3.638 .04

1.954 .01

1.190002

2.890004

$9.854=06$ 


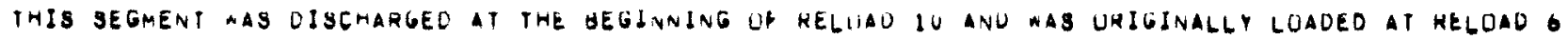

NUCLIDE AND GAMMA ENERGY IN MEY

10

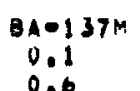

0.6

TUTAL

$B A=140$

0.1

0.3

TOTAL

BARIUM

0.1

0.3

IOTAL

$\frac{1}{\sigma}$

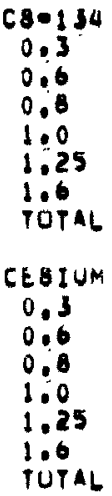

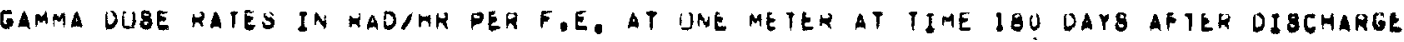

$$
\begin{aligned}
& \text { 23RS RECYCLE UEZ33 F.E. } \\
& \text { FERTILEPILE FISSILE OTL. }
\end{aligned}
$$

$1.358+00$

$3.585+02$

1.169002

2.550002

1.007001

$1.370+00$

$2.550=02$

$3.580+02$

$3.000+02$

6.9190 .02

$5.110+02$

$5.302+02$

$7.241+00$

$2.412+01$

$1.093+03$

$0.917-02$

$5.110+02$

$5.302+02$

$7.241+00$

$4.412+01$

$2.442=01$
$2.209+00$

$5.831+02$
$5.853+02$

3.620003

7.896003

3.120002

., 271002

$2.213+00$

$5.831+02$
$5.853+02$

1.638001

$1.210+03$

$1.255+03$

$1.715+01$

$1.045+02$

$2.588+03$

1.638001

$1,210+03$

$1.255+03$

$1.715+01$

$1.045+02$

5,781001

$2,598+03$
25RS RECYCLE U 235 P.E.

FERTILE PTL FISSILE PTL.

$\begin{array}{ll}1.358+00 & 1.522+00 \\ 3.585+02 & 4.016+02 \\ 3.509+02 & 4.032+02 \\ & \\ 1.109002 & 2.260003 \\ 2.550002 & 4.929003 \\ 1.007-01 & 1.947002 \\ 1.379001 & 2.606002\end{array}$

$1.370400 \quad 1.524400$

$2.550=02 \quad 4.920003$

$3.586+02 \quad 4.016+02$

$\begin{array}{ll}3.586+02 & 4.016+02 \\ 3.000+02 & 4.032+02\end{array}$

0.919002

$0.919002 \quad 1.354001$

$5.110+02 \quad 9.998402$

$5.302+02 \quad 1.037+03$

$7.241+00 \quad 1.417+01$

$4.412+01 \quad 8.633+08$

$2.44201 \quad 4.777001$

$1.003+03 \quad 2.138+03$

$0.919-02 \quad 1.354001$

$5.110+02 \quad 9.990+02$

$5.302+02 \quad 1.037 .03$

$\$ .241+00 \quad 1.417+01$

$4.412+01 \quad 8.633+01$

$2.442=01 \quad 4.777-01$ 


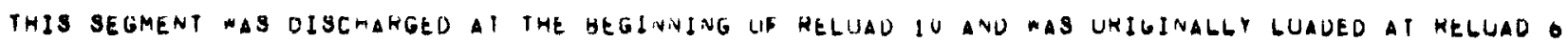
NUCLIDE AND GAMMA ENERGY IN MEV

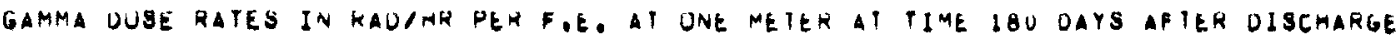

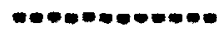

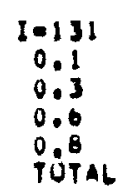

IOOINE

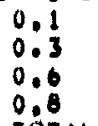

TÖTAL

$\stackrel{\overrightarrow{1}}{\sigma}$

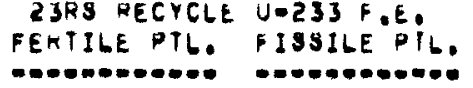

$4.878=00$ $4.244=04$ 0.226005 $1.041=05$
$5.079=04$

$$
\begin{aligned}
& 1.424000 \\
& 1.239004 \\
& 1.818005 \\
& 4.791000 \\
& 1.483004
\end{aligned}
$$

\section{$4.878=00$} 4.244004
0.220 .05

0.220 .05
1.041 .05

$1.641-05$
5.079004

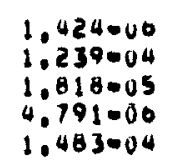

4.192002

$5.440+00$

$1.055+00$
$9.287-002$

$9.287-02$

2.075002

$7.250+00$

$4.471-01$

4.018001

$4.379+00$

$2.460-01$

$8.929=01$

$3.458-02$

$6.402+00$

$4.89 v=01$

4.018001

$9.820+00$

$1.901+00$

9.858001

5.533002

$1.305+01$
1.088002

$1.412+00$

4.29400

2.41000

5,384005

$1,802+00$

1.160001

1.043001

1.136000

$6.383-02$

$2,317=01$

$1.061+00$

1.209001

1.043001

$2.548+00$

4,932001

2.558001

1.430002

$3.543+00$

\begin{tabular}{|c|c|}
\hline $\begin{array}{l}4.878=06 \\
4.244004 \\
0.226005 \\
1.641005 \\
5.079004\end{array}$ & $\begin{array}{l}7.771=07 \\
0.761003 \\
9.919000 \\
2.615000 \\
8.092005\end{array}$ \\
\hline $\begin{array}{l}4.878-06 \\
4.244004 \\
6.220 .05 \\
1.641 .05 \\
5.0790 .04\end{array}$ & $\begin{array}{l}7.771=01 \\
0.701=05 \\
9.919=00 \\
2.615=00 \\
8.092=05\end{array}$ \\
\hline
\end{tabular}

25RS RECYCLE U-235 F.E.

FERTILE HTL. FISSILE PIL.

C....................

4.192002

3.864003

$5.440+00$

$1.055+00$
9.289 .02

$2.075=02$

$7.250+00$

5.015001

1.525001

$0.562=03$

6.684001

4.471 .01

4.018001

4.122002

3.704002

4.037 .01

2.268 .02

8,232002

3.180 .03

$5,902-01$

3.458002

$4.508-02$

$\begin{array}{ll}4.890001 & 4.508002 \\ 4.018001 & 3.704002\end{array}$

$\begin{array}{ll}4.018001 & 3.704002 \\ 9.020+00 & 9.053001\end{array}$

$9.020+00$
$1.901+00$

$9.858-01 \quad 9.088-02$

$5.533 .02 \quad 5.101003$

$1.365+01 \quad 1.259+00$ 


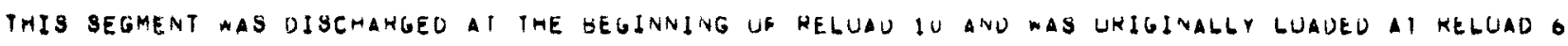
NUCLIDE AND
GAMMA ENERGY IN MEV

GAMTA EnEgr

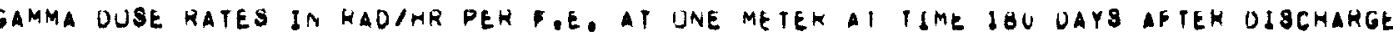

\section{$88=124$}

$\underset{\infty}{\stackrel{\emptyset}{\sigma}}$

0.6

1.0

1.25

iótal

$98-125$

0.1

0.3

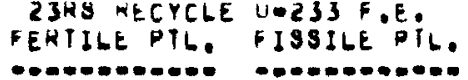

7.568 .03

2.036003

3.830003

5.089 .02

$6.704=03$

., $757=03$

$1.225+00$

$1.162+0$

$3.329+01$

$1.225+00$

$2.162+01$

$2.333+01$

$7.568-03$

2.638 .03

$3,830=03$

.089002

6.704003

$3.625+01$

1.140 .03

5,549004

1,701003

1.140 .03

5.549004
1.701003

$5.988 .0 \mathrm{~d}$

1.028002

3.564003

6.016002

0111003

$1.572=01$

$1.442+00$

$1.369+01$

$2,743+01$

$4.255+01$

$1.442+00$

$1.369+01$

1.02000

1,028002
3.584003

5,205005

0,916002

$0.111-03$

$4.271+01$
5,006004

2.423004
$7.430=04$

5,006004

2.423004
7.430004
1.0

TUTAL

IOIAL
FERTILE PTLE PISIILE PIL.

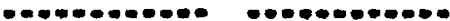

$\begin{array}{ll}4.400002 & 2.598002 \\ 1.508003 & 4.402003 \\ 2.038003 & 1.555003 \\ 3.830003 & 2.258003 \\ 5.089002 & 3.000002 \\ 6.704 .03 & 3.953003 \\ 1.157-01 & 6.821002\end{array}$

$\begin{array}{ll}1.225+00 & 2.029-01 \\ 1.102+01 & 2.605+00 \\ 2.329+01 & 5.300+00\end{array}$

2.329+01 $5.380+00$

$1.225+00 \quad 2.829001$

$1.162+01 \quad 2.605400$

$2.353+01 \quad 5.400400$

$3.830003 \quad 2.258003$

$5.089002 \quad 3.000002$

$6.704=03 \quad 3.953=0$

$3.025+01 \quad 8.416+00$

$1.146003 \quad 3.184000$

$\begin{array}{ll}5.549004 & 1.541=04 \\ 1.701003 & 4.726004\end{array}$

$1.146003 \quad 3.184004$

$5.549004 \quad 1.541 .04$

$1.701 .03 \quad 4.726004$ 


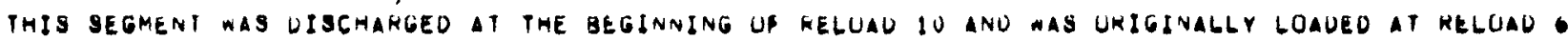

NUCLIOE ANO GAMMA ENERGY IN MEV

IN Men

\section{$10=108 M$}

0.1
0.3
0.6
0.8
1.0
rótal

$160110 \mathrm{M}$

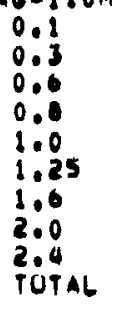

$\frac{\emptyset}{a}$

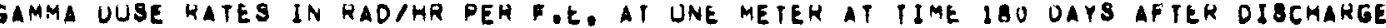

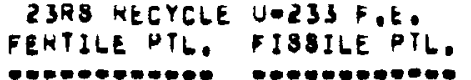

1.130000

9.149005
1.371 .04

1.533004

2.081 .06

3.871004

$1.232-04$

1.030002
5.088001

$0.778-01$

2.012001

2.221001

1.697 .01

1.067 .03

1.104003

$92+00$

1.243004

1.040002

$0.780-01$

$2.012=01$

2.221-01

$1.097-01$

$1.104-03$

$1.793+00$

$1.545-01$

$4.482+01$

$.805=01$

$3.957+00$

$1.317+00$

$.088-01$

$3.352-01$

$3.350-01$

5.502 .02

$5.207+01$

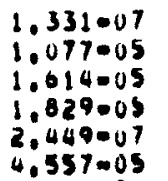

2,449007
$4,557.05$

$$
\begin{aligned}
& 2.035=04 \\
& 1.112002 \\
& 8.406001 \\
& 1.120000 \\
& 3.324=01 \\
& 3.669001 \\
& 2.803001 \\
& 1.703003 \\
& 1.824003
\end{aligned}
$$

$2,961+00$

$2.036-04$

1.713002

$8.406-01$

$1.120+00$

3. 324001

3.669001

$2,803=0$

1.763003

$2.961+00$

1.402001

$4.241+01$

9.277001

$3.744+00$

$1.246+00$

8,599001

$3,172=0$

$3,170=0$

5,206002
25AS RECYCLE U-235 FOE

FERTILE PTL, FISSILEPIL,

$\begin{array}{ll}1.130006 & 2.790-07 \\ 0.199005 & 2.258-05 \\ 1.371004 & 3.303=05 \\ 1.553004 & 3.834005 \\ 2.091006 & 5.135007 \\ 3.871004 & 9.554005\end{array}$

$1.232004 \quad 1.243004$

$1.036002 \quad 1.045002$

$5.080 .01 \quad 5.132 .01$

$0.770001 \quad 6.037=01$

$2.012=01 \quad 2.030001$

$2.221 .01 \quad 2.200001$

$1.697001 \quad 1.711=01$

$1.007003 \quad 1.076003$

$1.104003 \quad 1.114=03$

$1.243004 \quad 1.245=04$

$1.046002 \quad 1.048002$

$5,090001 \quad 5.133001$

$0.780001 \quad 6.837 .01$

$2.012=01 \quad 2.030=01$

$2.221001 \quad 2.240001$

$1.097001 \quad 1.71100$

1.076003

$1.104003 \quad 1.11400$

$1.800+00$

$\begin{array}{ll}1.545001 & 1.405001 \\ 4.482+01 & 4.249+01 \\ 9.80501 & 9.293001 \\ 3.957+00 & 3.750+00 \\ 1.317+00 & 1.248+00 \\ 9.088001 & 8.614001 \\ 3.35201 & 3.177-01 \\ 3.350001 & 3.175001 \\ 5.502-02 & 5.215002 \\ 5.287+01 & 5.011+01\end{array}$




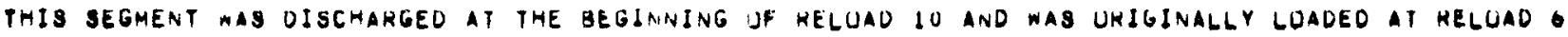

NUELIDE AND GARMA ENERGY IN MẸ

RHUDIUM

0.3

0.0

0.8

$1: 05$

1.6

2.0

2.4

TUTAL

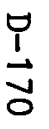

aUe 103

0.1

0.6

RUTHENIUM

0.1

TƯTAL

N8.95

POP

NIUE IUM

90.8

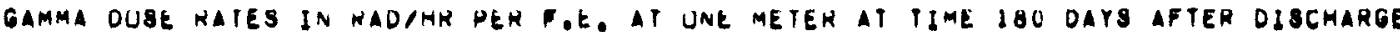

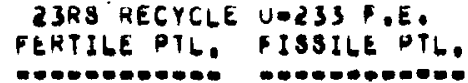

$1.545-01$

$4.462+01$

$9.805-01$

$3.957+00$

$1,317+00$

.088001

3.352 .01

$3.350-01$

5.502002

$5,287+01$

2.794002

$3.720-01$

$? .315+01$

$2.402=01$

$4.241+01$

$9.277=01$

$.744+00$

$.246+00$

$8,399=01$

3.172001

$3,170.01$

.206002

$5,002+01$

1.222002

$1.627=01$

$3.199+01$

$2.704=02$

3.720001

$7.315+01$

$7.315+01$

$1,222=02$

1.627001

$3.199+81$

$3.217+01$

$2.728+03$

$2.728+03$

$9.964+02$

$.964+02$

$2.728+03$

$2.728+03$

$9.964+02$

$9: 964+02$

25RS RECYCLE UNZZ35 F.K.

PERTILE PTL. PI8SILE PTL.

anconaces

$\begin{array}{ll}1.545001 & 1.405001 \\ 4.482+01 & 4.249+01 \\ 9.805001 & 9.293001 \\ 3.957+00 & 3.750+00 \\ 1.317+00 & 1.248400 \\ 9.088001 & 0.614001 \\ 3.352001 & 3.177001 \\ 3.350001 & 3.175001 \\ 5.502002 & 5.215002 \\ 5.267+01 & 5.011+01\end{array}$

$2.794002 \quad 1.003=02$

1.416001

$7.355+01 \quad 2.784+01$

$2.794=02 \quad 1.003002$

$3.720001 \quad 1.416001$

$\begin{array}{ll}7.315+01 & 2.764001 \\ 7.355+01 & 2.799+01\end{array}$

$2.726+03 \quad 0.504+02$

$\begin{array}{ll}2.120+03 & 0.504+02 \\ 2.720+03 & 6.504602\end{array}$ 
THIS SEGMENT WAS OISCHAKGED AT THE GEGINNING OF RELUAD IO ANO WAS UKIGINAGLY LUADED aT RELOAO O NUCLIDE AND
OARMA ENERGY IN MEY

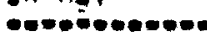

\section{$3 R=95$
0.3}

TOTAL

2IRGUNIUM

0.3

TÜTAL

ne-86

tótal

RUBIOIUM

Gótal

co-58

0.6

TUPAL

60060
1.25

YOPAL

cusab?

0.0

0.85

TOTAL

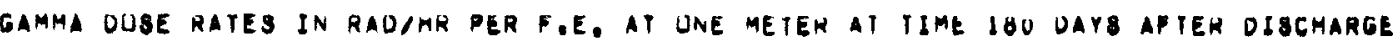

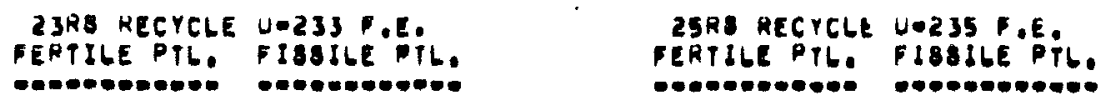

\section{$2.402+00$ \\ $8,003=01$}

$1.294+03$
$1.297+03$

$4.681+02$
4.690002

$2.462+00$

$1.204+03$

\subsection{1}

$4.681+02$

$4,690+02$

$\begin{array}{ll}1.000003 & 1.245003 \\ 1.060003 & 1.245003\end{array}$

1.00000

1.860003
1.860003

$1.245=03$
1.245003

9.115005

4.725004

$5,367=06$

$4.840=00$

2.512005

$2.054=07$

5.090004

3.025005

6.366004

$3.404-05$

$0.366-04$

3.404 .05

$0.115 \cdot 05$

$4.725-04$

0.366004

$5,367-06$
$1,206.03$

4,040000

2,512005

$3.404 \cdot 0$

2.854007
6.429 .05
TIbe PTL. Plaste PTL.

\section{$2.402+00 \quad 5.797 .01$}

$\begin{array}{ll}2.294+03 & 3.048+02 \\ 1.297003 & 3.053+02\end{array}$

$2.462+00 \quad 3.797-01$

1.29403
$1.297+03$

$1.860003 \quad 4.808=04$

$1.660003 \quad 4.502004$

$1.860003 \quad 4.502000$

$9.115005 \quad 1.010005$

$\begin{array}{ll}9.725004 & 5.267005 \\ 5.367-06 & 5.963-07\end{array}$

$\begin{array}{ll}5.367006 & 5,963=07 \\ 5.690-04 & 6.343=05\end{array}$

$0.306004 \quad 7.137005$

$6.360004 \quad 7.137=05$

$9.115005 \quad 1.016005$

$4.725004 \quad 5.267 .05$

$0.366=04 \quad 7.137=05$

$\begin{array}{ll}5.307 .06 & 5.983007 \\ 1.200003 & 1.348004\end{array}$
FERTILE PYL FI8BILE PTL 
THIS SEGMENT WAS DISCHARGEU AT THE BEGLNAING UF RELUAD IO ANO WAB UKIGINALLY LUADEO AT RELUAD D

NUCLIDE AND

GATMMA ENERGY

IN HEV

ALL ELEMENTS

0.1
0.10
0.0
0.0
1.0
1.25
1.0
$2: 0$
$2: 4$
2.9
3.5
POTAL

GAMMA DOSE RATES IN RADIHR PEA F.E. AT ONE METER AT TIME 180 DAYS AFTER DISCHARGE

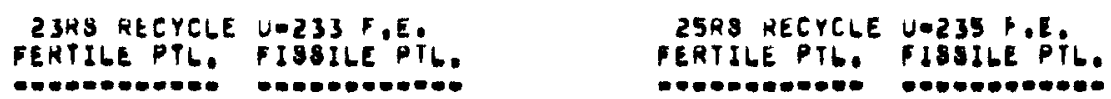

$1.024+02$

$0.718+02$

1.081003

$4.562+03$

$1.820+0$

$5.035+0$

2.00640

$6.063+01$

$8.320=01$

5.651 .02

5.691002

$6.567+03$

$4.750+00$

$1.670+01$

$1.905+03$

$2.731+01$

$2.922+01$

$1.150+02$

$1.007+01$

4.067401

$1.172+00$

5.252 .05

\begin{tabular}{|c|c|}
\hline $\begin{array}{l}1.024+02 \\
0.718+02 \\
1.001+03 \\
4.562+03 \\
1.020+01 \\
5.035+01 \\
2.006001 \\
6.063+01 \\
0.220=01 \\
3.651=02 \\
5.096=05 \\
6.567+03\end{array}$ & $\begin{array}{l}2.004+00 \\
4.964+00 \\
1.517+03 \\
2.002+03 \\
3.580+01 \\
9.654+01 \\
1.320+01 \\
3.011+01 \\
3.400001 \\
5.204=02 \\
9.054000 \\
3.700+03\end{array}$ \\
\hline
\end{tabular}

1.379065

$4.005+u j$ 
THIS SEgMENT MAS OISCHARGED AT IHE BEgINNING UH RELUAO IO ANU WAS UKIGINALLY LUADCO AT KELOAD O NUCLIDE AND GAMMA ENERGY IN MEV

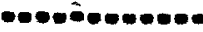

GAMMA DDSE RATES IN RADIHR PER F.E. AT UNE METEK AT IIME I VEAR AFTER DISCHARGE

U.235

0.1

tótal

UAANIUM

0.1

TUTAL

23RS HECYCLE U-233 F.E.

FENTILEPTL, FISSILEPIL.

3.810007

1.016006

1.356000

3.610000

$3.810=07$

1.010006

1.350000

$3.610=06$

PA-233

$0: 1$

$5.635+00$
$6.485+00$

1.745009

1.150008

010234

$\frac{\forall}{\omega}$

5.720005
5.6310 .05

$5.631=05$
$2.808-05$

$2.860-05$

3.092 .05

9.471 .05

8.149004

2.173011

$2.213=11$

1.089011

$2.034-10$

$1.515-11$
3.594011

3.093 .10

$0.494-01$

$5.635+00$

$5.831 \cdot 05$

$2.068=05$

$5.360=04$

$3.992-05$

0.471005

1.745009

1.160000

$2.213-11$

$1.009-11$

$2,034-10$

$1,5,5011$

$3,594-11$

1.6

$6.485+00$

$1.303-06$

25RS RECYCLE U-2SS P.E.
FERTILE PTLE FJOSILE PIL.
-

$3.810007 \quad 1.916000$

$1.016006 \quad 5.103006$

7.010006

$\begin{array}{ll}3.016007 & 1.916006 \\ 1.016006 & 5.103=06\end{array}$

$\begin{array}{ll}1.016006 & 5.103=06 \\ 1.390-06 & 7.019006\end{array}$

$\begin{array}{ll}8.494001 & 9.192009 \\ 5.635+00 & 0.098000\end{array}$

$\begin{array}{ll}5.635+00 & 0.098 \times 00 \\ 6.485+00 & 7.017000\end{array}$

$5.726 .05 \quad 1.861006$

$5.831005 \quad 1.895000$

$2.660005 \quad 9.322007$

5.360 .04

$3.992-05 \quad 1.297-06$

$9.471005 \quad 3.078006$

$8.149004 \quad 2.648 .05$

$8.494001 \quad 9.192009$

$5.035+00 \quad 1,922=00$

$5.631005 \quad 1.895=06$

$2.860 .05 \quad 9.322=07$

5.360004

0471005

$0,485+00$

1.742005

1.297006

2.078006 


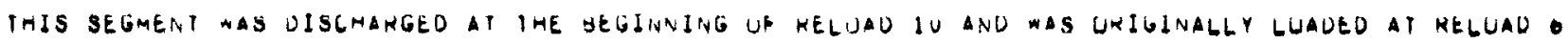
NUELIOE AND
GAMMA ENERGY IN MEV

GAMMA DUSE RATES IN KAOIMH PER F.E
ZSHS HECYCLE UEZ33 F.E.

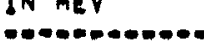

FEHTILE PTLE FISSILE PIL.

$1 C=228$

0.3
0.6
0.8

0.6

1.0

1.0
2.0
rolat

cIINIUM

ACIINIUM
0.3
0.6
0.8
1.0
1.0
2.0
Türab

$\stackrel{i}{i}$

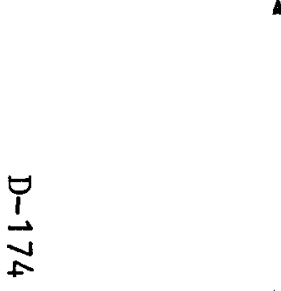

\section{$K A-223$}

0.1

TUTAL

RADIUN

0.1

TUTAL

KNa.219

PUTAL

RADUN

POY TAL

\section{$5.273-12$ \\ $2.685-16$ \\ $0.003-12$ \\ $5.949-11$}

$\begin{array}{ll}9.043005 & 1.861-11 \\ 7.010006 & 4.290-12 \\ 1.586-04 & 9.095-11\end{array}$

$\begin{array}{ll}9.043005 & 1.861-11 \\ 7.010006 & 4.290-12 \\ 1.586004 & 9.095-11\end{array}$

$8,625=06$

4.392 .06

$9.730-05$

$3.043=05$

$7.016-00$

$5,273=12$

$2,685-12$

6.003012

$5.949-11$

$1.861-11$

$1.861-11$
$4.290-12$

$9.695-11$

$2.317-00$

$6.169=06$
$8.485=06$

3.007000

$8.007=00$

1.201007

2.317006

0.169006
$8.485=00$

3.007 .08

$8.007=00$

1.101007

0.109000

8.007006

8,507008

0.109006

$8,007=00$
8,007000
2SRS RECYCLE U.235 .E.

PEHILE PTL. FISSILE PIL.

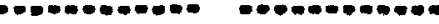

$8.025 .06 \quad 0.000$

$4.392-06 \quad 0.000$

9.0000005

$3.043-05 \quad 0.000$

$7.016-060.000$

1.0160000 .000

$0.025=00 \quad 0.000$

$4.392006 \quad 0.000$

1.080005

$9.730 .05 \quad 0.000$

$3.043 .05 \quad 0.000$

$\begin{array}{ll}7.016000 & 0.000 \\ 1.586004 & 0.000\end{array}$

$2.317000 \quad 0.008010$

$0.109006 \quad 1.600009$

$8.485000 \quad 2.200009$

$2.317 .00 \quad 0.008-10$

$\begin{array}{ll}6.109=06 & 1.000009 \\ 8.485000 & 2.200009\end{array}$

$0.169000 \quad 1.600009$

$0.169000 \quad 1.000009$

$0.109=00 \quad 1.600=09$ 


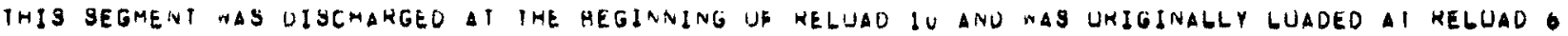

NUCLIDE ANO GAMMAA ENERGY IN MEV ENERGY

\section{$B I-2 ! 1$}

TOTAL

$8]-212$

0.8

1.0

Tóral

0.0 .313

0.3

TUTAL

$\vec{v}$

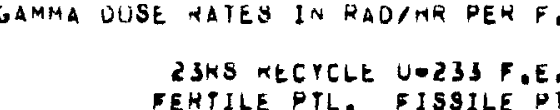

0.109006

4.300002
6.974003

6.974 .03

1.922002
6.9050 .02

2.890005

1.982006

$3.089=05$

$3.507=05$

4.366002

1.922002

0.989002

3.741 .06

$3.700-06$
7.409006

8.716002
8.710002

8.7160 .02

3.708006

8.710002
6.007048

8.007006

0,436002

1,092002

3.01000
1.09400

\subsection{5}

1.526 .06
2.378 .05

2.233005
6.836002
1.092002
3.010002
1.094001

$1.094-01$

4.804008

$4.813=08$

$1.365=0$

$1,365=01$
$1,305=01$

$1.365=01$

4.813000

$1.365-01$
I yeah after dischakge

0.3
0.8
TUTAL

rutal 


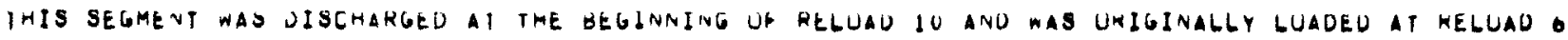

IN MEV ENERGY

$T 6=208$

0.3
0.6
0.8
2.04
TUTAL

THALLIUM

0.3

0.3
0.6
0.8

Tóta

$\frac{i}{\sigma}$

$16-158$

0.3

1.0 NUCLIUE AND GAMMA ENERGY

GAMMA UUSE RATES IN RADUH

$23 H S$ KECYCLE J-233 FEE
FERIILEPIL. FISSILEPIL

FERIILE PIL. FISSILE PIL.

5,202005

1.042001

3.127 .02

1.202001
3.195001

8.189003

2.585001

.922000

0.177001

$1.134+00$

TUTAL

$T B=100$

0.3

1.00

TUTAL

TERBJUM

0.3

0.8
1.0
1.25

TUIAL
5.2020113

$1.642-01$
$3.127-02$

$3.127-02$

$7.202=01$

$8,189=03$

2.585001

$4.922=02$

$8.177-01$

$1.134+00$

$9.343-10$

8,008009

6.130000

1.930 .04

7.217 .00

4.320008

5.087 .08

$4.854-05$

$1: 175=02$

1.204002

$4.589=03$

1.138002

0.531063

$9.023=03$

$4.580-03$

$1.111=02$

1.138002
$8.531=03$

$4.854-0.3$

$1.175=02$

1.204002

$3.767=02$
3.561 .02
1 YEAR AF IEK DISCHARGE

25RS HECYCLE U-235 F.E

FERTILE PTLE FISSILE PIL.

\begin{tabular}{|c|c|}
\hline $\begin{array}{l}5.262 .05 \\
1.042001 \\
3.127 .02 \\
5.195=01 \\
7.202 .01\end{array}$ & $\begin{array}{l}1.960-00 \\
6.208005 \\
1.182005 \\
1.964=04 \\
2.722-04\end{array}$ \\
\hline $\begin{array}{l}5.202003 \\
1.642001 \\
3.127=02 \\
5.195001 \\
7.202=01\end{array}$ & $\begin{array}{l}1.900000 \\
6.208005 \\
1.182005 \\
1.904004 \\
2.722004\end{array}$ \\
\hline
\end{tabular}

$\begin{array}{ll}0.585010 & 9.014011 \\ 5.644009 & 7.7260110 \\ 4.320008 & 5.914009 \\ 1.360009 & 1.0620110 \\ 5.087 .08 & 0.963009\end{array}$

$4.589003 \quad 5.238003$

$1.111=02 \quad 1.260$

1.138002
8.531 .03

$3.501 .02 \quad 4.065 .02$

$4.589003 \quad 3.238003$

$1.111 .02 \quad 1.268002$

$1.138002 \quad 1.299002$

3.501002

9.758003
4.065002 


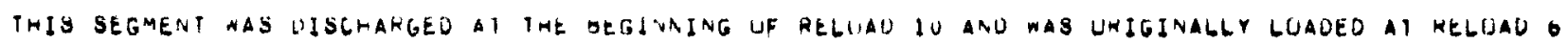

NUGLIUE ANU IN MEV

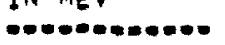

EU.
0.152
0.3
0.6
0.8
1.0
1.25
1.0
TUTAL

\section{EU⿻ 154}

0.1

0.6

0.6

1.0

$\underset{⿱}{\ominus}$

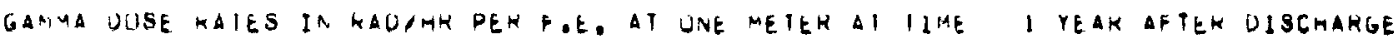

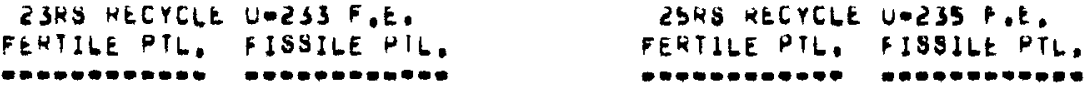

CSRS HECYCLE U.233 F.E.

FEHTILE PTL, FISSILE PIL.

C............

2SKS RECYCLE U-235 . .E.

FERTILE PTL. FISSILE PTL,

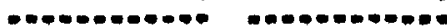

$1.640003 \quad 1.975001$

$4.369003 \quad 5.236-113$

$1.137 .04 \quad 8.553 .04$

1.302002

$1.238=02$

1.228002

$\begin{array}{ll}3.731=04 & 4.472=04 \\ 3,490-02 & 4.182=02\end{array}$

$4.382-01$

$1.904-01$

5,00000

$2.724+00$

$2.505+00$

$3.969+00$

$3.005-01$
$1.071+01$

9.011-01

$3.914=01$
$1.030+00$

$5.602+00$

$5.311+00$

$8.162+00$

0,303001

$2,203+01$

$2.566=06$

$7.572-60$

$6.773-40$

1.780005

$1.908-05$

$1.928=06$
$5.031=05$

5.178000

1.528 .05

$1.367-05$
3.592 .05

3.971 .05

3.8910100

$1,137.04$

4.395001

$1.947-01$

$5.014-01$

$2.729+00$

$2.596+00$

$3.979+00$

3.069 .01

$1.968-05$

$1,928-60$

$1.075+u 1$

.030001

$3.967-01$

$1.030+00$

$5.608+00$

$5.327+40$

6.307 .01

3.971 .05

$2,207+01$

\begin{tabular}{|c|c|}
\hline $\begin{array}{r}1.648003 \\
4.369003 \\
7.137004 \\
4.520003 \\
1.302002 \\
1.025002 \\
3,731004 \\
3,490002\end{array}$ & $\begin{array}{l}1.927003 \\
5.109003 \\
8.306=04 \\
5.290003 \\
1.523=02 \\
1.198002 \\
4.364=04 \\
4.081=02\end{array}$ \\
\hline
\end{tabular}

$0.382-01 \quad 8.908001$

$1.904001 \quad 3.870001$

$5.006-01 \quad 1.016+00$

$2.724+00 \quad 5.539+00$

$2.565+00 \quad 5.251+00$

3.969600

$3,065-01 \quad 6.231=01$

2.178 .01

$2.560006 \quad 5.119000$

$7.572 .06 \quad 1.511005$

$1.700005 \quad 3.551 .05$

$1.968-05 \quad 3.925005$

$5.631 .05 \quad 1.123004$

$\begin{array}{ll}4.398001 & 6.928001 \\ 1.947001 & 3.921001\end{array}$

$5.014001 \quad 1.019+00$

$2.729+00 \quad 3.544000$

$2.596+00 \quad 5.266+00$

$3.979+00 \quad 8.082+00$

$3.069001 \quad 0.236001$

$1.968 .05 \quad 3.925-05$

$1.928006 \quad 3.840000$

$1.075+01 \quad 2.102+01$
$6.773006 \quad 1.351 .05$

$1.928000 \quad 3.846006$ 


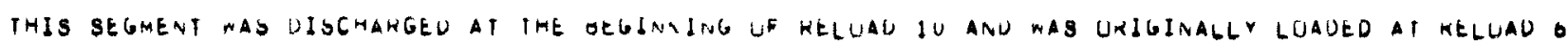

NUCLIDE AND GAMMA ENEKGY IN MEV

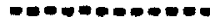

$P M=148 M$

0.1

0.3
0.0
0.8
1.0

TUTAL

PRUMETHIUM

0.1

0.3

0.6

1.0

IUTAL

$\stackrel{\varphi}{\infty}$

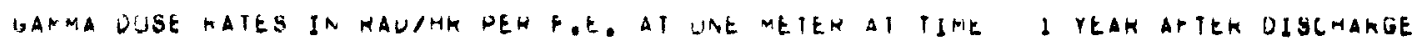

23RS RECYCLE UOZ33 F.E.
FEKTILE PTL. FISSILE PIL.

.......... .

$\begin{array}{ll}2.010-03 & 1.491-03 \\ 5.798-02 & 9.287-02 \\ 4.625-01 & 3.420-01 \\ 8.435-02 & 0.237-02 \\ 1.334-01 & 9.861-02 \\ 7.402-01 & 5.493-01\end{array}$

$2.416=03$ $4,025=01$

$4.025=01$
$8.435-02$

0.035002

1.334001
7.402001

1.491003

$4.287-02$

3.420001

6.237 .02

9.861 .02

NO.147
0.1
0.3
0.6
TUTAL

NEUOYMIUM

0.1

0.3

TUTAL

$P R=144$

0.6
0.8
1.25
1.0
2.0
10141

0.0

1.25

TUTAL
1. $\cup 18=08$

$6.447-09$

$2.865=08$
$4,527=08$

3.634009

$2.302-0$

$1.023=00$

1.016008

$0.447-09$

2.605000

$3.634=09$

2.302009

$1.023=00$

1.010000

$3.100+01$

5.824001

$10.103+01$

$3.831+01$

$8.127+01$
$2,384+01$

$4.467-01$

$2.072=01$

$8.456+0$

$2,438+0$
ZSKS RECYCLE LOE3S F,E

FERIILE PTL. PISSILE PTL.

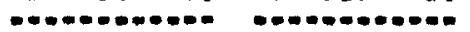

$\begin{array}{ll}2.010003 & 1.288003 \\ 5.798002 & 3.705002 \\ 0.025001 & 2.955-01 \\ 0.435002 & 5.390002 \\ 1.334001 & 8.521=02 \\ 7.402001 & 4.730001 \\ & \\ 2.010003 & 1.288003 \\ 5.798002 & 3.705-02 \\ 4.02501 & 2.955001 \\ 8.435002 & 5.390002 \\ 1.334001 & 8.521002 \\ 7.402001 & 4.730001\end{array}$

$1.018008 \quad 2.642000$

$0.447-09 \quad 1.073=09$

$\begin{array}{ll}2.865-08 & 7.434009 \\ 4.527008 & 1.175008\end{array}$

$1.018008 \quad 2.642009$

$6.447 .09 \quad \frac{1}{1.673009}$

$2.865=00 \quad 7.434=09$

$3.108+01 \quad 1.943+01$

5.802010100

$2.103+01 \quad 1.689-01$

$3.831+010.39400$

$\begin{array}{ll}3.127+01 & 5.394+01 \\ 8.1279+01 & \end{array}$ 


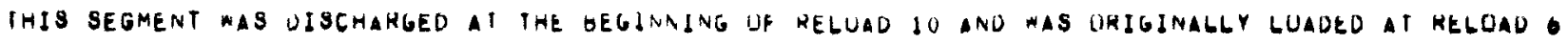
NUCLIDE AND

GAMMA UUSE KATES IN RAOIHK PER F.E. AT LNE MEIER AT IIME

I VEAF AFTER UIBChaAge

IN MEY

PRABEODYMIUM

0.6

1.25

1.0

TOTAL

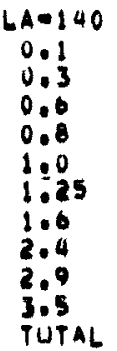

GANTHANUM

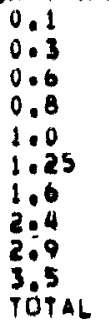
GAMMA ENERGY IN MEV

$23 K S$ RECYCLE U-233 F.E. FERTILE PTLE FISSILE PIL.

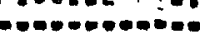

$$
\begin{aligned}
& 3.108+01 \\
& 5.824-01 \\
& 2.702-01 \\
& 1.103+01 \\
& 3.831+01 \\
& 8.127+01
\end{aligned}
$$

\begin{tabular}{|c|c|}
\hline $\begin{array}{l}4.959-08 \\
2.792-06 \\
7.539006 \\
8.686006 \\
3.061-06 \\
8.283-08 \\
4.449-05 \\
2.723=06 \\
0.581-08 \\
2.244009 \\
0.949-05\end{array}$ & $\begin{array}{l}1.536=08 \\
8,648=07 \\
2,335000 \\
2,690006 \\
9,481=07 \\
2,565=08 \\
1.378=05 \\
8,432=07 \\
2,038=08 \\
6,948=10 \\
2,152005\end{array}$ \\
\hline $\begin{array}{l}4.959=08 \\
2.792000 \\
7.539=06 \\
8.680006 \\
3.061=06 \\
6.283=08 \\
4.449=05 \\
2.723=06 \\
0.581=08 \\
2.244009 \\
6.949=05\end{array}$ & $\begin{array}{l}1.536=00 \\
8.648=07 \\
2.335=06 \\
2.090=00 \\
9.481=07 \\
2.565=00 \\
1.378=05 \\
8.432=07 \\
2.038=00 \\
6.948=10 \\
2.152005\end{array}$ \\
\hline
\end{tabular}

$$
\begin{aligned}
& 2.364+01 \\
& 0.407-01 \\
& 2.072001 \\
& 0.456+00 \\
& 2.938+01 \\
& 0,233+01
\end{aligned}
$$

$\underset{v}{\nabla}$
25 RS RECYCLE UE 235 . .E. FERIILE PTL. FISSILE PIL.

$\begin{array}{ll}3.106+01 & 1.943+01 \\ 5.824001 & 3.640001 \\ 2.702001 & 1.08901 \\ 1.103+01 & 6.891+00 \\ 3.831+01 & 2.309+01 \\ 8.127+01 & 5.079401\end{array}$

$4.959008 \quad 9.586009$

$2.792 .06 \quad 5.398=07$

$7.539060 \quad 1.457-06$

$8.686006 \quad 5.679006$

$3.001006 \quad 5.918 .07$
8.283008
0.040005

4.449 .05

$2.723 .06 \quad 5.263 .07$

$0.581 .08 \quad 1.272008$

$2.344=09 \quad 1.272000$

0.949 .05

$4.959008 \quad 9.586009$

$2.792 .06 \quad 5.398007$

$7.539006 \quad 1.457006$

$8.686006 \quad 1.679006$

$3.061 .06 \quad 5.918007$

Q.283.08 $\quad 1.601 .08$

$0.409005 \quad 0.601006$

$2.723=06 \quad 5.263007$

$0.501 .08 \quad 1.272008$

$\begin{array}{ll}2,204009 & 4.337-10 \\ 0,949-05 & 1.343005\end{array}$ 


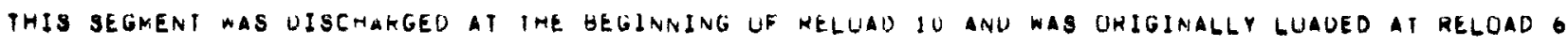
NUCLIDE AND

gamma duse kates in raujhm pek p.e. at une metek al time i reah aptek discharge

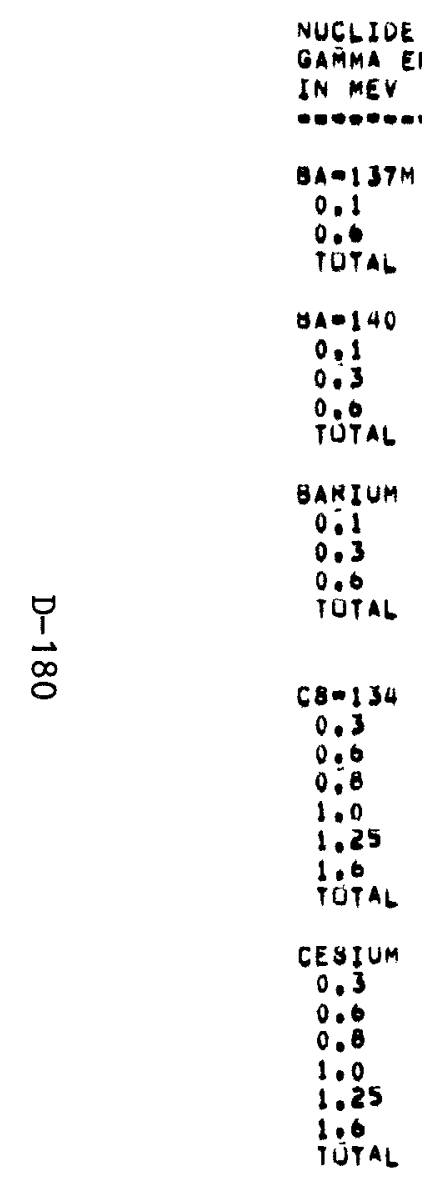
GAMMA ENERGY IN MEV

Z3RS MECYCLE UEZ3SFOE.
FERTILE PTL. FISSILE PIL

PERTILE PTL, FISSILE HIL.

$0.137 M$

0.1

TOTA

TÜTAL

0.1

0.3

$\cos \{34$

0.6

1.0

TA

SIU

0.6

TUYAL

\section{1. $343+00$ \\ $2.183+00$}

$3.543+02$

$3.557+02$

$5.785+02$

5.145007

$1.122=06$

4.433006

1.593007

3.475007

1.373000

$1.343+00$

$1.122=06$

$3.543+02$

$2,183+00$

$3.475-07$

$5.763+02$

$5.785+02$

$5.827-02$

$4.303+02$
$4.465+02$

$4.065+02$
$0.098+00$

$3.716+01$

$3.710+01$
$2.056=01$

$9.204+02$

5.827 .02

$4.303+02$

$.465+02$

$0.098+00$

3.716401

$2.056-01$

$9.204+02$
1.380001

$1.019+0$

$1.057+03$

1.00401

8.79040

$0.869=01$
$2.179+03$

1.380001

$1.019+05$

$1.057+03$

1.444001
$8.798+01$

?.179+03
25RS RECYCLE U-235 PE.

-

$1.343+00 \quad 1.504+00$

$\begin{array}{ll}3.543+02 & 3.970+02 \\ 3.557+02 & 3.985+02\end{array}$

$5.145007 \quad 9.945000$

1.122006

$0.070-06$

$1.343+00 \quad 1.504+00$

$1.122-06$

$3.543+02$

$3.985+02$

$5.827-02 \quad 1.240001$

$.303+02 \quad 8.421+02$

$4.465+02 \quad 8.737+02$

$6.096+00 \quad 1.193+01$

$3.716+01 \quad 7.270+01$

$\begin{array}{ll}2.056001 & 4.023001 \\ 0.204+02 & 1.001+03\end{array}$

$5.827-02 \quad 1.140-01$

$4.303+02 \quad 6.421+02$

$4.665+02 \quad 0.737+02$

$0.096+00$

$3.710+01 \quad 7.270+0$

$\begin{array}{ll}2.050=01 & 4.023-01 \\ 9.2114+02 & 1.801+03\end{array}$ 


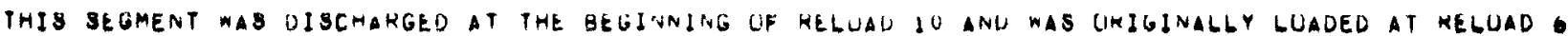

NUCLIDE ANO IN MEV

0.0000000

$\stackrel{\ominus}{!}$

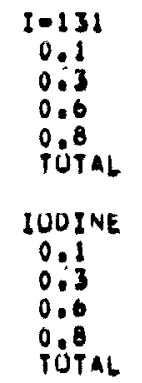
GAMMA ENERGY

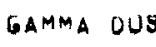

GAMMA DUSE
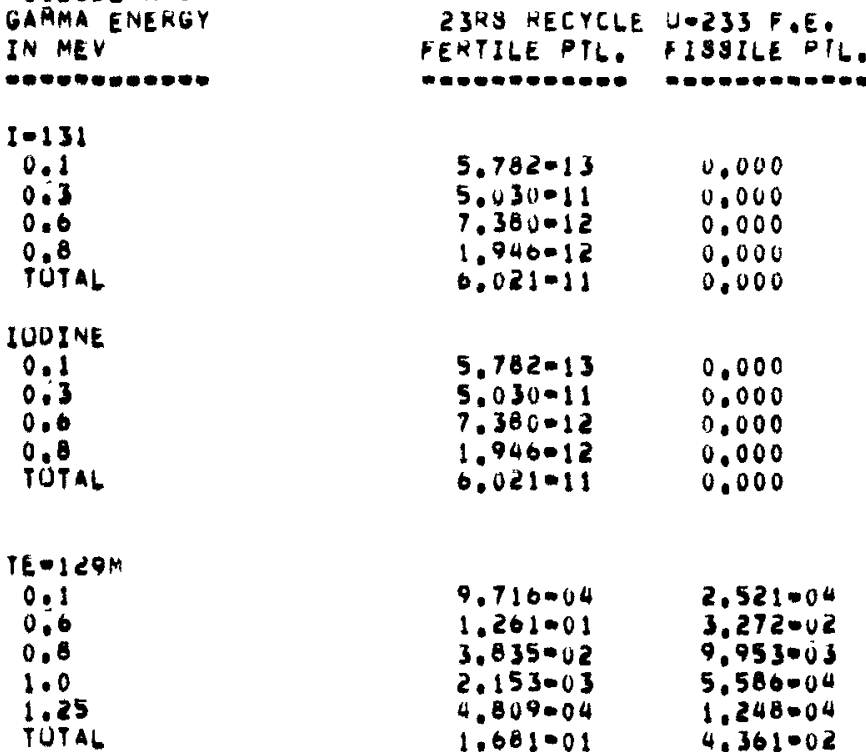

$T E=129$

0.1

0.3

0.6

1.0

TUTA

TELLURIUM

0.1

0.0

0.8

1.0

TUTAL
$5.782-13$

$5.030-11$

$1.940-12$
$0.021-11$

$5.782-13$

$5.030-11$

$360-12$
$1.946-12$

$6.021-11$

0.000

0.000

0.000

0.000

0.000

0.000

0.000
0.000

0.000

0,000

9.716004

1.261001

$3.835=02$

$2.153-03$

4.809 .04

1.681001

9.312003

$5.015=0 !$

0.070002

$8.015=04$

1.484001

5.521 .04

3.272002

5.586004

1.2480 .04

4.361 .02

$2.689=03$

1.479003

5.371 .03

5.371003

3.851 .02

$1.134-02$
9.312003

$2.276-01$

4.405002

$2.285=02$

1.282003
3.160 .01

$2.417-03$

5.907 .02

$1,143=02$

$5,930 \cdot 01$

$3,328-u$

8,212002
1.030002
25HS RECYCLE U-235 F.E.

FERTILE PTL, FIS\$ILE PIL.

$\begin{array}{ll}5.782-13 & 0.000 \\ 5.030-11 & 0.000 \\ 7.380-12 & 0.000 \\ 1.040-12 & 0.000 \\ 6.021-11 & 0.000\end{array}$

$5.742-13 \quad 0.000$

$\begin{array}{ll}5.030 .11 & 0.000 \\ 7.300012 & 0.000\end{array}$

$1.940-12 \quad 0.000$

$0.021-11 \quad 0.000$

$9.716004 \quad 0.957-05$

$1.261 .01 \quad 1.162002$

$3.035002 \quad 3.530003$

$4.809 .04 \quad 4.433005$

$1.681 .01 \quad 1.549-02$

$1.036-02$

9.312 .03

$1.015=01$

$5.701=03$

$2,070=02$

8.01500

9.354004

8.585004

9.356003

5.256004

1.908 .03
7.389 .05

1.360 .02

$1.134 .02 \quad 1.045=03$

$9.312-03 \quad 0.585-04$

2.276001

4.405002

2.205 .02

$1.282-0$

2.098 .02

$4.001=03$

$2.107=03$

$2.107=03$

1.182004
$2.917-02$ 


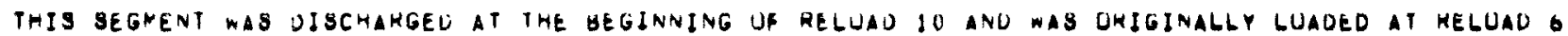
NUCLIDE AND

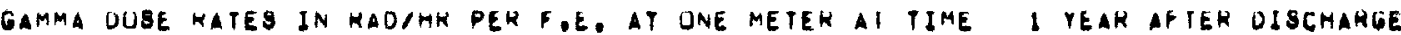
ENERGY IN MEV

IN MEV

$232 S$ RECYCLE U-233 F.E. FERTILE PIL, FI83ILE PIL.

$88=124$
0.6
0.8
1.0
1.25
1.0
2.0
TOTAL

$38-125$

0.3

PÜTAL

$\forall$
$\stackrel{D}{\infty}$
$N$
5.220 .03

8.977 .04

$3.126-04$

$4.543=04$

$6.636-113$

7.95206

$1.372=02$

$1.076+00$

$1.021+01$

$2.040+01$

$1.070+00$

$1.021+01$

2.046401

$8,077-04$

3.128004

$4.543-04$

0.036003

$3.175+01$

$5.794-05$

$2.805=05$

$8.598-05$

$5.794=05$

$2.805=05$

8.598 .05
$1.102=03$

$1.220-03$

4.251 .04

6.173004

1.081 .03

$1.865=02$

$1.207+00$

$1.202+01$

$2.409+01$

$1.267+00$

$1.202+01$

$2.410+01$

1.220003

$.173-04$

1.08100

34000

53005

1.225005

$3.755=0.5$

$2.530=05$

$1.225=05$

$3,755-05$
25RS RECYCLE UE235 F.E.

FERTILE PIL, +1SSILE PIL.

$\begin{array}{ll}5.220003 & 3.081003 \\ 8.977004 & 5.202004 \\ 3.128004 & 1.844004 \\ 4.543004 & 2.670004 \\ 6.036003 & 3.559003 \\ 7.952009 & 4.680004 \\ 1.372002 & 6.090003\end{array}$

1.076000

$1.021+01$

$2.046+0$

$3,174+01$

2.485001

$2.350+00$

$4.725+00$
$7.332+00$

$1.076+00$

$1.021+01$

$2.046+01$

$0.977=04$

3.128004
4.543004

$6.036=03$

$7.952=04$

$3.175+01$

2.405002

$2.358+00$

$4.720+00$

$5.292=04$

$1.044=04$

2.678004

2.670004

$3.559-03$
$4.686-04$

$7.340+00$

$5.794005 \quad 1.609005$

$\begin{array}{ll}2.805005 & 7.790=06 \\ 8.590-05 & 2.388=05\end{array}$

$\begin{array}{ll}5.794-05 & 1.609005 \\ 2.805005 & 7.790006\end{array}$

$\begin{array}{ll}2.805005 & 7.790006 \\ 8.598 .05 & 2.388=05\end{array}$ 


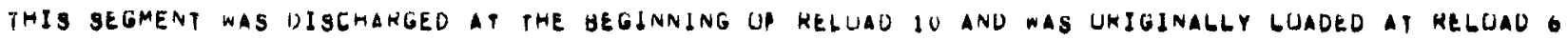

NUCLIDE ANO GAPMA ENERGY

IN MEV

\section{AG-108M}

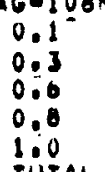

TUTAL

$A G=110 M$

0.1

0.3

0.0

$1: 0$

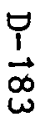

2.0

TÓTAL

SILVER

0.3

0.6

1.0

1.25

$1: 0$
2.0
2.4

TUTAL

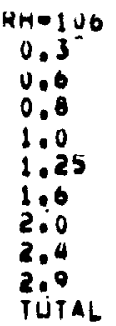

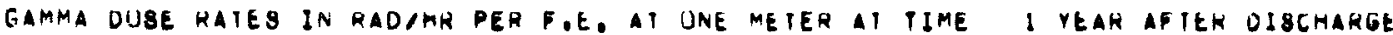

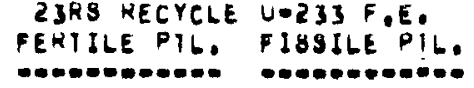

PEHILE PIL:

FISSILE P!L.

$\begin{array}{ll}1.127-00 & 1.327-07 \\ 0.125005 & 1.074005 \\ 1.367-04 & 1.009=05 \\ 1.549-04 & 1.824005 \\ 2.075-00 & 2.443=07 \\ 3.861-04 & 0.545=05\end{array}$

$7.440-05$

$6.265-03$

3.070001

4.097 .01

1.210001

1.342001

1.026001

.451 .04

$0.075=06$

$1.083+00$

$7.559-05$

$0.356=03$

3. $077-01$

$4.099-01$

$1.210-01$

1.342001

1.026 .01

$0.451-04$

$0.075=04$

$1.004+00$

$1.090-01$

$3.163+01$

$0.918-01$

2.792400

$9.290=01$

$6.412-01$

2. $365=01$

2. 360001

3.730401
1.230004

$1.035-02$

$5.081-01$
$6.769-01$

$2.009=01$

2.009001
2.218001
1.090001

$1.694-001$

1.066003

1.103003

1.790000

1.231004

1.036002

5.081 .01

$0.769=01$

$2.009-01$

2.21000

$1.060-03$

1.103003

$1.790+00$

$1.032-01$

$2.993+01$

0.54600

$8.791-01$

0.79100

0.0700

2.23800

2.2370.

3.673002
$3.530+101$
$25 R 3$ RECYCLE U.235 F.E.

FENIILE PTLO PISSILE PTLO

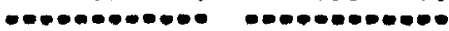

$\begin{array}{ll}1.127000 & 2.782007 \\ 9.125005 & 2.252=05 \\ 1.307004 & 3.374=05 \\ 1.549=04 & 3.824=05 \\ 2.075=06 & 5.121=09 \\ 3.801004 & 9.528=05\end{array}$

$7.446=05$

7.511008

$6.319=0.3$

$3.103=01$

$4,135=01$

1.227001

1.354001

$\begin{array}{ll}1.020001 & 1.035001 \\ 6.451-04 & 6.507004 \\ 0.675004 & 0.733004 \\ 1.0034000 & 1.093400\end{array}$

$\begin{array}{ll}1.020001 & 1.035001 \\ 6.451-04 & 6.507004 \\ 0.675004 & 0.733004 \\ 1.0034000 & 1.093400\end{array}$

$\begin{array}{ll}6.675004 & 0.733004 \\ 1.003+00 & 1.093+00\end{array}$

$7.539-05$

$0.356003 \quad 7.539005$

6.34200.

$3.077001 \quad 3.103=0$

4.099 .01

1.21000

$1.342-01$

1.026001

$.051=04$

0.675004

$1.084+00$

4.133001

1.227 .01

1.354 .01

1.035001

6.507 .04

6.733004

$\begin{array}{ll}1.090-01 & 1.033001 \\ 3.163+01 & 2.998+01 \\ 6.918-01 & 0.557001 \\ 2.792+00 & 2.646+000 \\ 9.290001 & 8.80501 \\ 6.412001 & 0.078001 \\ 2.305001 & 2.242001 \\ 2.364001 & 2.240001 \\ 3.882-02 & 3.679002 \\ 3.730+01 & 3.535+01\end{array}$




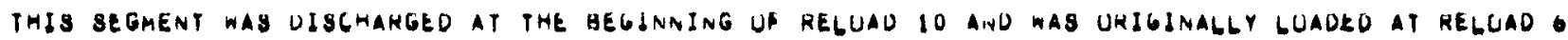
NUCLIDE AND
GAMMA ENERGY IN MEV

GAMMA UUSE RATES IA RAUIMK PER Fot AI UNE METEH AI TIME

I YEAK AFTEH DISCHARGE

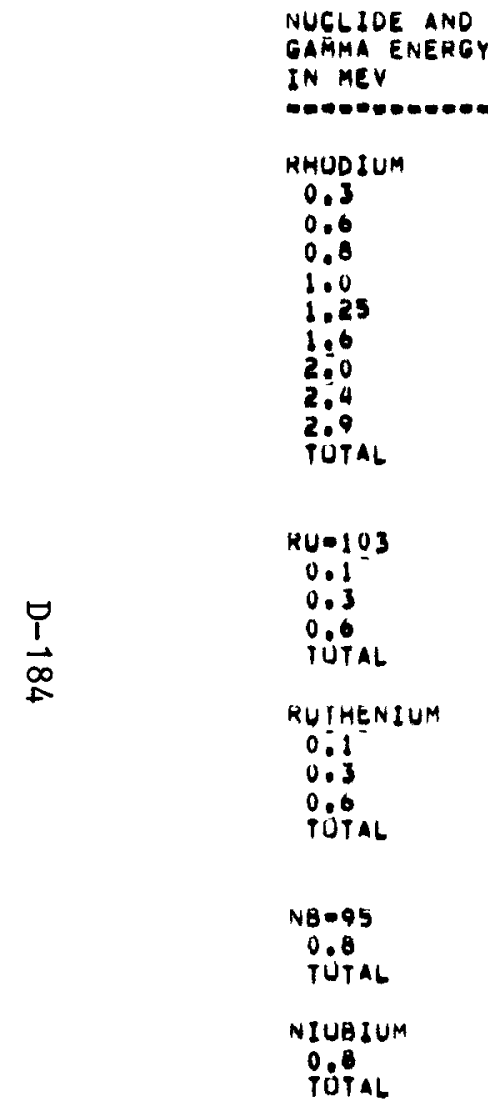

$23 R S$ RECYCLE U-233 F,E. FERTILE PTLO PISSILE PIL

-

$1.090-01$

$3.163+01$

$0.918-01$

$2.792+00$

9.29000

$6.412=01$

2.36500

2.364 .01

3.882002

$3.730+01$

1.032001

$2,993+01$

$6.546-01$

$2.641+00$
$0.791-01$

6.067001

2.230 .01

2.237 .01

$3.673=02$

3.530401

$1.109=03$

1.476002

$2.903+00$

$4.850=04$

$0.456-03$

$1.270+00$

$2.919+00$

$1.277+00$

1.109 .03

1.476002

$2.903+00$

4.850004

0.456003

$1.270+00$

$1.277+00$

$4.239+02$

$4.239+02$

$1.535+02$

$1.535+02$

$4.239+02$

$4.239+02$

1.535 .02

$1.535+02$
25RS RECYCLE U-235 F.E.

FEHILE PIL. FISSILE PIL.

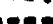

$\begin{array}{ll}1.090-01 & 1.033001 \\ 3.163+01 & 2.690+01 \\ 6.918001 & 6.557001 \\ 2.792+00 & 2.640+00 \\ 9.290001 & 0.80501 \\ 6.412001 & 0.076001 \\ 2.365001 & 2.242001 \\ 2.304001 & 2.240001 \\ 3.882002 & 3.679002 \\ 3.730+01 & 3.535+01\end{array}$

$1.109003 \quad 4.220004$

$1.476002 \quad 5.018-03$

$2.903+00 \quad \quad 1.105000$

$1.111+00$

1.109003

1.476002

$4.220=04$

5.6200003

$1.105+00$

$1.111+00$

$4.239+02 \quad 1.000+02$

$4.239+02 \quad 1.000+02$

$\begin{array}{ll}4.239+02 & 1.000402 \\ 4.230+02 & 1.000402\end{array}$ 
THIS SEGMENT WAS DISGMARGED AT THE BEGINNING JF KELUAD IO ANO MAB UNIGINALLY LUADED AT KELGAO G

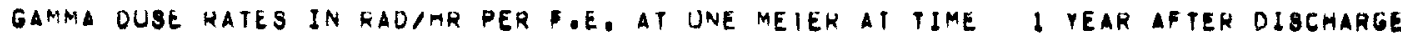

NUCLJDE AND

IN MEY

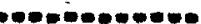

$2 R=95$

0.3

TOTAL

IIHCONIUM

0.3

TớTAL

$\$ 8-80$

TƯTAL

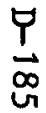

NUBIOIUM

†ÜTAL
23RS KECYCLE UE233 F.E. FERTILE PTL. FISSILE PIL.

\section{$3.471-01$}

$1.825+02$

$1.828+02$

$3.471-01$

$1.825+0$

$1.828+02$

1.908006

C0.58

0.6

Pútal

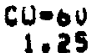

TUTAL

CUSALT

0.6

0.8
1.25
1.0

TÜTAL
$1.908=00$

1.908006

$1.277-00$
$1.277-00$

$1.508=05$

$7.817-05$

$8.880=07$

9.414005

$5.954-04$

5.954 .04

$1.508-05$

$7.817-05$

$5.954=04$

$0.895-04$

$4.157=06$

4.722000

$5.006=00$

$3.184-05$
$3.184-05$

$0.019-01$

$4.157-00$

3.184005

4.722008

$3.664-05$
25HS RECYCLE UEZSS F.E.

PERTILE PTL. FISJILE PTL.
$1.255-01$
$0,599+01$

$0.012+01$

1.255001
$6.509+01$

$0.599+01$
$6.612+01$

$1.277=00$

\section{$3.471001 \quad 0.173002$}

$1.825+02 \quad 4.299+0$

$1.828+02 \quad 4.305+0$

$\begin{array}{ll}3.471001 & 0.173=02 \\ 1.825+02 & 4.297+01\end{array}$

$\begin{array}{ll}1.825+02 & 4.297+01 \\ 1.028+02 & 4.305+01\end{array}$

$\begin{array}{ll}1.900006 & 4.700007 \\ 1.908006 & 4.700007\end{array}$

$\begin{array}{ll}1.908006 & 4.700007 \\ 1.908006 & 4.700007\end{array}$

$\begin{array}{ll}1.508-05 & 1.691006 \\ 7.817 .05 & 8.715006 \\ 8.880-07 & 9.900008 \\ 9.414005 & 1.050005\end{array}$

$5.954004 \quad 0.676005$

$5.954=04 \quad 6.670005$

$\begin{array}{ll}1.500005 & 1.681=00 \\ 7.817005 & 8.715006 \\ 5.954004 & 6.670005 \\ 8.880007 & 9.900008 \\ 6.895004 & 7.725005\end{array}$


THIS SEGMENT WAS UISCHARGEU AT TME GEGJUNING JF RELUAD IU ANU MAS UKIGINALLY LUADED AT KELUAD O NUGLSDE AND

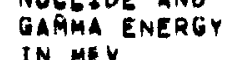

IN MEV

IN MEN

ALL ELEMENIS

0.1

0.3

0.6

0.8

1.25

1.6

2.4

2.9
3.5

$\not$
$\vec{\infty}$
$\sigma$

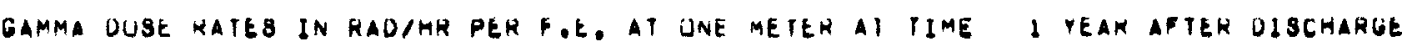

$23 R S$ RECYCLE U-233 F.E

FENTILE PTL FIBSILE PIL.

25RS RECYCLE UA235 F.E

FERTILE PTL. FI8BILE PTL

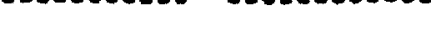

$\begin{array}{ll}3.722+00 & 4.358+00 \\ 1.074+01 & 1.300401 \\ 8.724+02 & 1.677+03 \\ 1.058+03 & 1.284+03 \\ 1.178+01 & 2.274+01 \\ 4.248+01 & 9.740+01 \\ 1.231+01 & 1.039+01 \\ 3.855+01 & 2.961+01 \\ 7.565001 & 1.043+00 \\ 3.882002 & 3.673002 \\ 2.244009 & 6.940010 \\ 2.056+03 & 3.100+01\end{array}$

$3.722+00$

$1.670+01$

8.724402

1.050 .03

$1.170+01$

$0.240+01$

$1.231+01$

$3.855+01$

7.565001

$3.002=02$

2.244009

$2.056+03$

948010
$.140+05$

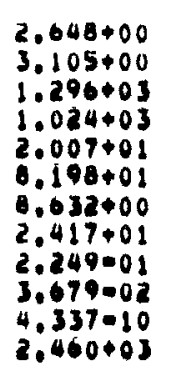




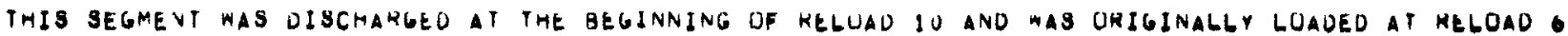

NUCLIOE AND
GAMMA ENERGY

IN MEV ENERG

U.235

0.1

9.3

URANIUM

0.1

TU. 3 AL

$P A=233$
$0.1^{2}$
0.3
TUTAL

$\frac{⿱}{\infty}$

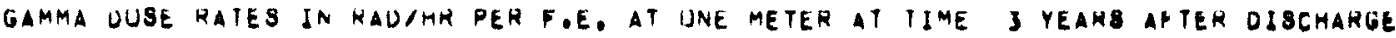

$$
\begin{aligned}
& \text { 23RS HECYCLE U-233 F.E. } \\
& \text { FERTILE PTL FISSILE PIL }
\end{aligned}
$$

$3.810-07$

1.016000

1.356000

$3.610=00$

3.810007

1.016000

1.356000

3.610000

$4.965-06$

$0.082=09$

$4.035=06$

$4.043=08$

0.000
0.000

0.000
0.000

1.6

PRUTACIINIUM

0.1

0.3

0.6

0.8
1.0
1.25

1.6
0.000

0.000

0.000

0.000

0.000

0.000

\section{$2.161-11$}

$2.201-11$

1.083018

$1.507-11$

$3.575=11$

$3.076-10$

$0.082=09$

$4.035=08$

0.000

0.000

0.000

0.000

0.000

$4.643=08$
0.000

$2.101-11$

$2.201-11$

$1.083-11$

$2,023-10$

$1,507-11$

$3.575-11$

$3.076-10$
$25 R 8$ KECYCLE U-235 F.E.

FENTILE PTL. FISSILE PTL.

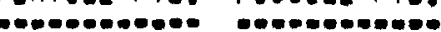

$3.810007 \quad 1.916006$

$1.016006 \quad 5.103006$

7.019006

$3.010007 \quad 1.910000$

$1.010006 \quad 5.103006$

$1.390006 \quad 9.019006$

6.002009
$4.035-08$

$\begin{array}{ll}4.035008 & 0.000 \\ 4.043000 & 0.060\end{array}$

0.000

0.000

0.000

0.000

0.000

0.000

0.000

$1.061=06$

$1.895-00$
$9.322-07$

1.742005

1.297006

3.078006

$0.082=09 \quad 0.000$

$4.03500 \quad \quad 1.861000$

$0.000 \quad 1.895=06$

0.000

0.000

0.000

0.000

9,322007

$1.897-06$

3.07000 
THIS SEGMENT WAS DISCHARGED AT THE BEGINAIAG JF RELUAU IU ANO WAS URIGINALLY GUADED AT RELOAD O

NUCLIDE AND

GAMMA ENEKGY

IN MEV

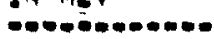

$A C-228$

0.3
0.6
0.6

0.6

1.0

2.00

†ับิ

ACIINIUM

$0: 3$

0.6

1.0

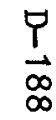

TữTAL

$N A=223$

0.1

Tutal

RAUIUM

0.1

TótAL

$R N-219$

TUTAL

RADON

Tútal

GAMMA DUSE RATES IN KADIMR PER F.E. AT ONE METER AT IIME 3 YEAHS ARIEN DISCMARGE

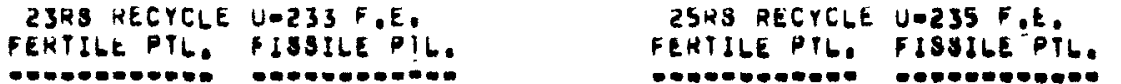

$1.048-05$

$5,337-06$

1.312 .05

$1.182=00$

3.698 .05

9.526006

$4.295 \cdot 12$

2.187 .12

5.379012

$4.646-11$

1.516011

18

1.040005

$5.337-06$

1.31200

$1.182=0$

$3.698=05$

0.520000

$1.927-04$

\section{$4.295=12$}

$2.187-12$

5.379012

4.04001

$1.516=11$

$.097+1$

2.173000

$5.785=06$

$0.335=00$

1.687 .07

2,320007

$2.173=00$

$5.785=00$

7.9580 .06

$0.335-08$

$1.687=07$

2.320007

$5.785=06$
$5.765=00$

$1,087=07$

1.607007

$5.785=06$

1.687 .07
$1.087-01$
FETIILE PTLE FISSILE PTL

\begin{tabular}{|c|}
\hline $\begin{array}{l}1.040=05 \\
5.337=06 \\
1.312=05 \\
1.182=04 \\
3.698=05 \\
8,526=06 \\
1.927=04\end{array}$ \\
\hline $\begin{array}{l}1.048005 \\
5.337006 \\
1.312005 \\
1.182004 \\
3.698005 \\
8.526006 \\
1.927-04\end{array}$ \\
\hline
\end{tabular}

$\begin{array}{ll}2.173006 & 1.212009 \\ 5.765006 & 3.226009\end{array}$

$\begin{array}{ll}5.785006 & 3.226009 \\ 7.958006 & 4.438009\end{array}$

$2.173006 \quad 1.212009$

$5.785006 \quad 3.226009$

$7.958-06 \quad 4.436-09$

$5.785=00 \quad 3.226009$

$5.785006 \quad 3.226009$
$5.785-06 \quad 3.220009$ 


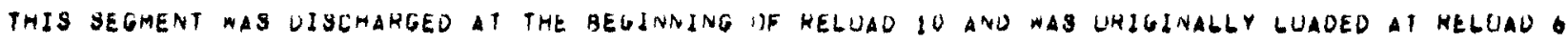
NUCLIDE ANO
GARMA ENERGY

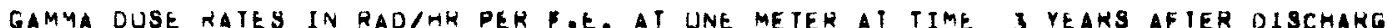

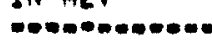

$8 I-211$

Tót

10.212

1.0

IOTAL

$[\cdot 213$

0.3

TUTAL

BISMUTH

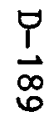
IN MEV

23RS RECYCLE UEZ3S FEE.
FEKTILE PTL. FISSILE PTL.

$5.785=06$

$5.785=06$

1.687007
1.087007

0.2780 .02

$1.003=02$
$2.764=02$

$1.004=01$

1.092002

$1.133=02$

$3.123=02$
1.135001

$3.774=05$

$3.960=06$

0.170005

2.687 .05

1.843000
2,872005

$6.353-05$

0.278 .02

$1.003-02$

$2.704-02$

$2.704=05$

7.092002

1.133002

1.135001

Tớlal

$P B=2+1$

0.3

TUTAL

$P B=212$

Pơtal

LEAD

0.3

TUTAL

$\begin{array}{ll}3.471 .06 & 1.012-01 \\ 3.479000 & 1.014007\end{array}$

$2.020=07$

$1.253-01 \quad 1.410001$

$1.253001 \quad 1.416-01$

$3.477 .06 \quad 1.01400$
CSHS RECYCLE U-235 R.E.

FERTILE PTL FISILEPTL.

\section{$\begin{array}{ll}5.785006 & 3.226009 \\ 5.785-06 & 3.226009\end{array}$}

$0.278 .02 \quad 2.370005$

1.003002
2.704002

$1.004-01 \quad 3.792-05$

$5.774005 \quad 1.136009$

$\begin{array}{ll}3.960006 & 7.790011 \\ 6.170005 & 1.214009\end{array}$

6.17000

$0.353 .05 \quad 4.362009$

2.370005

$\begin{array}{ll}1.003002 & 3.780006 \\ 3.764002 & 1.003005\end{array}$

$1.005-01 \quad 3.792005$

$3.471-06 \quad 1.936009$

1.939009

$1.253001 \quad 4.731005$
$1.253001 \quad 4.731-05$

1,410001

$1.253-01$

$3.477-06$

$1.253=01$

4.732 .05

$1.939=09$

$4.732=05$ 
THIS SEgMENT WAS UISCHAKGEU AT TAE BEgINNING UF KELIAL IO ANU WAS URIGINAGLY LUADEO AT HELUAD G NUCLIDE AND GAMMMA ENERGY IN MEV

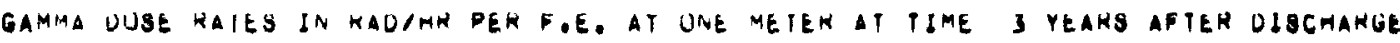

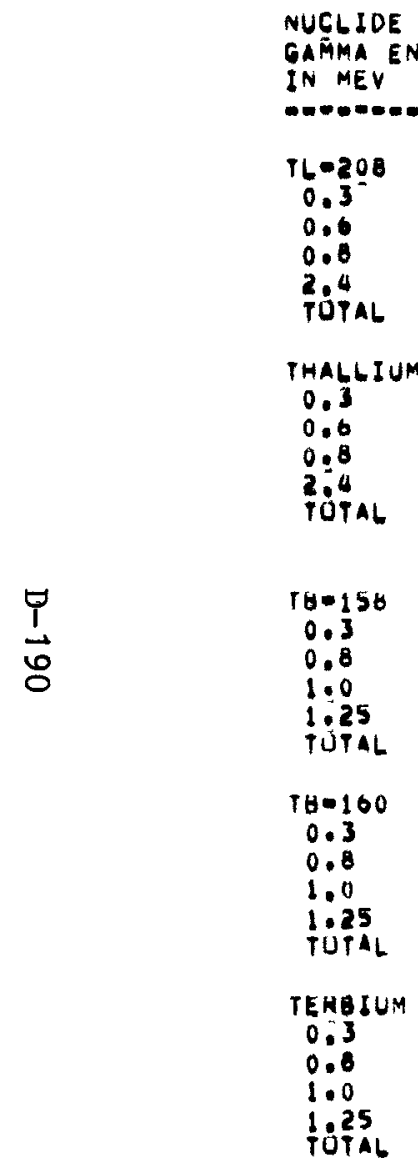

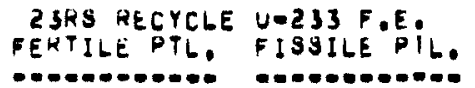

7.508 .03

$2.370-01$

7.497 .01

1.039400

7.500 .03

$2.370=01$

$4.513=02$

$7.497-01$

$1.039+00$

$6.524-10$

5.592009

.251.00

1.348009

$050=0$

$9,804=06$

$1.004-05$

.5280 .06

$3.143=05$

$4.050-00$

$9.810=06$

1.009005

$7,530=06$

3. $148-05$
$8.495-03$

2.682001

5.106002

8.483001

$1.176+00$

0.495005

$2.082=01$

$5.106-02$

$8.483-01$

$1.176+00$

$9.257-10$

$7.934=09$

1.912009

$7.151=08$

$4.283=00$

$1.037-05$

1.062 .05

$7.963=06$
$3.324-05$

4.284000

$1,038=05$

$1.068=05$

$7.965-16$
$3.331=05$

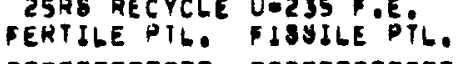

$\begin{array}{ll}7.508003 & 2.854006 \\ 2.370001 & 0.946-05 \\ 4.513002 & 1.703005 \\ 7.497 .01 & 2.830004\end{array}$

$1.098000 \quad 2.830000$

$7.508003 \quad 2.834006$

$2.370-01 \quad 8.946=05$

$4.517001 \quad 1.103005$

$1.039+00 \quad 3.923004$

$\begin{array}{ll}0.524-10 & 0.931-11 \\ 5.592009 & 7.655010 \\ 4.201008 & 5.860009 \\ 1.348009 & 1.8450110 \\ 5.040 .08 & 6.899000\end{array}$

5.100000

6.899000

$4.050-06 \quad 4.623=06$

$.004006 \quad 1.119005$

$1.004005 \quad 1.147 .05$

$\begin{array}{ll}1.004005 & 6.5940006 \\ 3.143005 & 3.587-05\end{array}$

$4.050006 \quad 4.623=06$

$9.810=00 \quad 1.119=05$

$1.009005 \quad 1.147 .05$

$7.530006 \quad 8.594=06$

3.100005 


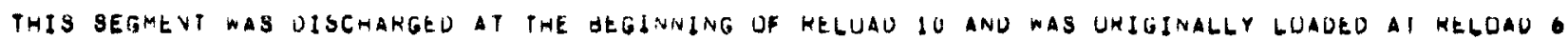

NUCLIDE AND GAMMA ENERGY

\section{EU० 152}

0.1

0.3

0.0

1.25

töTAL

EU- 154

0.1

0

$\frac{1}{1}$

1.0

TótAL

EUROPIUM

0.1

0.8

1.0

1.25

1.6

$P M=148 M$

0.1

0.3

0.6

TứLL

GAMMA DUSE KAIES IN RAUIHR PER FOE. AT UNE METER AI TIME 3 YEARS AFTER DISCHARGE

23RS KECYCLE U-233 F.E.

FEKTILE PTL. FISSILE PIL.

1.478003

3.917 .03

$0.399-04$

4.000003

$1.167-v a$

$9.187-03$

3.340004
3.129002

$1.771-03$

4.095003

7.669 .04

4,800003

1,399002

1.101002

4.010004

3,750002

$4.018=01$

$4.740=01$
1.7591001

$2.499+00$

$2.369+00$

$3.640+00$

$2.811-01$

$4.033=01$

1.785001

$4.598-01$

$2.503+00$

$2.380+00$

$3.049+00$

$2.814=0 !$

$0.203=01$

$3,590=01$

9.441001

$5.138+00$

4.870000
$7.485+00$

$7.485+00$

5.780001

$2.020+0$

$8.281=01$

$3,037-01$

9.449 .01

$5.143+00$

7.490400

$5.784=01$

$024+01$

1.105008

$3.177=07$

$2.334=06$

$0.622=07$

$7.307=07$
$4.056-00$

16900

2,349007

1.874000

$3,417.07$

$5,403-07$

2,999006
25RS AECYCLE U-23S F.E.

FERTILE PIL. F18SILE PTL.

$\begin{array}{ll}1.478003 & 1.728003 \\ 3.917-03 & 4.581003 \\ 6.390004 & 7.483004 \\ 4.000-03 & 4.748003 \\ 1.167-02 & 1.365002 \\ 9.187003 & 1.074002 \\ 3.346004 & 3.913004 \\ 3.129002 & 3.659002\end{array}$

$4.018 .01 \quad 8.169001$

$1.746001 \quad 3,549=0$

$4.591-01 \quad 9.334001$

$2.499400 \quad 5.099400$

$2.369+00 \quad 4.815400$

$3.640400 \quad 7.400+00$

$2.811001 \quad 5.715001$

$4.053=01 \quad 8.187001$

$1.785001 \quad 3.595-01$

$4.590 .01 \quad 9.341001$

$2.503+00 \quad 5.084+00$

$3.649+00$

3.61400100

$2.814001 \quad 5.718001$

$1.105008 \quad 9.059009$

$3.177-07 \quad 2.030=07$

$2.534006 \quad 1.619000$

$4.022 .07 \quad 2.953007$

$9.307007 \quad 4.669007$

2.592006 


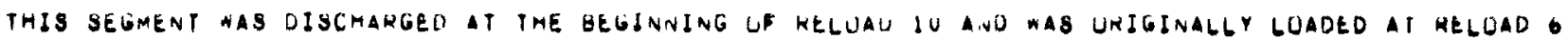

NUCLIDE AND GAMMA ENERGY JN MEV

IN MEV

PROMETHIUM

0.1

0.3

0.6
0.8
1.0

TUTAL

$00-144$

0.6

0.8

1.25

$\frac{0}{1}$

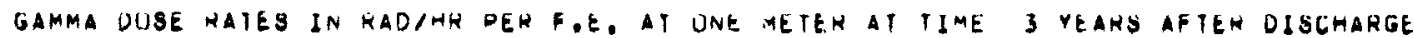

23RS NECYCLE U-233 .E.
FERTILE PTL OISILE PIL.
-

$1.105-08$

$3.177-01$

2.534000

4.0220 .07

$7.307-07$
4.056006

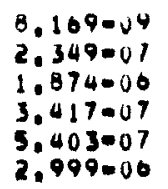

$5.227+00$

$9.794-02$
4.544002

$1.854+00$

$0.442+00$

$1.367+01$

$7.511-0.2$

$3,484-02$

$1.422+00$
$4.040+00$

$1.048+01$

$5.227+00$

$9.794-02$

$4.544=02$

$1.854+00$

$0.442+00$

$4.008+00$

$7.511-02$

3.462002

$1.922+000$

$1.048+01$

$1.282+00$
$3.383+02$

$2.085+00$

$A=137 M$

0.1

TÓTAL

BARIUM

0.1

TUTAL
$3.390+02$

$5.503+02$

$1.282+00$

$3.383+02$

$3.390+02$
$2.085+0$ $5,503+02$
$5,524+02$
25RS RECYLLE UAZSS F.E

FERTILE PTL. FISSILE PTL.

$\begin{array}{ll} & \\ 1.105000 & 7.059=09 \\ 3.177-07 & 2.030007 \\ 2.534000 & 1.619=00 \\ 4.622007 & 2.953007 \\ 7.307007 & 9.069007 \\ 4.056-06 & 2.592006\end{array}$

$5.227+00 \quad 3.267+00$

$\begin{array}{ll}9.794002 & 0.121002 \\ 4.544-02 & 2.840002\end{array}$

$1.854+00 \quad 1.159+00$

$\begin{array}{ll}0.442+00 & 4.020+00 \\ 1.367+01 & 8.541000\end{array}$

$5.227+00 \quad 3.207+00$

$9.794 .02 \quad 6.121 .02$

$4.540002 \quad 2.840002$

$1.054+00 \quad 1.159+00$

$1.369+01 \quad 0.541600$

$\begin{array}{ll}1.282+00 & 1.436+00 \\ 3.383+02 & 3.790+02\end{array}$

$\begin{array}{ll}3.383+02 & 3.790+02 \\ 3.396+02 & 3.805+02\end{array}$

$1.282000 \quad 1.456400$

$3.383+02 \quad 3.790+02$

$3,390+02 \quad 3,005+02$ 


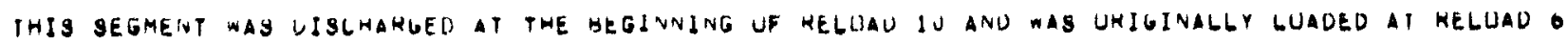

NUCLIDE ANO GAMMA ENEKGY IN MEV

C....

\section{$68=134$}

0.3

0.0

1.0
1.25

tootal

CESIUM

0.3

0.8

1.05

$\varphi$
$\dot{1}$
$\omega$

fuital

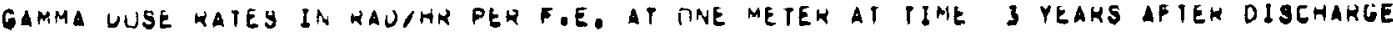

$$
\begin{aligned}
& 23 \text { HS KECYCLE U-233 F.E. } \\
& \text { FEKTILE PIL. FISSILE PIL. }
\end{aligned}
$$

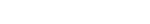

2.900002

$2.180+02$

$2.208+02$

$3.098+00$

$1.606+01$

$1.045=01$
$4.676+02$

7.010002

$5.197+02$

$5.371+02$

$7.336+00$

$4.470+01$

$1.107+03$

TE-129M

0.1

0.8

1.0

IUTAL

$16-129$

0.1

0.3

0.8

1.0
TOTAL
2.900002

2.16640

2.200402

$3,098+00$

$1.868+0$

4.076402

7.010002

$5.177+02$

$5,371+02$

$7.336+00$

$4.470+01$

$2.473=01$

$1.107+03$

$3,468-10$

$4.501-06$

$1.309-08$

$7.683=10$

$1.717-10$

$0,999-11$

$1.168-05$

3.553009

4.094010

4,454011
$1,557-08$

$3.699=09$

3.324009

$3.623=08$

$2.035=09$

$1.388=09$

$2.801-10$

$2.861=10$
$5.290=08$

$9.599-10$

$8,626-10$

9.402009

$5,281=10$

$1,917=09$

7,424011

1,374000
25HS RECYCLE U -235 \& EE

FERTILE PTL. FISSILE PTL.

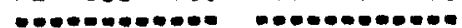

$\begin{array}{ll}2.960002 & 5.792=02 \\ 2.186+02 & 4.278+02 \\ 2.268+02 & 4.438+02 \\ 3.090+00 & 0.002+00 \\ 1.888+01 & 3.693+01 \\ 1.045001 & 2.044001 \\ 4.676+02 & 9.148+02\end{array}$

$2.960002 \quad 5.792002$

$2.100402 \quad 4.270+02$

$4.436+02$

$3.090+00 \quad 0.062+00$

$1.886001 \quad 3.693401$

$1.045001 \quad 2.044001$

$4.676+02 \quad 9.146+02$

$3.468 \cdot 10$

4.501000

1.369008

$7.083-10$

5.999000

$3.197 \cdot 11$

$4.150-09$

1.262009

7.084 .11

$1.583 \cdot 11$

3.699009

3.324009

3.023008

2.035009
$7.380-09$

$2.801-10$
$5.290-08$

3.410010

3,065010

3. 340009

$1.876-10$

$0.811-10$

$2.034-11$

$4.883=09$ 


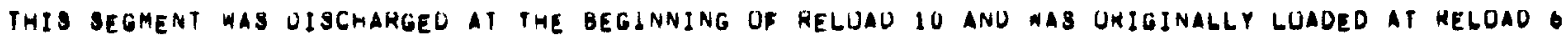
NUCLIDE AND
GAPMMA ENERGY IN MEY

(1)

\section{TELLURTUM}

0.1

0.3

0.0

100

TUTAL

$88 \cdot 124$

0.6
$0: 0$
100
$1: 25$
100
200

TOTAL

$38-125$

0.1

0.0

0.6

ANIIMUNY

0.1
0.3
0.6
0.0
1.0
1.25
1.6
2.00
TOMTAL

GAMMA DUSE RATES IN RAOJMR PEH F OE AT ONE METER AT TIME J YEAHS AFIER JIOCMAHGE

$23 K 8$ HECYCLE U-233 F.E

FERIILE PTL, FISBILE TL.

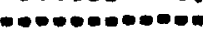

4.040009

3,324009

1.124000

1.572008

4.577 .10

1.130007

$\frac{1}{2.160000}$

6.092009

1.014007

$1.347-06$

$1.175=07$

$3.063=06$

6.451 .01

$0.121+00$

$1.227+01$

1.903001

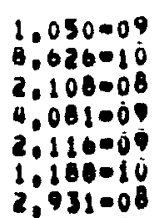

$0.451=01$

$0.121+00$

$1.227+01$

$2,003=07$

$6,062=08$

$1.014-007$

1.347006

$1.775=07$

$1.903+01$
1.505006
2.722007

9.488000

1,378007

1.831000

2,412009

$7.597-01$

$7.209+00$

$2,241+01$

$7.397-01$

1.209000

$1.444+01$
$2,722=07$

9.486008

1.378 .07

1.831000

2.412007

2.241002
25R8 HECYCLE UOZ3S DE.

FENTILE PTL. F188ILE PTL.

$\begin{array}{ll}4.040009 & 3.730010 \\ 3.324009 & 3.0650110 \\ 8.124008 & 7.490009 \\ 1.572000 & 1.450009 \\ 6.156009 & 7.519010 \\ 4.577-10 & 4.220011 \\ 1.130007 & 1.041000\end{array}$

$1.160000 \quad 0.077007$

1.101007

$1.014007 \quad 5.970008$

$1.347 .06 \quad 7.942007$

$1.978009 \quad 1.046007$

$3.063=06 \quad 1.00600$

$0.451 .01 \quad 1.490001$

$0.121+00 \quad 1.414+00$

$1.227+01 \quad 2.833+00$

$1.027+01 \quad 2.833+00$

$\begin{array}{ll}0.451001 & 1.490001 \\ 6.121+00 \quad 1.414000\end{array}$

$1.227+01 \quad 2.033000$

$0.003007 \quad 1.181007$

6.9820004 .116000

$1.010007 \quad 5.976000$

$1.347006 \quad 9.942=07$

$1.775007 \quad 1.046007$

$1.903+01 \quad 4.397+00$ 


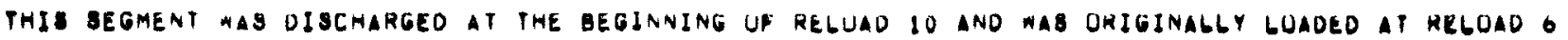

NUGLJDE ANO GAMMA ENERGY

IN MEY

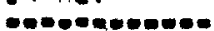

$\cos 115 M$

1.0

Total

CADMTUM

1.0

toTAL

$A G \cdot 108 \mathrm{M}$

0.1

0.3

0.6

$\frac{1}{0}$

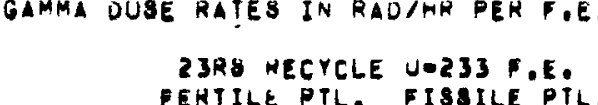

PENTILE PTL. FISSILE PIL,

$4.472-10$

$2.165=10$
$6.637-10$

$4.472-10$

$2.165=10$
$0.637=10$

1.115000

9.028002

$1.352=04$

$1.933=04$

2.053006

3.820004

$1.023 \cdot 05$

8.606004

4.225002

$5.628-02$

$1.671=02$

$1.844=02$

1.009002

8.862005

9.109 .05

1.488001

1.134 .05

1.50800
4.238 .02

$9.238=02$
$5.644=02$
1.671 .002

$1.644=02$
$1.671-02$

$1.671=02$

$1.844=02$
$1.409=02$

$1.409=02$

8.802005

9.169005
$1.953-10$

2.898 .10

$1.953-10$
$9.454-1 i$

$9.454-11$
$2.808-10$

1,313007

1.063005

$1.804-05$

$2,417-07$

$4.497=05$

$1.090-05$

1.422003

$6.980-02$

9.299002

2,760002

$3.040-02$

$3 ., 040-02$

1.51500

2,459001

$1.703-05$

1.432005

$6.962-02$

9.300002

2,760002

3.006002

2.327 .02

$1.464=04$

$1.515=04$

2.459001
25R8 RECYCLE U-235 F.E.

FEHTILE PTL FI83LLE PTL

PJSILE PTL.

$\begin{array}{ll}4.472-10 & 1.242-10 \\ 2.165-10 & 0.013=11 \\ 6.637-10 & 1.844-10 \\ & \\ 4.472-10 & 1.242=10 \\ 2.165-10 & 6.013=11 \\ 6.637-10 & 1.844-10\end{array}$

$1.115=0$

9.020005

$1.352=00$

1.533004

2.053006

$3.020=04$

2.753007

2.230005

$3: 33005$

5.067007

9.428005

$1.023-05 \quad 1.032005$

$0.006004 \quad 0.680004$

$4.225002 \quad 4.202002$

$5.628002 \quad 5.677 .02$

$1.671=02 \quad 1.685=02$

$1.844 .02 \quad 1.860 .02$

$1.409002 \quad 1.421002$

$0.062005 \quad 0.939005$

$9.109005 \quad 0.248 .05$

$1.488001 \quad 1.501001$

\begin{tabular}{|c|c|}
\hline $\begin{array}{l}1.134005 \\
9.506-04 \\
4.236-02 \\
5.644002 \\
1.671-02 \\
1.844-02 \\
1.409-02 \\
.862-05 \\
9.169-05 \\
1.492-011\end{array}$ & $\begin{array}{l}1.059=05 \\
8.903-04 \\
4.265-02 \\
5.641-02 \\
1.605-02 \\
1.660002 \\
1.421002 \\
8.939-05 \\
9.248-05 \\
1.502-01\end{array}$ \\
\hline
\end{tabular}




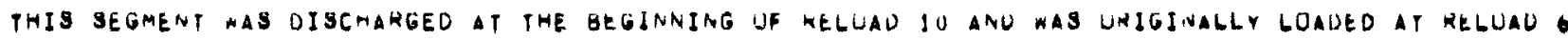
NUCLIDE AND

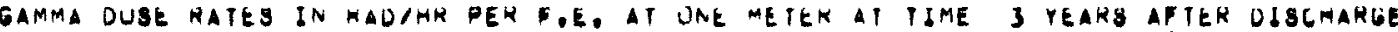
GAMMA ENERGY

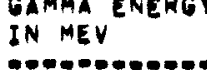

AH० 100

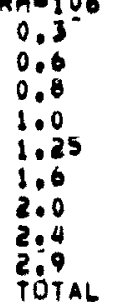

RHUOTUM

0.3

0.6

0.8

1.0

1.25

1.0

2.0

2.0
2.4
2.9

rótal

$R U=103$

0.1

TOTAL

RUIHENIUM

0.1

0.6

TUTAL

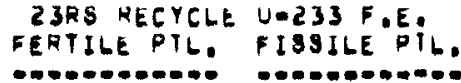

$2.750=02$

$7.993+00$

$\$ .740001$

7.056001

2.348001

$1.621-01$

$5.978-02$

$.970-02$

$9.428+00$

2.756002

$7.993+00$

$1.748-01$

$1.056-01$
$2.348-01$

1.021 .01

., 976002

.974002

9.811003

$9.428+00$

$3.300-09$
$4.393-08$

8.641000

8.687 .06

3. $300=09$

4.393000

$8.640=06$

$8.687-00$
$2.007 \cdot 02$

$7.564+00$

1.654001

$0.676 * 01$

2.22200

1.53300.

5.657002

$8.921+00$

2.607 .02

$7.564+00$

$1.654-01$

6,07000

$2,222=0$

1.533001

5,659002

$5.653=02$

9.284003

$8.921+00$

1.443009

3,799006

1.443004

$1.921=00$

3.779 .06

3,799006
25RS RECYCLE UEZ3S P.E.
PERTILE PTLE FISSILE PIL.

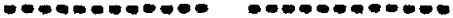

$\begin{array}{ll}2.750002 & 2.012002 \\ 7.993+00 & 7.576000 \\ 1.740001 & 1.057001 \\ 7.056001 & 6.687001 \\ 2.348001 & 2.226001 \\ 1.621001 & 1.536001 \\ 5.976002 & 5.660002 \\ 5.974002 & 3.063002 \\ 9.811003 & 9.300003 \\ 9.428+00 & 0.936000\end{array}$

$2.756002 \quad 2.612002$

1.99300

$1.748001 \quad 1.657=0$

$7.056001 \quad 0.087=0$

2.22600

$1.621001 \quad 1.53600$

$5,970.02$

$5.974002 \quad 3.663=0$

$9.811=03 \quad 9.300003$

$9.426400 \quad 8.936+00$

$3.300009 \quad 1.256009$

$4.393 .08 \quad 1.672=08$

$\begin{array}{ll}8.640006 & 3.280006 \\ 8.687-06 & 3.306006\end{array}$

$3.300009 \quad 1.256009$

$4.393008 \quad 1.072008$

$8.640006 \quad 3.288000$

$8.687006 \quad 3.306000$ 
THIS SEGMENT MAS DISCMARGED AT THE BEGINNING UF RELOAD IO ANO NAS UKIGINALLY GUADED MT KELOAD O

NUCLIDE ANU

IN MEY

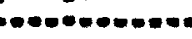

NB $=95$

Total

NIUB IUM

TOTAL

$6 R \cdot 95$

0.3

rótab

$\frac{\varphi}{v}$

\section{ZIRCUNIUM}

$21 R C$
0.3
0.8

TUTAL

CO-5o

0.6

1.6

culou

T.25
TOTAL

COBALT

0.6

0.8

1.25

1.6

GAMMA DUSE RATES IN RADIHK PER F,E. AI UNE METER AI TIME J YEARS AF FER DISCHARGE

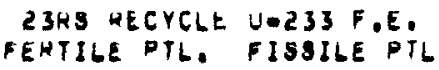

FEHTILE PTL. FISSILE PTL.

$1.909-01$

1.909001

0,903002
0.903002

1.909008

1,909001

0,003002

6.903002

$1.532=04$
$8.053-02$
0.069002

$8.069=02$

$5.539-05$

$2.912=02$
$3.018=02$

1.532004

$8.053=02$

8.069 .02

5.539005

2.918002

1.251008

6.48500

7.310 .08

$6.652-10$

$4.152=09$

4.570 .04

4.574004

2,446005

$2.446=05$

$6.652-10$

3.448 .09

0.485000

0.485009
4.574004

7.367 .10

4.5740 .04

2.9170011

$2,446-05$
ZSKS AECYCLE U-235 F.E.

FERTILE PTL. FISSILE PTL.

$1.909-01 \quad 4.444002$

$1.909001 \quad 4.494002$

$\begin{array}{ll}1.909001 & 4.494002 \\ 1.909001 & 4.494002\end{array}$

$\begin{array}{ll}1.532-04 & 3.600005 \\ 8.053002 & 1.890002\end{array}$

$6.069002 \quad 1.900002$

$\begin{array}{ll}1.532004 & 3.606005 \\ 8.053002 & 1.896002\end{array}$

$0.069002 \quad 1.900002$

$1.251 .06 \quad 1.395009$

$7.367-10 \quad 8.213011$

$4.574004 \quad 5.128005$

$4.574004 \quad 5.126=05$

$1.251008 \quad 1.395009$

7.230 .09

$4.574004 \quad 5.128005$

$7.574=04$ 


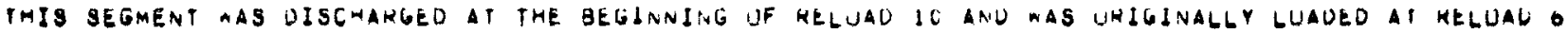
NUCLIDE ANO GAMMMA ENERGY

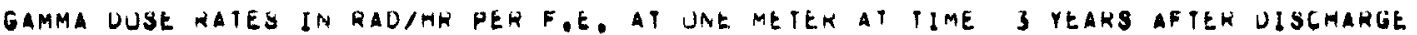

IN MEV ENERG

ALL ELEMENTS

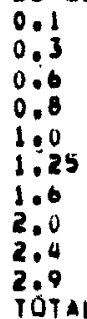

OTAL
$23 R S$ RECYCLE
FEHTILE PTL.

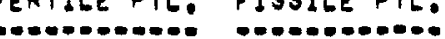

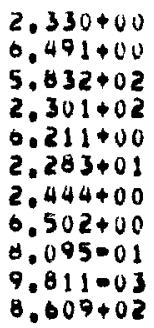

$3.673+100$

$7.020+0.5$

$1.095+03$

$.428+02$

$1.293+01$

$5,248+01$

2.456000

$4.997+00$

.050001

9.284003

$1.723+03$

25RS RECYCLE U 235 F.E.

FERTILE PTL. FISSILE PIL.

FERTILE PTLE FIBSILE OTL.

$\underset{\infty}{\grave{D}}$ 


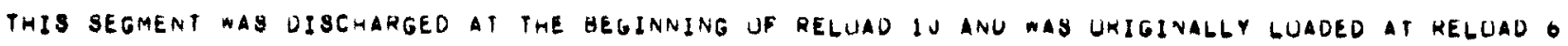
NUCLIDE AND GAMMA ENERGY

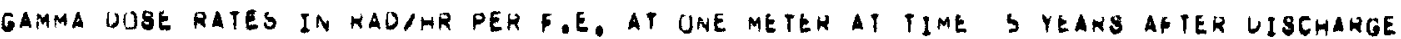

IN MEV

U.235

0.1
0.3
TOTAL

UAANIUM

0.1

rotat

$P A=234$

0.3

0.6

0.8

1.25

1.25

TútAL

PRUTACTINIUM

0.3

0.6

0.8

1.0

IUTAL

$A C=228$

0.3
0.6
0.8
1.0
1.00
2.0
TOTAL
23HS HECYCLE UA233P.E.

FERTILE PTL. FISSILE PIL.

(1)

3.010007

1.016006

1.356000

3,416007

$1.010=00$

1.356000

$4.905=00$

0.000

0.000

0.000
0.000

0.000

0.000

0.000

0.000

0.000

0.000

0.000

0.000

$$
\begin{aligned}
& 2,101-11 \\
& 2,201-11 \\
& 1.003-11 \\
& 2.023-10 \\
& 1.507-11 \\
& 3.575-11 \\
& 3.076010
\end{aligned}
$$

$2.161-11$

2.201011

1.083 .11

$2.023-10$

$1.507-11$

$3.076-10$

$1.199-05$

0.105000

$1.501=05$

1.353 .04

4.231 .05

9.7540 .06
2.2040 .14
$3.501-12$
$1.782-12$
$4.384-12$
$3.949-11$
$1.235-11$
$2.848-12$
$6.436-11$
25RS RECYCLE U-2S5 P.E.

FERTILE PIL. FIS3ILE PTL

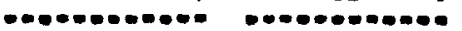

\section{$\quad 3.810007 \quad 1.410000$}

$1.010006 \quad 5.103006$

$1.398-0601019000$

$3.010007 \quad 1.910000$

$1.010006 \quad 5.103000$

7.019006

0.000

0.000
0.000

0.000
0.000

0.000

0,000

1.895000

9.322007

1.742005
1.297006

3.297006

2.048005

0,000

0.000

0,000

0,000

0,000

0.000

1.801006

1.895006

$9.322=07$

1.742005

$1.297-06$

3.078006

2.648005

$\begin{array}{ll}1.199005 & 0.000 \\ 6.105006 & 0.000 \\ 1.501 .05 & 0.000 \\ 1.353004 & 0.000 \\ 4.231 .05 & 0.000 \\ 0.754 .06 & 0.000 \\ 2.204004 & 0.000\end{array}$




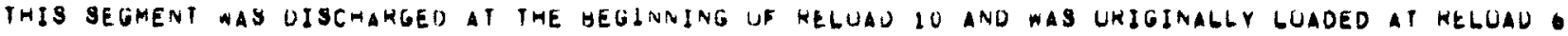

NUCLIDE AND GAMMA ENERG IN MEV

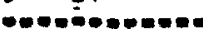

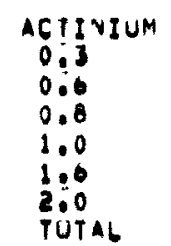

$14-223$

0.1
0.3
TOTA

0
1
$N$
0

0.1

TOTAL

RNA 219

0.3

rutal

TUTAL

$4-211$

POTAL

$81 \cdot 212$

0.6

1.0

1.6
TUTAL

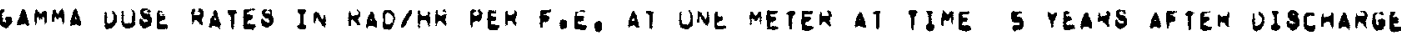

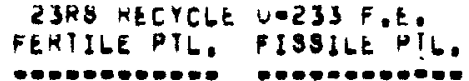

FERTILE PTL.

CISTILE IL,

$1.199-45$

6.105006

$1.501=05$
1.353004

1.353004
4.231005

4.231005
$. .754=06$

2.204004

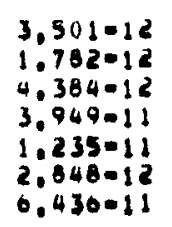

$2.038-06$

5.425006

7.403006

9.664000
2.573007

$2.573-07$
$3.539-07$

2.038000

5.425046

$9.604-00$
$2.573=07$
$3.539-07$

$5.425=06$

$2.573-07$
$2.573-07$

$5.425=00$

$5.425=06$

2.573007
2.573007

$5.425-00$
$5.425-00$

2.573007
$2,573-07$

$7.123=02$

1.138 .02

3.130002

1.100001

$7.092-02$
$1.133-02$

$1.133-02$

3,123002
1.135001
25RS RECYCLE U.235 P. E

FENIILE PTL. FISSILE PTL.

$\begin{array}{ll}1.199 .05 & 0.000 \\ 0.105006 & 0.000 \\ 1.501 .05 & 0.000 \\ 1.353004 & 0.000 \\ 4.231 .05 & 0.000 \\ 9.754 .06 & 0.000 \\ 2.204004 & 0.000\end{array}$

\section{$2.036000 \quad 1.707-09$}

$\begin{array}{ll}5.425006 & 4.756009 \\ 7.463006 & 0.345009\end{array}$

$2.038006 \quad 1.767-09$

$5.425006 \quad 4.758-09$

$\begin{array}{ll}5.4250 .06 & 4.758009 \\ 5.425006 & 4.758009\end{array}$

$\begin{array}{ll}5.425006 & 4.758-09 \\ 5.425-06 & 0.758009\end{array}$

$5.425006 \quad 4.758009$

$5.425006 \quad 4.758 .09$

$7.123002 \quad 2.609=05$

$1.138002 \quad 4.295006$

$\begin{array}{ll}3.130002 & 1.184=05 \\ 1.140001 & 4.302-05\end{array}$ 


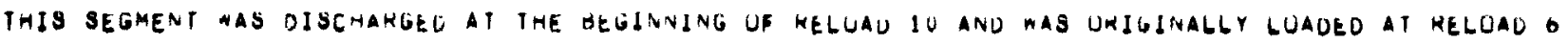
NUCLIDE ANO GAMMA ENERGY IN MEV

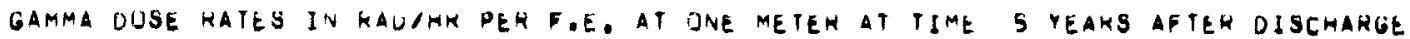

$81-2+3$

0.3

ToTAL

BIBMUTH

0.3

$1: 0$

OTAL

$P 8=2 ! 1$

0.3

TOTAL

$P B=212$

Pútal

LEAO
0.3

$0: 8$

$16=208$

0.3

0.8

2.4
23RS HECYCLE UN233 F.E.

FENIILE PIL. FISSILE PIL.

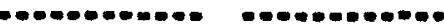

$8.657 .05 \quad 3.149=05$

$5.937=06 \quad 2.160 .00$

$3.365-05$

$9.200=05$

$7.123-15$

$3,136=0$

1.14100

3.175005

$1.092=02$

13300

1.135001

$3.255=00$

$3.261=06$

$6.516=00$

$1.422-01$
1.422001

1.42200

1.422001

3.201006

0.527 .03

2.602001

$5.125-02$

8,515001

$1.180+00$
1.544007

1.547 .07

3.090007

1.416001
1.416001

$1.416-01$

$1.547=07$

6.495003

$2,082-01$

5,106002

$8,483-01$

$1.176+00$
25RS RECYCLE U-235 RE.

FERIILE PTL. FISSILE PIL

-...........

$8.057=05 \quad 1.137-09$

$5.937 .06 \quad 7.797-11$

$9.251 .05 \quad 1.215=04$

$9.200005 \quad 5.895009$

$7.123=02 \quad 2.689=05$

$1.136002 \quad 4.295006$

$\begin{array}{ll}3.136 .02 & 1.184=05 \\ 1.141 .01 & 4.302=05\end{array}$

\section{$3.255=06 \quad 2.855009$}

$3.261006 \quad 2.860009$

$0.516000 \quad 5.714=00$

$\begin{array}{ll}1.422001 & 5.367005 \\ 1.422001 & 5.367005\end{array}$

$1.422001 \quad 5.368005$

$3.261006 \quad 2.860009$

$5,368 \cdot 05$

$\begin{array}{ll}8.527-03 & 3.219000 \\ 2.692-01 & 1.016004 \\ 5.125-02 & 1.935005 \\ 0.515001 & 3.214004 \\ 1.100000 & 4.450004\end{array}$

$1.100+00 \quad 4.450004$ 


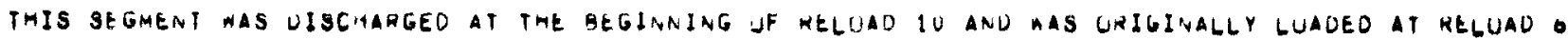

NUGLIDE AND GAMMA ENERGY IN MEV

IN MEV

THALLIUM

0.3

0.6

TULAL

$r B=158$

0.3

0.8

$1: 0$

TUTAL

$\ominus$
$N$
$N$

$0.3^{\circ}$

1.25

PÜTAL

TERBIUM

0.3

0.8

1.25

TUTAL

$E v=152$

0.1

$0: 8$

1.05

i.0.

TUTAL

GAMMA DUSE RAIES IN RADIHR PER F.E. AT UNE METEK AT TIME 5 YEAHS AFTER DISCMARgE

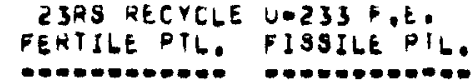

$0.527 \cdot 03$

$2.092-01$

5.125002

. 515001

$1.180+00$

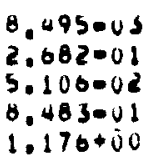

$0.405-10$

$5.541=09$

4.241008

1.3350 .49
4.994008

4

$3.575=09$

$8.656-09$

$0.808-09$

$0.047-09$
$2.775-08$

$4.222-09$

$1.020=08$

$5.128=06$

5.128008
$7.982-09$

7.982009

1. $325-03$

$3.512=03$

5.738004

3.641 .03

1.047002

$8.238-03$

3,000004
$2,000=02$
$9.103-10$
7.854009

0.012000

1.893009

7.078000

3.782009

$9.156=09$

$9.380-09$

$7.030=09$

$2.935=08$

4.098009

$1.701=00$

$6.950-08$

8.923009

$1.001=07$

$1.588-03$

$4.20900 \mathrm{~s}$

$0.876-04$

$4.363-03$

1.255002

.072-03

3.363 .02
2SRS HECYCLE W-235 F.E.

PERTILE PTL. FISSILE PTL.

$\begin{array}{ll}0.527 .03 & 1.219006 \\ 2.692001 & 1.016004 \\ 5.125002 & 1.935003 \\ 8.513001 & 3.214004 \\ 1.180+00 & 4.456004\end{array}$

$0.465-10$

5.541009

4.241000

$4.990-06$

0.640 .11

$7.505-10$

$5.806=09$

$1.028-10$

0.836009

$3.575=09$

$8.656-09$

8.808009

$0.647-09$

$2.775-00$

4.081009

$9.881-09$

1.012000

$7.387=09$

$3.167=08$

$\begin{array}{ll}4.222000 & 4.170009 \\ 1.420008 & 1.064008 \\ 5.128008 & 1.593008 \\ 7.982009 & 7.770009 \\ 7.760008 & 3.851008\end{array}$

1.325003

3,512003

5.738004

3.641 .03

1.047 .02

$8.238=03$

$3.000=04$
$2.806=02$

1.549 .03

4.108003

6.710004

4.258 .03

1.224002

$9.634=03$

3.506 .04
$3.281=02$ 


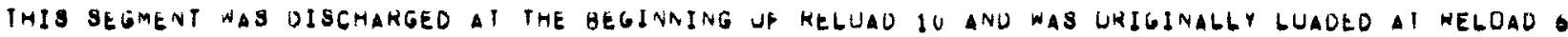
NUCLIUE AND

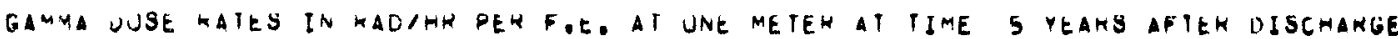
GAMMA ENERGY

IN MEV

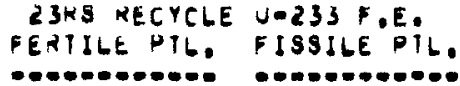

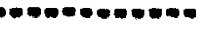

EU-154

0.1

0.3

0.8

1.0

1.25

Tó̀

EUROPIUM

0.1

0.3
0.0
0.8

1.0

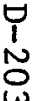

1.25

TUTAL

$P R=144$

0.6

0.8

1.23

1.6

TUTAL

PRABEOUYMIUM

0.6

1.25

1.25

2.0

TOTAL

$\begin{array}{ll}3.605-01 & 7.378001 \\ 1.601-01 & 3.292001 \\ 9.21001 & 8.65801 \\ 2.291+00 & 4.712+00 \\ 2.172+00 & 4.467+00 \\ 3.338+00 & 6.864000 \\ 2.578-01 & 5.301=01 \\ 9.009+00 & 1.853+01\end{array}$

$3.098-01$

$1.636-01$

4.216001

$2.295+00$

$2.182+00$

$3.340+00$

2.581001

$9,037+00$

$0.790-01$

$1.047-02$

3.118001

$1.083+00$

$2.298+00$

$9.790-01$
1.647002

$1.647-02$
$3.041-03$

$1.083+00$

7.504001

$3,334=01$

4.710000

4.470400

$6.874+00$

$1.856+01$

6.741 .01

1.263002

2.391.01

8.308001

$1.763+00$

6.741 .01

$1.203-02$

5.860003

2,391001

$0.308 \cdot 01$

0.30001
$1.703+00$
25RS RECYCLE UEZSS P.E.

FEKTILE PTL, FISSILE PIL.

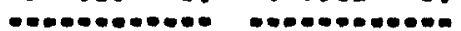

\begin{tabular}{|c|c|}
\hline $\begin{array}{l}3.685001 \\
1.601001 \\
4.210001 \\
2.291+00 \\
2.172400 \\
3.338000 \\
2.578001 \\
9.009+00\end{array}$ & $\begin{array}{l}7.492001 \\
3.255001 \\
8.560=01 \\
4.656400 \\
4.416000 \\
6.787+00 \\
5.241001 \\
1.032001\end{array}$ \\
\hline
\end{tabular}

3.698001

$7.507-01$

$1.036001 \quad 3.296001$

$4.210001 \quad 8.56700$

$2.295400 \quad 4.663+0$

$2.162+00 \quad 4.426+00$

$3.346+00 \quad 6.796+00$

$2.581001 \quad 5.24400$

$9.037000 \quad 1.835001$

$\begin{array}{ll}8.790 .01 & 9.494001 \\ 1.047 .02 & 1.029002 \\ 7.641 .03 & 4.776003 \\ 3.118001 & 1.949001 \\ 1.083000 & 0.771001 \\ 2.298000 & 1.436000\end{array}$

$2.298000 \quad 1.436000$

$\begin{array}{ll}8.790 .01 & 5.404001 \\ 1.647-02 & 1.029002 \\ 7.641 .03 & 4.770003 \\ 3.118001 & 1.949-01 \\ 1.083+00 & 6.771-01 \\ 2.298+00 & 1.436+00\end{array}$




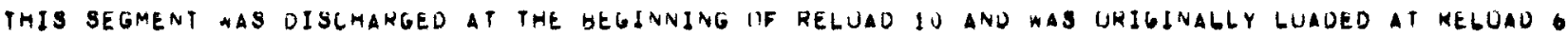
NUCLIDE AND GAMMA ENERGY IN MEV

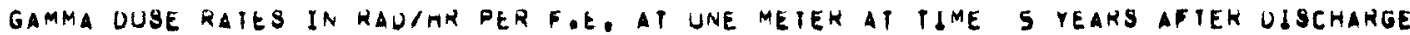

IN REV

\section{$B A=137 \mathrm{M}$ \\ 0.1 \\ TUTAL}

BAKIUM

0.1

TútAL

$c s \cdot 134$

0.6

0.6

1.0

IOTAL

CESIUM

0.3
0.0
0.8
1.0
1.25
1.0
TUTAL

TUTAL

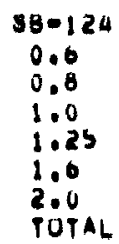

$23 k S$ RECYCLE J-233 F.E.

FEAIILE PTL. ISSILE PIL.

\section{$1.224+00$ \\ $1.991+00$
$5.254+02$}

$3.231+02$
$3.2+3+02$

$5.274+02$

\section{$1.224+00$}

$3,231+02$

$3.243+02$

$1.991+00$

$5.254+02$
$5.274+02$

1.504002

$1.111+02$

$1.152+02$
$1.574+00$

$9.590+00$

$5.307-02$

2. $375+02$

1.504002

$1.111+02$

$1.152+02$
1.574400

$9.590+00$

$5.307-02$

$2.375+02$

$.005-10$

$.474-11$

$1.554-11$

$2.204-11$

$3.008-10$

$3.963-11$

0.839 .10
$3,561=02$

$2.630+02$

$3.727+00$

$2.271+01$

1.257001

$5.625+02$

$3.561-02$

$2.630+02$

$2,729+02$

$3.727+00$

1.257 .01

$5.625+02$

$3,539-10$

$0.079-1$

$2.119-11$

3.077-1

$4.088-10$

$5.385-11$

$9.293-10$
25NS RECYCLE UELSS P.E.

LERIILE PTL. FISSILE PTL.

$\begin{array}{ll}1.224+00 & 1.371+00 \\ 3.231+02 & 3.619+02\end{array}$

$\begin{array}{ll}3.23 !+02 & 3.019+02 \\ 3.243+02 & 3.633+02\end{array}$

$\begin{array}{ll}1.220+00 & 1.371+00 \\ 3.231+02 & 3.619+02\end{array}$

$3.243+02 \quad 3.633+02$

$1.504002 \quad 2.943002$

$1.111+02 \quad 2.173+02$

$1.152+02 \quad 2.255402$

$1.574+00 \quad 3.079+00$

$5.307-02 \quad 1.876001$

$2.375+02 \quad 4.648+02$

$1.504 .02 \quad 2.943002$

$1.111+02 \quad 2.173+02$

$\begin{array}{ll}1.152+02 & 2.175+02 \\ 1.255+02 & 3.079+00\end{array}$

$1.574+00 \quad 3.079+00$

$1.876+01$

$5.307 .02 \quad 1.038001$

$2.005-10 \quad 1.536-10$

$4.474 .11 \quad 2.638-11$

$1.559 .1 ! \quad 9.192 .12$

2.204 .11

1.7740 .10

$3.963-11$

$0.839010 \quad 4.032-10$ 


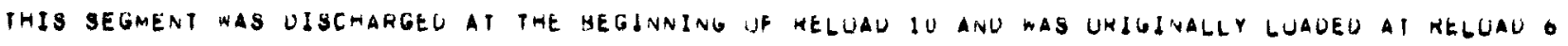

NUCLIDE AND GAMMMA ENERGY IN MEY

IN Mevenome.

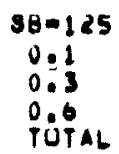

0.1
0.13
0.6

TUTAL

ANI I MONY

0.1

0.6

1.0

1.25

1.6

TUTAL

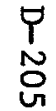

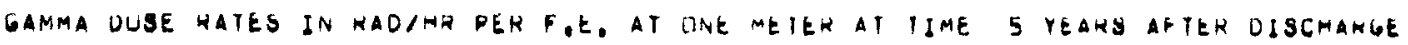

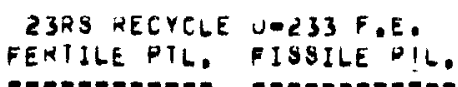

$3.808-01$
$3.071+00$
$7.355+00$
$1.141+01$

1.0

$A G=110 M$

0.1

0.3

0.6

1.0
1.25

1.6
2.0

2.4

TUTAL
3.8680 .11

$3.671+00$

$7,355+00$

$4.474-11$

$1.554=11$

$3.008-10$

$3.003-1$

$1.141+01$

$1.104=00$

$8.932=05$

1.338 .04

1.517004

$2.031=06$
3.779000

ISSILE Dil.

\section{$4.555-u 1$ \\ $4.323+00$ \\ $8.602+05$}

$4.555-01$

$4.323+00$

$0.062+00$

$2.119-11$

$3.077=11$

$4.088-10$

5.385011

$1.344+01$

$1.299-07$

$1.052=05$

1.575005

1.785005

.

$4.449=0.5$

$1.405-06$

$1.182=0$

5.804003

7.732 .03

2.295003

$2.533-03$

$1.935=u 3$

1.217005

$1.260=05$

2.322000

1.95300

9.590003

1.278002

$3.792-03$

$4.185-33$

5.198003

2.011 .05

2.081005

3,378002
$25 R S$ RECYCLE L-235 F.E.

FERTILE HTLO FISSILE PIL.

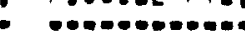

$3.008=01$

$3.671+00$

$7.355+00$

8.936 .02

8.479 .01

$1.699+00$

$1.141+01 \quad 2.630000$

$3.060 .01 \quad 8.936002$

$3.671+00 \quad 8.479001$

$7.355+00 \quad 1.099+00$

$\begin{array}{ll}4.474-11 & 2.030-11 \\ 1.559-11 & 9.102012\end{array}$

$2,204-11 \quad 9.192012$

$3.006-10 \quad 1.379011$

$3.963-11$

3.96301120 .301000

$1.104006 \quad 2.724007$

$8.932-05 \quad 2.205-05$

$1.338 .04 \quad 3.303005$

$1.517004 \quad 3.743005$

$2.031 .06 \quad 5.013007$

9.328005

$1.405-06$

$1.182-04$

5.804003

$7.732-03$

$2.295=03$

2.533 .03

$1.935=03$

1.217 .05

1.260005

2.044002
1.417000

1.193004

5.855003

7.800 .03

$2.315=03$

$2.555-03$

$1.052=05$

1.228 .05

$1.271-05$

$2.002-02$ 


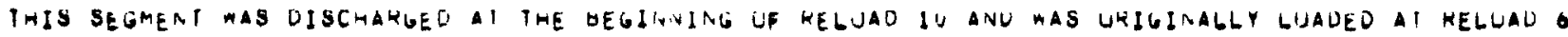

NUCLIDE ANO

GAMMA ENERGY

IN MEV

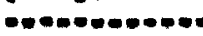

\section{SILVER}

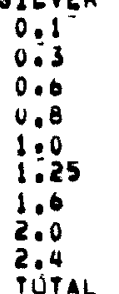

0
1
0
0

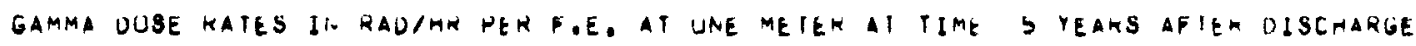

23 ST HECYCLE UE233 P.E.

FEKTILE PTL. FISSILE PIL.

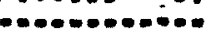

2.509000

2.075004

$5.939-03$

$2.297=03$

2.533 .03

$1.935 \cdot 03$

$1.217-05$

$1.260=05$

2.452000

2.058 .04

$9.005-03$

1.279002

3.793 .03

$3.198-03$

2.011005

$2,081.05$

3. $382=02$

$0.966=03$

$2.021+00$

$4.420=02$

1.783001
$5.935-02$

4.697 .02

$1.511=02$

1.510002

1.510002
2.480003

$2.383+00$

$0.966-03$

$2.021+00$

4.420002

1.783001

$5.935-02$

4.097 .02

1.511 .02

$1.510-02$

$2,480-03$
$2.383+00$
6.591003
$1.912+00$
4.182002
$1.688=01$
5.616002
3.876002
1.430002
$1.429-02$
$2.347-03$
$2.255+00$

$6.591 \cdot 03$

$1.912+00$

$4.182=02$

$5.610-02$

$3.876-02$

$1.430=0$

1.429 .0

$2.347-05$

$2.255+00$
ZSRS RECYCLE U.ZZ3S FE.

FEHTILE PIL PISSILE PIL.

$\begin{array}{ll}2.509000 & 1.090000 \\ 2.075004 & 1.413004 \\ 5.938003 & 5.888003 \\ 7.884003 & 7.837-03 \\ 2.297003 & 2.316003 \\ 2.533003 & 2.555003 \\ 1.935003 & 1.952003 \\ 1.217005 & 1.228005 \\ 1.200-05 & 1.271005 \\ 2.082-02 & 2.072-02\end{array}$

$\begin{array}{ll}0.906003 & 0.602003 \\ 2.021+00 & 1.915+00 \\ 4.420002 & 4.189002 \\ 1.783001 & 1.690001 \\ 5.935002 & 5.626002 \\ 4.097002 & 3.883002 \\ 1.511002 & 1.432002 \\ 1.510002 & 1.431002 \\ 2.480-03 & 2.351003 \\ 2.383+00 & 2.259+00\end{array}$

0.960003

$2,021+00$

$4.420=02$

1.783001

5.935002

$4.097-02$

1.511 .02

1.510002

$2,480.03$

$2.383+00$
$0.602-03$
$1.915+00$
$4.189=02$
$1.690-01$
5.620002
$3.883-02$
1.432002
$1.431=02$
$2.351-03$
$2.259+00$ 


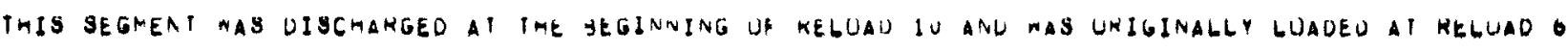
NUCLIOE ANU

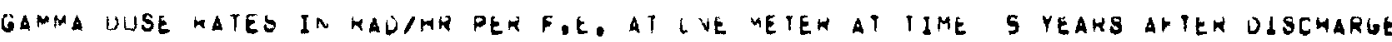
GAMMMA ENERGY IN MEV V

000000000

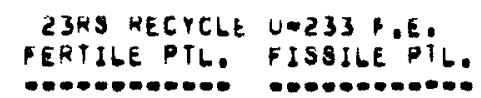

NBe95

Total

NIUBIUM

Pótal

2R-95

0.3
0.8

TOTAL

ZIRCONIUM

0,3

TUTAL

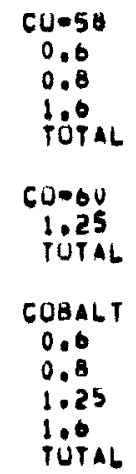

FERTILE PTL, FISSILEPIL.

$0.429 .05 \quad 3.048005$

$8.429005 \quad 3.048005$

$0.429=05$

8,429005

$3.048-05$
3.048 .05

$6.763=00$

3.556005

2.440000

$1.280-05$

$.763=00$

$0,763=08$
$3,556=05$

$3.562=05$

\subsection{0}

.286005

.288005

$1.038-11$

$5.382-11$

$0,114-13$

0.000

0.000

0.000

0.000

$\begin{array}{ll}3.513004 & 1.879005 \\ 3.513004 & 1.879005\end{array}$

$1.038-11$

$5.362-11$

$3.513-04$

$6.114-13$

0.000

1.000

1.879005

0.000

$1.879=05$
25NS REGYCLE UOZS5 F.E.

PERTILE PTL, PISSILE PTL.

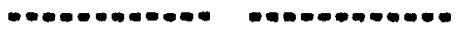

$\begin{array}{ll}0.429005 & 1.984005 \\ 8.429005 & 1.9840005\end{array}$

$8.429-05 \quad 1.984005$

$0.429005 \quad 1.984005$

$0.703-08 \quad 1.592008$

$\begin{array}{ll}3,556005 & 8.371=06 \\ 3,502-05 & 0.367=00\end{array}$

$\begin{array}{ll}0.763-08 & 1.592=08 \\ 3.550-05 & 8.371006\end{array}$

$\begin{array}{ll}3,550-05 & 8.371006 \\ 3,502-05 & 8.387 .06\end{array}$

$\begin{array}{ll}1.038-11 & 0.000 \\ 5.382-11 & 0.000 \\ 0.114-13 & 0.000 \\ 0.482-11 & 0.000\end{array}$

$\begin{array}{ll}3.513004 & 3.939=05 \\ 3.513004 & 3.039005\end{array}$

$1.038011 \quad 0.000$

$5.382-11 \quad 0.000$

$3.513004 \quad 3.939005$

$6.114013 \quad 0.000$ 


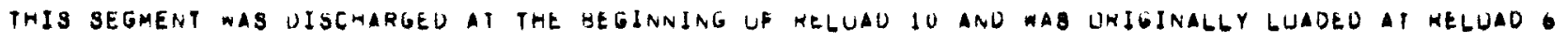

NUCLIOE AND GAMMMA ENERGY IN MEV

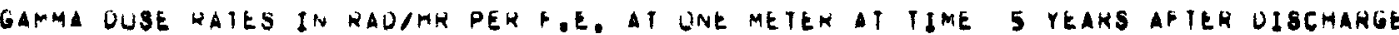

23RS RECYCLE UE233 POE.
FENIILE PTL. FISSILE PIL.
25RS RECYCLE U.235 P.E.

PERTILE PTLO DOSSILE PTL
En

ALL ELEMENTS

0.1

0.6

1.0

1.25

2.0

2.4

2.9

FutAL

$\begin{array}{ll}1.901+00 & 3.206+00 \\ 4.607+01 & 1.049+00 \\ 4.451+02 & 8.006402 \\ 1.177+02 & 2.778+02 \\ 3.949+00 & 8.390+00 \\ 1.301+01 & 2.965+01 \\ 6.973-01 & 9.684001 \\ 1.098+00 & 8.452001 \\ 8.006-01 & 8.626001 \\ 2.480-03 & 2.347003 \\ 5.064+02 & 1.127+03\end{array}$




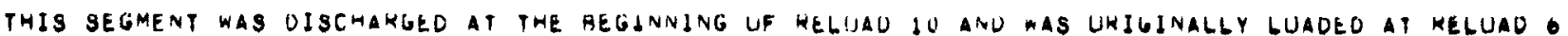

NUCLIDE ANO GAMMA ENERGY IN MEV

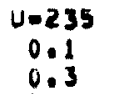

TUTAL

URANIUM

0.1

TUTAL

$P A-234$

0.3
0.6
0.8
1.0
1.25
1.6
TOTAL

PRUTACTINIUM

0.3
0.6
0.8
1.0
1.25
1.6
TOTAL

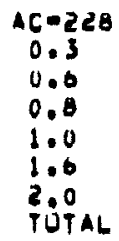

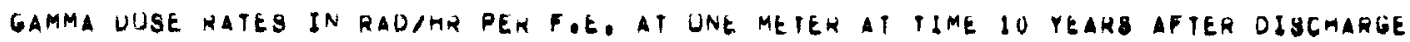

$23 R 3$ RECYCLE UEZ33 F.E.

FERIILE PTL. FISSILE PIL.

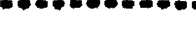

3.810007

1.016000
1,398000

$1.356-00$
3.610000

3,610000
4.965000

3.816007

1.010006
$1.398=06$

1.356000
3.610000

$4.905=06$

0.000

0.000

0.000

0.000

0.000

0.000

0.000

0.000

0.000
0.000

0.000
0.000

y. 000

0.000

$1.404-05$

$7.455-00$
1.833005

$1.833=05$

$1.652=04$

$5.166=05$

$1.191=05$
$2.161-11$

$2.201-11$

$1.083-11$

$2.023-10$

$1.507-11$

$3.575=11$

$2.101-11$

$2.101-11$
$2.201-11$

$2.201=11$

$2.023-10$

1.507 .11

$3.575-11$

$3.076-10$

\subsection{0}

0.000

0.000

0.000

0.000

0.000

0.000
25RS AECYCLE U.ZZ35 P.E.

FERTILE PTL. FISSILE PIL.

3.810007

$1.016-00 \quad 1.910000$

$1.398000 \quad 9.019000$

$3.016007 \quad 1.916=00$
1.016006

$1.398006 \quad 9.010006$

$\begin{array}{ll}0.000 & 1.861=00 \\ 0.000 & 1.095000 \\ 0.000 & 9.322007 \\ 0.000 & 1.742005 \\ 0.000 & 1.297=00 \\ 0.000 & 5.078000 \\ 0.000 & 2.648005\end{array}$

0.000

0.000

0.000

0,000

0.000

0.000

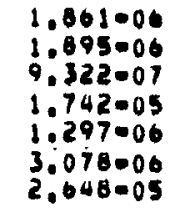

$1.404=05$

$7.455=06$

$1.033=05$

1.65200

5.106005

1.191 .05

0.000

0.000

0.000

0.000

0.000

0.000

0.000 


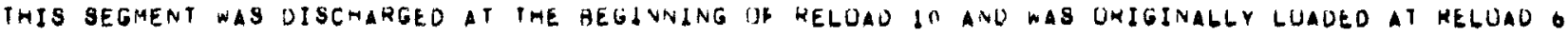
NUCLJDE AND GAMMA ENERGY IN MEV GamMa DUSE KATES IN RaOIMR PEK P.E. AT UNE MEIEH AI TIME IO YEARS AFTER DISCMARGE

ACIINIUM
0.3
0.6
0.8
1.0
1.6
2.0
TOTAL

$H A=223$

0.1

TÜTAL

1
0
0

RAIUM

0.1

$0^{2}=510$

0.3

TUTAL

MAOON

IOTAL

a $1-51]$

0.3
TOTAL

$\theta 1 \cdot 212$

0.8

1.0

IUTAL
23RS RECYCLE U-233 F.E.

FERTILE PIL. FISSILE PILO

$\begin{array}{ll}1.404 .05 & 0.000 \\ 7.455-06 & 0.000 \\ 1.033005 & 0.000 \\ 1.052004 & 0.000 \\ 5.160 .05 & 0.000 \\ 1.191 .05 & 0.000 \\ 2.092-04 & 0.000\end{array}$

$\begin{array}{ll}1.191 .05 & 0.000 \\ 2.092 .04 & 0.000\end{array}$

$1.736000 \quad 1.675007$

$\begin{array}{ll}1.621-00 & 1.075007\end{array}$

0.135007

1.736000

4.621000

$1.675=07$
$4.400=07$

$4.400=07$
$0.135=07$

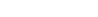

$4.621=06$

4.6210 .06

$4.021-06$

$4.621=00$

4.460007

4.460001

$4.400=07$
$4.460-07$

4.021000

$4.621=06$

$7.534=02$
$1.203-02$

$3.317-02$

$1.205-01$
25RS RECYCLE U-235 F.E.

PERTILE PTL. FISSILE PIL.

\begin{tabular}{|c|c|}
\hline $\begin{array}{l}1.464005 \\
7.455006 \\
1.833005 \\
1.652-04 \\
5.106005 \\
1.191 .05 \\
2.092004\end{array}$ & $\begin{array}{l}0.000 \\
0.000 \\
0.000 \\
0.000 \\
0.000 \\
0.000 \\
0.000\end{array}$ \\
\hline $\begin{array}{l}1.736006 \\
4.621006 \\
6.357006\end{array}$ & $\begin{array}{l}3.084009 \\
8.212009 \\
1.130000\end{array}$ \\
\hline $\begin{array}{l}1.736000 \\
0.621=00 \\
6.357=06\end{array}$ & $\begin{array}{l}3.084000 \\
0.212009 \\
1.130000\end{array}$ \\
\hline $\begin{array}{l}1.621000 \\
4.621000\end{array}$ & $\begin{array}{l}0.212009 \\
8.212009\end{array}$ \\
\hline $\begin{array}{l}4.021=06 \\
4.621=06\end{array}$ & $\begin{array}{l}8.212=09 \\
0.212=09\end{array}$ \\
\hline $\begin{array}{l}4.021=00 \\
4.021=06\end{array}$ & $\begin{array}{l}0.212009 \\
8.212009\end{array}$ \\
\hline $\begin{array}{l}7.534-02 \\
1,203-02 \\
3.317=02 \\
1.205001\end{array}$ & $\begin{array}{l}2.843=05 \\
4.541-06 \\
1.252=05 \\
4.549-05\end{array}$ \\
\hline
\end{tabular}




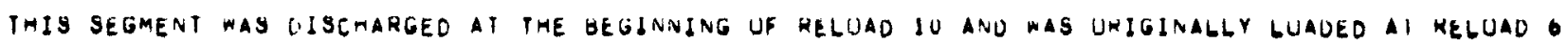
NUCLIDE ANU IN MEY

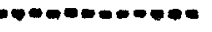

$81-2+3$

0.3

TUTAL

BI SMUTH

0.3

0.8

1.0

TOTAL

\section{$P B-211$}

0.3

TUTAL

$-B=212$

TUTAL

0.3
0.3

0.3

TUTAL

$16=208$

0.6

0.8

IUTAL

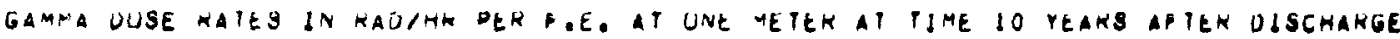

23RS RECYCLE J-233*.,E.

FERTILE PTL, PISSILE PIL.

(1)

$1.380 \cdot 04$

1.088005

$4.304-05$

2.952006
4.590 .05

1.095004

4.349 .05

0.773002

1.085002

$2,982-02$

1.0840 .01

$3.317=02$

$\begin{array}{ll}2.773000 & 2.676007 \\ 2.778006 & 2.081007\end{array}$

$\begin{array}{ll}2.778006 & 2.081=07 \\ 5.550006 & 5.357007\end{array}$

1.504001

1.504001

$1.352-01$
$1.352-01$

$1.504=01$

2.778006

$1.504-01$

$1.352=01$
$2.681=07$

2.681007
1.3520 .01

9.024003

2.649001

9.424002

$1.249+00$

$0.112=05$
2.561001

$4.876=02$

$0.101=01$

$1.223+00$

25A8 RECYCLE U-2SS F.E.

PERTILE PTL. PI8SJLE PTLO

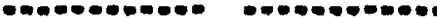

$1.580004 \quad 1.139=09$

$1.008005 \quad 7.014-11$

$1.032000 \quad 9.351009$

$9.534002 \quad 2.843008$

3.9702

$\begin{array}{ll}1.317002 & 1.252=05 \\ 1.207001 & 4.550005\end{array}$

$\begin{array}{ll}2.773006 & 4.927009 \\ 2.778006 & 4.936009\end{array}$

$.530000 \quad 9.863000$

$1.504001 \quad 5.670005$

$1.504001 \quad 5.676009$

$1.504-01 \quad 5.670005$

$1,504001 \quad 5.677008$

$\begin{array}{ll}9.024003 & 3.406006 \\ 2.849001 & 1.075004 \\ 5.424002 & 2.047005 \\ 9.012001 & 3.401004 \\ 1.249400 & 4.715004\end{array}$




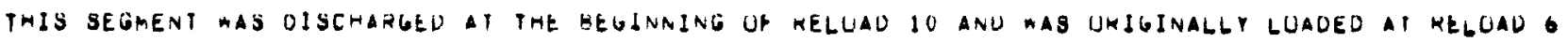

GUCLIDE ANU

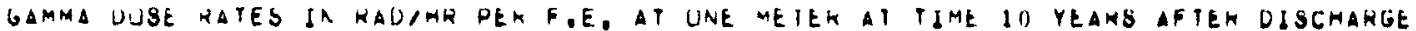

IN MEV

THALLIUMA

THALLIUM
0.3
0.6
0.8
2.4
TOTAL

\section{$T B=158$}

0.3

1.0

1.25

TERBjUM

$\underset{N}{N}$
23RS HECYCLE UEZ33 P.E.

FERIILE PIL. FISSILE PIL.

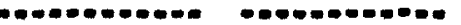

$\begin{array}{ll}9.024003 & 8.112-03 \\ 2.849-01 & 2.561-01 \\ 5.024=02 & 4.070-02 \\ 9.012=01 & 0.101-01 \\ 1.249+00 & 1.123+00\end{array}$

$0.317-10$

5. $414=09$

4.144000
1.305009

$4.879=08$

$8.962-10$

7.682009

5.880008
$1.851=09$

$1.851=09$
$6.923=08$

$0.311-10$

5,414009

4.144008

$4.879-06$

\section{$0.962-10$ \\ 7,682009 \\ $5,880=06$ \\ 1.851009 \\ $0,923=00$}

1.009003
2.674003

$2.772=03$

$7.970-03$

$6.272-03$

2.284004

$2.908-01$

1.289001

$3.391-01$

1.805000

$1.749+00$

$2.089+00$

2.0090001

2.076001
$7.256+00$
$1.209=03$

$3.205=03$

$5.235=04$

3.32200

7.510003

$2.737=04$

2.560002

0.103001

2.651 .01

$6.973=01$

$3.795+00$

$3,597+00$

$5,528+00$

4,269001
$1,492+01$
25RS RECYCLE UEZJS P.E.

FERIILE PTL. FISIILE PTLO

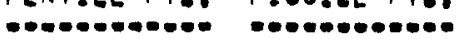

$\begin{array}{ll}9.024003 & 3.400006 \\ 2.849001 & 1.075004 \\ 5,424002 & 2.047005 \\ 9.012001 & 3.401004 \\ 1.249+00 & 4.715=04\end{array}$

$0.317-10$

5.414000

4.144000

1.305009
4.899008

$6.647-11$

$7.412-10$

5.073000

$1.780-10$

6.680000

$0.317-10$

5,414009

$4.144=08$

1.305009
4.879000

$0.047-11$

7.422010

5.073009

$1.786-10$

0.680009

1.009003

2.674003

4.368000

2.772003

$7.970-03$

0.272003

2.284004

$2,130.02$

1.100003

3.127 .03

5.100004

3.242003

$9.320-03$

7.334003

2.671004

2.498002

2.968001

0.034001

$1.289-01$

$1.845+00$

$1.845+00$

$1.749+00$

$2.089+00$

2.070001

$2.021-01$

0.094001

$3.752+00$

$3.557+00$
$5.466+00$

$5.466+00$

$4.221 \cdot 01$ 


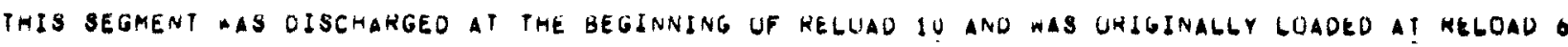
NUCLIDE ANU IN MEV

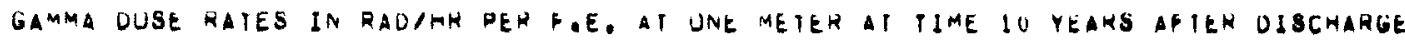

EUnOPIUM

0.13

0.6

1.0
1.25
1.6

IUTAL

$P R=144$

0.6

1.25

1.6
2.0

TÚTAL

prasevoYmiUm

0.6

0.8

1.25
1.0
2.0
1074

TOTAL

$B A+137 M$

0.1

TUTAL

BAKIUM

$0: 1$

TÓTAL
23RS RECYCLE U.233 P.E.
FERTILEPTLE FISIILEPTL.

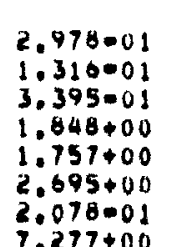

$0.115=01$

2.683001

$0.978=01$

$3.790+00$

$3.607+00$

$5.536+00$

$1.495+01$

$.277+00$

1.911004

$8.864-05$

$3.017=03$

$2.666=02$

1.020002

1.020002
$1.911=04$

$8.864-05$

$3.617-03$

$1.257=02$

$2.066=02$

$1.091+00$
$2.879+02$

$2.879+02$
$2.889+02$

$4.682+02$

$4.699+02$

$1.091+00$

$2.879+02$

$2.889+02$

7,019003

1.465004
0.797005

0.797005

$0.637-0.5$

2.044002

7,819003

$1.463=04$

0.797005

:. 173700

2.044002

$1,774+00$

$9.682+02$
$4.099+02$

25RS RECYCLE UOZ35 F.E.
FERTILE PTL FIBSILE PTL,

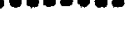

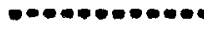

$\begin{array}{ll}2.978001 & 6.040001 \\ 1.310001 & 2.653001 \\ 3.395001 & 6.89901 \\ 1.048+00 & 3.755+00 \\ 1.757+00 & 3.566+00 \\ 2.695+00 & 5.473+00 \\ 2.078001 & 4.224001 \\ 7.277+00 & 1.478+01\end{array}$

$1.020002 \quad 6.372003$

$1.011004 \quad 1.194004$

$8.064005 \quad 5.539005$

$3.017 .03 \quad 2.261 .03$

$1.257002 \quad 7.694=03$

$2.660002 \quad 1.666002$

$\begin{array}{ll}1.020002 & 6.372003 \\ 1.911004 & 1.194004 \\ 8.864 .05 & 5.539005 \\ 3.617003 & 2.261003 \\ 1.257002 & 7.654003 \\ 2.060 .02 & 1.640002\end{array}$

$2.060002 \quad 7.05003$

.660 .02

$1.091+00 \quad 1.222+00$

$2.879+02 \quad 3.225+02$

$2,809+02 \quad 3.237+02$

$1.091+00 \quad 1.222+00$

$\begin{array}{ll}2.879+02 & 3.225+02 \\ 2.889+02 & 3.237+02\end{array}$ 


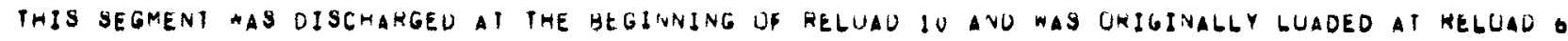
NUCLIDE ANU
GAMMA ENERGY

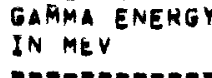

IN MEV

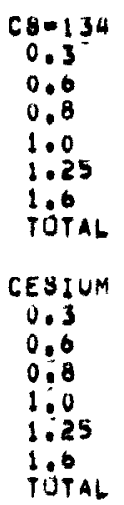

$\underset{⿱}{\stackrel{ }{\sim}}$

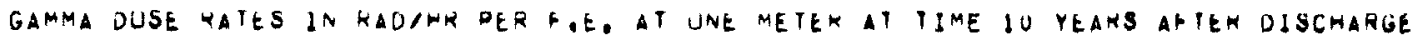

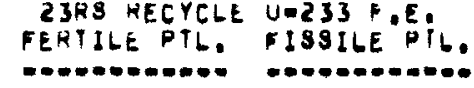

$2.043+01$

$2.120+01$

2.89501

$1.764+00$

$9.763-63$

$4.370+01$

2.707 .03

$2.043+01$

$2.120+01$

2.895001

$1.764+00$

$9.763-03$

$4.370+01$

$1.077-01$

$1.022+00$

2. $648+00$

$3.178+00$

$1.077-01$

$1.022+00$

2. $048+00$

$3.178+00$

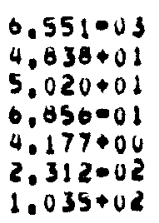

.551003

$4.836+01$
$.020+01$

$9.020+01$
0.850001

$4.179+00$

$4.177+00$

$1.035+02$

$1.268-01$

$1.204+00$

$2.412+00$

ANI IMONY

0.1

0.6

AG०I UBM

0.1

0.6

TUTAL
$1.075=00$

1.303004

1.377004

1.477000

$3.080=04$
$1.268-01$

1.204400

$2.412+00$

$3.742+0$ ?

1,205007

1.020 .05

$1.534=05$

$1.730-05$

$2,328-07$
$4,332-05$
2598 RECYCLE U-235 P.E.

FERTILE PTL, FIS3ILE PTL.

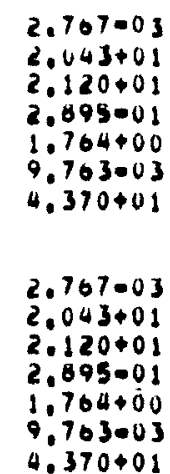

$3.413=03$

$4,148+01$

5.605001

$3.452+00$

1.910002
8.550001

$5,4,3003$

3.998001

$4.148+01$

5,065-01

$3.452+00$

$1.910=02$

$8,550 \cdot 01$

$\begin{array}{ll}1.077001 & 2.486002 \\ 1.022+00 & 2.301 .01 \\ 2.048+00 & 4.731001 \\ 3.178+00 & 9.341001\end{array}$

$3.178+00$

7,341001

$\begin{array}{ll}1.077-01 & 2.480002 \\ 1.022+00 & 2.361-01 \\ 2.048+00 & 4.731-01 \\ 3.170400 & 7.341001\end{array}$

1.079000

$8.697-05$

$1.303=04$

$1.477=04$

1.976006
3.680004
2.147005

3.645005

4.881 .09

9,082005
$2,652=07$ 


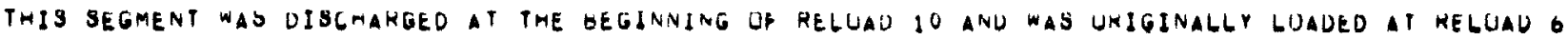

NUCLIDE ANO GAMMMA ENERGY IN MEV

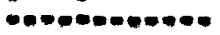

$4 G 0110 \mathrm{M}$

0.1

0.3

0.8

1.25

1.6

2.0

TOTAL

兑

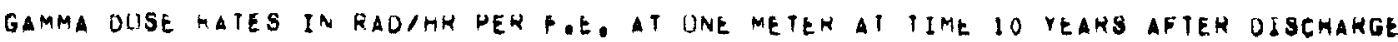

$$
\begin{aligned}
& \text { ZJRS KECYCLE U-Z33F.E. } \\
& \text { FERTILE PIL, FISSILEPIL. }
\end{aligned}
$$

$9.832=09$

$8.272=07$

4.061 .05

5.410005

1.600005

$1.772=05$

1.354005

$6.519=0$

8.814008

$1.431=04$

$1.084=06$

$8.780=05$

1.70900

2.018004

$1.804-05$

$1.772=05$

$1.354=05$
$8.519=08$

8.814000

TOTAL

RHE 106

$2 H=106$
0.3
0.06
0.8
1.0
1.25
1.0
2.0
2.4
2.9
TOTAL

1.024000

1.367000

0.710005

8.938005

$2.653-05$

2.92005

2.237005

1.407007

$2,363=04$

$1.427-07$

1.161 .05

8.243005

$1.008=04$

2.677005

$2.928=05$

$2.237-05$

$1.407-07$

$1.450-07$

2.797 .04

$2.117-04$

0.142002

$5.421=03$

1,804003

1.245003

4.593004

4.591 .04

$7.539-05$

7.244002
25HS REEYCLE W-2J5 F.E.

FERTILE PTL. FISSILE PIL.

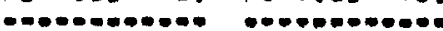

$\begin{array}{ll}9.832-09 & 9.915009 \\ 8.272-07 & 8.344007 \\ 4.061-05 & 4.097005 \\ 5.410005 & 5.457005 \\ 1.000005 & 1.620005 \\ 1.772005 & 1.706005 \\ 1.354005 & 1.366005 \\ 8.519008 & 8.593008 \\ 8.814=08 & 8.891008 \\ 1.431=04 & 1.443-04\end{array}$

\section{$1.084000 \quad 2.751007$}

$8.780 .05 \quad 2.230005$

$1.709004 \quad 7.313005$

$2.018 .04 \quad 9.102005$

$\begin{array}{ll}1.804-05 & 1.669-05 \\ 1.772-05 & 1.780-05\end{array}$

1.354005

$8.519006 \quad 8.593008$

$8.814008 \quad 8.891000$

$5.110 .04 \quad 2.351004$

$2.238004 \quad 2.121004$

$\begin{array}{ll}2.238004 & 2.121000 \\ 0.491-02 & 0.132002\end{array}$

$1.420 .03 \quad 1: 346003$

$5.729003 \quad 5.430003$

$1.907 .03 \quad 1.807003$

$1.316003 \quad 1.247=03$

4.601 .04

$4.852004 \quad 4.598004$

$7.967-05 \quad 7.552005$

7.250002 


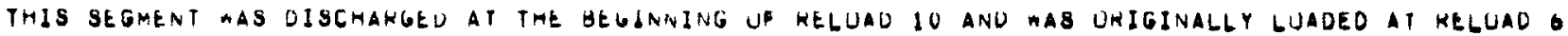
NUCLIUE ANO GATMA ENERGY IN MEV

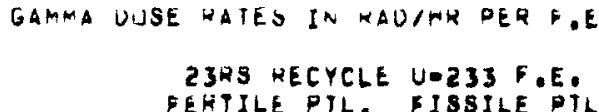

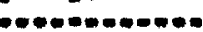

EEHTILE PTL. FISSILE PTL.

\section{RHUDIUM}

0.3
0.6
0.8
1.0
1.25
1.6
2.0
2.0
2.9
TUTAL

COM60
1.25
TOTAL
CUEALT
1.25
$909 A 6$

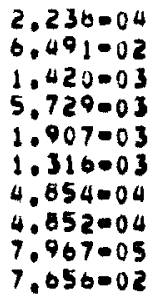

$$
\begin{aligned}
& 2.117004 \\
& 6.142-02 \\
& 1.343003 \\
& 5.421003 \\
& 1.804003 \\
& 1.245003 \\
& 4.593004 \\
& 4.591004 \\
& 7.539003 \\
& 7.244002
\end{aligned}
$$

$\{.817=04$

9.710006

$1.817-04$

9.718000

1.817004

$9.718-06$

1.496400

1.310400

$2.318+01$

$2.318+01$

4.401 .00

2.55800

1.306002

9.010001

$7.967=05$

$3.447+02$

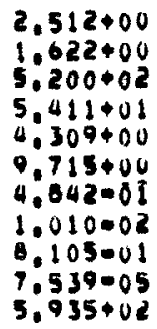

$2,512+00$

$1.622+00$

$5.411+01$

$0.715+00$

.042001

8.105001

$5.935+02$

25RS AECYCLE U-235 E.E.

FERTILE PTL. PISOILE PIL.

$\begin{array}{ll}2.238004 & 2.121004 \\ 0.091002 & 0.152002 \\ 1.420003 & 1.346003 \\ 5.729003 & 5.430003 \\ 1.907003 & 1.007003 \\ 1.310003 & 1.247003 \\ 4.854004 & 4.001004 \\ 0.852004 & 4.998004 \\ 7.967005 & 7.552005 \\ 7.056002 & 7.250002\end{array}$

$\begin{array}{ll}1.817004 & 2.038005 \\ 1.817004 & 2.030005\end{array}$

$\begin{array}{ll}1.817004 & 2.038005 \\ 1.817004 & 2.038005\end{array}$

$\begin{array}{ll}1.406+00 & 1.051+00 \\ 1.316+00 & 5.071001 \\ 3.110+02 & 3.637+02 \\ 2.318001 & 4.524+01 \\ 2.065+00 & 4.138+00 \\ 4.461+00 & 8.927+00 \\ 2.558001 & 4.450001 \\ 1.306002 & 8.314003 \\ 9.016001 & 8.000004 \\ 7.967-05 & 7.352005 \\ 3.447+02 & 4.240002\end{array}$


TABLE D-8

GAMMA DOSE RATES FOR FUEL ELEMENTS DISCHARGED AT BEGINNING OF RELOAD 13 AND ORIGINALLY LOADED AT RELOAD 9

NOTE: Table D-8 consists of 58 pages of computer print-out (pages D-219 through D-276). 
•

-

0 


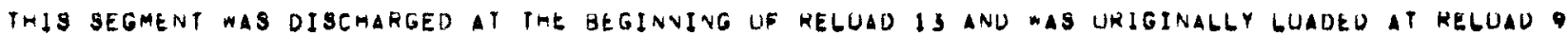

NUCLIDE AND GAMMA ENERGY IN MEY

$0+\infty=0$

$\cup \cdot 235$
0.1

TÓTAL

URANIUM

0.1

Tutal

$P A=233$

0.1

TUTAL

\begin{tabular}{l} 
N \\
\hline 1
\end{tabular}
MS FRESH U.ZZ3S F.E. FERTILE PTL, FISSILE PTL

3.717-07

$9.898=07$
$1.302=00$

$2.605=00$

7.409 .06

1.027 .05

$3.717-07$

$9.898=07$
1.362000

$2.805=06$

7.469000
1.027005

$9.945+01$

$0.598+02$
$7.592+02$

1.312007

$1,002=06$

1.207 .02
1.229 .02

5.364007

$5,40300 ?$

2.087 .07

$1.130=01$

1.9960 .02

5.021006

$3.740=07$

$1.717=01$

7.634006

$9.945+01$

$0.598+02$

1.229 .02

$0.045-03$

$1.150-01$

8.413 .03

1.996002

$7.594+02$

$1.312=07$

1.407000

5.403 .07

2.687 .07

$5.021=06$

3.740 .07

8.872 .07

0.030006
23RS RECYCLE U-235 FE.

ERTILE PTL. FISSILE PTL.

.....

$3.717 \cdot 07$

$9.898=07$

1.302000

$1.501=00$

3.624006
4,985000

$3.717-09$

9,898007

1.301000

$3.624=06$

$9.945+01$

$6,598+02$

$1.037=06$

6.879000

$1.207-02$

$1.229-02$

$6.045-03$

1.130001

8,413003

1.990002

$9.945+01$

$0.598+02$

1.229002

0.045003

$1.130=01$

$8.413-03$

$1.594+02$

1.498010

$1.526-10$

$7.504=11$

1.402009

$1.044-10$

2.478010

2.076010

$1.504-1$

$1.402-09$

1.044010

$2.078-10$
258 RECYCLE

PEHTILE PTL OISSILE PTL

3.717007

9.898 .07

$1.302-06$

5.193000

$5.840=06$

$8.033=00$

$3.717-07$

9.898007

1.302006

$2.193=06$

5.640006

$9.945 \times 01$

$6.596+02$

2.627006

1.743005

1.207002

1,229002

1. $130=01$

0.41300

0.09000

1.996000

4.002000

4.075006

2.004006

$3.746-05$

2.790000

$6.619-00$

$1.037000 \quad 9.045+01$

6.598402
1.229002
9.045002

0.045003

0.045003

$0.413=03$

1.996002

$7.594+02$

2.027000

2.143005

0.075000

$2.004=00$

3.700005

$2.790=00$

$6.619=06$

$7.741=05$ 


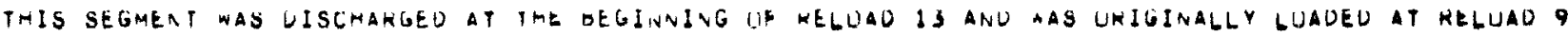
GAMMA OOSE HATES IN WAUIMN HER F.E. AT UNE METER AT IIME 180 DAYS APTER DISCHARGE

NUGL IDE AND

GAMMA ENERGY

IN MEV

$1 C=228$

0.3

0.0

1.6

TỨ AL

ACIINIUM

AC $1210 \mathrm{M}$
0.3
0.0
0.8
1.0
1.0
2.00
$T U T 4 L$

N
$N$
0
MS PRESH LO-235 F.E. FERTILE PIL. FISSILE PTL.

$0.111-00$

4.130000

1.010005

$9.150=05$

0.598 .00

1.491 .04

$0.111=00$

4.130000

$1.010-05$

9.150 .05

2.862 .05

0.598 .06

$1.491-04$

2.402 .06

0.394 .00

$8.795=0$

TUTAL

RAUIUM

POTAL

$2.402=06$

0.394 .06

$8.795-06$

$R N=219$

0.3

IUTAL

RAUUN

TUTAL
0.394006

$0,594=06$

$0.394=00$
$6.394=00$
0.000

0.000

0.000

0.000

0.000

0.000

0.000

0.000

0.000

0.000

0.000

0.000

0.000

0.000

$1.971 \cdot 10$

$5.248 \cdot 10$
$7.219 \cdot 10$

$1.971 \cdot 10$
$3.248 \cdot 10$

$3.248-10$
$7.219-10$

23 HS HECYCLE UDESS F.E.

FERTILE PIL. FISSILE PIL.

-.0.0.0.

8. 111.06

$4.130=00$

$1.010=05$

$9.150-05$

2.662 .05

1.091004

$2.877-11$

$1.405-11$

3.245010

$1.015-10$

$2.340-11$

8.111000

$4.130-06$

1.016005

$9.150=05$

$2,862.05$

0.598006

2.677 .11

$1.405-11$

$3,002-11$

$3.245-10$

1.015010

$2.340-11$

1.491004

2.402006

0.394006

$8.795=06$

1.202007

3.202007

$4.404=07$

$2.402=06$

$0.394=06$

1.202007

$3.202=07$

4.404007

5.248 .10

5.248 .10

$5.248-10$
$5.208-10$

0.394 .06

3.20200

3.202007

$3.202 \cdot 07$
$3.202 \cdot 09$

0.394000
25KS RECYCLE U=C3S P. E

FEMIILE PIL. FISSILE PIL.

$\begin{array}{ll}0.111-06 & 0.000 \\ 4.130006 & 0.000 \\ 1.010005 & 0.000 \\ 9.150005 & 0.000 \\ 2.862005 & 0.000 \\ 0.598006 & 0.000 \\ 1.491004 & 0.000\end{array}$

$8.111 .00 \quad 0.000$

$4.130006 \quad 0.000$

0.000

$0.862-05 \quad 0.000$

$0.598-00$

$1.401 .04 \quad 0.000$

\section{$2.402=06$}

0.394006

8.795006

0.200010

2.104009

$3.004=09$

2.402000

0.394000

$8.795=00$

$0.204=10$

2.184009

$3.004=09$

$0.394=00$

0.394 .06

2.184 .09

2.184009

0.394000

0.394000

2.184009
2.184009 


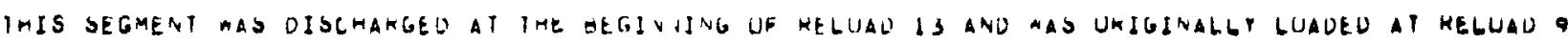

NUCLIDE AND GAMMA ENERGY IN MEV

(N)

8I-211

0.3

010.515

0.8

0.8

TútAL

$8 I-213$

0.3

TUTAL

0
$N$
$N$

GISMUTH

0.3

0.8

tüTAL

$P B-211$

0.21
0.3
0.8

iutAL

P8-2 12

TUTAL

LEAO

0.5

TUTAL

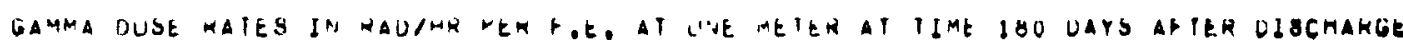

MS RRESH U-235 F.E.

FERIILE PIL. FISSILE PIL.

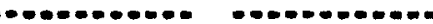

$0.394-10 \quad 5.248 \cdot 10$

5.248 .10

3.869 .00

9.376007

2.584000
9.391006

5.769 .05

1.590002

$2.102-05$

1.483006

$4.845-10$

$3.296-11$
$5.135 \cdot 10$

$1.005-09$

$9.869=00$

9.376009
2.584 .06

$2.584=06$
$9.392=00$

3.012002

5.770003

$1.590-02$
5.781 .02

0.392000

$3.830=06$

$3,843=06$

3.149 .10

$3.154-10$

$6.343 \cdot 10$

23HS KECYCLE U-23SF.E.
FERTILE PTL. PSSILE PIL

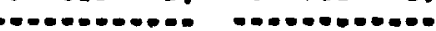

$0.394 \cdot 06$

$0.394=00$

3.202007

3.202007

3.012002

1.5900 .02

7.475002

1.194002

3.291002

1.190001

2.1020 .15

1.483006

$9.325 \cdot 05$

$0.395=00$

$2.801=05$

$3.612=02$

$5.770-0$

1.390002

$9,357.05$

9.475002

$1.195=02$

$3.291=02$

$1.197-01$

$3.843=00$

7.080006

$1.921=07$

1.924007

$1.924=07$
$3.845=07$

$1.172-05$
1.172005

7.210002
7.210002

1.492001

$1.492-01$

7.210002

1.172005

7.211002

9.211 .02

3.154010

3.845006
7.211 .02

1.092001

1.924007

1.492001
2SKS HECYCLE UA235 F.E.

PERIJLE PTL. FISSILE PIL.

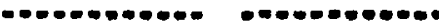

$0.394000 \quad 2.104009$

3.012003

5.769005

1.590002

$2.4<7=05$

$3.804=05$

$2.102 \cdot 05$

$1.483006 \quad 1.357-10$

2.801005

3.612 .02

5.770003

1.590 .02

1.590002
5.701002

2.427009

3.077000

0.069005

.664005

1.310009

3.843 .06

1.313000

$\$ .080000$

.623000

$7.210=02$

7.210002

4.840 .05

4.646005

7.210002

3.843000

$4.846=05$
$1.313=09$

1.313009
4.840005 


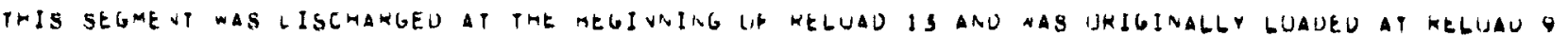

NUCLIOE ANO
GAMMA ENERGY

GAMMA ENERGY

$T L=208$
0.3
0.0
0.8
2.4
TUTAL

THALGIUM

0.3
0.6

0.6
0.8

TOTAL

$18-156$

0.3

0.0

1.0

rutal

$T 8=100$

0.3

0.8

1.0

TEKBIUM

0.3
0.8

1.0

1.0
GAMMA DLSE NAIES DS

MS FRESH U-235+.E. FEKTILE PIL. PSSILt PIL.

\section{$4.103=03$ \\ $1.320=01$ \\ $2.514=02$ \\ $4.177=01$
$5.790=01$}

2.140005

4.000000

0.788 .05

$9.410 \cdot 05$

$4.183 \cdot 03$

$1.320=01$

2.514 .02

4.177 .01

5.790001

$0.798=07$

0.790007
$2.100-05$
0.700000

0.788005

9.410005

$0.479-10$

4.251006

$4.251=08$
1.338 .09

$5.005=08$

$1.149 \cdot 10$

9.848 .10

$7.538-09$

$2.373-10$

$2.712=02$

$0.542-02$

0.902002
5.024 .02

2.797 .01

3.322002

8.042 .02

0.239002

$2.702-02$

$6.542-02$

0.712 .02

5.024002

2.397001

\subsection{2 .02}

0.239 .02

$0.175=02$
$2.576-01$
CSRS KECYCLE UN-235 F.E.

FERTILE PTL. BDSSILE HTL.

$$
\begin{aligned}
& 4.183003 \\
& 1.320001 \\
& 2.514002 \\
& 4.177 .01 \\
& 5.790 .01
\end{aligned}
$$

9.9540 .33

2.027 .01

3.302002

$8.942=01$

$1.240+00$

4.185 .03

1.320001

2.514002

4.177001

0.454 .03

2.527001

5.302002

8.942001

$1.200+00$

$0.479=10$

5.554009

1.330 .00

$5.005=08$

$0.158-10$

0.993009

5.353000
1.005 .009

1.005009

$2.702-02$

6.545002

0.702002

$2.097-11$

$2.991=02$

$7.241=02$

5.410 .02

2. $521=01$

2.702 .02

$0.542=02$

$0.702-02$

$5.024-112$
$2.097-01$

$2.941=02$

3.241 .02

$1.410-02$

$5.500=02$

2.321001
ZSRS RECYCLE UAZ35 .E.

FERTILE PTL. FISSILEPIL.

$\begin{array}{ll}4.103003 & 2.810000 \\ 1.320011 & 8.873005 \\ 2.514002 & 1.009005 \\ 4.177001 & 2.800004 \\ 5.790001 & 3.891004 \\ & \\ 4.103003 & 2.810006 \\ 1.320001 & 0.813005 \\ 2.514002 & 1.609005 \\ 4.177001 & 2.800004 \\ 5.790001 & 5.891004\end{array}$

$0.479-10$

4.251 .00

5.00500

2.102002
0.542002
0.702002
5.024002

2.097 .01

1.216010

1.095009
$8.383=09$

$2.639-10$

2.639010
9.810004

5.673 .02

1.374001

1.407 .01

1.055001

$4.403=01$

2.702002

5.073002

0.542 .02

6.702 .02

5.024 .0

$2.097-01$

1.407 .01

$1.055-01$

$4.403=01$ 


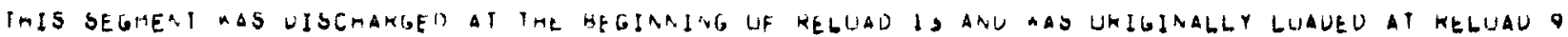

NUCLIUE AND GIMMA ENERGY IN MEV

\section{EU-152}

0.15
0.1
0.0
0.8
0.8
1.0
1.25
1.0
TOTAL

EU. 154

0.1

0.3

0.0

0.8
1.0
1.25
1.0

IUTALL

$E U=156$

$E U=156$
0.6
0.8
1.0
1.25
2.0
2.4
$T U T A L$

EUROPJUM

0.1
0.3
0.0
0.6
1.0
1.25
1.0
2.0
2.4
TUTAL

0.1

0.0

0.6

1.25

10

TUTAL
MS FHESH U-235 F.E. Z3RS RECYCLE U-235 FEKTILE PTL. FISSILE PIL. PETILE PIL. FISSILE PTL.

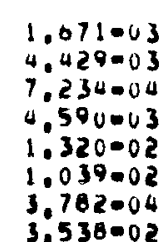

3.939004

3.538 .02

$4.340-61$

$4.960-01$

5. $7 \cup 2+00$

$2.562+00$

$3.957+00$

3.040001

1.057 .02

$1.057=02$
$3.119=02$

2.790 .02

7.332002
$.100-02$

$7.100-02$

$2.320-01$

$4.303=01$

$1.932=01$

$5.078=01$

$2.738+00$

$2.003+00$

$.021+00$

$3.044=01$

$0.100-v 2$

7.942003

\subsection{3 \\ 7.23200 \\ $1.181-03$ \\ $0.497-03$ \\ $1.090=02$ \\ $0.177=04$}

$1.227+00$

$5.331=01$

$1.402+00$

$7.035+00$

1.111001

1.111 .01
3.583 .01

$3.000+01$

$2.925=02$

8.033002
7.723002

2.029 .01

2.243 .01

$2.198-0$

$0.421-01$

$1.230+00$

5.403001

$1.432+00$

$7.723+00$

$7.331+00$

$1.133+01$

$2.243=0$

2.198002

$3.070+01$

\subsection{3 \\ 4.926003
$8.047-04$ \\ 5.04000 \\ 2.100003 \\ 1.155 .02 \\ 4.207004
3.935002

0.159001
3.530001
9.290001
$5.060+00$
$4.197+00$
$7.372+00$
5.693001
$1.990+01$

4.3460 .01

1.888001

4.966001

$2,562+00$

$3.937+00$

3.040001

$1.062+01$

1.057 .02

3.119002
2.790 .02

9.332 .02

$8.106-02$

$7.942=03$

2.320001

$4.305=01$

1.932 .01
5.078 .01

1.078 .01
2.734000

$2.734+00$

.

$3.004=01$

3.004001

8.106002
$1.942-03$

$1.089+01$

$1.942 \cdot 02$

$5.732=02$

1.34700

1.459002

$4.203=01$

6.157001

3.50500

9.50100

$5.123+00$

4.803400

$7.510+00$

$5.097 \cdot 0$.

$1.489=0$

1.459 .02

c. 036401
2SHS RECYCLE U-235 F.E.

FERTILE PTL. FISSILE PTL

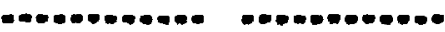

\begin{tabular}{|c|c|}
\hline $\begin{array}{l}1.671=03 \\
4.429005 \\
7.254004 \\
4.590003 \\
1.320002 \\
1.039002 \\
3.782004 \\
3.538002\end{array}$ & $\begin{array}{l}2.147005 \\
5.640003 \\
9.295004 \\
3.898003 \\
1.696=02 \\
1.355002 \\
4.800004 \\
4.546002\end{array}$ \\
\hline $\begin{array}{l}4.340001 \\
1.888001 \\
1.966001 \\
2.702+00 \\
2.562+00 \\
3.937+00 \\
3.040 .01 \\
1.002+01\end{array}$ & $\begin{array}{l}9.354-01 \\
4.055001 \\
1.067+00 \\
5.004+00 \\
9.502+00 \\
0.056+00 \\
0.520001 \\
2.262+01\end{array}$ \\
\hline
\end{tabular}

1.057 .02

3.110002

3.790002

7.332002

1.100002

2.320001

$\begin{array}{ll}4.303001 & 9.356001 \\ 1.932001 & 4.112001 \\ 5.078001 & 1.090+00 \\ 2.738+00 & 5.876+00 \\ 2.003+00 & 5.578+00 \\ 4.021+00 & 8.624+00 \\ 3.044001 & 0.534001 \\ 8.106002 & 1.710001 \\ 7.942003 & 1.675002 \\ 1.089+01 & 2.355+01\end{array}$

2.229 .02

6.579000

5.885002

$1.547-01$

1.71000

$1.675=02$

$843=0$

$2.355+01$ 


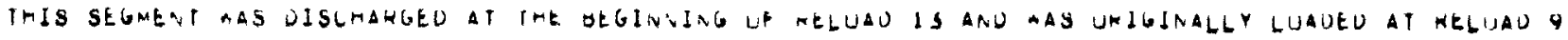

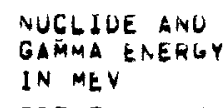

IN ME

\section{$P M=148 M$}

0.1

0.3

0.0

1.0

TUTAL

DRUMETHIUM

0.1

0.0

I.0

$P$
$N$
$N$

0.1

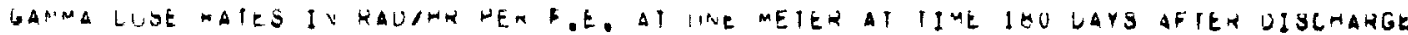

MS FQESH U-235 F,E.

FERTILE DTL. FISSILE PTL.

$4.310-42$

$1.240+60$

$9.896+0$

$1.805+00^{\prime}$

$2.853+01$

$1.580+01$

$4.314-82$

$1.240+00$

$9.896+00$

$1.805+00$

$1.853+00$
$1.584+01$

$1.156-1) 3$

7.196004

$3.197-63$

$5.053=03$

$1.136=03$

1.196004

5.153 .03

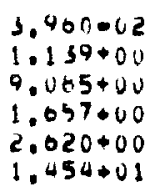

3.900002

$1.139+00$

$9.085+00$

$1.657+00$

$1.454+0$

0.3

TUTAL

NEUOYMIUM

0.1

0.6

TUTAL

PR- 144

0.6

0.8
1.25

1.25

2.0

TUTAL

$4.382-04$

2.775004

1.2330 .03

1.949003

4.3820 .04

$1.233=0$

1.949 .01

$4.87 \mathrm{~s}+01$

$9.132=01$

$.236-01$

$1.729+0$

$6.000+01$

$1.274+02$

$4.34 v+01$

$0.133=0$

$3.773=0$

$1.540+0$

$5.349+01$
$23 R S$ KECYCLE U-235 F.E.

FENIILE PIL. FISSILE PIL.

-............

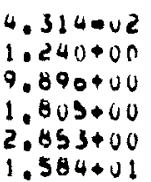

4.314002

$1.24 v+0 v$

$9.800+00$

$1.805+00$

$2.053+00$

$1.584+01$

1.130003

$7.190-04$

$3.197=03$

$5.053=03$
1.130003

3.19700

5.653 .03

$3.945=04$
2.494004
1.110003
1.754 .03

$4.873+01$

$0.152=01$

$4.236=01$

$1.729+01$

$1.0006+01$
$1.274+02$

$3.471+01$

0,504001

3.017001

$4.278+01$

$9.075+01$
$2.942 \div 02$

0.005 .01

$0.005+00$

$1.252+00$

$1.090+01$

\section{$2.992-112$}

$0.005=01$

1.252000

$1.979+00$

$1.099+01$

3.945004

2.498004

$1.110-03$
$1.754=03$

25HS KECYCLE WOCJ5 P.E.

ERTILE PTL. FIS3ILE PIL.

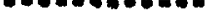

4.314002

1.240400

$9.890+00$

$1.005+00$

$2.853+00$

2.974002 0.552001 $0.022+00$

1.204000

$1.967+00$

$1.042+01$

4.314 .02

1.240400

$9.840+00$

$1.805+00$

$2.853+00$

0.974002

0.352001

$6.822+00$

$1.244+00$

1.907 .00

1.042001

1.150003

7.196004

$3.197-03$

3.354 .04

2.120004

$1002=03$

$1.136-03$

$7.196004 \quad 2.124004$

$3.197 .03 \quad 9.439 .04$

$5.053=03 \quad 1.402 .03$

3.250402

0.101001

2.830001

$1.135+01$

$\begin{array}{ll}0.000+01 & 4.013+01 \\ 1.274+112 & 0.513+01\end{array}$ 


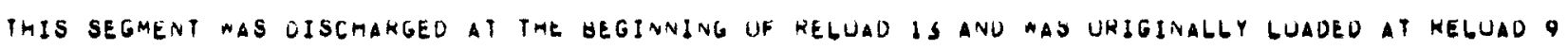

NUCLIOE ANU GAMMA ENERGY IN MES

inom.not

PRABEUDYMLUM

0.6

1.25

2.0

TUTAL

$L A=140$

10.140
0.1
0.3
0.6
0.8
100
1.25
1.0
2.4
2.9
3.5
$10 T 4$

LANTIANUM

0.1
$0: 3$
0.6
0.8
1.0
1.25
1.0
$2: 0$
$2: 9$
3.5
TUTAL

MS FRESH UNZ35 F.E. FERTILE PIL. FISSILE PTL.

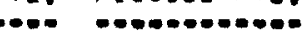

$4.873+01$
9.132001
$4.236-01$
$1.729+01$
$0.000+01$
$1.274+02$

$4.300+01$ 0.13300

$3.773-01$

$1.940+01$
$5.349+01$

$1.135+02$

$1.145-03$

$0.432=02$

1.737 .01

2.001 .01
7.051 .02

$1.908-03$

$1.025+00$

$0.271-02$

1.516003

$5.168 \cdot 05$

$5.168-05$
$1.001+00$

$1.142=03$

$6.432=0$

1.757 .01

$2.001=01$

$1.051=52$

$1.9,08=03$

$1.025+00$
0.271002

0.871002

$1.510=03$
$5.168-05$

$1.001+00$

$$
\begin{aligned}
& 3.317004 \\
& 1.808-02 \\
& 5.043=02 \\
& 5.810002 \\
& 2.048002 \\
& 5.541-04 \\
& 2.976001 \\
& 1.821002 \\
& 4.002004 \\
& 1.501-05 \\
& 4.649-01
\end{aligned}
$$

$3,317=04$

1.808002

5.0430 .02

$5.810=02$

2.048002

5.541004

$2.970-6$

1.821002

4.402004

$1.501=05$
$4.649-01$
23 RS RECYCLE UAZ3S F.E. FERTILE PIL. FISSILE PTL.

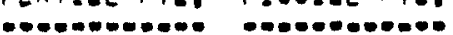

$\begin{array}{ll}4.073+01 & 3.471+01 \\ 9.132001 & 0.304001 \\ 4.230001 & 5.017001 \\ 1.729+01 & 1.231+01 \\ 6.000+01 & 4.278+01 \\ 1.274002 & 9.075+01\end{array}$

$1.274+02$

$9.075+01$
1.142003

$0.432=02$

$1,737=01$

2.001001

1.908003

$1.025+00$

$6.271-02$

51003

. 108005

$1.601+00$

0.432002

1.737 .01

$2.001=01$

$7.051=02$

1.908003

$1.025+00$

0.271 .02

$1.516=03$

5,108005

$1,601+00$
$1.142=03$
3.443004

1.039002

5.235002

0.032002

5,752004

5,752000

1.890002

1,890002

1.558005

4.826001

3.4430 .04

939002

5.235002

6.032 .02

2.126002

3,069001

1.890 .02

$4.570-0$

1.558005
4.026001
25RS RECYCLE U $235 \mathrm{~F}$. FERTILE PTL. FISSILE PIL.

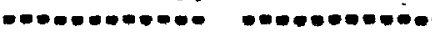

$\begin{array}{ll}4.873+01 & 1.250+01 \\ 9.132001 & 6.101001 \\ 4.230001 & 2.830001 \\ 1.729+01 & 1.155+01 \\ 6.006+01 & 4.013+01 \\ 1.274+02 & 0.513+01\end{array}$

0.432002

$1.737=01$

$2.001=01$

1.908003

$1.025+00$

$0.071=05$

516003

1.516003

$\$ .168005$

2.528004

3.424002

$3,844=02$

4.429002
1.501002

1.501002

$4.223=04$
$2.208 \cdot 01$

1.385000

3.355004

$3.14=0$

3.1443001

1.142003
6.432002

$1.737=01$

1.001001
3.001009

7.051002

$1.908=03$

$1.025+00$

.271002

1.516003

.168005

$1.601+00$

1.424002

3.844002

4.429 .02

1.501 .02

$4.223=04$

2.268001

1.388002

3.355004

1.144 .05

3.54301 


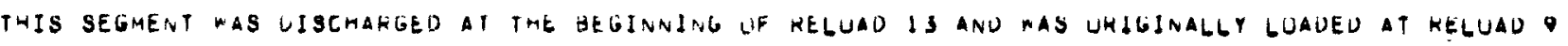

NUCLIOE AND GAMMA ENERGY

$B A-137 M$

0.1

TUTAL

$\forall A-140$

0.1

0.1
0.6
0

BAKIUM

0.1

0.3

$\Theta$
$N$
$N$
WS FRESA UAZ35 F.E.

FERTILE PTL. FISSILE PTL.

\section{$1.332+v 0$}

$3.515+02$

$2.007+00$

$5.454+02$

$5.475+42$

$1.185=02$
$2.585=02$

$1.021-01$

$3.441-03$

7.500003

2.906002

$4.060 \cdot 02$

$1.344+00$

$2.585-02$

$3,529+02$

2. $090+00$

7.506003

$5.455+02$
$5.475+02$

$0.710-02$

$4.955+02$

$5.142+02$

$7.022+00$

$4.279+01$

$2.308-01$
$1.060+03$

IUTAL

CES IUM

0.6

0.8

0.8
1.0
1.25

1:0

$0.710-02$

$4.955+02$

$7.022+00$

$+.279+01$

$2.368=01$

1.019001

. 344+05

$1.394+03$

$1.904+01$

$1.160+02$

$2.874+03$

1.819001

$1.344+03$

$1.904+01$

$1.100+02$

$0.425=01$
$2.874+03$

23RS HEGYCLE U-23S E.E.

PRTILE PTL. FISSILE PTL.

CSRS RECYCLE U-235 F.E.

FEKIILE PIL. DSSILE PIL.

\section{$1.332+00$}

$3.515+02$

$1.039+00$

$5.119+02$

$1.352+00$
$3.515+02$

$1.542+00$

$3.526+02$

$5,138+02$

$3.526+02$

$4.008+02$
$4.084+02$

$1.185 \cdot 02$
$2.585=02$

3.572003

7.792003

$3,076=02$
4.215002

1.185002

2.505002

1.021001

$1.021=01$

2.625003
5.721003

2.200002
3.095002

$1.344+00$
$2.585=02$
$3.516+02$

1.943000

7.792003

$5.138+02$

1.344000
2.565002

1.544000

2.565002

$5.721=01$

$4.064+02$

$3.529+02$

$4,955+02$

$5.142+02$

$7.022+00$

$4.279+01$

2.368001
$1.000+03$

1.428 .01

1.055403

$1.094+03$

$1.495+01$

$9.107+01$

5.039001
$2.256+03$

$0.710-02$

$1.397 \cdot 01$

$4.955+02$

1.002003

$5.142+02$

$4.279+01$

2.36800

1.060 .03

1.020601

0.031001

1.000105

$1.428-01$

0.71000

$4.955+02$

$5.142+02$

$7.022+00$
$4.279+01$

2.368001

$1.055+03$

$1.094+03$

$1.495+01$

$9.107+01$

5.039001

$2.250+03$

0.710002

$4.955+02$

5.142 .02

$7.022+00$
4.279 .01

$2.308=01$

$1.000+03$

2.143003

$1.357 \cdot 01$

$1.002+03$

1.420401

$8.651+01$

$4.707-01$

$2.143+03$ 


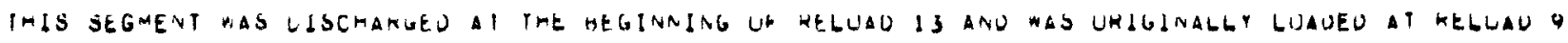

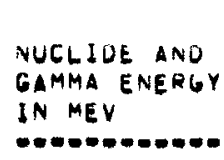

\section{$I=131$}

0.1
0.3
0.0

TUtal

IUUINE

0.1
0.3
0.0

0.0

TÓTAL

I
$N$

\section{TE $=1294$}

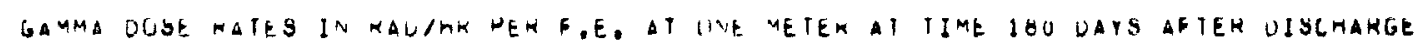

MS FRESH UD-235 F OE FEKIILE PIL. FISSILE WTL. -OA-O..

$4.945=00$
$0.302=04$
$6.312=05$
$1.064=05$
5.150004
1.186000
1.054004 1.510005

$3.997=00$
$1.237=04$

4.945006 $+.302004$

$0.312-05$

1.6040 .05

$$
\begin{aligned}
& 1.188000 \\
& 1.034004 \\
& 1.510005 \\
& 3.997000 \\
& 1.237000
\end{aligned}
$$

$T E=1204$
0.1
0.0
0.8
1.0
TUTS
TULA

TUTAL

$T E=129$

0.1
0.3
0.0
0.8
1.0
1.25
TUTAL

IELLURIUM

0.1

0.3

0.3
0.6
0.8

1.0

TUTAL

$0.052=03$

7.855001

$2.380-0$

3.341 .02

$1.047+00$

$2.105-02$

$7.357+00$

$4.537-01$
$4.070-01$
$4.444+00$
$2.490-01$
$9.000-11$
$3.509-02$
$0.490+00$

$0.456-02$

5.801 .02

0.323001

3.55100

1.289001

$9.243=01$

$\begin{array}{ll}4.962-01 & 7.001=02 \\ 4.070-01 & 5.801002 \\ 9.903+06 & 1.418+00 \\ 1.928+00 & 2.744=01 \\ 1.010+00 & 1.423001 \\ 5.614002 & 7.989-03 \\ 1.385+01 & 1.971+00\end{array}$

23HS RECYCLE UAZSS F.E. FERTILE PTL. FISSILE PIL

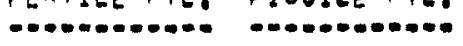

\section{$4.945-06$} $4.302-04$ $0.312-05$

5.004005

1.400000
1.218004
1.787005
4.710000
1.458004

4.945000 $4.302-04$

0.312 .05

1.000 .05
5.150004

$1.400=06$
$1.218=04$
1.787005
$4.710=06$
1.458004

$5.520+00$

$1.679+00$

$9.423=02$

$2.105=02$

$4.537=01$

$4.070-01$

$4.444+00$

4.090001
9,401001

9.601 .01
3.5090 .02

$0.496+00$

$4.962-01$

4.070001

$9.963+00$

$1.928+00$

$5.014=02$

$1.385+01$

$$
\begin{aligned}
& 1.134-01 \\
& 1.019-01 \\
& 1.111+00 \\
& 0.238002 \\
& 2.265=01 \\
& 0.709-03 \\
& 1.024+00
\end{aligned}
$$

1.240 .01

1.014001

2.490400
4.820001

2.50000

1.40300

$3,402+00$
C5KS HECYCLE U-235 R.K.

PENILE PIL. FISSILE PIL

$\begin{array}{lll}1.005002 & 4.253002 & 4.400003 \\ 1.380+00 & 5.520+00 & 5.815001 \\ 4.100001 & 1.079+00 & 1.769001 \\ 2.355002 & 9.423002 & 9.926003 \\ 5.26203 & 2.105002 & 2.218003 \\ 1.030000 & 7.357+00 & 7.750001\end{array}$

4.537001
4.070001
4.404000
2.496001
9.060001
3.509002
0.490400

$4.779-02$

4.204 .0

4.081001

2.629002

9.544002

$0.843=01$

$3.227-02$

4.2440 .02

$1.050+00$

2.052 .01

1.054001

$\begin{array}{ll}1.000+00 & 1.054001 \\ 1.305+01 & 5.914003 \\ 1.459+00\end{array}$ 


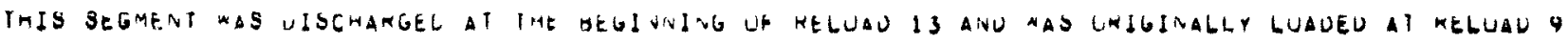

VUCLIOE ANO IN MEV

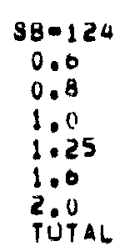

$\$ 8 \cdot 125$

0.1

0.6

$\underbrace{N}_{\infty}$ GAMMA ENERGY

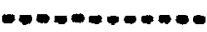

MS FRESH U-235 FOE FEHTILE PIL. FISSILE PIL.

CD-115H

$C D=115 \mathrm{H}$

1.0

TUTAL

CAOMIUM

1.0

TUTAL

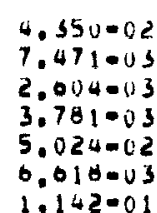

$1.142-01$

$1.208+00$ $1.140+01$ 2. $297+01$

$505+0$

$1.208+00$

1.146001

2. 3uz+01

$7,491-05$

$2,004-03$

$3.781-03$

$5.024=02$

$5.024=02$

0.018003
$3.570+01$

\subsection{2 \\ $5.043-03$ \\ 1.907 .03 \\ 2.856005 \\ 3.794 .02 \\ $4.999-0) 3$}

.026002

$3.951-01$

$5.750+00$

$7.514+00$

$1.100+01$

3.951 .01

$3.750+00$

7.540400
5.043003

5.043003

1.967 .03

$2.050-03$

3.7900112

$1.174+01$

$1.158-13$

5.600004
$1.719=03$

$1.158-03$
$5.608-00$

$1.719-03$
23 H KECYCLE U.23S F.E.

FEKTILE PTL. FISSILEPTL.

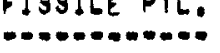

$4.350-02$

7.471 .03

2.004003

3.701 .03

$5.024=02$

0.018 .03

1.142001

$1.200+00$

$1.146+01$

$2.297+01$

$5.377=02$
9.230003
$3.219=03$
4.070003
0.210002
0.101003
1.412001

$1.204+00$

$1.200+01$

$2.404+01$

$3.730+01$

$1.208+00$

$1.106+01$

$5.305+01$

$7.471=03$

$2.004=03$

$3.701=03$

3.050 .02

$0.610=03$
$3.570+01$

$1.204+00$

$1.200 \div 01$

9.236003

9.236003

$3.219=03$

0.01400

$0.210=02$

$3.744+01$

$1.158-01$

1.719003

$4.752-04$

$2.300=04$

$7.053=04$

$1.158=03$

$5.008 \cdot 04$
1.719 .03

$4.752-04$

$2.300=04$
25KS NECYCLE U-235 P.E. PEKTILEPIL. FIS3ILE PIL.

$\begin{array}{ll}4.350 .02 & 3.454002 \\ 7.471 .03 & 5.896003 \\ 2.004 .03 & 2.050 .03 \\ 3.781 .03 & 2.985003 \\ 5.024002 & 3.966 .02 \\ 0.618 .03 & 5.225005 \\ 1.142001 & 9.017002\end{array}$

$1.208+00 \quad 2.902001$

$1.140+01 \quad 2.754400$

$2.297+01$
$3.565+01$

$\begin{array}{ll}1.208+00 & 2.902001 \\ 1.146001 & 2.754+00 \\ 2.302+01 & 3.552+00 \\ 7.471003 & 5.898003 \\ 2.004003 & 2.050003 \\ 3.781003 & 2.905003 \\ 5.024002 & 3.906002 \\ 0.018003 & 5.225003 \\ 3.576+01 & 8.052+00\end{array}$

1.15800

5.00600

1.719003

3.537 .04

5.249004

$1.158-03$

5.008004

1.719003
3.537 .04

1.712004
5.249 .04

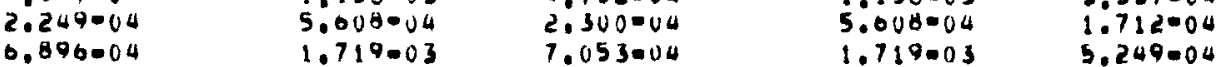




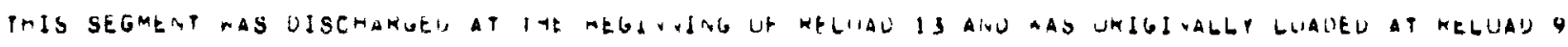

NUGLIDE ANO GAMMA ENERGY IN MEV

-OA.-.........

160108,4

0.1
0.3
0.0

0.0

I.:0

$A G=110 \mathrm{M}$

0.1

0.13
0.0
0.8

0.6

1.0

1.25
1.0
2.0
2.0

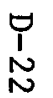

TUYAL

SILVER

0.1

0.3

0.0

1.0

1.25

3.0

2.4

IUTAL

$R R=100$
0.3
0.0
0.8
1.00
1.25
1.00
2.0
2.4
2.9
10941

GAMMA DUSE HATES IV

FEKTILE PTL, FISSILE PIL.

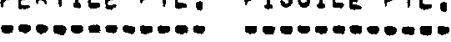

1.112000
8.999005
1.348002
1.528004
2.046006
3.800004

1.208004

1.010002

4.940001

6.647001

1.973001

$1.004-41$

$1.004-61$

$1.083-03$

1.083003
$1.758+00$

1.219004

$1.025-02$
4.991001

0.649001

$1.973-01$

$2.178-01$

$1.064-01$
1.047 .03

$1.047-03$

1.003003
$1.758+00$

$1.536-01$

$4.456+01$

$3.933+00$

$1.369+00$

$9.034=01$

$3.332=01$

$3.330=01$

$5.255+01$

$1.040-07$
$1.327-05$
$1.909-05$
2.2540 .05
$3.018-07$
5.010005

5.016 .05

1.092004

1.424002

0.990001

0.311001

2.764001

$3.050=0$

0460003

1.460003
1.317 .03

1.517 .03
$2.462+00$

$1.094=04$

1.425002

$\therefore 9000$

931100

$2.704-01$

3.05000

2.331 .01

$1.060=03$

1.517003
$2.462+00$

2. $555-01$

$5.901+01$

$1.3194+00$

$1.751+00$

$1.751+00$

$4,458=0$

$4.450=0$

$7.317=02$

$1.031+01$

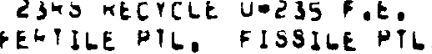

a.n.

$\begin{array}{ll}1.112000 & 2.450=07 \\ 0.999005 & 1.907-05 \\ 1.348-04 & 2.940005 \\ 1.320004 & 3.339005 \\ 2.046000 & 0.475007 \\ 3.808004 & 0.322005\end{array}$

1.200004

- 1110002

$4.990-01$

0.049001

1.973001
$2.178-01$

2.178001

$1.004-01$

1.04003

1.085003

1.219 .04

$1.025-02$

4.991001

0.649001

$1.973-01$

$2.178-01$

1.004001

1.047 .03

1.063003

$1.758+00$

1.530001

$4.450+01$

$9.740=01$

$3.935+00$

$1.309+00$

$3.332=01$

3.330011

$5.469-112$

b. $255+11$

$1.797-04$
$1.512=02$
7.422001
$9.807-01$
2.935001
3.239001
2.475001
1.557003
1.011003
2.014000

2.014000

1.190 .04

1.514002

?. 422001

$2,935=01$

3.239001

2.475001

$1.557 \cdot 03$

1.011003
$2.014+0.03$

1.347001

$3,408+01$

$3.449+00$

$1.140+0.1$

7,423001

2,923001

$2.921-01$

$4.069+0 !$
25NS HECYCLE INE235 F.EE
FEKTILE PTL. PSSSILEPIL.

Ge0nd

$\begin{array}{ll}1.112010 & 9.354007 \\ 0.999005 & 7.555005 \\ 1.548004 & 1.152004 \\ 1.520004 & 1.203004 \\ 2.046000 & 1.718000 \\ 3.800004 & 3.197-04\end{array}$

1.208004

$1.010=02$

4.990001

0.647 .01

$1.973=01$

2.178 .01

1.004 .01

1.083003

$1.758+00$

$1.271=04$

1.069002

5.549001

30001

1.020001
3.070001

2.291 .01
$1.750-01$

1.101003

1.101 .03

$1.849+00$

1.219004

1.025002

4.991 .01

0.649001

1.973001

$2.178=01$

$1.604=01$

$1.047-113$

1.003003

$1.758+00$

1.200004

$1.077-02$

5.250001
0.994 .01

2.070001

2.291001

1.750001

$1.161=05$

$1.139=03$

$1.530-01$

$450+01$
9.740001

$3.933+100$

$1.309+011$

$9.034=01$

3. $332-01$

$3.330-11$

5.069002

$5.255+01$
$1.542=01$

$4.42+01$
$9.791-01$

$3.947+00$

$1.314+110$

$9000=01$

3.342801

3.3409.02

$5.274+01$ 


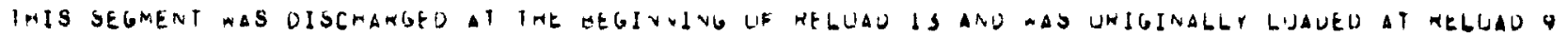

VUGLIUE AND GAMMA ENEKG N MEV

\section{RHUDIUM}

0.3

0.0

0.8

1.25

2.0

2.4

TUTAL

$90-103$

0.1

IOTAL

RUIHENIUM

0.1

0.3

IUTAL

NG $=95$

TUिAL

NIU日 IUM

TUTAL

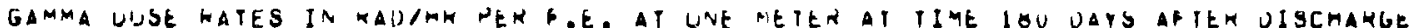

MS FNESH LO235 F.E

FENIILE PTL. FISSILE PIL.
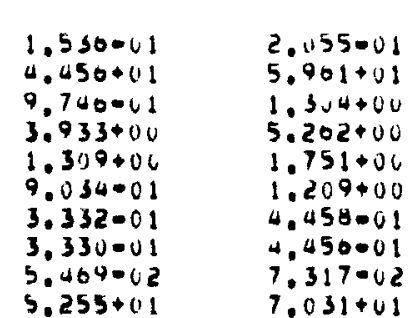

2.831002

3.708 .01

$7.411+01$

$2.831-02$

3.700001
$7.411+01$

$7.411+01$
$7.452+01$

$2.704+03$

$2,760+03$

$2.764+03$

$2,760+63$

2.182 .01

$4.292+01$

1.039002

$2.182-01$

4.510001

$1.011+03$

$1.011+03$

$1.011+03$

$1.011+03$
23HS KECYCLE U-23S R.E. FERTILE PIL. FISSILE PILO

25NS HELYCLE U-235 t.E.

PERTILE PTL, FISSILE PIL.
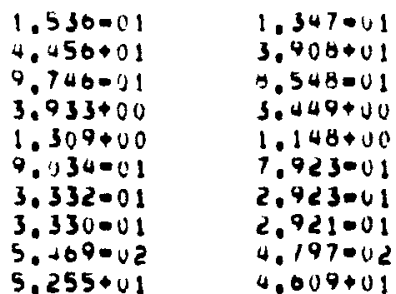

1.214002

1.010001

5.176001

5.190+01

3.70000

$7.411+01$

2.831 .02

3.708001
7.411001

$1.214-02$

1.016001

$3.190+01$

$7.452+01$

(19.0.

$2.704+03$

$2.704+03$
$9.800+02$

$9.808+02$

$9.008+02$

$9.808+02$
1.5300011

$4.450+0$

$9.7+0=0$

$3.933+40$

1.309400

9.03000

$3.332=0$

$3.330=0$

5.409 .0

$5.235+01$

1.542001

$4.472+01$

9.741 .01

$3.947+00$

1.314000

9.000001

3.344 .01

3.342 .01

5.489 .02

5.270001

$2.831=02 \quad 1.228002$

$7.452+01$

$2.6310 n 2 \quad 1.228002$

$3.768001 \quad 1.654001$

$\begin{array}{ll}7.411+01 & 3.214+01 \\ 7.452+01 & 3.231+01\end{array}$

$\begin{array}{ll}2.764+03 & 7.491+02 \\ 2.764+1.3 & 7.491+02\end{array}$

$\begin{array}{ll}2.764+03 & 7.491+02 \\ 2.704+03 & 7.441+02\end{array}$
$7.411+01 \quad 1.654001$ 


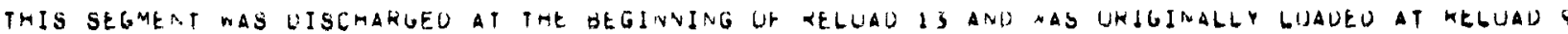

NUCLIUE ANO MA ENERGY IN MEV

Mev

$2 R=95$

0.3
0.8

TUTAL

2IKCUNIUM

0.3

TU์TAL

KO-80

lou

RUEIUIUM

I.0

$Q$
$\omega$
$\sim$

$00-58$

0.6
0.8

0.8
1.6
TUTAL

$c u-60$
1.25

TORAL

CUBALT

0.6
0.8
1.25

1.25

IUTAL

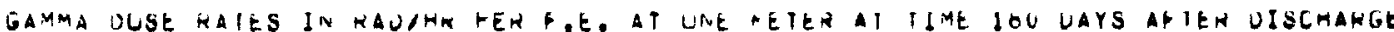

13 FKESH UI-Z35 F.E. Z3KS RECYCLE U-Z3S F.E. FEKTILE PTL. FISSILEPIL, FERIILE PTL. FISSILE PIL.

25HS RECYCLE U-235 +.E.

BETILE PTL.

$13316 t$ PIL.

$2.495+00$

$1.312+03$

9.018001

$4.7+1+02$

$2.495+10$

0.707001

$4.009+02$

$2.495+00$

$0.061=01$

$1.314+03$

$4.018+02$

$1.312+03$

$3.512+02$

9010001

$2.493+00$

$8.707-01$

$9.009+02$
$4.018+02$

$2.495+00$

. $59+02$

$2.495+00$

$4.01+1+02$

$1: 312+03$

$1.314+03$

0.061 .01

$1.314+03$

$4.750+02$

4.610402

1.874003

$0.538=04$

1.874003
1.874003

1.119003

$\left\{\begin{array}{l}1.874003 \\ 1.87400\}\end{array}\right.$

$3.519+42$

$1.874=03$

0.538 .04

1.119003

I. Bracos

$1.874003 \quad 1.119003$

1.814005
.874003

$1.874=03$

6.5380 .04

1.119003

a.

4.006004

$9.204=05$

$4.771=04$

$5.420=00$

0.131006

$3.178-05$

$3.010-07$

9.204005

9.067006

$4.710=05$
$5.350=07$

9.204005

4.771004

$4.771=04$

$5: 746=04$

$5.672=05$

5.420006

$3.491=05$

1.809004

$2.179=04$

$0.100=04$

$4.127=05$

0.160004

$0.117=05$

$0.100=04$

350004

$9.204-05$

$4.771=04$

$6.160=04$

5.426 .00

$0.131=00$

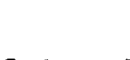

o.117avs

$0.100-04$

2.350004

3.178 .05

$4.127-05$

3.610 .07

9.2040 .05

$4.771=04$

$0.160=06$

9.087 .06

$4.110-05$

$6.117-05$

5.350 .07

$9.204=05$

$4.771-04$

0.100004

$3.420=00$

3.401 .05

1.809004
2.350004

$1.191=03$

1.179004

$1.141=03$

$2.055-06$

4.529004 


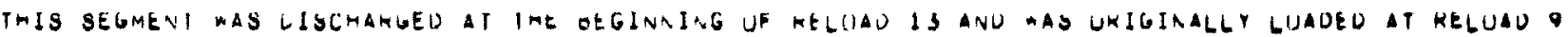

YUCLIDE ANO GAMMA ENERGY IN MEV

IN MEV

ALL ELEMENTS

$A L L E L E S ~$
0.1
0.3
0.0

0.0

1.0

1.25

1.6

2.9

2.9
3.5
TUTAL

MS FHESH U-Z35 FOE. MS FHESH U-235 F.E.
FERTILE FTL. FISSILE PTL.

23NS RECYCLE UECZSF OE. FERTILE PTL. FISSILE PTL.

Y AFIEN UISCHARGE

$1.030+05$

$6,704+02$

$1.059+03$

$4.599+03$

$1.787+01$

$4.888+01$

$2.001+01$

$0.049+08$

$0.224=01$

$5.021=02$

$5.108 \cdot 05$

$0.585+03$

$3.823+00$

7.068 .00

$2.055+03$

$2.893+03$

$3.470+01$

$1.299+02$

$.060+01$

$.873-01$

$7.301=02$
1.501 .05

$5,107+03$

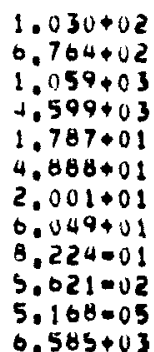

$4.190+00$

$1.480+01$

.700403

2.546003

$2,500.01$

1.004002

1.485001

$4,323+01$

$1.221+00$

$4.842=02$

$1.558-05$

$4,458+03$

$0,585+03$

TERT RECYCLE U-235 . E.

FERTILE NTL. FISSILE PTL.

-........... 


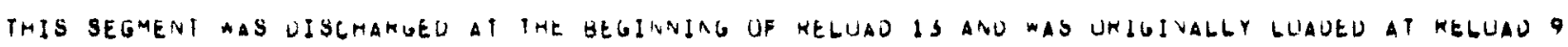
NUCLIDE AND IN MEV

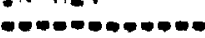

\section{$u=235$}

0.1
0.3
TUTAL

URANIUM

0.1

TÚTAL

$P A=Z S 3$

0.1

TUTAL

N
$\omega$

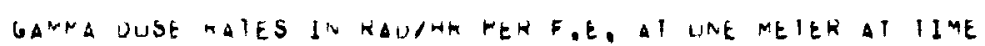

MS FHESM UIOZSS F.E.

FERTILE PTL. FISSILE PTL.

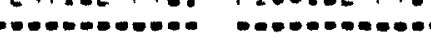

\section{$3.717-07$}

$9.898=07$

$2.805=00$
$9.409-00$

9.409000
1.027005

1.027005

$3.717=07$
$9.898-07$
1.0362000

$1.362=00$

2.805006
7.469000

$7.469=06$
$1.027=05$

$23 R S$ RECYCLE U-233 F.E.
FERTILE PTL, FISSILE PT

FERTILE PTLE FISSILE PTL.

1 reat AFIEH UJSCHAKGE

\subsection{7 .07
9.0980 .07}

1.302000

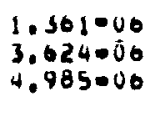

$3.717=07$
9.898007

1.362006

1.301000
3.024006

4,985006

9.890007
1.362006

$6.919=09$
5.917008

5.917008
6.809008

$5.676+00$
$6.531+00$

$5.676+00$
$0.531+00$

1.129009

6.610009

5.3640 .07

$5.462=07$

2.687 .07

$3.021=06$

$8.872=07$

$7.033=0$

$4.083=05$

$8.335=04$

PRUTACTINIUM

0.1
0.3
0.0
0.8
1.0
1.25
1.0
TUTAL

$0.555=01$
$5.676+00$
$5.905=05$
2.934005
$5.083-04$
$4.083=05$
$9.088-05$
$0.532+00$

1.129 .09

$5.439=07$

$5.462-07$

$5.007=07$

$5.021=06$

3.739007

$0.872=07$
$7.042=00$
25HS RECYCLE U-235 F.K.

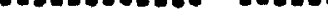

$3.717 \div 07$

9.698007

2.400011

2.450011

$1.205-11$

$2.252-10$

$1.677-11$

$3.080-11$

$3.424 \cdot 10$

0.555001

$0.511+00$

$5.857-05$

$2.934=05$
$5.483=04$

4.403 .05

.083005
$0.088-05$

8.315004

\section{$0.919=09$}

$2.450-11$

8.555001
5.676000

$5.076+00$

$5.965-05$

5.93004

5.483004

4.003005

0.688005

$2.252-10$

1.077 .11

$3.980-11$

$0,843=08$

$2.934=65$

5.463004

0.063005

$0.532+00$

FEKTILE PTL. PISSILE PTL

2.193006

5.840006
0.033000

2.143006

5.840000

2.260008

1.499007

2.00400

2.004006
3.946005

$3.740-05$

2.789006

5.0440 .05

2.200008

4.151006
4.775006

2. 775000

2.004006

3.709000

6.018000

5.712 .05 


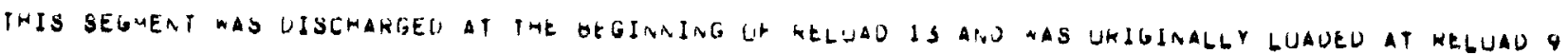

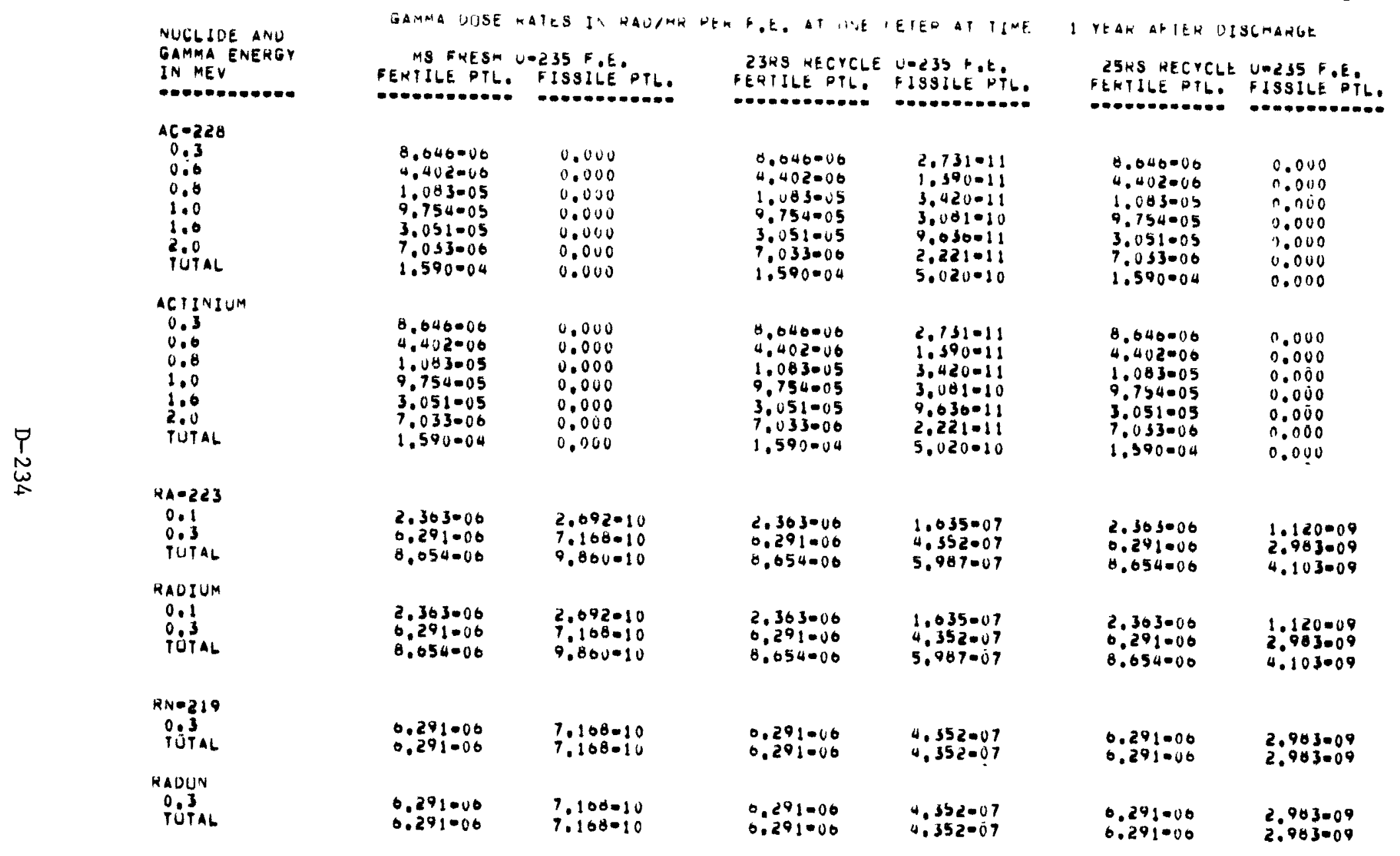




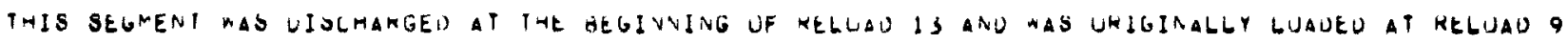

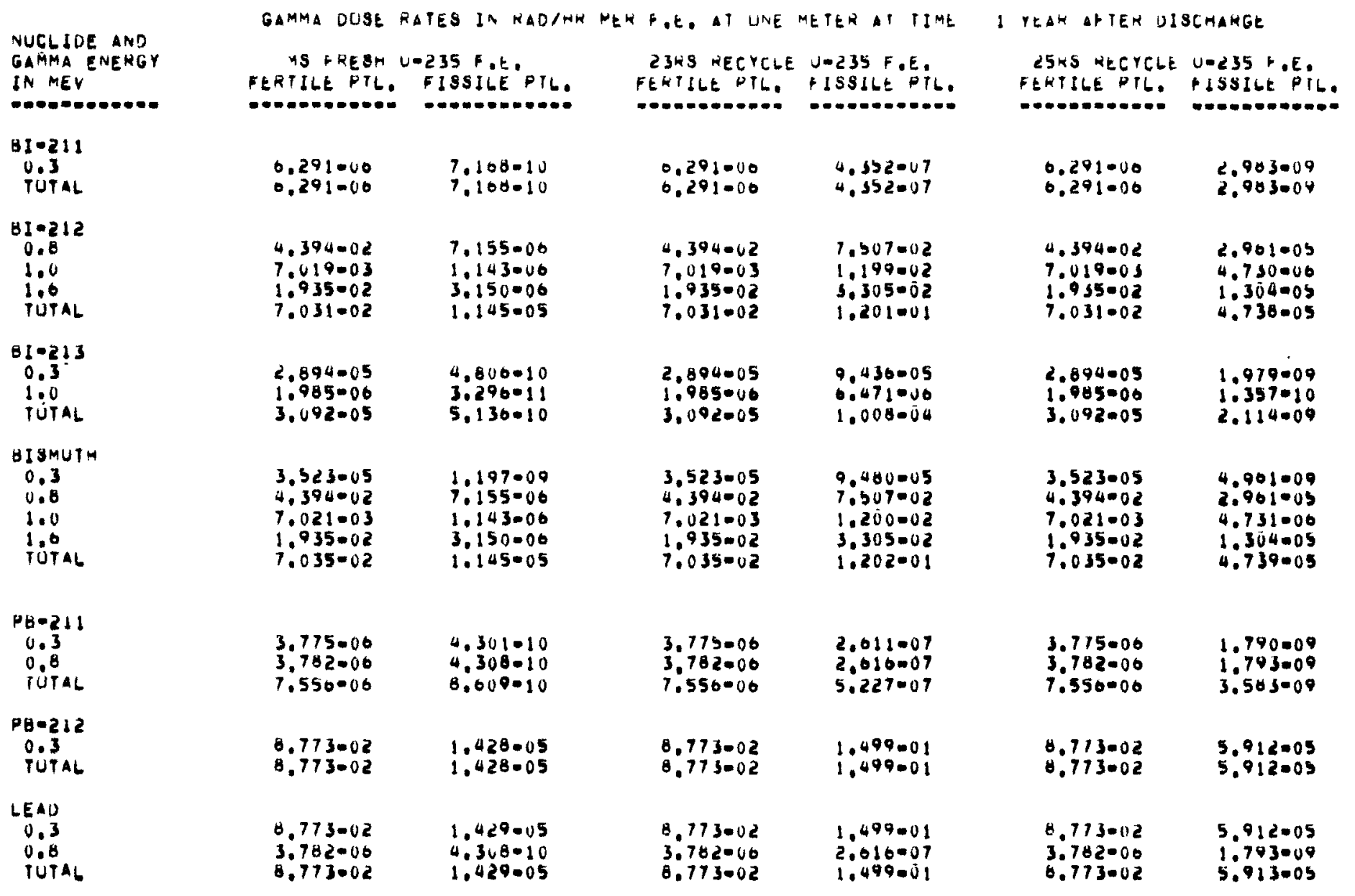




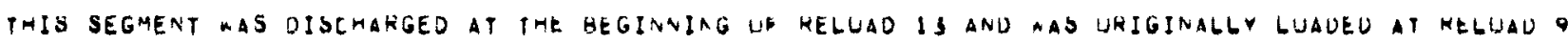

NUCLIDE AND GAMMA ENEKGY IN MEV

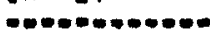

$T L=208$

TLe.2U
0.3
0.6
0.8

2.4

THALLIUM

0,3

0.6

0.8

TOTAL

$\underset{\sigma}{\omega}$
MS FKEYM U-235

FERTILE PIL, ISSILE PIL

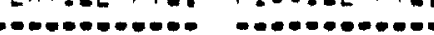

$5.236=0$
$1.053=01$

TUTAL

$T B=100$

0.3

0.8

0.8
1.0
1.25

1.25
TUTAL

TEKSIUM

0.3
0.8

0.8

1.25

TuTAL
$5.230-03$

$1.053=01$

5.229001
$7.249-01$

5.236003

5.22900

$7.249=01$

$0.405-10$

5.541 .0

$1.335=09$
$4.994=08$

\subsection{7 \\ $2.092-05$ \\ 5.125006 \\ .514005 \\ 1.280004 \\ 8,520007 \\ 2.692 .05 \\ $5.125=0$}

1.18000

$1.140-10$

$925-10$
7.521009

7.521 .09

$8.855=09$

$4.542=03$

1.100002

$1.127-02$

$8.443=03$

$5.583-03$

1.352002

1.038 .02

1.036002
$. .333=02$

5.583003

1.352002

1.385-02

4,542003

$1.100=02$

$8.443-03$
$3.525=02$
1.038 .02

23H8 RECYCLE UEZ3S F.E.

$5.230=03$
1.053001
3,147002
5,229001
7.249001

5,236003

1.6530 .01

3.107002

5.229001
7.249 .01

$0.465=10$

5.541 .09

4.241008
$1.335-09$

$4.994=08$

$4.542=03$

1.100002

$1.127=02$

8.443003
4.542003

1.100002

$1.127-02$

$8.443=03$
0.995003

2.839001

3,405002

0.980001

\section{$8.993=03$}

$2,839=01$

$5.405=02$

8.98000

$1,245+00$

$8.139-10$

0.976009

$5,340-08$

$1.681=09$
0.287000

5.027 .03

1.217 .02
1.247 .02

$9.345=03$

$3,901-02$

I TEAR ABTER UISLMAKGE

25RS HECYCLE U-Z35 P.EE

$\begin{array}{ll}5.236003 & 5.529000 \\ 1.053001 & 1.114004 \\ 3.147002 & 2.121005 \\ 3.229001 & 3.524004 \\ 7.249001 & 4.865004\end{array}$

5.256003

1.653001

5.229001

3.529000

1.114004

2.121 .05

.824004
.805004

$0.465=10$

$5.541=09$

4.241008

1.335009

4.994008

$4.542 \cdot 03$

1.100 .02

1.127 .02

3.525002

$5.027=03$

$1.217=02$

$9.345=03$

$3: 401.02$
$4.542=03$

1.100 .02

1.121 .02

$8.443=03$
$3.525-02$
1.043009
8.364009

$2.613-10$

9.847009

$0.535 \cdot 03$

2.309 .02

2.365002

1.773002

$9.53500 \mathrm{~s}$

2.309002

1.713002
$1.275=10$ 


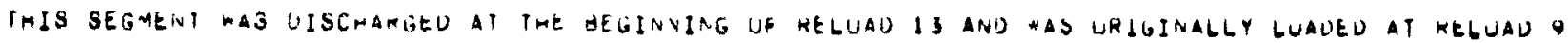

NUELIDE AND GAMMA ENERGY IN MEV

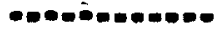

EU०152

0.1
0.3
0.0
0.8
1.0
1.25
1.6
ToTAL

EU. 154

0.1

0.3

0.6

1.0

1.25

$\forall$
$\omega$
$\omega$
GAMMA UUSE KAIES IN KAD

FERIILE PTL. PISSILE PTL

EU-156

0.6

$1: 0$

2.0

TÚTAL

EURUPIUM

0.1
0.3
0.6
0.8
1.0
1.25
1.0
2.0
2.04
10746

TƯ TAL
$1.625-03$

4.358 .03

4.037 .04
4.405 .03

1.284002

$1.010-02$

3.441 .02

$4.252-01$

1.847 .0

$4.858-0$

$2.644+0$

$2.506+00$

$3.851+00$

2.974001

$$
\begin{aligned}
& 2.654=03 \\
& 7.035=03 \\
& 1.149003 \\
& 7.292003 \\
& 2.097-02 \\
& 1.050002 \\
& 6.009004 \\
& 5.620002
\end{aligned}
$$

$1.200+00$

$5.215=01$

$1.371+00$

$7.464+00$

$1.075+00$

$1.087+01$

$2.935+01$

$2.529-00$

$7.465=06$

$6.677=00$

$1.755=05$

$1.940-05$

$1.9,00=06$

7.000006

2.066005

1.848 .05

4.856005

$5.368=05$

$5.200=00$
1.536004

4.268 .01

1.890 .01

$2.048+00$

$3.519+00$

$3.802+00$

1.978001

$1.940-05$

1.900006
$1.045+01$

\section{$1.203+00$}

$5.285=01$

$1.375+00$

$7.471+00$

$1000+01$

$1.089+01$

5. 30005

500000

$2,940+01$
23NS RECYCLE U-235 F OE

PERTILE PTL. FISSILE PIL

C........

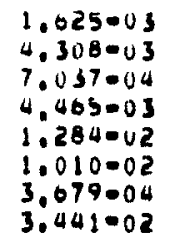

$4.792-03$

$7.627=04$

4.967 .03

$1.428=02$

$1.124=02$

0.093004
3.828002

4,25200

1.849 .01

4.856001

$.644+00$

$.506+00$

$3.851+00$

$1.039+01$

2.529006

$7.465=06$

0.677000

$1.755-05$

1,900006

$1,900-06$
$5,551-05$

$4.208=01$

$1.890=0$

0.86500

2.640400

$3.519+00$

$3.862+00$

2.978001

$1.940-05$

$1.900=06$

$1.043+01$

\subsection{1}

3.459001

9.097001

$4.950+00$

$4.693+00$

$7.212+00$

5,509001

4.048006

1.372005

1.227005

$3,504-05$

3.492000

3.492000
1.020004

7.900001

3.507001

$9.105=01$

$4.955+00$

$4.707 \div 00$

$7.224+00$

$5,574=01$

3.564005

$3.492=06$

$1.950+01$
I YEAK AFIEE OISCTARGE

2SRS RECYCLE U-Z3S F,E

FERIILE PIL. FJSYILE DIL

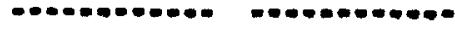

$4.300=03$

$7.037=04$

$4.465=03$

1.284 .02

1.010 .02

3.679004

3.441 .02

2.008003

5.535003

$0.042=04$

$5.737=03$

1.650002

1.298002

4.728004

$1.847001 \quad 3,967.01$

4.858001

$2.644+00 \quad 5.678+00$

$2.506000 \quad 5.343400$

$3.851+00 \quad 8.272+00$

$2.074=01 \quad 0.380 .01$

$1.039+01 \quad 2.232+01$

$2.520006 \quad 5.335000$

$7.465006 \quad 1.574005$

$0.077-06 \quad 1: 408.05$

$1.755=05 \quad 3.701 .05$

$.940 .05 \quad 4.091005$

$1.900=06 \quad 4.008006$

$4.268001 \quad 9.153001$

$.890 .01 \quad 4.022=01$

$.605001 \quad 1.044+00$

$2.648400 \quad 5.604400$

$2.519+00 \quad 3.399+00$

$3.862+00 \quad 0.285+00$

$2.978-01 \quad 0.39201$

$1.940005 \quad 4.042005$

$1.900006 \quad 4.008006$

$1.043+01$

$2.237+01$ 


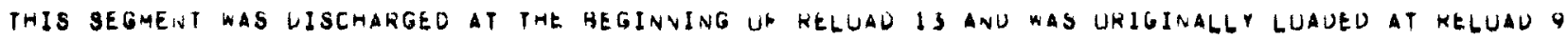

NUCLIUE ANO GAMMA ENERG IN MEV

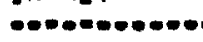

$P M=148 \mathrm{~m}$

0.1
0.3
0.0
0.8
1.0
TOTAL

PRUMETHIUM

0.1

0.6

TUTAL

$\underset{\infty}{\omega}$

0.1

0.3
0.6
TOIAL

NEUUYMIUM

0.1

0.3

TUTAL

$P R=144$
0.6

0.8

PUTAL

GAMMA JUSE KATES IN RADIHR PER F OE AT ONE METEK AT IIME

MS STESH 1-235 F.E FEKTILE PTL. FISSILE PTL.

$23 R S$ HECYCLE U-ZSS F.E. PERTILE PTL. PISSILE RTL.

(1)...

5.270002

1.670002

$1.214=0$

7.337 .01

$1.990-03$

$5,747=02$

$4.585=01$

$1.322-01$
$7.337-01$

1.3350 .03

5,276002

4.209001

1.214001

6,736001

$1.031-08$

0,532009

$2,003=06$

3.078009

2.520009

1.120008

$.587=08$

$1.031=08$
$6.532=09$

$2.903-08$

$2.903=08$
4.587 .08

3.978-09

$1.120=08$

1.769 .08

$3.101+01$

0.01

2.096-01

$1.100+01$

$3,822+01$

$2.762+01$

5.175001

$2.461=01$

$9.797+00$

$3.404+01$
$7.221+01$
$1.999-43$

3.747002

$4.505-01$

1.3200

7.337 .0 !

$1.980-03$

3.987002

3.180001

5.000 .02

9.170002

5.090001

$1.999-03$

5.747 .02

4.585001

$8,361.02$

1.322001

1.340003

3.987 .02

3.180001

170002

5.090001

1.031 .08

$0.532=09$
$2.903=08$

2.903008
$4.587-08$

3.501009

2.268009

$1.008=08$

1.593008

$1.031=08$
$0.532=09$

2.903 .08

$2.903=08$
$4,587=08$

$3.581-09$

1.008008
$1.593=08$

3.101+01

$5,810=0$

$3,822+01$

$8.208+01$

2.200401

$1.920=01$

$7.835+00$

$2.722+01$

$5.774+01$
YEAY AFTEK UISCMARGE

2SRS RECYCLE UVZSS F.E. FENTILE PIL. PISSILE PIL

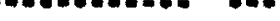

\begin{tabular}{|c|c|}
\hline $\begin{array}{l}1.999-03 \\
5.747-02 \\
4.585001 \\
0.561 .02 \\
1.322001 \\
7.357-01\end{array}$ & $\begin{array}{l}1.378=03 \\
3.902=02 \\
3.101=01 \\
5.704=02 \\
9.113=02 \\
5.059-01\end{array}$ \\
\hline $\begin{array}{l}1.999003 \\
5.747002 \\
4.585001 \\
8.301=02 \\
1.322001\end{array}$ & $\begin{array}{l}1.318=05 \\
3.902-02 \\
3.101-01 \\
5.704002 \\
9.113002 \\
5.059001\end{array}$ \\
\hline
\end{tabular}

$1.031=08$
$0.532=09$
$2.903=08$

3.045009

1.929009

8.509009

4.587008

1.354000

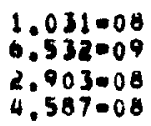

3.045009

1.029009

$8.569-09$

i. 354000

.903008
.587008

$3.101+01$

5.010001

2.696001

$1.100+01$

$3.822+01$
$8.108+01$

$2.072+01$

3.802001

$1.001-01$

$7.349+00$

$2.553+02$

$5.417+01$ 


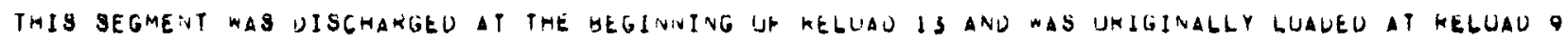

NUCLIDE AND GAMMA ENEKGY IN MEV

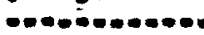

PRABEUDYMIUM

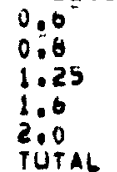

TUTAL

$64=140$

$0: 1$

0

0.8

0.8
1.0
1.25

1.25

1,6

2.4

3.5

TUTAL

GANTHANUM

0.1

0.3

0.0
0.8
1.0

1.0

1.25

1.6

2.9

3.5

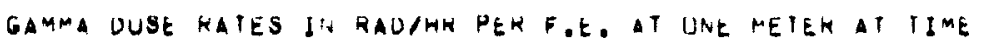

MS GKESH U-235 P. E.

FERIILE PIL, FISSILE PIL,

23WS RECYCLE UC233 P.E.

FERTILE PTL. FISSILE PIL

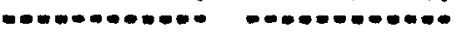

$3.101+01$

5.810001
2.090001

$3.822+01$

$$
\begin{aligned}
& 2.702+01 \\
& 5.175=01 \\
& 2.401=01 \\
& 9.797+00 \\
& 3.404+01 \\
& 7.221+01
\end{aligned}
$$

$5.027=08$

$2.831=00$

$7.643=00$

3.104000

4.511005

$2.760-00$

$0.672=08$

$0.075-00$

$2.275=09$
$7.045-05$

$5.027-08$

$2.831-00$

..043=06

$8.800=06$

$3.308=0$

$.311=05$

0.511005

2.700000

$0.672=08$

$2.275=09$
$7.045=05$
1.400008

8.221007

$2.220=06$

$2.557-06$

2.0439 .08

2.439 .08

$0.015=07$

$0.005-10$

1.400008

1.460 .08
8.221007

8.221007
2.220006

$2,557.06$

9.01200

$.439=0$

1.310005

$6.015=07$

1.937 .00

$0.005-10$
$2.040=05$

$\begin{array}{ll}3.101+01 & 2.208+01 \\ 5.810001 & 4.138001 \\ 2.090-01 & 1.920001 \\ 1.100+01 & 7.835+00 \\ 3.822+01 & 2.722+01 \\ 0.108+01 & 3.774+01\end{array}$

$5.027-08$

2.831 .00

$7.643-06$

8.800000

3.104006

$4 ., 511-05$

2.760 .06

2.760006

3.2750 .09

$9.045=05$

$2.027-08$

$5,027.08$
2.831 .06

7.043006

$8.800-06$

$3.104=06$

$8.396-08$

$\because 511005$

2.760006

$0.672=08$

$2.275=09$
$7.045-05$

1.515008

8.533007

$2,304=06$

$2.655-00$
$9,355.07$

2.532008

$1.360=05$

1.360 .05
8.320007

1.320007
2.011008

$0.857-10$

$2.124=05$

1.515008

$0.533=07$

2.304 .06

$2.655-00$
$9.355-07$

2,532008

1.500005

8.320007

2.011008

$2,124=05$
I year aften dischange

ZSRS KECYCLE UTZZS F.E.

FEKTILE PTL. FISSILE PIL

.

$\begin{array}{ll}3.101+01 & 2.072+01 \\ 5.810001 & 3.802001 \\ 2.096001 & 1.801001 \\ 1.100+01 & 7.349+00 \\ 3.822001 & 2.553+01 \\ 8.108001 & 5.417+01\end{array}$

$5.027 .08 \quad 1.113008$

$2.831 .06 \quad 0.266=07$

$7.643006 \quad 1.692006$

7.806006
$3.104-06$$\quad 1.949006$

$8.390 .08 \quad 900000$

4.511005

2.760006

$0.672008 \quad 0.497008$

$\begin{array}{ll}2.275=09 & 5.084-10 \\ 7.045005 & 1.559005\end{array}$

$5.027008 \quad 1.113008$

$2.831=06 \quad 0.266007$

1.692006

$1.006006 \quad 1.949000$

.

1.359000

$.511=05$

$2.760006 \quad 0.109007$

$6.672000 \quad 1.477000$

$\begin{array}{ll}2.275=09 & 5.034010 \\ 7.045005 & 1.5590 .05\end{array}$ 


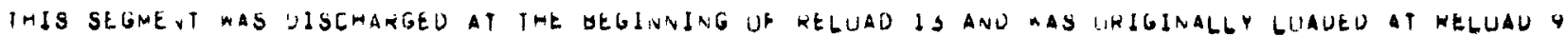

NUCLIOE AND GAMMA ENERGY IN MEV

(1).............

$B A=137$ it

0.1

TUTAL

BA-140

0.1

0.3
TÓTAL

GARIUM

0.1

0.3

TU์Tล

1
$N$
0
GAMMA DUSE KATES IN HADIMK HER F.E. AT UNE MEIER AT IIME

MS FRESh $1.235 \%$.

PERTILE PTL. FISSILE PTL.

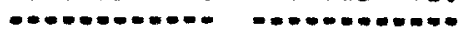

\section{$1.316+00$}

3.474002

2. $043+00$

$5.31+02$
$.411+02$

$5.210=07$

1.1380 .06

1.159000
$. .154-00$

$1.515-07$
$3.304-07$
1.305006
$1.789-06$

$1.310+00$

1.138006

$3.487+02$

$2.043+00$

$3.304=07$

$5,391+02$
$5,411+02$

5.651002

4.174002

$5.914+00$

3.604001

1.994001

8.920 .02

$5.051-02$

$4.174+02$

$130+02$

$30,4+01$

$3.034+01$
$1.994=01$
$8.926+02$

23 JRS FECYCLE UTZ33 F.E.

FEHTILE PILE FISSILE PTL.

C..........

\section{$1.310+00$}

$3.487+02$

$1.917+00$

$5.078+0$

5.216007
1.130 .06
4.495006

1.572007

3.430 .07

0.154006

$1.310+00$

1.138006
3.494002

$3,487+02$

$1.917+00$

$3.430-07$

$5.059+02$
$5.070+02$

5.651 .02

$4.174+02$

$4.330+02$

$5.914+00$

3.000 .01

$8.920+02$

$9.771+01$

$5.407=01$

\section{$1.532=01$}

$1.132+03$

$1.174+03$

1.0040

$5.407-01$

$5.407-01$
$2.420+03$

\subsection{1 .02 \\ 4.174402 \\ $5.914+00$ \\ 3,004001 \\ 1.994001
$0.920+02$}

1.555006

TEAN AFTER DISCHANGE

25RS RECYCLE U-235 F.E.

FERTILE PTL. FISSILE PTL.

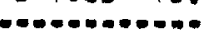

Hislle Pr.

$1.310+00$

3. $4 \$ 7+0$ ?

$1.523+00$

$4.021+02$

$4.056+02$

$5.216=07$
1.138000

$1.154=07$

$2.518=07$

1.362006

0.154000

$1.523+00$

$1.316+100$

1.138000
$3.474+02$

$3.474+02$
$3.487+02$

2.516007

$4.021+02$
$4.036+02$

1.203001
$8.803+02$
$9.217+02$
$1.250+01$
$7.670+01$
$4.244=01$
$1.900+03$
$1.203=01$
$8.083+02$
$9.217+02$
$1.259+01$
1.670001
4.244001
$1.900+03$

5.651002

$4.174+02$

$5.330+02$

$3.604+01$

1.094001

$8.926+02$

$1.143=01$

$8.755+02$

$0.755+02$
$1.190+01$

$7.286+01$

$7.206+01$

$1.805+03$

5.651002

$4.174+02$

$4.330+02$

$5.914+00$

$3.604+01$

1.994001

1.994001
$8.926+0 ?$

$1.143=01$
$8.438+02$

$8.755+02$

1.196401

4.206401

$4.052=01$
$1.805+03$
$8.926+02$ 


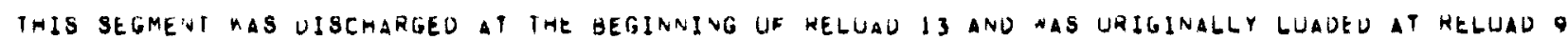

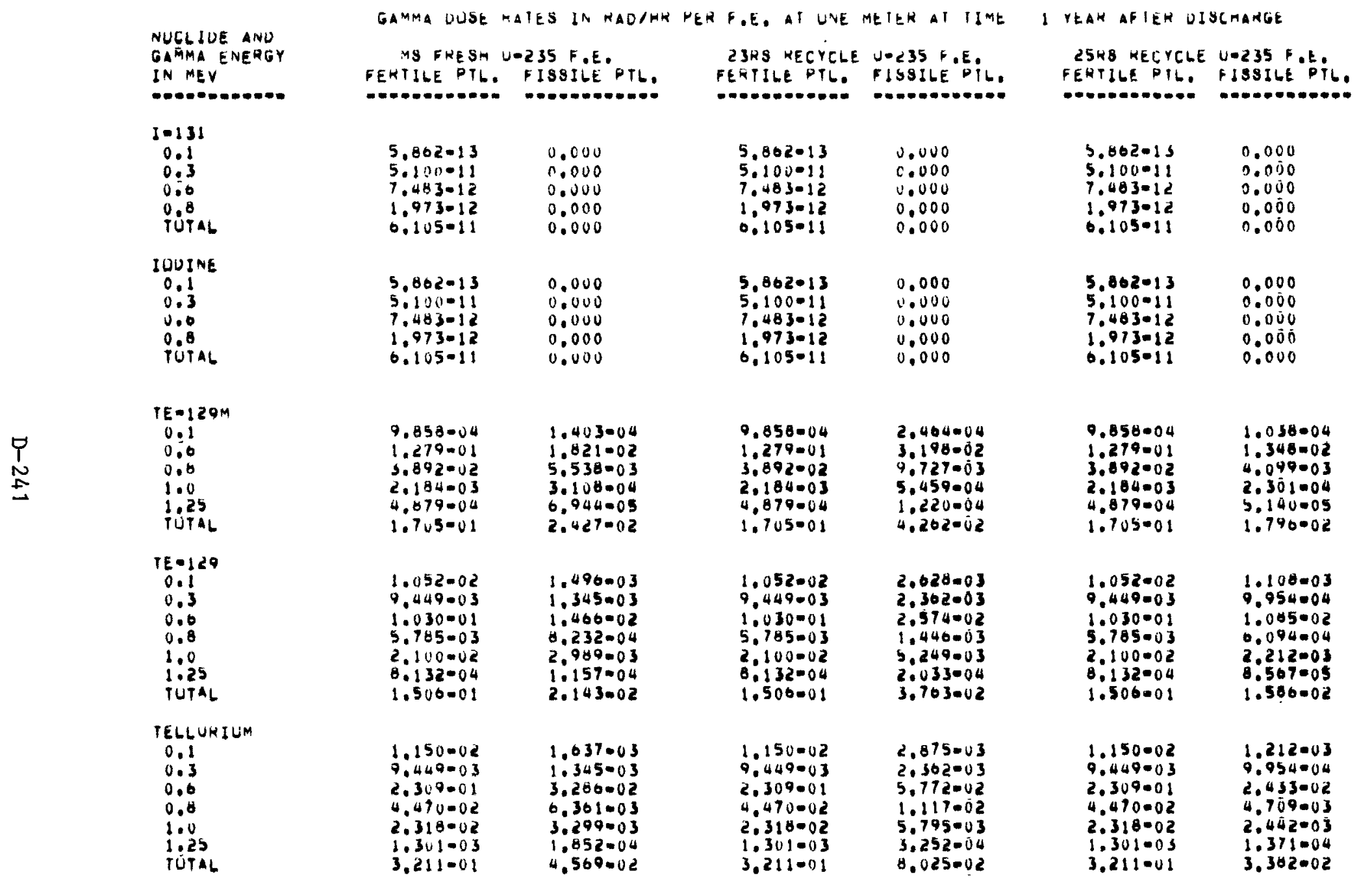




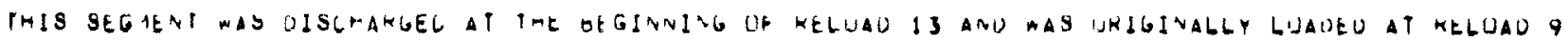
GUCLIOE AND IN MEV

-...-.........

$96-124$

0.0

1.0

1.25

1.0

TUTAL

$3+=1<5$

0.1

TÜTAL

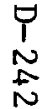

GaMMA DHOE RATES IN

MS FHEST U-235 FE.

FERTILE PIL, FISSILE PIL.
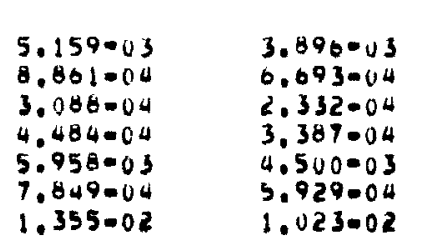

$1.001+00$

$1.007+0$

$2.018+01$
$3.131+01$

$1.001+00$

$1.001+00$

$2.018+01$

$8.861-04$
$3.088-04$

$4.484=04$

$5.958-03$

7.049004

$3.132+01$

$5.855-05$

2.830005

$8.089-05$

$5.855-05$

$2.834=05$
$8.689-05$
3.471001

$3.294+00$

$0.600+00$

$1.024+01$

$3.471 \cdot 01$

$3.294+00$

$6.604+00$

$0.693-04$

3.387 .04

4.500003

5.920 .04

$1.025+01$

2.348005

2.340 .05
1.137 .05
$3.485-05$

$2.348-05$

1.137 .05

3.4850 .05
33KS NECYCLE U-Z35 505

FERIILE PIL, SISSILE PIL.

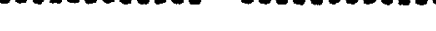

5.159003
$8.861-04$
3.080 .04
4.484004
5.958003
7.849004
1.355002

$1.001+00$

$1.007+01$

$2,010+01$

$3.131+01$

$1.001+00$

$1.007+01$

$2.018+01$

$8.86 ! 004$

3.088004

$4.484=04$
5.958003

7.849 .04

$3.132+01$

$5.855=05$

2.834 .05

$.089-05$

5.455 .05

$2,834.05$

8.089005

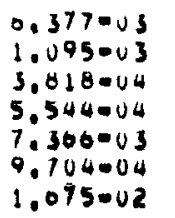

1.075002

$1,110+00$

$1.054+01$

2. $111+01$

. 270401

$1.110+00$

$1.054+01$

$2.112+01$

1.095003

5.544004

$7.300=03$

9.70400

$3.278+01$

$2.402=05$

1.163005

$3.564=05$

2. 4i) 2-05

$3.103-05$
I YEAK AFTEK DISCHAKGE

CSKS KECYCLE UEC3S P.E.

PEHIILE PIL. FISSILE PIL.

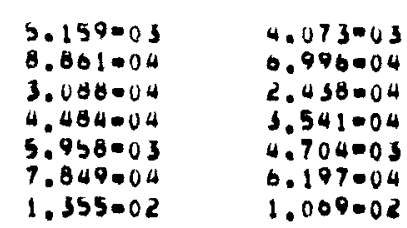

$1.001+00$

$1.007+01$

$3,131+01$

2.549002

$2.419+00$

4.847000
$7.521+00$

$1.001+00$

$1.007+01$

$2.018+01$

3.861004

3.008 .04

5.958003

7.849004

$3,132+01$

2.549001

$2.419+00$
$4.851+00$

0.090004

$6.996-04$
2.438 .04

$3,541=04$

4.904003

0.197000

$0.197+00$
$7.531+00$

$5.855 \cdot 05$

2.834005

1.787 .05

8.684 .05

8.652000

5.8550 .05

2.834005
$8.089=05$ 


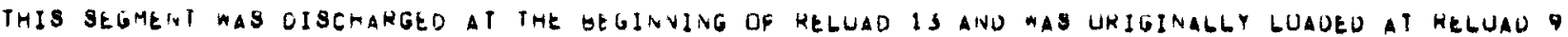

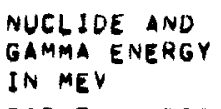

IN MEV

\section{AG-1 08M}

0.1

0.0

0.8

TUTAL

16-110M

0.1

0.3

0.8

1.0
1.25

1.0

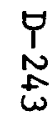

2.00
2.4

Tútal

\section{SILVER}

0.1

0.6

0.6

1.0

1.25

1.0
2.0

TUTAL

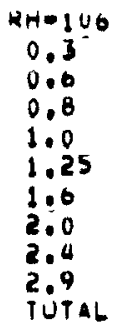

GAMMA JOSE RATES IN HADGHK PER F EE AT ONE MEIEK AT IIME

MS FRESH U. 235 F.E. FERTILE PTL. FISSILE PIL.

$$
\begin{aligned}
& 1.109=00 \\
& 8.975=05 \\
& 1.345=04 \\
& 1.524=04 \\
& 2.041=00 \\
& 3.797=04
\end{aligned}
$$

$7.302-05$

0.144003

3. 016001

4.018001

$1.193=01$

1.310001

$1.006-01$

$0.320-06$

$1.062+00$

$7.413=03$

0.233 .03

$3.018=01$

4.020 .01

1.19300

1.31600

1.00600

$0.320-04$

0.540004

$1.063+00$

$1.034-01$

3.14440

$6.876=01$

$2.775+00$

$9.235=01$

$2.351=01$

$2.350=01$

3.859 .02

$3.706+01$
1.036 .07

$1.324-05$

.083005

$2.248-05$

3.010007

$5.001=05$

1.023004

$8.0116=03$

$5.028-01$

$.671-01$

$1.844=01$

$1.409=01$

$8.802-04$

9.109004

$1.488+00$

$1.024-04$

8.019 .03

4.225001

5.628 .01

1.071001

1.844001

$1.409-01$

$.002-04$

$1.488+00$

$1.450=01$

$4.206+01$

.200001

$3.713+00$

$1.236+00$

8.528001

3.140001

3.144001

5.103 .02

$4.901+41$
2385 HECYCLE UD235 to

FERTILE PTLE FISSILE PIL

DERTL

FISSILE DTL.

1.109006
8.975005
1.345004
1.524004
2.049006
3.797004

2.425007

1.902005

$2.939-05$

$3.330-05$

8.209005

$7.302 \cdot 05$

0.144003

3.016001

4.010001

1.195001

1.31600

6.326004

$0.546=04$
$1.062+00$

$7.413-05$

$0.233=03$

3.018001

$4.020=01$

1.31600

1.006001

0.540000

$1.063+00$

1.006004

$9.138=03$

4.486001

5.976001

1.774001

9.410004

9.730004

$1,580+00$

1.084 .04

9.158003

$5.977-01$

$1.774-01$

1.938001

1.490001

$9.410=04$

.500000

$1.084=01$

$3.144+01$

0.876001

$2.775+00$

$9,235=01$

$6.374=0$

$2.351-01$

$2.350=0$

$3,859-02$

$3.708+01$

9.500002

$2.757+01$

$0.031=01$

$2.434+00$

8.099001

5.590001

2.062001

2.001001

3,384002

$3,252+01$
I YEAR AFIEK UISCMARGE

25K8 RECYCLE U.235 P.E. FENIILE PTL. FISSILE PTL.

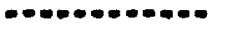

(t)

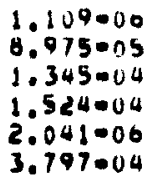

.309001

7.535005

2.129004

1.279004

1.713006

$3.797 .04 \quad 3.188004$

7.302005

0.144003

$3.016=01$

4.018001

1.193001

1.316001

6,326004

0.546004

7.682 .05

0.463003

3.173001

$4.227-01$

1.255001

1.385001

1.058001

$0.655 \cdot 04$

$0.886=04$

$1.002+00$

7.775005

7.413005

0.233003

3.018001

$4.020=0$

1.103001

1.316008

$1.006=01$

$0.326=0$

$1.063+00$

6.538003

.228001

1.255001

$1.365=01$

$1.090=01$

0.655004

$1.118+00$
$1.084=01$

$3.144+01$

6.876001

$2.775+00$

9.23500

$0.374=0$

$2.351=0$

2.35000

$3.859-02$

$3.708+01$
1.008001

$3.135+01$

$6.901-01$

$2.785+00$

9.208001

$0.347=01$

2.360001

2.350001

$3.873=02$

$3.721+01$ 


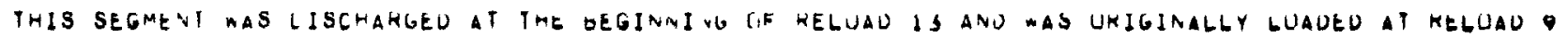

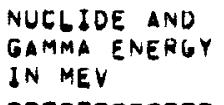

IN MEV

\section{RMUDIUM}

0.3

0.8

1.00

1.25

1.6

2.0

TUTAL

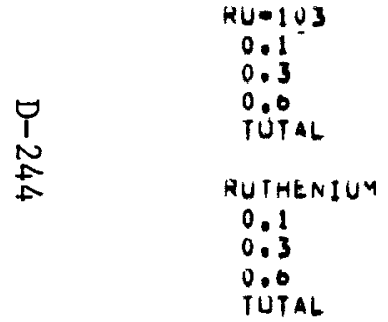

N6-95

TUिTLL

NIUB IUM

TƯTAL

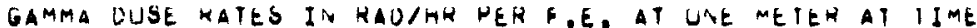

I YEAK ARTEN UISCHAKGE.

MS FRESH LE235 F.E.

FERTILE PIL. FISSILE PIL.

$23 K 3$ KECYCLE U-235 F.E.

PERIILE PIL. FISSILE PIL.

CSHS RECYCLE

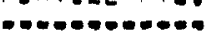

ISSILE PIL.

$\begin{array}{ll}1.084-01 & 1.450-01 \\ 3.144+01 & 4.200+01 \\ 0.870-01 & 9.200001 \\ 2.775+00 & 3.713+00 \\ 9.235-01 & 1.250+00 \\ 0.374-01 & 8.528001 \\ 2.351 .01 & 3.140001 \\ 2.350 .01 & 3.144001 \\ 3.859-02 & 5.103002 \\ 3.708+01 & 4.901+01\end{array}$

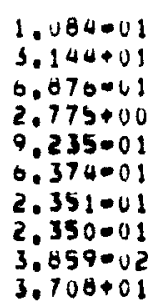

0.350 .05

$2.757+01$

$0.031=01$

$2.434+00$

8.099001

$5.590=0$

$2.002=0$

$2.061=0$

$3,384=0$

$3.252+01$

3.859002
1.004001
3.14040
6.870001
$2.775+00$
9.235001
0.374 .01
2.351 .01
2.350001
3.859002
$3.708+01$

1.080001

$3.155+01$

0.901001

$2.785+00$

$9.268=01$

6.397 .0 !

2.300001

2. 358001

$3.873 \cdot 02$

$\$, 721.01$
4.018000

0.414003

$1.261+00$

$1.268+00$

$1.490=02 \quad 8.601=03$

$2.957+00 \quad 1.703+00$

1,490002

$2.941+00$

$2.957+00$

$1.123 \cdot 03$

$1,490=02$

$2,941+00$

4.018004

0.414003

$1.201+00$

$1.200+00$

$1.490-02 \quad 8.001=03$

$2.941+00 \quad 1.703+00$

$2,957+00$

$4.295+02$

$1.556+02$

$4.295+02$

$4,295+02$

$1.512+42$

$1.512+02$

$4.295+02$

$1.512+02$

$1.512+02$

\section{$1.123003 \quad 4.872004$ \\ 2.941 .00}

$\begin{array}{ll}1.941+00 & 1.275+00 \\ 2.957+00 & 1.262+00\end{array}$

$1.123003 \quad 4.872004$

$1.496002 \quad 0.485003$

$2.941+00 \quad 1.275+00$

$1.262+00$

$\begin{array}{ll}4.295+02 & 1.556+02 \\ 4.295+02 & 1.556+02\end{array}$

$4.295+02$
$4.295+02 \quad 1.133+02$

$4.295+02 \quad 1.153+02$
$4.205+02 \quad 1.133+02$ 


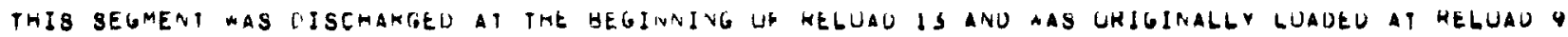

NUCLIUE AND GAMMA ENERGY IN MEV ENERGY

2H-95

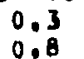

TUTAL

ZIKCUNIUM

0.3

Tutal

R8-60

TOOTAL

RUEIDIUM

TUTA

$\underset{N}{N}$

CU.58
0.0
0.8
TUTAL

CU日GU
I.25
TUTAL

CUBALT

0.6

1.25

1.25

TUTAL
GAMMA DUSE RATES IS KADIHE RER F.E. AI UNE MEIER AT IIME

MS FRESH U-23S F.E.

FERTILE PIL. FISSILE PIL.

$\begin{array}{ll}3.517-01 & 1.271-01 \\ 1.849+02 & 0.084+01 \\ 1.853+02 & 0.697+01\end{array}$

$3.517=0$

$1.849+02$

$1.853+02$

\subsection{1}

$6.684+01$

$6.697+01$

1.922000
$1.922=06$

0.705007

$0.705=07$

0.705007

1.922006

0.70500

1.015000

5.259000

$0.333=00$

$0.907-07$

$9.967-07$

8.967 .07

0.333006

$5.762-04$

3.800005

$5.762=04$
$5.762=04$

1.523005

1.015006

5.259006
3.860005

3.800005
$5.974=08$

$7.894=05$

3.702004

$0.713=04$

4.494005

7.894 .05

5,702004

0.713004
$5,72 !=05$

7.793006

5.721005

$0.050-65$

YEAR AFIER UISCHARGE

C5KS NECYCLE U-Z35 +.E.

FERIILE PIL. FISSILE PTL.

CET

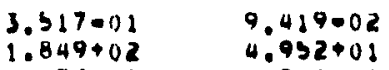

$\begin{array}{ll}1.849+02 & 4.932+01 \\ 1.053+02 & 4.901+01\end{array}$

$\begin{array}{ll}3.517-01 & 9.419002 \\ 1.849+02 & 4.952+01\end{array}$

$\begin{array}{ll}1.849+02 & 4.952+01 \\ 1.853+02 & 4.961+01\end{array}$

$\begin{array}{ll}1.922000 & 4.806007 \\ 1.922006 & 4.806007\end{array}$

$1.922000 \quad 4.800007$

$\begin{array}{lll}1.148000 & 1.922000 & 4.800007 \\ 1.148000 & 1.922000 & 4.806007\end{array}$

$1.503=06$

$453-08$

$5.776=06$

$2.994-05$

$\begin{array}{ll}8.967-07 & 3.401=07 \\ 9.500005 & 3.005005\end{array}$

$\begin{array}{ll}5.762004 & 2.198004 \\ 5.702004 & 2.198004\end{array}$

$1.523=05$
7.894005
$5.762=04$
$8.967=07$
$0.713=04$

3.770006

$2.944=05$

2.198004

3.401007

2.558000 


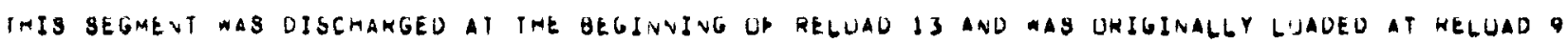

NUCLJUE AND

IN MEY

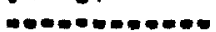

ALL ELEMENIS

0.1
0.3
0.0
0.8
1.0
1.25
1.0
2.0
2.4
2.9
3.5
TUTAL

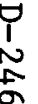

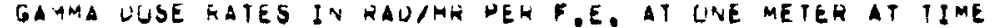

MS FREST U-235 F.E.

FERTILE PTL. FISSILE PTL.

$23 \mathrm{KS}$ HECYCLE UNZ3S D.E. FERTILE PTL. FISSILE PTL.

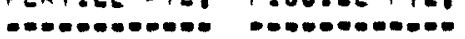

YEAR AFTER DISGMARGE

$3.07+00$
$1.004+01$
$0.519+02$
$1.052+03$
$1.150+01$
$4.123+01$
$1.220+01$
$3.846+01$
7.585001
3.859002
2.275009
$2.029+03$

$3,597+00$

$4.324+00$

$1.751+03$

1.40603

$2.915+01$

$1.103+02$

$1.218+01$

$3,435+01$

3.154001

$6.605 \cdot 10$

$3,349+03$
25KS KECYCLE UOZS5 P.E. FEFTILE PTL PISBILE PTL

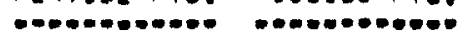

$3.074+00$
1.004001
0.519002
1.052003
$1.150+01$
$4.123+01$
$1.226+01$
3.846001
7.585001
3.859002
2.275009
$2.029+03$

$2.097 \bullet 00$

$3.202+00$

$1.306+03$

$2.030+01$

$0.241+01$

9.142400

2.577001

2.369 .01

$3.673=02$

5.034010
$2.409+03$ 
THIS SEGMENT WAS UIBCHAKGEO AT THE GEGINNING OF RELUAO I3 ANO WAS ORIGINALLY LUAUED AT RELUAO 9 NUGLIDE AND IN MEV

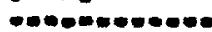

Ue235

0.1

Tutal

URANIUM

0.1

POTAL

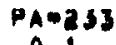

iOt

是
MS FHESH U-235 F.E.

PERTILE PTL. FISSILE PTL

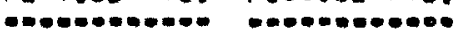

$3.717=07$
$9.898-07$
$1.302=06$
$3.717=07$
$9.898=07$
$1.362=06$

$6.126=09$
$4.064=08$
$4.677=08$
0.000
0.000
0.000
0.000
0.000
0.000
0.000

$6.126-09$

4.060008

0.000

0,000

0.000

0.000

0.000

$4,077.08$

\subsection{0 \\ 1.069 .06 \\ $2.605=06$ \\ $7.469-06$
$1.027-05$}

0.000

0.000

$5.364=07$

5.364007
$5.462=07$

2,687007

$5.021=06$

$3.739-07$

8.872007
7.033000

0.000

$5.364=07$
$5.462=07$

$5,462-07$

$2.687-07$

5.021000

$3.739-07$
$8.872-07$

$7.633=00$
23RS HECYCLE U-235 F.E.

PERTILE PTLO FISSILE PIL.

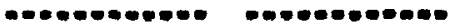

$3.717=07$
9.808007
1.362000
$3.717=07$
$9.808=07$
1.362006

6.120009
4.064006
4.677000

0.000
0.000
0.000
0.000
0.000
0.000
0.000

$1.361=06$

3.024006
4.985000

1.301000

3.024006

0.000

9.000

$2.345-11$

$2.345-11$

$1.174-11$

$2.105-10$

$1.635-11$

$3.878=11$

$0.126=09$

4.064008

0,000

0,000

0,000

0,000

0.000

$4.077-08$
0.000

2.345-i!

i. 174011

2.195010

$1.635-1$

$3.878-1$

$3,337-10$
25AS RECYCLE U-235 POLP
FERTILE PTLE FISSILE PYL.

\begin{tabular}{|c|c|}
\hline $\begin{array}{l}3.717007 \\
9.890-07 \\
1.362006\end{array}$ & $\begin{array}{l}2.193006 \\
5.840006 \\
8.033006\end{array}$ \\
\hline $\begin{array}{l}3.717=07 \\
9.898=07 \\
1.362=06\end{array}$ & $\begin{array}{l}2.193=00 \\
5.840=06 \\
6.033=06\end{array}$ \\
\hline $\begin{array}{l}6.126000 \\
4.064009 \\
4.677000\end{array}$ & $\begin{array}{l}0.000 \\
0.000 \\
0.080\end{array}$ \\
\hline $\begin{array}{l}0.000 \\
0.000 \\
0.000 \\
0.000 \\
0.000 \\
0.000 \\
0.000\end{array}$ & $\begin{array}{l}4.001=00 \\
4.075006 \\
2.004000 \\
3.766=05 \\
2.749006 \\
6.618000 \\
5.694005\end{array}$ \\
\hline
\end{tabular}

0.000

$4.064000 \quad 4.001006$

0.000

0,000

0.000

0.000

0.000

4.077008

$4.075=06$

$3.004=06$

2.760000

6.610000

5,694008 
THIS SEGMENT WAS DISCHARGED AT THE GEGINNING UF RELUAD 13 AND WAS URIGINALLY LUADEU AT RELUAU NUGLIDE AND IN MEV

IN MEV Exaror

\section{$46-228$}

0.3
0.6
0.8
1.0
1.0
2.0
TOTAL

ACTINIUM

0.3

$0: 0$

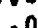

$1: 6$

200

$\forall$
1
$\infty$

GAMMA DUSE KATES IN RADIHR PER F.E. AT UNE METER AT TIME J YEARS APIER OJSGMARge

MS FRESH U-235 F.E. BERTILE PTL. FISSILE PIL. $23 K 8$ RECYCLE U-235 F.E. FERTILE PTLO FI8SILE PTL,

1.050005

5.347000

$1.315=05$

1.185004

3.706005

, 503006

0.000

0.000

0.000

0.000

0.000

0.000

0.000

$1.050=05$

$5.347=06$

$1,315=05$

$1.165=04$

$3.706=09$

8.543006

$1.931=06$

0.000

0.000

0.000

0.000

0.000

$2.210-06$

8.116006

$5.448 \cdot 10$

1.451 .09

1.995009

2.216000

$5.900=00$

$8,116=00$

$5.448-10$

1.451009

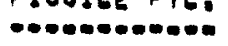

25 HS RECYCLE U⿴囗235 D.E.

FEKIJLE PIL. FIBSILE PTL.

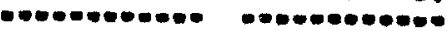

RAOTUM

rotal

$R N=219$

TÜTAL

RADON

TÓTAL

5.900006

5,900006

1.451009

$5,900=06$
5,900006

1.451009

$\begin{array}{ll}1.050=05 & 2.224-11 \\ 5.347-06 & 1.132-11 \\ 1.315005 & 2.705-11 \\ 1.185004 & 2.509010 \\ 3.706005 & 7.847011 \\ 8.543006 & 1.809-11 \\ 1.931004 & 4.009010\end{array}$

$\begin{array}{ll}1.050005 & 0.000 \\ 3.347006 & 0.000 \\ 1.315005 & 0.000 \\ 1.165004 & 0.000 \\ 3.706005 & 0.000 \\ 8.543006 & 0.000 \\ 1.931004 & 0.000\end{array}$

1.050005

5.347 .06

1.313005

$1.105-04$

$8.543-06$

$1.931=04$

$2,224-11$

$1.132-11$

$2.765-11$

$3.509-10$

$7.847-1$

7.809011
4.089010

5.347006

1.315005

1.185004

3.706005

.543006

1.931004

0.000

0.000

0.060

0.000

0.000

0.000

2.216000

5.90000

3,410007

9.096007

2.216000

$3.900006 \quad 2.259009$

$0.116006 \quad 8,274009$

2.210006

5.900006

3.416007

$9.096-07$

2.216006

5.900006

2.259009

8.116006

$0.274=0$

5.90000

5,900000

9.096007

5.900006

6.015009

5.900006

9.090007

1.900000

6.015008

5,900000

9.096007

5.900006

0.015009

$3.900006 \quad 6.01500$ 
THIS SEGMENT MAS OISCHARgED at THE GEgINNING OF KELUAD IS AIVU MAS URIGINALLY LUADEU aT HELUAD 9

NUCLIDE AND GAMMA ENERGY IN MEV

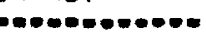

0.211
0.3

Total

$B 1-212$

0.8

1.0

TUTAL

$3\{-213$

0.3

Tútal

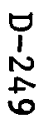

GAMMA DUSE KATES IN RADIHR PE

MS FRESH UTESS F.E. FERTILE PIL. FISSILE PTL.

\section{$5.900 \cdot 60$ \\ 5.900000}

0.337 .02

1.012002
$2.790-02$

$1.014=01$

$5.779-05$

3.964006

6.176 .05

0.369005

$0,357-02$

1.013002
$2.790-02$

1.015001

3.540 .00

3.540 .00

3.540000
$7.087=06$

$1.205-01$

PQ-212

TUTAL

LEAD
0.3
0.8

Putal

3.54600

$1.265=01$
$1.451=09$
$1.451=09$

$1.035-05$

1.653000

4,556006

1.656005

$4.810-10$

5.140010

$1.932=09$

1.053006

1.653006
., 556000

$1.650=05$

.

$8.703-10$

$8.719-10$
1.742009

0.06005

2.006005

$2.066-05$

$8.719-10$

2.066005
$23 F S$ RECYCLE J-233 E OLO FEHILE PTL. FISSILE PIL.

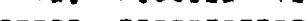

$5,900=00$

$5,900=00$

$0.337-42$ $1.012=02$
2.790002

$1.014=01$

$5.779-05$

$0.176=05$

$0,369=05$

$0.337-02$

$1.013=02$
2.790002

$1.015=01$

$3,540=06$

3,546006

$7.007=06$

$1.265=0$

$1.205=01$

$1.265-01$

$3.546-00$
$1.265-01$

$9.965=05$
9.096007

7.703002

1.240002

3,418002
1,242001

9.874005

1.0550 .04

3.418002

3,418002

1.243001

5.458007

5,468007

$1.093=00$

1.550001

1,950001

1.550 .01

5,468007
1,550001
I YEARS AFTER UISCMARGE

25RS RECYCLE U-235 F.E.

FERTILE PTL. PISSILE PTLO

5.900000

5.900006

$0.015=09$

6.015009

$0.337-02$

$1.012=02$

1.014001

.268005

0.850000

1.808005

0.802005

5.779005

$3.064=06$

1.981009

$1.350 \cdot 10$

2. 11000

0.369005

6.337 .02

$1.013=02$

2.79000

$4: 268005$

. .850006

1.086005

$6.86 z=05$
3.540006

3.009009
3.610009

7.224009

$1.205=0$

8.561 .05

1.265001

.561005

1.265001

3.546006

$1.205=01$

0.561005

3.616009
8.561009 


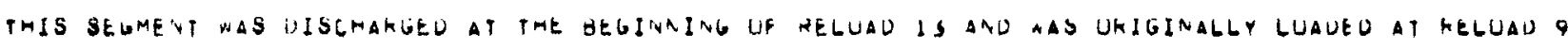

NUCLIDE ANO GAMMA ENERGY IN MEV

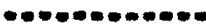

$T L=208$

$0.3^{-10}$

0,6

2,4

TUTAL

THALLIUM

0.3

0.8

TÜTAL

$\underbrace{1}$
un
0

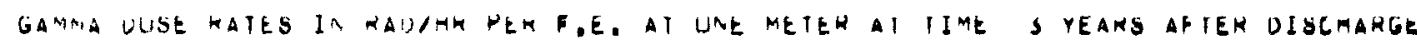

FERTILE PTL, FISSILE PTL.

7.578 .05

$2.392-01$

4.555002

7.567 .01

$1.049+00$

7.576 .03

2.392 .01

$4.555-02$

$6.405-10$

5,490009
4.202008

.202008
1.323009

$4,947=08$

4.008000

9.704000

$1.451=00$

$3.110-05$

$4.009-00$

9.709000

$9.963-00$

$7.452=00$
$3.115=05$

1,236004
1.713004

$1.237-00$

3.900005

. 437.06

1.230004

1.713004

$1.130-10$

$9.734=10$

$7.452=09$
$2.340-10$

$2.340=10$
$0.773=0$

$4.927=06$

$1.193-05$

$1.222-05$

$3.023=05$

$4.927-00$

1.193005

$1.223-05$

$9.160=06$
$3.024=05$

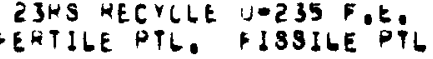

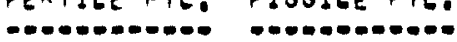

$7,578-103$
2.392001

4.555002

$7.567-61$

$1.009+00$

2,936001

$5,589.02$

9.285001

$1.287+00$

9.598 .03

$2.392-01$

4507001

.507 .01

9.299 .03

2.956001

$0.505=01$

9.285001
$1.287+00$

0.405 .10

$5,490=09$

$4.202=08$

1.323009
$4.947-08$

$0.005-10$

0.912009
5.291000

5.291008

$0.230=08$

4.008000

9.704006

9.941 .06

$7.451=06$

$.030=06$

1.074 .05

3.442 .05

4.009000

$9.709-06$

$9,963=06$

$7.452=00$

4.437 .06

$1.075=05$

$1.106-05$

8.246000
3.449005
25KS KECYCLE U-235 DE.

CHTLE PRLO EISBDLETL.

$\begin{array}{ll}7.578003 & 5.128000 \\ 2.392001 & 1.019004 \\ 4.555002 & 3.002005 \\ 7.567-01 & 5.120004 \\ 1.049+00 & 7.049004\end{array}$

1.578003

2.392 .01

4.555 .02

7.507 .01

$1.049+00$

3.128000

1.019004

3.082005

5.120004

.099004

$0.405 \cdot 10$

5.490009

4.202008

$1.323=09$
4.947 .08

$1.203=10$

$.083=09$
.267009

$2.609 \cdot 10$

2.609010
9.756009

4.008006

9.704000

$9,941=06$

7.451 .06

$0.415=06$

$2.037=05$

2.087 .05

1.504005

0.530 .05

4.009006

9.709006
9.983006

7.452 .06

0.015006

$2.057-05$

2.000 .05

1.504005

1.554005 


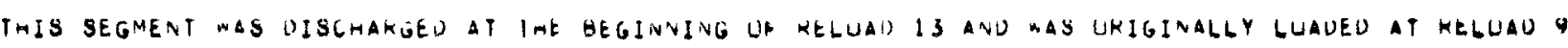

NUCLIDE AND GAMMA ENERGY IN MEV

$E U=1 \$ 2$

0.1
0.3
0.06
0.8
1.0
1.25
1.0
$109 A L$

TÜ TOL

Eu- 154

0.1
0.3
0.6
0.8
1.0
1.25
1.0
TOTAL

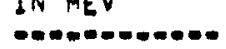

gamma UUSE MATES IA haDJHM PEW .

MS FRESM $U-235$ F.E.

EETILE PIL. FISSILE PIL

EURLPIUM

0.1
0.3
0.6
0.0
1.0
1.25
1.0

IUTAL

$P M=148 M$

0.1
0.3
0.6
0.0
1.0
TUTAL

$$
\begin{aligned}
& 1.057=03 \\
& 3.802=03 \\
& 0.310=04 \\
& 4.004013 \\
& 1.151=02 \\
& 9.059=03 \\
& 3.299=04 \\
& 3.085=02
\end{aligned}
$$

3.899 .01

$1.094=01$

$4.455=01$

$2.424+01)$

$2.298+00$

$3 ., 535+00$
$3.5288+00$

$2.728=01$

$9.532+00$

$$
\begin{aligned}
& 2.379003 \\
& 0.308-03 \\
& 1.030003 \\
& 0.538003 \\
& 1.089002 \\
& 1.479002 \\
& 5.388004 \\
& 5.059002 \\
& \\
& 1.101+00 \\
& 4.782=01 \\
& 1.258+00 \\
& 6.844+00 \\
& 0.488+00 \\
& 9.972+00 \\
& 7.700=01 \\
& 2.091+01
\end{aligned}
$$

$3,914-01$

$1.733=0 !$

4.40100

$5.928+00$

$2.310+00$

$3.541+00$

$2.731-0.1$
$9.503+110$

$1.103+00$

$4.845-01$

$1.259+00$

$6.851+00$

$0.507+00$

$9.986+00$

$7.706-01$

$1.095=08$

3.149007

$2.512=06$

$4.581=07$
$7.243=07$

$7.243=07$
4.020000
$2.696+01$
$1.005=08$

$2.891-07$

2,306006

4.206007

6.650 .07

$3.091=06$
23NS HECYCLE UO235 *E.
FETILE PTL. FISSILE PTL.

$$
\begin{aligned}
& 1.457-03 \\
& 3.862003 \\
& 0.310014 \\
& 0.004003 \\
& 1.151002 \\
& 9.059003 \\
& 3.299004 \\
& 3.085002
\end{aligned}
$$

3.090001

1.094001

$4.055-01$

$2.424+00$

2.298400

$3.532+00$

2.726 .01

$2.726=01$
$9.532+00$

3.914001

$1.733=0$.

$2.428+00$

$2.310+00$

$3.541+00$

$9.563+00$

$1.021=03$
4.200003
7.018004
$4.453=03$
1.200002
1.008002
$3.690=04$
3.452002

3.432002

7. 302001

$3,172=01$

0.543001
$0.540+00$

$0.540+00$

0.610400

0.610400

5,108001
$1,785+01$

7.310001

3.215001

0.35001

$4,544+00$

$4,317+00$

$0.624 * 00$

5.111 .01

$1.708+01$

$1.095-08$

3.149007

$2.512 \cdot 00$

4.581 .07

$7.243=07$

7.597009

2.184007

1.743006
3.178007

2.769006
$25 \mathrm{kB}$ RECYCLE UO235 P.E. FERTILE PIL. FISSILE PIL.

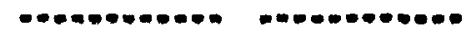

$\begin{array}{ll}1.457-03 & 1.812003 \\ 3.802003 & 4.903003 \\ 0.310004 & 0.107004 \\ 4.000003 & 5.144003 \\ 1.151002 & 1.479002 \\ 9.059003 & 1.104002 \\ 3.299004 & 4.239004 \\ 3.005002 & 3.905002\end{array}$

$\begin{array}{ll}3.899001 & 8.375001 \\ 1.694001 & 3.630001 \\ 4.455001 & 9.500001 \\ 2.424000 & 5.207+00 \\ 2.290000 & 4.936+00 \\ 3.532+00 & 7.586+00 \\ 2.728001 & 5.834001 \\ 9.532+00 & 2.047+01\end{array}$

3.914001

$1.733=01$

4.401001

$2.428+00$

$2.310+00$

$3,541+00$

$2.731=0$

$9.563+00$

$0.343-01$

3.058001

.516001

$5.212+00$

$4.951+00$

$7.598+00$

$3.862 \cdot 01$

$2.051+01$

$1.095=06$

3.140007

$2.512=06$

4.581 .01

$7.243-07$

4.020006

7.530000 2.171 .07 1.752006 3.158007 4.944007 


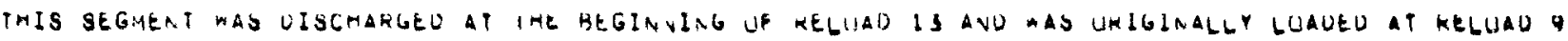

NUGLIDE AND GAMMA ENERGY IN MEY

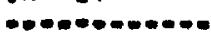

PRUMETHIUM

0.1

0.1
0.3
0.8

0.0
0.8
1.0

TUTAL

$P R=144$

0.6

0.8

1.25

IOUTAL

$\forall$
$N$

PRASEODYMIUM

0.6

1.25

TOTAL

$B A-137 M$

0.1

PUTAL

BAKIUM

0.1

TÜTAL

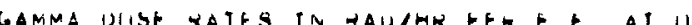

MS FRESH U-235 F.E.

FEKTILE PIL. FISSILE PTL.

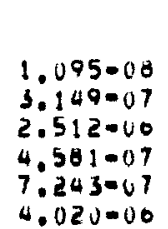

1. $005=00$

2.891 .07

2.360 .000

., 206007

0.050 .07

3.091 .06

23WS KECYCLE U:23S F.K.

FERTILE PIL. PISSILE PIL

COC.........

$1.095-08$

3.149 .07

2.512 .00

4.581 .07

$7.243=07$

1.597009

2.184009

1.743006

$3.178=07$

5.025007

2,109000

$8.702=02$

$9.214+00$

$4.533=02$

$1.850+00$

$6.427+00$

4.037 .02

$5.724+00$

$1.214+01$

$5.21 a+06$

$9.771=02$

4.533002

$0427+00$

1. $363+01$

$4.044+00$

$8.702=02$

0.037 .02

$1.047+00$

$1.210+01$

$5.214+00$

$. .733=02$

$1.850+00$

$0.427+011$

$1.303+01$

0.959002

3.22000

$1.357+00$
$4.577+00$

$9.110+00$

$5.214+00$

$9.771-02$

4.533002

$1.850+00$

3.714000

$6.959=02$

$3.228=02$

$1.317+00$

$4.577+00$

$1.363+01$

$0.720+00$

$1.257+00$

$3.317+02$

$1.950+00$
$5.147+02$

$5.147+02$
$5.107+02$

1.830400

$3,317+62$

$3.329+02$

$1.950+00$

$1.257+00$

$3.317+02$
$3,329+12$

$5.147+02$
$5.167+02$

\section{$1.257+00$}

3. $317+02$

$3.329+02$

$1.830+00$
$4.831+02$
$4.849+02$

$3.317+02$

3. $329+02$

$7.350=09$

$2.171=07$

$1.732=06$

3.158007

$4.994-07$

2.772006

$\begin{array}{ll}5.214+00 & 3.404+00 \\ 9.771-02 & 6.527 .02 \\ 4.533002 & 3.026002 \\ 1.850+00 & 1.236+00 \\ 0.427+00 & 4.293+00 \\ 1.303+01 & 9.100+00\end{array}$

$3.214+00$
$9.771+02$
$4.533+02$
$1.050+00$
$0.427+00$
$1.363+01$

$3.484+00$

0.527 .02

3.028 .02

1.256000

$9.108+00$

$1.257+00$
$3.317+02$

$1.455+00$

$3.839+02$

$1.455+00$

$3.89+00$

$3.854+02$ 


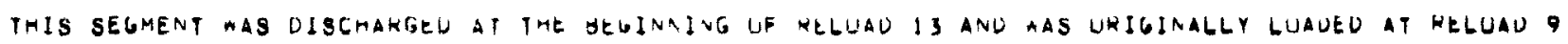

NUCLIUE AND GAMMA ENERGY IN MEV

IN MEV

\section{$68 \cdot 134$}

0.3

0.6

1.0

1.6

TUTAL

CESIUM
0.3
0.6
0.8
1.0
1.25
1.0
1 UTAL

0
1
$\omega$
$\omega$
MS FKESH U-233 P.E.

FERTILE PTL, FISSILE PTL.

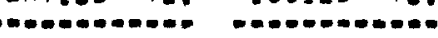

$2.871-02$
$2.120+02$
$2.200+02$
$3.004+00$
$1.831+01$
$1.013-01$
$4.534+02$

3.871002

$5.120+05$

$2.200+02$

$3.004+00$

$1.831+01$

$1.013=01$

$4.534+02$

$3.519-10$

$4.567=08$

$1.389-08$

$7.795-10$

$7.795-10$
$1.742-10$
$0.000-08$

3.753009

3.372 .09

3.670008

2.065009
7.096009

2.903010

$5.374=04$

1.784002

$5,749+02$
$5,965+02$

$8.146+00$

$4.904+01$

2.747001

$1.229+03$

\section{$7.784-02$}

$5.749+02$

$8.146+00$

$4.964+01$

2.747 .01

$1.229+03$

$5.007-11$

0.499 .09

$1090-10$

$2.478-11$

$8.061=09$

$5.341-10$

$4.799 \cdot 10$

$5.232-09$

$2.938-10$

1.067009

$4.131=11$
$7.047-09$
$E=129$

0.3

0.8

1.25
23HS KECYCLE U-233 F.E.

FESTILE PTL. FISSILE PTL

2SHS RECYCLE U-235 P.L.

FEKTILE PTL. FISSILE PIL.

$$
\begin{aligned}
& 2.871=02 \\
& 2.120+02 \\
& 2.200+02 \\
& 3.004+00 \\
& 1.831+01 \\
& 1.013=01 \\
& 4.534+02
\end{aligned}
$$

$0.110-02$
$4.512+02$
$4.082+02$
$0.394+00$
$3.896+01$
2.156001
$9.050+02$

2.871 .02
$5.120+05$

$3.2004+05$
5.000

$1.831+01$

1.013001

$4.534+02$

$0.110=02$

$4,315+05$

$0.394+00$

$3.696+0$

2.156001

$9.650+02$

$3.519-10$

4.567 .08

1.389 .68

$7.795-10$

$1.742-10$

$0.795-11$

$1.141=08$

$3.072=09$

$4.353-11$

$1.521=08$

3.153009

3.372009

3.076006

2.065009

$2.903-10$
$5.374-68$

$9.381=10$

$8.450-10$

1010 10

3.161010

$1.255-11$

$1.255-11$
1.543008
$2.871-02$
$2.120+02$
$2.200+02$
$3.004+00$
$1.831+01$
1.013001
$4.554+02$

5.604002

$4.287+02$

$4.448+02$

$0.074 \$ 00$

$3.701+01$

2.048 .01

$4.554+02 \quad 9.168+02$

$2.871002 \quad 5.804002$

$2.120+02 \quad 4.287+02$

$2.200402 \quad 4.448402$

$3.004+00 \quad 6.074+00$

$1.631+01 \quad 3.701+01$

$\begin{array}{ll}1.0133001 & 2.006001 \\ 4.534002 & 9.100+02\end{array}$

$3.519 \cdot 10$

$4.567-08$

1.389008

7.795 .10

$1.742-10$

$1.403=09$

$1.403=09$
$8.212=11$

$8.212=11$

6.411009

3.753009

$3.372=09$

$3.676=08$

2.065009

$2.903-10$

$3.374-08$
$3.954-10$

3.533010

3.67300

$2.175-10$

3.050

$3.058-11$ 
THIS SEGMENT WAS LISCMARGED AT THE GEGINNING UF RELUAD IS AND HAB UMIGINALLY LUAUEU AT KELUAU 9

NUCLIDE AND GAMMA ENERGY IN MEV

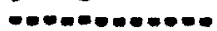

TELLURIUM

0.1
0.3
0.6
0.8
1.0
1.25
TUTAL

$S H \oplus 124$

0.6

0.8

1.0

i
N

1.0

TUTAL

$58 \cdot 125$

0.1

$0: 3$

NIIMUNY

0.1
0.3
0.3
0.0
0.8
1.0
1023
1.6
2.0
TUTAL

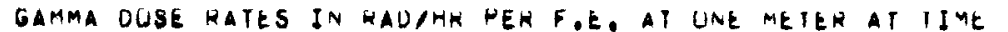

$23 R S$ KECYCLE U-235 F.E.

FERTILE PTL. FISSILE PTL.

MS FKESM U-Z3S FEE TILT
FEKTILE PTL. FISSILE PTL

FEKTILE PTL: FISSILE PTL.

$4.109=09$
9.372009
0.243008
$1.595=00$
$8.275=09$
$4.644-10$
1.146007

$$
\begin{aligned}
& 5.042010 \\
& 4.799-10 \\
& 1.173=08 \\
& 2.270-09 \\
& 1.178=09 \\
& 0.609011 \\
& 1.031=08
\end{aligned}
$$

$1.151 \cdot 00$

1.978007

0,892008

1.041 .07

1.330000

$1,752-07$

3.023=06

$6.363-01$

$6.038+00$

1.210401

$1,877+01$

$6.363-01$

$0.038+0$

$1.210+01$

0.89200

$0.89=00$

$1.001=07$

$1.330-06$

1.752007
$1.877+01$
$8.090=07$

1.494007

5.206008

$7.560-08$

$1.323=0$

$2.283-06$

2.061 .01

$1.975+00$

$0.140+00$

$2.081=01$

2.081001
$1.975+00$

$3.957+00$

1.494007

5.206000

$7.560-00$

1.004 .06

$1.323 \cdot 07$

$0.140+00$

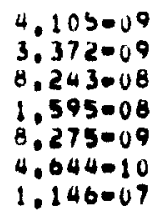

$8.430 \cdot 10$

2.000000

$3,988=09$

$2.008=0$

$1.101=10$
2.864000

1.151000

1.978007
6.092008

1.001 .07

1.330006

$1.752-07$

3.023000

$0.363-01$

$0.038+00$

$1,877+01$

$6.363=01$

$0.038+00$

$1.210+01$

$0.892-08$

$1.001=07$

1.330006

$1.752-07$

$1.752-07$
$1.877+01$

1.425000

2.445007
8.520 .08

$8.520=08$

$1.237=07$

2.10000

2.160007

0.058 .01

$0.318+00$

$1.200+01$

1.964001
CSHS RECYCLE UOCSS F.E

FEKTILE PTL, P\$SSILE PIL

$\begin{array}{ll}4.105009 & 4.324010 \\ 3.312009 & 3.353010 \\ 8.243008 & 8.003009 \\ 1.595008 & 1.081009 \\ 8.275-09 & 8.717-110 \\ 4.044-10 & 4.842011 \\ 1.140009 & 1.207008\end{array}$

1.976 .07

0.092008

1.001007

1.330 .06

3.023006

6.363 .01

.038400

$1.210+01$

$1.077+01$

$9.040=07$

$1.561=07$

$5.442=00$

1.050006

1.363 .07

$2: 387000$

0.363001

$6.036+00$

$1.210+01$

$1.978-07$

0.092008

1.001 .07

1.330 .06

$\begin{array}{ll}1.60000 & 1.330006 \\ 2.100007 & 1.752007 \\ 1.904001 & 1.877001\end{array}$

1.528001

$1.050+00$

$4.510+00$

$$
\begin{aligned}
& 1.520001 \\
& 1.450000 \\
& 2.906400 \\
& 1.561007 \\
& 5.442008 \\
& 7.902008 \\
& 1.050006 \\
& 1.353=07 \\
& 4.510400
\end{aligned}
$$




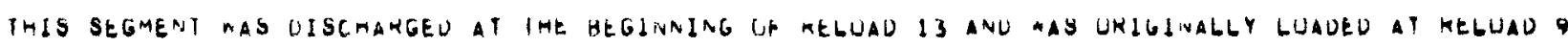

NUCLIDE AND GAMMA ENERGY IN MEV

(n)

CD-115M

1.0

$1: 25$

CAUMIUA

$1: 0$
$1: 25$
1074

AGe1084

$A G 0108 M$
$0.1^{\circ}$
0.3
0.6
0.8
1.0
TOTAL

$A G=110 \mathrm{M}$

0.1

0.3

0.6

1.0

1.25

1.6
2.0

TUTAL

SILVER

0.1
0.3
0.6
0.8
1.0
1.25
1.6
2.0
2.4
TUTAL

TUTAL

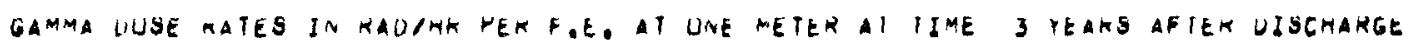

MS FKESH HO235 P.E.

FERTILE PTL. FISSILE PTL.

FERTILE PTLO FISSILE PTLO

$4.519=10$

$2.188 \cdot 10$

$1.813-10$

$2.090-10$

$4.519-10$

$2.188-10$

$1.813-10$

$2.090-10$

$1.097-06$

8.880005

1.5300004
1.508004

$2.019=00$
$3.757=04$

$1.003-05$

8.439004

$9.143=02$
5,52000

1.639002

1.808 .02

1.382 .02

$8.690-05$

$1.459-61$

\section{$1.618=07$ \\ 1.310005 \\ $1.962=05$
$2.224-05$ \\ 2.978007 \\ 2.978007
5.542005}

$1.405-05$

$1.182-03$
5.804002

$7.732-02$

2.295002

2.533002

$1.935-02$

$1.217-04$

$2.044=01$

23HS NECYCLE U-235 FE,

PENIILE PIL, FISSILE PIL.

$4,519=10$

$2.188-10$

$4.519=10$

$0 ., 188.10$

$1.097=00$

8.800005

$1.330=04$

$2,019=00$

2,019000
$3,757-04$

1.003005

$8.439=04$

4.143002

$5,520.02$

1.639002
1.808002

1.808002
1.382002

8.690 .05

$8.992-05$

$1.459 \cdot 01$

1.421 .05

$1.195-03$
$5.806-02$

$5.806=02$

$7.734-02$
$2.295-02$

2.295002

$2.533=02$

$1.935-62$

$1.260=04$

2.045001
1.113005

9.327 .04
$4,157.02$

$5,535=02$

$1.639=02$

$1.800-02$

1.382002

$8.690-05$

$8.992=05$

1.403001
$1.054-10$

$8.974-11$
$2.751-10$

$1.854-10$
8.974011

2.751010

2SKS RECYCLE UT235 F.E.

PERTILE PTL. FISSILE PIL.

ond

$4.519-10 \quad 1.560-10$ $0.707 .10 \quad 2.048010$

$\begin{array}{ll}4.519-10 & 1.300-10 \\ 2.0188-10 & 0.679-111 \\ 6.707 .10 & 2.048010\end{array}$

2.398 .07

1.941005

$2.907-05$

4.413007

4.413007
$8.211=05$

1.097 .06

1.330004

1.508004

$2.019=06$

1.455005

1.117004

1.200004

1.095006

1.492005

1.003005

$8.439=0$

4.14300

5.520002

1.630002

1.808002

1.362002

$8.690-05$

$\begin{array}{lll}1.337 .04 & 8.992-05 & 9.459005 \\ 2.171 .01 & 1.450 .01 & 1.535001\end{array}$

$1.055=05$

8.878004

4.358 .02

5.806002
1.724002

1.724002

1.902002

1.453002

9.142005

$\begin{array}{lll}1.337 .04 & 8.992-05 & 9.459005 \\ 2.171 .01 & 1.450 .01 & 1.535001\end{array}$

1.516005

1.275003

6.166002

2.437 .02

2.690 .02

$2.055=02$

$1.293=04$

1.337 .04

1.113005

$9,327=04$

$4.157=02$

5.535002

$1.659=02$

1.808002

$1.382=02$
$0.090-05$

0.690005

$0.992=05$

$1.147-05$

9.023004

4.370002

5.819002

1.724002

1.002002

$1.453=02$

$9.142-05$

9.459005

1.538001 


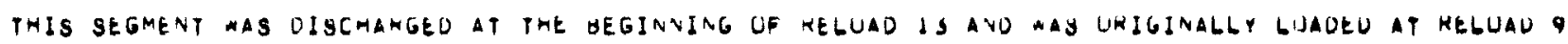

NUCLIDE AND GAPMA ENERG IN MEV

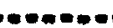

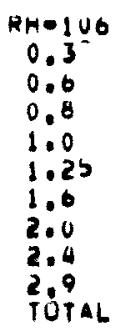

RMUOLUM

0.3
0.0
0.8
1.0
1.25
1.6
2.0
2.4
2.0
TUTAL

RU $1 \cup 3$

0.1

TUंTL

RUIHENIUM

0.1

TUTAL

GAMMA DUSE KATES IN RAOIAK FER F,E. AT ONE MEIER AT IIME 3 YEARS APIER DISCHAHGE

MS FFESH UAZ235 F.E. FERTILE PTL. FISSILE PIL.

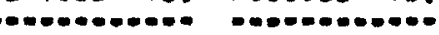

$$
\begin{aligned}
& 2.739-02 \\
& 7.946000 \\
& 1.738-01 \\
& 7.014-01 \\
& 2.334=01 \\
& 1.611=01 \\
& 5.942002 \\
& 5.939=02 \\
& 9.753-03 \\
& 9.371+00
\end{aligned}
$$

$2.739=02$
$7.946+00$

$1.738-01$

7.014001

2.334001

$1.611=01$

5.942 .02

5.939 .02

9,753003

$9.371+00$

3.344009

4.451 .08

$8.75+006$
$8.8 \times 2=00$

$3,344=09$

4.451 .08

$8.754=06$
8.802006
$3.005-02$

$1.063+01$

$9.364=01$

3.123 .01

2.155 .01

7.051 .02

7.940 .02

$1.305-02$

$1.254+01$

3.605002

$1.063+01$

2., $325=01$

9.384001

2.155001

7.040002

$1.305=02$

$1.305=02$
$1.254+01$

$1.936=09$

2.578 .08

$5,097=06$

$1.930=09$

$2,578=08$

$5,069.06$

$5,097=06$
23KS RECYCLE U-235 F.E.

FERTILE PTL. FISSILE PTL.

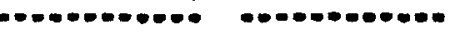

2.739002

$7.940+00$

$1,730-01$

7.014001

2.334 .01

$1.611-01$

5.942 .02

5.939002

9.753003

$9.371+00$

2.739002

$.940+00$

7.730001

$? .014001$

1.011001
5.043002

$5.939=02$

$9,753=03$

$9 ., 371+00$

2.403002

0.909400

1.324001

0.151 .01

2.047 .01

1.413001

$5.212=02$

5.209 .02

8.554 .03

$8.210+00$

$2.403=02$

$.969+00$

6.131 .01

1.131001
2.047 .01

. 13001

$1.413=01$

. 12002

$8.554=03$

. 19000

3,344009

4.451 .08

$8.754=06$
$8.802=06$

1.434009

3.754000

$3.775=06$

$3,344=09$

4.451 .00

8.754 .06

8,802006

1.909008

3.754000

3,775006
ESHS HECYCLE U-23S .E.

REKTILE PTL. HISSILE PTL.

$$
\begin{aligned}
& 2.739002 \\
& 7.946+00 \\
& 1.798001 \\
& 7.014001 \\
& 2.334=01 \\
& 1.611=01 \\
& 6.942002 \\
& 5.939-02 \\
& 9.753=03 \\
& 9.371+00
\end{aligned}
$$

2.749002

7.974400

$1.744=01$

$7.039=01$

$2.342=01$

1.617001

5.904002

$5.960-02$

9.788003

$9.405+00$

2.739002

$7.946+00$

$1.738-01$

$7.014=01$

1.011001

$5.942=02$

$5.930=02$

0.75300

9.371000

2.749002

7.974400

1.744001

2.34201

1.617001

5.060002

$9.788-03$

9.780003

3. $344=09$

$4.051=08$

$8.754=06$

8,002006

$1.450=09$

197000

3.796006
3.817006

3. 344009

$4.451=06$

8.75400

8.602006

1.450009

1.930008

3.796006
3.817000 


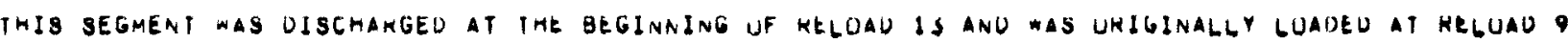
GAMMA DOSE RATES IN RADIHR PER F.E. AT DNE MEIER AT IIME S YEARS ARTER UISCMARGE

NUCLIDE ANO GAMMA ENERGY IN MEV

IN MEV

NG $=95$

POTAL

NIUBIUM

0.8

2k-95

0.3

TORAL.

2IKCUNIUM

0.3

Tútal

$\cos 5 \mathrm{C}^{\circ}$

0.6

TÓTAL

$c 0.60$
1.25

TUTAL

COBALT

0.6
0.6
0.8
1.25

1.25

TUTAL
MS RESH U-235 F.E.

FERTILE FTL. FIS8ILE PTL.

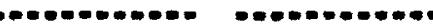

23KS RECYCLLE U.235 F.E.

FERTILE PTL. FISSILE PTL.

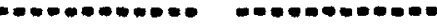

1.934001

0.992 .02

$6,992=02$

1.934001

1.934001

0.798002
0,798002

1.934001

6,992002

0.794002
$0.798-02$

1.552 .04

8.160002
8.176002

$5.455=05$

2.868002

2.073002

1.552004

8.160002

0.176002

5.455005

2,868002

2.873002

8.160002
8.170002

5.610005
2.950002

2.955 .02

1.203008
6.549008

$7.439 \cdot 1$

7.886008

1.247009
$0.405=0$

$7.340=11$

4.426004
4.426004

$4,395=05$
$4,395=05$

$2,965=05$

0.426004

$8.416=10$

$4.362-09$

$2.965-05$

$4.955-11$

2.966005
$1.263=08$

0.549008

4.426004

$7.439-10$
4.420000

$4.427-04$
1.247009

6,465009

$4.395 \cdot 09$

7.344011

$4.395-05$
$0.465=09$

$25 R 3$ RECYCLE UW235 P.E.

FERTILE PTL. FISSILE PTL.

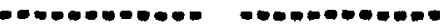

\section{$1.934001 \quad 5.180002$ \\ 1.934001 \\ 3.100002 \\ $1.954001 \quad 5.100002$ \\ $1.034001 \quad 5.180-02$}

$1.552004 \quad 4.156005$

$8.100-02 \quad 2.105002$

$0.176002 \quad 2.109002$

$1.552004 \quad 4.156005$

$8.160002 \quad 2.185002$

2.109002

$\begin{array}{ll}1.263008 & 4.791009 \\ 6.549008 & 2.484008\end{array}$

$\begin{array}{ll}6.549 .08 & 2.484008 \\ 7.450-10 & 2.821 .10\end{array}$

$\begin{array}{ll}7.439-10 & 2.821 .10 \\ 7.806-08 & 2.991008\end{array}$

$4.426004 \quad 1.608004$

$4.426004 \quad 1.608004$

1.263008
6.549000
$4.426-04$
$7.439-10$

4.427 .04
4.791009

2.484000

1.608004

$2.821-10$

1.608004 


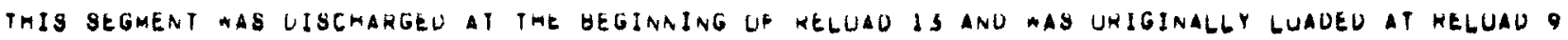

NUCLLDE ANU GAMMA ENERGY IN MEY

ALG ELEMENIS

0.1

0.6

0.6

1.0

1.25

1.06

2.0

3.9

TUTAL
GAMMa DUSE HATES IN RADIHK DER F.E. AT UNE METER AT IIME

MS FRESH UAZ35 F.E. FEKIILEPTL. FISSILE PTL. 23RS RECYCLE U. 235 F.E PERIILE PTL. FISSILE NTL -0.0.0.0.0.

$2.284+00$
$6.403+00$
$5.697+02$
$2.231+02$
$6.042+00$
$2.214+01$
$2.427+00$
$6.486+00$
9.162001
$9.753=03$
$8.394+02$

$3,228+00$

$0.890+00$

$4.589+02$

4.733002
$1.136+01$

$4.385+0.1$

$2.240+00$

$4.629+00$

9.808001

0.5540 .03

$0.762-01$

$8.394+02$

$1.305-02$

$8,304+02$
YEARB AF TER DISCHAHGE

25HS RECYCLE UA235 P.E. FEKTILE PTL. FISSILE PIL

$2.284+00$
$0.403+00$
$5.097+02$
$2.231+02$
$0.042+00$
2.214001
$2.427+00$
$0.486+00$
0.102001
9.753003
$0.394+02$

$2.447+00$

1.000000

$8.200+02$

$4.504+02$

$1.175+01$

$4.409+01$

$2.203+00$

$4.353+00$

6.021 .02

$0.760=03$

$1.346+03$ 


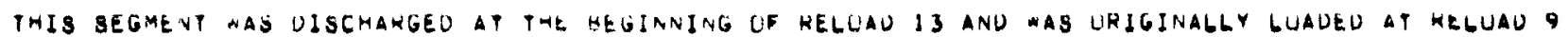

NUCLIOE ANO IN MEV

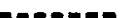
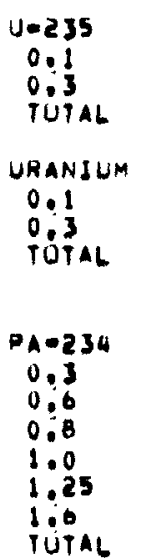

\section{PRUTACTINIUM}

0.3

0.8

0.8
1.0
1.25

TÓTAL

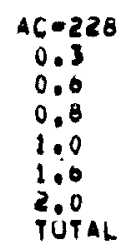

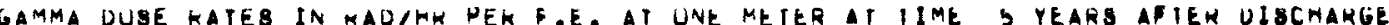

MS FRESM UMZZ35 F.E. Z3RS RECTCLE UEC35 F.E.

FERIILE PTL. PISSILE PIL. TERTILEPIL, FISSILEPTL.

\section{$3.717-07$} 9.898 .07 $1.362-00$

$3.717=07$ $9.898=07$ $1.362=00$ 7.469 .06 $1.027=05$

0.000

0,000

0.000

0.000

0.000

0.000

0.000

0.000

0.0000

0.000

0.000

0.000

0.000

$1.201=05$
$0.116=00$

1.504005

$1: 355=04$

$4.238-05$

$0.770=06$

$.770=06$
$2.208=04$

$5,364=07$
$5,462-07$
$2.087-07$
$5,021=06$
$3,730=07$
$8.872-07$
$7.633-00$

7.033000

5.364007

$5.462-07$

$5,021=06$

$3.739=07$

8.872007

$7.633-00$

0.000
0.000
0.000
0.000
0.000
0.000
0.000

\section{$3.717-07$} 9.898 .07
$1.362-06$

3.717 .07

9.898007

1.362006

\subsection{024006 \\ 3.624006
$4.965=00$}

$1.361=00$
3.624000

$3,61,4=06$
$4,985=00$

0.000

0.000

0.000

0,000

0,000

0.000

0.000

0.000

0.000

0.000

0.006

0.000

0.000

0.000

1.201005

0.110000

$1,504=05$

$1.355=04$

4.2380 .05

$9.770-06$

$2,208=04$
25RS KECYCLE UD2S5 PE

PENTILE PTL. PISSILE PIL.

$\begin{array}{ll} & \\ 3.117007 & 2.193000 \\ 9.898007 & 5.840006 \\ 1.362006 & 8.053000 \\ & \\ 3.717007 & 2.143006 \\ 9.898007 & 5.840006 \\ 1.302006 & 8.033000\end{array}$

\begin{tabular}{|c|c|c|}
\hline $\begin{array}{l}2,345-11 \\
2,386-11 \\
1,174=11 \\
2,195-10 \\
1,635-11 \\
3,878-11 \\
3,337 \cdot 10\end{array}$ & $\begin{array}{l}0.000 \\
0.000 \\
0.000 \\
0.000 \\
0.000 \\
0.000 \\
0.000\end{array}$ & $\begin{array}{l}4.001=00 \\
4.075000 \\
2.004000 \\
3.706=05 \\
2.789000 \\
0.018000 \\
9.694005\end{array}$ \\
\hline $\begin{array}{l}2,345-11 \\
2,380-11 \\
1.174011 \\
2,195-10 \\
1,635-11 \\
3,478-11 \\
3,357-10\end{array}$ & $\begin{array}{l}0.000 \\
0.000 \\
0.000 \\
0.000 \\
0.000 \\
0.000 \\
0.000\end{array}$ & $\begin{array}{l}4.001006 \\
4.075006 \\
2.004000 \\
3.740005 \\
2.709006 \\
6.618006 \\
5.094005\end{array}$ \\
\hline
\end{tabular}

$1.812011 \quad 1.201005 \quad 0.000$

$6.116000 \quad 0.000$

$1.504005 \quad 0.000$

$0.395-11 \quad 4.238005 \quad 0.000$

$1.474-11 \quad 9.790006$

$3.332010 \quad 2.208004 \quad 0.000$ 


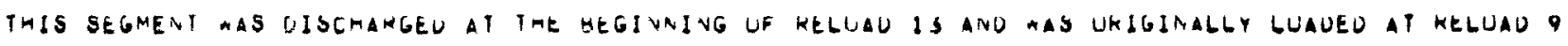

NUGLIDE AND GAMMA ENEKGY IN MEV

OA.

\section{ACTINIUM}

0.0
0.0

0.8

0.8
1.0
1.0

1.6
2.0

TUTAL

$H A-223$

0.1

TUTAL

RAUIUM

$0: 1$
$0: 3$
TOTAL

RN-219

TUTAL

RauUN

TUPAL

B. 0211

TUTAL

$\forall I-212$
0.8

0.8

TUTAL
GAMMA DOSE FATES IN RADIHK HER F.E. AT UNE MEIER AT IIME MS GKEOM LES 35 F,E. FEHTILE PTL. FISSILE PIL. 23KS KECYCLE U-Z35 FEE.
FERTILE PIL. FIS3ILE TIL.

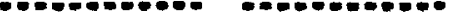

$1.201=05$

$0.110=00$

$1.504-05$

$1.355=04$

4.238 .05

$9.770=06$

$2.208 \cdot 04$

$2.078=00$

$5.533=06$

2,078006

$2,078=06$
$5,533=00$
$7.612=00$

0.000
0.000
0.000
0.000
0.000
0.000
0.000

1.201 .05

0.116006

1.504005

$4.238-05$

9.770000

2.200 .04

\section{$1.812-11$
$0.227=12$ \\ 2.209018 \\ $2,044-10$ \\ $6.395-11$ \\ $1.474-11$ \\ $3,332-10$}

2.078006

5.533006
7.012006

1.384006

2.149009
2,956009

$0.070-10$

2.149000
2.950009

$5.533=06$

9.199007

1.384006

$1 ., 904=06$

5.535006

5,535000

1.384006

2,149009

$2.149-09$

2.149009

5.533006

5.533006

$1.384=06$

1.380006

5.533006
5.533006

1.384006

$1.384=06$

7.763002

7.197-02

1.150002
$3.169-02$

1.152001

1.240002

3.418002

3.150000

1.170005
1.079006

5.179006
$1.882=05$

$1.152=01$
3.199007

2.078006

25KS RECYCLE U-235 F.E.

PEATILE PIL. NISSILE PIL.

$\begin{array}{ll}1.201005 & 0.000 \\ 0.116006 & 0.000 \\ 1.504005 & 0.000 \\ 1.355004 & 0.000 \\ 4.238005 & 0.000 \\ 9.770006 & 0.000 \\ 2.208014 & 0.000\end{array}$

0.000

\section{$2.076006 \quad 3.350009$}

7.012006 . 1.220000

1.2420 .1

$\begin{array}{ll}2.078000 & 3.330009 \\ 5.533000 & 8.866009\end{array}$

1.220006

1.900.0.




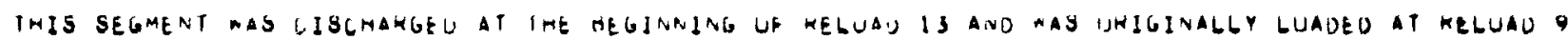

NUELIDE AND GAMMA ENERGY IN MEV ENERG

$81-213$

0

TÜTAL

B IBMUTH

0.3

0.8

1.0

IUTAL

$p$
$N$
$a$

$P B=211$
0.3
0.8

TUTAL

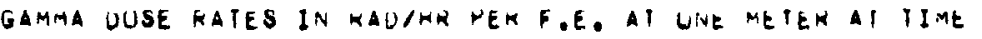

YEAHS ARIEN DISCHAHGE

NS FRESM U-Z3S P.E. FERIILE PIL. FISSILE PIL.

23RS HECYCLE U-235 R.E. GENTILE PIL. FISSILE PTL.

25HS RECYCLE U-235 F.L. PEKIILE PIL. FISSILE PIL.

$\begin{array}{ll} & \\ 0.005-05 & 4.813-10 \\ 5.942000 & 3.301011 \\ 9.259005 & 5.143010 \\ & \\ 9.218005 & 2.630009 \\ 7.197-02 & 1.170-05 \\ 1.150-02 & 1.879-06 \\ 3.169-02 & 5.179-00 \\ 1.153-01 & 1.882005\end{array}$

\subsection{5}

5.942000

$7.071=00$

1.102004

0.210005

7.197002

1.045004

7.763002

1.130002

3.169002

1.241 .02

3.418 .02

1.243001

$P Q=212$

PUTAL

LEAO

0.3

TUTAL

$16=208$
0.3
0.6
0.8
2.4

3.320006

3,3260116
0,640000

1.269 .09

1.291 .09

$2.581=00$

1.437 .01
1.437 .01

$2.348=05$
$2.348-05$

3,320000

$0.300=08$

0.321007

1.063000

$1.437-01$

$1.550=01$
1.55000

550.0

1.437 .01

1.437 .01

$2,348.05$

1.291 .09

$1.437=01$

3.326006

$1.550=0$
$8.321=0$

1.55000

$8.015-03$

$2.720-01$

$5.176-02$

$.603=01$

4.446 .05

8.466006

1.006000

$0.015-03$

$2.720-01$

$5.178=02$

$.603=01$

2.930001

5.589 .02

$9.285=01$

$1.287+00$

\section{$0.605=05$ \\ 1.963009}

.94200

.259005

$1.360 \cdot 10$

2.11900

9.218005

7.197002

1.150002

3.169 .0

1.153001

$1.085=00$

4.816005

7.768006

2.147 .05

$7.802 \cdot 05$

3.320006

3,326006
0,046000

3.319009

3.329009

$1.065=08$

$\begin{array}{ll}1.457001 & 9.7340 .05 \\ 1.437001 & 9.734005\end{array}$

$1.437-01$

9.754005

$5: 329009$

1.437 .01

9.735005

6.615003

2.720 .01

5.836000

.17800

$1.103+00$

842004

$3,508.05$

5.827 .04 


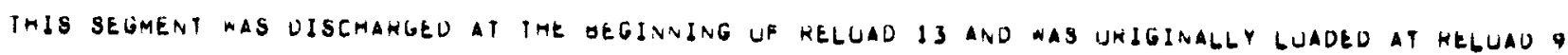

NUGLIDE ANO GAMMA ENERGY IN MEV

(N)

THALLIUM

0.3

0.0
0.8
2.4

Tutal

$18=158$

0.150
0.3
0.8
1.0

0.0
1.0
1.25

TUTAL

$18 \cdot 100$

0.3

0.8

1.0

TUTAL

TENGIUM
0.3

0.8
1.0

1.25

YUTAL

cu- 152

0.1
0.13
0.0
0.8
1.0
1.25
1.00
IUTAL

0.1

0.0

1.0

TUTAL

gamma duse kates in maujhk per f.e. at une meter at lime g yeakg afiek uischarge

MS FRESH L-235 F.E
FERTILE PTL, FISSILE PIL.

$$
\begin{aligned}
& 8.615003 \\
& 2.720001 \\
& 5.178002 \\
& 8.003-01 \\
& 1.193+00
\end{aligned}
$$

1.400000
4.440005
$8.400-06$
1.400004
$1.950-04$

$0.340-10$

3.439 .09

1.30
4.90200

$9.645-10$

2.324 .10

2.324010
8,693009

1.539009

$8,567-09$

$0.777-09$

0.579009
2.746008

4.350 .09

1.053 .08

$1.079=08$

8.087 .09

$3.370-08$

$4.173-09$

1.401 .04

5.041 .00

7.809009

7.048008

4.462009

$1.150=08$

$1.817=08$
$8.319=09$

$4.245=08$

$1.300-03$

$3,403-03$

$3.657-04$

3,590063

8.123 .03

$2.958-04$

$2.767=02$

2.134003

5.656003

9.2390 .04

1.683003

1.686002

$4.851=04$

4.518 .02
$23 R S$ HECYCLE U-235 F.E.

FERTILE PTL. FISSILE PTL.

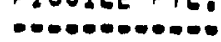

FERTILE PTb.

Fis

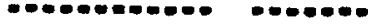

ISSILE PILO

$\begin{array}{ll}8.015003 & 9.299003 \\ 2.720001 & 2.936001 \\ 5.178002 & 5.589002 \\ 8.605001 & 9.285001 \\ 1.193+00 & 1.287+00\end{array}$

6.015003

2.720001

3.176002

$8.603=01$

$1.193+00$

5.830000

$1.842-04$

3.508005

$5.827-04$

$0.01000 \mathrm{M}$

$0,346-10$
$5,439-09$
$4.164-08$
1.311000
4,902008

7.990010
6.849009

6.849000

5.242008

1.631000
6.172008

6. 346010

5.439 .09

4.104008

1.311009

4.902008

1.251 .10

$1.013=09$

0.210009

$2.545-10$

3.539009

$8.567=09$

$0.777-09$

3.916009

9.485000

9.714000

$7.281 \cdot 09$
3.030 .00

3.539009

8.567009

0.777 .09

0.579000

2.746008

7.420009

1.799000

1.843008

5.706008

$4.173-09$

1.401008

5.041008

7.089009

4.715009

1.033000

8.931000

$0.2120 j b$

4.173009

1.401008

5.041 .00

7.889009

7.555009

1.006008

2.664000

$1.407-08$

$1.300-03$

1.453 .03

$3,852=03$

6.293004

6.293004
3.993003

$1.148=02$

$3.590-03$

. $123=03$

3.020004

$\begin{array}{ll}2.958 .04 & \$ .290004 \\ 2.707-02 & 3.077 .02\end{array}$

1.300003

$3.403=03$

$5.657-04$

3.580003

1.032002

. 123003

2.709002

1.679 .03

4.450003

7.200004

$4.613=03$

1.326002

1.044002

$3.801=04$

$3.555=02$ 
THIS SEGMENT MAS UISCMAKGED AT THE BEGIVNING UF RELUAD I3 ANU MAS UMIGINALLY GUAUEU AT HELUAD 9

NUCLIDE ANO GAMMA ENERGY IN MEV

IN MEV

EU. 154

0.1

0.3

0.0

0.0

1.05

IU⿴囗十⺝木

EUKUPIUM

0.1
0.3
0.6
0.8
1.0
1.25
100
1074

N

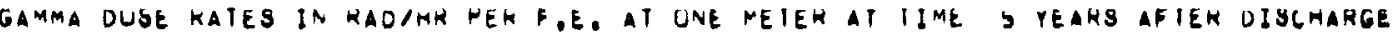

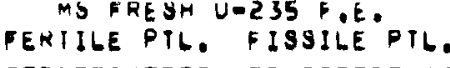

$\begin{array}{ll}3.570001 & 1.010+00 \\ 1.553001 & 4.380001 \\ 4.080001 & 1.153+00 \\ 2.223+00 & 6.277+00 \\ 2.108+00 & 5.950+00 \\ 3.230+00 & 9.145+00 \\ 2.501-01 & 7.062001 \\ 8.742+00 & 2.468+01\end{array}$

$3.589-01$

$1.588=0$

$3.357+00$

50

1.000

$0,504=01$

2.50401

.769 .01

1.643002

$7.023 \cdot 03$

$3.111-01$

$1.081+00$
$2.293+00$

$0.769-01$

1.643 .02

.023-03

3.111 .01

$1.081+00$

$2.293+00$

$1.012+00$

4.442001

$1.154+00$
$0.283+00$

$6.283+00$

$9.967+00$

$7.007=01$

$7.067=01$

$1.463-02$

$2.771=01$

$9.026=01$
$2.042+00$

$7.810-01$

1.403 .02

0.789003

$2.771-01$

9.626001

$2.042+00$
23RS KECYCLE U-235 PE.
PERTILE PTL. ISSILE PIL.

Coconomaco

$\begin{array}{ll}3.570001 & 6.040001 \\ 1.553001 & 2.909001 \\ 4.086001 & 7.051=01 \\ 2.223+00 & 4.105400 \\ 2.108000 & 3.947000 \\ 3.239000 & 0.060000 \\ 2.501001 & 4.084=01 \\ 8.742+00 & 1.037+01\end{array}$

$\begin{array}{ll}3.589001 & 0.711001 \\ 1.588001 & 2.948001 \\ 4.091001 & 7.657001 \\ 2.227000 & 4.167000 \\ 2.118000 & 5.958000 \\ 3.247000 & 0.075000 \\ 2.504001 & 4.607001 \\ 8.770000 & 1.640001\end{array}$

$\begin{array}{ll}8.769=01 & 0.246001 \\ 1.643002 & 1.170002 \\ 7.623003 & 5.429003 \\ 3.11101 & 2.210001 \\ 1.081+00 & 7.690001 \\ 2.293+00 & 1.633+00\end{array}$

$8,769-01$

1.043 .02

7.623003

3.111 .01

$1.061+00$

0,246001

$1.170=02$

5.429003

2,210001

7,69800

$1,033+00$ 25RS RECYCLE UDZ35 R.ER
FEKTILE PIL. FISSILEPIL.

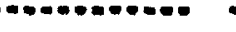

$\begin{array}{ll}3.570001 & 7.600001 \\ 1.553001 & 3.356001 \\ 4.00001 & 5.775001 \\ 2.223+00 & 4.775+00 \\ 2.108+00 & 4.527+00 \\ 3.239+00 & 0.957+00 \\ 2.501001 & 5.372001 \\ 8.742+00 & 1.878+01\end{array}$

3.589 .01

1.588001

$4.091-01$

$2.227+00$

$2.118+00$

3.247400

2.504001

$0.790+00$

7.607 .01

3.381001

4.702001

$4.540+00$

$4.540+00$
$0.967+00$

$: .97400$

$1.861+01$

5.859001

$1.043002 \quad 1.098002$

.023003

3.111 .01

$1.061+00$
$2.203+00$

2.070 .01

$7.221=0 !$
$1.532+00$

0.709001

5.859001

1.643 .02

7.623003

3.111 .01

$1.081+00$

1.098002

5.093-03

2. $090-01$

7.221001

$1.532+00$ 


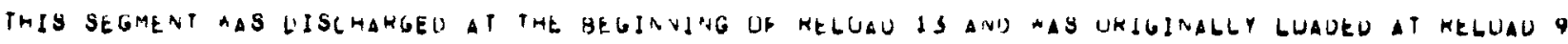

NUCLIDE AND GAAMA ENERGY IN MEY

IN MEV

\section{$840.137 M$}

0.6

TUTAL

GARIUM

0.1
0.0
PUTAL

Cs- 134

$C 8-134$
0.3
0.6
0.8
1.0
1.25
1.0
$T O T A L$

CESI JM

$$
\begin{aligned}
& \text { CESIJM } \\
& 0.3 \\
& 0.0 \\
& 0.0 \\
& 1.0 \\
& 1.25
\end{aligned}
$$

TƯTAL

$38=124$

0.0
0.8
1.0
1.25

1.25

1.00
1.00
TUTAL

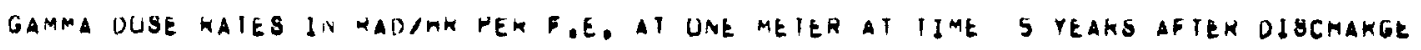

$$
\begin{aligned}
& \text { YS RRESH L-23S F,E. } \\
& \text { FERTILE PTL. FISSILE PIL. }
\end{aligned}
$$

FERTILE PTL. FIV

$1.2 \div 0+00$

$3.107+02$
$3.179+02$

$1.802+00$

$4,915+02$

$1.200+00$

$3.107+02$

$1.802+00$

$4.915+02$

.930 .02

1.4580 .02

$1.077+02$

$1.118+02$

$1.520+00$

$9.3 \cup(1)+00$
$5.140-02$

5,146002

$1.458-02$

$1.077+02$

$1.118+02$

$1.526+00$

$0.30 v+0$

$5.140=02$
$2.304+02$

3.954 .02

$2,920 \bullet 02$
$3,030+02$

$3.030+02$

$2.522 \div 01$

$1.395-01$

$6.200+02$

$3.954-02$

$2.920+02$

$3.030+02$

$4.139+00$

$1.395-01$

$1.305-01$
$0.246+02$

$2.571-10$

$4.416-11$

$1.539-11$

$2.235-11$

$2.970-10$

$3.912-11$
$0.751-10$

$1.942-10$

$3.336-11$

$1.163-11$

1.688 .11

$2.243-10$

$2.955-11$
$5.099-10$
23RS WECYCLE UO235 F.E.

PERIILE PILA FISSILE PIL.

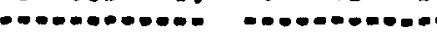

$3.200+00$
$3.167+62$

$3,167+02$
$3,179+02$

$1.746+00$

$4.613+02$
$4.030+02$

$1.200+00$

$3,107+42$
$3,179+02$

$1.748+00$

$4.013+02$

4.030402

3.104002

$2.292+02$
$2.379+02$
$3.249+00$

$3.249+00$

1.079001

1.979001

$4.003+02$

$2.300+02$

1.458002

$1.077+02$

$1.118+02$

$1.526+00$

$9.300+00$

5.146002

$2,304+02$

$2.571=10$

$4.410-1$

$1.539-11$

$2.235-11$

$2.970-10$

$3.912-11$

$0.751=10$

\section{3,104002 \\ $2.292+02$ \\ $3.249+00$ \\ $1.979+01$}

$4.903+02$

$3.179-10$

$5.459-11$

$1.903-11$

$2.763-11$

$3.671-10$

$4.836-1$

$4.346-10$
L5KS RECYCLE UOZ35 F.E.
FEKTILE PTLE PISILE PTL.

-oonoconomon

$1.200+00$

$3.167+02$

$1.340+00$

$3.000+02$

$1.200+00$

$3.167+02$

$1.300+00$

3.6060 .02

1.458002

$1.077+02$

$1.118+02$

1.526000

$0.300+00$

5.146002

1.458002
$1.077+02$

$1.077+02$
$1.118+02$

$1,118+02$

1.

$9.300+00$
5.106002

$2.304+02$

2.949002

2.176002

2.260002

3.086000

1.040001

1.040001
$4.657+02$

\subsection{2}

$2.178+02$

$2.260+02$

3.006400

$1.800+01$

1.040001

$4.657+02$

$2.571-10$
$4.916-11$
$1.539-11$
$2.235-11$
$2.970-10$
$3.912-11$
$6.751-10$

2.030 .10

$3.487-11$

1.215011

$1.705-11$

2. 345-10

3.069-11

$5.350-10$ 


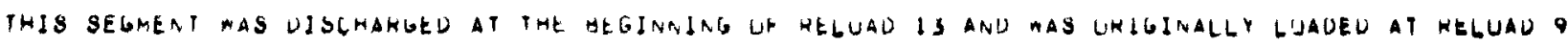

NUCLIDE AND GAMMA ENEKGY IN MEV

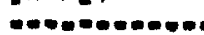

\section{$58 \cdot 125$}

0.13

TUTAL

ANIIMONY

0.1

0.3
0.6
0.8

1.00

1.25

2.0

$\theta$
N
on

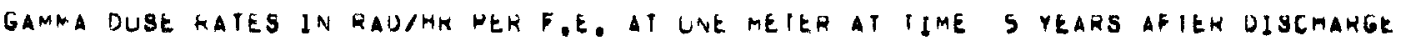

MS FKEgH U-235 P.E. FERTILE PIL. FISSILE PTL. 23HS KECYCLE UEZ3S FOE.
FERTILE PTLE PISSILE PTL.

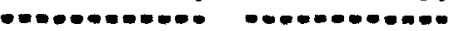

$3.810-01$
$3.021+00$
$1.255+00$

$3.810-01$

$3.621+00$

$7.255+00$

$4.416-11$

2.235-11

$2.970-10$

3.91201

$1.120+01$

$$
\begin{aligned}
& 1.248-01 \\
& 1.184+00 \\
& 2.373+00 \\
& 3.336-11 \\
& 1.163-11 \\
& 1.688-11 \\
& 2.243-10 \\
& 2.955-11 \\
& 3.682+00
\end{aligned}
$$

8.786005

1.310004

1.998000

$3.717-04$

1.378000

$1.159-04$
$5.092-03$

$5.692-03$
$7.583-03$

$7.583-03$
2.251003

$.484=03$

$.898-03$

1.194 .05

$1.235-05$
$2,005-02$
$1.248-111$

$1.184+00$

$3.682+00$

$1.001=07$
$1.296=05$
1.941005
$2,200=05$
$2,947=07$
$5.483=05$

$1.083=06$
8.786005
1.316004
$1.492=04$
$1.998=06$
3.717004

3.816001
$3.621+00$
$7.255+00$
$1.120+01$

$1.126+111$

3.816001
$3.021+00$
7.255000
4.410011
1.539011
2.235011
2.970011
$3.912=11$
$1.120+01$

$1.083=06$
8.786005
1.316004
1.492004
1.998006
3.717004

1.930000

$9.624=04$

7.9740 .03

1.002002

$3.153=03$

$3,480-03$

$2,059=03$

1.672005

$1.730-05$
2.809002
1.378006

$1.159=04$

5.692 .03

7.563003
2.251003

2.484 .03

1.098 .03

1.1940 .05

$1.235=05$

2,005002
$3.993-61$
$3.789+00$

7.392400

$1.178+01$

$3.993-01$

$3.789+00$

$5,459-11$

1.003011

$2.763=11$

$3.071-10$

4.836011

1.170001

2.372007
1.920005

$2.877-05$

$4.300-07$

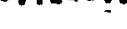

2.050006

1.725004

4.407 .03

1.128002

3.348003

$3.695-03$

$2.823=03$

1.770005

1.037005

2,982002 PERIILE PTL. FISSILE PTL

nomonomon

$3.021+00$

$8.120+01$

9.165002

$1.743+00$

$2.704+00$

3.816001

$3.621+00$

4.416011

1.539011

2.235012

2.970010

3.91201

$1,120+01$

$9.105 \cdot 02$

0.649001

$1.743+00$

$1.743+00$
$3.087-11$

$1.015-11$

$1.705=11$

$345=10$

3.009011

$3.704+00$

$1.005000 \quad 9.113007$

8.786005

1.31600

50

1.376005

1.105004

$1.077=00$

1.677 .06

$3.717-04$

1.450000

1.378000

1.159004
5.692003

1.220004

$5.988=03$

7.977 .03

2.308003

2.613003

1.907003

$1.256-05$

1.89800

1.194005

1.300005

2.005002 


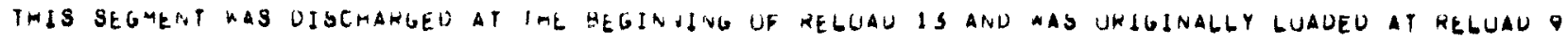
NUCLIUE AND
GAMMA ENERGY IN MEV

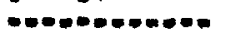

SILVER
0.1
0.3
0.6
0.8
1.0
1.25
1.6
2.0
2.04
TUTAL

0
N
a
GAMMA DUSE RAIES IN KAUT

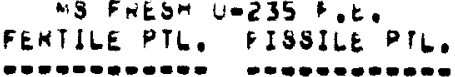

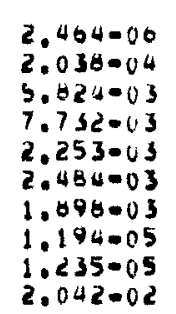

$0.924 \cdot 03$

$2.000+00$

$4.393=02$

$5,900=02$

$4.072-02$

$1.502=02$

$1.501-02$

$2.465=03$

$2.369+00$

$0.924-03$

$2.008+00$

$2.098+00$
4.393 .02

1.773001

5.900002

$4.072=02$
$1.502-02$

1.541002

$2.465-03$

$2.309+00$

\section{$2.091-00$ \\ $1.754=04$ \\ 7.995063 \\ $1.064-02$ \\ 3.154003 \\ $2.059=03$ \\ 1.672005 \\ $1.730=05$
2.814002 \\ 2.810002}

$9.204=03$

$2.687+00$

5.878 .02

$2.372-01$

5.448 .02

2.010002

2.010 .02

3.008002
3.598003

$3.160+00$

9.264003

$2.687+00$
5.878002

$2.372=01$

1.093002

$5,448.02$

$2.010-02$

2.008002

3.298003
$3.169+00$
23RS WECYCLE UEZ3S F.E. PERTILE PIL. FISSILE PTL.

$2.404-00$
$2.038-04$
$5.024-03$
7.732003
$2.253-03$
2.484003
1.898003
1.194005
$1.235=05$
2.042002

$2.000+00$

1.773001

$5.90,002$

4.072002

1.502002

1.501002

$2.300+00$

$0.924-03$

2.008400

1.773001

5.900002

4.072002
4.002005

4.072002
1.502002

1.501 .02

2.465003

$2,369+00$

\subsection{0 \\ 1.917004 \\ $8.495-03$ \\ 1.131002 \\ 3.349003 \\ $2.823=0$ \\ 1.796005 \\ $1.837-05$
2.090002}

0.075003

$1.702+00$

$3.853-02$

1.355001
5.174 .02

5.174002

3.571 .02

1.317002

1.317002

$2.078+00$

0.073003

$1.762+00$
$3.853=02$

1.555001

5.174002

$3.571=02$

1.317002

1.317 .02

2.162003
2.078000
S reAHS AFIER UISCMARGE

L5HS HECYCLE UO2SS H.E. FEHIILE NTL. FISSILE PIL.

2.464006
2.058004
5.824003
7.732005
2.253003
2.484003
1.898003
1.194005
1.235005
2.042002

2.361000

$1.937=04$

6.099003
8.102003

8.102003

2.370003
2.613003

1.04700

1.2560 .05

1.300005

2.140 .02

0.924003

$2.008+00$

4.393002

1.773001

4.072002

4.072002

1.502002

$1.501=02$

$2.360+00$

6.949003

$2.010+00$

4.409002
$1.779-01$

5.779001

9.907 .02

4.047 .02

$1.507-02$

$1.507-02$

2.474003

$0.924=03$

$2.008+10$

4.393002

1.773001

5.900002

4.072002

.502002

$1.501-02$

2.465003

$2.369+00$
0.949 .03

$2.016+00$

4.40900

5. $921=02$

$4.087=02$

1.507 .02

1.507 .02

2.377400 


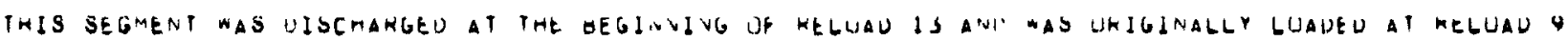

NUCL DOE AND
GAMMA ENERGY GAMMA ENERG

IN $\operatorname{mev}$

NQD-QS

TOTAL

NIU⿴囗十⺝M

YUTAL

\section{$2 R=95$}

0.3

PÚTAL

ZIKCUNIUM

0.3

$\vartheta$
Na
a

GAMMA UUSE HATES IN RADIHK PER F.E. AI HNE MEIEH AT IIME S YEAHS AFTER DJSCHARGE

MS FRESM U-a35 OE.

arion

8.5400115

8.540005

$3.087-05$
$3.087-05$

3.087005

0.540005
6.540005

\section{$2.477-00$}

$1.302-05$

$3.610=05$

$0.853-08$

3.003005

2.477 .00

$1.302-05$

$1.305-05$

$1.048-11$
$5.435-11$

$0.174-13$

$0.545-11$

0.000

0.000

0.000

2.278 .05

3.400004

$2.278-05$

$1.048-11$

$5.435-1$

$3.400=0$

0.174013
3.400004

0.000
0.000

0.000
2.278005

0.000

0.000
2.278 .05
FEKTILE PTL, FISSILE PIL.

$3.067-05$
$3.087-05$
Z3RS RECYCLE JOESS FOE.

FERTILE VIL, FISSILE NIL,

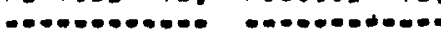

$8.500-05$
$0.540-05$

3.001005
3.001005

0.540 .05
8.540005

3.001005

3.001.05

2.400000

1.206005

3,603005

3.610 .05

0.853 .08

3.003005

2.400000

1.200005
1.269005

$1.048-11$

5.435011

$0.174-11$

$0.545-11$

0.000

0.000

0.000

3.400004

3.370005

$1.048-11$

$5.435-1$

$3,400=0$

$0,174-13$
$3,400=04$
25RS HECYCLE UEZ35 F.E. FERTILE PIL. FISSILE PIL.

0.540005

8.500005

2.207 .05

2.207 .05

0.540005
8.540005

2.207 .05

2.207005

1.25

TUTAL

(2) 


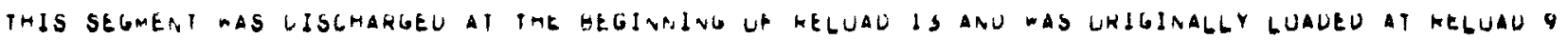

NUCLIUE ANO GAMMA ENEKGY IN ME

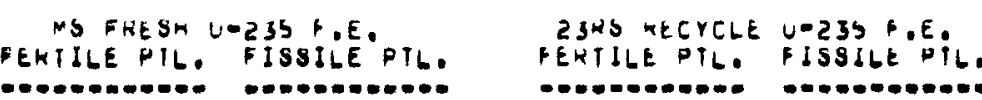
S TEARS AF IEK DISCHARGE ALL ELEMENTS

0.1

0.0
0.8
1.0

1.25

1.65

2.0

2.9

TUTAL
$1.940+00$
3.954000
$4.353+02$
$1.142+02$
$3.030+00$
$1.202+61$
$6.073-01$
$1.096+00$
$0.753=01$
$2.405=03$
$5.744+02$

$2.999+00$
$1.077+00$
$7.900+02$
$3.094+02$
$1.035+01$
$3.446+01$
$1.180+00$
$9.827-01$
$2.024=02$
$3.298-03$
$1.152+03$

$1.940+00$
$3.954+00$
$4.353+02$
$1.142+02$
$3.830+00$
1.262401
$6.873-01$
$1.096+00$
$0.753-61$
2.465003
$5.744+02$

2.018400

$4.205+00$

$7.010+02$

$7.425+02$
$7.370+00$

2.593401

8.72500

9.417001

$2.162=03$
$9.808+02$

1.940400
3.954000
9.353002
1.142002
3.856000
$1.262+01$
6.873001
$1.096+00$
8.753001
$2.465=03$
$5.744+02$

$2.250+00$

1.245000

$5.896+0 \mathrm{~d}$

$2.308+02$

7.866000

2.584001

8.924001

$7.372-01$

1.506002

2.474 .03

$8.592+02$
2SKS RECYCLF U-235 +.E. FERTILE PTL. PISSILE PILO 


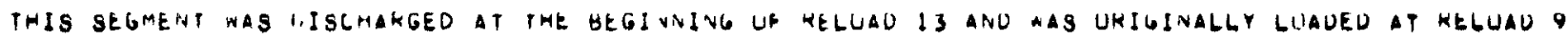

NUCLIDE AND GAMMA ENERGY IN MEV

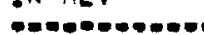

U. 235

$0: \frac{1}{3}$

TUTAL

URANIUM

0.1

TUTAL

\section{PA-234}

0.3

0.0

0.8

$P$
N

1.25

TUTAL

PKUTACIINIUM

0.3

0.6

0.8

1.25

Tútal

$A C-22 B$

0.3
0.6
0.8
1.0
1.6
2.0
TUTAL

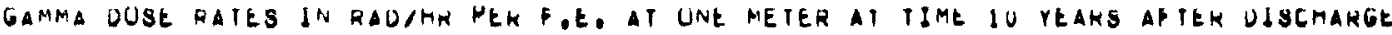

MS FRESH U-235 P.E.

FERTILE PIL. FISSILE PIL.

$\begin{array}{ll}3.717-07 & 2.805=06 \\ 0.896007 & 7.469-00 \\ 1.362006 & 1.027 .05\end{array}$

$5.717=07$
$9.898=07$

2.805000

$1.302=06$

$1.027=05$

0.000

0.000

0.000
0.000

0.000
0.000

0.000

0.000

0.000

0.000

0.060

0.000

0.000

0.000

0.000

$$
\begin{aligned}
& 5.364-07 \\
& 5.402-07 \\
& 2.087-07 \\
& 5.021-00 \\
& 3.739-07 \\
& 8.872007 \\
& 7.633-00
\end{aligned}
$$

$.466-05$

1.465000

.850005

5.173 .05

$1.193=05$
$2.695=04$
2325 HECYCLE U-235 F,

FERTILE PTL. BJSSILE

$\begin{array}{ll}3.717007 & 1.361000 \\ 9.898007 & 3.024006 \\ 1.302000 & 4.985000 \\ & \\ 3.717 .07 & 1.361000 \\ 9.898007 & 3.624000 \\ 1.302006 & 4.905006\end{array}$

25RS RECYCLE U-235 P.t.

FEKTILE PTLE FISSILE PILO

\section{$3.717=117 \quad 2.193=06$ \\ 3.89807
$1.302006 \quad 5.840006$

$\begin{array}{ll}3.717 .07 & 2.193006 \\ 9.898007 & 5.840006\end{array}$ \\ $9.898007 \quad 5.040006$}

$5.364=07$

$5,462=0$

2.687 .07

$5.021-06$

3.739007

8.872007

0.000
0.000
0,000
0,000
0,000
0,000
0.000

0.000

0,000

0,000

0.000

\begin{tabular}{|c|c|c|}
\hline $\begin{array}{l}2.345=11 \\
2.388-11 \\
1.174=11 \\
2.195-10 \\
1.035=11 \\
3.878=11 \\
3.337=10\end{array}$ & $\begin{array}{l}0.000 \\
0.000 \\
0.000 \\
0.000 \\
0.000 \\
0.000 \\
0.000\end{array}$ & $\begin{array}{l}4.001=06 \\
4.075000 \\
2.004006 \\
3.740-05 \\
2.789006 \\
6.618006 \\
5.694005\end{array}$ \\
\hline $\begin{array}{l}2.345-11 \\
2.388-11 \\
1.174011 \\
2.195-10 \\
1.635-11 \\
3.878011 \\
1.357-10\end{array}$ & $\begin{array}{l}0.000 \\
0.000 \\
0.000 \\
0.000 \\
0.000 \\
0.000 \\
0.000\end{array}$ & $\begin{array}{l}4.041006 \\
4.075006 \\
2.004006 \\
3.706005 \\
2.709006 \\
6.018006 \\
5.694005\end{array}$ \\
\hline
\end{tabular}

0,000

0,000

0,000

$$
\begin{aligned}
& 0.000 \\
& 0.000 \\
& 0.000 \\
& 0.000 \\
& 0.000 \\
& 0.000 \\
& 0.000
\end{aligned}
$$

$1,460=05$
.065006

1.830005

5.173005

1.193005

$1.193=05$
$2.695=04$

$\begin{array}{ll}1.466005 & 0.000 \\ 7.405006 & 0.000 \\ 1.036005 & 0.000 \\ 1.654004 & 0.000 \\ 5.173005 & 0.000 \\ 1.193005 & 0.000 \\ 2.695004 & 0.000\end{array}$




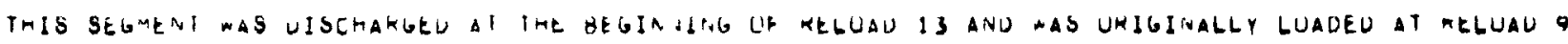

NUCLIDE ANO GAMMA ENERGY IN MEY

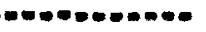

ACIINIUM

AC19NIUM
$0: 3$
0.6
0.8
1.0
1.0
2.0
TUTAL

TUTAL

$R A-223$

0.1

TUTAL

$\forall$
$N$
0

TOTAL

GAMM DUSE RATES IN HADIHK PER F.E. AT UNE METER AT IIME IU YEAKS AFIEK UISCMARGE

MS FHESH J.235 F.E.

FEHTILE PTL. FISSILE PIL.

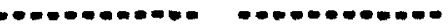

$1.466-05$

$7.465-06$

$1.036-05$

1.054 .04

$5.173=05$

1.193 .05

$2.095=04$

$$
\begin{aligned}
& 0.000 \\
& 0.000 \\
& 0.000 \\
& 0.000 \\
& 0.000 \\
& 0.000 \\
& 0.000
\end{aligned}
$$

\section{$1.770=00$}

$4.713-06$

$1.409-09$
3.751 .09

5.160009

1.770000

$4.713=00$

1.409 .09
3.751 .00

3.751009
5.160009

23HS KECRELE U-235 F.E.

FEKIILE PTLE IISBILE RTL.

4.713000
6.483006

23XS KECYCLE U-235 F.E.

PERTILE PIL. FISSILE PIL,

$R N=219$

TUTA:

RADUN

TUTAL

010211

0.3

BI-212

0.8

1.0

TUTAL
$4.713000 \quad 3.751 .09$

$4.713=00$

$3.751=09$

$4.713=00$

3,751009

4.713006

2.394006

2.394 .06

4.713006

2. 394006

$2.304=00$

4.713006
4.713000

2.394000

$4.713=06$

$3.751=09$
3.751009

7.010 .02

1.217 .02

$3.353=02$

$1.245-05$

1.989 .05

$5,083-06$

1,902005

4.713000

7.616002

3.217 .02

3.353002
$1.219-01$

7.411002

1.164002
$3.263=02$

1.180001

$\begin{array}{ll}1.400005 & 1.009-11 \\ 7.405006 & 5.545-12 \\ 1.030005 & 1.304-11 \\ 1.050004 & 1.220-11 \\ 5.175005 & 3.842-11 \\ 1.103005 & 8.858-12 \\ 2.095004 & 2.002-10\end{array}$

$2,002-10$

$1.770=06$
4.713000

8.993007
2.394000

2.394000
3,294000

1.770006
4.713006

$4.713=06$
$6.483=06$

$0.993=07$
2.394006

2.394006
3.294006

$\begin{array}{ll}1.400005 & 0.000 \\ 9.405000 & 0.000 \\ 1.050005 & 0.000 \\ 1.654004 & 0.000 \\ 5.173005 & 0.000 \\ 1.103005 & 0.000 \\ 2.0950 .04 & 0.000\end{array}$

$2.095=04$

0,000

5.757009

$4.713000 \quad 1.527008$

$0.483=00$

2.101000

1.770000

4.713000

3.737 .09

1.527000

$2.101 \cdot 08$

6.035000

$4.713=00$

1.527000

0

.527 .08

$4.713=06$

$1.527-08$

$1.527-08$
$1.527-00$

1.217 .02

$3.353=02$

1.219 .01

5.103005

4.247 .06

2.273005
8.201005 
THIS SEgMENT WAS DISCHAKGEO AI THE HEGINNING UP RELHAD IJ AND WAS URIGINALLY LUAUED MT HELUAD 9

NUCL DDE ANU GAMMA ENEKGY IN MEV (t)

$B 1-213$
0.3
1.0

TUTAL

BI BMUTH

0.3

1.0

1.0

PQ-2!1

0.3

$\stackrel{\ominus}{N}$

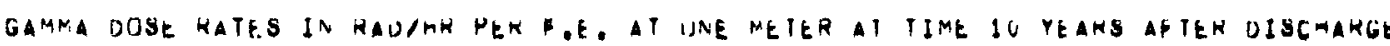
FENTILE FTL. FISSILE PTL.

$1.587=0.0$ 1.009 .05

\section{$4.922-10$} $3.307-11$

$1.635=04$

$7.010-02$

$1.218=02$
$3.353-02$

4.234009

1.205005
1.989006

$5.483-00$

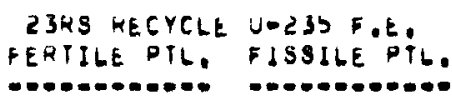

1.587 .04
1.089 .05

1.090004

1.140004
1.620000

1.218004

$1.635=04$

$1.018-02$

3.353 .02

1.220001

1.104004
7.411002

1.185002

3,203002

1.169001

$2.820-06$
$2.833-06$

$5.061=06$

$2.251=09$

$2,255=09$
$0,506=09$

2.828006
$2.833=06$

$5.833=06$

1.437006

1.439006
2.876006

1.520001

2.486005

$1.520=01$
$1.520=01$

2.4860 .05

1.520001

2.486005

$.255=09$

$1.520-01$

2.533006
1.520 .01

2.687 .05

1.520001
2.833006

1.520001

1,480001
1,400001

1.480001

.430006

1.480001

0.122003

$5.880=01$

5.483002

$9.109-111$

0,074003

2,003001

$5.336-02$

$2.680=01$

9.1129001

1.708005
8.964000

$1.489=04$

2:005-04
MS FRESH U-235 \&.E.

8.805001

25KS RECYCLE U-235 \&.E. FERTILE PIL. FISSILE PIL.

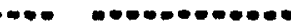

$1.587=04$

1.000005

1.696004

1.035004
7.016002

1.218002

$3.353-02$

1.220001

2.828000

$2.633=06$

5.061006

1.520001

$1.520=0$.

$1.520=01$

2.833006

1.520001

9.122003

2.80000

$5.483=02$

$9.109=01$

$1.263+00$
1.988009

1.303010

$2.124=09$

1.720000

5.163005

8.247006

$0.205=05$

\subsection{9}

101009

1.835008

$1.031=04$

1.031004

1.031 .04

9.181 .09

$1.031=04$

$0.184=00$ 1.952004
3.717 .05 0.175004 8.501004 


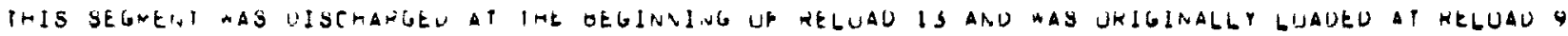

NUCLIUE ANU GAMMA ENEKGY IN MEV

THALLIUM

0.3
0.0
0.8

0.8

TUTAL

$T B=158$

$18=150$
0.3
0.8
1.0

$1: 0$

10 T.

TERBIUM

0.3

0.8

1.25

TUTAL

$E U-152$

0.1

0.3

0.0

1.0

1.25

IUTAL

EL. 154

0.1

0.0

1.25

IUít

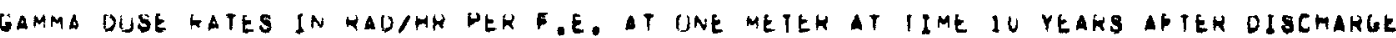

MS FKESH U-235 P.E. FEKTILE PIL. FISSILE PIL.

9.122003

$3.880-61$

$3.463-02$

$1.203+06$

1.491 .00

4.708005

1.489 .04

2,005004

$0.201-10$

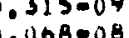

1.081 .09

4.790 .08

$0.425=10$
.215009

7.215009

$0,494.09$

$0.201=10$

$1.100-10$

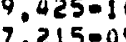

2.271010

$8.494=09$

$9.946-04$

2.637 .03

$4.307-04$

2.733003

.858 .03

.154 .03

2.252 .04

2.100 .02

2.880001

$1.251-01$
$3.290-01$

3.290001

$1.791+0 r_{1}$

1.697400
2.609400

$2.014=01$

$7.040+0 \mathrm{v}$

1.010002

3.44000

6.131 .01

$3.532=01$

9.290001
$5.055+00$

$4.792+00$

$7.365+00$

$5.687=01$
$1.988+01$
23KS RECYCLE U-235 F.E. FEKIILE PTL. FISSILE PIL. .000000-0.

9.122 .03

0.070003

2.803001

0.865001

$5.483=02$

$9.109-01$

$1.205+00$

$1.229+00$

$5,315=09$

4.068008

$4.790-08$

0.092009

$1.0,13000$
$0.123=06$

0.032000

$6.201-10$

1.06000

4.790008

$7.800-10$

$0.692=09$

5.123006

1.613009

$0.032=08$

$9.946=04$
$2,637.03$

4.307 .04

2.733003

7.858 .03

0.184 .03

2.252004

2.100002

1.106003

$2.933=03$

3. 040003

$8.741=03$

0.8780 .03

0.505004

$2.343=02$

$2.880-01$ 1.251001

$3.29 v-U 1$
$1.791+00$

$1.791+00$

$1.097+00$
$2.009+00$

2.014001

$7.040+00$

5.393001
2.343001
$0.102=01$
$3.353+00$
$3.179+00$
$4.885+00$
$3.772=01$
$1.318+01$

$1,318+01$
25KS NECYCLE U-235 F.E.

FERTILE PTL. FISSILE PIL.

$9.122=03$

2.880 .01

5.483 .02

9.109001

$1.263+00$

1.952000

8.501004

$0.201=10 \quad 1.223=10$

5.315 .09

4.068008

4.790000

.048009

0.023000

2.520010

9.44600 ?

\section{$0.201-10$ \\ 4.00800 \\ $1.281=08$ \\ $1.790=0$}

$1.223-10$

0.0300

$950-10$

$\begin{array}{ll}9.946004 & 1.218003 \\ 2.057003 & 3.388003 \\ 4.307004 & 5.594004 \\ 2.733003 & 3.512003 \\ 7.858003 & 1.010002 \\ 0.184003 & 7.940005 \\ 2.252004 & 2.894004 \\ 2.106002 & 2.706002\end{array}$

$0.185=01$

2.880001

1.251 .01

3.290 .01

1.791000

$1.697+00$

$2.607-01$

$3.846+00$

3.646000

$2.609+00 \quad 5.603+00$

$2.014001 \quad 4.327-01$

$1.512+01$ 


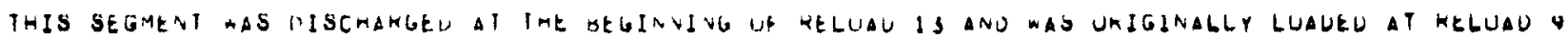

NUCLIDE ANO GAMMA ENERGY IN MEV ENERGY

\section{EUKUPIUM}

0.1

$0: 3$

0.6

0.8

1.00

1.6

TUTAL

$P R=144$

0.6

1.5

1.6

2.0

完

RABEUDYMIUM

0.6

1.25

1.6

TUTAL

$B A-137 M$

0.1

TUTAL

GAKJUM

OAKIUM
0.1
0.0

TUTAL

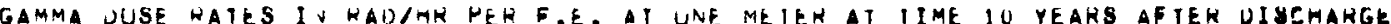

MS FRESH U-235 FOE.

23HS RECYCLE U-23S F.E. FERIILE PIL. FISSILE PTL

FERTILE PTL. FISSILE PTL.

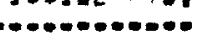

TSKS RECYCLE UAZ3S P.E FERIILE PIL HISSILE PIL

rISSILE OIL.

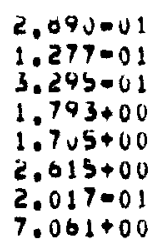

$8.147=01$

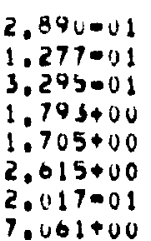

3.404001

$2.372=01$

0.106001

$3.356+00$

$3.187+00$

$4.892+00$

$3.775=0$.

$1,321+01$

2.890001

1.277001

3.295001

0.148001

3.650400

$7.375+00$

$5.091=01$
$1.991+01$

$7.061+00$

$7.061+00$

4.350001
$1.515+01$

\section{$1.017=02$}

$1.900-04$

3.009 .03

1.254002

9.060003

1.098 .04

7.876005

$3.214=03$

$1.111=02$

1.017002

1.245003

$1.357 \cdot 04$

0.2980 .05

8.842 .05

3.009003

1.250002

1.894002

.906000

8.842 .05

3.609003

2.060002

$0.00 \dot{B}=03$
$1.073=00$

2.411 .01

8.376005

$1.017-02$

$9.060-03$

1.098004

$3.21=0$

2.369002

$1.017-02$

$8.906=04$

3.609003

2.060 .02

7.245003

1.357 .04

0.2980 .05

$2.570=03$

8.929003

$1.250=0$

1.894002

1.017002

1.906004
$8.802-05$

3.600 03

$3.659-03$

$1.250-02$

$0.79000 s$

$1.273=04$

$2.411=03$
8.376003

$1.777-02$

$1.069+00$
$.822+02$

$1.659+00$

$4.379+02$

$1.069+00$

$1.557+00$

$4.110+02$

$4.125+02$

$1.069+00$

$2.822+02$

$2.833+02$

$1.059+00$

$1.009+00$

$2.822+02$
$2.833 \div 02$

$4.379+02$

$4.390+02$

$1.069+00$

2.822402

$2.833+42$

$1.557+00$

$4.110+02$
$4.125+02$

$1.009+0$

$2.822+02$
$2.833+02$
$1.218+00$

$3.270+0$

$1.258+00$
$3.267+02$

$3.267+02$
$3.279+02$ 


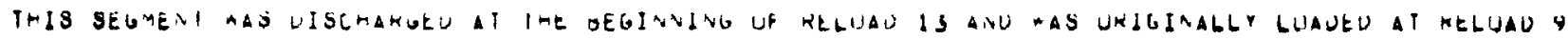

NUCLLIDE AND IN MEY

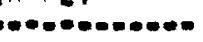

$C 8=134$
0.3
0.6
0.8
1.0
1.25
1.0
$10 T a 6$

CE\&IUM

0.3
0.3
0.6
0.8
1.0
1.25
1.6
10146

N
N GAMNA UUSE RATES IA RAUIHH PEK F.E. AT UNE MEIER AT IIME IO TEAKS AFIER DISCMAKGE GAMMA ENERG

US ERESH U-23S F.E. FEKIILE PTL. FISSILE PIL.

$2.003=03$ $1.981+01$ $2.050+01$ $2.808-01$ $1.711+00$ $9.467-03$ $4.238+01$

$2.083-03$ $1.901+01$ $2.1156+01$ $2.80,8=01$ $1.711+06$

$9.467-05$

$4.238+01$

1.003001
1.000000 $1.000+00$
$3.020+00$ $3.135+00$

$7.275-03$
$5.373+01$
$5,575+01$
$7.613-01$
$4.039+00$
$2,507-02$
$1.149+02$

$7,275-03$ $5.373+01$ $5.575+01$ 7.013001 $4.639+00$ $3.567-02$ 2.569002

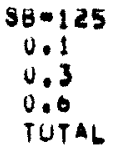

ANIIMONY

0.1

TUTAL

$1.003=01$ $1.006+00$

$46-106 \mathrm{M}$

0.1

0.3

0.6

1.0

IUTAL

3.475 .02

$3.297=01$

$1.025+00$

3.4750 .02

\section{$3.297-01$}

$0.607-01$
$1.025+00$

1.057000
$8.595=05$

$1.282-1.4$

$1.452=04$

$1.945=00$

3.019 .04 $2.020+00$
$3.135+00$

23RS KECYCLE U-23S F.E. FERTILE PTL, FISBILE PTL.

\section{$2.083-03$}

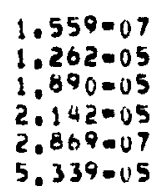

$5,339-05$

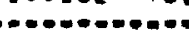

$1.981+01$

$2.056+01$

$2,808-01$

$1.711+00$

$9.467-03$

$4.238+01$

$2.085=03$

$1.981+01$

$2.056+01$

2.808001

$1.711+00$

$9.467-03$

$4.238+01$

$1.063-01$

1. $0008+00$

$3.135+00$

1.063001
$1.008+00$
$2.020+00$
$3.135+00$

5.710003
$4.217+01$
$4.370+01$
3.976001
$3.041+00$
2.015002
$9.010+01$

5.710003

$4.217+01$

5,976001

5,976001

3.041500

2.015002

$1.112-01$

$1.055+00$

$3.260+00$

$1.112=01$

$1.055+00$

$2.110+00$
$3,280+00$

$1.057-06$

$8.555-05$

$1,282=04$

\subsection{7} 1.870005 3.174005 4.252007 $7,910-05$
$25 \mathrm{kS}$ RECYCLE U-235 F.E. FERTILE PTL. FISSILEP IL

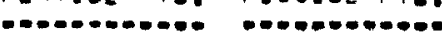

\section{$2.603=09$ $1.981+01$ \\ $2.050+01$ \\ 2.806001 \\ 9.407 .03}

$3.424=05$

$4.238+01$

4.156001 5.677001

$3.459+00$

1.914002

0.508001

$\begin{array}{ll}2.083003 & 5.424003 \\ 1.981+01 & 4.000001 \\ 2.056+01 & 4.156001 \\ 2.008001 & 5.617001 \\ 1.911+00 & 3.459000 \\ 9.467 .03 & 1.914002 \\ 4.230+01 & 8.508001\end{array}$

$4.238+01$

8.568001

1.003001

1.008400

$3.135+00$

2.5320 .02 2.422001 .053001 .520 .01

$1.003=01$

$1.008+00$

$2.020+00$
$3.135+00$

2.552 .02
2.422 .01

4.853001

7.529001

$1.052=00$

$3.019=04$
1.057000

8.555005

$1.262=04$

1.452004

$3.019=04$
4.813007 7.162005
1.076004 1.219004 1.653006 3.059.04 


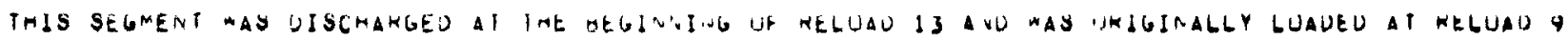

NUGLIDE AND AAMA ENERGY IN MEV

\section{$16 \cdot 110 \mathrm{M}$}

0.1

0.3

0.6

$1: 0$

1.6

2.0

$2: 4$

ILVER

0.11

0.0

1.025

1.6

2.0

TUTAL

$R H=106$
0.3
0.0
0.8
1.0
1.25
1.0
2.0
2.0
2.9
TUTAL

GAMMA OUSE RATES IN RADIMK HEM F

MS FRESH U. 235 F.E. FERIILE PIL. FISSILE PIL.

$9.042=09$
$8.112=07$
$3.983-05$
$5.306-05$
$1.575=05$
$1.738-05$
$1.328=05$
$8.354=08$
$8.643=08$
$1.403-04$

1.007 .06

0.036 .05

1.080 .04

$.983-64$

1.770005

$1.738=05$

.328 .05

0.354 .08

0.043008

2.224004

$0.452-02$

1.411 .03

$.695=03$

.095003

$4.825-04$

$4.835=04$

7.920005

$1.351=08$
1.136000
$5.579-05$
$1.432-05$
$2.216-05$
2.435005
$1.860-05$
1.170007
$1.211-07$
1.965 .04

$1.965-04$

$1.094-07$
$1.375-05$
$7.409-05$
$9.574-05$
$2.235-05$
$2.435-05$
$1.800-05$
$1.170-07$
1.211007
$2.499-04$

2.210907

2.976004

8.633002

$1.886-03$

7.020003

$2.536=03$

1.750003

6.456004

$0.452-04$

$1.000=04$

$1.018-01$
23RS RECYCLE UEZJS F.E

EERTILE PTL. FISSILE PIL.

F.

9.042009

8.112007

$3.963-05$

5,306005

1.575005

1.738 .05

$1.328=05$

8.3540 .08

8.043006

1.403004

$1.007-06$

0.636005

1.080004

1.963 .04

1.770005

1.738005

$.328-05$

0.04008

8.645008
5.422 .04

2.224004

$0.452-02$

$1.011-03$

$5.695=03$

1.895005

1,308003

4.825004

4.823006

$7.920-05$
1.034008

$1.207-06$

5.924005

$2: 343005$

2.585005

1.975005

1.243007

1.286007

$2.087-04$

2.453007

1.990005
8.7250 .05

1.107 .04

$3,385=05$

2.585005

1.975005

1,243007

1.280007

1.280007
3.878004

5.659002

1.236003

$4.995-03$

$1.602-03$

$1.147-03$

4.232004

4.230004

0.946005
ZSRS RECYCLE UACZS F.E.

FERIILE PIL. FISSILE PTL.

$\begin{array}{ll}9.642009 & 1.014000 \\ 8.112007 & 0.534007 \\ 3.963005 & 4.190005 \\ 5.306005 & 5.542005 \\ 1.575005 & 1.657005 \\ 1.736005 & 1.829005 \\ 1.328005 & 1.397005 \\ 8.354008 & 8.768000 \\ 8.643000 & 9.093008 \\ 1.403004 & 1.470004\end{array}$

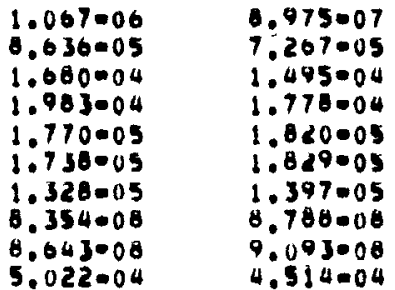

5.022004

4.514 .04

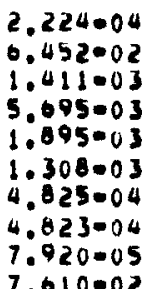

2.252 .04

0.475002

1.416003

5.716003

$1.902 \circ 03$

. $313=03$

4.843004

4.640004

1.948 .05

$7.037=02$ 


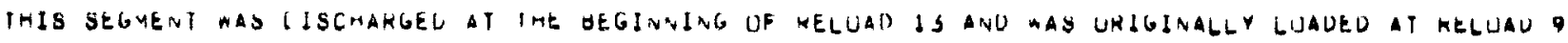

NUCLIOE AND GAMMA ENERGY IN MEV

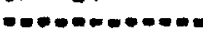

\section{RHUDIUM}

$240010 M$
0.3
0.0
0.8
1.0
1.25
1.6
2.0
2.4
2.0

60.60
1.25

TUTAL

CUGALT
TOZSE
TUTAL

ALL ELEMENTS

0.1
0.3
0.6
0.8
1.0
1.25
1.0
2.0
2.4
200
TUTAL

GAMMA DUSE KATES IN RADIHK PER F.E. AT UVE METEH I IIME IU YEARS GFTEK UISCHARGE

MS FRESM U-235 E.E. FENTILE PTL. FISSILE PTL. COATILE PTL. FIOSILE PTL

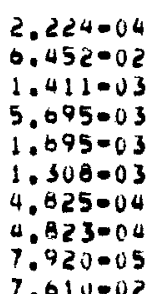

$7.010=02$
0.900

$1.759-04$
1.759 .04

1.759004

1.7590 .04

$1.178=05$
$1.178-05$

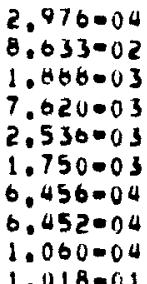

1.018 .01

$1.178=05$

1.178 .05

$1.404+00$

$1.300+00$

$3.047+02$
c. $249+01$

2. $249+01$
$2.004+00$

$4.328+00$

2.490 .01

1.303 .02

9.114001

$7.92 u=05$
$3.375+02$

$2.509+00$

6.949001

$4.933+02$

$0.081+01$

$5,574+00$

$1,202+01$

5,996001

1.181 .02

7.943004

$5.060=04$
23RS RECYCLE UNZZS FE,

FERTILE PTL. FISSILE PTL.

-......

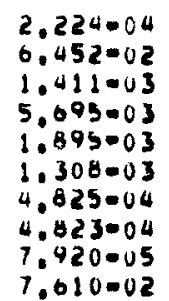

1.051004

5.059002

1.258003

$4.995-0 \hat{3}$

1.062003

4.23200

$4.230=04$

$6.940=05$

0.074002

1.759 .04

1.746005
$1.740-05$

1.7460 .05

1.740005

$1.759=00$
1.759 .04
$1.464+00$

$1.300+00$

$3.047+02$

$2.249+01$
$2.004+00$

$4.328+00$

$2.490=01$

1.303 .02

9.114001

3. $375+02$
$2.209+00$

$1.455+00$
$4.562+02$

$4.562+02$
4.724 .01

3.802400

$8.535+00$

4.340001
0.352003

0.86900

$0.946-05$

$5.208+002$
ZSKS RECYCLE U-235 F.E. FEHTILE PTL. FISSILE PTL.

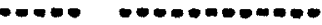

$\begin{array}{ll}2.224004 & 2.252004 \\ 0.052002 & 6.475=02 \\ 1.411003 & 1.410003 \\ 5.005003 & 5.710005 \\ 1.095005 & 1.902005 \\ 1.308003 & 1.315003 \\ 4.825004 & 4.843004 \\ 4.623004 & 4.840004 \\ 7.920005 & 7.948005 \\ 1.010002 & 7.637002\end{array}$

$1.739=04$

0.700005

1.759004

\section{$0.708 \cdot 05$}

0.700005

$1.464+00$
$1.300+00$
$3.047+02$
$2.249+01$
$2.004+00$
$4.328+00$
2.496001
1.303002
9.114001
7.920005
$3.375+02$

$1.863+00$

5.201 .01

$3.000 \bullet 02$

$4.542+01$

$4.229+00$

$0.072+00$

4.559001
8.860003

1.102003

7.9080 .05

$4.290+02$ 
NEUTRON SOURCE STRENGTHS 

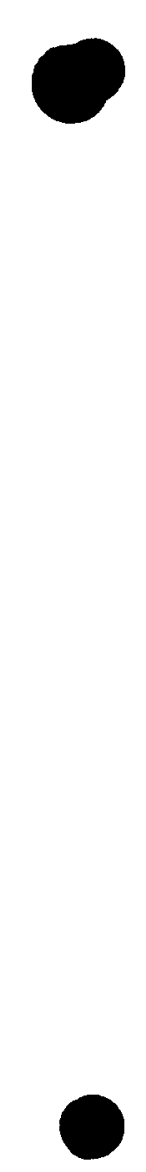

0 
TABLE D-9

NEUTRON SOURCE STRENGTHS FOR FUEL ELEMENTS DISCHARGED AT BEGINNING OF RELOAD 1 AND ORIGINALLY LOADED AT RELOAD 0

\author{
and \\ TABLE D-10 \\ NEUTRON SOURCE STRENGTHS FOR FUEL ELEMENTS DISCHARGED AT BEGINNING \\ OF RELOAD 5 AND ORIGINALLY LOADED AT RELOAD 1
}

NOTE: Tables $D-9$ and $D-10$ consist of 10 pages of computer print-out, with the two tables appearing side by side (pages D-279 through D-288). 
0

-

8 
THIS SEGOENT MAS IISCMARGED AT

THE GEGIANING UF RELUAD I ANU MAS

URIGINALGY LOADED AT RELUAD O

NEUTROA SUUHCE IN N/BEC RER B. AT TIME IOU DAYS AFTER DISCMARGE
IHIS SEGMENT MAS UISEAARGED AT IHE HEGINN ING UF RELUAU S ANO WAS URJGINALLY LUADED AI RELUAD I

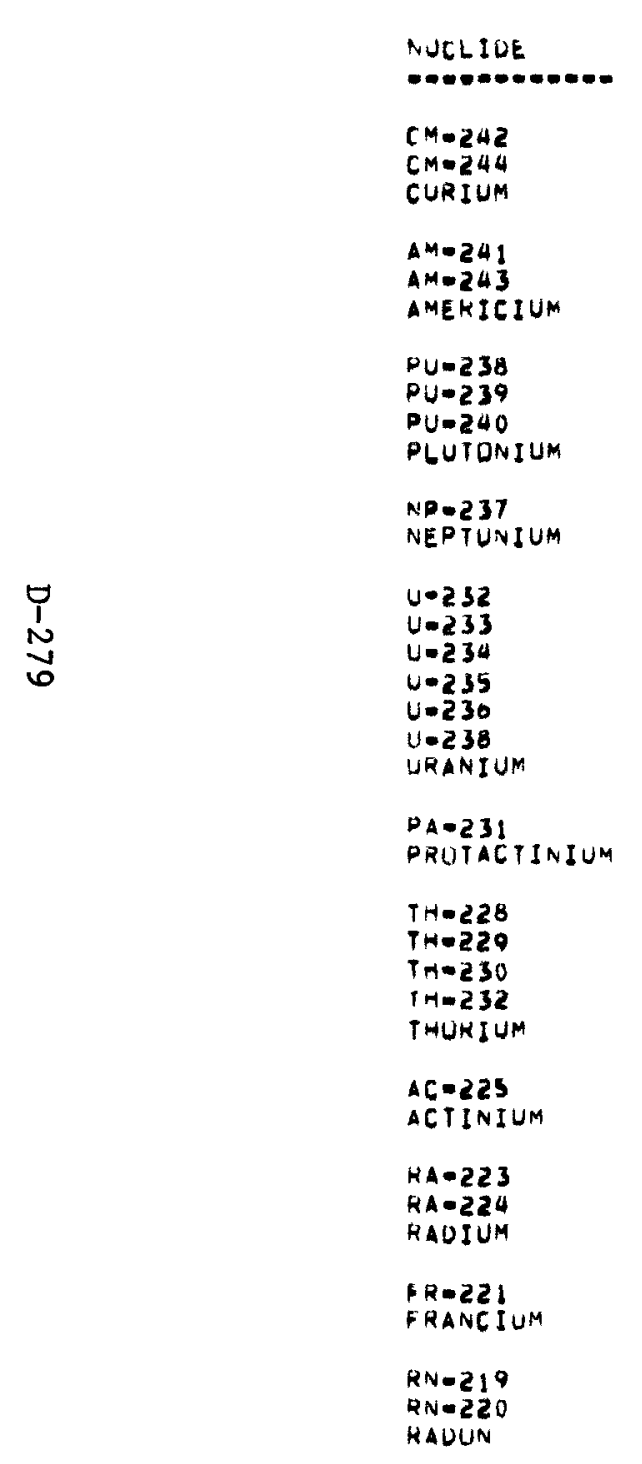

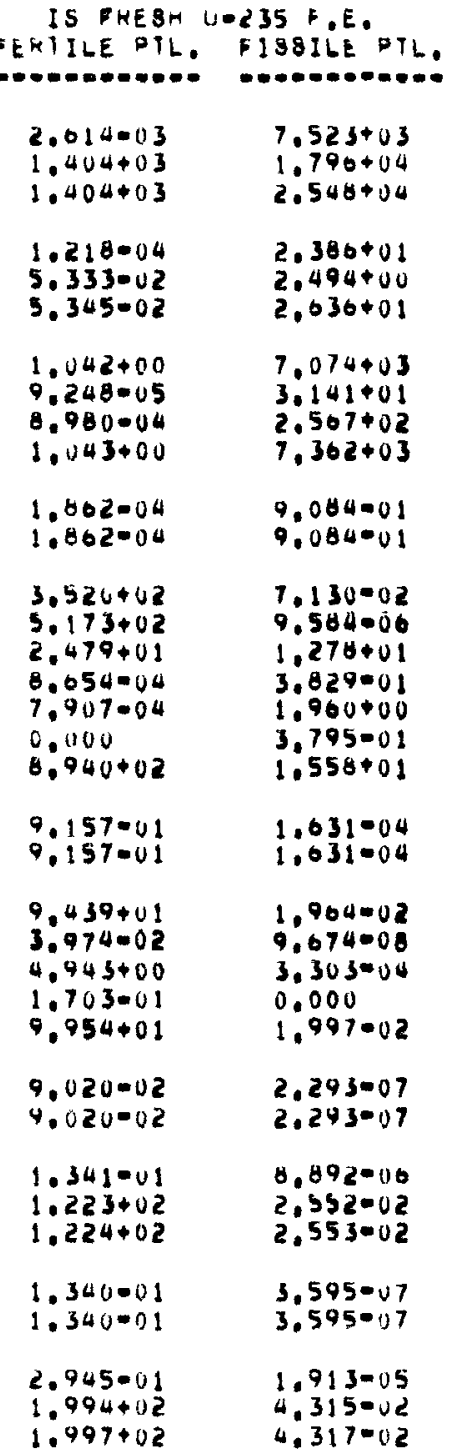

MS FHESH UNC3S F.E. FERTILE PIL. FIS8ILE PTL.

\begin{tabular}{|c|c|}
\hline $\begin{array}{l}3.042+01 \\
7.225+02 \\
7.009+02\end{array}$ & $\begin{array}{l}1.461+05 \\
1.137+06 \\
1.283+06\end{array}$ \\
\hline $\begin{array}{l}4.2710 .02 \\
5,344003 \\
4.806002\end{array}$ & $\begin{array}{l}1.203+02 \\
4.656+01 \\
1.668+02\end{array}$ \\
\hline $\begin{array}{l}4.309+02 \\
8.239+02 \\
8.732001 \\
4.519+02\end{array}$ & $\begin{array}{l}1.807+05 \\
9.072+01 \\
7.808+02 \\
1.816+05\end{array}$ \\
\hline $\begin{array}{l}5.109-02 \\
3.109002\end{array}$ & $\begin{array}{l}6.600+00 \\
6.600+00\end{array}$ \\
\hline $\begin{array}{l}1.067+03 \\
8.077+02 \\
1.124+02 \\
1.091-02 \\
4.001002 \\
0.000 \\
2.587+03\end{array}$ & $\begin{array}{l}5.140=01 \\
1.959-05 \\
1.092+01 \\
0.748002 \\
4.005+00 \\
5.067-01 \\
1.684+01\end{array}$ \\
\hline $\begin{array}{l}1.287+00 \\
1.281+00\end{array}$ & $\begin{array}{l}3.548004 \\
3.548004\end{array}$ \\
\hline $\begin{array}{l}7.980+02 \\
1.245=01 \\
2.023+00 \\
1.446001 \\
8.003+02\end{array}$ & $\begin{array}{l}2.567=01 \\
5.937000 \\
4.076004 \\
0.000 \\
2.572002\end{array}$ \\
\hline $\begin{array}{l}2.820001 \\
2.620001\end{array}$ & $\begin{array}{l}1.407=05 \\
1.407=05\end{array}$ \\
\hline $\begin{array}{l}2.122001 \\
1.034+03 \\
1.034+03\end{array}$ & $\begin{array}{l}3.025=05 \\
3.335001 \\
3.336001\end{array}$ \\
\hline $\begin{array}{l}4.196001 \\
4.196001\end{array}$ & $\begin{array}{l}2.200=05 \\
2.200005\end{array}$ \\
\hline $\begin{array}{l}4.059001 \\
1.680+03 \\
1.080+03\end{array}$ & $\begin{array}{l}0.508005 \\
5.640001 \\
5.641001\end{array}$ \\
\hline
\end{tabular}


THIS SELKENT WAS UISCHARGED AT

THE DEGINNING UF KELUAU I ANO WAS

UKIGINALLY LUAUEO AT RELUAD O

nEUTKUA SUURCE IN N/SEC PEM F.E. AT IIME IOU DAYS AFTKR DISCMATGE
IMIS SEGMENT WAS DISCMARGEU AT

INE GEGINNDING OF KELLUAD 5 ANO HAS

URIGINALLY LOAUED AI HELUAD I

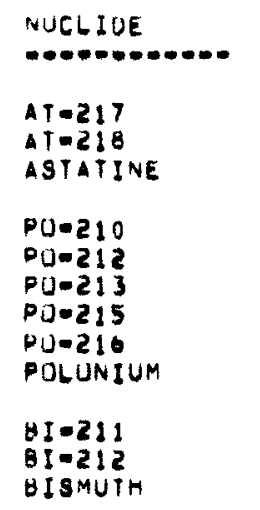

ALL ELEMENTS
19 FRESH U- 235 F.E.

FERTILE PTL. FISSILE PIL.

2.393.01

7.840060

5.606007

$2.390-01$

0.600

$4.256-01$

$5.957+02$

$2.394-11$

0.767 .01

$2.885+02$

$8.897-05$

$9.634=02$

1.194000

$3.007=05$

1,354001

$2.741=01$

$0.120+01$

$1.778-05$
1.276002

1.270005
1.276002
1.2780 .02

$3.670+03$

3,288404

MS FKESH UEd35 P.E. FERTILE PTL, PISSILE PIL.

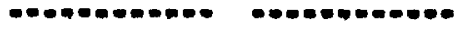
1.498001
0.090000 $\begin{array}{ll}0.898006 & 3.000 \\ 7.498001 & 3.613 .05\end{array}$

\section{$4.057001 \quad 1.030004$}

$5.288+03 \quad 1.322+00$ $1.690+00 \quad 7.329=05$ $7.530403 \quad 1.025004$ $\begin{array}{ll}7.730+03 & 7.7093+00\end{array}$

$\begin{array}{ll}4.336001 & 6.050005 \\ 5.182+02 & 1.668001\end{array}$
$\begin{array}{ll}5.182+02 & 1.668001 \\ 5.160+02 & 1.068001\end{array}$

$1.557+04 \quad 1.465+06$ 
IMIS SEgmeAT MAS DISCHARGEO AT URIGINALLY LUADED AI RELUAD O

REUTRUR SIJURCE IN N/SEC PEN F.E. AT TIME

IMIS SEGMENI WAS ISISCHANGED AT

ITE OEGINAING UF KELUAO S ANU WAS

URIGINALLY LUAUED AT KELOAD

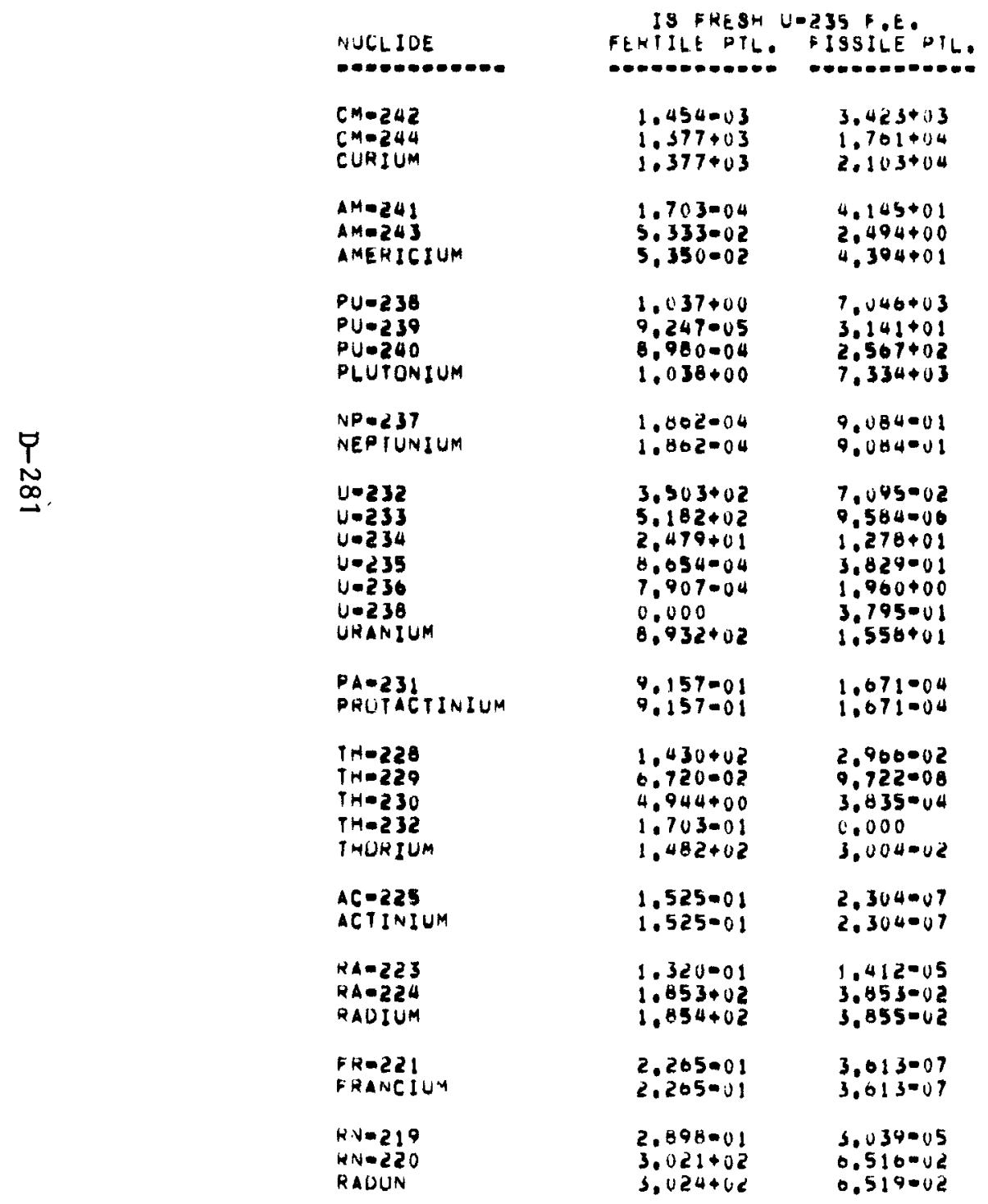
MS FHE $8 H$ UAZ35 F.E. FERIILE PTL. FISSILENTL. O.OOO-

$1.751+01 \quad 0.047+04$ $\begin{array}{ll}7.084002 & 1.113006 \\ 7.250602 & 1.181+06\end{array}$

$7.258002 \quad 1.905+02$ $\begin{array}{ll}5.344003 & 4.656+01 \\ 7.792002 & 2.371002\end{array}$

$4.491+02 \quad 1.799+05$ $8.239002 \quad 9.071001$ $0.731001 \quad 9.068+02$

$3.109002 \quad 0.600400$ $3.104002 \quad 0,000000$

$1.059+03 \quad 5.115001$

$8.085+02 \quad 1.950-05$

$1.124402 \quad 1.092+01$

$1.091 .02 \quad 8.748 .02$

$4.001002 \quad 4.005+00$

$\begin{array}{ll}0.000 & 5.067 .01 \\ 2.580003 & 1.683+01\end{array}$

$1.287+00 \quad 3.557004$

$9.092402 \quad 3.090001$

$1.673001 \quad 5.938006$

$2.023400 \quad 5.131 .04$

$\begin{array}{ll}1.446001 & 0.000 \\ 9.110002 & 3.201 .01\end{array}$

$3.798 .01 \quad 1.407 .05$

$3.798001 \quad 1.407005$

$2.088001 \quad 4.136005$

$\begin{array}{ll}1.256+03 & 4.022001 \\ 1.256+03 & 4.022001\end{array}$

$5.041001 \quad 2.200009$

$4.584001 \quad 8.099005$

$2.048+03 \quad 6.801=01$

$2.048+03 \quad 6.802001$ 
IHIS SEGMERT HAS DISCHARUED AT

TME GEGIINNING JF RELIJAU I AVD NAS

UHIGIN

NEUIRUN SLUKCE IN NASEC PER F.E. AI TIME

HIS SEGMEINT MAS UISCHARGEO AT

WE GEGLINNING UT KELUAD 5 AND MAS

A KELUAD 1

I YEAK AFTER UISCHARGE

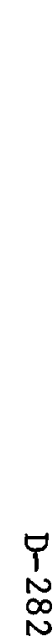

NUCLIUE

$4 T-217$
$A-216$

ASTATINE

Puez10

PU. 212

Pu- 213

PUण 215

$P U-216$

POLUNIUM

BI-21!

$81-212$

BI BMUTH

ALL ELEMENTS
FEHIS FKESH UEZ35 F.E.

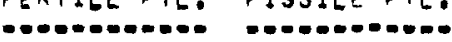

$4.047-01$

8.901006

$4.947-01$

$5.917 \cdot 07$

5.060

$1.697=01$

$9.698+02$

$9.121=01$

4.091001

$4.370+02$

$4.031=05$

1.564001

$1.200=00$

$4.777-05$

$8.897-02$

2.454001

$2.097=01$

$9.265+01$

$2.825=05$

$9,312+01$

$1.927=1.12$
$1.929=112$

$4.411+03$

$2.843+04$

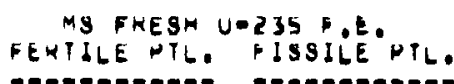

$1.008400 \quad 1.014005$

$\begin{array}{ll}1.353056 & 0.000 \\ 1.008+00 & 3.614005\end{array}$

$1.914001 \quad 8.542005$

$6.003+03 \quad 1.640+00$

$2.271000 \quad 7.330005$

$7.421 .01 \quad 1.399=04$

$2.902+03 \quad 9.287001$

$9.571+03 \quad 2.569+00$

$0.200001 \quad 8.271=05$

$0.293+02 \quad 2.011-0$

$0.299+02 \quad 2.022001$

$1.624+04 \quad 1.362+00$ 


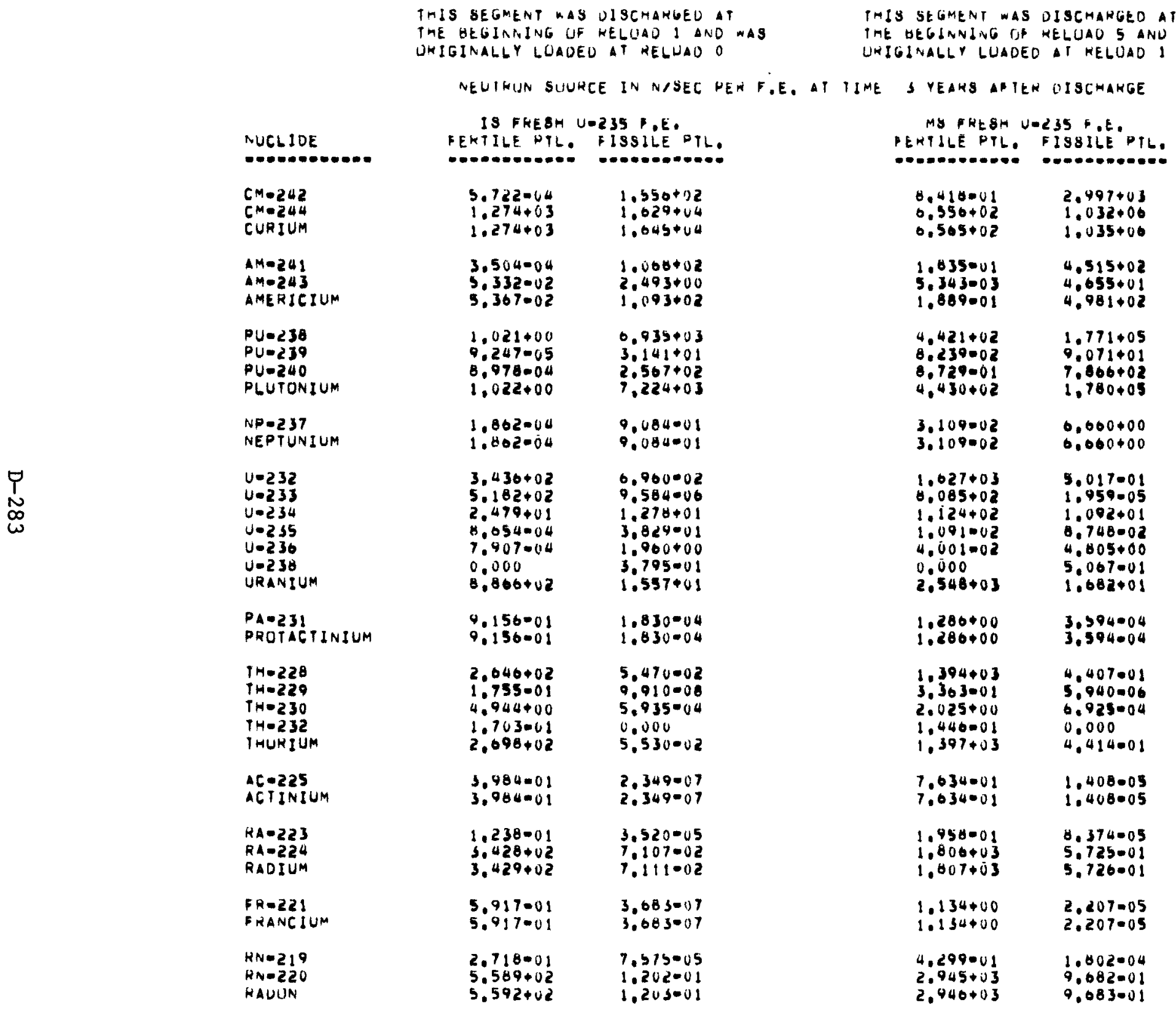


THIS SEGMENT WAS OI GCMARGED AT

THE HEGIUNING OF RELGAD I ANU AAS

HKIGINALLY LUADED AT RELUAU O

NEUTHUN SUURCE IN N/SEC PER F.E. AT TIME

N:JCLIUE

NOCLIUE

4 AT-217

AT 19218

$P 0=210$

PO- 212

$P 0-213$

PO- 215

PU=510

POLUNIUM

$B]-211$

$B I-215$

BISMUTH

ALL ELEMENTS

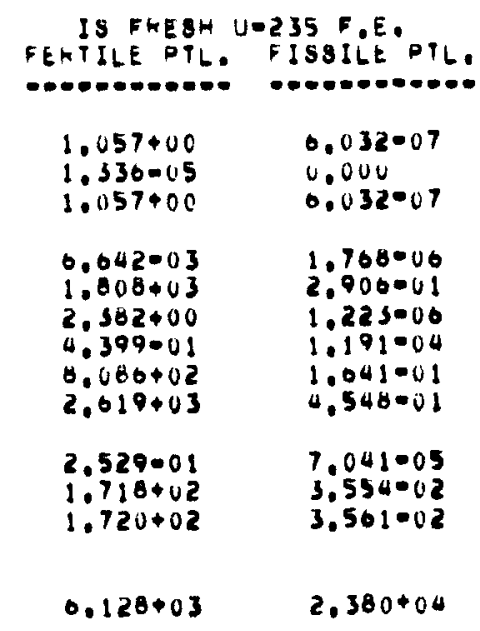

PHIS SEGMENT HAS UISCMAMGED AT

IME GEGIVNING OF RELUAU 5 ANU WAS

URIGINALLY LUAUED AT RELUAOO

MS FRESH UD 355 F. FERTILE PIL. FISSILE PIL.

$\begin{array}{ll}2.020+00 & 3.010005 \\ 9.144000 & 0.000 \\ 2.026000 & 3.610005 \\ 1.016002 & 8.077000 \\ 9.535+03 & 2.343+00 \\ 4.565+00 & 1.334005 \\ 0.900-01 & 2.832004 \\ 4.261+03 & 1.322+00 \\ 1.360+04 & 5.065+00 \\ 4.001001 & 1.075004 \\ 9.052402 & 2.863001 \\ 9.056+02 & 2.064001 \\ & \\ 2.451+04 & 1.213+06\end{array}$


THIS SEGMENT WAS UISCHAKGEU AT TME GEGINNING OF RELUAD I ANO WAS

THIS SEGMENT NAB UISCHAKGED AT UKIGINALLY LGADED II RELUAO O

BEGINNING OF RELUAD 5 ANO WAS

NEUTHUN SUURCE IN N/SEL PER F,E. AT IIME 5 YEAKS ABTER UIBCMARGE

\begin{tabular}{|c|c|c|}
\hline $\begin{array}{l}C M=242 \\
C M=244 \\
\text { CURIUM }\end{array}$ & $\begin{array}{l}5.071=04 \\
1.179+03 \\
1.179+03\end{array}$ & $\begin{array}{l}9.371+00 \\
1.500+04 \\
1.509+04\end{array}$ \\
\hline $\begin{array}{l}\triangle M-24 I \\
A M=2 A 3 \\
A M E R I C I U M\end{array}$ & $\begin{array}{l}5,140=04 \\
5,331=02 \\
5,382=02\end{array}$ & $\begin{array}{l}1.661+02 \\
2.493+00 \\
1.086+02\end{array}$ \\
\hline $\begin{array}{l}P U=238 \\
P U=239 \\
P U=240 \\
P L U T D N I U M\end{array}$ & $\begin{array}{l}1.005+00 \\
9.246-05 \\
8.970-04 \\
1.000+00\end{array}$ & $\begin{array}{l}0.827+03 \\
3.141+01 \\
2.560+02 \\
7.115+03\end{array}$ \\
\hline $\begin{array}{l}\text { NPE-237 } \\
\text { NEPTUNIUM }\end{array}$ & $\begin{array}{l}1.862=04 \\
1.862=04\end{array}$ & $\begin{array}{l}9,004=01 \\
9,004=01\end{array}$ \\
\hline $\begin{array}{l}U-232 \\
U=233 \\
U=234 \\
U-235 \\
U-236 \\
U-238 \\
\text { URANIUM }\end{array}$ & $\begin{array}{l}3.370+02 \\
5.182+02 \\
2.479+02 \\
0.054=04 \\
7.907-04 \\
0.000 \\
0.800+02\end{array}$ & $\begin{array}{l}0.827-02 \\
9.584-06 \\
1.278+01 \\
3.829001 \\
1.960+00 \\
3.795-01 \\
1.557+01\end{array}$ \\
\hline $\begin{array}{l}\text { PA- } 231 \\
\text { PRUTACIINIUM }\end{array}$ & $\begin{array}{l}9.156001 \\
9.156001\end{array}$ & $\begin{array}{l}1.989000 \\
1.989004\end{array}$ \\
\hline $\begin{array}{l}T H=228 \\
T H=229 \\
T H=230 \\
T H=232 \\
T H O K J U M\end{array}$ & $\begin{array}{l}3.197+02 \\
2.838001 \\
4.944+00 \\
1.703001 \\
5.251+02\end{array}$ & $\begin{array}{l}0.005-02 \\
1.010008 \\
6.035004 \\
0.000 \\
0.680-02\end{array}$ \\
\hline $\begin{array}{l}A C=225 \\
A C T I N I U M\end{array}$ & $\begin{array}{l}0.442=01 \\
0.442=01\end{array}$ & $\begin{array}{l}2.394007 \\
2,394007\end{array}$ \\
\hline $\begin{array}{l}R A-223 \\
R A-224 \\
\text { RADIUM }\end{array}$ & $\begin{array}{l}1.101-01 \\
4.142+02 \\
4.143+02\end{array}$ & $\begin{array}{l}5.697=05 \\
8.582-02 \\
8.588=02\end{array}$ \\
\hline $\begin{array}{l}\text { FR-22I } \\
\text { FRANCIUM }\end{array}$ & $\begin{array}{l}9.507=01 \\
9.507=01\end{array}$ & $\begin{array}{l}3.7550 .07 \\
5.7530 .07\end{array}$ \\
\hline $\begin{array}{l}R N=219 \\
R N=220 \\
R A D U N\end{array}$ & $\begin{array}{l}2.549001 \\
0.753+02 \\
0.750+02\end{array}$ & $\begin{array}{l}1.220=04 \\
1.451=01 \\
1.452-01\end{array}$ \\
\hline
\end{tabular}

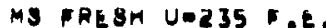
FERT ILE PTL. PISIILE PTL

\begin{tabular}{|c|c|}
\hline $\begin{array}{l}0.530002 \\
0.068+02 \\
0.069+02\end{array}$ & $\begin{array}{l}1.568+02 \\
9.549+05 \\
0.551+05\end{array}$ \\
\hline $\begin{array}{l}2.843001 \\
5.342003 \\
2.896001\end{array}$ & $\begin{array}{l}0.085+02 \\
4.054 .01 \\
7.351+02\end{array}$ \\
\hline $\begin{array}{l}4.352+02 \\
8.238002 \\
8.727001 \\
4.361+02\end{array}$ & $\begin{array}{l}1.745+05 \\
9.070+01 \\
7.805+08 \\
1.752+05\end{array}$ \\
\hline $\begin{array}{l}3.109002 \\
3.109002\end{array}$ & $\begin{array}{l}0.660000 \\
0.660000\end{array}$ \\
\hline $\begin{array}{l}1.590+03 \\
8.085+02 \\
1.124+02 \\
1.091002 \\
4.001002 \\
0.000 \\
2.517+03\end{array}$ & $\begin{array}{l}4.921-01 \\
1.959=05 \\
1.092+01 \\
8.748002 \\
4.005+00 \\
5.067=01 \\
1.001+01\end{array}$ \\
\hline $\begin{array}{l}1.286+00 \\
1.206+00\end{array}$ & $\begin{array}{l}3.630000 \\
3.630004\end{array}$ \\
\hline $\begin{array}{l}1.582+03 \\
5.053001 \\
2.027+00 \\
1.446001 \\
1.585+03\end{array}$ & $\begin{array}{l}4.980=01 \\
5.943006 \\
0,719=04 \\
0.000 \\
4.994=01\end{array}$ \\
\hline $\begin{array}{l}1.147+00 \\
1.147+00\end{array}$ & $\begin{array}{l}1.409005 \\
1.409005\end{array}$ \\
\hline $\begin{array}{l}1.030-01 \\
2.050+03 \\
2.050+03\end{array}$ & $\begin{array}{l}1.230004 \\
6.498001 \\
0.479001\end{array}$ \\
\hline $\begin{array}{l}1.703+00 \\
1.703+00\end{array}$ & $\begin{array}{l}2.208=05 \\
2.208=05\end{array}$ \\
\hline $\begin{array}{l}4.032001 \\
3.342+03 \\
3.343+03\end{array}$ & $\begin{array}{l}2.067004 \\
1.095+00 \\
1.090 \$ 00\end{array}$ \\
\hline
\end{tabular}


ITIS SEGMENT WAS OISCHAKGEO AI

THE EEGINNING UF RELUAD I ANU WAS

UHIGINALLY LUAUED T RELUAD O

NEUThUN SUURCE IN N/SEC PER F.E. AI TIME

HE DEGINNING UP HELUAD 5 ANO WAS

URIGINALLY LUAUEO MI KELUAD I

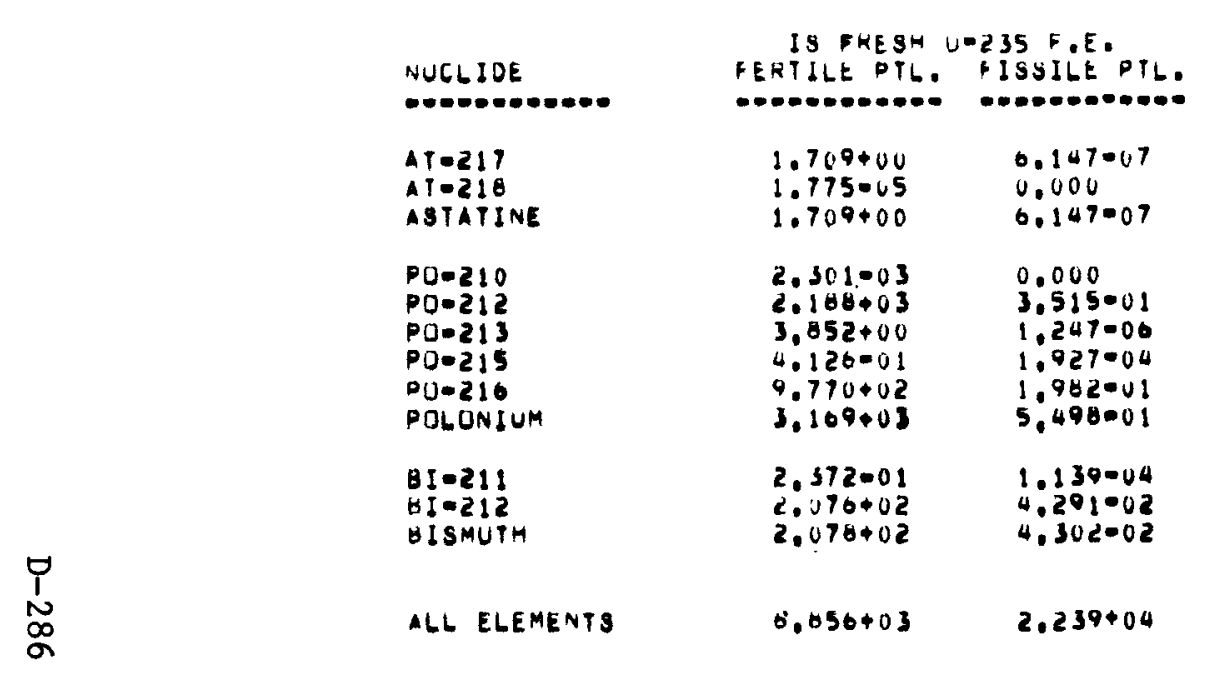

MS GRESH U 235 OR. FERTILE PTL. ISSILE PIL.

$\begin{array}{ll}3.043+00 & 3.017005 \\ 1.004005 & 0.000 \\ 3.043+00 & 3.619005 \\ 1.092002 & 0.000 \\ 1.083+04 & 2.653+00 \\ 6.058+00 & 7.337005 \\ 0.527001 & 4.192004 \\ 4.035+03 & 1.496+00 \\ 1.566+04 & 4.150+00 \\ 3.752001 & 2.478004 \\ 1.027+03 & 3.239001 \\ 1.020+03 & 3.241=01 \\ & \\ 2.725+04 & 1.131+00\end{array}$

$2.725+04 \quad 1.131+06$ 
TMIS SEGMENT WAS DISCHAKGEO AT

THE BEGLNNIVG UF RELUAU I AND WAS

okiginaliy lUaded a r heluado

NEUTRUN SUURCE IN NJGEC PEH F E AT TIME IU YEAHS AETER DISCAARGE
TMIS SEGMENI AAS DISCMAKGED AI THE BEGINNING UF WELUAD S AND WAS

URIGINALLY LUADEL T KELUAO I
NUCLIOE

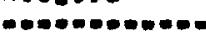

$C M-242$

$C M=244$

CURIUA

AME 241

AMPRI

PU-238

$P U=239$
$P U=240$

PUD240

NP-237

NEPTUNIUM

\[ \]
1
$N$
$N$

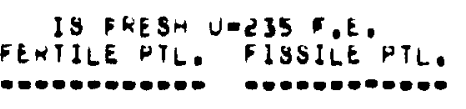

$5.543004 \quad 2.922+00$

$9.717+02 \quad 1.243+04$

$9.717+02$

0.599004

5,328002
5,414002

9.603001

9.245005

$8.971-04$
$9.073-01$

$1.802=04$

$3.212+02$

$5.182+02$

$2.479+01$

8.054004

7.907 .001000

$0.042+02$

0.155001
9.155001

$3.510+02$
5.545001

$4.945+00$

$1.703-01$

$3.567+02$

$1.258+00$

$1.258+00$

9.087 .02

$9.548+02$
$4.549+02$

$1.069+60$

$2.171=0$

$7.416+02$
$7.418+62$
$1.203+04$

$2.910+02$

$2.491+00$

$6.563+03$

$3.141+01$

$2.565+02$
$6.851+03$

9.084001

0.507002

9.563006

$1.278+01$

3.829001

3.960000

$1.557+01$

2.367000

2.387 .04

$7.251=02$
$1.057=07$

1.057007
1.328003
0.0000

1.32800
0.000

7.384002

$2.500=07$
$2.5100=07$

1.141 .04

$9.421=02$

3.920007

$3,928=07$

$2.450-04$

1.593001
1.590001
1,590001

$R N-219$

RAUUN

MS REBH HedzS

PERTILE HTLE FISSILE PTL.

\begin{tabular}{|c|c|}
\hline $\begin{array}{l}7.001-02 \\
4.999+02 \\
5.000+02\end{array}$ & $\begin{array}{l}2.748+01 \\
7.008+05 \\
7.000+05\end{array}$ \\
\hline $\begin{array}{l}0.974001 \\
5,339003 \\
5.020001\end{array}$ & $\begin{array}{l}1.190+03 \\
4.052+01 \\
1.236+03\end{array}$ \\
\hline $\begin{array}{l}4.183+02 \\
0,237=02 \\
0,723=01 \\
4,193+02\end{array}$ & $\begin{array}{l}1.676+05 \\
9.069+01 \\
7.060+02 \\
1.065+05\end{array}$ \\
\hline $\begin{array}{l}3,109002 \\
3.109=02\end{array}$ & $\begin{array}{l}0.660+00 \\
6.660+00\end{array}$ \\
\hline $\begin{array}{l}1.521+03 \\
8.085+02 \\
1.124+02 \\
1.091=02 \\
4.001002 \\
0.000 \\
2.442+03\end{array}$ & $\begin{array}{l}4.690-01 \\
1.959-05 \\
1.092+01 \\
8.748-02 \\
4.005+00 \\
5.067-01 \\
1.079+01\end{array}$ \\
\hline $\begin{array}{l}1.286+00 \\
1.286+00\end{array}$ & $\begin{array}{l}3,720=04 \\
3,720=04\end{array}$ \\
\hline $\begin{array}{l}1.073+03 \\
9.270=01 \\
2.031+00 \\
1.446001 \\
1.677+03\end{array}$ & $\begin{array}{l}5.203001 \\
5.950000 \\
1.320003 \\
0.000 \\
5.270-01\end{array}$ \\
\hline $\begin{array}{l}2.105+00 \\
2.105+00\end{array}$ & $\begin{array}{l}1.410005 \\
1.410005\end{array}$ \\
\hline $\begin{array}{l}1,364001 \\
2,168+03 \\
2,169+03\end{array}$ & $\begin{array}{l}2.158000 \\
0.838001 \\
0.840=01\end{array}$ \\
\hline $\begin{array}{l}3.127+00 \\
3.127+00\end{array}$ & $\begin{array}{l}2.211=05 \\
2.211=05\end{array}$ \\
\hline $\begin{array}{l}5.434001 \\
3.535003 \\
3.530003\end{array}$ & $\begin{array}{l}4.043004 \\
1.156000 \\
1.157+00\end{array}$ \\
\hline
\end{tabular}


IHIS SEGMENT A AS UISCMAHGEO AT

TME BEGINNING OH KELLUA I ANU WAS

URIGINALLY LUALEO AT RELUAU O

IMIS SEGMENI AAS UISCMAKGED AT

THE UEGINNING UF NELUAD 5 AND WAS

URIGINALLY LUADED AT RELOADI

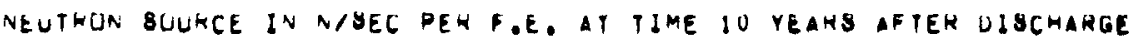

RUCLIUE

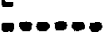

$A T=217$
$T=2 I B$

ATISIB
ASTATINE

PO- 210

$P U=212$

PO- 213

Pu=215

PO-21:

DOLUNIUM

$\forall 1=211$

BI-ZIZ
GISMUIH

ALL ELEMENTS
IS FREBM U-235 F.E. FEKTILE PTL. PISSILEPTL.

\section{$3,339+00$}

2,871005

$3.339+00$

0,434007

0.000

0.434007

$1.848-03$

1.040003
$7.505+03$

3.514001

$1.073+03$

0.000

3,801001
1.305006

$1.305=06$
$3,860=04$

2.175001

0,040001

2.020001

$2.279+02$
$2.261+02$

2,283004
4,710002

4.7100 .02
4.7330 .02

$7.111+03$

$1.959+04$
ME PRESH U-235 .E. PERTILE HTL. FISSILE PTL.

$\begin{array}{ll}5.586+00 & 3.621005 \\ 1.545005 & 0.000 \\ 5.506+00 & 3.021005 \\ 0.110003 & 0.000 \\ 1.140004 & 2.803+00 \\ 1.259+01 & 7.346005 \\ 5.559001 & 7.290004 \\ 5.114+03 & 1.579+00 \\ 1.059+04 & 4.583+00 \\ 3.196001 & 4.316004 \\ 1.087+03 & 3.419001 \\ 1.087+03 & 3.423001 \\ & \\ 2.843+04 & 9.560+05\end{array}$


TABLE D-11

NEUTRON SOURCE STRENGTHS FOR FUEL ELEMENTS DISCHARGED AT BEGINNING OF RELOAD 10 AND ORIGINALLY LOADED AT RELOAD 6

NOTE: Table D-11 consists of 10 pages of computer print-out (pages D-291 through $\mathrm{D}-300)$. 
O

-

0 


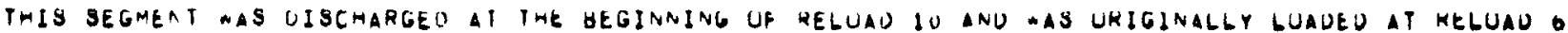

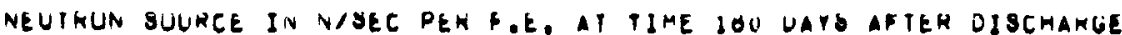

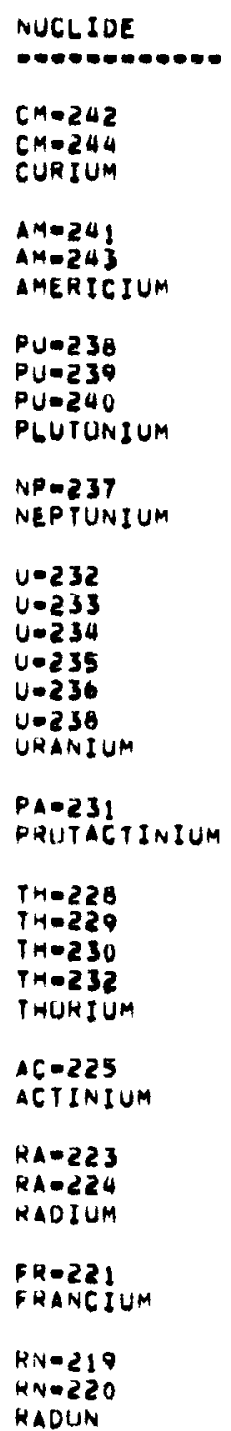

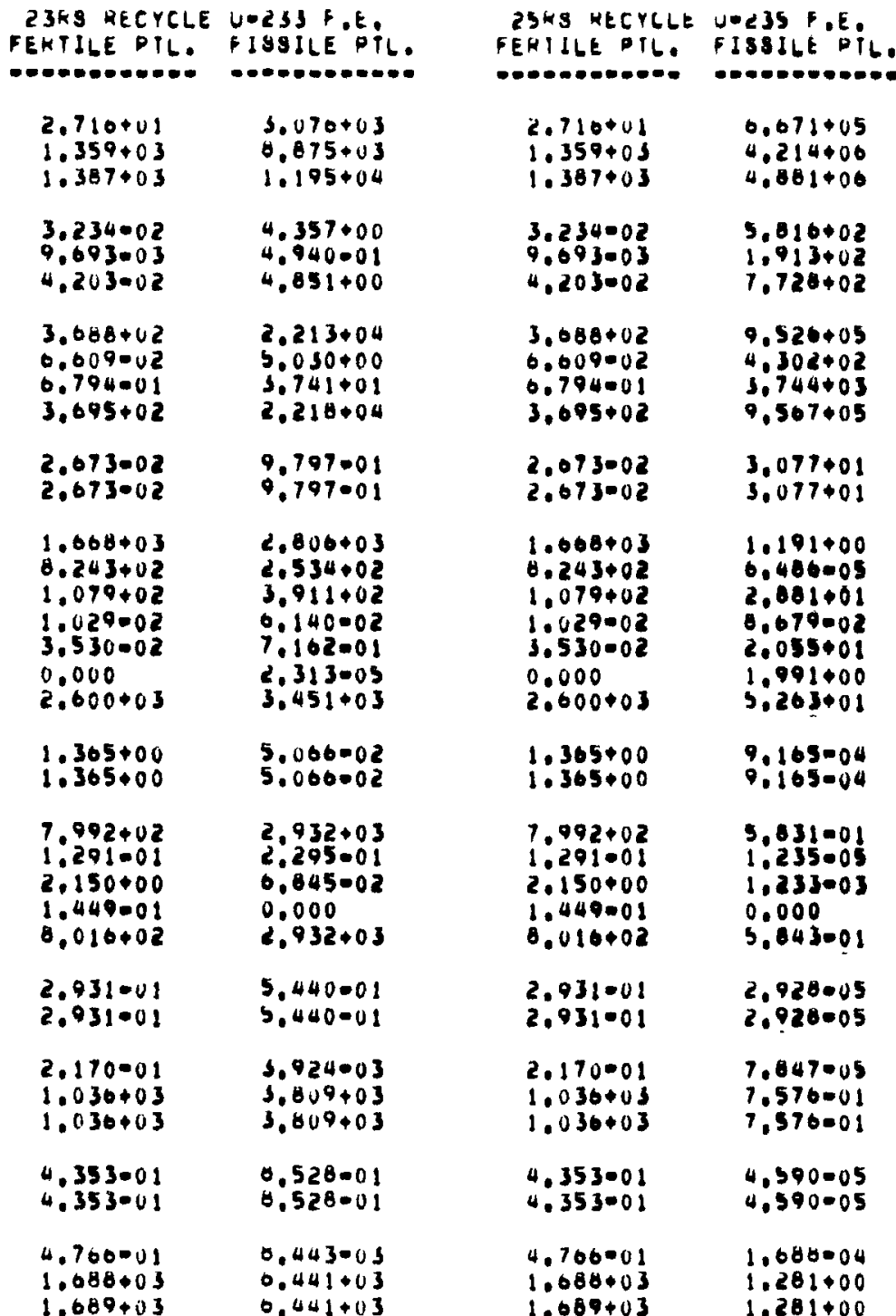




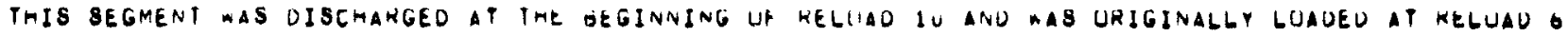

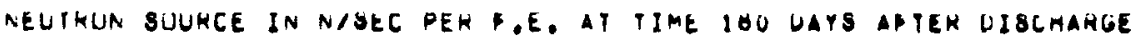

NUCLIDE

NUCLIDE

$4 T-217$

Pu- 210

$P 0=212$

$P U=213$

PU. 15

POLONIUA

$8 !-2 ! !$
$\forall I-2 ! 2$

BI-
BI 8 MUT

N
N

ALL ELEMENTS

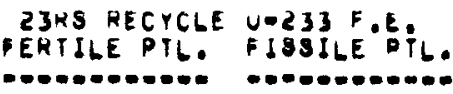

\section{$7.778=01$}

7.258 .00

$1.397+00$

.778 .01

0.000
$1.397+00$

$4.409-01$

$5.295+03$

$1.753+00$

$7.715=01$

$2.443+03$

$7.741+03$

1.600001
$1.502+04$

$.8302+04$
$2.833+00$

$2.833+00$

1.327002
$0.795+03$

$1.795+03$
., $442+04$

$4.435=0$

$5.189+02$

$5.193+02$

7.840 .03

$1.905+03$

$1.615+04$

7.709004

2SHS RECYCLE

FERIILE PTL.

\section{$7.778-01$}

7.258000

7.77800

V-235 F.E.

LESILE PIL.

4.409001
5.295003

$1.753+00$

7.71500

$2.443+03$

$7.741+03$

1,318005

0.000

7,518005

$4.227=04$

3.003000

1.525004

2.054004

$1.749+00$

$4.753+00$

4.43500

5.180402

$3.193+02$

$\$ .700001$

$3.789=01$

$1.615+04$

$5.639+00$

$1.619+04$


THIS SEgmevi mas oISChargev at tMe beginning of keluad iv anu mas likiginably luadeu at heluad o NEUTHON SUURCE IN N/SEC PER F.E, AT IIME I TEAT AFTER DIBCAARGE

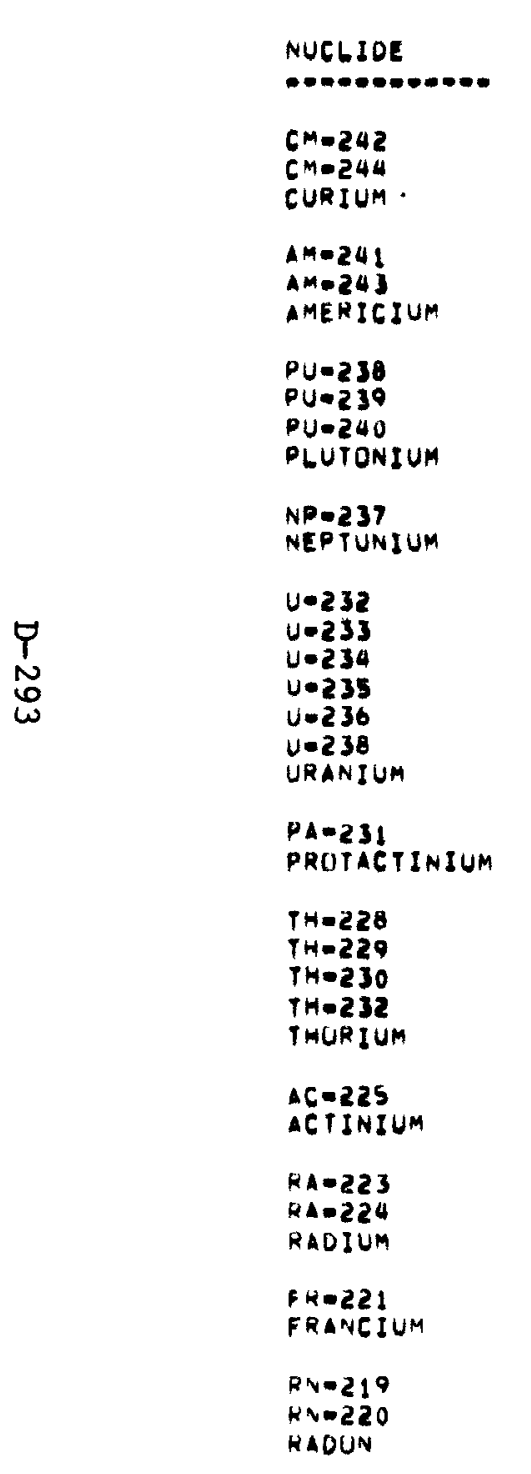

\begin{tabular}{|c|c|c|c|}
\hline $\begin{array}{l}2398 \text { RECYCLE } \\
\text { FERTILE PTL. } \\
\text { W-O }\end{array}$ & 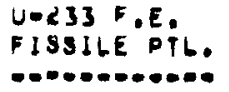 & 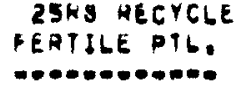 & $\begin{array}{l}\text { U.235 P.E. } \\
\text { ISSILE PTL } \\
0.0-0.0-0.00\end{array}$ \\
\hline $\begin{array}{l}1.238+01 \\
1.333+03 \\
1.345+03\end{array}$ & $\begin{array}{l}1.309+03 \\
0.702+03 \\
1.010+04\end{array}$ & $\begin{array}{l}1.238+01 \\
1.335+03 \\
1.345+03\end{array}$ & $\begin{array}{l}3.035+05 \\
4.132+06 \\
0.436+06\end{array}$ \\
\hline $\begin{array}{l}5.480=02 \\
9.693003 \\
0.449 .02\end{array}$ & $\begin{array}{l}7.108+00 \\
4.900-01 \\
7.602+00\end{array}$ & $\begin{array}{l}5.400=02 \\
9.093=03 \\
6.449002\end{array}$ & $\begin{array}{l}9.145+02 \\
1.915+02 \\
1.106+03\end{array}$ \\
\hline $\begin{array}{l}3.673+02 \\
6.609-02 \\
6.794001 \\
3.680+02\end{array}$ & $\begin{array}{l}2.205+04 \\
5.030+00 \\
5.741+01 \\
2.209+04\end{array}$ & $\begin{array}{l}3.073+02 \\
6.609002 \\
6.794001 \\
3.660+02\end{array}$ & $\begin{array}{l}9.487+05 \\
4.302+02 \\
3.944+03 \\
9.529+05\end{array}$ \\
\hline $\begin{array}{l}2.673002 \\
2.673=02\end{array}$ & $\begin{array}{l}9.797 .01 \\
9.797 .01\end{array}$ & $\begin{array}{l}2.673002 \\
2.673-02\end{array}$ & $\begin{array}{l}3.077+01 \\
3.077+01\end{array}$ \\
\hline $\begin{array}{l}1.600+03 \\
8.251+02 \\
1.079+02 \\
1.020-02 \\
3.530-02 \\
0.000 \\
2.593+03\end{array}$ & $\begin{array}{l}2.792+03 \\
2.534+02 \\
3.911+02 \\
0.140002 \\
7.162 .01 \\
2.313005 \\
3.437+03\end{array}$ & $\begin{array}{l}1.060+03 \\
8.251+02 \\
1.079+02 \\
1.029002 \\
3.530-02 \\
0.000 \\
2.593+03\end{array}$ & $\begin{array}{l}1.165+00 \\
6.467-05 \\
2,861+01 \\
6.679002 \\
2.055+01 \\
1.091+00 \\
5.262+01\end{array}$ \\
\hline $\begin{array}{l}1.365+00 \\
1.365+00\end{array}$ & $\begin{array}{l}5.0660 .02 \\
3.006 .02\end{array}$ & $\begin{array}{l}1.365+00 \\
1.365+00\end{array}$ & $\begin{array}{l}0.175004 \\
9.175004\end{array}$ \\
\hline $\begin{array}{l}9.703+02 \\
1.729-01 \\
2.150+00 \\
1.449-01 \\
9.728+02\end{array}$ & $\begin{array}{l}2.932+03 \\
2,422=01 \\
7.007-02 \\
0.000 \\
2.932+03\end{array}$ & $\begin{array}{l}9.105+02 \\
1.729001 \\
2.150+00 \\
1.449-01 \\
9.728+02\end{array}$ & $\begin{array}{l}7.075001 \\
1.235005 \\
1.355003 \\
0.000 \\
7.000001\end{array}$ \\
\hline $\begin{array}{l}3.924=01 \\
3.924001\end{array}$ & $\begin{array}{l}5.742=01 \\
5.742001\end{array}$ & $\begin{array}{l}3.924001 \\
3.920001\end{array}$ & $\begin{array}{l}2,929=05 \\
2,929005\end{array}$ \\
\hline $\begin{array}{l}2.136-01 \\
1.257+03 \\
1.257+03\end{array}$ & $\begin{array}{l}3.363 \cdot 03 \\
3.809+03 \\
3.809+03\end{array}$ & $\begin{array}{l}2.136=01 \\
1.257+03 \\
1.257+03\end{array}$ & $\begin{array}{l}1.071004 \\
9.192001 \\
9.103=01\end{array}$ \\
\hline $\begin{array}{l}5.827=01 \\
5.827=01\end{array}$ & $\begin{array}{l}9.002-01 \\
9.002=01\end{array}$ & $\begin{array}{l}5.827-01 \\
5.827-01\end{array}$ & $\begin{array}{l}4.591-05 \\
4.591-05\end{array}$ \\
\hline $\begin{array}{l}4.089+01 \\
20050+03 \\
2.050+03\end{array}$ & $\begin{array}{l}1.154-02 \\
0.441+1) 3 \\
0.441+03\end{array}$ & $\begin{array}{l}4.689001 \\
2.050+03 \\
2.050+03\end{array}$ & $\begin{array}{l}2,300=04 \\
1.554+00 \\
1.555+00\end{array}$ \\
\hline
\end{tabular}


IHIS SEGMENT WAS UISCHAKGED AT THE BEGINNING UF RELUAD IO ANU MAS UKIGINALLY LUADEU AT RELUAD O

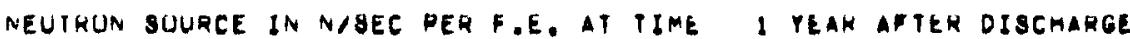

NUCLIDE

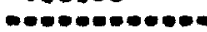

$A T-217$
$A T-218$

ATEZIO

$0=210$

Pu=2is

$P O=213$
$P O=215$

$P Q=216$

POLONIUM

$0\{-211$

BI-2Iz
BIsmuTn

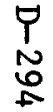

ALL ELEMENTS
23RS RECYCLE UN233 F.E.
FERTILE PILL FISILE PTL

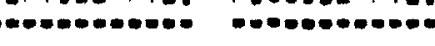

$1.041+00$
$7.742-06$

$1.742-06$
$1.041+00$

$1.474+00$

0.000

$1.474+00$

$1.820=01$
$.0613+03$

$0.613+03$

2.346000
7.501 .01

$9,982+03$

4.364 .01

6.300402

$6.304+02$

$1.800+04$

\subsection{2
$1.562+04$}

$1.562+04$

1.014002

$0.795+03$

$2,442+04$

1.073002

$1.905+03$
25RS RECYCLE UE235 F.E.

ERTILE PTL, FISBILE PTLO

1.041000

7.742006

$1,041+00$

7,320005

7,520005

$1.820=01$
$6.013+03$

$0.613+03$

7.591001

$2.906 * 03$

1.902004

$3.746+00$

1.525004

3,624004

$2.122+00$
5.071000

4.364008

$6.300 \div 02$

$0.304+02$

$2.143=04$

4.596061

$1.800+04$

$5.390+06$

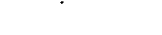




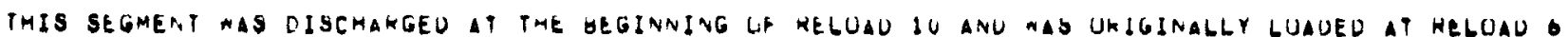

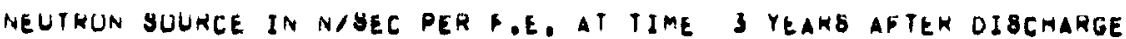

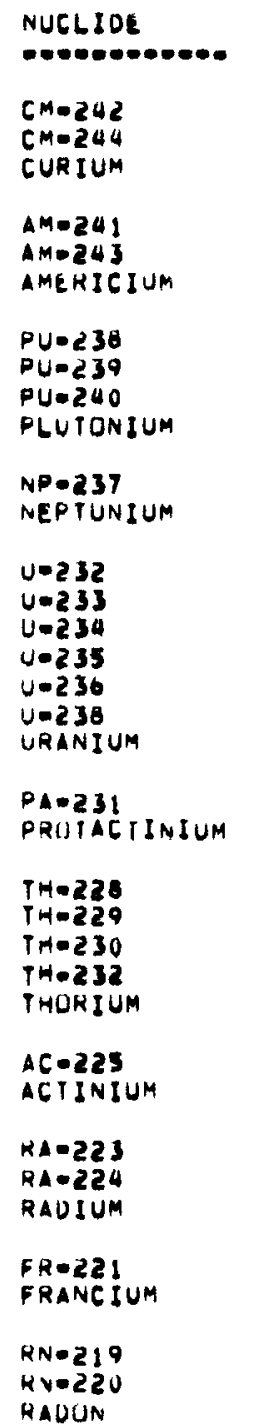

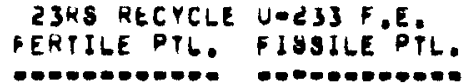

5.982001

$1.234+03$

$0.332+01$
$0.053+03$

$0.053+03$

$1,733+01$

1.382001

9.691 .03

$1.479-01$

$1,782+01$

$3.615+02$

6.009002

6.792 .01
$3.623+02$

$3.170+04$

$5.029+00$

$3.7 \$ 040$

$2,174+04$

2.673 .02

2.673 .02

$9.797-01$

$1.628+03$

$8.251+02$

$1.079+02$

1.029002

0.000

$2.561+03$

$1.365+00$

$1.395+03$

$1.395+03$
3.453001

$2.152+00$

$1.440-01$

$1.398+03$

7.836001

7.838 .01

$2.003-01$
$1.808+03$
$1.800+03$

$1.808+03$
$1.808+03$

$1.104+00$

$1.104+00$

4.398001

$2.948+03$
$25 \mathrm{Kg}$ HECYCLE U-235 P.E.

FERTILE PTL. HIS8ILE PTL.

\begin{tabular}{|c|c|c|}
\hline $\begin{array}{l}0.332+01 \\
0.053+03 \\
0.117+03\end{array}$ & $\begin{array}{l}5.962001 \\
1.234003 \\
1.234+03\end{array}$ & $\begin{array}{l}1,370+04 \\
3,820+06 \\
3,838+00\end{array}$ \\
\hline $\begin{array}{l}1.733+01 \\
4,939-01 \\
1,782+01\end{array}$ & $\begin{array}{l}1.362001 \\
9.691001 \\
1.490001\end{array}$ & $\begin{array}{l}2,151+03 \\
1.912002 \\
2.342+03\end{array}$ \\
\hline $\begin{array}{l}2.170+04 \\
5.029+00 \\
3.740 \$ 01 \\
2.174 \$ 04\end{array}$ & $\begin{array}{l}3.615+02 \\
6.609002 \\
6.192001 \\
3.023+02\end{array}$ & $\begin{array}{l}9.339+05 \\
4.302+02 \\
3.743+03 \\
9.301+05\end{array}$ \\
\hline $\begin{array}{l}9.797-01 \\
9.799001\end{array}$ & $\begin{array}{l}2.673=02 \\
2.673002\end{array}$ & $\begin{array}{l}3,077+08 \\
3.077+01\end{array}$ \\
\hline $\begin{array}{l}2,730+03 \\
2,534+02 \\
3,9111+02 \\
0,140002 \\
7,162001 \\
2,313005 \\
3,364+03\end{array}$ & $\begin{array}{l}1.028+03 \\
8.251002 \\
1.070+02 \\
1.029002 \\
3.530002 \\
0.000 \\
2.561+03\end{array}$ & $\begin{array}{l}1.163+00 \\
6.081+05 \\
2.081+01 \\
8.679002 \\
2.035 \times 01 \\
1.991+00 \\
5.260+01\end{array}$ \\
\hline $\begin{array}{l}5,0660.02 \\
5,066002\end{array}$ & $\begin{array}{l}1.365+00 \\
1.365+00\end{array}$ & $\begin{array}{l}9.210004 \\
9,210004\end{array}$ \\
\hline $\begin{array}{l}3.041 .03 \\
2,0250.01 \\
7.050002 \\
0.000 \\
3,042.03\end{array}$ & $\begin{array}{l}1.395 \times 03 \\
3.453001 \\
2.152+00 \\
1.449001 \\
1.396403\end{array}$ & $\begin{array}{l}1.016+00 \\
1.237005 \\
1.827003 \\
0.000 \\
1.018 \times 00\end{array}$ \\
\hline $\begin{array}{l}0.9340 .01 \\
0.9340 .01\end{array}$ & $\begin{array}{l}7.838-01 \\
7.8380 .01\end{array}$ & $\begin{array}{l}2.931005 \\
2.931005\end{array}$ \\
\hline $\begin{array}{l}1.130002 \\
3.952+03 \\
3.952+03\end{array}$ & $\begin{array}{l}2.003001 \\
1.800403 \\
1.808+03\end{array}$ & $\begin{array}{l}2.161=04 \\
1.321+00 \\
1.321+00\end{array}$ \\
\hline $\begin{array}{l}1.087+00 \\
1.087+00\end{array}$ & $\begin{array}{l}1.164+00 \\
1.164+00\end{array}$ & $\begin{array}{l}4.595=05 \\
4.595=05\end{array}$ \\
\hline $\begin{array}{l}2.431=02 \\
0.082+03 \\
0.682+03\end{array}$ & $\begin{array}{l}4,398-01 \\
2,948+03 \\
2,948+03\end{array}$ & $\begin{array}{l}4.050004 \\
2.233+00 \\
2.235+00\end{array}$ \\
\hline
\end{tabular}




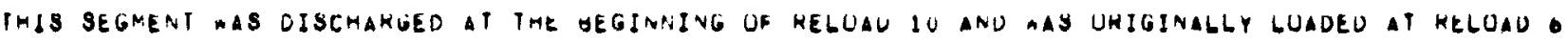
NEUTRUN SUUKCE IN N/SEC PER P.t. AT TIME S YEARS AFTER OJSCharge

\begin{tabular}{|c|c|c|c|}
\hline $\begin{array}{l}\text { 23RS RECYCLE } \\
\text { FERIILE PYLE }\end{array}$ & $\begin{array}{l}U-233 F \cdot E \text {. } \\
\text { FISSILE PIL. }\end{array}$ & $\begin{array}{l}\text { 25RS RECYCLE } \\
\text { PERTILE PTL }\end{array}$ & 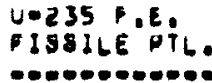 \\
\hline $\begin{array}{l}2.080+00 \\
9.651=06 \\
2.060+00\end{array}$ & $\begin{array}{l}1.781+00 \\
0.000 \\
1.781+00\end{array}$ & $\begin{array}{l}2.080+00 \\
9.651=00 \\
2.080+00\end{array}$ & $\begin{array}{l}7.526005 \\
0.000 \\
7.520005\end{array}$ \\
\hline $\begin{array}{l}1.641-02 \\
9.543+03 \\
4.687+00 \\
7.119001 \\
4.264+03 \\
1.361+04\end{array}$ & $\begin{array}{l}5,958003 \\
1.020+04 \\
3,612+00 \\
3.821002 \\
9.124+03 \\
2,533+04\end{array}$ & $\begin{array}{l}1.641=02 \\
9.543+03 \\
4.087+00 \\
7.119001 \\
4.264+03 \\
1.381004\end{array}$ & $\begin{array}{l}2.003005 \\
5.004000 \\
1.527004 \\
7.308004 \\
3.049000 \\
8.454000\end{array}$ \\
\hline $\begin{array}{l}4.093001 \\
9.059+02 \\
9.063+02\end{array}$ & $\begin{array}{l}2.259002 \\
1.970 \circ 03 \\
1.976+03\end{array}$ & $\begin{array}{l}4.093001 \\
9.059+02 \\
9.063+02\end{array}$ & $\begin{array}{l}4.322=04 \\
0.603001 \\
6.609=01\end{array}$ \\
\hline $2.504+04$ & $7.425+04$ & $2.504+04$ & $4.778+00$ \\
\hline
\end{tabular}

NUCLIOE

$A T-217$

$A T-218$

ASTATINE

PU-210

$0 u=512$

PU- 113

$P D=215$

$P U=216$

PULONIUA

BI -211

SISMUTH

ALL ELEMENTS
$23 R S$ RECYCLE U-233 F.E.

4.778000

2SHS HECYCLE UDESS P.E.

., 520005

-10
0.003009

$454+00$

0.003001

$009=01$ 


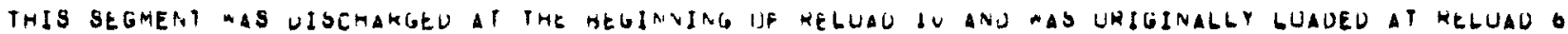

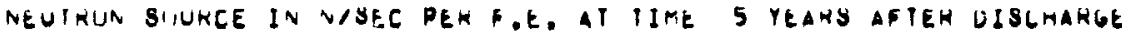

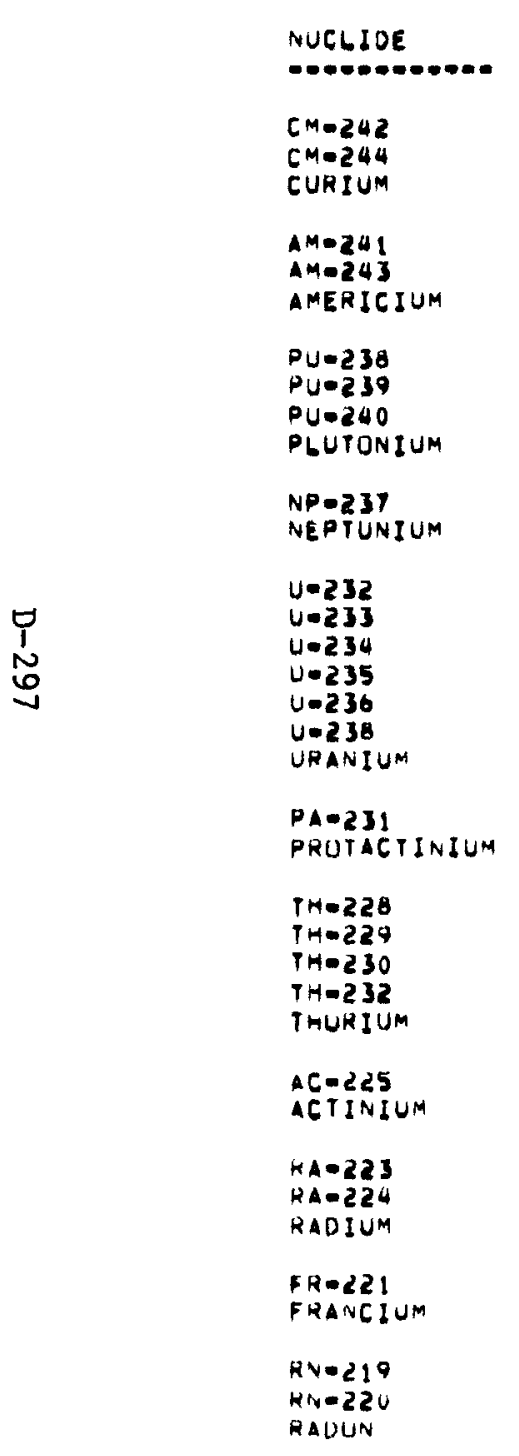

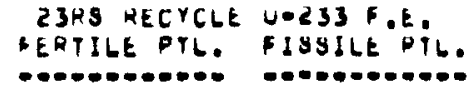

$7.040=02$

$1.142+03$

$1.142+03$

$3.517+00$

$7.453+03$
$7.457+03$

$2.061+01$

2.140001

9.690 .03

2.239001

4.938001
$2.710+01$

$3.559+02$

6.008 .02

$0.791=01$
$3.500+02$

$2.136+04$

$5.029+00$
$3.739+01$

$3.739+01$
$2.140+04$

2.673002

$2.673-02$

4.797 .01
$9.797-01$

$1.597+03$

$8.251+02$

$1.079+02$

1.029002

0.500

$2.530+03$

$1.305+00$

$1.365+00$

$1.583+03$

$2.154+00$

$1.449=01$

1.449001

$1.175+00$

$1.175+00$

1.870001

$2.051+03$
$2.052+03$

$1.745+00$

$1: 745+00$

$4.124-01$

$3.345+03$
$3.345+03$

$2.006+03$

$2.534+02$

3.911 .02

$0.140=02$

2.313005

$3.332+03$

3.000002

$3.041+03$

$0.292-02$

0.000

0.000
$3.042+03$

$0.127-01$
0.127011

$3.952+03$

$5.952+03$

$1.274+00$

3.706002

$0.082+03$

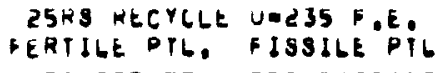

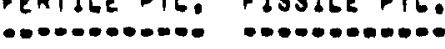

7. v40002 1.250+02

$1.102+03 \quad 5.539+00$

$1.142403 \quad 3.540400$

$\begin{array}{ll}2.140001 & 3.274003 \\ 9.690005 & 1.912002\end{array}$

$\begin{array}{ll}9.690005 & 1.912002 \\ 2.237001 & 3,460003\end{array}$

$3.359+02 \quad 9.195+05$

6.608 .02

$\begin{array}{ll}0.791-01 & 5.742+03 \\ 3.506+02 & 9.234+05\end{array}$

$2.673002 \quad 3.077401$

$2.673=02 \quad 3.077+01$

$1.597+03$

$8.251+02$

$1.079+02$

$3.029=02$

$2,530+03$

$1.140+00$

$6,487-05$

$2.081+01$

8.679002

$2.055+01$

$1.991+00$

$5.258+01$

$1.365+00 \quad 9.246004$

$1.365+00 \quad 9.246004$

$1,583+03$
5,170001

$2.154+00$

1.449001

1.580003

$1,153+00$

$2,300=03$

0.000

$1,155+00$

$1.175+00$

$1.175+00$

2.934005

1.878001

$1.498+00$

$1.745+00$

4.5990 .05

0.090000

$0.857=04$

$\begin{array}{ll}3,345+03 & 2.5354,10 \\ 3,345+03 & 2,534+00\end{array}$ 


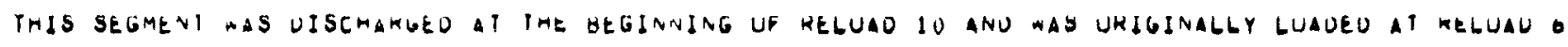

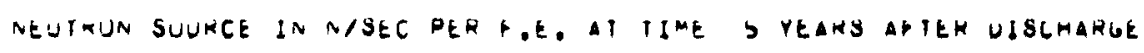

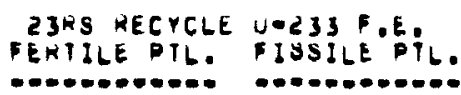

$3.118+00$

$1.156=05$

$3.110+00$

1.1390 .02

$1.084+04$

$7.028+00$

6.676001

$0.839+03$

3,838001

$1.028+03$

$1.028+03$

$2.773+04$

\section{$2.087+00$} $+.197 .07$ $2.087+00$

0.000

$1.020+04$

$4.233+00$

5.829002

$9.124+03$

$2.533+04$

$\$ .447 .02$

$1.970+03$

1.976403

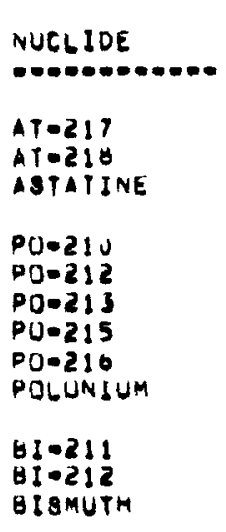

ALL ELEMENTS
25KS KECYCLE HE235 POE
FERIILE PIL PISSILEPIL.

\section{$3.118+00 \quad 7.353=05$}

$\begin{array}{ll}3.156003 & 0.000 \\ 3.118+00 & 7.533005\end{array}$

$1.139002 \quad 0.000$

$1.084004 \quad 6.137000$

$7.028+00 \quad 1.528000$

$0.670001 \quad 1.078003$

4.839403

$1.569+04 \quad 9.597+00$

$3.838 .01 \quad 0.374004$

$1.028403 \quad 9.400=01$

$1.028+03 \quad 7.497001$

$7.320+04$
$2.773+04$$\quad 4.467000$ 


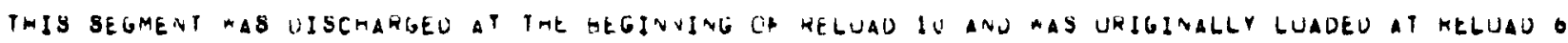
NEUTKUR SUUHCE IN N/SEC PEK P. E. AT IIME IU YEAKS AFIER UISCMARGE

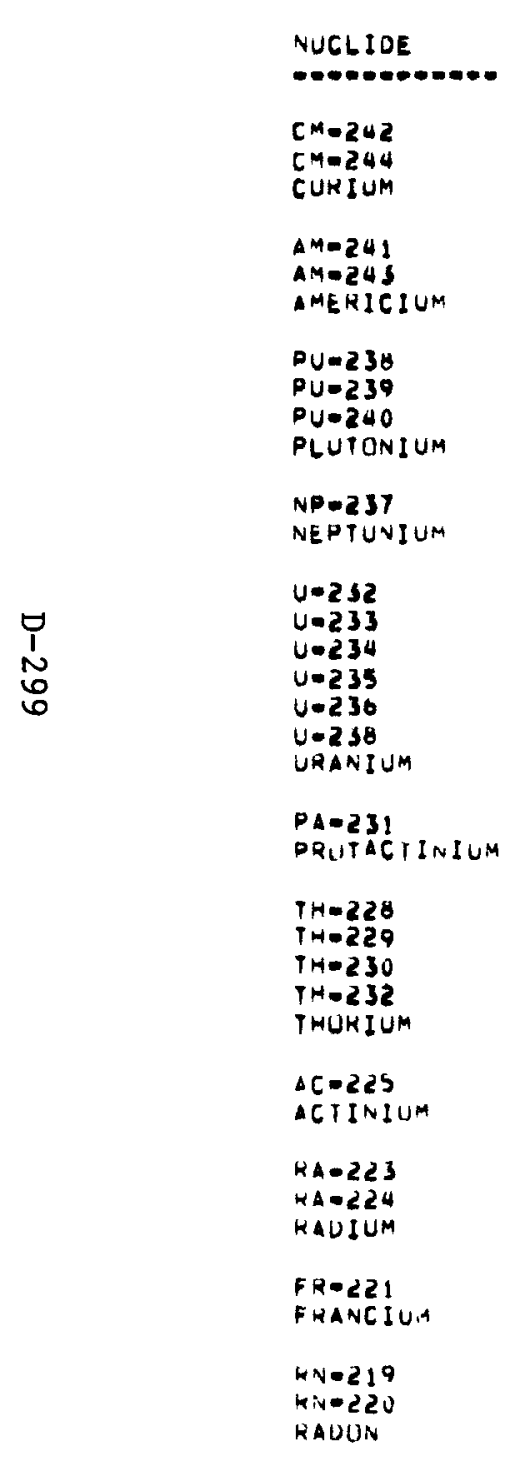

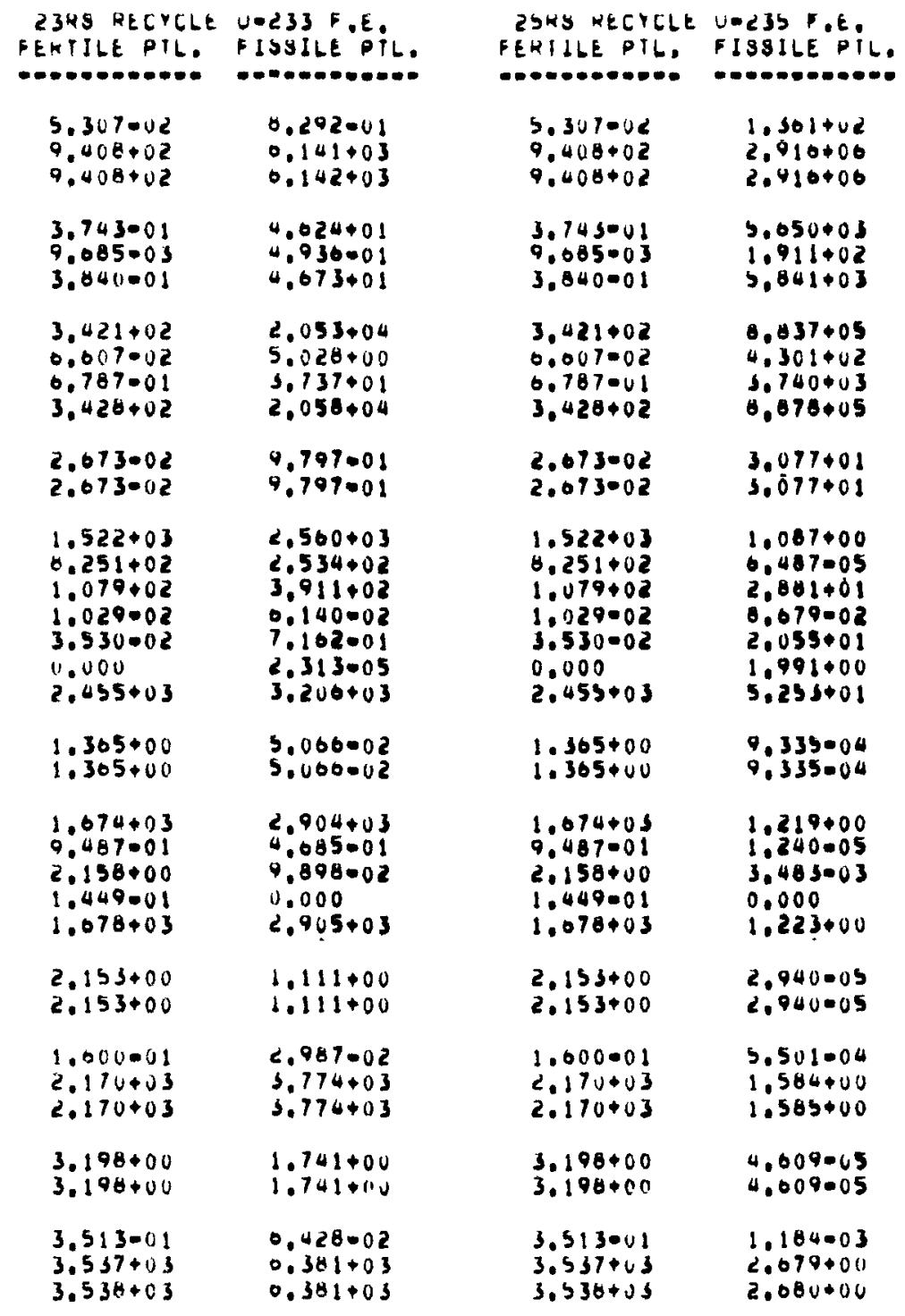




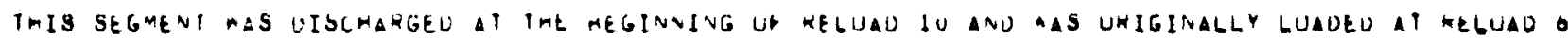

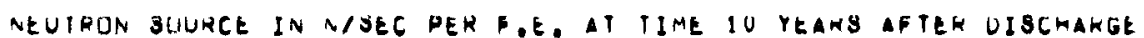

NUCLIDE

NUCLIOE

$A T-217$
$A T=216$

ASTATINE

Pu- 210

$P 0-212$

$P Q-213$

PU 215

PU. 110

POLUAIUM

$\operatorname{lin}(1)$

B $\$-212$

BISMUTH

ALL ELEMENTS
Z3KS KECYCLE UER33 FEE ERTILE PIL. FISSILEPIL.

$5.713+00$

$1.053=05$

5.713000

$8.852+00$

$2.852+00$

$1.167+04$

$1.208+0$

$5.687=01$

$5.118+05$

$1.600+04$

3.26900

$1.087+0$

$1.088+03$

$2.883+04$
0.000

$1.547+04$

$5.784+00$

1.010001

$0.713+03$

$2.419+04$

3.975 .02

$\{.887+0\}$

$0.911+04$
2SKS KECYCLE UE233 F.E,

FERTILE NTL. FISSILE NIL.

\begin{tabular}{|c|c|}
\hline $\begin{array}{l}5.713+00 \\
1.033005 \\
5.713+00\end{array}$ & $\begin{array}{l}7.5440 .05 \\
0.000 \\
7.549=05\end{array}$ \\
\hline $\begin{array}{l}9.514001 \\
1.147+04 \\
1.288+01 \\
5.087=01 \\
5.118+03 \\
1.060+04\end{array}$ & $\begin{array}{l}0.000 \\
0.093+00 \\
1.531004 \\
1.860003 \\
3.656+00 \\
1.015+01\end{array}$ \\
\hline $\begin{array}{l}3.269001 \\
1.087+03 \\
1.080+03\end{array}$ & $\begin{array}{l}1.100003 \\
7.920001 \\
1.931001\end{array}$ \\
\hline $2.863+04$ & 5.610000 \\
\hline
\end{tabular}


TABLE D-12

NEUTRON SOURCE STRENGTH FOR FUEL ELEMENTS DISCHARGED AT BEGINNING OF RELOAD 13 AND ORIGINALLY LOADED AT RELOAD 9

NOTE: Table D-12 consists of 10 pages of computer read-out (pages D-303 through D-312). 
0

-

0 


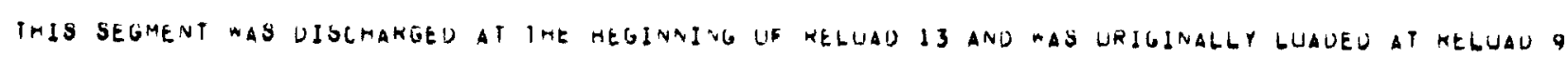

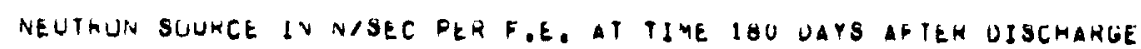

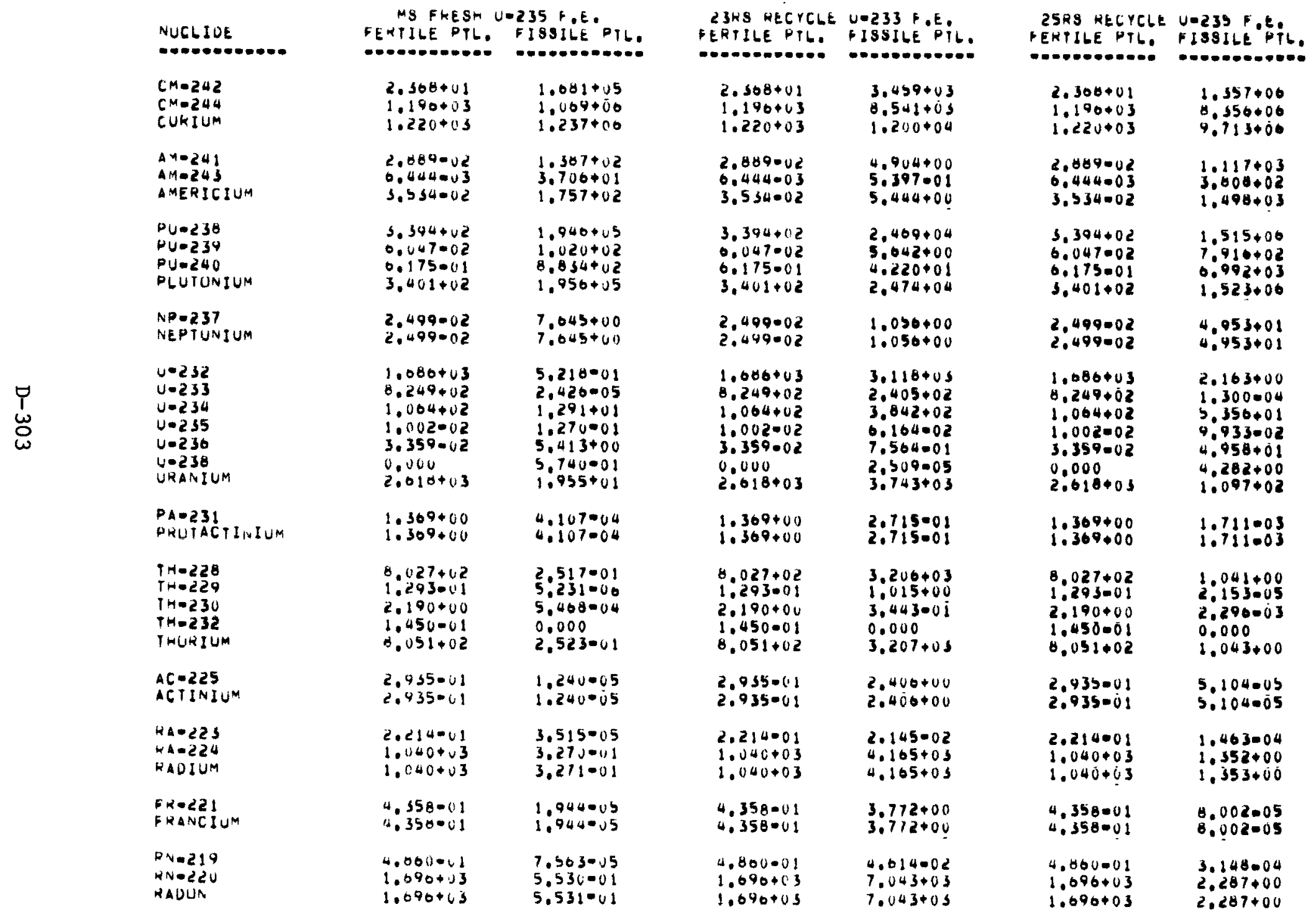




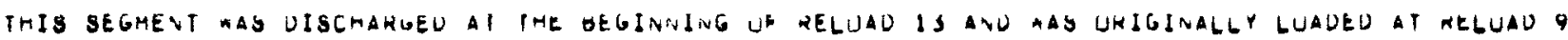

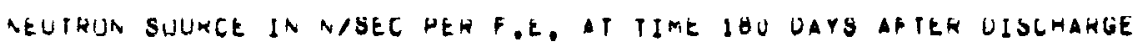

\begin{tabular}{|c|c|c|c|c|c|c|}
\hline $\begin{array}{l}\text { NUCLIDE } \\
-\end{array}$ & $\begin{array}{l}\text { MS BKESH } \\
\text { FEKIILE PIL. }\end{array}$ & 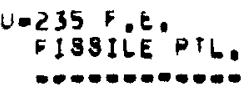 & $\begin{array}{l}\text { 23KS RECYCLE } \\
\text { FERIILE PTL. } \\
\text { - }\end{array}$ & 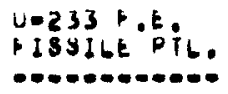 & $\begin{array}{l}\text { 2SRS KECYCLE } \\
\text { PERTILE PTL. } \\
\text { - O-OA- }\end{array}$ & 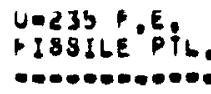 \\
\hline $\begin{array}{l}\text { AT-217 } \\
\text { ATERI8 } \\
\text { ASTATINE }\end{array}$ & $\begin{array}{l}7,787-01 \\
7,302=06 \\
7,787-01\end{array}$ & $\begin{array}{l}3.104005 \\
0.000 \\
3.104005\end{array}$ & $\begin{array}{l}7.787-01 \\
7.302-00 \\
7.787-01\end{array}$ & $\begin{array}{l}0.179+00 \\
1.094=06 \\
0.179+00\end{array}$ & $\begin{array}{l}7.781=01 \\
7.582000 \\
7.787=01\end{array}$ & $\begin{array}{l}1.511=04 \\
0.000 \\
1.511=04\end{array}$ \\
\hline $\begin{array}{l}P U=21 U \\
P U=212 \\
P U=213 \\
P U=215 \\
P I J=210 \\
\text { PULUNIUM }\end{array}$ & $\begin{array}{l}4.427-01 \\
5.310+03 \\
1.755+00 \\
7.008001 \\
2.453+03 \\
7.773+03\end{array}$ & $\begin{array}{l}1.800-04 \\
1.296+00 \\
6.458005 \\
1.180-04 \\
7.551=01 \\
2.052+00\end{array}$ & $\begin{array}{l}9.427=01 \\
5.310+03 \\
1.755+00 \\
7.808=01 \\
2.453+03 \\
7.773+03\end{array}$ & $\begin{array}{l}8.507-01 \\
1.708+04 \\
1.253+01 \\
7.253002 \\
9.017+03 \\
2.671+04\end{array}$ & $\begin{array}{l}4.427=01 \\
5.310003 \\
1.755+00 \\
7.808-01 \\
2.455+03 \\
7.775+03\end{array}$ & $\begin{array}{l}7.072=04 \\
5.360000 \\
2.658=04 \\
4.948=04 \\
5.125+00 \\
8.484+00\end{array}$ \\
\hline $\begin{array}{l}B I-21 I \\
\text { BI-2II } \\
\text { BISMUTH }\end{array}$ & $\begin{array}{l}0.523=01 \\
5.212+02 \\
5.216+02\end{array}$ & $\begin{array}{l}7.030005 \\
1.035001 \\
1.636001\end{array}$ & $\begin{array}{l}4.523+01 \\
5.212+02 \\
5.216+02\end{array}$ & $\begin{array}{l}4.289-02 \\
2.083+03 \\
2.003+03\end{array}$ & $\begin{array}{l}4.523001 \\
5.212+02 \\
5.216+02\end{array}$ & $\begin{array}{l}2.920=04 \\
6.762=01 \\
0.765=01\end{array}$ \\
\hline ALL ELEMENTS & $1.002+04$ & 1.035406 & $1.002+04$ & $8.371+04$ & 1.002004 & $1.124+07$ \\
\hline
\end{tabular}




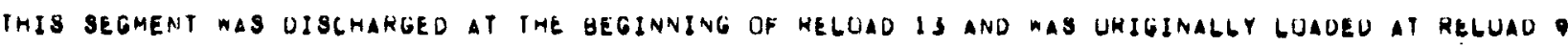
NEUTRUN SUUKCE IN IN/SEC PER F.t. AT TIME I YEAR AFTER DISCMARGE

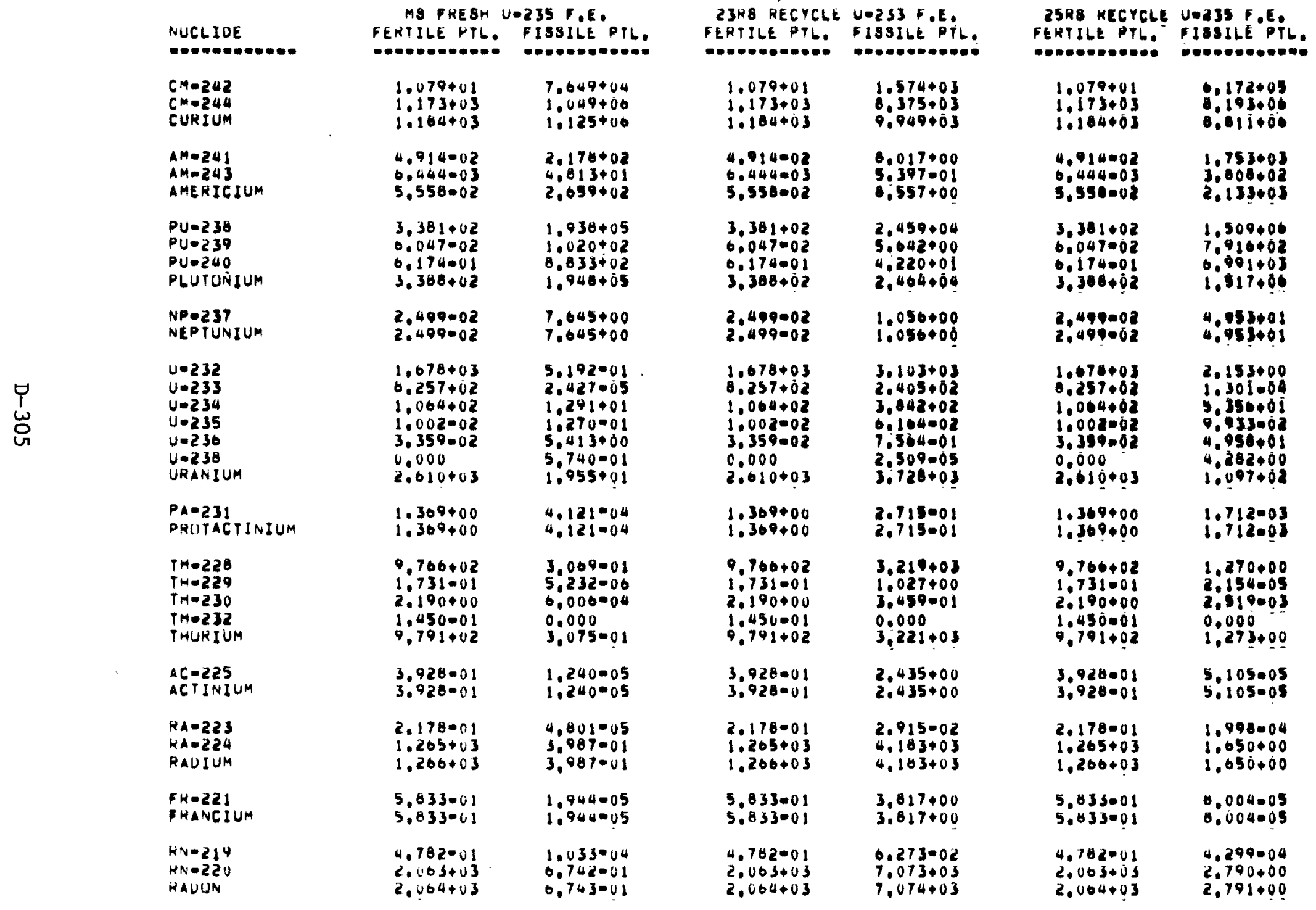




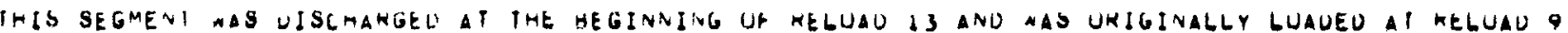
neutrun suUkCe in n/bec per f.e. at time I yeak aptek discmarge

\begin{tabular}{|c|c|c|c|c|c|c|}
\hline $\begin{array}{l}\text { NUCLIUE } \\
\text { D. }\end{array}$ & 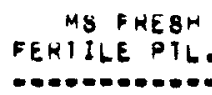 & $\begin{array}{l}35 \text { FEE. } \\
\text { ISSILE PTL. }\end{array}$ & $\begin{array}{l}\text { 23HS RECYCLE } \\
\text { FERIILE PTLE } \\
\text { WO- }\end{array}$ & 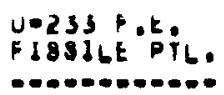 & 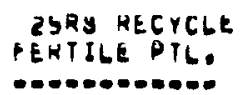 & 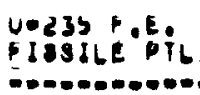 \\
\hline $\begin{array}{l}\text { AT-21? } \\
\text { ATE-2I8 } \\
\text { ASTATINE }\end{array}$ & $\begin{array}{l}1.042+00 \\
7.875-00 \\
1.042+00\end{array}$ & $\begin{array}{l}3.105 \cdot 05 \\
0.000 \\
3.185 \cdot 05\end{array}$ & $\begin{array}{l}1.042+00 \\
7.875006 \\
1.042+00\end{array}$ & $\begin{array}{l}0.252+00 \\
1.173000 \\
0.252+00\end{array}$ & $\begin{array}{l}1.042+00 \\
7.075006 \\
1.042+00\end{array}$ & $\begin{array}{l}1.311004 \\
0.000 \\
1.311004\end{array}$ \\
\hline $\begin{array}{l}P D=210 \\
P U=212 \\
P U-213 \\
P D=215 \\
P U=216 \\
P O L O N I U M\end{array}$ & $\begin{array}{l}1.069001 \\
6.055003 \\
2.349+00 \\
7.742001 \\
2.965403 \\
9.043003\end{array}$ & $\begin{array}{l}8.855=05 \\
1.626+00 \\
0.459-05 \\
1.024004 \\
9.200-01 \\
2.541+00\end{array}$ & $\begin{array}{l}1.829001 \\
6.653+03 \\
2.349+00 \\
7.742001 \\
2.905+03 \\
9.643+03\end{array}$ & $\begin{array}{l}3.549001 \\
1.715+04 \\
1.208+01 \\
9.000002 \\
9.058+03 \\
2.002+04\end{array}$ & $\begin{array}{l}1.629001 \\
0.655+03 \\
2.349+00 \\
7.742001 \\
2.985+03 \\
9.643+03\end{array}$ & $\begin{array}{l}3.690004 \\
6.728+00 \\
2.059004 \\
6.787004 \\
3.810+00 \\
1.054+01\end{array}$ \\
\hline $\begin{array}{l}\forall I-211 \\
\forall I-212 \\
\text { GISMUTM }\end{array}$ & $\begin{array}{l}4.451-01 \\
6.341+02 \\
6.346+02\end{array}$ & $\begin{array}{l}9.002005 \\
1.993001 \\
1.994-01\end{array}$ & $\begin{array}{l}4.451=01 \\
6.341+02 \\
6.346+02\end{array}$ & $\begin{array}{l}5.831=02 \\
2.091+03 \\
2.062+03\end{array}$ & $\begin{array}{l}4.451001 \\
0.341+02 \\
0.346+02\end{array}$ & $\begin{array}{l}3.996004 \\
: 250=01 \\
.254=01\end{array}$ \\
\hline ALL ELEMENTS & $1.872+0.4$ & $1.320+06$ & $1.872+04$ & $8.172+04$ & $1.872+04$ & $1.033+07$ \\
\hline
\end{tabular}




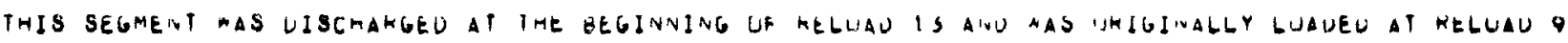

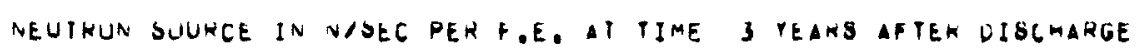

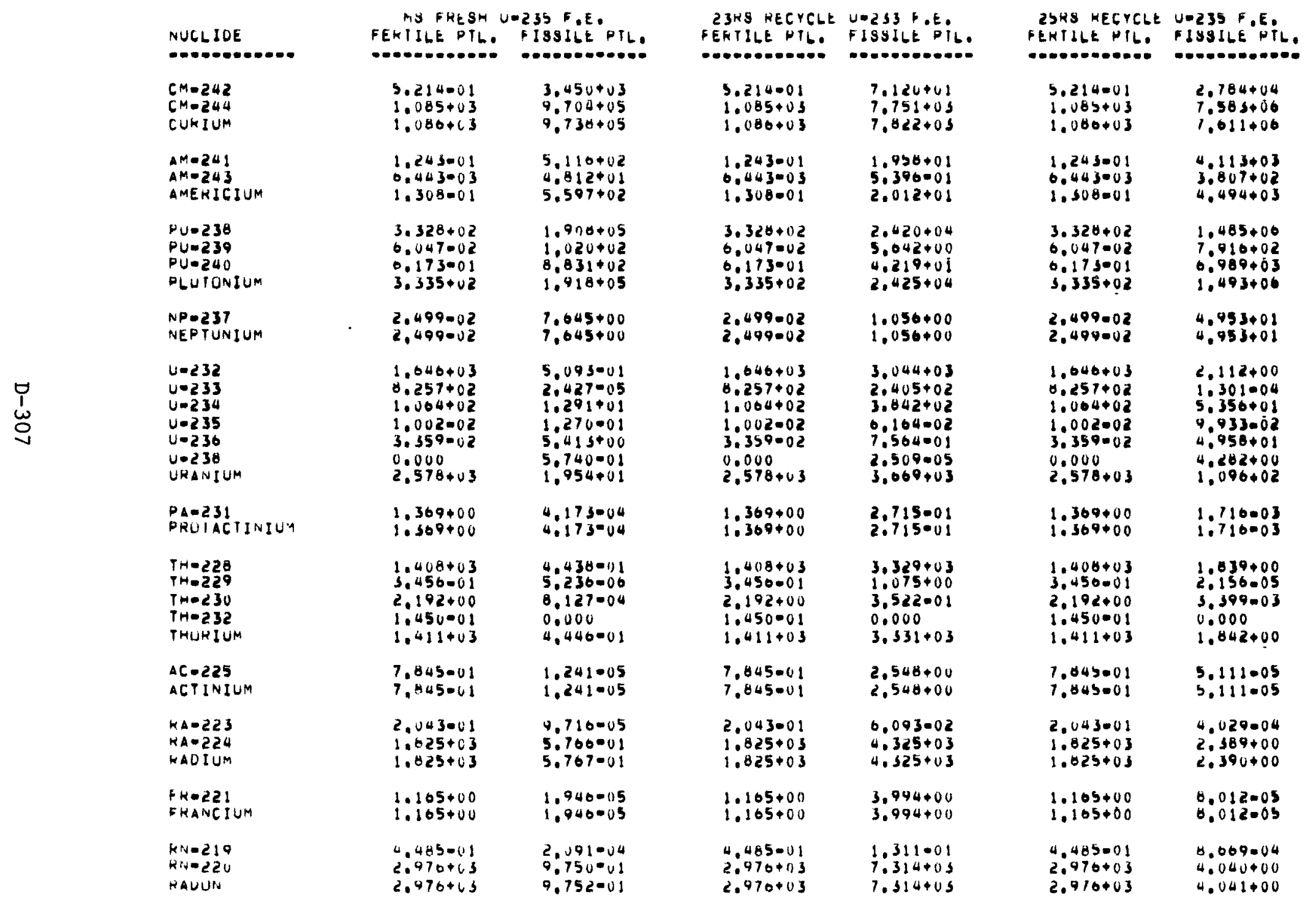




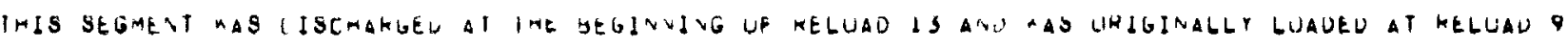

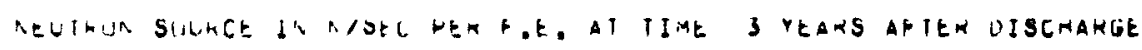

\begin{tabular}{|c|c|c|c|c|c|c|}
\hline $\begin{array}{l}\text { NUCLIOE } \\
\text { WOA }\end{array}$ & $\begin{array}{l}\text { MS FKESH } \\
\text { FEMIILE PIL. }\end{array}$ & 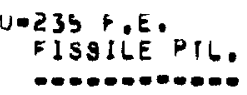 & $\begin{array}{l}\text { ZZHS RECYCLE } \\
\text { FERIILE PTL. } \\
\text { W. }\end{array}$ & $\begin{array}{l}\text { U-23S P.E. } \\
\text { FISSILE PTL. }\end{array}$ & $\begin{array}{l}\text { 25HS KECYCLE } \\
\text { FEKTILE PTL. } \\
\text {-OE- }\end{array}$ & 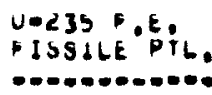 \\
\hline $\begin{array}{l}A T=217 \\
A T-218 \\
A S T A T I N E\end{array}$ & $\begin{array}{l}2.062+00 \\
9.820-00 \\
2.002+100\end{array}$ & $\begin{array}{l}3.187 .05 \\
0.000 \\
3.187 .05\end{array}$ & $\begin{array}{l}2.062+00 \\
9.820000 \\
2.082+100\end{array}$ & $\begin{array}{l}0.542+00 \\
1.408 .06 \\
0.542+00\end{array}$ & $\begin{array}{l}2.082+00 \\
9.820006 \\
2.002+018\end{array}$ & $\begin{array}{l}1.312004 \\
0.000 \\
1.512004\end{array}$ \\
\hline $\begin{array}{l}P U-210 \\
P U=212 \\
P O-213 \\
P U-215 \\
P U=210 \\
P O L U N I U M\end{array}$ & $\begin{array}{l}1.679-02 \\
9.032+03 \\
4.691+00 \\
7.200001 \\
4.305+03 \\
1.394+04\end{array}$ & $\begin{array}{l}9.104000 \\
2.354+00 \\
0.464=05 \\
3.280-04 \\
1.331+00 \\
3.691+00\end{array}$ & $\begin{array}{l}1.679002 \\
9.632+03 \\
4.691+00 \\
7.200001 \\
4.305+03 \\
1.394+04\end{array}$ & $\begin{array}{l}3.257-02 \\
1.773+04 \\
1.327+01 \\
2.001=01 \\
9.987+03 \\
2.773+04\end{array}$ & $\begin{array}{l}1.679002 \\
9.632+03 \\
4.601000 \\
7.200=01 \\
4.305+03 \\
1.394+04\end{array}$ & $\begin{array}{l}3.800005 \\
9.777+00 \\
2.662004 \\
1.363003 \\
5.517+00 \\
1.530+01\end{array}$ \\
\hline $\begin{array}{l}\forall I-211 \\
\text { BI-2IL } \\
\text { BISMUIH }\end{array}$ & $\begin{array}{l}4.174001 \\
9.145+02 \\
9.149+02\end{array}$ & $\begin{array}{l}1.943004 \\
2.883-01 \\
2.885-01\end{array}$ & $\begin{array}{l}4.174=01 \\
9.145+02 \\
9.149+02\end{array}$ & $\begin{array}{l}1.219-01 \\
2.163+03 \\
2.103+03\end{array}$ & $\begin{array}{l}4.174001 \\
9.145+02 \\
9.149+02\end{array}$ & $\begin{array}{l}0.058004 \\
1.195+00 \\
1.195+00\end{array}$ \\
\hline ALL ELEMENTS & $2.5 \cup 7+04$ & $1.100+00$ & $2.507+04$ & $0.064+04$ & $2.507+04$ & 9.100000 \\
\hline
\end{tabular}




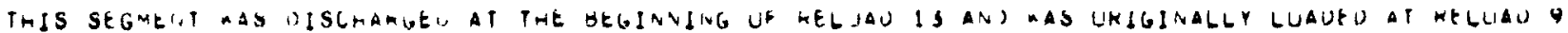
NEUTRUN SIJURE IN NIOEC HER F.E. AI TIME S YEAHS ARTER DISCMARGE

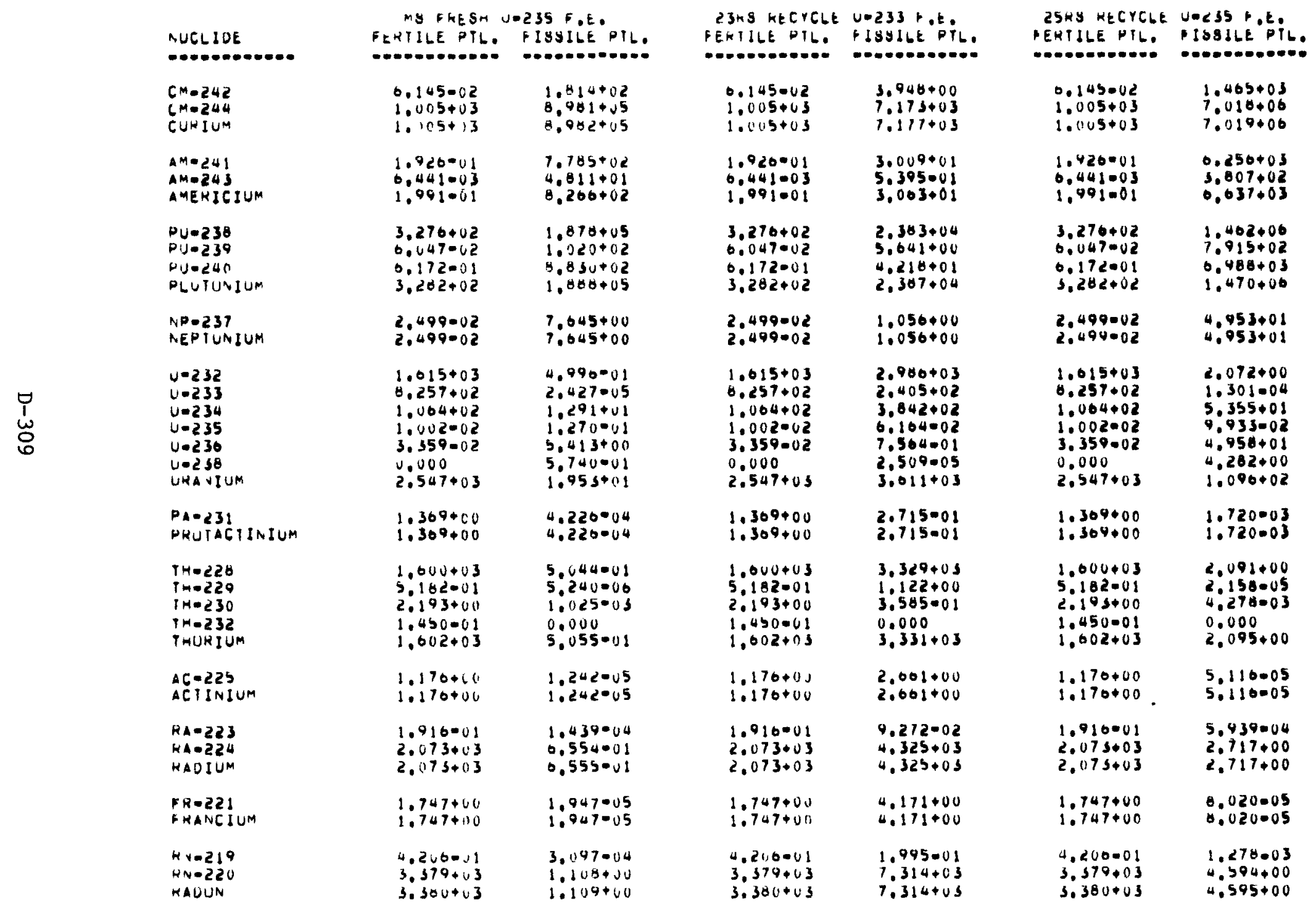




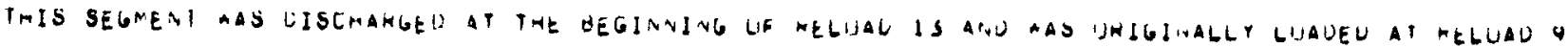

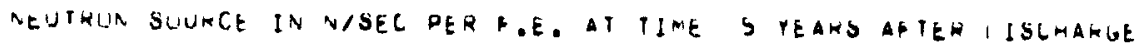

\begin{tabular}{|c|}
\hline $\begin{array}{l}A T-217 \\
A T-216 \\
A S T A T I N E\end{array}$ \\
\hline $\begin{array}{l}P U=210 \\
P O-212 \\
O J=213 \\
P U-215 \\
P U-216 \\
P U L U N I U M\end{array}$ \\
\hline $\begin{array}{l}H I-211 \\
\forall I-212 \\
b I S M U I n\end{array}$ \\
\hline
\end{tabular}

MS FRESM U-235 \&.E. FEHIILE PIL. FISSILE PTL.

\section{$3.121+60$}

$3.189-05$ PETILE PIL. FISSILE PIL.

$3.121+00$ 0.000
3.1840 .05

\section{$3.121+0.5$}

$1.170-05$

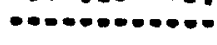

$1.172=02$

0.000

$1.095+04$

7.034000

$2.085+00$

0.400005

$0.809=01$

$4.867=04$

$1.585+u 4$

$1.515+00$
$4.198+00$

$3.915-01$

2.878004

$1.039+03$
$1.039+03$

$3.277-01$

$3.121+00$

\section{$0.832+00$}

1.813 .06

$1.172 \cdot 62$

$9.095+04$

0.809001

$4.889+03$

$1.505+04$

$032+00$

0.000

$1.773+04$

$1.386+01$

3.136001

$9.987+03$

$3.915=01$

$1.039+03$

$2.773+04$

2SES KECYCLE UE235 F.

FEHTILEPTL. PISSILE'PIL.

ALL ELEMENTS

$2.785+64$

$1.088+06$

$2,783+04$

1.054001

0
$03+03$

$\begin{array}{ll}3.121+00 & 1.314004 \\ 1.176005 & 0.000 \\ 3.121+00 & 1.314004 \\ 1.178002 & 0.000 \\ 1.095+04 & 1.113+01 \\ 7.034+00 & 2.064=04 \\ 0.809001 & 2.008003 \\ 4.889+03 & 0.273+00 \\ 1.585+04 & 1.740+01 \\ 1.915001 & 1.288003 \\ 1.039+03 & 1.358+00 \\ 1.039+03 & 1.360+00 \\ & \\ 2.783+04 & 8.496+06\end{array}$

$7.957+04$

2.785000

$.496+06$ 


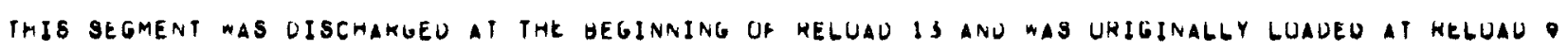

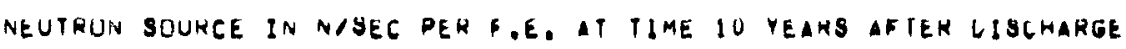

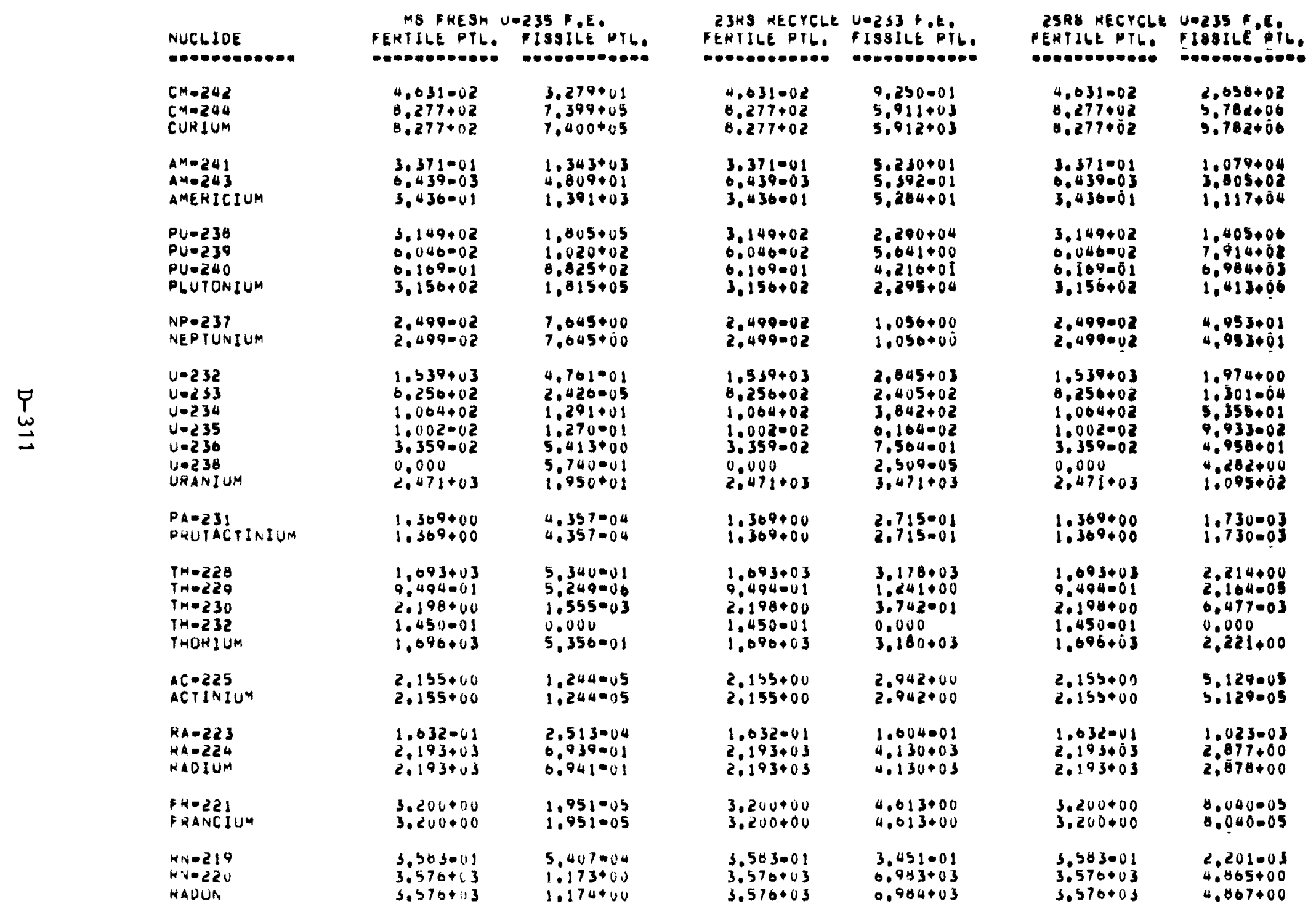




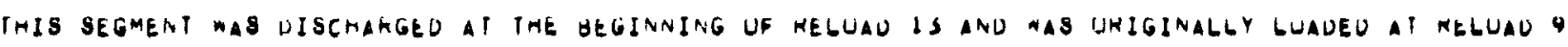
NeUTRUA SUUKCE IN N/SEC PER P. E. AT TIME IO YEAKS AFTER DISCHARGE

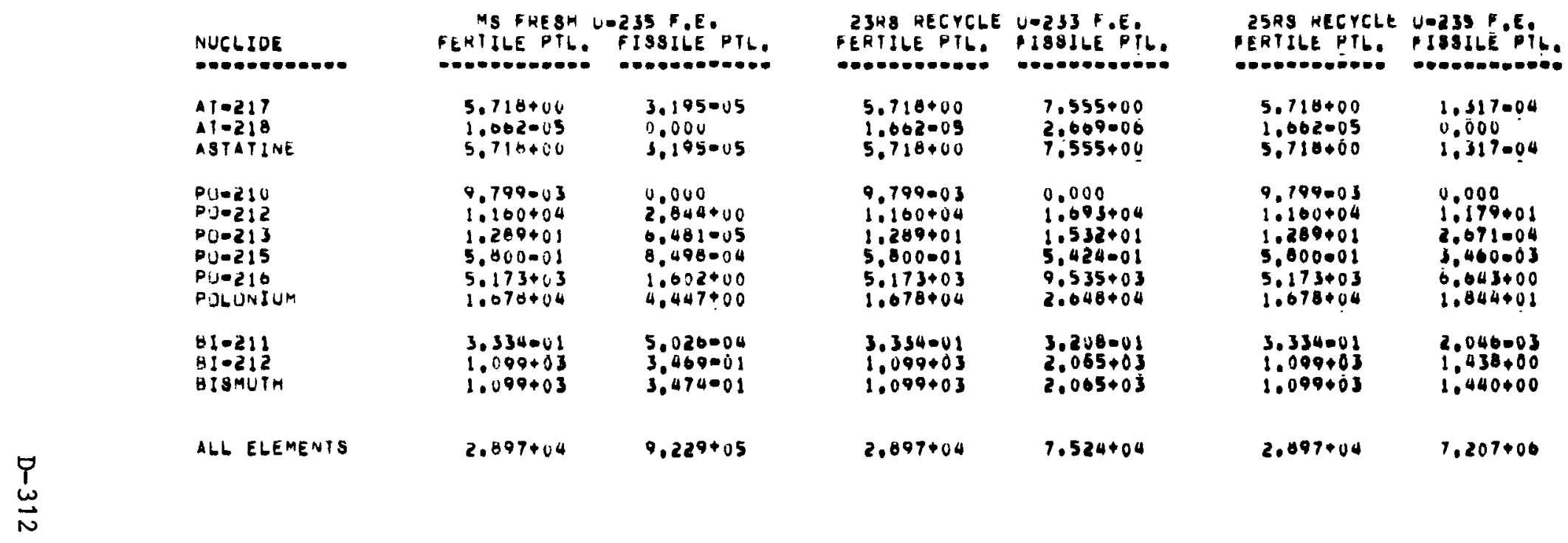


IMPORTANT ISOTOPIC CONTRIBUTORS

TO DECAY HEAT, GAMMA DOSE RATES, AND NEUTRON SOURCE STRENGTHS 
-

-

0 
TABLE D-13

IMPORTANT ISOTOPIC CONTRIBUTORS TO DECAY HEAT

\begin{abstract}
NOTE: Table 13 consists of 20 pages of computer print-out (pages D-315 through $\mathrm{D}-334$ ).
\end{abstract}

CONTENTS OF TABLE D-13

Fuel Elements discharged at the beginning of reload 1 and origi-

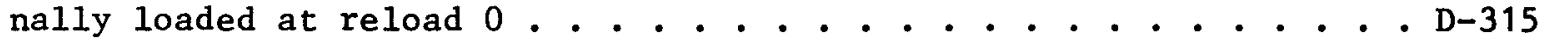
Fuel Elements discharged at the beginning of reload 5 and originally loaded at reload 1.............. . . . . . . . . . . . . . . . Fuel Elements discharged at the beginning of reload 10 and origi-

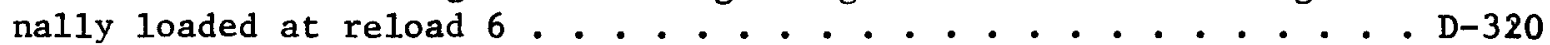
Fuel Elements discharged at the beginning of reload 13 and originally loaded at reload 9... . . . . . . . . . . D-325 
-

-

0 


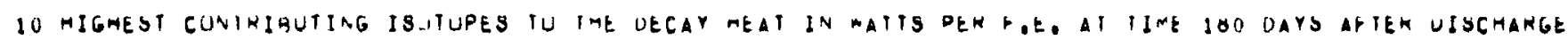

(PERCENI CUNTRIGUTIUN TU TJTAL HEATING FAIE IV PAREVTMESES)

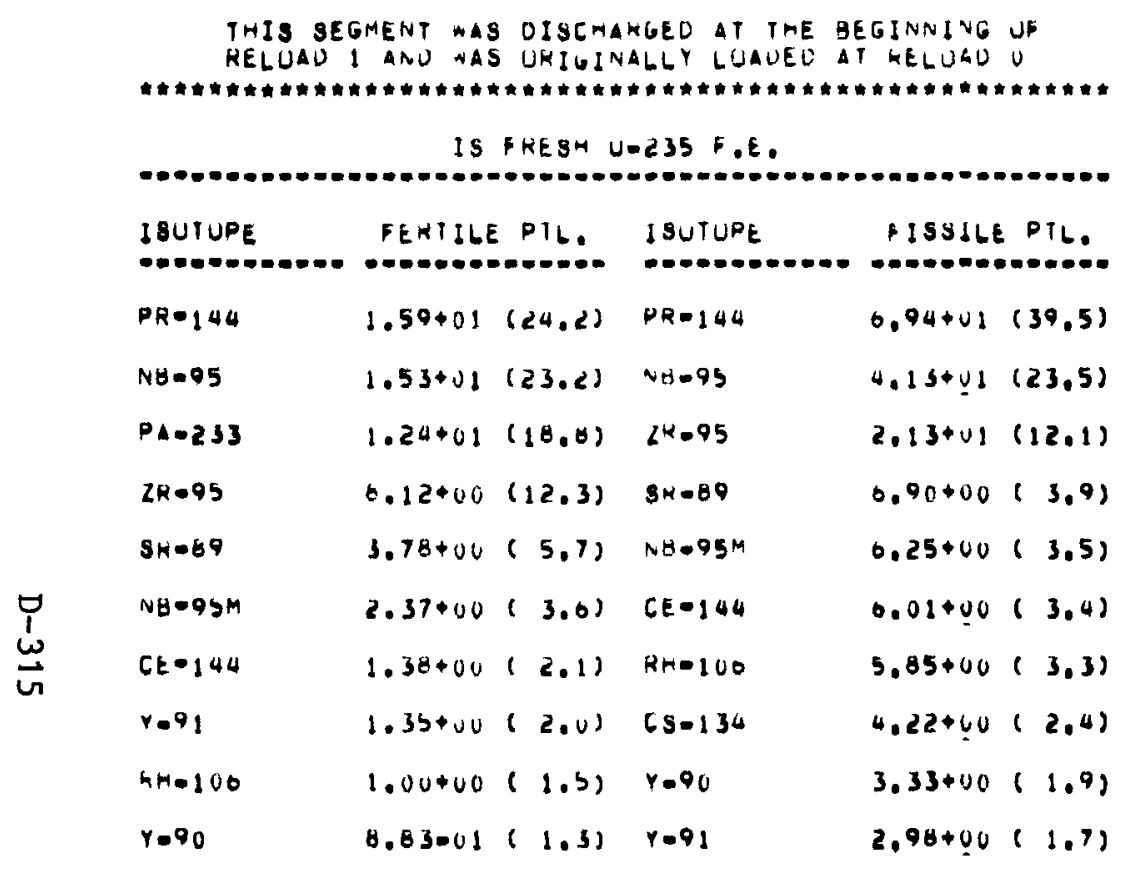

\begin{tabular}{|c|c|c|c|c|}
\hline 1 SUTUPE & 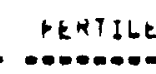 & erth. & I SUTONE & FISYILE PIL, \\
\hline$P A=144$ & $0.00+01$ & $(30.0)$ & PKoI\&4 & $4.81+01(30,3)$ \\
\hline$v \forall-95$ & $3.35+01$ & $(17.5)$ & $\csc 134$ & $3.29+01(24.8)$ \\
\hline $2^{2}-95$ & $1.73+01$ & $(0.1)$ & $r=90$ & $9.05+00(0.0)$ \\
\hline$c 9-134$ & $1.45+01$ & $(7.0)$ & NE-95 & $0.71+00(0.0)$ \\
\hline$P 4=233$ & $1.0<+01$ & $(5.4)$ & $B A-137 M$ & $0.37+00(4.0)$ \\
\hline Sheyg & 7.44000 & $(3.9)$ & $K N=100$ & $5.28 \times 00(4.0)$ \\
\hline$r=90$ & $1.59+00$ & $(3.9)$ & $2 K-95$ & $4.47400(5.4)$ \\
\hline$C E-144$ & $5.93+00$ & $(3.1)$ & CE-14U & $4.16+00(3.1)$ \\
\hline$N 8.95 \mathrm{M}$ & $3.07+00$ & $(2.7)$ & PU.230 & $3.60+00(2.7)$ \\
\hline TH=100 & $4.95+00$ & $(2.6)$ & SHEOO & $1,92+00(1,5)$ \\
\hline
\end{tabular}




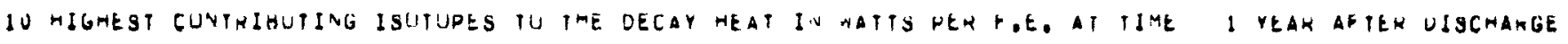
(PERCENT CUNTRIBUTISV TU TUTAL TEATING RATE IV PAKENTMESES)

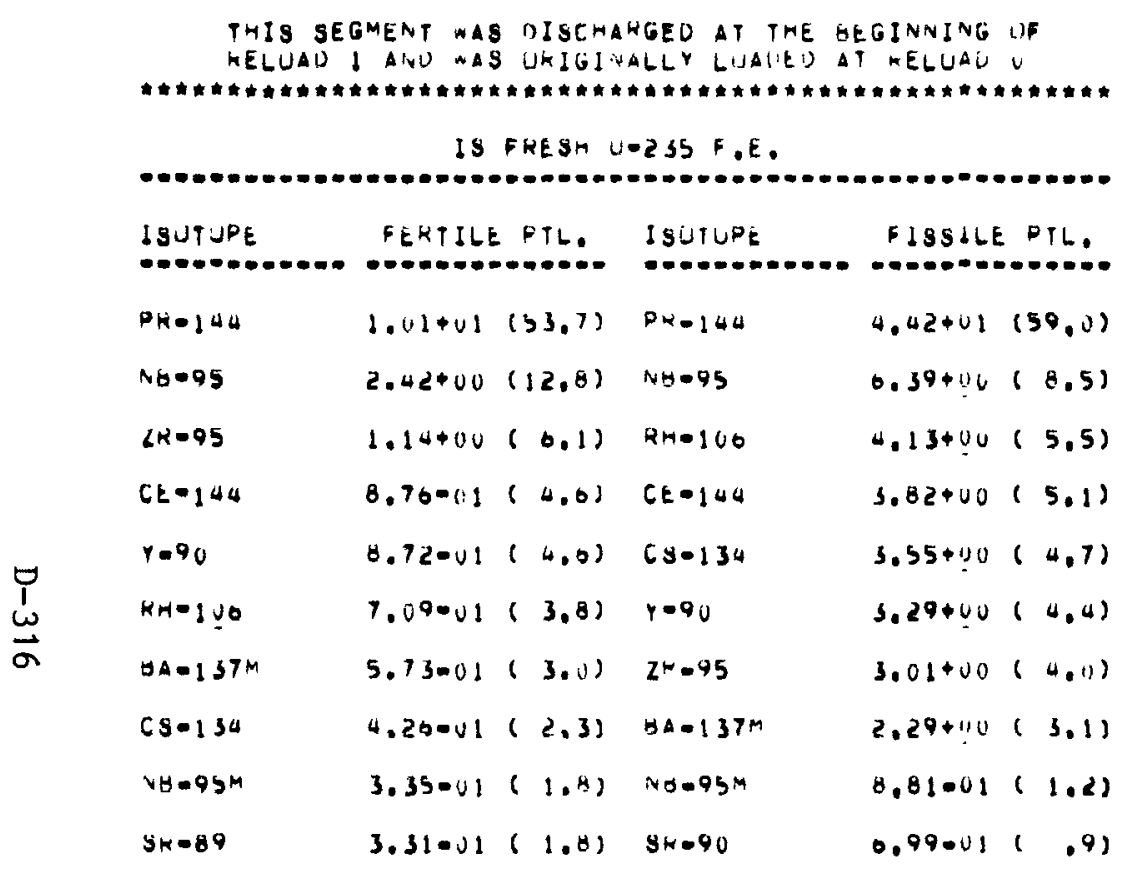

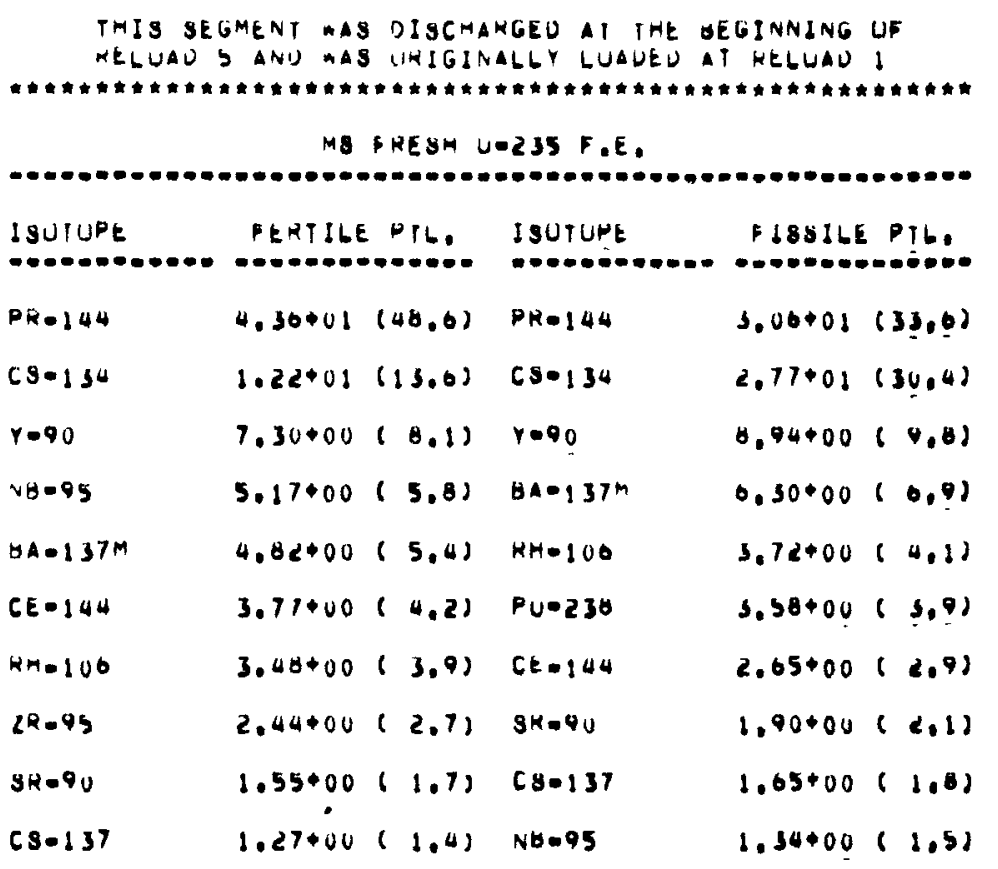




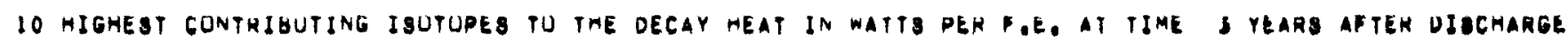

(PEREENI CONTRIBUTIUN TO TOTAL HEATIVG RATE IN PARENTHESES)

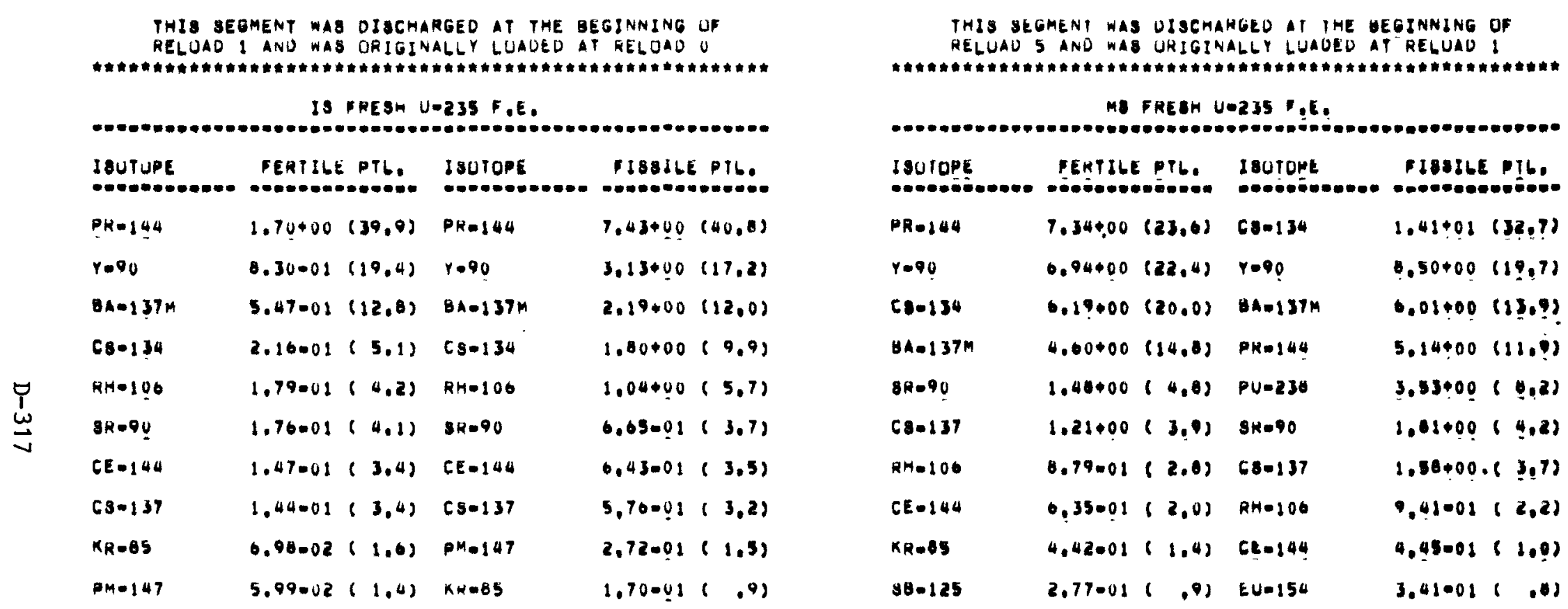


10 MIGHEST CUNTRIBUTING ISUTUPES TO TAE DECAY HEAT IN MATTS DER Fo. AT TIME S YEARS AFTEN UISCMARGE

(PERCENT CONTRIBUTIUN TU TOTAL NEAIJUG RATE IN PAHENTHESEB)

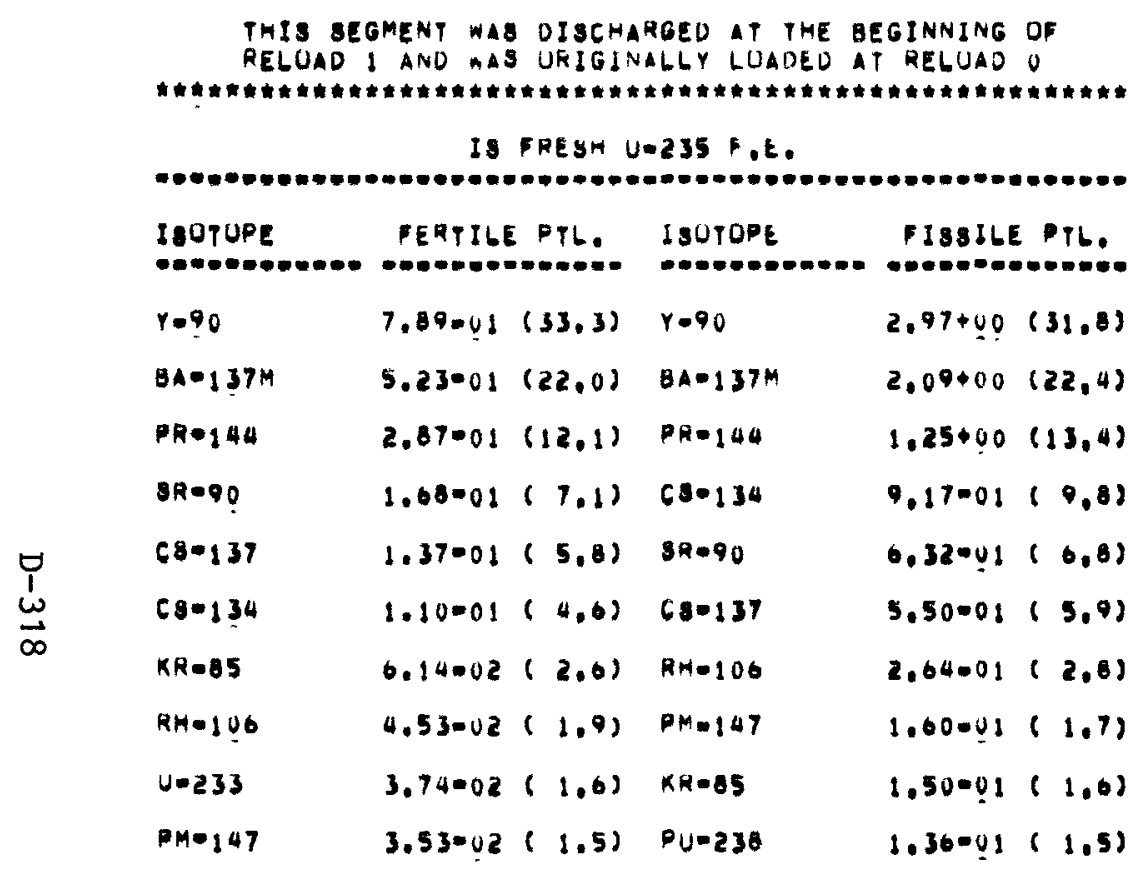

TMIS BEGMENT WAS DIBCHARGEO AT THE GEGINNING OF RELUAU 5 ANO WAS URIGJNALGY LUADEU AT RELUAU 1

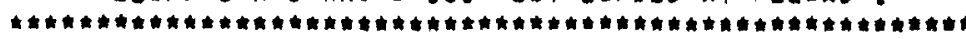

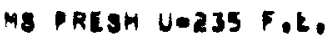

\begin{tabular}{|c|c|c|c|c|c|}
\hline I8UTOPE & PEHTILE & PYL. & 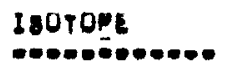 & F188165 & PTL. \\
\hline$Y=0$ & $0.60 \div 00$ & $(33,4)$ & $r=90$ & $0.00+00$ & $(2 ! \cdot 1)$ \\
\hline$B A=137 M$ & $4.40 \bullet 00$ & $(22,2)$ & $\operatorname{cse} 134$ & $7.16 \div 00$ & $(24,0)$ \\
\hline $68 \cdot 134$ & $3.15+00$ & $(15.9)$ & $94-137 m$ & $5.74+00$ & $(10,2)$ \\
\hline$S R=90$ & $1.40+00$ & $(7.1)$ & $P \cup=0236$ & $3.47 \div 00$ & $(11,0)$ \\
\hline$P R=144$ & $1.23+00$ & $(0.2)$ & $8 k=90$ & $1.72+00$ & $(5.6)$ \\
\hline $\cos 137$ & $1.10+00$ & $(5,0)$ & $68-137$ & $1.51 \div 00$ & $(0.1)$ \\
\hline$K R=85$ & $3,80.01$ & $(2.0)$ & $P K=144$ & $0.05=01$ & $(2,0)$ \\
\hline$R H=106$ & $2.22=01$ & $(1.1)$ & EU-154 & 3.120 .01 & $(1.00)$ \\
\hline $89-125$ & 1.60001 & $(.0)$ & $K H=85$ & 2.19001 & 1.01 \\
\hline$P M \bullet 147$ & 1.39001 & .71 & RHE 106 & $2.30-01$ & .01 \\
\hline
\end{tabular}




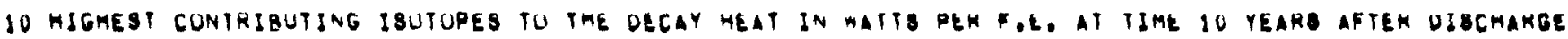

(PERCENT CUNTRIBUTIUN TO TOTAL MEAIING hatL IN PARENTMESES)

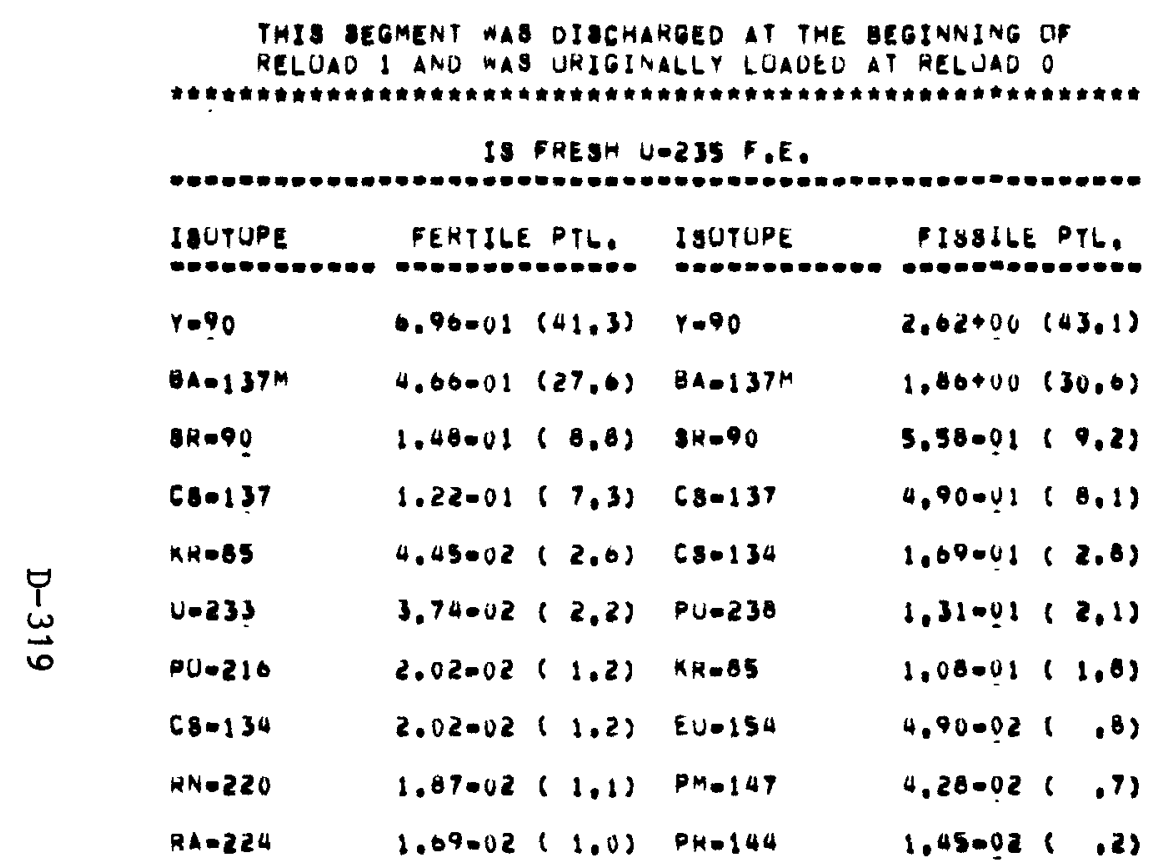

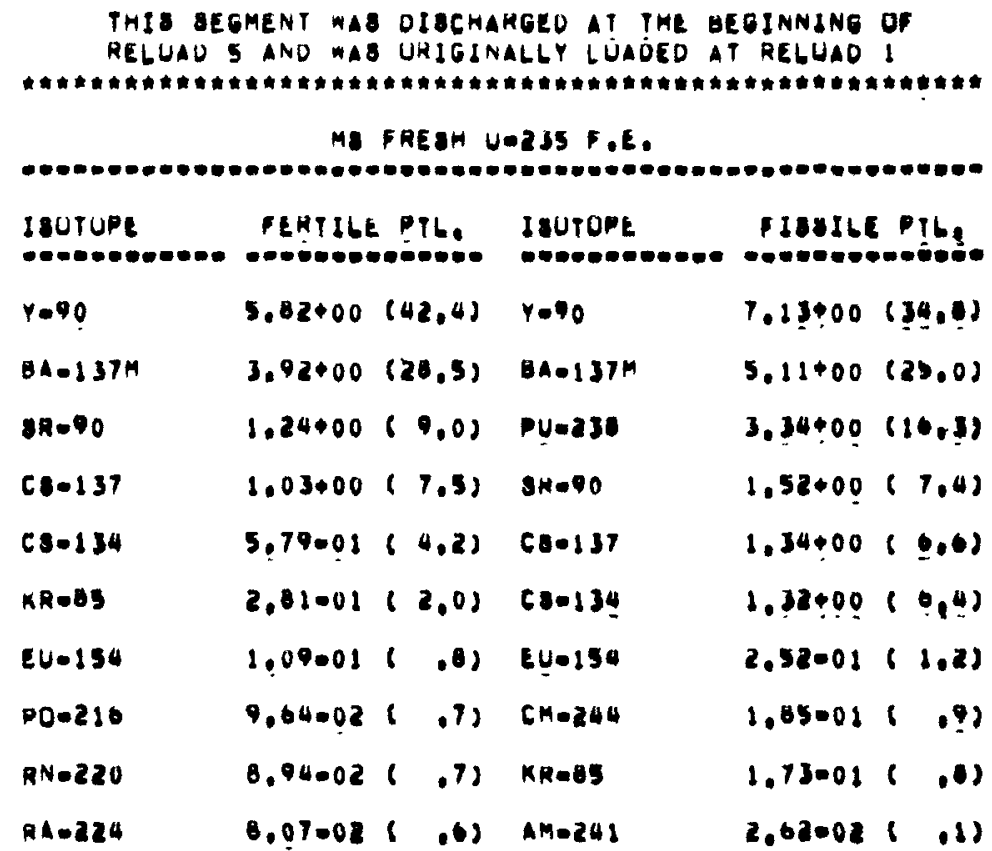


10 HIGHEST CUNTKIGUTING ISUTUPES TU THE DECAY GEAT IN WATTS PEH FoE. AT IIME IOO DAYS AFTEN UISGHARGE

(PERCENT CUNTRIBUTIUN IU TUTAL MEAIING HATE IN PARENTHESES)

IHIS GEGMENT WAS DISCHAKGEO AT THE BEGINNING DF RELUAO 10 AND MAB OHIGINALLY LOADED AT HELUAD 6

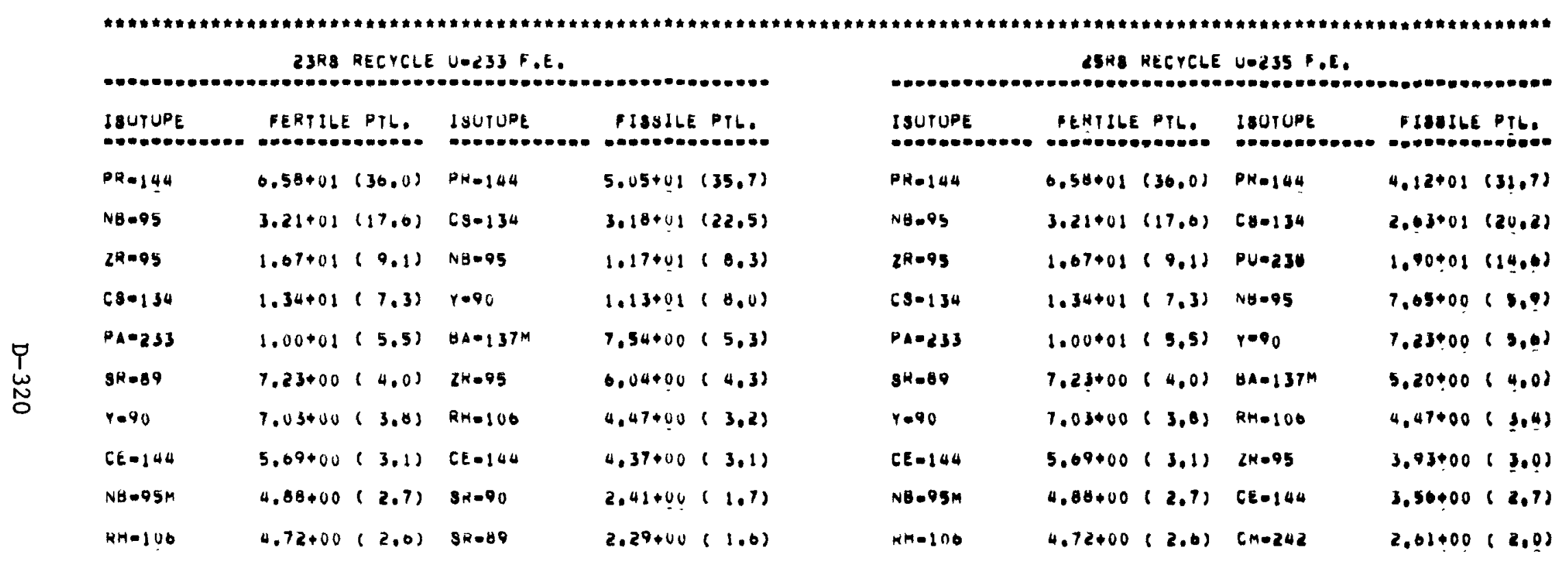




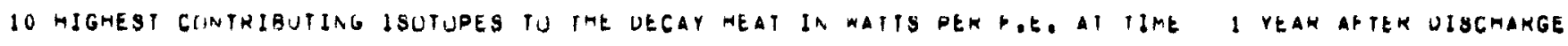

(PEKCENT CUNTRIOUTIUN TU TUTAL MEAIING RAIE IN RAKETTMESES)

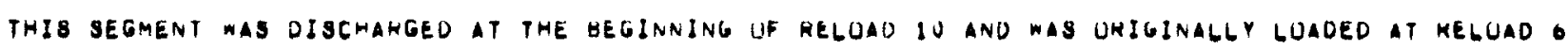

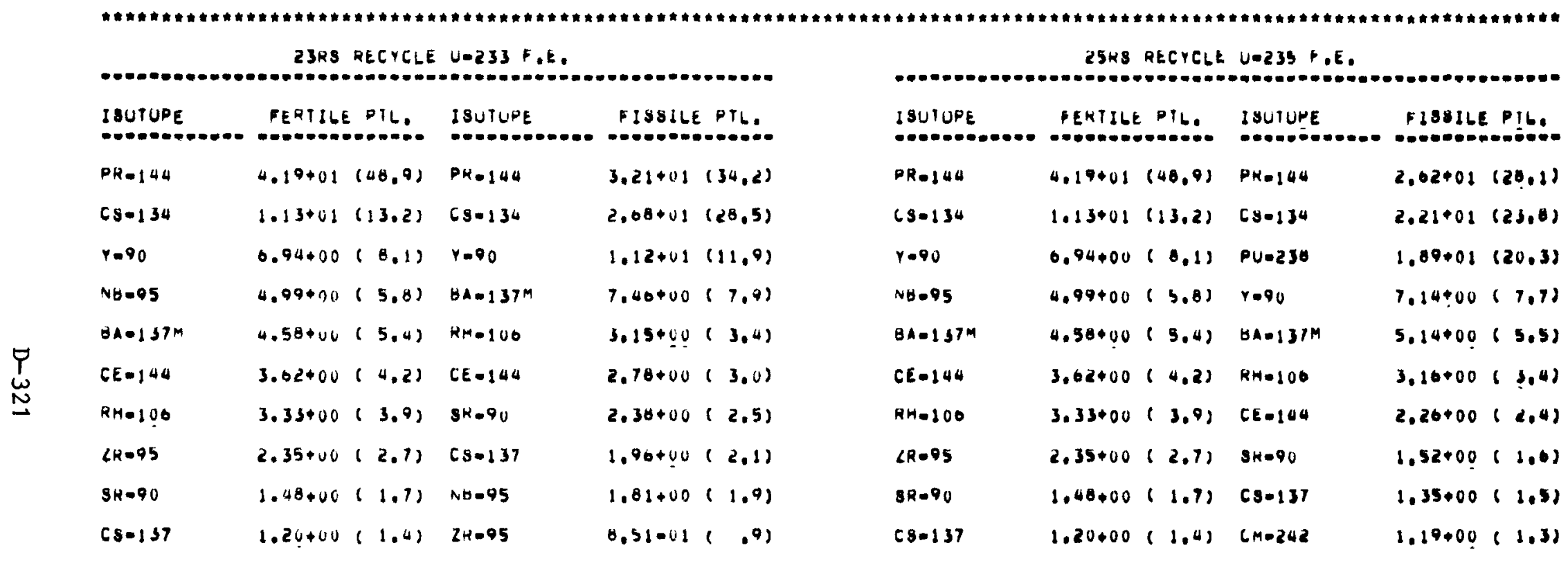




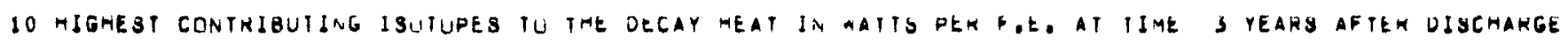
(DERCEAT CUNTRIOUTIUN TU TUTAL MEATING RATE IN PARENTHEBES)

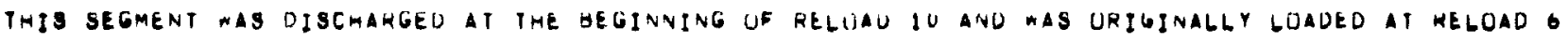

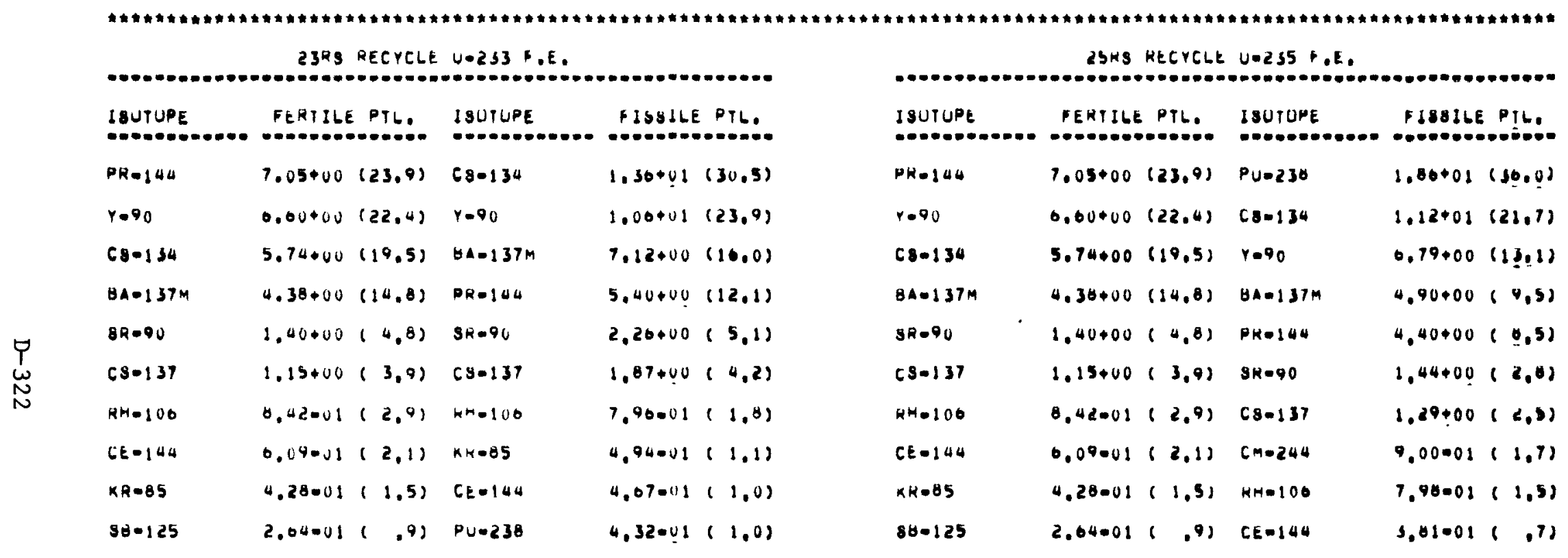




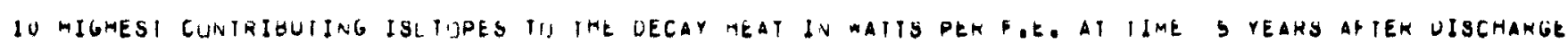
(PERCENT CUNTKIQUTJUN IU TUTAL REAIING KAIE IN PARENTHESES)

THIS SEGMENI MAS DISCMARGEU AT THE BEgINNING UF HELUAU IU ANO MAS URIGINALLY LOADED AT KELUAD 6

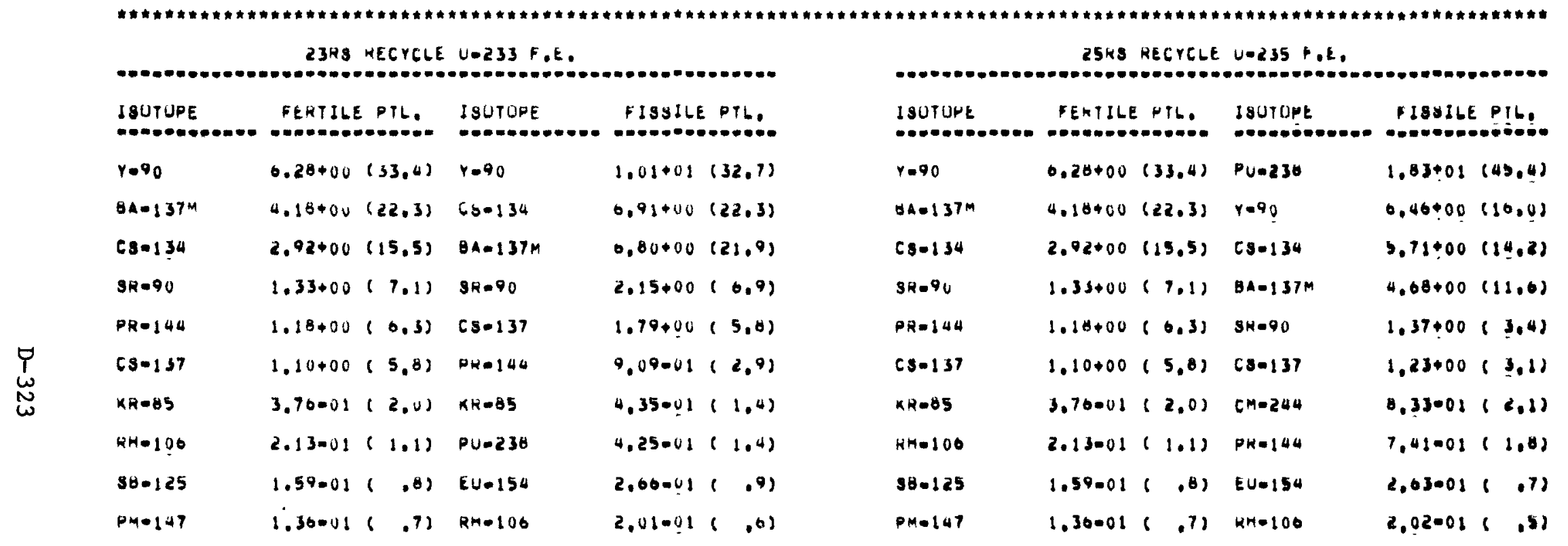




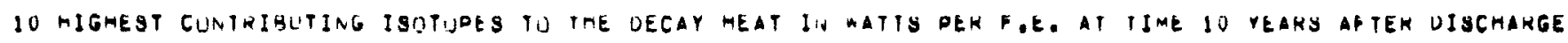

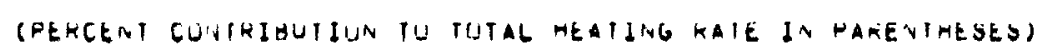

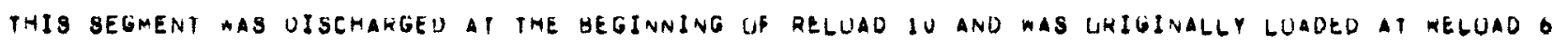

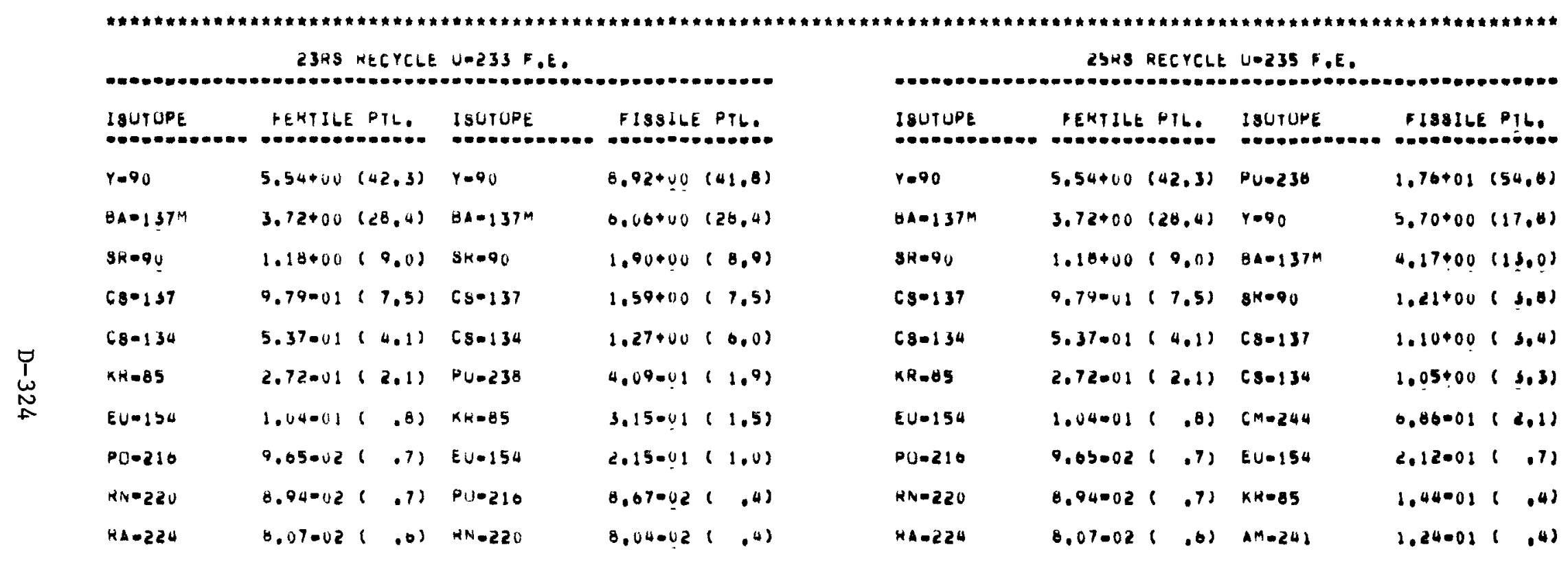


10 HIGHEST CUNIRIBUTING ISUTUPEB TO TME DECAY MEAT IN WATTS PER Fe. AT TIME 180 DAYS AFTEN UIYCHAKGE

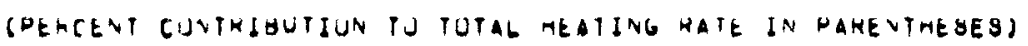

TMIS AEGMENT WAS OISCHAHGED A THE BEgINNING UF RELOAD IJ ANO WAS UKIGINALLY LOADEO AT HELUAD

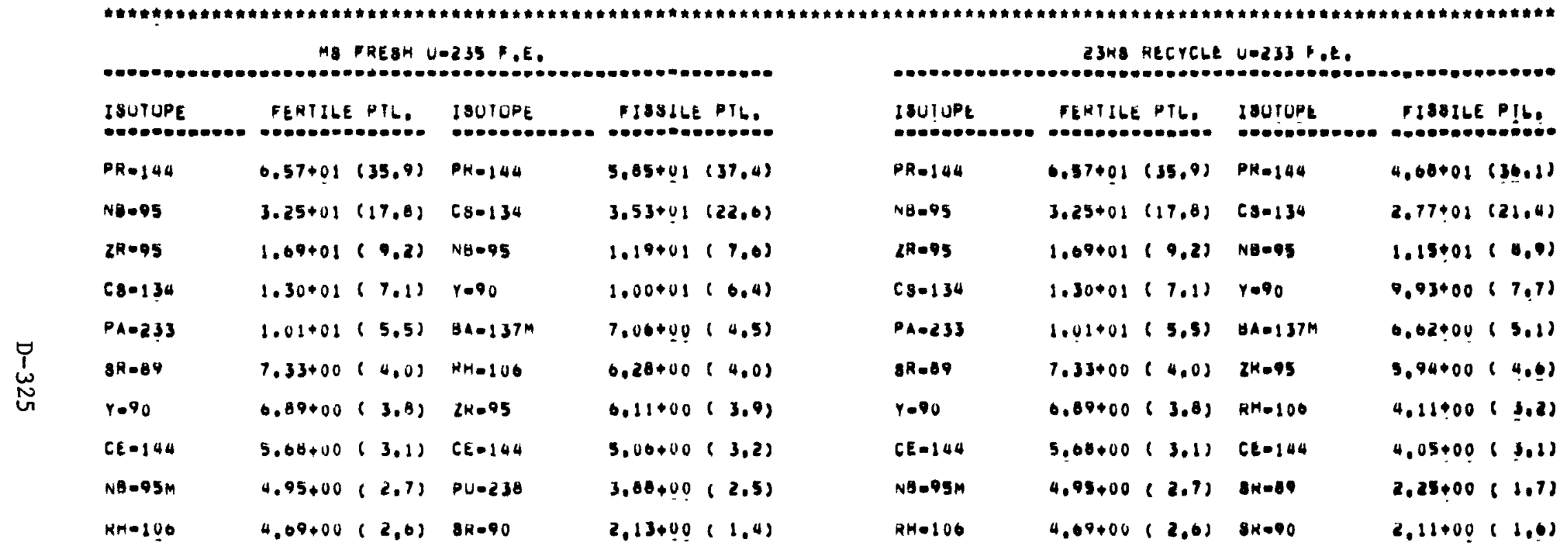




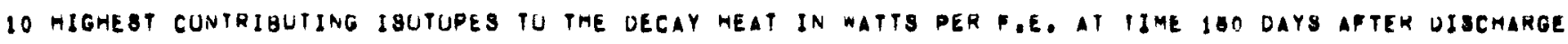
(PEKCENT CONTRJUUTIUN TO TUTAL MEATING HATE IN HAMENTHESES)

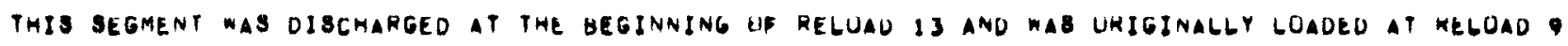

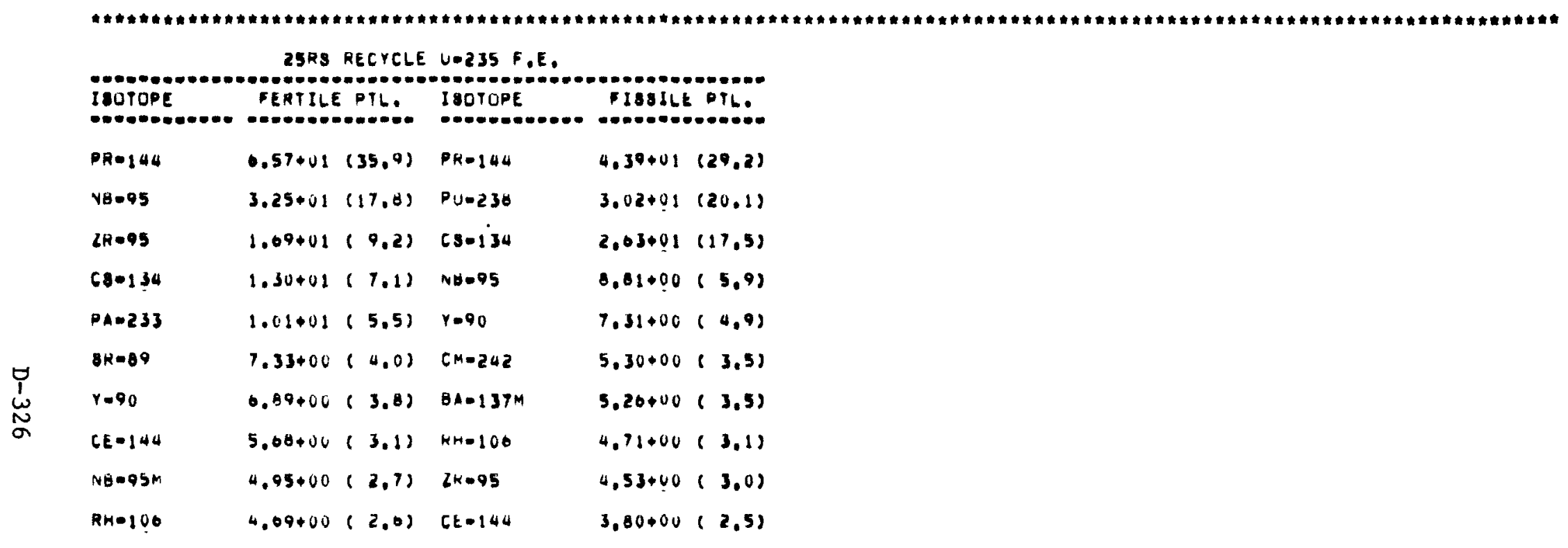




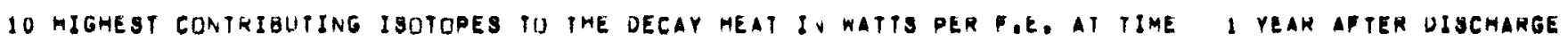

(PERCENT CONTRJUUTIUN TU TOTAL MEATING Rate IA PARENIHESES)

THIS BEgMENT MAS UISCHAhGEU AT THE BEGINNING OF RELOAD 13 ANO WAS URIGINALLY LUADEU AT hELUAD 9

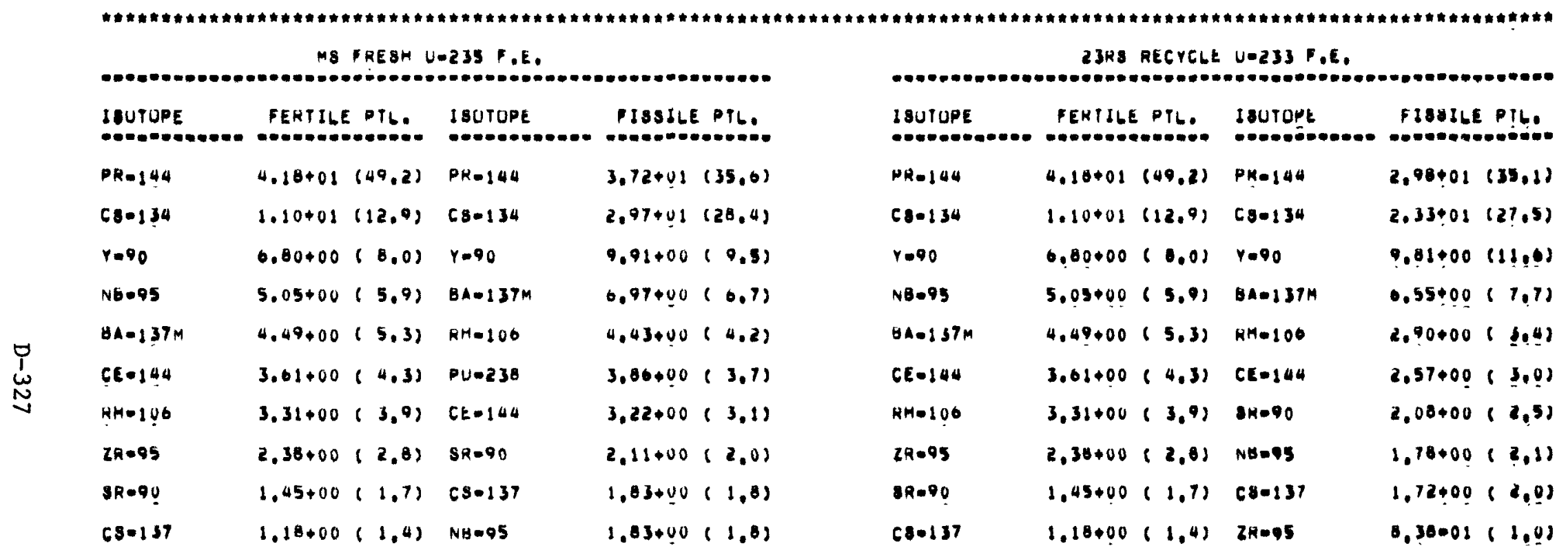




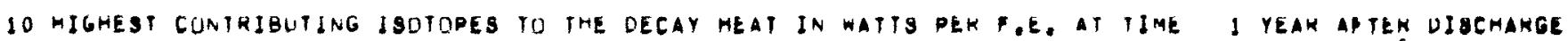
(PERCENT CUNTKIBUTIUN TO TUTAL heating hate in Pahentheges)

THIS SEgMENT HAS DISCHARGED AT THE BEgINNING OF RELUAD 13 AND WAS URIGINALLY LOADED AT KELUAD

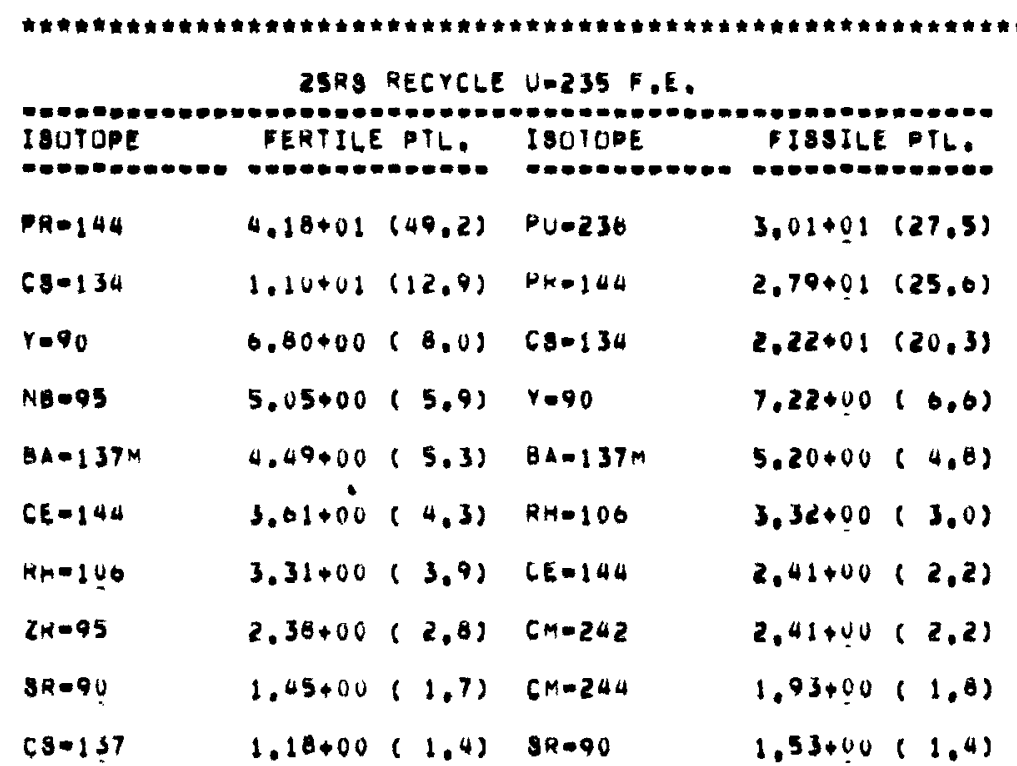




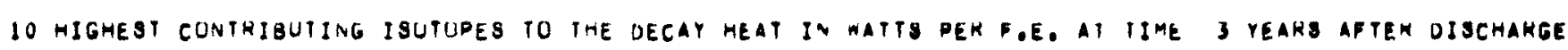
(PERCENT CUNTRIBUTIUN TU TUTAL HEAIJNG Rate IN PANENIMESES)

THIS SEGMENT WAS DISCMAKGEO AT TME HEGINNING OF RELOAD IS AND MAS UNIGINALLY LUADED AT RELOAD

\begin{tabular}{|c|c|c|c|c|c|c|c|c|}
\hline \multicolumn{4}{|c|}{ MS FRESH UA235 ..E. } & \multicolumn{5}{|c|}{$23 H 8$ RECYCLE UA233 F.E. } \\
\hline I8UTUPE & FERTILE PTL. & ISUTUPE & Flo81LE PTL. & 280TUPL & $\begin{array}{l}\text { FERTILE } \\
\text { OOEOEOE }\end{array}$ & E PTL. & I80TUME & FISALEE PIL. \\
\hline$P A-144$ & $7.03+00(24.2)$ & $C 8-134$ & $1.51+0.1(31.5)$ & $P R=144$ & $7.03+00$ & $(24.2)$ & $C 8=134$ & $1.10+01(30.0)$ \\
\hline$Y=90$ & $6.47+00(22.3)$ & $r \circ 90$ & $9,42+00(19,6)$ & $r \circ \theta_{0}$ & $0.47+00$ & $(22,3)$ & $r=90$ & $9.35 \div 00 \quad(2 \leq .6)$ \\
\hline$c 5 \cdot 134$ & $5.57+00(19.2)$ & $\theta A-137 M$ & $0.060 .00(13.9)$ & $68-134$ & $5.57+00$ & $(19.2)$ & $8 A-137 M$ & $0.25+00(18.9)$ \\
\hline$B A=137 M$ & $4.29+00(14.8)$ & $P k-144$ & $0,20+00(13,1)$ & $y A-157 \mathrm{~m}$ & $4.2^{9}+00$ & $(14,8)$ & PK=144 & $9.01400(12.7)$ \\
\hline$s R=9 \underline{0}$ & $1.38+00(4.7)$ & $P U=230$ & $3.80+00(7.9)$ & $8 R=90$ & $1.38+00$ & $(4.7)$ & $8 K=90$ & $1.98+00(5,0)$ \\
\hline$C 8-137$ & $1.13+00(3.9)$ & $8 \pi-90$ & $2.00000(4.2)$ & $68-137$ & $1.13+00$ & $(3.9)$ & $C 8-137$ & $1.04 \div 00(4.2)$ \\
\hline$R M=106$ & $8.37001(2.9)$ & $60-137$ & $1.75+00(3.6)$ & $A H=106$ & 0.370 .01 & $(2.9)$ & An-106 & $7.34001 \div 1,97$ \\
\hline$C E-144$ & $0.08 .01(2.1)$ & $R H=100$ & $1,12+00(2,3)$ & CE. 144 & 0.080 .01 & 12.11 & $P U=230$ & $4.02-01(1.2)$ \\
\hline$K R=85$ & $4.23 .01(1.5)$ & $C E-144$ & $5.410 .01(1.1)$ & KR.AS & 4.250 .01 & $(1.5)$ & $K R=.85$ & $0.43-01(1.1)$ \\
\hline$S B \cdot 125$ & $2.01 .01(.9)$ & $E \cup-154$ & $3.87 .02(.0)$ & $s 8-125$ & 2.01001 & $(.9)$ & $C E=144$ & $4.33001(1.1)$ \\
\hline
\end{tabular}




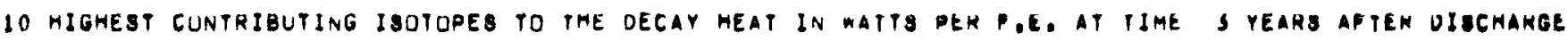
(PERCENI CONTKIGUTJUN TO TUTAL MEATING KaIE IN PARENTMESES)

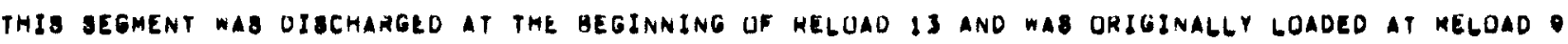

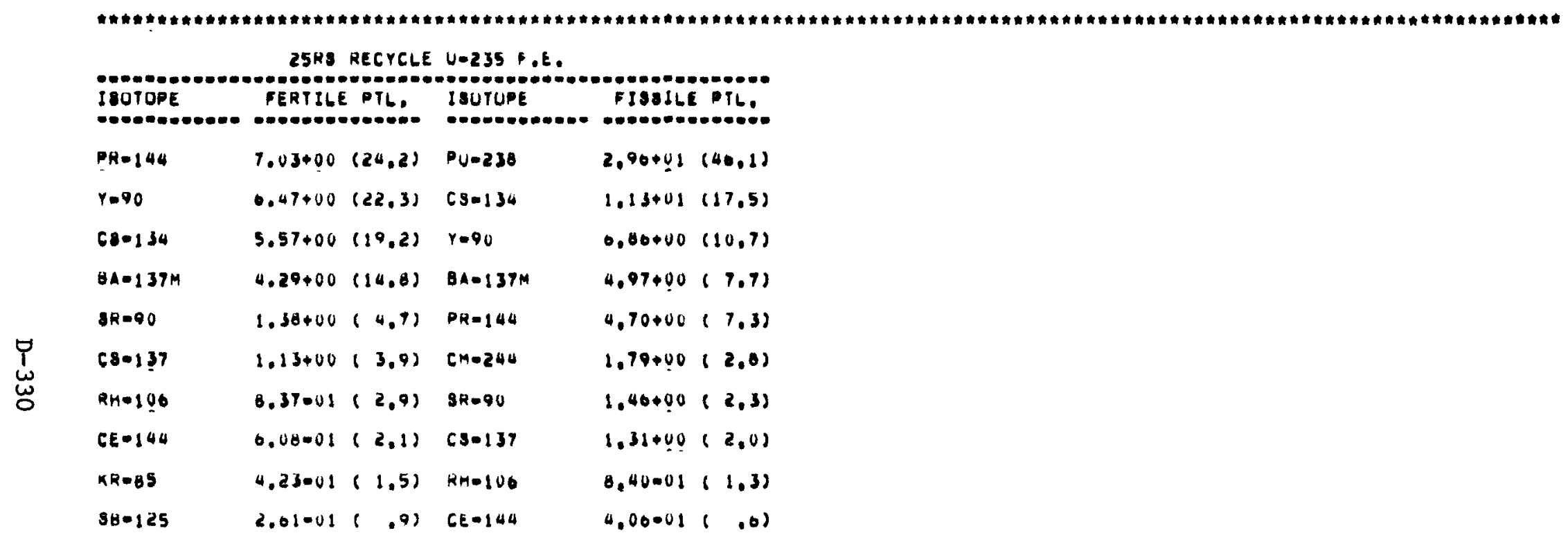


10 HIGHEST CONTRIBUTING ISUTUPES TU THE DECAY MEAT IN WATTS PER Fo. AT IIME S YEAKS APIEN UISCHARGE (PERCENT CUNTKIGUTIUN IL TUTAL MEATING RAIE IN HAKENTMESES)

THIS SEGMENT HAS DISCHARGEO AT THE BEGINNING UR RELUAD I3 AND WAS UKIGINALGY LOADED AI KELOAD

\begin{tabular}{|c|c|c|c|c|c|c|c|c|c|}
\hline IEUTUPE & $\begin{array}{l}\text { FERTILE } \\
\text { WOOWOEGE }\end{array}$ & E 916. & I SUTUPE & FI883LE PTL. & I8UTUPE & PERTILE & PThe. & IsuTupt & Fiskste P16. \\
\hline$Y=90$ & $0.15+00$ & $(33,4)$ & $r=90$ & $0.96+00(27.3)$ & $r=90$ & 0.15000 & $(35,4)$ & $r \cdot 90$ & $0.87+00 \quad(32,5)$ \\
\hline$B A-137 M$ & $4.10+00$ & $(22.2)$ & $\operatorname{css} 134$ & $7.67+00(23,4)$ & $010137 M$ & $4.10+00$ & $(22.2)$ & $c 8-134$ & $0.02+00(21.0)$ \\
\hline $68-134$ & $2.83+00$ & $(15,3)$ & $B A-137 M$ & $0.36+00(19.4)$ & $\operatorname{css} 134$ & 2,83400 & $(15.3)$ & BA-137M & $9.97+00(21.0)$ \\
\hline $3 R=90$ & $1.31+40$ & $(7,1)$ & $P \cup-238$ & $3,74+00(11.4)$ & $8 k-90$ & $1,31+00$ & $(7.1)$ & $8 N-90$ & $1.09+00(0.9)$ \\
\hline PK-144 & $1.18+00$ & $(0.4)$ & Sk-9O & $1.91+00 \quad(5.8)$ & $P R=144$ & $1.18+00$ & $(0.4)$ & $c 8-131$ & $3.57+00(5.7)$ \\
\hline$[3-137$ & $1.08+00$ & $(5,8)$ & $\operatorname{cs}-137$ & $1.67+000(5.1)$ & $c 8-137$ & 1.00000 & $(5.0)$ & PR=144 & $0.42001(3.2)$ \\
\hline$K K=85$ & $3.72=01$ & $(2.0)$ & $P K=144$ & $1.05+00(3.2)$ & $K R-85$ & 3.72001 & $(2,0)$ & $p \cup-230$ & $4.75001(1.7)$ \\
\hline RHel 00 & 2.11 .01 & $(1.1)$ & $E U-154$ & $3.55001(1.1)$ & $R M=106$ & 2.11001 & $(1.1)$ & $K R=85$ & $3.89001(1.4)$ \\
\hline $38 \cdot 125$ & 1.56001 & $(.8)$ & $k+-85$ & $2.83=011.02$ & $88-125$ & 1.56001 & $(.8)$ & $E V-154$ & $2,35001(0)$ \\
\hline$P M=149$ & $1.30-01$ & $.7)$ & $R n=100$ & $2,83-011$ & $P M-147$ & 1.30001 & $.7)$ & $R H=106$ & $1.85=011$ \\
\hline
\end{tabular}




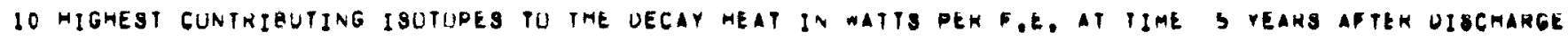

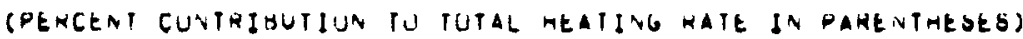

THIS SEGMENT WAS DISCHAHGED AT THE BEgINNING OF RELOAO IJ ANO MAS UKIGINALLY LOADED AT MELUAD

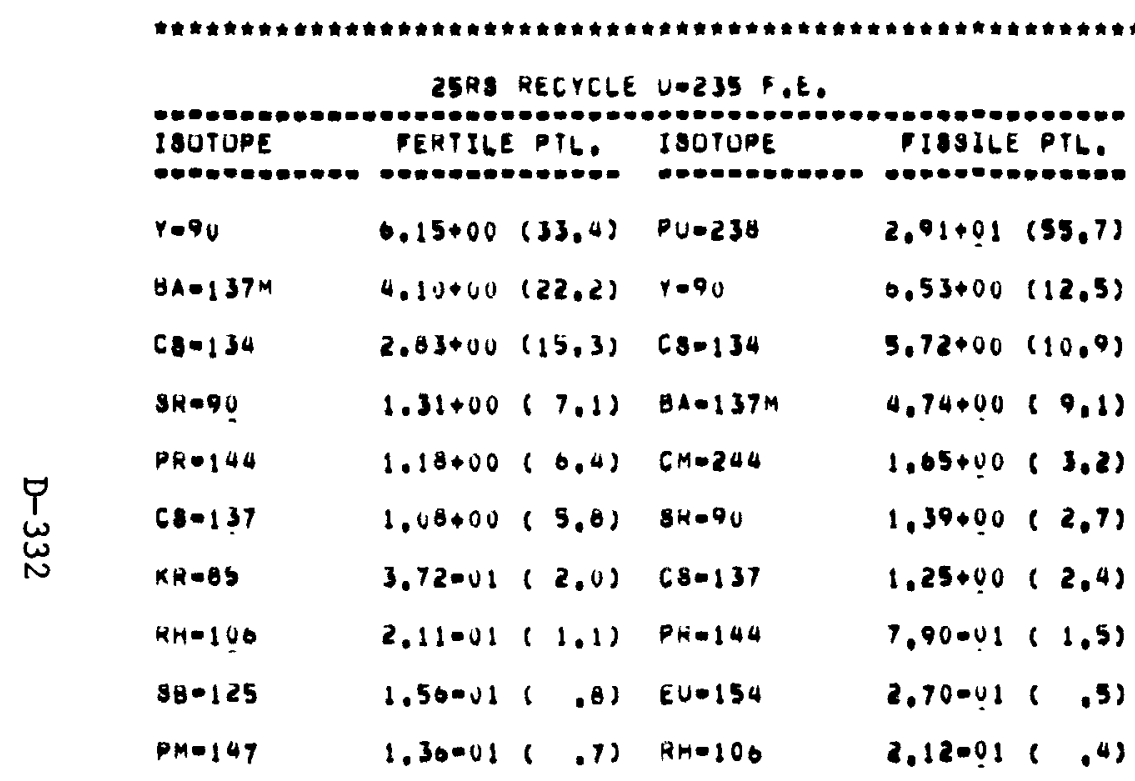




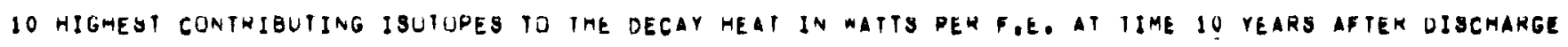

(PERCENi CUNTRIGUTIUN TU TLTAL MEATING RATE J' FAHENTNESES)

THIS SEGMENT MAS DIBCHAKGED AT THE HEGINNING UF RELUAO 13 AND MAS OHIGINALGY LOADEU AT KELOAD 9

\begin{tabular}{|c|c|c|c|c|c|c|c|c|c|}
\hline I8UTUPE & PERTILE & PTh, & ISUTOPE & FI8SILE PTL, & ISUTUPE & $\begin{array}{l}\text { PERTILE } \\
\text { DOOEOOE }\end{array}$ & PTL. & 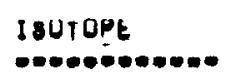 & FISAJLE Ttb. \\
\hline$Y=90$ & $5.43+00$ & $(42.3)$ & $r=90$ & $7.91400(35.1)$ & $Y=\varnothing 0$ & $5.43+00$ & $(42,3)$ & $r \bullet 90$ & $7.03 \div 00(48.4)$ \\
\hline$B A-137 M$ & $3.65+00$ & $(28.4)$ & $B A-137 m$ & $5.67+60(25.1)$ & $9 A-137 m$ & $3.65+00$ & $(20,4)$ & $8 A-137 m$ & $5,32000 \quad(20,1)$ \\
\hline $3 H=90$ & $2.15+00$ & $(9.0)$ & Pu-238 & $3,00000(16,0)$ & $S R=90$ & $1.15+00$ & $(0.0)$ & $9 R=90$ & $1,00+00(0.0)$ \\
\hline$c 8-137$ & 9.00001 & $(7.5)$ & $8 k=90$ & $1.68+000(9.5)$ & 680137 & $9.00=01$ & $(7,5)$ & $\cos 137$ & $1.40+00(7.4)$ \\
\hline $\cos =134$ & 5.20001 & $(4.6)$ & $c s=137$ & $1.40+000(6.6)$ & $\cos 134$ & 5.20001 & $(4.0)$ & $\operatorname{cse} 134$ & $1,12000(0,9)$ \\
\hline$K R=85$ & $2.69-01$ & $(2,1)$ & $\cos 134$ & $1.41+00(0.3)$ & KROES & 2.69001 & $(2,2)$ & Pu-236 & $4.56001(2,0)$ \\
\hline$E U=154$ & 1.01001 & $(.8)$ & $E \cup-154$ & $2.86001(1.3)$ & $E U-154$ & 1.01001 & $(.8)$ & $K R=85$ & $2.82001(1.5)$ \\
\hline$-0 \cdot 216$ & $9.75 \cdot 02$ & .81 & $K K=85$ & $2,05=01(.9)$ & 90.216 & $9.75-02$ & .01 & $E U=154$ & $1.90001(1.0)$ \\
\hline$H N=220$ & $9.04-02$ & .71 & $C M-244$ & $1.74=0.1(.8)$ & $H N=220$ & $0.04=02$ & .71 & $P U=216$ & $9.49002(.5)$ \\
\hline $9 A-224$ & 0.10002 & $(.0)$ & $P M=147$ & $3.150 .02(.1)$ & $14-224$ & 0.10002 & .01 & AM-220 & $0.00002(.5)$ \\
\hline
\end{tabular}




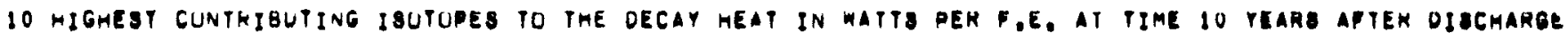
(PEKCENT CONTRIGUTIUN TU TUTAL MEATING RAIE IN PARENTHEgES)

TMIS SEGMENT WAS DISGHAREED AT THE BEgINNING OF RELDAD IJ ANO WAS ORIGINALGY GOADEO AT RELOAD

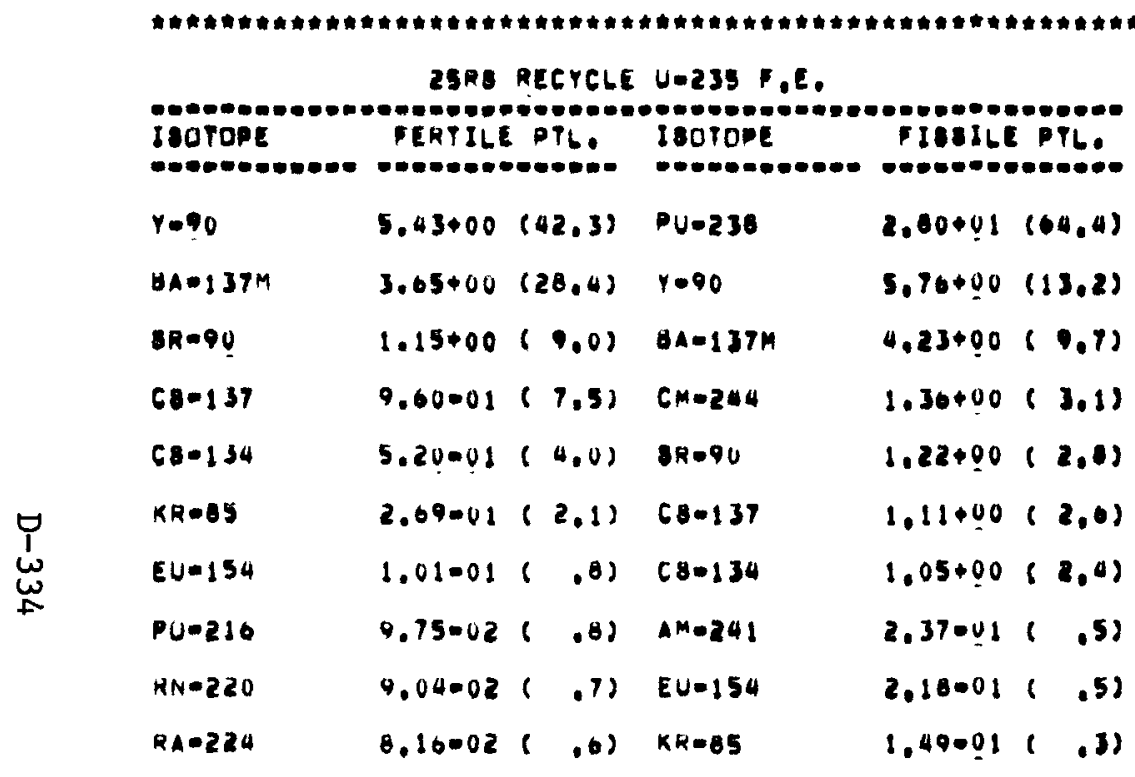


TABLE D-14

IMPORTANT ISOTOPIC CONTRIBUTORS TO GAMMA DOSE RATE

NOTE: Table D-14 consists of 20 pages of computer print-out (pages D-337 through D-356).

CONTENTS OF TABLE D-14

Fuel Elements discharged at the beginning of reload 1 and origi-

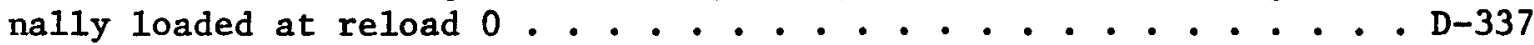

Fuel Elements discharged at the beginning of reload 5 and origi-

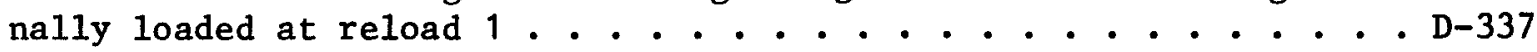

Fuel Elements discharged at the beginning of reload 10 and origi-

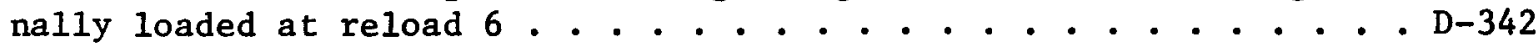

Fuel Elements discharged at the beginning of reload 13 and originally loaded at reload 9... . . . . . . . . . . . D-347 
-

-

0 


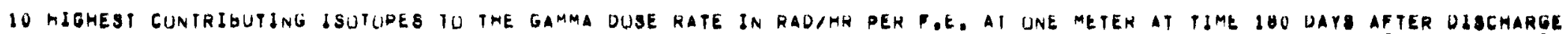
(PERLENT CONTRIOUTIUN TO TOTAL DUSE RATE IN PANENTHEBES)

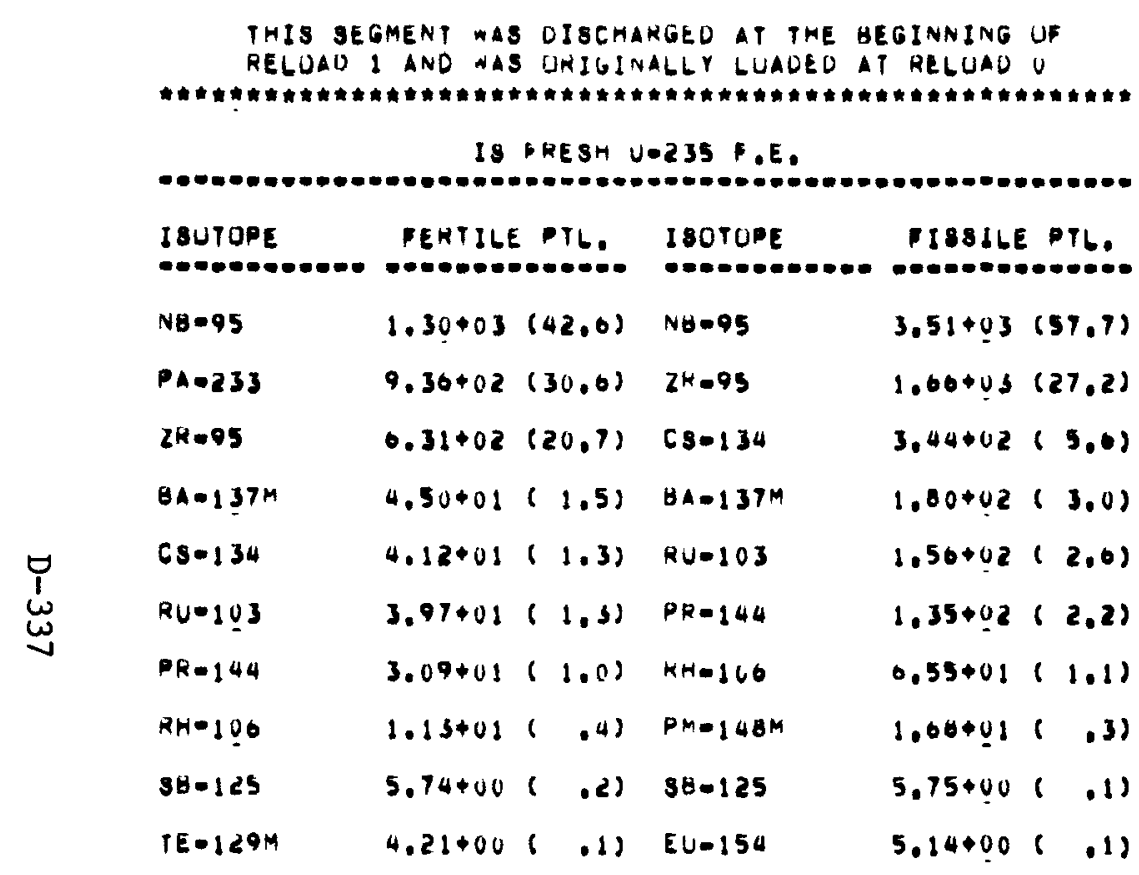

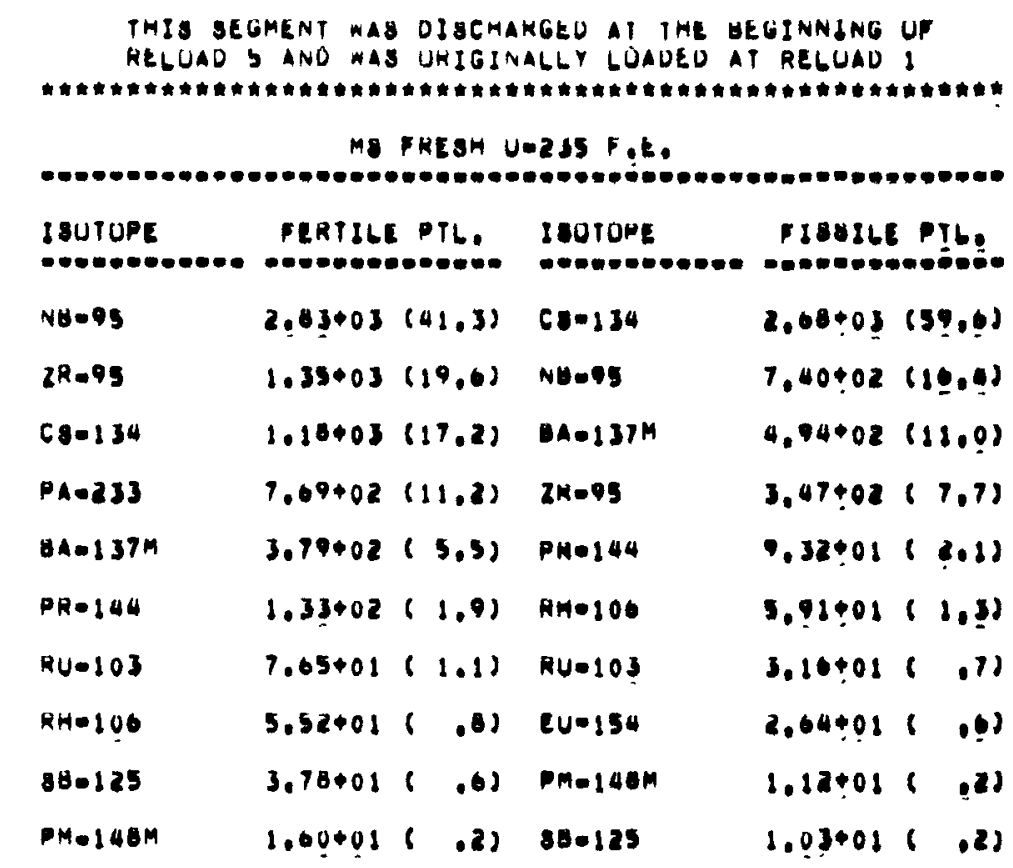




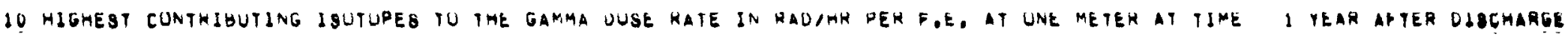
(PERCENT CUNTKIUUTION TU TUTAL DOSE hatE IN PAKENTMESES)

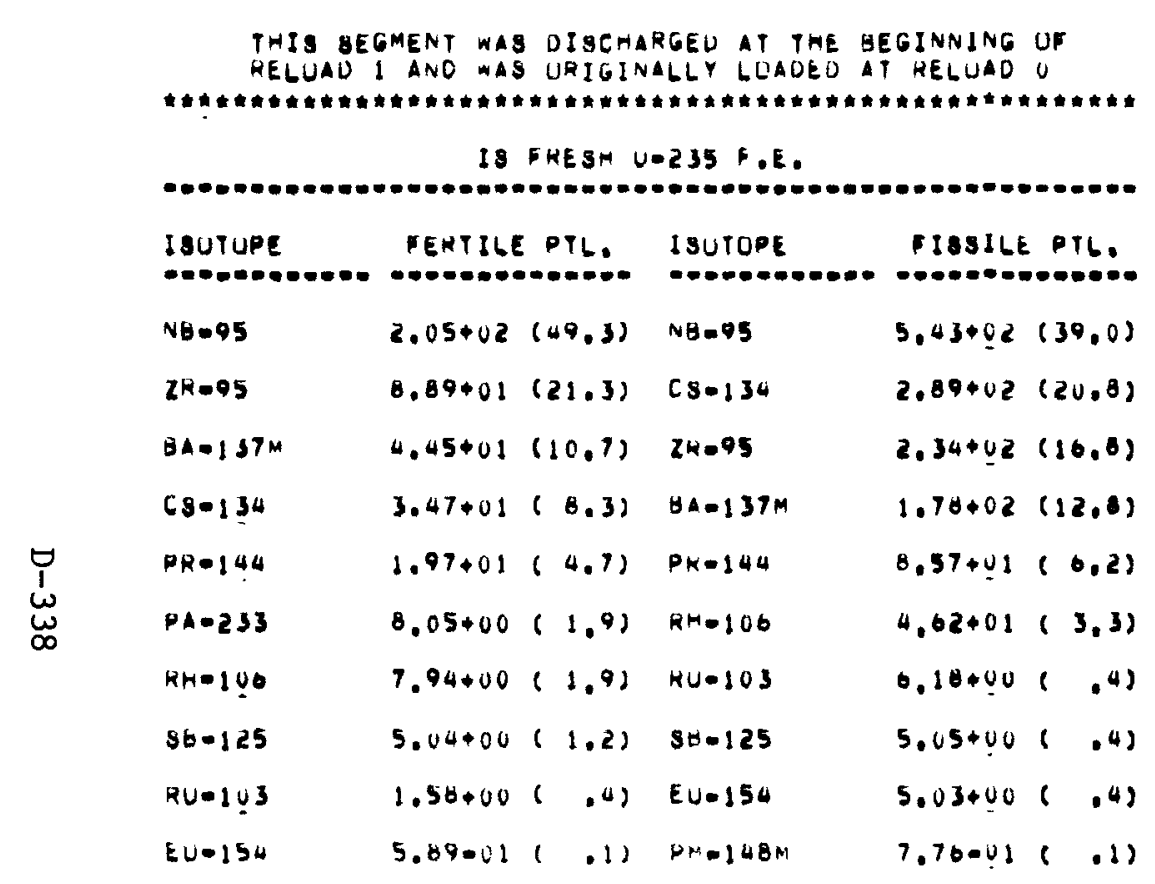

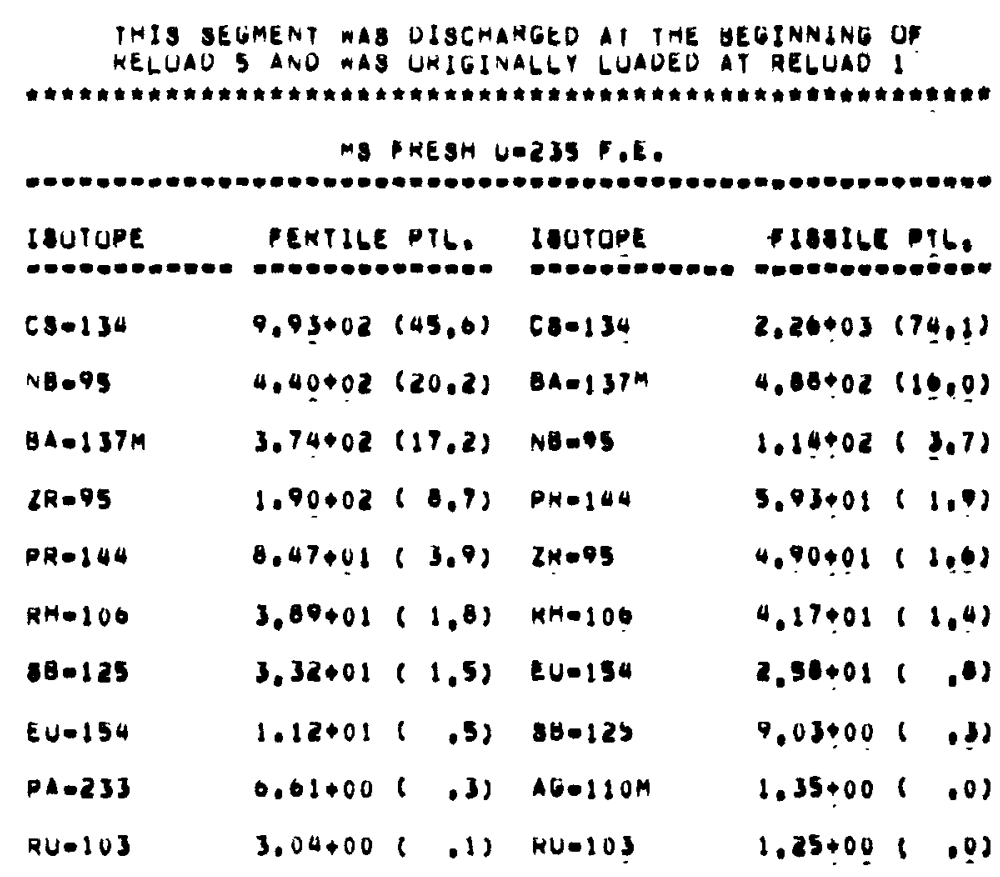




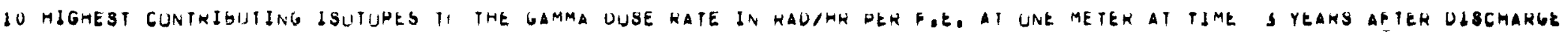
(PERCENT CUNTRJOUTIUN TU TUTAL DUSE RATE IN PARENTHESES)

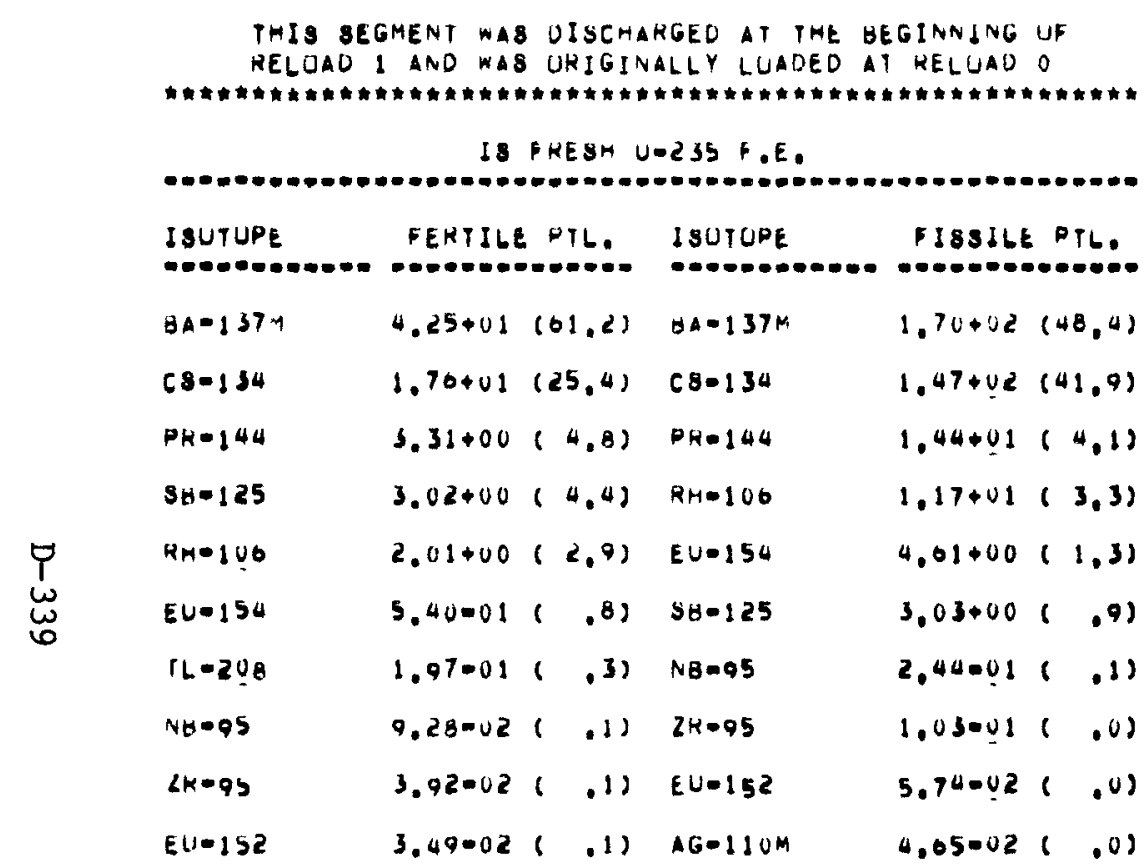

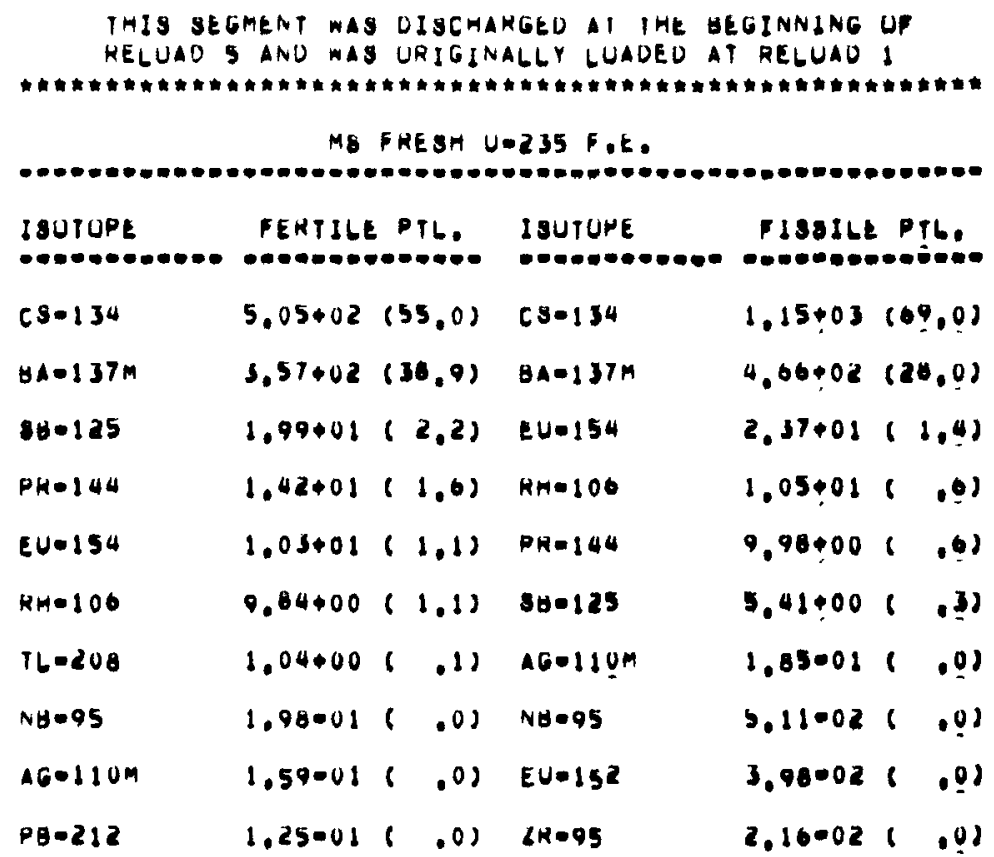




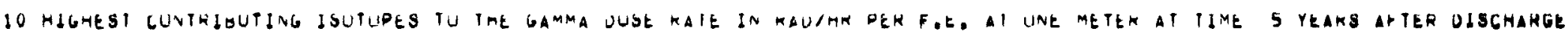

(PERCENT CUNTHIOUTIUN TU TUTAL gUSE Kate in PAKENTHESES)

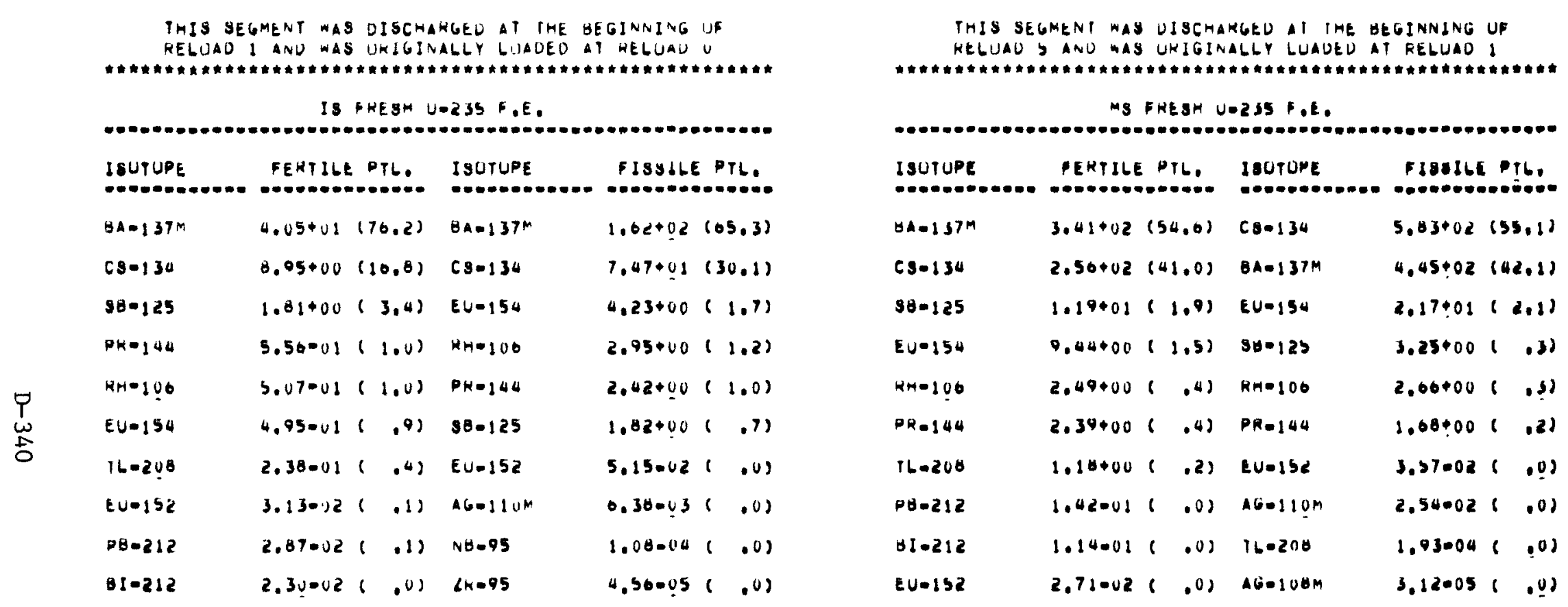




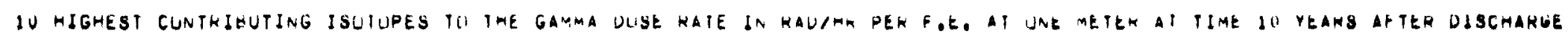
(PERGENT CUMTRIGUIJON TO TUIAL DUSE kaIE IN PANENTHESEY)

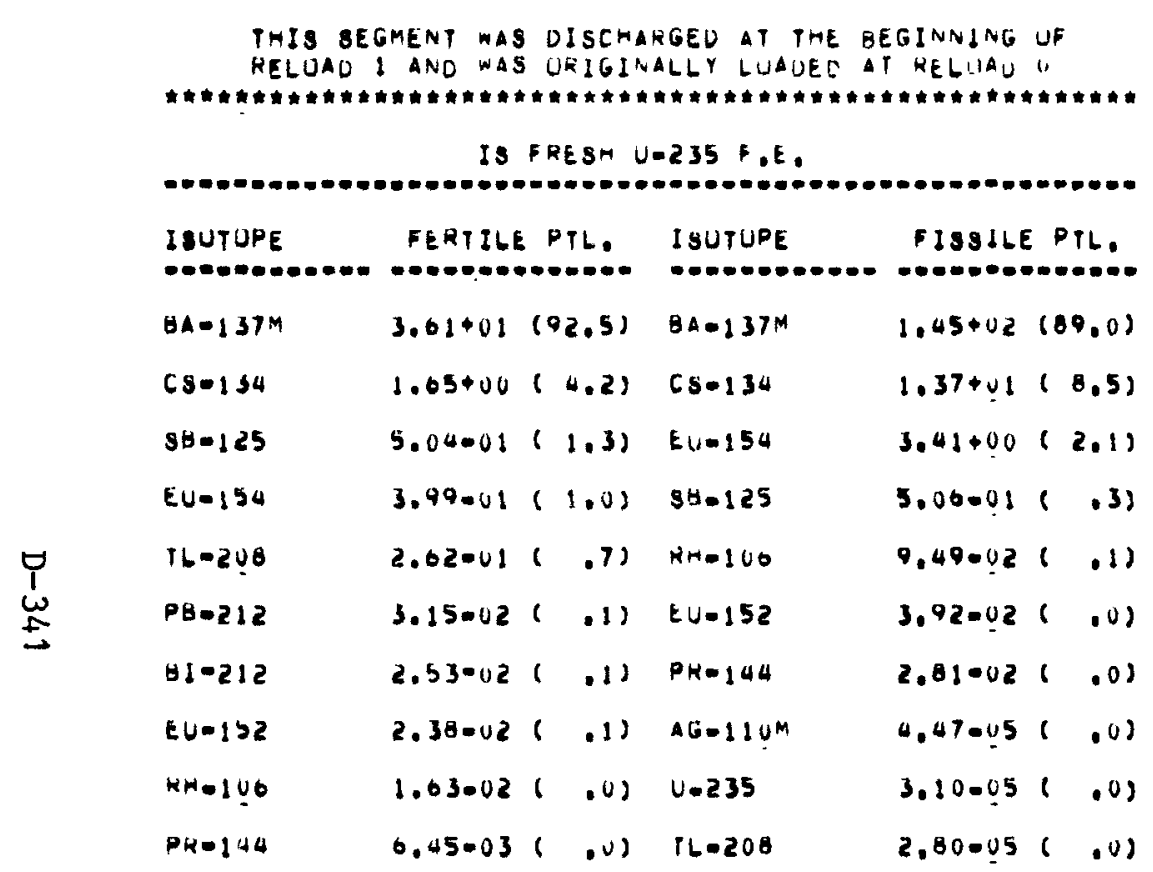

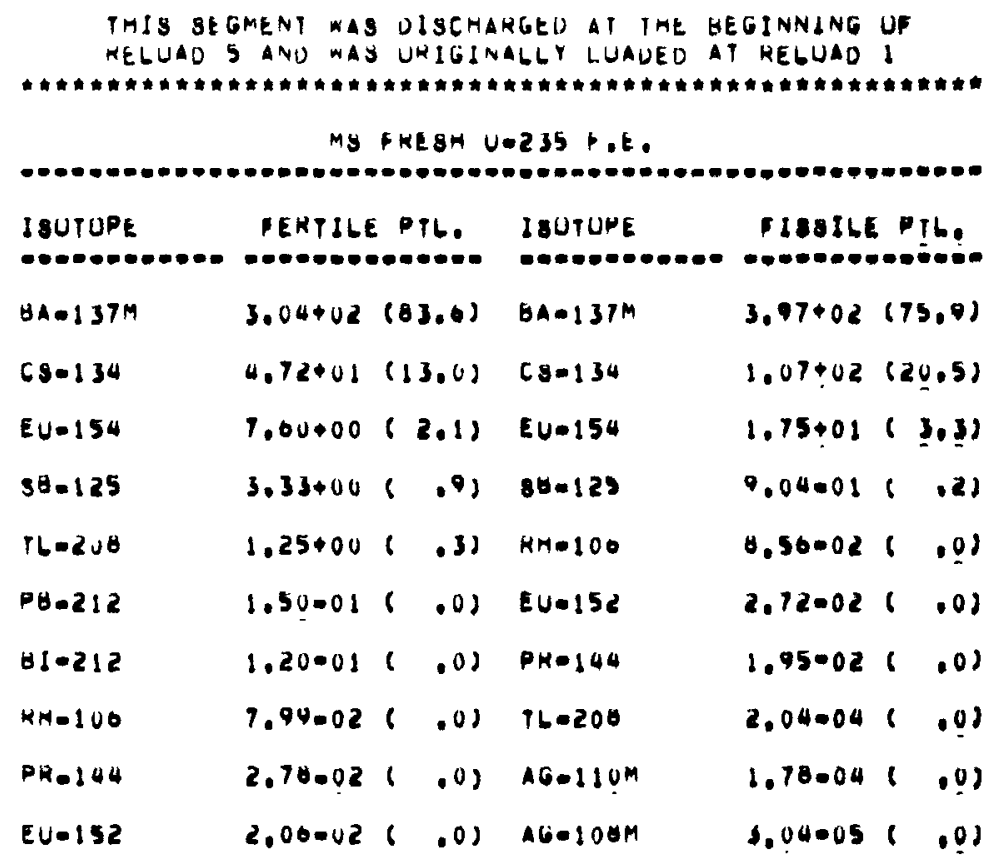




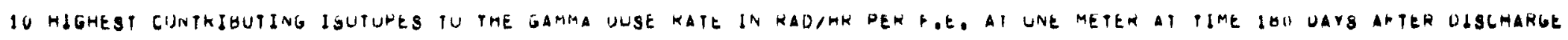
(PEKCENT CONTKIOUTION TII TITAL DUSE RATE IN PAKENTMESES)

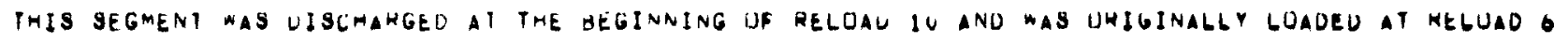

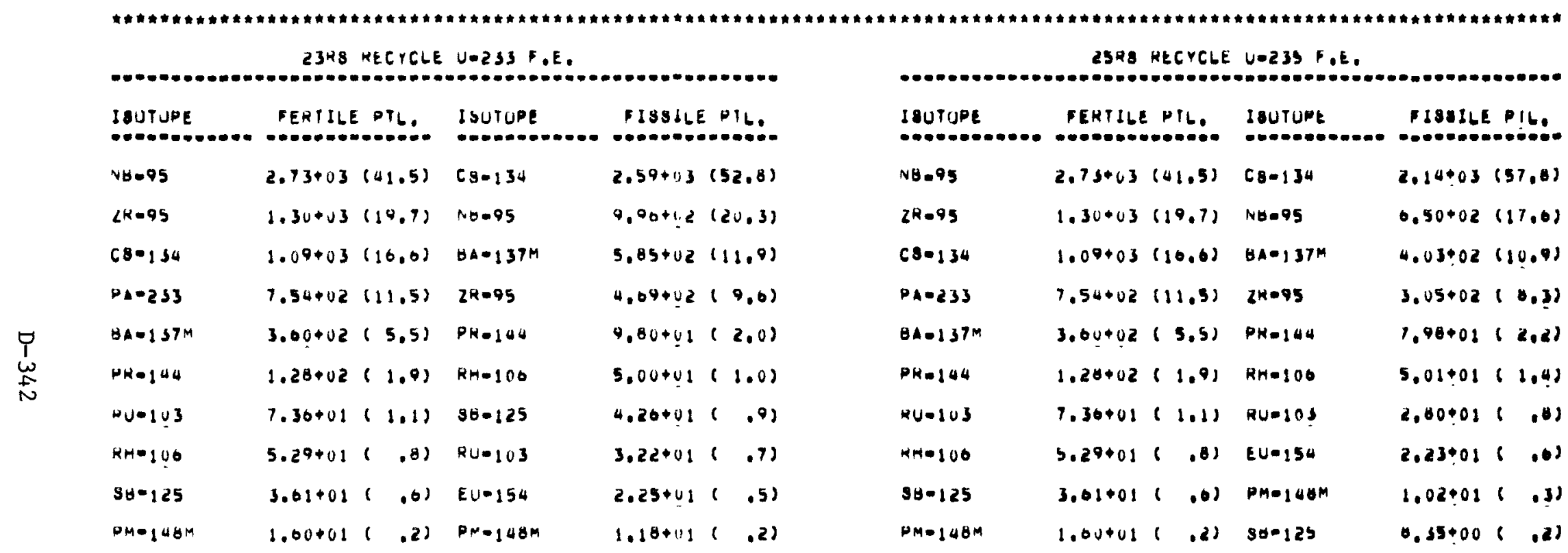




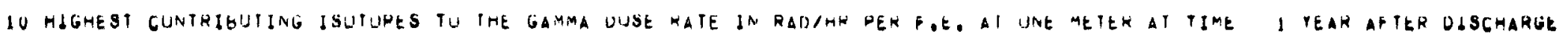

(PERCENT CUNTKIOUTIUN TIJ TOTAL UUSE RATE IN PAMENTHESES)

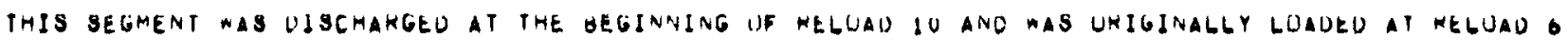

$23 K S$ RECYCLE U-233 F.E.

\begin{tabular}{|c|c|c|c|c|c|}
\hline $\begin{array}{l}\text { I SUTUPE } \\
\text { SUTU }\end{array}$ & FEKTILE & PTL. & 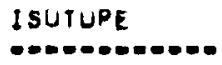 & DISSILE & PIL. \\
\hline$C 8 \cdot 134$ & $9.20+02$ & $(40.6)$ & $C S=134$ & $2.18 \cdot 0,3$ & $(09,4)$ \\
\hline$N G=95$ & $4.24+02$ & $(20.0)$ & $B A-137 \mathrm{~m}$ & $5.78+02$ & $(18.4)$ \\
\hline$B A=157 M$ & $3.50+02$ & $(17,3)$ & $N 8.95$ & $1.54+02$ & $(4.9)$ \\
\hline $2 R=95$ & $1.83+02$ & $(8.9)$ & $2 k-05$ & $0.01+01$ & $(2.1)$ \\
\hline$P R \cdot 144$ & $0.13+01$ & $(4.0)$ & $P_{K=144}$ & $0.23+\underline{0.1}$ & $(2,0)$ \\
\hline$H H=100$ & $3.73+01$ & $(1.8)$ & $58-125$ & $3,7 a+01$ & $(1,2)$ \\
\hline $38-125$ & $3.17+0.1$ & $(1.5)$ & $H H=I \cup O$ & $3.53+01$ & $(1,1)$ \\
\hline$E U=154$ & $1.07 \$ 01$ & $.5)$ & $E U=154$ & 2.20001 & $.7)$ \\
\hline$P A=233$ & $0.48+00$ & .31 & $A G-110 M$ & $1.79+00$ & .11 \\
\hline$R U=1 \cup 3$ & $2.92+00$ & .11 & $R U=1 \cup 3$ & $1.20+00$ & .01 \\
\hline
\end{tabular}

25AS RECYCLE U-235 P.E.

\begin{tabular}{|c|c|c|c|c|c|}
\hline I8UTUPE & FEKTILE & PTL. & ISUTOPE & F10s & PIL. \\
\hline $\operatorname{cs}=134$ & $9.20+102$ & $(44.8)$ & $\operatorname{css}=134$ & $1.80 \div 03$ & $(7 \leq, 2)$ \\
\hline NB. 95 & $4.24+02$ & $(20.6)$ & $B A-1374$ & $3.98+02$ & $(10,2)$ \\
\hline$\forall 4=137 \mathrm{~m}$ & $3.50+02$ & $(17.3)$ & $N \forall=95$ & $1.00+02$ & $(4.2)$ \\
\hline $2 R .95$ & $1.83+02$ & $(6.9)$ & $P H=144$ & $5.06+01$ & $(2,1)$ \\
\hline$P R=144$ & $0.15+01$ & $(4.0)$ & $2 k 0.95$ & $4.30+01$ & $(1.7)$ \\
\hline$B H=106$ & $3.73+01$ & $(1.8)$ & $R H=100$ & $1.54+01$ & $(1.4)$ \\
\hline $80 \cdot 125$ & $3.17+01$ & $(1.5)$ & $E U=154$ & $2.16 \div 01$ & .48 \\
\hline$E U=154$ & $1.07 \$ 01$ & .53 & $36=125$ & $7.33 \div 00$ & .3) \\
\hline$p 4-233$ & $0.48+00$ & .33 & RUP $10 \$$ & $1.21+00$ & .08 \\
\hline RUD 103 & $2,92 \$ 00$ & .12 & $A G \cdot 110 M$ & $1.09+00$ & .08 \\
\hline
\end{tabular}




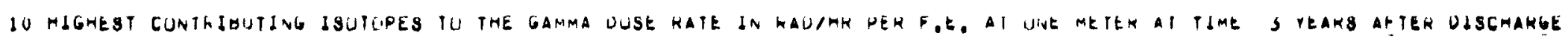
(PERCENT CUNTKIOUTIUN IU TUTAL DUSE RAIE IV PARENTHESESJ

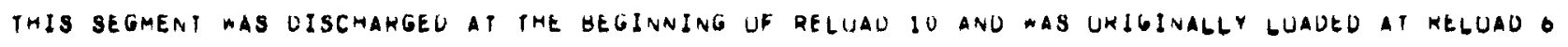

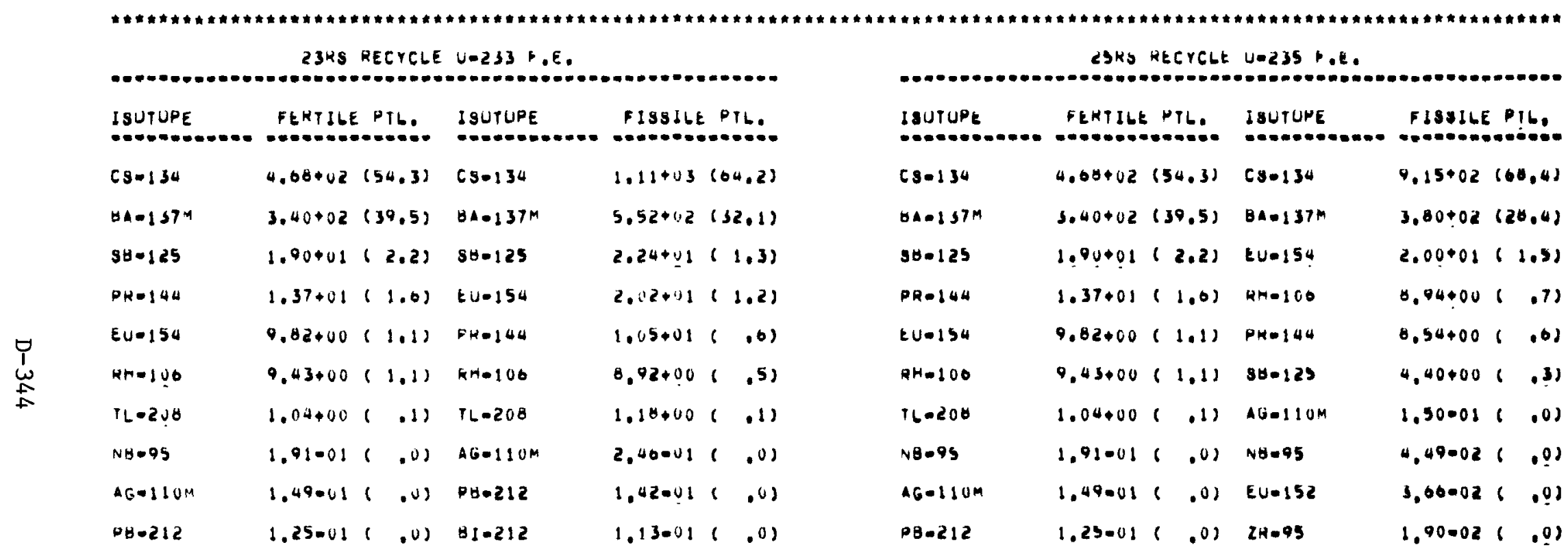




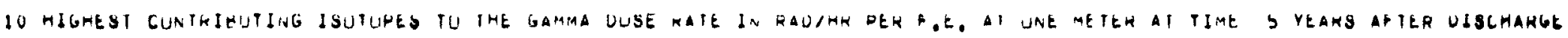

(PERCENT CUNTHINUTIJN TO TUIAL OUSE KaTE IN HAKETIMESES)

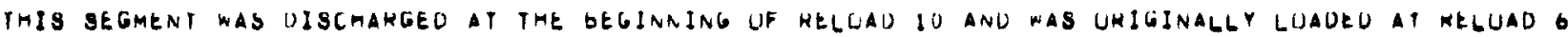

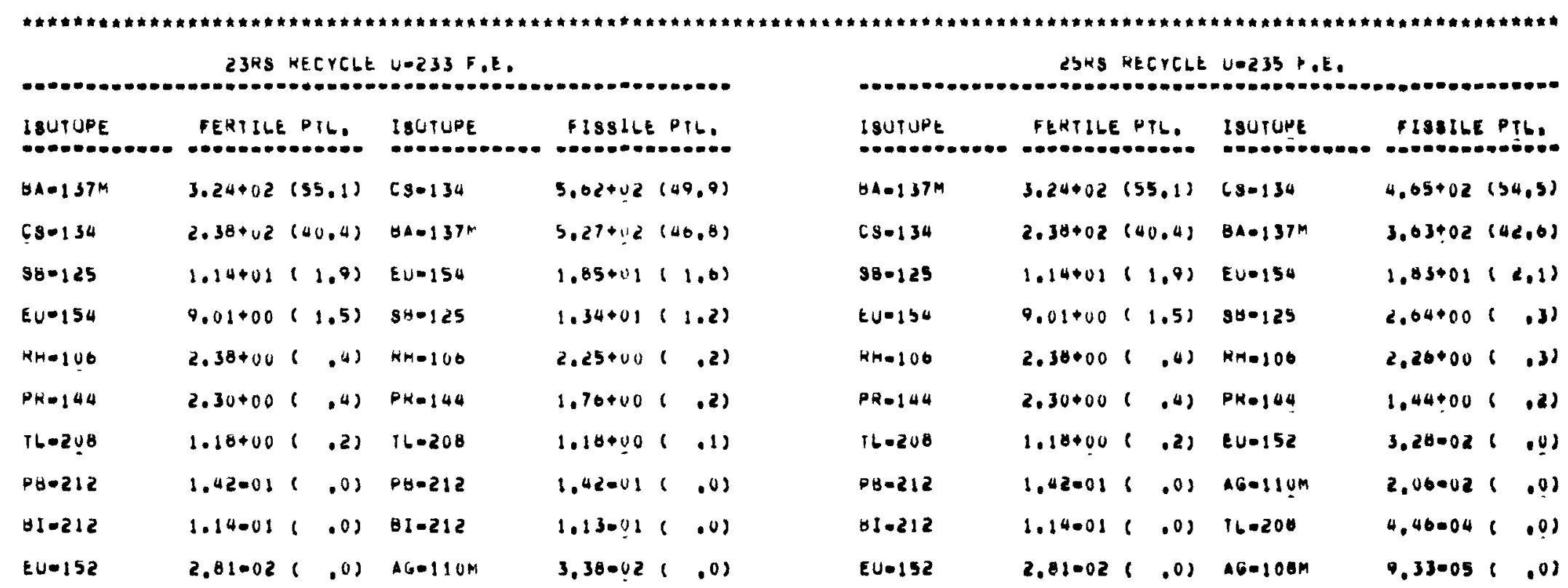




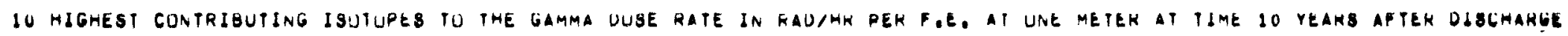
(PERCENT CUNTKIBUTIUN TU TUTAL DUSE HATE IN MAKENTHESES)

THIS SEGMENT MAS DIBCHARGED AT THE HEGINNING OF RELOAD IU AND WAS UKIGINALLY LOADEO AT MELUAD O

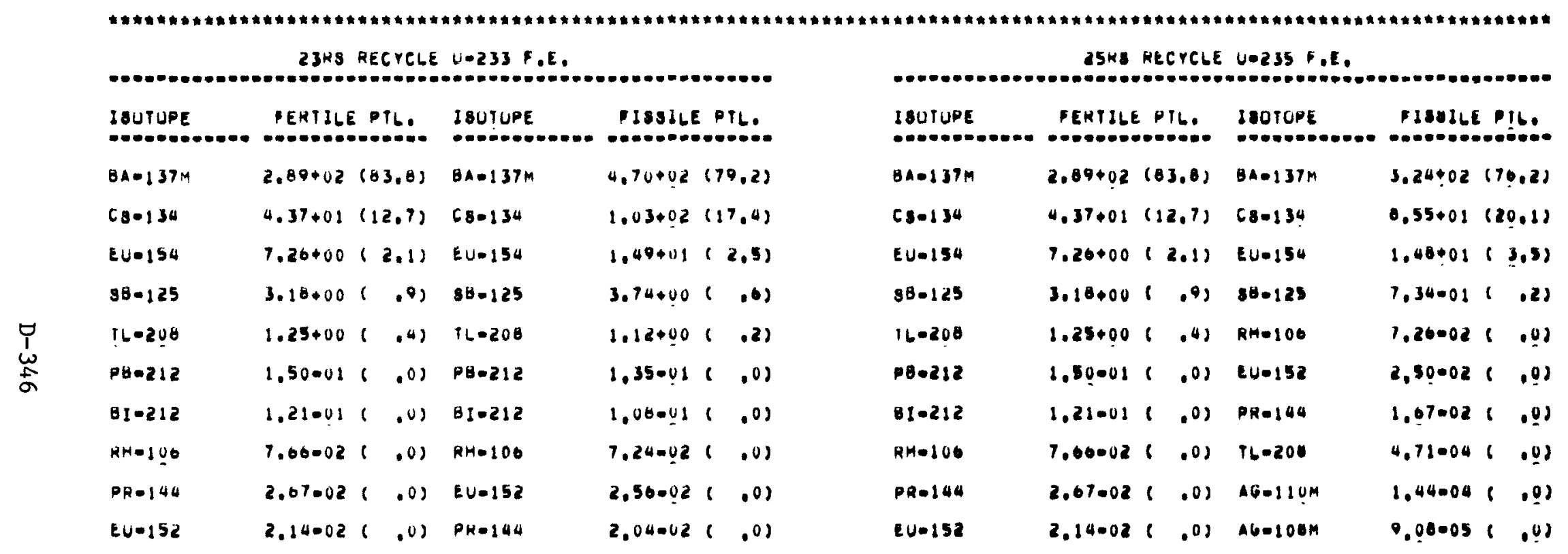




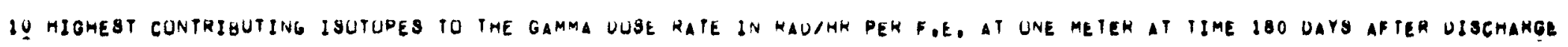

(PERCENT CONTRIOUTIUN TU TUTAL UUBE RATE IN PARENTHESES)

THIS BEGMENT WAS UISCHARGED AI TME BEGINNING UF RELUAD I3 ANU WAB UKIGINALLY LOADED AT KELOAD O

\begin{tabular}{|c|c|c|c|c|c|c|c|c|c|c|}
\hline I8UTUPE & $\begin{array}{l}\text { FERTILE } \\
\text { MOSOADOE }\end{array}$ & $=P T L$. & ISUTUPE & FI8SILE PTL. & j8UTOPt & FERT ILE & PTL. & I NOTUPE & 180165 & PIb: \\
\hline$N 8.95$ & $2.76+03$ & $(42.0)$ & 68.134 & $2.87+03 \quad(55.3)$ & NB.95 & 2,76003 & $(42.0)$ & $\cos 130$ & $2.20 \div 03$ & $(50,0)$ \\
\hline $2 H 095$ & $1.31+03$ & $(20.0)$ & NO.9S & $1.02+03(19.5)$ & 28.05 & 1.31003 & $(20.0)$ & NG-0S & $9.81+02$ & $(22,0)$ \\
\hline $\cos -134$ & $1.00+03$ & $(10,1)$ & $B A=137 M$ & $5.47 .02(10.5)$ & $\cos 134$ & $1,06+03$ & $(10.1)$ & $B A-137 M$ & 5.24002 & $(86,5)$ \\
\hline$P A 0.233$ & $7.59+02$ & $(11,5)$ & $2 k=95$ & $4.75+02(9.1)$ & $P A=233$ & $7.59+02$ & $(12.5)$ & $2 H=95$ & $4.02 \div 02$ & $(10,4)$ \\
\hline$B A \cdot 157 M$ & $3.53+02$ & $(5.4)$ & $P K=144$ & $1.13+02(2.2)$ & $8 A-137 M$ & $3,53+02$ & $(5.4)$ & PHOINU & 9.08001 & $(2,0)$ \\
\hline$P R=144$ & $1.27+02$ & $(1.0)$ & $R H=100$ & $7.03+01(1.4)$ & $P 9=144$ & $1.27+0.2$ & $(1.0)$ & RH $=100$ & $4.01 \div 01$ & $(1,0)$ \\
\hline$R \cup=103$ & $7.45+01$ & $(1.1)$ & $R \cup=103$ & $4.32+01(.8)$ & $R U=103$ & $7.45+01$ & $(1.1)$ & $80-125$ & $3.73 \times 02$ & 1.01 \\
\hline$R M=100$ & $5.20+01$ & $(.8)$ & $\varepsilon \cup-154$ & $3.00+01(0.0)$ & $K M=800$ & $3.26+01$ & $(.0)$ & Ru-10! & $3.20 \div 08$ & $(9)$ \\
\hline $88-125$ & $3,56+01$ & .51 & $P M=148 M$ & $1.45+011$ & $38-125$ & 3.56001 & $(.5)$ & $E \cup-154$ & $1.09+01$ & 1.41 \\
\hline$P M \cdot 148 M$ & $1.58+01$ & $.2)$ & $88-125$ & $1.17+011$ & PMo140M & 1.50001 & $(.2)$ & $P M-140 M$ & $1.10 \div 01$ & .21 \\
\hline
\end{tabular}




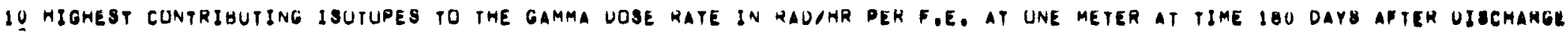
(PERCENT CONTHIUUTIUN TU TUTAL DUSE RATE IN PAKENTHEBEg)

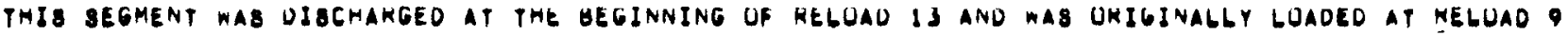

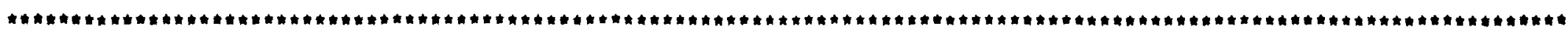
25Rg RECYGLE VEZ 35 F.E.

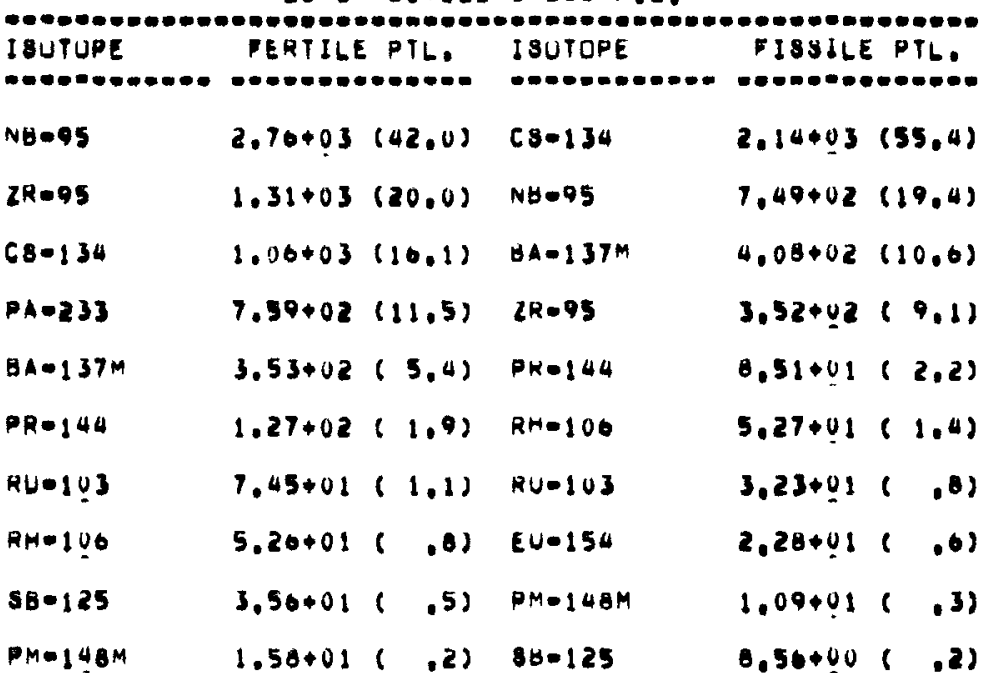




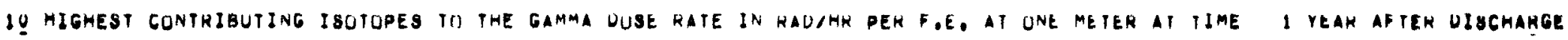
(PENCENT CONTKIGUTION TO TUTAL DOSE KATE IN PAKENTHESES)

THIS SEGMENT WAS UISCHAHGEO AT THE GEGINNING UF RELUAO 13 ANU MAS UKIGINALLY LOADED AT KELUAD 9

\begin{tabular}{|c|c|c|c|c|c|c|c|c|c|}
\hline \multicolumn{5}{|c|}{ MS FRESH UND35 F.E. } & \multicolumn{5}{|c|}{ CJRS RECYCLE U-233 FEE. } \\
\hline I8UTUPE & $\begin{array}{l}\text { FERTILE } \\
\text { DOAODOOE }\end{array}$ & PIL. & ISUTUPE & FISSILE PTL. & \BUTUPE & 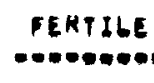 & PTL. & 1RUTUPE & BIS8ILE PIt! \\
\hline $\cos -134$ & $8.93+02$ & $(44.0)$ & $C 8-134$ & $2.42+0.3(72.3)$ & $68 \cdot 134$ & $0.95+02$ & $(44.0)$ & $c \$-134$ & $1.90 \div 03(60.5)$ \\
\hline N8-95 & $4.30+02$ & $(21.2)$ & $\forall A-137 M$ & $5.41+02(16.2)$ & $N G 0.95$ & $4.3 v+02$ & $(21.2)$ & $B A-137 m$ & $5,08+02(10.3)$ \\
\hline$B A-137 M$ & $3.49+02$ & $(17,2)$ & $N B=95$ & $1.50+02(4.0)$ & $B A-137 M$ & 3,49002 & $(17.2)$ & NB.95 & $1,51+02(5,5)$ \\
\hline $2 R=95$ & $1.85+02$ & $(9,1)$ & $P K=144$ & $1.22+01(2,2)$ & $2 R=95$ & $1.85+02$ & $(9.1)$ & $28-95$ & $0.51 \div 01(2,3)$ \\
\hline$P R=144$ & $8.11+01$ & $(4.0)$ & $2 k=95$ & $0.70001(2.0)$ & $P A=144$ & 0.11001 & $(4.0)$ & PHE 144 & $5.77+01(2,1)$ \\
\hline$R H=10_{6}$ & $3.71+01$ & $(1.8)$ & $R M=100$ & $4.96401(1.5)$ & $R H=100$ & 3.71001 & $(1.8)$ & $84-125$ & $3,20 * 01(1,2)$ \\
\hline $98-125$ & $3.13+01$ & $(1.5)$ & $E U=154$ & $2.93+01 \quad(.9)$ & $38-125$ & $3,13+01$ & $(1.5)$ & RMO109 & $3.25001(1.2)$ \\
\hline$E U \cdot 154$ & $1.04+01$ & 6.53 & $84-125$ & $1.02+011$ & $E U \cdot 154$ & $1.04+01$ & $(.5)$ & $E v-154$ & $1.95 \div 01(.71$ \\
\hline$P A=233$ & $0.53+00$ & $(.3)$ & $R \cup=103$ & $1.71+001$ & $P A=233$ & $0.53+00$ & .31 & $\triangle G=11$ UM & 1.580006 \\
\hline$R U=103$ & $2.96+00$ & $(.1)$ & $16-110 M$ & $1.49+001$ & HUDIOJ & $2.96,00$ & .11 & $R U=103$ & $1,37 \div 001$ \\
\hline
\end{tabular}




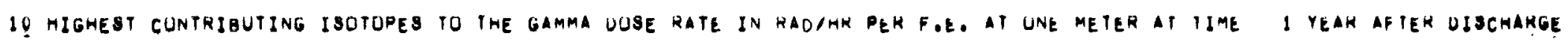
(PERCENT CUNTKIUUTIUN TO TUTAL DUSE hatE in PAKENTMESES)

THIS SEGMENT WAS DISGMAKGEO AT THE GEgINNING UF RELUAD IS ANU WAS UNIGINALGY GOADED AT KELOAD

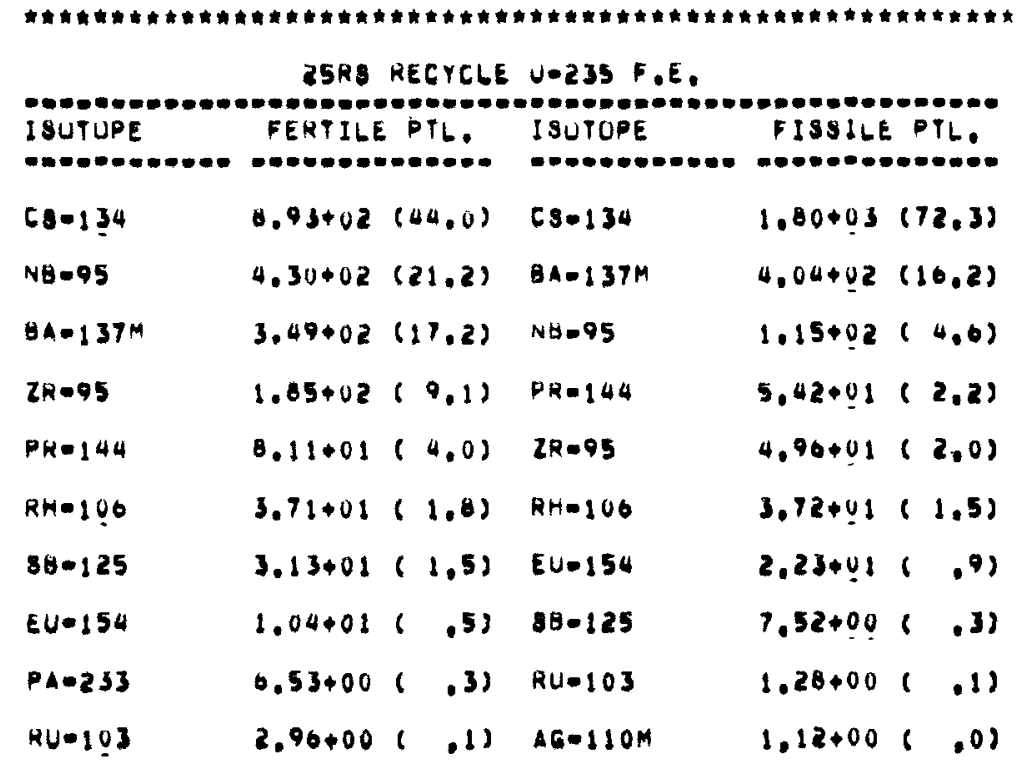




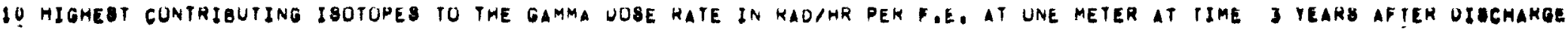
(PERGENT CUNTRIBUTIUN TU TOTAL DUSE Rate IN PARENTAEgES)

THIS BEGMENT MAS DISCMARGEU AT THE BEGINNING UF RELOAD IJ AND MAS URIGINALLY LOAOEO AT MELDAD

\begin{tabular}{|c|c|c|c|c|c|c|c|c|c|c|c|}
\hline I8OTUPE & FERTILE & PTL. & I8OTUPE & 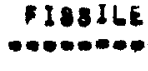 & PTLO & I8010PE & BEKTILE & PT6. & IEUTOPG & P100145 & PIton. \\
\hline $68=134$ & $4.53+02$ & $(50.0)$ & $\operatorname{csm} 134$ & $1.23+0.3$ & $(00.1)$ & $\operatorname{cs}-134$ & $4.53+02$ & $(54.0)$ & $68=134$ & $9.05 \div 02$ & $(0,0.0)$ \\
\hline$B A=137 M$ & $3.33+\cup 2$ & $(39.7)$ & $B A-137 M$ & $5.17+0.2$ & $(28,0)$ & $B A-137 M$ & $3.33+02$ & $(39.7)$ & $B A=137 M$ & $4.85+02$ & $(32,2)$ \\
\hline $58-125$ & $1.80+01$ & $(2.2)$ & $E U=154$ & $2.69+0.1$ & $(1.5)$ & $88 \cdot 125$ & $1.08+01$ & $(2.2)$ & $86 \cdot 125$ & $1.90+01$ & $(2,3)$ \\
\hline$P K=144$ & $1.36+01$ & $(1.6)$ & RHe 100 & $1.25+01$ & $(.7)$ & $P R=144$ & $1.30+01$ & $(1.0)$ & Eve154 & $1.79+08$ & $(1,2)$ \\
\hline$E U \cdot 154$ & $9.53+00$ & $(1.1)$ & $P R=144$ & $1.21+0 ! 1$ & .71 & $E U-154$ & $9.55+00$ & $(1.1)$ & $P H=144$ & $9.71 \% 00$ & .01 \\
\hline$R H=1 \cup b$ & $9.37+00$ & $(1.1)$ & $88-125$ & $0.24+0.0$ & .31 & RHOI & $9.37+00$ & $(1.1)$ & $R H=100$ & $0.22 \div 00$ & .51 \\
\hline$T L=208$ & $1.05+00$ & $(.1)$ & $A G=110 \mathrm{M}$ & $2,04=01$ & .01 & $T L=200$ & $1.05+00$ & .12 & $16=200$ & $1.24+00$ & .13 \\
\hline NB $=95$ & 1.05001 & $(.0)$ & $N 8=95$ & 6.990 .02 & .01 & $N \forall-9 S$ & $1,93=01$ & .01 & $A Q=110 \mathrm{M}$ & $2.17 \cdot 01$ & .91 \\
\hline$A G=110 M$ & 1.40001 & $(.0)$ & $E \cup-152$ & 5,04002 & .01 & $16-110 \mathrm{~m}$ & 1.40001 & .01 & $P 8=212$ & $1,55=01$ & $(\quad 09)$ \\
\hline$P 8=212$ & $1.27=01$ & $(.0)$ & $2 R=95$ & 2,96002 & .02 & $P B=212$ & $1,27=01$ & $(.0)$ & $B I \cdot 212$ & 1.24001 & $(10)$ \\
\hline
\end{tabular}




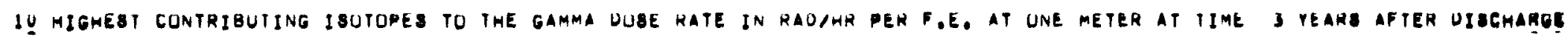
(PERCENT CUNTHIGUTION TU TUTAL DUSE RATE IN PAKENTHEsES)

TMIS SEGMENT WAS DISCHARGEO AT THE BEGINNING OF RELOAU IS ANO WAB URIGINALLY LOADEO AT KELGUAO

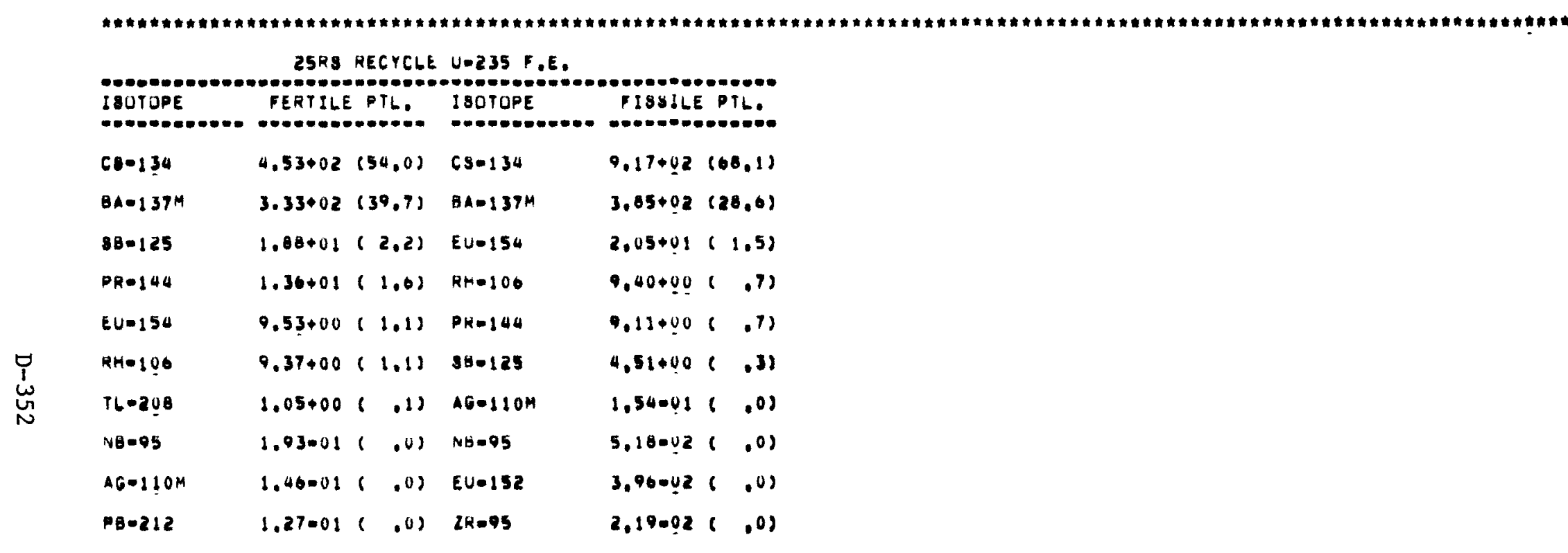




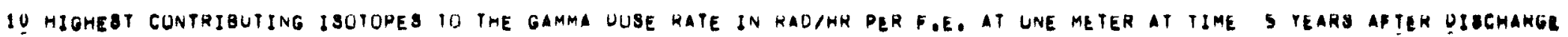
(PERCENT CUNTKIGUTION TU TOTAL DOSE RATE IN PARENTMESES)

THIS SEGMENI MAB UISCMAHGED AT THE gEgINNING UF HELUAD IJ AND MAB UHIGINALLY LOADED AT KELUAD

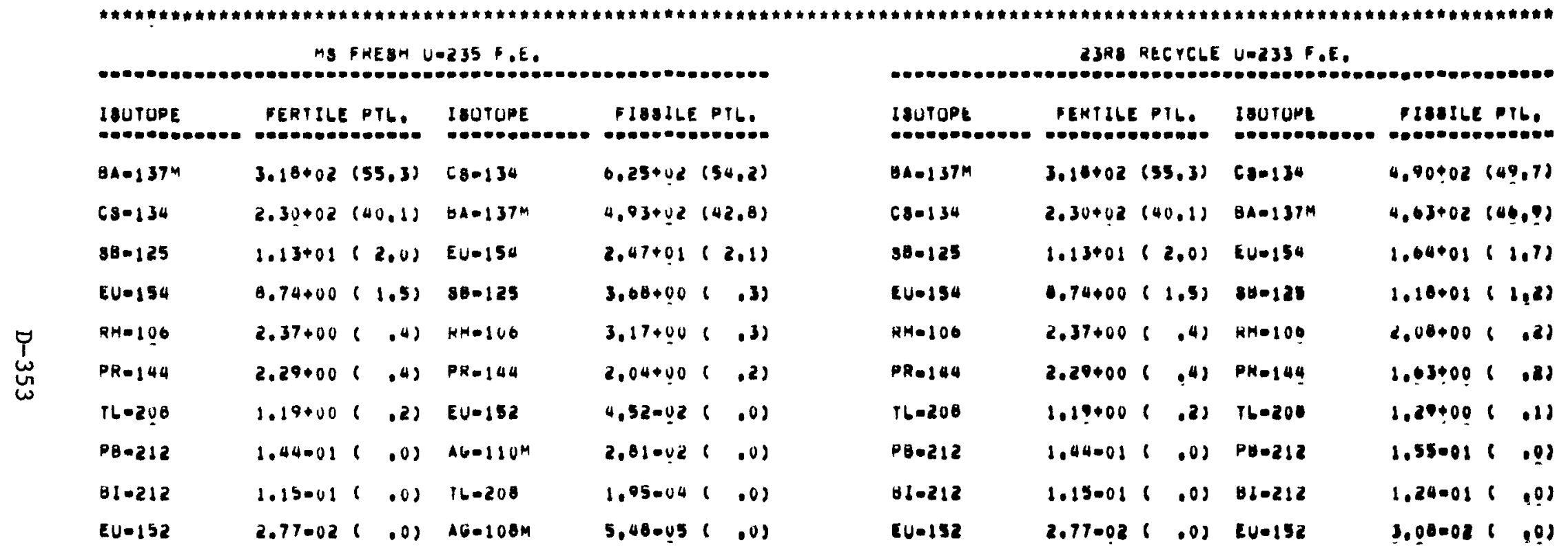




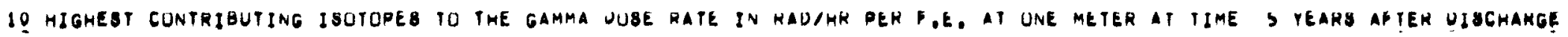
(PERCENT CONTKIQUTIUN TO TUTAL dOBE hate in Pakentheses)

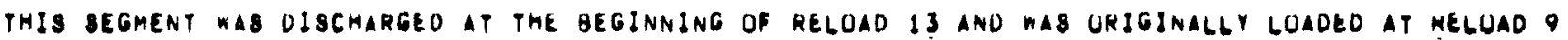

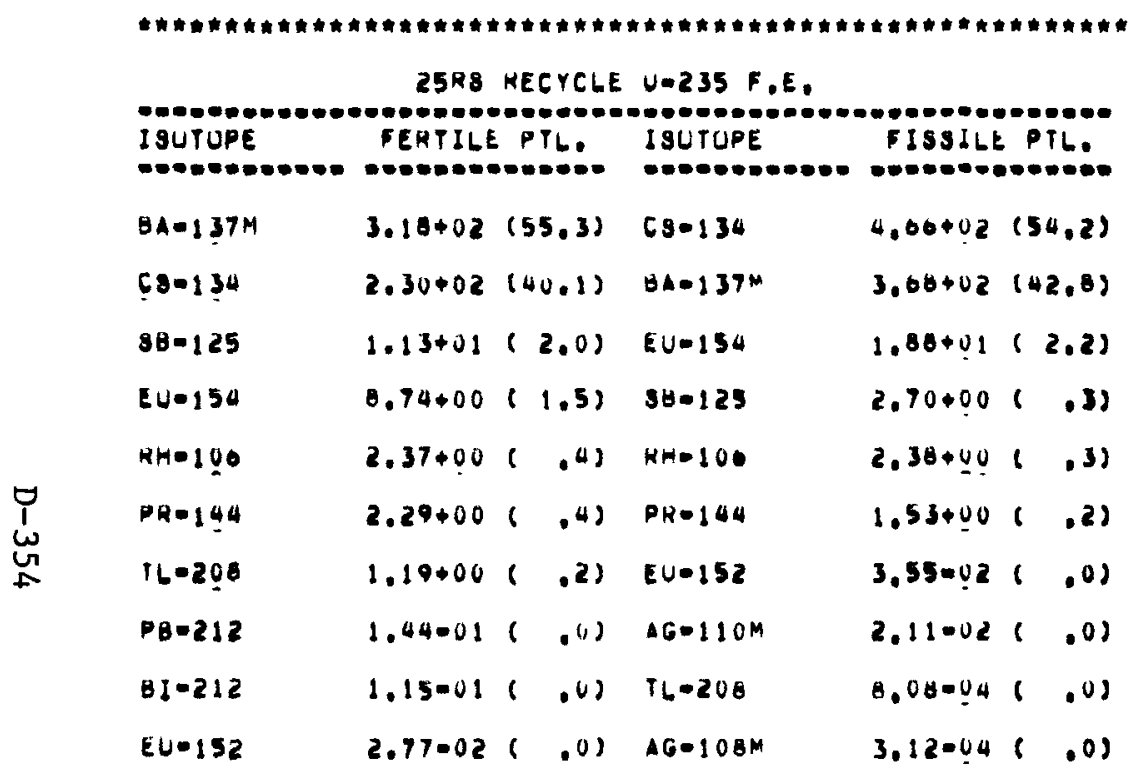




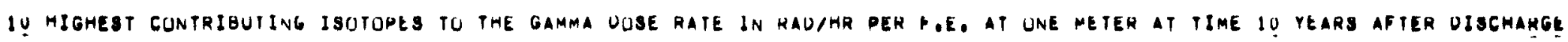
(PERCENT CUNTHIBUTIUN TO TUTAL DOSE RATE IN PAKENTMEBES)

THIS SEGMENT WAS DISGMARGEO AT THE GEGINNING UF KELOAD IS ANO MAS URIGINALLY LUAUED AT HELOAD

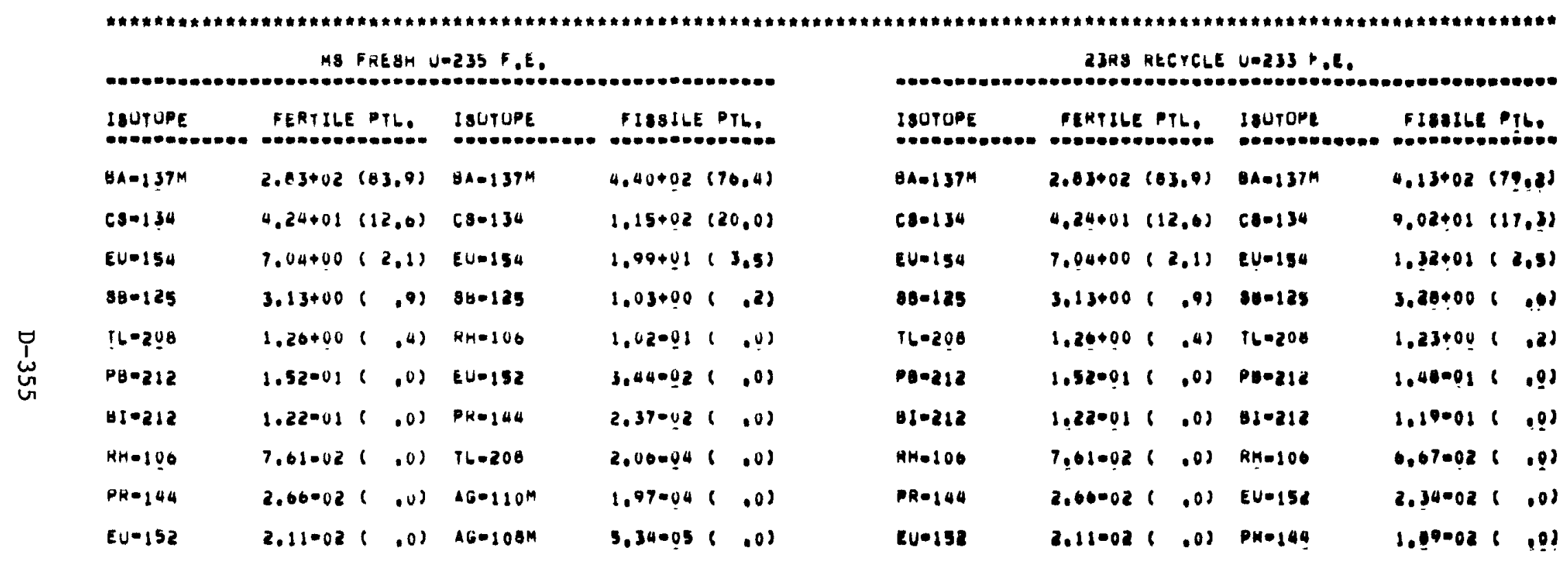




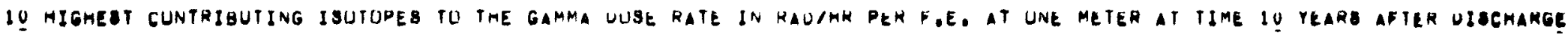
(PERCENT CONTRIOUTION IO TUTAL DOSE KaTE dU PAKENTMESES)

THIS SEGMENT MAS DISCMARGEU AT THE GEGINNING UR RELUAD IS AND MAS UHIGINALGY LUADEO AI GELUAD

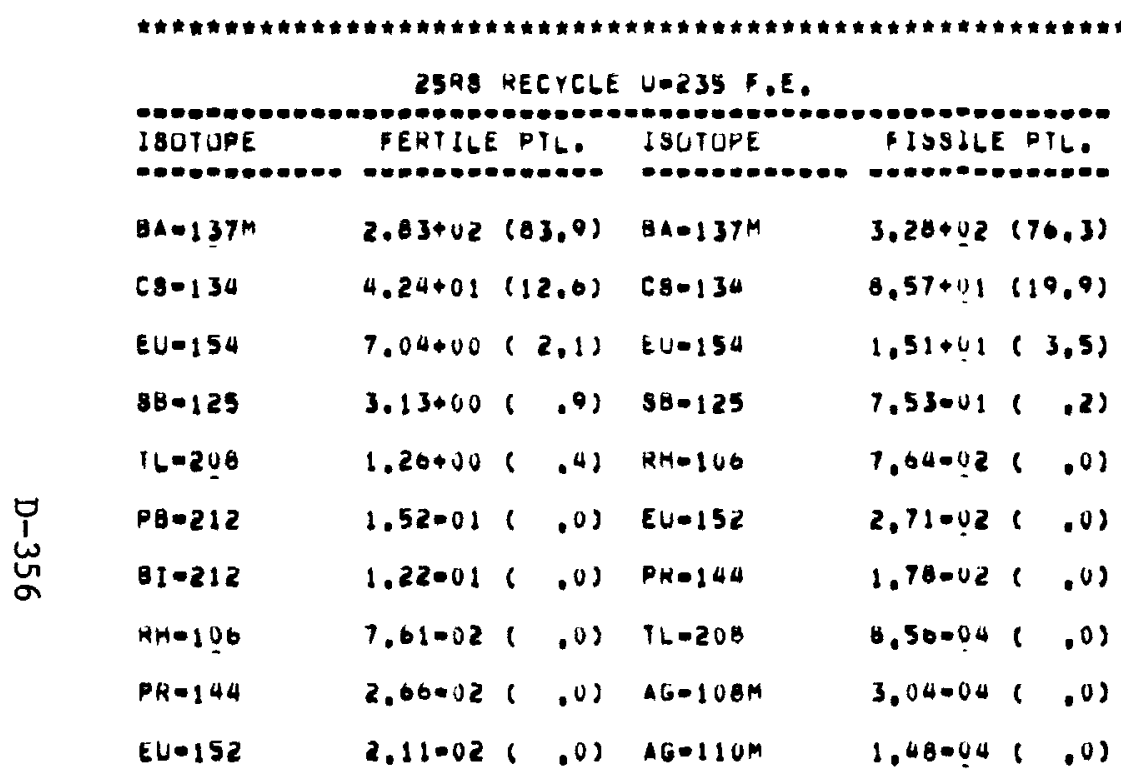


TABLE D-15

IMPORTANT ISOTOPIC CONTRIBUTORS TO NEUTRON SOURCE STRENGTH

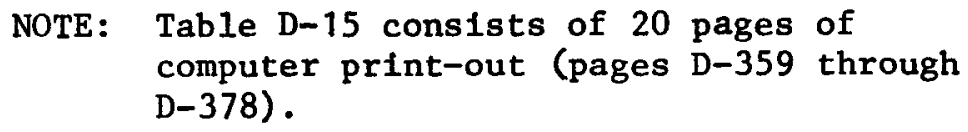

CONTENTS OF TABLE D-15

Fuel Elements discharged at the beginning of reload 1 and origi-

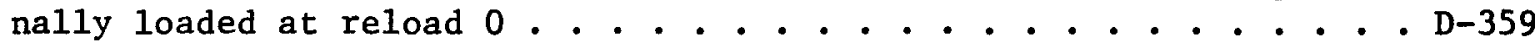

Fuel Elements discharged at the beginning of reload 5 and originally loaded at reload 1... . . . . . . . . . . . D-359 Fuel Elements discharged at the beginning of reload 10 and originally loaded at reload 6................ . . . . . . . . . . . . . . . . . . . .

Fuel Elements discharged at the beginning of reload 13 and originally loaded at reload 9... . . . . . . . . . . . . . D-369 


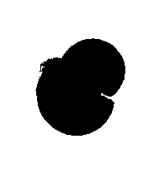

-

0 
10 HIGHEST CONTRIBUTING ISUTUPES TU THE NEUTHUN SUUHCE IN N/SEC PER F.E. AF TIME 100 DAYS AFTEK UISCHARGE

(PERCENT CONTHIGUTIUN TO TUTAL NEUIRUN SUUNCE IN PAHENTHESES

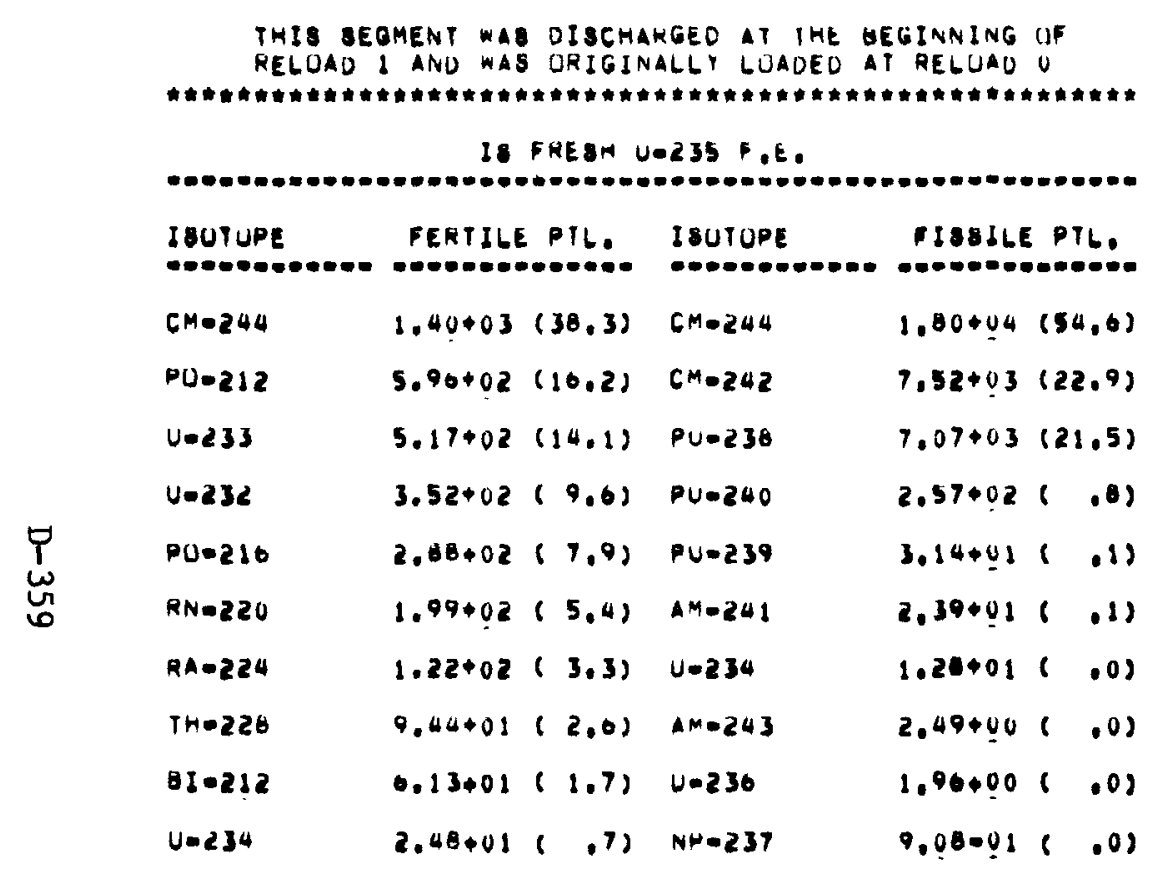

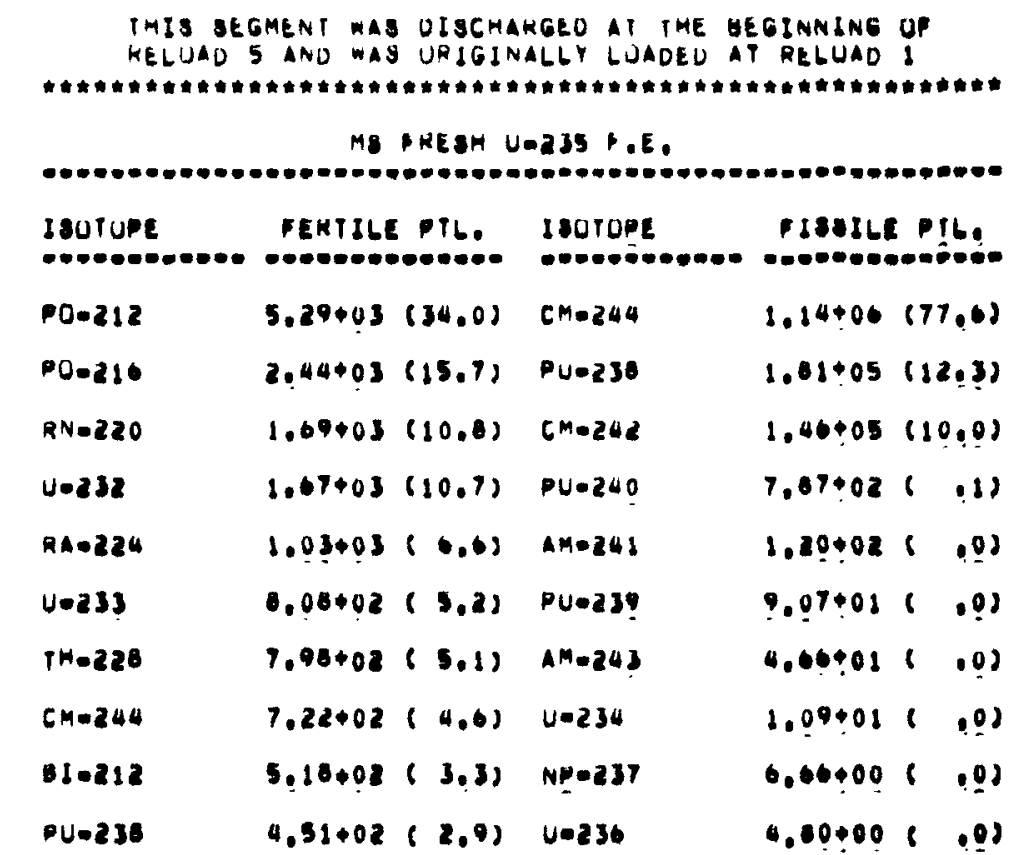


10 HIGHEST CONTRIBUTING ISUTUPES TU THE NEUTRUN SUURCE IN N/SEC HER F.e. AT TIME I YEAH AFIEK UISCMAHGE (PERCENT CUNTRIGUTIUN TU TOTAL NEUTKUN SUUHCE IN PAHEATMEBES)

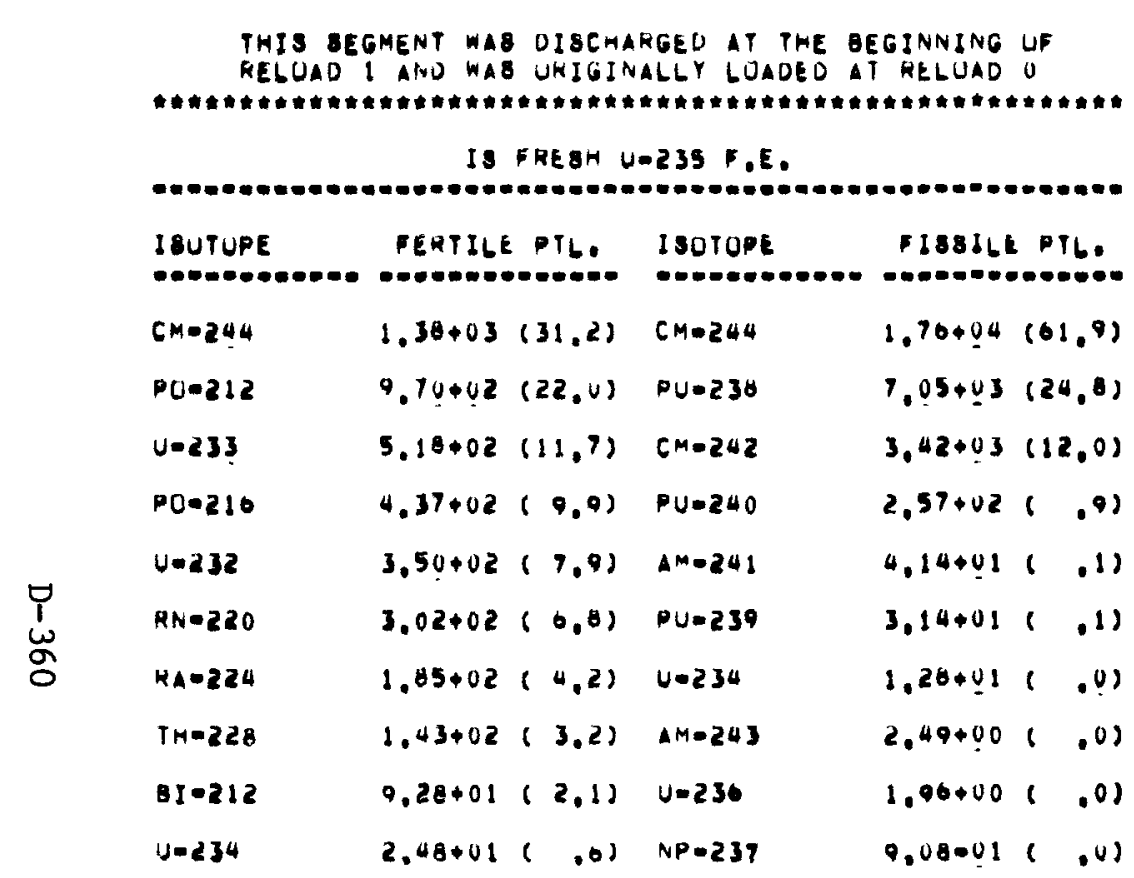

THIS BEGMENT WAO OISCHARGED AT THE BEGINNING UF

Is FREBH U-235 F.E.
THIS SEGMENT MAS DISCHAKGEU AT THE BEGINNING OF

HELUAD 5 ANO WAS URIGINALLY LUAUEU at RELUAD I

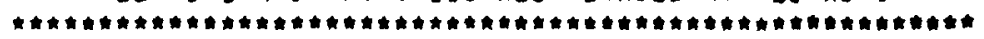

MS FRESH U.235 P.E.

\begin{tabular}{|c|c|c|c|c|}
\hline IBUTUPE & FENTILE PTL. & 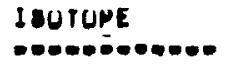 & 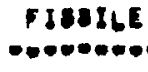 & It: \\
\hline$P 0.212$ & $0.01 .03(30,2)$ & $C M=244$ & $1.11+06$ & $(01,8)$ \\
\hline $90-216$ & $2,90+03(10,2)$ & $P \cup=230$ & $1.80+05$ & $(13,2)$ \\
\hline$R N=220$ & $2.05+03(11.2)$ & $C M-242$ & $0.05+04$ & $(4.9)$ \\
\hline $4=232$ & $1.06+03(0.1)$ & $P U=240$ & $7.67 \cdot 02$ & .11 \\
\hline$R A-224$ & $1.20+03(0.9)$ & $A M=241$ & $1.91 \div 02$ & .07 \\
\hline$T H=220$ & $9.69+02(5.3)$ & PU-23? & $9.07 \div 01$ & .08 \\
\hline v.233 & $0.09+02(4.4)$ & $4 M=243$ & $4.06+02$ & .00 \\
\hline$C M=244$ & $7.00+02(3.0)$ & $4 \cdot 234$ & $2.00+01$ & .01 \\
\hline $81-212$ & $6.29402(3.5)$ & $N W=23 Y$ & $6.60 \div 00$ & 01 \\
\hline$P \cup=238$ & $4.49+02(2.5)$ & $v=236$ & $4.80 \div 00$ & .07 \\
\hline
\end{tabular}


IO HIGHEST CONTRIBUTING IgOTUPES TU TME NEUTRUN SUURCE IN N/SEC PER P.E, AT TIME 3 YEARB AFIEK DIBCHAFGE

[PERCENT CONTRIBUTIUN TU TUTAL NEUTRUN SOUHCE IN PAHENTHESES]

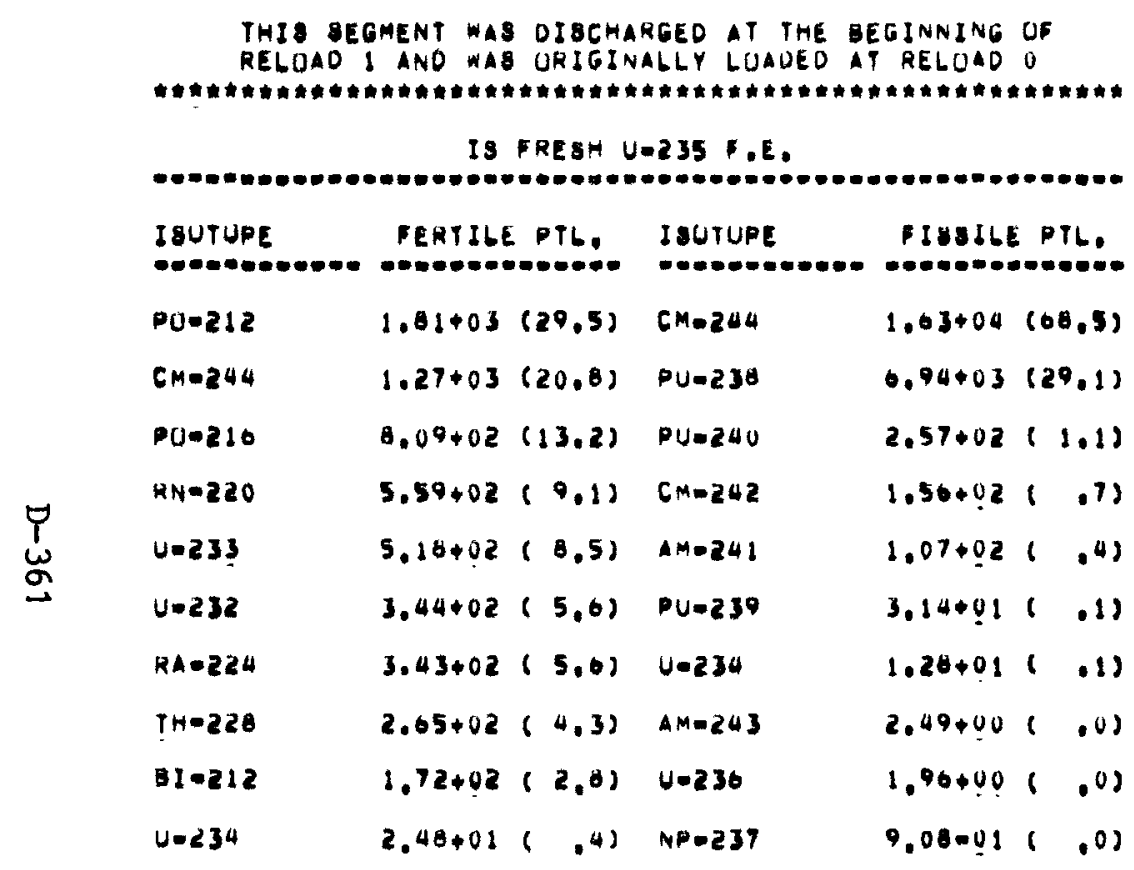

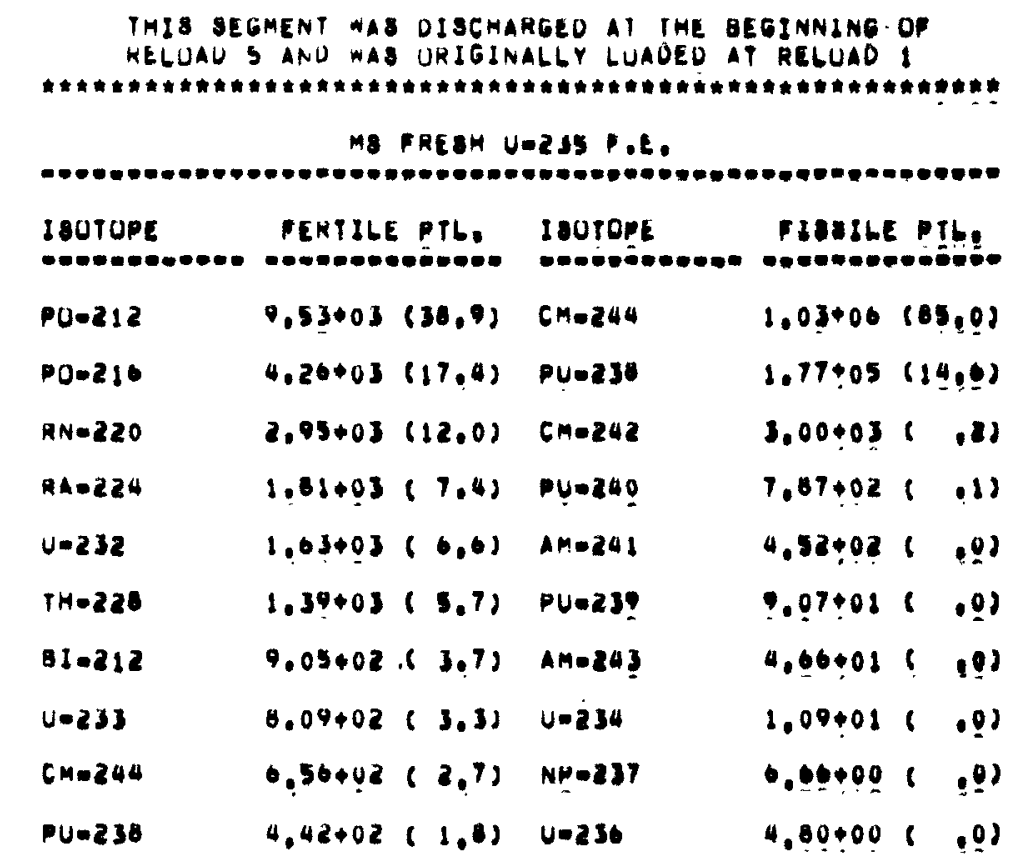




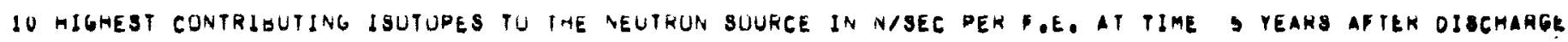
(PERCENT CONTRIGUTIUN TU IUTAL NEUTRUN SUUHCE IN PAHENTHESES)

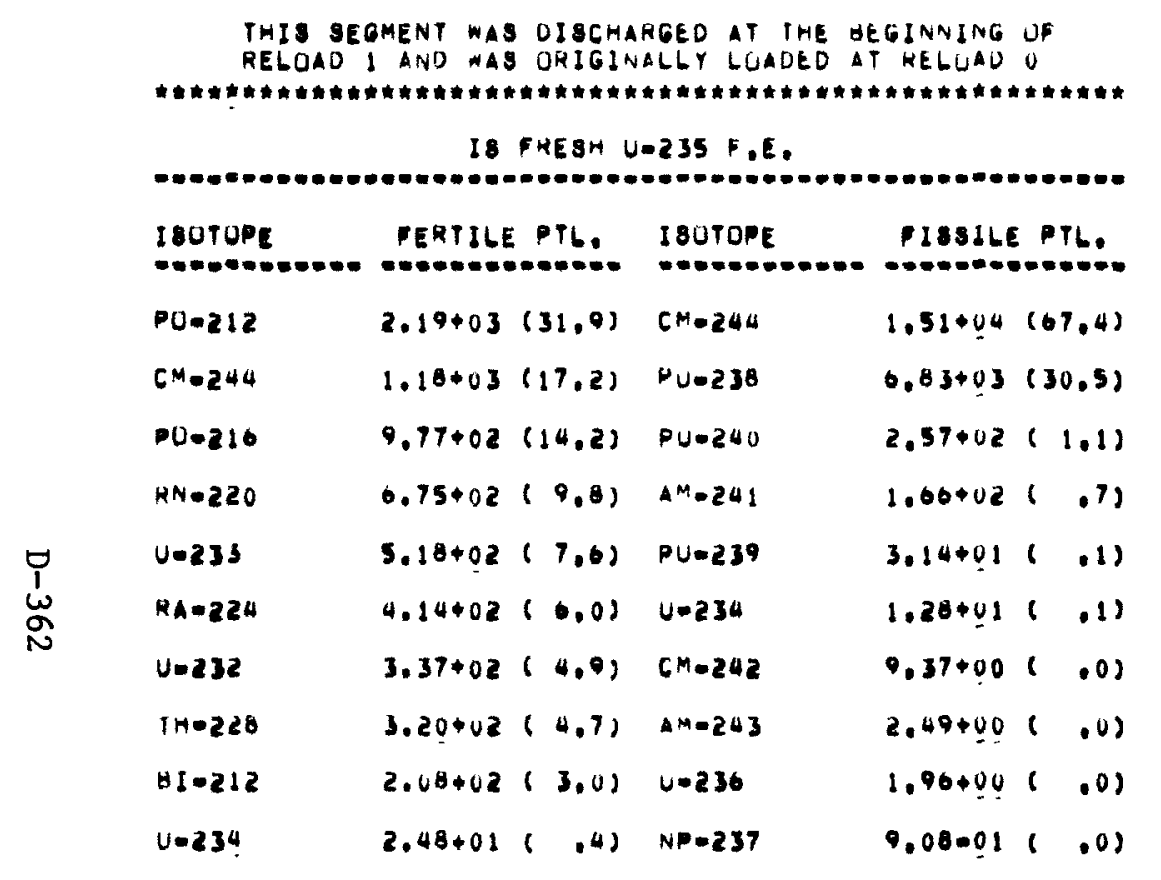

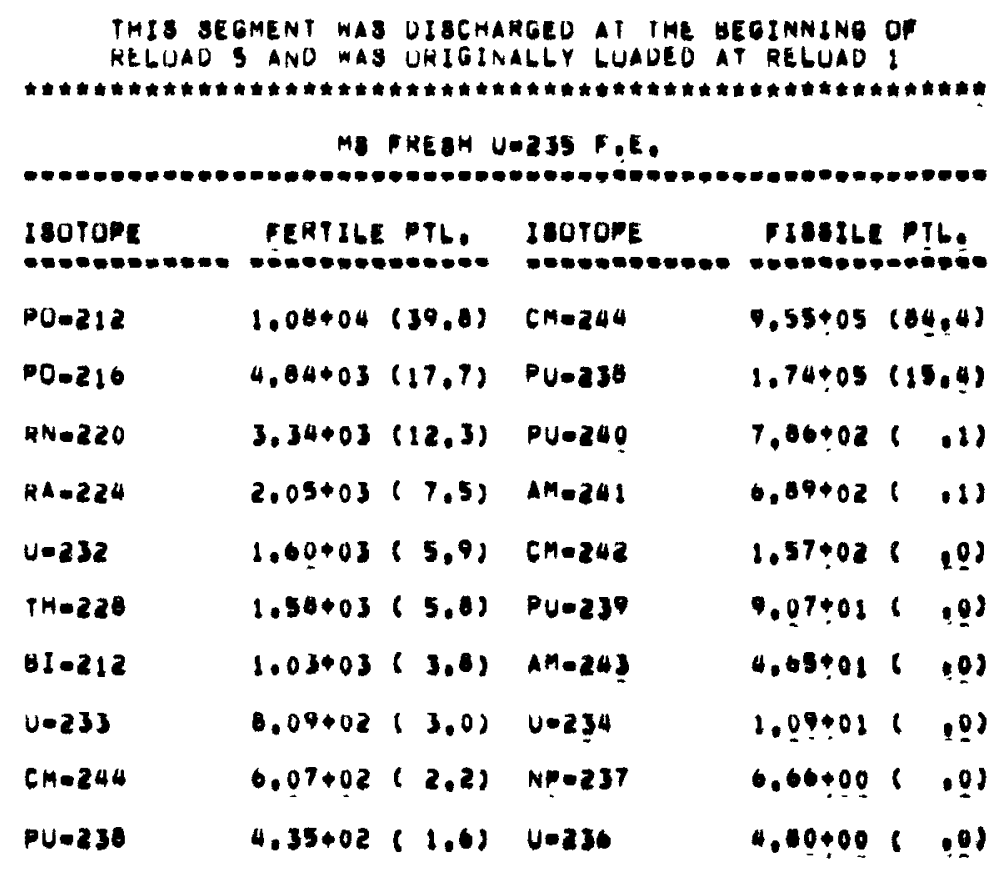




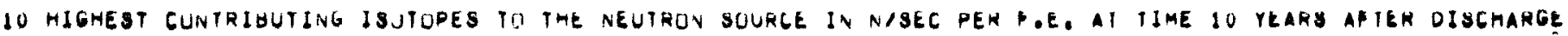
(PERCENT CONTRIGUIJUN TU TUTAL NEUTRUN SUUHCE IN PAFENTHEBES)

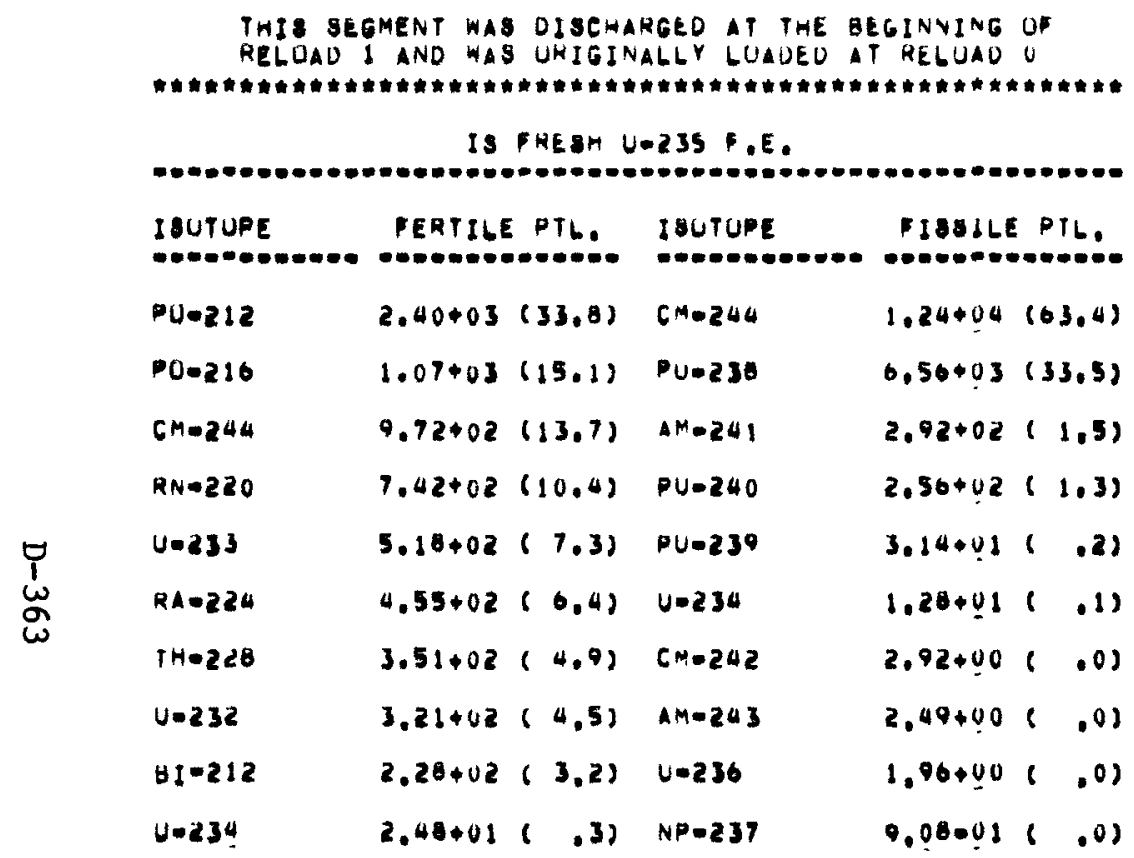

THIS SEGMENT WAS DISCHARGED AT THE BEGINYING OF

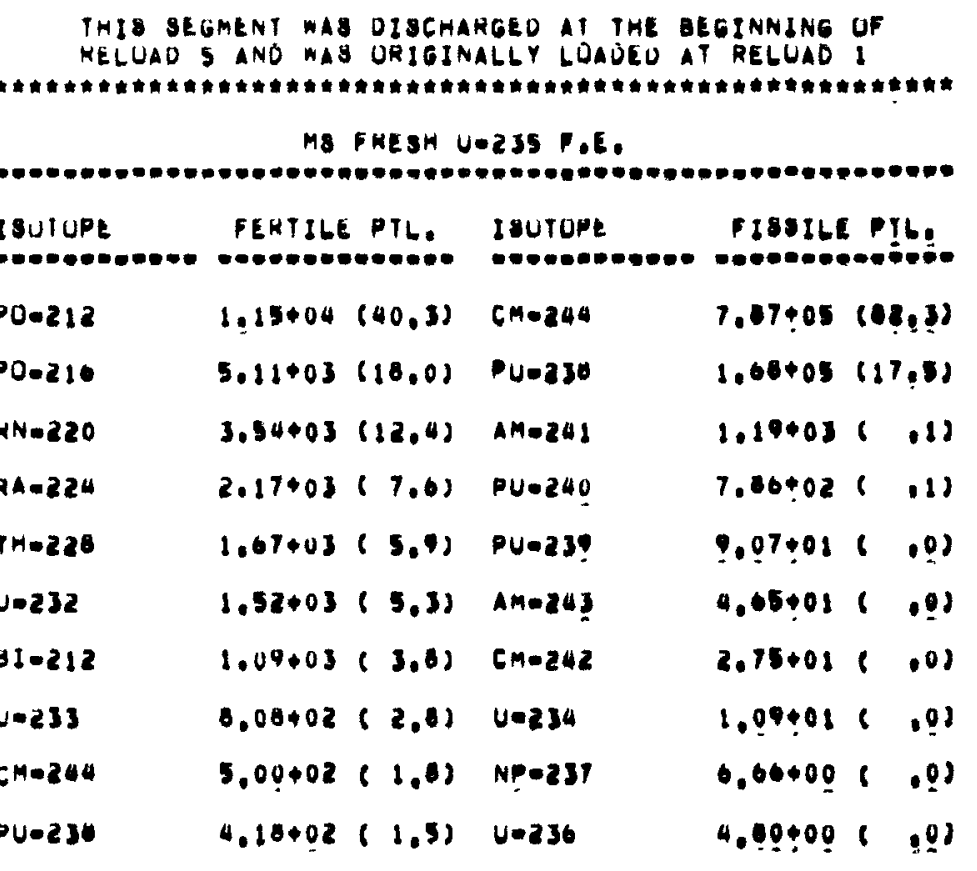


10 MIGHEST CONTRIGUTING ISUTUPES TU THE NEUTRUN SUURGE IN N/GEC PER F.E. AT TIME IOO DAYS AFTEK OISGMAREE (PERCENT CUNTRIBUTIUN TO TOTAL NEUTRUN SUUHCE IN PARENTHEBES)

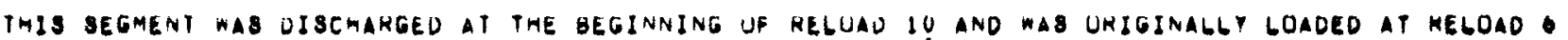

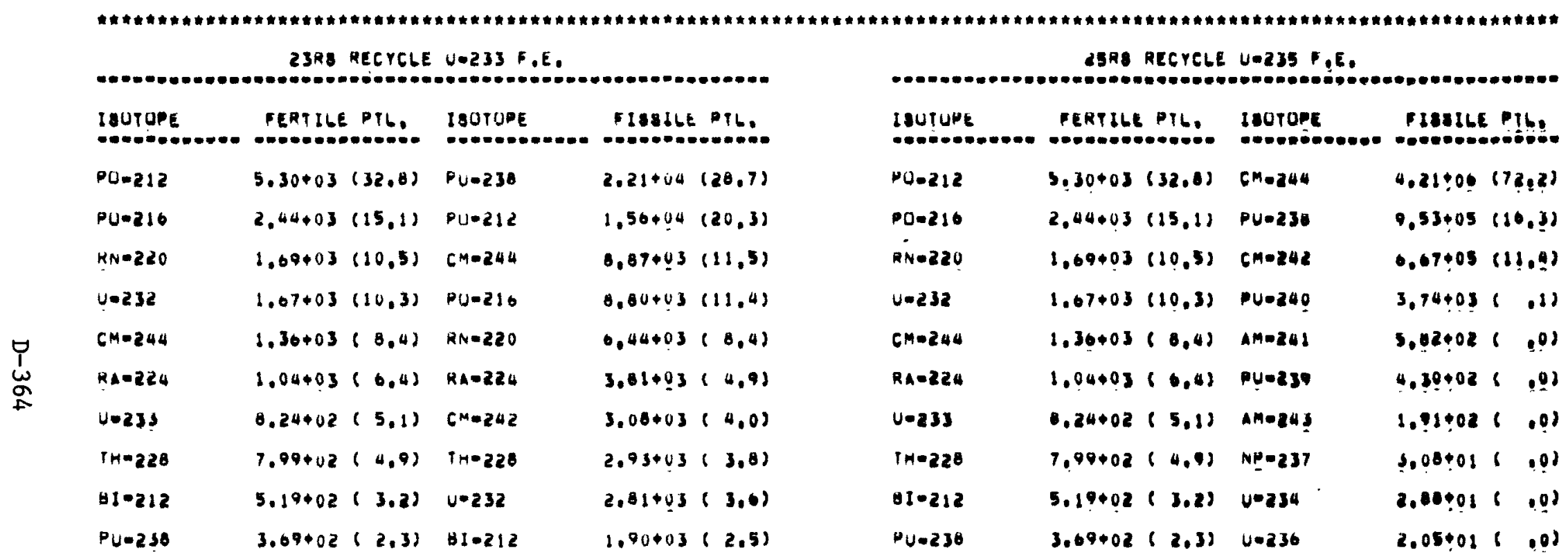




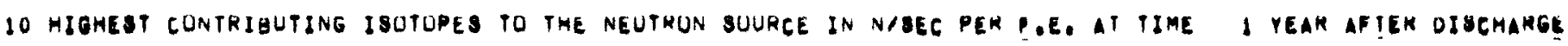
(PERCENT CONTRIBUTION TO TUTAL NEUTHUN SUURCE IN PARENTHEJES)

IHIS SEGMENT WAS DISGMAKGED AT THE BEGINNING OF RELOAD IU AND MAS URIGINALLY LOADED AT HELOAD O

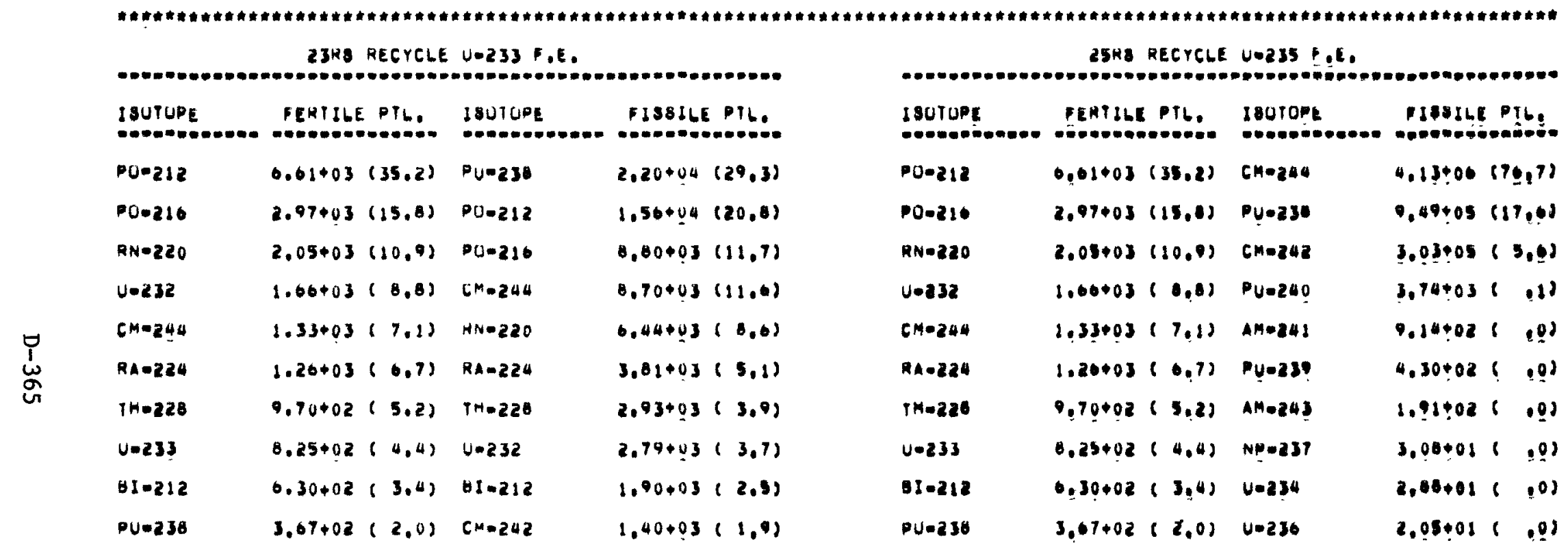


10 HIGHEST CUNTRIBUTING ISOTOPES TO THE NEUTRON BUURCE IN N/SEC PEK F.E. AT TIME 3 YEAKB AFTEK DISCMARGE (PERCENT CUNTRIUUTIUN TO TOTAL NEUTRON BUUKCE IN PAKENTHEOEB)

THJS SEGMENT WAS DISCMARGED AT THE GEGINVING UF RELUAD IO AND MAS URIGINALGY LOADED MT KELUAD G

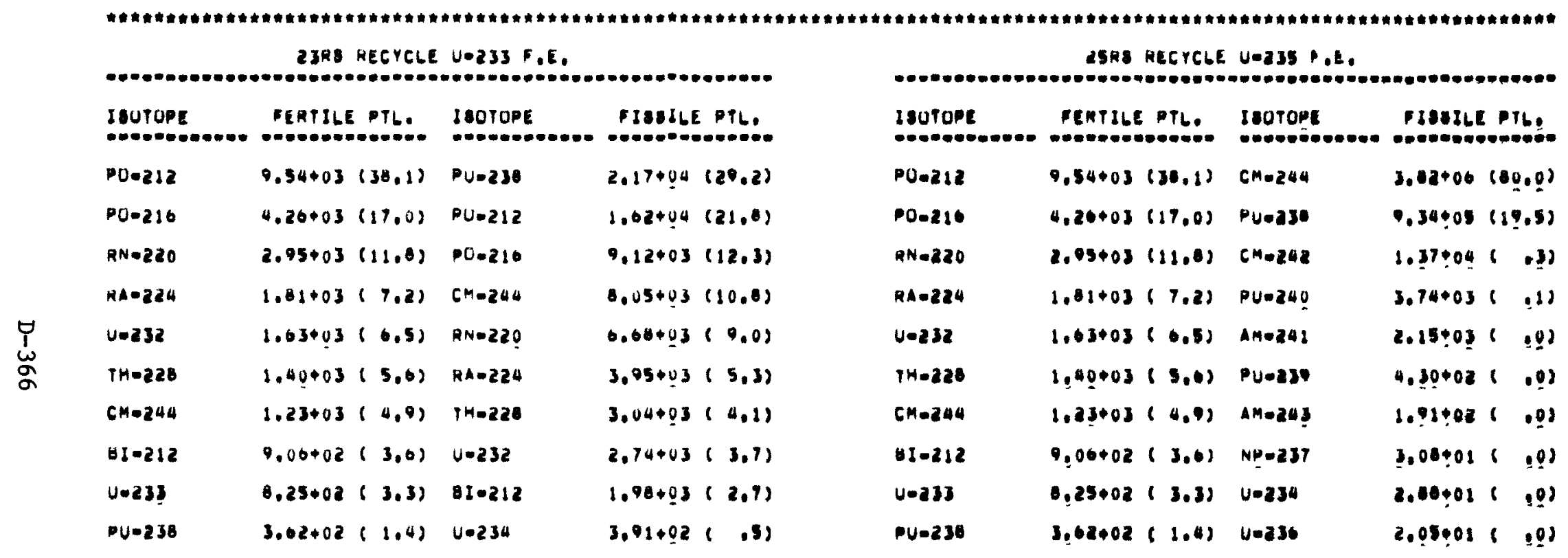




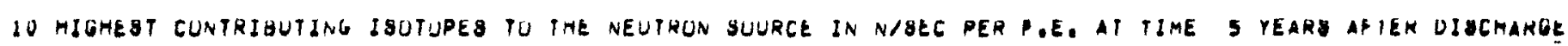

(PERCENT CUNTRIBUTION TO TUTAL NEUTRUN BUUKCE IN PAKENTHESES)

THIS SEGMENT WAS OISCHAHGED AT THE GEGINNING OF RELOAD IO ANO WAS URIGINALLY LOADED AI KELUAD O

\begin{tabular}{|c|c|c|c|c|c|c|c|c|c|c|c|}
\hline IOUTUPE & $\begin{array}{l}\text { FERTILE } \\
\text { DOO- }\end{array}$ & ETtb. & I80TOPE & FISSILE PTL, & I8OPUPE & $\begin{array}{l}\text { FERTILE } \\
\text { OAOAOEOE }\end{array}$ & EPb. & 18010pE & 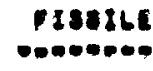 & 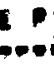 & $16=$ \\
\hline$P 0-212$ & 1.00404 & $(39.1)$ & $P U=238$ & $2.14+0.4 \quad(29.2)$ & $00-212$ & $1,08+04$ & $(30.1)$ & $\mathrm{CH}-244$ & $3.54+00$ & & $9(2)$ \\
\hline$P 0-216$ & $4.84+03$ & $(17.4)$ & $P \cup=212$ & $1.62+04(22.1)$ & $00-216$ & $4.84 * 03$ & $(17.4)$ & Pụ-230 & $9.19+05$ & & 0.01 \\
\hline$R N=220$ & $3.34+03$ & $(12.1)$ & $P 0-216$ & $9,12+0.3(12,5)$ & $R N=220$ & $3.34+03$ & $(12.1)$ & PU-240 & $3.7 \leqslant \bullet 03$ & 1 & $1 !$ \\
\hline$R A=224$ & $2.05+03$ & $(7.4)$ & $6 m-244$ & $7.45+03(10.2)$ & $94-224$ & $2.05 \$ 03$ & $(7,4)$ & $A M-241$ & $3.27 \div 03$ & 1 & .11 \\
\hline U.232 & $1.00+03$ & $(5,8)$ & $R N=220$ & $6.68+0.3(9.1)$ & U.232 & 1.00003 & $(5.8)$ & $C M-242$ & $7.25 \div 02$ & 1 & .08 \\
\hline$T H=228$ & $1.58+03$ & $(5.7)$ & $R 4-224$ & $3,95003(5,4)$ & $T M=228$ & 1.50403 & $(5.7)$ & Pueasu & $4.30 \div 02$ & 1 & .98 \\
\hline$C M=244$ & $1.14+03$ & $(4.1)$ & $T H=228$ & $1,04+0.3(4.2)$ & $C M-244$ & 1.14403 & $(4.1)$ & $A M=24 s$ & $1.91 \div 02$ & 6 & .08 \\
\hline$\forall I=212$ & $1.03+03$ & $(3.7)$ & $u=232$ & $2.00+03(3.7)$ & $y 1-212$ & $1.03+03$ & $(3,7)$ & $N P=237$ & $3.08+08$ & 6 & .09 \\
\hline$u=233$ & $8.25+02$ & $(3.0)$ & $81-212$ & $1,98+0,3(2,7)$ & $v=233$ & $8,25+02$ & $(3.0)$ & $u=23 u$ & $2.80 \div 08$ & 6 & .92 \\
\hline$P U=230$ & $3.56+02$ & $(1,3)$ & $U=234$ & $3.01+0.2(.5)$ & $P U=238$ & $3,56+02$ & $(1.3)$ & U-230 & $2.05 \div 02$ & l & .00 \\
\hline
\end{tabular}




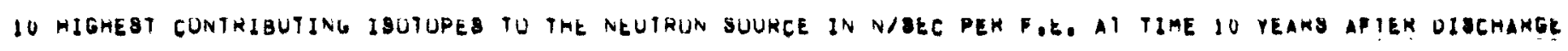
(PERCENT CONTRIGUIJON TU TUTAL NEUTRUN SOUKCE IN PAKENTMESES)

THIS SEGMENT WAS DISCMAKGED AT THE BEgINNING UF RELUAD IU AND MAS UKJGINAGLY LOADED AT RELUAD G

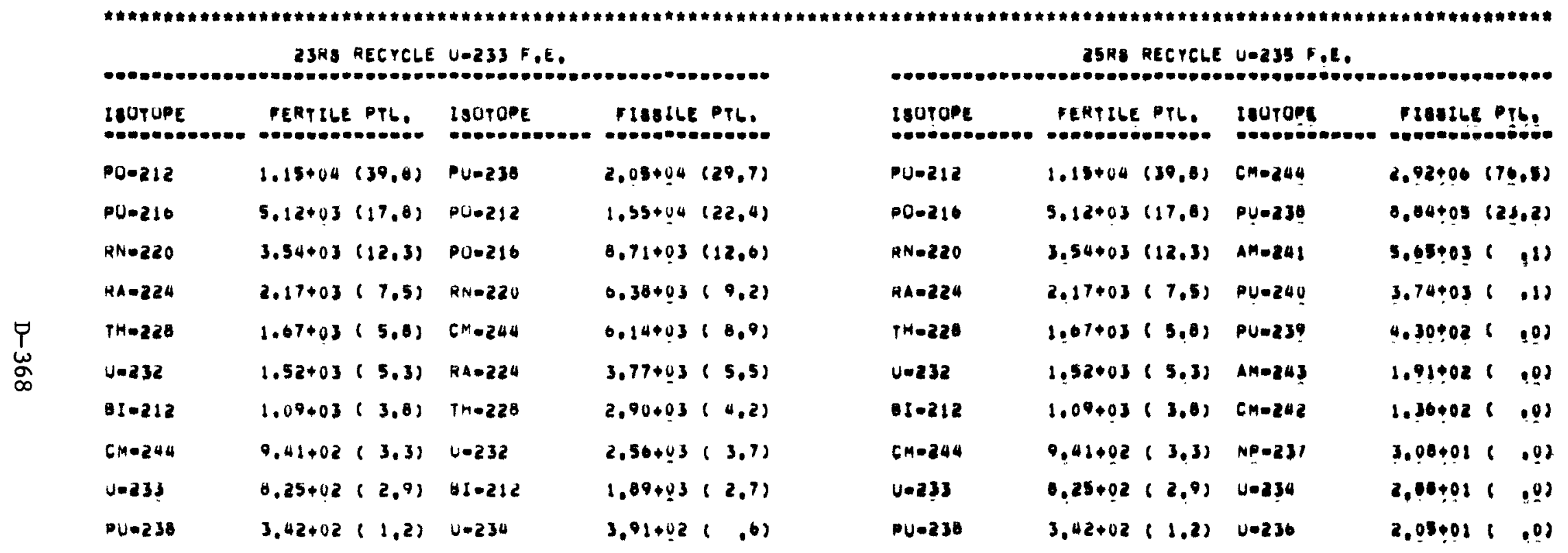




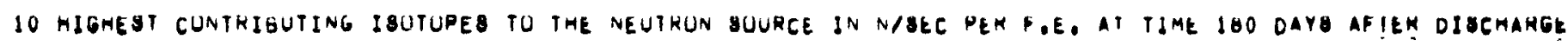

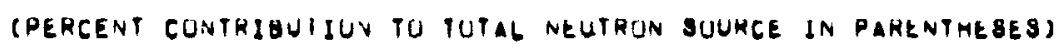

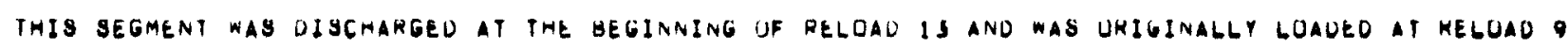

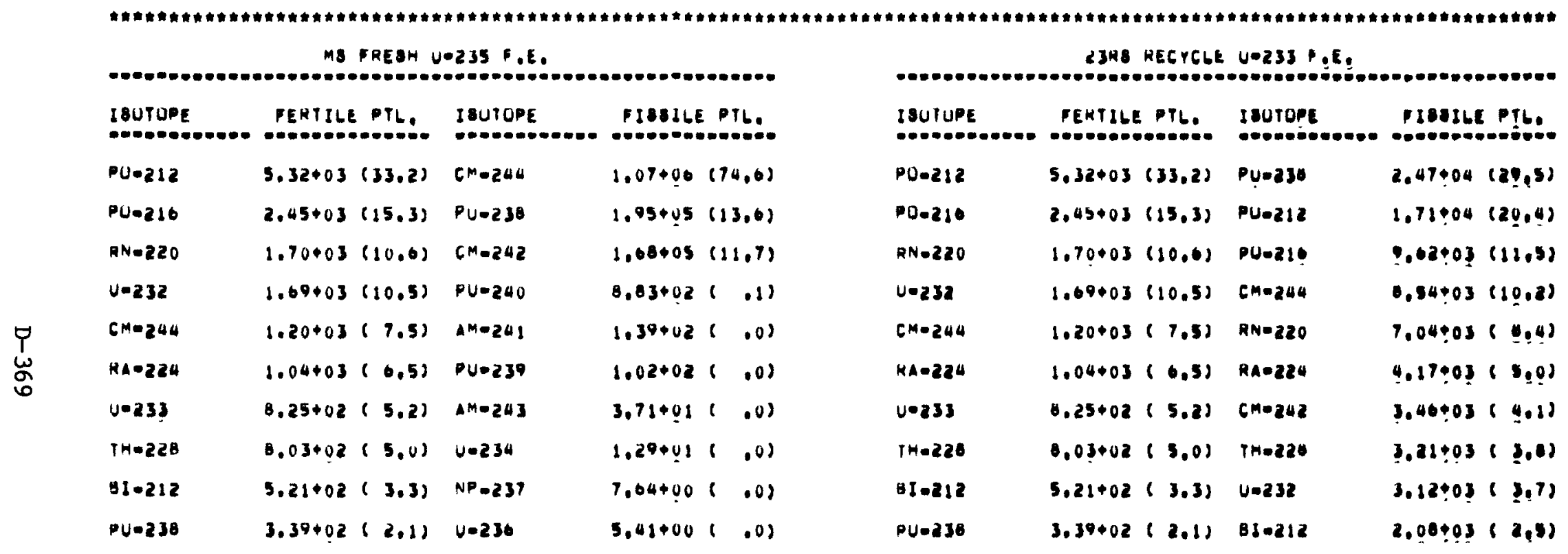


10 HIGHEST CUNTKIGUIING ISUTLPES TU THE NEUTRUN SUUKCE IN N/BEE PER F.E. AT TIME IOO DAYS AFTEK UISCHAKGE (PERCENT CONTRIQUTIUN TU TUTAL NEUTRUN BOUKCE IN PARENTMESES)

THIS SEGMENT WAS DISCMARGED AT THE BEGINNING OF RELOAO I3 ANO WAS UKIGINALLY LOAMED AT KELOAD

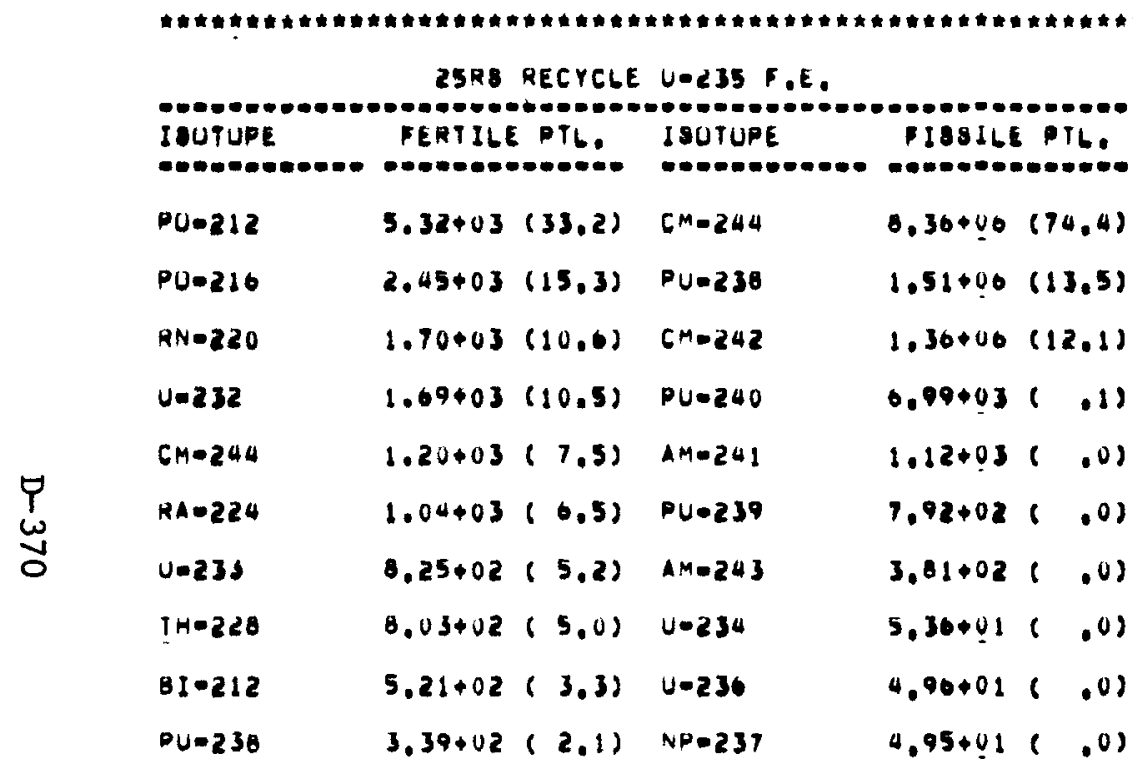


10 HIGHEST CONTRIAUTING ISUTUPES TU THE NEUTRUN BUURCE IN N/BEC PER F.E. AT TIME I YEAK AFIEK UISCHARGE (PERCENT CONTRIBUTIUN TU TUTAL NEUTHUN SUUKCE IN PARENTHEBES)

TMIS SEGMENT WAS DIBCHAHGED AT THE HEGINNING OF RELUAU I3 AND WAB UHJGINALLY LUADEO aT RELOAD

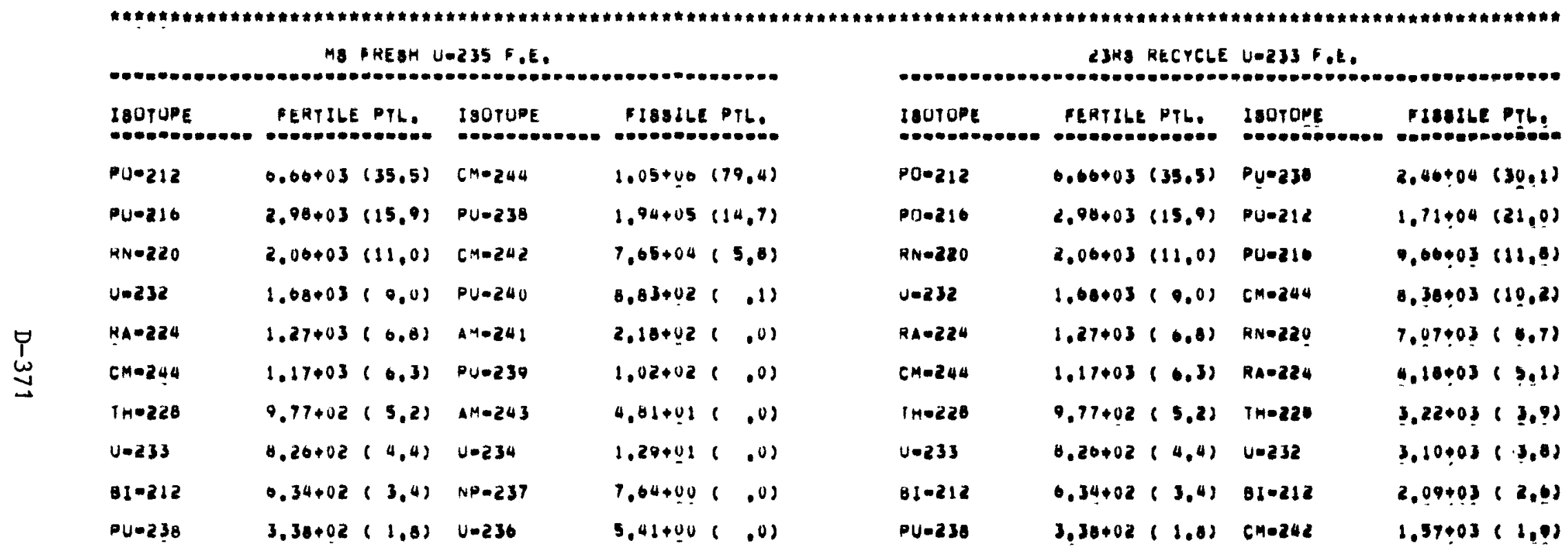




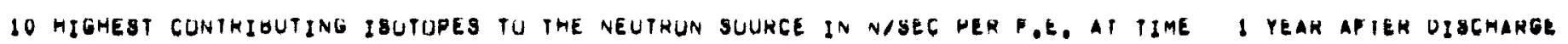
(PERCENT CUNTRIBUIJON IO TUTAL NEUTKON BOURCE IN PARENTMEBES)

THIS SEGMENT WAS OISCHARGED AT THE GEGINNING UF hELOAD I3 AND WAS ONIGINALLY LOAGED AT RELOAD

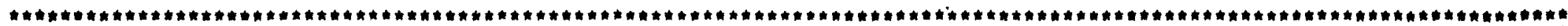
$25 R 8$ RECYCLE U.235 F.E.

\begin{tabular}{|c|c|c|c|c|c|c|}
\hline poea12 & 6.66403 & $(35,5)$ & $C M=244$ & $0.19+06$ & & 19.31 \\
\hline$v-216$ & $2.98+03$ & $(15.9)$ & $P U=238$ & $1,51+06$ & & $(4.6)$ \\
\hline$R N-220$ & 2.00403 & $(11.0)$ & $C M-242$ & $0.17+05$ & 1 & $0.0)$ \\
\hline$v-232$ & $1.08+03$ & $(9.0)$ & PUEZ240 & $0.09+03$ & 1 & .11 \\
\hline$R A=224$ & $1.27+03$ & $(0,0)$ & $A M-241$ & $1.75+03$ & 1 & .05 \\
\hline$C M=244$ & $1.17+03$ & $(0,3)$ & $P U=239$ & 7.92 .02 & 6 & .01 \\
\hline$T H=220$ & $9.77+02$ & $(5,2)$ & $A M=243$ & $3.81+02$ & 6 & .01 \\
\hline$v=23\}$ & $8.26+02$ & $(4.4)$ & $v-234$ & $5,36+01$ & 6 & .01 \\
\hline$\forall I-212$ & $0.34+02$ & $(3.4)$ & $v-256$ & $4.90+01$ & 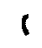 & .01 \\
\hline$P \cup=238$ & $3.38+02$ & $(1,8)$ & $N P=237$ & $4.95+01$ & C & .01 \\
\hline
\end{tabular}




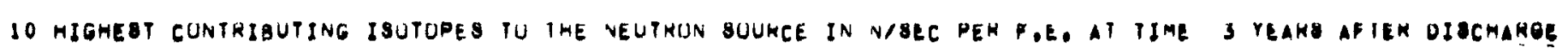
(PERCENT CUNTKIBUTIUIN TO TUTAL NEUTRUN SUUKCE IN PAHENTMEBES)

THIS SEGMENT MAS UISGMARGED AT THE GEGINNING OF RELOAD I3 AND NAB ORIGINALLY LOADED AT MELOAD 9

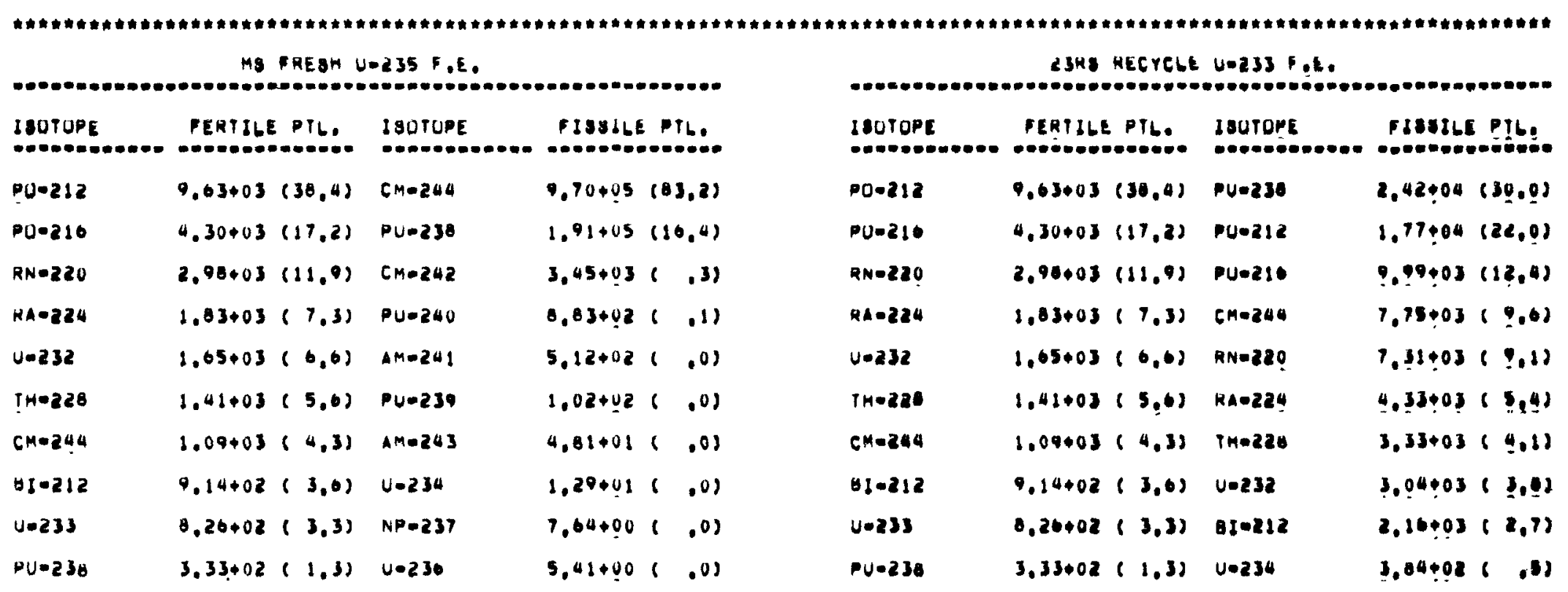




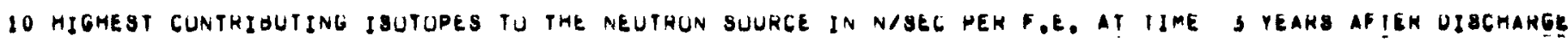
(PERCENT CUNTKIBUIION TO TUTAL NEUTKUN BUURCE IN PARENTHESES)

THIS SEGMENT WAS OIBCMAKGEU aT THE BEgINNING UF RELUAD IS AND WAS UKIGINALLY LOAHEU AT RLLOAD 9

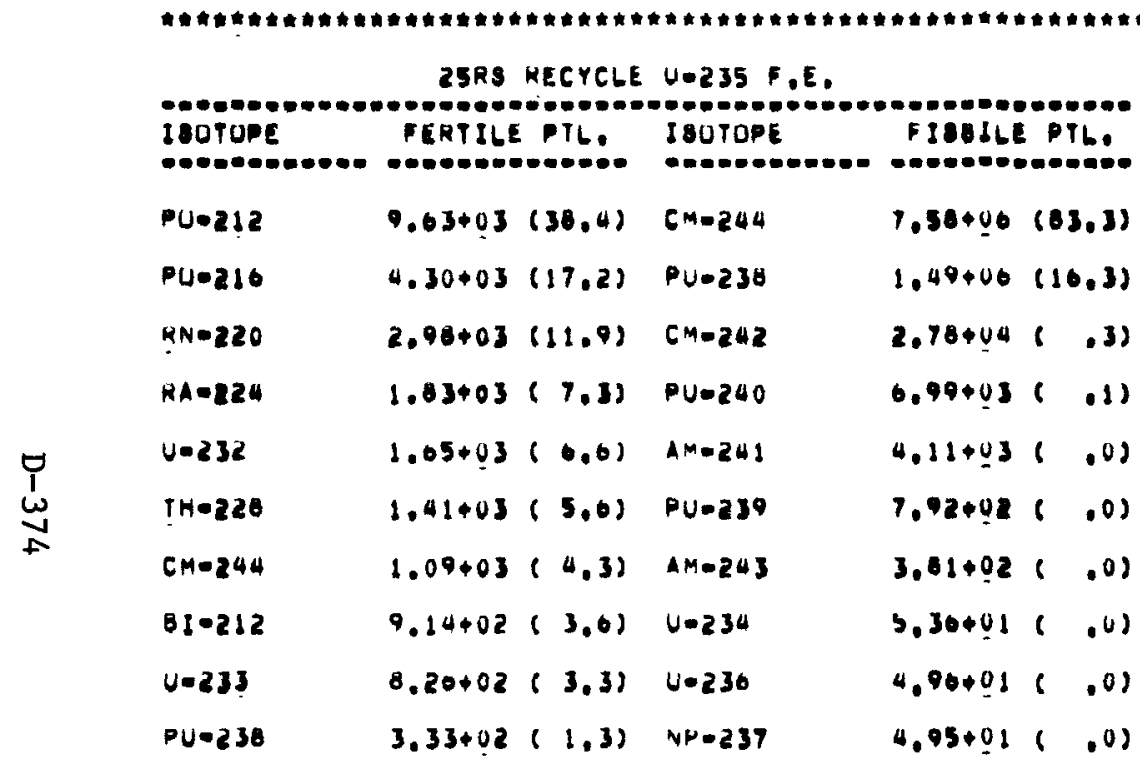




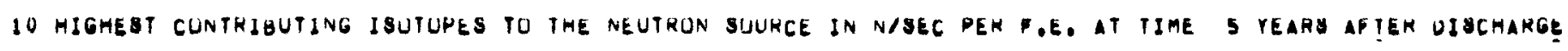
(PERGENT CUNTRIGUTION TU TUTAL NEUIRUN SUUACE IN PARENTHESES)

THIS SEGMENT WAS UISCMARGED AT TME BEGINNING OF RELUAO I3 AND MAS OKIGINALLY LOADEO AT KELUAD

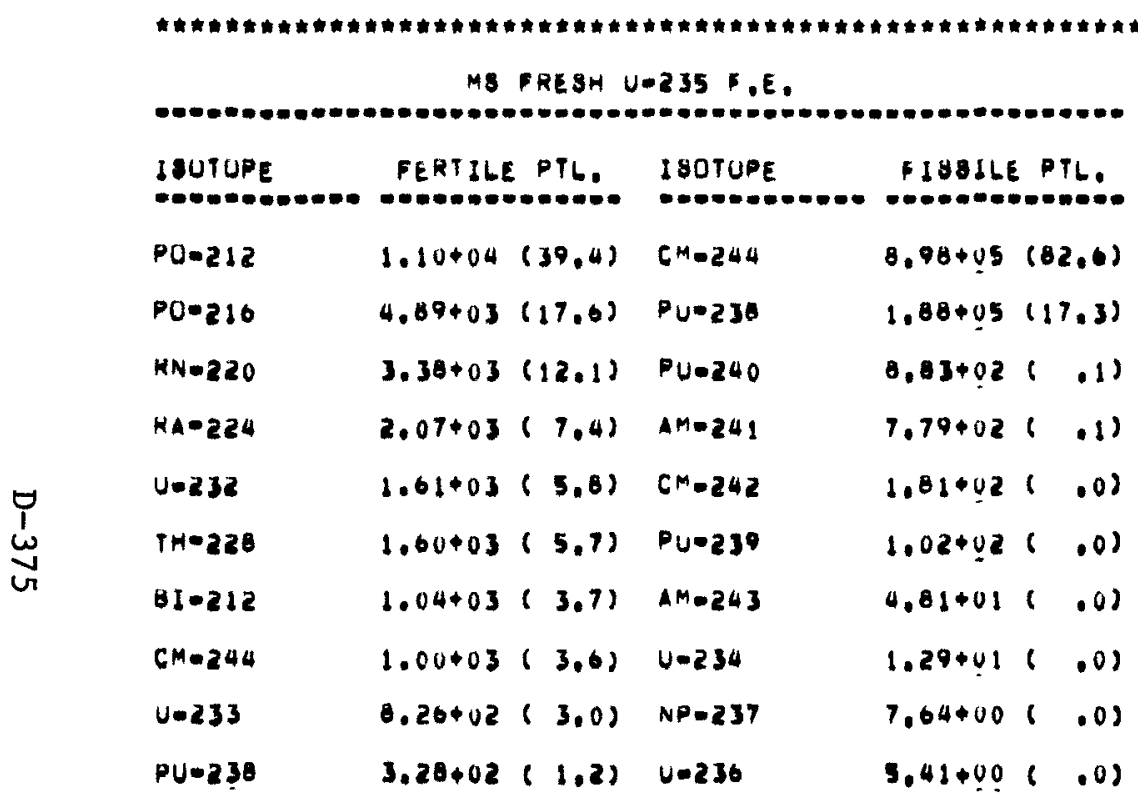

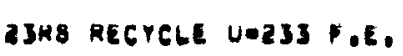

\begin{tabular}{|c|c|c|c|c|}
\hline I 801 UPE & FERTILE & E PYb. & IBUTOPE & FI80ILE PILE \\
\hline po-212 & $1.10+04$ & $(39.4)$ & Pu-230 & $2,30+04(29,0)$ \\
\hline $90 \cdot 216$ & $4.89+03$ & $(17.6)$ & PUe 212 & $1.77+04(22,3)$ \\
\hline$K N=220$ & $3,36+03$ & $122.1)$ & Pund16 & $9.09+03(12.6)$ \\
\hline$R A=224$ & $2.07+03$ & $(7.4)$ & AN-220 & $7.31+03(9.2)$ \\
\hline $4 \cdot 232$ & $1.61+0.3$ & $(5.0)$ & $C M=244$ & $7.17 \div 03(2.0)$ \\
\hline$T H=220$ & $1.60+03$ & $(5.7)$ & RA-224 & $4.33 \div 03(0.4)$ \\
\hline$B 1-212$ & $1.04+03$ & $(3.7)$ & THeato & $3.33 \div 03(4,2)$ \\
\hline$C M-204$ & 1.00003 & $(3.0)$ & $v=232$ & $2.99 \div 05(\$, 4)$ \\
\hline$v-233$ & $0.20+02$ & 13.03 & $81-212$ & $2.10403(2.7)$ \\
\hline$P U=236$ & $3.28+02$ & $(1,2)$ & $v=234$ & $3.64002(.5)$ \\
\hline
\end{tabular}


10 HIGHEST CONTRIGUTING IBUTOPES TO THE NEUTRUN SOURCE IN N/8EC PER P.E. AI TIME S YEAKB AR!ER UISCHARGE (PERCENT CUNTRIBUIION TO TOTAL NEUTRUN SOURCE IN PARENTHESES)

THIS SEGMENT WAS DISCHARGEO AT THE BEGINNING OF RELOAD I3 AND WAB ORIGINALLY LOAMEO AT MELOAD

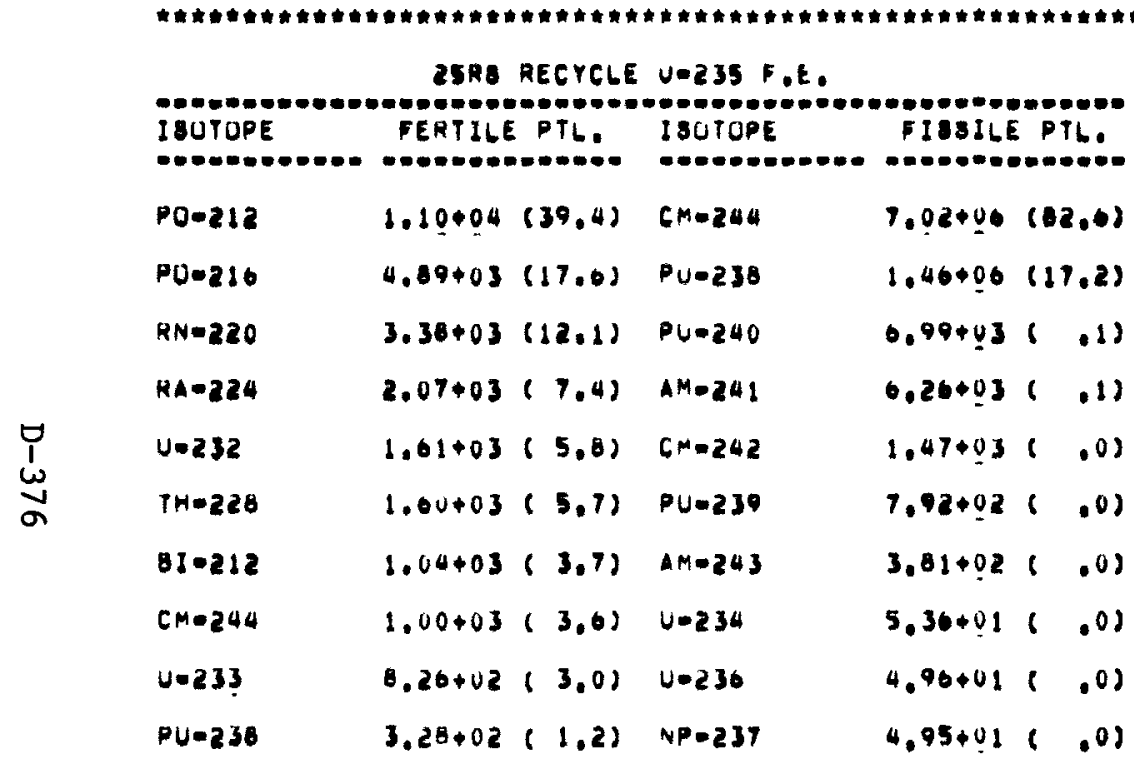


I0 MIGHEST CONTKIBUTING ISUTOPES TO THE NEUTRON SUURCE IN N/SEC PEK F.E. AT TIME IO YEAKS AF!EK UISGMAKGE (PERCENT CONTRIBUTIUN IU TUTAL NEUTHUN SUURCE IN PAKENTMESES)

THIS SEGMENT MAS DIBGHARGED AT THE BEgINNING UF RELOAO 13 anO WAS UHIGINALLY LUADED AT RELOAD 9

\begin{tabular}{|c|c|c|c|c|c|c|c|c|c|c|}
\hline ISUTOPE & FERTILE & $=76$ & IOUTOPE & 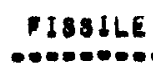 & PTLE & 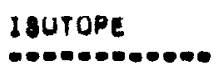 & FERTILE & PTL. & IBUTUPE & FI88ILE PIL. \\
\hline$P 0=212$ & $1.16+04$ & $(40.0)$ & $C M=244$ & $7.40+05$ & $(00,2)$ & $P 0-212$ & $1.10+04$ & $(40,0)$ & PUez30 & $2.20 \div 04(30,4)$ \\
\hline$P 0-216$ & $5.17+03$ & $(17.9)$ & $P U=230$ & $1.81+05$ & $(10,0)$ & P0.216 & $5.17+03$ & $(17.9)$ & P0-212 & $1.60+04(22.5)$ \\
\hline$R N=220$ & $3.58+03$ & $(12.3)$ & $A M=241$ & $1.34+03$ & .11 & $R N-220$ & $3.58+03$ & $(12,3)$ & PUle210 & $9.54403(12,7)$ \\
\hline$K A-224$ & $2.19+03$ & $(1.6)$ & PUe 240 & $4,82+02$ & .11 & RA-224 & $2.19 \bullet 03$ & $(7.6)$ & RN-220 & $0.90+03(9.3)$ \\
\hline $\mathrm{TH} \cdot 228$ & 1.69 .03 & $(5.6)$ & $P U=239$ & $1.62+\underline{.02}$ & .05 & $T H=226$ & $1.69+03$ & $(5,0)$ & $C M=244$ & $5.98 \div 03(7.9)$ \\
\hline U.232 & $1.54+0.3$ & $(5,3)$ & $4 M-243$ & $4.01+91$ & .01 & U-232 & $1.54+0.3$ & $(5.3)$ & $R A=224$ & $4.13+03(5.5)$ \\
\hline$\forall I \cdot 212$ & $1.10+03$ & $(3.6)$ & $C M-242$ & $3.28+01$ & .01 & $81-212$ & $1.10 * 03$ & $(3,8)$ & PHo220 & $3,10+03(4,2)$ \\
\hline$C M=244$ & $8.28+02$ & $(2,9)$ & $v=234$ & $1.29+01$ & .01 & $C M=244$ & $0.28+02$ & $(2,9)$ & u=asz & $2,65+03(3,0)$ \\
\hline$\underline{y}=233$ & $0.26+0.2$ & $(2,8)$ & $N P=237$ & $7.64+00$ & .01 & $v=233$ & $6.20+0.2$ & $(2.6)$ & $81-212$ & $2.00+03(2,7)$ \\
\hline$P \cup=238$ & $3.15+02$ & $(1.1)$ & $v=236$ & $5.41+00$ & .01 & $P \cup=238$ & $3.15+02$ & $(1.1)$ & $4=234$ & $3.84+026$ \\
\hline
\end{tabular}




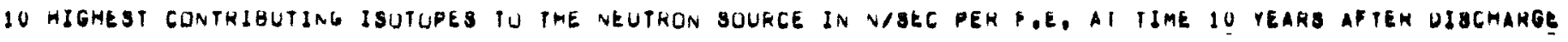
(PERCENT CONTRIBUTION TO TUTAL NEUIRON SUURCE IN PARENTMEBES)

THIS SEGMENT MAB UISGHARGEU AT THE BEgINNING OF RELUAU IJ AND WAS DRIGINALLY LUAMED AT KELOAD

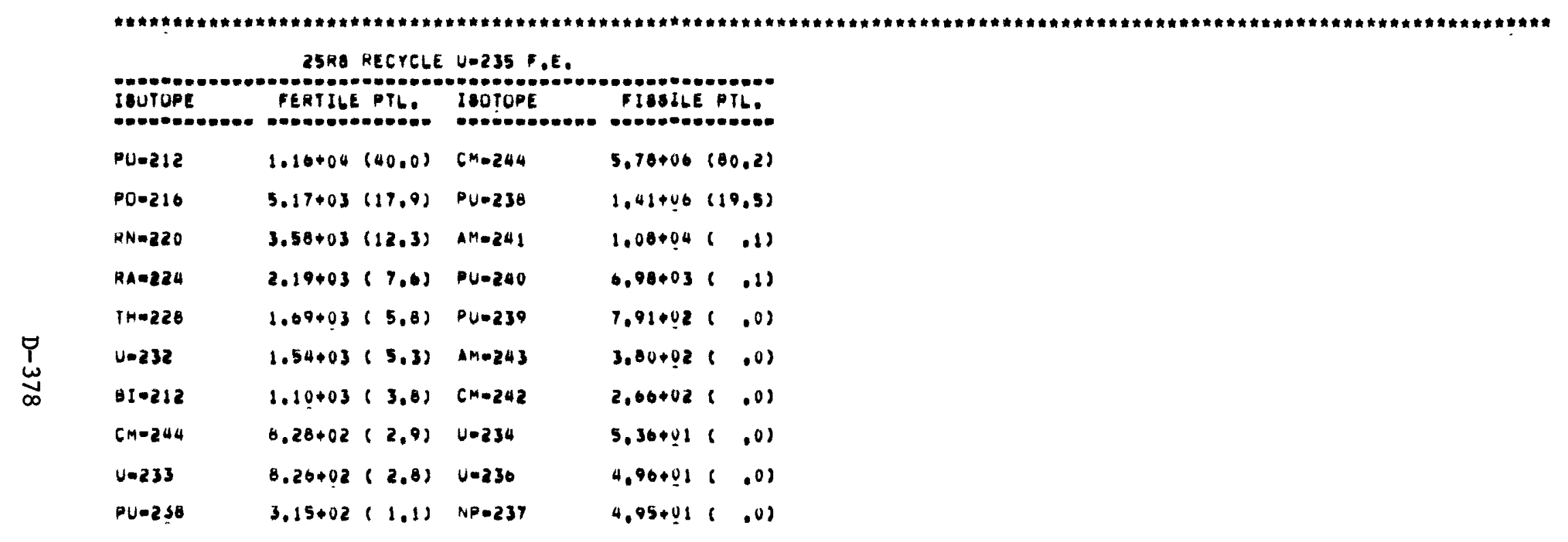

